\title{
Geochemical Study of Grab Samples Collected From UPR 200-E- 86 Investigative Site
}

Michael Lindberg

June 2008

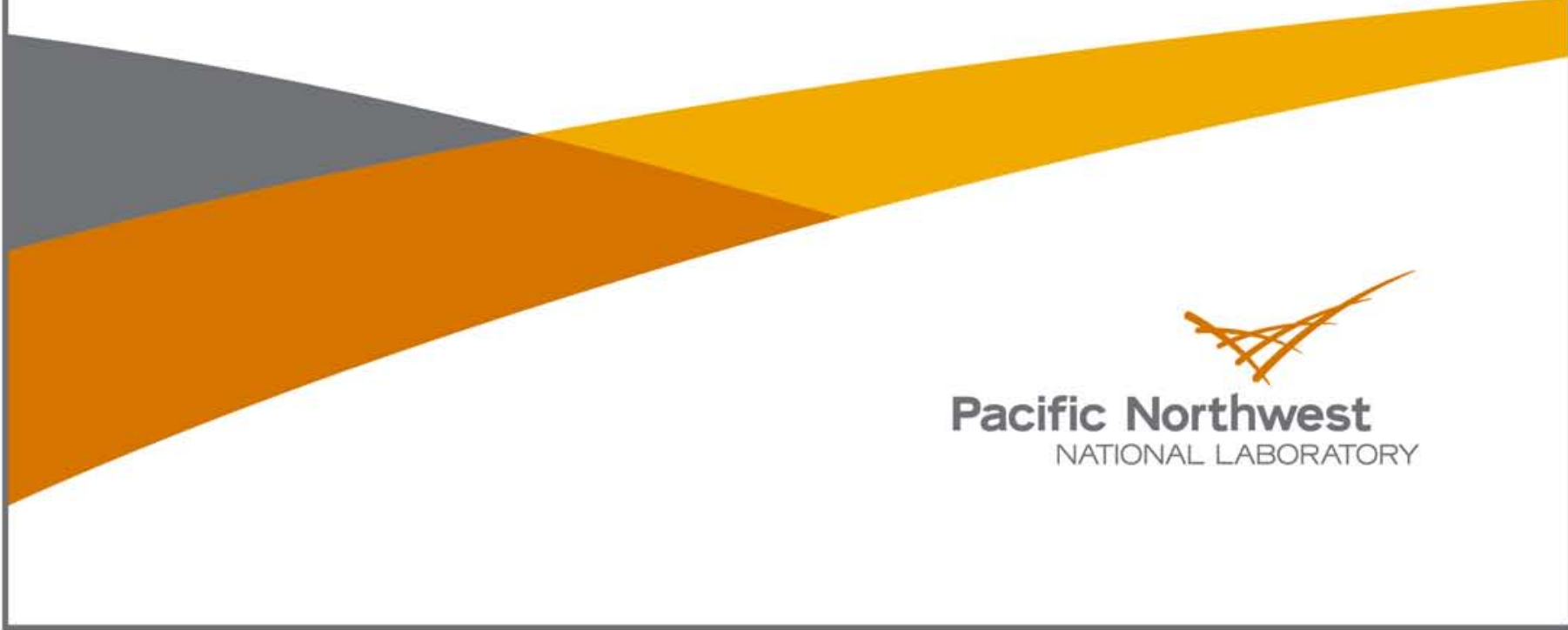




\section{Pacific Northwest \\ National Laboratory}

Operated by Battelle for the

U.S. Department of Energy

$06 / 11 / 08$

PNNL Report: $\quad$ PNNL-60937

To: Fredrick Mann

From: Michael J. Lindberg

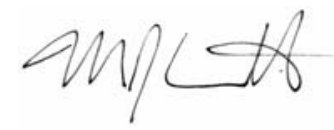

Environmental Sciences Laboratory

Subject: Geochemical Study of Grab Samples Collected From UPR 200-E-86 Investigative Site, Sample Delivery Group ESL080009, SAF Number V08-001

This letter contains the following information for sample delivery group ESL080009

- Cover Sheet

- Narrative

- Analytical Results

- Quality Control

- Chain of Custodies 


\section{Introduction}

\section{Matrix sample Dates project name for geochemical studies}

Between December 17, 2007 and March 3, 2008 soil cores and grab samples were received from UPR 200 -E-86 Investigative Site for geochemical studies.

\section{Analytical Results/Methodology}

The analyses for this project were performed at the 325 building located in the 300 Area of the Hanford Site. The analyses were performed according to Pacific Northwest National Laboratory (PNNL) approved procedures and/or nationally recognized test procedures. The data sets include the sample identification numbers, analytical results, estimated quantification limits (EQL), and quality control data.

\section{Quality Control}

The preparatory and analytical quality control requirements, calibration requirements, acceptance criteria, and failure actions are defined in the on-line QA plan “Conducting Analytical Work in Support of Regulatory Programs” (CAW). This QA plan implements the Hanford Analytical Services Quality Assurance Requirements Documents (HASQARD) for PNNL.

\section{Definitions}

Dup Duplicate

RPD Relative Percent Difference

\section{Sample Receipt}

Samples were received with a chain of custody (COC) and were analyzed according to the sample identification numbers supplied by the client. All Samples were refrigerated upon receipt until prepared for analysis.

All samples were received with custody seals intact unless noted in the Case Narrative.

\section{Holding Times}

Holding time is defined as the time from sample preparation to the time of analyses. The prescribed holding times were met for all analytes unless noted in the Case Narrative.

\section{Analytical Results}

All reported analytical results meet the requirements of the CAW or client specified SOW unless noted in the case narrative. 


\section{Case Narrative Report}

\section{Test reports/summary forms for blank samples}

QC Sample 8D01004-BLK1 failed criteria for Specific Conductance (EC) in Conductivity-WE.

$\mathrm{MDL}=0.0100 \mathrm{mS} / \mathrm{cm}$

$\mathrm{MRL}=0.0100 \mathrm{mS} / \mathrm{cm}$

Result $=0.0110 \mathrm{mS} / \mathrm{cm}$

Criterion $=1 \times \mathrm{MRL}$

Sample results were greater than 5 times the blank results; therefore, there should be no impact to data.

QC Sample 8D03004-BLK1 failed criteria for Specific Conductance (EC) in Conductivity-WE.

$\mathrm{MDL}=0.0100 \mathrm{mS} / \mathrm{cm}$

$\mathrm{MRL}=0.0100 \mathrm{mS} / \mathrm{cm}$

Result $=0.0100 \mathrm{mS} / \mathrm{cm}$

Criterion = $1 \mathrm{x}$ MRL

Sample results were greater than 5 times the blank results; therefore, there should be no impact to data.

QC Sample 8D03004-BLK2 failed criteria for Specific Conductance (EC) in Conductivity-WE.

$\mathrm{MDL}=0.0100 \mathrm{mS} / \mathrm{cm}$

MRL $=0.0100 \mathrm{mS} / \mathrm{cm}$

Result $=0.0100 \mathrm{mS} / \mathrm{cm}$

Criterion $=1 \times$ MRL

Sample results were greater than 5 times the blank results; therefore, there should be no impact to data.

QC Sample 8E12001-BLK2 failed criteria for Zinc in ICP-OES Vadose-AE.

$\mathrm{MDL}=0.077 \mathrm{ug} / \mathrm{g}$

MRL $=0.077 \mathrm{ug} / \mathrm{g}$

Result $=0.181 \mathrm{ug} / \mathrm{g}$

Criterion = $1 \mathrm{x}$ MRL

Sample results were greater than 5 times the blank results; therefore, there should be no impact to data.

\section{Laboratory control samples (LCS):}

LCS Recovery for Silver (59.1\%) was outside acceptance limits (80-120) in 8E12001-BS1 for ICP-OES Vadose-AE Samples results were not reported.

LCS Recovery for Silver (45.0\%) was outside acceptance limits (80-120) in 8E12001-BS2 for ICP-OES Vadose-AE Samples results were not reported.

LCS Recovery for Silver (58.3\%) was outside acceptance limits (80-120) in 8E12001-BS3 for ICP-OES Vadose-AE Samples results were not reported.

\section{Post spike (PS) and post spike duplicate (PSD) data}

Post-Spike Recovery for Aluminum (3130\%) was outside acceptance limits (75-125) in 8E12001-PS1 for ICP-OES Vadose-AE. 


\section{Case Narrative Report}

\section{Post spike (PS) and post spike duplicate (PSD) data}

Sample Concentration was greater than 10 times the spiked concentration, data is reported as valid.

Post-Spike Recovery for Barium (141\%) was outside acceptance limits (75-125) in 8E12001-PS1 for ICP-OES Vadose-AE. Sample Concentration was greater than 10 times the spiked concentration, data is reported as valid.

Post-Spike Recovery for Calcium (5110\%) was outside acceptance limits (75-125) in 8E12001-PS1 for ICP-OES Vadose-AE. Sample Concentration was greater than 10 times the spiked concentration, data is reported as valid.

Post-Spike Recovery for Iron (7580\%) was outside acceptance limits (75-125) in 8E12001-PS1 for ICP-OES Vadose-AE. Sample Concentration was greater than 10 times the spiked concentration, data is reported as valid.

Post-Spike Recovery for Magnesium (2420\%) was outside acceptance limits (75-125) in 8E12001-PS1 for ICP-OES Vadose-AE. Sample Concentration was greater than 10 times the spiked concentration, data is reported as valid.

Post-Spike Recovery for Manganese (387\%) was outside acceptance limits (75-125) in 8E12001-PS1 for ICP-OES Vadose-AE. Sample Concentration was greater than 10 times the spiked concentration, data is reported as valid.

Post-Spike Recovery for Phosphorus (218\%) was outside acceptance limits (75-125) in 8E12001-PS1 for ICP-OES Vadose-AE. Sample Concentration was greater than 10 times the spiked concentration, data is reported as valid.

Post-Spike Recovery for Potassium (259\%) was outside acceptance limits (75-125) in 8E12001-PS1 for ICP-OES Vadose-AE. Sample Concentration was greater than 10 times the spiked concentration, data is reported as valid.

Post-Spike Recovery for Sodium (199\%) was outside acceptance limits (75-125) in 8E12001-PS1 for ICP-OES Vadose-AE. Sample Concentration was greater than 10 times the spiked concentration, data is reported as valid.

Post-Spike Recovery for Titanium (810\%) was outside acceptance limits (75-125) in 8E12001-PS1 for ICP-OES Vadose-AE. Sample Concentration was greater than 10 times the spiked concentration, data is reported as valid.

Post-Spike Recovery for Zinc (128\%) was outside acceptance limits (75-125) in 8E12001-PS1 for ICP-OES Vadose-AE. Sample Concentration was greater than 10 times the spiked concentration, data is reported as valid.

Post-Spike Recovery for Aluminum (2890\%) was outside acceptance limits (75-125) in 8E12001-PS2 for ICP-OES Vadose-AE. Sample Concentration was greater than 10 times the spiked concentration, data is reported as valid.

Post-Spike Recovery for Barium (145\%) was outside acceptance limits (75-125) in 8E12001-PS2 for ICP-OES Vadose-AE. Sample Concentration was greater than 10 times the spiked concentration, data is reported as valid.

Post-Spike Recovery for Calcium (5180\%) was outside acceptance limits (75-125) in 8E12001-PS2 for ICP-OES Vadose-AE. Sample Concentration was greater than 10 times the spiked concentration, data is reported as valid.

Post-Spike Recovery for Chromium (126\%) was outside acceptance limits (75-125) in 8E12001-PS2 for ICP-OES Vadose-AE. Sample Concentration was greater than 10 times the spiked concentration, data is reported as valid.

Post-Spike Recovery for Iron (6930\%) was outside acceptance limits (75-125) in 8E12001-PS2 for ICP-OES Vadose-AE. Sample Concentration was greater than 10 times the spiked concentration, data is reported as valid.

Post-Spike Recovery for Magnesium (2440\%) was outside acceptance limits (75-125) in 8E12001-PS2 for ICP-OES Vadose-AE. Sample Concentration was greater than 10 times the spiked concentration, data is reported as valid.

Post-Spike Recovery for Manganese (382\%) was outside acceptance limits (75-125) in 8E12001-PS2 for ICP-OES Vadose-AE. Sample Concentration was greater than 10 times the spiked concentration, data is reported as valid.

Post-Spike Recovery for Phosphorus (220\%) was outside acceptance limits (75-125) in 8E12001-PS2 for ICP-OES Vadose-AE. Sample Concentration was greater than 10 times the spiked concentration, data is reported as valid.

Post-Spike Recovery for Potassium (269\%) was outside acceptance limits (75-125) in 8E12001-PS2 for ICP-OES Vadose-AE. 


\section{Case Narrative Report}

\section{Post spike (PS) and post spike duplicate (PSD) data}

Sample Concentration was greater than 10 times the spiked concentration, data is reported as valid.

Post-Spike Recovery for Sodium (175\%) was outside acceptance limits (75-125) in 8E12001-PS2 for ICP-OES Vadose-AE. Sample Concentration was greater than 10 times the spiked concentration, data is reported as valid.

Post-Spike Recovery for Titanium (683\%) was outside acceptance limits (75-125) in 8E12001-PS2 for ICP-OES Vadose-AE. Sample Concentration was greater than 10 times the spiked concentration, data is reported as valid.

Post-Spike Recovery for Zinc (131\%) was outside acceptance limits (75-125) in 8E12001-PS2 for ICP-OES Vadose-AE. Sample Concentration was greater than 10 times the spiked concentration, data is reported as valid.

Post-Spike Recovery for Aluminum (2900\%) was outside acceptance limits (75-125) in 8E12001-PS3 for ICP-OES Vadose-AE. Sample Concentration was greater than 10 times the spiked concentration, data is reported as valid.

Post-Spike Recovery for Barium (151\%) was outside acceptance limits (75-125) in 8E12001-PS3 for ICP-OES Vadose-AE. Sample Concentration was greater than 10 times the spiked concentration, data is reported as valid.

Post-Spike Recovery for Calcium (5440\%) was outside acceptance limits (75-125) in 8E12001-PS3 for ICP-OES Vadose-AE. Sample Concentration was greater than 10 times the spiked concentration, data is reported as valid.

Post-Spike Recovery for Iron (6840\%) was outside acceptance limits (75-125) in 8E12001-PS3 for ICP-OES Vadose-AE. Sample Concentration was greater than 10 times the spiked concentration, data is reported as valid.

Post-Spike Recovery for Magnesium (2120\%) was outside acceptance limits (75-125) in 8E12001-PS3 for ICP-OES Vadose-AE. Sample Concentration was greater than 10 times the spiked concentration, data is reported as valid.

Post-Spike Recovery for Manganese (346\%) was outside acceptance limits (75-125) in 8E12001-PS3 for ICP-OES Vadose-AE. Sample Concentration was greater than 10 times the spiked concentration, data is reported as valid.

Post-Spike Recovery for Phosphorus (229\%) was outside acceptance limits (75-125) in 8E12001-PS3 for ICP-OES Vadose-AE. Sample Concentration was greater than 10 times the spiked concentration, data is reported as valid.

Post-Spike Recovery for Potassium (260\%) was outside acceptance limits (75-125) in 8E12001-PS3 for ICP-OES Vadose-AE. Sample Concentration was greater than 10 times the spiked concentration, data is reported as valid.

Post-Spike Recovery for Sodium (200\%) was outside acceptance limits (75-125) in 8E12001-PS3 for ICP-OES Vadose-AE. Sample Concentration was greater than 10 times the spiked concentration, data is reported as valid.

Post-Spike Recovery for Titanium (739\%) was outside acceptance limits (75-125) in 8E12001-PS3 for ICP-OES Vadose-AE. Sample Concentration was greater than 10 times the spiked concentration, data is reported as valid.

Post-Spike Recovery for Zinc (131\%) was outside acceptance limits (75-125) in 8E12001-PS3 for ICP-OES Vadose-AE. Sample Concentration was greater than 10 times the spiked concentration, data is reported as valid.

Post-Spike Recovery for Sulfate (129\%) was outside acceptance limits (75-125) in 8D02003-PS1 for Anions by IC-WE. Sample Concentration was greater than 10 times the spiked concentration, data is reported as valid.

Post-Spike Recovery for Sulfur (43.0\%) was outside acceptance limits (75-125) in 8D28005-PS1 for ICP-OES Vadose-WE. Sample Concentration was greater than 10 times the spiked concentration, data is reported as valid.

Post-Spike Recovery for Calcium (192\%) was outside acceptance limits (75-125) in 8D28006-PS1 for ICP-OES Vadose-WE. Sample Concentration was greater than 10 times the spiked concentration, data is reported as valid.

Post-Spike Recovery for Sodium (170\%) was outside acceptance limits (75-125) in 8D28006-PS1 for ICP-OES Vadose-WE. Sample Concentration was greater than 10 times the spiked concentration, data is reported as valid.

Post-Spike Recovery for Calcium (141\%) was outside acceptance limits (75-125) in 8D28006-PS2 for ICP-OES Vadose-WE. Sample Concentration was greater than 10 times the spiked concentration, data is reported as valid. 


\section{Case Narrative Report}

\section{Post spike (PS) and post spike duplicate (PSD) data}

Sample Concentration was greater than 10 times the spiked concentration, data is reported as valid.

\section{$\underline{\text { Analytical duplicate data }}$}

Duplicate RPD for Uranium 238 (46.1\%) was above the acceptance limit (20) in 8D16002-DUP1 for ICPMS-Tc_U-AE.

All other duplicates and QC associated with the batch were in limits. Duplicate failure may be due to sample heterogeneity. There should be no impact to sample data as reported.

Duplicate RPD for Barium (21.4\%) was above the acceptance limit (20) in 8E12001-DUP3 for ICP-OES Vadose-AE.

All other duplicates and QC associated with the batch were in limits. Duplicate failure may be due to sample heterogeneity. There should be no impact to sample data as reported.

Duplicate RPD for Sulfate (62.5\%) was above the acceptance limit (20) in 8D03001-DUP2 for Anions by IC-WE.

All other duplicates and QC associated with the batch were in limits. Duplicate failure may be due to sample heterogeneity. There should be no impact to sample data as reported.

Duplicate RPD for Specific Conductance (EC) (43.1\%) was above the acceptance limit (20) in 8D03004-DUP2 for Conductivity-WE. All other duplicates and QC associated with the batch were in limits. Duplicate failure may be due to sample heterogeneity. There should be no impact to sample data as reported.

Duplicate RPD for Calcium (61.2\%) was above the acceptance limit (20) in 8D28006-DUP2 for ICP-OES Vadose-WE. All other duplicates and QC associated with the batch were in limits. Duplicate failure may be due to sample heterogeneity. There should be no impact to sample data as reported.

Duplicate RPD for Magnesium (51.7\%) was above the acceptance limit (20) in 8D28006-DUP2 for ICP-OES Vadose-WE.

All other duplicates and QC associated with the batch were in limits. Duplicate failure may be due to sample heterogeneity. There should be no impact to sample data as reported.

Duplicate RPD for Strontium (43.0\%) was above the acceptance limit (20) in 8D28006-DUP2 for ICP-OES Vadose-WE.

All other duplicates and QC associated with the batch were in limits. Duplicate failure may be due to sample heterogeneity. There should be no impact to sample data as reported.

Duplicate RPD for Sulfur (62.0\%) was above the acceptance limit (20) in 8D28006-DUP2 for ICP-OES Vadose-WE.

All other duplicates and QC associated with the batch were in limits. Duplicate failure may be due to sample heterogeneity. There should be no impact to sample data as reported.

\section{Hold Time Report}

All hold times were met. 


\section{DISCLAIMER}

This report was prepared as an account of work sponsored by an agency of the United States Government. Neither the United States Government nor any agency thereof, nor Battelle Memorial Institute, nor any of their employees, makes any warranty, express or implied, or assumes any legal liability or responsibility for the accuracy, completeness, or usefulness of any information, apparatus, product, or process disclosed, or represents that its use would not infringe privately owned rights. Reference herein to any specific commercial product, process, or service by trade name, trademark, manufacturer, or otherwise does not necessarily constitute or imply its endorsement, recommendation, or favoring by the United States Government or any agency thereof, or Battelle Memorial Institute. The views and opinions of authors expressed herein do not necessarily state or reflect those of the United States Government or any agency thereof. 


\section{C-Farm Direct Push C5952}

\begin{tabular}{|c|c|c|}
\hline $\begin{array}{l}\text { HEIS No. } \\
\text { B1RTF8 }\end{array}$ & $\begin{array}{l}\text { Laboratory ID } \\
0803001-01\end{array}$ & $\begin{array}{l}\text { Matrix } \\
\text { SOIL }\end{array}$ \\
\hline B1RTF9A & 0803001-02 & SOIL \\
\hline B1RTH0 & 0803012-01 & SOIL \\
\hline B1RTH1A & 0803012-02 & SOIL \\
\hline B1RTH1 & 0803012-03 & SOIL \\
\hline B1RTH2C & 0803012-04 & SOIL \\
\hline B1RTH2B & 0803012-05 & SOIL \\
\hline B1RTH2A & 0803012-06 & SOIL \\
\hline B1RTH2 & 0803012-07 & SOIL \\
\hline B1RTH3C & 0803012-08 & SOIL \\
\hline B1RTH3B & 0803012-09 & SOIL \\
\hline B1RTH3A & 0803012-10 & SOIL \\
\hline B1RTH3 & 0803012-11 & SOIL \\
\hline B1RYR7C & 0803012-12 & SOIL \\
\hline B1RYR7B & 0803012-13 & SOIL \\
\hline B1RYR7A & 0803012-14 & SOIL \\
\hline B1RYR7 & 0803012-15 & SOIL \\
\hline B1RYR8C & 0803012-16 & SOIL \\
\hline B1RYR8B & 0803012-17 & SOIL \\
\hline B1RYR8A & 0803012-18 & SOIL \\
\hline B1RYR8 & 0803012-19 & SOIL \\
\hline B1RYR9C & 0803012-20 & SOIL \\
\hline B1RYR9B & 0803012-21 & SOIL \\
\hline B1RYR9A & 0803012-22 & SOIL \\
\hline B1RYR9 & 0803012-23 & SOIL \\
\hline B1RYT0B & 0803014-01 & SOIL \\
\hline B1RYT0A & 0803014-02 & SOIL \\
\hline B1RYT0 & 0803014-03 & SOIL \\
\hline B1T2P1C & 0803014-04 & SOIL \\
\hline В1Т2Р1B & 0803014-05 & SOIL \\
\hline B1T2P1A & 0803014-06 & SOIL \\
\hline B1T2P1 & 0803014-07 & SOIL \\
\hline B1T2P2C & 0803014-08 & SOIL \\
\hline В1Т2P2B & 0803014-09 & SOIL \\
\hline B1T2P2A & 0803014-10 & SOIL \\
\hline B1T2P2 & 0803014-11 & SOIL \\
\hline В1Т2Р3С & 0803014-12 & SOIL \\
\hline В1T2Р3B & 0803014-13 & SOIL \\
\hline B1T2P3A & 0803014-14 & SOIL \\
\hline B1T2P4C & 0803014-15 & SOIL \\
\hline B1T2P4B & 0803014-16 & SOIL \\
\hline B1T2P4A & 0803014-17 & SOIL \\
\hline B1T2P4 & 0803014-18 & SOIL \\
\hline B1T2P5C & 0803014-19 & SOIL \\
\hline B1T2P5B & 0803014-20 & SOIL \\
\hline
\end{tabular}

\begin{tabular}{|c|c|}
\hline $\begin{array}{l}\text { Date Collected } \\
12 / 12 / 07 \quad 10: 02\end{array}$ & $\begin{array}{l}\text { Date Received } \\
\text { 12/1/07 09:51 }\end{array}$ \\
\hline $12 / 12 / 07 \quad 10: 55$ & 12/1/07 09:51 \\
\hline 12/13/07 13:15 & 3/14/08 14:49 \\
\hline 12/17/07 09:14 & $\begin{array}{lll}3 / 14 / 08 & 14: 49\end{array}$ \\
\hline 12/17/07 09:14 & 3/14/08 $14: 49$ \\
\hline 12/20/07 09:00 & 3/14/08 $\quad 14: 49$ \\
\hline 12/20/07 09:00 & $3 / 14 / 08 \quad 14: 49$ \\
\hline 12/20/07 09:00 & 3/14/08 $14: 49$ \\
\hline 12/20/07 09:00 & $\begin{array}{lll}3 / 14 / 08 & 14: 49\end{array}$ \\
\hline 12/21/07 08:55 & 3/14/08 14:49 \\
\hline 12/21/07 08:55 & 3/14/08 14:49 \\
\hline 12/21/07 08:55 & 3/14/08 14:49 \\
\hline 12/21/07 08:55 & $3 / 14 / 08 \quad 14: 49$ \\
\hline $\begin{array}{lll}12 / 31 / 07 & 10: 30\end{array}$ & 3/14/08 14:49 \\
\hline 12/31/07 10:30 & $3 / 14 / 08 \quad 14: 49$ \\
\hline $12 / 31 / 07 \quad 10: 30$ & 3/14/08 14:49 \\
\hline 12/31/07 10:30 & $\begin{array}{lll}3 / 14 / 08 & 14: 49\end{array}$ \\
\hline 1/3/08 $10: 40$ & $3 / 14 / 08 \quad 14: 49$ \\
\hline 1/3/08 $\quad 10: 40$ & $3 / 14 / 08 \quad 14: 49$ \\
\hline 1/3/08 $10: 40$ & $3 / 14 / 08 \quad 14: 49$ \\
\hline 1/3/08 10:40 & $3 / 14 / 08 \quad 14: 49$ \\
\hline 1/7/08 09:15 & $3 / 14 / 08 \quad 14: 49$ \\
\hline 1/7/08 09:15 & $3 / 14 / 08 \quad 14: 49$ \\
\hline 1/7/08 09:15 & $3 / 14 / 08 \quad 14: 49$ \\
\hline 1/7/08 09:15 & $3 / 14 / 08 \quad 14: 49$ \\
\hline 1/8/08 11:20 & 3/1/08 12:09 \\
\hline 1/8/08 11:20 & 3/1/08 12:09 \\
\hline 1/8/08 11:20 & 3/1/08 12:09 \\
\hline 1/10/08 09:15 & 3/1/08 12:09 \\
\hline 1/10/08 09:15 & 3/1/08 12:09 \\
\hline 1/10/08 09:15 & 3/1/08 12:09 \\
\hline 1/10/08 09:15 & 3/1/08 12:09 \\
\hline 1/15/08 08:30 & 3/1/08 12:09 \\
\hline 1/15/08 08:30 & 3/1/08 12:09 \\
\hline 1/15/08 08:30 & 3/1/08 12:09 \\
\hline 1/15/08 08:30 & 3/1/08 12:09 \\
\hline 1/16/08 08:45 & 3/1/08 12:09 \\
\hline 1/16/08 08:45 & 3/1/08 12:09 \\
\hline 1/16/08 08:45 & 3/1/08 12:09 \\
\hline 1/17/08 09:20 & 3/1/08 12:09 \\
\hline 1/17/08 09:20 & 3/1/08 12:09 \\
\hline 1/17/08 09:20 & 3/1/08 12:09 \\
\hline 1/17/08 09:20 & 3/1/08 12:09 \\
\hline 1/17/08 11:05 & 3/1/08 12:09 \\
\hline 1/17/08 11:05 & 3/1/08 12:09 \\
\hline
\end{tabular}




\section{C-Farm Direct Push C5958}

\begin{tabular}{|c|c|c|}
\hline $\begin{array}{l}\text { HEIS No. } \\
\text { B1T2P5A }\end{array}$ & $\begin{array}{l}\text { Laboratory ID } \\
0803014-21\end{array}$ & $\begin{array}{l}\text { Matrix } \\
\text { SOIL }\end{array}$ \\
\hline B1T2P5 & 0803014-22 & SOIL \\
\hline В1T2P6C & 0803014-23 & SOIL \\
\hline В1Т2Р6B & 0803014-24 & SOIL \\
\hline B1T2P6A & 0803014-25 & SOIL \\
\hline B1T2P6 & 0803014-26 & SOIL \\
\hline B1T2P7C & 0803015-01 & SOIL \\
\hline B1T2P7B & 0803015-02 & SOIL \\
\hline B1T2P7A & 0803015-03 & SOIL \\
\hline B1T2P7 & 0803015-04 & SOIL \\
\hline В1T2P8C & 0803015-05 & SOIL \\
\hline В1T2P8B & 0803015-06 & SOIL \\
\hline B1T2P8A & 0803015-07 & SOIL \\
\hline B1T2P8 & 0803015-08 & SOIL \\
\hline B1T2P9C & 0803015-09 & SOIL \\
\hline В1Т2Р9В & 0803015-10 & SOIL \\
\hline B1T2P9A & 0803015-11 & SOIL \\
\hline В1Т2P9 & 0803015-12 & SOIL \\
\hline B1T2R0C & 0803015-13 & SOIL \\
\hline B1T2R0B & 0803015-14 & SOIL \\
\hline B1T2R0A & 0803015-15 & SOIL \\
\hline B1T2R0 & 0803015-16 & SOIL \\
\hline B1TNK6C & 0803015-17 & SOIL \\
\hline B1TNK6B & 0803015-18 & SOIL \\
\hline B1TNK6A & 0803015-19 & SOIL \\
\hline B1TNK6 & 0803015-20 & SOIL \\
\hline B1TNK7C & 0803015-21 & SOIL \\
\hline B1TNK7B & 0803015-22 & SOIL \\
\hline B1TNK7A & 0803015-23 & SOIL \\
\hline B1TNK7 & 0803015-24 & SOIL \\
\hline B1TNK8C & 0803015-25 & SOIL \\
\hline B1TNK8B & 0803015-26 & SOIL \\
\hline B1TNK8A & 0803015-27 & SOIL \\
\hline B1TNK8 & 0803015-28 & SOIL \\
\hline B1TNK9C & 0803015-29 & SOIL \\
\hline B1TNK9B & 0803015-30 & SOIL \\
\hline B1TNK9A & 0803015-31 & SOIL \\
\hline B1TNK9 & 0803015-32 & SOIL \\
\hline
\end{tabular}

\begin{tabular}{|c|c|}
\hline Date Collected & Date Received \\
\hline 1/17/08 11:05 & 3/1/08 12:09 \\
\hline 1/17/08 $11: 05$ & $\begin{array}{ll}3 / 1 / 08 & 12: 09\end{array}$ \\
\hline 1/21/08 $09: 40$ & $\begin{array}{ll}3 / 1 / 08 & 12: 09\end{array}$ \\
\hline 1/21/08 $09: 40$ & $\begin{array}{ll}3 / 1 / 08 & 12: 09\end{array}$ \\
\hline 1/21/08 $09: 40$ & 3/1/08 12:09 \\
\hline 1/21/08 $09: 40$ & 3/1/08 12:09 \\
\hline 2/4/08 13:00 & 3/14/08 $\quad 12: 59$ \\
\hline 2/4/08 13:00 & 3/14/08 $\quad 12: 59$ \\
\hline 2/4/08 13:00 & $3 / 14 / 08 \quad 12: 59$ \\
\hline 2/4/08 13:00 & 3/14/08 $\quad 12: 59$ \\
\hline 2/5/08 09:40 & $3 / 14 / 08 \quad 12: 59$ \\
\hline 2/5/08 09:40 & 3/14/08 $\quad 12: 59$ \\
\hline 2/5/08 09:40 & 3/14/08 $\quad 12: 59$ \\
\hline 2/5/08 09:40 & 3/14/08 $\quad 12: 59$ \\
\hline 2/6/08 09:20 & $\begin{array}{ll}3 / 14 / 08 & 12: 59\end{array}$ \\
\hline 2/6/08 09:20 & $3 / 14 / 08 \quad 12: 59$ \\
\hline 2/6/08 09:20 & $3 / 14 / 08 \quad 12: 59$ \\
\hline 2/6/08 09:20 & 3/14/08 $\quad 12: 59$ \\
\hline 2/26/08 $09: 20$ & 3/14/08 $\quad 12: 59$ \\
\hline 2/26/08 09:20 & 3/14/08 $\quad 12: 59$ \\
\hline 2/26/08 $09: 20$ & $3 / 14 / 08 \quad 12: 59$ \\
\hline 2/26/08 09:20 & 3/14/08 $\quad 12: 59$ \\
\hline 2/27/08 09:15 & 3/14/08 $\quad 12: 59$ \\
\hline 2/27/08 09:15 & 3/14/08 $12: 59$ \\
\hline 2/27/08 09:15 & $3 / 14 / 08 \quad 12: 59$ \\
\hline 2/27/08 09:15 & $3 / 14 / 08 \quad 12: 59$ \\
\hline $\begin{array}{lll}2 / 28 / 08 & 10: 15\end{array}$ & $\begin{array}{lll}3 / 14 / 08 & 12: 59\end{array}$ \\
\hline 2/28/08 10:15 & $3 / 14 / 08 \quad 12: 59$ \\
\hline $\begin{array}{lll}2 / 28 / 08 & 10: 15\end{array}$ & $3 / 14 / 08 \quad 12: 59$ \\
\hline $\begin{array}{lll}2 / 28 / 08 & 10: 15\end{array}$ & $3 / 14 / 08 \quad 12: 59$ \\
\hline 2/28/08 $11: 45$ & 3/14/08 $\quad 12: 59$ \\
\hline 2/28/08 11:45 & $3 / 14 / 08 \quad 12: 59$ \\
\hline 2/28/08 $11: 45$ & $3 / 14 / 08 \quad 12: 59$ \\
\hline 2/28/08 11:45 & $3 / 14 / 08 \quad 12: 59$ \\
\hline 3/3/08 09:00 & 3/14/08 $\quad 12: 59$ \\
\hline 3/3/08 09:00 & 3/14/08 $\quad 12: 59$ \\
\hline 3/3/08 09:00 & $\begin{array}{lll}3 / 14 / 08 & 12: 59\end{array}$ \\
\hline 3/3/08 09:00 & $\begin{array}{ll}3 / 14 / 08 & 12: 59\end{array}$ \\
\hline
\end{tabular}


The following analyses were performed on the following samples included in this report:

Metals 1:1 DI Water Extract by ICPMS

Metals Acid Extract by ICPMS

1:1 DI Water Extract

Alkalinity, Titrimetic ( $\mathrm{pH} 4.5)$

Anions By Ion Chromatography

Carbon, Total, Combustion or Oxidation

GEA No Preparation

geological description

Inorganic Carbon, Total, Combustion or Oxidation

Metals 1:1 Water Extract by ICPOES

Metals Acid Extract by ICPOES

Moisture Content

Nitric Acid Digestion

Percent Solids

$\mathrm{pH}$ of Waters By Electrode

Specific Conductance

Tc_U Acid Extract by ICPMS

Tc_U 1:1 DI Water Extract by ICPMS

Total Alpha Total Beta 1:1 DI Water Extract By LSC

Total Alpha Total Beta Acid Extract By LSC

Total Organic Carbon NP 


\begin{tabular}{|c|c|c|}
\hline $\begin{array}{l}\text { HEIS No. } \\
\text { B1RTF8 }\end{array}$ & $\begin{array}{l}\text { Laboratory ID } \\
0803001-01\end{array}$ & $\begin{array}{l}\text { Matrix } \\
\text { SOIL }\end{array}$ \\
\hline B1RTF9A & 0803001-02 & SOIL \\
\hline B1RTH0 & 0803012-01 & SOIL \\
\hline B1RTH1A & 0803012-02 & SOIL \\
\hline B1RTH1 & 0803012-03 & SOIL \\
\hline B1RTH2C & 0803012-04 & SOIL \\
\hline B1RTH2B & 0803012-05 & SOIL \\
\hline B1RTH2A & 0803012-06 & SOIL \\
\hline B1RTH2 & 0803012-07 & SOIL \\
\hline B1RTH3C & 0803012-08 & SOIL \\
\hline B1RTH3B & 0803012-09 & SOIL \\
\hline B1RTH3A & 0803012-10 & SOIL \\
\hline B1RTH3 & 0803012-11 & SOIL \\
\hline B1RYR7C & 0803012-12 & SOIL \\
\hline B1RYR7B & 0803012-13 & SOIL \\
\hline B1RYR7A & 0803012-14 & SOIL \\
\hline B1RYR7 & 0803012-15 & SOIL \\
\hline B1RYR8C & 0803012-16 & SOIL \\
\hline B1RYR8B & 0803012-17 & SOIL \\
\hline B1RYR8A & 0803012-18 & SOIL \\
\hline B1RYR8 & 0803012-19 & SOIL \\
\hline B1RYR9C & 0803012-20 & SOIL \\
\hline B1RYR9B & 0803012-21 & SOIL \\
\hline B1RYR9A & 0803012-22 & SOIL \\
\hline B1RYR9 & 0803012-23 & SOIL \\
\hline B1RYT0B & 0803014-01 & SOIL \\
\hline B1RYT0A & 0803014-02 & SOIL \\
\hline B1RYT0 & 0803014-03 & SOIL \\
\hline B1T2P1C & 0803014-04 & SOIL \\
\hline В1T2P1B & 0803014-05 & SOIL \\
\hline B1T2P1A & 0803014-06 & SOIL \\
\hline B1T2P1 & 0803014-07 & SOIL \\
\hline B1T2P2C & 0803014-08 & SOIL \\
\hline В1Т2Р2B & 0803014-09 & SOIL \\
\hline B1T2P2A & 0803014-10 & SOIL \\
\hline B1T2P2 & 0803014-11 & SOIL \\
\hline В1Т2Р3С & 0803014-12 & SOIL \\
\hline В1Т2Р3В & 0803014-13 & SOIL \\
\hline В1Т2Р3А & 0803014-14 & SOIL \\
\hline B1T2P4C & 0803014-15 & SOIL \\
\hline В1Т2Р4B & 0803014-16 & SOIL \\
\hline B1T2P4A & 0803014-17 & SOIL \\
\hline B1T2P4 & 0803014-18 & SOIL \\
\hline B1T2P5C & 0803014-19 & SOIL \\
\hline В1Т2Р5B & 0803014-20 & SOIL \\
\hline B1T2P5A & 0803014-21 & SOIL \\
\hline
\end{tabular}

\begin{tabular}{|c|c|}
\hline $\begin{array}{l}\text { Date Collected } \\
\text { 12/12/07 10:02 }\end{array}$ & $\begin{array}{l}\text { Date Received } \\
12 / 17 / 07 \quad 14: 00\end{array}$ \\
\hline 12/12/07 10:55 & $12 / 17 / 07 \quad 14: 00$ \\
\hline 12/13/07 13:15 & $12 / 17 / 07 \quad 14: 00$ \\
\hline 12/17/07 09:14 & $12 / 17 / 07 \quad 14: 00$ \\
\hline 12/17/07 09:14 & $12 / 17 / 07 \quad 14: 00$ \\
\hline 12/20/07 09:00 & 12/20/07 12:35 \\
\hline 12/20/07 09:00 & 12/20/07 12:35 \\
\hline 12/20/07 09:00 & 12/20/07 12:35 \\
\hline 12/20/07 09:00 & 12/20/07 12:35 \\
\hline 12/21/07 08:55 & $12 / 21 / 07 \quad 11: 50$ \\
\hline 12/21/07 08:55 & $12 / 21 / 07 \quad 11: 50$ \\
\hline 12/21/07 08:55 & $12 / 21 / 07 \quad 11: 50$ \\
\hline 12/21/07 08:55 & 12/21/07 11:50 \\
\hline 12/31/07 10:30 & 1/2/08 10:45 \\
\hline 12/31/07 10:30 & 1/2/08 10:45 \\
\hline 12/31/07 10:30 & 1/2/08 $10: 45$ \\
\hline 12/31/07 10:30 & 1/2/08 10:45 \\
\hline 1/3/08 10:40 & 1/4/08 13:05 \\
\hline 1/3/08 $10: 40$ & 1/4/08 13:05 \\
\hline 1/3/08 $\quad 10: 40$ & 1/4/08 13:05 \\
\hline 1/3/08 10:40 & 1/4/08 13:05 \\
\hline 1/7/08 09:15 & 1/7/08 12:05 \\
\hline 1/7/08 09:15 & 1/7/08 12:05 \\
\hline 1/7/08 09:15 & 1/7/08 12:05 \\
\hline 1/7/08 09:15 & 1/7/08 12:05 \\
\hline 1/8/08 11:20 & $1 / 14 / 08 \quad 10: 30$ \\
\hline 1/8/08 11:20 & $1 / 14 / 08 \quad 10: 30$ \\
\hline 1/8/08 11:20 & 1/14/08 $10: 30$ \\
\hline 1/10/08 09:15 & $1 / 14 / 08 \quad 10: 30$ \\
\hline 1/10/08 09:15 & $1 / 14 / 08 \quad 10: 30$ \\
\hline 1/10/08 09:15 & $1 / 14 / 08 \quad 10: 30$ \\
\hline 1/10/08 09:15 & $1 / 14 / 08 \quad 10: 30$ \\
\hline 1/15/08 08:30 & 1/16/08 12:42 \\
\hline 1/15/08 08:30 & 1/16/08 $12: 42$ \\
\hline 1/15/08 08:30 & 1/16/08 12:42 \\
\hline 1/15/08 08:30 & 1/16/08 12:42 \\
\hline 1/16/08 08:45 & 1/16/08 12:42 \\
\hline 1/16/08 08:45 & $1 / 16 / 08 \quad 12: 42$ \\
\hline 1/16/08 08:45 & 1/16/08 12:42 \\
\hline 1/17/08 09:20 & $1 / 21 / 08 \quad 13: 35$ \\
\hline 1/17/08 09:20 & 1/21/08 13:35 \\
\hline 1/17/08 09:20 & 1/21/08 13:35 \\
\hline 1/17/08 09:20 & 1/21/08 13:35 \\
\hline 1/17/08 11:05 & 1/21/08 13:35 \\
\hline 1/17/08 11:05 & $1 / 21 / 08 \quad 13: 35$ \\
\hline 1/17/08 11:05 & 1/21/08 $13: 35$ \\
\hline
\end{tabular}




\begin{tabular}{|c|c|c|}
\hline $\begin{array}{l}\text { HEIS No. } \\
\text { B1T2P5 }\end{array}$ & $\begin{array}{l}\text { Laboratory ID } \\
0803014-22\end{array}$ & $\begin{array}{l}\text { Matrix } \\
\text { SOIL }\end{array}$ \\
\hline B1T2P6C & 0803014-23 & SOIL \\
\hline В1Т2Р6B & 0803014-24 & SOIL \\
\hline B1T2P6A & 0803014-25 & SOIL \\
\hline B1T2P6 & 0803014-26 & SOIL \\
\hline B1T2P7C & 0803015-01 & SOIL \\
\hline B1T2P7B & 0803015-02 & SOIL \\
\hline B1T2P7A & 0803015-03 & SOIL \\
\hline B1T2P7 & 0803015-04 & SOIL \\
\hline B1T2P8C & 0803015-05 & SOIL \\
\hline В1Т2Р8B & 0803015-06 & SOIL \\
\hline B1T2P8A & 0803015-07 & SOIL \\
\hline B1T2P8 & 0803015-08 & SOIL \\
\hline B1T2P9C & 0803015-09 & SOIL \\
\hline В1Т2Р9В & 0803015-10 & SOIL \\
\hline В1T2P9A & 0803015-11 & SOIL \\
\hline В1Т2Р9 & 0803015-12 & SOIL \\
\hline B1T2R0C & 0803015-13 & SOIL \\
\hline B1T2R0B & 0803015-14 & SOIL \\
\hline B1T2R0A & 0803015-15 & SOIL \\
\hline B1T2R0 & 0803015-16 & SOIL \\
\hline B1TNK6C & 0803015-17 & SOIL \\
\hline B1TNK6B & 0803015-18 & SOIL \\
\hline B1TNK6A & 0803015-19 & SOIL \\
\hline B1TNK6 & 0803015-20 & SOIL \\
\hline B1TNK7C & 0803015-21 & SOIL \\
\hline B1TNK7B & 0803015-22 & SOIL \\
\hline B1TNK7A & 0803015-23 & SOIL \\
\hline B1TNK7 & 0803015-24 & SOIL \\
\hline B1TNK8C & 0803015-25 & SOIL \\
\hline B1TNK8B & 0803015-26 & SOIL \\
\hline B1TNK8A & 0803015-27 & SOIL \\
\hline B1TNK8 & 0803015-28 & SOIL \\
\hline B1TNK9C & 0803015-29 & SOIL \\
\hline B1TNK9B & 0803015-30 & SOIL \\
\hline B1TNK9A & 0803015-31 & SOIL \\
\hline B1TNK9 & 0803015-32 & SOIL \\
\hline
\end{tabular}

\begin{tabular}{|c|c|}
\hline Date Collected & Date Received \\
\hline 1/17/08 11:05 & 1/21/08 $13: 35$ \\
\hline 1/21/08 $09: 40$ & $1 / 21 / 08 \quad 13: 35$ \\
\hline 1/21/08 09:40 & 1/21/08 $13: 35$ \\
\hline 1/21/08 09:40 & 1/21/08 13:35 \\
\hline 1/21/08 09:40 & 1/21/08 13:35 \\
\hline 2/4/08 13:00 & 2/5/08 13:50 \\
\hline 2/4/08 13:00 & 2/5/08 13:50 \\
\hline 2/4/08 13:00 & 2/5/08 $13: 50$ \\
\hline 2/4/08 13:00 & 2/5/08 $13: 50$ \\
\hline 2/5/08 09:40 & 2/5/08 $13: 50$ \\
\hline 2/5/08 $09: 40$ & 2/5/08 $13: 50$ \\
\hline 2/5/08 09:40 & 2/5/08 $13: 50$ \\
\hline 2/5/08 09:40 & 2/5/08 13:50 \\
\hline 2/6/08 09:20 & $\begin{array}{ll}2 / 7 / 08 & 10: 30\end{array}$ \\
\hline 2/6/08 09:20 & 2/7/08 $10: 30$ \\
\hline 2/6/08 09:20 & $\begin{array}{ll}2 / 7 / 08 & 10: 30\end{array}$ \\
\hline 2/6/08 09:20 & 2/7/08 $10: 30$ \\
\hline 2/26/08 09:20 & 2/28/08 13:50 \\
\hline 2/26/08 09:20 & 2/28/08 13:50 \\
\hline 2/26/08 09:20 & 2/28/08 13:50 \\
\hline 2/26/08 09:20 & 2/28/08 13:50 \\
\hline 2/27/08 09:15 & $2 / 28 / 08 \quad 13: 50$ \\
\hline 2/27/08 09:15 & $2 / 28 / 08 \quad 13: 50$ \\
\hline 2/27/08 09:15 & $2 / 28 / 08 \quad 13: 50$ \\
\hline 2/27/08 09:15 & $2 / 28 / 08 \quad 13: 50$ \\
\hline 2/28/08 10:15 & $2 / 28 / 08 \quad 13: 50$ \\
\hline 2/28/08 10:15 & $2 / 28 / 08 \quad 13: 50$ \\
\hline 2/28/08 10:15 & $2 / 28 / 08 \quad 13: 50$ \\
\hline 2/28/08 10:15 & $2 / 28 / 08 \quad 13: 50$ \\
\hline 2/28/08 11:45 & $2 / 28 / 08 \quad 13: 50$ \\
\hline 2/28/08 11:45 & $2 / 28 / 08 \quad 13: 50$ \\
\hline 2/28/08 $11: 45$ & $2 / 28 / 08 \quad 13: 50$ \\
\hline 2/28/08 11:45 & $2 / 28 / 08 \quad 13: 50$ \\
\hline 3/3/08 09:00 & 3/3/08 11:05 \\
\hline 3/3/08 09:00 & 3/3/08 11:05 \\
\hline 3/3/08 09:00 & 3/3/08 11:05 \\
\hline 3/3/08 09:00 & 3/3/08 11:05 \\
\hline
\end{tabular}




\section{Wet Chemistry}

\begin{tabular}{|c|c|c|c|c|c|}
\hline $\begin{array}{l}\text { Alkalinity } \\
\text { Lab ID }\end{array}$ & $\begin{array}{l}\mathrm{CaCO} 3 \text { (u } \\
\text { HEIS No. }\end{array}$ & $\begin{array}{l}\text { Metho } \\
\text { Results }\end{array}$ & EQL & Analyzed & Batch \\
\hline 0803001-01 & B1RTF8 & 67.7 & 23.5 & $4 / 07 / 08$ & 8D04002 \\
\hline 0803001-02 & B1RTF9A & 68.4 & 23.5 & $4 / 07 / 08$ & 8D04002 \\
\hline 0803012-02 & B1RTH1A & 41.3 & 23.7 & $4 / 07 / 08$ & 8D04002 \\
\hline 0803012-03 & B1RTH1 & 73.5 & 23.7 & $4 / 07 / 08$ & 8D04002 \\
\hline 0803012-05 & B1RTH2B & 37.2 & 23.5 & $4 / 07 / 08$ & 8D04002 \\
\hline 0803012-06 & B1RTH2A & 35.7 & 23.5 & $4 / 07 / 08$ & 8D04002 \\
\hline 0803012-09 & B1RTH3B & 40.3 & 23.5 & $4 / 07 / 08$ & 8D04002 \\
\hline 0803012-10 & B1RTH3A & 39.5 & 23.5 & $4 / 07 / 08$ & 8D04002 \\
\hline 0803012-13 & B1RYR7B & 40.3 & 23.5 & $4 / 07 / 08$ & 8D04002 \\
\hline 0803012-14 & B1RYR7A & 39.5 & 23.5 & $4 / 07 / 08$ & 8D04002 \\
\hline 0803012-17 & B1RYR8B & 34.9 & 23.5 & $4 / 07 / 08$ & 8D04002 \\
\hline 0803012-18 & B1RYR8A & 40.8 & 24.7 & $4 / 07 / 08$ & 8D04002 \\
\hline 0803012-21 & B1RYR9B & 47.1 & 23.5 & $4 / 07 / 08$ & 8D04002 \\
\hline 0803012-22 & B1RYR9A & 46.4 & 23.5 & $4 / 07 / 08$ & 8D04002 \\
\hline 0803014-01 & B1RYT0B & 96.6 & 23.5 & $4 / 07 / 08$ & 8D04002 \\
\hline 0803014-02 & B1RYT0A & 80.7 & 23.6 & $4 / 07 / 08$ & 8D04002 \\
\hline 0803014-05 & B1T2P1B & 36.5 & 23.5 & $4 / 14 / 08$ & 8D04003 \\
\hline 0803014-06 & B1T2P1A & 42.6 & 23.5 & $4 / 14 / 08$ & 8D04003 \\
\hline 0803014-09 & В1Т2Р2B & 30.8 & 23.8 & $4 / 14 / 08$ & 8D04003 \\
\hline 0803014-10 & B1T2P2A & 27.4 & 23.5 & $4 / 14 / 08$ & 8D04003 \\
\hline 0803014-13 & В1Т2Р3В & 27.6 & 20.9 & $4 / 14 / 08$ & 8D04003 \\
\hline 0803014-14 & B1T2P3A & 35.8 & 18.2 & $4 / 14 / 08$ & 8D04003 \\
\hline 0803014-16 & В1Т2Р4B & 38.8 & 23.5 & $4 / 14 / 08$ & 8D04003 \\
\hline 0803014-17 & B1T2P4A & 42.7 & 23.6 & $4 / 14 / 08$ & 8D04003 \\
\hline 0803014-20 & В1Т2Р5B & 38.8 & 23.5 & $4 / 14 / 08$ & 8D04003 \\
\hline 0803014-21 & B1T2P5A & 35.7 & 23.5 & $4 / 14 / 08$ & 8D04003 \\
\hline 0803014-24 & В1Т2Р6B & 38.8 & 23.5 & $4 / 14 / 08$ & 8D04003 \\
\hline 0803014-25 & В1T2Р6A & 38.8 & 23.5 & $4 / 14 / 08$ & 8D04003 \\
\hline 0803015-02 & B1T2P7B & 47.1 & 23.5 & $4 / 14 / 08$ & 8D04003 \\
\hline 0803015-03 & B1T2P7A & 44.1 & 23.5 & $4 / 14 / 08$ & 8D04003 \\
\hline 0803015-06 & B1T2P8B & 32.7 & 23.5 & $4 / 14 / 08$ & 8D04003 \\
\hline 0803015-07 & В1T2P8A & 30.4 & 23.5 & $4 / 14 / 08$ & 8D04003 \\
\hline 0803015-10 & В1Т2Р9В & 37.2 & 23.5 & $4 / 14 / 08$ & 8D04003 \\
\hline 0803015-11 & В1T2P9A & 32.7 & 23.5 & $4 / 14 / 08$ & 8D04003 \\
\hline 0803015-14 & B1T2R0B & 34.2 & 23.5 & $4 / 14 / 08$ & 8D04003 \\
\hline 0803015-15 & B1T2R0A & 32.9 & 23.6 & $4 / 14 / 08$ & 8D04003 \\
\hline 0803015-18 & B1TNK6B & 35.7 & 23.5 & $4 / 14 / 08$ & 8D04003 \\
\hline 0803015-19 & B1TNK6A & 37.0 & 23.8 & $4 / 14 / 08$ & 8D04003 \\
\hline 0803015-22 & B1TNK7B & 35.0 & 23.5 & $4 / 14 / 08$ & 8D04003 \\
\hline 0803015-23 & B1TNK7A & 35.0 & 23.5 & $4 / 14 / 08$ & 8D04003 \\
\hline 0803015-26 & B1TNK8B & 37.2 & 23.5 & $4 / 14 / 08$ & 8D04003 \\
\hline 0803015-27 & B1TNK8A & 31.2 & 23.5 & $4 / 14 / 08$ & 8D04003 \\
\hline 0803015-30 & B1TNK9B & 41.3 & 24.6 & $4 / 14 / 08$ & 8D04003 \\
\hline 0803015-31 & B1TNK9A & 32.7 & 23.5 & $4 / 14 / 08$ & 8D04003 \\
\hline
\end{tabular}




\section{Wet Chemistry}

\begin{tabular}{|c|c|c|c|c|c|}
\hline \multicolumn{6}{|c|}{ Specific Conductance (EC) (mS/cm) by EPA 120.1} \\
\hline Lab ID & HEIS No. & Results & EQL & Analyzed & Batch \\
\hline 0803001-01 & B1RTF8 & 0.217 & 0.00974 & $4 / 02 / 08$ & 8D01004 \\
\hline 0803001-02 & B1RTF9A & 0.170 & 0.00972 & $4 / 02 / 08$ & 8D01004 \\
\hline 0803012-02 & B1RTH1A & 0.108 & 0.00488 & $4 / 02 / 08$ & 8D01004 \\
\hline 0803012-03 & B1RTH1 & 0.237 & 0.00465 & $4 / 02 / 08$ & 8D01004 \\
\hline 0803012-05 & B1RTH2B & 0.303 & 0.00479 & $4 / 02 / 08$ & 8D01004 \\
\hline 0803012-06 & B1RTH2A & 0.242 & 0.00485 & $4 / 02 / 08$ & 8D01004 \\
\hline 0803012-09 & B1RTH3B & 0.259 & 0.00487 & $4 / 02 / 08$ & 8D01004 \\
\hline 0803012-10 & B1RTH3A & 0.282 & 0.00485 & $4 / 02 / 08$ & 8D01004 \\
\hline 0803012-13 & B1RYR7B & 0.300 & 0.00479 & $4 / 02 / 08$ & 8D01004 \\
\hline 0803012-14 & B1RYR7A & 0.253 & 0.00482 & $4 / 02 / 08$ & 8D01004 \\
\hline 0803012-17 & B1RYR8B & 0.319 & 0.00474 & $4 / 02 / 08$ & 8D01004 \\
\hline 0803012-18 & B1RYR8A & 0.179 & 0.00513 & $4 / 02 / 08$ & 8D01004 \\
\hline 0803012-21 & B1RYR9B & 0.180 & 0.00492 & $4 / 02 / 08$ & 8D01004 \\
\hline 0803012-22 & B1RYR9A & 0.168 & 0.00492 & $4 / 02 / 08$ & 8D01004 \\
\hline 0803014-01 & B1RYT0B & 0.231 & 0.00877 & $4 / 02 / 08$ & 8D01004 \\
\hline 0803014-02 & B1RYT0A & 0.210 & 0.00897 & $4 / 02 / 08$ & 8D01004 \\
\hline 0803014-05 & B1T2P1B & 0.255 & 0.00970 & $4 / 03 / 08$ & 8D03004 \\
\hline 0803014-06 & $\mathrm{B} 1 \mathrm{~T} 2 \mathrm{P} 1 \mathrm{~A}$ & 0.239 & 0.00974 & $4 / 03 / 08$ & 8D03004 \\
\hline 0803014-09 & B1T2P2B & 0.389 & 0.00979 & $4 / 03 / 08$ & 8D03004 \\
\hline 0803014-10 & B1T2P2A & 0.270 & 0.00975 & $4 / 03 / 08$ & 8D03004 \\
\hline 0803014-13 & В1Т2Р3В & 0.184 & 0.00891 & $4 / 03 / 08$ & 8D03004 \\
\hline 0803014-14 & B1T2P3A & 0.210 & 0.00754 & $4 / 03 / 08$ & 8D03004 \\
\hline 0803014-16 & В1Т2Р4B & 0.267 & 0.00965 & $4 / 03 / 08$ & 8D03004 \\
\hline 0803014-17 & B1T2P4A & 0.271 & 0.00969 & $4 / 03 / 08$ & 8D03004 \\
\hline 0803014-20 & В1T2Р5B & 0.328 & 0.00954 & $4 / 03 / 08$ & 8D03004 \\
\hline 0803014-21 & B1T2P5A & 0.367 & 0.00934 & $4 / 03 / 08$ & 8D03004 \\
\hline 0803014-24 & В1Т2Р6B & 0.231 & 0.00973 & $4 / 03 / 08$ & 8D03004 \\
\hline 0803014-25 & B1T2P6A & 0.195 & 0.00978 & $4 / 03 / 08$ & 8D03004 \\
\hline 0803015-02 & B1T2P7B & 0.464 & 0.00916 & $4 / 03 / 08$ & 8D03004 \\
\hline 0803015-03 & B1T2P7A & 0.326 & 0.00946 & $4 / 03 / 08$ & 8D03004 \\
\hline 0803015-06 & B1T2P8B & 0.208 & 0.00974 & 4/03/08 & 8D03004 \\
\hline 0803015-07 & В1T2P8A & 0.253 & 0.00966 & $4 / 03 / 08$ & 8D03004 \\
\hline 0803015-10 & В1Т2Р9B & 0.222 & 0.00978 & $4 / 03 / 08$ & 8D03004 \\
\hline 0803015-11 & В1Т2Р9А & 0.225 & 0.00977 & $4 / 03 / 08$ & 8D03004 \\
\hline 0803015-14 & B1T2R0B & 0.363 & 0.00960 & $4 / 03 / 08$ & 8D03004 \\
\hline 0803015-15 & B1T2R0A & 0.452 & 0.00967 & $4 / 03 / 08$ & 8D03004 \\
\hline 0803015-18 & B1TNK6B & 0.242 & 0.00967 & $4 / 03 / 08$ & 8D03004 \\
\hline 0803015-19 & B1TNK6A & 0.191 & 0.00988 & $4 / 03 / 08$ & 8D03004 \\
\hline 0803015-22 & B1TNK7B & 0.173 & 0.00979 & $4 / 03 / 08$ & 8D03004 \\
\hline 0803015-23 & B1TNK7A & 0.165 & 0.00982 & $4 / 03 / 08$ & 8D03004 \\
\hline 0803015-26 & B1TNK8B & 0.265 & 0.00981 & $4 / 03 / 08$ & 8D03004 \\
\hline 0803015-27 & B1TNK8A & 0.388 & 0.00980 & $4 / 03 / 08$ & 8D03004 \\
\hline 0803015-30 & B1TNK9B & 0.212 & 0.0102 & $4 / 03 / 08$ & 8D03004 \\
\hline 0803015-31 & B1TNK9A & 0.377 & 0.00976 & 4/03/08 & 8D03004 \\
\hline
\end{tabular}




\section{Wet Chemistry}

\begin{tabular}{|c|c|c|c|c|c|}
\hline $\begin{array}{l}\text { Moisture } \\
\text { Lab ID }\end{array}$ & $\begin{array}{l}\text { tent (\% b } \\
\text { HEIS No. }\end{array}$ & $\begin{array}{l}\text { C-001 } \\
\text { Results }\end{array}$ & EQL & Analyzed & Batch \\
\hline 0803001-01 & B1RTF8 & 2.73 & & $3 / 25 / 08$ & 8C17007 \\
\hline 0803001-02 & B1RTF9A & 2.88 & & $3 / 25 / 08$ & 8C17007 \\
\hline 0803012-01 & B1RTH0 & 2.12 & & $3 / 25 / 08$ & 8C17007 \\
\hline 0803012-02 & B1RTH1A & 3.16 & & $3 / 25 / 08$ & 8C17007 \\
\hline 0803012-03 & B1RTH1 & 8.31 & & $3 / 25 / 08$ & 8C17007 \\
\hline 0803012-04 & B1RTH2C & 7.23 & & $3 / 25 / 08$ & 8C17007 \\
\hline 0803012-05 & B1RTH2B & 4.41 & & $3 / 25 / 08$ & 8C17007 \\
\hline 0803012-06 & B1RTH2A & 3.07 & & $3 / 25 / 08$ & 8C17007 \\
\hline 0803012-07 & B1RTH2 & 3.04 & & $3 / 25 / 08$ & 8C17007 \\
\hline 0803012-08 & B1RTH3C & 4.02 & & $3 / 25 / 08$ & 8C17007 \\
\hline 0803012-09 & B1RTH3B & 2.76 & & $3 / 25 / 08$ & 8C17007 \\
\hline 0803012-10 & B1RTH3A & 3.02 & & $3 / 25 / 08$ & 8C17007 \\
\hline 0803012-11 & B1RTH3 & 2.50 & & $3 / 25 / 08$ & 8C17007 \\
\hline 0803012-12 & B1RYR7C & 5.80 & & $3 / 25 / 08$ & 8C17007 \\
\hline 0803012-13 & B1RYR7B & 4.43 & & $3 / 25 / 08$ & 8C17007 \\
\hline 0803012-14 & B1RYR7A & 3.59 & & $3 / 25 / 08$ & 8C17007 \\
\hline 0803012-15 & B1RYR7 & 3.27 & & $3 / 25 / 08$ & 8C17007 \\
\hline 0803012-16 & B1RYR8C & 9.21 & & $3 / 25 / 08$ & 8C17007 \\
\hline 0803012-17 & B1RYR8B & 5.35 & & $3 / 25 / 08$ & 8C17007 \\
\hline 0803012-18 & B1RYR8A & 2.45 & & $3 / 25 / 08$ & 8C17007 \\
\hline 0803012-19 & B1RYR8 & 1.59 & & $3 / 25 / 08$ & 8C17007 \\
\hline 0803012-20 & B1RYR9C & 1.75 & & $3 / 25 / 08$ & 8C17007 \\
\hline 0803012-21 & B1RYR9B & 1.59 & & $3 / 25 / 08$ & 8C17007 \\
\hline 0803012-22 & B1RYR9A & 1.60 & & $3 / 25 / 08$ & 8C17007 \\
\hline 0803012-23 & B1RYR9 & 1.07 & & $3 / 25 / 08$ & 8C17007 \\
\hline 0803014-01 & B1RYT0B & 14.2 & & $3 / 25 / 08$ & 8C17007 \\
\hline 0803014-02 & B1RYT0A & 11.7 & & $3 / 25 / 08$ & 8C17007 \\
\hline 0803014-03 & B1RYT0 & 4.83 & & $3 / 25 / 08$ & 8C17007 \\
\hline 0803014-04 & B1T2P1C & 4.75 & & $3 / 25 / 08$ & 8C17007 \\
\hline 0803014-05 & B1T2P1B & 3.12 & & $3 / 25 / 08$ & 8C17007 \\
\hline 0803014-06 & B1T2P1A & 2.66 & & $3 / 25 / 08$ & 8C17007 \\
\hline 0803014-07 & B1T2P1 & 2.51 & & $3 / 25 / 08$ & 8C17007 \\
\hline 0803014-08 & B1T2P2C & 6.38 & & $3 / 25 / 08$ & 8C17007 \\
\hline 0803014-09 & В1T2Р2B & 3.40 & & $3 / 25 / 08$ & 8C17007 \\
\hline 0803014-10 & B1T2P2A & 2.53 & & $3 / 25 / 08$ & 8C17007 \\
\hline 0803014-11 & B1T2P2 & 2.28 & & $3 / 25 / 08$ & 8C17007 \\
\hline 0803014-12 & B1T2P3C & 5.51 & & $3 / 25 / 08$ & 8C17007 \\
\hline 0803014-13 & В1Т2Р3В & 2.75 & & $3 / 25 / 08$ & 8C17007 \\
\hline 0803014-14 & В1T2P3A & 2.57 & & $3 / 25 / 08$ & 8C17007 \\
\hline 0803014-15 & B1T2P4C & 4.88 & & $3 / 25 / 08$ & 8C17007 \\
\hline 0803014-16 & B1T2P4B & 3.70 & & $3 / 25 / 08$ & 8C17007 \\
\hline 0803014-17 & B1T2P4A & 3.51 & & $3 / 25 / 08$ & 8C17007 \\
\hline 0803014-18 & B1T2P4 & 2.75 & & $3 / 25 / 08$ & 8C17007 \\
\hline 0803014-19 & B1T2P5C & 4.88 & & $3 / 25 / 08$ & 8C17007 \\
\hline 0803014-20 & B1T2P5B & 4.92 & & $3 / 25 / 08$ & 8C17007 \\
\hline 0803014-21 & B1T2P5A & 7.09 & & $3 / 25 / 08$ & 8C17007 \\
\hline 0803014-22 & B1T2P5 & 2.26 & & $3 / 25 / 08$ & 8C17007 \\
\hline
\end{tabular}




\begin{tabular}{|c|c|c|c|c|c|}
\hline & & Wet & & & \\
\hline Moisture & atent (\% by & /C-001 & & & \\
\hline Lab ID & HEIS No. & Results & EQL & Analyzed & Batch \\
\hline 0803014-23 & B1T2P6C & 4.67 & & $3 / 25 / 08$ & 8C17007 \\
\hline 0803014-24 & В1T2P6B & 2.79 & & $3 / 25 / 08$ & 8C17007 \\
\hline 0803014-25 & B1T2P6A & 2.33 & & $3 / 25 / 08$ & 8C17007 \\
\hline 0803014-26 & B1T2P6 & 1.45 & & $3 / 25 / 08$ & 8C17007 \\
\hline 0803015-01 & B1T2P7C & 6.72 & & $3 / 25 / 08$ & 8C17007 \\
\hline 0803015-02 & В1T2Р7B & 9.16 & & $3 / 25 / 08$ & 8C17007 \\
\hline 0803015-03 & B1T2P7A & 5.79 & & $3 / 25 / 08$ & 8C17007 \\
\hline 0803015-04 & B1T2P7 & 7.67 & & $3 / 25 / 08$ & 8C17007 \\
\hline 0803015-05 & B1T2P8C & 3.53 & & $3 / 25 / 08$ & 8C17007 \\
\hline 0803015-06 & B1T2P8B & 2.83 & & $3 / 25 / 08$ & 8C17007 \\
\hline 0803015-07 & B1T2P8A & 3.50 & & $3 / 25 / 08$ & 8C17007 \\
\hline 0803015-08 & B1T2P8 & 2.60 & & $3 / 25 / 08$ & 8C17007 \\
\hline 0803015-09 & B1T2P9C & 3.98 & & $3 / 25 / 08$ & 8C17007 \\
\hline 0803015-10 & В1Т2Р9В & 2.24 & & $3 / 25 / 08$ & 8C17007 \\
\hline 0803015-11 & B1T2P9A & 2.37 & & $3 / 25 / 08$ & 8C17007 \\
\hline 0803015-12 & В1T2P9 & 1.84 & & $3 / 25 / 08$ & 8C17007 \\
\hline 0803015-13 & B1T2R0C & 8.08 & & $3 / 25 / 08$ & 8C17007 \\
\hline 0803015-14 & B1T2R0B & 4.17 & & $3 / 25 / 08$ & 8C17007 \\
\hline 0803015-15 & B1T2R0A & 4.08 & & $3 / 25 / 08$ & 8C17007 \\
\hline 0803015-16 & B1T2R0 & 2.99 & & $3 / 25 / 08$ & 8C17007 \\
\hline 0803015-17 & B1TNK6C & 4.11 & & $3 / 25 / 08$ & 8C17007 \\
\hline 0803015-18 & B1TNK6B & 3.37 & & $3 / 25 / 08$ & 8C17007 \\
\hline 0803015-19 & B1TNK6A & 2.66 & & $3 / 25 / 08$ & 8C17007 \\
\hline 0803015-20 & B1TNK6 & 1.97 & & $3 / 25 / 08$ & 8C17007 \\
\hline 0803015-21 & B1TNK7C & 4.21 & & $3 / 25 / 08$ & 8C17007 \\
\hline 0803015-22 & B1TNK7B & 2.21 & & $3 / 25 / 08$ & 8C17007 \\
\hline 0803015-23 & B1TNK7A & 1.96 & & $3 / 25 / 08$ & 8C17007 \\
\hline 0803015-24 & B1TNK7 & 1.48 & & $3 / 25 / 08$ & 8C17007 \\
\hline 0803015-25 & B1TNK8C & 2.01 & & $3 / 25 / 08$ & 8C17007 \\
\hline 0803015-26 & B1TNK8B & 2.01 & & $3 / 25 / 08$ & 8C17007 \\
\hline 0803015-27 & B1TNK8A & 2.08 & & $3 / 25 / 08$ & 8C17007 \\
\hline 0803015-28 & B1TNK8 & 1.81 & & $3 / 25 / 08$ & 8C17007 \\
\hline 0803015-29 & B1TNK9C & 5.02 & & $3 / 25 / 08$ & 8C17007 \\
\hline 0803015-30 & B1TNK9B & 2.76 & & $3 / 25 / 08$ & 8C17007 \\
\hline 0803015-31 & B1TNK9A & 2.46 & & $3 / 25 / 08$ & 8C17007 \\
\hline 0803015-32 & B1TNK9 & 1.86 & & $3 / 25 / 08$ & 8C17007 \\
\hline
\end{tabular}


pH (pH Units) by AGG-pH-001

\begin{tabular}{|c|c|c|c|c|c|}
\hline Lab ID & HEIS No. & Results & EQL & Analyzed & Batch \\
\hline 0803001-01 & B1RTF8 & 8.78 & & $4 / 02 / 08$ & 8D01004 \\
\hline 0803001-02 & B1RTF9A & 9.02 & & $4 / 02 / 08$ & 8D01004 \\
\hline 0803012-02 & B1RTH1A & 8.55 & & $4 / 02 / 08$ & 8D01004 \\
\hline 0803012-03 & B1RTH1 & 8.63 & & $4 / 02 / 08$ & 8D01004 \\
\hline 0803012-05 & B1RTH2B & 7.84 & & 4/02/08 & 8D01004 \\
\hline 0803012-06 & B1RTH2A & 8.11 & & 4/02/08 & 8D01004 \\
\hline 0803012-09 & B1RTH3B & 7.88 & & $4 / 02 / 08$ & 8D01004 \\
\hline 0803012-10 & B1RTH3A & 7.89 & & $4 / 02 / 08$ & 8D01004 \\
\hline 0803012-13 & B1RYR7B & 7.93 & & $4 / 02 / 08$ & 8D01004 \\
\hline 0803012-14 & B1RYR7A & 7.92 & & $4 / 02 / 08$ & 8D01004 \\
\hline 0803012-17 & B1RYR8B & 7.83 & & $4 / 02 / 08$ & 8D01004 \\
\hline 0803012-18 & B1RYR8A & 8.22 & & $4 / 02 / 08$ & 8D01004 \\
\hline 0803012-21 & B1RYR9B & 8.06 & & $4 / 02 / 08$ & 8D01004 \\
\hline 0803012-22 & B1RYR9A & 8.24 & & 4/02/08 & 8D01004 \\
\hline 0803014-01 & B1RYT0B & 8.93 & & $4 / 02 / 08$ & 8D01004 \\
\hline 0803014-02 & B1RYT0A & 8.80 & & 4/02/08 & 8D01004 \\
\hline 0803014-05 & B1T2P1B & 7.84 & & 4/03/08 & 8D03004 \\
\hline 0803014-06 & B1T2P1A & 7.78 & & 4/03/08 & 8D03004 \\
\hline 0803014-09 & В1T2Р2B & 7.56 & & 4/03/08 & 8D03004 \\
\hline 0803014-10 & B1T2P2A & 7.67 & & $4 / 03 / 08$ & 8D03004 \\
\hline 0803014-13 & B1T2P3B & 7.91 & & 4/03/08 & 8D03004 \\
\hline 0803014-14 & В1Т2Р3А & 7.81 & & 4/03/08 & 8D03004 \\
\hline 0803014-16 & B1T2P4B & 7.85 & & 4/03/08 & 8D03004 \\
\hline 0803014-17 & B1T2P4A & 7.81 & & $4 / 03 / 08$ & 8D03004 \\
\hline 0803014-20 & B1T2P5B & 7.85 & & 4/03/08 & 8D03004 \\
\hline 0803014-21 & B1T2P5A & 7.61 & & 4/03/08 & 8D03004 \\
\hline 0803014-24 & B1T2P6B & 7.85 & & 4/03/08 & 8D03004 \\
\hline 0803014-25 & B1T2P6A & 7.90 & & 4/03/08 & 8D03004 \\
\hline 0803015-02 & В1Т2Р7B & 7.79 & & $4 / 03 / 08$ & 8D03004 \\
\hline 0803015-03 & B1T2P7A & 7.88 & & 4/03/08 & 8D03004 \\
\hline 0803015-06 & В1T2P8B & 7.63 & & 4/03/08 & 8D03004 \\
\hline 0803015-07 & B1T2P8A & 7.77 & & 4/03/08 & 8D03004 \\
\hline 0803015-10 & В1Т2Р9B & 7.87 & & $4 / 03 / 08$ & 8D03004 \\
\hline 0803015-11 & B1T2P9A & 7.82 & & 4/03/08 & 8D03004 \\
\hline 0803015-14 & B1T2R0B & 7.62 & & 4/03/08 & 8D03004 \\
\hline 0803015-15 & B1T2R0A & 7.55 & & 4/03/08 & 8D03004 \\
\hline 0803015-18 & B1TNK6B & 7.76 & & 4/03/08 & 8D03004 \\
\hline 0803015-19 & B1TNK6A & 7.88 & & $4 / 03 / 08$ & 8D03004 \\
\hline 0803015-22 & B1TNK7B & 7.88 & & 4/03/08 & 8D03004 \\
\hline 0803015-23 & B1TNK7A & 7.79 & & 4/03/08 & 8D03004 \\
\hline 0803015-26 & B1TNK8B & 7.71 & & 4/03/08 & 8D03004 \\
\hline 0803015-27 & B1TNK8A & 7.61 & & 4/03/08 & 8D03004 \\
\hline 0803015-30 & B1TNK9B & 7.83 & & 4/03/08 & 8D03004 \\
\hline 0803015-31 & B1TNK9A & 7.66 & & 4/03/08 & 8D03004 \\
\hline
\end{tabular}




\section{Anions by Ion Chromatography}

\begin{tabular}{|c|c|c|c|c|c|c|c|}
\hline CAS \# & Analyte & Results & Units & EQL & Analyzed & Batch & Method \\
\hline HEIS No. & B1RTF8 & \multicolumn{3}{|c|}{ Lab ID: $\quad$ 0803001-01 } & & & \\
\hline 16984-48-8 & Fluoride & 0.719 & ug/g dry & 0.200 & 4/02/08 & 8D02003 & AGG-IC-001 \\
\hline $16887-00-6$ & Chloride & 0.744 & ug/g dry & 0.500 & $4 / 02 / 08$ & 8D02003 & AGG-IC-001 \\
\hline $14797-65-0$ & Nitrite & $<1.00$ & ug/g dry & 1.00 & $4 / 02 / 08$ & 8D02003 & AGG-IC-001 \\
\hline 24959-67-9 & Bromide & $<1.00$ & ug/g dry & 1.00 & $4 / 02 / 08$ & 8D02003 & AGG-IC-001 \\
\hline $14797-55-8$ & Nitrate & 1.95 & ug/g dry & 1.00 & $4 / 02 / 08$ & 8D02003 & AGG-IC-001 \\
\hline $14808-79-8$ & Sulfate & 36.3 & ug/g dry & 1.50 & $4 / 02 / 08$ & 8D02003 & AGG-IC-001 \\
\hline $14265-44-2$ & Phosphate & $<1.50$ & ug/g dry & 1.50 & $4 / 02 / 08$ & 8D02003 & AGG-IC-001 \\
\hline HEIS No. & B1RTF9A & \multicolumn{3}{|c|}{ Lab ID: $\quad$ 0803001-02 } & & & \\
\hline $16984-48-8$ & Fluoride & 0.721 & ug/g dry & 0.200 & $4 / 02 / 08$ & 8D02003 & AGG-IC-001 \\
\hline $16887-00-6$ & Chloride & $<0.500$ & ug/g dry & 0.500 & $4 / 02 / 08$ & 8D02003 & AGG-IC-001 \\
\hline $14797-65-0$ & Nitrite & $<1.00$ & ug/g dry & 1.00 & $4 / 02 / 08$ & 8D02003 & AGG-IC-001 \\
\hline 24959-67-9 & Bromide & $<1.00$ & ug/g dry & 1.00 & $4 / 02 / 08$ & 8D02003 & AGG-IC-001 \\
\hline $14797-55-8$ & Nitrate & 3.68 & ug/g dry & 1.00 & $4 / 02 / 08$ & 8D02003 & AGG-IC-001 \\
\hline $14808-79-8$ & Sulfate & 16.8 & ug/g dry & 1.50 & $4 / 02 / 08$ & 8D02003 & AGG-IC-001 \\
\hline $14265-44-2$ & Phosphate & $<1.50$ & ug/g dry & 1.50 & $4 / 02 / 08$ & 8D02003 & AGG-IC-001 \\
\hline HEIS No. & B1RTH1A & \multicolumn{3}{|c|}{ Lab ID: $\quad$ 0803012-02 } & & & \\
\hline $16984-48-8$ & Fluoride & 0.310 & ug/g dry & 0.201 & $4 / 02 / 08$ & 8D02003 & AGG-IC-001 \\
\hline $16887-00-6$ & Chloride & 0.516 & ug/g dry & 0.503 & $4 / 02 / 08$ & 8D02003 & AGG-IC-001 \\
\hline $14797-65-0$ & Nitrite & $<1.01$ & ug/g dry & 1.01 & $4 / 02 / 08$ & 8D02003 & AGG-IC-001 \\
\hline 24959-67-9 & Bromide & $<1.01$ & ug/g dry & 1.01 & $4 / 02 / 08$ & 8D02003 & AGG-IC-001 \\
\hline $14797-55-8$ & Nitrate & 2.33 & ug/g dry & 1.01 & $4 / 02 / 08$ & 8D02003 & AGG-IC-001 \\
\hline $14808-79-8$ & Sulfate & 14.5 & ug/g dry & 1.51 & $4 / 02 / 08$ & 8D02003 & AGG-IC-001 \\
\hline $14265-44-2$ & Phosphate & $<1.51$ & ug/g dry & 1.51 & $4 / 02 / 08$ & 8D02003 & AGG-IC-001 \\
\hline HEIS No. & B1RTH1 & \multicolumn{3}{|c|}{ Lab ID: $\quad$ 0803012-03 } & & & \\
\hline $16984-48-8$ & Fluoride & 1.08 & ug/g dry & 0.201 & $4 / 02 / 08$ & 8D02003 & AGG-IC-001 \\
\hline $16887-00-6$ & Chloride & 1.09 & ug/g dry & 0.503 & $4 / 02 / 08$ & 8D02003 & AGG-IC-001 \\
\hline $14797-65-0$ & Nitrite & $<1.01$ & ug/g dry & 1.01 & $4 / 02 / 08$ & 8D02003 & AGG-IC-001 \\
\hline 24959-67-9 & Bromide & $<1.01$ & ug/g dry & 1.01 & $4 / 02 / 08$ & 8D02003 & AGG-IC-001 \\
\hline $14797-55-8$ & Nitrate & 9.55 & ug/g dry & 1.01 & $4 / 02 / 08$ & 8D02003 & AGG-IC-001 \\
\hline $14808-79-8$ & Sulfate & 37.1 & ug/g dry & 1.51 & $4 / 02 / 08$ & 8D02003 & AGG-IC-001 \\
\hline 14265-44-2 & Phosphate & $<1.51$ & ug/g dry & 1.51 & $4 / 02 / 08$ & 8D02003 & AGG-IC-001 \\
\hline HEIS No. & B1RTH2B & \multicolumn{3}{|c|}{ Lab ID: $\quad$ 0803012-05 } & & & \\
\hline $16984-48-8$ & Fluoride & 0.294 & ug/g dry & 0.200 & $4 / 02 / 08$ & 8D02003 & AGG-IC-001 \\
\hline $16887-00-6$ & Chloride & 6.56 & ug/g dry & 0.500 & $4 / 02 / 08$ & 8D02003 & AGG-IC-001 \\
\hline $14797-65-0$ & Nitrite & $<1.00$ & ug/g dry & 1.00 & $4 / 02 / 08$ & 8D02003 & AGG-IC-001 \\
\hline 24959-67-9 & Bromide & $<1.00$ & ug/g dry & 1.00 & $4 / 02 / 08$ & 8D02003 & AGG-IC-001 \\
\hline $14797-55-8$ & Nitrate & 5.91 & ug/g dry & 1.00 & $4 / 02 / 08$ & 8D02003 & AGG-IC-001 \\
\hline 14808-79-8 & Sulfate & 98.2 & ug/g dry & 1.50 & $4 / 02 / 08$ & 8D02003 & AGG-IC-001 \\
\hline $14265-44-2$ & Phosphate & $<1.50$ & ug/g dry & 1.50 & $4 / 02 / 08$ & 8D02003 & AGG-IC-001 \\
\hline HEIS No. & B1RTH2A & \multicolumn{3}{|c|}{ Lab ID: $\quad$ 0803012-06 } & & & \\
\hline $16984-48-8$ & Fluoride & 0.337 & ug/g dry & 0.200 & $4 / 02 / 08$ & 8D02003 & AGG-IC-001 \\
\hline $16887-00-6$ & Chloride & 4.21 & ug/g dry & 0.500 & $4 / 02 / 08$ & 8D02003 & AGG-IC-001 \\
\hline $14797-65-0$ & Nitrite & $<1.00$ & ug/g dry & 1.00 & $4 / 02 / 08$ & 8D02003 & AGG-IC-001 \\
\hline 24959-67-9 & Bromide & $<1.00$ & ug/g dry & 1.00 & $4 / 02 / 08$ & 8D02003 & AGG-IC-001 \\
\hline $14797-55-8$ & Nitrate & 4.96 & ug/g dry & 1.00 & $4 / 02 / 08$ & 8D02003 & AGG-IC-001 \\
\hline $14808-79-8$ & Sulfate & 68.6 & ug/g dry & 1.50 & $4 / 02 / 08$ & 8D02003 & AGG-IC-001 \\
\hline $14265-44-2$ & Phosphate & $<1.50$ & ug/g dry & 1.50 & $4 / 02 / 08$ & 8D02003 & AGG-IC-001 \\
\hline HEIS No. & B1RTH3B & \multicolumn{2}{|c|}{ Lab ID: } & 3012-09 & & & \\
\hline
\end{tabular}




\section{Anions by Ion Chromatography}

\begin{tabular}{|c|c|c|c|c|c|c|c|}
\hline CAS \# & Analyte & Results & Units & EQL & Analyzed & Batch & Method \\
\hline HEIS No. & B1RTH3B & \multicolumn{3}{|c|}{ Lab ID: $\quad$ 0803012-09 } & & & \\
\hline 16984-48-8 & Fluoride & 0.312 & ug/g dry & 0.200 & 4/02/08 & 8D02003 & AGG-IC-001 \\
\hline $16887-00-6$ & Chloride & 21.7 & ug/g dry & 0.500 & $4 / 02 / 08$ & 8D02003 & AGG-IC-001 \\
\hline $14797-65-0$ & Nitrite & $<1.00$ & ug/g dry & 1.00 & $4 / 02 / 08$ & 8D02003 & AGG-IC-001 \\
\hline 24959-67-9 & Bromide & $<1.00$ & ug/g dry & 1.00 & $4 / 02 / 08$ & 8D02003 & AGG-IC-001 \\
\hline $14797-55-8$ & Nitrate & 15.8 & ug/g dry & 1.00 & $4 / 02 / 08$ & 8D02003 & AGG-IC-001 \\
\hline $14808-79-8$ & Sulfate & 45.3 & ug/g dry & 1.50 & $4 / 02 / 08$ & 8D02003 & AGG-IC-001 \\
\hline $14265-44-2$ & Phosphate & $<1.50$ & ug/g dry & 1.50 & $4 / 02 / 08$ & 8D02003 & AGG-IC-001 \\
\hline HEIS No. & B1RTH3A & \multicolumn{3}{|c|}{ Lab ID: $\quad$ 0803012-10 } & & & \\
\hline $16984-48-8$ & Fluoride & 0.320 & ug/g dry & 0.200 & $4 / 02 / 08$ & 8D02003 & AGG-IC-001 \\
\hline $16887-00-6$ & Chloride & 23.4 & ug/g dry & 0.500 & $4 / 02 / 08$ & 8D02003 & AGG-IC-001 \\
\hline $14797-65-0$ & Nitrite & $<1.00$ & ug/g dry & 1.00 & $4 / 02 / 08$ & 8D02003 & AGG-IC-001 \\
\hline 24959-67-9 & Bromide & $<1.00$ & ug/g dry & 1.00 & $4 / 02 / 08$ & 8D02003 & AGG-IC-001 \\
\hline $14797-55-8$ & Nitrate & 15.7 & ug/g dry & 1.00 & $4 / 02 / 08$ & 8D02003 & AGG-IC-001 \\
\hline $14808-79-8$ & Sulfate & 53.1 & ug/g dry & 1.50 & $4 / 02 / 08$ & 8D02003 & AGG-IC-001 \\
\hline $14265-44-2$ & Phosphate & $<1.50$ & ug/g dry & 1.50 & $4 / 02 / 08$ & 8D02003 & AGG-IC-001 \\
\hline HEIS No. & B1RYR7B & \multicolumn{3}{|c|}{ Lab ID: $\quad$ 0803012-13 } & & & \\
\hline $16984-48-8$ & Fluoride & 0.367 & ug/g dry & 0.200 & $4 / 02 / 08$ & 8D02003 & AGG-IC-001 \\
\hline $16887-00-6$ & Chloride & 20.7 & ug/g dry & 0.500 & $4 / 02 / 08$ & 8D02003 & AGG-IC-001 \\
\hline $14797-65-0$ & Nitrite & $<1.00$ & ug/g dry & 1.00 & $4 / 02 / 08$ & 8D02003 & AGG-IC-001 \\
\hline 24959-67-9 & Bromide & $<1.00$ & ug/g dry & 1.00 & $4 / 02 / 08$ & 8D02003 & AGG-IC-001 \\
\hline $14797-55-8$ & Nitrate & 4.16 & ug/g dry & 1.00 & $4 / 02 / 08$ & 8D02003 & AGG-IC-001 \\
\hline $14808-79-8$ & Sulfate & 73.3 & ug/g dry & 1.50 & $4 / 02 / 08$ & 8D02003 & AGG-IC-001 \\
\hline $14265-44-2$ & Phosphate & $<1.50$ & ug/g dry & 1.50 & $4 / 02 / 08$ & 8D02003 & AGG-IC-001 \\
\hline HEIS No. & B1RYR7A & \multicolumn{3}{|c|}{ Lab ID: $\quad$ 0803012-14 } & & & \\
\hline $16984-48-8$ & Fluoride & 0.344 & ug/g dry & 0.200 & $4 / 02 / 08$ & 8D02003 & AGG-IC-001 \\
\hline $16887-00-6$ & Chloride & 3.21 & ug/g dry & 0.500 & $4 / 02 / 08$ & 8D02003 & AGG-IC-001 \\
\hline $14797-65-0$ & Nitrite & $<1.00$ & ug/g dry & 1.00 & $4 / 02 / 08$ & 8D02003 & AGG-IC-001 \\
\hline 24959-67-9 & Bromide & $<1.00$ & ug/g dry & 1.00 & $4 / 02 / 08$ & 8D02003 & AGG-IC-001 \\
\hline $14797-55-8$ & Nitrate & 9.95 & ug/g dry & 1.00 & $4 / 02 / 08$ & 8D02003 & AGG-IC-001 \\
\hline $14808-79-8$ & Sulfate & 71.4 & ug/g dry & 1.50 & $4 / 02 / 08$ & 8D02003 & AGG-IC-001 \\
\hline 14265-44-2 & Phosphate & $<1.50$ & ug/g dry & 1.50 & $4 / 02 / 08$ & 8D02003 & AGG-IC-001 \\
\hline HEIS No. & B1RYR8B & \multicolumn{3}{|c|}{ Lab ID: $\quad$ 0803012-17 } & & & \\
\hline $16984-48-8$ & Fluoride & 0.353 & ug/g dry & 0.200 & $4 / 02 / 08$ & 8D02003 & AGG-IC-001 \\
\hline $16887-00-6$ & Chloride & 2.74 & ug/g dry & 0.500 & $4 / 02 / 08$ & 8D02003 & AGG-IC-001 \\
\hline $14797-65-0$ & Nitrite & $<0.999$ & ug/g dry & 0.999 & $4 / 02 / 08$ & 8D02003 & AGG-IC-001 \\
\hline 24959-67-9 & Bromide & $<0.999$ & ug/g dry & 0.999 & $4 / 02 / 08$ & 8D02003 & AGG-IC-001 \\
\hline $14797-55-8$ & Nitrate & 13.5 & ug/g dry & 0.999 & $4 / 02 / 08$ & 8D02003 & AGG-IC-001 \\
\hline $14808-79-8$ & Sulfate & 103 & ug/g dry & 1.50 & $4 / 02 / 08$ & 8D02003 & AGG-IC-001 \\
\hline $14265-44-2$ & Phosphate & $<1.50$ & ug/g dry & 1.50 & $4 / 02 / 08$ & 8D02003 & AGG-IC-001 \\
\hline HEIS No. & B1RYR8A & \multicolumn{3}{|c|}{ Lab ID: $\quad$ 0803012-18 } & & & \\
\hline $16984-48-8$ & Fluoride & 0.331 & ug/g dry & 0.210 & $4 / 02 / 08$ & 8D02003 & AGG-IC-001 \\
\hline $16887-00-6$ & Chloride & 1.17 & ug/g dry & 0.526 & $4 / 02 / 08$ & 8D02003 & AGG-IC-001 \\
\hline $14797-65-0$ & Nitrite & $<1.05$ & ug/g dry & 1.05 & $4 / 02 / 08$ & 8D02003 & AGG-IC-001 \\
\hline 24959-67-9 & Bromide & $<1.05$ & ug/g dry & 1.05 & $4 / 02 / 08$ & 8D02003 & AGG-IC-001 \\
\hline $14797-55-8$ & Nitrate & 6.26 & ug/g dry & 1.05 & $4 / 02 / 08$ & 8D02003 & AGG-IC-001 \\
\hline $14808-79-8$ & Sulfate & 44.2 & ug/g dry & 1.58 & $4 / 02 / 08$ & 8D02003 & AGG-IC-001 \\
\hline $14265-44-2$ & Phosphate & $<1.58$ & ug/g dry & 1.58 & $4 / 02 / 08$ & 8D02003 & AGG-IC-001 \\
\hline HEIS No. & B1RYR9B & \multicolumn{2}{|c|}{ Lab ID: } & 3012-21 & & & \\
\hline
\end{tabular}




\section{Anions by Ion Chromatography}

\begin{tabular}{|c|c|c|c|c|c|c|c|}
\hline CAS \# & Analyte & Results & Units & EQL & Analyzed & Batch & Method \\
\hline HEIS No. & B1RYR9B & \multicolumn{3}{|c|}{ Lab ID: $\quad$ 0803012-21 } & & & \\
\hline 16984-48-8 & Fluoride & 0.574 & ug/g dry & 0.200 & 4/02/08 & 8D02003 & AGG-IC-001 \\
\hline $16887-00-6$ & Chloride & 1.17 & ug/g dry & 0.500 & $4 / 02 / 08$ & 8D02003 & AGG-IC-001 \\
\hline $14797-65-0$ & Nitrite & $<1.00$ & ug/g dry & 1.00 & $4 / 02 / 08$ & 8D02003 & AGG-IC-001 \\
\hline 24959-67-9 & Bromide & $<1.00$ & ug/g dry & 1.00 & $4 / 02 / 08$ & 8D02003 & AGG-IC-001 \\
\hline $14797-55-8$ & Nitrate & 4.98 & ug/g dry & 1.00 & $4 / 02 / 08$ & 8D02003 & AGG-IC-001 \\
\hline $14808-79-8$ & Sulfate & 33.8 & ug/g dry & 1.50 & $4 / 02 / 08$ & 8D02003 & AGG-IC-001 \\
\hline $14265-44-2$ & Phosphate & $<1.50$ & ug/g dry & 1.50 & $4 / 02 / 08$ & 8D02003 & AGG-IC-001 \\
\hline HEIS No. & B1RYR9A & \multicolumn{3}{|c|}{ Lab ID: $\quad$ 0803012-22 } & & & \\
\hline $16984-48-8$ & Fluoride & 0.476 & ug/g dry & 0.200 & $4 / 02 / 08$ & 8D02003 & AGG-IC-001 \\
\hline $16887-00-6$ & Chloride & 1.02 & ug/g dry & 0.500 & $4 / 02 / 08$ & 8D02003 & AGG-IC-001 \\
\hline $14797-65-0$ & Nitrite & $<1.00$ & ug/g dry & 1.00 & $4 / 02 / 08$ & 8D02003 & AGG-IC-001 \\
\hline 24959-67-9 & Bromide & $<1.00$ & ug/g dry & 1.00 & $4 / 02 / 08$ & 8D02003 & AGG-IC-001 \\
\hline $14797-55-8$ & Nitrate & 4.41 & ug/g dry & 1.00 & $4 / 02 / 08$ & 8D02003 & AGG-IC-001 \\
\hline $14808-79-8$ & Sulfate & 31.9 & ug/g dry & 1.50 & $4 / 02 / 08$ & 8D02003 & AGG-IC-001 \\
\hline $14265-44-2$ & Phosphate & $<1.50$ & ug/g dry & 1.50 & $4 / 02 / 08$ & 8D02003 & AGG-IC-001 \\
\hline HEIS No. & B1RYT0B & \multicolumn{3}{|c|}{ Lab ID: $\quad$ 0803014-01 } & & & \\
\hline $16984-48-8$ & Fluoride & 1.18 & ug/g dry & 0.200 & $4 / 02 / 08$ & 8D02003 & AGG-IC-001 \\
\hline $16887-00-6$ & Chloride & 0.806 & ug/g dry & 0.501 & $4 / 02 / 08$ & 8D02003 & AGG-IC-001 \\
\hline $14797-65-0$ & Nitrite & $<1.00$ & ug/g dry & 1.00 & $4 / 02 / 08$ & 8D02003 & AGG-IC-001 \\
\hline 24959-67-9 & Bromide & $<1.00$ & ug/g dry & 1.00 & $4 / 02 / 08$ & 8D02003 & AGG-IC-001 \\
\hline $14797-55-8$ & Nitrate & 6.56 & ug/g dry & 1.00 & $4 / 02 / 08$ & 8D02003 & AGG-IC-001 \\
\hline $14808-79-8$ & Sulfate & 14.3 & ug/g dry & 1.50 & $4 / 02 / 08$ & 8D02003 & AGG-IC-001 \\
\hline $14265-44-2$ & Phosphate & $<1.50$ & ug/g dry & 1.50 & $4 / 02 / 08$ & 8D02003 & AGG-IC-001 \\
\hline HEIS No. & B1RYT0A & \multicolumn{3}{|c|}{ Lab ID: $\quad$ 0803014-02 } & & & \\
\hline $16984-48-8$ & Fluoride & 1.24 & ug/g dry & 0.200 & $4 / 02 / 08$ & 8D02003 & AGG-IC-001 \\
\hline $16887-00-6$ & Chloride & 1.12 & ug/g dry & 0.501 & $4 / 02 / 08$ & 8D02003 & AGG-IC-001 \\
\hline $14797-65-0$ & Nitrite & $<1.00$ & ug/g dry & 1.00 & $4 / 02 / 08$ & 8D02003 & AGG-IC-001 \\
\hline 24959-67-9 & Bromide & $<1.00$ & ug/g dry & 1.00 & $4 / 02 / 08$ & 8D02003 & AGG-IC-001 \\
\hline $14797-55-8$ & Nitrate & 7.91 & ug/g dry & 1.00 & $4 / 02 / 08$ & 8D02003 & AGG-IC-001 \\
\hline $14808-79-8$ & Sulfate & 16.1 & ug/g dry & 1.50 & $4 / 02 / 08$ & 8D02003 & AGG-IC-001 \\
\hline 14265-44-2 & Phosphate & $<1.50$ & ug/g dry & 1.50 & $4 / 02 / 08$ & 8D02003 & AGG-IC-001 \\
\hline HEIS No. & B1T2P1B & \multicolumn{3}{|c|}{ Lab ID: $\quad$ 0803014-05 } & & & \\
\hline $16984-48-8$ & Fluoride & 0.344 & ug/g dry & 0.200 & $4 / 03 / 08$ & 8D03001 & AGG-IC-001 \\
\hline $16887-00-6$ & Chloride & 8.04 & ug/g dry & 0.500 & $4 / 03 / 08$ & 8D03001 & AGG-IC-001 \\
\hline $14797-65-0$ & Nitrite & $<1.00$ & ug/g dry & 1.00 & 4/03/08 & 8D03001 & AGG-IC-001 \\
\hline 24959-67-9 & Bromide & $<1.00$ & ug/g dry & 1.00 & $4 / 03 / 08$ & 8D03001 & AGG-IC-001 \\
\hline $14797-55-8$ & Nitrate & 4.95 & ug/g dry & 1.00 & 4/03/08 & 8D03001 & AGG-IC-001 \\
\hline 14808-79-8 & Sulfate & 70.5 & ug/g dry & 1.50 & 4/03/08 & 8D03001 & AGG-IC-001 \\
\hline $14265-44-2$ & Phosphate & $<1.50$ & ug/g dry & 1.50 & $4 / 03 / 08$ & 8D03001 & AGG-IC-001 \\
\hline HEIS No. & B1T2P1A & \multicolumn{3}{|c|}{ Lab ID: $\quad$ 0803014-06 } & & & \\
\hline $16984-48-8$ & Fluoride & 0.311 & ug/g dry & 0.200 & $4 / 03 / 08$ & 8D03001 & AGG-IC-001 \\
\hline $16887-00-6$ & Chloride & 6.99 & ug/g dry & 0.500 & $4 / 03 / 08$ & 8D03001 & AGG-IC-001 \\
\hline $14797-65-0$ & Nitrite & $<1.00$ & ug/g dry & 1.00 & 4/03/08 & 8D03001 & AGG-IC-001 \\
\hline 24959-67-9 & Bromide & $<1.00$ & ug/g dry & 1.00 & $4 / 03 / 08$ & 8D03001 & AGG-IC-001 \\
\hline $14797-55-8$ & Nitrate & 3.89 & ug/g dry & 1.00 & $4 / 03 / 08$ & 8D03001 & AGG-IC-001 \\
\hline $14808-79-8$ & Sulfate & 64.6 & ug/g dry & 1.50 & 4/03/08 & 8D03001 & AGG-IC-001 \\
\hline $14265-44-2$ & Phosphate & $<1.50$ & ug/g dry & 1.50 & 4/03/08 & 8D03001 & AGG-IC-001 \\
\hline HEIS No. & В1T2P2B & \multicolumn{2}{|c|}{ Lab ID: } & 3014-09 & & & \\
\hline
\end{tabular}




\section{Anions by Ion Chromatography}

\begin{tabular}{|c|c|c|c|c|c|c|c|}
\hline CAS \# & Analyte & Results & Units & EQL & Analyzed & Batch & Method \\
\hline HEIS No. & B1T2P2B & \multicolumn{3}{|c|}{ Lab ID: $\quad$ 0803014-09 } & & & \\
\hline 16984-48-8 & Fluoride & 0.283 & ug/g dry & 0.203 & 4/03/08 & 8D03001 & AGG-IC-001 \\
\hline $16887-00-6$ & Chloride & 43.7 & ug/g dry & 0.506 & $4 / 03 / 08$ & 8D03001 & AGG-IC-001 \\
\hline $14797-65-0$ & Nitrite & $<1.01$ & ug/g dry & 1.01 & $4 / 03 / 08$ & 8D03001 & AGG-IC-001 \\
\hline 24959-67-9 & Bromide & $<1.01$ & ug/g dry & 1.01 & $4 / 03 / 08$ & 8D03001 & AGG-IC-001 \\
\hline $14797-55-8$ & Nitrate & 14.4 & ug/g dry & 1.01 & 4/03/08 & 8D03001 & AGG-IC-001 \\
\hline $14808-79-8$ & Sulfate & 84.9 & ug/g dry & 1.52 & $4 / 03 / 08$ & 8D03001 & AGG-IC-001 \\
\hline $14265-44-2$ & Phosphate & $<1.52$ & ug/g dry & 1.52 & $4 / 03 / 08$ & 8D03001 & AGG-IC-001 \\
\hline HEIS No. & B1T2P2A & \multicolumn{3}{|c|}{ Lab ID: $\quad$ 0803014-10 } & & & \\
\hline $16984-48-8$ & Fluoride & 0.282 & ug/g dry & 0.200 & 4/03/08 & 8D03001 & AGG-IC-001 \\
\hline $16887-00-6$ & Chloride & 28.1 & ug/g dry & 0.500 & $4 / 03 / 08$ & 8D03001 & AGG-IC-001 \\
\hline $14797-65-0$ & Nitrite & $<1.00$ & ug/g dry & 1.00 & $4 / 03 / 08$ & 8D03001 & AGG-IC-001 \\
\hline 24959-67-9 & Bromide & $<1.00$ & ug/g dry & 1.00 & 4/03/08 & 8D03001 & AGG-IC-001 \\
\hline $14797-55-8$ & Nitrate & 11.1 & ug/g dry & 1.00 & $4 / 03 / 08$ & 8D03001 & AGG-IC-001 \\
\hline $14808-79-8$ & Sulfate & 90.3 & ug/g dry & 1.50 & 4/03/08 & 8D03001 & AGG-IC-001 \\
\hline $14265-44-2$ & Phosphate & $<1.50$ & ug/g dry & 1.50 & $4 / 03 / 08$ & 8D03001 & AGG-IC-001 \\
\hline HEIS No. & В1T2P3B & \multicolumn{3}{|c|}{ Lab ID: $\quad$ 0803014-13 } & & & \\
\hline $16984-48-8$ & Fluoride & 0.346 & ug/g dry & 0.178 & $4 / 03 / 08$ & 8D03001 & AGG-IC-001 \\
\hline $16887-00-6$ & Chloride & 1.11 & ug/g dry & 0.446 & 4/03/08 & 8D03001 & AGG-IC-001 \\
\hline $14797-65-0$ & Nitrite & $<0.891$ & ug/g dry & 0.891 & $4 / 03 / 08$ & 8D03001 & AGG-IC-001 \\
\hline 24959-67-9 & Bromide & $<0.891$ & ug/g dry & 0.891 & 4/03/08 & 8D03001 & AGG-IC-001 \\
\hline $14797-55-8$ & Nitrate & 6.21 & ug/g dry & 0.891 & 4/03/08 & 8D03001 & AGG-IC-001 \\
\hline $14808-79-8$ & Sulfate & 42.2 & ug/g dry & 1.34 & $4 / 03 / 08$ & 8D03001 & AGG-IC-001 \\
\hline $14265-44-2$ & Phosphate & $<1.34$ & ug/g dry & 1.34 & $4 / 03 / 08$ & 8D03001 & AGG-IC-001 \\
\hline HEIS No. & B1T2P3A & \multicolumn{3}{|c|}{ Lab ID: $\quad$ 0803014-14 } & & & \\
\hline $16984-48-8$ & Fluoride & 0.274 & ug/g dry & 0.155 & $4 / 03 / 08$ & 8D03001 & AGG-IC-001 \\
\hline $16887-00-6$ & Chloride & 1.19 & ug/g dry & 0.386 & 4/03/08 & 8D03001 & AGG-IC-001 \\
\hline $14797-65-0$ & Nitrite & $<0.773$ & ug/g dry & 0.773 & 4/03/08 & 8D03001 & AGG-IC-001 \\
\hline 24959-67-9 & Bromide & $<0.773$ & ug/g dry & 0.773 & 4/03/08 & 8D03001 & AGG-IC-001 \\
\hline $14797-55-8$ & Nitrate & 6.78 & ug/g dry & 0.773 & 4/03/08 & 8D03001 & AGG-IC-001 \\
\hline $14808-79-8$ & Sulfate & 46.8 & ug/g dry & 1.16 & 4/03/08 & 8D03001 & AGG-IC-001 \\
\hline 14265-44-2 & Phosphate & $<1.16$ & ug/g dry & 1.16 & $4 / 03 / 08$ & 8D03001 & AGG-IC-001 \\
\hline HEIS No. & B1T2P4B & \multicolumn{3}{|c|}{ Lab ID: $\quad$ 0803014-16 } & & & \\
\hline $16984-48-8$ & Fluoride & 0.434 & ug/g dry & 0.200 & $4 / 03 / 08$ & 8D03001 & AGG-IC-001 \\
\hline $16887-00-6$ & Chloride & 2.16 & ug/g dry & 0.500 & $4 / 03 / 08$ & 8D03001 & AGG-IC-001 \\
\hline $14797-65-0$ & Nitrite & $<1.00$ & ug/g dry & 1.00 & 4/03/08 & 8D03001 & AGG-IC-001 \\
\hline 24959-67-9 & Bromide & $<1.00$ & ug/g dry & 1.00 & $4 / 03 / 08$ & 8D03001 & AGG-IC-001 \\
\hline $14797-55-8$ & Nitrate & 10.7 & ug/g dry & 1.00 & 4/03/08 & 8D03001 & AGG-IC-001 \\
\hline 14808-79-8 & Sulfate & 77.6 & ug/g dry & 1.50 & 4/03/08 & 8D03001 & AGG-IC-001 \\
\hline $14265-44-2$ & Phosphate & $<1.50$ & ug/g dry & 1.50 & $4 / 03 / 08$ & 8D03001 & AGG-IC-001 \\
\hline HEIS No. & B1T2P4A & \multicolumn{3}{|c|}{ Lab ID: $\quad$ 0803014-17 } & & & \\
\hline $16984-48-8$ & Fluoride & 0.445 & ug/g dry & 0.201 & $4 / 03 / 08$ & 8D03001 & AGG-IC-001 \\
\hline $16887-00-6$ & Chloride & 2.21 & ug/g dry & 0.502 & $4 / 03 / 08$ & 8D03001 & AGG-IC-001 \\
\hline $14797-65-0$ & Nitrite & $<1.00$ & ug/g dry & 1.00 & 4/03/08 & 8D03001 & AGG-IC-001 \\
\hline 24959-67-9 & Bromide & $<1.00$ & ug/g dry & 1.00 & $4 / 03 / 08$ & 8D03001 & AGG-IC-001 \\
\hline $14797-55-8$ & Nitrate & 11.1 & ug/g dry & 1.00 & 4/03/08 & 8D03001 & AGG-IC-001 \\
\hline $14808-79-8$ & Sulfate & 78.3 & ug/g dry & 1.50 & 4/03/08 & 8D03001 & AGG-IC-001 \\
\hline $14265-44-2$ & Phosphate & $<1.50$ & ug/g dry & 1.50 & 4/03/08 & 8D03001 & AGG-IC-001 \\
\hline HEIS No. & B1T2P5B & \multicolumn{2}{|c|}{ Lab ID: } & $3014-20$ & & & \\
\hline
\end{tabular}




\section{Anions by Ion Chromatography}

\begin{tabular}{|c|c|c|c|c|c|c|c|}
\hline CAS \# & Analyte & Results & Units & EQL & Analyzed & Batch & Method \\
\hline HEIS No. & В1T2P5B & \multicolumn{3}{|c|}{ Lab ID: $\quad$ 0803014-20 } & & & \\
\hline 16984-48-8 & Fluoride & 0.484 & ug/g dry & 0.200 & 4/03/08 & 8D03001 & AGG-IC-001 \\
\hline $16887-00-6$ & Chloride & 2.84 & ug/g dry & 0.500 & $4 / 03 / 08$ & 8D03001 & AGG-IC-001 \\
\hline $14797-65-0$ & Nitrite & $<1.00$ & ug/g dry & 1.00 & $4 / 03 / 08$ & 8D03001 & AGG-IC-001 \\
\hline 24959-67-9 & Bromide & $<1.00$ & ug/g dry & 1.00 & $4 / 03 / 08$ & 8D03001 & AGG-IC-001 \\
\hline $14797-55-8$ & Nitrate & 14.6 & ug/g dry & 1.00 & 4/03/08 & 8D03001 & AGG-IC-001 \\
\hline $14808-79-8$ & Sulfate & 105 & ug/g dry & 1.50 & $4 / 03 / 08$ & 8D03001 & AGG-IC-001 \\
\hline $14265-44-2$ & Phosphate & $<1.50$ & ug/g dry & 1.50 & $4 / 03 / 08$ & 8D03001 & AGG-IC-001 \\
\hline HEIS No. & B1T2P5A & \multicolumn{3}{|c|}{ Lab ID: $\quad$ 0803014-21 } & & & \\
\hline $16984-48-8$ & Fluoride & 0.423 & ug/g dry & 0.200 & 4/03/08 & 8D03001 & AGG-IC-001 \\
\hline $16887-00-6$ & Chloride & 3.88 & ug/g dry & 0.500 & $4 / 03 / 08$ & 8D03001 & AGG-IC-001 \\
\hline $14797-65-0$ & Nitrite & $<1.00$ & ug/g dry & 1.00 & $4 / 03 / 08$ & 8D03001 & AGG-IC-001 \\
\hline 24959-67-9 & Bromide & $<1.00$ & ug/g dry & 1.00 & 4/03/08 & 8D03001 & AGG-IC-001 \\
\hline $14797-55-8$ & Nitrate & 16.5 & ug/g dry & 1.00 & $4 / 03 / 08$ & 8D03001 & AGG-IC-001 \\
\hline $14808-79-8$ & Sulfate & 149 & ug/g dry & 1.50 & 4/03/08 & 8D03001 & AGG-IC-001 \\
\hline $14265-44-2$ & Phosphate & $<1.50$ & ug/g dry & 1.50 & $4 / 03 / 08$ & 8D03001 & AGG-IC-001 \\
\hline HEIS No. & B1T2P6B & \multicolumn{3}{|c|}{ Lab ID: $\quad$ 0803014-24 } & & & \\
\hline $16984-48-8$ & Fluoride & 0.513 & ug/g dry & 0.200 & $4 / 03 / 08$ & 8D03001 & AGG-IC-001 \\
\hline $16887-00-6$ & Chloride & 1.86 & ug/g dry & 0.500 & $4 / 03 / 08$ & 8D03001 & AGG-IC-001 \\
\hline $14797-65-0$ & Nitrite & $<1.00$ & ug/g dry & 1.00 & $4 / 03 / 08$ & 8D03001 & AGG-IC-001 \\
\hline 24959-67-9 & Bromide & $<1.00$ & ug/g dry & 1.00 & $4 / 03 / 08$ & 8D03001 & AGG-IC-001 \\
\hline $14797-55-8$ & Nitrate & 9.13 & ug/g dry & 1.00 & 4/03/08 & 8D03001 & AGG-IC-001 \\
\hline $14808-79-8$ & Sulfate & 59.7 & ug/g dry & 1.50 & $4 / 03 / 08$ & 8D03001 & AGG-IC-001 \\
\hline $14265-44-2$ & Phosphate & $<1.50$ & ug/g dry & 1.50 & $4 / 03 / 08$ & 8D03001 & AGG-IC-001 \\
\hline HEIS No. & B1T2P6A & \multicolumn{3}{|c|}{ Lab ID: $\quad$ 0803014-25 } & & & \\
\hline $16984-48-8$ & Fluoride & 0.420 & ug/g dry & 0.200 & $4 / 03 / 08$ & 8D03001 & AGG-IC-001 \\
\hline $16887-00-6$ & Chloride & 1.47 & ug/g dry & 0.500 & 4/03/08 & 8D03001 & AGG-IC-001 \\
\hline $14797-65-0$ & Nitrite & $<1.00$ & ug/g dry & 1.00 & 4/03/08 & 8D03001 & AGG-IC-001 \\
\hline 24959-67-9 & Bromide & $<1.00$ & ug/g dry & 1.00 & 4/03/08 & 8D03001 & AGG-IC-001 \\
\hline $14797-55-8$ & Nitrate & 7.44 & ug/g dry & 1.00 & 4/03/08 & 8D03001 & AGG-IC-001 \\
\hline $14808-79-8$ & Sulfate & 45.1 & ug/g dry & 1.50 & 4/03/08 & 8D03001 & AGG-IC-001 \\
\hline 14265-44-2 & Phosphate & $<1.50$ & ug/g dry & 1.50 & $4 / 03 / 08$ & 8D03001 & AGG-IC-001 \\
\hline HEIS No. & B1T2P7B & \multicolumn{3}{|c|}{ Lab ID: $\quad$ 0803015-02 } & & & \\
\hline $16984-48-8$ & Fluoride & 0.677 & ug/g dry & 0.200 & $4 / 03 / 08$ & 8D03001 & AGG-IC-001 \\
\hline $16887-00-6$ & Chloride & 3.13 & ug/g dry & 0.500 & $4 / 03 / 08$ & 8D03001 & AGG-IC-001 \\
\hline $14797-65-0$ & Nitrite & $<1.00$ & ug/g dry & 1.00 & 4/03/08 & 8D03001 & AGG-IC-001 \\
\hline 24959-67-9 & Bromide & $<1.00$ & ug/g dry & 1.00 & $4 / 03 / 08$ & 8D03001 & AGG-IC-001 \\
\hline $14797-55-8$ & Nitrate & 21.4 & ug/g dry & 1.00 & 4/03/08 & 8D03001 & AGG-IC-001 \\
\hline 14808-79-8 & Sulfate & 195 & ug/g dry & 15.0 & $4 / 04 / 08$ & 8D03001 & AGG-IC-001 \\
\hline $14265-44-2$ & Phosphate & $<1.50$ & ug/g dry & 1.50 & $4 / 03 / 08$ & 8D03001 & AGG-IC-001 \\
\hline HEIS No. & B1T2P7A & \multicolumn{3}{|c|}{ Lab ID: $\quad$ 0803015-03 } & & & \\
\hline $16984-48-8$ & Fluoride & 0.546 & ug/g dry & 0.200 & $4 / 03 / 08$ & 8D03001 & AGG-IC-001 \\
\hline $16887-00-6$ & Chloride & 2.00 & ug/g dry & 0.500 & $4 / 03 / 08$ & 8D03001 & AGG-IC-001 \\
\hline $14797-65-0$ & Nitrite & $<1.00$ & ug/g dry & 1.00 & 4/03/08 & 8D03001 & AGG-IC-001 \\
\hline 24959-67-9 & Bromide & $<1.00$ & ug/g dry & 1.00 & $4 / 03 / 08$ & 8D03001 & AGG-IC-001 \\
\hline $14797-55-8$ & Nitrate & $<1.00$ & ug/g dry & 1.00 & 4/03/08 & 8D03001 & AGG-IC-001 \\
\hline $14808-79-8$ & Sulfate & 125 & ug/g dry & 1.50 & 4/03/08 & 8D03001 & AGG-IC-001 \\
\hline $14265-44-2$ & Phosphate & $<1.50$ & ug/g dry & 1.50 & 4/03/08 & 8D03001 & AGG-IC-001 \\
\hline HEIS No. & В1T2P8B & \multicolumn{2}{|c|}{ Lab ID: } & 3015-06 & & & \\
\hline
\end{tabular}




\section{Anions by Ion Chromatography}

\begin{tabular}{|c|c|c|c|c|c|c|c|}
\hline CAS \# & Analyte & Results & Units & EQL & Analyzed & Batch & Method \\
\hline HEIS No. & B1T2P8B & \multicolumn{3}{|c|}{ Lab ID: $\quad$ 0803015-06 } & & & \\
\hline 16984-48-8 & Fluoride & 0.269 & ug/g dry & 0.200 & 4/03/08 & 8D03001 & AGG-IC-001 \\
\hline $16887-00-6$ & Chloride & 1.76 & ug/g dry & 0.501 & $4 / 03 / 08$ & 8D03001 & AGG-IC-001 \\
\hline $14797-65-0$ & Nitrite & $<1.00$ & ug/g dry & 1.00 & $4 / 03 / 08$ & 8D03001 & AGG-IC-001 \\
\hline 24959-67-9 & Bromide & $<1.00$ & ug/g dry & 1.00 & $4 / 03 / 08$ & 8D03001 & AGG-IC-001 \\
\hline $14797-55-8$ & Nitrate & 7.38 & ug/g dry & 1.00 & 4/03/08 & 8D03001 & AGG-IC-001 \\
\hline $14808-79-8$ & Sulfate & 58.6 & ug/g dry & 1.50 & $4 / 03 / 08$ & 8D03001 & AGG-IC-001 \\
\hline $14265-44-2$ & Phosphate & $<1.50$ & ug/g dry & 1.50 & $4 / 03 / 08$ & 8D03001 & AGG-IC-001 \\
\hline HEIS No. & B1T2P8A & \multicolumn{3}{|c|}{ Lab ID: $\quad$ 0803015-07 } & & & \\
\hline $16984-48-8$ & Fluoride & 0.249 & ug/g dry & 0.200 & $4 / 04 / 08$ & 8D03001 & AGG-IC-001 \\
\hline $16887-00-6$ & Chloride & 2.11 & ug/g dry & 0.500 & $4 / 04 / 08$ & 8D03001 & AGG-IC-001 \\
\hline $14797-65-0$ & Nitrite & $<1.00$ & ug/g dry & 1.00 & $4 / 04 / 08$ & 8D03001 & AGG-IC-001 \\
\hline 24959-67-9 & Bromide & $<1.00$ & ug/g dry & 1.00 & $4 / 04 / 08$ & 8D03001 & AGG-IC-001 \\
\hline $14797-55-8$ & Nitrate & 8.64 & ug/g dry & 1.00 & $4 / 04 / 08$ & 8D03001 & AGG-IC-001 \\
\hline $14808-79-8$ & Sulfate & 78.4 & ug/g dry & 1.50 & $4 / 04 / 08$ & 8D03001 & AGG-IC-001 \\
\hline 14265-44-2 & Phosphate & $<1.50$ & ug/g dry & 1.50 & $4 / 04 / 08$ & 8D03001 & AGG-IC-001 \\
\hline HEIS No. & В1T2P9B & \multicolumn{3}{|c|}{ Lab ID: $\quad$ 0803015-10 } & & & \\
\hline $16984-48-8$ & Fluoride & 0.335 & ug/g dry & 0.200 & $4 / 04 / 08$ & 8D03001 & AGG-IC-001 \\
\hline $16887-00-6$ & Chloride & 9.08 & ug/g dry & 0.500 & $4 / 04 / 08$ & 8D03001 & AGG-IC-001 \\
\hline $14797-65-0$ & Nitrite & $<1.00$ & ug/g dry & 1.00 & $4 / 04 / 08$ & 8D03001 & AGG-IC-001 \\
\hline 24959-67-9 & Bromide & $<1.00$ & ug/g dry & 1.00 & $4 / 04 / 08$ & 8D03001 & AGG-IC-001 \\
\hline $14797-55-8$ & Nitrate & 7.37 & ug/g dry & 1.00 & $4 / 04 / 08$ & 8D03001 & AGG-IC-001 \\
\hline $14808-79-8$ & Sulfate & 51.4 & ug/g dry & 1.50 & $4 / 04 / 08$ & 8D03001 & AGG-IC-001 \\
\hline $14265-44-2$ & Phosphate & $<1.50$ & ug/g dry & 1.50 & $4 / 04 / 08$ & 8D03001 & AGG-IC-001 \\
\hline HEIS No. & B1T2P9A & \multicolumn{3}{|c|}{ Lab ID: $\quad$ 0803015-11 } & & & \\
\hline $16984-48-8$ & Fluoride & 0.312 & ug/g dry & 0.200 & $4 / 04 / 08$ & 8D03001 & AGG-IC-001 \\
\hline $16887-00-6$ & Chloride & 8.58 & ug/g dry & 0.500 & $4 / 04 / 08$ & 8D03001 & AGG-IC-001 \\
\hline $14797-65-0$ & Nitrite & $<1.00$ & ug/g dry & 1.00 & $4 / 04 / 08$ & 8D03001 & AGG-IC-001 \\
\hline 24959-67-9 & Bromide & $<1.00$ & ug/g dry & 1.00 & $4 / 04 / 08$ & 8D03001 & AGG-IC-001 \\
\hline $14797-55-8$ & Nitrate & 7.04 & ug/g dry & 1.00 & $4 / 04 / 08$ & 8D03001 & AGG-IC-001 \\
\hline $14808-79-8$ & Sulfate & 54.2 & ug/g dry & 1.50 & $4 / 04 / 08$ & 8D03001 & AGG-IC-001 \\
\hline 14265-44-2 & Phosphate & $<1.50$ & ug/g dry & 1.50 & $4 / 04 / 08$ & 8D03001 & AGG-IC-001 \\
\hline HEIS No. & B1T2R0B & \multicolumn{3}{|c|}{ Lab ID: $\quad$ 0803015-14 } & & & \\
\hline $16984-48-8$ & Fluoride & 0.272 & ug/g dry & 0.200 & $4 / 04 / 08$ & 8D03001 & AGG-IC-001 \\
\hline $16887-00-6$ & Chloride & 33.7 & ug/g dry & 0.500 & $4 / 04 / 08$ & 8D03001 & AGG-IC-001 \\
\hline $14797-65-0$ & Nitrite & $<1.00$ & ug/g dry & 1.00 & $4 / 04 / 08$ & 8D03001 & AGG-IC-001 \\
\hline 24959-67-9 & Bromide & $<1.00$ & ug/g dry & 1.00 & $4 / 04 / 08$ & 8D03001 & AGG-IC-001 \\
\hline $14797-55-8$ & Nitrate & 21.2 & ug/g dry & 1.00 & $4 / 04 / 08$ & 8D03001 & AGG-IC-001 \\
\hline 14808-79-8 & Sulfate & 79.6 & ug/g dry & 1.50 & $4 / 04 / 08$ & 8D03001 & AGG-IC-001 \\
\hline $14265-44-2$ & Phosphate & $<1.50$ & ug/g dry & 1.50 & $4 / 04 / 08$ & 8D03001 & AGG-IC-001 \\
\hline HEIS No. & B1T2R0A & \multicolumn{3}{|c|}{ Lab ID: $\quad$ 0803015-15 } & & & \\
\hline $16984-48-8$ & Fluoride & 0.272 & ug/g dry & 0.201 & $4 / 04 / 08$ & 8D03001 & AGG-IC-001 \\
\hline $16887-00-6$ & Chloride & 30.6 & ug/g dry & 0.503 & $4 / 04 / 08$ & 8D03001 & AGG-IC-001 \\
\hline $14797-65-0$ & Nitrite & $<1.01$ & ug/g dry & 1.01 & $4 / 04 / 08$ & 8D03001 & AGG-IC-001 \\
\hline 24959-67-9 & Bromide & $<1.01$ & ug/g dry & 1.01 & $4 / 04 / 08$ & 8D03001 & AGG-IC-001 \\
\hline $14797-55-8$ & Nitrate & 20.1 & ug/g dry & 1.01 & $4 / 04 / 08$ & 8D03001 & AGG-IC-001 \\
\hline $14808-79-8$ & Sulfate & 136 & ug/g dry & 1.51 & $4 / 04 / 08$ & 8D03001 & AGG-IC-001 \\
\hline $14265-44-2$ & Phosphate & $<1.51$ & ug/g dry & 1.51 & $4 / 04 / 08$ & 8D03001 & AGG-IC-001 \\
\hline HEIS No. & B1TNK6B & \multicolumn{2}{|c|}{ Lab ID: } & 3015-18 & & & \\
\hline
\end{tabular}




\section{Anions by Ion Chromatography}

\begin{tabular}{|c|c|c|c|c|c|c|c|}
\hline CAS \# & Analyte & Results & Units & EQL & Analyzed & Batch & Method \\
\hline HEIS No. & B1TNK6B & \multicolumn{3}{|c|}{ Lab ID: $\quad$ 0803015-18 } & & & \\
\hline 16984-48-8 & Fluoride & 0.340 & ug/g dry & 0.200 & 4/04/08 & 8D03001 & AGG-IC-001 \\
\hline $16887-00-6$ & Chloride & 1.60 & ug/g dry & 0.500 & $4 / 04 / 08$ & 8D03001 & AGG-IC-001 \\
\hline $14797-65-0$ & Nitrite & $<1.00$ & ug/g dry & 1.00 & $4 / 04 / 08$ & 8D03001 & AGG-IC-001 \\
\hline 24959-67-9 & Bromide & $<1.00$ & ug/g dry & 1.00 & $4 / 04 / 08$ & 8D03001 & AGG-IC-001 \\
\hline $14797-55-8$ & Nitrate & 8.58 & ug/g dry & 1.00 & $4 / 04 / 08$ & 8D03001 & AGG-IC-001 \\
\hline $14808-79-8$ & Sulfate & 66.6 & ug/g dry & 1.50 & $4 / 04 / 08$ & 8D03001 & AGG-IC-001 \\
\hline $14265-44-2$ & Phosphate & $<1.50$ & ug/g dry & 1.50 & $4 / 04 / 08$ & 8D03001 & AGG-IC-001 \\
\hline HEIS No. & B1TNK6A & \multicolumn{3}{|c|}{ Lab ID: $\quad$ 0803015-19 } & & & \\
\hline $16984-48-8$ & Fluoride & 0.279 & ug/g dry & 0.203 & $4 / 04 / 08$ & 8D03001 & AGG-IC-001 \\
\hline $16887-00-6$ & Chloride & 1.20 & ug/g dry & 0.507 & $4 / 04 / 08$ & 8D03001 & AGG-IC-001 \\
\hline $14797-65-0$ & Nitrite & $<1.01$ & ug/g dry & 1.01 & $4 / 04 / 08$ & 8D03001 & AGG-IC-001 \\
\hline 24959-67-9 & Bromide & $<1.01$ & ug/g dry & 1.01 & $4 / 04 / 08$ & 8D03001 & AGG-IC-001 \\
\hline $14797-55-8$ & Nitrate & 6.21 & ug/g dry & 1.01 & $4 / 04 / 08$ & 8D03001 & AGG-IC-001 \\
\hline $14808-79-8$ & Sulfate & 48.6 & ug/g dry & 1.52 & $4 / 04 / 08$ & 8D03001 & AGG-IC-001 \\
\hline $14265-44-2$ & Phosphate & $<1.52$ & ug/g dry & 1.52 & $4 / 04 / 08$ & 8D03001 & AGG-IC-001 \\
\hline HEIS No. & B1TNK7B & \multicolumn{3}{|c|}{ Lab ID: $\quad$ 0803015-22 } & & & \\
\hline $16984-48-8$ & Fluoride & 0.291 & ug/g dry & 0.200 & $4 / 04 / 08$ & 8D03001 & AGG-IC-001 \\
\hline $16887-00-6$ & Chloride & 1.23 & ug/g dry & 0.500 & $4 / 04 / 08$ & 8D03001 & AGG-IC-001 \\
\hline $14797-65-0$ & Nitrite & $<1.00$ & ug/g dry & 1.00 & $4 / 04 / 08$ & 8D03001 & AGG-IC-001 \\
\hline 24959-67-9 & Bromide & $<1.00$ & ug/g dry & 1.00 & $4 / 04 / 08$ & 8D03001 & AGG-IC-001 \\
\hline $14797-55-8$ & Nitrate & 6.51 & ug/g dry & 1.00 & $4 / 04 / 08$ & 8D03001 & AGG-IC-001 \\
\hline $14808-79-8$ & Sulfate & 38.4 & ug/g dry & 1.50 & $4 / 04 / 08$ & 8D03001 & AGG-IC-001 \\
\hline $14265-44-2$ & Phosphate & $<1.50$ & ug/g dry & 1.50 & $4 / 04 / 08$ & 8D03001 & AGG-IC-001 \\
\hline HEIS No. & B1TNK7A & \multicolumn{3}{|c|}{ Lab ID: $\quad$ 0803015-23 } & & & \\
\hline $16984-48-8$ & Fluoride & 0.254 & ug/g dry & 0.200 & $4 / 04 / 08$ & 8D03001 & AGG-IC-001 \\
\hline $16887-00-6$ & Chloride & 1.32 & ug/g dry & 0.501 & $4 / 04 / 08$ & 8D03001 & AGG-IC-001 \\
\hline $14797-65-0$ & Nitrite & $<1.00$ & ug/g dry & 1.00 & $4 / 04 / 08$ & 8D03001 & AGG-IC-001 \\
\hline 24959-67-9 & Bromide & $<1.00$ & ug/g dry & 1.00 & $4 / 04 / 08$ & 8D03001 & AGG-IC-001 \\
\hline $14797-55-8$ & Nitrate & 6.71 & ug/g dry & 1.00 & $4 / 04 / 08$ & 8D03001 & AGG-IC-001 \\
\hline $14808-79-8$ & Sulfate & 39.6 & ug/g dry & 1.50 & $4 / 04 / 08$ & 8D03001 & AGG-IC-001 \\
\hline 14265-44-2 & Phosphate & $<1.50$ & ug/g dry & 1.50 & $4 / 04 / 08$ & 8D03001 & AGG-IC-001 \\
\hline HEIS No. & B1TNK8B & \multicolumn{3}{|c|}{ Lab ID: $\quad$ 0803015-26 } & & & \\
\hline $16984-48-8$ & Fluoride & 0.383 & ug/g dry & 0.200 & $4 / 04 / 08$ & 8D03001 & AGG-IC-001 \\
\hline $16887-00-6$ & Chloride & 1.59 & ug/g dry & 0.500 & $4 / 04 / 08$ & 8D03001 & AGG-IC-001 \\
\hline $14797-65-0$ & Nitrite & $<1.00$ & ug/g dry & 1.00 & $4 / 04 / 08$ & 8D03001 & AGG-IC-001 \\
\hline 24959-67-9 & Bromide & $<1.00$ & ug/g dry & 1.00 & $4 / 04 / 08$ & 8D03001 & AGG-IC-001 \\
\hline $14797-55-8$ & Nitrate & 6.82 & ug/g dry & 1.00 & $4 / 04 / 08$ & 8D03001 & AGG-IC-001 \\
\hline 14808-79-8 & Sulfate & 82.0 & ug/g dry & 1.50 & $4 / 04 / 08$ & 8D03001 & AGG-IC-001 \\
\hline $14265-44-2$ & Phosphate & $<1.50$ & ug/g dry & 1.50 & $4 / 04 / 08$ & 8D03001 & AGG-IC-001 \\
\hline HEIS No. & B1TNK8A & \multicolumn{3}{|c|}{ Lab ID: $\quad$ 0803015-27 } & & & \\
\hline $16984-48-8$ & Fluoride & 0.304 & ug/g dry & 0.200 & $4 / 04 / 08$ & 8D03001 & AGG-IC-001 \\
\hline $16887-00-6$ & Chloride & 1.79 & ug/g dry & 0.500 & $4 / 04 / 08$ & 8D03001 & AGG-IC-001 \\
\hline $14797-65-0$ & Nitrite & $<1.00$ & ug/g dry & 1.00 & $4 / 04 / 08$ & 8D03001 & AGG-IC-001 \\
\hline 24959-67-9 & Bromide & $<1.00$ & ug/g dry & 1.00 & $4 / 04 / 08$ & 8D03001 & AGG-IC-001 \\
\hline $14797-55-8$ & Nitrate & 7.47 & ug/g dry & 1.00 & $4 / 04 / 08$ & 8D03001 & AGG-IC-001 \\
\hline $14808-79-8$ & Sulfate & 149 & ug/g dry & 1.50 & $4 / 04 / 08$ & 8D03001 & AGG-IC-001 \\
\hline $14265-44-2$ & Phosphate & $<1.50$ & ug/g dry & 1.50 & $4 / 04 / 08$ & 8D03001 & AGG-IC-001 \\
\hline HEIS No. & B1TNK9B & \multicolumn{2}{|c|}{ Lab ID: } & 3015-30 & & & \\
\hline
\end{tabular}




\section{Anions by Ion Chromatography}

\begin{tabular}{|c|c|c|c|c|c|c|c|}
\hline CAS \# & Analyte & Results & Units & EQL & Analyzed & Batch & Method \\
\hline HEIS No. & B1TNK9B & \multicolumn{3}{|c|}{ Lab ID: $\quad$ 0803015-30 } & & & \\
\hline $16984-48-8$ & Fluoride & 0.385 & ug/g dry & 0.209 & $4 / 04 / 08$ & 8D03001 & AGG-IC-001 \\
\hline $16887-00-6$ & Chloride & 6.83 & ug/g dry & 0.523 & $4 / 04 / 08$ & 8D03001 & AGG-IC-001 \\
\hline $14797-65-0$ & Nitrite & $<1.05$ & ug/g dry & 1.05 & $4 / 04 / 08$ & 8D03001 & AGG-IC-001 \\
\hline $24959-67-9$ & Bromide & $<1.05$ & ug/g dry & 1.05 & $4 / 04 / 08$ & 8D03001 & AGG-IC-001 \\
\hline $14797-55-8$ & Nitrate & 6.15 & ug/g dry & 1.05 & 4/04/08 & 8D03001 & AGG-IC-001 \\
\hline $14808-79-8$ & Sulfate & 49.3 & ug/g dry & 1.57 & $4 / 04 / 08$ & 8D03001 & AGG-IC-001 \\
\hline $14265-44-2$ & Phosphate & $<1.57$ & ug/g dry & 1.57 & 4/04/08 & 8D03001 & AGG-IC-001 \\
\hline HEIS No. & B1TNK9A & \multicolumn{3}{|c|}{ Lab ID: $\quad 0803015-31$} & & & \\
\hline $16984-48-8$ & Fluoride & 0.371 & ug/g dry & 0.200 & 4/04/08 & 8D03001 & AGG-IC-001 \\
\hline 16887-00-6 & Chloride & 6.27 & ug/g dry & 0.500 & $4 / 04 / 08$ & 8D03001 & AGG-IC-001 \\
\hline $14797-65-0$ & Nitrite & $<1.00$ & ug/g dry & 1.00 & 4/04/08 & 8D03001 & AGG-IC-001 \\
\hline 24959-67-9 & Bromide & $<1.00$ & ug/g dry & 1.00 & 4/04/08 & 8D03001 & AGG-IC-001 \\
\hline $14797-55-8$ & Nitrate & 5.72 & ug/g dry & 1.00 & $4 / 04 / 08$ & 8D03001 & AGG-IC-001 \\
\hline 14808-79-8 & Sulfate & 137 & ug/g dry & 1.50 & $4 / 04 / 08$ & 8D03001 & AGG-IC-001 \\
\hline $14265-44-2$ & Phosphate & $<1.50$ & ug/g dry & 1.50 & 4/04/08 & 8D03001 & AGG-IC-001 \\
\hline
\end{tabular}


Total Metals by SW846 6010B/Water Extract

\begin{tabular}{|c|c|c|c|c|c|c|c|}
\hline CAS \# & Analyte & Results & Units & EQL & Analyzed & Batch & Method \\
\hline HEIS No. & B1RTF8 & \multicolumn{3}{|c|}{ Lab ID: $\quad$ 0803001-01 } & & & \\
\hline 7429-90-5 & Aluminum & 0.624 & ug/g dry & 0.086 & $5 / 07 / 08$ & 8D28005 & PNNL-AGG-ICP-AES \\
\hline $7440-38-2$ & Arsenic & $<0.360$ & ug/g dry & 0.360 & $5 / 07 / 08$ & 8D28005 & PNNL-AGG-ICP-AES \\
\hline $7440-42-8$ & Boron & $<1.94$ & ug/g dry & 1.94 & $5 / 07 / 08$ & 8D28005 & PNNL-AGG-ICP-AES \\
\hline $7440-39-3$ & Barium & 0.072 & ug/g dry & 0.009 & $5 / 07 / 08$ & 8D28005 & PNNL-AGG-ICP-AES \\
\hline $7440-41-7$ & Beryllium & $<0.028$ & ug/g dry & 0.028 & $5 / 07 / 08$ & 8D28005 & PNNL-AGG-ICP-AES \\
\hline 7440-69-9 & Bismuth & $<0.188$ & ug/g dry & 0.188 & $5 / 07 / 08$ & 8D28005 & PNNL-AGG-ICP-AES \\
\hline $7440-70-2$ & Calcium & 3.45 & ug/g dry & 0.387 & $5 / 07 / 08$ & 8D28005 & PNNL-AGG-ICP-AES \\
\hline $7440-43-9$ & Cadmium & $<0.027$ & ug/g dry & 0.027 & $5 / 07 / 08$ & 8D28005 & PNNL-AGG-ICP-AES \\
\hline $7440-48-4$ & Cobalt & $<0.096$ & ug/g dry & 0.096 & $5 / 07 / 08$ & 8D28005 & PNNL-AGG-ICP-AES \\
\hline $7440-47-3$ & Chromium & $<0.033$ & ug/g dry & 0.033 & $5 / 07 / 08$ & 8D28005 & PNNL-AGG-ICP-AES \\
\hline $7440-50-8$ & Copper & $<0.080$ & ug/g dry & 0.080 & $5 / 07 / 08$ & 8D28005 & PNNL-AGG-ICP-AES \\
\hline 7439-89-6 & Iron & 1.14 & ug/g dry & 0.142 & $5 / 07 / 08$ & 8D28005 & PNNL-AGG-ICP-AES \\
\hline 7440-09-7 & Potassium & 4.00 & ug/g dry & 2.33 & $5 / 07 / 08$ & 8D28005 & PNNL-AGG-ICP-AES \\
\hline 7439-93-2 & Lithium & $<0.540$ & ug/g dry & 0.540 & $5 / 07 / 08$ & 8D28005 & PNNL-AGG-ICP-AES \\
\hline 7439-95-4 & Magnesium & 1.03 & ug/g dry & 0.083 & $5 / 07 / 08$ & 8D28005 & PNNL-AGG-ICP-AES \\
\hline 7439-96-5 & Manganese & $<0.017$ & ug/g dry & 0.017 & $5 / 07 / 08$ & 8D28005 & PNNL-AGG-ICP-AES \\
\hline 7439-98-7 & Molybdenum & $<0.139$ & ug/g dry & 0.139 & $5 / 07 / 08$ & 8D28005 & PNNL-AGG-ICP-AES \\
\hline $7440-02-0$ & Nickel & $<0.093$ & ug/g dry & 0.093 & $5 / 07 / 08$ & 8D28005 & PNNL-AGG-ICP-AES \\
\hline $7723-14-0$ & Phosphorus & $<1.03$ & ug/g dry & 1.03 & $5 / 07 / 08$ & 8D28005 & PNNL-AGG-ICP-AES \\
\hline $7439-92-1$ & Lead & $<0.432$ & ug/g dry & 0.432 & $5 / 07 / 08$ & 8D28005 & PNNL-AGG-ICP-AES \\
\hline $7782-49-2$ & Selenium & $<1.95$ & ug/g dry & 1.95 & $5 / 07 / 08$ & 8D28005 & PNNL-AGG-ICP-AES \\
\hline $7440-24-6$ & Strontium & $<0.052$ & ug/g dry & 0.052 & $5 / 07 / 08$ & 8D28005 & PNNL-AGG-ICP-AES \\
\hline $7440-28-0$ & Thallium & $<1.03$ & ug/g dry & 1.03 & $5 / 07 / 08$ & 8D28005 & PNNL-AGG-ICP-AES \\
\hline $7440-62-2$ & Vanadium & $<0.045$ & ug/g dry & 0.045 & $5 / 07 / 08$ & 8D28005 & PNNL-AGG-ICP-AES \\
\hline $7440-66-6$ & Zinc & $<0.093$ & ug/g dry & 0.093 & $5 / 07 / 08$ & 8D28005 & PNNL-AGG-ICP-AES \\
\hline $7440-23-5$ & Sodium & 39.0 & ug/g dry & 0.669 & $5 / 07 / 08$ & 8D28005 & PNNL-AGG-ICP-AES \\
\hline $7440-21-3$ & Silicon & 11.4 & ug/g dry & 1.50 & $5 / 07 / 08$ & 8D28005 & PNNL-AGG-ICP-AES \\
\hline 7704-34-9 & Sulfur & 12.9 & ug/g dry & 3.08 & $5 / 07 / 08$ & 8D28005 & PNNL-AGG-ICP-AES \\
\hline $7440-32-6$ & Titanium & 0.050 & ug/g dry & 0.009 & $5 / 07 / 08$ & 8D28005 & PNNL-AGG-ICP-AES \\
\hline $7440-67-7$ & Zirconium & $<0.150$ & ug/g dry & 0.150 & $5 / 07 / 08$ & 8D28005 & PNNL-AGG-ICP-AES \\
\hline $7440-22-4$ & Silver & $<0.077$ & ug/g dry & 0.077 & $5 / 07 / 08$ & 8D28005 & PNNL-AGG-ICP-AES \\
\hline $7440-15-5$ & Rhenium & $<0.152$ & ug/g dry & 0.152 & $5 / 07 / 08$ & 8D28005 & PNNL-AGG-ICP-AES \\
\hline $7440-36-0$ & Antimony & $<0.633$ & ug/g dry & 0.633 & $5 / 07 / 08$ & 8D28005 & PNNL-AGG-ICP-AES \\
\hline HEIS No. & B1RTF9A & & b ID: & 3001-02 & & & \\
\hline $7429-90-5$ & Aluminum & 1.22 & ug/g dry & 0.086 & $5 / 07 / 08$ & 8D28005 & PNNL-AGG-ICP-AES \\
\hline $7440-38-2$ & Arsenic & $<0.360$ & ug/g dry & 0.360 & $5 / 07 / 08$ & 8D28005 & PNNL-AGG-ICP-AES \\
\hline $7440-42-8$ & Boron & $<1.94$ & ug/g dry & 1.94 & $5 / 07 / 08$ & 8D28005 & PNNL-AGG-ICP-AES \\
\hline $7440-39-3$ & Barium & 0.022 & ug/g dry & 0.009 & $5 / 07 / 08$ & 8D28005 & PNNL-AGG-ICP-AES \\
\hline $7440-41-7$ & Beryllium & $<0.028$ & ug/g dry & 0.028 & $5 / 07 / 08$ & 8D28005 & PNNL-AGG-ICP-AES \\
\hline 7440-69-9 & Bismuth & $<0.188$ & ug/g dry & 0.188 & $5 / 07 / 08$ & 8D28005 & PNNL-AGG-ICP-AES \\
\hline $7440-70-2$ & Calcium & 3.00 & ug/g dry & 0.387 & $5 / 07 / 08$ & 8D28005 & PNNL-AGG-ICP-AES \\
\hline $7440-43-9$ & Cadmium & $<0.027$ & ug/g dry & 0.027 & $5 / 07 / 08$ & 8D28005 & PNNL-AGG-ICP-AES \\
\hline $7440-48-4$ & Cobalt & $<0.096$ & ug/g dry & 0.096 & $5 / 07 / 08$ & 8D28005 & PNNL-AGG-ICP-AES \\
\hline $7440-47-3$ & Chromium & $<0.033$ & ug/g dry & 0.033 & $5 / 07 / 08$ & 8D28005 & PNNL-AGG-ICP-AES \\
\hline $7440-50-8$ & Copper & $<0.080$ & ug/g dry & 0.080 & $5 / 07 / 08$ & 8D28005 & PNNL-AGG-ICP-AES \\
\hline 7439-89-6 & Iron & 2.43 & ug/g dry & 0.142 & $5 / 07 / 08$ & 8D28005 & PNNL-AGG-ICP-AES \\
\hline 7440-09-7 & Potassium & 2.85 & ug/g dry & 2.33 & $5 / 07 / 08$ & 8D28005 & PNNL-AGG-ICP-AES \\
\hline 7439-93-2 & Lithium & $<0.540$ & ug/g dry & 0.540 & $5 / 07 / 08$ & 8D28005 & PNNL-AGG-ICP-AES \\
\hline
\end{tabular}


Total Metals by SW846 6010B/Water Extract

\begin{tabular}{|c|c|c|c|c|c|c|c|}
\hline CAS \# & Analyte & Results & Units & EQL & Analyzed & Batch & Method \\
\hline HEIS No. & B1RTF9A & \multicolumn{3}{|c|}{ Lab ID: $\quad$ 0803001-02 } & & & \\
\hline 7439-95-4 & Magnesium & 0.973 & ug/g dry & 0.083 & $5 / 07 / 08$ & 8D28005 & PNNL-AGG-ICP-AES \\
\hline 7439-96-5 & Manganese & 0.019 & ug/g dry & 0.017 & $5 / 07 / 08$ & 8D28005 & PNNL-AGG-ICP-AES \\
\hline 7439-98-7 & Molybdenum & $<0.139$ & ug/g dry & 0.139 & $5 / 07 / 08$ & 8D28005 & PNNL-AGG-ICP-AES \\
\hline $7440-02-0$ & Nickel & $<0.093$ & ug/g dry & 0.093 & $5 / 07 / 08$ & 8D28005 & PNNL-AGG-ICP-AES \\
\hline $7723-14-0$ & Phosphorus & $<1.03$ & ug/g dry & 1.03 & $5 / 07 / 08$ & 8D28005 & PNNL-AGG-ICP-AES \\
\hline 7439-92-1 & Lead & $<0.432$ & ug/g dry & 0.432 & $5 / 07 / 08$ & 8D28005 & PNNL-AGG-ICP-AES \\
\hline $7782-49-2$ & Selenium & $<1.95$ & ug/g dry & 1.95 & $5 / 07 / 08$ & 8D28005 & PNNL-AGG-ICP-AES \\
\hline $7440-24-6$ & Strontium & $<0.052$ & ug/g dry & 0.052 & $5 / 07 / 08$ & 8D28005 & PNNL-AGG-ICP-AES \\
\hline $7440-28-0$ & Thallium & $<1.03$ & ug/g dry & 1.03 & $5 / 07 / 08$ & 8D28005 & PNNL-AGG-ICP-AES \\
\hline $7440-62-2$ & Vanadium & 0.046 & ug/g dry & 0.045 & $5 / 07 / 08$ & 8D28005 & PNNL-AGG-ICP-AES \\
\hline $7440-66-6$ & Zinc & $<0.093$ & ug/g dry & 0.093 & $5 / 07 / 08$ & 8D28005 & PNNL-AGG-ICP-AES \\
\hline $7440-23-5$ & Sodium & 30.4 & ug/g dry & 0.669 & $5 / 07 / 08$ & 8D28005 & PNNL-AGG-ICP-AES \\
\hline $7440-21-3$ & Silicon & 14.4 & ug/g dry & 1.50 & $5 / 07 / 08$ & 8D28005 & PNNL-AGG-ICP-AES \\
\hline $7704-34-9$ & Sulfur & 6.16 & ug/g dry & 3.08 & $5 / 07 / 08$ & 8D28005 & PNNL-AGG-ICP-AES \\
\hline $7440-32-6$ & Titanium & 0.100 & ug/g dry & 0.009 & $5 / 07 / 08$ & 8D28005 & PNNL-AGG-ICP-AES \\
\hline $7440-67-7$ & Zirconium & $<0.150$ & ug/g dry & 0.150 & $5 / 07 / 08$ & 8D28005 & PNNL-AGG-ICP-AES \\
\hline $7440-22-4$ & Silver & $<0.077$ & ug/g dry & 0.077 & $5 / 07 / 08$ & 8D28005 & PNNL-AGG-ICP-AES \\
\hline $7440-15-5$ & Rhenium & $<0.152$ & ug/g dry & 0.152 & $5 / 07 / 08$ & 8D28005 & PNNL-AGG-ICP-AES \\
\hline $7440-36-0$ & Antimony & $<0.633$ & ug/g dry & 0.633 & $5 / 07 / 08$ & 8D28005 & PNNL-AGG-ICP-AES \\
\hline HEIS No. & B1RTH1A & \multicolumn{3}{|c|}{ Lab ID: $\quad$ 0803012-02 } & & & \\
\hline $7429-90-5$ & Aluminum & 0.343 & ug/g dry & 0.086 & $5 / 07 / 08$ & 8D28005 & PNNL-AGG-ICP-AES \\
\hline $7440-38-2$ & Arsenic & $<0.362$ & ug/g dry & 0.362 & $5 / 07 / 08$ & 8D28005 & PNNL-AGG-ICP-AES \\
\hline $7440-42-8$ & Boron & $<1.95$ & ug/g dry & 1.95 & $5 / 07 / 08$ & 8D28005 & PNNL-AGG-ICP-AES \\
\hline $7440-39-3$ & Barium & 0.019 & ug/g dry & 0.009 & $5 / 07 / 08$ & 8D28005 & PNNL-AGG-ICP-AES \\
\hline $7440-41-7$ & Beryllium & $<0.029$ & ug/g dry & 0.029 & $5 / 07 / 08$ & 8D28005 & PNNL-AGG-ICP-AES \\
\hline 7440-69-9 & Bismuth & $<0.189$ & ug/g dry & 0.189 & $5 / 07 / 08$ & 8D28005 & PNNL-AGG-ICP-AES \\
\hline $7440-70-2$ & Calcium & 6.99 & ug/g dry & 0.390 & $5 / 07 / 08$ & 8D28005 & PNNL-AGG-ICP-AES \\
\hline $7440-43-9$ & Cadmium & $<0.027$ & ug/g dry & 0.027 & $5 / 07 / 08$ & 8D28005 & PNNL-AGG-ICP-AES \\
\hline $7440-48-4$ & Cobalt & $<0.097$ & ug/g dry & 0.097 & $5 / 07 / 08$ & 8D28005 & PNNL-AGG-ICP-AES \\
\hline $7440-47-3$ & Chromium & $<0.034$ & ug/g dry & 0.034 & $5 / 07 / 08$ & 8D28005 & PNNL-AGG-ICP-AES \\
\hline $7440-50-8$ & Copper & $<0.081$ & ug/g dry & 0.081 & $5 / 07 / 08$ & 8D28005 & PNNL-AGG-ICP-AES \\
\hline 7439-89-6 & Iron & 0.721 & ug/g dry & 0.143 & $5 / 07 / 08$ & 8D28005 & PNNL-AGG-ICP-AES \\
\hline 7440-09-7 & Potassium & 2.73 & ug/g dry & 2.34 & $5 / 07 / 08$ & 8D28005 & PNNL-AGG-ICP-AES \\
\hline $7439-93-2$ & Lithium & $<0.544$ & ug/g dry & 0.544 & $5 / 07 / 08$ & 8D28005 & PNNL-AGG-ICP-AES \\
\hline 7439-95-4 & Magnesium & 1.44 & ug/g dry & 0.084 & $5 / 07 / 08$ & 8D28005 & PNNL-AGG-ICP-AES \\
\hline $7439-96-5$ & Manganese & $<0.017$ & ug/g dry & 0.017 & $5 / 07 / 08$ & 8D28005 & PNNL-AGG-ICP-AES \\
\hline 7439-98-7 & Molybdenum & $<0.140$ & ug/g dry & 0.140 & $5 / 07 / 08$ & 8D28005 & PNNL-AGG-ICP-AES \\
\hline $7440-02-0$ & Nickel & $<0.094$ & ug/g dry & 0.094 & $5 / 07 / 08$ & 8D28005 & PNNL-AGG-ICP-AES \\
\hline $7723-14-0$ & Phosphorus & $<1.04$ & ug/g dry & 1.04 & $5 / 07 / 08$ & 8D28005 & PNNL-AGG-ICP-AES \\
\hline 7439-92-1 & Lead & $<0.435$ & ug/g dry & 0.435 & $5 / 07 / 08$ & 8D28005 & PNNL-AGG-ICP-AES \\
\hline $7782-49-2$ & Selenium & $<1.96$ & ug/g dry & 1.96 & $5 / 07 / 08$ & 8D28005 & PNNL-AGG-ICP-AES \\
\hline $7440-24-6$ & Strontium & $<0.053$ & ug/g dry & 0.053 & $5 / 07 / 08$ & 8D28005 & PNNL-AGG-ICP-AES \\
\hline $7440-28-0$ & Thallium & $<1.03$ & ug/g dry & 1.03 & $5 / 07 / 08$ & 8D28005 & PNNL-AGG-ICP-AES \\
\hline $7440-62-2$ & Vanadium & $<0.046$ & ug/g dry & 0.046 & $5 / 07 / 08$ & 8D28005 & PNNL-AGG-ICP-AES \\
\hline $7440-66-6$ & Zinc & $<0.093$ & ug/g dry & 0.093 & $5 / 07 / 08$ & 8D28005 & PNNL-AGG-ICP-AES \\
\hline $7440-23-5$ & Sodium & 9.44 & ug/g dry & 0.674 & $5 / 07 / 08$ & 8D28005 & PNNL-AGG-ICP-AES \\
\hline $7440-21-3$ & Silicon & 8.87 & ug/g dry & 1.51 & $5 / 07 / 08$ & 8D28005 & PNNL-AGG-ICP-AES \\
\hline 7704-34-9 & Sulfur & 5.21 & ug/g dry & 3.10 & $5 / 07 / 08$ & 8D28005 & PNNL-AGG-ICP-AES \\
\hline
\end{tabular}


Total Metals by SW846 6010B/Water Extract

\begin{tabular}{|c|c|c|c|c|c|c|c|}
\hline CAS \# & Analyte & Results & Units & EQL & Analyzed & Batch & Method \\
\hline HEIS No. & B1RTH1A & \multicolumn{3}{|c|}{ Lab ID: $\quad$ 0803012-02 } & & & \\
\hline $7440-32-6$ & Titanium & 0.026 & ug/g dry & 0.009 & $5 / 07 / 08$ & 8D28005 & PNNL-AGG-ICP-AES \\
\hline $7440-67-7$ & Zirconium & $<0.151$ & ug/g dry & 0.151 & $5 / 07 / 08$ & 8D28005 & PNNL-AGG-ICP-AES \\
\hline $7440-22-4$ & Silver & $<0.078$ & ug/g dry & 0.078 & $5 / 07 / 08$ & 8D28005 & PNNL-AGG-ICP-AES \\
\hline $7440-15-5$ & Rhenium & $<0.153$ & ug/g dry & 0.153 & $5 / 07 / 08$ & 8D28005 & PNNL-AGG-ICP-AES \\
\hline $7440-36-0$ & Antimony & $<0.637$ & ug/g dry & 0.637 & $5 / 07 / 08$ & 8D28005 & PNNL-AGG-ICP-AES \\
\hline HEIS No. & B1RTH1 & \multicolumn{3}{|c|}{ Lab ID: $\quad$ 0803012-03 } & & & \\
\hline $7429-90-5$ & Aluminum & 0.387 & ug/g dry & 0.086 & $5 / 07 / 08$ & 8D28005 & PNNL-AGG-ICP-AES \\
\hline $7440-38-2$ & Arsenic & $<0.362$ & ug/g dry & 0.362 & $5 / 07 / 08$ & 8D28005 & PNNL-AGG-ICP-AES \\
\hline $7440-42-8$ & Boron & $<1.95$ & ug/g dry & 1.95 & $5 / 07 / 08$ & 8D28005 & PNNL-AGG-ICP-AES \\
\hline $7440-39-3$ & Barium & $<0.009$ & ug/g dry & 0.009 & $5 / 07 / 08$ & 8D28005 & PNNL-AGG-ICP-AES \\
\hline $7440-41-7$ & Beryllium & $<0.029$ & ug/g dry & 0.029 & $5 / 07 / 08$ & 8D28005 & PNNL-AGG-ICP-AES \\
\hline $7440-69-9$ & Bismuth & $<0.189$ & ug/g dry & 0.189 & $5 / 07 / 08$ & 8D28005 & PNNL-AGG-ICP-AES \\
\hline $7440-70-2$ & Calcium & 3.58 & ug/g dry & 0.390 & $5 / 07 / 08$ & 8D28005 & PNNL-AGG-ICP-AES \\
\hline $7440-43-9$ & Cadmium & $<0.027$ & ug/g dry & 0.027 & $5 / 07 / 08$ & 8D28005 & PNNL-AGG-ICP-AES \\
\hline $7440-48-4$ & Cobalt & $<0.097$ & ug/g dry & 0.097 & $5 / 07 / 08$ & 8D28005 & PNNL-AGG-ICP-AES \\
\hline $7440-47-3$ & Chromium & $<0.034$ & ug/g dry & 0.034 & $5 / 07 / 08$ & 8D28005 & PNNL-AGG-ICP-AES \\
\hline $7440-50-8$ & Copper & $<0.081$ & ug/g dry & 0.081 & $5 / 07 / 08$ & 8D28005 & PNNL-AGG-ICP-AES \\
\hline 7439-89-6 & Iron & 0.666 & ug/g dry & 0.143 & $5 / 07 / 08$ & 8D28005 & PNNL-AGG-ICP-AES \\
\hline 7440-09-7 & Potassium & 2.69 & ug/g dry & 2.34 & $5 / 07 / 08$ & 8D28005 & PNNL-AGG-ICP-AES \\
\hline $7439-93-2$ & Lithium & $<0.544$ & ug/g dry & 0.544 & $5 / 07 / 08$ & 8D28005 & PNNL-AGG-ICP-AES \\
\hline $7439-95-4$ & Magnesium & 0.744 & ug/g dry & 0.084 & $5 / 07 / 08$ & 8D28005 & PNNL-AGG-ICP-AES \\
\hline $7439-96-5$ & Manganese & $<0.017$ & ug/g dry & 0.017 & $5 / 07 / 08$ & 8D28005 & PNNL-AGG-ICP-AES \\
\hline 7439-98-7 & Molybdenum & $<0.140$ & ug/g dry & 0.140 & $5 / 07 / 08$ & 8D28005 & PNNL-AGG-ICP-AES \\
\hline $7440-02-0$ & Nickel & $<0.094$ & ug/g dry & 0.094 & $5 / 07 / 08$ & 8D28005 & PNNL-AGG-ICP-AES \\
\hline $7723-14-0$ & Phosphorus & $<1.04$ & ug/g dry & 1.04 & $5 / 07 / 08$ & 8D28005 & PNNL-AGG-ICP-AES \\
\hline 7439-92-1 & Lead & $<0.435$ & ug/g dry & 0.435 & $5 / 07 / 08$ & 8D28005 & PNNL-AGG-ICP-AES \\
\hline $7782-49-2$ & Selenium & $<1.96$ & ug/g dry & 1.96 & $5 / 07 / 08$ & 8D28005 & PNNL-AGG-ICP-AES \\
\hline $7440-24-6$ & Strontium & $<0.053$ & ug/g dry & 0.053 & $5 / 07 / 08$ & 8D28005 & PNNL-AGG-ICP-AES \\
\hline $7440-28-0$ & Thallium & $<1.03$ & ug/g dry & 1.03 & $5 / 07 / 08$ & 8D28005 & PNNL-AGG-ICP-AES \\
\hline $7440-62-2$ & Vanadium & 0.070 & ug/g dry & 0.046 & $5 / 07 / 08$ & 8D28005 & PNNL-AGG-ICP-AES \\
\hline $7440-66-6$ & Zinc & $<0.093$ & ug/g dry & 0.093 & $5 / 07 / 08$ & 8D28005 & PNNL-AGG-ICP-AES \\
\hline $7440-23-5$ & Sodium & 44.2 & ug/g dry & 0.674 & $5 / 07 / 08$ & 8D28005 & PNNL-AGG-ICP-AES \\
\hline $7440-21-3$ & Silicon & 15.6 & ug/g dry & 1.51 & $5 / 07 / 08$ & 8D28005 & PNNL-AGG-ICP-AES \\
\hline $7704-34-9$ & Sulfur & 12.8 & ug/g dry & 3.10 & $5 / 07 / 08$ & 8D28005 & PNNL-AGG-ICP-AES \\
\hline $7440-32-6$ & Titanium & 0.034 & ug/g dry & 0.009 & $5 / 07 / 08$ & 8D28005 & PNNL-AGG-ICP-AES \\
\hline $7440-67-7$ & Zirconium & $<0.151$ & ug/g dry & 0.151 & $5 / 07 / 08$ & 8D28005 & PNNL-AGG-ICP-AES \\
\hline $7440-22-4$ & Silver & $<0.078$ & ug/g dry & 0.078 & $5 / 07 / 08$ & 8D28005 & PNNL-AGG-ICP-AES \\
\hline $7440-15-5$ & Rhenium & $<0.153$ & ug/g dry & 0.153 & $5 / 07 / 08$ & 8D28005 & PNNL-AGG-ICP-AES \\
\hline $7440-36-0$ & Antimony & $<0.637$ & ug/g dry & 0.637 & $5 / 07 / 08$ & 8D28005 & PNNL-AGG-ICP-AES \\
\hline HEIS No. & B1RTH2B & & b ID: & 3012-05 & & & \\
\hline $7429-90-5$ & Aluminum & 0.094 & ug/g dry & 0.086 & $5 / 07 / 08$ & 8D28005 & PNNL-AGG-ICP-AES \\
\hline $7440-38-2$ & Arsenic & $<0.360$ & ug/g dry & 0.360 & $5 / 07 / 08$ & 8D28005 & PNNL-AGG-ICP-AES \\
\hline $7440-42-8$ & Boron & $<1.94$ & ug/g dry & 1.94 & $5 / 07 / 08$ & 8D28005 & PNNL-AGG-ICP-AES \\
\hline $7440-39-3$ & Barium & 0.024 & ug/g dry & 0.009 & $5 / 07 / 08$ & 8D28005 & PNNL-AGG-ICP-AES \\
\hline $7440-41-7$ & Beryllium & $<0.028$ & ug/g dry & 0.028 & $5 / 07 / 08$ & 8D28005 & PNNL-AGG-ICP-AES \\
\hline $7440-69-9$ & Bismuth & $<0.188$ & ug/g dry & 0.188 & $5 / 07 / 08$ & 8D28005 & PNNL-AGG-ICP-AES \\
\hline $7440-70-2$ & Calcium & 22.9 & ug/g dry & 0.387 & $5 / 07 / 08$ & 8D28005 & PNNL-AGG-ICP-AES \\
\hline $7440-43-9$ & Cadmium & $<0.027$ & ug/g dry & 0.027 & $5 / 07 / 08$ & 8D28005 & PNNL-AGG-ICP-AES \\
\hline
\end{tabular}


Total Metals by SW846 6010B/Water Extract

\begin{tabular}{|c|c|c|c|c|c|c|c|}
\hline CAS \# & Analyte & Results & Units & EQL & Analyzed & Batch & Method \\
\hline HEIS No. & B1RTH2B & \multicolumn{3}{|c|}{ Lab ID: $\quad$ 0803012-05 } & & & \\
\hline $7440-48-4$ & Cobalt & $<0.096$ & ug/g dry & 0.096 & $5 / 07 / 08$ & 8D28005 & PNNL-AGG-ICP-AES \\
\hline $7440-47-3$ & Chromium & $<0.033$ & ug/g dry & 0.033 & $5 / 07 / 08$ & 8D28005 & PNNL-AGG-ICP-AES \\
\hline $7440-50-8$ & Copper & $<0.080$ & ug/g dry & 0.080 & $5 / 07 / 08$ & 8D28005 & PNNL-AGG-ICP-AES \\
\hline 7439-89-6 & Iron & $<0.142$ & ug/g dry & 0.142 & $5 / 07 / 08$ & 8D28005 & PNNL-AGG-ICP-AES \\
\hline 7440-09-7 & Potassium & 7.25 & ug/g dry & 2.33 & $5 / 07 / 08$ & 8D28005 & PNNL-AGG-ICP-AES \\
\hline 7439-93-2 & Lithium & $<0.540$ & ug/g dry & 0.540 & $5 / 07 / 08$ & 8D28005 & PNNL-AGG-ICP-AES \\
\hline 7439-95-4 & Magnesium & 8.50 & ug/g dry & 0.083 & $5 / 07 / 08$ & 8D28005 & PNNL-AGG-ICP-AES \\
\hline $7439-96-5$ & Manganese & $<0.017$ & ug/g dry & 0.017 & $5 / 07 / 08$ & 8D28005 & PNNL-AGG-ICP-AES \\
\hline 7439-98-7 & Molybdenum & $<0.139$ & ug/g dry & 0.139 & $5 / 07 / 08$ & 8D28005 & PNNL-AGG-ICP-AES \\
\hline $7440-02-0$ & Nickel & $<0.093$ & ug/g dry & 0.093 & $5 / 07 / 08$ & 8D28005 & PNNL-AGG-ICP-AES \\
\hline 7723-14-0 & Phosphorus & $<1.03$ & ug/g dry & 1.03 & $5 / 07 / 08$ & 8D28005 & PNNL-AGG-ICP-AES \\
\hline 7439-92-1 & Lead & $<0.432$ & ug/g dry & 0.432 & $5 / 07 / 08$ & 8D28005 & PNNL-AGG-ICP-AES \\
\hline $7782-49-2$ & Selenium & $<1.95$ & ug/g dry & 1.95 & $5 / 07 / 08$ & 8D28005 & PNNL-AGG-ICP-AES \\
\hline $7440-24-6$ & Strontium & 0.095 & ug/g dry & 0.052 & $5 / 07 / 08$ & 8D28005 & PNNL-AGG-ICP-AES \\
\hline $7440-28-0$ & Thallium & $<1.03$ & ug/g dry & 1.03 & $5 / 07 / 08$ & 8D28005 & PNNL-AGG-ICP-AES \\
\hline $7440-62-2$ & Vanadium & $<0.045$ & ug/g dry & 0.045 & $5 / 07 / 08$ & 8D28005 & PNNL-AGG-ICP-AES \\
\hline $7440-66-6$ & Zinc & 0.825 & ug/g dry & 0.093 & $5 / 07 / 08$ & 8D28005 & PNNL-AGG-ICP-AES \\
\hline $7440-23-5$ & Sodium & 19.0 & ug/g dry & 0.669 & $5 / 07 / 08$ & 8D28005 & PNNL-AGG-ICP-AES \\
\hline $7440-21-3$ & Silicon & 5.75 & ug/g dry & 1.50 & $5 / 07 / 08$ & 8D28005 & PNNL-AGG-ICP-AES \\
\hline 7704-34-9 & Sulfur & 35.1 & ug/g dry & 3.08 & $5 / 07 / 08$ & 8D28005 & PNNL-AGG-ICP-AES \\
\hline $7440-32-6$ & Titanium & $<0.009$ & ug/g dry & 0.009 & $5 / 07 / 08$ & 8D28005 & PNNL-AGG-ICP-AES \\
\hline $7440-67-7$ & Zirconium & $<0.150$ & ug/g dry & 0.150 & $5 / 07 / 08$ & 8D28005 & PNNL-AGG-ICP-AES \\
\hline $7440-22-4$ & Silver & $<0.077$ & ug/g dry & 0.077 & $5 / 07 / 08$ & 8D28005 & PNNL-AGG-ICP-AES \\
\hline $7440-15-5$ & Rhenium & $<0.152$ & ug/g dry & 0.152 & $5 / 07 / 08$ & 8D28005 & PNNL-AGG-ICP-AES \\
\hline 7440-36-0 & Antimony & $<0.633$ & ug/g dry & 0.633 & $5 / 07 / 08$ & 8D28005 & PNNL-AGG-ICP-AES \\
\hline HEIS No. & B1RTH2A & \multicolumn{3}{|c|}{ Lab ID: $\quad$ 0803012-06 } & & & \\
\hline $7429-90-5$ & Aluminum & 0.132 & ug/g dry & 0.086 & $5 / 07 / 08$ & 8D28005 & PNNL-AGG-ICP-AES \\
\hline $7440-38-2$ & Arsenic & $<0.360$ & ug/g dry & 0.360 & $5 / 07 / 08$ & 8D28005 & PNNL-AGG-ICP-AES \\
\hline $7440-42-8$ & Boron & $<1.94$ & ug/g dry & 1.94 & $5 / 07 / 08$ & 8D28005 & PNNL-AGG-ICP-AES \\
\hline $7440-39-3$ & Barium & 0.040 & ug/g dry & 0.009 & $5 / 07 / 08$ & 8D28005 & PNNL-AGG-ICP-AES \\
\hline $7440-41-7$ & Beryllium & $<0.028$ & ug/g dry & 0.028 & $5 / 07 / 08$ & 8D28005 & PNNL-AGG-ICP-AES \\
\hline $7440-69-9$ & Bismuth & $<0.188$ & ug/g dry & 0.188 & $5 / 07 / 08$ & 8D28005 & PNNL-AGG-ICP-AES \\
\hline $7440-70-2$ & Calcium & 16.6 & ug/g dry & 0.387 & $5 / 07 / 08$ & 8D28005 & PNNL-AGG-ICP-AES \\
\hline $7440-43-9$ & Cadmium & $<0.027$ & ug/g dry & 0.027 & $5 / 07 / 08$ & 8D28005 & PNNL-AGG-ICP-AES \\
\hline $7440-48-4$ & Cobalt & $<0.096$ & ug/g dry & 0.096 & $5 / 07 / 08$ & 8D28005 & PNNL-AGG-ICP-AES \\
\hline $7440-47-3$ & Chromium & $<0.033$ & ug/g dry & 0.033 & $5 / 07 / 08$ & 8D28005 & PNNL-AGG-ICP-AES \\
\hline $7440-50-8$ & Copper & $<0.080$ & ug/g dry & 0.080 & $5 / 07 / 08$ & 8D28005 & PNNL-AGG-ICP-AES \\
\hline 7439-89-6 & Iron & $<0.142$ & ug/g dry & 0.142 & $5 / 07 / 08$ & 8D28005 & PNNL-AGG-ICP-AES \\
\hline 7440-09-7 & Potassium & 5.95 & ug/g dry & 2.33 & $5 / 07 / 08$ & 8D28005 & PNNL-AGG-ICP-AES \\
\hline $7439-93-2$ & Lithium & $<0.540$ & ug/g dry & 0.540 & $5 / 07 / 08$ & 8D28005 & PNNL-AGG-ICP-AES \\
\hline 7439-95-4 & Magnesium & 6.31 & ug/g dry & 0.083 & $5 / 07 / 08$ & 8D28005 & PNNL-AGG-ICP-AES \\
\hline 7439-96-5 & Manganese & $<0.017$ & ug/g dry & 0.017 & $5 / 07 / 08$ & 8D28005 & PNNL-AGG-ICP-AES \\
\hline 7439-98-7 & Molybdenum & $<0.139$ & ug/g dry & 0.139 & $5 / 07 / 08$ & 8D28005 & PNNL-AGG-ICP-AES \\
\hline $7440-02-0$ & Nickel & $<0.093$ & ug/g dry & 0.093 & $5 / 07 / 08$ & 8D28005 & PNNL-AGG-ICP-AES \\
\hline 7723-14-0 & Phosphorus & $<1.03$ & ug/g dry & 1.03 & $5 / 07 / 08$ & 8D28005 & PNNL-AGG-ICP-AES \\
\hline $7439-92-1$ & Lead & $<0.432$ & ug/g dry & 0.432 & $5 / 07 / 08$ & 8D28005 & PNNL-AGG-ICP-AES \\
\hline $7782-49-2$ & Selenium & $<1.95$ & ug/g dry & 1.95 & $5 / 07 / 08$ & 8D28005 & PNNL-AGG-ICP-AES \\
\hline $7440-24-6$ & Strontium & 0.076 & ug/g dry & 0.052 & $5 / 07 / 08$ & 8D28005 & PNNL-AGG-ICP-AES \\
\hline
\end{tabular}


Total Metals by SW846 6010B/Water Extract

\begin{tabular}{|c|c|c|c|c|c|c|c|}
\hline CAS \# & Analyte & Results & Units & EQL & Analyzed & Batch & Method \\
\hline HEIS No. & B1RTH2A & \multicolumn{3}{|c|}{ Lab ID: $\quad$ 0803012-06 } & & & \\
\hline $7440-28-0$ & Thallium & $<1.03$ & ug/g dry & 1.03 & $5 / 07 / 08$ & 8D28005 & PNNL-AGG-ICP-AES \\
\hline $7440-62-2$ & Vanadium & $<0.045$ & ug/g dry & 0.045 & $5 / 07 / 08$ & 8D28005 & PNNL-AGG-ICP-AES \\
\hline $7440-66-6$ & Zinc & $<0.093$ & ug/g dry & 0.093 & $5 / 07 / 08$ & 8D28005 & PNNL-AGG-ICP-AES \\
\hline $7440-23-5$ & Sodium & 15.0 & ug/g dry & 0.669 & $5 / 07 / 08$ & 8D28005 & PNNL-AGG-ICP-AES \\
\hline $7440-21-3$ & Silicon & 7.19 & ug/g dry & 1.50 & $5 / 07 / 08$ & 8D28005 & PNNL-AGG-ICP-AES \\
\hline 7704-34-9 & Sulfur & 24.4 & ug/g dry & 3.08 & $5 / 07 / 08$ & 8D28005 & PNNL-AGG-ICP-AES \\
\hline $7440-32-6$ & Titanium & $<0.009$ & ug/g dry & 0.009 & $5 / 07 / 08$ & 8D28005 & PNNL-AGG-ICP-AES \\
\hline $7440-67-7$ & Zirconium & $<0.150$ & ug/g dry & 0.150 & $5 / 07 / 08$ & 8D28005 & PNNL-AGG-ICP-AES \\
\hline $7440-22-4$ & Silver & $<0.077$ & ug/g dry & 0.077 & $5 / 07 / 08$ & 8D28005 & PNNL-AGG-ICP-AES \\
\hline $7440-15-5$ & Rhenium & $<0.152$ & ug/g dry & 0.152 & $5 / 07 / 08$ & 8D28005 & PNNL-AGG-ICP-AES \\
\hline $7440-36-0$ & Antimony & $<0.633$ & ug/g dry & 0.633 & $5 / 07 / 08$ & 8D28005 & PNNL-AGG-ICP-AES \\
\hline HEIS No. & B1RTH3B & \multicolumn{3}{|c|}{ Lab ID: $\quad$ 0803012-09 } & & & \\
\hline $7429-90-5$ & Aluminum & 0.135 & ug/g dry & 0.086 & $5 / 07 / 08$ & 8D28005 & PNNL-AGG-ICP-AES \\
\hline $7440-38-2$ & Arsenic & $<0.360$ & ug/g dry & 0.360 & $5 / 07 / 08$ & 8D28005 & PNNL-AGG-ICP-AES \\
\hline $7440-42-8$ & Boron & $<1.94$ & ug/g dry & 1.94 & $5 / 07 / 08$ & 8D28005 & PNNL-AGG-ICP-AES \\
\hline $7440-39-3$ & Barium & 0.057 & ug/g dry & 0.009 & $5 / 07 / 08$ & 8D28005 & PNNL-AGG-ICP-AES \\
\hline $7440-41-7$ & Beryllium & $<0.028$ & ug/g dry & 0.028 & $5 / 07 / 08$ & 8D28005 & PNNL-AGG-ICP-AES \\
\hline 7440-69-9 & Bismuth & $<0.188$ & ug/g dry & 0.188 & $5 / 07 / 08$ & 8D28005 & PNNL-AGG-ICP-AES \\
\hline $7440-70-2$ & Calcium & 18.1 & ug/g dry & 0.387 & $5 / 07 / 08$ & 8D28005 & PNNL-AGG-ICP-AES \\
\hline $7440-43-9$ & Cadmium & $<0.027$ & ug/g dry & 0.027 & $5 / 07 / 08$ & 8D28005 & PNNL-AGG-ICP-AES \\
\hline $7440-48-4$ & Cobalt & $<0.096$ & ug/g dry & 0.096 & $5 / 07 / 08$ & 8D28005 & PNNL-AGG-ICP-AES \\
\hline $7440-47-3$ & Chromium & $<0.033$ & ug/g dry & 0.033 & $5 / 07 / 08$ & 8D28005 & PNNL-AGG-ICP-AES \\
\hline $7440-50-8$ & Copper & $<0.080$ & ug/g dry & 0.080 & $5 / 07 / 08$ & 8D28005 & PNNL-AGG-ICP-AES \\
\hline 7439-89-6 & Iron & $<0.142$ & ug/g dry & 0.142 & $5 / 07 / 08$ & 8D28005 & PNNL-AGG-ICP-AES \\
\hline $7440-09-7$ & Potassium & 6.60 & ug/g dry & 2.33 & $5 / 07 / 08$ & 8D28005 & PNNL-AGG-ICP-AES \\
\hline 7439-93-2 & Lithium & $<0.540$ & ug/g dry & 0.540 & $5 / 07 / 08$ & 8D28005 & PNNL-AGG-ICP-AES \\
\hline $7439-95-4$ & Magnesium & 7.20 & ug/g dry & 0.083 & $5 / 07 / 08$ & 8D28005 & PNNL-AGG-ICP-AES \\
\hline 7439-96-5 & Manganese & $<0.017$ & ug/g dry & 0.017 & $5 / 07 / 08$ & 8D28005 & PNNL-AGG-ICP-AES \\
\hline 7439-98-7 & Molybdenum & $<0.139$ & ug/g dry & 0.139 & $5 / 07 / 08$ & 8D28005 & PNNL-AGG-ICP-AES \\
\hline $7440-02-0$ & Nickel & $<0.093$ & ug/g dry & 0.093 & $5 / 07 / 08$ & 8D28005 & PNNL-AGG-ICP-AES \\
\hline 7723-14-0 & Phosphorus & $<1.03$ & ug/g dry & 1.03 & $5 / 07 / 08$ & 8D28005 & PNNL-AGG-ICP-AES \\
\hline $7439-92-1$ & Lead & $<0.432$ & ug/g dry & 0.432 & $5 / 07 / 08$ & 8D28005 & PNNL-AGG-ICP-AES \\
\hline $7782-49-2$ & Selenium & $<1.95$ & ug/g dry & 1.95 & $5 / 07 / 08$ & 8D28005 & PNNL-AGG-ICP-AES \\
\hline $7440-24-6$ & Strontium & 0.098 & ug/g dry & 0.052 & $5 / 07 / 08$ & 8D28005 & PNNL-AGG-ICP-AES \\
\hline $7440-28-0$ & Thallium & $<1.03$ & ug/g dry & 1.03 & $5 / 07 / 08$ & 8D28005 & PNNL-AGG-ICP-AES \\
\hline $7440-62-2$ & Vanadium & $<0.045$ & ug/g dry & 0.045 & $5 / 07 / 08$ & 8D28005 & PNNL-AGG-ICP-AES \\
\hline $7440-66-6$ & Zinc & 0.255 & ug/g dry & 0.093 & $5 / 07 / 08$ & 8D28005 & PNNL-AGG-ICP-AES \\
\hline $7440-23-5$ & Sodium & 12.6 & ug/g dry & 0.669 & $5 / 07 / 08$ & 8D28005 & PNNL-AGG-ICP-AES \\
\hline $7440-21-3$ & Silicon & 6.04 & ug/g dry & 1.50 & $5 / 07 / 08$ & 8D28005 & PNNL-AGG-ICP-AES \\
\hline 7704-34-9 & Sulfur & 15.5 & ug/g dry & 3.07 & $5 / 07 / 08$ & 8D28005 & PNNL-AGG-ICP-AES \\
\hline $7440-32-6$ & Titanium & $<0.009$ & ug/g dry & 0.009 & $5 / 07 / 08$ & 8D28005 & PNNL-AGG-ICP-AES \\
\hline $7440-67-7$ & Zirconium & $<0.150$ & ug/g dry & 0.150 & $5 / 07 / 08$ & 8D28005 & PNNL-AGG-ICP-AES \\
\hline $7440-22-4$ & Silver & $<0.077$ & ug/g dry & 0.077 & $5 / 07 / 08$ & 8D28005 & PNNL-AGG-ICP-AES \\
\hline $7440-15-5$ & Rhenium & $<0.152$ & ug/g dry & 0.152 & $5 / 07 / 08$ & 8D28005 & PNNL-AGG-ICP-AES \\
\hline 7440-36-0 & Antimony & $<0.633$ & ug/g dry & 0.633 & $5 / 07 / 08$ & 8D28005 & PNNL-AGG-ICP-AES \\
\hline HEIS No. & B1RTH3A & & b ID: & $3012-10$ & & & \\
\hline $7429-90-5$ & Aluminum & 0.112 & ug/g dry & 0.086 & $5 / 07 / 08$ & 8D28005 & PNNL-AGG-ICP-AES \\
\hline $7440-38-2$ & Arsenic & $<0.360$ & ug/g dry & 0.360 & $5 / 07 / 08$ & 8D28005 & PNNL-AGG-ICP-AES \\
\hline
\end{tabular}


Total Metals by SW846 6010B/Water Extract

\begin{tabular}{|c|c|c|c|c|c|c|c|}
\hline CAS \# & Analyte & Results & Units & EQL & Analyzed & Batch & Method \\
\hline HEIS No. & B1RTH3A & \multicolumn{3}{|c|}{ Lab ID: $\quad$ 0803012-10 } & & & \\
\hline $7440-42-8$ & Boron & $<1.94$ & ug/g dry & 1.94 & $5 / 07 / 08$ & 8D28005 & PNNL-AGG-ICP-AES \\
\hline $7440-39-3$ & Barium & 0.017 & ug/g dry & 0.009 & $5 / 07 / 08$ & 8D28005 & PNNL-AGG-ICP-AES \\
\hline $7440-41-7$ & Beryllium & $<0.028$ & ug/g dry & 0.028 & $5 / 07 / 08$ & 8D28005 & PNNL-AGG-ICP-AES \\
\hline 7440-69-9 & Bismuth & $<0.188$ & ug/g dry & 0.188 & $5 / 07 / 08$ & 8D28005 & PNNL-AGG-ICP-AES \\
\hline $7440-70-2$ & Calcium & 20.5 & ug/g dry & 0.387 & $5 / 07 / 08$ & 8D28005 & PNNL-AGG-ICP-AES \\
\hline $7440-43-9$ & Cadmium & $<0.027$ & ug/g dry & 0.027 & $5 / 07 / 08$ & 8D28005 & PNNL-AGG-ICP-AES \\
\hline $7440-48-4$ & Cobalt & $<0.096$ & ug/g dry & 0.096 & $5 / 07 / 08$ & 8D28005 & PNNL-AGG-ICP-AES \\
\hline $7440-47-3$ & Chromium & $<0.033$ & ug/g dry & 0.033 & $5 / 07 / 08$ & 8D28005 & PNNL-AGG-ICP-AES \\
\hline $7440-50-8$ & Copper & $<0.080$ & ug/g dry & 0.080 & $5 / 07 / 08$ & 8D28005 & PNNL-AGG-ICP-AES \\
\hline 7439-89-6 & Iron & $<0.142$ & ug/g dry & 0.142 & $5 / 07 / 08$ & 8D28005 & PNNL-AGG-ICP-AES \\
\hline 7440-09-7 & Potassium & 6.76 & ug/g dry & 2.33 & $5 / 07 / 08$ & 8D28005 & PNNL-AGG-ICP-AES \\
\hline $7439-93-2$ & Lithium & $<0.540$ & ug/g dry & 0.540 & $5 / 07 / 08$ & 8D28005 & PNNL-AGG-ICP-AES \\
\hline 7439-95-4 & Magnesium & 8.09 & ug/g dry & 0.083 & $5 / 07 / 08$ & 8D28005 & PNNL-AGG-ICP-AES \\
\hline 7439-96-5 & Manganese & $<0.017$ & ug/g dry & 0.017 & $5 / 07 / 08$ & 8D28005 & PNNL-AGG-ICP-AES \\
\hline 7439-98-7 & Molybdenum & $<0.139$ & ug/g dry & 0.139 & $5 / 07 / 08$ & 8D28005 & PNNL-AGG-ICP-AES \\
\hline $7440-02-0$ & Nickel & $<0.093$ & ug/g dry & 0.093 & $5 / 07 / 08$ & 8D28005 & PNNL-AGG-ICP-AES \\
\hline $7723-14-0$ & Phosphorus & $<1.03$ & ug/g dry & 1.03 & $5 / 07 / 08$ & 8D28005 & PNNL-AGG-ICP-AES \\
\hline 7439-92-1 & Lead & $<0.432$ & ug/g dry & 0.432 & $5 / 07 / 08$ & 8D28005 & PNNL-AGG-ICP-AES \\
\hline $7782-49-2$ & Selenium & $<1.95$ & ug/g dry & 1.95 & $5 / 07 / 08$ & 8D28005 & PNNL-AGG-ICP-AES \\
\hline $7440-24-6$ & Strontium & 0.103 & ug/g dry & 0.052 & $5 / 07 / 08$ & 8D28005 & PNNL-AGG-ICP-AES \\
\hline $7440-28-0$ & Thallium & $<1.03$ & ug/g dry & 1.03 & $5 / 07 / 08$ & 8D28005 & PNNL-AGG-ICP-AES \\
\hline $7440-62-2$ & Vanadium & $<0.045$ & ug/g dry & 0.045 & $5 / 07 / 08$ & 8D28005 & PNNL-AGG-ICP-AES \\
\hline $7440-66-6$ & Zinc & $<0.093$ & ug/g dry & 0.093 & $5 / 07 / 08$ & 8D28005 & PNNL-AGG-ICP-AES \\
\hline $7440-23-5$ & Sodium & 13.1 & ug/g dry & 0.669 & $5 / 07 / 08$ & $8 \mathrm{D} 28005$ & PNNL-AGG-ICP-AES \\
\hline $7440-21-3$ & Silicon & 6.63 & ug/g dry & 1.50 & $5 / 07 / 08$ & 8D28005 & PNNL-AGG-ICP-AES \\
\hline $7704-34-9$ & Sulfur & 18.7 & ug/g dry & 3.07 & $5 / 07 / 08$ & 8D28005 & PNNL-AGG-ICP-AES \\
\hline $7440-32-6$ & Titanium & $<0.009$ & ug/g dry & 0.009 & $5 / 07 / 08$ & 8D28005 & PNNL-AGG-ICP-AES \\
\hline $7440-67-7$ & Zirconium & $<0.150$ & ug/g dry & 0.150 & $5 / 07 / 08$ & 8D28005 & PNNL-AGG-ICP-AES \\
\hline $7440-22-4$ & Silver & $<0.077$ & ug/g dry & 0.077 & $5 / 07 / 08$ & 8D28005 & PNNL-AGG-ICP-AES \\
\hline $7440-15-5$ & Rhenium & $<0.152$ & ug/g dry & 0.152 & $5 / 07 / 08$ & 8D28005 & PNNL-AGG-ICP-AES \\
\hline $7440-36-0$ & Antimony & $<0.633$ & ug/g dry & 0.633 & $5 / 07 / 08$ & 8D28005 & PNNL-AGG-ICP-AES \\
\hline HEIS No. & B1RYR7B & \multicolumn{3}{|c|}{ Lab ID: $\quad$ 0803012-13 } & & & \\
\hline $7429-90-5$ & Aluminum & 0.103 & ug/g dry & 0.086 & $5 / 07 / 08$ & 8D28005 & PNNL-AGG-ICP-AES \\
\hline $7440-38-2$ & Arsenic & $<0.360$ & ug/g dry & 0.360 & $5 / 07 / 08$ & 8D28005 & PNNL-AGG-ICP-AES \\
\hline $7440-42-8$ & Boron & $<1.94$ & ug/g dry & 1.94 & $5 / 07 / 08$ & 8D28005 & PNNL-AGG-ICP-AES \\
\hline 7440-39-3 & Barium & $<0.009$ & ug/g dry & 0.009 & $5 / 07 / 08$ & 8D28005 & PNNL-AGG-ICP-AES \\
\hline $7440-41-7$ & Beryllium & $<0.028$ & ug/g dry & 0.028 & $5 / 07 / 08$ & 8D28005 & PNNL-AGG-ICP-AES \\
\hline $7440-69-9$ & Bismuth & $<0.188$ & ug/g dry & 0.188 & $5 / 07 / 08$ & 8D28005 & PNNL-AGG-ICP-AES \\
\hline $7440-70-2$ & Calcium & 21.9 & ug/g dry & 0.387 & $5 / 07 / 08$ & 8D28005 & PNNL-AGG-ICP-AES \\
\hline $7440-43-9$ & Cadmium & $<0.027$ & ug/g dry & 0.027 & $5 / 07 / 08$ & 8D28005 & PNNL-AGG-ICP-AES \\
\hline $7440-48-4$ & Cobalt & $<0.096$ & ug/g dry & 0.096 & $5 / 07 / 08$ & 8D28005 & PNNL-AGG-ICP-AES \\
\hline $7440-47-3$ & Chromium & $<0.033$ & ug/g dry & 0.033 & $5 / 07 / 08$ & 8D28005 & PNNL-AGG-ICP-AES \\
\hline $7440-50-8$ & Copper & $<0.080$ & ug/g dry & 0.080 & $5 / 07 / 08$ & 8D28005 & PNNL-AGG-ICP-AES \\
\hline 7439-89-6 & Iron & $<0.142$ & ug/g dry & 0.142 & $5 / 07 / 08$ & 8D28005 & PNNL-AGG-ICP-AES \\
\hline 7440-09-7 & Potassium & 6.84 & ug/g dry & 2.33 & $5 / 07 / 08$ & 8D28005 & PNNL-AGG-ICP-AES \\
\hline $7439-93-2$ & Lithium & $<0.540$ & ug/g dry & 0.540 & $5 / 07 / 08$ & 8D28005 & PNNL-AGG-ICP-AES \\
\hline 7439-95-4 & Magnesium & 7.59 & ug/g dry & 0.083 & $5 / 07 / 08$ & 8D28005 & PNNL-AGG-ICP-AES \\
\hline $7439-96-5$ & Manganese & $<0.017$ & ug/g dry & 0.017 & $5 / 07 / 08$ & 8D28005 & PNNL-AGG-ICP-AES \\
\hline
\end{tabular}


Total Metals by SW846 6010B/Water Extract

\begin{tabular}{|c|c|c|c|c|c|c|c|}
\hline CAS \# & Analyte & Results & Units & EQL & Analyzed & Batch & Method \\
\hline HEIS No. & B1RYR7B & \multicolumn{3}{|c|}{ Lab ID: $\quad$ 0803012-13 } & & & \\
\hline 7439-98-7 & Molybdenum & $<0.139$ & ug/g dry & 0.139 & $5 / 07 / 08$ & 8D28005 & PNNL-AGG-ICP-AES \\
\hline 7440-02-0 & Nickel & $<0.093$ & ug/g dry & 0.093 & $5 / 07 / 08$ & 8D28005 & PNNL-AGG-ICP-AES \\
\hline $7723-14-0$ & Phosphorus & $<1.03$ & ug/g dry & 1.03 & $5 / 07 / 08$ & 8D28005 & PNNL-AGG-ICP-AES \\
\hline 7439-92-1 & Lead & $<0.432$ & ug/g dry & 0.432 & $5 / 07 / 08$ & 8D28005 & PNNL-AGG-ICP-AES \\
\hline $7782-49-2$ & Selenium & $<1.95$ & ug/g dry & 1.95 & $5 / 07 / 08$ & 8D28005 & PNNL-AGG-ICP-AES \\
\hline $7440-24-6$ & Strontium & 0.102 & ug/g dry & 0.052 & $5 / 07 / 08$ & 8D28005 & PNNL-AGG-ICP-AES \\
\hline $7440-28-0$ & Thallium & $<1.03$ & ug/g dry & 1.03 & $5 / 07 / 08$ & 8D28005 & PNNL-AGG-ICP-AES \\
\hline $7440-62-2$ & Vanadium & $<0.045$ & ug/g dry & 0.045 & $5 / 07 / 08$ & 8D28005 & PNNL-AGG-ICP-AES \\
\hline $7440-66-6$ & Zinc & $<0.093$ & ug/g dry & 0.093 & $5 / 07 / 08$ & 8D28005 & PNNL-AGG-ICP-AES \\
\hline $7440-23-5$ & Sodium & 15.9 & ug/g dry & 0.669 & $5 / 07 / 08$ & 8D28005 & PNNL-AGG-ICP-AES \\
\hline $7440-21-3$ & Silicon & 6.44 & ug/g dry & 1.50 & $5 / 07 / 08$ & 8D28005 & PNNL-AGG-ICP-AES \\
\hline $7704-34-9$ & Sulfur & 25.0 & ug/g dry & 3.08 & $5 / 07 / 08$ & 8D28005 & PNNL-AGG-ICP-AES \\
\hline $7440-32-6$ & Titanium & $<0.009$ & ug/g dry & 0.009 & $5 / 07 / 08$ & 8D28005 & PNNL-AGG-ICP-AES \\
\hline $7440-67-7$ & Zirconium & $<0.150$ & ug/g dry & 0.150 & $5 / 07 / 08$ & 8D28005 & PNNL-AGG-ICP-AES \\
\hline $7440-22-4$ & Silver & $<0.077$ & ug/g dry & 0.077 & $5 / 07 / 08$ & 8D28005 & PNNL-AGG-ICP-AES \\
\hline $7440-15-5$ & Rhenium & $<0.152$ & ug/g dry & 0.152 & $5 / 07 / 08$ & 8D28005 & PNNL-AGG-ICP-AES \\
\hline $7440-36-0$ & Antimony & $<0.633$ & ug/g dry & 0.633 & $5 / 07 / 08$ & 8D28005 & PNNL-AGG-ICP-AES \\
\hline HEIS No. & B1RYR7A & \multicolumn{3}{|c|}{ Lab ID: $\quad$ 0803012-14 } & & & \\
\hline $7429-90-5$ & Aluminum & 0.167 & ug/g dry & 0.086 & $5 / 07 / 08$ & 8D28005 & PNNL-AGG-ICP-AES \\
\hline $7440-38-2$ & Arsenic & $<0.360$ & ug/g dry & 0.360 & $5 / 07 / 08$ & 8D28005 & PNNL-AGG-ICP-AES \\
\hline $7440-42-8$ & Boron & $<1.94$ & ug/g dry & 1.94 & $5 / 07 / 08$ & 8D28005 & PNNL-AGG-ICP-AES \\
\hline $7440-39-3$ & Barium & $<0.009$ & ug/g dry & 0.009 & $5 / 07 / 08$ & 8D28005 & PNNL-AGG-ICP-AES \\
\hline $7440-41-7$ & Beryllium & $<0.028$ & ug/g dry & 0.028 & $5 / 07 / 08$ & 8D28005 & PNNL-AGG-ICP-AES \\
\hline $7440-69-9$ & Bismuth & $<0.188$ & ug/g dry & 0.188 & $5 / 07 / 08$ & 8D28005 & PNNL-AGG-ICP-AES \\
\hline $7440-70-2$ & Calcium & 18.7 & ug/g dry & 0.387 & $5 / 07 / 08$ & 8D28005 & PNNL-AGG-ICP-AES \\
\hline $7440-43-9$ & Cadmium & $<0.027$ & ug/g dry & 0.027 & $5 / 07 / 08$ & 8D28005 & PNNL-AGG-ICP-AES \\
\hline $7440-48-4$ & Cobalt & $<0.096$ & ug/g dry & 0.096 & $5 / 07 / 08$ & 8D28005 & PNNL-AGG-ICP-AES \\
\hline $7440-47-3$ & Chromium & $<0.033$ & ug/g dry & 0.033 & $5 / 07 / 08$ & 8D28005 & PNNL-AGG-ICP-AES \\
\hline $7440-50-8$ & Copper & $<0.080$ & ug/g dry & 0.080 & $5 / 07 / 08$ & 8D28005 & PNNL-AGG-ICP-AES \\
\hline 7439-89-6 & Iron & $<0.142$ & ug/g dry & 0.142 & $5 / 07 / 08$ & 8D28005 & PNNL-AGG-ICP-AES \\
\hline 7440-09-7 & Potassium & 6.39 & ug/g dry & 2.33 & $5 / 07 / 08$ & 8D28005 & PNNL-AGG-ICP-AES \\
\hline $7439-93-2$ & Lithium & $<0.540$ & ug/g dry & 0.540 & $5 / 07 / 08$ & 8D28005 & PNNL-AGG-ICP-AES \\
\hline $7439-95-4$ & Magnesium & 6.35 & ug/g dry & 0.083 & $5 / 07 / 08$ & 8D28005 & PNNL-AGG-ICP-AES \\
\hline $7439-96-5$ & Manganese & $<0.017$ & ug/g dry & 0.017 & $5 / 07 / 08$ & 8D28005 & PNNL-AGG-ICP-AES \\
\hline 7439-98-7 & Molybdenum & $<0.139$ & ug/g dry & 0.139 & $5 / 07 / 08$ & 8D28005 & PNNL-AGG-ICP-AES \\
\hline $7440-02-0$ & Nickel & $<0.093$ & ug/g dry & 0.093 & $5 / 07 / 08$ & 8D28005 & PNNL-AGG-ICP-AES \\
\hline 7723-14-0 & Phosphorus & $<1.03$ & ug/g dry & 1.03 & $5 / 07 / 08$ & 8D28005 & PNNL-AGG-ICP-AES \\
\hline $7439-92-1$ & Lead & $<0.432$ & ug/g dry & 0.432 & $5 / 07 / 08$ & 8D28005 & PNNL-AGG-ICP-AES \\
\hline $7782-49-2$ & Selenium & $<1.95$ & ug/g dry & 1.95 & $5 / 07 / 08$ & 8D28005 & PNNL-AGG-ICP-AES \\
\hline $7440-24-6$ & Strontium & 0.086 & ug/g dry & 0.052 & $5 / 07 / 08$ & 8D28005 & PNNL-AGG-ICP-AES \\
\hline $7440-28-0$ & Thallium & $<1.03$ & ug/g dry & 1.03 & $5 / 07 / 08$ & 8D28005 & PNNL-AGG-ICP-AES \\
\hline $7440-62-2$ & Vanadium & $<0.045$ & ug/g dry & 0.045 & $5 / 07 / 08$ & 8D28005 & PNNL-AGG-ICP-AES \\
\hline $7440-66-6$ & Zinc & $<0.093$ & ug/g dry & 0.093 & $5 / 07 / 08$ & 8D28005 & PNNL-AGG-ICP-AES \\
\hline $7440-23-5$ & Sodium & 12.8 & ug/g dry & 0.669 & $5 / 07 / 08$ & 8D28005 & PNNL-AGG-ICP-AES \\
\hline $7440-21-3$ & Silicon & 6.44 & ug/g dry & 1.50 & $5 / 07 / 08$ & 8D28005 & PNNL-AGG-ICP-AES \\
\hline $7704-34-9$ & Sulfur & 24.2 & ug/g dry & 3.07 & $5 / 07 / 08$ & 8D28005 & PNNL-AGG-ICP-AES \\
\hline $7440-32-6$ & Titanium & $<0.009$ & ug/g dry & 0.009 & $5 / 07 / 08$ & 8D28005 & PNNL-AGG-ICP-AES \\
\hline $7440-67-7$ & Zirconium & $<0.150$ & ug/g dry & 0.150 & $5 / 07 / 08$ & 8D28005 & PNNL-AGG-ICP-AES \\
\hline
\end{tabular}


Total Metals by SW846 6010B/Water Extract

\begin{tabular}{|c|c|c|c|c|c|c|c|}
\hline CAS \# & Analyte & Results & Units & EQL & Analyzed & Batch & Method \\
\hline HEIS No. & B1RYR7A & \multicolumn{3}{|c|}{ Lab ID: $\quad$ 0803012-14 } & & & \\
\hline $7440-22-4$ & Silver & $<0.077$ & ug/g dry & 0.077 & $5 / 07 / 08$ & 8D28005 & PNNL-AGG-ICP-AES \\
\hline $7440-15-5$ & Rhenium & $<0.152$ & ug/g dry & 0.152 & $5 / 07 / 08$ & 8D28005 & PNNL-AGG-ICP-AES \\
\hline $7440-36-0$ & Antimony & $<0.633$ & ug/g dry & 0.633 & $5 / 07 / 08$ & 8D28005 & PNNL-AGG-ICP-AES \\
\hline HEIS No. & B1RYR8B & \multicolumn{3}{|c|}{ Lab ID: $\quad$ 0803012-17 } & & & \\
\hline 7429-90-5 & Aluminum & $<0.086$ & ug/g dry & 0.086 & $5 / 07 / 08$ & 8D28005 & PNNL-AGG-ICP-AES \\
\hline $7440-38-2$ & Arsenic & $<0.360$ & ug/g dry & 0.360 & $5 / 07 / 08$ & 8D28005 & PNNL-AGG-ICP-AES \\
\hline $7440-42-8$ & Boron & $<1.94$ & ug/g dry & 1.94 & $5 / 07 / 08$ & 8D28005 & PNNL-AGG-ICP-AES \\
\hline $7440-39-3$ & Barium & $<0.009$ & ug/g dry & 0.009 & $5 / 07 / 08$ & 8D28005 & PNNL-AGG-ICP-AES \\
\hline $7440-41-7$ & Beryllium & $<0.028$ & ug/g dry & 0.028 & $5 / 07 / 08$ & 8D28005 & PNNL-AGG-ICP-AES \\
\hline 7440-69-9 & Bismuth & $<0.188$ & ug/g dry & 0.188 & $5 / 07 / 08$ & 8D28005 & PNNL-AGG-ICP-AES \\
\hline $7440-70-2$ & Calcium & 24.3 & ug/g dry & 0.387 & $5 / 07 / 08$ & 8D28005 & PNNL-AGG-ICP-AES \\
\hline $7440-43-9$ & Cadmium & $<0.027$ & ug/g dry & 0.027 & $5 / 07 / 08$ & 8D28005 & PNNL-AGG-ICP-AES \\
\hline $7440-48-4$ & Cobalt & $<0.096$ & ug/g dry & 0.096 & $5 / 07 / 08$ & 8D28005 & PNNL-AGG-ICP-AES \\
\hline $7440-47-3$ & Chromium & $<0.033$ & ug/g dry & 0.033 & $5 / 07 / 08$ & 8D28005 & PNNL-AGG-ICP-AES \\
\hline $7440-50-8$ & Copper & $<0.080$ & ug/g dry & 0.080 & $5 / 07 / 08$ & 8D28005 & PNNL-AGG-ICP-AES \\
\hline 7439-89-6 & Iron & $<0.142$ & ug/g dry & 0.142 & $5 / 07 / 08$ & 8D28005 & PNNL-AGG-ICP-AES \\
\hline $7440-09-7$ & Potassium & 7.86 & ug/g dry & 2.33 & $5 / 07 / 08$ & 8D28005 & PNNL-AGG-ICP-AES \\
\hline 7439-93-2 & Lithium & $<0.540$ & ug/g dry & 0.540 & $5 / 07 / 08$ & 8D28005 & PNNL-AGG-ICP-AES \\
\hline 7439-95-4 & Magnesium & 8.99 & ug/g dry & 0.083 & $5 / 07 / 08$ & 8D28005 & PNNL-AGG-ICP-AES \\
\hline $7439-96-5$ & Manganese & $<0.017$ & ug/g dry & 0.017 & $5 / 07 / 08$ & 8D28005 & PNNL-AGG-ICP-AES \\
\hline 7439-98-7 & Molybdenum & $<0.139$ & ug/g dry & 0.139 & $5 / 07 / 08$ & 8D28005 & PNNL-AGG-ICP-AES \\
\hline $7440-02-0$ & Nickel & $<0.093$ & ug/g dry & 0.093 & $5 / 07 / 08$ & 8D28005 & PNNL-AGG-ICP-AES \\
\hline $7723-14-0$ & Phosphorus & $<1.03$ & ug/g dry & 1.03 & $5 / 07 / 08$ & 8D28005 & PNNL-AGG-ICP-AES \\
\hline 7439-92-1 & Lead & $<0.432$ & ug/g dry & 0.432 & $5 / 07 / 08$ & 8D28005 & PNNL-AGG-ICP-AES \\
\hline $7782-49-2$ & Selenium & $<1.95$ & ug/g dry & 1.95 & $5 / 07 / 08$ & 8D28005 & PNNL-AGG-ICP-AES \\
\hline $7440-24-6$ & Strontium & 0.114 & ug/g dry & 0.052 & $5 / 07 / 08$ & 8D28005 & PNNL-AGG-ICP-AES \\
\hline $7440-28-0$ & Thallium & $<1.03$ & ug/g dry & 1.03 & $5 / 07 / 08$ & 8D28005 & PNNL-AGG-ICP-AES \\
\hline $7440-62-2$ & Vanadium & $<0.045$ & ug/g dry & 0.045 & $5 / 07 / 08$ & 8D28005 & PNNL-AGG-ICP-AES \\
\hline $7440-66-6$ & Zinc & $<0.093$ & ug/g dry & 0.093 & $5 / 07 / 08$ & 8D28005 & PNNL-AGG-ICP-AES \\
\hline $7440-23-5$ & Sodium & 16.9 & ug/g dry & 0.669 & $5 / 07 / 08$ & 8D28005 & PNNL-AGG-ICP-AES \\
\hline $7440-21-3$ & Silicon & 6.35 & ug/g dry & 1.50 & $5 / 07 / 08$ & $8 D 28005$ & PNNL-AGG-ICP-AES \\
\hline $7704-34-9$ & Sulfur & 35.6 & ug/g dry & 3.07 & $5 / 07 / 08$ & 8D28005 & PNNL-AGG-ICP-AES \\
\hline $7440-32-6$ & Titanium & $<0.009$ & ug/g dry & 0.009 & $5 / 07 / 08$ & 8D28005 & PNNL-AGG-ICP-AES \\
\hline 7440-67-7 & Zirconium & $<0.150$ & ug/g dry & 0.150 & $5 / 07 / 08$ & 8D28005 & PNNL-AGG-ICP-AES \\
\hline $7440-22-4$ & Silver & $<0.077$ & ug/g dry & 0.077 & $5 / 07 / 08$ & 8D28005 & PNNL-AGG-ICP-AES \\
\hline $7440-15-5$ & Rhenium & $<0.152$ & ug/g dry & 0.152 & $5 / 07 / 08$ & 8D28005 & PNNL-AGG-ICP-AES \\
\hline $7440-36-0$ & Antimony & $<0.633$ & ug/g dry & 0.633 & $5 / 07 / 08$ & 8D28005 & PNNL-AGG-ICP-AES \\
\hline HEIS No. & B1RYR8A & & b ID: & 3012-18 & & & \\
\hline $7429-90-5$ & Aluminum & 0.222 & ug/g dry & 0.090 & $5 / 07 / 08$ & 8D28005 & PNNL-AGG-ICP-AES \\
\hline $7440-38-2$ & Arsenic & $<0.379$ & ug/g dry & 0.379 & $5 / 07 / 08$ & 8D28005 & PNNL-AGG-ICP-AES \\
\hline $7440-42-8$ & Boron & $<2.04$ & ug/g dry & 2.04 & $5 / 07 / 08$ & 8D28005 & PNNL-AGG-ICP-AES \\
\hline $7440-39-3$ & Barium & $<0.009$ & ug/g dry & 0.009 & $5 / 07 / 08$ & 8D28005 & PNNL-AGG-ICP-AES \\
\hline $7440-41-7$ & Beryllium & $<0.030$ & ug/g dry & 0.030 & $5 / 07 / 08$ & 8D28005 & PNNL-AGG-ICP-AES \\
\hline $7440-69-9$ & Bismuth & $<0.198$ & ug/g dry & 0.198 & $5 / 07 / 08$ & 8D28005 & PNNL-AGG-ICP-AES \\
\hline $7440-70-2$ & Calcium & 11.4 & ug/g dry & 0.407 & $5 / 07 / 08$ & 8D28005 & PNNL-AGG-ICP-AES \\
\hline $7440-43-9$ & Cadmium & $<0.028$ & ug/g dry & 0.028 & $5 / 07 / 08$ & 8D28005 & PNNL-AGG-ICP-AES \\
\hline $7440-48-4$ & Cobalt & $<0.101$ & ug/g dry & 0.101 & $5 / 07 / 08$ & 8D28005 & PNNL-AGG-ICP-AES \\
\hline $7440-47-3$ & Chromium & $<0.035$ & ug/g dry & 0.035 & $5 / 07 / 08$ & 8D28005 & PNNL-AGG-ICP-AES \\
\hline
\end{tabular}


Total Metals by SW846 6010B/Water Extract

\begin{tabular}{|c|c|c|c|c|c|c|c|}
\hline CAS \# & Analyte & Results & Units & EQL & Analyzed & Batch & Method \\
\hline HEIS No. & B1RYR8A & \multicolumn{3}{|c|}{ Lab ID: $\quad$ 0803012-18 } & & & \\
\hline $7440-50-8$ & Copper & $<0.085$ & ug/g dry & 0.085 & $5 / 07 / 08$ & 8D28005 & PNNL-AGG-ICP-AES \\
\hline 7439-89-6 & Iron & $<0.150$ & ug/g dry & 0.150 & $5 / 07 / 08$ & 8D28005 & PNNL-AGG-ICP-AES \\
\hline 7440-09-7 & Potassium & 5.75 & ug/g dry & 2.45 & $5 / 07 / 08$ & 8D28005 & PNNL-AGG-ICP-AES \\
\hline 7439-93-2 & Lithium & $<0.568$ & ug/g dry & 0.568 & $5 / 07 / 08$ & 8D28005 & PNNL-AGG-ICP-AES \\
\hline 7439-95-4 & Magnesium & 4.39 & ug/g dry & 0.088 & $5 / 07 / 08$ & 8D28005 & PNNL-AGG-ICP-AES \\
\hline 7439-96-5 & Manganese & $<0.018$ & ug/g dry & 0.018 & $5 / 07 / 08$ & 8D28005 & PNNL-AGG-ICP-AES \\
\hline 7439-98-7 & Molybdenum & $<0.146$ & ug/g dry & 0.146 & $5 / 07 / 08$ & 8D28005 & PNNL-AGG-ICP-AES \\
\hline $7440-02-0$ & Nickel & $<0.098$ & ug/g dry & 0.098 & $5 / 07 / 08$ & 8D28005 & PNNL-AGG-ICP-AES \\
\hline 7723-14-0 & Phosphorus & $<1.09$ & ug/g dry & 1.09 & $5 / 07 / 08$ & 8D28005 & PNNL-AGG-ICP-AES \\
\hline $7439-92-1$ & Lead & $<0.454$ & ug/g dry & 0.454 & $5 / 07 / 08$ & 8D28005 & PNNL-AGG-ICP-AES \\
\hline $7782-49-2$ & Selenium & $<2.05$ & ug/g dry & 2.05 & $5 / 07 / 08$ & 8D28005 & PNNL-AGG-ICP-AES \\
\hline $7440-24-6$ & Strontium & 0.058 & ug/g dry & 0.055 & $5 / 07 / 08$ & 8D28005 & PNNL-AGG-ICP-AES \\
\hline $7440-28-0$ & Thallium & $<1.08$ & ug/g dry & 1.08 & $5 / 07 / 08$ & 8D28005 & PNNL-AGG-ICP-AES \\
\hline $7440-62-2$ & Vanadium & $<0.048$ & ug/g dry & 0.048 & $5 / 07 / 08$ & 8D28005 & PNNL-AGG-ICP-AES \\
\hline $7440-66-6$ & Zinc & $<0.097$ & ug/g dry & 0.097 & $5 / 07 / 08$ & 8D28005 & PNNL-AGG-ICP-AES \\
\hline $7440-23-5$ & Sodium & 11.9 & ug/g dry & 0.703 & $5 / 07 / 08$ & 8D28005 & PNNL-AGG-ICP-AES \\
\hline $7440-21-3$ & Silicon & 6.26 & ug/g dry & 1.58 & $5 / 07 / 08$ & 8D28005 & PNNL-AGG-ICP-AES \\
\hline $7704-34-9$ & Sulfur & 15.6 & ug/g dry & 3.23 & $5 / 07 / 08$ & 8D28005 & PNNL-AGG-ICP-AES \\
\hline $7440-32-6$ & Titanium & $<0.009$ & ug/g dry & 0.009 & $5 / 07 / 08$ & 8D28005 & PNNL-AGG-ICP-AES \\
\hline $7440-67-7$ & Zirconium & $<0.158$ & ug/g dry & 0.158 & $5 / 07 / 08$ & 8D28005 & PNNL-AGG-ICP-AES \\
\hline $7440-22-4$ & Silver & $<0.081$ & ug/g dry & 0.081 & $5 / 07 / 08$ & 8D28005 & PNNL-AGG-ICP-AES \\
\hline $7440-15-5$ & Rhenium & $<0.160$ & ug/g dry & 0.160 & $5 / 07 / 08$ & 8D28005 & PNNL-AGG-ICP-AES \\
\hline $7440-36-0$ & Antimony & $<0.666$ & ug/g dry & 0.666 & $5 / 07 / 08$ & 8D28005 & PNNL-AGG-ICP-AES \\
\hline HEIS No. & B1RYR9B & \multicolumn{3}{|c|}{ Lab ID: $\quad$ 0803012-21 } & & & \\
\hline $7429-90-5$ & Aluminum & 0.283 & ug/g dry & 0.086 & $5 / 07 / 08$ & 8D28005 & PNNL-AGG-ICP-AES \\
\hline $7440-38-2$ & Arsenic & $<0.360$ & ug/g dry & 0.360 & $5 / 07 / 08$ & 8D28005 & PNNL-AGG-ICP-AES \\
\hline $7440-42-8$ & Boron & $<1.94$ & ug/g dry & 1.94 & $5 / 07 / 08$ & 8D28005 & PNNL-AGG-ICP-AES \\
\hline $7440-39-3$ & Barium & $<0.009$ & ug/g dry & 0.009 & $5 / 07 / 08$ & 8D28005 & PNNL-AGG-ICP-AES \\
\hline $7440-41-7$ & Beryllium & $<0.028$ & ug/g dry & 0.028 & $5 / 07 / 08$ & 8D28005 & PNNL-AGG-ICP-AES \\
\hline $7440-69-9$ & Bismuth & $<0.188$ & ug/g dry & 0.188 & $5 / 07 / 08$ & 8D28005 & PNNL-AGG-ICP-AES \\
\hline $7440-70-2$ & Calcium & 9.64 & ug/g dry & 0.387 & $5 / 07 / 08$ & 8D28005 & PNNL-AGG-ICP-AES \\
\hline $7440-43-9$ & Cadmium & $<0.027$ & ug/g dry & 0.027 & $5 / 07 / 08$ & 8D28005 & PNNL-AGG-ICP-AES \\
\hline $7440-48-4$ & Cobalt & $<0.096$ & ug/g dry & 0.096 & $5 / 07 / 08$ & 8D28005 & PNNL-AGG-ICP-AES \\
\hline $7440-47-3$ & Chromium & $<0.033$ & ug/g dry & 0.033 & $5 / 07 / 08$ & 8D28005 & PNNL-AGG-ICP-AES \\
\hline $7440-50-8$ & Copper & $<0.080$ & ug/g dry & 0.080 & $5 / 07 / 08$ & 8D28005 & PNNL-AGG-ICP-AES \\
\hline 7439-89-6 & Iron & 0.177 & ug/g dry & 0.142 & $5 / 07 / 08$ & 8D28005 & PNNL-AGG-ICP-AES \\
\hline 7440-09-7 & Potassium & 6.72 & ug/g dry & 2.33 & $5 / 07 / 08$ & 8D28005 & PNNL-AGG-ICP-AES \\
\hline $7439-93-2$ & Lithium & $<0.540$ & ug/g dry & 0.540 & $5 / 07 / 08$ & 8D28005 & PNNL-AGG-ICP-AES \\
\hline $7439-95-4$ & Magnesium & 3.59 & ug/g dry & 0.083 & $5 / 07 / 08$ & 8D28005 & PNNL-AGG-ICP-AES \\
\hline $7439-96-5$ & Manganese & $<0.017$ & ug/g dry & 0.017 & $5 / 07 / 08$ & 8D28005 & PNNL-AGG-ICP-AES \\
\hline 7439-98-7 & Molybdenum & $<0.139$ & ug/g dry & 0.139 & $5 / 07 / 08$ & 8D28005 & PNNL-AGG-ICP-AES \\
\hline $7440-02-0$ & Nickel & $<0.093$ & ug/g dry & 0.093 & $5 / 07 / 08$ & 8D28005 & PNNL-AGG-ICP-AES \\
\hline $7723-14-0$ & Phosphorus & $<1.03$ & ug/g dry & 1.03 & $5 / 07 / 08$ & 8D28005 & PNNL-AGG-ICP-AES \\
\hline 7439-92-1 & Lead & $<0.432$ & ug/g dry & 0.432 & $5 / 07 / 08$ & 8D28005 & PNNL-AGG-ICP-AES \\
\hline 7782-49-2 & Selenium & $<1.95$ & ug/g dry & 1.95 & $5 / 07 / 08$ & 8D28005 & PNNL-AGG-ICP-AES \\
\hline $7440-24-6$ & Strontium & $<0.052$ & ug/g dry & 0.052 & $5 / 07 / 08$ & 8D28005 & PNNL-AGG-ICP-AES \\
\hline $7440-28-0$ & Thallium & $<1.03$ & ug/g dry & 1.03 & $5 / 07 / 08$ & 8D28005 & PNNL-AGG-ICP-AES \\
\hline $7440-62-2$ & Vanadium & $<0.045$ & ug/g dry & 0.045 & $5 / 07 / 08$ & 8D28005 & PNNL-AGG-ICP-AES \\
\hline
\end{tabular}


Total Metals by SW846 6010B/Water Extract

\begin{tabular}{|c|c|c|c|c|c|c|c|}
\hline CAS \# & Analyte & Results & Units & EQL & Analyzed & Batch & Method \\
\hline HEIS No. & B1RYR9B & \multicolumn{3}{|c|}{ Lab ID: $\quad$ 0803012-21 } & & & \\
\hline $7440-66-6$ & Zinc & $<0.093$ & ug/g dry & 0.093 & $5 / 07 / 08$ & 8D28005 & PNNL-AGG-ICP-AES \\
\hline $7440-23-5$ & Sodium & 14.8 & ug/g dry & 0.669 & 5/07/08 & 8D28005 & PNNL-AGG-ICP-AES \\
\hline $7440-21-3$ & Silicon & 7.26 & ug/g dry & 1.50 & $5 / 07 / 08$ & 8D28005 & PNNL-AGG-ICP-AES \\
\hline 7704-34-9 & Sulfur & 12.1 & ug/g dry & 3.08 & $5 / 07 / 08$ & 8D28005 & PNNL-AGG-ICP-AES \\
\hline $7440-32-6$ & Titanium & 0.011 & ug/g dry & 0.009 & $5 / 07 / 08$ & 8D28005 & PNNL-AGG-ICP-AES \\
\hline $7440-67-7$ & Zirconium & $<0.150$ & ug/g dry & 0.150 & $5 / 07 / 08$ & 8D28005 & PNNL-AGG-ICP-AES \\
\hline $7440-22-4$ & Silver & $<0.077$ & ug/g dry & 0.077 & $5 / 07 / 08$ & 8D28005 & PNNL-AGG-ICP-AES \\
\hline $7440-15-5$ & Rhenium & $<0.152$ & ug/g dry & 0.152 & $5 / 07 / 08$ & 8D28005 & PNNL-AGG-ICP-AES \\
\hline $7440-36-0$ & Antimony & $<0.633$ & ug/g dry & 0.633 & $5 / 07 / 08$ & 8D28005 & PNNL-AGG-ICP-AES \\
\hline HEIS No. & B1RYR9A & \multicolumn{3}{|c|}{ Lab ID: $\quad$ 0803012-22 } & & & \\
\hline $7429-90-5$ & Aluminum & 0.236 & ug/g dry & 0.086 & $5 / 07 / 08$ & 8D28005 & PNNL-AGG-ICP-AES \\
\hline $7440-38-2$ & Arsenic & $<0.360$ & ug/g dry & 0.360 & $5 / 07 / 08$ & 8D28005 & PNNL-AGG-ICP-AES \\
\hline $7440-42-8$ & Boron & $<1.94$ & ug/g dry & 1.94 & $5 / 07 / 08$ & 8D28005 & PNNL-AGG-ICP-AES \\
\hline 7440-39-3 & Barium & $<0.009$ & ug/g dry & 0.009 & $5 / 07 / 08$ & 8D28005 & PNNL-AGG-ICP-AES \\
\hline $7440-41-7$ & Beryllium & $<0.028$ & ug/g dry & 0.028 & $5 / 07 / 08$ & 8D28005 & PNNL-AGG-ICP-AES \\
\hline 7440-69-9 & Bismuth & $<0.188$ & ug/g dry & 0.188 & $5 / 07 / 08$ & 8D28005 & PNNL-AGG-ICP-AES \\
\hline $7440-70-2$ & Calcium & 9.22 & ug/g dry & 0.387 & $5 / 07 / 08$ & 8D28005 & PNNL-AGG-ICP-AES \\
\hline $7440-43-9$ & Cadmium & $<0.027$ & ug/g dry & 0.027 & $5 / 07 / 08$ & 8D28005 & PNNL-AGG-ICP-AES \\
\hline $7440-48-4$ & Cobalt & $<0.096$ & ug/g dry & 0.096 & $5 / 07 / 08$ & 8D28005 & PNNL-AGG-ICP-AES \\
\hline $7440-47-3$ & Chromium & $<0.033$ & ug/g dry & 0.033 & $5 / 07 / 08$ & 8D28005 & PNNL-AGG-ICP-AES \\
\hline $7440-50-8$ & Copper & $<0.080$ & ug/g dry & 0.080 & $5 / 07 / 08$ & 8D28005 & PNNL-AGG-ICP-AES \\
\hline 7439-89-6 & Iron & 0.154 & ug/g dry & 0.142 & $5 / 07 / 08$ & 8D28005 & PNNL-AGG-ICP-AES \\
\hline 7440-09-7 & Potassium & 5.89 & ug/g dry & 2.33 & $5 / 07 / 08$ & 8D28005 & PNNL-AGG-ICP-AES \\
\hline 7439-93-2 & Lithium & $<0.540$ & ug/g dry & 0.540 & $5 / 07 / 08$ & 8D28005 & PNNL-AGG-ICP-AES \\
\hline 7439-95-4 & Magnesium & 3.30 & ug/g dry & 0.083 & 5/07/08 & 8D28005 & PNNL-AGG-ICP-AES \\
\hline 7439-96-5 & Manganese & $<0.017$ & ug/g dry & 0.017 & $5 / 07 / 08$ & 8D28005 & PNNL-AGG-ICP-AES \\
\hline 7439-98-7 & Molybdenum & $<0.139$ & ug/g dry & 0.139 & $5 / 07 / 08$ & 8D28005 & PNNL-AGG-ICP-AES \\
\hline $7440-02-0$ & Nickel & $<0.093$ & ug/g dry & 0.093 & $5 / 07 / 08$ & 8D28005 & PNNL-AGG-ICP-AES \\
\hline 7723-14-0 & Phosphorus & $<1.03$ & ug/g dry & 1.03 & $5 / 07 / 08$ & 8D28005 & PNNL-AGG-ICP-AES \\
\hline 7439-92-1 & Lead & $<0.432$ & ug/g dry & 0.432 & $5 / 07 / 08$ & 8D28005 & PNNL-AGG-ICP-AES \\
\hline $7782-49-2$ & Selenium & $<1.95$ & ug/g dry & 1.95 & $5 / 07 / 08$ & 8D28005 & PNNL-AGG-ICP-AES \\
\hline $7440-24-6$ & Strontium & $<0.052$ & ug/g dry & 0.052 & $5 / 07 / 08$ & 8D28005 & PNNL-AGG-ICP-AES \\
\hline $7440-28-0$ & Thallium & $<1.03$ & ug/g dry & 1.03 & $5 / 07 / 08$ & 8D28005 & PNNL-AGG-ICP-AES \\
\hline $7440-62-2$ & Vanadium & $<0.045$ & ug/g dry & 0.045 & $5 / 07 / 08$ & 8D28005 & PNNL-AGG-ICP-AES \\
\hline $7440-66-6$ & Zinc & $<0.093$ & ug/g dry & 0.093 & $5 / 07 / 08$ & 8D28005 & PNNL-AGG-ICP-AES \\
\hline $7440-23-5$ & Sodium & 13.0 & ug/g dry & 0.669 & 5/07/08 & 8D28005 & PNNL-AGG-ICP-AES \\
\hline $7440-21-3$ & Silicon & 6.93 & ug/g dry & 1.50 & $5 / 07 / 08$ & 8D28005 & PNNL-AGG-ICP-AES \\
\hline $7704-34-9$ & Sulfur & 11.0 & ug/g dry & 3.08 & $5 / 07 / 08$ & 8D28005 & PNNL-AGG-ICP-AES \\
\hline $7440-32-6$ & Titanium & 0.010 & ug/g dry & 0.009 & $5 / 07 / 08$ & 8D28005 & PNNL-AGG-ICP-AES \\
\hline $7440-67-7$ & Zirconium & $<0.150$ & ug/g dry & 0.150 & $5 / 07 / 08$ & 8D28005 & PNNL-AGG-ICP-AES \\
\hline $7440-22-4$ & Silver & $<0.077$ & ug/g dry & 0.077 & $5 / 07 / 08$ & 8D28005 & PNNL-AGG-ICP-AES \\
\hline $7440-15-5$ & Rhenium & $<0.152$ & ug/g dry & 0.152 & $5 / 07 / 08$ & 8D28005 & PNNL-AGG-ICP-AES \\
\hline $7440-36-0$ & Antimony & $<0.633$ & ug/g dry & 0.633 & $5 / 07 / 08$ & 8D28005 & PNNL-AGG-ICP-AES \\
\hline HEIS No. & B1RYT0B & & b ID: & 3014-01 & & & \\
\hline 7429-90-5 & Aluminum & 0.560 & ug/g dry & 0.086 & $5 / 07 / 08$ & 8D28005 & PNNL-AGG-ICP-AES \\
\hline $7440-38-2$ & Arsenic & $<0.361$ & ug/g dry & 0.361 & $5 / 07 / 08$ & 8D28005 & PNNL-AGG-ICP-AES \\
\hline $7440-42-8$ & Boron & $<1.94$ & ug/g dry & 1.94 & $5 / 07 / 08$ & 8D28005 & PNNL-AGG-ICP-AES \\
\hline $7440-39-3$ & Barium & $<0.009$ & ug/g dry & 0.009 & 5/07/08 & 8D28005 & PNNL-AGG-ICP-AES \\
\hline
\end{tabular}


Total Metals by SW846 6010B/Water Extract

\begin{tabular}{|c|c|c|c|c|c|c|c|}
\hline CAS \# & Analyte & Results & Units & EQL & Analyzed & Batch & Method \\
\hline HEIS No. & B1RYT0B & \multicolumn{3}{|c|}{ Lab ID: $\quad$ 0803014-01 } & & & \\
\hline $7440-41-7$ & Beryllium & $<0.028$ & ug/g dry & 0.028 & $5 / 07 / 08$ & 8D28005 & PNNL-AGG-ICP-AES \\
\hline 7440-69-9 & Bismuth & $<0.188$ & ug/g dry & 0.188 & $5 / 07 / 08$ & 8D28005 & PNNL-AGG-ICP-AES \\
\hline $7440-70-2$ & Calcium & 3.01 & ug/g dry & 0.388 & $5 / 07 / 08$ & 8D28005 & PNNL-AGG-ICP-AES \\
\hline $7440-43-9$ & Cadmium & $<0.027$ & ug/g dry & 0.027 & $5 / 07 / 08$ & 8D28005 & PNNL-AGG-ICP-AES \\
\hline $7440-48-4$ & Cobalt & $<0.096$ & ug/g dry & 0.096 & $5 / 07 / 08$ & 8D28005 & PNNL-AGG-ICP-AES \\
\hline $7440-47-3$ & Chromium & $<0.033$ & ug/g dry & 0.033 & $5 / 07 / 08$ & 8D28005 & PNNL-AGG-ICP-AES \\
\hline $7440-50-8$ & Copper & $<0.081$ & ug/g dry & 0.081 & $5 / 07 / 08$ & 8D28005 & PNNL-AGG-ICP-AES \\
\hline 7439-89-6 & Iron & 0.719 & ug/g dry & 0.142 & $5 / 07 / 08$ & 8D28005 & PNNL-AGG-ICP-AES \\
\hline 7440-09-7 & Potassium & 2.89 & ug/g dry & 2.33 & $5 / 07 / 08$ & 8D28005 & PNNL-AGG-ICP-AES \\
\hline $7439-93-2$ & Lithium & $<0.541$ & ug/g dry & 0.541 & $5 / 07 / 08$ & 8D28005 & PNNL-AGG-ICP-AES \\
\hline $7439-95-4$ & Magnesium & 0.778 & ug/g dry & 0.084 & $5 / 07 / 08$ & 8D28005 & PNNL-AGG-ICP-AES \\
\hline 7439-96-5 & Manganese & $<0.017$ & ug/g dry & 0.017 & $5 / 07 / 08$ & 8D28005 & PNNL-AGG-ICP-AES \\
\hline 7439-98-7 & Molybdenum & $<0.139$ & ug/g dry & 0.139 & $5 / 07 / 08$ & 8D28005 & PNNL-AGG-ICP-AES \\
\hline $7440-02-0$ & Nickel & $<0.093$ & ug/g dry & 0.093 & $5 / 07 / 08$ & 8D28005 & PNNL-AGG-ICP-AES \\
\hline $7723-14-0$ & Phosphorus & $<1.03$ & ug/g dry & 1.03 & $5 / 07 / 08$ & 8D28005 & PNNL-AGG-ICP-AES \\
\hline 7439-92-1 & Lead & $<0.433$ & ug/g dry & 0.433 & $5 / 07 / 08$ & 8D28005 & PNNL-AGG-ICP-AES \\
\hline 7782-49-2 & Selenium & $<1.95$ & ug/g dry & 1.95 & $5 / 07 / 08$ & 8D28005 & PNNL-AGG-ICP-AES \\
\hline $7440-24-6$ & Strontium & $<0.052$ & ug/g dry & 0.052 & $5 / 07 / 08$ & 8D28005 & PNNL-AGG-ICP-AES \\
\hline $7440-28-0$ & Thallium & $<1.03$ & ug/g dry & 1.03 & $5 / 07 / 08$ & 8D28005 & PNNL-AGG-ICP-AES \\
\hline $7440-62-2$ & Vanadium & 0.074 & ug/g dry & 0.045 & $5 / 07 / 08$ & 8D28005 & PNNL-AGG-ICP-AES \\
\hline $7440-66-6$ & Zinc & $<0.093$ & ug/g dry & 0.093 & $5 / 07 / 08$ & 8D28005 & PNNL-AGG-ICP-AES \\
\hline $7440-23-5$ & Sodium & 45.8 & ug/g dry & 0.670 & $5 / 07 / 08$ & 8D28005 & PNNL-AGG-ICP-AES \\
\hline $7440-21-3$ & Silicon & 16.5 & ug/g dry & 1.50 & $5 / 07 / 08$ & 8D28005 & PNNL-AGG-ICP-AES \\
\hline $7704-34-9$ & Sulfur & 5.77 & ug/g dry & 3.08 & $5 / 07 / 08$ & 8D28005 & PNNL-AGG-ICP-AES \\
\hline $7440-32-6$ & Titanium & 0.028 & ug/g dry & 0.009 & $5 / 07 / 08$ & 8D28005 & PNNL-AGG-ICP-AES \\
\hline $7440-67-7$ & Zirconium & $<0.150$ & ug/g dry & 0.150 & $5 / 07 / 08$ & 8D28005 & PNNL-AGG-ICP-AES \\
\hline $7440-22-4$ & Silver & $<0.077$ & ug/g dry & 0.077 & $5 / 07 / 08$ & 8D28005 & PNNL-AGG-ICP-AES \\
\hline $7440-15-5$ & Rhenium & $<0.152$ & ug/g dry & 0.152 & $5 / 07 / 08$ & 8D28005 & PNNL-AGG-ICP-AES \\
\hline $7440-36-0$ & Antimony & $<0.634$ & ug/g dry & 0.634 & $5 / 07 / 08$ & 8D28005 & PNNL-AGG-ICP-AES \\
\hline HEIS No. & B1RYT0A & \multicolumn{3}{|c|}{ Lab ID: $\quad$ 0803014-02 } & & & \\
\hline $7429-90-5$ & Aluminum & 0.291 & ug/g dry & 0.086 & $5 / 07 / 08$ & 8D28005 & PNNL-AGG-ICP-AES \\
\hline $7440-38-2$ & Arsenic & $<0.361$ & ug/g dry & 0.361 & $5 / 07 / 08$ & 8D28005 & PNNL-AGG-ICP-AES \\
\hline $7440-42-8$ & Boron & $<1.95$ & ug/g dry & 1.95 & $5 / 07 / 08$ & 8D28005 & PNNL-AGG-ICP-AES \\
\hline $7440-39-3$ & Barium & $<0.009$ & ug/g dry & 0.009 & $5 / 07 / 08$ & 8D28005 & PNNL-AGG-ICP-AES \\
\hline $7440-41-7$ & Beryllium & $<0.028$ & ug/g dry & 0.028 & $5 / 07 / 08$ & 8D28005 & PNNL-AGG-ICP-AES \\
\hline $7440-69-9$ & Bismuth & $<0.189$ & ug/g dry & 0.189 & $5 / 07 / 08$ & 8D28005 & PNNL-AGG-ICP-AES \\
\hline $7440-70-2$ & Calcium & 2.25 & ug/g dry & 0.388 & $5 / 07 / 08$ & 8D28005 & PNNL-AGG-ICP-AES \\
\hline $7440-43-9$ & Cadmium & $<0.027$ & ug/g dry & 0.027 & $5 / 07 / 08$ & 8D28005 & PNNL-AGG-ICP-AES \\
\hline $7440-48-4$ & Cobalt & $<0.096$ & ug/g dry & 0.096 & $5 / 07 / 08$ & 8D28005 & PNNL-AGG-ICP-AES \\
\hline $7440-47-3$ & Chromium & $<0.033$ & ug/g dry & 0.033 & $5 / 07 / 08$ & 8D28005 & PNNL-AGG-ICP-AES \\
\hline $7440-50-8$ & Copper & $<0.081$ & ug/g dry & 0.081 & $5 / 07 / 08$ & 8D28005 & PNNL-AGG-ICP-AES \\
\hline 7439-89-6 & Iron & 0.557 & ug/g dry & 0.143 & $5 / 07 / 08$ & 8D28005 & PNNL-AGG-ICP-AES \\
\hline 7440-09-7 & Potassium & 2.70 & ug/g dry & 2.33 & $5 / 07 / 08$ & 8D28005 & PNNL-AGG-ICP-AES \\
\hline $7439-93-2$ & Lithium & $<0.541$ & ug/g dry & 0.541 & $5 / 07 / 08$ & 8D28005 & PNNL-AGG-ICP-AES \\
\hline 7439-95-4 & Magnesium & 0.671 & ug/g dry & 0.084 & $5 / 07 / 08$ & 8D28005 & PNNL-AGG-ICP-AES \\
\hline $7439-96-5$ & Manganese & $<0.017$ & ug/g dry & 0.017 & $5 / 07 / 08$ & 8D28005 & PNNL-AGG-ICP-AES \\
\hline 7439-98-7 & Molybdenum & $<0.140$ & ug/g dry & 0.140 & $5 / 07 / 08$ & 8D28005 & PNNL-AGG-ICP-AES \\
\hline 7440-02-0 & Nickel & $<0.094$ & ug/g dry & 0.094 & $5 / 07 / 08$ & 8D28005 & PNNL-AGG-ICP-AES \\
\hline
\end{tabular}


Total Metals by SW846 6010B/Water Extract

\begin{tabular}{|c|c|c|c|c|c|c|c|}
\hline CAS \# & Analyte & Results & Units & EQL & Analyzed & Batch & Method \\
\hline HEIS No. & B1RYT0A & \multicolumn{3}{|c|}{ Lab ID: $\quad$ 0803014-02 } & & & \\
\hline 7723-14-0 & Phosphorus & $<1.03$ & ug/g dry & 1.03 & $5 / 07 / 08$ & 8D28005 & PNNL-AGG-ICP-AES \\
\hline 7439-92-1 & Lead & $<0.433$ & ug/g dry & 0.433 & $5 / 07 / 08$ & 8D28005 & PNNL-AGG-ICP-AES \\
\hline $7782-49-2$ & Selenium & $<1.95$ & ug/g dry & 1.95 & $5 / 07 / 08$ & 8D28005 & PNNL-AGG-ICP-AES \\
\hline $7440-24-6$ & Strontium & $<0.052$ & ug/g dry & 0.052 & $5 / 07 / 08$ & 8D28005 & PNNL-AGG-ICP-AES \\
\hline $7440-28-0$ & Thallium & $<1.03$ & ug/g dry & 1.03 & $5 / 07 / 08$ & 8D28005 & PNNL-AGG-ICP-AES \\
\hline $7440-62-2$ & Vanadium & 0.084 & ug/g dry & 0.045 & $5 / 07 / 08$ & 8D28005 & PNNL-AGG-ICP-AES \\
\hline $7440-66-6$ & Zinc & $<0.093$ & ug/g dry & 0.093 & $5 / 07 / 08$ & 8D28005 & PNNL-AGG-ICP-AES \\
\hline $7440-23-5$ & Sodium & 39.5 & ug/g dry & 0.670 & $5 / 07 / 08$ & 8D28005 & PNNL-AGG-ICP-AES \\
\hline $7440-21-3$ & Silicon & 15.8 & ug/g dry & 1.50 & $5 / 07 / 08$ & 8D28005 & PNNL-AGG-ICP-AES \\
\hline 7704-34-9 & Sulfur & 5.68 & ug/g dry & 3.08 & $5 / 07 / 08$ & 8D28005 & PNNL-AGG-ICP-AES \\
\hline $7440-32-6$ & Titanium & 0.026 & ug/g dry & 0.009 & $5 / 07 / 08$ & 8D28005 & PNNL-AGG-ICP-AES \\
\hline $7440-67-7$ & Zirconium & $<0.150$ & ug/g dry & 0.150 & $5 / 07 / 08$ & 8D28005 & PNNL-AGG-ICP-AES \\
\hline $7440-22-4$ & Silver & $<0.077$ & ug/g dry & 0.077 & $5 / 07 / 08$ & 8D28005 & PNNL-AGG-ICP-AES \\
\hline $7440-15-5$ & Rhenium & $<0.152$ & ug/g dry & 0.152 & $5 / 07 / 08$ & 8D28005 & PNNL-AGG-ICP-AES \\
\hline $7440-36-0$ & Antimony & $<0.634$ & ug/g dry & 0.634 & $5 / 07 / 08$ & 8D28005 & PNNL-AGG-ICP-AES \\
\hline HEIS No. & B1T2P1B & \multicolumn{3}{|c|}{ Lab ID: $\quad$ 0803014-05 } & & & \\
\hline 7429-90-5 & Aluminum & 0.205 & ug/g dry & 0.086 & $5 / 07 / 08$ & 8D28006 & PNNL-AGG-ICP-AES \\
\hline $7440-38-2$ & Arsenic & $<0.360$ & ug/g dry & 0.360 & $5 / 07 / 08$ & 8D28006 & PNNL-AGG-ICP-AES \\
\hline $7440-42-8$ & Boron & $<1.94$ & ug/g dry & 1.94 & $5 / 07 / 08$ & 8D28006 & PNNL-AGG-ICP-AES \\
\hline $7440-39-3$ & Barium & $<0.009$ & ug/g dry & 0.009 & $5 / 07 / 08$ & 8D28006 & PNNL-AGG-ICP-AES \\
\hline $7440-41-7$ & Beryllium & $<0.028$ & ug/g dry & 0.028 & $5 / 07 / 08$ & 8D28006 & PNNL-AGG-ICP-AES \\
\hline $7440-69-9$ & Bismuth & $<0.188$ & ug/g dry & 0.188 & $5 / 07 / 08$ & 8D28006 & PNNL-AGG-ICP-AES \\
\hline $7440-70-2$ & Calcium & 17.4 & ug/g dry & 0.387 & $5 / 07 / 08$ & 8D28006 & PNNL-AGG-ICP-AES \\
\hline $7440-43-9$ & Cadmium & $<0.027$ & ug/g dry & 0.027 & $5 / 07 / 08$ & 8D28006 & PNNL-AGG-ICP-AES \\
\hline $7440-48-4$ & Cobalt & $<0.096$ & ug/g dry & 0.096 & $5 / 07 / 08$ & 8D28006 & PNNL-AGG-ICP-AES \\
\hline $7440-47-3$ & Chromium & $<0.033$ & ug/g dry & 0.033 & $5 / 07 / 08$ & 8D28006 & PNNL-AGG-ICP-AES \\
\hline $7440-50-8$ & Copper & $<0.080$ & ug/g dry & 0.080 & $5 / 07 / 08$ & 8D28006 & PNNL-AGG-ICP-AES \\
\hline 7439-89-6 & Iron & $<0.142$ & ug/g dry & 0.142 & $5 / 07 / 08$ & 8D28006 & PNNL-AGG-ICP-AES \\
\hline 7440-09-7 & Potassium & 6.93 & ug/g dry & 2.33 & $5 / 07 / 08$ & 8D28006 & PNNL-AGG-ICP-AES \\
\hline $7439-93-2$ & Lithium & $<0.540$ & ug/g dry & 0.540 & $5 / 07 / 08$ & 8D28006 & PNNL-AGG-ICP-AES \\
\hline 7439-95-4 & Magnesium & 6.30 & ug/g dry & 0.083 & $5 / 07 / 08$ & 8D28006 & PNNL-AGG-ICP-AES \\
\hline 7439-96-5 & Manganese & $<0.017$ & ug/g dry & 0.017 & $5 / 07 / 08$ & 8D28006 & PNNL-AGG-ICP-AES \\
\hline 7439-98-7 & Molybdenum & $<0.139$ & ug/g dry & 0.139 & $5 / 07 / 08$ & 8D28006 & PNNL-AGG-ICP-AES \\
\hline $7440-02-0$ & Nickel & $<0.093$ & ug/g dry & 0.093 & $5 / 07 / 08$ & 8D28006 & PNNL-AGG-ICP-AES \\
\hline 7723-14-0 & Phosphorus & $<1.03$ & ug/g dry & 1.03 & $5 / 07 / 08$ & 8D28006 & PNNL-AGG-ICP-AES \\
\hline 7439-92-1 & Lead & $<0.432$ & ug/g dry & 0.432 & $5 / 07 / 08$ & 8D28006 & PNNL-AGG-ICP-AES \\
\hline $7782-49-2$ & Selenium & $<1.95$ & ug/g dry & 1.95 & $5 / 07 / 08$ & 8D28006 & PNNL-AGG-ICP-AES \\
\hline $7440-24-6$ & Strontium & 0.075 & ug/g dry & 0.052 & $5 / 07 / 08$ & 8D28006 & PNNL-AGG-ICP-AES \\
\hline $7440-28-0$ & Thallium & $<1.03$ & ug/g dry & 1.03 & $5 / 07 / 08$ & 8D28006 & PNNL-AGG-ICP-AES \\
\hline $7440-62-2$ & Vanadium & $<0.045$ & ug/g dry & 0.045 & $5 / 07 / 08$ & 8D28006 & PNNL-AGG-ICP-AES \\
\hline $7440-23-5$ & Sodium & 15.2 & ug/g dry & 0.669 & $5 / 07 / 08$ & 8D28006 & PNNL-AGG-ICP-AES \\
\hline $7440-21-3$ & Silicon & 6.15 & ug/g dry & 1.50 & $5 / 07 / 08$ & 8D28006 & PNNL-AGG-ICP-AES \\
\hline 7704-34-9 & Sulfur & 23.7 & ug/g dry & 3.08 & $5 / 07 / 08$ & 8D28006 & PNNL-AGG-ICP-AES \\
\hline $7440-32-6$ & Titanium & 0.010 & ug/g dry & 0.009 & $5 / 07 / 08$ & 8D28006 & PNNL-AGG-ICP-AES \\
\hline $7440-67-7$ & Zirconium & $<0.150$ & ug/g dry & 0.150 & $5 / 07 / 08$ & 8D28006 & PNNL-AGG-ICP-AES \\
\hline $7440-22-4$ & Silver & $<0.077$ & ug/g dry & 0.077 & $5 / 07 / 08$ & 8D28006 & PNNL-AGG-ICP-AES \\
\hline $7440-15-5$ & Rhenium & $<0.152$ & ug/g dry & 0.152 & $5 / 07 / 08$ & 8D28006 & PNNL-AGG-ICP-AES \\
\hline $7440-36-0$ & Antimony & $<0.633$ & ug/g dry & 0.633 & $5 / 07 / 08$ & 8D28006 & PNNL-AGG-ICP-AES \\
\hline
\end{tabular}


Total Metals by SW846 6010B/Water Extract

\begin{tabular}{|c|c|c|c|c|c|c|c|}
\hline CAS \# & Analyte & Results & Units & EQL & Analyzed & Batch & Method \\
\hline HEIS No. & B1T2P1A & \multicolumn{3}{|c|}{ Lab ID: $\quad$ 0803014-06 } & & & \\
\hline 7429-90-5 & Aluminum & 0.178 & ug/g dry & 0.086 & $5 / 07 / 08$ & 8D28006 & PNNL-AGG-ICP-AES \\
\hline $7440-38-2$ & Arsenic & $<0.360$ & ug/g dry & 0.360 & $5 / 07 / 08$ & 8D28006 & PNNL-AGG-ICP-AES \\
\hline $7440-42-8$ & Boron & $<1.94$ & ug/g dry & 1.94 & $5 / 07 / 08$ & 8D28006 & PNNL-AGG-ICP-AES \\
\hline $7440-39-3$ & Barium & $<0.009$ & ug/g dry & 0.009 & $5 / 07 / 08$ & 8D28006 & PNNL-AGG-ICP-AES \\
\hline $7440-41-7$ & Beryllium & $<0.028$ & ug/g dry & 0.028 & $5 / 07 / 08$ & 8D28006 & PNNL-AGG-ICP-AES \\
\hline 7440-69-9 & Bismuth & $<0.188$ & ug/g dry & 0.188 & $5 / 07 / 08$ & 8D28006 & PNNL-AGG-ICP-AES \\
\hline $7440-70-2$ & Calcium & 15.9 & ug/g dry & 0.387 & $5 / 07 / 08$ & 8D28006 & PNNL-AGG-ICP-AES \\
\hline $7440-43-9$ & Cadmium & $<0.027$ & ug/g dry & 0.027 & $5 / 07 / 08$ & 8D28006 & PNNL-AGG-ICP-AES \\
\hline $7440-48-4$ & Cobalt & $<0.096$ & ug/g dry & 0.096 & $5 / 07 / 08$ & 8D28006 & PNNL-AGG-ICP-AES \\
\hline $7440-47-3$ & Chromium & $<0.033$ & ug/g dry & 0.033 & $5 / 07 / 08$ & 8D28006 & PNNL-AGG-ICP-AES \\
\hline $7440-50-8$ & Copper & $<0.080$ & ug/g dry & 0.080 & $5 / 07 / 08$ & 8D28006 & PNNL-AGG-ICP-AES \\
\hline 7439-89-6 & Iron & $<0.142$ & ug/g dry & 0.142 & $5 / 07 / 08$ & 8D28006 & PNNL-AGG-ICP-AES \\
\hline 7440-09-7 & Potassium & 6.12 & ug/g dry & 2.33 & $5 / 07 / 08$ & 8D28006 & PNNL-AGG-ICP-AES \\
\hline 7439-93-2 & Lithium & $<0.540$ & ug/g dry & 0.540 & $5 / 07 / 08$ & 8D28006 & PNNL-AGG-ICP-AES \\
\hline $7439-95-4$ & Magnesium & 5.73 & ug/g dry & 0.083 & $5 / 07 / 08$ & 8D28006 & PNNL-AGG-ICP-AES \\
\hline 7439-96-5 & Manganese & $<0.017$ & ug/g dry & 0.017 & $5 / 07 / 08$ & 8D28006 & PNNL-AGG-ICP-AES \\
\hline 7439-98-7 & Molybdenum & $<0.139$ & ug/g dry & 0.139 & $5 / 07 / 08$ & 8D28006 & PNNL-AGG-ICP-AES \\
\hline $7440-02-0$ & Nickel & $<0.093$ & ug/g dry & 0.093 & $5 / 07 / 08$ & 8D28006 & PNNL-AGG-ICP-AES \\
\hline $7723-14-0$ & Phosphorus & $<1.03$ & ug/g dry & 1.03 & $5 / 07 / 08$ & 8D28006 & PNNL-AGG-ICP-AES \\
\hline 7439-92-1 & Lead & $<0.432$ & ug/g dry & 0.432 & $5 / 07 / 08$ & 8D28006 & PNNL-AGG-ICP-AES \\
\hline $7782-49-2$ & Selenium & $<1.95$ & ug/g dry & 1.95 & $5 / 07 / 08$ & 8D28006 & PNNL-AGG-ICP-AES \\
\hline $7440-24-6$ & Strontium & 0.067 & ug/g dry & 0.052 & $5 / 07 / 08$ & 8D28006 & PNNL-AGG-ICP-AES \\
\hline $7440-28-0$ & Thallium & $<1.03$ & ug/g dry & 1.03 & $5 / 07 / 08$ & 8D28006 & PNNL-AGG-ICP-AES \\
\hline $7440-62-2$ & Vanadium & $<0.045$ & ug/g dry & 0.045 & $5 / 07 / 08$ & 8D28006 & PNNL-AGG-ICP-AES \\
\hline $7440-23-5$ & Sodium & 13.6 & ug/g dry & 0.669 & $5 / 07 / 08$ & 8D28006 & PNNL-AGG-ICP-AES \\
\hline $7440-21-3$ & Silicon & 5.77 & ug/g dry & 1.50 & $5 / 07 / 08$ & 8D28006 & PNNL-AGG-ICP-AES \\
\hline 7704-34-9 & Sulfur & 21.7 & ug/g dry & 3.07 & $5 / 07 / 08$ & 8D28006 & PNNL-AGG-ICP-AES \\
\hline $7440-32-6$ & Titanium & $<0.009$ & ug/g dry & 0.009 & $5 / 07 / 08$ & 8D28006 & PNNL-AGG-ICP-AES \\
\hline $7440-67-7$ & Zirconium & $<0.150$ & ug/g dry & 0.150 & $5 / 07 / 08$ & 8D28006 & PNNL-AGG-ICP-AES \\
\hline $7440-22-4$ & Silver & $<0.077$ & ug/g dry & 0.077 & $5 / 07 / 08$ & 8D28006 & PNNL-AGG-ICP-AES \\
\hline $7440-15-5$ & Rhenium & $<0.152$ & ug/g dry & 0.152 & $5 / 07 / 08$ & 8D28006 & PNNL-AGG-ICP-AES \\
\hline $7440-36-0$ & Antimony & $<0.633$ & ug/g dry & 0.633 & $5 / 07 / 08$ & 8D28006 & PNNL-AGG-ICP-AES \\
\hline HEIS No. & B1T2P2B & & b ID: & 3014-09 & & & \\
\hline $7429-90-5$ & Aluminum & $<0.087$ & ug/g dry & 0.087 & $5 / 07 / 08$ & 8D28006 & PNNL-AGG-ICP-AES \\
\hline $7440-38-2$ & Arsenic & $<0.365$ & ug/g dry & 0.365 & $5 / 07 / 08$ & 8D28006 & PNNL-AGG-ICP-AES \\
\hline $7440-42-8$ & Boron & $<1.97$ & ug/g dry & 1.97 & $5 / 07 / 08$ & 8D28006 & PNNL-AGG-ICP-AES \\
\hline $7440-39-3$ & Barium & 0.016 & ug/g dry & 0.009 & $5 / 07 / 08$ & 8D28006 & PNNL-AGG-ICP-AES \\
\hline $7440-41-7$ & Beryllium & $<0.029$ & ug/g dry & 0.029 & $5 / 07 / 08$ & $8 \mathrm{D} 28006$ & PNNL-AGG-ICP-AES \\
\hline 7440-69-9 & Bismuth & $<0.190$ & ug/g dry & 0.190 & $5 / 07 / 08$ & 8D28006 & PNNL-AGG-ICP-AES \\
\hline $7440-70-2$ & Calcium & 34.1 & ug/g dry & 0.392 & $5 / 07 / 08$ & 8D28006 & PNNL-AGG-ICP-AES \\
\hline $7440-43-9$ & Cadmium & $<0.027$ & ug/g dry & 0.027 & $5 / 07 / 08$ & 8D28006 & PNNL-AGG-ICP-AES \\
\hline $7440-48-4$ & Cobalt & $<0.097$ & ug/g dry & 0.097 & $5 / 07 / 08$ & 8D28006 & PNNL-AGG-ICP-AES \\
\hline $7440-47-3$ & Chromium & $<0.034$ & ug/g dry & 0.034 & $5 / 07 / 08$ & 8D28006 & PNNL-AGG-ICP-AES \\
\hline $7440-50-8$ & Copper & $<0.081$ & ug/g dry & 0.081 & $5 / 07 / 08$ & 8D28006 & PNNL-AGG-ICP-AES \\
\hline 7439-89-6 & Iron & $<0.144$ & ug/g dry & 0.144 & $5 / 07 / 08$ & 8D28006 & PNNL-AGG-ICP-AES \\
\hline $7440-09-7$ & Potassium & 8.52 & ug/g dry & 2.36 & $5 / 07 / 08$ & 8D28006 & PNNL-AGG-ICP-AES \\
\hline 7439-93-2 & Lithium & $<0.547$ & ug/g dry & 0.547 & $5 / 07 / 08$ & 8D28006 & PNNL-AGG-ICP-AES \\
\hline 7439-95-4 & Magnesium & 11.9 & ug/g dry & 0.084 & $5 / 07 / 08$ & 8D28006 & PNNL-AGG-ICP-AES \\
\hline
\end{tabular}


Total Metals by SW846 6010B/Water Extract

\begin{tabular}{|c|c|c|c|c|c|c|c|}
\hline CAS \# & Analyte & Results & Units & EQL & Analyzed & Batch & Method \\
\hline HEIS No. & B1T2P2B & \multicolumn{3}{|c|}{ Lab ID: $\quad$ 0803014-09 } & & & \\
\hline 7439-96-5 & Manganese & $<0.017$ & ug/g dry & 0.017 & $5 / 07 / 08$ & 8D28006 & PNNL-AGG-ICP-AES \\
\hline 7439-98-7 & Molybdenum & $<0.141$ & ug/g dry & 0.141 & $5 / 07 / 08$ & 8D28006 & PNNL-AGG-ICP-AES \\
\hline $7440-02-0$ & Nickel & $<0.094$ & ug/g dry & 0.094 & $5 / 07 / 08$ & 8D28006 & PNNL-AGG-ICP-AES \\
\hline 7723-14-0 & Phosphorus & $<1.05$ & ug/g dry & 1.05 & $5 / 07 / 08$ & 8D28006 & PNNL-AGG-ICP-AES \\
\hline 7439-92-1 & Lead & $<0.437$ & ug/g dry & 0.437 & $5 / 07 / 08$ & 8D28006 & PNNL-AGG-ICP-AES \\
\hline $7782-49-2$ & Selenium & $<1.97$ & ug/g dry & 1.97 & $5 / 07 / 08$ & 8D28006 & PNNL-AGG-ICP-AES \\
\hline $7440-24-6$ & Strontium & 0.147 & ug/g dry & 0.053 & $5 / 07 / 08$ & 8D28006 & PNNL-AGG-ICP-AES \\
\hline $7440-28-0$ & Thallium & $<1.04$ & ug/g dry & 1.04 & $5 / 07 / 08$ & 8D28006 & PNNL-AGG-ICP-AES \\
\hline $7440-62-2$ & Vanadium & $<0.046$ & ug/g dry & 0.046 & $5 / 07 / 08$ & 8D28006 & PNNL-AGG-ICP-AES \\
\hline $7440-23-5$ & Sodium & 16.0 & ug/g dry & 0.677 & $5 / 07 / 08$ & 8D28006 & PNNL-AGG-ICP-AES \\
\hline $7440-21-3$ & Silicon & 6.00 & ug/g dry & 1.52 & $5 / 07 / 08$ & 8D28006 & PNNL-AGG-ICP-AES \\
\hline $7704-34-9$ & Sulfur & 29.2 & ug/g dry & 3.11 & $5 / 07 / 08$ & 8D28006 & PNNL-AGG-ICP-AES \\
\hline $7440-32-6$ & Titanium & $<0.009$ & ug/g dry & 0.009 & $5 / 07 / 08$ & 8D28006 & PNNL-AGG-ICP-AES \\
\hline $7440-67-7$ & Zirconium & $<0.152$ & ug/g dry & 0.152 & $5 / 07 / 08$ & 8D28006 & PNNL-AGG-ICP-AES \\
\hline $7440-22-4$ & Silver & $<0.078$ & ug/g dry & 0.078 & $5 / 07 / 08$ & 8D28006 & PNNL-AGG-ICP-AES \\
\hline $7440-15-5$ & Rhenium & $<0.154$ & ug/g dry & 0.154 & $5 / 07 / 08$ & 8D28006 & PNNL-AGG-ICP-AES \\
\hline $7440-36-0$ & Antimony & $<0.641$ & ug/g dry & 0.641 & $5 / 07 / 08$ & 8D28006 & PNNL-AGG-ICP-AES \\
\hline HEIS No. & B1T2P2A & \multicolumn{3}{|c|}{ Lab ID: $\quad$ 0803014-10 } & & & \\
\hline $7429-90-5$ & Aluminum & $<0.086$ & ug/g dry & 0.086 & $5 / 07 / 08$ & 8D28006 & PNNL-AGG-ICP-AES \\
\hline $7440-38-2$ & Arsenic & $<0.360$ & ug/g dry & 0.360 & $5 / 07 / 08$ & 8D28006 & PNNL-AGG-ICP-AES \\
\hline $7440-42-8$ & Boron & $<1.94$ & ug/g dry & 1.94 & $5 / 07 / 08$ & 8D28006 & PNNL-AGG-ICP-AES \\
\hline $7440-39-3$ & Barium & $<0.009$ & ug/g dry & 0.009 & $5 / 07 / 08$ & 8D28006 & PNNL-AGG-ICP-AES \\
\hline $7440-41-7$ & Beryllium & $<0.028$ & ug/g dry & 0.028 & $5 / 07 / 08$ & 8D28006 & PNNL-AGG-ICP-AES \\
\hline $7440-69-9$ & Bismuth & $<0.188$ & ug/g dry & 0.188 & $5 / 07 / 08$ & 8D28006 & PNNL-AGG-ICP-AES \\
\hline $7440-70-2$ & Calcium & 30.0 & ug/g dry & 0.387 & $5 / 07 / 08$ & 8D28006 & PNNL-AGG-ICP-AES \\
\hline $7440-43-9$ & Cadmium & $<0.027$ & ug/g dry & 0.027 & $5 / 07 / 08$ & 8D28006 & PNNL-AGG-ICP-AES \\
\hline $7440-48-4$ & Cobalt & $<0.096$ & ug/g dry & 0.096 & $5 / 07 / 08$ & 8D28006 & PNNL-AGG-ICP-AES \\
\hline $7440-47-3$ & Chromium & $<0.033$ & ug/g dry & 0.033 & $5 / 07 / 08$ & 8D28006 & PNNL-AGG-ICP-AES \\
\hline $7440-50-8$ & Copper & $<0.080$ & ug/g dry & 0.080 & $5 / 07 / 08$ & 8D28006 & PNNL-AGG-ICP-AES \\
\hline 7439-89-6 & Iron & $<0.142$ & ug/g dry & 0.142 & $5 / 07 / 08$ & 8D28006 & PNNL-AGG-ICP-AES \\
\hline 7440-09-7 & Potassium & 7.83 & ug/g dry & 2.33 & $5 / 07 / 08$ & 8D28006 & PNNL-AGG-ICP-AES \\
\hline $7439-93-2$ & Lithium & $<0.540$ & ug/g dry & 0.540 & $5 / 07 / 08$ & 8D28006 & PNNL-AGG-ICP-AES \\
\hline $7439-95-4$ & Magnesium & 10.2 & ug/g dry & 0.083 & $5 / 07 / 08$ & 8D28006 & PNNL-AGG-ICP-AES \\
\hline $7439-96-5$ & Manganese & $<0.017$ & ug/g dry & 0.017 & $5 / 07 / 08$ & 8D28006 & PNNL-AGG-ICP-AES \\
\hline 7439-98-7 & Molybdenum & $<0.139$ & ug/g dry & 0.139 & $5 / 07 / 08$ & 8D28006 & PNNL-AGG-ICP-AES \\
\hline $7440-02-0$ & Nickel & $<0.093$ & ug/g dry & 0.093 & $5 / 07 / 08$ & 8D28006 & PNNL-AGG-ICP-AES \\
\hline 7723-14-0 & Phosphorus & $<1.03$ & ug/g dry & 1.03 & $5 / 07 / 08$ & 8D28006 & PNNL-AGG-ICP-AES \\
\hline $7439-92-1$ & Lead & $<0.432$ & ug/g dry & 0.432 & $5 / 07 / 08$ & 8D28006 & PNNL-AGG-ICP-AES \\
\hline $7782-49-2$ & Selenium & $<1.95$ & ug/g dry & 1.95 & $5 / 07 / 08$ & 8D28006 & PNNL-AGG-ICP-AES \\
\hline $7440-24-6$ & Strontium & 0.125 & ug/g dry & 0.052 & $5 / 07 / 08$ & 8D28006 & PNNL-AGG-ICP-AES \\
\hline $7440-28-0$ & Thallium & $<1.03$ & ug/g dry & 1.03 & $5 / 07 / 08$ & 8D28006 & PNNL-AGG-ICP-AES \\
\hline $7440-62-2$ & Vanadium & $<0.045$ & ug/g dry & 0.045 & $5 / 07 / 08$ & 8D28006 & PNNL-AGG-ICP-AES \\
\hline $7440-23-5$ & Sodium & 13.6 & ug/g dry & 0.669 & $5 / 07 / 08$ & 8D28006 & PNNL-AGG-ICP-AES \\
\hline $7440-21-3$ & Silicon & 5.68 & ug/g dry & 1.50 & $5 / 07 / 08$ & 8D28006 & PNNL-AGG-ICP-AES \\
\hline 7704-34-9 & Sulfur & 30.3 & ug/g dry & 3.08 & $5 / 07 / 08$ & 8D28006 & PNNL-AGG-ICP-AES \\
\hline $7440-32-6$ & Titanium & $<0.009$ & ug/g dry & 0.009 & $5 / 07 / 08$ & 8D28006 & PNNL-AGG-ICP-AES \\
\hline $7440-67-7$ & Zirconium & $<0.150$ & ug/g dry & 0.150 & $5 / 07 / 08$ & 8D28006 & PNNL-AGG-ICP-AES \\
\hline $7440-22-4$ & Silver & $<0.077$ & ug/g dry & 0.077 & $5 / 07 / 08$ & 8D28006 & PNNL-AGG-ICP-AES \\
\hline
\end{tabular}


Total Metals by SW846 6010B/Water Extract

\begin{tabular}{|c|c|c|c|c|c|c|c|}
\hline CAS \# & Analyte & Results & Units & EQL & Analyzed & Batch & Method \\
\hline HEIS No. & B1T2P2A & \multicolumn{3}{|c|}{ Lab ID: $\quad$ 0803014-10 } & & & \\
\hline $7440-15-5$ & Rhenium & $<0.152$ & ug/g dry & 0.152 & $5 / 07 / 08$ & 8D28006 & PNNL-AGG-ICP-AES \\
\hline $7440-36-0$ & Antimony & $<0.633$ & ug/g dry & 0.633 & $5 / 07 / 08$ & 8D28006 & PNNL-AGG-ICP-AES \\
\hline HEIS No. & В1Т2Р3В & \multicolumn{3}{|c|}{ Lab ID: $\quad$ 0803014-13 } & & & \\
\hline $7429-90-5$ & Aluminum & 0.219 & ug/g dry & 0.076 & $5 / 07 / 08$ & 8D28006 & PNNL-AGG-ICP-AES \\
\hline $7440-38-2$ & Arsenic & $<0.321$ & ug/g dry & 0.321 & $5 / 07 / 08$ & 8D28006 & PNNL-AGG-ICP-AES \\
\hline $7440-42-8$ & Boron & $<1.73$ & ug/g dry & 1.73 & $5 / 07 / 08$ & 8D28006 & PNNL-AGG-ICP-AES \\
\hline $7440-39-3$ & Barium & $<0.008$ & ug/g dry & 0.008 & $5 / 07 / 08$ & 8D28006 & PNNL-AGG-ICP-AES \\
\hline $7440-41-7$ & Beryllium & $<0.025$ & ug/g dry & 0.025 & $5 / 07 / 08$ & 8D28006 & PNNL-AGG-ICP-AES \\
\hline $7440-69-9$ & Bismuth & $<0.168$ & ug/g dry & 0.168 & $5 / 07 / 08$ & 8D28006 & PNNL-AGG-ICP-AES \\
\hline $7440-70-2$ & Calcium & 11.8 & ug/g dry & 0.345 & $5 / 07 / 08$ & 8D28006 & PNNL-AGG-ICP-AES \\
\hline $7440-43-9$ & Cadmium & $<0.024$ & ug/g dry & 0.024 & $5 / 07 / 08$ & 8D28006 & PNNL-AGG-ICP-AES \\
\hline $7440-48-4$ & Cobalt & $<0.086$ & ug/g dry & 0.086 & $5 / 07 / 08$ & 8D28006 & PNNL-AGG-ICP-AES \\
\hline $7440-47-3$ & Chromium & $<0.030$ & ug/g dry & 0.030 & $5 / 07 / 08$ & 8D28006 & PNNL-AGG-ICP-AES \\
\hline $7440-50-8$ & Copper & $<0.072$ & ug/g dry & 0.072 & $5 / 07 / 08$ & 8D28006 & PNNL-AGG-ICP-AES \\
\hline 7439-89-6 & Iron & 0.168 & ug/g dry & 0.127 & $5 / 07 / 08$ & 8D28006 & PNNL-AGG-ICP-AES \\
\hline 7440-09-7 & Potassium & 4.53 & ug/g dry & 2.08 & $5 / 07 / 08$ & 8D28006 & PNNL-AGG-ICP-AES \\
\hline 7439-93-2 & Lithium & $<0.481$ & ug/g dry & 0.481 & $5 / 07 / 08$ & 8D28006 & PNNL-AGG-ICP-AES \\
\hline 7439-95-4 & Magnesium & 4.17 & ug/g dry & 0.074 & $5 / 07 / 08$ & 8D28006 & PNNL-AGG-ICP-AES \\
\hline 7439-96-5 & Manganese & $<0.015$ & ug/g dry & 0.015 & $5 / 07 / 08$ & 8D28006 & PNNL-AGG-ICP-AES \\
\hline 7439-98-7 & Molybdenum & $<0.124$ & ug/g dry & 0.124 & $5 / 07 / 08$ & 8D28006 & PNNL-AGG-ICP-AES \\
\hline $7440-02-0$ & Nickel & $<0.083$ & ug/g dry & 0.083 & $5 / 07 / 08$ & 8D28006 & PNNL-AGG-ICP-AES \\
\hline $7723-14-0$ & Phosphorus & $<0.920$ & ug/g dry & 0.920 & $5 / 07 / 08$ & 8D28006 & PNNL-AGG-ICP-AES \\
\hline 7439-92-1 & Lead & $<0.385$ & ug/g dry & 0.385 & $5 / 07 / 08$ & 8D28006 & PNNL-AGG-ICP-AES \\
\hline $7782-49-2$ & Selenium & $<1.74$ & ug/g dry & 1.74 & $5 / 07 / 08$ & 8D28006 & PNNL-AGG-ICP-AES \\
\hline 7440-24-6 & Strontium & 0.059 & ug/g dry & 0.047 & $5 / 07 / 08$ & 8D28006 & PNNL-AGG-ICP-AES \\
\hline $7440-28-0$ & Thallium & $<0.915$ & ug/g dry & 0.915 & $5 / 07 / 08$ & 8D28006 & PNNL-AGG-ICP-AES \\
\hline $7440-62-2$ & Vanadium & $<0.040$ & ug/g dry & 0.040 & $5 / 07 / 08$ & 8D28006 & PNNL-AGG-ICP-AES \\
\hline $7440-23-5$ & Sodium & 8.43 & ug/g dry & 0.596 & $5 / 07 / 08$ & 8D28006 & PNNL-AGG-ICP-AES \\
\hline $7440-21-3$ & Silicon & 5.52 & ug/g dry & 1.34 & $5 / 07 / 08$ & 8D28006 & PNNL-AGG-ICP-AES \\
\hline 7704-34-9 & Sulfur & 14.0 & ug/g dry & 2.74 & $5 / 07 / 08$ & 8D28006 & PNNL-AGG-ICP-AES \\
\hline $7440-32-6$ & Titanium & 0.010 & ug/g dry & 0.008 & $5 / 07 / 08$ & 8D28006 & PNNL-AGG-ICP-AES \\
\hline $7440-67-7$ & Zirconium & $<0.134$ & ug/g dry & 0.134 & $5 / 07 / 08$ & 8D28006 & PNNL-AGG-ICP-AES \\
\hline $7440-22-4$ & Silver & $<0.069$ & ug/g dry & 0.069 & $5 / 07 / 08$ & 8D28006 & PNNL-AGG-ICP-AES \\
\hline $7440-15-5$ & Rhenium & $<0.135$ & ug/g dry & 0.135 & $5 / 07 / 08$ & 8D28006 & PNNL-AGG-ICP-AES \\
\hline $7440-36-0$ & Antimony & $<0.564$ & ug/g dry & 0.564 & $5 / 07 / 08$ & 8D28006 & PNNL-AGG-ICP-AES \\
\hline HEIS No. & В1T2P3A & & b ID: & 3014-14 & & & \\
\hline $7429-90-5$ & Aluminum & 0.129 & ug/g dry & 0.066 & $5 / 07 / 08$ & 8D28006 & PNNL-AGG-ICP-AES \\
\hline $7440-38-2$ & Arsenic & $<0.278$ & ug/g dry & 0.278 & $5 / 07 / 08$ & 8D28006 & PNNL-AGG-ICP-AES \\
\hline $7440-42-8$ & Boron & $<1.50$ & ug/g dry & 1.50 & $5 / 07 / 08$ & 8D28006 & PNNL-AGG-ICP-AES \\
\hline $7440-39-3$ & Barium & $<0.007$ & ug/g dry & 0.007 & $5 / 07 / 08$ & 8D28006 & PNNL-AGG-ICP-AES \\
\hline $7440-41-7$ & Beryllium & $<0.022$ & ug/g dry & 0.022 & $5 / 07 / 08$ & 8D28006 & PNNL-AGG-ICP-AES \\
\hline $7440-69-9$ & Bismuth & $<0.145$ & ug/g dry & 0.145 & $5 / 07 / 08$ & 8D28006 & PNNL-AGG-ICP-AES \\
\hline $7440-70-2$ & Calcium & 12.5 & ug/g dry & 0.299 & $5 / 07 / 08$ & 8D28006 & PNNL-AGG-ICP-AES \\
\hline $7440-43-9$ & Cadmium & $<0.021$ & ug/g dry & 0.021 & $5 / 07 / 08$ & 8D28006 & PNNL-AGG-ICP-AES \\
\hline $7440-48-4$ & Cobalt & $<0.074$ & ug/g dry & 0.074 & $5 / 07 / 08$ & 8D28006 & PNNL-AGG-ICP-AES \\
\hline $7440-47-3$ & Chromium & $<0.026$ & ug/g dry & 0.026 & $5 / 07 / 08$ & 8D28006 & PNNL-AGG-ICP-AES \\
\hline $7440-50-8$ & Copper & $<0.062$ & ug/g dry & 0.062 & $5 / 07 / 08$ & 8D28006 & PNNL-AGG-ICP-AES \\
\hline 7439-89-6 & Iron & $<0.110$ & ug/g dry & 0.110 & $5 / 07 / 08$ & 8D28006 & PNNL-AGG-ICP-AES \\
\hline
\end{tabular}


Total Metals by SW846 6010B/Water Extract

\begin{tabular}{|c|c|c|c|c|c|c|c|}
\hline CAS \# & Analyte & Results & Units & EQL & Analyzed & Batch & Method \\
\hline HEIS No. & В1T2P3A & \multicolumn{3}{|c|}{ Lab ID: $\quad$ 0803014-14 } & & & \\
\hline 7440-09-7 & Potassium & 4.55 & ug/g dry & 1.80 & $5 / 07 / 08$ & 8D28006 & PNNL-AGG-ICP-AES \\
\hline 7439-93-2 & Lithium & $<0.417$ & ug/g dry & 0.417 & $5 / 07 / 08$ & 8D28006 & PNNL-AGG-ICP-AES \\
\hline 7439-95-4 & Magnesium & 4.47 & ug/g dry & 0.064 & $5 / 07 / 08$ & 8D28006 & PNNL-AGG-ICP-AES \\
\hline 7439-96-5 & Manganese & $<0.013$ & ug/g dry & 0.013 & $5 / 07 / 08$ & 8D28006 & PNNL-AGG-ICP-AES \\
\hline 7439-98-7 & Molybdenum & $<0.108$ & ug/g dry & 0.108 & $5 / 07 / 08$ & 8D28006 & PNNL-AGG-ICP-AES \\
\hline 7440-02-0 & Nickel & $<0.072$ & ug/g dry & 0.072 & $5 / 07 / 08$ & 8D28006 & PNNL-AGG-ICP-AES \\
\hline $7723-14-0$ & Phosphorus & $<0.798$ & ug/g dry & 0.798 & $5 / 07 / 08$ & 8D28006 & PNNL-AGG-ICP-AES \\
\hline 7439-92-1 & Lead & $<0.334$ & ug/g dry & 0.334 & $5 / 07 / 08$ & 8D28006 & PNNL-AGG-ICP-AES \\
\hline $7782-49-2$ & Selenium & $<1.51$ & ug/g dry & 1.51 & $5 / 07 / 08$ & 8D28006 & PNNL-AGG-ICP-AES \\
\hline $7440-24-6$ & Strontium & 0.066 & ug/g dry & 0.040 & $5 / 07 / 08$ & 8D28006 & PNNL-AGG-ICP-AES \\
\hline $7440-28-0$ & Thallium & $<0.793$ & ug/g dry & 0.793 & $5 / 07 / 08$ & 8D28006 & PNNL-AGG-ICP-AES \\
\hline $7440-62-2$ & Vanadium & $<0.035$ & ug/g dry & 0.035 & $5 / 07 / 08$ & 8D28006 & PNNL-AGG-ICP-AES \\
\hline $7440-23-5$ & Sodium & 9.11 & ug/g dry & 0.517 & $5 / 07 / 08$ & 8D28006 & PNNL-AGG-ICP-AES \\
\hline $7440-21-3$ & Silicon & 5.70 & ug/g dry & 1.16 & $5 / 07 / 08$ & 8D28006 & PNNL-AGG-ICP-AES \\
\hline $7704-34-9$ & Sulfur & 15.4 & ug/g dry & 2.38 & $5 / 07 / 08$ & 8D28006 & PNNL-AGG-ICP-AES \\
\hline $7440-32-6$ & Titanium & $<0.007$ & ug/g dry & 0.007 & $5 / 07 / 08$ & 8D28006 & PNNL-AGG-ICP-AES \\
\hline $7440-67-7$ & Zirconium & $<0.116$ & ug/g dry & 0.116 & $5 / 07 / 08$ & 8D28006 & PNNL-AGG-ICP-AES \\
\hline $7440-22-4$ & Silver & $<0.060$ & ug/g dry & 0.060 & $5 / 07 / 08$ & 8D28006 & PNNL-AGG-ICP-AES \\
\hline $7440-15-5$ & Rhenium & $<0.117$ & ug/g dry & 0.117 & $5 / 07 / 08$ & 8D28006 & PNNL-AGG-ICP-AES \\
\hline $7440-36-0$ & Antimony & $<0.489$ & ug/g dry & 0.489 & $5 / 07 / 08$ & 8D28006 & PNNL-AGG-ICP-AES \\
\hline HEIS No. & B1T2P4B & \multicolumn{3}{|c|}{ Lab ID: $\quad$ 0803014-16 } & & & \\
\hline $7429-90-5$ & Aluminum & 0.176 & ug/g dry & 0.086 & $5 / 07 / 08$ & 8D28006 & PNNL-AGG-ICP-AES \\
\hline $7440-38-2$ & Arsenic & $<0.360$ & ug/g dry & 0.360 & $5 / 07 / 08$ & 8D28006 & PNNL-AGG-ICP-AES \\
\hline $7440-42-8$ & Boron & $<1.94$ & ug/g dry & 1.94 & $5 / 07 / 08$ & 8D28006 & PNNL-AGG-ICP-AES \\
\hline $7440-39-3$ & Barium & $<0.009$ & ug/g dry & 0.009 & $5 / 07 / 08$ & 8D28006 & PNNL-AGG-ICP-AES \\
\hline $7440-41-7$ & Beryllium & $<0.028$ & ug/g dry & 0.028 & $5 / 07 / 08$ & 8D28006 & PNNL-AGG-ICP-AES \\
\hline $7440-69-9$ & Bismuth & $<0.188$ & ug/g dry & 0.188 & $5 / 07 / 08$ & 8D28006 & PNNL-AGG-ICP-AES \\
\hline $7440-70-2$ & Calcium & 17.7 & ug/g dry & 0.387 & $5 / 07 / 08$ & 8D28006 & PNNL-AGG-ICP-AES \\
\hline $7440-43-9$ & Cadmium & $<0.027$ & ug/g dry & 0.027 & $5 / 07 / 08$ & 8D28006 & PNNL-AGG-ICP-AES \\
\hline $7440-48-4$ & Cobalt & $<0.096$ & ug/g dry & 0.096 & $5 / 07 / 08$ & 8D28006 & PNNL-AGG-ICP-AES \\
\hline $7440-47-3$ & Chromium & $<0.033$ & ug/g dry & 0.033 & $5 / 07 / 08$ & 8D28006 & PNNL-AGG-ICP-AES \\
\hline $7440-50-8$ & Copper & $<0.080$ & ug/g dry & 0.080 & $5 / 07 / 08$ & 8D28006 & PNNL-AGG-ICP-AES \\
\hline 7439-89-6 & Iron & $<0.142$ & ug/g dry & 0.142 & $5 / 07 / 08$ & 8D28006 & PNNL-AGG-ICP-AES \\
\hline $7440-09-7$ & Potassium & 7.45 & ug/g dry & 2.33 & $5 / 07 / 08$ & 8D28006 & PNNL-AGG-ICP-AES \\
\hline $7439-93-2$ & Lithium & $<0.540$ & ug/g dry & 0.540 & $5 / 07 / 08$ & 8D28006 & PNNL-AGG-ICP-AES \\
\hline $7439-95-4$ & Magnesium & 6.93 & ug/g dry & 0.083 & $5 / 07 / 08$ & 8D28006 & PNNL-AGG-ICP-AES \\
\hline 7439-96-5 & Manganese & $<0.017$ & ug/g dry & 0.017 & $5 / 07 / 08$ & 8D28006 & PNNL-AGG-ICP-AES \\
\hline 7439-98-7 & Molybdenum & $<0.139$ & ug/g dry & 0.139 & $5 / 07 / 08$ & 8D28006 & PNNL-AGG-ICP-AES \\
\hline $7440-02-0$ & Nickel & $<0.093$ & ug/g dry & 0.093 & $5 / 07 / 08$ & 8D28006 & PNNL-AGG-ICP-AES \\
\hline $7723-14-0$ & Phosphorus & $<1.03$ & ug/g dry & 1.03 & $5 / 07 / 08$ & 8D28006 & PNNL-AGG-ICP-AES \\
\hline $7439-92-1$ & Lead & $<0.432$ & ug/g dry & 0.432 & $5 / 07 / 08$ & 8D28006 & PNNL-AGG-ICP-AES \\
\hline $7782-49-2$ & Selenium & $<1.95$ & ug/g dry & 1.95 & $5 / 07 / 08$ & 8D28006 & PNNL-AGG-ICP-AES \\
\hline $7440-24-6$ & Strontium & 0.087 & ug/g dry & 0.052 & $5 / 07 / 08$ & 8D28006 & PNNL-AGG-ICP-AES \\
\hline $7440-28-0$ & Thallium & $<1.03$ & ug/g dry & 1.03 & $5 / 07 / 08$ & 8D28006 & PNNL-AGG-ICP-AES \\
\hline $7440-62-2$ & Vanadium & $<0.045$ & ug/g dry & 0.045 & $5 / 07 / 08$ & 8D28006 & PNNL-AGG-ICP-AES \\
\hline $7440-23-5$ & Sodium & 15.8 & ug/g dry & 0.670 & $5 / 07 / 08$ & 8D28006 & PNNL-AGG-ICP-AES \\
\hline $7440-21-3$ & Silicon & 6.62 & ug/g dry & 1.50 & $5 / 07 / 08$ & 8D28006 & PNNL-AGG-ICP-AES \\
\hline 7704-34-9 & Sulfur & 26.3 & ug/g dry & 3.08 & $5 / 07 / 08$ & 8D28006 & PNNL-AGG-ICP-AES \\
\hline
\end{tabular}


Total Metals by SW846 6010B/Water Extract

\begin{tabular}{|c|c|c|c|c|c|c|c|}
\hline CAS \# & Analyte & Results & Units & EQL & Analyzed & Batch & Method \\
\hline HEIS No. & В1T2P4B & \multicolumn{3}{|c|}{ Lab ID: $\quad$ 0803014-16 } & & & \\
\hline $7440-32-6$ & Titanium & $<0.009$ & ug/g dry & 0.009 & $5 / 07 / 08$ & 8D28006 & PNNL-AGG-ICP-AES \\
\hline $7440-67-7$ & Zirconium & $<0.150$ & ug/g dry & 0.150 & $5 / 07 / 08$ & 8D28006 & PNNL-AGG-ICP-AES \\
\hline $7440-22-4$ & Silver & $<0.077$ & ug/g dry & 0.077 & $5 / 07 / 08$ & 8D28006 & PNNL-AGG-ICP-AES \\
\hline $7440-15-5$ & Rhenium & $<0.152$ & ug/g dry & 0.152 & $5 / 07 / 08$ & 8D28006 & PNNL-AGG-ICP-AES \\
\hline $7440-36-0$ & Antimony & $<0.634$ & ug/g dry & 0.634 & $5 / 07 / 08$ & 8D28006 & PNNL-AGG-ICP-AES \\
\hline HEIS No. & B1T2P4A & \multicolumn{3}{|c|}{ Lab ID: $\quad$ 0803014-17 } & & & \\
\hline 7429-90-5 & Aluminum & 0.156 & ug/g dry & 0.086 & $5 / 07 / 08$ & 8D28006 & PNNL-AGG-ICP-AES \\
\hline $7440-38-2$ & Arsenic & $<0.361$ & ug/g dry & 0.361 & $5 / 07 / 08$ & 8D28006 & PNNL-AGG-ICP-AES \\
\hline $7440-42-8$ & Boron & $<1.95$ & ug/g dry & 1.95 & $5 / 07 / 08$ & 8D28006 & PNNL-AGG-ICP-AES \\
\hline $7440-39-3$ & Barium & $<0.009$ & ug/g dry & 0.009 & 5/07/08 & 8D28006 & PNNL-AGG-ICP-AES \\
\hline $7440-41-7$ & Beryllium & $<0.028$ & ug/g dry & 0.028 & $5 / 07 / 08$ & 8D28006 & PNNL-AGG-ICP-AES \\
\hline 7440-69-9 & Bismuth & $<0.189$ & ug/g dry & 0.189 & $5 / 07 / 08$ & 8D28006 & PNNL-AGG-ICP-AES \\
\hline $7440-70-2$ & Calcium & 17.9 & ug/g dry & 0.388 & $5 / 07 / 08$ & 8D28006 & PNNL-AGG-ICP-AES \\
\hline $7440-43-9$ & Cadmium & $<0.027$ & ug/g dry & 0.027 & $5 / 07 / 08$ & 8D28006 & PNNL-AGG-ICP-AES \\
\hline $7440-48-4$ & Cobalt & $<0.096$ & ug/g dry & 0.096 & $5 / 07 / 08$ & 8D28006 & PNNL-AGG-ICP-AES \\
\hline $7440-47-3$ & Chromium & $<0.033$ & ug/g dry & 0.033 & $5 / 07 / 08$ & 8D28006 & PNNL-AGG-ICP-AES \\
\hline $7440-50-8$ & Copper & $<0.081$ & ug/g dry & 0.081 & $5 / 07 / 08$ & 8D28006 & PNNL-AGG-ICP-AES \\
\hline 7439-89-6 & Iron & $<0.143$ & ug/g dry & 0.143 & $5 / 07 / 08$ & 8D28006 & PNNL-AGG-ICP-AES \\
\hline $7440-09-7$ & Potassium & 7.69 & ug/g dry & 2.34 & $5 / 07 / 08$ & 8D28006 & PNNL-AGG-ICP-AES \\
\hline 7439-93-2 & Lithium & $<0.542$ & ug/g dry & 0.542 & $5 / 07 / 08$ & 8D28006 & PNNL-AGG-ICP-AES \\
\hline 7439-95-4 & Magnesium & 7.09 & ug/g dry & 0.084 & $5 / 07 / 08$ & 8D28006 & PNNL-AGG-ICP-AES \\
\hline 7439-96-5 & Manganese & $<0.017$ & ug/g dry & 0.017 & $5 / 07 / 08$ & 8D28006 & PNNL-AGG-ICP-AES \\
\hline 7439-98-7 & Molybdenum & $<0.140$ & ug/g dry & 0.140 & $5 / 07 / 08$ & 8D28006 & PNNL-AGG-ICP-AES \\
\hline 7440-02-0 & Nickel & $<0.094$ & ug/g dry & 0.094 & $5 / 07 / 08$ & 8D28006 & PNNL-AGG-ICP-AES \\
\hline 7723-14-0 & Phosphorus & $<1.04$ & ug/g dry & 1.04 & $5 / 07 / 08$ & 8D28006 & PNNL-AGG-ICP-AES \\
\hline 7439-92-1 & Lead & $<0.433$ & ug/g dry & 0.433 & $5 / 07 / 08$ & 8D28006 & PNNL-AGG-ICP-AES \\
\hline $7782-49-2$ & Selenium & $<1.96$ & ug/g dry & 1.96 & $5 / 07 / 08$ & 8D28006 & PNNL-AGG-ICP-AES \\
\hline $7440-24-6$ & Strontium & 0.086 & ug/g dry & 0.052 & $5 / 07 / 08$ & 8D28006 & PNNL-AGG-ICP-AES \\
\hline $7440-28-0$ & Thallium & $<1.03$ & ug/g dry & 1.03 & $5 / 07 / 08$ & 8D28006 & PNNL-AGG-ICP-AES \\
\hline $7440-62-2$ & Vanadium & $<0.045$ & ug/g dry & 0.045 & $5 / 07 / 08$ & 8D28006 & PNNL-AGG-ICP-AES \\
\hline $7440-23-5$ & Sodium & 16.8 & ug/g dry & 0.671 & $5 / 07 / 08$ & 8D28006 & PNNL-AGG-ICP-AES \\
\hline $7440-21-3$ & Silicon & 7.03 & ug/g dry & 1.50 & $5 / 07 / 08$ & 8D28006 & PNNL-AGG-ICP-AES \\
\hline $7704-34-9$ & Sulfur & 26.2 & ug/g dry & 3.08 & $5 / 07 / 08$ & 8D28006 & PNNL-AGG-ICP-AES \\
\hline $7440-32-6$ & Titanium & $<0.009$ & ug/g dry & 0.009 & $5 / 07 / 08$ & 8D28006 & PNNL-AGG-ICP-AES \\
\hline $7440-67-7$ & Zirconium & $<0.150$ & ug/g dry & 0.150 & $5 / 07 / 08$ & 8D28006 & PNNL-AGG-ICP-AES \\
\hline $7440-22-4$ & Silver & $<0.077$ & ug/g dry & 0.077 & $5 / 07 / 08$ & 8D28006 & PNNL-AGG-ICP-AES \\
\hline $7440-15-5$ & Rhenium & $<0.152$ & ug/g dry & 0.152 & $5 / 07 / 08$ & 8D28006 & PNNL-AGG-ICP-AES \\
\hline $7440-36-0$ & Antimony & $<0.635$ & ug/g dry & 0.635 & $5 / 07 / 08$ & 8D28006 & PNNL-AGG-ICP-AES \\
\hline HEIS No. & B1T2P5B & \multicolumn{3}{|c|}{ Lab ID: $\quad$ 0803014-20 } & & & \\
\hline 7429-90-5 & Aluminum & 0.101 & ug/g dry & 0.086 & $5 / 07 / 08$ & 8D28006 & PNNL-AGG-ICP-AES \\
\hline $7440-38-2$ & Arsenic & $<0.360$ & ug/g dry & 0.360 & $5 / 07 / 08$ & 8D28006 & PNNL-AGG-ICP-AES \\
\hline $7440-42-8$ & Boron & $<1.94$ & ug/g dry & 1.94 & $5 / 07 / 08$ & 8D28006 & PNNL-AGG-ICP-AES \\
\hline 7440-39-3 & Barium & $<0.009$ & ug/g dry & 0.009 & $5 / 07 / 08$ & 8D28006 & PNNL-AGG-ICP-AES \\
\hline $7440-41-7$ & Beryllium & $<0.028$ & ug/g dry & 0.028 & $5 / 07 / 08$ & 8D28006 & PNNL-AGG-ICP-AES \\
\hline $7440-69-9$ & Bismuth & $<0.188$ & ug/g dry & 0.188 & $5 / 07 / 08$ & 8D28006 & PNNL-AGG-ICP-AES \\
\hline $7440-70-2$ & Calcium & 24.4 & ug/g dry & 0.387 & $5 / 07 / 08$ & 8D28006 & PNNL-AGG-ICP-AES \\
\hline $7440-43-9$ & Cadmium & $<0.027$ & ug/g dry & 0.027 & $5 / 07 / 08$ & 8D28006 & PNNL-AGG-ICP-AES \\
\hline $7440-48-4$ & Cobalt & $<0.096$ & ug/g dry & 0.096 & $5 / 07 / 08$ & 8D28006 & PNNL-AGG-ICP-AES \\
\hline
\end{tabular}


Total Metals by SW846 6010B/Water Extract

\begin{tabular}{|c|c|c|c|c|c|c|c|}
\hline CAS \# & Analyte & Results & Units & EQL & Analyzed & Batch & Method \\
\hline HEIS No. & B1T2P5B & \multicolumn{3}{|c|}{ Lab ID: $\quad$ 0803014-20 } & & & \\
\hline $7440-47-3$ & Chromium & $<0.033$ & ug/g dry & 0.033 & $5 / 07 / 08$ & 8D28006 & PNNL-AGG-ICP-AES \\
\hline $7440-50-8$ & Copper & $<0.080$ & ug/g dry & 0.080 & $5 / 07 / 08$ & 8D28006 & PNNL-AGG-ICP-AES \\
\hline 7439-89-6 & Iron & $<0.142$ & ug/g dry & 0.142 & $5 / 07 / 08$ & 8D28006 & PNNL-AGG-ICP-AES \\
\hline 7440-09-7 & Potassium & 8.37 & ug/g dry & 2.33 & $5 / 07 / 08$ & 8D28006 & PNNL-AGG-ICP-AES \\
\hline 7439-93-2 & Lithium & $<0.540$ & ug/g dry & 0.540 & $5 / 07 / 08$ & 8D28006 & PNNL-AGG-ICP-AES \\
\hline 7439-95-4 & Magnesium & 9.30 & ug/g dry & 0.083 & $5 / 07 / 08$ & 8D28006 & PNNL-AGG-ICP-AES \\
\hline $7439-96-5$ & Manganese & $<0.017$ & ug/g dry & 0.017 & $5 / 07 / 08$ & 8D28006 & PNNL-AGG-ICP-AES \\
\hline 7439-98-7 & Molybdenum & $<0.139$ & ug/g dry & 0.139 & $5 / 07 / 08$ & 8D28006 & PNNL-AGG-ICP-AES \\
\hline 7440-02-0 & Nickel & $<0.093$ & ug/g dry & 0.093 & $5 / 07 / 08$ & 8D28006 & PNNL-AGG-ICP-AES \\
\hline 7723-14-0 & Phosphorus & $<1.03$ & ug/g dry & 1.03 & $5 / 07 / 08$ & 8D28006 & PNNL-AGG-ICP-AES \\
\hline 7439-92-1 & Lead & $<0.432$ & ug/g dry & 0.432 & $5 / 07 / 08$ & 8D28006 & PNNL-AGG-ICP-AES \\
\hline $7782-49-2$ & Selenium & $<1.95$ & ug/g dry & 1.95 & $5 / 07 / 08$ & 8D28006 & PNNL-AGG-ICP-AES \\
\hline $7440-24-6$ & Strontium & 0.115 & ug/g dry & 0.052 & $5 / 07 / 08$ & 8D28006 & PNNL-AGG-ICP-AES \\
\hline $7440-28-0$ & Thallium & $<1.03$ & ug/g dry & 1.03 & $5 / 07 / 08$ & 8D28006 & PNNL-AGG-ICP-AES \\
\hline $7440-62-2$ & Vanadium & $<0.045$ & ug/g dry & 0.045 & $5 / 07 / 08$ & 8D28006 & PNNL-AGG-ICP-AES \\
\hline $7440-23-5$ & Sodium & 18.5 & ug/g dry & 0.669 & $5 / 07 / 08$ & 8D28006 & PNNL-AGG-ICP-AES \\
\hline $7440-21-3$ & Silicon & 7.70 & ug/g dry & 1.50 & $5 / 07 / 08$ & 8D28006 & PNNL-AGG-ICP-AES \\
\hline $7704-34-9$ & Sulfur & 34.1 & ug/g dry & 3.08 & $5 / 07 / 08$ & 8D28006 & PNNL-AGG-ICP-AES \\
\hline $7440-32-6$ & Titanium & $<0.009$ & ug/g dry & 0.009 & $5 / 07 / 08$ & 8D28006 & PNNL-AGG-ICP-AES \\
\hline $7440-67-7$ & Zirconium & $<0.150$ & ug/g dry & 0.150 & $5 / 07 / 08$ & 8D28006 & PNNL-AGG-ICP-AES \\
\hline $7440-22-4$ & Silver & $<0.077$ & ug/g dry & 0.077 & $5 / 07 / 08$ & 8D28006 & PNNL-AGG-ICP-AES \\
\hline $7440-15-5$ & Rhenium & $<0.152$ & ug/g dry & 0.152 & $5 / 07 / 08$ & 8D28006 & PNNL-AGG-ICP-AES \\
\hline $7440-36-0$ & Antimony & $<0.633$ & ug/g dry & 0.633 & $5 / 07 / 08$ & 8D28006 & PNNL-AGG-ICP-AES \\
\hline HEIS No. & B1T2P5A & \multicolumn{3}{|c|}{ Lab ID: $\quad$ 0803014-21 } & & & \\
\hline 7429-90-5 & Aluminum & $<0.086$ & ug/g dry & 0.086 & $5 / 07 / 08$ & 8D28006 & PNNL-AGG-ICP-AES \\
\hline $7440-38-2$ & Arsenic & $<0.360$ & ug/g dry & 0.360 & $5 / 07 / 08$ & 8D28006 & PNNL-AGG-ICP-AES \\
\hline $7440-42-8$ & Boron & $<1.94$ & ug/g dry & 1.94 & $5 / 07 / 08$ & 8D28006 & PNNL-AGG-ICP-AES \\
\hline $7440-39-3$ & Barium & $<0.009$ & ug/g dry & 0.009 & $5 / 07 / 08$ & 8D28006 & PNNL-AGG-ICP-AES \\
\hline $7440-41-7$ & Beryllium & $<0.028$ & ug/g dry & 0.028 & $5 / 07 / 08$ & 8D28006 & PNNL-AGG-ICP-AES \\
\hline $7440-69-9$ & Bismuth & $<0.188$ & ug/g dry & 0.188 & $5 / 07 / 08$ & 8D28006 & PNNL-AGG-ICP-AES \\
\hline $7440-70-2$ & Calcium & 33.6 & ug/g dry & 0.387 & $5 / 07 / 08$ & 8D28006 & PNNL-AGG-ICP-AES \\
\hline $7440-43-9$ & Cadmium & $<0.027$ & ug/g dry & 0.027 & $5 / 07 / 08$ & 8D28006 & PNNL-AGG-ICP-AES \\
\hline $7440-48-4$ & Cobalt & $<0.096$ & ug/g dry & 0.096 & $5 / 07 / 08$ & 8D28006 & PNNL-AGG-ICP-AES \\
\hline $7440-47-3$ & Chromium & $<0.033$ & ug/g dry & 0.033 & $5 / 07 / 08$ & 8D28006 & PNNL-AGG-ICP-AES \\
\hline $7440-50-8$ & Copper & $<0.080$ & ug/g dry & 0.080 & $5 / 07 / 08$ & 8D28006 & PNNL-AGG-ICP-AES \\
\hline 7439-89-6 & Iron & $<0.142$ & ug/g dry & 0.142 & $5 / 07 / 08$ & 8D28006 & PNNL-AGG-ICP-AES \\
\hline 7440-09-7 & Potassium & 9.28 & ug/g dry & 2.33 & $5 / 07 / 08$ & 8D28006 & PNNL-AGG-ICP-AES \\
\hline 7439-93-2 & Lithium & $<0.540$ & ug/g dry & 0.540 & $5 / 07 / 08$ & 8D28006 & PNNL-AGG-ICP-AES \\
\hline 7439-95-4 & Magnesium & 12.6 & ug/g dry & 0.083 & $5 / 07 / 08$ & 8D28006 & PNNL-AGG-ICP-AES \\
\hline 7439-96-5 & Manganese & $<0.017$ & ug/g dry & 0.017 & $5 / 07 / 08$ & 8D28006 & PNNL-AGG-ICP-AES \\
\hline 7439-98-7 & Molybdenum & $<0.139$ & ug/g dry & 0.139 & $5 / 07 / 08$ & 8D28006 & PNNL-AGG-ICP-AES \\
\hline $7440-02-0$ & Nickel & $<0.093$ & ug/g dry & 0.093 & $5 / 07 / 08$ & 8D28006 & PNNL-AGG-ICP-AES \\
\hline 7723-14-0 & Phosphorus & $<1.03$ & ug/g dry & 1.03 & $5 / 07 / 08$ & 8D28006 & PNNL-AGG-ICP-AES \\
\hline $7439-92-1$ & Lead & $<0.432$ & ug/g dry & 0.432 & $5 / 07 / 08$ & 8D28006 & PNNL-AGG-ICP-AES \\
\hline $7782-49-2$ & Selenium & $<1.95$ & ug/g dry & 1.95 & $5 / 07 / 08$ & 8D28006 & PNNL-AGG-ICP-AES \\
\hline $7440-24-6$ & Strontium & 0.154 & ug/g dry & 0.052 & $5 / 07 / 08$ & 8D28006 & PNNL-AGG-ICP-AES \\
\hline $7440-28-0$ & Thallium & $<1.03$ & ug/g dry & 1.03 & $5 / 07 / 08$ & 8D28006 & PNNL-AGG-ICP-AES \\
\hline $7440-62-2$ & Vanadium & $<0.045$ & ug/g dry & 0.045 & $5 / 07 / 08$ & 8D28006 & PNNL-AGG-ICP-AES \\
\hline
\end{tabular}


Total Metals by SW846 6010B/Water Extract

\begin{tabular}{|c|c|c|c|c|c|c|c|}
\hline CAS \# & Analyte & Results & Units & EQL & Analyzed & Batch & Method \\
\hline HEIS No. & B1T2P5A & \multicolumn{3}{|c|}{ Lab ID: $\quad$ 0803014-21 } & & & \\
\hline $7440-23-5$ & Sodium & 20.6 & ug/g dry & 0.669 & $5 / 07 / 08$ & 8D28006 & PNNL-AGG-ICP-AES \\
\hline $7440-21-3$ & Silicon & 7.40 & ug/g dry & 1.50 & $5 / 07 / 08$ & 8D28006 & PNNL-AGG-ICP-AES \\
\hline 7704-34-9 & Sulfur & 49.0 & ug/g dry & 3.08 & $5 / 07 / 08$ & 8D28006 & PNNL-AGG-ICP-AES \\
\hline $7440-32-6$ & Titanium & $<0.009$ & ug/g dry & 0.009 & $5 / 07 / 08$ & 8D28006 & PNNL-AGG-ICP-AES \\
\hline $7440-67-7$ & Zirconium & $<0.150$ & ug/g dry & 0.150 & $5 / 07 / 08$ & 8D28006 & PNNL-AGG-ICP-AES \\
\hline $7440-22-4$ & Silver & $<0.077$ & ug/g dry & 0.077 & $5 / 07 / 08$ & 8D28006 & PNNL-AGG-ICP-AES \\
\hline $7440-15-5$ & Rhenium & $<0.152$ & ug/g dry & 0.152 & $5 / 07 / 08$ & 8D28006 & PNNL-AGG-ICP-AES \\
\hline $7440-36-0$ & Antimony & $<0.633$ & ug/g dry & 0.633 & $5 / 07 / 08$ & 8D28006 & PNNL-AGG-ICP-AES \\
\hline HEIS No. & В1T2P6B & \multicolumn{3}{|c|}{ Lab ID: $\quad$ 0803014-24 } & & & \\
\hline $7429-90-5$ & Aluminum & 0.272 & ug/g dry & 0.086 & $5 / 07 / 08$ & 8D28006 & PNNL-AGG-ICP-AES \\
\hline $7440-38-2$ & Arsenic & $<0.360$ & ug/g dry & 0.360 & $5 / 07 / 08$ & 8D28006 & PNNL-AGG-ICP-AES \\
\hline $7440-42-8$ & Boron & $<1.94$ & ug/g dry & 1.94 & $5 / 07 / 08$ & 8D28006 & PNNL-AGG-ICP-AES \\
\hline $7440-39-3$ & Barium & $<0.009$ & ug/g dry & 0.009 & $5 / 07 / 08$ & 8D28006 & PNNL-AGG-ICP-AES \\
\hline $7440-41-7$ & Beryllium & $<0.028$ & ug/g dry & 0.028 & $5 / 07 / 08$ & 8D28006 & PNNL-AGG-ICP-AES \\
\hline $7440-69-9$ & Bismuth & $<0.188$ & ug/g dry & 0.188 & $5 / 07 / 08$ & 8D28006 & PNNL-AGG-ICP-AES \\
\hline $7440-70-2$ & Calcium & 13.2 & ug/g dry & 0.387 & $5 / 07 / 08$ & 8D28006 & PNNL-AGG-ICP-AES \\
\hline 7440-43-9 & Cadmium & $<0.027$ & ug/g dry & 0.027 & $5 / 07 / 08$ & 8D28006 & PNNL-AGG-ICP-AES \\
\hline $7440-48-4$ & Cobalt & $<0.096$ & ug/g dry & 0.096 & $5 / 07 / 08$ & 8D28006 & PNNL-AGG-ICP-AES \\
\hline $7440-47-3$ & Chromium & $<0.033$ & ug/g dry & 0.033 & $5 / 07 / 08$ & 8D28006 & PNNL-AGG-ICP-AES \\
\hline $7440-50-8$ & Copper & $<0.080$ & ug/g dry & 0.080 & $5 / 07 / 08$ & 8D28006 & PNNL-AGG-ICP-AES \\
\hline 7439-89-6 & Iron & 0.186 & ug/g dry & 0.142 & $5 / 07 / 08$ & 8D28006 & PNNL-AGG-ICP-AES \\
\hline $7440-09-7$ & Potassium & 7.76 & ug/g dry & 2.33 & $5 / 07 / 08$ & 8D28006 & PNNL-AGG-ICP-AES \\
\hline 7439-93-2 & Lithium & $<0.540$ & ug/g dry & 0.540 & $5 / 07 / 08$ & 8D28006 & PNNL-AGG-ICP-AES \\
\hline 7439-95-4 & Magnesium & 5.15 & ug/g dry & 0.083 & $5 / 07 / 08$ & 8D28006 & PNNL-AGG-ICP-AES \\
\hline 7439-96-5 & Manganese & $<0.017$ & ug/g dry & 0.017 & $5 / 07 / 08$ & 8D28006 & PNNL-AGG-ICP-AES \\
\hline 7439-98-7 & Molybdenum & $<0.139$ & ug/g dry & 0.139 & $5 / 07 / 08$ & 8D28006 & PNNL-AGG-ICP-AES \\
\hline $7440-02-0$ & Nickel & $<0.093$ & ug/g dry & 0.093 & $5 / 07 / 08$ & 8D28006 & PNNL-AGG-ICP-AES \\
\hline 7723-14-0 & Phosphorus & $<1.03$ & ug/g dry & 1.03 & $5 / 07 / 08$ & 8D28006 & PNNL-AGG-ICP-AES \\
\hline 7439-92-1 & Lead & $<0.432$ & ug/g dry & 0.432 & $5 / 07 / 08$ & 8D28006 & PNNL-AGG-ICP-AES \\
\hline $7782-49-2$ & Selenium & $<1.95$ & ug/g dry & 1.95 & $5 / 07 / 08$ & 8D28006 & PNNL-AGG-ICP-AES \\
\hline $7440-24-6$ & Strontium & 0.063 & ug/g dry & 0.052 & $5 / 07 / 08$ & 8D28006 & PNNL-AGG-ICP-AES \\
\hline $7440-28-0$ & Thallium & $<1.03$ & ug/g dry & 1.03 & $5 / 07 / 08$ & 8D28006 & PNNL-AGG-ICP-AES \\
\hline $7440-62-2$ & Vanadium & $<0.045$ & ug/g dry & 0.045 & $5 / 07 / 08$ & 8D28006 & PNNL-AGG-ICP-AES \\
\hline $7440-23-5$ & Sodium & 16.6 & ug/g dry & 0.669 & $5 / 07 / 08$ & 8D28006 & PNNL-AGG-ICP-AES \\
\hline $7440-21-3$ & Silicon & 6.97 & ug/g dry & 1.50 & $5 / 07 / 08$ & 8D28006 & PNNL-AGG-ICP-AES \\
\hline 7704-34-9 & Sulfur & 19.6 & ug/g dry & 3.07 & $5 / 07 / 08$ & 8D28006 & PNNL-AGG-ICP-AES \\
\hline $7440-32-6$ & Titanium & 0.012 & ug/g dry & 0.009 & $5 / 07 / 08$ & 8D28006 & PNNL-AGG-ICP-AES \\
\hline $7440-67-7$ & Zirconium & $<0.150$ & ug/g dry & 0.150 & $5 / 07 / 08$ & $8 \mathrm{D} 28006$ & PNNL-AGG-ICP-AES \\
\hline $7440-22-4$ & Silver & $<0.077$ & ug/g dry & 0.077 & $5 / 07 / 08$ & 8D28006 & PNNL-AGG-ICP-AES \\
\hline $7440-15-5$ & Rhenium & $<0.152$ & ug/g dry & 0.152 & $5 / 07 / 08$ & 8D28006 & PNNL-AGG-ICP-AES \\
\hline $7440-36-0$ & Antimony & $<0.633$ & ug/g dry & 0.633 & $5 / 07 / 08$ & 8D28006 & PNNL-AGG-ICP-AES \\
\hline HEIS No. & B1T2P6A & & b ID: & 3014-25 & & & \\
\hline $7429-90-5$ & Aluminum & 0.216 & ug/g dry & 0.086 & $5 / 07 / 08$ & 8D28006 & PNNL-AGG-ICP-AES \\
\hline $7440-38-2$ & Arsenic & $<0.360$ & ug/g dry & 0.360 & $5 / 07 / 08$ & 8D28006 & PNNL-AGG-ICP-AES \\
\hline $7440-42-8$ & Boron & $<1.94$ & ug/g dry & 1.94 & $5 / 07 / 08$ & 8D28006 & PNNL-AGG-ICP-AES \\
\hline 7440-39-3 & Barium & $<0.009$ & ug/g dry & 0.009 & $5 / 07 / 08$ & 8D28006 & PNNL-AGG-ICP-AES \\
\hline $7440-41-7$ & Beryllium & $<0.028$ & ug/g dry & 0.028 & $5 / 07 / 08$ & 8D28006 & PNNL-AGG-ICP-AES \\
\hline 7440-69-9 & Bismuth & $<0.188$ & ug/g dry & 0.188 & $5 / 07 / 08$ & 8D28006 & PNNL-AGG-ICP-AES \\
\hline
\end{tabular}


Total Metals by SW846 6010B/Water Extract

\begin{tabular}{|c|c|c|c|c|c|c|c|}
\hline CAS \# & Analyte & Results & Units & EQL & Analyzed & Batch & Method \\
\hline HEIS No. & B1T2P6A & \multicolumn{3}{|c|}{ Lab ID: $\quad$ 0803014-25 } & & & \\
\hline $7440-70-2$ & Calcium & 11.0 & ug/g dry & 0.387 & $5 / 07 / 08$ & 8D28006 & PNNL-AGG-ICP-AES \\
\hline 7440-43-9 & Cadmium & $<0.027$ & ug/g dry & 0.027 & $5 / 07 / 08$ & 8D28006 & PNNL-AGG-ICP-AES \\
\hline $7440-48-4$ & Cobalt & $<0.096$ & ug/g dry & 0.096 & $5 / 07 / 08$ & 8D28006 & PNNL-AGG-ICP-AES \\
\hline $7440-47-3$ & Chromium & $<0.033$ & ug/g dry & 0.033 & $5 / 07 / 08$ & 8D28006 & PNNL-AGG-ICP-AES \\
\hline $7440-50-8$ & Copper & $<0.080$ & ug/g dry & 0.080 & $5 / 07 / 08$ & 8D28006 & PNNL-AGG-ICP-AES \\
\hline 7439-89-6 & Iron & 0.145 & ug/g dry & 0.142 & $5 / 07 / 08$ & 8D28006 & PNNL-AGG-ICP-AES \\
\hline 7440-09-7 & Potassium & 6.48 & ug/g dry & 2.33 & $5 / 07 / 08$ & 8D28006 & PNNL-AGG-ICP-AES \\
\hline $7439-93-2$ & Lithium & $<0.540$ & ug/g dry & 0.540 & $5 / 07 / 08$ & 8D28006 & PNNL-AGG-ICP-AES \\
\hline 7439-95-4 & Magnesium & 4.16 & ug/g dry & 0.083 & $5 / 07 / 08$ & 8D28006 & PNNL-AGG-ICP-AES \\
\hline 7439-96-5 & Manganese & $<0.017$ & ug/g dry & 0.017 & $5 / 07 / 08$ & 8D28006 & PNNL-AGG-ICP-AES \\
\hline 7439-98-7 & Molybdenum & $<0.139$ & ug/g dry & 0.139 & $5 / 07 / 08$ & 8D28006 & PNNL-AGG-ICP-AES \\
\hline $7440-02-0$ & Nickel & $<0.093$ & ug/g dry & 0.093 & $5 / 07 / 08$ & 8D28006 & PNNL-AGG-ICP-AES \\
\hline 7723-14-0 & Phosphorus & $<1.03$ & ug/g dry & 1.03 & $5 / 07 / 08$ & 8D28006 & PNNL-AGG-ICP-AES \\
\hline 7439-92-1 & Lead & $<0.432$ & ug/g dry & 0.432 & $5 / 07 / 08$ & 8D28006 & PNNL-AGG-ICP-AES \\
\hline $7782-49-2$ & Selenium & $<1.95$ & ug/g dry & 1.95 & $5 / 07 / 08$ & 8D28006 & PNNL-AGG-ICP-AES \\
\hline $7440-24-6$ & Strontium & 0.056 & ug/g dry & 0.052 & $5 / 07 / 08$ & 8D28006 & PNNL-AGG-ICP-AES \\
\hline $7440-28-0$ & Thallium & $<1.03$ & ug/g dry & 1.03 & $5 / 07 / 08$ & 8D28006 & PNNL-AGG-ICP-AES \\
\hline $7440-62-2$ & Vanadium & $<0.045$ & ug/g dry & 0.045 & $5 / 07 / 08$ & 8D28006 & PNNL-AGG-ICP-AES \\
\hline $7440-23-5$ & Sodium & 14.4 & ug/g dry & 0.669 & $5 / 07 / 08$ & 8D28006 & PNNL-AGG-ICP-AES \\
\hline $7440-21-3$ & Silicon & 6.94 & ug/g dry & 1.50 & $5 / 07 / 08$ & 8D28006 & PNNL-AGG-ICP-AES \\
\hline $7704-34-9$ & Sulfur & 15.5 & ug/g dry & 3.08 & $5 / 07 / 08$ & 8D28006 & PNNL-AGG-ICP-AES \\
\hline $7440-32-6$ & Titanium & 0.010 & ug/g dry & 0.009 & $5 / 07 / 08$ & 8D28006 & PNNL-AGG-ICP-AES \\
\hline $7440-67-7$ & Zirconium & $<0.150$ & ug/g dry & 0.150 & $5 / 07 / 08$ & 8D28006 & PNNL-AGG-ICP-AES \\
\hline $7440-22-4$ & Silver & $<0.077$ & ug/g dry & 0.077 & $5 / 07 / 08$ & 8D28006 & PNNL-AGG-ICP-AES \\
\hline $7440-15-5$ & Rhenium & $<0.152$ & ug/g dry & 0.152 & $5 / 07 / 08$ & 8D28006 & PNNL-AGG-ICP-AES \\
\hline $7440-36-0$ & Antimony & $<0.633$ & ug/g dry & 0.633 & $5 / 07 / 08$ & 8D28006 & PNNL-AGG-ICP-AES \\
\hline HEIS No. & В1T2P7B & \multicolumn{3}{|c|}{ Lab ID: $\quad$ 0803015-02 } & & & \\
\hline 7429-90-5 & Aluminum & $<0.086$ & ug/g dry & 0.086 & $5 / 07 / 08$ & 8D28006 & PNNL-AGG-ICP-AES \\
\hline $7440-38-2$ & Arsenic & $<0.360$ & ug/g dry & 0.360 & $5 / 07 / 08$ & 8D28006 & PNNL-AGG-ICP-AES \\
\hline $7440-42-8$ & Boron & $<1.94$ & ug/g dry & 1.94 & $5 / 07 / 08$ & 8D28006 & PNNL-AGG-ICP-AES \\
\hline $7440-39-3$ & Barium & $<0.009$ & ug/g dry & 0.009 & $5 / 07 / 08$ & 8D28006 & PNNL-AGG-ICP-AES \\
\hline $7440-41-7$ & Beryllium & $<0.028$ & ug/g dry & 0.028 & $5 / 07 / 08$ & 8D28006 & PNNL-AGG-ICP-AES \\
\hline $7440-69-9$ & Bismuth & $<0.188$ & ug/g dry & 0.188 & $5 / 07 / 08$ & 8D28006 & PNNL-AGG-ICP-AES \\
\hline $7440-70-2$ & Calcium & 28.9 & ug/g dry & 0.387 & $5 / 07 / 08$ & 8D28006 & PNNL-AGG-ICP-AES \\
\hline $7440-43-9$ & Cadmium & $<0.027$ & ug/g dry & 0.027 & $5 / 07 / 08$ & 8D28006 & PNNL-AGG-ICP-AES \\
\hline $7440-48-4$ & Cobalt & $<0.096$ & ug/g dry & 0.096 & $5 / 07 / 08$ & 8D28006 & PNNL-AGG-ICP-AES \\
\hline $7440-47-3$ & Chromium & $<0.033$ & ug/g dry & 0.033 & $5 / 07 / 08$ & 8D28006 & PNNL-AGG-ICP-AES \\
\hline $7440-50-8$ & Copper & $<0.080$ & ug/g dry & 0.080 & $5 / 07 / 08$ & 8D28006 & PNNL-AGG-ICP-AES \\
\hline 7439-89-6 & Iron & $<0.142$ & ug/g dry & 0.142 & $5 / 07 / 08$ & 8D28006 & PNNL-AGG-ICP-AES \\
\hline 7440-09-7 & Potassium & 6.76 & ug/g dry & 2.33 & $5 / 07 / 08$ & 8D28006 & PNNL-AGG-ICP-AES \\
\hline $7439-93-2$ & Lithium & $<0.540$ & ug/g dry & 0.540 & $5 / 07 / 08$ & 8D28006 & PNNL-AGG-ICP-AES \\
\hline 7439-95-4 & Magnesium & 5.66 & ug/g dry & 0.083 & $5 / 07 / 08$ & 8D28006 & PNNL-AGG-ICP-AES \\
\hline $7439-96-5$ & Manganese & $<0.017$ & ug/g dry & 0.017 & $5 / 07 / 08$ & 8D28006 & PNNL-AGG-ICP-AES \\
\hline 7439-98-7 & Molybdenum & $<0.139$ & ug/g dry & 0.139 & $5 / 07 / 08$ & 8D28006 & PNNL-AGG-ICP-AES \\
\hline $7440-02-0$ & Nickel & $<0.093$ & ug/g dry & 0.093 & $5 / 07 / 08$ & 8D28006 & PNNL-AGG-ICP-AES \\
\hline $7723-14-0$ & Phosphorus & $<1.03$ & ug/g dry & 1.03 & $5 / 07 / 08$ & 8D28006 & PNNL-AGG-ICP-AES \\
\hline 7439-92-1 & Lead & $<0.432$ & ug/g dry & 0.432 & $5 / 07 / 08$ & 8D28006 & PNNL-AGG-ICP-AES \\
\hline $7782-49-2$ & Selenium & $<1.95$ & ug/g dry & 1.95 & $5 / 07 / 08$ & 8D28006 & PNNL-AGG-ICP-AES \\
\hline
\end{tabular}


Total Metals by SW846 6010B/Water Extract

\begin{tabular}{|c|c|c|c|c|c|c|c|}
\hline CAS \# & Analyte & Results & Units & EQL & Analyzed & Batch & Method \\
\hline HEIS No. & B1T2P7B & \multicolumn{3}{|c|}{ Lab ID: $\quad$ 0803015-02 } & & & \\
\hline $7440-24-6$ & Strontium & 0.095 & ug/g dry & 0.052 & $5 / 07 / 08$ & 8D28006 & PNNL-AGG-ICP-AES \\
\hline $7440-28-0$ & Thallium & $<1.03$ & ug/g dry & 1.03 & 5/07/08 & 8D28006 & PNNL-AGG-ICP-AES \\
\hline $7440-62-2$ & Vanadium & $<0.045$ & ug/g dry & 0.045 & $5 / 07 / 08$ & 8D28006 & PNNL-AGG-ICP-AES \\
\hline $7440-23-5$ & Sodium & 75.3 & ug/g dry & 0.669 & $5 / 07 / 08$ & 8D28006 & PNNL-AGG-ICP-AES \\
\hline $7440-21-3$ & Silicon & 10.8 & ug/g dry & 1.50 & $5 / 07 / 08$ & 8D28006 & PNNL-AGG-ICP-AES \\
\hline 7704-34-9 & Sulfur & 68.7 & ug/g dry & 3.08 & $5 / 07 / 08$ & 8D28006 & PNNL-AGG-ICP-AES \\
\hline $7440-32-6$ & Titanium & $<0.009$ & ug/g dry & 0.009 & $5 / 07 / 08$ & 8D28006 & PNNL-AGG-ICP-AES \\
\hline $7440-67-7$ & Zirconium & $<0.150$ & ug/g dry & 0.150 & $5 / 07 / 08$ & 8D28006 & PNNL-AGG-ICP-AES \\
\hline $7440-22-4$ & Silver & $<0.077$ & ug/g dry & 0.077 & $5 / 07 / 08$ & 8D28006 & PNNL-AGG-ICP-AES \\
\hline $7440-15-5$ & Rhenium & $<0.152$ & ug/g dry & 0.152 & $5 / 07 / 08$ & 8D28006 & PNNL-AGG-ICP-AES \\
\hline $7440-36-0$ & Antimony & $<0.633$ & ug/g dry & 0.633 & $5 / 07 / 08$ & 8D28006 & PNNL-AGG-ICP-AES \\
\hline HEIS No. & B1T2P7A & \multicolumn{3}{|c|}{ Lab ID: $\quad 0803015-03$} & & & \\
\hline $7429-90-5$ & Aluminum & 0.160 & ug/g dry & 0.086 & $5 / 07 / 08$ & 8D28006 & PNNL-AGG-ICP-AES \\
\hline $7440-38-2$ & Arsenic & $<0.360$ & ug/g dry & 0.360 & $5 / 07 / 08$ & 8D28006 & PNNL-AGG-ICP-AES \\
\hline $7440-42-8$ & Boron & $<1.94$ & ug/g dry & 1.94 & $5 / 07 / 08$ & 8D28006 & PNNL-AGG-ICP-AES \\
\hline $7440-39-3$ & Barium & $<0.009$ & ug/g dry & 0.009 & $5 / 07 / 08$ & 8D28006 & PNNL-AGG-ICP-AES \\
\hline 7440-41-7 & Beryllium & $<0.028$ & ug/g dry & 0.028 & $5 / 07 / 08$ & 8D28006 & PNNL-AGG-ICP-AES \\
\hline $7440-69-9$ & Bismuth & $<0.188$ & ug/g dry & 0.188 & 5/07/08 & 8D28006 & PNNL-AGG-ICP-AES \\
\hline $7440-70-2$ & Calcium & 15.4 & ug/g dry & 0.387 & $5 / 07 / 08$ & 8D28006 & PNNL-AGG-ICP-AES \\
\hline $7440-43-9$ & Cadmium & $<0.027$ & ug/g dry & 0.027 & $5 / 07 / 08$ & 8D28006 & PNNL-AGG-ICP-AES \\
\hline $7440-48-4$ & Cobalt & $<0.096$ & ug/g dry & 0.096 & $5 / 07 / 08$ & 8D28006 & PNNL-AGG-ICP-AES \\
\hline $7440-47-3$ & Chromium & $<0.033$ & ug/g dry & 0.033 & $5 / 07 / 08$ & 8D28006 & PNNL-AGG-ICP-AES \\
\hline $7440-50-8$ & Copper & $<0.080$ & ug/g dry & 0.080 & $5 / 07 / 08$ & 8D28006 & PNNL-AGG-ICP-AES \\
\hline 7439-89-6 & Iron & $<0.142$ & ug/g dry & 0.142 & $5 / 07 / 08$ & 8D28006 & PNNL-AGG-ICP-AES \\
\hline 7440-09-7 & Potassium & 4.39 & ug/g dry & 2.33 & 5/07/08 & 8D28006 & PNNL-AGG-ICP-AES \\
\hline 7439-93-2 & Lithium & $<0.540$ & ug/g dry & 0.540 & $5 / 07 / 08$ & 8D28006 & PNNL-AGG-ICP-AES \\
\hline 7439-95-4 & Magnesium & 2.86 & ug/g dry & 0.083 & $5 / 07 / 08$ & 8D28006 & PNNL-AGG-ICP-AES \\
\hline 7439-96-5 & Manganese & $<0.017$ & ug/g dry & 0.017 & $5 / 07 / 08$ & 8D28006 & PNNL-AGG-ICP-AES \\
\hline 7439-98-7 & Molybdenum & $<0.139$ & ug/g dry & 0.139 & $5 / 07 / 08$ & 8D28006 & PNNL-AGG-ICP-AES \\
\hline $7440-02-0$ & Nickel & $<0.093$ & ug/g dry & 0.093 & $5 / 07 / 08$ & 8D28006 & PNNL-AGG-ICP-AES \\
\hline 7723-14-0 & Phosphorus & $<1.03$ & ug/g dry & 1.03 & $5 / 07 / 08$ & 8D28006 & PNNL-AGG-ICP-AES \\
\hline 7439-92-1 & Lead & $<0.432$ & ug/g dry & 0.432 & $5 / 07 / 08$ & 8D28006 & PNNL-AGG-ICP-AES \\
\hline 7782-49-2 & Selenium & $<1.95$ & ug/g dry & 1.95 & $5 / 07 / 08$ & 8D28006 & PNNL-AGG-ICP-AES \\
\hline $7440-24-6$ & Strontium & 0.057 & ug/g dry & 0.052 & $5 / 07 / 08$ & 8D28006 & PNNL-AGG-ICP-AES \\
\hline $7440-28-0$ & Thallium & $<1.03$ & ug/g dry & 1.03 & $5 / 07 / 08$ & 8D28006 & PNNL-AGG-ICP-AES \\
\hline $7440-62-2$ & Vanadium & $<0.045$ & ug/g dry & 0.045 & $5 / 07 / 08$ & 8D28006 & PNNL-AGG-ICP-AES \\
\hline 7440-23-5 & Sodium & 51.0 & ug/g dry & 0.669 & $5 / 07 / 08$ & 8D28006 & PNNL-AGG-ICP-AES \\
\hline $7440-21-3$ & Silicon & 8.16 & ug/g dry & 1.50 & $5 / 07 / 08$ & 8D28006 & PNNL-AGG-ICP-AES \\
\hline 7704-34-9 & Sulfur & 41.8 & ug/g dry & 3.08 & $5 / 07 / 08$ & 8D28006 & PNNL-AGG-ICP-AES \\
\hline $7440-32-6$ & Titanium & 0.009 & ug/g dry & 0.009 & $5 / 07 / 08$ & 8D28006 & PNNL-AGG-ICP-AES \\
\hline $7440-67-7$ & Zirconium & $<0.150$ & ug/g dry & 0.150 & $5 / 07 / 08$ & 8D28006 & PNNL-AGG-ICP-AES \\
\hline $7440-22-4$ & Silver & $<0.077$ & ug/g dry & 0.077 & $5 / 07 / 08$ & 8D28006 & PNNL-AGG-ICP-AES \\
\hline $7440-15-5$ & Rhenium & $<0.152$ & ug/g dry & 0.152 & $5 / 07 / 08$ & 8D28006 & PNNL-AGG-ICP-AES \\
\hline $7440-36-0$ & Antimony & $<0.633$ & ug/g dry & 0.633 & $5 / 07 / 08$ & 8D28006 & PNNL-AGG-ICP-AES \\
\hline HEIS No. & B1T2P8B & & b ID: & 3015-06 & & & \\
\hline $7429-90-5$ & Aluminum & 0.202 & ug/g dry & 0.086 & $5 / 07 / 08$ & 8D28006 & PNNL-AGG-ICP-AES \\
\hline $7440-38-2$ & Arsenic & $<0.361$ & ug/g dry & 0.361 & $5 / 07 / 08$ & 8D28006 & PNNL-AGG-ICP-AES \\
\hline $7440-42-8$ & Boron & $<1.94$ & ug/g dry & 1.94 & 5/07/08 & 8D28006 & PNNL-AGG-ICP-AES \\
\hline
\end{tabular}


Total Metals by SW846 6010B/Water Extract

\begin{tabular}{|c|c|c|c|c|c|c|c|}
\hline CAS \# & Analyte & Results & Units & EQL & Analyzed & Batch & Method \\
\hline HEIS No. & B1T2P8B & \multicolumn{3}{|c|}{ Lab ID: $\quad$ 0803015-06 } & & & \\
\hline $7440-39-3$ & Barium & $<0.009$ & ug/g dry & 0.009 & $5 / 07 / 08$ & 8D28006 & PNNL-AGG-ICP-AES \\
\hline $7440-41-7$ & Beryllium & $<0.028$ & ug/g dry & 0.028 & $5 / 07 / 08$ & 8D28006 & PNNL-AGG-ICP-AES \\
\hline 7440-69-9 & Bismuth & $<0.188$ & ug/g dry & 0.188 & $5 / 07 / 08$ & 8D28006 & PNNL-AGG-ICP-AES \\
\hline $7440-70-2$ & Calcium & 14.5 & ug/g dry & 0.388 & $5 / 07 / 08$ & 8D28006 & PNNL-AGG-ICP-AES \\
\hline $7440-43-9$ & Cadmium & $<0.027$ & ug/g dry & 0.027 & $5 / 07 / 08$ & 8D28006 & PNNL-AGG-ICP-AES \\
\hline $7440-48-4$ & Cobalt & $<0.096$ & ug/g dry & 0.096 & $5 / 07 / 08$ & 8D28006 & PNNL-AGG-ICP-AES \\
\hline $7440-47-3$ & Chromium & $<0.033$ & ug/g dry & 0.033 & $5 / 07 / 08$ & 8D28006 & PNNL-AGG-ICP-AES \\
\hline $7440-50-8$ & Copper & $<0.081$ & ug/g dry & 0.081 & $5 / 07 / 08$ & 8D28006 & PNNL-AGG-ICP-AES \\
\hline 7439-89-6 & Iron & $<0.142$ & ug/g dry & 0.142 & $5 / 07 / 08$ & 8D28006 & PNNL-AGG-ICP-AES \\
\hline 7440-09-7 & Potassium & 4.28 & ug/g dry & 2.33 & $5 / 07 / 08$ & 8D28006 & PNNL-AGG-ICP-AES \\
\hline 7439-93-2 & Lithium & $<0.541$ & ug/g dry & 0.541 & $5 / 07 / 08$ & 8D28006 & PNNL-AGG-ICP-AES \\
\hline 7439-95-4 & Magnesium & 5.23 & ug/g dry & 0.084 & $5 / 07 / 08$ & 8D28006 & PNNL-AGG-ICP-AES \\
\hline $7439-96-5$ & Manganese & $<0.017$ & ug/g dry & 0.017 & $5 / 07 / 08$ & 8D28006 & PNNL-AGG-ICP-AES \\
\hline 7439-98-7 & Molybdenum & $<0.139$ & ug/g dry & 0.139 & $5 / 07 / 08$ & 8D28006 & PNNL-AGG-ICP-AES \\
\hline 7440-02-0 & Nickel & $<0.093$ & ug/g dry & 0.093 & $5 / 07 / 08$ & 8D28006 & PNNL-AGG-ICP-AES \\
\hline $7723-14-0$ & Phosphorus & $<1.03$ & ug/g dry & 1.03 & $5 / 07 / 08$ & 8D28006 & PNNL-AGG-ICP-AES \\
\hline 7439-92-1 & Lead & $<0.433$ & ug/g dry & 0.433 & $5 / 07 / 08$ & 8D28006 & PNNL-AGG-ICP-AES \\
\hline $7782-49-2$ & Selenium & $<1.95$ & ug/g dry & 1.95 & $5 / 07 / 08$ & 8D28006 & PNNL-AGG-ICP-AES \\
\hline $7440-24-6$ & Strontium & 0.065 & ug/g dry & 0.052 & $5 / 07 / 08$ & 8D28006 & PNNL-AGG-ICP-AES \\
\hline $7440-28-0$ & Thallium & $<1.03$ & ug/g dry & 1.03 & $5 / 07 / 08$ & 8D28006 & PNNL-AGG-ICP-AES \\
\hline $7440-62-2$ & Vanadium & $<0.045$ & ug/g dry & 0.045 & $5 / 07 / 08$ & 8D28006 & PNNL-AGG-ICP-AES \\
\hline $7440-23-5$ & Sodium & 12.5 & ug/g dry & 0.670 & $5 / 07 / 08$ & 8D28006 & PNNL-AGG-ICP-AES \\
\hline $7440-21-3$ & Silicon & 6.38 & ug/g dry & 1.50 & $5 / 07 / 08$ & 8D28006 & PNNL-AGG-ICP-AES \\
\hline $7704-34-9$ & Sulfur & 19.6 & ug/g dry & 3.08 & $5 / 07 / 08$ & 8D28006 & PNNL-AGG-ICP-AES \\
\hline $7440-32-6$ & Titanium & 0.009 & ug/g dry & 0.009 & $5 / 07 / 08$ & 8D28006 & PNNL-AGG-ICP-AES \\
\hline $7440-67-7$ & Zirconium & $<0.150$ & ug/g dry & 0.150 & $5 / 07 / 08$ & 8D28006 & PNNL-AGG-ICP-AES \\
\hline $7440-22-4$ & Silver & $<0.077$ & ug/g dry & 0.077 & $5 / 07 / 08$ & 8D28006 & PNNL-AGG-ICP-AES \\
\hline $7440-15-5$ & Rhenium & $<0.152$ & ug/g dry & 0.152 & $5 / 07 / 08$ & 8D28006 & PNNL-AGG-ICP-AES \\
\hline $7440-36-0$ & Antimony & $<0.634$ & ug/g dry & 0.634 & $5 / 07 / 08$ & 8D28006 & PNNL-AGG-ICP-AES \\
\hline HEIS No. & B1T2P8A & \multicolumn{3}{|c|}{ Lab ID: $\quad$ 0803015-07 } & & & \\
\hline 7429-90-5 & Aluminum & 0.146 & ug/g dry & 0.086 & $5 / 07 / 08$ & 8D28006 & PNNL-AGG-ICP-AES \\
\hline $7440-38-2$ & Arsenic & $<0.360$ & ug/g dry & 0.360 & $5 / 07 / 08$ & 8D28006 & PNNL-AGG-ICP-AES \\
\hline $7440-42-8$ & Boron & $<1.94$ & ug/g dry & 1.94 & $5 / 07 / 08$ & 8D28006 & PNNL-AGG-ICP-AES \\
\hline $7440-39-3$ & Barium & $<0.009$ & ug/g dry & 0.009 & $5 / 07 / 08$ & 8D28006 & PNNL-AGG-ICP-AES \\
\hline $7440-41-7$ & Beryllium & $<0.028$ & ug/g dry & 0.028 & $5 / 07 / 08$ & 8D28006 & PNNL-AGG-ICP-AES \\
\hline $7440-69-9$ & Bismuth & $<0.188$ & ug/g dry & 0.188 & $5 / 07 / 08$ & 8D28006 & PNNL-AGG-ICP-AES \\
\hline $7440-70-2$ & Calcium & 20.5 & ug/g dry & 0.387 & $5 / 07 / 08$ & 8D28006 & PNNL-AGG-ICP-AES \\
\hline $7440-43-9$ & Cadmium & $<0.027$ & ug/g dry & 0.027 & $5 / 07 / 08$ & 8D28006 & PNNL-AGG-ICP-AES \\
\hline $7440-48-4$ & Cobalt & $<0.096$ & ug/g dry & 0.096 & $5 / 07 / 08$ & 8D28006 & PNNL-AGG-ICP-AES \\
\hline $7440-47-3$ & Chromium & $<0.033$ & ug/g dry & 0.033 & $5 / 07 / 08$ & 8D28006 & PNNL-AGG-ICP-AES \\
\hline $7440-50-8$ & Copper & $<0.080$ & ug/g dry & 0.080 & $5 / 07 / 08$ & 8D28006 & PNNL-AGG-ICP-AES \\
\hline 7439-89-6 & Iron & $<0.142$ & ug/g dry & 0.142 & $5 / 07 / 08$ & 8D28006 & PNNL-AGG-ICP-AES \\
\hline 7440-09-7 & Potassium & 4.70 & ug/g dry & 2.33 & $5 / 07 / 08$ & 8D28006 & PNNL-AGG-ICP-AES \\
\hline $7439-93-2$ & Lithium & $<0.540$ & ug/g dry & 0.540 & $5 / 07 / 08$ & 8D28006 & PNNL-AGG-ICP-AES \\
\hline 7439-95-4 & Magnesium & 7.30 & ug/g dry & 0.083 & $5 / 07 / 08$ & 8D28006 & PNNL-AGG-ICP-AES \\
\hline $7439-96-5$ & Manganese & $<0.017$ & ug/g dry & 0.017 & $5 / 07 / 08$ & 8D28006 & PNNL-AGG-ICP-AES \\
\hline 7439-98-7 & Molybdenum & $<0.139$ & ug/g dry & 0.139 & $5 / 07 / 08$ & 8D28006 & PNNL-AGG-ICP-AES \\
\hline 7440-02-0 & Nickel & $<0.093$ & ug/g dry & 0.093 & $5 / 07 / 08$ & 8D28006 & PNNL-AGG-ICP-AES \\
\hline
\end{tabular}


Total Metals by SW846 6010B/Water Extract

\begin{tabular}{|c|c|c|c|c|c|c|c|}
\hline CAS \# & Analyte & Results & Units & EQL & Analyzed & Batch & Method \\
\hline HEIS No. & B1T2P8A & \multicolumn{3}{|c|}{ Lab ID: $\quad$ 0803015-07 } & & & \\
\hline 7723-14-0 & Phosphorus & $<1.03$ & ug/g dry & 1.03 & $5 / 07 / 08$ & 8D28006 & PNNL-AGG-ICP-AES \\
\hline 7439-92-1 & Lead & $<0.432$ & ug/g dry & 0.432 & $5 / 07 / 08$ & 8D28006 & PNNL-AGG-ICP-AES \\
\hline $7782-49-2$ & Selenium & $<1.95$ & ug/g dry & 1.95 & $5 / 07 / 08$ & 8D28006 & PNNL-AGG-ICP-AES \\
\hline $7440-24-6$ & Strontium & 0.085 & ug/g dry & 0.052 & $5 / 07 / 08$ & 8D28006 & PNNL-AGG-ICP-AES \\
\hline $7440-28-0$ & Thallium & $<1.03$ & ug/g dry & 1.03 & $5 / 07 / 08$ & 8D28006 & PNNL-AGG-ICP-AES \\
\hline $7440-62-2$ & Vanadium & $<0.045$ & ug/g dry & 0.045 & $5 / 07 / 08$ & 8D28006 & PNNL-AGG-ICP-AES \\
\hline $7440-23-5$ & Sodium & 12.9 & ug/g dry & 0.669 & $5 / 07 / 08$ & 8D28006 & PNNL-AGG-ICP-AES \\
\hline $7440-21-3$ & Silicon & 7.16 & ug/g dry & 1.50 & $5 / 07 / 08$ & 8D28006 & PNNL-AGG-ICP-AES \\
\hline $7704-34-9$ & Sulfur & 26.9 & ug/g dry & 3.08 & $5 / 07 / 08$ & 8D28006 & PNNL-AGG-ICP-AES \\
\hline $7440-32-6$ & Titanium & $<0.009$ & ug/g dry & 0.009 & $5 / 07 / 08$ & 8D28006 & PNNL-AGG-ICP-AES \\
\hline $7440-67-7$ & Zirconium & $<0.150$ & ug/g dry & 0.150 & $5 / 07 / 08$ & 8D28006 & PNNL-AGG-ICP-AES \\
\hline $7440-22-4$ & Silver & $<0.077$ & ug/g dry & 0.077 & $5 / 07 / 08$ & 8D28006 & PNNL-AGG-ICP-AES \\
\hline $7440-15-5$ & Rhenium & $<0.152$ & ug/g dry & 0.152 & $5 / 07 / 08$ & 8D28006 & PNNL-AGG-ICP-AES \\
\hline $7440-36-0$ & Antimony & $<0.633$ & ug/g dry & 0.633 & $5 / 07 / 08$ & 8D28006 & PNNL-AGG-ICP-AES \\
\hline HEIS No. & B1T2P9B & \multicolumn{3}{|c|}{ Lab ID: $\quad$ 0803015-10 } & & & \\
\hline $7429-90-5$ & Aluminum & 0.192 & ug/g dry & 0.086 & $5 / 07 / 08$ & 8D28006 & PNNL-AGG-ICP-AES \\
\hline $7440-38-2$ & Arsenic & $<0.360$ & ug/g dry & 0.360 & $5 / 07 / 08$ & 8D28006 & PNNL-AGG-ICP-AES \\
\hline $7440-42-8$ & Boron & $<1.94$ & ug/g dry & 1.94 & $5 / 07 / 08$ & 8D28006 & PNNL-AGG-ICP-AES \\
\hline $7440-39-3$ & Barium & 0.097 & ug/g dry & 0.009 & $5 / 07 / 08$ & 8D28006 & PNNL-AGG-ICP-AES \\
\hline $7440-41-7$ & Beryllium & $<0.028$ & ug/g dry & 0.028 & $5 / 07 / 08$ & 8D28006 & PNNL-AGG-ICP-AES \\
\hline $7440-69-9$ & Bismuth & $<0.188$ & ug/g dry & 0.188 & $5 / 07 / 08$ & 8D28006 & PNNL-AGG-ICP-AES \\
\hline $7440-70-2$ & Calcium & 15.9 & ug/g dry & 0.387 & $5 / 07 / 08$ & 8D28006 & PNNL-AGG-ICP-AES \\
\hline $7440-43-9$ & Cadmium & $<0.027$ & ug/g dry & 0.027 & $5 / 07 / 08$ & 8D28006 & PNNL-AGG-ICP-AES \\
\hline $7440-48-4$ & Cobalt & $<0.096$ & ug/g dry & 0.096 & $5 / 07 / 08$ & 8D28006 & PNNL-AGG-ICP-AES \\
\hline $7440-47-3$ & Chromium & $<0.033$ & ug/g dry & 0.033 & $5 / 07 / 08$ & 8D28006 & PNNL-AGG-ICP-AES \\
\hline $7440-50-8$ & Copper & $<0.080$ & ug/g dry & 0.080 & $5 / 07 / 08$ & 8D28006 & PNNL-AGG-ICP-AES \\
\hline 7439-89-6 & Iron & $<0.142$ & ug/g dry & 0.142 & $5 / 07 / 08$ & 8D28006 & PNNL-AGG-ICP-AES \\
\hline 7440-09-7 & Potassium & 6.14 & ug/g dry & 2.33 & $5 / 07 / 08$ & 8D28006 & PNNL-AGG-ICP-AES \\
\hline $7439-93-2$ & Lithium & $<0.540$ & ug/g dry & 0.540 & $5 / 07 / 08$ & 8D28006 & PNNL-AGG-ICP-AES \\
\hline 7439-95-4 & Magnesium & 5.69 & ug/g dry & 0.083 & $5 / 07 / 08$ & 8D28006 & PNNL-AGG-ICP-AES \\
\hline 7439-96-5 & Manganese & $<0.017$ & ug/g dry & 0.017 & $5 / 07 / 08$ & 8D28006 & PNNL-AGG-ICP-AES \\
\hline $7439-98-7$ & Molybdenum & $<0.139$ & ug/g dry & 0.139 & $5 / 07 / 08$ & 8D28006 & PNNL-AGG-ICP-AES \\
\hline $7440-02-0$ & Nickel & $<0.093$ & ug/g dry & 0.093 & $5 / 07 / 08$ & 8D28006 & PNNL-AGG-ICP-AES \\
\hline 7723-14-0 & Phosphorus & $<1.03$ & ug/g dry & 1.03 & $5 / 07 / 08$ & 8D28006 & PNNL-AGG-ICP-AES \\
\hline $7439-92-1$ & Lead & $<0.432$ & ug/g dry & 0.432 & $5 / 07 / 08$ & 8D28006 & PNNL-AGG-ICP-AES \\
\hline $7782-49-2$ & Selenium & $<1.95$ & ug/g dry & 1.95 & $5 / 07 / 08$ & 8D28006 & PNNL-AGG-ICP-AES \\
\hline $7440-24-6$ & Strontium & 0.074 & ug/g dry & 0.052 & $5 / 07 / 08$ & 8D28006 & PNNL-AGG-ICP-AES \\
\hline $7440-28-0$ & Thallium & $<1.03$ & ug/g dry & 1.03 & $5 / 07 / 08$ & 8D28006 & PNNL-AGG-ICP-AES \\
\hline $7440-62-2$ & Vanadium & $<0.045$ & ug/g dry & 0.045 & $5 / 07 / 08$ & 8D28006 & PNNL-AGG-ICP-AES \\
\hline $7440-23-5$ & Sodium & 13.1 & ug/g dry & 0.669 & $5 / 07 / 08$ & 8D28006 & PNNL-AGG-ICP-AES \\
\hline $7440-21-3$ & Silicon & 6.19 & ug/g dry & 1.50 & $5 / 07 / 08$ & 8D28006 & PNNL-AGG-ICP-AES \\
\hline 7704-34-9 & Sulfur & 17.9 & ug/g dry & 3.08 & $5 / 07 / 08$ & 8D28006 & PNNL-AGG-ICP-AES \\
\hline $7440-32-6$ & Titanium & $<0.009$ & ug/g dry & 0.009 & $5 / 07 / 08$ & 8D28006 & PNNL-AGG-ICP-AES \\
\hline $7440-67-7$ & Zirconium & $<0.150$ & ug/g dry & 0.150 & $5 / 07 / 08$ & 8D28006 & PNNL-AGG-ICP-AES \\
\hline $7440-22-4$ & Silver & $<0.077$ & ug/g dry & 0.077 & $5 / 07 / 08$ & 8D28006 & PNNL-AGG-ICP-AES \\
\hline $7440-15-5$ & Rhenium & $<0.152$ & ug/g dry & 0.152 & $5 / 07 / 08$ & 8D28006 & PNNL-AGG-ICP-AES \\
\hline $7440-36-0$ & Antimony & $<0.633$ & ug/g dry & 0.633 & $5 / 07 / 08$ & 8D28006 & PNNL-AGG-ICP-AES \\
\hline HEIS No. & B1T2P9A & La & b ID: & 3015-11 & & & \\
\hline
\end{tabular}


Total Metals by SW846 6010B/Water Extract

\begin{tabular}{|c|c|c|c|c|c|c|c|}
\hline CAS \# & Analyte & Results & Units & EQL & Analyzed & Batch & Method \\
\hline HEIS No. & B1T2P9A & \multicolumn{3}{|c|}{ Lab ID: $\quad$ 0803015-11 } & & & \\
\hline 7429-90-5 & Aluminum & 0.206 & ug/g dry & 0.086 & $5 / 07 / 08$ & 8D28006 & PNNL-AGG-ICP-AES \\
\hline $7440-38-2$ & Arsenic & $<0.360$ & ug/g dry & 0.360 & $5 / 07 / 08$ & 8D28006 & PNNL-AGG-ICP-AES \\
\hline $7440-42-8$ & Boron & $<1.94$ & ug/g dry & 1.94 & $5 / 07 / 08$ & 8D28006 & PNNL-AGG-ICP-AES \\
\hline $7440-39-3$ & Barium & 0.012 & ug/g dry & 0.009 & $5 / 07 / 08$ & 8D28006 & PNNL-AGG-ICP-AES \\
\hline $7440-41-7$ & Beryllium & $<0.028$ & ug/g dry & 0.028 & $5 / 07 / 08$ & 8D28006 & PNNL-AGG-ICP-AES \\
\hline 7440-69-9 & Bismuth & $<0.188$ & ug/g dry & 0.188 & $5 / 07 / 08$ & 8D28006 & PNNL-AGG-ICP-AES \\
\hline $7440-70-2$ & Calcium & 15.9 & ug/g dry & 0.387 & $5 / 07 / 08$ & 8D28006 & PNNL-AGG-ICP-AES \\
\hline $7440-43-9$ & Cadmium & $<0.027$ & ug/g dry & 0.027 & $5 / 07 / 08$ & 8D28006 & PNNL-AGG-ICP-AES \\
\hline $7440-48-4$ & Cobalt & $<0.096$ & ug/g dry & 0.096 & $5 / 07 / 08$ & 8D28006 & PNNL-AGG-ICP-AES \\
\hline $7440-47-3$ & Chromium & $<0.033$ & ug/g dry & 0.033 & $5 / 07 / 08$ & 8D28006 & PNNL-AGG-ICP-AES \\
\hline $7440-50-8$ & Copper & $<0.080$ & ug/g dry & 0.080 & $5 / 07 / 08$ & 8D28006 & PNNL-AGG-ICP-AES \\
\hline 7439-89-6 & Iron & $<0.142$ & ug/g dry & 0.142 & $5 / 07 / 08$ & 8D28006 & PNNL-AGG-ICP-AES \\
\hline 7440-09-7 & Potassium & 5.84 & ug/g dry & 2.33 & $5 / 07 / 08$ & 8D28006 & PNNL-AGG-ICP-AES \\
\hline 7439-93-2 & Lithium & $<0.540$ & ug/g dry & 0.540 & $5 / 07 / 08$ & 8D28006 & PNNL-AGG-ICP-AES \\
\hline $7439-95-4$ & Magnesium & 5.58 & ug/g dry & 0.083 & $5 / 07 / 08$ & 8D28006 & PNNL-AGG-ICP-AES \\
\hline 7439-96-5 & Manganese & $<0.017$ & ug/g dry & 0.017 & $5 / 07 / 08$ & 8D28006 & PNNL-AGG-ICP-AES \\
\hline 7439-98-7 & Molybdenum & $<0.139$ & ug/g dry & 0.139 & $5 / 07 / 08$ & 8D28006 & PNNL-AGG-ICP-AES \\
\hline $7440-02-0$ & Nickel & $<0.093$ & ug/g dry & 0.093 & $5 / 07 / 08$ & 8D28006 & PNNL-AGG-ICP-AES \\
\hline $7723-14-0$ & Phosphorus & $<1.03$ & ug/g dry & 1.03 & $5 / 07 / 08$ & 8D28006 & PNNL-AGG-ICP-AES \\
\hline 7439-92-1 & Lead & $<0.432$ & ug/g dry & 0.432 & $5 / 07 / 08$ & 8D28006 & PNNL-AGG-ICP-AES \\
\hline $7782-49-2$ & Selenium & $<1.95$ & ug/g dry & 1.95 & $5 / 07 / 08$ & 8D28006 & PNNL-AGG-ICP-AES \\
\hline $7440-24-6$ & Strontium & 0.071 & ug/g dry & 0.052 & $5 / 07 / 08$ & 8D28006 & PNNL-AGG-ICP-AES \\
\hline $7440-28-0$ & Thallium & $<1.03$ & ug/g dry & 1.03 & $5 / 07 / 08$ & 8D28006 & PNNL-AGG-ICP-AES \\
\hline $7440-62-2$ & Vanadium & $<0.045$ & ug/g dry & 0.045 & $5 / 07 / 08$ & 8D28006 & PNNL-AGG-ICP-AES \\
\hline $7440-23-5$ & Sodium & 12.4 & ug/g dry & 0.669 & $5 / 07 / 08$ & 8D28006 & PNNL-AGG-ICP-AES \\
\hline $7440-21-3$ & Silicon & 6.08 & ug/g dry & 1.50 & $5 / 07 / 08$ & 8D28006 & PNNL-AGG-ICP-AES \\
\hline 7704-34-9 & Sulfur & 17.8 & ug/g dry & 3.08 & $5 / 07 / 08$ & 8D28006 & PNNL-AGG-ICP-AES \\
\hline $7440-32-6$ & Titanium & $<0.009$ & ug/g dry & 0.009 & $5 / 07 / 08$ & 8D28006 & PNNL-AGG-ICP-AES \\
\hline $7440-67-7$ & Zirconium & $<0.150$ & ug/g dry & 0.150 & $5 / 07 / 08$ & 8D28006 & PNNL-AGG-ICP-AES \\
\hline $7440-22-4$ & Silver & $<0.077$ & ug/g dry & 0.077 & $5 / 07 / 08$ & 8D28006 & PNNL-AGG-ICP-AES \\
\hline $7440-15-5$ & Rhenium & $<0.152$ & ug/g dry & 0.152 & $5 / 07 / 08$ & 8D28006 & PNNL-AGG-ICP-AES \\
\hline $7440-36-0$ & Antimony & $<0.633$ & ug/g dry & 0.633 & $5 / 07 / 08$ & 8D28006 & PNNL-AGG-ICP-AES \\
\hline HEIS No. & B1T2R0B & & b ID: & 3015-14 & & & \\
\hline $7429-90-5$ & Aluminum & $<0.086$ & ug/g dry & 0.086 & $5 / 07 / 08$ & 8D28006 & PNNL-AGG-ICP-AES \\
\hline $7440-38-2$ & Arsenic & $<0.360$ & ug/g dry & 0.360 & $5 / 07 / 08$ & 8D28006 & PNNL-AGG-ICP-AES \\
\hline $7440-42-8$ & Boron & $<1.94$ & ug/g dry & 1.94 & $5 / 07 / 08$ & 8D28006 & PNNL-AGG-ICP-AES \\
\hline $7440-39-3$ & Barium & $<0.009$ & ug/g dry & 0.009 & $5 / 07 / 08$ & 8D28006 & PNNL-AGG-ICP-AES \\
\hline $7440-41-7$ & Beryllium & $<0.028$ & ug/g dry & 0.028 & $5 / 07 / 08$ & $8 \mathrm{D} 28006$ & PNNL-AGG-ICP-AES \\
\hline 7440-69-9 & Bismuth & $<0.188$ & ug/g dry & 0.188 & $5 / 07 / 08$ & 8D28006 & PNNL-AGG-ICP-AES \\
\hline $7440-70-2$ & Calcium & 28.7 & ug/g dry & 0.387 & $5 / 07 / 08$ & 8D28006 & PNNL-AGG-ICP-AES \\
\hline $7440-43-9$ & Cadmium & $<0.027$ & ug/g dry & 0.027 & $5 / 07 / 08$ & 8D28006 & PNNL-AGG-ICP-AES \\
\hline $7440-48-4$ & Cobalt & $<0.096$ & ug/g dry & 0.096 & $5 / 07 / 08$ & 8D28006 & PNNL-AGG-ICP-AES \\
\hline $7440-47-3$ & Chromium & $<0.033$ & ug/g dry & 0.033 & $5 / 07 / 08$ & 8D28006 & PNNL-AGG-ICP-AES \\
\hline $7440-50-8$ & Copper & $<0.080$ & ug/g dry & 0.080 & $5 / 07 / 08$ & 8D28006 & PNNL-AGG-ICP-AES \\
\hline 7439-89-6 & Iron & $<0.142$ & ug/g dry & 0.142 & $5 / 07 / 08$ & 8D28006 & PNNL-AGG-ICP-AES \\
\hline $7440-09-7$ & Potassium & 8.05 & ug/g dry & 2.33 & $5 / 07 / 08$ & 8D28006 & PNNL-AGG-ICP-AES \\
\hline 7439-93-2 & Lithium & $<0.540$ & ug/g dry & 0.540 & $5 / 07 / 08$ & 8D28006 & PNNL-AGG-ICP-AES \\
\hline $7439-95-4$ & Magnesium & 10.7 & ug/g dry & 0.083 & $5 / 07 / 08$ & 8D28006 & PNNL-AGG-ICP-AES \\
\hline
\end{tabular}


Total Metals by SW846 6010B/Water Extract

\begin{tabular}{|c|c|c|c|c|c|c|c|}
\hline CAS \# & Analyte & Results & Units & EQL & Analyzed & Batch & Method \\
\hline HEIS No. & B1T2R0B & \multicolumn{3}{|c|}{ Lab ID: $\quad$ 0803015-14 } & & & \\
\hline 7439-96-5 & Manganese & $<0.017$ & ug/g dry & 0.017 & $5 / 07 / 08$ & 8D28006 & PNNL-AGG-ICP-AES \\
\hline 7439-98-7 & Molybdenum & $<0.139$ & ug/g dry & 0.139 & $5 / 07 / 08$ & 8D28006 & PNNL-AGG-ICP-AES \\
\hline $7440-02-0$ & Nickel & $<0.093$ & ug/g dry & 0.093 & $5 / 07 / 08$ & 8D28006 & PNNL-AGG-ICP-AES \\
\hline 7723-14-0 & Phosphorus & $<1.03$ & ug/g dry & 1.03 & $5 / 07 / 08$ & 8D28006 & PNNL-AGG-ICP-AES \\
\hline 7439-92-1 & Lead & $<0.432$ & ug/g dry & 0.432 & $5 / 07 / 08$ & 8D28006 & PNNL-AGG-ICP-AES \\
\hline $7782-49-2$ & Selenium & $<1.95$ & ug/g dry & 1.95 & $5 / 07 / 08$ & 8D28006 & PNNL-AGG-ICP-AES \\
\hline $7440-24-6$ & Strontium & 0.132 & ug/g dry & 0.052 & $5 / 07 / 08$ & 8D28006 & PNNL-AGG-ICP-AES \\
\hline $7440-28-0$ & Thallium & $<1.03$ & ug/g dry & 1.03 & $5 / 07 / 08$ & 8D28006 & PNNL-AGG-ICP-AES \\
\hline $7440-62-2$ & Vanadium & $<0.045$ & ug/g dry & 0.045 & $5 / 07 / 08$ & 8D28006 & PNNL-AGG-ICP-AES \\
\hline $7440-23-5$ & Sodium & 16.6 & ug/g dry & 0.669 & $5 / 07 / 08$ & 8D28006 & PNNL-AGG-ICP-AES \\
\hline $7440-21-3$ & Silicon & 5.64 & ug/g dry & 1.50 & $5 / 07 / 08$ & 8D28006 & PNNL-AGG-ICP-AES \\
\hline $7704-34-9$ & Sulfur & 26.4 & ug/g dry & 3.08 & $5 / 07 / 08$ & 8D28006 & PNNL-AGG-ICP-AES \\
\hline $7440-32-6$ & Titanium & $<0.009$ & ug/g dry & 0.009 & $5 / 07 / 08$ & 8D28006 & PNNL-AGG-ICP-AES \\
\hline $7440-67-7$ & Zirconium & $<0.150$ & ug/g dry & 0.150 & $5 / 07 / 08$ & 8D28006 & PNNL-AGG-ICP-AES \\
\hline $7440-22-4$ & Silver & $<0.077$ & ug/g dry & 0.077 & $5 / 07 / 08$ & 8D28006 & PNNL-AGG-ICP-AES \\
\hline $7440-15-5$ & Rhenium & $<0.152$ & ug/g dry & 0.152 & $5 / 07 / 08$ & 8D28006 & PNNL-AGG-ICP-AES \\
\hline $7440-36-0$ & Antimony & $<0.633$ & ug/g dry & 0.633 & $5 / 07 / 08$ & 8D28006 & PNNL-AGG-ICP-AES \\
\hline HEIS No. & B1T2R0A & \multicolumn{3}{|c|}{ Lab ID: $\quad$ 0803015-15 } & & & \\
\hline $7429-90-5$ & Aluminum & $<0.086$ & ug/g dry & 0.086 & $5 / 07 / 08$ & 8D28006 & PNNL-AGG-ICP-AES \\
\hline $7440-38-2$ & Arsenic & $<0.362$ & ug/g dry & 0.362 & $5 / 07 / 08$ & 8D28006 & PNNL-AGG-ICP-AES \\
\hline $7440-42-8$ & Boron & $<1.95$ & ug/g dry & 1.95 & $5 / 07 / 08$ & 8D28006 & PNNL-AGG-ICP-AES \\
\hline $7440-39-3$ & Barium & $<0.009$ & ug/g dry & 0.009 & $5 / 07 / 08$ & 8D28006 & PNNL-AGG-ICP-AES \\
\hline $7440-41-7$ & Beryllium & $<0.029$ & ug/g dry & 0.029 & $5 / 07 / 08$ & 8D28006 & PNNL-AGG-ICP-AES \\
\hline $7440-69-9$ & Bismuth & $<0.189$ & ug/g dry & 0.189 & $5 / 07 / 08$ & 8D28006 & PNNL-AGG-ICP-AES \\
\hline $7440-70-2$ & Calcium & 44.8 & ug/g dry & 0.389 & $5 / 07 / 08$ & 8D28006 & PNNL-AGG-ICP-AES \\
\hline $7440-43-9$ & Cadmium & $<0.027$ & ug/g dry & 0.027 & $5 / 07 / 08$ & 8D28006 & PNNL-AGG-ICP-AES \\
\hline $7440-48-4$ & Cobalt & $<0.097$ & ug/g dry & 0.097 & $5 / 07 / 08$ & 8D28006 & PNNL-AGG-ICP-AES \\
\hline $7440-47-3$ & Chromium & $<0.034$ & ug/g dry & 0.034 & $5 / 07 / 08$ & 8D28006 & PNNL-AGG-ICP-AES \\
\hline $7440-50-8$ & Copper & $<0.081$ & ug/g dry & 0.081 & $5 / 07 / 08$ & 8D28006 & PNNL-AGG-ICP-AES \\
\hline 7439-89-6 & Iron & $<0.143$ & ug/g dry & 0.143 & $5 / 07 / 08$ & 8D28006 & PNNL-AGG-ICP-AES \\
\hline 7440-09-7 & Potassium & 8.66 & ug/g dry & 2.34 & $5 / 07 / 08$ & 8D28006 & PNNL-AGG-ICP-AES \\
\hline $7439-93-2$ & Lithium & $<0.543$ & ug/g dry & 0.543 & $5 / 07 / 08$ & 8D28006 & PNNL-AGG-ICP-AES \\
\hline $7439-95-4$ & Magnesium & 14.1 & ug/g dry & 0.084 & $5 / 07 / 08$ & 8D28006 & PNNL-AGG-ICP-AES \\
\hline $7439-96-5$ & Manganese & $<0.017$ & ug/g dry & 0.017 & $5 / 07 / 08$ & 8D28006 & PNNL-AGG-ICP-AES \\
\hline 7439-98-7 & Molybdenum & $<0.140$ & ug/g dry & 0.140 & $5 / 07 / 08$ & 8D28006 & PNNL-AGG-ICP-AES \\
\hline $7440-02-0$ & Nickel & $<0.094$ & ug/g dry & 0.094 & $5 / 07 / 08$ & 8D28006 & PNNL-AGG-ICP-AES \\
\hline 7723-14-0 & Phosphorus & $<1.04$ & ug/g dry & 1.04 & $5 / 07 / 08$ & 8D28006 & PNNL-AGG-ICP-AES \\
\hline $7439-92-1$ & Lead & $<0.435$ & ug/g dry & 0.435 & $5 / 07 / 08$ & 8D28006 & PNNL-AGG-ICP-AES \\
\hline $7782-49-2$ & Selenium & $<1.96$ & ug/g dry & 1.96 & $5 / 07 / 08$ & 8D28006 & PNNL-AGG-ICP-AES \\
\hline $7440-24-6$ & Strontium & 0.180 & ug/g dry & 0.053 & $5 / 07 / 08$ & 8D28006 & PNNL-AGG-ICP-AES \\
\hline $7440-28-0$ & Thallium & $<1.03$ & ug/g dry & 1.03 & $5 / 07 / 08$ & 8D28006 & PNNL-AGG-ICP-AES \\
\hline $7440-62-2$ & Vanadium & $<0.046$ & ug/g dry & 0.046 & $5 / 07 / 08$ & 8D28006 & PNNL-AGG-ICP-AES \\
\hline $7440-23-5$ & Sodium & 15.9 & ug/g dry & 0.673 & $5 / 07 / 08$ & 8D28006 & PNNL-AGG-ICP-AES \\
\hline $7440-21-3$ & Silicon & 6.08 & ug/g dry & 1.51 & $5 / 07 / 08$ & 8D28006 & PNNL-AGG-ICP-AES \\
\hline 7704-34-9 & Sulfur & 44.4 & ug/g dry & 3.09 & $5 / 07 / 08$ & 8D28006 & PNNL-AGG-ICP-AES \\
\hline $7440-32-6$ & Titanium & $<0.009$ & ug/g dry & 0.009 & $5 / 07 / 08$ & 8D28006 & PNNL-AGG-ICP-AES \\
\hline $7440-67-7$ & Zirconium & $<0.151$ & ug/g dry & 0.151 & $5 / 07 / 08$ & 8D28006 & PNNL-AGG-ICP-AES \\
\hline $7440-22-4$ & Silver & $<0.078$ & ug/g dry & 0.078 & $5 / 07 / 08$ & 8D28006 & PNNL-AGG-ICP-AES \\
\hline
\end{tabular}


Total Metals by SW846 6010B/Water Extract

\begin{tabular}{|c|c|c|c|c|c|c|c|}
\hline CAS \# & Analyte & Results & Units & EQL & Analyzed & Batch & Method \\
\hline HEIS No. & B1T2R0A & \multicolumn{3}{|c|}{ Lab ID: $\quad$ 0803015-15 } & & & \\
\hline $7440-15-5$ & Rhenium & $<0.153$ & ug/g dry & 0.153 & $5 / 07 / 08$ & 8D28006 & PNNL-AGG-ICP-AES \\
\hline $7440-36-0$ & Antimony & $<0.637$ & ug/g dry & 0.637 & $5 / 07 / 08$ & 8D28006 & PNNL-AGG-ICP-AES \\
\hline HEIS No. & B1TNK6B & \multicolumn{3}{|c|}{ Lab ID: $\quad$ 0803015-18 } & & & \\
\hline $7429-90-5$ & Aluminum & 0.194 & ug/g dry & 0.086 & $5 / 07 / 08$ & 8D28006 & PNNL-AGG-ICP-AES \\
\hline $7440-38-2$ & Arsenic & $<0.360$ & ug/g dry & 0.360 & $5 / 07 / 08$ & 8D28006 & PNNL-AGG-ICP-AES \\
\hline $7440-42-8$ & Boron & $<1.94$ & ug/g dry & 1.94 & $5 / 07 / 08$ & 8D28006 & PNNL-AGG-ICP-AES \\
\hline $7440-39-3$ & Barium & $<0.009$ & ug/g dry & 0.009 & $5 / 07 / 08$ & 8D28006 & PNNL-AGG-ICP-AES \\
\hline $7440-41-7$ & Beryllium & $<0.028$ & ug/g dry & 0.028 & $5 / 07 / 08$ & 8D28006 & PNNL-AGG-ICP-AES \\
\hline $7440-69-9$ & Bismuth & $<0.188$ & ug/g dry & 0.188 & $5 / 07 / 08$ & 8D28006 & PNNL-AGG-ICP-AES \\
\hline $7440-70-2$ & Calcium & 17.2 & ug/g dry & 0.387 & $5 / 07 / 08$ & 8D28006 & PNNL-AGG-ICP-AES \\
\hline $7440-43-9$ & Cadmium & $<0.027$ & ug/g dry & 0.027 & $5 / 07 / 08$ & 8D28006 & PNNL-AGG-ICP-AES \\
\hline $7440-48-4$ & Cobalt & $<0.096$ & ug/g dry & 0.096 & $5 / 07 / 08$ & 8D28006 & PNNL-AGG-ICP-AES \\
\hline $7440-47-3$ & Chromium & $<0.033$ & ug/g dry & 0.033 & $5 / 07 / 08$ & 8D28006 & PNNL-AGG-ICP-AES \\
\hline $7440-50-8$ & Copper & $<0.080$ & ug/g dry & 0.080 & $5 / 07 / 08$ & 8D28006 & PNNL-AGG-ICP-AES \\
\hline 7439-89-6 & Iron & $<0.142$ & ug/g dry & 0.142 & $5 / 07 / 08$ & 8D28006 & PNNL-AGG-ICP-AES \\
\hline 7440-09-7 & Potassium & 6.14 & ug/g dry & 2.33 & $5 / 07 / 08$ & 8D28006 & PNNL-AGG-ICP-AES \\
\hline 7439-93-2 & Lithium & $<0.540$ & ug/g dry & 0.540 & $5 / 07 / 08$ & 8D28006 & PNNL-AGG-ICP-AES \\
\hline 7439-95-4 & Magnesium & 6.10 & ug/g dry & 0.083 & $5 / 07 / 08$ & 8D28006 & PNNL-AGG-ICP-AES \\
\hline 7439-96-5 & Manganese & $<0.017$ & ug/g dry & 0.017 & $5 / 07 / 08$ & 8D28006 & PNNL-AGG-ICP-AES \\
\hline 7439-98-7 & Molybdenum & $<0.139$ & ug/g dry & 0.139 & $5 / 07 / 08$ & 8D28006 & PNNL-AGG-ICP-AES \\
\hline $7440-02-0$ & Nickel & $<0.093$ & ug/g dry & 0.093 & $5 / 07 / 08$ & 8D28006 & PNNL-AGG-ICP-AES \\
\hline $7723-14-0$ & Phosphorus & $<1.03$ & ug/g dry & 1.03 & $5 / 07 / 08$ & 8D28006 & PNNL-AGG-ICP-AES \\
\hline 7439-92-1 & Lead & $<0.432$ & ug/g dry & 0.432 & $5 / 07 / 08$ & 8D28006 & PNNL-AGG-ICP-AES \\
\hline $7782-49-2$ & Selenium & $<1.95$ & ug/g dry & 1.95 & $5 / 07 / 08$ & 8D28006 & PNNL-AGG-ICP-AES \\
\hline 7440-24-6 & Strontium & 0.079 & ug/g dry & 0.052 & $5 / 07 / 08$ & 8D28006 & PNNL-AGG-ICP-AES \\
\hline $7440-28-0$ & Thallium & $<1.03$ & ug/g dry & 1.03 & $5 / 07 / 08$ & 8D28006 & PNNL-AGG-ICP-AES \\
\hline $7440-62-2$ & Vanadium & $<0.045$ & ug/g dry & 0.045 & $5 / 07 / 08$ & 8D28006 & PNNL-AGG-ICP-AES \\
\hline $7440-23-5$ & Sodium & 12.5 & ug/g dry & 0.669 & $5 / 07 / 08$ & 8D28006 & PNNL-AGG-ICP-AES \\
\hline $7440-21-3$ & Silicon & 6.98 & ug/g dry & 1.50 & $5 / 07 / 08$ & 8D28006 & PNNL-AGG-ICP-AES \\
\hline 7704-34-9 & Sulfur & 21.9 & ug/g dry & 3.07 & $5 / 07 / 08$ & 8D28006 & PNNL-AGG-ICP-AES \\
\hline $7440-32-6$ & Titanium & $<0.009$ & ug/g dry & 0.009 & $5 / 07 / 08$ & 8D28006 & PNNL-AGG-ICP-AES \\
\hline $7440-67-7$ & Zirconium & $<0.150$ & ug/g dry & 0.150 & $5 / 07 / 08$ & 8D28006 & PNNL-AGG-ICP-AES \\
\hline $7440-22-4$ & Silver & $<0.077$ & ug/g dry & 0.077 & $5 / 07 / 08$ & 8D28006 & PNNL-AGG-ICP-AES \\
\hline $7440-15-5$ & Rhenium & $<0.152$ & ug/g dry & 0.152 & $5 / 07 / 08$ & 8D28006 & PNNL-AGG-ICP-AES \\
\hline $7440-36-0$ & Antimony & $<0.633$ & ug/g dry & 0.633 & $5 / 07 / 08$ & 8D28006 & PNNL-AGG-ICP-AES \\
\hline HEIS No. & B1TNK6A & & b ID: & 3015-19 & & & \\
\hline $7429-90-5$ & Aluminum & 0.240 & ug/g dry & 0.087 & $5 / 07 / 08$ & 8D28006 & PNNL-AGG-ICP-AES \\
\hline $7440-38-2$ & Arsenic & $<0.365$ & ug/g dry & 0.365 & $5 / 07 / 08$ & 8D28006 & PNNL-AGG-ICP-AES \\
\hline $7440-42-8$ & Boron & $<1.97$ & ug/g dry & 1.97 & $5 / 07 / 08$ & 8D28006 & PNNL-AGG-ICP-AES \\
\hline $7440-39-3$ & Barium & $<0.009$ & ug/g dry & 0.009 & $5 / 07 / 08$ & 8D28006 & PNNL-AGG-ICP-AES \\
\hline $7440-41-7$ & Beryllium & $<0.029$ & ug/g dry & 0.029 & $5 / 07 / 08$ & 8D28006 & PNNL-AGG-ICP-AES \\
\hline $7440-69-9$ & Bismuth & $<0.191$ & ug/g dry & 0.191 & $5 / 07 / 08$ & 8D28006 & PNNL-AGG-ICP-AES \\
\hline $7440-70-2$ & Calcium & 13.8 & ug/g dry & 0.393 & $5 / 07 / 08$ & 8D28006 & PNNL-AGG-ICP-AES \\
\hline $7440-43-9$ & Cadmium & $<0.027$ & ug/g dry & 0.027 & $5 / 07 / 08$ & 8D28006 & PNNL-AGG-ICP-AES \\
\hline $7440-48-4$ & Cobalt & $<0.097$ & ug/g dry & 0.097 & $5 / 07 / 08$ & 8D28006 & PNNL-AGG-ICP-AES \\
\hline $7440-47-3$ & Chromium & $<0.034$ & ug/g dry & 0.034 & $5 / 07 / 08$ & 8D28006 & PNNL-AGG-ICP-AES \\
\hline $7440-50-8$ & Copper & $<0.082$ & ug/g dry & 0.082 & $5 / 07 / 08$ & 8D28006 & PNNL-AGG-ICP-AES \\
\hline 7439-89-6 & Iron & $<0.144$ & ug/g dry & 0.144 & $5 / 07 / 08$ & 8D28006 & PNNL-AGG-ICP-AES \\
\hline
\end{tabular}


Total Metals by SW846 6010B/Water Extract

\begin{tabular}{|c|c|c|c|c|c|c|c|}
\hline CAS \# & Analyte & Results & Units & EQL & Analyzed & Batch & Method \\
\hline HEIS No. & B1TNK6A & \multicolumn{3}{|c|}{ Lab ID: $\quad$ 0803015-19 } & & & \\
\hline 7440-09-7 & Potassium & 5.38 & ug/g dry & 2.36 & $5 / 07 / 08$ & 8D28006 & PNNL-AGG-ICP-AES \\
\hline 7439-93-2 & Lithium & $<0.548$ & ug/g dry & 0.548 & $5 / 07 / 08$ & 8D28006 & PNNL-AGG-ICP-AES \\
\hline 7439-95-4 & Magnesium & 4.57 & ug/g dry & 0.085 & $5 / 07 / 08$ & 8D28006 & PNNL-AGG-ICP-AES \\
\hline 7439-96-5 & Manganese & $<0.017$ & ug/g dry & 0.017 & $5 / 07 / 08$ & 8D28006 & PNNL-AGG-ICP-AES \\
\hline 7439-98-7 & Molybdenum & $<0.141$ & ug/g dry & 0.141 & $5 / 07 / 08$ & 8D28006 & PNNL-AGG-ICP-AES \\
\hline 7440-02-0 & Nickel & $<0.095$ & ug/g dry & 0.095 & $5 / 07 / 08$ & 8D28006 & PNNL-AGG-ICP-AES \\
\hline $7723-14-0$ & Phosphorus & $<1.05$ & ug/g dry & 1.05 & $5 / 07 / 08$ & 8D28006 & PNNL-AGG-ICP-AES \\
\hline 7439-92-1 & Lead & $<0.438$ & ug/g dry & 0.438 & $5 / 07 / 08$ & 8D28006 & PNNL-AGG-ICP-AES \\
\hline $7782-49-2$ & Selenium & $<1.98$ & ug/g dry & 1.98 & $5 / 07 / 08$ & 8D28006 & PNNL-AGG-ICP-AES \\
\hline $7440-24-6$ & Strontium & 0.068 & ug/g dry & 0.053 & $5 / 07 / 08$ & 8D28006 & PNNL-AGG-ICP-AES \\
\hline $7440-28-0$ & Thallium & $<1.04$ & ug/g dry & 1.04 & $5 / 07 / 08$ & 8D28006 & PNNL-AGG-ICP-AES \\
\hline $7440-62-2$ & Vanadium & $<0.046$ & ug/g dry & 0.046 & $5 / 07 / 08$ & 8D28006 & PNNL-AGG-ICP-AES \\
\hline $7440-23-5$ & Sodium & 11.3 & ug/g dry & 0.679 & $5 / 07 / 08$ & 8D28006 & PNNL-AGG-ICP-AES \\
\hline $7440-21-3$ & Silicon & 6.78 & ug/g dry & 1.52 & $5 / 07 / 08$ & 8D28006 & PNNL-AGG-ICP-AES \\
\hline $7704-34-9$ & Sulfur & 16.1 & ug/g dry & 3.12 & $5 / 07 / 08$ & 8D28006 & PNNL-AGG-ICP-AES \\
\hline $7440-32-6$ & Titanium & $<0.009$ & ug/g dry & 0.009 & $5 / 07 / 08$ & 8D28006 & PNNL-AGG-ICP-AES \\
\hline $7440-67-7$ & Zirconium & $<0.152$ & ug/g dry & 0.152 & $5 / 07 / 08$ & 8D28006 & PNNL-AGG-ICP-AES \\
\hline $7440-22-4$ & Silver & $<0.078$ & ug/g dry & 0.078 & $5 / 07 / 08$ & 8D28006 & PNNL-AGG-ICP-AES \\
\hline $7440-15-5$ & Rhenium & $<0.154$ & ug/g dry & 0.154 & $5 / 07 / 08$ & 8D28006 & PNNL-AGG-ICP-AES \\
\hline $7440-36-0$ & Antimony & $<0.642$ & ug/g dry & 0.642 & $5 / 07 / 08$ & 8D28006 & PNNL-AGG-ICP-AES \\
\hline HEIS No. & B1TNK7B & \multicolumn{3}{|c|}{ Lab ID: $\quad$ 0803015-22 } & & & \\
\hline $7429-90-5$ & Aluminum & 0.232 & ug/g dry & 0.086 & $5 / 07 / 08$ & 8D28006 & PNNL-AGG-ICP-AES \\
\hline $7440-38-2$ & Arsenic & $<0.360$ & ug/g dry & 0.360 & $5 / 07 / 08$ & 8D28006 & PNNL-AGG-ICP-AES \\
\hline $7440-42-8$ & Boron & $<1.94$ & ug/g dry & 1.94 & $5 / 07 / 08$ & 8D28006 & PNNL-AGG-ICP-AES \\
\hline $7440-39-3$ & Barium & $<0.009$ & ug/g dry & 0.009 & $5 / 07 / 08$ & 8D28006 & PNNL-AGG-ICP-AES \\
\hline $7440-41-7$ & Beryllium & $<0.028$ & ug/g dry & 0.028 & $5 / 07 / 08$ & 8D28006 & PNNL-AGG-ICP-AES \\
\hline $7440-69-9$ & Bismuth & $<0.188$ & ug/g dry & 0.188 & $5 / 07 / 08$ & 8D28006 & PNNL-AGG-ICP-AES \\
\hline $7440-70-2$ & Calcium & 11.2 & ug/g dry & 0.387 & $5 / 07 / 08$ & 8D28006 & PNNL-AGG-ICP-AES \\
\hline $7440-43-9$ & Cadmium & $<0.027$ & ug/g dry & 0.027 & $5 / 07 / 08$ & 8D28006 & PNNL-AGG-ICP-AES \\
\hline $7440-48-4$ & Cobalt & $<0.096$ & ug/g dry & 0.096 & $5 / 07 / 08$ & 8D28006 & PNNL-AGG-ICP-AES \\
\hline $7440-47-3$ & Chromium & $<0.033$ & ug/g dry & 0.033 & $5 / 07 / 08$ & 8D28006 & PNNL-AGG-ICP-AES \\
\hline $7440-50-8$ & Copper & $<0.080$ & ug/g dry & 0.080 & $5 / 07 / 08$ & 8D28006 & PNNL-AGG-ICP-AES \\
\hline 7439-89-6 & Iron & $<0.142$ & ug/g dry & 0.142 & $5 / 07 / 08$ & 8D28006 & PNNL-AGG-ICP-AES \\
\hline $7440-09-7$ & Potassium & 5.40 & ug/g dry & 2.33 & $5 / 07 / 08$ & 8D28006 & PNNL-AGG-ICP-AES \\
\hline $7439-93-2$ & Lithium & $<0.540$ & ug/g dry & 0.540 & $5 / 07 / 08$ & 8D28006 & PNNL-AGG-ICP-AES \\
\hline $7439-95-4$ & Magnesium & 4.04 & ug/g dry & 0.083 & $5 / 07 / 08$ & 8D28006 & PNNL-AGG-ICP-AES \\
\hline 7439-96-5 & Manganese & $<0.017$ & ug/g dry & 0.017 & $5 / 07 / 08$ & 8D28006 & PNNL-AGG-ICP-AES \\
\hline 7439-98-7 & Molybdenum & $<0.139$ & ug/g dry & 0.139 & $5 / 07 / 08$ & 8D28006 & PNNL-AGG-ICP-AES \\
\hline $7440-02-0$ & Nickel & $<0.093$ & ug/g dry & 0.093 & $5 / 07 / 08$ & 8D28006 & PNNL-AGG-ICP-AES \\
\hline $7723-14-0$ & Phosphorus & $<1.03$ & ug/g dry & 1.03 & $5 / 07 / 08$ & 8D28006 & PNNL-AGG-ICP-AES \\
\hline $7439-92-1$ & Lead & $<0.432$ & ug/g dry & 0.432 & $5 / 07 / 08$ & 8D28006 & PNNL-AGG-ICP-AES \\
\hline $7782-49-2$ & Selenium & $<1.95$ & ug/g dry & 1.95 & $5 / 07 / 08$ & 8D28006 & PNNL-AGG-ICP-AES \\
\hline $7440-24-6$ & Strontium & 0.057 & ug/g dry & 0.052 & $5 / 07 / 08$ & 8D28006 & PNNL-AGG-ICP-AES \\
\hline $7440-28-0$ & Thallium & $<1.03$ & ug/g dry & 1.03 & $5 / 07 / 08$ & 8D28006 & PNNL-AGG-ICP-AES \\
\hline $7440-62-2$ & Vanadium & $<0.045$ & ug/g dry & 0.045 & $5 / 07 / 08$ & 8D28006 & PNNL-AGG-ICP-AES \\
\hline $7440-23-5$ & Sodium & 11.3 & ug/g dry & 0.669 & $5 / 07 / 08$ & 8D28006 & PNNL-AGG-ICP-AES \\
\hline $7440-21-3$ & Silicon & 5.96 & ug/g dry & 1.50 & $5 / 07 / 08$ & 8D28006 & PNNL-AGG-ICP-AES \\
\hline 7704-34-9 & Sulfur & 13.3 & ug/g dry & 3.08 & $5 / 07 / 08$ & 8D28006 & PNNL-AGG-ICP-AES \\
\hline
\end{tabular}


Total Metals by SW846 6010B/Water Extract

\begin{tabular}{|c|c|c|c|c|c|c|c|}
\hline CAS \# & Analyte & Results & Units & EQL & Analyzed & Batch & Method \\
\hline HEIS No. & B1TNK7B & \multicolumn{3}{|c|}{ Lab ID: $\quad$ 0803015-22 } & & & \\
\hline $7440-32-6$ & Titanium & 0.009 & ug/g dry & 0.009 & $5 / 07 / 08$ & 8D28006 & PNNL-AGG-ICP-AES \\
\hline $7440-67-7$ & Zirconium & $<0.150$ & ug/g dry & 0.150 & $5 / 07 / 08$ & 8D28006 & PNNL-AGG-ICP-AES \\
\hline $7440-22-4$ & Silver & $<0.077$ & ug/g dry & 0.077 & $5 / 07 / 08$ & 8D28006 & PNNL-AGG-ICP-AES \\
\hline $7440-15-5$ & Rhenium & $<0.152$ & ug/g dry & 0.152 & $5 / 07 / 08$ & 8D28006 & PNNL-AGG-ICP-AES \\
\hline $7440-36-0$ & Antimony & $<0.633$ & ug/g dry & 0.633 & $5 / 07 / 08$ & 8D28006 & PNNL-AGG-ICP-AES \\
\hline HEIS No. & B1TNK7A & \multicolumn{3}{|c|}{ Lab ID: $\quad$ 0803015-23 } & & & \\
\hline 7429-90-5 & Aluminum & 0.166 & ug/g dry & 0.086 & $5 / 07 / 08$ & 8D28006 & PNNL-AGG-ICP-AES \\
\hline $7440-38-2$ & Arsenic & $<0.361$ & ug/g dry & 0.361 & $5 / 07 / 08$ & 8D28006 & PNNL-AGG-ICP-AES \\
\hline $7440-42-8$ & Boron & $<1.94$ & ug/g dry & 1.94 & $5 / 07 / 08$ & 8D28006 & PNNL-AGG-ICP-AES \\
\hline $7440-39-3$ & Barium & $<0.009$ & ug/g dry & 0.009 & $5 / 07 / 08$ & 8D28006 & PNNL-AGG-ICP-AES \\
\hline $7440-41-7$ & Beryllium & $<0.028$ & ug/g dry & 0.028 & $5 / 07 / 08$ & 8D28006 & PNNL-AGG-ICP-AES \\
\hline 7440-69-9 & Bismuth & $<0.188$ & ug/g dry & 0.188 & $5 / 07 / 08$ & 8D28006 & PNNL-AGG-ICP-AES \\
\hline $7440-70-2$ & Calcium & 10.5 & ug/g dry & 0.388 & $5 / 07 / 08$ & 8D28006 & PNNL-AGG-ICP-AES \\
\hline $7440-43-9$ & Cadmium & $<0.027$ & ug/g dry & 0.027 & $5 / 07 / 08$ & 8D28006 & PNNL-AGG-ICP-AES \\
\hline $7440-48-4$ & Cobalt & $<0.096$ & ug/g dry & 0.096 & $5 / 07 / 08$ & 8D28006 & PNNL-AGG-ICP-AES \\
\hline $7440-47-3$ & Chromium & $<0.033$ & ug/g dry & 0.033 & $5 / 07 / 08$ & 8D28006 & PNNL-AGG-ICP-AES \\
\hline $7440-50-8$ & Copper & $<0.081$ & ug/g dry & 0.081 & $5 / 07 / 08$ & 8D28006 & PNNL-AGG-ICP-AES \\
\hline 7439-89-6 & Iron & $<0.142$ & ug/g dry & 0.142 & $5 / 07 / 08$ & 8D28006 & PNNL-AGG-ICP-AES \\
\hline $7440-09-7$ & Potassium & 4.79 & ug/g dry & 2.33 & $5 / 07 / 08$ & 8D28006 & PNNL-AGG-ICP-AES \\
\hline 7439-93-2 & Lithium & $<0.541$ & ug/g dry & 0.541 & $5 / 07 / 08$ & 8D28006 & PNNL-AGG-ICP-AES \\
\hline $7439-95-4$ & Magnesium & 3.95 & ug/g dry & 0.084 & $5 / 07 / 08$ & 8D28006 & PNNL-AGG-ICP-AES \\
\hline 7439-96-5 & Manganese & $<0.017$ & ug/g dry & 0.017 & $5 / 07 / 08$ & 8D28006 & PNNL-AGG-ICP-AES \\
\hline 7439-98-7 & Molybdenum & $<0.139$ & ug/g dry & 0.139 & $5 / 07 / 08$ & 8D28006 & PNNL-AGG-ICP-AES \\
\hline $7440-02-0$ & Nickel & $<0.093$ & ug/g dry & 0.093 & $5 / 07 / 08$ & 8D28006 & PNNL-AGG-ICP-AES \\
\hline 7723-14-0 & Phosphorus & $<1.03$ & ug/g dry & 1.03 & $5 / 07 / 08$ & 8D28006 & PNNL-AGG-ICP-AES \\
\hline 7439-92-1 & Lead & $<0.433$ & ug/g dry & 0.433 & $5 / 07 / 08$ & 8D28006 & PNNL-AGG-ICP-AES \\
\hline $7782-49-2$ & Selenium & $<1.95$ & ug/g dry & 1.95 & $5 / 07 / 08$ & 8D28006 & PNNL-AGG-ICP-AES \\
\hline $7440-24-6$ & Strontium & $<0.052$ & ug/g dry & 0.052 & $5 / 07 / 08$ & 8D28006 & PNNL-AGG-ICP-AES \\
\hline $7440-28-0$ & Thallium & $<1.03$ & ug/g dry & 1.03 & $5 / 07 / 08$ & 8D28006 & PNNL-AGG-ICP-AES \\
\hline $7440-62-2$ & Vanadium & $<0.045$ & ug/g dry & 0.045 & $5 / 07 / 08$ & 8D28006 & PNNL-AGG-ICP-AES \\
\hline $7440-23-5$ & Sodium & 9.86 & ug/g dry & 0.670 & $5 / 07 / 08$ & 8D28006 & PNNL-AGG-ICP-AES \\
\hline $7440-21-3$ & Silicon & 5.52 & ug/g dry & 1.50 & $5 / 07 / 08$ & 8D28006 & PNNL-AGG-ICP-AES \\
\hline $7704-34-9$ & Sulfur & 12.9 & ug/g dry & 3.08 & $5 / 07 / 08$ & 8D28006 & PNNL-AGG-ICP-AES \\
\hline $7440-32-6$ & Titanium & $<0.009$ & ug/g dry & 0.009 & $5 / 07 / 08$ & 8D28006 & PNNL-AGG-ICP-AES \\
\hline $7440-67-7$ & Zirconium & $<0.150$ & ug/g dry & 0.150 & $5 / 07 / 08$ & 8D28006 & PNNL-AGG-ICP-AES \\
\hline $7440-22-4$ & Silver & $<0.077$ & ug/g dry & 0.077 & $5 / 07 / 08$ & 8D28006 & PNNL-AGG-ICP-AES \\
\hline $7440-15-5$ & Rhenium & $<0.152$ & ug/g dry & 0.152 & $5 / 07 / 08$ & 8D28006 & PNNL-AGG-ICP-AES \\
\hline $7440-36-0$ & Antimony & $<0.634$ & ug/g dry & 0.634 & $5 / 07 / 08$ & 8D28006 & PNNL-AGG-ICP-AES \\
\hline HEIS No. & B1TNK8B & \multicolumn{3}{|c|}{ Lab ID: $\quad$ 0803015-26 } & & & \\
\hline $7429-90-5$ & Aluminum & 0.136 & ug/g dry & 0.086 & $5 / 07 / 08$ & 8D28006 & PNNL-AGG-ICP-AES \\
\hline $7440-38-2$ & Arsenic & $<0.360$ & ug/g dry & 0.360 & $5 / 07 / 08$ & 8D28006 & PNNL-AGG-ICP-AES \\
\hline $7440-42-8$ & Boron & $<1.94$ & ug/g dry & 1.94 & $5 / 07 / 08$ & 8D28006 & PNNL-AGG-ICP-AES \\
\hline 7440-39-3 & Barium & $<0.009$ & ug/g dry & 0.009 & $5 / 07 / 08$ & 8D28006 & PNNL-AGG-ICP-AES \\
\hline $7440-41-7$ & Beryllium & $<0.028$ & ug/g dry & 0.028 & $5 / 07 / 08$ & 8D28006 & PNNL-AGG-ICP-AES \\
\hline $7440-69-9$ & Bismuth & $<0.188$ & ug/g dry & 0.188 & $5 / 07 / 08$ & 8D28006 & PNNL-AGG-ICP-AES \\
\hline $7440-70-2$ & Calcium & 20.3 & ug/g dry & 0.387 & $5 / 07 / 08$ & 8D28006 & PNNL-AGG-ICP-AES \\
\hline $7440-43-9$ & Cadmium & $<0.027$ & ug/g dry & 0.027 & $5 / 07 / 08$ & 8D28006 & PNNL-AGG-ICP-AES \\
\hline $7440-48-4$ & Cobalt & $<0.096$ & ug/g dry & 0.096 & $5 / 07 / 08$ & 8D28006 & PNNL-AGG-ICP-AES \\
\hline
\end{tabular}


Total Metals by SW846 6010B/Water Extract

\begin{tabular}{|c|c|c|c|c|c|c|c|}
\hline CAS \# & Analyte & Results & Units & EQL & Analyzed & Batch & Method \\
\hline HEIS No. & B1TNK8B & \multicolumn{3}{|c|}{ Lab ID: $\quad$ 0803015-26 } & & & \\
\hline $7440-47-3$ & Chromium & $<0.033$ & ug/g dry & 0.033 & $5 / 07 / 08$ & 8D28006 & PNNL-AGG-ICP-AES \\
\hline $7440-50-8$ & Copper & $<0.080$ & ug/g dry & 0.080 & $5 / 07 / 08$ & 8D28006 & PNNL-AGG-ICP-AES \\
\hline 7439-89-6 & Iron & $<0.142$ & ug/g dry & 0.142 & $5 / 07 / 08$ & 8D28006 & PNNL-AGG-ICP-AES \\
\hline 7440-09-7 & Potassium & 7.57 & ug/g dry & 2.33 & $5 / 07 / 08$ & 8D28006 & PNNL-AGG-ICP-AES \\
\hline $7439-93-2$ & Lithium & $<0.540$ & ug/g dry & 0.540 & $5 / 07 / 08$ & 8D28006 & PNNL-AGG-ICP-AES \\
\hline 7439-95-4 & Magnesium & 7.39 & ug/g dry & 0.083 & $5 / 07 / 08$ & 8D28006 & PNNL-AGG-ICP-AES \\
\hline $7439-96-5$ & Manganese & $<0.017$ & ug/g dry & 0.017 & $5 / 07 / 08$ & 8D28006 & PNNL-AGG-ICP-AES \\
\hline 7439-98-7 & Molybdenum & $<0.139$ & ug/g dry & 0.139 & $5 / 07 / 08$ & 8D28006 & PNNL-AGG-ICP-AES \\
\hline 7440-02-0 & Nickel & $<0.093$ & ug/g dry & 0.093 & $5 / 07 / 08$ & 8D28006 & PNNL-AGG-ICP-AES \\
\hline $7723-14-0$ & Phosphorus & $<1.03$ & ug/g dry & 1.03 & $5 / 07 / 08$ & 8D28006 & PNNL-AGG-ICP-AES \\
\hline 7439-92-1 & Lead & $<0.432$ & ug/g dry & 0.432 & $5 / 07 / 08$ & 8D28006 & PNNL-AGG-ICP-AES \\
\hline $7782-49-2$ & Selenium & $<1.95$ & ug/g dry & 1.95 & $5 / 07 / 08$ & 8D28006 & PNNL-AGG-ICP-AES \\
\hline $7440-24-6$ & Strontium & 0.091 & ug/g dry & 0.052 & $5 / 07 / 08$ & 8D28006 & PNNL-AGG-ICP-AES \\
\hline $7440-28-0$ & Thallium & $<1.03$ & ug/g dry & 1.03 & $5 / 07 / 08$ & 8D28006 & PNNL-AGG-ICP-AES \\
\hline $7440-62-2$ & Vanadium & $<0.045$ & ug/g dry & 0.045 & $5 / 07 / 08$ & 8D28006 & PNNL-AGG-ICP-AES \\
\hline $7440-23-5$ & Sodium & 13.3 & ug/g dry & 0.669 & $5 / 07 / 08$ & 8D28006 & PNNL-AGG-ICP-AES \\
\hline $7440-21-3$ & Silicon & 5.38 & ug/g dry & 1.50 & $5 / 07 / 08$ & 8D28006 & PNNL-AGG-ICP-AES \\
\hline $7704-34-9$ & Sulfur & 27.2 & ug/g dry & 3.08 & $5 / 07 / 08$ & 8D28006 & PNNL-AGG-ICP-AES \\
\hline $7440-32-6$ & Titanium & $<0.009$ & ug/g dry & 0.009 & $5 / 07 / 08$ & 8D28006 & PNNL-AGG-ICP-AES \\
\hline $7440-67-7$ & Zirconium & $<0.150$ & ug/g dry & 0.150 & $5 / 07 / 08$ & 8D28006 & PNNL-AGG-ICP-AES \\
\hline $7440-22-4$ & Silver & $<0.077$ & ug/g dry & 0.077 & $5 / 07 / 08$ & 8D28006 & PNNL-AGG-ICP-AES \\
\hline $7440-15-5$ & Rhenium & $<0.152$ & ug/g dry & 0.152 & $5 / 07 / 08$ & 8D28006 & PNNL-AGG-ICP-AES \\
\hline $7440-36-0$ & Antimony & $<0.633$ & ug/g dry & 0.633 & $5 / 07 / 08$ & 8D28006 & PNNL-AGG-ICP-AES \\
\hline HEIS No. & B1TNK8A & \multicolumn{3}{|c|}{ Lab ID: $\quad$ 0803015-27 } & & & \\
\hline $7429-90-5$ & Aluminum & $<0.086$ & ug/g dry & 0.086 & $5 / 07 / 08$ & 8D28006 & PNNL-AGG-ICP-AES \\
\hline $7440-38-2$ & Arsenic & $<0.360$ & ug/g dry & 0.360 & $5 / 07 / 08$ & 8D28006 & PNNL-AGG-ICP-AES \\
\hline $7440-42-8$ & Boron & $<1.94$ & ug/g dry & 1.94 & $5 / 07 / 08$ & 8D28006 & PNNL-AGG-ICP-AES \\
\hline $7440-39-3$ & Barium & $<0.009$ & ug/g dry & 0.009 & $5 / 07 / 08$ & 8D28006 & PNNL-AGG-ICP-AES \\
\hline $7440-41-7$ & Beryllium & $<0.028$ & ug/g dry & 0.028 & $5 / 07 / 08$ & 8D28006 & PNNL-AGG-ICP-AES \\
\hline $7440-69-9$ & Bismuth & $<0.188$ & ug/g dry & 0.188 & $5 / 07 / 08$ & 8D28006 & PNNL-AGG-ICP-AES \\
\hline $7440-70-2$ & Calcium & 36.1 & ug/g dry & 0.387 & $5 / 07 / 08$ & 8D28006 & PNNL-AGG-ICP-AES \\
\hline $7440-43-9$ & Cadmium & $<0.027$ & ug/g dry & 0.027 & $5 / 07 / 08$ & 8D28006 & PNNL-AGG-ICP-AES \\
\hline $7440-48-4$ & Cobalt & $<0.096$ & ug/g dry & 0.096 & $5 / 07 / 08$ & 8D28006 & PNNL-AGG-ICP-AES \\
\hline $7440-47-3$ & Chromium & $<0.033$ & ug/g dry & 0.033 & $5 / 07 / 08$ & 8D28006 & PNNL-AGG-ICP-AES \\
\hline $7440-50-8$ & Copper & $<0.080$ & ug/g dry & 0.080 & $5 / 07 / 08$ & 8D28006 & PNNL-AGG-ICP-AES \\
\hline 7439-89-6 & Iron & $<0.142$ & ug/g dry & 0.142 & $5 / 07 / 08$ & 8D28006 & PNNL-AGG-ICP-AES \\
\hline 7440-09-7 & Potassium & 7.99 & ug/g dry & 2.33 & $5 / 07 / 08$ & 8D28006 & PNNL-AGG-ICP-AES \\
\hline $7439-93-2$ & Lithium & $<0.540$ & ug/g dry & 0.540 & $5 / 07 / 08$ & 8D28006 & PNNL-AGG-ICP-AES \\
\hline $7439-95-4$ & Magnesium & 12.0 & ug/g dry & 0.083 & $5 / 07 / 08$ & 8D28006 & PNNL-AGG-ICP-AES \\
\hline $7439-96-5$ & Manganese & $<0.017$ & ug/g dry & 0.017 & $5 / 07 / 08$ & 8D28006 & PNNL-AGG-ICP-AES \\
\hline 7439-98-7 & Molybdenum & $<0.139$ & ug/g dry & 0.139 & $5 / 07 / 08$ & 8D28006 & PNNL-AGG-ICP-AES \\
\hline $7440-02-0$ & Nickel & $<0.093$ & ug/g dry & 0.093 & $5 / 07 / 08$ & 8D28006 & PNNL-AGG-ICP-AES \\
\hline $7723-14-0$ & Phosphorus & $<1.03$ & ug/g dry & 1.03 & $5 / 07 / 08$ & 8D28006 & PNNL-AGG-ICP-AES \\
\hline 7439-92-1 & Lead & $<0.432$ & ug/g dry & 0.432 & $5 / 07 / 08$ & 8D28006 & PNNL-AGG-ICP-AES \\
\hline 7782-49-2 & Selenium & $<1.95$ & ug/g dry & 1.95 & $5 / 07 / 08$ & 8D28006 & PNNL-AGG-ICP-AES \\
\hline $7440-24-6$ & Strontium & 0.142 & ug/g dry & 0.052 & $5 / 07 / 08$ & 8D28006 & PNNL-AGG-ICP-AES \\
\hline $7440-28-0$ & Thallium & $<1.03$ & ug/g dry & 1.03 & $5 / 07 / 08$ & 8D28006 & PNNL-AGG-ICP-AES \\
\hline $7440-62-2$ & Vanadium & $<0.045$ & ug/g dry & 0.045 & $5 / 07 / 08$ & 8D28006 & PNNL-AGG-ICP-AES \\
\hline
\end{tabular}


Total Metals by SW846 6010B/Water Extract

\begin{tabular}{|c|c|c|c|c|c|c|c|}
\hline CAS \# & Analyte & Results & Units & EQL & Analyzed & Batch & Method \\
\hline HEIS No. & B1TNK8A & \multicolumn{3}{|c|}{ Lab ID: $\quad$ 0803015-27 } & & & \\
\hline $7440-23-5$ & Sodium & 13.0 & ug/g dry & 0.669 & $5 / 07 / 08$ & 8D28006 & PNNL-AGG-ICP-AES \\
\hline $7440-21-3$ & Silicon & 5.43 & ug/g dry & 1.50 & $5 / 07 / 08$ & 8D28006 & PNNL-AGG-ICP-AES \\
\hline 7704-34-9 & Sulfur & 47.2 & ug/g dry & 3.08 & $5 / 07 / 08$ & 8D28006 & PNNL-AGG-ICP-AES \\
\hline $7440-32-6$ & Titanium & $<0.009$ & ug/g dry & 0.009 & $5 / 07 / 08$ & 8D28006 & PNNL-AGG-ICP-AES \\
\hline 7440-67-7 & Zirconium & $<0.150$ & ug/g dry & 0.150 & $5 / 07 / 08$ & 8D28006 & PNNL-AGG-ICP-AES \\
\hline $7440-22-4$ & Silver & $<0.077$ & ug/g dry & 0.077 & $5 / 07 / 08$ & 8D28006 & PNNL-AGG-ICP-AES \\
\hline $7440-15-5$ & Rhenium & $<0.152$ & ug/g dry & 0.152 & $5 / 07 / 08$ & 8D28006 & PNNL-AGG-ICP-AES \\
\hline $7440-36-0$ & Antimony & $<0.633$ & ug/g dry & 0.633 & $5 / 07 / 08$ & 8D28006 & PNNL-AGG-ICP-AES \\
\hline HEIS No. & B1TNK9B & \multicolumn{3}{|c|}{ Lab ID: $\quad$ 0803015-30 } & & & \\
\hline $7429-90-5$ & Aluminum & 0.136 & ug/g dry & 0.090 & $5 / 07 / 08$ & 8D28006 & PNNL-AGG-ICP-AES \\
\hline $7440-38-2$ & Arsenic & $<0.377$ & ug/g dry & 0.377 & $5 / 07 / 08$ & 8D28006 & PNNL-AGG-ICP-AES \\
\hline $7440-42-8$ & Boron & $<2.03$ & ug/g dry & 2.03 & $5 / 07 / 08$ & 8D28006 & PNNL-AGG-ICP-AES \\
\hline $7440-39-3$ & Barium & $<0.009$ & ug/g dry & 0.009 & $5 / 07 / 08$ & 8D28006 & PNNL-AGG-ICP-AES \\
\hline $7440-41-7$ & Beryllium & $<0.030$ & ug/g dry & 0.030 & $5 / 07 / 08$ & 8D28006 & PNNL-AGG-ICP-AES \\
\hline $7440-69-9$ & Bismuth & $<0.197$ & ug/g dry & 0.197 & $5 / 07 / 08$ & 8D28006 & PNNL-AGG-ICP-AES \\
\hline $7440-70-2$ & Calcium & 14.4 & ug/g dry & 0.405 & $5 / 07 / 08$ & 8D28006 & PNNL-AGG-ICP-AES \\
\hline 7440-43-9 & Cadmium & $<0.028$ & ug/g dry & 0.028 & $5 / 07 / 08$ & 8D28006 & PNNL-AGG-ICP-AES \\
\hline $7440-48-4$ & Cobalt & $<0.100$ & ug/g dry & 0.100 & $5 / 07 / 08$ & 8D28006 & PNNL-AGG-ICP-AES \\
\hline $7440-47-3$ & Chromium & $<0.035$ & ug/g dry & 0.035 & $5 / 07 / 08$ & 8D28006 & PNNL-AGG-ICP-AES \\
\hline $7440-50-8$ & Copper & $<0.084$ & ug/g dry & 0.084 & $5 / 07 / 08$ & 8D28006 & PNNL-AGG-ICP-AES \\
\hline 7439-89-6 & Iron & $<0.149$ & ug/g dry & 0.149 & $5 / 07 / 08$ & 8D28006 & PNNL-AGG-ICP-AES \\
\hline $7440-09-7$ & Potassium & 6.82 & ug/g dry & 2.43 & $5 / 07 / 08$ & 8D28006 & PNNL-AGG-ICP-AES \\
\hline 7439-93-2 & Lithium & $<0.565$ & ug/g dry & 0.565 & $5 / 07 / 08$ & 8D28006 & PNNL-AGG-ICP-AES \\
\hline 7439-95-4 & Magnesium & 4.65 & ug/g dry & 0.087 & $5 / 07 / 08$ & 8D28006 & PNNL-AGG-ICP-AES \\
\hline 7439-96-5 & Manganese & $<0.018$ & ug/g dry & 0.018 & $5 / 07 / 08$ & 8D28006 & PNNL-AGG-ICP-AES \\
\hline 7439-98-7 & Molybdenum & $<0.146$ & ug/g dry & 0.146 & $5 / 07 / 08$ & 8D28006 & PNNL-AGG-ICP-AES \\
\hline $7440-02-0$ & Nickel & $<0.098$ & ug/g dry & 0.098 & $5 / 07 / 08$ & 8D28006 & PNNL-AGG-ICP-AES \\
\hline 7723-14-0 & Phosphorus & $<1.08$ & ug/g dry & 1.08 & $5 / 07 / 08$ & 8D28006 & PNNL-AGG-ICP-AES \\
\hline 7439-92-1 & Lead & $<0.452$ & ug/g dry & 0.452 & $5 / 07 / 08$ & 8D28006 & PNNL-AGG-ICP-AES \\
\hline $7782-49-2$ & Selenium & $<2.04$ & ug/g dry & 2.04 & $5 / 07 / 08$ & 8D28006 & PNNL-AGG-ICP-AES \\
\hline $7440-24-6$ & Strontium & 0.064 & ug/g dry & 0.055 & $5 / 07 / 08$ & 8D28006 & PNNL-AGG-ICP-AES \\
\hline $7440-28-0$ & Thallium & $<1.07$ & ug/g dry & 1.07 & $5 / 07 / 08$ & 8D28006 & PNNL-AGG-ICP-AES \\
\hline $7440-62-2$ & Vanadium & $<0.047$ & ug/g dry & 0.047 & $5 / 07 / 08$ & 8D28006 & PNNL-AGG-ICP-AES \\
\hline $7440-23-5$ & Sodium & 14.2 & ug/g dry & 0.700 & $5 / 07 / 08$ & 8D28006 & PNNL-AGG-ICP-AES \\
\hline $7440-21-3$ & Silicon & 6.23 & ug/g dry & 1.57 & $5 / 07 / 08$ & 8D28006 & PNNL-AGG-ICP-AES \\
\hline 7704-34-9 & Sulfur & 16.0 & ug/g dry & 3.22 & $5 / 07 / 08$ & 8D28006 & PNNL-AGG-ICP-AES \\
\hline $7440-32-6$ & Titanium & $<0.009$ & ug/g dry & 0.009 & $5 / 07 / 08$ & 8D28006 & PNNL-AGG-ICP-AES \\
\hline $7440-67-7$ & Zirconium & $<0.157$ & ug/g dry & 0.157 & $5 / 07 / 08$ & 8D28006 & PNNL-AGG-ICP-AES \\
\hline $7440-22-4$ & Silver & $<0.081$ & ug/g dry & 0.081 & $5 / 07 / 08$ & 8D28006 & PNNL-AGG-ICP-AES \\
\hline $7440-15-5$ & Rhenium & $<0.159$ & ug/g dry & 0.159 & $5 / 07 / 08$ & 8D28006 & PNNL-AGG-ICP-AES \\
\hline $7440-36-0$ & Antimony & $<0.662$ & ug/g dry & 0.662 & $5 / 07 / 08$ & 8D28006 & PNNL-AGG-ICP-AES \\
\hline HEIS No. & B1TNK9A & & b ID: & 3015-31 & & & \\
\hline $7429-90-5$ & Aluminum & $<0.086$ & ug/g dry & 0.086 & $5 / 07 / 08$ & 8D28006 & PNNL-AGG-ICP-AES \\
\hline $7440-38-2$ & Arsenic & $<0.360$ & ug/g dry & 0.360 & $5 / 07 / 08$ & 8D28006 & PNNL-AGG-ICP-AES \\
\hline $7440-42-8$ & Boron & $<1.94$ & ug/g dry & 1.94 & $5 / 07 / 08$ & 8D28006 & PNNL-AGG-ICP-AES \\
\hline 7440-39-3 & Barium & 0.009 & ug/g dry & 0.009 & $5 / 07 / 08$ & 8D28006 & PNNL-AGG-ICP-AES \\
\hline $7440-41-7$ & Beryllium & $<0.028$ & ug/g dry & 0.028 & $5 / 07 / 08$ & 8D28006 & PNNL-AGG-ICP-AES \\
\hline 7440-69-9 & Bismuth & $<0.188$ & ug/g dry & 0.188 & $5 / 07 / 08$ & 8D28006 & PNNL-AGG-ICP-AES \\
\hline
\end{tabular}


Total Metals by SW846 6010B/Water Extract

\begin{tabular}{|c|c|c|c|c|c|c|c|}
\hline CAS \# & Analyte & Results & Units & EQL & Analyzed & Batch & Method \\
\hline HEIS No. & B1TNK9A & \multicolumn{3}{|c|}{ Lab ID: $\quad$ 0803015-31 } & & & \\
\hline $7440-70-2$ & Calcium & 35.1 & ug/g dry & 0.387 & $5 / 07 / 08$ & 8D28006 & PNNL-AGG-ICP-AES \\
\hline 7440-43-9 & Cadmium & $<0.027$ & ug/g dry & 0.027 & $5 / 07 / 08$ & 8D28006 & PNNL-AGG-ICP-AES \\
\hline $7440-48-4$ & Cobalt & $<0.096$ & ug/g dry & 0.096 & $5 / 07 / 08$ & 8D28006 & PNNL-AGG-ICP-AES \\
\hline $7440-47-3$ & Chromium & $<0.033$ & ug/g dry & 0.033 & $5 / 07 / 08$ & 8D28006 & PNNL-AGG-ICP-AES \\
\hline $7440-50-8$ & Copper & $<0.080$ & ug/g dry & 0.080 & $5 / 07 / 08$ & 8D28006 & PNNL-AGG-ICP-AES \\
\hline 7439-89-6 & Iron & $<0.142$ & ug/g dry & 0.142 & $5 / 07 / 08$ & 8D28006 & PNNL-AGG-ICP-AES \\
\hline 7440-09-7 & Potassium & 8.96 & ug/g dry & 2.33 & $5 / 07 / 08$ & 8D28006 & PNNL-AGG-ICP-AES \\
\hline 7439-93-2 & Lithium & $<0.540$ & ug/g dry & 0.540 & $5 / 07 / 08$ & 8D28006 & PNNL-AGG-ICP-AES \\
\hline 7439-95-4 & Magnesium & 9.94 & ug/g dry & 0.083 & $5 / 07 / 08$ & 8D28006 & PNNL-AGG-ICP-AES \\
\hline $7439-96-5$ & Manganese & $<0.017$ & ug/g dry & 0.017 & $5 / 07 / 08$ & 8D28006 & PNNL-AGG-ICP-AES \\
\hline 7439-98-7 & Molybdenum & $<0.139$ & ug/g dry & 0.139 & $5 / 07 / 08$ & 8D28006 & PNNL-AGG-ICP-AES \\
\hline $7440-02-0$ & Nickel & $<0.093$ & ug/g dry & 0.093 & $5 / 07 / 08$ & 8D28006 & PNNL-AGG-ICP-AES \\
\hline $7723-14-0$ & Phosphorus & $<1.03$ & ug/g dry & 1.03 & $5 / 07 / 08$ & 8D28006 & PNNL-AGG-ICP-AES \\
\hline 7439-92-1 & Lead & $<0.432$ & ug/g dry & 0.432 & $5 / 07 / 08$ & 8D28006 & PNNL-AGG-ICP-AES \\
\hline 7782-49-2 & Selenium & $<1.95$ & ug/g dry & 1.95 & $5 / 07 / 08$ & 8D28006 & PNNL-AGG-ICP-AES \\
\hline $7440-24-6$ & Strontium & 0.129 & ug/g dry & 0.052 & $5 / 07 / 08$ & 8D28006 & PNNL-AGG-ICP-AES \\
\hline $7440-28-0$ & Thallium & $<1.03$ & ug/g dry & 1.03 & $5 / 07 / 08$ & 8D28006 & PNNL-AGG-ICP-AES \\
\hline $7440-62-2$ & Vanadium & $<0.045$ & ug/g dry & 0.045 & $5 / 07 / 08$ & 8D28006 & PNNL-AGG-ICP-AES \\
\hline $7440-23-5$ & Sodium & 16.6 & ug/g dry & 0.669 & $5 / 07 / 08$ & 8D28006 & PNNL-AGG-ICP-AES \\
\hline $7440-21-3$ & Silicon & 6.03 & ug/g dry & 1.50 & $5 / 07 / 08$ & 8D28006 & PNNL-AGG-ICP-AES \\
\hline 7704-34-9 & Sulfur & 44.5 & ug/g dry & 3.07 & $5 / 07 / 08$ & 8D28006 & PNNL-AGG-ICP-AES \\
\hline $7440-32-6$ & Titanium & $<0.009$ & ug/g dry & 0.009 & $5 / 07 / 08$ & 8D28006 & PNNL-AGG-ICP-AES \\
\hline 7440-67-7 & Zirconium & $<0.150$ & ug/g dry & 0.150 & $5 / 07 / 08$ & 8D28006 & PNNL-AGG-ICP-AES \\
\hline $7440-22-4$ & Silver & $<0.077$ & ug/g dry & 0.077 & $5 / 07 / 08$ & 8D28006 & PNNL-AGG-ICP-AES \\
\hline $7440-15-5$ & Rhenium & $<0.152$ & ug/g dry & 0.152 & $5 / 07 / 08$ & 8D28006 & PNNL-AGG-ICP-AES \\
\hline $7440-36-0$ & Antimony & $<0.633$ & ug/g dry & 0.633 & $5 / 07 / 08$ & 8D28006 & PNNL-AGG-ICP-AES \\
\hline
\end{tabular}


Total Metals by SW846 6010B/Acid Extract

\begin{tabular}{|c|c|c|c|c|c|c|c|}
\hline CAS \# & Analyte & Results & Units & EQL & Analyzed & Batch & Method \\
\hline HEIS No. & B1RTF8 & \multicolumn{3}{|c|}{ Lab ID: $\quad$ 0803001-01 } & & & \\
\hline 7429-90-5 & Aluminum & 4420 & ug/g dry & 31.8 & $5 / 22 / 08$ & 8E12001 & PNNL-AGG-ICP-AES \\
\hline $7440-38-2$ & Arsenic & $<77.2$ & ug/g dry & 77.2 & $5 / 22 / 08$ & 8E12001 & PNNL-AGG-ICP-AES \\
\hline $7440-42-8$ & Boron & $<672$ & ug/g dry & 672 & $5 / 22 / 08$ & 8E12001 & PNNL-AGG-ICP-AES \\
\hline $7440-39-3$ & Barium & 50.1 & ug/g dry & 3.03 & $5 / 22 / 08$ & 8E12001 & PNNL-AGG-ICP-AES \\
\hline $7440-41-7$ & Beryllium & $<1.20$ & ug/g dry & 1.20 & $5 / 22 / 08$ & 8E12001 & PNNL-AGG-ICP-AES \\
\hline 7440-69-9 & Bismuth & $<16.0$ & ug/g dry & 16.0 & $5 / 22 / 08$ & 8E12001 & PNNL-AGG-ICP-AES \\
\hline $7440-70-2$ & Calcium & 5770 & ug/g dry & 118 & $5 / 22 / 08$ & 8E12001 & PNNL-AGG-ICP-AES \\
\hline $7440-43-9$ & Cadmium & $<1.80$ & ug/g dry & 1.80 & $5 / 22 / 08$ & 8E12001 & PNNL-AGG-ICP-AES \\
\hline $7440-48-4$ & Cobalt & 8.58 & ug/g dry & 6.59 & $5 / 22 / 08$ & 8E12001 & PNNL-AGG-ICP-AES \\
\hline $7440-47-3$ & Chromium & 6.29 & ug/g dry & 2.64 & $5 / 22 / 08$ & 8E12001 & PNNL-AGG-ICP-AES \\
\hline $7440-50-8$ & Copper & $<28.1$ & ug/g dry & 28.1 & $5 / 22 / 08$ & 8E12001 & PNNL-AGG-ICP-AES \\
\hline 7439-89-6 & Iron & 16800 & ug/g dry & 161 & $5 / 22 / 08$ & 8E12001 & PNNL-AGG-ICP-AES \\
\hline 7440-09-7 & Potassium & 610 & ug/g dry & 74.9 & $5 / 22 / 08$ & 8E12001 & PNNL-AGG-ICP-AES \\
\hline 7439-93-2 & Lithium & $<11.5$ & ug/g dry & 11.5 & $5 / 22 / 08$ & 8E12001 & PNNL-AGG-ICP-AES \\
\hline $7439-95-4$ & Magnesium & 3970 & ug/g dry & 25.0 & $5 / 22 / 08$ & 8E12001 & PNNL-AGG-ICP-AES \\
\hline 7439-96-5 & Manganese & 263 & ug/g dry & 0.946 & $5 / 22 / 08$ & 8E12001 & PNNL-AGG-ICP-AES \\
\hline 7439-98-7 & Molybdenum & $<17.9$ & ug/g dry & 17.9 & $5 / 22 / 08$ & 8E12001 & PNNL-AGG-ICP-AES \\
\hline 7440-02-0 & Nickel & 7.80 & ug/g dry & 6.50 & $5 / 22 / 08$ & 8E12001 & PNNL-AGG-ICP-AES \\
\hline $7723-14-0$ & Phosphorus & 974 & ug/g dry & 59.5 & $5 / 22 / 08$ & 8E12001 & PNNL-AGG-ICP-AES \\
\hline $7439-92-1$ & Lead & $<25.1$ & ug/g dry & 25.1 & $5 / 22 / 08$ & 8E12001 & PNNL-AGG-ICP-AES \\
\hline $7782-49-2$ & Selenium & $<110$ & ug/g dry & 110 & $5 / 22 / 08$ & 8E12001 & PNNL-AGG-ICP-AES \\
\hline $7440-24-6$ & Strontium & 16.9 & ug/g dry & 1.15 & $5 / 27 / 08$ & 8E12001 & PNNL-AGG-ICP-AES \\
\hline $7440-28-0$ & Thallium & $<35.7$ & ug/g dry & 35.7 & $5 / 22 / 08$ & 8E12001 & PNNL-AGG-ICP-AES \\
\hline $7440-62-2$ & Vanadium & 22.3 & ug/g dry & 2.94 & $5 / 22 / 08$ & 8E12001 & PNNL-AGG-ICP-AES \\
\hline $7440-66-6$ & Zinc & 29.9 & ug/g dry & 4.95 & $5 / 22 / 08$ & 8E12001 & PNNL-AGG-ICP-AES \\
\hline $7440-23-5$ & Sodium & $<847$ & ug/g dry & 847 & $5 / 22 / 08$ & 8E12001 & PNNL-AGG-ICP-AES \\
\hline $7440-21-3$ & Silicon & $<1100$ & ug/g dry & 1100 & $5 / 22 / 08$ & 8E12001 & PNNL-AGG-ICP-AES \\
\hline 7704-34-9 & Sulfur & $<187$ & ug/g dry & 187 & $5 / 22 / 08$ & 8E12001 & PNNL-AGG-ICP-AES \\
\hline $7440-32-6$ & Titanium & 848 & ug/g dry & 24.9 & $5 / 22 / 08$ & 8E12001 & PNNL-AGG-ICP-AES \\
\hline $7440-67-7$ & Zirconium & $<16.1$ & ug/g dry & 16.1 & $5 / 22 / 08$ & 8E12001 & PNNL-AGG-ICP-AES \\
\hline $7440-15-5$ & Rhenium & $<13.9$ & ug/g dry & 13.9 & $5 / 22 / 08$ & 8E12001 & PNNL-AGG-ICP-AES \\
\hline $7440-36-0$ & Antimony & $<88.4$ & ug/g dry & 88.4 & $5 / 22 / 08$ & 8E12001 & PNNL-AGG-ICP-AES \\
\hline HEIS No. & B1RTF9A & \multicolumn{3}{|c|}{ Lab ID: $\quad$ 0803001-02 } & & & \\
\hline $7429-90-5$ & Aluminum & 4300 & ug/g dry & 27.8 & $5 / 22 / 08$ & 8E12001 & PNNL-AGG-ICP-AES \\
\hline $7440-38-2$ & Arsenic & $<67.6$ & ug/g dry & 67.6 & $5 / 22 / 08$ & 8E12001 & PNNL-AGG-ICP-AES \\
\hline $7440-42-8$ & Boron & $<589$ & ug/g dry & 589 & $5 / 22 / 08$ & 8E12001 & PNNL-AGG-ICP-AES \\
\hline $7440-39-3$ & Barium & 60.8 & ug/g dry & 2.65 & $5 / 22 / 08$ & 8E12001 & PNNL-AGG-ICP-AES \\
\hline $7440-41-7$ & Beryllium & $<1.05$ & ug/g dry & 1.05 & $5 / 22 / 08$ & 8E12001 & PNNL-AGG-ICP-AES \\
\hline 7440-69-9 & Bismuth & $<14.1$ & ug/g dry & 14.1 & $5 / 22 / 08$ & 8E12001 & PNNL-AGG-ICP-AES \\
\hline $7440-70-2$ & Calcium & 7140 & ug/g dry & 103 & $5 / 22 / 08$ & 8E12001 & PNNL-AGG-ICP-AES \\
\hline $7440-43-9$ & Cadmium & $<1.58$ & ug/g dry & 1.58 & $5 / 22 / 08$ & 8E12001 & PNNL-AGG-ICP-AES \\
\hline $7440-48-4$ & Cobalt & 7.98 & ug/g dry & 5.78 & $5 / 22 / 08$ & 8E12001 & PNNL-AGG-ICP-AES \\
\hline $7440-47-3$ & Chromium & 4.93 & ug/g dry & 2.31 & $5 / 22 / 08$ & 8E12001 & PNNL-AGG-ICP-AES \\
\hline $7440-50-8$ & Copper & $<24.7$ & ug/g dry & 24.7 & $5 / 22 / 08$ & 8E12001 & PNNL-AGG-ICP-AES \\
\hline 7439-89-6 & Iron & 16300 & ug/g dry & 141 & $5 / 22 / 08$ & 8E12001 & PNNL-AGG-ICP-AES \\
\hline $7440-09-7$ & Potassium & 513 & ug/g dry & 65.6 & $5 / 22 / 08$ & 8E12001 & PNNL-AGG-ICP-AES \\
\hline 7439-93-2 & Lithium & $<10.0$ & ug/g dry & 10.0 & $5 / 22 / 08$ & 8E12001 & PNNL-AGG-ICP-AES \\
\hline $7439-95-4$ & Magnesium & 3130 & ug/g dry & 21.9 & $5 / 22 / 08$ & 8E12001 & PNNL-AGG-ICP-AES \\
\hline
\end{tabular}


Total Metals by SW846 6010B/Acid Extract

\begin{tabular}{|c|c|c|c|c|c|c|c|}
\hline CAS \# & Analyte & Results & Units & EQL & Analyzed & Batch & Method \\
\hline HEIS No. & B1RTF9A & \multicolumn{3}{|c|}{ Lab ID: $\quad$ 0803001-02 } & & & \\
\hline 7439-96-5 & Manganese & 243 & ug/g dry & 0.828 & $5 / 22 / 08$ & 8E12001 & PNNL-AGG-ICP-AES \\
\hline 7439-98-7 & Molybdenum & $<15.7$ & ug/g dry & 15.7 & $5 / 22 / 08$ & 8E12001 & PNNL-AGG-ICP-AES \\
\hline $7440-02-0$ & Nickel & 5.84 & ug/g dry & 5.69 & $5 / 22 / 08$ & 8E12001 & PNNL-AGG-ICP-AES \\
\hline 7723-14-0 & Phosphorus & 953 & ug/g dry & 52.1 & $5 / 22 / 08$ & 8E12001 & PNNL-AGG-ICP-AES \\
\hline 7439-92-1 & Lead & $<22.0$ & ug/g dry & 22.0 & $5 / 22 / 08$ & 8E12001 & PNNL-AGG-ICP-AES \\
\hline 7782-49-2 & Selenium & $<96.6$ & ug/g dry & 96.6 & $5 / 22 / 08$ & 8E12001 & PNNL-AGG-ICP-AES \\
\hline $7440-24-6$ & Strontium & 22.3 & ug/g dry & 1.00 & $5 / 27 / 08$ & 8E12001 & PNNL-AGG-ICP-AES \\
\hline $7440-28-0$ & Thallium & $<31.3$ & ug/g dry & 31.3 & $5 / 22 / 08$ & 8E12001 & PNNL-AGG-ICP-AES \\
\hline $7440-62-2$ & Vanadium & 24.0 & ug/g dry & 2.58 & $5 / 22 / 08$ & 8E12001 & PNNL-AGG-ICP-AES \\
\hline $7440-66-6$ & Zinc & 26.6 & ug/g dry & 4.34 & $5 / 22 / 08$ & 8E12001 & PNNL-AGG-ICP-AES \\
\hline $7440-23-5$ & Sodium & $<742$ & ug/g dry & 742 & $5 / 22 / 08$ & 8E12001 & PNNL-AGG-ICP-AES \\
\hline $7440-21-3$ & Silicon & $<967$ & ug/g dry & 967 & $5 / 22 / 08$ & 8E12001 & PNNL-AGG-ICP-AES \\
\hline $7704-34-9$ & Sulfur & $<164$ & ug/g dry & 164 & $5 / 22 / 08$ & 8E12001 & PNNL-AGG-ICP-AES \\
\hline $7440-32-6$ & Titanium & 829 & ug/g dry & 21.8 & $5 / 22 / 08$ & 8E12001 & PNNL-AGG-ICP-AES \\
\hline $7440-67-7$ & Zirconium & 14.6 & ug/g dry & 14.1 & $5 / 22 / 08$ & 8E12001 & PNNL-AGG-ICP-AES \\
\hline $7440-15-5$ & Rhenium & $<12.2$ & ug/g dry & 12.2 & $5 / 22 / 08$ & 8E12001 & PNNL-AGG-ICP-AES \\
\hline $7440-36-0$ & Antimony & $<77.5$ & ug/g dry & 77.5 & $5 / 22 / 08$ & 8E12001 & PNNL-AGG-ICP-AES \\
\hline HEIS No. & B1RTH1A & \multicolumn{3}{|c|}{ Lab ID: $\quad$ 0803012-02 } & & & \\
\hline $7429-90-5$ & Aluminum & 3430 & ug/g dry & 26.5 & $5 / 22 / 08$ & 8E12001 & PNNL-AGG-ICP-AES \\
\hline $7440-38-2$ & Arsenic & $<64.4$ & ug/g dry & 64.4 & $5 / 22 / 08$ & 8E12001 & PNNL-AGG-ICP-AES \\
\hline $7440-42-8$ & Boron & $<561$ & ug/g dry & 561 & $5 / 22 / 08$ & 8E12001 & PNNL-AGG-ICP-AES \\
\hline $7440-39-3$ & Barium & 51.2 & ug/g dry & 2.52 & $5 / 22 / 08$ & 8E12001 & PNNL-AGG-ICP-AES \\
\hline $7440-41-7$ & Beryllium & $<1.00$ & ug/g dry & 1.00 & $5 / 22 / 08$ & 8E12001 & PNNL-AGG-ICP-AES \\
\hline $7440-69-9$ & Bismuth & $<13.4$ & ug/g dry & 13.4 & $5 / 22 / 08$ & 8E12001 & PNNL-AGG-ICP-AES \\
\hline $7440-70-2$ & Calcium & 5030 & ug/g dry & 98.5 & $5 / 22 / 08$ & 8E12001 & PNNL-AGG-ICP-AES \\
\hline $7440-43-9$ & Cadmium & $<1.51$ & ug/g dry & 1.51 & $5 / 22 / 08$ & 8E12001 & PNNL-AGG-ICP-AES \\
\hline $7440-48-4$ & Cobalt & 8.79 & ug/g dry & 5.50 & $5 / 22 / 08$ & 8E12001 & PNNL-AGG-ICP-AES \\
\hline $7440-47-3$ & Chromium & 2.24 & ug/g dry & 2.20 & $5 / 22 / 08$ & 8E12001 & PNNL-AGG-ICP-AES \\
\hline $7440-50-8$ & Copper & $<23.5$ & ug/g dry & 23.5 & $5 / 22 / 08$ & 8E12001 & PNNL-AGG-ICP-AES \\
\hline 7439-89-6 & Iron & 15700 & ug/g dry & 135 & $5 / 22 / 08$ & 8E12001 & PNNL-AGG-ICP-AES \\
\hline 7440-09-7 & Potassium & 419 & ug/g dry & 62.5 & $5 / 22 / 08$ & 8E12001 & PNNL-AGG-ICP-AES \\
\hline $7439-93-2$ & Lithium & $<9.55$ & ug/g dry & 9.55 & $5 / 22 / 08$ & 8E12001 & PNNL-AGG-ICP-AES \\
\hline $7439-95-4$ & Magnesium & 2940 & ug/g dry & 20.8 & $5 / 22 / 08$ & 8E12001 & PNNL-AGG-ICP-AES \\
\hline $7439-96-5$ & Manganese & 237 & ug/g dry & 0.789 & $5 / 22 / 08$ & 8E12001 & PNNL-AGG-ICP-AES \\
\hline 7439-98-7 & Molybdenum & $<14.9$ & ug/g dry & 14.9 & $5 / 22 / 08$ & 8E12001 & PNNL-AGG-ICP-AES \\
\hline $7440-02-0$ & Nickel & 5.45 & ug/g dry & 5.42 & $5 / 22 / 08$ & 8E12001 & PNNL-AGG-ICP-AES \\
\hline 7723-14-0 & Phosphorus & 1070 & ug/g dry & 49.6 & $5 / 22 / 08$ & 8E12001 & PNNL-AGG-ICP-AES \\
\hline $7439-92-1$ & Lead & $<21.0$ & ug/g dry & 21.0 & $5 / 22 / 08$ & 8E12001 & PNNL-AGG-ICP-AES \\
\hline $7782-49-2$ & Selenium & $<92.0$ & ug/g dry & 92.0 & $5 / 22 / 08$ & 8E12001 & PNNL-AGG-ICP-AES \\
\hline $7440-24-6$ & Strontium & 13.3 & ug/g dry & 0.955 & $5 / 22 / 08$ & 8E12001 & PNNL-AGG-ICP-AES \\
\hline $7440-28-0$ & Thallium & $<29.8$ & ug/g dry & 29.8 & $5 / 22 / 08$ & 8E12001 & PNNL-AGG-ICP-AES \\
\hline $7440-62-2$ & Vanadium & 22.5 & ug/g dry & 2.45 & $5 / 22 / 08$ & 8E12001 & PNNL-AGG-ICP-AES \\
\hline $7440-66-6$ & Zinc & 26.3 & ug/g dry & 4.13 & $5 / 22 / 08$ & 8E12001 & PNNL-AGG-ICP-AES \\
\hline $7440-23-5$ & Sodium & $<706$ & ug/g dry & 706 & $5 / 22 / 08$ & 8E12001 & PNNL-AGG-ICP-AES \\
\hline $7440-21-3$ & Silicon & $<921$ & ug/g dry & 921 & $5 / 22 / 08$ & 8E12001 & PNNL-AGG-ICP-AES \\
\hline 7704-34-9 & Sulfur & $<156$ & ug/g dry & 156 & $5 / 22 / 08$ & 8E12001 & PNNL-AGG-ICP-AES \\
\hline $7440-32-6$ & Titanium & 823 & ug/g dry & 20.8 & $5 / 22 / 08$ & 8E12001 & PNNL-AGG-ICP-AES \\
\hline $7440-67-7$ & Zirconium & 15.4 & ug/g dry & 13.4 & $5 / 22 / 08$ & 8E12001 & PNNL-AGG-ICP-AES \\
\hline
\end{tabular}


Total Metals by SW846 6010B/Acid Extract

\begin{tabular}{|c|c|c|c|c|c|c|c|}
\hline CAS \# & Analyte & Results & Units & EQL & Analyzed & Batch & Method \\
\hline HEIS No. & B1RTH1A & \multicolumn{3}{|c|}{ Lab ID: $\quad$ 0803012-02 } & & & \\
\hline $7440-15-5$ & Rhenium & $<11.6$ & ug/g dry & 11.6 & $5 / 22 / 08$ & 8E12001 & PNNL-AGG-ICP-AES \\
\hline $7440-36-0$ & Antimony & $<73.8$ & ug/g dry & 73.8 & $5 / 22 / 08$ & 8E12001 & PNNL-AGG-ICP-AES \\
\hline HEIS No. & B1RTH1 & \multicolumn{3}{|c|}{ Lab ID: $\quad 0803012-03$} & & & \\
\hline $7429-90-5$ & Aluminum & 5320 & ug/g dry & 28.5 & $5 / 22 / 08$ & 8E12001 & PNNL-AGG-ICP-AES \\
\hline $7440-38-2$ & Arsenic & $<69.3$ & ug/g dry & 69.3 & $5 / 22 / 08$ & 8E12001 & PNNL-AGG-ICP-AES \\
\hline $7440-42-8$ & Boron & $<604$ & ug/g dry & 604 & $5 / 22 / 08$ & 8E12001 & PNNL-AGG-ICP-AES \\
\hline $7440-39-3$ & Barium & 64.3 & ug/g dry & 2.72 & $5 / 22 / 08$ & 8E12001 & PNNL-AGG-ICP-AES \\
\hline $7440-41-7$ & Beryllium & $<1.08$ & ug/g dry & 1.08 & $5 / 22 / 08$ & 8E12001 & PNNL-AGG-ICP-AES \\
\hline $7440-69-9$ & Bismuth & $<14.4$ & ug/g dry & 14.4 & $5 / 22 / 08$ & 8E12001 & PNNL-AGG-ICP-AES \\
\hline $7440-70-2$ & Calcium & 7500 & ug/g dry & 106 & $5 / 22 / 08$ & 8E12001 & PNNL-AGG-ICP-AES \\
\hline $7440-43-9$ & Cadmium & $<1.62$ & ug/g dry & 1.62 & $5 / 22 / 08$ & 8E12001 & PNNL-AGG-ICP-AES \\
\hline $7440-48-4$ & Cobalt & 8.61 & ug/g dry & 5.92 & $5 / 22 / 08$ & 8E12001 & PNNL-AGG-ICP-AES \\
\hline $7440-47-3$ & Chromium & 2.99 & ug/g dry & 2.37 & $5 / 22 / 08$ & 8E12001 & PNNL-AGG-ICP-AES \\
\hline $7440-50-8$ & Copper & $<25.3$ & ug/g dry & 25.3 & $5 / 22 / 08$ & 8E12001 & PNNL-AGG-ICP-AES \\
\hline 7439-89-6 & Iron & 15000 & ug/g dry & 145 & $5 / 22 / 08$ & 8E12001 & PNNL-AGG-ICP-AES \\
\hline $7440-09-7$ & Potassium & 596 & ug/g dry & 67.3 & $5 / 22 / 08$ & 8E12001 & PNNL-AGG-ICP-AES \\
\hline $7439-93-2$ & Lithium & $<10.3$ & ug/g dry & 10.3 & $5 / 22 / 08$ & 8E12001 & PNNL-AGG-ICP-AES \\
\hline 7439-95-4 & Magnesium & 3370 & ug/g dry & 22.4 & $5 / 22 / 08$ & 8E12001 & PNNL-AGG-ICP-AES \\
\hline $7439-96-5$ & Manganese & 286 & ug/g dry & 0.849 & $5 / 22 / 08$ & 8E12001 & PNNL-AGG-ICP-AES \\
\hline 7439-98-7 & Molybdenum & $<16.1$ & ug/g dry & 16.1 & $5 / 22 / 08$ & 8E12001 & PNNL-AGG-ICP-AES \\
\hline $7440-02-0$ & Nickel & 6.25 & ug/g dry & 5.84 & $5 / 22 / 08$ & 8E12001 & PNNL-AGG-ICP-AES \\
\hline $7723-14-0$ & Phosphorus & 774 & ug/g dry & 53.4 & $5 / 22 / 08$ & 8E12001 & PNNL-AGG-ICP-AES \\
\hline 7439-92-1 & Lead & $<22.6$ & ug/g dry & 22.6 & $5 / 22 / 08$ & 8E12001 & PNNL-AGG-ICP-AES \\
\hline $7782-49-2$ & Selenium & $<99.1$ & ug/g dry & 99.1 & $5 / 22 / 08$ & 8E12001 & PNNL-AGG-ICP-AES \\
\hline $7440-24-6$ & Strontium & 19.8 & ug/g dry & 1.03 & $5 / 22 / 08$ & 8E12001 & PNNL-AGG-ICP-AES \\
\hline $7440-28-0$ & Thallium & $<32.1$ & ug/g dry & 32.1 & $5 / 22 / 08$ & 8E12001 & PNNL-AGG-ICP-AES \\
\hline $7440-62-2$ & Vanadium & 22.1 & ug/g dry & 2.64 & $5 / 22 / 08$ & 8E12001 & PNNL-AGG-ICP-AES \\
\hline $7440-66-6$ & Zinc & 24.5 & ug/g dry & 4.45 & $5 / 22 / 08$ & 8E12001 & PNNL-AGG-ICP-AES \\
\hline $7440-23-5$ & Sodium & $<760$ & ug/g dry & 760 & $5 / 22 / 08$ & 8E12001 & PNNL-AGG-ICP-AES \\
\hline $7440-21-3$ & Silicon & $<991$ & ug/g dry & 991 & $5 / 22 / 08$ & 8E12001 & PNNL-AGG-ICP-AES \\
\hline $7704-34-9$ & Sulfur & $<168$ & ug/g dry & 168 & $5 / 22 / 08$ & 8E12001 & PNNL-AGG-ICP-AES \\
\hline $7440-32-6$ & Titanium & 736 & ug/g dry & 22.4 & $5 / 22 / 08$ & 8E12001 & PNNL-AGG-ICP-AES \\
\hline $7440-67-7$ & Zirconium & $<14.4$ & ug/g dry & 14.4 & $5 / 22 / 08$ & 8E12001 & PNNL-AGG-ICP-AES \\
\hline $7440-15-5$ & Rhenium & $<12.5$ & ug/g dry & 12.5 & $5 / 22 / 08$ & 8E12001 & PNNL-AGG-ICP-AES \\
\hline $7440-36-0$ & Antimony & $<79.4$ & ug/g dry & 79.4 & $5 / 22 / 08$ & 8E12001 & PNNL-AGG-ICP-AES \\
\hline HEIS No. & B1RTH2B & \multicolumn{3}{|c|}{ Lab ID: $\quad$ 0803012-05 } & & & \\
\hline $7429-90-5$ & Aluminum & 4560 & ug/g dry & 29.7 & $5 / 22 / 08$ & 8E12001 & PNNL-AGG-ICP-AES \\
\hline $7440-38-2$ & Arsenic & $<72.1$ & ug/g dry & 72.1 & $5 / 22 / 08$ & 8E12001 & PNNL-AGG-ICP-AES \\
\hline $7440-42-8$ & Boron & $<628$ & ug/g dry & 628 & $5 / 22 / 08$ & 8E12001 & PNNL-AGG-ICP-AES \\
\hline $7440-39-3$ & Barium & 39.8 & ug/g dry & 2.83 & $5 / 22 / 08$ & 8E12001 & PNNL-AGG-ICP-AES \\
\hline $7440-41-7$ & Beryllium & $<1.12$ & ug/g dry & 1.12 & $5 / 22 / 08$ & 8E12001 & PNNL-AGG-ICP-AES \\
\hline $7440-69-9$ & Bismuth & $<15.0$ & ug/g dry & 15.0 & $5 / 22 / 08$ & 8E12001 & PNNL-AGG-ICP-AES \\
\hline $7440-70-2$ & Calcium & 7330 & ug/g dry & 110 & $5 / 22 / 08$ & 8E12001 & PNNL-AGG-ICP-AES \\
\hline $7440-43-9$ & Cadmium & $<1.68$ & ug/g dry & 1.68 & $5 / 22 / 08$ & 8E12001 & PNNL-AGG-ICP-AES \\
\hline $7440-48-4$ & Cobalt & $<6.16$ & ug/g dry & 6.16 & $5 / 22 / 08$ & 8E12001 & PNNL-AGG-ICP-AES \\
\hline $7440-47-3$ & Chromium & 7.03 & ug/g dry & 2.47 & $5 / 22 / 08$ & 8E12001 & PNNL-AGG-ICP-AES \\
\hline $7440-50-8$ & Copper & $<26.3$ & ug/g dry & 26.3 & $5 / 22 / 08$ & 8E12001 & PNNL-AGG-ICP-AES \\
\hline 7439-89-6 & Iron & 11200 & ug/g dry & 75.4 & $5 / 22 / 08$ & 8E12001 & PNNL-AGG-ICP-AES \\
\hline
\end{tabular}


Total Metals by SW846 6010B/Acid Extract

\begin{tabular}{|c|c|c|c|c|c|c|c|}
\hline CAS \# & Analyte & Results & Units & EQL & Analyzed & Batch & Method \\
\hline HEIS No. & B1RTH2B & \multicolumn{3}{|c|}{ Lab ID: $\quad$ 0803012-05 } & & & \\
\hline 7440-09-7 & Potassium & 669 & ug/g dry & 70.0 & $5 / 22 / 08$ & 8E12001 & PNNL-AGG-ICP-AES \\
\hline 7439-93-2 & Lithium & $<10.7$ & ug/g dry & 10.7 & $5 / 22 / 08$ & 8E12001 & PNNL-AGG-ICP-AES \\
\hline 7439-95-4 & Magnesium & 3480 & ug/g dry & 23.3 & $5 / 22 / 08$ & 8E12001 & PNNL-AGG-ICP-AES \\
\hline 7439-96-5 & Manganese & 217 & ug/g dry & 0.883 & $5 / 22 / 08$ & 8E12001 & PNNL-AGG-ICP-AES \\
\hline 7439-98-7 & Molybdenum & $<16.7$ & ug/g dry & 16.7 & $5 / 22 / 08$ & 8E12001 & PNNL-AGG-ICP-AES \\
\hline 7440-02-0 & Nickel & 7.64 & ug/g dry & 6.07 & $5 / 22 / 08$ & 8E12001 & PNNL-AGG-ICP-AES \\
\hline $7723-14-0$ & Phosphorus & 453 & ug/g dry & 55.5 & $5 / 22 / 08$ & 8E12001 & PNNL-AGG-ICP-AES \\
\hline 7439-92-1 & Lead & $<23.4$ & ug/g dry & 23.4 & $5 / 22 / 08$ & 8E12001 & PNNL-AGG-ICP-AES \\
\hline $7782-49-2$ & Selenium & $<103$ & ug/g dry & 103 & $5 / 22 / 08$ & 8E12001 & PNNL-AGG-ICP-AES \\
\hline $7440-24-6$ & Strontium & 20.4 & ug/g dry & 1.07 & $5 / 22 / 08$ & 8E12001 & PNNL-AGG-ICP-AES \\
\hline $7440-28-0$ & Thallium & $<33.3$ & ug/g dry & 33.3 & $5 / 22 / 08$ & 8E12001 & PNNL-AGG-ICP-AES \\
\hline $7440-62-2$ & Vanadium & 13.6 & ug/g dry & 2.74 & $5 / 22 / 08$ & 8E12001 & PNNL-AGG-ICP-AES \\
\hline $7440-66-6$ & Zinc & 23.8 & ug/g dry & 4.62 & $5 / 22 / 08$ & 8E12001 & PNNL-AGG-ICP-AES \\
\hline $7440-23-5$ & Sodium & $<790$ & ug/g dry & 790 & $5 / 22 / 08$ & 8E12001 & PNNL-AGG-ICP-AES \\
\hline $7440-21-3$ & Silicon & $<1030$ & ug/g dry & 1030 & $5 / 22 / 08$ & 8E12001 & PNNL-AGG-ICP-AES \\
\hline $7704-34-9$ & Sulfur & $<175$ & ug/g dry & 175 & $5 / 22 / 08$ & 8E12001 & PNNL-AGG-ICP-AES \\
\hline $7440-32-6$ & Titanium & 537 & ug/g dry & 23.3 & $5 / 22 / 08$ & 8E12001 & PNNL-AGG-ICP-AES \\
\hline $7440-67-7$ & Zirconium & $<15.0$ & ug/g dry & 15.0 & $5 / 22 / 08$ & 8E12001 & PNNL-AGG-ICP-AES \\
\hline $7440-15-5$ & Rhenium & $<13.0$ & ug/g dry & 13.0 & $5 / 22 / 08$ & 8E12001 & PNNL-AGG-ICP-AES \\
\hline $7440-36-0$ & Antimony & $<82.6$ & ug/g dry & 82.6 & $5 / 22 / 08$ & 8E12001 & PNNL-AGG-ICP-AES \\
\hline HEIS No. & B1RTH2A & \multicolumn{3}{|c|}{ Lab ID: $\quad$ 0803012-06 } & & & \\
\hline $7429-90-5$ & Aluminum & 4530 & ug/g dry & 32.9 & $5 / 22 / 08$ & 8E12001 & PNNL-AGG-ICP-AES \\
\hline $7440-38-2$ & Arsenic & $<80.0$ & ug/g dry & 80.0 & $5 / 22 / 08$ & 8E12001 & PNNL-AGG-ICP-AES \\
\hline $7440-42-8$ & Boron & $<696$ & ug/g dry & 696 & $5 / 22 / 08$ & 8E12001 & PNNL-AGG-ICP-AES \\
\hline $7440-39-3$ & Barium & 53.5 & ug/g dry & 3.14 & $5 / 22 / 08$ & 8E12001 & PNNL-AGG-ICP-AES \\
\hline $7440-41-7$ & Beryllium & $<1.24$ & ug/g dry & 1.24 & $5 / 22 / 08$ & 8E12001 & PNNL-AGG-ICP-AES \\
\hline $7440-69-9$ & Bismuth & $<16.6$ & ug/g dry & 16.6 & $5 / 22 / 08$ & 8E12001 & PNNL-AGG-ICP-AES \\
\hline $7440-70-2$ & Calcium & 7950 & ug/g dry & 122 & $5 / 22 / 08$ & 8E12001 & PNNL-AGG-ICP-AES \\
\hline $7440-43-9$ & Cadmium & $<1.87$ & ug/g dry & 1.87 & $5 / 22 / 08$ & 8E12001 & PNNL-AGG-ICP-AES \\
\hline $7440-48-4$ & Cobalt & $<6.83$ & ug/g dry & 6.83 & $5 / 22 / 08$ & 8E12001 & PNNL-AGG-ICP-AES \\
\hline $7440-47-3$ & Chromium & 6.31 & ug/g dry & 2.74 & $5 / 22 / 08$ & 8E12001 & PNNL-AGG-ICP-AES \\
\hline $7440-50-8$ & Copper & $<29.2$ & ug/g dry & 29.2 & $5 / 22 / 08$ & 8E12001 & PNNL-AGG-ICP-AES \\
\hline 7439-89-6 & Iron & 10500 & ug/g dry & 83.6 & $5 / 22 / 08$ & 8E12001 & PNNL-AGG-ICP-AES \\
\hline $7440-09-7$ & Potassium & 697 & ug/g dry & 77.6 & $5 / 22 / 08$ & 8E12001 & PNNL-AGG-ICP-AES \\
\hline $7439-93-2$ & Lithium & $<11.9$ & ug/g dry & 11.9 & $5 / 22 / 08$ & 8E12001 & PNNL-AGG-ICP-AES \\
\hline 7439-95-4 & Magnesium & 3420 & ug/g dry & 25.9 & $5 / 22 / 08$ & 8E12001 & PNNL-AGG-ICP-AES \\
\hline 7439-96-5 & Manganese & 212 & ug/g dry & 0.980 & $5 / 22 / 08$ & 8E12001 & PNNL-AGG-ICP-AES \\
\hline 7439-98-7 & Molybdenum & $<18.6$ & ug/g dry & 18.6 & $5 / 22 / 08$ & 8E12001 & PNNL-AGG-ICP-AES \\
\hline $7440-02-0$ & Nickel & 8.30 & ug/g dry & 6.73 & $5 / 22 / 08$ & 8E12001 & PNNL-AGG-ICP-AES \\
\hline $7723-14-0$ & Phosphorus & 473 & ug/g dry & 61.6 & $5 / 22 / 08$ & 8E12001 & PNNL-AGG-ICP-AES \\
\hline $7439-92-1$ & Lead & $<26.0$ & ug/g dry & 26.0 & $5 / 22 / 08$ & 8E12001 & PNNL-AGG-ICP-AES \\
\hline $7782-49-2$ & Selenium & $<114$ & ug/g dry & 114 & $5 / 22 / 08$ & 8E12001 & PNNL-AGG-ICP-AES \\
\hline $7440-24-6$ & Strontium & 24.8 & ug/g dry & 1.19 & $5 / 22 / 08$ & 8E12001 & PNNL-AGG-ICP-AES \\
\hline $7440-28-0$ & Thallium & $<37.0$ & ug/g dry & 37.0 & $5 / 22 / 08$ & 8E12001 & PNNL-AGG-ICP-AES \\
\hline $7440-62-2$ & Vanadium & 13.7 & ug/g dry & 3.05 & $5 / 22 / 08$ & 8E12001 & PNNL-AGG-ICP-AES \\
\hline $7440-66-6$ & Zinc & 26.1 & ug/g dry & 5.13 & $5 / 22 / 08$ & 8E12001 & PNNL-AGG-ICP-AES \\
\hline $7440-23-5$ & Sodium & $<877$ & ug/g dry & 877 & $5 / 22 / 08$ & 8E12001 & PNNL-AGG-ICP-AES \\
\hline $7440-21-3$ & Silicon & $<1140$ & ug/g dry & 1140 & $5 / 22 / 08$ & 8E12001 & PNNL-AGG-ICP-AES \\
\hline
\end{tabular}


Total Metals by SW846 6010B/Acid Extract

\begin{tabular}{|c|c|c|c|c|c|c|c|}
\hline CAS \# & Analyte & Results & Units & EQL & Analyzed & Batch & Method \\
\hline HEIS No. & B1RTH2A & \multicolumn{3}{|c|}{ Lab ID: $\quad$ 0803012-06 } & & & \\
\hline 7704-34-9 & Sulfur & $<194$ & ug/g dry & 194 & $5 / 22 / 08$ & 8E12001 & PNNL-AGG-ICP-AES \\
\hline $7440-32-6$ & Titanium & 536 & ug/g dry & 25.8 & $5 / 22 / 08$ & 8E12001 & PNNL-AGG-ICP-AES \\
\hline $7440-67-7$ & Zirconium & $<16.7$ & ug/g dry & 16.7 & $5 / 22 / 08$ & 8E12001 & PNNL-AGG-ICP-AES \\
\hline $7440-15-5$ & Rhenium & $<14.4$ & ug/g dry & 14.4 & $5 / 22 / 08$ & 8E12001 & PNNL-AGG-ICP-AES \\
\hline $7440-36-0$ & Antimony & $<91.6$ & ug/g dry & 91.6 & $5 / 22 / 08$ & 8E12001 & PNNL-AGG-ICP-AES \\
\hline HEIS No. & B1RTH3B & \multicolumn{3}{|c|}{ Lab ID: $\quad$ 0803012-09 } & & & \\
\hline $7429-90-5$ & Aluminum & 4240 & ug/g dry & 31.0 & $5 / 22 / 08$ & 8E12001 & PNNL-AGG-ICP-AES \\
\hline $7440-38-2$ & Arsenic & $<75.4$ & ug/g dry & 75.4 & $5 / 22 / 08$ & 8E12001 & PNNL-AGG-ICP-AES \\
\hline $7440-42-8$ & Boron & $<657$ & ug/g dry & 657 & $5 / 22 / 08$ & 8E12001 & PNNL-AGG-ICP-AES \\
\hline $7440-39-3$ & Barium & 42.9 & ug/g dry & 2.96 & $5 / 22 / 08$ & 8E12001 & PNNL-AGG-ICP-AES \\
\hline $7440-41-7$ & Beryllium & $<1.17$ & ug/g dry & 1.17 & $5 / 22 / 08$ & 8E12001 & PNNL-AGG-ICP-AES \\
\hline 7440-69-9 & Bismuth & $<15.7$ & ug/g dry & 15.7 & $5 / 22 / 08$ & 8E12001 & PNNL-AGG-ICP-AES \\
\hline $7440-70-2$ & Calcium & 6780 & ug/g dry & 115 & $5 / 22 / 08$ & 8E12001 & PNNL-AGG-ICP-AES \\
\hline $7440-43-9$ & Cadmium & $<1.76$ & ug/g dry & 1.76 & $5 / 22 / 08$ & 8E12001 & PNNL-AGG-ICP-AES \\
\hline $7440-48-4$ & Cobalt & $<6.44$ & ug/g dry & 6.44 & $5 / 22 / 08$ & 8E12001 & PNNL-AGG-ICP-AES \\
\hline $7440-47-3$ & Chromium & 5.12 & ug/g dry & 2.58 & $5 / 22 / 08$ & 8E12001 & PNNL-AGG-ICP-AES \\
\hline $7440-50-8$ & Copper & $<27.5$ & ug/g dry & 27.5 & $5 / 22 / 08$ & 8E12001 & PNNL-AGG-ICP-AES \\
\hline 7439-89-6 & Iron & 11100 & ug/g dry & 78.9 & $5 / 22 / 08$ & 8E12001 & PNNL-AGG-ICP-AES \\
\hline $7440-09-7$ & Potassium & 644 & ug/g dry & 73.2 & $5 / 22 / 08$ & 8E12001 & PNNL-AGG-ICP-AES \\
\hline 7439-93-2 & Lithium & $<11.2$ & ug/g dry & 11.2 & $5 / 22 / 08$ & 8E12001 & PNNL-AGG-ICP-AES \\
\hline $7439-95-4$ & Magnesium & 3260 & ug/g dry & 24.4 & $5 / 22 / 08$ & 8E12001 & PNNL-AGG-ICP-AES \\
\hline 7439-96-5 & Manganese & 205 & ug/g dry & 0.924 & $5 / 22 / 08$ & 8E12001 & PNNL-AGG-ICP-AES \\
\hline 7439-98-7 & Molybdenum & $<17.5$ & ug/g dry & 17.5 & $5 / 22 / 08$ & 8E12001 & PNNL-AGG-ICP-AES \\
\hline $7440-02-0$ & Nickel & 7.05 & ug/g dry & 6.35 & $5 / 22 / 08$ & 8E12001 & PNNL-AGG-ICP-AES \\
\hline $7723-14-0$ & Phosphorus & 530 & ug/g dry & 58.1 & $5 / 22 / 08$ & 8E12001 & PNNL-AGG-ICP-AES \\
\hline 7439-92-1 & Lead & $<24.5$ & ug/g dry & 24.5 & $5 / 22 / 08$ & 8E12001 & PNNL-AGG-ICP-AES \\
\hline $7782-49-2$ & Selenium & $<108$ & ug/g dry & 108 & $5 / 22 / 08$ & 8E12001 & PNNL-AGG-ICP-AES \\
\hline $7440-24-6$ & Strontium & 21.5 & ug/g dry & 1.12 & $5 / 22 / 08$ & 8E12001 & PNNL-AGG-ICP-AES \\
\hline $7440-28-0$ & Thallium & $<34.9$ & ug/g dry & 34.9 & $5 / 22 / 08$ & 8E12001 & PNNL-AGG-ICP-AES \\
\hline $7440-62-2$ & Vanadium & 14.6 & ug/g dry & 2.87 & $5 / 22 / 08$ & 8E12001 & PNNL-AGG-ICP-AES \\
\hline $7440-66-6$ & Zinc & 25.3 & ug/g dry & 4.84 & $5 / 22 / 08$ & 8E12001 & PNNL-AGG-ICP-AES \\
\hline $7440-23-5$ & Sodium & $<827$ & ug/g dry & 827 & $5 / 22 / 08$ & 8E12001 & PNNL-AGG-ICP-AES \\
\hline $7440-21-3$ & Silicon & $<1080$ & ug/g dry & 1080 & $5 / 22 / 08$ & 8E12001 & PNNL-AGG-ICP-AES \\
\hline $7704-34-9$ & Sulfur & $<183$ & ug/g dry & 183 & $5 / 22 / 08$ & 8E12001 & PNNL-AGG-ICP-AES \\
\hline $7440-32-6$ & Titanium & 606 & ug/g dry & 24.4 & $5 / 22 / 08$ & 8E12001 & PNNL-AGG-ICP-AES \\
\hline $7440-67-7$ & Zirconium & $<15.7$ & ug/g dry & 15.7 & $5 / 22 / 08$ & 8E12001 & PNNL-AGG-ICP-AES \\
\hline $7440-15-5$ & Rhenium & $<13.6$ & ug/g dry & 13.6 & $5 / 22 / 08$ & 8E12001 & PNNL-AGG-ICP-AES \\
\hline $7440-36-0$ & Antimony & $<86.4$ & ug/g dry & 86.4 & $5 / 22 / 08$ & 8E12001 & PNNL-AGG-ICP-AES \\
\hline HEIS No. & B1RTH3A & \multicolumn{3}{|c|}{ Lab ID: $\quad$ 0803012-10 } & & & \\
\hline $7429-90-5$ & Aluminum & 4910 & ug/g dry & 32.2 & $5 / 22 / 08$ & 8E12001 & PNNL-AGG-ICP-AES \\
\hline $7440-38-2$ & Arsenic & $<78.3$ & ug/g dry & 78.3 & $5 / 22 / 08$ & 8E12001 & PNNL-AGG-ICP-AES \\
\hline $7440-42-8$ & Boron & $<682$ & ug/g dry & 682 & $5 / 22 / 08$ & 8E12001 & PNNL-AGG-ICP-AES \\
\hline $7440-39-3$ & Barium & 47.6 & ug/g dry & 3.07 & $5 / 22 / 08$ & 8E12001 & PNNL-AGG-ICP-AES \\
\hline $7440-41-7$ & Beryllium & $<1.22$ & ug/g dry & 1.22 & $5 / 22 / 08$ & 8E12001 & PNNL-AGG-ICP-AES \\
\hline $7440-69-9$ & Bismuth & $<16.3$ & ug/g dry & 16.3 & $5 / 22 / 08$ & 8E12001 & PNNL-AGG-ICP-AES \\
\hline $7440-70-2$ & Calcium & 7640 & ug/g dry & 120 & $5 / 22 / 08$ & 8E12001 & PNNL-AGG-ICP-AES \\
\hline $7440-43-9$ & Cadmium & $<1.83$ & ug/g dry & 1.83 & $5 / 22 / 08$ & 8E12001 & PNNL-AGG-ICP-AES \\
\hline $7440-48-4$ & Cobalt & $<6.69$ & ug/g dry & 6.69 & $5 / 22 / 08$ & 8E12001 & PNNL-AGG-ICP-AES \\
\hline
\end{tabular}


Total Metals by SW846 6010B/Acid Extract

\begin{tabular}{|c|c|c|c|c|c|c|c|}
\hline CAS \# & Analyte & Results & Units & EQL & Analyzed & Batch & Method \\
\hline HEIS No. & B1RTH3A & \multicolumn{3}{|c|}{ Lab ID: $\quad$ 0803012-10 } & & & \\
\hline $7440-47-3$ & Chromium & 4.59 & ug/g dry & 2.68 & $5 / 22 / 08$ & 8E12001 & PNNL-AGG-ICP-AES \\
\hline $7440-50-8$ & Copper & $<28.5$ & ug/g dry & 28.5 & $5 / 22 / 08$ & 8E12001 & PNNL-AGG-ICP-AES \\
\hline 7439-89-6 & Iron & 12400 & ug/g dry & 81.9 & $5 / 22 / 08$ & 8E12001 & PNNL-AGG-ICP-AES \\
\hline 7440-09-7 & Potassium & 626 & ug/g dry & 76.0 & $5 / 22 / 08$ & 8E12001 & PNNL-AGG-ICP-AES \\
\hline $7439-93-2$ & Lithium & $<11.6$ & ug/g dry & 11.6 & $5 / 22 / 08$ & 8E12001 & PNNL-AGG-ICP-AES \\
\hline 7439-95-4 & Magnesium & 3740 & ug/g dry & 25.3 & $5 / 22 / 08$ & 8E12001 & PNNL-AGG-ICP-AES \\
\hline $7439-96-5$ & Manganese & 216 & ug/g dry & 0.959 & $5 / 22 / 08$ & 8E12001 & PNNL-AGG-ICP-AES \\
\hline 7439-98-7 & Molybdenum & $<18.2$ & ug/g dry & 18.2 & $5 / 22 / 08$ & 8E12001 & PNNL-AGG-ICP-AES \\
\hline 7440-02-0 & Nickel & 9.46 & ug/g dry & 6.59 & $5 / 22 / 08$ & 8E12001 & PNNL-AGG-ICP-AES \\
\hline $7723-14-0$ & Phosphorus & 606 & ug/g dry & 60.3 & $5 / 22 / 08$ & 8E12001 & PNNL-AGG-ICP-AES \\
\hline 7439-92-1 & Lead & $<25.5$ & ug/g dry & 25.5 & $5 / 22 / 08$ & 8E12001 & PNNL-AGG-ICP-AES \\
\hline $7782-49-2$ & Selenium & $<112$ & ug/g dry & 112 & $5 / 22 / 08$ & 8E12001 & PNNL-AGG-ICP-AES \\
\hline $7440-24-6$ & Strontium & 21.9 & ug/g dry & 1.16 & $5 / 22 / 08$ & 8E12001 & PNNL-AGG-ICP-AES \\
\hline $7440-28-0$ & Thallium & $<36.2$ & ug/g dry & 36.2 & $5 / 22 / 08$ & 8E12001 & PNNL-AGG-ICP-AES \\
\hline $7440-62-2$ & Vanadium & 14.5 & ug/g dry & 2.98 & $5 / 22 / 08$ & 8E12001 & PNNL-AGG-ICP-AES \\
\hline $7440-66-6$ & Zinc & 26.9 & ug/g dry & 5.02 & $5 / 22 / 08$ & 8E12001 & PNNL-AGG-ICP-AES \\
\hline $7440-23-5$ & Sodium & $<859$ & ug/g dry & 859 & $5 / 22 / 08$ & 8E12001 & PNNL-AGG-ICP-AES \\
\hline $7440-21-3$ & Silicon & $<1120$ & ug/g dry & 1120 & $5 / 22 / 08$ & 8E12001 & PNNL-AGG-ICP-AES \\
\hline $7704-34-9$ & Sulfur & $<190$ & ug/g dry & 190 & $5 / 22 / 08$ & 8E12001 & PNNL-AGG-ICP-AES \\
\hline $7440-32-6$ & Titanium & 617 & ug/g dry & 25.3 & $5 / 22 / 08$ & 8E12001 & PNNL-AGG-ICP-AES \\
\hline $7440-67-7$ & Zirconium & $<16.3$ & ug/g dry & 16.3 & $5 / 22 / 08$ & 8E12001 & PNNL-AGG-ICP-AES \\
\hline $7440-15-5$ & Rhenium & $<14.1$ & ug/g dry & 14.1 & $5 / 22 / 08$ & 8E12001 & PNNL-AGG-ICP-AES \\
\hline $7440-36-0$ & Antimony & $<89.7$ & ug/g dry & 89.7 & $5 / 22 / 08$ & 8E12001 & PNNL-AGG-ICP-AES \\
\hline HEIS No. & B1RYR7B & \multicolumn{3}{|c|}{ Lab ID: $\quad$ 0803012-13 } & & & \\
\hline $7429-90-5$ & Aluminum & 4380 & ug/g dry & 31.7 & $5 / 22 / 08$ & 8E12001 & PNNL-AGG-ICP-AES \\
\hline $7440-38-2$ & Arsenic & $<76.9$ & ug/g dry & 76.9 & $5 / 22 / 08$ & 8E12001 & PNNL-AGG-ICP-AES \\
\hline $7440-42-8$ & Boron & $<670$ & ug/g dry & 670 & $5 / 22 / 08$ & 8E12001 & PNNL-AGG-ICP-AES \\
\hline $7440-39-3$ & Barium & 42.5 & ug/g dry & 3.02 & $5 / 22 / 08$ & 8E12001 & PNNL-AGG-ICP-AES \\
\hline $7440-41-7$ & Beryllium & $<1.20$ & ug/g dry & 1.20 & $5 / 22 / 08$ & 8E12001 & PNNL-AGG-ICP-AES \\
\hline $7440-69-9$ & Bismuth & $<16.0$ & ug/g dry & 16.0 & $5 / 22 / 08$ & 8E12001 & PNNL-AGG-ICP-AES \\
\hline $7440-70-2$ & Calcium & 6460 & ug/g dry & 118 & $5 / 22 / 08$ & 8E12001 & PNNL-AGG-ICP-AES \\
\hline $7440-43-9$ & Cadmium & $<1.80$ & ug/g dry & 1.80 & $5 / 22 / 08$ & 8E12001 & PNNL-AGG-ICP-AES \\
\hline $7440-48-4$ & Cobalt & $<6.57$ & ug/g dry & 6.57 & $5 / 22 / 08$ & 8E12001 & PNNL-AGG-ICP-AES \\
\hline $7440-47-3$ & Chromium & 7.39 & ug/g dry & 2.63 & $5 / 22 / 08$ & 8E12001 & PNNL-AGG-ICP-AES \\
\hline $7440-50-8$ & Copper & $<28.0$ & ug/g dry & 28.0 & $5 / 22 / 08$ & 8E12001 & PNNL-AGG-ICP-AES \\
\hline 7439-89-6 & Iron & 10600 & ug/g dry & 80.4 & $5 / 22 / 08$ & 8E12001 & PNNL-AGG-ICP-AES \\
\hline 7440-09-7 & Potassium & 718 & ug/g dry & 74.7 & $5 / 22 / 08$ & 8E12001 & PNNL-AGG-ICP-AES \\
\hline $7439-93-2$ & Lithium & $<11.4$ & ug/g dry & 11.4 & $5 / 22 / 08$ & 8E12001 & PNNL-AGG-ICP-AES \\
\hline $7439-95-4$ & Magnesium & 3190 & ug/g dry & 24.9 & $5 / 22 / 08$ & 8E12001 & PNNL-AGG-ICP-AES \\
\hline $7439-96-5$ & Manganese & 212 & ug/g dry & 0.942 & $5 / 22 / 08$ & 8E12001 & PNNL-AGG-ICP-AES \\
\hline 7439-98-7 & Molybdenum & $<17.8$ & ug/g dry & 17.8 & $5 / 22 / 08$ & 8E12001 & PNNL-AGG-ICP-AES \\
\hline $7440-02-0$ & Nickel & 8.39 & ug/g dry & 6.47 & $5 / 22 / 08$ & 8E12001 & PNNL-AGG-ICP-AES \\
\hline $7723-14-0$ & Phosphorus & 469 & ug/g dry & 59.3 & $5 / 22 / 08$ & 8E12001 & PNNL-AGG-ICP-AES \\
\hline $7439-92-1$ & Lead & $<25.0$ & ug/g dry & 25.0 & $5 / 22 / 08$ & 8E12001 & PNNL-AGG-ICP-AES \\
\hline 7782-49-2 & Selenium & $<110$ & ug/g dry & 110 & $5 / 22 / 08$ & 8E12001 & PNNL-AGG-ICP-AES \\
\hline $7440-24-6$ & Strontium & 20.7 & ug/g dry & 1.14 & $5 / 22 / 08$ & 8E12001 & PNNL-AGG-ICP-AES \\
\hline $7440-28-0$ & Thallium & $<35.6$ & ug/g dry & 35.6 & $5 / 22 / 08$ & 8E12001 & PNNL-AGG-ICP-AES \\
\hline $7440-62-2$ & Vanadium & 13.4 & ug/g dry & 2.93 & $5 / 22 / 08$ & 8E12001 & PNNL-AGG-ICP-AES \\
\hline
\end{tabular}


Total Metals by SW846 6010B/Acid Extract

\begin{tabular}{|c|c|c|c|c|c|c|c|}
\hline CAS \# & Analyte & Results & Units & EQL & Analyzed & Batch & Method \\
\hline HEIS No. & B1RYR7B & \multicolumn{3}{|c|}{ Lab ID: $\quad$ 0803012-13 } & & & \\
\hline $7440-66-6$ & Zinc & 23.1 & ug/g dry & 4.93 & $5 / 22 / 08$ & 8E12001 & PNNL-AGG-ICP-AES \\
\hline $7440-23-5$ & Sodium & $<843$ & ug/g dry & 843 & $5 / 22 / 08$ & 8E12001 & PNNL-AGG-ICP-AES \\
\hline $7440-21-3$ & Silicon & $<1100$ & ug/g dry & 1100 & $5 / 22 / 08$ & 8E12001 & PNNL-AGG-ICP-AES \\
\hline 7704-34-9 & Sulfur & $<187$ & ug/g dry & 187 & $5 / 22 / 08$ & 8E12001 & PNNL-AGG-ICP-AES \\
\hline 7440-32-6 & Titanium & 489 & ug/g dry & 24.8 & $5 / 22 / 08$ & 8E12001 & PNNL-AGG-ICP-AES \\
\hline $7440-67-7$ & Zirconium & $<16.0$ & ug/g dry & 16.0 & $5 / 22 / 08$ & 8E12001 & PNNL-AGG-ICP-AES \\
\hline $7440-15-5$ & Rhenium & $<13.9$ & ug/g dry & 13.9 & $5 / 22 / 08$ & 8E12001 & PNNL-AGG-ICP-AES \\
\hline $7440-36-0$ & Antimony & $<88.1$ & ug/g dry & 88.1 & $5 / 22 / 08$ & 8E12001 & PNNL-AGG-ICP-AES \\
\hline HEIS No. & B1RYR7A & \multicolumn{3}{|c|}{ Lab ID: $\quad$ 0803012-14 } & & & \\
\hline $7429-90-5$ & Aluminum & 4740 & ug/g dry & 33.3 & $5 / 22 / 08$ & 8E12001 & PNNL-AGG-ICP-AES \\
\hline $7440-38-2$ & Arsenic & $<81.0$ & ug/g dry & 81.0 & $5 / 22 / 08$ & 8E12001 & PNNL-AGG-ICP-AES \\
\hline $7440-42-8$ & Boron & $<705$ & ug/g dry & 705 & $5 / 22 / 08$ & 8E12001 & PNNL-AGG-ICP-AES \\
\hline $7440-39-3$ & Barium & 45.0 & ug/g dry & 3.17 & $5 / 22 / 08$ & 8E12001 & PNNL-AGG-ICP-AES \\
\hline $7440-41-7$ & Beryllium & $<1.26$ & ug/g dry & 1.26 & $5 / 22 / 08$ & 8E12001 & PNNL-AGG-ICP-AES \\
\hline $7440-69-9$ & Bismuth & $<16.8$ & ug/g dry & 16.8 & $5 / 22 / 08$ & 8E12001 & PNNL-AGG-ICP-AES \\
\hline $7440-70-2$ & Calcium & 7780 & ug/g dry & 124 & $5 / 22 / 08$ & 8E12001 & PNNL-AGG-ICP-AES \\
\hline 7440-43-9 & Cadmium & $<1.89$ & ug/g dry & 1.89 & $5 / 22 / 08$ & 8E12001 & PNNL-AGG-ICP-AES \\
\hline $7440-48-4$ & Cobalt & $<6.92$ & ug/g dry & 6.92 & $5 / 22 / 08$ & 8E12001 & PNNL-AGG-ICP-AES \\
\hline $7440-47-3$ & Chromium & 8.91 & ug/g dry & 2.77 & $5 / 22 / 08$ & 8E12001 & PNNL-AGG-ICP-AES \\
\hline $7440-50-8$ & Copper & $<29.5$ & ug/g dry & 29.5 & $5 / 22 / 08$ & 8E12001 & PNNL-AGG-ICP-AES \\
\hline 7439-89-6 & Iron & 11500 & ug/g dry & 84.7 & $5 / 22 / 08$ & 8E12001 & PNNL-AGG-ICP-AES \\
\hline $7440-09-7$ & Potassium & 767 & ug/g dry & 78.6 & $5 / 22 / 08$ & 8E12001 & PNNL-AGG-ICP-AES \\
\hline 7439-93-2 & Lithium & $<12.0$ & ug/g dry & 12.0 & $5 / 22 / 08$ & 8E12001 & PNNL-AGG-ICP-AES \\
\hline $7439-95-4$ & Magnesium & 3760 & ug/g dry & 26.2 & $5 / 22 / 08$ & 8E12001 & PNNL-AGG-ICP-AES \\
\hline 7439-96-5 & Manganese & 231 & ug/g dry & 0.992 & $5 / 22 / 08$ & 8E12001 & PNNL-AGG-ICP-AES \\
\hline 7439-98-7 & Molybdenum & $<18.8$ & ug/g dry & 18.8 & $5 / 22 / 08$ & 8E12001 & PNNL-AGG-ICP-AES \\
\hline $7440-02-0$ & Nickel & 8.93 & ug/g dry & 6.81 & $5 / 22 / 08$ & 8E12001 & PNNL-AGG-ICP-AES \\
\hline 7723-14-0 & Phosphorus & 494 & ug/g dry & 62.4 & $5 / 22 / 08$ & 8E12001 & PNNL-AGG-ICP-AES \\
\hline 7439-92-1 & Lead & $<26.3$ & ug/g dry & 26.3 & $5 / 22 / 08$ & 8E12001 & PNNL-AGG-ICP-AES \\
\hline $7782-49-2$ & Selenium & $<116$ & ug/g dry & 116 & $5 / 22 / 08$ & 8E12001 & PNNL-AGG-ICP-AES \\
\hline $7440-24-6$ & Strontium & 24.5 & ug/g dry & 1.20 & $5 / 22 / 08$ & 8E12001 & PNNL-AGG-ICP-AES \\
\hline $7440-28-0$ & Thallium & $<37.4$ & ug/g dry & 37.4 & $5 / 22 / 08$ & 8E12001 & PNNL-AGG-ICP-AES \\
\hline $7440-62-2$ & Vanadium & 14.0 & ug/g dry & 3.08 & $5 / 22 / 08$ & 8E12001 & PNNL-AGG-ICP-AES \\
\hline $7440-66-6$ & Zinc & 25.2 & ug/g dry & 5.20 & $5 / 22 / 08$ & 8E12001 & PNNL-AGG-ICP-AES \\
\hline $7440-23-5$ & Sodium & $<888$ & ug/g dry & 888 & $5 / 22 / 08$ & 8E12001 & PNNL-AGG-ICP-AES \\
\hline $7440-21-3$ & Silicon & $<1160$ & ug/g dry & 1160 & $5 / 22 / 08$ & 8E12001 & PNNL-AGG-ICP-AES \\
\hline $7704-34-9$ & Sulfur & $<196$ & ug/g dry & 196 & $5 / 22 / 08$ & 8E12001 & PNNL-AGG-ICP-AES \\
\hline $7440-32-6$ & Titanium & 550 & ug/g dry & 26.1 & $5 / 22 / 08$ & 8E12001 & PNNL-AGG-ICP-AES \\
\hline $7440-67-7$ & Zirconium & $<16.9$ & ug/g dry & 16.9 & $5 / 22 / 08$ & 8E12001 & PNNL-AGG-ICP-AES \\
\hline $7440-15-5$ & Rhenium & $<14.6$ & ug/g dry & 14.6 & $5 / 22 / 08$ & 8E12001 & PNNL-AGG-ICP-AES \\
\hline $7440-36-0$ & Antimony & $<92.8$ & ug/g dry & 92.8 & $5 / 22 / 08$ & 8E12001 & PNNL-AGG-ICP-AES \\
\hline HEIS No. & B1RYR8B & & b ID: & 3012-17 & & & \\
\hline $7429-90-5$ & Aluminum & 5110 & ug/g dry & 36.7 & $5 / 22 / 08$ & 8E12001 & PNNL-AGG-ICP-AES \\
\hline $7440-38-2$ & Arsenic & $<89.0$ & ug/g dry & 89.0 & $5 / 22 / 08$ & 8E12001 & PNNL-AGG-ICP-AES \\
\hline $7440-42-8$ & Boron & $<775$ & ug/g dry & 775 & $5 / 22 / 08$ & 8E12001 & PNNL-AGG-ICP-AES \\
\hline $7440-39-3$ & Barium & 46.7 & ug/g dry & 3.49 & $5 / 22 / 08$ & 8E12001 & PNNL-AGG-ICP-AES \\
\hline $7440-41-7$ & Beryllium & $<1.38$ & ug/g dry & 1.38 & $5 / 22 / 08$ & 8E12001 & PNNL-AGG-ICP-AES \\
\hline $7440-69-9$ & Bismuth & $<18.5$ & ug/g dry & 18.5 & $5 / 22 / 08$ & 8E12001 & PNNL-AGG-ICP-AES \\
\hline
\end{tabular}


Total Metals by SW846 6010B/Acid Extract

\begin{tabular}{|c|c|c|c|c|c|c|c|}
\hline CAS \# & Analyte & Results & Units & EQL & Analyzed & Batch & Method \\
\hline HEIS No. & B1RYR8B & \multicolumn{3}{|c|}{ Lab ID: $\quad$ 0803012-17 } & & & \\
\hline $7440-70-2$ & Calcium & 8030 & ug/g dry & 136 & $5 / 22 / 08$ & 8E12001 & PNNL-AGG-ICP-AES \\
\hline 7440-43-9 & Cadmium & $<2.08$ & ug/g dry & 2.08 & $5 / 22 / 08$ & 8E12001 & PNNL-AGG-ICP-AES \\
\hline $7440-48-4$ & Cobalt & $<7.61$ & ug/g dry & 7.61 & $5 / 22 / 08$ & 8E12001 & PNNL-AGG-ICP-AES \\
\hline $7440-47-3$ & Chromium & 6.57 & ug/g dry & 3.05 & $5 / 22 / 08$ & 8E12001 & PNNL-AGG-ICP-AES \\
\hline $7440-50-8$ & Copper & $<32.5$ & ug/g dry & 32.5 & $5 / 22 / 08$ & 8E12001 & PNNL-AGG-ICP-AES \\
\hline 7439-89-6 & Iron & 11100 & ug/g dry & 93.1 & $5 / 22 / 08$ & 8E12001 & PNNL-AGG-ICP-AES \\
\hline 7440-09-7 & Potassium & 874 & ug/g dry & 86.4 & $5 / 22 / 08$ & 8E12001 & PNNL-AGG-ICP-AES \\
\hline $7439-93-2$ & Lithium & $<13.2$ & ug/g dry & 13.2 & $5 / 22 / 08$ & 8E12001 & PNNL-AGG-ICP-AES \\
\hline 7439-95-4 & Magnesium & 4080 & ug/g dry & 28.8 & $5 / 22 / 08$ & 8E12001 & PNNL-AGG-ICP-AES \\
\hline 7439-96-5 & Manganese & 245 & ug/g dry & 1.09 & $5 / 22 / 08$ & 8E12001 & PNNL-AGG-ICP-AES \\
\hline 7439-98-7 & Molybdenum & $<20.7$ & ug/g dry & 20.7 & $5 / 22 / 08$ & 8E12001 & PNNL-AGG-ICP-AES \\
\hline $7440-02-0$ & Nickel & 9.96 & ug/g dry & 7.49 & $5 / 22 / 08$ & 8E12001 & PNNL-AGG-ICP-AES \\
\hline 7723-14-0 & Phosphorus & 424 & ug/g dry & 68.6 & $5 / 22 / 08$ & 8E12001 & PNNL-AGG-ICP-AES \\
\hline 7439-92-1 & Lead & $<29.0$ & ug/g dry & 29.0 & $5 / 22 / 08$ & 8E12001 & PNNL-AGG-ICP-AES \\
\hline $7782-49-2$ & Selenium & $<127$ & ug/g dry & 127 & $5 / 22 / 08$ & 8E12001 & PNNL-AGG-ICP-AES \\
\hline $7440-24-6$ & Strontium & 26.2 & ug/g dry & 1.32 & $5 / 22 / 08$ & 8E12001 & PNNL-AGG-ICP-AES \\
\hline $7440-28-0$ & Thallium & $<41.2$ & ug/g dry & 41.2 & $5 / 22 / 08$ & 8E12001 & PNNL-AGG-ICP-AES \\
\hline $7440-62-2$ & Vanadium & 11.8 & ug/g dry & 3.39 & $5 / 22 / 08$ & 8E12001 & PNNL-AGG-ICP-AES \\
\hline $7440-66-6$ & Zinc & 25.0 & ug/g dry & 5.71 & $5 / 22 / 08$ & 8E12001 & PNNL-AGG-ICP-AES \\
\hline $7440-23-5$ & Sodium & $<976$ & ug/g dry & 976 & $5 / 22 / 08$ & 8E12001 & PNNL-AGG-ICP-AES \\
\hline $7440-21-3$ & Silicon & $<1270$ & ug/g dry & 1270 & $5 / 22 / 08$ & 8E12001 & PNNL-AGG-ICP-AES \\
\hline 7704-34-9 & Sulfur & $<216$ & ug/g dry & 216 & $5 / 22 / 08$ & 8E12001 & PNNL-AGG-ICP-AES \\
\hline $7440-32-6$ & Titanium & 478 & ug/g dry & 28.8 & $5 / 22 / 08$ & 8E12001 & PNNL-AGG-ICP-AES \\
\hline $7440-67-7$ & Zirconium & $<18.5$ & ug/g dry & 18.5 & $5 / 22 / 08$ & 8E12001 & PNNL-AGG-ICP-AES \\
\hline $7440-15-5$ & Rhenium & $<16.1$ & ug/g dry & 16.1 & $5 / 22 / 08$ & 8E12001 & PNNL-AGG-ICP-AES \\
\hline $7440-36-0$ & Antimony & $<102$ & ug/g dry & 102 & $5 / 22 / 08$ & 8E12001 & PNNL-AGG-ICP-AES \\
\hline HEIS No. & B1RYR8A & \multicolumn{3}{|c|}{ Lab ID: $\quad$ 0803012-18 } & & & \\
\hline 7429-90-5 & Aluminum & 4240 & ug/g dry & 29.5 & $5 / 22 / 08$ & 8E12001 & PNNL-AGG-ICP-AES \\
\hline $7440-38-2$ & Arsenic & $<71.6$ & ug/g dry & 71.6 & $5 / 22 / 08$ & 8E12001 & PNNL-AGG-ICP-AES \\
\hline $7440-42-8$ & Boron & $<624$ & ug/g dry & 624 & $5 / 22 / 08$ & 8E12001 & PNNL-AGG-ICP-AES \\
\hline $7440-39-3$ & Barium & 40.0 & ug/g dry & 2.81 & $5 / 22 / 08$ & 8E12001 & PNNL-AGG-ICP-AES \\
\hline $7440-41-7$ & Beryllium & $<1.11$ & ug/g dry & 1.11 & $5 / 22 / 08$ & 8E12001 & PNNL-AGG-ICP-AES \\
\hline $7440-69-9$ & Bismuth & $<14.9$ & ug/g dry & 14.9 & $5 / 22 / 08$ & 8E12001 & PNNL-AGG-ICP-AES \\
\hline $7440-70-2$ & Calcium & 7490 & ug/g dry & 110 & $5 / 22 / 08$ & 8E12001 & PNNL-AGG-ICP-AES \\
\hline $7440-43-9$ & Cadmium & $<1.67$ & ug/g dry & 1.67 & $5 / 22 / 08$ & 8E12001 & PNNL-AGG-ICP-AES \\
\hline $7440-48-4$ & Cobalt & $<6.12$ & ug/g dry & 6.12 & $5 / 22 / 08$ & 8E12001 & PNNL-AGG-ICP-AES \\
\hline $7440-47-3$ & Chromium & 7.37 & ug/g dry & 2.45 & $5 / 22 / 08$ & 8E12001 & PNNL-AGG-ICP-AES \\
\hline $7440-50-8$ & Copper & $<26.1$ & ug/g dry & 26.1 & $5 / 22 / 08$ & 8E12001 & PNNL-AGG-ICP-AES \\
\hline 7439-89-6 & Iron & 9870 & ug/g dry & 74.9 & $5 / 22 / 08$ & 8E12001 & PNNL-AGG-ICP-AES \\
\hline $7440-09-7$ & Potassium & 684 & ug/g dry & 69.5 & $5 / 22 / 08$ & 8E12001 & PNNL-AGG-ICP-AES \\
\hline $7439-93-2$ & Lithium & $<10.6$ & ug/g dry & 10.6 & $5 / 22 / 08$ & 8E12001 & PNNL-AGG-ICP-AES \\
\hline 7439-95-4 & Magnesium & 3550 & ug/g dry & 23.2 & $5 / 22 / 08$ & 8E12001 & PNNL-AGG-ICP-AES \\
\hline $7439-96-5$ & Manganese & 228 & ug/g dry & 0.878 & $5 / 22 / 08$ & 8E12001 & PNNL-AGG-ICP-AES \\
\hline 7439-98-7 & Molybdenum & $<16.6$ & ug/g dry & 16.6 & $5 / 22 / 08$ & 8E12001 & PNNL-AGG-ICP-AES \\
\hline $7440-02-0$ & Nickel & 8.91 & ug/g dry & 6.03 & $5 / 22 / 08$ & 8E12001 & PNNL-AGG-ICP-AES \\
\hline $7723-14-0$ & Phosphorus & 444 & ug/g dry & 55.2 & $5 / 22 / 08$ & 8E12001 & PNNL-AGG-ICP-AES \\
\hline 7439-92-1 & Lead & $<23.3$ & ug/g dry & 23.3 & $5 / 22 / 08$ & 8E12001 & PNNL-AGG-ICP-AES \\
\hline $7782-49-2$ & Selenium & $<102$ & ug/g dry & 102 & $5 / 22 / 08$ & 8E12001 & PNNL-AGG-ICP-AES \\
\hline
\end{tabular}


Total Metals by SW846 6010B/Acid Extract

\begin{tabular}{|c|c|c|c|c|c|c|c|}
\hline CAS \# & Analyte & Results & Units & EQL & Analyzed & Batch & Method \\
\hline HEIS No. & B1RYR8A & \multicolumn{3}{|c|}{ Lab ID: $\quad$ 0803012-18 } & & & \\
\hline $7440-24-6$ & Strontium & 22.3 & ug/g dry & 1.06 & $5 / 22 / 08$ & 8E12001 & PNNL-AGG-ICP-AES \\
\hline $7440-28-0$ & Thallium & $<33.1$ & ug/g dry & 33.1 & $5 / 22 / 08$ & 8E12001 & PNNL-AGG-ICP-AES \\
\hline $7440-62-2$ & Vanadium & 11.1 & ug/g dry & 2.73 & $5 / 22 / 08$ & 8E12001 & PNNL-AGG-ICP-AES \\
\hline $7440-66-6$ & Zinc & 21.4 & ug/g dry & 4.60 & $5 / 22 / 08$ & 8E12001 & PNNL-AGG-ICP-AES \\
\hline $7440-23-5$ & Sodium & $<786$ & ug/g dry & 786 & $5 / 22 / 08$ & 8E12001 & PNNL-AGG-ICP-AES \\
\hline $7440-21-3$ & Silicon & $<1020$ & ug/g dry & 1020 & $5 / 22 / 08$ & 8E12001 & PNNL-AGG-ICP-AES \\
\hline 7704-34-9 & Sulfur & $<174$ & ug/g dry & 174 & $5 / 22 / 08$ & 8E12001 & PNNL-AGG-ICP-AES \\
\hline $7440-32-6$ & Titanium & 443 & ug/g dry & 23.1 & $5 / 22 / 08$ & 8E12001 & PNNL-AGG-ICP-AES \\
\hline $7440-67-7$ & Zirconium & $<14.9$ & ug/g dry & 14.9 & $5 / 22 / 08$ & 8E12001 & PNNL-AGG-ICP-AES \\
\hline $7440-15-5$ & Rhenium & $<12.9$ & ug/g dry & 12.9 & $5 / 22 / 08$ & 8E12001 & PNNL-AGG-ICP-AES \\
\hline $7440-36-0$ & Antimony & $<82.1$ & ug/g dry & 82.1 & $5 / 22 / 08$ & 8E12001 & PNNL-AGG-ICP-AES \\
\hline HEIS No. & B1RYR9B & \multicolumn{3}{|c|}{ Lab ID: $\quad 0803012-21$} & & & \\
\hline 7429-90-5 & Aluminum & 4860 & ug/g dry & 31.4 & $5 / 22 / 08$ & 8E12001 & PNNL-AGG-ICP-AES \\
\hline $7440-38-2$ & Arsenic & $<76.2$ & ug/g dry & 76.2 & 5/22/08 & 8E12001 & PNNL-AGG-ICP-AES \\
\hline $7440-42-8$ & Boron & $<664$ & ug/g dry & 664 & $5 / 22 / 08$ & 8E12001 & PNNL-AGG-ICP-AES \\
\hline $7440-39-3$ & Barium & 42.9 & ug/g dry & 2.99 & $5 / 22 / 08$ & 8E12001 & PNNL-AGG-ICP-AES \\
\hline 7440-41-7 & Beryllium & $<1.18$ & ug/g dry & 1.18 & $5 / 22 / 08$ & 8E12001 & PNNL-AGG-ICP-AES \\
\hline $7440-69-9$ & Bismuth & $<15.9$ & ug/g dry & 15.9 & $5 / 22 / 08$ & 8E12001 & PNNL-AGG-ICP-AES \\
\hline $7440-70-2$ & Calcium & 7160 & ug/g dry & 117 & $5 / 22 / 08$ & 8E12001 & PNNL-AGG-ICP-AES \\
\hline $7440-43-9$ & Cadmium & $<1.78$ & ug/g dry & 1.78 & $5 / 22 / 08$ & 8E12001 & PNNL-AGG-ICP-AES \\
\hline $7440-48-4$ & Cobalt & $<6.51$ & ug/g dry & 6.51 & $5 / 22 / 08$ & 8E12001 & PNNL-AGG-ICP-AES \\
\hline $7440-47-3$ & Chromium & 11.4 & ug/g dry & 2.61 & $5 / 22 / 08$ & 8E12001 & PNNL-AGG-ICP-AES \\
\hline $7440-50-8$ & Copper & $<27.8$ & ug/g dry & 27.8 & $5 / 22 / 08$ & 8E12001 & PNNL-AGG-ICP-AES \\
\hline 7439-89-6 & Iron & 10800 & ug/g dry & 79.7 & $5 / 22 / 08$ & 8E12001 & PNNL-AGG-ICP-AES \\
\hline 7440-09-7 & Potassium & 807 & ug/g dry & 74.0 & 5/22/08 & 8E12001 & PNNL-AGG-ICP-AES \\
\hline 7439-93-2 & Lithium & $<11.3$ & ug/g dry & 11.3 & $5 / 22 / 08$ & 8E12001 & PNNL-AGG-ICP-AES \\
\hline 7439-95-4 & Magnesium & 3660 & ug/g dry & 24.7 & $5 / 22 / 08$ & 8E12001 & PNNL-AGG-ICP-AES \\
\hline 7439-96-5 & Manganese & 213 & ug/g dry & 0.934 & $5 / 22 / 08$ & 8E12001 & PNNL-AGG-ICP-AES \\
\hline 7439-98-7 & Molybdenum & $<17.7$ & ug/g dry & 17.7 & $5 / 22 / 08$ & 8E12001 & PNNL-AGG-ICP-AES \\
\hline $7440-02-0$ & Nickel & 15.7 & ug/g dry & 6.42 & $5 / 22 / 08$ & 8E12001 & PNNL-AGG-ICP-AES \\
\hline 7723-14-0 & Phosphorus & 458 & ug/g dry & 58.8 & $5 / 22 / 08$ & 8E12001 & PNNL-AGG-ICP-AES \\
\hline 7439-92-1 & Lead & $<24.8$ & ug/g dry & 24.8 & $5 / 22 / 08$ & 8E12001 & PNNL-AGG-ICP-AES \\
\hline $7782-49-2$ & Selenium & $<109$ & ug/g dry & 109 & $5 / 22 / 08$ & 8E12001 & PNNL-AGG-ICP-AES \\
\hline $7440-24-6$ & Strontium & 21.4 & ug/g dry & 1.13 & $5 / 22 / 08$ & 8E12001 & PNNL-AGG-ICP-AES \\
\hline $7440-28-0$ & Thallium & $<35.3$ & ug/g dry & 35.3 & $5 / 22 / 08$ & 8E12001 & PNNL-AGG-ICP-AES \\
\hline $7440-62-2$ & Vanadium & 12.6 & ug/g dry & 2.90 & $5 / 22 / 08$ & 8E12001 & PNNL-AGG-ICP-AES \\
\hline 7440-66-6 & Zinc & 24.5 & ug/g dry & 4.89 & $5 / 22 / 08$ & 8E12001 & PNNL-AGG-ICP-AES \\
\hline $7440-23-5$ & Sodium & $<836$ & ug/g dry & 836 & $5 / 22 / 08$ & 8E12001 & PNNL-AGG-ICP-AES \\
\hline $7440-21-3$ & Silicon & $<1090$ & ug/g dry & 1090 & $5 / 22 / 08$ & 8E12001 & PNNL-AGG-ICP-AES \\
\hline 7704-34-9 & Sulfur & $<185$ & ug/g dry & 185 & 5/22/08 & 8E12001 & PNNL-AGG-ICP-AES \\
\hline 7440-32-6 & Titanium & 477 & ug/g dry & 24.6 & $5 / 22 / 08$ & 8E12001 & PNNL-AGG-ICP-AES \\
\hline $7440-67-7$ & Zirconium & $<15.9$ & ug/g dry & 15.9 & $5 / 22 / 08$ & 8E12001 & PNNL-AGG-ICP-AES \\
\hline $7440-15-5$ & Rhenium & $<13.8$ & ug/g dry & 13.8 & $5 / 22 / 08$ & 8E12001 & PNNL-AGG-ICP-AES \\
\hline $7440-36-0$ & Antimony & $<87.4$ & ug/g dry & 87.4 & $5 / 22 / 08$ & 8E12001 & PNNL-AGG-ICP-AES \\
\hline HEIS No. & B1RYR9A & \multicolumn{3}{|c|}{ Lab ID: $\quad$ 0803012-22 } & & & \\
\hline $7429-90-5$ & Aluminum & 4610 & ug/g dry & 30.3 & $5 / 22 / 08$ & 8E12001 & PNNL-AGG-ICP-AES \\
\hline $7440-38-2$ & Arsenic & $<73.6$ & ug/g dry & 73.6 & $5 / 22 / 08$ & 8E12001 & PNNL-AGG-ICP-AES \\
\hline $7440-42-8$ & Boron & $<641$ & ug/g dry & 641 & 5/22/08 & 8E12001 & PNNL-AGG-ICP-AES \\
\hline
\end{tabular}


Total Metals by SW846 6010B/Acid Extract

\begin{tabular}{|c|c|c|c|c|c|c|c|}
\hline CAS \# & Analyte & Results & Units & EQL & Analyzed & Batch & Method \\
\hline HEIS No. & B1RYR9A & \multicolumn{3}{|c|}{ Lab ID: $\quad$ 0803012-22 } & & & \\
\hline $7440-39-3$ & Barium & 38.3 & ug/g dry & 2.88 & $5 / 22 / 08$ & 8E12001 & PNNL-AGG-ICP-AES \\
\hline $7440-41-7$ & Beryllium & $<1.14$ & ug/g dry & 1.14 & $5 / 22 / 08$ & 8E12001 & PNNL-AGG-ICP-AES \\
\hline 7440-69-9 & Bismuth & $<15.3$ & ug/g dry & 15.3 & $5 / 22 / 08$ & 8E12001 & PNNL-AGG-ICP-AES \\
\hline $7440-70-2$ & Calcium & 6630 & ug/g dry & 112 & $5 / 22 / 08$ & 8E12001 & PNNL-AGG-ICP-AES \\
\hline $7440-43-9$ & Cadmium & $<1.72$ & ug/g dry & 1.72 & $5 / 22 / 08$ & 8E12001 & PNNL-AGG-ICP-AES \\
\hline $7440-48-4$ & Cobalt & $<6.28$ & ug/g dry & 6.28 & $5 / 22 / 08$ & 8E12001 & PNNL-AGG-ICP-AES \\
\hline $7440-47-3$ & Chromium & 9.91 & ug/g dry & 2.52 & $5 / 22 / 08$ & 8E12001 & PNNL-AGG-ICP-AES \\
\hline $7440-50-8$ & Copper & $<26.8$ & ug/g dry & 26.8 & $5 / 22 / 08$ & 8E12001 & PNNL-AGG-ICP-AES \\
\hline 7439-89-6 & Iron & 10800 & ug/g dry & 76.9 & $5 / 22 / 08$ & 8E12001 & PNNL-AGG-ICP-AES \\
\hline 7440-09-7 & Potassium & 734 & ug/g dry & 71.4 & $5 / 22 / 08$ & 8E12001 & PNNL-AGG-ICP-AES \\
\hline 7439-93-2 & Lithium & $<10.9$ & ug/g dry & 10.9 & $5 / 22 / 08$ & 8E12001 & PNNL-AGG-ICP-AES \\
\hline 7439-95-4 & Magnesium & 3670 & ug/g dry & 23.8 & $5 / 22 / 08$ & 8E12001 & PNNL-AGG-ICP-AES \\
\hline $7439-96-5$ & Manganese & 225 & ug/g dry & 0.901 & $5 / 22 / 08$ & 8E12001 & PNNL-AGG-ICP-AES \\
\hline 7439-98-7 & Molybdenum & $<17.1$ & ug/g dry & 17.1 & $5 / 22 / 08$ & 8E12001 & PNNL-AGG-ICP-AES \\
\hline 7440-02-0 & Nickel & 11.2 & ug/g dry & 6.19 & $5 / 22 / 08$ & 8E12001 & PNNL-AGG-ICP-AES \\
\hline $7723-14-0$ & Phosphorus & 460 & ug/g dry & 56.7 & $5 / 22 / 08$ & 8E12001 & PNNL-AGG-ICP-AES \\
\hline 7439-92-1 & Lead & $<23.9$ & ug/g dry & 23.9 & $5 / 22 / 08$ & 8E12001 & PNNL-AGG-ICP-AES \\
\hline $7782-49-2$ & Selenium & $<105$ & ug/g dry & 105 & $5 / 22 / 08$ & 8E12001 & PNNL-AGG-ICP-AES \\
\hline $7440-24-6$ & Strontium & 21.0 & ug/g dry & 1.09 & $5 / 22 / 08$ & 8E12001 & PNNL-AGG-ICP-AES \\
\hline $7440-28-0$ & Thallium & $<34.0$ & ug/g dry & 34.0 & $5 / 22 / 08$ & 8E12001 & PNNL-AGG-ICP-AES \\
\hline $7440-62-2$ & Vanadium & 12.9 & ug/g dry & 2.80 & $5 / 22 / 08$ & 8E12001 & PNNL-AGG-ICP-AES \\
\hline $7440-66-6$ & Zinc & 23.4 & ug/g dry & 4.72 & $5 / 22 / 08$ & 8E12001 & PNNL-AGG-ICP-AES \\
\hline $7440-23-5$ & Sodium & $<807$ & ug/g dry & 807 & $5 / 22 / 08$ & 8E12001 & PNNL-AGG-ICP-AES \\
\hline $7440-21-3$ & Silicon & $<1050$ & ug/g dry & 1050 & $5 / 22 / 08$ & 8E12001 & PNNL-AGG-ICP-AES \\
\hline $7704-34-9$ & Sulfur & $<178$ & ug/g dry & 178 & $5 / 22 / 08$ & 8E12001 & PNNL-AGG-ICP-AES \\
\hline $7440-32-6$ & Titanium & 491 & ug/g dry & 23.8 & $5 / 22 / 08$ & 8E12001 & PNNL-AGG-ICP-AES \\
\hline $7440-67-7$ & Zirconium & $<15.3$ & ug/g dry & 15.3 & $5 / 22 / 08$ & 8E12001 & PNNL-AGG-ICP-AES \\
\hline $7440-15-5$ & Rhenium & $<13.3$ & ug/g dry & 13.3 & $5 / 22 / 08$ & 8E12001 & PNNL-AGG-ICP-AES \\
\hline $7440-36-0$ & Antimony & $<84.3$ & ug/g dry & 84.3 & $5 / 22 / 08$ & 8E12001 & PNNL-AGG-ICP-AES \\
\hline HEIS No. & B1RYT0B & \multicolumn{3}{|c|}{ Lab ID: $\quad$ 0803014-01 } & & & \\
\hline 7429-90-5 & Aluminum & 7060 & ug/g dry & 36.6 & $5 / 22 / 08$ & 8E12001 & PNNL-AGG-ICP-AES \\
\hline $7440-38-2$ & Arsenic & $<88.9$ & ug/g dry & 88.9 & $5 / 22 / 08$ & 8E12001 & PNNL-AGG-ICP-AES \\
\hline $7440-42-8$ & Boron & $<774$ & ug/g dry & 774 & $5 / 22 / 08$ & 8E12001 & PNNL-AGG-ICP-AES \\
\hline 7440-39-3 & Barium & 77.0 & ug/g dry & 3.49 & $5 / 22 / 08$ & 8E12001 & PNNL-AGG-ICP-AES \\
\hline $7440-41-7$ & Beryllium & $<1.38$ & ug/g dry & 1.38 & $5 / 22 / 08$ & 8E12001 & PNNL-AGG-ICP-AES \\
\hline $7440-69-9$ & Bismuth & $<18.5$ & ug/g dry & 18.5 & $5 / 22 / 08$ & 8E12001 & PNNL-AGG-ICP-AES \\
\hline $7440-70-2$ & Calcium & 11800 & ug/g dry & 136 & $5 / 22 / 08$ & 8E12001 & PNNL-AGG-ICP-AES \\
\hline $7440-43-9$ & Cadmium & $<2.08$ & ug/g dry & 2.08 & $5 / 22 / 08$ & 8E12001 & PNNL-AGG-ICP-AES \\
\hline $7440-48-4$ & Cobalt & 10.2 & ug/g dry & 7.60 & $5 / 22 / 08$ & 8E12001 & PNNL-AGG-ICP-AES \\
\hline $7440-47-3$ & Chromium & 5.38 & ug/g dry & 3.04 & $5 / 22 / 08$ & 8E12001 & PNNL-AGG-ICP-AES \\
\hline $7440-50-8$ & Copper & $<32.4$ & ug/g dry & 32.4 & $5 / 22 / 08$ & 8E12001 & PNNL-AGG-ICP-AES \\
\hline 7439-89-6 & Iron & 18400 & ug/g dry & 93.0 & $5 / 22 / 08$ & 8E12001 & PNNL-AGG-ICP-AES \\
\hline 7440-09-7 & Potassium & 1160 & ug/g dry & 86.3 & $5 / 22 / 08$ & 8E12001 & PNNL-AGG-ICP-AES \\
\hline $7439-93-2$ & Lithium & $<13.2$ & ug/g dry & 13.2 & $5 / 22 / 08$ & 8E12001 & PNNL-AGG-ICP-AES \\
\hline 7439-95-4 & Magnesium & 5740 & ug/g dry & 28.8 & $5 / 22 / 08$ & 8E12001 & PNNL-AGG-ICP-AES \\
\hline $7439-96-5$ & Manganese & 392 & ug/g dry & 1.09 & $5 / 22 / 08$ & 8E12001 & PNNL-AGG-ICP-AES \\
\hline 7439-98-7 & Molybdenum & $<20.6$ & ug/g dry & 20.6 & $5 / 22 / 08$ & 8E12001 & PNNL-AGG-ICP-AES \\
\hline 7440-02-0 & Nickel & 9.47 & ug/g dry & 7.48 & $5 / 22 / 08$ & 8E12001 & PNNL-AGG-ICP-AES \\
\hline
\end{tabular}


Total Metals by SW846 6010B/Acid Extract

\begin{tabular}{|c|c|c|c|c|c|c|c|}
\hline CAS \# & Analyte & Results & Units & EQL & Analyzed & Batch & Method \\
\hline HEIS No. & B1RYT0B & \multicolumn{3}{|c|}{ Lab ID: $\quad$ 0803014-01 } & & & \\
\hline 7723-14-0 & Phosphorus & 861 & ug/g dry & 68.5 & $5 / 22 / 08$ & 8E12001 & PNNL-AGG-ICP-AES \\
\hline 7439-92-1 & Lead & $<28.9$ & ug/g dry & 28.9 & $5 / 22 / 08$ & 8E12001 & PNNL-AGG-ICP-AES \\
\hline $7782-49-2$ & Selenium & $<127$ & ug/g dry & 127 & $5 / 22 / 08$ & 8E12001 & PNNL-AGG-ICP-AES \\
\hline $7440-24-6$ & Strontium & 22.3 & ug/g dry & 1.32 & $5 / 22 / 08$ & 8E12001 & PNNL-AGG-ICP-AES \\
\hline $7440-28-0$ & Thallium & $<41.1$ & ug/g dry & 41.1 & $5 / 22 / 08$ & 8E12001 & PNNL-AGG-ICP-AES \\
\hline $7440-62-2$ & Vanadium & 20.6 & ug/g dry & 3.39 & $5 / 22 / 08$ & 8E12001 & PNNL-AGG-ICP-AES \\
\hline $7440-66-6$ & Zinc & 44.5 & ug/g dry & 5.71 & $5 / 22 / 08$ & 8E12001 & PNNL-AGG-ICP-AES \\
\hline $7440-23-5$ & Sodium & $<975$ & ug/g dry & 975 & $5 / 22 / 08$ & 8E12001 & PNNL-AGG-ICP-AES \\
\hline $7440-21-3$ & Silicon & $<1270$ & ug/g dry & 1270 & $5 / 22 / 08$ & 8E12001 & PNNL-AGG-ICP-AES \\
\hline $7704-34-9$ & Sulfur & $<216$ & ug/g dry & 216 & $5 / 22 / 08$ & 8E12001 & PNNL-AGG-ICP-AES \\
\hline $7440-32-6$ & Titanium & 718 & ug/g dry & 28.7 & $5 / 22 / 08$ & 8E12001 & PNNL-AGG-ICP-AES \\
\hline $7440-67-7$ & Zirconium & $<18.5$ & ug/g dry & 18.5 & $5 / 22 / 08$ & 8E12001 & PNNL-AGG-ICP-AES \\
\hline $7440-15-5$ & Rhenium & $<16.0$ & ug/g dry & 16.0 & $5 / 22 / 08$ & 8E12001 & PNNL-AGG-ICP-AES \\
\hline $7440-36-0$ & Antimony & $<102$ & ug/g dry & 102 & $5 / 22 / 08$ & 8E12001 & PNNL-AGG-ICP-AES \\
\hline HEIS No. & B1RYT0A & \multicolumn{3}{|c|}{ Lab ID: $\quad$ 0803014-02 } & & & \\
\hline $7429-90-5$ & Aluminum & 5670 & ug/g dry & 34.1 & $5 / 22 / 08$ & 8E12001 & PNNL-AGG-ICP-AES \\
\hline $7440-38-2$ & Arsenic & $<82.9$ & ug/g dry & 82.9 & $5 / 22 / 08$ & 8E12001 & PNNL-AGG-ICP-AES \\
\hline $7440-42-8$ & Boron & $<722$ & ug/g dry & 722 & $5 / 22 / 08$ & 8E12001 & PNNL-AGG-ICP-AES \\
\hline $7440-39-3$ & Barium & 70.7 & ug/g dry & 3.25 & $5 / 22 / 08$ & 8E12001 & PNNL-AGG-ICP-AES \\
\hline $7440-41-7$ & Beryllium & $<1.29$ & ug/g dry & 1.29 & $5 / 22 / 08$ & 8E12001 & PNNL-AGG-ICP-AES \\
\hline $7440-69-9$ & Bismuth & $<17.2$ & ug/g dry & 17.2 & $5 / 22 / 08$ & 8E12001 & PNNL-AGG-ICP-AES \\
\hline $7440-70-2$ & Calcium & 6230 & ug/g dry & 127 & $5 / 22 / 08$ & 8E12001 & PNNL-AGG-ICP-AES \\
\hline $7440-43-9$ & Cadmium & $<1.94$ & ug/g dry & 1.94 & $5 / 22 / 08$ & 8E12001 & PNNL-AGG-ICP-AES \\
\hline $7440-48-4$ & Cobalt & 9.99 & ug/g dry & 7.08 & $5 / 22 / 08$ & 8E12001 & PNNL-AGG-ICP-AES \\
\hline $7440-47-3$ & Chromium & $<2.84$ & ug/g dry & 2.84 & $5 / 22 / 08$ & 8E12001 & PNNL-AGG-ICP-AES \\
\hline $7440-50-8$ & Copper & $<30.2$ & ug/g dry & 30.2 & $5 / 22 / 08$ & 8E12001 & PNNL-AGG-ICP-AES \\
\hline 7439-89-6 & Iron & 17900 & ug/g dry & 173 & $5 / 22 / 08$ & 8E12001 & PNNL-AGG-ICP-AES \\
\hline $7440-09-7$ & Potassium & 756 & ug/g dry & 80.5 & $5 / 22 / 08$ & 8E12001 & PNNL-AGG-ICP-AES \\
\hline $7439-93-2$ & Lithium & $<12.3$ & ug/g dry & 12.3 & $5 / 22 / 08$ & 8E12001 & PNNL-AGG-ICP-AES \\
\hline $7439-95-4$ & Magnesium & 4160 & ug/g dry & 26.8 & $5 / 22 / 08$ & 8E12001 & PNNL-AGG-ICP-AES \\
\hline $7439-96-5$ & Manganese & 327 & ug/g dry & 1.02 & $5 / 22 / 08$ & 8E12001 & PNNL-AGG-ICP-AES \\
\hline $7439-98-7$ & Molybdenum & $<19.2$ & ug/g dry & 19.2 & $5 / 22 / 08$ & 8E12001 & PNNL-AGG-ICP-AES \\
\hline $7440-02-0$ & Nickel & 7.38 & ug/g dry & 6.98 & $5 / 22 / 08$ & 8E12001 & PNNL-AGG-ICP-AES \\
\hline 7723-14-0 & Phosphorus & 858 & ug/g dry & 63.9 & $5 / 22 / 08$ & 8E12001 & PNNL-AGG-ICP-AES \\
\hline $7439-92-1$ & Lead & $<27.0$ & ug/g dry & 27.0 & $5 / 22 / 08$ & 8E12001 & PNNL-AGG-ICP-AES \\
\hline $7782-49-2$ & Selenium & $<119$ & ug/g dry & 119 & $5 / 22 / 08$ & 8E12001 & PNNL-AGG-ICP-AES \\
\hline $7440-24-6$ & Strontium & 17.8 & ug/g dry & 1.23 & $5 / 22 / 08$ & 8E12001 & PNNL-AGG-ICP-AES \\
\hline $7440-28-0$ & Thallium & $<38.3$ & ug/g dry & 38.3 & $5 / 22 / 08$ & 8E12001 & PNNL-AGG-ICP-AES \\
\hline $7440-62-2$ & Vanadium & 26.1 & ug/g dry & 3.16 & $5 / 22 / 08$ & 8E12001 & PNNL-AGG-ICP-AES \\
\hline $7440-66-6$ & Zinc & 32.6 & ug/g dry & 5.32 & $5 / 22 / 08$ & 8E12001 & PNNL-AGG-ICP-AES \\
\hline $7440-23-5$ & Sodium & $<909$ & ug/g dry & 909 & $5 / 22 / 08$ & 8E12001 & PNNL-AGG-ICP-AES \\
\hline $7440-21-3$ & Silicon & $<1190$ & ug/g dry & 1190 & $5 / 22 / 08$ & 8E12001 & PNNL-AGG-ICP-AES \\
\hline $7704-34-9$ & Sulfur & $<201$ & ug/g dry & 201 & $5 / 22 / 08$ & 8E12001 & PNNL-AGG-ICP-AES \\
\hline $7440-32-6$ & Titanium & 900 & ug/g dry & 26.8 & $5 / 22 / 08$ & 8E12001 & PNNL-AGG-ICP-AES \\
\hline $7440-67-7$ & Zirconium & $<17.3$ & ug/g dry & 17.3 & $5 / 22 / 08$ & 8E12001 & PNNL-AGG-ICP-AES \\
\hline $7440-15-5$ & Rhenium & $<15.0$ & ug/g dry & 15.0 & $5 / 22 / 08$ & 8E12001 & PNNL-AGG-ICP-AES \\
\hline $7440-36-0$ & Antimony & $<95.0$ & ug/g dry & 95.0 & $5 / 22 / 08$ & 8E12001 & PNNL-AGG-ICP-AES \\
\hline HEIS No. & B1T2P1B & $\mathbf{L a}$ & b ID: & 3014-05 & & & \\
\hline
\end{tabular}


Total Metals by SW846 6010B/Acid Extract

\begin{tabular}{|c|c|c|c|c|c|c|c|}
\hline CAS \# & Analyte & Results & Units & EQL & Analyzed & Batch & Method \\
\hline HEIS No. & B1T2P1B & \multicolumn{3}{|c|}{ Lab ID: $\quad$ 0803014-05 } & & & \\
\hline 7429-90-5 & Aluminum & 4760 & ug/g dry & 32.7 & $5 / 22 / 08$ & 8E12001 & PNNL-AGG-ICP-AES \\
\hline $7440-38-2$ & Arsenic & $<79.5$ & ug/g dry & 79.5 & $5 / 22 / 08$ & 8E12001 & PNNL-AGG-ICP-AES \\
\hline $7440-42-8$ & Boron & $<692$ & ug/g dry & 692 & $5 / 22 / 08$ & 8E12001 & PNNL-AGG-ICP-AES \\
\hline $7440-39-3$ & Barium & 43.4 & ug/g dry & 3.12 & $5 / 22 / 08$ & 8E12001 & PNNL-AGG-ICP-AES \\
\hline $7440-41-7$ & Beryllium & $<1.24$ & ug/g dry & 1.24 & $5 / 22 / 08$ & 8E12001 & PNNL-AGG-ICP-AES \\
\hline 7440-69-9 & Bismuth & $<16.5$ & ug/g dry & 16.5 & $5 / 22 / 08$ & 8E12001 & PNNL-AGG-ICP-AES \\
\hline $7440-70-2$ & Calcium & 8140 & ug/g dry & 122 & $5 / 22 / 08$ & 8E12001 & PNNL-AGG-ICP-AES \\
\hline 7440-43-9 & Cadmium & $<1.86$ & ug/g dry & 1.86 & $5 / 22 / 08$ & 8E12001 & PNNL-AGG-ICP-AES \\
\hline $7440-48-4$ & Cobalt & $<6.79$ & ug/g dry & 6.79 & $5 / 22 / 08$ & 8E12001 & PNNL-AGG-ICP-AES \\
\hline $7440-47-3$ & Chromium & 13.4 & ug/g dry & 2.72 & $5 / 22 / 08$ & 8E12001 & PNNL-AGG-ICP-AES \\
\hline $7440-50-8$ & Copper & $<29.0$ & ug/g dry & 29.0 & $5 / 22 / 08$ & 8E12001 & PNNL-AGG-ICP-AES \\
\hline 7439-89-6 & Iron & 11600 & ug/g dry & 83.1 & $5 / 22 / 08$ & 8E12001 & PNNL-AGG-ICP-AES \\
\hline 7440-09-7 & Potassium & 764 & ug/g dry & 77.2 & $5 / 22 / 08$ & 8E12001 & PNNL-AGG-ICP-AES \\
\hline 7439-93-2 & Lithium & $<11.8$ & ug/g dry & 11.8 & $5 / 22 / 08$ & 8E12001 & PNNL-AGG-ICP-AES \\
\hline $7439-95-4$ & Magnesium & 3980 & ug/g dry & 25.7 & $5 / 22 / 08$ & 8E12001 & PNNL-AGG-ICP-AES \\
\hline 7439-96-5 & Manganese & 233 & ug/g dry & 0.974 & $5 / 22 / 08$ & 8E12001 & PNNL-AGG-ICP-AES \\
\hline 7439-98-7 & Molybdenum & $<18.4$ & ug/g dry & 18.4 & $5 / 22 / 08$ & 8E12001 & PNNL-AGG-ICP-AES \\
\hline 7440-02-0 & Nickel & 20.1 & ug/g dry & 6.69 & $5 / 22 / 08$ & 8E12001 & PNNL-AGG-ICP-AES \\
\hline $7723-14-0$ & Phosphorus & 504 & ug/g dry & 61.3 & $5 / 22 / 08$ & 8E12001 & PNNL-AGG-ICP-AES \\
\hline $7439-92-1$ & Lead & $<25.9$ & ug/g dry & 25.9 & $5 / 22 / 08$ & 8E12001 & PNNL-AGG-ICP-AES \\
\hline $7782-49-2$ & Selenium & $<114$ & ug/g dry & 114 & $5 / 22 / 08$ & 8E12001 & PNNL-AGG-ICP-AES \\
\hline $7440-24-6$ & Strontium & 22.2 & ug/g dry & 1.18 & $5 / 22 / 08$ & 8E12001 & PNNL-AGG-ICP-AES \\
\hline $7440-28-0$ & Thallium & $<36.8$ & ug/g dry & 36.8 & $5 / 22 / 08$ & 8E12001 & PNNL-AGG-ICP-AES \\
\hline $7440-62-2$ & Vanadium & 12.6 & ug/g dry & 3.03 & $5 / 22 / 08$ & 8E12001 & PNNL-AGG-ICP-AES \\
\hline $7440-66-6$ & Zinc & 26.7 & ug/g dry & 5.10 & $5 / 22 / 08$ & 8E12001 & PNNL-AGG-ICP-AES \\
\hline $7440-23-5$ & Sodium & $<872$ & ug/g dry & 872 & $5 / 22 / 08$ & 8E12001 & PNNL-AGG-ICP-AES \\
\hline $7440-21-3$ & Silicon & $<1140$ & ug/g dry & 1140 & $5 / 22 / 08$ & 8E12001 & PNNL-AGG-ICP-AES \\
\hline 7704-34-9 & Sulfur & $<193$ & ug/g dry & 193 & $5 / 22 / 08$ & 8E12001 & PNNL-AGG-ICP-AES \\
\hline $7440-32-6$ & Titanium & 499 & ug/g dry & 25.7 & $5 / 22 / 08$ & 8E12001 & PNNL-AGG-ICP-AES \\
\hline $7440-67-7$ & Zirconium & $<16.6$ & ug/g dry & 16.6 & $5 / 22 / 08$ & 8E12001 & PNNL-AGG-ICP-AES \\
\hline $7440-15-5$ & Rhenium & $<14.3$ & ug/g dry & 14.3 & $5 / 22 / 08$ & 8E12001 & PNNL-AGG-ICP-AES \\
\hline $7440-36-0$ & Antimony & $<91.1$ & ug/g dry & 91.1 & $5 / 22 / 08$ & 8E12001 & PNNL-AGG-ICP-AES \\
\hline HEIS No. & B1T2P1A & \multicolumn{3}{|c|}{ Lab ID: $\quad$ 0803014-06 } & & & \\
\hline $7429-90-5$ & Aluminum & 4760 & ug/g dry & 31.9 & $5 / 22 / 08$ & 8E12001 & PNNL-AGG-ICP-AES \\
\hline $7440-38-2$ & Arsenic & $<77.5$ & ug/g dry & 77.5 & $5 / 22 / 08$ & 8E12001 & PNNL-AGG-ICP-AES \\
\hline $7440-42-8$ & Boron & $<675$ & ug/g dry & 675 & $5 / 22 / 08$ & 8E12001 & PNNL-AGG-ICP-AES \\
\hline $7440-39-3$ & Barium & 47.6 & ug/g dry & 3.04 & $5 / 22 / 08$ & 8E12001 & PNNL-AGG-ICP-AES \\
\hline $7440-41-7$ & Beryllium & $<1.20$ & ug/g dry & 1.20 & $5 / 22 / 08$ & 8E12001 & PNNL-AGG-ICP-AES \\
\hline 7440-69-9 & Bismuth & $<16.1$ & ug/g dry & 16.1 & $5 / 22 / 08$ & 8E12001 & PNNL-AGG-ICP-AES \\
\hline $7440-70-2$ & Calcium & 8160 & ug/g dry & 119 & $5 / 22 / 08$ & 8E12001 & PNNL-AGG-ICP-AES \\
\hline $7440-43-9$ & Cadmium & $<1.81$ & ug/g dry & 1.81 & $5 / 22 / 08$ & 8E12001 & PNNL-AGG-ICP-AES \\
\hline $7440-48-4$ & Cobalt & $<6.62$ & ug/g dry & 6.62 & $5 / 22 / 08$ & 8E12001 & PNNL-AGG-ICP-AES \\
\hline $7440-47-3$ & Chromium & 9.83 & ug/g dry & 2.65 & $5 / 22 / 08$ & 8E12001 & PNNL-AGG-ICP-AES \\
\hline $7440-50-8$ & Copper & $<28.3$ & ug/g dry & 28.3 & $5 / 22 / 08$ & 8E12001 & PNNL-AGG-ICP-AES \\
\hline 7439-89-6 & Iron & 11200 & ug/g dry & 81.1 & $5 / 22 / 08$ & 8E12001 & PNNL-AGG-ICP-AES \\
\hline $7440-09-7$ & Potassium & 677 & ug/g dry & 75.3 & $5 / 22 / 08$ & 8E12001 & PNNL-AGG-ICP-AES \\
\hline 7439-93-2 & Lithium & $<11.5$ & ug/g dry & 11.5 & $5 / 22 / 08$ & 8E12001 & PNNL-AGG-ICP-AES \\
\hline $7439-95-4$ & Magnesium & 3860 & ug/g dry & 25.1 & $5 / 22 / 08$ & 8E12001 & PNNL-AGG-ICP-AES \\
\hline
\end{tabular}


Total Metals by SW846 6010B/Acid Extract

\begin{tabular}{|c|c|c|c|c|c|c|c|}
\hline CAS \# & Analyte & Results & Units & EQL & Analyzed & Batch & Method \\
\hline HEIS No. & B1T2P1A & \multicolumn{3}{|c|}{ Lab ID: $\quad$ 0803014-06 } & & & \\
\hline 7439-96-5 & Manganese & 223 & ug/g dry & 0.950 & $5 / 22 / 08$ & 8E12001 & PNNL-AGG-ICP-AES \\
\hline 7439-98-7 & Molybdenum & $<18.0$ & ug/g dry & 18.0 & $5 / 22 / 08$ & 8E12001 & PNNL-AGG-ICP-AES \\
\hline $7440-02-0$ & Nickel & 10.9 & ug/g dry & 6.52 & $5 / 22 / 08$ & 8E12001 & PNNL-AGG-ICP-AES \\
\hline 7723-14-0 & Phosphorus & 489 & ug/g dry & 59.8 & $5 / 22 / 08$ & 8E12001 & PNNL-AGG-ICP-AES \\
\hline 7439-92-1 & Lead & $<25.2$ & ug/g dry & 25.2 & $5 / 22 / 08$ & 8E12001 & PNNL-AGG-ICP-AES \\
\hline 7782-49-2 & Selenium & $<111$ & ug/g dry & 111 & $5 / 22 / 08$ & 8E12001 & PNNL-AGG-ICP-AES \\
\hline $7440-24-6$ & Strontium & 23.0 & ug/g dry & 1.15 & $5 / 22 / 08$ & 8E12001 & PNNL-AGG-ICP-AES \\
\hline $7440-28-0$ & Thallium & $<35.9$ & ug/g dry & 35.9 & $5 / 22 / 08$ & 8E12001 & PNNL-AGG-ICP-AES \\
\hline $7440-62-2$ & Vanadium & 11.9 & ug/g dry & 2.95 & $5 / 22 / 08$ & 8E12001 & PNNL-AGG-ICP-AES \\
\hline $7440-66-6$ & Zinc & 30.7 & ug/g dry & 4.97 & $5 / 22 / 08$ & 8E12001 & PNNL-AGG-ICP-AES \\
\hline $7440-23-5$ & Sodium & $<850$ & ug/g dry & 850 & $5 / 22 / 08$ & 8E12001 & PNNL-AGG-ICP-AES \\
\hline $7440-21-3$ & Silicon & $<1110$ & ug/g dry & 1110 & $5 / 22 / 08$ & 8E12001 & PNNL-AGG-ICP-AES \\
\hline $7704-34-9$ & Sulfur & $<188$ & ug/g dry & 188 & $5 / 22 / 08$ & 8E12001 & PNNL-AGG-ICP-AES \\
\hline $7440-32-6$ & Titanium & 485 & ug/g dry & 25.0 & $5 / 22 / 08$ & 8E12001 & PNNL-AGG-ICP-AES \\
\hline $7440-67-7$ & Zirconium & $<16.2$ & ug/g dry & 16.2 & $5 / 22 / 08$ & 8E12001 & PNNL-AGG-ICP-AES \\
\hline $7440-15-5$ & Rhenium & $<14.0$ & ug/g dry & 14.0 & $5 / 22 / 08$ & 8E12001 & PNNL-AGG-ICP-AES \\
\hline $7440-36-0$ & Antimony & $<88.8$ & ug/g dry & 88.8 & $5 / 22 / 08$ & 8E12001 & PNNL-AGG-ICP-AES \\
\hline HEIS No. & B1T2P2B & \multicolumn{3}{|c|}{ Lab ID: $\quad$ 0803014-09 } & & & \\
\hline $7429-90-5$ & Aluminum & 5390 & ug/g dry & 37.2 & $5 / 22 / 08$ & 8E12001 & PNNL-AGG-ICP-AES \\
\hline $7440-38-2$ & Arsenic & $<90.5$ & ug/g dry & 90.5 & $5 / 22 / 08$ & 8E12001 & PNNL-AGG-ICP-AES \\
\hline $7440-42-8$ & Boron & $<788$ & ug/g dry & 788 & $5 / 22 / 08$ & 8E12001 & PNNL-AGG-ICP-AES \\
\hline $7440-39-3$ & Barium & 48.4 & ug/g dry & 3.55 & $5 / 22 / 08$ & 8E12001 & PNNL-AGG-ICP-AES \\
\hline $7440-41-7$ & Beryllium & $<1.41$ & ug/g dry & 1.41 & $5 / 22 / 08$ & 8E12001 & PNNL-AGG-ICP-AES \\
\hline $7440-69-9$ & Bismuth & $<18.8$ & ug/g dry & 18.8 & $5 / 22 / 08$ & 8E12001 & PNNL-AGG-ICP-AES \\
\hline $7440-70-2$ & Calcium & 7810 & ug/g dry & 138 & $5 / 22 / 08$ & 8E12001 & PNNL-AGG-ICP-AES \\
\hline $7440-43-9$ & Cadmium & $<2.11$ & ug/g dry & 2.11 & $5 / 22 / 08$ & 8E12001 & PNNL-AGG-ICP-AES \\
\hline $7440-48-4$ & Cobalt & $<7.73$ & ug/g dry & 7.73 & $5 / 22 / 08$ & 8E12001 & PNNL-AGG-ICP-AES \\
\hline $7440-47-3$ & Chromium & 8.19 & ug/g dry & 3.09 & $5 / 22 / 08$ & 8E12001 & PNNL-AGG-ICP-AES \\
\hline $7440-50-8$ & Copper & $<33.0$ & ug/g dry & 33.0 & $5 / 22 / 08$ & 8E12001 & PNNL-AGG-ICP-AES \\
\hline 7439-89-6 & Iron & 12600 & ug/g dry & 94.6 & $5 / 22 / 08$ & 8E12001 & PNNL-AGG-ICP-AES \\
\hline 7440-09-7 & Potassium & 762 & ug/g dry & 87.8 & $5 / 22 / 08$ & 8E12001 & PNNL-AGG-ICP-AES \\
\hline $7439-93-2$ & Lithium & $<13.4$ & ug/g dry & 13.4 & $5 / 22 / 08$ & 8E12001 & PNNL-AGG-ICP-AES \\
\hline $7439-95-4$ & Magnesium & 4160 & ug/g dry & 29.3 & $5 / 22 / 08$ & 8E12001 & PNNL-AGG-ICP-AES \\
\hline $7439-96-5$ & Manganese & 222 & ug/g dry & 1.11 & $5 / 22 / 08$ & 8E12001 & PNNL-AGG-ICP-AES \\
\hline 7439-98-7 & Molybdenum & $<21.0$ & ug/g dry & 21.0 & $5 / 22 / 08$ & 8E12001 & PNNL-AGG-ICP-AES \\
\hline $7440-02-0$ & Nickel & 9.57 & ug/g dry & 7.61 & $5 / 22 / 08$ & 8E12001 & PNNL-AGG-ICP-AES \\
\hline 7723-14-0 & Phosphorus & 507 & ug/g dry & 69.7 & $5 / 22 / 08$ & 8E12001 & PNNL-AGG-ICP-AES \\
\hline $7439-92-1$ & Lead & $<29.4$ & ug/g dry & 29.4 & $5 / 22 / 08$ & 8E12001 & PNNL-AGG-ICP-AES \\
\hline $7782-49-2$ & Selenium & $<129$ & ug/g dry & 129 & $5 / 22 / 08$ & 8E12001 & PNNL-AGG-ICP-AES \\
\hline $7440-24-6$ & Strontium & 25.1 & ug/g dry & 1.34 & $5 / 22 / 08$ & 8E12001 & PNNL-AGG-ICP-AES \\
\hline $7440-28-0$ & Thallium & $<41.8$ & ug/g dry & 41.8 & $5 / 22 / 08$ & 8E12001 & PNNL-AGG-ICP-AES \\
\hline $7440-62-2$ & Vanadium & 16.8 & ug/g dry & 3.44 & $5 / 22 / 08$ & 8E12001 & PNNL-AGG-ICP-AES \\
\hline $7440-66-6$ & Zinc & 27.3 & ug/g dry & 5.80 & $5 / 22 / 08$ & 8E12001 & PNNL-AGG-ICP-AES \\
\hline $7440-23-5$ & Sodium & $<992$ & ug/g dry & 992 & $5 / 22 / 08$ & 8E12001 & PNNL-AGG-ICP-AES \\
\hline $7440-21-3$ & Silicon & $<1290$ & ug/g dry & 1290 & $5 / 22 / 08$ & 8E12001 & PNNL-AGG-ICP-AES \\
\hline 7704-34-9 & Sulfur & $<219$ & ug/g dry & 219 & $5 / 22 / 08$ & 8E12001 & PNNL-AGG-ICP-AES \\
\hline $7440-32-6$ & Titanium & 619 & ug/g dry & 29.2 & $5 / 22 / 08$ & 8E12001 & PNNL-AGG-ICP-AES \\
\hline $7440-67-7$ & Zirconium & $<18.8$ & ug/g dry & 18.8 & $5 / 22 / 08$ & 8E12001 & PNNL-AGG-ICP-AES \\
\hline
\end{tabular}


Total Metals by SW846 6010B/Acid Extract

\begin{tabular}{|c|c|c|c|c|c|c|c|}
\hline CAS \# & Analyte & Results & Units & EQL & Analyzed & Batch & Method \\
\hline HEIS No. & В1T2P2B & \multicolumn{3}{|c|}{ Lab ID: $\quad$ 0803014-09 } & & & \\
\hline $7440-15-5$ & Rhenium & $<16.3$ & ug/g dry & 16.3 & $5 / 22 / 08$ & 8E12001 & PNNL-AGG-ICP-AES \\
\hline $7440-36-0$ & Antimony & $<104$ & ug/g dry & 104 & $5 / 22 / 08$ & 8E12001 & PNNL-AGG-ICP-AES \\
\hline HEIS No. & B1T2P2A & \multicolumn{3}{|c|}{ Lab ID: $\quad$ 0803014-10 } & & & \\
\hline $7429-90-5$ & Aluminum & 4950 & ug/g dry & 36.6 & $5 / 22 / 08$ & 8E12001 & PNNL-AGG-ICP-AES \\
\hline $7440-38-2$ & Arsenic & $<88.9$ & ug/g dry & 88.9 & $5 / 22 / 08$ & 8E12001 & PNNL-AGG-ICP-AES \\
\hline $7440-42-8$ & Boron & $<774$ & ug/g dry & 774 & $5 / 22 / 08$ & 8E12001 & PNNL-AGG-ICP-AES \\
\hline $7440-39-3$ & Barium & 49.3 & ug/g dry & 3.49 & $5 / 22 / 08$ & 8E12001 & PNNL-AGG-ICP-AES \\
\hline $7440-41-7$ & Beryllium & $<1.38$ & ug/g dry & 1.38 & $5 / 22 / 08$ & 8E12001 & PNNL-AGG-ICP-AES \\
\hline $7440-69-9$ & Bismuth & $<18.5$ & ug/g dry & 18.5 & $5 / 22 / 08$ & 8E12001 & PNNL-AGG-ICP-AES \\
\hline $7440-70-2$ & Calcium & 8250 & ug/g dry & 136 & $5 / 22 / 08$ & 8E12001 & PNNL-AGG-ICP-AES \\
\hline $7440-43-9$ & Cadmium & $<2.08$ & ug/g dry & 2.08 & $5 / 22 / 08$ & 8E12001 & PNNL-AGG-ICP-AES \\
\hline $7440-48-4$ & Cobalt & $<7.59$ & ug/g dry & 7.59 & $5 / 22 / 08$ & 8E12001 & PNNL-AGG-ICP-AES \\
\hline $7440-47-3$ & Chromium & 7.32 & ug/g dry & 3.04 & $5 / 22 / 08$ & 8E12001 & PNNL-AGG-ICP-AES \\
\hline $7440-50-8$ & Copper & $<32.4$ & ug/g dry & 32.4 & $5 / 22 / 08$ & 8E12001 & PNNL-AGG-ICP-AES \\
\hline 7439-89-6 & Iron & 12500 & ug/g dry & 93.0 & $5 / 22 / 08$ & 8E12001 & PNNL-AGG-ICP-AES \\
\hline $7440-09-7$ & Potassium & 717 & ug/g dry & 86.3 & $5 / 22 / 08$ & 8E12001 & PNNL-AGG-ICP-AES \\
\hline $7439-93-2$ & Lithium & $<13.2$ & ug/g dry & 13.2 & $5 / 22 / 08$ & 8E12001 & PNNL-AGG-ICP-AES \\
\hline 7439-95-4 & Magnesium & 3910 & ug/g dry & 28.8 & $5 / 22 / 08$ & 8E12001 & PNNL-AGG-ICP-AES \\
\hline $7439-96-5$ & Manganese & 224 & ug/g dry & 1.09 & $5 / 22 / 08$ & 8E12001 & PNNL-AGG-ICP-AES \\
\hline 7439-98-7 & Molybdenum & $<20.6$ & ug/g dry & 20.6 & $5 / 22 / 08$ & 8E12001 & PNNL-AGG-ICP-AES \\
\hline $7440-02-0$ & Nickel & 9.12 & ug/g dry & 7.48 & $5 / 22 / 08$ & 8E12001 & PNNL-AGG-ICP-AES \\
\hline $7723-14-0$ & Phosphorus & 563 & ug/g dry & 68.5 & $5 / 22 / 08$ & 8E12001 & PNNL-AGG-ICP-AES \\
\hline 7439-92-1 & Lead & $<28.9$ & ug/g dry & 28.9 & $5 / 22 / 08$ & 8E12001 & PNNL-AGG-ICP-AES \\
\hline $7782-49-2$ & Selenium & $<127$ & ug/g dry & 127 & $5 / 22 / 08$ & 8E12001 & PNNL-AGG-ICP-AES \\
\hline $7440-24-6$ & Strontium & 24.6 & ug/g dry & 1.32 & $5 / 22 / 08$ & 8E12001 & PNNL-AGG-ICP-AES \\
\hline $7440-28-0$ & Thallium & $<41.1$ & ug/g dry & 41.1 & $5 / 22 / 08$ & 8E12001 & PNNL-AGG-ICP-AES \\
\hline $7440-62-2$ & Vanadium & 15.7 & ug/g dry & 3.39 & $5 / 22 / 08$ & 8E12001 & PNNL-AGG-ICP-AES \\
\hline $7440-66-6$ & Zinc & 27.6 & ug/g dry & 5.70 & $5 / 22 / 08$ & 8E12001 & PNNL-AGG-ICP-AES \\
\hline $7440-23-5$ & Sodium & $<975$ & ug/g dry & 975 & $5 / 22 / 08$ & 8E12001 & PNNL-AGG-ICP-AES \\
\hline $7440-21-3$ & Silicon & $<1270$ & ug/g dry & 1270 & $5 / 22 / 08$ & 8E12001 & PNNL-AGG-ICP-AES \\
\hline $7704-34-9$ & Sulfur & $<216$ & ug/g dry & 216 & $5 / 22 / 08$ & 8E12001 & PNNL-AGG-ICP-AES \\
\hline $7440-32-6$ & Titanium & 606 & ug/g dry & 28.7 & $5 / 22 / 08$ & 8E12001 & PNNL-AGG-ICP-AES \\
\hline $7440-67-7$ & Zirconium & $<18.5$ & ug/g dry & 18.5 & $5 / 22 / 08$ & 8E12001 & PNNL-AGG-ICP-AES \\
\hline $7440-15-5$ & Rhenium & $<16.0$ & ug/g dry & 16.0 & $5 / 22 / 08$ & 8E12001 & PNNL-AGG-ICP-AES \\
\hline $7440-36-0$ & Antimony & $<102$ & ug/g dry & 102 & $5 / 22 / 08$ & 8E12001 & PNNL-AGG-ICP-AES \\
\hline HEIS No. & B1T2P3B & & b ID: & 0803014-13 & & & \\
\hline $7429-90-5$ & Aluminum & 4120 & ug/g dry & 32.3 & $5 / 22 / 08$ & 8E12001 & PNNL-AGG-ICP-AES \\
\hline $7440-38-2$ & Arsenic & $<78.5$ & ug/g dry & 78.5 & $5 / 22 / 08$ & 8E12001 & PNNL-AGG-ICP-AES \\
\hline $7440-42-8$ & Boron & $<684$ & ug/g dry & 684 & $5 / 22 / 08$ & 8E12001 & PNNL-AGG-ICP-AES \\
\hline $7440-39-3$ & Barium & 42.1 & ug/g dry & 3.08 & $5 / 22 / 08$ & 8E12001 & PNNL-AGG-ICP-AES \\
\hline $7440-41-7$ & Beryllium & $<1.22$ & ug/g dry & 1.22 & $5 / 22 / 08$ & 8E12001 & PNNL-AGG-ICP-AES \\
\hline $7440-69-9$ & Bismuth & $<16.3$ & ug/g dry & 16.3 & $5 / 22 / 08$ & 8E12001 & PNNL-AGG-ICP-AES \\
\hline $7440-70-2$ & Calcium & 6790 & ug/g dry & 120 & $5 / 22 / 08$ & 8E12001 & PNNL-AGG-ICP-AES \\
\hline $7440-43-9$ & Cadmium & $<1.84$ & ug/g dry & 1.84 & $5 / 22 / 08$ & 8E12001 & PNNL-AGG-ICP-AES \\
\hline $7440-48-4$ & Cobalt & $<6.71$ & ug/g dry & 6.71 & $5 / 22 / 08$ & 8E12001 & PNNL-AGG-ICP-AES \\
\hline $7440-47-3$ & Chromium & 5.30 & ug/g dry & 2.69 & $5 / 22 / 08$ & 8E12001 & PNNL-AGG-ICP-AES \\
\hline $7440-50-8$ & Copper & $<28.6$ & ug/g dry & 28.6 & $5 / 22 / 08$ & 8E12001 & PNNL-AGG-ICP-AES \\
\hline 7439-89-6 & Iron & 11700 & ug/g dry & 82.1 & $5 / 22 / 08$ & 8E12001 & PNNL-AGG-ICP-AES \\
\hline
\end{tabular}


Total Metals by SW846 6010B/Acid Extract

\begin{tabular}{|c|c|c|c|c|c|c|c|}
\hline CAS \# & Analyte & Results & Units & EQL & Analyzed & Batch & Method \\
\hline HEIS No. & В1T2P3B & \multicolumn{3}{|c|}{ Lab ID: $\quad$ 0803014-13 } & & & \\
\hline 7440-09-7 & Potassium & 621 & ug/g dry & 76.2 & $5 / 22 / 08$ & 8E12001 & PNNL-AGG-ICP-AES \\
\hline 7439-93-2 & Lithium & $<11.6$ & ug/g dry & 11.6 & $5 / 22 / 08$ & 8E12001 & PNNL-AGG-ICP-AES \\
\hline 7439-95-4 & Magnesium & 3260 & ug/g dry & 25.4 & $5 / 22 / 08$ & 8E12001 & PNNL-AGG-ICP-AES \\
\hline 7439-96-5 & Manganese & 203 & ug/g dry & 0.962 & $5 / 22 / 08$ & 8E12001 & PNNL-AGG-ICP-AES \\
\hline 7439-98-7 & Molybdenum & $<18.2$ & ug/g dry & 18.2 & $5 / 22 / 08$ & 8E12001 & PNNL-AGG-ICP-AES \\
\hline 7440-02-0 & Nickel & 6.79 & ug/g dry & 6.61 & $5 / 22 / 08$ & 8E12001 & PNNL-AGG-ICP-AES \\
\hline $7723-14-0$ & Phosphorus & 567 & ug/g dry & 60.5 & $5 / 22 / 08$ & 8E12001 & PNNL-AGG-ICP-AES \\
\hline 7439-92-1 & Lead & $<25.6$ & ug/g dry & 25.6 & $5 / 22 / 08$ & 8E12001 & PNNL-AGG-ICP-AES \\
\hline $7782-49-2$ & Selenium & $<112$ & ug/g dry & 112 & $5 / 22 / 08$ & 8E12001 & PNNL-AGG-ICP-AES \\
\hline $7440-24-6$ & Strontium & 21.1 & ug/g dry & 1.16 & $5 / 22 / 08$ & 8E12001 & PNNL-AGG-ICP-AES \\
\hline $7440-28-0$ & Thallium & $<36.3$ & ug/g dry & 36.3 & $5 / 22 / 08$ & 8E12001 & PNNL-AGG-ICP-AES \\
\hline $7440-62-2$ & Vanadium & 16.4 & ug/g dry & 2.99 & $5 / 22 / 08$ & 8E12001 & PNNL-AGG-ICP-AES \\
\hline $7440-66-6$ & Zinc & 27.2 & ug/g dry & 5.04 & $5 / 22 / 08$ & 8E12001 & PNNL-AGG-ICP-AES \\
\hline $7440-23-5$ & Sodium & $<861$ & ug/g dry & 861 & $5 / 22 / 08$ & 8E12001 & PNNL-AGG-ICP-AES \\
\hline $7440-21-3$ & Silicon & $<1120$ & ug/g dry & 1120 & $5 / 22 / 08$ & 8E12001 & PNNL-AGG-ICP-AES \\
\hline $7704-34-9$ & Sulfur & $<190$ & ug/g dry & 190 & $5 / 22 / 08$ & 8E12001 & PNNL-AGG-ICP-AES \\
\hline $7440-32-6$ & Titanium & 550 & ug/g dry & 25.4 & $5 / 22 / 08$ & 8E12001 & PNNL-AGG-ICP-AES \\
\hline $7440-67-7$ & Zirconium & $<16.4$ & ug/g dry & 16.4 & $5 / 22 / 08$ & 8E12001 & PNNL-AGG-ICP-AES \\
\hline $7440-15-5$ & Rhenium & $<14.2$ & ug/g dry & 14.2 & $5 / 22 / 08$ & 8E12001 & PNNL-AGG-ICP-AES \\
\hline $7440-36-0$ & Antimony & $<90.0$ & ug/g dry & 90.0 & $5 / 22 / 08$ & 8E12001 & PNNL-AGG-ICP-AES \\
\hline HEIS No. & B1T2P3A & \multicolumn{3}{|c|}{ Lab ID: $\quad$ 0803014-14 } & & & \\
\hline $7429-90-5$ & Aluminum & 4770 & ug/g dry & 33.0 & $5 / 22 / 08$ & 8E12001 & PNNL-AGG-ICP-AES \\
\hline $7440-38-2$ & Arsenic & $<80.2$ & ug/g dry & 80.2 & $5 / 22 / 08$ & 8E12001 & PNNL-AGG-ICP-AES \\
\hline $7440-42-8$ & Boron & $<698$ & ug/g dry & 698 & $5 / 22 / 08$ & 8E12001 & PNNL-AGG-ICP-AES \\
\hline $7440-39-3$ & Barium & 55.8 & ug/g dry & 3.14 & $5 / 22 / 08$ & 8E12001 & PNNL-AGG-ICP-AES \\
\hline $7440-41-7$ & Beryllium & $<1.25$ & ug/g dry & 1.25 & $5 / 22 / 08$ & 8E12001 & PNNL-AGG-ICP-AES \\
\hline $7440-69-9$ & Bismuth & $<16.7$ & ug/g dry & 16.7 & $5 / 22 / 08$ & 8E12001 & PNNL-AGG-ICP-AES \\
\hline $7440-70-2$ & Calcium & 6530 & ug/g dry & 123 & $5 / 22 / 08$ & 8E12001 & PNNL-AGG-ICP-AES \\
\hline $7440-43-9$ & Cadmium & $<1.87$ & ug/g dry & 1.87 & $5 / 22 / 08$ & 8E12001 & PNNL-AGG-ICP-AES \\
\hline $7440-48-4$ & Cobalt & $<6.85$ & ug/g dry & 6.85 & $5 / 22 / 08$ & 8E12001 & PNNL-AGG-ICP-AES \\
\hline $7440-47-3$ & Chromium & 5.82 & ug/g dry & 2.74 & $5 / 22 / 08$ & 8E12001 & PNNL-AGG-ICP-AES \\
\hline $7440-50-8$ & Copper & $<29.2$ & ug/g dry & 29.2 & $5 / 22 / 08$ & 8E12001 & PNNL-AGG-ICP-AES \\
\hline 7439-89-6 & Iron & 13100 & ug/g dry & 83.8 & $5 / 22 / 08$ & 8E12001 & PNNL-AGG-ICP-AES \\
\hline 7440-09-7 & Potassium & 661 & ug/g dry & 77.8 & $5 / 22 / 08$ & 8E12001 & PNNL-AGG-ICP-AES \\
\hline $7439-93-2$ & Lithium & $<11.9$ & ug/g dry & 11.9 & $5 / 22 / 08$ & 8E12001 & PNNL-AGG-ICP-AES \\
\hline 7439-95-4 & Magnesium & 3760 & ug/g dry & 26.0 & $5 / 22 / 08$ & 8E12001 & PNNL-AGG-ICP-AES \\
\hline 7439-96-5 & Manganese & 222 & ug/g dry & 0.982 & $5 / 22 / 08$ & 8E12001 & PNNL-AGG-ICP-AES \\
\hline 7439-98-7 & Molybdenum & $<18.6$ & ug/g dry & 18.6 & $5 / 22 / 08$ & 8E12001 & PNNL-AGG-ICP-AES \\
\hline $7440-02-0$ & Nickel & 7.69 & ug/g dry & 6.75 & $5 / 22 / 08$ & 8E12001 & PNNL-AGG-ICP-AES \\
\hline $7723-14-0$ & Phosphorus & 631 & ug/g dry & 61.8 & $5 / 22 / 08$ & 8E12001 & PNNL-AGG-ICP-AES \\
\hline $7439-92-1$ & Lead & $<26.1$ & ug/g dry & 26.1 & $5 / 22 / 08$ & 8E12001 & PNNL-AGG-ICP-AES \\
\hline $7782-49-2$ & Selenium & $<115$ & ug/g dry & 115 & $5 / 22 / 08$ & 8E12001 & PNNL-AGG-ICP-AES \\
\hline $7440-24-6$ & Strontium & 23.5 & ug/g dry & 1.19 & $5 / 22 / 08$ & 8E12001 & PNNL-AGG-ICP-AES \\
\hline $7440-28-0$ & Thallium & $<37.1$ & ug/g dry & 37.1 & $5 / 22 / 08$ & 8E12001 & PNNL-AGG-ICP-AES \\
\hline $7440-62-2$ & Vanadium & 19.1 & ug/g dry & 3.05 & $5 / 22 / 08$ & 8E12001 & PNNL-AGG-ICP-AES \\
\hline $7440-66-6$ & Zinc & 29.7 & ug/g dry & 5.14 & $5 / 22 / 08$ & 8E12001 & PNNL-AGG-ICP-AES \\
\hline $7440-23-5$ & Sodium & $<879$ & ug/g dry & 879 & $5 / 22 / 08$ & 8E12001 & PNNL-AGG-ICP-AES \\
\hline $7440-21-3$ & Silicon & $<1150$ & ug/g dry & 1150 & $5 / 22 / 08$ & 8E12001 & PNNL-AGG-ICP-AES \\
\hline
\end{tabular}


Total Metals by SW846 6010B/Acid Extract

\begin{tabular}{|c|c|c|c|c|c|c|c|}
\hline CAS \# & Analyte & Results & Units & EQL & Analyzed & Batch & Method \\
\hline HEIS No. & В1T2P3A & \multicolumn{3}{|c|}{ Lab ID: $\quad$ 0803014-14 } & & & \\
\hline 7704-34-9 & Sulfur & $<194$ & ug/g dry & 194 & $5 / 22 / 08$ & 8E12001 & PNNL-AGG-ICP-AES \\
\hline $7440-32-6$ & Titanium & 693 & ug/g dry & 25.9 & $5 / 22 / 08$ & 8E12001 & PNNL-AGG-ICP-AES \\
\hline $7440-67-7$ & Zirconium & $<16.7$ & ug/g dry & 16.7 & $5 / 22 / 08$ & 8E12001 & PNNL-AGG-ICP-AES \\
\hline $7440-15-5$ & Rhenium & $<14.5$ & ug/g dry & 14.5 & $5 / 22 / 08$ & 8E12001 & PNNL-AGG-ICP-AES \\
\hline $7440-36-0$ & Antimony & $<91.9$ & ug/g dry & 91.9 & $5 / 22 / 08$ & 8E12001 & PNNL-AGG-ICP-AES \\
\hline HEIS No. & B1T2P4B & \multicolumn{3}{|c|}{ Lab ID: $\quad$ 0803014-16 } & & & \\
\hline $7429-90-5$ & Aluminum & 4760 & ug/g dry & 34.4 & $5 / 22 / 08$ & 8E12001 & PNNL-AGG-ICP-AES \\
\hline $7440-38-2$ & Arsenic & $<83.4$ & ug/g dry & 83.4 & $5 / 22 / 08$ & 8E12001 & PNNL-AGG-ICP-AES \\
\hline $7440-42-8$ & Boron & $<727$ & ug/g dry & 727 & $5 / 22 / 08$ & 8E12001 & PNNL-AGG-ICP-AES \\
\hline $7440-39-3$ & Barium & 49.0 & ug/g dry & 3.27 & $5 / 22 / 08$ & 8E12001 & PNNL-AGG-ICP-AES \\
\hline $7440-41-7$ & Beryllium & $<1.30$ & ug/g dry & 1.30 & $5 / 22 / 08$ & 8E12001 & PNNL-AGG-ICP-AES \\
\hline 7440-69-9 & Bismuth & $<17.4$ & ug/g dry & 17.4 & $5 / 22 / 08$ & 8E12001 & PNNL-AGG-ICP-AES \\
\hline $7440-70-2$ & Calcium & 8080 & ug/g dry & 128 & $5 / 22 / 08$ & 8E12001 & PNNL-AGG-ICP-AES \\
\hline $7440-43-9$ & Cadmium & $<1.95$ & ug/g dry & 1.95 & $5 / 22 / 08$ & 8E12001 & PNNL-AGG-ICP-AES \\
\hline $7440-48-4$ & Cobalt & $<7.13$ & ug/g dry & 7.13 & $5 / 22 / 08$ & 8E12001 & PNNL-AGG-ICP-AES \\
\hline $7440-47-3$ & Chromium & 11.3 & ug/g dry & 2.85 & $5 / 22 / 08$ & 8E12001 & PNNL-AGG-ICP-AES \\
\hline $7440-50-8$ & Copper & $<30.4$ & ug/g dry & 30.4 & $5 / 22 / 08$ & 8E12001 & PNNL-AGG-ICP-AES \\
\hline 7439-89-6 & Iron & 10000 & ug/g dry & 87.3 & $5 / 22 / 08$ & 8E12001 & PNNL-AGG-ICP-AES \\
\hline $7440-09-7$ & Potassium & 888 & ug/g dry & 81.0 & $5 / 22 / 08$ & 8E12001 & PNNL-AGG-ICP-AES \\
\hline 7439-93-2 & Lithium & $<12.4$ & ug/g dry & 12.4 & $5 / 22 / 08$ & 8E12001 & PNNL-AGG-ICP-AES \\
\hline $7439-95-4$ & Magnesium & 3660 & ug/g dry & 27.0 & $5 / 22 / 08$ & 8E12001 & PNNL-AGG-ICP-AES \\
\hline 7439-96-5 & Manganese & 222 & ug/g dry & 1.02 & $5 / 22 / 08$ & 8E12001 & PNNL-AGG-ICP-AES \\
\hline 7439-98-7 & Molybdenum & $<19.4$ & ug/g dry & 19.4 & $5 / 22 / 08$ & 8E12001 & PNNL-AGG-ICP-AES \\
\hline $7440-02-0$ & Nickel & 10.2 & ug/g dry & 7.02 & $5 / 22 / 08$ & 8E12001 & PNNL-AGG-ICP-AES \\
\hline 7723-14-0 & Phosphorus & 411 & ug/g dry & 64.3 & $5 / 22 / 08$ & 8E12001 & PNNL-AGG-ICP-AES \\
\hline 7439-92-1 & Lead & $<27.2$ & ug/g dry & 27.2 & $5 / 22 / 08$ & 8E12001 & PNNL-AGG-ICP-AES \\
\hline $7782-49-2$ & Selenium & $<119$ & ug/g dry & 119 & $5 / 22 / 08$ & 8E12001 & PNNL-AGG-ICP-AES \\
\hline $7440-24-6$ & Strontium & 28.0 & ug/g dry & 1.24 & $5 / 22 / 08$ & 8E12001 & PNNL-AGG-ICP-AES \\
\hline $7440-28-0$ & Thallium & $<38.6$ & ug/g dry & 38.6 & $5 / 22 / 08$ & 8E12001 & PNNL-AGG-ICP-AES \\
\hline $7440-62-2$ & Vanadium & 10.8 & ug/g dry & 3.18 & $5 / 22 / 08$ & 8E12001 & PNNL-AGG-ICP-AES \\
\hline $7440-66-6$ & Zinc & 24.6 & ug/g dry & 5.35 & $5 / 22 / 08$ & 8E12001 & PNNL-AGG-ICP-AES \\
\hline $7440-23-5$ & Sodium & $<915$ & ug/g dry & 915 & $5 / 22 / 08$ & 8E12001 & PNNL-AGG-ICP-AES \\
\hline $7440-21-3$ & Silicon & $<1190$ & ug/g dry & 1190 & $5 / 22 / 08$ & 8E12001 & PNNL-AGG-ICP-AES \\
\hline $7704-34-9$ & Sulfur & $<202$ & ug/g dry & 202 & $5 / 22 / 08$ & 8E12001 & PNNL-AGG-ICP-AES \\
\hline $7440-32-6$ & Titanium & 433 & ug/g dry & 26.9 & $5 / 22 / 08$ & 8E12001 & PNNL-AGG-ICP-AES \\
\hline $7440-67-7$ & Zirconium & $<17.4$ & ug/g dry & 17.4 & $5 / 22 / 08$ & 8E12001 & PNNL-AGG-ICP-AES \\
\hline $7440-15-5$ & Rhenium & $<15.1$ & ug/g dry & 15.1 & $5 / 22 / 08$ & 8E12001 & PNNL-AGG-ICP-AES \\
\hline $7440-36-0$ & Antimony & $<95.6$ & ug/g dry & 95.6 & $5 / 22 / 08$ & 8E12001 & PNNL-AGG-ICP-AES \\
\hline HEIS No. & B1T2P4A & \multicolumn{3}{|c|}{ Lab ID: $\quad$ 0803014-17 } & & & \\
\hline $7429-90-5$ & Aluminum & 4540 & ug/g dry & 33.8 & $5 / 22 / 08$ & 8E12001 & PNNL-AGG-ICP-AES \\
\hline $7440-38-2$ & Arsenic & $<82.0$ & ug/g dry & 82.0 & $5 / 22 / 08$ & 8E12001 & PNNL-AGG-ICP-AES \\
\hline $7440-42-8$ & Boron & $<714$ & ug/g dry & 714 & $5 / 22 / 08$ & 8E12001 & PNNL-AGG-ICP-AES \\
\hline $7440-39-3$ & Barium & 43.8 & ug/g dry & 3.21 & $5 / 22 / 08$ & 8E12001 & PNNL-AGG-ICP-AES \\
\hline $7440-41-7$ & Beryllium & $<1.27$ & ug/g dry & 1.27 & $5 / 22 / 08$ & 8E12001 & PNNL-AGG-ICP-AES \\
\hline $7440-69-9$ & Bismuth & $<17.0$ & ug/g dry & 17.0 & $5 / 22 / 08$ & 8E12001 & PNNL-AGG-ICP-AES \\
\hline $7440-70-2$ & Calcium & 7470 & ug/g dry & 125 & $5 / 22 / 08$ & 8E12001 & PNNL-AGG-ICP-AES \\
\hline $7440-43-9$ & Cadmium & $<1.92$ & ug/g dry & 1.92 & $5 / 22 / 08$ & 8E12001 & PNNL-AGG-ICP-AES \\
\hline $7440-48-4$ & Cobalt & $<7.00$ & ug/g dry & 7.00 & $5 / 22 / 08$ & 8E12001 & PNNL-AGG-ICP-AES \\
\hline
\end{tabular}


Total Metals by SW846 6010B/Acid Extract

\begin{tabular}{|c|c|c|c|c|c|c|c|}
\hline CAS \# & Analyte & Results & Units & EQL & Analyzed & Batch & Method \\
\hline HEIS No. & В1T2P4A & \multicolumn{3}{|c|}{ Lab ID: $\quad$ 0803014-17 } & & & \\
\hline $7440-47-3$ & Chromium & 11.3 & ug/g dry & 2.80 & $5 / 22 / 08$ & 8E12001 & PNNL-AGG-ICP-AES \\
\hline $7440-50-8$ & Copper & $<29.9$ & ug/g dry & 29.9 & $5 / 22 / 08$ & 8E12001 & PNNL-AGG-ICP-AES \\
\hline 7439-89-6 & Iron & 9600 & ug/g dry & 85.7 & $5 / 22 / 08$ & 8E12001 & PNNL-AGG-ICP-AES \\
\hline 7440-09-7 & Potassium & 884 & ug/g dry & 79.6 & $5 / 22 / 08$ & 8E12001 & PNNL-AGG-ICP-AES \\
\hline $7439-93-2$ & Lithium & $<12.2$ & ug/g dry & 12.2 & $5 / 22 / 08$ & 8E12001 & PNNL-AGG-ICP-AES \\
\hline 7439-95-4 & Magnesium & 3550 & ug/g dry & 26.5 & $5 / 22 / 08$ & 8E12001 & PNNL-AGG-ICP-AES \\
\hline $7439-96-5$ & Manganese & 211 & ug/g dry & 1.00 & $5 / 22 / 08$ & 8E12001 & PNNL-AGG-ICP-AES \\
\hline 7439-98-7 & Molybdenum & $<19.0$ & ug/g dry & 19.0 & $5 / 22 / 08$ & 8E12001 & PNNL-AGG-ICP-AES \\
\hline 7440-02-0 & Nickel & 9.88 & ug/g dry & 6.90 & $5 / 22 / 08$ & 8E12001 & PNNL-AGG-ICP-AES \\
\hline $7723-14-0$ & Phosphorus & 368 & ug/g dry & 63.2 & $5 / 22 / 08$ & 8E12001 & PNNL-AGG-ICP-AES \\
\hline 7439-92-1 & Lead & $<26.7$ & ug/g dry & 26.7 & $5 / 22 / 08$ & 8E12001 & PNNL-AGG-ICP-AES \\
\hline $7782-49-2$ & Selenium & $<117$ & ug/g dry & 117 & $5 / 22 / 08$ & 8E12001 & PNNL-AGG-ICP-AES \\
\hline $7440-24-6$ & Strontium & 23.5 & ug/g dry & 1.22 & $5 / 22 / 08$ & 8E12001 & PNNL-AGG-ICP-AES \\
\hline $7440-28-0$ & Thallium & $<37.9$ & ug/g dry & 37.9 & $5 / 22 / 08$ & 8E12001 & PNNL-AGG-ICP-AES \\
\hline $7440-62-2$ & Vanadium & 10.7 & ug/g dry & 3.12 & $5 / 22 / 08$ & 8E12001 & PNNL-AGG-ICP-AES \\
\hline $7440-66-6$ & Zinc & 24.3 & ug/g dry & 5.26 & $5 / 22 / 08$ & 8E12001 & PNNL-AGG-ICP-AES \\
\hline $7440-23-5$ & Sodium & $<899$ & ug/g dry & 899 & $5 / 22 / 08$ & 8E12001 & PNNL-AGG-ICP-AES \\
\hline $7440-21-3$ & Silicon & $<1170$ & ug/g dry & 1170 & $5 / 22 / 08$ & 8E12001 & PNNL-AGG-ICP-AES \\
\hline $7704-34-9$ & Sulfur & $<199$ & ug/g dry & 199 & $5 / 22 / 08$ & 8E12001 & PNNL-AGG-ICP-AES \\
\hline $7440-32-6$ & Titanium & 390 & ug/g dry & 26.5 & $5 / 22 / 08$ & 8E12001 & PNNL-AGG-ICP-AES \\
\hline $7440-67-7$ & Zirconium & $<17.1$ & ug/g dry & 17.1 & $5 / 22 / 08$ & 8E12001 & PNNL-AGG-ICP-AES \\
\hline $7440-15-5$ & Rhenium & $<14.8$ & ug/g dry & 14.8 & $5 / 22 / 08$ & 8E12001 & PNNL-AGG-ICP-AES \\
\hline $7440-36-0$ & Antimony & $<93.9$ & ug/g dry & 93.9 & $5 / 22 / 08$ & 8E12001 & PNNL-AGG-ICP-AES \\
\hline HEIS No. & B1T2P5B & \multicolumn{3}{|c|}{ Lab ID: $\quad$ 0803014-20 } & & & \\
\hline $7429-90-5$ & Aluminum & 4880 & ug/g dry & 36.7 & $5 / 22 / 08$ & 8E12001 & PNNL-AGG-ICP-AES \\
\hline $7440-38-2$ & Arsenic & $<89.1$ & ug/g dry & 89.1 & $5 / 22 / 08$ & 8E12001 & PNNL-AGG-ICP-AES \\
\hline $7440-42-8$ & Boron & $<776$ & ug/g dry & 776 & $5 / 22 / 08$ & 8E12001 & PNNL-AGG-ICP-AES \\
\hline $7440-39-3$ & Barium & 53.9 & ug/g dry & 3.49 & $5 / 22 / 08$ & 8E12001 & PNNL-AGG-ICP-AES \\
\hline $7440-41-7$ & Beryllium & $<1.38$ & ug/g dry & 1.38 & $5 / 22 / 08$ & 8E12001 & PNNL-AGG-ICP-AES \\
\hline $7440-69-9$ & Bismuth & $<18.5$ & ug/g dry & 18.5 & $5 / 22 / 08$ & 8E12001 & PNNL-AGG-ICP-AES \\
\hline $7440-70-2$ & Calcium & 7750 & ug/g dry & 136 & $5 / 22 / 08$ & 8E12001 & PNNL-AGG-ICP-AES \\
\hline $7440-43-9$ & Cadmium & $<2.08$ & ug/g dry & 2.08 & $5 / 22 / 08$ & 8E12001 & PNNL-AGG-ICP-AES \\
\hline $7440-48-4$ & Cobalt & $<7.61$ & ug/g dry & 7.61 & $5 / 22 / 08$ & 8E12001 & PNNL-AGG-ICP-AES \\
\hline $7440-47-3$ & Chromium & 13.6 & ug/g dry & 3.05 & $5 / 22 / 08$ & 8E12001 & PNNL-AGG-ICP-AES \\
\hline $7440-50-8$ & Copper & $<32.5$ & ug/g dry & 32.5 & $5 / 22 / 08$ & 8E12001 & PNNL-AGG-ICP-AES \\
\hline 7439-89-6 & Iron & 9870 & ug/g dry & 93.1 & $5 / 22 / 08$ & 8E12001 & PNNL-AGG-ICP-AES \\
\hline 7440-09-7 & Potassium & 1140 & ug/g dry & 86.5 & $5 / 22 / 08$ & 8E12001 & PNNL-AGG-ICP-AES \\
\hline $7439-93-2$ & Lithium & $<13.2$ & ug/g dry & 13.2 & $5 / 22 / 08$ & 8E12001 & PNNL-AGG-ICP-AES \\
\hline $7439-95-4$ & Magnesium & 3710 & ug/g dry & 28.8 & $5 / 22 / 08$ & 8E12001 & PNNL-AGG-ICP-AES \\
\hline $7439-96-5$ & Manganese & 235 & ug/g dry & 1.09 & $5 / 22 / 08$ & 8E12001 & PNNL-AGG-ICP-AES \\
\hline 7439-98-7 & Molybdenum & $<20.7$ & ug/g dry & 20.7 & $5 / 22 / 08$ & 8E12001 & PNNL-AGG-ICP-AES \\
\hline $7440-02-0$ & Nickel & 11.1 & ug/g dry & 7.50 & $5 / 22 / 08$ & 8E12001 & PNNL-AGG-ICP-AES \\
\hline $7723-14-0$ & Phosphorus & 335 & ug/g dry & 68.7 & $5 / 22 / 08$ & 8E12001 & PNNL-AGG-ICP-AES \\
\hline $7439-92-1$ & Lead & $<29.0$ & ug/g dry & 29.0 & $5 / 22 / 08$ & 8E12001 & PNNL-AGG-ICP-AES \\
\hline 7782-49-2 & Selenium & $<127$ & ug/g dry & 127 & $5 / 22 / 08$ & 8E12001 & PNNL-AGG-ICP-AES \\
\hline $7440-24-6$ & Strontium & 27.5 & ug/g dry & 1.32 & $5 / 22 / 08$ & 8E12001 & PNNL-AGG-ICP-AES \\
\hline $7440-28-0$ & Thallium & $<41.2$ & ug/g dry & 41.2 & $5 / 22 / 08$ & 8E12001 & PNNL-AGG-ICP-AES \\
\hline $7440-62-2$ & Vanadium & 10.6 & ug/g dry & 3.39 & $5 / 22 / 08$ & 8E12001 & PNNL-AGG-ICP-AES \\
\hline
\end{tabular}


Total Metals by SW846 6010B/Acid Extract

\begin{tabular}{|c|c|c|c|c|c|c|c|}
\hline CAS \# & Analyte & Results & Units & EQL & Analyzed & Batch & Method \\
\hline HEIS No. & B1T2P5B & \multicolumn{3}{|c|}{ Lab ID: $\quad$ 0803014-20 } & & & \\
\hline $7440-66-6$ & Zinc & 29.6 & ug/g dry & 5.71 & $5 / 22 / 08$ & 8E12001 & PNNL-AGG-ICP-AES \\
\hline $7440-23-5$ & Sodium & $<977$ & ug/g dry & 977 & $5 / 22 / 08$ & 8E12001 & PNNL-AGG-ICP-AES \\
\hline $7440-21-3$ & Silicon & $<1270$ & ug/g dry & 1270 & $5 / 22 / 08$ & 8E12001 & PNNL-AGG-ICP-AES \\
\hline 7704-34-9 & Sulfur & $<216$ & ug/g dry & 216 & $5 / 22 / 08$ & 8E12001 & PNNL-AGG-ICP-AES \\
\hline 7440-32-6 & Titanium & 392 & ug/g dry & 28.8 & $5 / 22 / 08$ & 8E12001 & PNNL-AGG-ICP-AES \\
\hline $7440-67-7$ & Zirconium & $<18.6$ & ug/g dry & 18.6 & $5 / 22 / 08$ & 8E12001 & PNNL-AGG-ICP-AES \\
\hline $7440-15-5$ & Rhenium & $<16.1$ & ug/g dry & 16.1 & $5 / 22 / 08$ & 8E12001 & PNNL-AGG-ICP-AES \\
\hline $7440-36-0$ & Antimony & $<102$ & ug/g dry & 102 & $5 / 22 / 08$ & 8E12001 & PNNL-AGG-ICP-AES \\
\hline HEIS No. & B1T2P5A & \multicolumn{3}{|c|}{ Lab ID: $\quad$ 0803014-21 } & & & \\
\hline $7429-90-5$ & Aluminum & 5340 & ug/g dry & 38.1 & $5 / 22 / 08$ & 8E12001 & PNNL-AGG-ICP-AES \\
\hline $7440-38-2$ & Arsenic & $<92.5$ & ug/g dry & 92.5 & $5 / 22 / 08$ & 8E12001 & PNNL-AGG-ICP-AES \\
\hline $7440-42-8$ & Boron & $<805$ & ug/g dry & 805 & $5 / 22 / 08$ & 8E12001 & PNNL-AGG-ICP-AES \\
\hline $7440-39-3$ & Barium & 52.8 & ug/g dry & 3.63 & $5 / 22 / 08$ & 8E12001 & PNNL-AGG-ICP-AES \\
\hline $7440-41-7$ & Beryllium & $<1.44$ & ug/g dry & 1.44 & $5 / 22 / 08$ & 8E12001 & PNNL-AGG-ICP-AES \\
\hline $7440-69-9$ & Bismuth & $<19.2$ & ug/g dry & 19.2 & $5 / 22 / 08$ & 8E12001 & PNNL-AGG-ICP-AES \\
\hline $7440-70-2$ & Calcium & 8070 & ug/g dry & 141 & $5 / 22 / 08$ & 8E12001 & PNNL-AGG-ICP-AES \\
\hline 7440-43-9 & Cadmium & $<2.16$ & ug/g dry & 2.16 & $5 / 22 / 08$ & 8E12001 & PNNL-AGG-ICP-AES \\
\hline $7440-48-4$ & Cobalt & $<7.90$ & ug/g dry & 7.90 & $5 / 22 / 08$ & 8E12001 & PNNL-AGG-ICP-AES \\
\hline $7440-47-3$ & Chromium & 8.20 & ug/g dry & 3.16 & $5 / 22 / 08$ & 8E12001 & PNNL-AGG-ICP-AES \\
\hline $7440-50-8$ & Copper & $<33.7$ & ug/g dry & 33.7 & $5 / 22 / 08$ & 8E12001 & PNNL-AGG-ICP-AES \\
\hline 7439-89-6 & Iron & 10700 & ug/g dry & 96.7 & $5 / 22 / 08$ & 8E12001 & PNNL-AGG-ICP-AES \\
\hline $7440-09-7$ & Potassium & 1110 & ug/g dry & 89.8 & $5 / 22 / 08$ & 8E12001 & PNNL-AGG-ICP-AES \\
\hline 7439-93-2 & Lithium & $<13.7$ & ug/g dry & 13.7 & $5 / 22 / 08$ & 8E12001 & PNNL-AGG-ICP-AES \\
\hline $7439-95-4$ & Magnesium & 3940 & ug/g dry & 29.9 & $5 / 22 / 08$ & 8E12001 & PNNL-AGG-ICP-AES \\
\hline 7439-96-5 & Manganese & 255 & ug/g dry & 1.13 & $5 / 22 / 08$ & 8E12001 & PNNL-AGG-ICP-AES \\
\hline 7439-98-7 & Molybdenum & $<21.5$ & ug/g dry & 21.5 & $5 / 22 / 08$ & 8E12001 & PNNL-AGG-ICP-AES \\
\hline $7440-02-0$ & Nickel & 10.1 & ug/g dry & 7.78 & $5 / 22 / 08$ & 8E12001 & PNNL-AGG-ICP-AES \\
\hline 7723-14-0 & Phosphorus & 409 & ug/g dry & 71.3 & $5 / 22 / 08$ & 8E12001 & PNNL-AGG-ICP-AES \\
\hline 7439-92-1 & Lead & $<30.1$ & ug/g dry & 30.1 & $5 / 22 / 08$ & 8E12001 & PNNL-AGG-ICP-AES \\
\hline $7782-49-2$ & Selenium & $<132$ & ug/g dry & 132 & $5 / 22 / 08$ & 8E12001 & PNNL-AGG-ICP-AES \\
\hline $7440-24-6$ & Strontium & 25.8 & ug/g dry & 1.37 & $5 / 22 / 08$ & 8E12001 & PNNL-AGG-ICP-AES \\
\hline $7440-28-0$ & Thallium & $<42.8$ & ug/g dry & 42.8 & $5 / 22 / 08$ & 8E12001 & PNNL-AGG-ICP-AES \\
\hline $7440-62-2$ & Vanadium & 12.5 & ug/g dry & 3.52 & $5 / 22 / 08$ & 8E12001 & PNNL-AGG-ICP-AES \\
\hline $7440-66-6$ & Zinc & 30.3 & ug/g dry & 5.93 & $5 / 22 / 08$ & 8E12001 & PNNL-AGG-ICP-AES \\
\hline $7440-23-5$ & Sodium & $<1010$ & ug/g dry & 1010 & $5 / 22 / 08$ & 8E12001 & PNNL-AGG-ICP-AES \\
\hline $7440-21-3$ & Silicon & $<1320$ & ug/g dry & 1320 & $5 / 22 / 08$ & 8E12001 & PNNL-AGG-ICP-AES \\
\hline $7704-34-9$ & Sulfur & $<224$ & ug/g dry & 224 & $5 / 22 / 08$ & 8E12001 & PNNL-AGG-ICP-AES \\
\hline $7440-32-6$ & Titanium & 437 & ug/g dry & 29.9 & $5 / 22 / 08$ & 8E12001 & PNNL-AGG-ICP-AES \\
\hline $7440-67-7$ & Zirconium & $<19.3$ & ug/g dry & 19.3 & $5 / 22 / 08$ & 8E12001 & PNNL-AGG-ICP-AES \\
\hline $7440-15-5$ & Rhenium & $<16.7$ & ug/g dry & 16.7 & $5 / 22 / 08$ & 8E12001 & PNNL-AGG-ICP-AES \\
\hline $7440-36-0$ & Antimony & $<106$ & ug/g dry & 106 & $5 / 22 / 08$ & 8E12001 & PNNL-AGG-ICP-AES \\
\hline HEIS No. & B1T2P6B & \multicolumn{3}{|c|}{ Lab ID: $\quad$ 0803014-24 } & & & \\
\hline $7429-90-5$ & Aluminum & 5260 & ug/g dry & 36.6 & $5 / 22 / 08$ & 8E12001 & PNNL-AGG-ICP-AES \\
\hline $7440-38-2$ & Arsenic & $<89.0$ & ug/g dry & 89.0 & $5 / 22 / 08$ & 8E12001 & PNNL-AGG-ICP-AES \\
\hline $7440-42-8$ & Boron & $<775$ & ug/g dry & 775 & $5 / 22 / 08$ & 8E12001 & PNNL-AGG-ICP-AES \\
\hline $7440-39-3$ & Barium & 45.5 & ug/g dry & 3.49 & $5 / 22 / 08$ & 8E12001 & PNNL-AGG-ICP-AES \\
\hline $7440-41-7$ & Beryllium & $<1.38$ & ug/g dry & 1.38 & $5 / 22 / 08$ & 8E12001 & PNNL-AGG-ICP-AES \\
\hline $7440-69-9$ & Bismuth & $<18.5$ & ug/g dry & 18.5 & $5 / 22 / 08$ & 8E12001 & PNNL-AGG-ICP-AES \\
\hline
\end{tabular}


Total Metals by SW846 6010B/Acid Extract

\begin{tabular}{|c|c|c|c|c|c|c|c|}
\hline CAS \# & Analyte & Results & Units & EQL & Analyzed & Batch & Method \\
\hline HEIS No. & B1T2P6B & \multicolumn{3}{|c|}{ Lab ID: $\quad$ 0803014-24 } & & & \\
\hline $7440-70-2$ & Calcium & 10400 & ug/g dry & 136 & $5 / 22 / 08$ & 8E12001 & PNNL-AGG-ICP-AES \\
\hline 7440-43-9 & Cadmium & $<2.08$ & ug/g dry & 2.08 & $5 / 22 / 08$ & 8E12001 & PNNL-AGG-ICP-AES \\
\hline $7440-48-4$ & Cobalt & $<7.60$ & ug/g dry & 7.60 & $5 / 22 / 08$ & 8E12001 & PNNL-AGG-ICP-AES \\
\hline $7440-47-3$ & Chromium & 19.5 & ug/g dry & 3.05 & $5 / 22 / 08$ & 8E12001 & PNNL-AGG-ICP-AES \\
\hline $7440-50-8$ & Copper & $<32.5$ & ug/g dry & 32.5 & $5 / 22 / 08$ & 8E12001 & PNNL-AGG-ICP-AES \\
\hline 7439-89-6 & Iron & 12600 & ug/g dry & 93.1 & $5 / 22 / 08$ & 8E12001 & PNNL-AGG-ICP-AES \\
\hline 7440-09-7 & Potassium & 877 & ug/g dry & 86.4 & $5 / 22 / 08$ & 8E12001 & PNNL-AGG-ICP-AES \\
\hline $7439-93-2$ & Lithium & $<13.2$ & ug/g dry & 13.2 & $5 / 22 / 08$ & 8E12001 & PNNL-AGG-ICP-AES \\
\hline 7439-95-4 & Magnesium & 4290 & ug/g dry & 28.8 & $5 / 22 / 08$ & 8E12001 & PNNL-AGG-ICP-AES \\
\hline 7439-96-5 & Manganese & 222 & ug/g dry & 1.09 & $5 / 22 / 08$ & 8E12001 & PNNL-AGG-ICP-AES \\
\hline 7439-98-7 & Molybdenum & $<20.7$ & ug/g dry & 20.7 & $5 / 22 / 08$ & 8E12001 & PNNL-AGG-ICP-AES \\
\hline $7440-02-0$ & Nickel & 17.6 & ug/g dry & 7.49 & $5 / 22 / 08$ & 8E12001 & PNNL-AGG-ICP-AES \\
\hline 7723-14-0 & Phosphorus & 548 & ug/g dry & 68.6 & $5 / 22 / 08$ & 8E12001 & PNNL-AGG-ICP-AES \\
\hline 7439-92-1 & Lead & $<29.0$ & ug/g dry & 29.0 & $5 / 22 / 08$ & 8E12001 & PNNL-AGG-ICP-AES \\
\hline $7782-49-2$ & Selenium & $<127$ & ug/g dry & 127 & $5 / 22 / 08$ & 8E12001 & PNNL-AGG-ICP-AES \\
\hline $7440-24-6$ & Strontium & 23.1 & ug/g dry & 1.32 & $5 / 22 / 08$ & 8E12001 & PNNL-AGG-ICP-AES \\
\hline $7440-28-0$ & Thallium & $<41.2$ & ug/g dry & 41.2 & $5 / 22 / 08$ & 8E12001 & PNNL-AGG-ICP-AES \\
\hline $7440-62-2$ & Vanadium & 15.3 & ug/g dry & 3.39 & $5 / 22 / 08$ & 8E12001 & PNNL-AGG-ICP-AES \\
\hline $7440-66-6$ & Zinc & 28.1 & ug/g dry & 5.71 & $5 / 22 / 08$ & 8E12001 & PNNL-AGG-ICP-AES \\
\hline $7440-23-5$ & Sodium & $<976$ & ug/g dry & 976 & $5 / 22 / 08$ & 8E12001 & PNNL-AGG-ICP-AES \\
\hline $7440-21-3$ & Silicon & $<1270$ & ug/g dry & 1270 & $5 / 22 / 08$ & 8E12001 & PNNL-AGG-ICP-AES \\
\hline 7704-34-9 & Sulfur & $<216$ & ug/g dry & 216 & $5 / 22 / 08$ & 8E12001 & PNNL-AGG-ICP-AES \\
\hline $7440-32-6$ & Titanium & 572 & ug/g dry & 28.7 & $5 / 22 / 08$ & 8E12001 & PNNL-AGG-ICP-AES \\
\hline $7440-67-7$ & Zirconium & $<18.5$ & ug/g dry & 18.5 & $5 / 22 / 08$ & 8E12001 & PNNL-AGG-ICP-AES \\
\hline $7440-15-5$ & Rhenium & $<16.1$ & ug/g dry & 16.1 & $5 / 22 / 08$ & 8E12001 & PNNL-AGG-ICP-AES \\
\hline $7440-36-0$ & Antimony & $<102$ & ug/g dry & 102 & $5 / 22 / 08$ & 8E12001 & PNNL-AGG-ICP-AES \\
\hline HEIS No. & B1T2P6A & \multicolumn{3}{|c|}{ Lab ID: $\quad$ 0803014-25 } & & & \\
\hline $7429-90-5$ & Aluminum & 5160 & ug/g dry & 41.4 & $5 / 22 / 08$ & 8E12001 & PNNL-AGG-ICP-AES \\
\hline $7440-38-2$ & Arsenic & $<101$ & ug/g dry & 101 & $5 / 22 / 08$ & 8E12001 & PNNL-AGG-ICP-AES \\
\hline $7440-42-8$ & Boron & $<876$ & ug/g dry & 876 & $5 / 22 / 08$ & 8E12001 & PNNL-AGG-ICP-AES \\
\hline $7440-39-3$ & Barium & 51.4 & ug/g dry & 3.94 & $5 / 22 / 08$ & 8E12001 & PNNL-AGG-ICP-AES \\
\hline $7440-41-7$ & Beryllium & $<1.56$ & ug/g dry & 1.56 & $5 / 22 / 08$ & 8E12001 & PNNL-AGG-ICP-AES \\
\hline $7440-69-9$ & Bismuth & $<20.9$ & ug/g dry & 20.9 & $5 / 22 / 08$ & 8E12001 & PNNL-AGG-ICP-AES \\
\hline $7440-70-2$ & Calcium & 7490 & ug/g dry & 154 & $5 / 22 / 08$ & 8E12001 & PNNL-AGG-ICP-AES \\
\hline $7440-43-9$ & Cadmium & $<2.35$ & ug/g dry & 2.35 & $5 / 22 / 08$ & 8E12001 & PNNL-AGG-ICP-AES \\
\hline $7440-48-4$ & Cobalt & $<8.59$ & ug/g dry & 8.59 & $5 / 22 / 08$ & 8E12001 & PNNL-AGG-ICP-AES \\
\hline $7440-47-3$ & Chromium & 16.8 & ug/g dry & 3.44 & $5 / 22 / 08$ & 8E12001 & PNNL-AGG-ICP-AES \\
\hline $7440-50-8$ & Copper & $<36.7$ & ug/g dry & 36.7 & $5 / 22 / 08$ & 8E12001 & PNNL-AGG-ICP-AES \\
\hline 7439-89-6 & Iron & 11900 & ug/g dry & 105 & $5 / 22 / 08$ & 8E12001 & PNNL-AGG-ICP-AES \\
\hline 7440-09-7 & Potassium & 867 & ug/g dry & 97.6 & $5 / 22 / 08$ & 8E12001 & PNNL-AGG-ICP-AES \\
\hline $7439-93-2$ & Lithium & $<14.9$ & ug/g dry & 14.9 & $5 / 22 / 08$ & 8E12001 & PNNL-AGG-ICP-AES \\
\hline 7439-95-4 & Magnesium & 3950 & ug/g dry & 32.6 & $5 / 22 / 08$ & 8E12001 & PNNL-AGG-ICP-AES \\
\hline $7439-96-5$ & Manganese & 236 & ug/g dry & 1.23 & $5 / 22 / 08$ & 8E12001 & PNNL-AGG-ICP-AES \\
\hline 7439-98-7 & Molybdenum & $<23.3$ & ug/g dry & 23.3 & $5 / 22 / 08$ & 8E12001 & PNNL-AGG-ICP-AES \\
\hline $7440-02-0$ & Nickel & 15.1 & ug/g dry & 8.47 & $5 / 22 / 08$ & 8E12001 & PNNL-AGG-ICP-AES \\
\hline $7723-14-0$ & Phosphorus & 487 & ug/g dry & 77.5 & $5 / 22 / 08$ & 8E12001 & PNNL-AGG-ICP-AES \\
\hline 7439-92-1 & Lead & $<32.7$ & ug/g dry & 32.7 & $5 / 22 / 08$ & 8E12001 & PNNL-AGG-ICP-AES \\
\hline $7782-49-2$ & Selenium & $<144$ & ug/g dry & 144 & $5 / 22 / 08$ & 8E12001 & PNNL-AGG-ICP-AES \\
\hline
\end{tabular}


Total Metals by SW846 6010B/Acid Extract

\begin{tabular}{|c|c|c|c|c|c|c|c|}
\hline CAS \# & Analyte & Results & Units & EQL & Analyzed & Batch & Method \\
\hline HEIS No. & B1T2P6A & \multicolumn{3}{|c|}{ Lab ID: $\quad$ 0803014-25 } & & & \\
\hline $7440-24-6$ & Strontium & 21.2 & ug/g dry & 1.49 & $5 / 22 / 08$ & 8E12001 & PNNL-AGG-ICP-AES \\
\hline $7440-28-0$ & Thallium & $<46.5$ & ug/g dry & 46.5 & $5 / 22 / 08$ & 8E12001 & PNNL-AGG-ICP-AES \\
\hline $7440-62-2$ & Vanadium & 15.2 & ug/g dry & 3.83 & $5 / 22 / 08$ & 8E12001 & PNNL-AGG-ICP-AES \\
\hline $7440-66-6$ & Zinc & 35.9 & ug/g dry & 6.45 & $5 / 22 / 08$ & 8E12001 & PNNL-AGG-ICP-AES \\
\hline $7440-23-5$ & Sodium & $<1100$ & ug/g dry & 1100 & $5 / 22 / 08$ & 8E12001 & PNNL-AGG-ICP-AES \\
\hline $7440-21-3$ & Silicon & $<1440$ & ug/g dry & 1440 & $5 / 22 / 08$ & 8E12001 & PNNL-AGG-ICP-AES \\
\hline 7704-34-9 & Sulfur & $<244$ & ug/g dry & 244 & $5 / 22 / 08$ & 8E12001 & PNNL-AGG-ICP-AES \\
\hline $7440-32-6$ & Titanium & 516 & ug/g dry & 32.5 & $5 / 22 / 08$ & 8E12001 & PNNL-AGG-ICP-AES \\
\hline $7440-67-7$ & Zirconium & $<21.0$ & ug/g dry & 21.0 & $5 / 22 / 08$ & 8E12001 & PNNL-AGG-ICP-AES \\
\hline $7440-15-5$ & Rhenium & $<18.1$ & ug/g dry & 18.1 & $5 / 22 / 08$ & 8E12001 & PNNL-AGG-ICP-AES \\
\hline $7440-36-0$ & Antimony & $<115$ & ug/g dry & 115 & $5 / 22 / 08$ & 8E12001 & PNNL-AGG-ICP-AES \\
\hline HEIS No. & B1T2P7B & \multicolumn{3}{|c|}{ Lab ID: $\quad$ 0803015-02 } & & & \\
\hline 7429-90-5 & Aluminum & 5400 & ug/g dry & 37.8 & $5 / 22 / 08$ & 8E12001 & PNNL-AGG-ICP-AES \\
\hline $7440-38-2$ & Arsenic & $<91.8$ & ug/g dry & 91.8 & 5/22/08 & 8E12001 & PNNL-AGG-ICP-AES \\
\hline $7440-42-8$ & Boron & $<800$ & ug/g dry & 800 & $5 / 22 / 08$ & 8E12001 & PNNL-AGG-ICP-AES \\
\hline $7440-39-3$ & Barium & 51.0 & ug/g dry & 3.60 & $5 / 22 / 08$ & 8E12001 & PNNL-AGG-ICP-AES \\
\hline 7440-41-7 & Beryllium & $<1.43$ & ug/g dry & 1.43 & $5 / 22 / 08$ & 8E12001 & PNNL-AGG-ICP-AES \\
\hline $7440-69-9$ & Bismuth & $<19.1$ & ug/g dry & 19.1 & $5 / 22 / 08$ & 8E12001 & PNNL-AGG-ICP-AES \\
\hline $7440-70-2$ & Calcium & 7870 & ug/g dry & 140 & $5 / 22 / 08$ & 8E12001 & PNNL-AGG-ICP-AES \\
\hline $7440-43-9$ & Cadmium & $<2.15$ & ug/g dry & 2.15 & $5 / 22 / 08$ & 8E12001 & PNNL-AGG-ICP-AES \\
\hline $7440-48-4$ & Cobalt & $<7.84$ & ug/g dry & 7.84 & $5 / 22 / 08$ & 8E12001 & PNNL-AGG-ICP-AES \\
\hline $7440-47-3$ & Chromium & 7.02 & ug/g dry & 3.14 & $5 / 22 / 08$ & 8E12001 & PNNL-AGG-ICP-AES \\
\hline $7440-50-8$ & Copper & $<33.5$ & ug/g dry & 33.5 & $5 / 22 / 08$ & 8E12001 & PNNL-AGG-ICP-AES \\
\hline 7439-89-6 & Iron & 10400 & ug/g dry & 96.0 & $5 / 22 / 08$ & 8E12001 & PNNL-AGG-ICP-AES \\
\hline 7440-09-7 & Potassium & 1070 & ug/g dry & 89.2 & 5/22/08 & 8E12001 & PNNL-AGG-ICP-AES \\
\hline 7439-93-2 & Lithium & $<13.6$ & ug/g dry & 13.6 & $5 / 22 / 08$ & 8E12001 & PNNL-AGG-ICP-AES \\
\hline 7439-95-4 & Magnesium & 3480 & ug/g dry & 29.7 & $5 / 22 / 08$ & 8E12001 & PNNL-AGG-ICP-AES \\
\hline 7439-96-5 & Manganese & 233 & ug/g dry & 1.12 & $5 / 22 / 08$ & 8E12001 & PNNL-AGG-ICP-AES \\
\hline 7439-98-7 & Molybdenum & $<21.3$ & ug/g dry & 21.3 & $5 / 22 / 08$ & 8E12001 & PNNL-AGG-ICP-AES \\
\hline $7440-02-0$ & Nickel & 7.92 & ug/g dry & 7.73 & $5 / 22 / 08$ & 8E12001 & PNNL-AGG-ICP-AES \\
\hline 7723-14-0 & Phosphorus & 444 & ug/g dry & 70.8 & $5 / 22 / 08$ & 8E12001 & PNNL-AGG-ICP-AES \\
\hline 7439-92-1 & Lead & $<29.9$ & ug/g dry & 29.9 & $5 / 22 / 08$ & 8E12001 & PNNL-AGG-ICP-AES \\
\hline $7782-49-2$ & Selenium & $<131$ & ug/g dry & 131 & $5 / 22 / 08$ & 8E12001 & PNNL-AGG-ICP-AES \\
\hline $7440-24-6$ & Strontium & 22.9 & ug/g dry & 1.36 & $5 / 22 / 08$ & 8E12001 & PNNL-AGG-ICP-AES \\
\hline $7440-28-0$ & Thallium & $<42.5$ & ug/g dry & 42.5 & $5 / 22 / 08$ & 8E12001 & PNNL-AGG-ICP-AES \\
\hline $7440-62-2$ & Vanadium & 12.9 & ug/g dry & 3.50 & $5 / 22 / 08$ & 8E12001 & PNNL-AGG-ICP-AES \\
\hline 7440-66-6 & Zinc & 31.5 & ug/g dry & 5.89 & $5 / 22 / 08$ & 8E12001 & PNNL-AGG-ICP-AES \\
\hline $7440-23-5$ & Sodium & $<1010$ & ug/g dry & 1010 & $5 / 22 / 08$ & 8E12001 & PNNL-AGG-ICP-AES \\
\hline $7440-21-3$ & Silicon & $<1310$ & ug/g dry & 1310 & $5 / 22 / 08$ & 8E12001 & PNNL-AGG-ICP-AES \\
\hline 7704-34-9 & Sulfur & $<223$ & ug/g dry & 223 & 5/22/08 & 8E12001 & PNNL-AGG-ICP-AES \\
\hline 7440-32-6 & Titanium & 400 & ug/g dry & 29.7 & $5 / 22 / 08$ & 8E12001 & PNNL-AGG-ICP-AES \\
\hline $7440-67-7$ & Zirconium & $<19.1$ & ug/g dry & 19.1 & $5 / 22 / 08$ & 8E12001 & PNNL-AGG-ICP-AES \\
\hline $7440-15-5$ & Rhenium & $<16.6$ & ug/g dry & 16.6 & $5 / 22 / 08$ & 8E12001 & PNNL-AGG-ICP-AES \\
\hline $7440-36-0$ & Antimony & $<105$ & ug/g dry & 105 & $5 / 22 / 08$ & 8E12001 & PNNL-AGG-ICP-AES \\
\hline HEIS No. & B1T2P7A & \multicolumn{3}{|c|}{ Lab ID: $\quad$ 0803015-03 } & & & \\
\hline $7429-90-5$ & Aluminum & 5350 & ug/g dry & 36.9 & $5 / 22 / 08$ & 8E12001 & PNNL-AGG-ICP-AES \\
\hline $7440-38-2$ & Arsenic & $<89.6$ & ug/g dry & 89.6 & $5 / 22 / 08$ & 8E12001 & PNNL-AGG-ICP-AES \\
\hline $7440-42-8$ & Boron & $<780$ & ug/g dry & 780 & 5/22/08 & 8E12001 & PNNL-AGG-ICP-AES \\
\hline
\end{tabular}


Total Metals by SW846 6010B/Acid Extract

\begin{tabular}{|c|c|c|c|c|c|c|c|}
\hline CAS \# & Analyte & Results & Units & EQL & Analyzed & Batch & Method \\
\hline HEIS No. & B1T2P7A & \multicolumn{3}{|c|}{ Lab ID: $\quad$ 0803015-03 } & & & \\
\hline $7440-39-3$ & Barium & 56.0 & ug/g dry & 3.51 & $5 / 22 / 08$ & 8E12001 & PNNL-AGG-ICP-AES \\
\hline $7440-41-7$ & Beryllium & $<1.39$ & ug/g dry & 1.39 & $5 / 22 / 08$ & 8E12001 & PNNL-AGG-ICP-AES \\
\hline 7440-69-9 & Bismuth & $<18.6$ & ug/g dry & 18.6 & $5 / 22 / 08$ & 8E12001 & PNNL-AGG-ICP-AES \\
\hline $7440-70-2$ & Calcium & 6220 & ug/g dry & 137 & $5 / 22 / 08$ & 8E12001 & PNNL-AGG-ICP-AES \\
\hline $7440-43-9$ & Cadmium & $<2.10$ & ug/g dry & 2.10 & $5 / 22 / 08$ & 8E12001 & PNNL-AGG-ICP-AES \\
\hline $7440-48-4$ & Cobalt & $<7.66$ & ug/g dry & 7.66 & $5 / 22 / 08$ & 8E12001 & PNNL-AGG-ICP-AES \\
\hline $7440-47-3$ & Chromium & 8.13 & ug/g dry & 3.07 & $5 / 22 / 08$ & 8E12001 & PNNL-AGG-ICP-AES \\
\hline $7440-50-8$ & Copper & $<32.7$ & ug/g dry & 32.7 & $5 / 22 / 08$ & 8E12001 & PNNL-AGG-ICP-AES \\
\hline 7439-89-6 & Iron & 14600 & ug/g dry & 93.7 & $5 / 22 / 08$ & 8E12001 & PNNL-AGG-ICP-AES \\
\hline 7440-09-7 & Potassium & 718 & ug/g dry & 87.0 & $5 / 22 / 08$ & 8E12001 & PNNL-AGG-ICP-AES \\
\hline 7439-93-2 & Lithium & $<13.3$ & ug/g dry & 13.3 & $5 / 22 / 08$ & 8E12001 & PNNL-AGG-ICP-AES \\
\hline 7439-95-4 & Magnesium & 3950 & ug/g dry & 29.0 & $5 / 22 / 08$ & 8E12001 & PNNL-AGG-ICP-AES \\
\hline $7439-96-5$ & Manganese & 256 & ug/g dry & 1.10 & $5 / 22 / 08$ & 8E12001 & PNNL-AGG-ICP-AES \\
\hline 7439-98-7 & Molybdenum & $<20.8$ & ug/g dry & 20.8 & $5 / 22 / 08$ & 8E12001 & PNNL-AGG-ICP-AES \\
\hline 7440-02-0 & Nickel & 11.8 & ug/g dry & 7.54 & $5 / 22 / 08$ & 8E12001 & PNNL-AGG-ICP-AES \\
\hline $7723-14-0$ & Phosphorus & 715 & ug/g dry & 69.1 & $5 / 22 / 08$ & 8E12001 & PNNL-AGG-ICP-AES \\
\hline 7439-92-1 & Lead & $<29.2$ & ug/g dry & 29.2 & $5 / 22 / 08$ & 8E12001 & PNNL-AGG-ICP-AES \\
\hline $7782-49-2$ & Selenium & $<128$ & ug/g dry & 128 & $5 / 22 / 08$ & 8E12001 & PNNL-AGG-ICP-AES \\
\hline $7440-24-6$ & Strontium & 22.4 & ug/g dry & 1.33 & $5 / 22 / 08$ & 8E12001 & PNNL-AGG-ICP-AES \\
\hline $7440-28-0$ & Thallium & $<41.5$ & ug/g dry & 41.5 & $5 / 22 / 08$ & 8E12001 & PNNL-AGG-ICP-AES \\
\hline $7440-62-2$ & Vanadium & 21.0 & ug/g dry & 3.41 & $5 / 22 / 08$ & 8E12001 & PNNL-AGG-ICP-AES \\
\hline $7440-66-6$ & Zinc & 27.8 & ug/g dry & 5.75 & $5 / 22 / 08$ & 8E12001 & PNNL-AGG-ICP-AES \\
\hline $7440-23-5$ & Sodium & $<983$ & ug/g dry & 983 & $5 / 22 / 08$ & 8E12001 & PNNL-AGG-ICP-AES \\
\hline $7440-21-3$ & Silicon & $<1280$ & ug/g dry & 1280 & $5 / 22 / 08$ & 8E12001 & PNNL-AGG-ICP-AES \\
\hline $7704-34-9$ & Sulfur & $<217$ & ug/g dry & 217 & $5 / 22 / 08$ & 8E12001 & PNNL-AGG-ICP-AES \\
\hline $7440-32-6$ & Titanium & 710 & ug/g dry & 28.9 & $5 / 22 / 08$ & 8E12001 & PNNL-AGG-ICP-AES \\
\hline $7440-67-7$ & Zirconium & $<18.7$ & ug/g dry & 18.7 & $5 / 22 / 08$ & 8E12001 & PNNL-AGG-ICP-AES \\
\hline $7440-15-5$ & Rhenium & $<16.2$ & ug/g dry & 16.2 & $5 / 22 / 08$ & 8E12001 & PNNL-AGG-ICP-AES \\
\hline $7440-36-0$ & Antimony & $<103$ & ug/g dry & 103 & $5 / 22 / 08$ & 8E12001 & PNNL-AGG-ICP-AES \\
\hline HEIS No. & B1T2P8B & \multicolumn{3}{|c|}{ Lab ID: $\quad$ 0803015-06 } & & & \\
\hline 7429-90-5 & Aluminum & 4740 & ug/g dry & 32.5 & $5 / 22 / 08$ & 8E12001 & PNNL-AGG-ICP-AES \\
\hline $7440-38-2$ & Arsenic & $<79.0$ & ug/g dry & 79.0 & $5 / 22 / 08$ & 8E12001 & PNNL-AGG-ICP-AES \\
\hline $7440-42-8$ & Boron & $<688$ & ug/g dry & 688 & $5 / 22 / 08$ & 8E12001 & PNNL-AGG-ICP-AES \\
\hline 7440-39-3 & Barium & 47.2 & ug/g dry & 3.10 & $5 / 22 / 08$ & 8E12001 & PNNL-AGG-ICP-AES \\
\hline $7440-41-7$ & Beryllium & $<1.23$ & ug/g dry & 1.23 & $5 / 22 / 08$ & 8E12001 & PNNL-AGG-ICP-AES \\
\hline $7440-69-9$ & Bismuth & $<16.4$ & ug/g dry & 16.4 & $5 / 22 / 08$ & 8E12001 & PNNL-AGG-ICP-AES \\
\hline $7440-70-2$ & Calcium & 7030 & ug/g dry & 121 & $5 / 22 / 08$ & 8E12001 & PNNL-AGG-ICP-AES \\
\hline $7440-43-9$ & Cadmium & $<1.85$ & ug/g dry & 1.85 & $5 / 22 / 08$ & 8E12001 & PNNL-AGG-ICP-AES \\
\hline $7440-48-4$ & Cobalt & $<6.74$ & ug/g dry & 6.74 & $5 / 22 / 08$ & 8E12001 & PNNL-AGG-ICP-AES \\
\hline $7440-47-3$ & Chromium & 6.71 & ug/g dry & 2.70 & $5 / 22 / 08$ & 8E12001 & PNNL-AGG-ICP-AES \\
\hline $7440-50-8$ & Copper & $<28.8$ & ug/g dry & 28.8 & $5 / 22 / 08$ & 8E12001 & PNNL-AGG-ICP-AES \\
\hline 7439-89-6 & Iron & 11800 & ug/g dry & 82.6 & $5 / 22 / 08$ & 8E12001 & PNNL-AGG-ICP-AES \\
\hline 7440-09-7 & Potassium & 650 & ug/g dry & 76.7 & $5 / 22 / 08$ & 8E12001 & PNNL-AGG-ICP-AES \\
\hline $7439-93-2$ & Lithium & $<11.7$ & ug/g dry & 11.7 & $5 / 22 / 08$ & 8E12001 & PNNL-AGG-ICP-AES \\
\hline 7439-95-4 & Magnesium & 3590 & ug/g dry & 25.6 & $5 / 22 / 08$ & 8E12001 & PNNL-AGG-ICP-AES \\
\hline $7439-96-5$ & Manganese & 196 & ug/g dry & 0.967 & $5 / 22 / 08$ & 8E12001 & PNNL-AGG-ICP-AES \\
\hline 7439-98-7 & Molybdenum & $<18.3$ & ug/g dry & 18.3 & $5 / 22 / 08$ & 8E12001 & PNNL-AGG-ICP-AES \\
\hline 7440-02-0 & Nickel & 7.66 & ug/g dry & 6.65 & $5 / 22 / 08$ & 8E12001 & PNNL-AGG-ICP-AES \\
\hline
\end{tabular}


Total Metals by SW846 6010B/Acid Extract

\begin{tabular}{|c|c|c|c|c|c|c|c|}
\hline CAS \# & Analyte & Results & Units & EQL & Analyzed & Batch & Method \\
\hline HEIS No. & B1T2P8B & \multicolumn{3}{|c|}{ Lab ID: $\quad$ 0803015-06 } & & & \\
\hline 7723-14-0 & Phosphorus & 541 & ug/g dry & 60.9 & $5 / 22 / 08$ & 8E12001 & PNNL-AGG-ICP-AES \\
\hline 7439-92-1 & Lead & $<25.7$ & ug/g dry & 25.7 & $5 / 22 / 08$ & 8E12001 & PNNL-AGG-ICP-AES \\
\hline $7782-49-2$ & Selenium & $<113$ & ug/g dry & 113 & $5 / 22 / 08$ & 8E12001 & PNNL-AGG-ICP-AES \\
\hline $7440-24-6$ & Strontium & 20.8 & ug/g dry & 1.17 & $5 / 22 / 08$ & 8E12001 & PNNL-AGG-ICP-AES \\
\hline $7440-28-0$ & Thallium & $<36.5$ & ug/g dry & 36.5 & $5 / 22 / 08$ & 8E12001 & PNNL-AGG-ICP-AES \\
\hline $7440-62-2$ & Vanadium & 14.2 & ug/g dry & 3.01 & $5 / 22 / 08$ & 8E12001 & PNNL-AGG-ICP-AES \\
\hline $7440-66-6$ & Zinc & 26.9 & ug/g dry & 5.07 & $5 / 22 / 08$ & 8E12001 & PNNL-AGG-ICP-AES \\
\hline $7440-23-5$ & Sodium & $<866$ & ug/g dry & 866 & $5 / 22 / 08$ & 8E12001 & PNNL-AGG-ICP-AES \\
\hline $7440-21-3$ & Silicon & $<1130$ & ug/g dry & 1130 & $5 / 22 / 08$ & 8E12001 & PNNL-AGG-ICP-AES \\
\hline $7704-34-9$ & Sulfur & $<191$ & ug/g dry & 191 & $5 / 22 / 08$ & 8E12001 & PNNL-AGG-ICP-AES \\
\hline $7440-32-6$ & Titanium & 590 & ug/g dry & 25.5 & $5 / 22 / 08$ & 8E12001 & PNNL-AGG-ICP-AES \\
\hline $7440-67-7$ & Zirconium & $<16.4$ & ug/g dry & 16.4 & $5 / 22 / 08$ & 8E12001 & PNNL-AGG-ICP-AES \\
\hline $7440-15-5$ & Rhenium & $<14.2$ & ug/g dry & 14.2 & $5 / 22 / 08$ & 8E12001 & PNNL-AGG-ICP-AES \\
\hline $7440-36-0$ & Antimony & $<90.5$ & ug/g dry & 90.5 & $5 / 22 / 08$ & 8E12001 & PNNL-AGG-ICP-AES \\
\hline HEIS No. & B1T2P8A & \multicolumn{3}{|c|}{ Lab ID: $\quad$ 0803015-07 } & & & \\
\hline $7429-90-5$ & Aluminum & 4760 & ug/g dry & 32.7 & $5 / 22 / 08$ & 8E12001 & PNNL-AGG-ICP-AES \\
\hline $7440-38-2$ & Arsenic & $<79.5$ & ug/g dry & 79.5 & $5 / 22 / 08$ & 8E12001 & PNNL-AGG-ICP-AES \\
\hline $7440-42-8$ & Boron & $<692$ & ug/g dry & 692 & $5 / 22 / 08$ & 8E12001 & PNNL-AGG-ICP-AES \\
\hline $7440-39-3$ & Barium & 55.7 & ug/g dry & 3.12 & $5 / 22 / 08$ & 8E12001 & PNNL-AGG-ICP-AES \\
\hline $7440-41-7$ & Beryllium & $<1.24$ & ug/g dry & 1.24 & $5 / 22 / 08$ & 8E12001 & PNNL-AGG-ICP-AES \\
\hline $7440-69-9$ & Bismuth & $<16.5$ & ug/g dry & 16.5 & $5 / 22 / 08$ & 8E12001 & PNNL-AGG-ICP-AES \\
\hline $7440-70-2$ & Calcium & 9190 & ug/g dry & 122 & $5 / 22 / 08$ & 8E12001 & PNNL-AGG-ICP-AES \\
\hline $7440-43-9$ & Cadmium & $<1.86$ & ug/g dry & 1.86 & $5 / 22 / 08$ & 8E12001 & PNNL-AGG-ICP-AES \\
\hline $7440-48-4$ & Cobalt & $<6.79$ & ug/g dry & 6.79 & $5 / 22 / 08$ & 8E12001 & PNNL-AGG-ICP-AES \\
\hline $7440-47-3$ & Chromium & 4.98 & ug/g dry & 2.72 & $5 / 22 / 08$ & 8E12001 & PNNL-AGG-ICP-AES \\
\hline $7440-50-8$ & Copper & $<29.0$ & ug/g dry & 29.0 & $5 / 22 / 08$ & 8E12001 & PNNL-AGG-ICP-AES \\
\hline 7439-89-6 & Iron & 11500 & ug/g dry & 83.1 & $5 / 22 / 08$ & 8E12001 & PNNL-AGG-ICP-AES \\
\hline $7440-09-7$ & Potassium & 692 & ug/g dry & 77.1 & $5 / 22 / 08$ & 8E12001 & PNNL-AGG-ICP-AES \\
\hline $7439-93-2$ & Lithium & $<11.8$ & ug/g dry & 11.8 & $5 / 22 / 08$ & 8E12001 & PNNL-AGG-ICP-AES \\
\hline $7439-95-4$ & Magnesium & 3430 & ug/g dry & 25.7 & $5 / 22 / 08$ & 8E12001 & PNNL-AGG-ICP-AES \\
\hline $7439-96-5$ & Manganese & 204 & ug/g dry & 0.973 & $5 / 22 / 08$ & 8E12001 & PNNL-AGG-ICP-AES \\
\hline $7439-98-7$ & Molybdenum & $<18.4$ & ug/g dry & 18.4 & $5 / 22 / 08$ & 8E12001 & PNNL-AGG-ICP-AES \\
\hline $7440-02-0$ & Nickel & 6.94 & ug/g dry & 6.69 & $5 / 22 / 08$ & 8E12001 & PNNL-AGG-ICP-AES \\
\hline 7723-14-0 & Phosphorus & 538 & ug/g dry & 61.3 & $5 / 22 / 08$ & 8E12001 & PNNL-AGG-ICP-AES \\
\hline $7439-92-1$ & Lead & $<25.9$ & ug/g dry & 25.9 & $5 / 22 / 08$ & 8E12001 & PNNL-AGG-ICP-AES \\
\hline $7782-49-2$ & Selenium & $<114$ & ug/g dry & 114 & $5 / 22 / 08$ & 8E12001 & PNNL-AGG-ICP-AES \\
\hline $7440-24-6$ & Strontium & 21.6 & ug/g dry & 1.18 & $5 / 22 / 08$ & 8E12001 & PNNL-AGG-ICP-AES \\
\hline $7440-28-0$ & Thallium & $<36.8$ & ug/g dry & 36.8 & $5 / 22 / 08$ & 8E12001 & PNNL-AGG-ICP-AES \\
\hline $7440-62-2$ & Vanadium & 15.4 & ug/g dry & 3.03 & $5 / 22 / 08$ & 8E12001 & PNNL-AGG-ICP-AES \\
\hline $7440-66-6$ & Zinc & 24.8 & ug/g dry & 5.10 & $5 / 22 / 08$ & 8E12001 & PNNL-AGG-ICP-AES \\
\hline $7440-23-5$ & Sodium & $<871$ & ug/g dry & 871 & $5 / 22 / 08$ & 8E12001 & PNNL-AGG-ICP-AES \\
\hline $7440-21-3$ & Silicon & $<1140$ & ug/g dry & 1140 & $5 / 22 / 08$ & 8E12001 & PNNL-AGG-ICP-AES \\
\hline $7704-34-9$ & Sulfur & $<193$ & ug/g dry & 193 & $5 / 22 / 08$ & 8E12001 & PNNL-AGG-ICP-AES \\
\hline $7440-32-6$ & Titanium & 548 & ug/g dry & 25.7 & $5 / 22 / 08$ & 8E12001 & PNNL-AGG-ICP-AES \\
\hline $7440-67-7$ & Zirconium & $<16.6$ & ug/g dry & 16.6 & $5 / 22 / 08$ & 8E12001 & PNNL-AGG-ICP-AES \\
\hline $7440-15-5$ & Rhenium & $<14.3$ & ug/g dry & 14.3 & $5 / 22 / 08$ & 8E12001 & PNNL-AGG-ICP-AES \\
\hline $7440-36-0$ & Antimony & $<91.1$ & ug/g dry & 91.1 & $5 / 22 / 08$ & 8E12001 & PNNL-AGG-ICP-AES \\
\hline HEIS No. & B1T2P9B & La & b ID: & 3015-10 & & & \\
\hline
\end{tabular}


Total Metals by SW846 6010B/Acid Extract

\begin{tabular}{|c|c|c|c|c|c|c|c|}
\hline CAS \# & Analyte & Results & Units & EQL & Analyzed & Batch & Method \\
\hline HEIS No. & В1T2P9B & \multicolumn{3}{|c|}{ Lab ID: $\quad$ 0803015-10 } & & & \\
\hline $7429-90-5$ & Aluminum & 4630 & ug/g dry & 30.4 & $5 / 22 / 08$ & 8E12001 & PNNL-AGG-ICP-AES \\
\hline $7440-38-2$ & Arsenic & $<73.9$ & ug/g dry & 73.9 & $5 / 22 / 08$ & 8E12001 & PNNL-AGG-ICP-AES \\
\hline $7440-42-8$ & Boron & $<644$ & ug/g dry & 644 & $5 / 22 / 08$ & 8E12001 & PNNL-AGG-ICP-AES \\
\hline $7440-39-3$ & Barium & 45.9 & ug/g dry & 2.90 & $5 / 22 / 08$ & 8E12001 & PNNL-AGG-ICP-AES \\
\hline $7440-41-7$ & Beryllium & $<1.15$ & ug/g dry & 1.15 & $5 / 22 / 08$ & 8E12001 & PNNL-AGG-ICP-AES \\
\hline 7440-69-9 & Bismuth & $<15.4$ & ug/g dry & 15.4 & $5 / 22 / 08$ & 8E12001 & PNNL-AGG-ICP-AES \\
\hline $7440-70-2$ & Calcium & 7050 & ug/g dry & 113 & $5 / 22 / 08$ & 8E12001 & PNNL-AGG-ICP-AES \\
\hline $7440-43-9$ & Cadmium & $<1.73$ & ug/g dry & 1.73 & $5 / 22 / 08$ & 8E12001 & PNNL-AGG-ICP-AES \\
\hline $7440-48-4$ & Cobalt & $<6.31$ & ug/g dry & 6.31 & $5 / 22 / 08$ & 8E12001 & PNNL-AGG-ICP-AES \\
\hline $7440-47-3$ & Chromium & 8.50 & ug/g dry & 2.53 & $5 / 22 / 08$ & 8E12001 & PNNL-AGG-ICP-AES \\
\hline $7440-50-8$ & Copper & $<27.0$ & ug/g dry & 27.0 & $5 / 22 / 08$ & 8E12001 & PNNL-AGG-ICP-AES \\
\hline 7439-89-6 & Iron & 11200 & ug/g dry & 77.3 & $5 / 22 / 08$ & 8E12001 & PNNL-AGG-ICP-AES \\
\hline 7440-09-7 & Potassium & 724 & ug/g dry & 71.8 & $5 / 22 / 08$ & 8E12001 & PNNL-AGG-ICP-AES \\
\hline 7439-93-2 & Lithium & $<11.0$ & ug/g dry & 11.0 & $5 / 22 / 08$ & 8E12001 & PNNL-AGG-ICP-AES \\
\hline $7439-95-4$ & Magnesium & 3640 & ug/g dry & 23.9 & $5 / 22 / 08$ & 8E12001 & PNNL-AGG-ICP-AES \\
\hline 7439-96-5 & Manganese & 209 & ug/g dry & 0.906 & $5 / 22 / 08$ & 8E12001 & PNNL-AGG-ICP-AES \\
\hline 7439-98-7 & Molybdenum & $<17.2$ & ug/g dry & 17.2 & $5 / 22 / 08$ & 8E12001 & PNNL-AGG-ICP-AES \\
\hline 7440-02-0 & Nickel & 9.19 & ug/g dry & 6.22 & $5 / 22 / 08$ & 8E12001 & PNNL-AGG-ICP-AES \\
\hline $7723-14-0$ & Phosphorus & 521 & ug/g dry & 57.0 & $5 / 22 / 08$ & 8E12001 & PNNL-AGG-ICP-AES \\
\hline 7439-92-1 & Lead & $<24.1$ & ug/g dry & 24.1 & $5 / 22 / 08$ & 8E12001 & PNNL-AGG-ICP-AES \\
\hline $7782-49-2$ & Selenium & $<106$ & ug/g dry & 106 & $5 / 22 / 08$ & 8E12001 & PNNL-AGG-ICP-AES \\
\hline $7440-24-6$ & Strontium & 21.3 & ug/g dry & 1.10 & $5 / 22 / 08$ & 8E12001 & PNNL-AGG-ICP-AES \\
\hline $7440-28-0$ & Thallium & $<34.2$ & ug/g dry & 34.2 & $5 / 22 / 08$ & 8E12001 & PNNL-AGG-ICP-AES \\
\hline $7440-62-2$ & Vanadium & 14.9 & ug/g dry & 2.82 & $5 / 22 / 08$ & 8E12001 & PNNL-AGG-ICP-AES \\
\hline $7440-66-6$ & Zinc & 26.8 & ug/g dry & 4.74 & $5 / 22 / 08$ & 8E12001 & PNNL-AGG-ICP-AES \\
\hline $7440-23-5$ & Sodium & $<811$ & ug/g dry & 811 & $5 / 22 / 08$ & 8E12001 & PNNL-AGG-ICP-AES \\
\hline $7440-21-3$ & Silicon & $<1060$ & ug/g dry & 1060 & $5 / 22 / 08$ & 8E12001 & PNNL-AGG-ICP-AES \\
\hline $7704-34-9$ & Sulfur & $<179$ & ug/g dry & 179 & $5 / 22 / 08$ & 8E12001 & PNNL-AGG-ICP-AES \\
\hline $7440-32-6$ & Titanium & 524 & ug/g dry & 23.9 & $5 / 22 / 08$ & 8E12001 & PNNL-AGG-ICP-AES \\
\hline $7440-67-7$ & Zirconium & $<15.4$ & ug/g dry & 15.4 & $5 / 22 / 08$ & 8E12001 & PNNL-AGG-ICP-AES \\
\hline $7440-15-5$ & Rhenium & $<13.3$ & ug/g dry & 13.3 & $5 / 22 / 08$ & 8E12001 & PNNL-AGG-ICP-AES \\
\hline $7440-36-0$ & Antimony & $<84.7$ & ug/g dry & 84.7 & $5 / 22 / 08$ & 8E12001 & PNNL-AGG-ICP-AES \\
\hline HEIS No. & B1T2P9A & \multicolumn{3}{|c|}{ Lab ID: $\quad$ 0803015-11 } & & & \\
\hline $7429-90-5$ & Aluminum & 4490 & ug/g dry & 28.8 & $5 / 22 / 08$ & 8E12001 & PNNL-AGG-ICP-AES \\
\hline $7440-38-2$ & Arsenic & $<69.9$ & ug/g dry & 69.9 & $5 / 22 / 08$ & 8E12001 & PNNL-AGG-ICP-AES \\
\hline $7440-42-8$ & Boron & $<609$ & ug/g dry & 609 & $5 / 22 / 08$ & 8E12001 & PNNL-AGG-ICP-AES \\
\hline $7440-39-3$ & Barium & 46.4 & ug/g dry & 2.74 & $5 / 22 / 08$ & 8E12001 & PNNL-AGG-ICP-AES \\
\hline $7440-41-7$ & Beryllium & $<1.09$ & ug/g dry & 1.09 & $5 / 22 / 08$ & 8E12001 & PNNL-AGG-ICP-AES \\
\hline 7440-69-9 & Bismuth & $<14.5$ & ug/g dry & 14.5 & $5 / 22 / 08$ & 8E12001 & PNNL-AGG-ICP-AES \\
\hline $7440-70-2$ & Calcium & 6580 & ug/g dry & 107 & $5 / 22 / 08$ & 8E12001 & PNNL-AGG-ICP-AES \\
\hline $7440-43-9$ & Cadmium & $<1.63$ & ug/g dry & 1.63 & $5 / 22 / 08$ & 8E12001 & PNNL-AGG-ICP-AES \\
\hline $7440-48-4$ & Cobalt & $<5.97$ & ug/g dry & 5.97 & $5 / 22 / 08$ & 8E12001 & PNNL-AGG-ICP-AES \\
\hline $7440-47-3$ & Chromium & 7.19 & ug/g dry & 2.39 & $5 / 22 / 08$ & 8E12001 & PNNL-AGG-ICP-AES \\
\hline $7440-50-8$ & Copper & $<25.5$ & ug/g dry & 25.5 & $5 / 22 / 08$ & 8E12001 & PNNL-AGG-ICP-AES \\
\hline 7439-89-6 & Iron & 10900 & ug/g dry & 73.1 & $5 / 22 / 08$ & 8E12001 & PNNL-AGG-ICP-AES \\
\hline $7440-09-7$ & Potassium & 713 & ug/g dry & 67.9 & $5 / 22 / 08$ & 8E12001 & PNNL-AGG-ICP-AES \\
\hline 7439-93-2 & Lithium & $<10.4$ & ug/g dry & 10.4 & $5 / 22 / 08$ & 8E12001 & PNNL-AGG-ICP-AES \\
\hline $7439-95-4$ & Magnesium & 3350 & ug/g dry & 22.6 & $5 / 22 / 08$ & 8E12001 & PNNL-AGG-ICP-AES \\
\hline
\end{tabular}


Total Metals by SW846 6010B/Acid Extract

\begin{tabular}{|c|c|c|c|c|c|c|c|}
\hline CAS \# & Analyte & Results & Units & EQL & Analyzed & Batch & Method \\
\hline HEIS No. & B1T2P9A & \multicolumn{3}{|c|}{ Lab ID: $\quad$ 0803015-11 } & & & \\
\hline 7439-96-5 & Manganese & 185 & ug/g dry & 0.857 & $5 / 22 / 08$ & 8E12001 & PNNL-AGG-ICP-AES \\
\hline 7439-98-7 & Molybdenum & $<16.2$ & ug/g dry & 16.2 & $5 / 22 / 08$ & 8E12001 & PNNL-AGG-ICP-AES \\
\hline $7440-02-0$ & Nickel & 7.94 & ug/g dry & 5.89 & $5 / 22 / 08$ & 8E12001 & PNNL-AGG-ICP-AES \\
\hline $7723-14-0$ & Phosphorus & 497 & ug/g dry & 53.9 & $5 / 22 / 08$ & 8E12001 & PNNL-AGG-ICP-AES \\
\hline 7439-92-1 & Lead & $<22.8$ & ug/g dry & 22.8 & $5 / 22 / 08$ & 8E12001 & PNNL-AGG-ICP-AES \\
\hline $7782-49-2$ & Selenium & $<99.9$ & ug/g dry & 99.9 & $5 / 22 / 08$ & 8E12001 & PNNL-AGG-ICP-AES \\
\hline $7440-24-6$ & Strontium & 21.0 & ug/g dry & 1.04 & $5 / 22 / 08$ & 8E12001 & PNNL-AGG-ICP-AES \\
\hline $7440-28-0$ & Thallium & $<32.3$ & ug/g dry & 32.3 & $5 / 22 / 08$ & 8E12001 & PNNL-AGG-ICP-AES \\
\hline $7440-62-2$ & Vanadium & 14.7 & ug/g dry & 2.66 & $5 / 22 / 08$ & 8E12001 & PNNL-AGG-ICP-AES \\
\hline $7440-66-6$ & Zinc & 29.4 & ug/g dry & 4.49 & $5 / 22 / 08$ & 8E12001 & PNNL-AGG-ICP-AES \\
\hline $7440-23-5$ & Sodium & $<767$ & ug/g dry & 767 & $5 / 22 / 08$ & 8E12001 & PNNL-AGG-ICP-AES \\
\hline $7440-21-3$ & Silicon & $<1000$ & ug/g dry & 1000 & $5 / 22 / 08$ & 8E12001 & PNNL-AGG-ICP-AES \\
\hline $7704-34-9$ & Sulfur & $<170$ & ug/g dry & 170 & $5 / 22 / 08$ & 8E12001 & PNNL-AGG-ICP-AES \\
\hline $7440-32-6$ & Titanium & 563 & ug/g dry & 22.6 & $5 / 22 / 08$ & 8E12001 & PNNL-AGG-ICP-AES \\
\hline $7440-67-7$ & Zirconium & $<14.6$ & ug/g dry & 14.6 & $5 / 22 / 08$ & 8E12001 & PNNL-AGG-ICP-AES \\
\hline $7440-15-5$ & Rhenium & $<12.6$ & ug/g dry & 12.6 & $5 / 22 / 08$ & 8E12001 & PNNL-AGG-ICP-AES \\
\hline $7440-36-0$ & Antimony & $<80.1$ & ug/g dry & 80.1 & $5 / 22 / 08$ & 8E12001 & PNNL-AGG-ICP-AES \\
\hline HEIS No. & B1T2R0B & \multicolumn{3}{|c|}{ Lab ID: $\quad$ 0803015-14 } & & & \\
\hline $7429-90-5$ & Aluminum & 5300 & ug/g dry & 42.2 & $5 / 22 / 08$ & 8E12001 & PNNL-AGG-ICP-AES \\
\hline $7440-38-2$ & Arsenic & $<103$ & ug/g dry & 103 & $5 / 22 / 08$ & 8E12001 & PNNL-AGG-ICP-AES \\
\hline $7440-42-8$ & Boron & $<893$ & ug/g dry & 893 & $5 / 22 / 08$ & 8E12001 & PNNL-AGG-ICP-AES \\
\hline $7440-39-3$ & Barium & 54.1 & ug/g dry & 4.02 & $5 / 22 / 08$ & 8E12001 & PNNL-AGG-ICP-AES \\
\hline $7440-41-7$ & Beryllium & $<1.59$ & ug/g dry & 1.59 & $5 / 22 / 08$ & 8E12001 & PNNL-AGG-ICP-AES \\
\hline $7440-69-9$ & Bismuth & $<21.3$ & ug/g dry & 21.3 & $5 / 22 / 08$ & 8E12001 & PNNL-AGG-ICP-AES \\
\hline $7440-70-2$ & Calcium & 8930 & ug/g dry & 157 & $5 / 22 / 08$ & 8E12001 & PNNL-AGG-ICP-AES \\
\hline $7440-43-9$ & Cadmium & $<2.40$ & ug/g dry & 2.40 & $5 / 22 / 08$ & 8E12001 & PNNL-AGG-ICP-AES \\
\hline $7440-48-4$ & Cobalt & $<8.76$ & ug/g dry & 8.76 & $5 / 22 / 08$ & 8E12001 & PNNL-AGG-ICP-AES \\
\hline $7440-47-3$ & Chromium & 5.28 & ug/g dry & 3.51 & $5 / 22 / 08$ & 8E12001 & PNNL-AGG-ICP-AES \\
\hline $7440-50-8$ & Copper & $<37.4$ & ug/g dry & 37.4 & $5 / 22 / 08$ & 8E12001 & PNNL-AGG-ICP-AES \\
\hline 7439-89-6 & Iron & 13800 & ug/g dry & 107 & $5 / 22 / 08$ & 8E12001 & PNNL-AGG-ICP-AES \\
\hline 7440-09-7 & Potassium & 765 & ug/g dry & 99.6 & $5 / 22 / 08$ & 8E12001 & PNNL-AGG-ICP-AES \\
\hline $7439-93-2$ & Lithium & $<15.2$ & ug/g dry & 15.2 & $5 / 22 / 08$ & 8E12001 & PNNL-AGG-ICP-AES \\
\hline 7439-95-4 & Magnesium & 4340 & ug/g dry & 33.2 & $5 / 22 / 08$ & 8E12001 & PNNL-AGG-ICP-AES \\
\hline $7439-96-5$ & Manganese & 241 & ug/g dry & 1.26 & $5 / 22 / 08$ & 8E12001 & PNNL-AGG-ICP-AES \\
\hline 7439-98-7 & Molybdenum & $<23.8$ & ug/g dry & 23.8 & $5 / 22 / 08$ & 8E12001 & PNNL-AGG-ICP-AES \\
\hline $7440-02-0$ & Nickel & $<8.63$ & ug/g dry & 8.63 & $5 / 22 / 08$ & 8E12001 & PNNL-AGG-ICP-AES \\
\hline $7723-14-0$ & Phosphorus & 595 & ug/g dry & 79.1 & $5 / 22 / 08$ & 8E12001 & PNNL-AGG-ICP-AES \\
\hline 7439-92-1 & Lead & $<33.4$ & ug/g dry & 33.4 & $5 / 22 / 08$ & 8E12001 & PNNL-AGG-ICP-AES \\
\hline $7782-49-2$ & Selenium & $<147$ & ug/g dry & 147 & $5 / 22 / 08$ & 8E12001 & PNNL-AGG-ICP-AES \\
\hline $7440-24-6$ & Strontium & 30.6 & ug/g dry & 1.52 & $5 / 22 / 08$ & 8E12001 & PNNL-AGG-ICP-AES \\
\hline $7440-28-0$ & Thallium & $<47.4$ & ug/g dry & 47.4 & $5 / 22 / 08$ & 8E12001 & PNNL-AGG-ICP-AES \\
\hline $7440-62-2$ & Vanadium & 18.0 & ug/g dry & 3.91 & $5 / 22 / 08$ & 8E12001 & PNNL-AGG-ICP-AES \\
\hline $7440-66-6$ & Zinc & 38.2 & ug/g dry & 6.58 & $5 / 22 / 08$ & 8E12001 & PNNL-AGG-ICP-AES \\
\hline $7440-23-5$ & Sodium & $<1120$ & ug/g dry & 1120 & $5 / 22 / 08$ & 8E12001 & PNNL-AGG-ICP-AES \\
\hline $7440-21-3$ & Silicon & $<1470$ & ug/g dry & 1470 & $5 / 22 / 08$ & 8E12001 & PNNL-AGG-ICP-AES \\
\hline $7704-34-9$ & Sulfur & $<249$ & ug/g dry & 249 & $5 / 22 / 08$ & 8E12001 & PNNL-AGG-ICP-AES \\
\hline $7440-32-6$ & Titanium & 656 & ug/g dry & 33.1 & $5 / 22 / 08$ & 8E12001 & PNNL-AGG-ICP-AES \\
\hline $7440-67-7$ & Zirconium & $<21.4$ & ug/g dry & 21.4 & $5 / 22 / 08$ & 8E12001 & PNNL-AGG-ICP-AES \\
\hline
\end{tabular}


Total Metals by SW846 6010B/Acid Extract

\begin{tabular}{|c|c|c|c|c|c|c|c|}
\hline CAS \# & Analyte & Results & Units & EQL & Analyzed & Batch & Method \\
\hline HEIS No. & B1T2R0B & \multicolumn{3}{|c|}{ Lab ID: $\quad$ 0803015-14 } & & & \\
\hline $7440-15-5$ & Rhenium & $<18.5$ & ug/g dry & 18.5 & $5 / 22 / 08$ & 8E12001 & PNNL-AGG-ICP-AES \\
\hline $7440-36-0$ & Antimony & $<118$ & ug/g dry & 118 & $5 / 22 / 08$ & 8E12001 & PNNL-AGG-ICP-AES \\
\hline HEIS No. & B1T2R0A & \multicolumn{3}{|c|}{ Lab ID: $\quad$ 0803015-15 } & & & \\
\hline $7429-90-5$ & Aluminum & 4590 & ug/g dry & 30.2 & $5 / 22 / 08$ & 8E12001 & PNNL-AGG-ICP-AES \\
\hline $7440-38-2$ & Arsenic & $<73.4$ & ug/g dry & 73.4 & $5 / 22 / 08$ & 8E12001 & PNNL-AGG-ICP-AES \\
\hline $7440-42-8$ & Boron & $<639$ & ug/g dry & 639 & $5 / 22 / 08$ & 8E12001 & PNNL-AGG-ICP-AES \\
\hline $7440-39-3$ & Barium & 43.5 & ug/g dry & 2.88 & $5 / 22 / 08$ & 8E12001 & PNNL-AGG-ICP-AES \\
\hline $7440-41-7$ & Beryllium & $<1.14$ & ug/g dry & 1.14 & $5 / 22 / 08$ & 8E12001 & PNNL-AGG-ICP-AES \\
\hline $7440-69-9$ & Bismuth & $<15.3$ & ug/g dry & 15.3 & $5 / 22 / 08$ & 8E12001 & PNNL-AGG-ICP-AES \\
\hline $7440-70-2$ & Calcium & 6960 & ug/g dry & 112 & $5 / 22 / 08$ & 8E12001 & PNNL-AGG-ICP-AES \\
\hline $7440-43-9$ & Cadmium & $<1.72$ & ug/g dry & 1.72 & $5 / 22 / 08$ & 8E12001 & PNNL-AGG-ICP-AES \\
\hline $7440-48-4$ & Cobalt & $<6.27$ & ug/g dry & 6.27 & $5 / 22 / 08$ & 8E12001 & PNNL-AGG-ICP-AES \\
\hline $7440-47-3$ & Chromium & 5.45 & ug/g dry & 2.51 & $5 / 22 / 08$ & 8E12001 & PNNL-AGG-ICP-AES \\
\hline $7440-50-8$ & Copper & $<26.8$ & ug/g dry & 26.8 & $5 / 22 / 08$ & 8E12001 & PNNL-AGG-ICP-AES \\
\hline 7439-89-6 & Iron & 10900 & ug/g dry & 76.7 & $5 / 22 / 08$ & 8E12001 & PNNL-AGG-ICP-AES \\
\hline $7440-09-7$ & Potassium & 678 & ug/g dry & 71.2 & $5 / 22 / 08$ & 8E12001 & PNNL-AGG-ICP-AES \\
\hline $7439-93-2$ & Lithium & $<10.9$ & ug/g dry & 10.9 & $5 / 22 / 08$ & 8E12001 & PNNL-AGG-ICP-AES \\
\hline 7439-95-4 & Magnesium & 3500 & ug/g dry & 23.8 & $5 / 22 / 08$ & 8E12001 & PNNL-AGG-ICP-AES \\
\hline $7439-96-5$ & Manganese & 209 & ug/g dry & 0.899 & $5 / 22 / 08$ & 8E12001 & PNNL-AGG-ICP-AES \\
\hline 7439-98-7 & Molybdenum & $<17.0$ & ug/g dry & 17.0 & $5 / 22 / 08$ & 8E12001 & PNNL-AGG-ICP-AES \\
\hline $7440-02-0$ & Nickel & 8.92 & ug/g dry & 6.18 & $5 / 22 / 08$ & 8E12001 & PNNL-AGG-ICP-AES \\
\hline $7723-14-0$ & Phosphorus & 509 & ug/g dry & 56.6 & $5 / 22 / 08$ & 8E12001 & PNNL-AGG-ICP-AES \\
\hline 7439-92-1 & Lead & $<23.9$ & ug/g dry & 23.9 & $5 / 22 / 08$ & 8E12001 & PNNL-AGG-ICP-AES \\
\hline $7782-49-2$ & Selenium & $<105$ & ug/g dry & 105 & $5 / 22 / 08$ & 8E12001 & PNNL-AGG-ICP-AES \\
\hline $7440-24-6$ & Strontium & 21.7 & ug/g dry & 1.09 & $5 / 22 / 08$ & 8E12001 & PNNL-AGG-ICP-AES \\
\hline $7440-28-0$ & Thallium & $<33.9$ & ug/g dry & 33.9 & $5 / 22 / 08$ & 8E12001 & PNNL-AGG-ICP-AES \\
\hline $7440-62-2$ & Vanadium & 15.3 & ug/g dry & 2.79 & $5 / 22 / 08$ & 8E12001 & PNNL-AGG-ICP-AES \\
\hline $7440-66-6$ & Zinc & 25.6 & ug/g dry & 4.71 & $5 / 22 / 08$ & 8E12001 & PNNL-AGG-ICP-AES \\
\hline $7440-23-5$ & Sodium & $<805$ & ug/g dry & 805 & $5 / 22 / 08$ & 8E12001 & PNNL-AGG-ICP-AES \\
\hline $7440-21-3$ & Silicon & $<1050$ & ug/g dry & 1050 & $5 / 22 / 08$ & 8E12001 & PNNL-AGG-ICP-AES \\
\hline $7704-34-9$ & Sulfur & $<178$ & ug/g dry & 178 & $5 / 22 / 08$ & 8E12001 & PNNL-AGG-ICP-AES \\
\hline $7440-32-6$ & Titanium & 533 & ug/g dry & 23.7 & $5 / 22 / 08$ & 8E12001 & PNNL-AGG-ICP-AES \\
\hline $7440-67-7$ & Zirconium & $<15.3$ & ug/g dry & 15.3 & $5 / 22 / 08$ & 8E12001 & PNNL-AGG-ICP-AES \\
\hline $7440-15-5$ & Rhenium & $<13.2$ & ug/g dry & 13.2 & $5 / 22 / 08$ & 8E12001 & PNNL-AGG-ICP-AES \\
\hline $7440-36-0$ & Antimony & $<84.1$ & ug/g dry & 84.1 & $5 / 22 / 08$ & 8E12001 & PNNL-AGG-ICP-AES \\
\hline HEIS No. & B1TNK6B & & b ID: & 0803015-18 & & & \\
\hline $7429-90-5$ & Aluminum & 4870 & ug/g dry & 36.0 & $5 / 22 / 08$ & 8E12001 & PNNL-AGG-ICP-AES \\
\hline $7440-38-2$ & Arsenic & $<87.3$ & ug/g dry & 87.3 & $5 / 22 / 08$ & 8E12001 & PNNL-AGG-ICP-AES \\
\hline $7440-42-8$ & Boron & $<761$ & ug/g dry & 761 & $5 / 22 / 08$ & 8E12001 & PNNL-AGG-ICP-AES \\
\hline $7440-39-3$ & Barium & 48.3 & ug/g dry & 3.42 & $5 / 22 / 08$ & 8E12001 & PNNL-AGG-ICP-AES \\
\hline $7440-41-7$ & Beryllium & $<1.36$ & ug/g dry & 1.36 & $5 / 22 / 08$ & 8E12001 & PNNL-AGG-ICP-AES \\
\hline $7440-69-9$ & Bismuth & $<18.2$ & ug/g dry & 18.2 & $5 / 22 / 08$ & 8E12001 & PNNL-AGG-ICP-AES \\
\hline $7440-70-2$ & Calcium & 7630 & ug/g dry & 134 & $5 / 22 / 08$ & 8E12001 & PNNL-AGG-ICP-AES \\
\hline $7440-43-9$ & Cadmium & $<2.04$ & ug/g dry & 2.04 & $5 / 22 / 08$ & 8E12001 & PNNL-AGG-ICP-AES \\
\hline $7440-48-4$ & Cobalt & $<7.46$ & ug/g dry & 7.46 & $5 / 22 / 08$ & 8E12001 & PNNL-AGG-ICP-AES \\
\hline $7440-47-3$ & Chromium & 5.75 & ug/g dry & 2.99 & $5 / 22 / 08$ & 8E12001 & PNNL-AGG-ICP-AES \\
\hline $7440-50-8$ & Copper & $<31.8$ & ug/g dry & 31.8 & $5 / 22 / 08$ & 8E12001 & PNNL-AGG-ICP-AES \\
\hline 7439-89-6 & Iron & 11500 & ug/g dry & 91.3 & $5 / 22 / 08$ & 8E12001 & PNNL-AGG-ICP-AES \\
\hline
\end{tabular}


Total Metals by SW846 6010B/Acid Extract

\begin{tabular}{|c|c|c|c|c|c|c|c|}
\hline CAS \# & Analyte & Results & Units & EQL & Analyzed & Batch & Method \\
\hline HEIS No. & B1TNK6B & \multicolumn{3}{|c|}{ Lab ID: $\quad$ 0803015-18 } & & & \\
\hline 7440-09-7 & Potassium & 809 & ug/g dry & 84.8 & $5 / 22 / 08$ & 8E12001 & PNNL-AGG-ICP-AES \\
\hline 7439-93-2 & Lithium & $<13.0$ & ug/g dry & 13.0 & $5 / 22 / 08$ & 8E12001 & PNNL-AGG-ICP-AES \\
\hline 7439-95-4 & Magnesium & 3810 & ug/g dry & 28.3 & $5 / 22 / 08$ & 8E12001 & PNNL-AGG-ICP-AES \\
\hline 7439-96-5 & Manganese & 230 & ug/g dry & 1.07 & $5 / 22 / 08$ & 8E12001 & PNNL-AGG-ICP-AES \\
\hline 7439-98-7 & Molybdenum & $<20.3$ & ug/g dry & 20.3 & $5 / 22 / 08$ & 8E12001 & PNNL-AGG-ICP-AES \\
\hline 7440-02-0 & Nickel & 8.10 & ug/g dry & 7.35 & $5 / 22 / 08$ & 8E12001 & PNNL-AGG-ICP-AES \\
\hline $7723-14-0$ & Phosphorus & 479 & ug/g dry & 67.3 & $5 / 22 / 08$ & 8E12001 & PNNL-AGG-ICP-AES \\
\hline 7439-92-1 & Lead & $<28.4$ & ug/g dry & 28.4 & $5 / 22 / 08$ & 8E12001 & PNNL-AGG-ICP-AES \\
\hline $7782-49-2$ & Selenium & $<125$ & ug/g dry & 125 & $5 / 22 / 08$ & 8E12001 & PNNL-AGG-ICP-AES \\
\hline $7440-24-6$ & Strontium & 25.0 & ug/g dry & 1.30 & $5 / 22 / 08$ & 8E12001 & PNNL-AGG-ICP-AES \\
\hline $7440-28-0$ & Thallium & $<40.4$ & ug/g dry & 40.4 & $5 / 22 / 08$ & 8E12001 & PNNL-AGG-ICP-AES \\
\hline $7440-62-2$ & Vanadium & 14.6 & ug/g dry & 3.33 & $5 / 22 / 08$ & 8E12001 & PNNL-AGG-ICP-AES \\
\hline $7440-66-6$ & Zinc & 25.4 & ug/g dry & 5.60 & $5 / 22 / 08$ & 8E12001 & PNNL-AGG-ICP-AES \\
\hline $7440-23-5$ & Sodium & $<958$ & ug/g dry & 958 & $5 / 22 / 08$ & 8E12001 & PNNL-AGG-ICP-AES \\
\hline $7440-21-3$ & Silicon & $<1250$ & ug/g dry & 1250 & $5 / 22 / 08$ & 8E12001 & PNNL-AGG-ICP-AES \\
\hline $7704-34-9$ & Sulfur & $<212$ & ug/g dry & 212 & $5 / 22 / 08$ & 8E12001 & PNNL-AGG-ICP-AES \\
\hline $7440-32-6$ & Titanium & 544 & ug/g dry & 28.2 & $5 / 22 / 08$ & 8E12001 & PNNL-AGG-ICP-AES \\
\hline $7440-67-7$ & Zirconium & $<18.2$ & ug/g dry & 18.2 & $5 / 22 / 08$ & 8E12001 & PNNL-AGG-ICP-AES \\
\hline $7440-15-5$ & Rhenium & $<15.8$ & ug/g dry & 15.8 & $5 / 22 / 08$ & 8E12001 & PNNL-AGG-ICP-AES \\
\hline $7440-36-0$ & Antimony & $<100$ & ug/g dry & 100 & $5 / 22 / 08$ & 8E12001 & PNNL-AGG-ICP-AES \\
\hline HEIS No. & B1TNK6A & \multicolumn{3}{|c|}{ Lab ID: $\quad$ 0803015-19 } & & & \\
\hline $7429-90-5$ & Aluminum & 4660 & ug/g dry & 34.6 & $5 / 22 / 08$ & 8E12001 & PNNL-AGG-ICP-AES \\
\hline $7440-38-2$ & Arsenic & $<83.9$ & ug/g dry & 83.9 & $5 / 22 / 08$ & 8E12001 & PNNL-AGG-ICP-AES \\
\hline $7440-42-8$ & Boron & $<731$ & ug/g dry & 731 & $5 / 22 / 08$ & 8E12001 & PNNL-AGG-ICP-AES \\
\hline $7440-39-3$ & Barium & 51.3 & ug/g dry & 3.29 & $5 / 22 / 08$ & 8E12001 & PNNL-AGG-ICP-AES \\
\hline $7440-41-7$ & Beryllium & $<1.30$ & ug/g dry & 1.30 & $5 / 22 / 08$ & 8E12001 & PNNL-AGG-ICP-AES \\
\hline $7440-69-9$ & Bismuth & $<17.5$ & ug/g dry & 17.5 & $5 / 22 / 08$ & 8E12001 & PNNL-AGG-ICP-AES \\
\hline $7440-70-2$ & Calcium & 7250 & ug/g dry & 128 & $5 / 22 / 08$ & 8E12001 & PNNL-AGG-ICP-AES \\
\hline $7440-43-9$ & Cadmium & $<1.96$ & ug/g dry & 1.96 & $5 / 22 / 08$ & 8E12001 & PNNL-AGG-ICP-AES \\
\hline $7440-48-4$ & Cobalt & $<7.17$ & ug/g dry & 7.17 & $5 / 22 / 08$ & 8E12001 & PNNL-AGG-ICP-AES \\
\hline $7440-47-3$ & Chromium & 6.51 & ug/g dry & 2.87 & $5 / 22 / 08$ & 8E12001 & PNNL-AGG-ICP-AES \\
\hline $7440-50-8$ & Copper & $<30.6$ & ug/g dry & 30.6 & $5 / 22 / 08$ & 8E12001 & PNNL-AGG-ICP-AES \\
\hline 7439-89-6 & Iron & 11700 & ug/g dry & 87.8 & $5 / 22 / 08$ & 8E12001 & PNNL-AGG-ICP-AES \\
\hline $7440-09-7$ & Potassium & 773 & ug/g dry & 81.5 & $5 / 22 / 08$ & 8E12001 & PNNL-AGG-ICP-AES \\
\hline $7439-93-2$ & Lithium & $<12.5$ & ug/g dry & 12.5 & $5 / 22 / 08$ & 8E12001 & PNNL-AGG-ICP-AES \\
\hline $7439-95-4$ & Magnesium & 3610 & ug/g dry & 27.2 & $5 / 22 / 08$ & 8E12001 & PNNL-AGG-ICP-AES \\
\hline 7439-96-5 & Manganese & 212 & ug/g dry & 1.03 & $5 / 22 / 08$ & 8E12001 & PNNL-AGG-ICP-AES \\
\hline 7439-98-7 & Molybdenum & $<19.5$ & ug/g dry & 19.5 & $5 / 22 / 08$ & 8E12001 & PNNL-AGG-ICP-AES \\
\hline $7440-02-0$ & Nickel & 8.43 & ug/g dry & 7.07 & $5 / 22 / 08$ & 8E12001 & PNNL-AGG-ICP-AES \\
\hline $7723-14-0$ & Phosphorus & 569 & ug/g dry & 64.7 & $5 / 22 / 08$ & 8E12001 & PNNL-AGG-ICP-AES \\
\hline $7439-92-1$ & Lead & $<27.3$ & ug/g dry & 27.3 & $5 / 22 / 08$ & 8E12001 & PNNL-AGG-ICP-AES \\
\hline $7782-49-2$ & Selenium & $<120$ & ug/g dry & 120 & $5 / 22 / 08$ & 8E12001 & PNNL-AGG-ICP-AES \\
\hline $7440-24-6$ & Strontium & 23.1 & ug/g dry & 1.25 & $5 / 22 / 08$ & 8E12001 & PNNL-AGG-ICP-AES \\
\hline $7440-28-0$ & Thallium & $<38.8$ & ug/g dry & 38.8 & $5 / 22 / 08$ & 8E12001 & PNNL-AGG-ICP-AES \\
\hline $7440-62-2$ & Vanadium & 16.6 & ug/g dry & 3.20 & $5 / 22 / 08$ & 8E12001 & PNNL-AGG-ICP-AES \\
\hline $7440-66-6$ & Zinc & 25.9 & ug/g dry & 5.39 & $5 / 22 / 08$ & 8E12001 & PNNL-AGG-ICP-AES \\
\hline $7440-23-5$ & Sodium & $<921$ & ug/g dry & 921 & $5 / 22 / 08$ & 8E12001 & PNNL-AGG-ICP-AES \\
\hline $7440-21-3$ & Silicon & $<1200$ & ug/g dry & 1200 & $5 / 22 / 08$ & 8E12001 & PNNL-AGG-ICP-AES \\
\hline
\end{tabular}


Total Metals by SW846 6010B/Acid Extract

\begin{tabular}{|c|c|c|c|c|c|c|c|}
\hline CAS \# & Analyte & Results & Units & EQL & Analyzed & Batch & Method \\
\hline HEIS No. & B1TNK6A & \multicolumn{3}{|c|}{ Lab ID: $\quad$ 0803015-19 } & & & \\
\hline 7704-34-9 & Sulfur & $<204$ & ug/g dry & 204 & $5 / 22 / 08$ & 8E12001 & PNNL-AGG-ICP-AES \\
\hline $7440-32-6$ & Titanium & 620 & ug/g dry & 27.1 & $5 / 22 / 08$ & 8E12001 & PNNL-AGG-ICP-AES \\
\hline $7440-67-7$ & Zirconium & $<17.5$ & ug/g dry & 17.5 & $5 / 22 / 08$ & 8E12001 & PNNL-AGG-ICP-AES \\
\hline $7440-15-5$ & Rhenium & $<15.1$ & ug/g dry & 15.1 & $5 / 22 / 08$ & 8E12001 & PNNL-AGG-ICP-AES \\
\hline $7440-36-0$ & Antimony & $<96.2$ & ug/g dry & 96.2 & $5 / 22 / 08$ & 8E12001 & PNNL-AGG-ICP-AES \\
\hline HEIS No. & B1TNK7B & \multicolumn{3}{|c|}{ Lab ID: $\quad$ 0803015-22 } & & & \\
\hline $7429-90-5$ & Aluminum & 4700 & ug/g dry & 33.1 & $5 / 22 / 08$ & 8E12001 & PNNL-AGG-ICP-AES \\
\hline $7440-38-2$ & Arsenic & $<80.3$ & ug/g dry & 80.3 & $5 / 22 / 08$ & 8E12001 & PNNL-AGG-ICP-AES \\
\hline $7440-42-8$ & Boron & $<699$ & ug/g dry & 699 & $5 / 22 / 08$ & 8E12001 & PNNL-AGG-ICP-AES \\
\hline $7440-39-3$ & Barium & 44.5 & ug/g dry & 3.15 & $5 / 22 / 08$ & 8E12001 & PNNL-AGG-ICP-AES \\
\hline $7440-41-7$ & Beryllium & $<1.25$ & ug/g dry & 1.25 & $5 / 22 / 08$ & 8E12001 & PNNL-AGG-ICP-AES \\
\hline 7440-69-9 & Bismuth & $<16.7$ & ug/g dry & 16.7 & $5 / 22 / 08$ & 8E12001 & PNNL-AGG-ICP-AES \\
\hline $7440-70-2$ & Calcium & 7930 & ug/g dry & 123 & $5 / 22 / 08$ & 8E12001 & PNNL-AGG-ICP-AES \\
\hline $7440-43-9$ & Cadmium & $<1.88$ & ug/g dry & 1.88 & $5 / 22 / 08$ & 8E12001 & PNNL-AGG-ICP-AES \\
\hline $7440-48-4$ & Cobalt & $<6.86$ & ug/g dry & 6.86 & $5 / 22 / 08$ & 8E12001 & PNNL-AGG-ICP-AES \\
\hline $7440-47-3$ & Chromium & 6.75 & ug/g dry & 2.75 & $5 / 22 / 08$ & 8E12001 & PNNL-AGG-ICP-AES \\
\hline $7440-50-8$ & Copper & $<29.3$ & ug/g dry & 29.3 & $5 / 22 / 08$ & 8E12001 & PNNL-AGG-ICP-AES \\
\hline 7439-89-6 & Iron & 11700 & ug/g dry & 84.0 & $5 / 22 / 08$ & 8E12001 & PNNL-AGG-ICP-AES \\
\hline $7440-09-7$ & Potassium & 728 & ug/g dry & 78.0 & $5 / 22 / 08$ & 8E12001 & PNNL-AGG-ICP-AES \\
\hline 7439-93-2 & Lithium & $<11.9$ & ug/g dry & 11.9 & $5 / 22 / 08$ & 8E12001 & PNNL-AGG-ICP-AES \\
\hline $7439-95-4$ & Magnesium & 3810 & ug/g dry & 26.0 & $5 / 22 / 08$ & 8E12001 & PNNL-AGG-ICP-AES \\
\hline 7439-96-5 & Manganese & 236 & ug/g dry & 0.984 & $5 / 22 / 08$ & 8E12001 & PNNL-AGG-ICP-AES \\
\hline 7439-98-7 & Molybdenum & $<18.6$ & ug/g dry & 18.6 & $5 / 22 / 08$ & 8E12001 & PNNL-AGG-ICP-AES \\
\hline $7440-02-0$ & Nickel & 9.04 & ug/g dry & 6.76 & $5 / 22 / 08$ & 8E12001 & PNNL-AGG-ICP-AES \\
\hline 7723-14-0 & Phosphorus & 540 & ug/g dry & 61.9 & $5 / 22 / 08$ & 8E12001 & PNNL-AGG-ICP-AES \\
\hline 7439-92-1 & Lead & $<26.1$ & ug/g dry & 26.1 & $5 / 22 / 08$ & 8E12001 & PNNL-AGG-ICP-AES \\
\hline $7782-49-2$ & Selenium & $<115$ & ug/g dry & 115 & $5 / 22 / 08$ & 8E12001 & PNNL-AGG-ICP-AES \\
\hline $7440-24-6$ & Strontium & 20.2 & ug/g dry & 1.19 & $5 / 22 / 08$ & 8E12001 & PNNL-AGG-ICP-AES \\
\hline $7440-28-0$ & Thallium & $<37.1$ & ug/g dry & 37.1 & $5 / 22 / 08$ & 8E12001 & PNNL-AGG-ICP-AES \\
\hline $7440-62-2$ & Vanadium & 15.3 & ug/g dry & 3.06 & $5 / 22 / 08$ & 8E12001 & PNNL-AGG-ICP-AES \\
\hline $7440-66-6$ & Zinc & 28.0 & ug/g dry & 5.15 & $5 / 22 / 08$ & 8E12001 & PNNL-AGG-ICP-AES \\
\hline $7440-23-5$ & Sodium & $<881$ & ug/g dry & 881 & $5 / 22 / 08$ & 8E12001 & PNNL-AGG-ICP-AES \\
\hline $7440-21-3$ & Silicon & $<1150$ & ug/g dry & 1150 & $5 / 22 / 08$ & 8E12001 & PNNL-AGG-ICP-AES \\
\hline $7704-34-9$ & Sulfur & $<195$ & ug/g dry & 195 & $5 / 22 / 08$ & 8E12001 & PNNL-AGG-ICP-AES \\
\hline $7440-32-6$ & Titanium & 555 & ug/g dry & 25.9 & $5 / 22 / 08$ & 8E12001 & PNNL-AGG-ICP-AES \\
\hline $7440-67-7$ & Zirconium & $<16.7$ & ug/g dry & 16.7 & $5 / 22 / 08$ & 8E12001 & PNNL-AGG-ICP-AES \\
\hline $7440-15-5$ & Rhenium & $<14.5$ & ug/g dry & 14.5 & $5 / 22 / 08$ & 8E12001 & PNNL-AGG-ICP-AES \\
\hline $7440-36-0$ & Antimony & $<92.0$ & ug/g dry & 92.0 & $5 / 22 / 08$ & 8E12001 & PNNL-AGG-ICP-AES \\
\hline HEIS No. & B1TNK7A & \multicolumn{3}{|c|}{ Lab ID: $\quad$ 0803015-23 } & & & \\
\hline $7429-90-5$ & Aluminum & 4700 & ug/g dry & 30.9 & $5 / 22 / 08$ & 8E12001 & PNNL-AGG-ICP-AES \\
\hline $7440-38-2$ & Arsenic & $<75.0$ & ug/g dry & 75.0 & $5 / 22 / 08$ & 8E12001 & PNNL-AGG-ICP-AES \\
\hline $7440-42-8$ & Boron & $<653$ & ug/g dry & 653 & $5 / 22 / 08$ & 8E12001 & PNNL-AGG-ICP-AES \\
\hline $7440-39-3$ & Barium & 37.2 & ug/g dry & 2.94 & $5 / 22 / 08$ & 8E12001 & PNNL-AGG-ICP-AES \\
\hline $7440-41-7$ & Beryllium & $<1.17$ & ug/g dry & 1.17 & $5 / 22 / 08$ & 8E12001 & PNNL-AGG-ICP-AES \\
\hline $7440-69-9$ & Bismuth & $<15.6$ & ug/g dry & 15.6 & $5 / 22 / 08$ & 8E12001 & PNNL-AGG-ICP-AES \\
\hline $7440-70-2$ & Calcium & 9010 & ug/g dry & 115 & $5 / 22 / 08$ & 8E12001 & PNNL-AGG-ICP-AES \\
\hline $7440-43-9$ & Cadmium & $<1.75$ & ug/g dry & 1.75 & $5 / 22 / 08$ & 8E12001 & PNNL-AGG-ICP-AES \\
\hline $7440-48-4$ & Cobalt & $<6.40$ & ug/g dry & 6.40 & $5 / 22 / 08$ & 8E12001 & PNNL-AGG-ICP-AES \\
\hline
\end{tabular}


Total Metals by SW846 6010B/Acid Extract

\begin{tabular}{|c|c|c|c|c|c|c|c|}
\hline CAS \# & Analyte & Results & Units & EQL & Analyzed & Batch & Method \\
\hline HEIS No. & B1TNK7A & \multicolumn{3}{|c|}{ Lab ID: $\quad$ 0803015-23 } & & & \\
\hline $7440-47-3$ & Chromium & 7.45 & ug/g dry & 2.57 & $5 / 22 / 08$ & 8E12001 & PNNL-AGG-ICP-AES \\
\hline $7440-50-8$ & Copper & $<27.3$ & ug/g dry & 27.3 & $5 / 22 / 08$ & 8E12001 & PNNL-AGG-ICP-AES \\
\hline 7439-89-6 & Iron & 10800 & ug/g dry & 78.4 & $5 / 22 / 08$ & 8E12001 & PNNL-AGG-ICP-AES \\
\hline 7440-09-7 & Potassium & 681 & ug/g dry & 72.8 & $5 / 22 / 08$ & 8E12001 & PNNL-AGG-ICP-AES \\
\hline $7439-93-2$ & Lithium & $<11.1$ & ug/g dry & 11.1 & $5 / 22 / 08$ & 8E12001 & PNNL-AGG-ICP-AES \\
\hline 7439-95-4 & Magnesium & 3950 & ug/g dry & 24.3 & $5 / 22 / 08$ & 8E12001 & PNNL-AGG-ICP-AES \\
\hline $7439-96-5$ & Manganese & 219 & ug/g dry & 0.919 & $5 / 22 / 08$ & 8E12001 & PNNL-AGG-ICP-AES \\
\hline 7439-98-7 & Molybdenum & $<17.4$ & ug/g dry & 17.4 & $5 / 22 / 08$ & 8E12001 & PNNL-AGG-ICP-AES \\
\hline $7440-02-0$ & Nickel & 9.89 & ug/g dry & 6.31 & $5 / 22 / 08$ & 8E12001 & PNNL-AGG-ICP-AES \\
\hline $7723-14-0$ & Phosphorus & 464 & ug/g dry & 57.8 & $5 / 22 / 08$ & 8E12001 & PNNL-AGG-ICP-AES \\
\hline 7439-92-1 & Lead & $<24.4$ & ug/g dry & 24.4 & $5 / 22 / 08$ & 8E12001 & PNNL-AGG-ICP-AES \\
\hline $7782-49-2$ & Selenium & $<107$ & ug/g dry & 107 & $5 / 22 / 08$ & 8E12001 & PNNL-AGG-ICP-AES \\
\hline $7440-24-6$ & Strontium & 21.9 & ug/g dry & 1.11 & $5 / 22 / 08$ & 8E12001 & PNNL-AGG-ICP-AES \\
\hline $7440-28-0$ & Thallium & $<34.7$ & ug/g dry & 34.7 & $5 / 22 / 08$ & 8E12001 & PNNL-AGG-ICP-AES \\
\hline $7440-62-2$ & Vanadium & 13.2 & ug/g dry & 2.86 & $5 / 22 / 08$ & 8E12001 & PNNL-AGG-ICP-AES \\
\hline $7440-66-6$ & Zinc & 24.3 & ug/g dry & 4.81 & $5 / 22 / 08$ & 8E12001 & PNNL-AGG-ICP-AES \\
\hline $7440-23-5$ & Sodium & $<822$ & ug/g dry & 822 & $5 / 22 / 08$ & 8E12001 & PNNL-AGG-ICP-AES \\
\hline $7440-21-3$ & Silicon & $<1070$ & ug/g dry & 1070 & $5 / 22 / 08$ & 8E12001 & PNNL-AGG-ICP-AES \\
\hline 7704-34-9 & Sulfur & $<182$ & ug/g dry & 182 & $5 / 22 / 08$ & 8E12001 & PNNL-AGG-ICP-AES \\
\hline $7440-32-6$ & Titanium & 475 & ug/g dry & 24.2 & $5 / 22 / 08$ & 8E12001 & PNNL-AGG-ICP-AES \\
\hline $7440-67-7$ & Zirconium & $<15.6$ & ug/g dry & 15.6 & $5 / 22 / 08$ & 8E12001 & PNNL-AGG-ICP-AES \\
\hline $7440-15-5$ & Rhenium & $<13.5$ & ug/g dry & 13.5 & $5 / 22 / 08$ & 8E12001 & PNNL-AGG-ICP-AES \\
\hline $7440-36-0$ & Antimony & $<85.9$ & ug/g dry & 85.9 & $5 / 22 / 08$ & 8E12001 & PNNL-AGG-ICP-AES \\
\hline HEIS No. & B1TNK8B & \multicolumn{3}{|c|}{ Lab ID: $\quad$ 0803015-26 } & & & \\
\hline $7429-90-5$ & Aluminum & 4860 & ug/g dry & 35.1 & $5 / 22 / 08$ & 8E12001 & PNNL-AGG-ICP-AES \\
\hline $7440-38-2$ & Arsenic & $<85.4$ & ug/g dry & 85.4 & $5 / 22 / 08$ & 8E12001 & PNNL-AGG-ICP-AES \\
\hline $7440-42-8$ & Boron & $<743$ & ug/g dry & 743 & $5 / 22 / 08$ & 8E12001 & PNNL-AGG-ICP-AES \\
\hline $7440-39-3$ & Barium & 45.8 & ug/g dry & 3.35 & $5 / 22 / 08$ & 8E12001 & PNNL-AGG-ICP-AES \\
\hline $7440-41-7$ & Beryllium & $<1.33$ & ug/g dry & 1.33 & $5 / 22 / 08$ & 8E12001 & PNNL-AGG-ICP-AES \\
\hline $7440-69-9$ & Bismuth & $<17.7$ & ug/g dry & 17.7 & $5 / 22 / 08$ & 8E12001 & PNNL-AGG-ICP-AES \\
\hline $7440-70-2$ & Calcium & 9460 & ug/g dry & 131 & $5 / 22 / 08$ & 8E12001 & PNNL-AGG-ICP-AES \\
\hline $7440-43-9$ & Cadmium & $<2.00$ & ug/g dry & 2.00 & $5 / 22 / 08$ & 8E12001 & PNNL-AGG-ICP-AES \\
\hline $7440-48-4$ & Cobalt & $<7.29$ & ug/g dry & 7.29 & $5 / 22 / 08$ & 8E12001 & PNNL-AGG-ICP-AES \\
\hline $7440-47-3$ & Chromium & 13.3 & ug/g dry & 2.92 & $5 / 22 / 08$ & 8E12001 & PNNL-AGG-ICP-AES \\
\hline $7440-50-8$ & Copper & $<31.1$ & ug/g dry & 31.1 & $5 / 22 / 08$ & 8E12001 & PNNL-AGG-ICP-AES \\
\hline 7439-89-6 & Iron & 11300 & ug/g dry & 89.3 & $5 / 22 / 08$ & 8E12001 & PNNL-AGG-ICP-AES \\
\hline $7440-09-7$ & Potassium & 725 & ug/g dry & 82.9 & $5 / 22 / 08$ & 8E12001 & PNNL-AGG-ICP-AES \\
\hline 7439-93-2 & Lithium & $<12.7$ & ug/g dry & 12.7 & $5 / 22 / 08$ & 8E12001 & PNNL-AGG-ICP-AES \\
\hline 7439-95-4 & Magnesium & 4170 & ug/g dry & 27.6 & $5 / 22 / 08$ & 8E12001 & PNNL-AGG-ICP-AES \\
\hline 7439-96-5 & Manganese & 238 & ug/g dry & 1.05 & $5 / 22 / 08$ & 8E12001 & PNNL-AGG-ICP-AES \\
\hline 7439-98-7 & Molybdenum & $<19.8$ & ug/g dry & 19.8 & $5 / 22 / 08$ & 8E12001 & PNNL-AGG-ICP-AES \\
\hline $7440-02-0$ & Nickel & 12.1 & ug/g dry & 7.18 & $5 / 22 / 08$ & 8E12001 & PNNL-AGG-ICP-AES \\
\hline $7723-14-0$ & Phosphorus & 446 & ug/g dry & 65.8 & $5 / 22 / 08$ & 8E12001 & PNNL-AGG-ICP-AES \\
\hline 7439-92-1 & Lead & $<27.8$ & ug/g dry & 27.8 & $5 / 22 / 08$ & 8E12001 & PNNL-AGG-ICP-AES \\
\hline $7782-49-2$ & Selenium & $<122$ & ug/g dry & 122 & $5 / 22 / 08$ & 8E12001 & PNNL-AGG-ICP-AES \\
\hline $7440-24-6$ & Strontium & 26.7 & ug/g dry & 1.27 & $5 / 22 / 08$ & 8E12001 & PNNL-AGG-ICP-AES \\
\hline $7440-28-0$ & Thallium & $<39.5$ & ug/g dry & 39.5 & $5 / 22 / 08$ & 8E12001 & PNNL-AGG-ICP-AES \\
\hline $7440-62-2$ & Vanadium & 12.6 & ug/g dry & 3.25 & $5 / 22 / 08$ & 8E12001 & PNNL-AGG-ICP-AES \\
\hline
\end{tabular}


Total Metals by SW846 6010B/Acid Extract

\begin{tabular}{|c|c|c|c|c|c|c|c|}
\hline CAS \# & Analyte & Results & Units & EQL & Analyzed & Batch & Method \\
\hline HEIS No. & B1TNK8B & \multicolumn{3}{|c|}{ Lab ID: $\quad$ 0803015-26 } & & & \\
\hline $7440-66-6$ & Zinc & 26.6 & ug/g dry & 5.48 & $5 / 22 / 08$ & 8E12001 & PNNL-AGG-ICP-AES \\
\hline $7440-23-5$ & Sodium & $<936$ & ug/g dry & 936 & $5 / 22 / 08$ & 8E12001 & PNNL-AGG-ICP-AES \\
\hline $7440-21-3$ & Silicon & $<1220$ & ug/g dry & 1220 & $5 / 22 / 08$ & 8E12001 & PNNL-AGG-ICP-AES \\
\hline 7704-34-9 & Sulfur & $<207$ & ug/g dry & 207 & $5 / 22 / 08$ & 8E12001 & PNNL-AGG-ICP-AES \\
\hline 7440-32-6 & Titanium & 457 & ug/g dry & 27.6 & $5 / 22 / 08$ & 8E12001 & PNNL-AGG-ICP-AES \\
\hline $7440-67-7$ & Zirconium & $<17.8$ & ug/g dry & 17.8 & $5 / 22 / 08$ & 8E12001 & PNNL-AGG-ICP-AES \\
\hline $7440-15-5$ & Rhenium & $<15.4$ & ug/g dry & 15.4 & $5 / 22 / 08$ & 8E12001 & PNNL-AGG-ICP-AES \\
\hline $7440-36-0$ & Antimony & $<97.8$ & ug/g dry & 97.8 & $5 / 22 / 08$ & 8E12001 & PNNL-AGG-ICP-AES \\
\hline HEIS No. & B1TNK8A & \multicolumn{3}{|c|}{ Lab ID: $\quad$ 0803015-27 } & & & \\
\hline $7429-90-5$ & Aluminum & 4900 & ug/g dry & 37.6 & $5 / 22 / 08$ & 8E12001 & PNNL-AGG-ICP-AES \\
\hline $7440-38-2$ & Arsenic & $<91.2$ & ug/g dry & 91.2 & $5 / 22 / 08$ & 8E12001 & PNNL-AGG-ICP-AES \\
\hline $7440-42-8$ & Boron & $<795$ & ug/g dry & 795 & $5 / 22 / 08$ & 8E12001 & PNNL-AGG-ICP-AES \\
\hline $7440-39-3$ & Barium & 41.7 & ug/g dry & 3.58 & $5 / 22 / 08$ & 8E12001 & PNNL-AGG-ICP-AES \\
\hline $7440-41-7$ & Beryllium & $<1.42$ & ug/g dry & 1.42 & $5 / 22 / 08$ & 8E12001 & PNNL-AGG-ICP-AES \\
\hline $7440-69-9$ & Bismuth & $<19.0$ & ug/g dry & 19.0 & $5 / 22 / 08$ & 8E12001 & PNNL-AGG-ICP-AES \\
\hline $7440-70-2$ & Calcium & 8050 & ug/g dry & 140 & $5 / 22 / 08$ & 8E12001 & PNNL-AGG-ICP-AES \\
\hline 7440-43-9 & Cadmium & $<2.13$ & ug/g dry & 2.13 & $5 / 22 / 08$ & 8E12001 & PNNL-AGG-ICP-AES \\
\hline $7440-48-4$ & Cobalt & $<7.79$ & ug/g dry & 7.79 & $5 / 22 / 08$ & 8E12001 & PNNL-AGG-ICP-AES \\
\hline $7440-47-3$ & Chromium & 10.2 & ug/g dry & 3.12 & $5 / 22 / 08$ & 8E12001 & PNNL-AGG-ICP-AES \\
\hline $7440-50-8$ & Copper & $<33.3$ & ug/g dry & 33.3 & $5 / 22 / 08$ & 8E12001 & PNNL-AGG-ICP-AES \\
\hline 7439-89-6 & Iron & 10500 & ug/g dry & 95.4 & $5 / 22 / 08$ & 8E12001 & PNNL-AGG-ICP-AES \\
\hline 7440-09-7 & Potassium & 847 & ug/g dry & 88.6 & $5 / 22 / 08$ & 8E12001 & PNNL-AGG-ICP-AES \\
\hline 7439-93-2 & Lithium & $<13.5$ & ug/g dry & 13.5 & $5 / 22 / 08$ & 8E12001 & PNNL-AGG-ICP-AES \\
\hline $7439-95-4$ & Magnesium & 3900 & ug/g dry & 29.5 & $5 / 22 / 08$ & 8E12001 & PNNL-AGG-ICP-AES \\
\hline 7439-96-5 & Manganese & 209 & ug/g dry & 1.12 & $5 / 22 / 08$ & 8E12001 & PNNL-AGG-ICP-AES \\
\hline 7439-98-7 & Molybdenum & $<21.2$ & ug/g dry & 21.2 & $5 / 22 / 08$ & 8E12001 & PNNL-AGG-ICP-AES \\
\hline $7440-02-0$ & Nickel & 9.75 & ug/g dry & 7.68 & $5 / 22 / 08$ & 8E12001 & PNNL-AGG-ICP-AES \\
\hline 7723-14-0 & Phosphorus & 431 & ug/g dry & 70.3 & $5 / 22 / 08$ & 8E12001 & PNNL-AGG-ICP-AES \\
\hline 7439-92-1 & Lead & $<29.7$ & ug/g dry & 29.7 & $5 / 22 / 08$ & 8E12001 & PNNL-AGG-ICP-AES \\
\hline $7782-49-2$ & Selenium & $<130$ & ug/g dry & 130 & $5 / 22 / 08$ & 8E12001 & PNNL-AGG-ICP-AES \\
\hline $7440-24-6$ & Strontium & 24.1 & ug/g dry & 1.35 & $5 / 22 / 08$ & 8E12001 & PNNL-AGG-ICP-AES \\
\hline $7440-28-0$ & Thallium & $<42.2$ & ug/g dry & 42.2 & $5 / 22 / 08$ & 8E12001 & PNNL-AGG-ICP-AES \\
\hline $7440-62-2$ & Vanadium & 11.6 & ug/g dry & 3.47 & $5 / 22 / 08$ & 8E12001 & PNNL-AGG-ICP-AES \\
\hline $7440-66-6$ & Zinc & 27.2 & ug/g dry & 5.85 & $5 / 22 / 08$ & 8E12001 & PNNL-AGG-ICP-AES \\
\hline $7440-23-5$ & Sodium & $<1000$ & ug/g dry & 1000 & $5 / 22 / 08$ & 8E12001 & PNNL-AGG-ICP-AES \\
\hline $7440-21-3$ & Silicon & $<1300$ & ug/g dry & 1300 & $5 / 22 / 08$ & 8E12001 & PNNL-AGG-ICP-AES \\
\hline $7704-34-9$ & Sulfur & $<221$ & ug/g dry & 221 & $5 / 22 / 08$ & 8E12001 & PNNL-AGG-ICP-AES \\
\hline $7440-32-6$ & Titanium & 439 & ug/g dry & 29.5 & $5 / 22 / 08$ & 8E12001 & PNNL-AGG-ICP-AES \\
\hline $7440-67-7$ & Zirconium & $<19.0$ & ug/g dry & 19.0 & $5 / 22 / 08$ & 8E12001 & PNNL-AGG-ICP-AES \\
\hline $7440-15-5$ & Rhenium & $<16.5$ & ug/g dry & 16.5 & $5 / 22 / 08$ & 8E12001 & PNNL-AGG-ICP-AES \\
\hline $7440-36-0$ & Antimony & $<105$ & ug/g dry & 105 & $5 / 22 / 08$ & 8E12001 & PNNL-AGG-ICP-AES \\
\hline HEIS No. & B1TNK9B & \multicolumn{3}{|c|}{ Lab ID: $\quad$ 0803015-30 } & & & \\
\hline $7429-90-5$ & Aluminum & 5260 & ug/g dry & 36.7 & $5 / 22 / 08$ & 8E12001 & PNNL-AGG-ICP-AES \\
\hline $7440-38-2$ & Arsenic & $<89.1$ & ug/g dry & 89.1 & $5 / 22 / 08$ & 8E12001 & PNNL-AGG-ICP-AES \\
\hline $7440-42-8$ & Boron & $<776$ & ug/g dry & 776 & $5 / 22 / 08$ & 8E12001 & PNNL-AGG-ICP-AES \\
\hline $7440-39-3$ & Barium & 52.6 & ug/g dry & 3.50 & $5 / 27 / 08$ & 8E12001 & PNNL-AGG-ICP-AES \\
\hline $7440-41-7$ & Beryllium & $<1.39$ & ug/g dry & 1.39 & $5 / 22 / 08$ & 8E12001 & PNNL-AGG-ICP-AES \\
\hline $7440-69-9$ & Bismuth & $<18.5$ & ug/g dry & 18.5 & $5 / 22 / 08$ & 8E12001 & PNNL-AGG-ICP-AES \\
\hline
\end{tabular}


Total Metals by SW846 6010B/Acid Extract

\begin{tabular}{|c|c|c|c|c|c|c|c|}
\hline CAS \# & Analyte & Results & Units & EQL & Analyzed & Batch & Method \\
\hline HEIS No. & B1TNK9B & \multicolumn{3}{|c|}{ Lab ID: $\quad$ 0803015-30 } & & & \\
\hline $7440-70-2$ & Calcium & 7540 & ug/g dry & 136 & $5 / 22 / 08$ & 8E12001 & PNNL-AGG-ICP-AES \\
\hline 7440-43-9 & Cadmium & $<2.08$ & ug/g dry & 2.08 & $5 / 22 / 08$ & 8E12001 & PNNL-AGG-ICP-AES \\
\hline $7440-48-4$ & Cobalt & $<7.61$ & ug/g dry & 7.61 & $5 / 22 / 08$ & 8E12001 & PNNL-AGG-ICP-AES \\
\hline $7440-47-3$ & Chromium & 10.3 & ug/g dry & 3.05 & $5 / 22 / 08$ & 8E12001 & PNNL-AGG-ICP-AES \\
\hline $7440-50-8$ & Copper & $<32.5$ & ug/g dry & 32.5 & $5 / 22 / 08$ & 8E12001 & PNNL-AGG-ICP-AES \\
\hline 7439-89-6 & Iron & 12100 & ug/g dry & 93.2 & $5 / 22 / 08$ & 8E12001 & PNNL-AGG-ICP-AES \\
\hline 7440-09-7 & Potassium & 871 & ug/g dry & 86.5 & $5 / 22 / 08$ & 8E12001 & PNNL-AGG-ICP-AES \\
\hline 7439-93-2 & Lithium & $<13.2$ & ug/g dry & 13.2 & $5 / 22 / 08$ & 8E12001 & PNNL-AGG-ICP-AES \\
\hline 7439-95-4 & Magnesium & 4080 & ug/g dry & 28.9 & $5 / 22 / 08$ & 8E12001 & PNNL-AGG-ICP-AES \\
\hline $7439-96-5$ & Manganese & 230 & ug/g dry & 1.09 & $5 / 22 / 08$ & 8E12001 & PNNL-AGG-ICP-AES \\
\hline 7439-98-7 & Molybdenum & $<20.7$ & ug/g dry & 20.7 & $5 / 22 / 08$ & 8E12001 & PNNL-AGG-ICP-AES \\
\hline $7440-02-0$ & Nickel & 11.1 & ug/g dry & 7.50 & $5 / 22 / 08$ & 8E12001 & PNNL-AGG-ICP-AES \\
\hline $7723-14-0$ & Phosphorus & 500 & ug/g dry & 68.7 & $5 / 22 / 08$ & 8E12001 & PNNL-AGG-ICP-AES \\
\hline 7439-92-1 & Lead & $<29.0$ & ug/g dry & 29.0 & $5 / 22 / 08$ & 8E12001 & PNNL-AGG-ICP-AES \\
\hline $7782-49-2$ & Selenium & $<127$ & ug/g dry & 127 & $5 / 22 / 08$ & 8E12001 & PNNL-AGG-ICP-AES \\
\hline $7440-24-6$ & Strontium & 21.8 & ug/g dry & 1.32 & $5 / 22 / 08$ & 8E12001 & PNNL-AGG-ICP-AES \\
\hline $7440-28-0$ & Thallium & $<41.2$ & ug/g dry & 41.2 & $5 / 22 / 08$ & 8E12001 & PNNL-AGG-ICP-AES \\
\hline $7440-62-2$ & Vanadium & 16.8 & ug/g dry & 3.40 & $5 / 22 / 08$ & 8E12001 & PNNL-AGG-ICP-AES \\
\hline $7440-66-6$ & Zinc & 34.1 & ug/g dry & 5.72 & $5 / 22 / 08$ & 8E12001 & PNNL-AGG-ICP-AES \\
\hline $7440-23-5$ & Sodium & $<978$ & ug/g dry & 978 & $5 / 22 / 08$ & 8E12001 & PNNL-AGG-ICP-AES \\
\hline $7440-21-3$ & Silicon & $<1270$ & ug/g dry & 1270 & $5 / 22 / 08$ & 8E12001 & PNNL-AGG-ICP-AES \\
\hline 7704-34-9 & Sulfur & $<216$ & ug/g dry & 216 & $5 / 22 / 08$ & 8E12001 & PNNL-AGG-ICP-AES \\
\hline $7440-32-6$ & Titanium & 575 & ug/g dry & 28.8 & $5 / 22 / 08$ & 8E12001 & PNNL-AGG-ICP-AES \\
\hline $7440-67-7$ & Zirconium & $<18.6$ & ug/g dry & 18.6 & $5 / 22 / 08$ & 8E12001 & PNNL-AGG-ICP-AES \\
\hline $7440-15-5$ & Rhenium & $<16.1$ & ug/g dry & 16.1 & $5 / 22 / 08$ & 8E12001 & PNNL-AGG-ICP-AES \\
\hline $7440-36-0$ & Antimony & $<102$ & ug/g dry & 102 & $5 / 22 / 08$ & 8E12001 & PNNL-AGG-ICP-AES \\
\hline HEIS No. & B1TNK9A & \multicolumn{3}{|c|}{ Lab ID: $\quad$ 0803015-31 } & & & \\
\hline $7429-90-5$ & Aluminum & 5590 & ug/g dry & 37.3 & $5 / 22 / 08$ & 8E12001 & PNNL-AGG-ICP-AES \\
\hline $7440-38-2$ & Arsenic & $<90.5$ & ug/g dry & 90.5 & $5 / 22 / 08$ & 8E12001 & PNNL-AGG-ICP-AES \\
\hline $7440-42-8$ & Boron & $<788$ & ug/g dry & 788 & $5 / 22 / 08$ & 8E12001 & PNNL-AGG-ICP-AES \\
\hline $7440-39-3$ & Barium & 50.6 & ug/g dry & 3.55 & $5 / 27 / 08$ & 8E12001 & PNNL-AGG-ICP-AES \\
\hline $7440-41-7$ & Beryllium & $<1.41$ & ug/g dry & 1.41 & $5 / 22 / 08$ & 8E12001 & PNNL-AGG-ICP-AES \\
\hline $7440-69-9$ & Bismuth & $<18.8$ & ug/g dry & 18.8 & $5 / 22 / 08$ & 8E12001 & PNNL-AGG-ICP-AES \\
\hline $7440-70-2$ & Calcium & 6850 & ug/g dry & 138 & $5 / 22 / 08$ & 8E12001 & PNNL-AGG-ICP-AES \\
\hline $7440-43-9$ & Cadmium & $<2.12$ & ug/g dry & 2.12 & $5 / 22 / 08$ & 8E12001 & PNNL-AGG-ICP-AES \\
\hline $7440-48-4$ & Cobalt & $<7.73$ & ug/g dry & 7.73 & $5 / 22 / 08$ & 8E12001 & PNNL-AGG-ICP-AES \\
\hline $7440-47-3$ & Chromium & 12.2 & ug/g dry & 3.10 & $5 / 22 / 08$ & 8E12001 & PNNL-AGG-ICP-AES \\
\hline $7440-50-8$ & Copper & $<33.0$ & ug/g dry & 33.0 & $5 / 22 / 08$ & 8E12001 & PNNL-AGG-ICP-AES \\
\hline 7439-89-6 & Iron & 13300 & ug/g dry & 94.7 & $5 / 22 / 08$ & 8E12001 & PNNL-AGG-ICP-AES \\
\hline $7440-09-7$ & Potassium & 834 & ug/g dry & 87.9 & $5 / 22 / 08$ & 8E12001 & PNNL-AGG-ICP-AES \\
\hline $7439-93-2$ & Lithium & $<13.4$ & ug/g dry & 13.4 & $5 / 22 / 08$ & 8E12001 & PNNL-AGG-ICP-AES \\
\hline 7439-95-4 & Magnesium & 4370 & ug/g dry & 29.3 & $5 / 22 / 08$ & 8E12001 & PNNL-AGG-ICP-AES \\
\hline $7439-96-5$ & Manganese & 246 & ug/g dry & 1.11 & $5 / 22 / 08$ & 8E12001 & PNNL-AGG-ICP-AES \\
\hline 7439-98-7 & Molybdenum & $<21.0$ & ug/g dry & 21.0 & $5 / 22 / 08$ & 8E12001 & PNNL-AGG-ICP-AES \\
\hline $7440-02-0$ & Nickel & 12.8 & ug/g dry & 7.62 & $5 / 22 / 08$ & 8E12001 & PNNL-AGG-ICP-AES \\
\hline $7723-14-0$ & Phosphorus & 514 & ug/g dry & 69.8 & $5 / 22 / 08$ & 8E12001 & PNNL-AGG-ICP-AES \\
\hline 7439-92-1 & Lead & $<29.5$ & ug/g dry & 29.5 & $5 / 22 / 08$ & 8E12001 & PNNL-AGG-ICP-AES \\
\hline $7782-49-2$ & Selenium & $<129$ & ug/g dry & 129 & $5 / 22 / 08$ & 8E12001 & PNNL-AGG-ICP-AES \\
\hline
\end{tabular}


Total Metals by SW846 6010B/Acid Extract

\begin{tabular}{|c|c|c|c|c|c|c|c|}
\hline CAS \# & Analyte & Results & Units & EQL & Analyzed & Batch & Method \\
\hline HEIS No. & B1TNK9A & \multicolumn{3}{|c|}{ Lab ID: $\quad$ 0803015-31 } & & & \\
\hline $7440-24-6$ & Strontium & 21.0 & ug/g dry & 1.34 & $5 / 22 / 08$ & 8E12001 & PNNL-AGG-ICP-AES \\
\hline 7440-28-0 & Thallium & $<41.9$ & ug/g dry & 41.9 & $5 / 22 / 08$ & 8E12001 & PNNL-AGG-ICP-AES \\
\hline $7440-62-2$ & Vanadium & 18.7 & ug/g dry & 3.45 & $5 / 22 / 08$ & 8E12001 & PNNL-AGG-ICP-AES \\
\hline $7440-66-6$ & Zinc & 30.4 & ug/g dry & 5.81 & $5 / 22 / 08$ & 8E12001 & PNNL-AGG-ICP-AES \\
\hline $7440-23-5$ & Sodium & $<993$ & ug/g dry & 993 & $5 / 22 / 08$ & 8E12001 & PNNL-AGG-ICP-AES \\
\hline $7440-21-3$ & Silicon & $<1290$ & ug/g dry & 1290 & $5 / 22 / 08$ & 8E12001 & PNNL-AGG-ICP-AES \\
\hline 7704-34-9 & Sulfur & $<219$ & ug/g dry & 219 & $5 / 22 / 08$ & 8E12001 & PNNL-AGG-ICP-AES \\
\hline $7440-32-6$ & Titanium & 643 & ug/g dry & 29.2 & $5 / 22 / 08$ & 8E12001 & PNNL-AGG-ICP-AES \\
\hline $7440-67-7$ & Zirconium & $<18.9$ & ug/g dry & 18.9 & $5 / 22 / 08$ & 8E12001 & PNNL-AGG-ICP-AES \\
\hline $7440-15-5$ & Rhenium & $<16.3$ & ug/g dry & 16.3 & $5 / 22 / 08$ & 8E12001 & PNNL-AGG-ICP-AES \\
\hline $7440-36-0$ & Antimony & $<104$ & ug/g dry & 104 & $5 / 22 / 08$ & 8E12001 & PNNL-AGG-ICP-AES \\
\hline
\end{tabular}




\section{Radionuclides by ICP-MS/Acid Extract}

\begin{tabular}{|c|c|c|c|c|c|c|c|}
\hline CAS \# & Analyte & Results & Units & EQL & Analyzed & Batch & Method \\
\hline HEIS No. & B1RTF8 & \multicolumn{3}{|c|}{ Lab ID: $\quad$ 0803001-01 } & & & \\
\hline \multirow[t]{2}{*}{ 14133-76-7 } & Technetium-99 & $<0.004$ & ug/g dry & 0.004 & 4/16/08 & 8D16002 & PNNL-AGG-415 \\
\hline & Uranium 238 & 0.330 & ug/g dry & 0.028 & 4/16/08 & 8D16002 & PNNL-AGG-415 \\
\hline HEIS No. & B1RTF9A & \multicolumn{3}{|c|}{ Lab ID: $\quad$ 0803001-02 } & & & \\
\hline \multirow[t]{2}{*}{$14133-76-7$} & Technetium-99 & $<0.003$ & ug/g dry & 0.003 & 4/16/08 & 8D16002 & PNNL-AGG-415 \\
\hline & Uranium 238 & 0.395 & ug/g dry & 0.024 & $4 / 16 / 08$ & 8D16002 & PNNL-AGG-415 \\
\hline HEIS No. & B1RTH1A & \multicolumn{3}{|c|}{ Lab ID: $\quad$ 0803012-02 } & & & \\
\hline \multirow[t]{2}{*}{$14133-76-7$} & Technetium-99 & $<0.003$ & ug/g dry & 0.003 & 4/16/08 & 8D16002 & PNNL-AGG-415 \\
\hline & Uranium 238 & 0.324 & ug/g dry & 0.023 & $4 / 16 / 08$ & 8D16002 & PNNL-AGG-415 \\
\hline HEIS No. & B1RTH1 & \multicolumn{3}{|c|}{ Lab ID: $\quad$ 0803012-03 } & & & \\
\hline \multirow[t]{2}{*}{$14133-76-7$} & Technetium-99 & $<0.003$ & ug/g dry & 0.003 & $4 / 16 / 08$ & 8D16002 & PNNL-AGG-415 \\
\hline & Uranium 238 & 1.47 & ug/g dry & 0.025 & $4 / 16 / 08$ & 8D16002 & PNNL-AGG-415 \\
\hline HEIS No. & B1RTH2B & \multicolumn{3}{|c|}{ Lab ID: $\quad$ 0803012-05 } & & & \\
\hline \multirow[t]{2}{*}{ 14133-76-7 } & Technetium-99 & $<0.004$ & ug/g dry & 0.004 & 4/16/08 & 8D16002 & PNNL-AGG-415 \\
\hline & Uranium 238 & 0.413 & ug/g dry & 0.026 & $4 / 16 / 08$ & 8D16002 & PNNL-AGG-415 \\
\hline HEIS No. & B1RTH2A & \multicolumn{3}{|c|}{ Lab ID: $\quad$ 0803012-06 } & & & \\
\hline \multirow[t]{2}{*}{ 14133-76-7 } & Technetium-99 & $<0.004$ & ug/g dry & 0.004 & 4/16/08 & 8D16002 & PNNL-AGG-415 \\
\hline & Uranium 238 & 0.375 & ug/g dry & 0.029 & $4 / 16 / 08$ & 8D16002 & PNNL-AGG-415 \\
\hline HEIS No. & B1RTH3B & \multicolumn{3}{|c|}{ Lab ID: $\quad$ 0803012-09 } & & & \\
\hline \multirow[t]{2}{*}{$14133-76-7$} & Technetium-99 & $<0.004$ & ug/g dry & 0.004 & 4/16/08 & 8D16002 & PNNL-AGG-415 \\
\hline & Uranium 238 & 0.379 & ug/g dry & 0.027 & $4 / 16 / 08$ & 8D16002 & PNNL-AGG-415 \\
\hline \multirow{3}{*}{$\begin{array}{l}\text { HEIS No. } \\
14133-76-7\end{array}$} & B1RTH3A & \multicolumn{3}{|c|}{ Lab ID: $\quad$ 0803012-10 } & & & \\
\hline & Technetium-99 & $<0.004$ & ug/g dry & 0.004 & 4/16/08 & 8D16002 & PNNL-AGG-415 \\
\hline & Uranium 238 & 0.385 & ug/g dry & 0.028 & $4 / 16 / 08$ & 8D16002 & PNNL-AGG-415 \\
\hline \multirow{3}{*}{$\begin{array}{l}\text { HEIS No. } \\
14133-76-7\end{array}$} & B1RYR7B & \multicolumn{3}{|c|}{ Lab ID: $\quad$ 0803012-13 } & & & \\
\hline & Technetium-99 & $<0.004$ & ug/g dry & 0.004 & $4 / 16 / 08$ & 8D16002 & PNNL-AGG-415 \\
\hline & Uranium 238 & 0.314 & ug/g dry & 0.028 & $4 / 16 / 08$ & 8D16002 & PNNL-AGG-415 \\
\hline \multirow{3}{*}{$\begin{array}{l}\text { HEIS No. } \\
14133-76-7\end{array}$} & B1RYR7A & \multicolumn{3}{|c|}{ Lab ID: $\quad$ 0803012-14 } & & & \\
\hline & Technetium-99 & $<0.004$ & ug/g dry & 0.004 & 4/16/08 & 8D16002 & PNNL-AGG-415 \\
\hline & Uranium 238 & 0.352 & ug/g dry & 0.029 & $4 / 16 / 08$ & 8D16002 & PNNL-AGG-415 \\
\hline \multirow{3}{*}{$\begin{array}{l}\text { HEIS No. } \\
14133-76-7\end{array}$} & B1RYR8B & & b ID: & 0803012-17 & & & \\
\hline & Technetium-99 & $<0.004$ & ug/g dry & 0.004 & $4 / 16 / 08$ & 8D16002 & PNNL-AGG-415 \\
\hline & Uranium 238 & 0.376 & ug/g dry & 0.032 & $4 / 16 / 08$ & 8D16002 & PNNL-AGG-415 \\
\hline HEIS No. & B1RYR8A & & b ID: & 0803012-18 & & & \\
\hline 14133-76-7 & Technetium-99 & $<0.004$ & ug/g dry & 0.004 & $4 / 16 / 08$ & 8D16002 & PNNL-AGG-415 \\
\hline & Uranium 238 & 0.690 & ug/g dry & 0.026 & $4 / 16 / 08$ & 8D16002 & PNNL-AGG-415 \\
\hline HEIS No. & B1RYR9B & & b ID: & 0803012-21 & & & \\
\hline 14133-76-7 & Technetium-99 & $<0.004$ & ug/g dry & 0.004 & 4/16/08 & 8D16002 & PNNL-AGG-415 \\
\hline & Uranium 238 & 0.358 & ug/g dry & 0.027 & $4 / 16 / 08$ & 8D16002 & PNNL-AGG-415 \\
\hline HEIS No. & B1RYR9A & & b ID: & 0803012-22 & & & \\
\hline $14133-76-7$ & Technetium-99 & $<0.004$ & ug/g dry & 0.004 & $4 / 16 / 08$ & 8D16002 & PNNL-AGG-415 \\
\hline & Uranium 238 & 0.328 & ug/g dry & 0.026 & 4/16/08 & 8D16002 & PNNL-AGG-415 \\
\hline HEIS No. & B1RYT0B & & b ID: & 0803014-01 & & & \\
\hline 14133-76-7 & Technetium-99 & $<0.004$ & ug/g dry & 0.004 & 4/16/08 & 8D16002 & PNNL-AGG-415 \\
\hline & Uranium 238 & 0.415 & ug/g dry & 0.032 & 4/16/08 & 8D16002 & PNNL-AGG-415 \\
\hline HEIS No. & B1RYT0A & & b ID: & 0803014-02 & & & \\
\hline $14133-76-7$ & Technetium-99 & $<0.004$ & ug/g dry & 0.004 & 4/16/08 & 8D16002 & PNNL-AGG-415 \\
\hline
\end{tabular}




\section{Radionuclides by ICP-MS/Acid Extract}

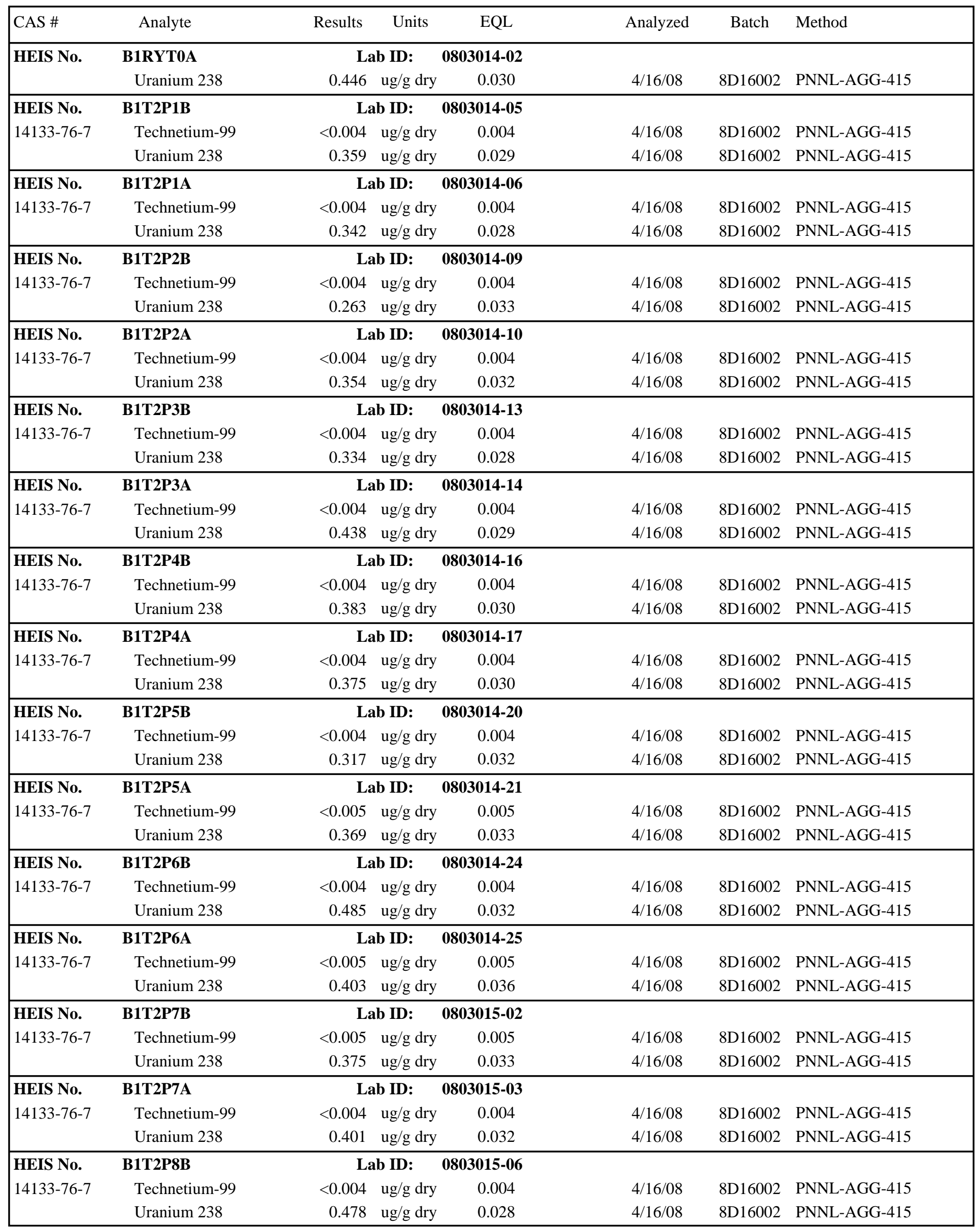




\section{Radionuclides by ICP-MS/Acid Extract}

\begin{tabular}{|c|c|c|c|c|c|c|c|}
\hline CAS \# & Analyte & Results & Units & EQL & Analyzed & Batch & Method \\
\hline HEIS No. & B1T2P8A & \multicolumn{3}{|c|}{ Lab ID: $\quad$ 0803015-07 } & & & \\
\hline \multirow[t]{2}{*}{ 14133-76-7 } & Technetium-99 & $<0.004$ & ug/g dry & 0.004 & $4 / 16 / 08$ & 8D16002 & PNNL-AGG-415 \\
\hline & Uranium 238 & 0.405 & ug/g dry & 0.029 & $4 / 16 / 08$ & 8D16002 & PNNL-AGG-415 \\
\hline HEIS No. & В1Т2Р9B & \multicolumn{3}{|c|}{ Lab ID: $\quad$ 0803015-10 } & & & \\
\hline \multirow[t]{2}{*}{$14133-76-7$} & Technetium-99 & $<0.004$ & ug/g dry & 0.004 & $4 / 16 / 08$ & 8D16002 & PNNL-AGG-415 \\
\hline & Uranium 238 & 0.346 & ug/g dry & 0.027 & $4 / 16 / 08$ & 8D16002 & PNNL-AGG-415 \\
\hline HEIS No. & B1T2P9A & \multicolumn{3}{|c|}{ Lab ID: $\quad$ 0803015-11 } & & & \\
\hline \multirow[t]{2}{*}{ 14133-76-7 } & Technetium-99 & $<0.003$ & ug/g dry & 0.003 & $4 / 16 / 08$ & 8D16002 & PNNL-AGG-415 \\
\hline & Uranium 238 & 0.367 & ug/g dry & 0.025 & $4 / 16 / 08$ & 8D16002 & PNNL-AGG-415 \\
\hline HEIS No. & B1T2R0B & \multicolumn{3}{|c|}{ Lab ID: $\quad$ 0803015-14 } & & & \\
\hline \multirow[t]{2}{*}{$14133-76-7$} & Technetium-99 & $<0.005$ & ug/g dry & 0.005 & $4 / 16 / 08$ & 8D16002 & PNNL-AGG-415 \\
\hline & Uranium 238 & 0.414 & ug/g dry & 0.037 & 4/16/08 & 8D16002 & PNNL-AGG-415 \\
\hline HEIS No. & B1T2R0A & \multicolumn{3}{|c|}{ Lab ID: $\quad$ 0803015-15 } & & & \\
\hline \multirow[t]{2}{*}{$14133-76-7$} & Technetium-99 & $<0.004$ & ug/g dry & 0.004 & 4/16/08 & 8D16002 & PNNL-AGG-415 \\
\hline & Uranium 238 & 0.435 & ug/g dry & 0.026 & $4 / 16 / 08$ & 8D16002 & PNNL-AGG-415 \\
\hline HEIS No. & B1TNK6B & \multicolumn{3}{|c|}{ Lab ID: $\quad$ 0803015-18 } & & & \\
\hline \multirow[t]{2}{*}{$14133-76-7$} & Technetium-99 & $<0.004$ & ug/g dry & 0.004 & 4/16/08 & 8D16002 & PNNL-AGG-415 \\
\hline & Uranium 238 & 0.355 & ug/g dry & 0.031 & $4 / 16 / 08$ & 8D16002 & PNNL-AGG-415 \\
\hline HEIS No. & B1TNK6A & \multicolumn{3}{|c|}{ Lab ID: $\quad$ 0803015-19 } & & & \\
\hline \multirow[t]{2}{*}{$14133-76-7$} & Technetium-99 & $<0.004$ & ug/g dry & 0.004 & 4/16/08 & 8D16002 & PNNL-AGG-415 \\
\hline & Uranium 238 & 0.298 & ug/g dry & 0.030 & 4/16/08 & 8D16002 & PNNL-AGG-415 \\
\hline HEIS No. & B1TNK7B & \multicolumn{3}{|c|}{ Lab ID: $\quad$ 0803015-22 } & & & \\
\hline \multirow[t]{2}{*}{ 14133-76-7 } & Technetium-99 & $<0.004$ & ug/g dry & 0.004 & $4 / 17 / 08$ & 8D16002 & PNNL-AGG-415 \\
\hline & Uranium 238 & 0.401 & ug/g dry & 0.029 & $4 / 17 / 08$ & 8D16002 & PNNL-AGG-415 \\
\hline HEIS No. & B1TNK7A & \multicolumn{3}{|c|}{ Lab ID: $\quad$ 0803015-23 } & & & \\
\hline \multirow[t]{2}{*}{$14133-76-7$} & Technetium-99 & $<0.004$ & ug/g dry & 0.004 & $4 / 17 / 08$ & 8D16002 & PNNL-AGG-415 \\
\hline & Uranium 238 & 0.368 & ug/g dry & 0.027 & $4 / 17 / 08$ & 8D16002 & PNNL-AGG-415 \\
\hline HEIS No. & B1TNK8B & \multicolumn{3}{|c|}{ Lab ID: $\quad$ 0803015-26 } & & & \\
\hline \multirow[t]{2}{*}{ 14133-76-7 } & Technetium-99 & $<0.004$ & ug/g dry & 0.004 & $4 / 17 / 08$ & 8D16002 & PNNL-AGG-415 \\
\hline & Uranium 238 & 0.351 & ug/g dry & 0.031 & $4 / 17 / 08$ & 8D16002 & PNNL-AGG-415 \\
\hline HEIS No. & B1TNK8A & \multicolumn{3}{|c|}{ Lab ID: $\quad$ 0803015-27 } & & & \\
\hline \multirow[t]{2}{*}{$14133-76-7$} & Technetium-99 & $<0.005$ & ug/g dry & 0.005 & $4 / 17 / 08$ & 8D16002 & PNNL-AGG-415 \\
\hline & Uranium 238 & 0.351 & ug/g dry & 0.033 & $4 / 17 / 08$ & 8D16002 & PNNL-AGG-415 \\
\hline HEIS No. & B1TNK9B & \multicolumn{3}{|c|}{ Lab ID: $\quad$ 0803015-30 } & & & \\
\hline \multirow[t]{2}{*}{$14133-76-7$} & Technetium-99 & $<0.004$ & ug/g dry & 0.004 & $4 / 17 / 08$ & 8D16002 & PNNL-AGG-415 \\
\hline & Uranium 238 & 0.381 & ug/g dry & 0.032 & $4 / 17 / 08$ & 8D16002 & PNNL-AGG-415 \\
\hline HEIS No. & B1TNK9A & \multicolumn{3}{|c|}{ Lab ID: $\quad$ 0803015-31 } & & & \\
\hline 14133-76-7 & Technetium-99 & $<0.004$ & ug/g dry & 0.004 & $4 / 17 / 08$ & 8D16002 & PNNL-AGG-415 \\
\hline & Uranium 238 & 0.432 & ug/g dry & 0.033 & $4 / 17 / 08$ & 8D16002 & PNNL-AGG-415 \\
\hline
\end{tabular}




\section{Radionuclides by ICP-MS/Water Extract}

\begin{tabular}{|c|c|c|c|c|c|c|c|}
\hline CAS \# & Analyte & Results & Units & EQL & Analyzed & Batch & Method \\
\hline \multirow{3}{*}{$\begin{array}{l}\text { HEIS No. } \\
14133-76-7\end{array}$} & B1RTF8 & \multicolumn{3}{|c|}{ Lab ID: $\quad$ 0803001-01 } & & & \\
\hline & Technetium-99 & $<0.00002$ & ug/g dry & 0.00002 & $4 / 10 / 08$ & 8D10003 & PNNL-AGG-415 \\
\hline & Uranium 238 & 0.0009 & ug/g dry & 0.0006 & $4 / 10 / 08$ & 8D10003 & PNNL-AGG-415 \\
\hline \multirow{3}{*}{$\begin{array}{l}\text { HEIS No. } \\
14133-76-7\end{array}$} & B1RTF9A & \multicolumn{3}{|c|}{ Lab ID: $\quad$ 0803001-02 } & & & \\
\hline & Technetium-99 & $<0.00002$ & ug/g dry & 0.00002 & $4 / 10 / 08$ & 8D10003 & PNNL-AGG-415 \\
\hline & Uranium 238 & 0.003 & ug/g dry & 0.0006 & $4 / 10 / 08$ & 8D10003 & PNNL-AGG-415 \\
\hline \multirow{3}{*}{$\begin{array}{l}\text { HEIS No. } \\
14133-76-7\end{array}$} & B1RTH1A & \multicolumn{3}{|c|}{ Lab ID: $\quad$ 0803012-02 } & & & \\
\hline & Technetium-99 & $<0.00002$ & ug/g dry & 0.00002 & $4 / 10 / 08$ & 8D10003 & PNNL-AGG-415 \\
\hline & Uranium 238 & $<0.0006$ & ug/g dry & 0.0006 & $4 / 10 / 08$ & 8D10003 & PNNL-AGG-415 \\
\hline \multirow{3}{*}{$\begin{array}{l}\text { HEIS No. } \\
14133-76-7\end{array}$} & B1RTH1 & \multicolumn{3}{|c|}{ Lab ID: $\quad$ 0803012-03 } & & & \\
\hline & Technetium-99 & $<0.00002$ & ug/g dry & 0.00002 & $4 / 10 / 08$ & 8D10003 & PNNL-AGG-415 \\
\hline & Uranium 238 & 0.046 & ug/g dry & 0.0006 & $4 / 10 / 08$ & 8D10003 & PNNL-AGG-415 \\
\hline \multirow{3}{*}{$\begin{array}{l}\text { HEIS No. } \\
14133-76-7\end{array}$} & B1RTH2B & \multicolumn{3}{|c|}{ Lab ID: $\quad$ 0803012-05 } & & & \\
\hline & Technetium-99 & $<0.00002$ & ug/g dry & 0.00002 & $4 / 10 / 08$ & 8D10003 & PNNL-AGG-415 \\
\hline & Uranium 238 & $<0.0006$ & ug/g dry & 0.0006 & $4 / 10 / 08$ & 8D10003 & PNNL-AGG-415 \\
\hline \multirow{3}{*}{$\begin{array}{l}\text { HEIS No. } \\
14133-76-7\end{array}$} & B1RTH2A & \multicolumn{3}{|c|}{ Lab ID: $\quad$ 0803012-06 } & & & \\
\hline & Technetium-99 & 0.00003 & ug/g dry & 0.00002 & $4 / 10 / 08$ & 8D10003 & PNNL-AGG-415 \\
\hline & Uranium 238 & $<0.0006$ & ug/g dry & 0.0006 & $4 / 10 / 08$ & 8D10003 & PNNL-AGG-415 \\
\hline \multirow{3}{*}{$\begin{array}{l}\text { HEIS No. } \\
14133-76-7\end{array}$} & B1RTH3B & \multicolumn{3}{|c|}{ Lab ID: $\quad$ 0803012-09 } & & & \\
\hline & Technetium-99 & $<0.00002$ & ug/g dry & 0.00002 & $4 / 10 / 08$ & 8D10003 & PNNL-AGG-415 \\
\hline & Uranium 238 & $<0.0006$ & ug/g dry & 0.0006 & $4 / 10 / 08$ & 8D10003 & PNNL-AGG-415 \\
\hline \multirow{3}{*}{$\begin{array}{l}\text { HEIS No. } \\
14133-76-7\end{array}$} & B1RTH3A & \multicolumn{3}{|c|}{ Lab ID: $\quad$ 0803012-10 } & & & \\
\hline & Technetium-99 & $<0.00002$ & ug/g dry & 0.00002 & $4 / 10 / 08$ & 8D10003 & PNNL-AGG-415 \\
\hline & Uranium 238 & $<0.0006$ & ug/g dry & 0.0006 & $4 / 10 / 08$ & 8D10003 & PNNL-AGG-415 \\
\hline \multirow{3}{*}{$\begin{array}{l}\text { HEIS No. } \\
14133-76-7\end{array}$} & B1RYR7B & \multicolumn{3}{|c|}{ Lab ID: $\quad$ 0803012-13 } & & & \\
\hline & Technetium-99 & $<0.00002$ & ug/g dry & 0.00002 & $4 / 10 / 08$ & 8D10003 & PNNL-AGG-415 \\
\hline & Uranium 238 & $<0.0006$ & ug/g dry & 0.0006 & $4 / 10 / 08$ & 8D10003 & PNNL-AGG-415 \\
\hline \multirow{3}{*}{$\begin{array}{l}\text { HEIS No. } \\
14133-76-7\end{array}$} & B1RYR7A & \multicolumn{3}{|c|}{$\begin{array}{ll}\text { Lab ID: } & \text { 0803012-14 }\end{array}$} & & & \\
\hline & Technetium-99 & $<0.00002$ & ug/g dry & 0.00002 & $4 / 10 / 08$ & 8D10003 & PNNL-AGG-415 \\
\hline & Uranium 238 & $<0.0006$ & ug/g dry & 0.0006 & $4 / 10 / 08$ & 8D10003 & PNNL-AGG-415 \\
\hline \multirow{3}{*}{$\begin{array}{l}\text { HEIS No. } \\
14133-76-7\end{array}$} & B1RYR8B & & b ID: & $3012-17$ & & & \\
\hline & Technetium-99 & $<0.00002$ & ug/g dry & 0.00002 & $4 / 10 / 08$ & 8D10003 & PNNL-AGG-415 \\
\hline & Uranium 238 & $<0.0006$ & ug/g dry & 0.0006 & $4 / 10 / 08$ & 8D10003 & PNNL-AGG-415 \\
\hline HEIS No. & B1RYR8A & & b ID: & $3012-18$ & & & \\
\hline $14133-76-7$ & Technetium-99 & $<0.00002$ & ug/g dry & 0.00002 & $4 / 10 / 08$ & 8D10003 & PNNL-AGG-415 \\
\hline & Uranium 238 & $<0.0006$ & ug/g dry & 0.0006 & $4 / 10 / 08$ & 8D10003 & PNNL-AGG-415 \\
\hline HEIS No. & B1RYR9B & & b ID: & $3012-21$ & & & \\
\hline 14133-76-7 & Technetium-99 & $<0.00002$ & ug/g dry & 0.00002 & $4 / 10 / 08$ & 8D10003 & PNNL-AGG-415 \\
\hline & Uranium 238 & $<0.0006$ & ug/g dry & 0.0006 & $4 / 10 / 08$ & 8D10003 & PNNL-AGG-415 \\
\hline HEIS No. & B1RYR9A & & b ID: & $3012-22$ & & & \\
\hline $14133-76-7$ & Technetium-99 & $<0.00002$ & ug/g dry & 0.00002 & $4 / 10 / 08$ & 8D10003 & PNNL-AGG-415 \\
\hline & Uranium 238 & $<0.0006$ & ug/g dry & 0.0006 & $4 / 10 / 08$ & 8D10003 & PNNL-AGG-415 \\
\hline HEIS No. & B1RYT0B & & b ID: & 3014-01 & & & \\
\hline $14133-76-7$ & Technetium-99 & $<0.00002$ & ug/g dry & 0.00002 & $4 / 10 / 08$ & 8D10003 & PNNL-AGG-415 \\
\hline & Uranium 238 & 0.007 & ug/g dry & 0.0006 & $4 / 10 / 08$ & 8D10003 & PNNL-AGG-415 \\
\hline HEIS No. & B1RYT0A & & b ID: & $3014-02$ & & & \\
\hline 14133-76-7 & Technetium-99 & $<0.00002$ & ug/g dry & 0.00002 & 4/10/08 & 8D10003 & PNNL-AGG-415 \\
\hline
\end{tabular}




\section{Radionuclides by ICP-MS/Water Extract}

\begin{tabular}{|c|c|c|c|c|c|c|c|}
\hline CAS \# & Analyte & Results & Units & EQL & Analyzed & Batch & Method \\
\hline \multirow[t]{2}{*}{ HEIS No. } & B1RYT0A & \multicolumn{3}{|c|}{ Lab ID: $\quad$ 0803014-02 } & & & \\
\hline & Uranium 238 & 0.003 & ug/g dry & 0.0006 & $4 / 10 / 08$ & 8D10003 & PNNL-AGG-415 \\
\hline \multirow{3}{*}{$\begin{array}{l}\text { HEIS No. } \\
\text { 14133-76-7 }\end{array}$} & B1T2P1B & \multicolumn{3}{|c|}{ Lab ID: $\quad$ 0803014-05 } & & & \\
\hline & Technetium-99 & 0.00006 & ug/g dry & 0.00002 & $4 / 10 / 08$ & 8D10004 & PNNL-AGG-415 \\
\hline & Uranium 238 & $<0.0006$ & ug/g dry & 0.0006 & $4 / 10 / 08$ & 8D10004 & PNNL-AGG-415 \\
\hline \multirow{3}{*}{$\begin{array}{l}\text { HEIS No. } \\
14133-76-7\end{array}$} & B1T2P1A & \multicolumn{3}{|c|}{ Lab ID: $\quad$ 0803014-06 } & & & \\
\hline & Technetium-99 & 0.00006 & ug/g dry & 0.00002 & $4 / 10 / 08$ & 8D10004 & PNNL-AGG-415 \\
\hline & Uranium 238 & $<0.0006$ & ug/g dry & 0.0006 & $4 / 10 / 08$ & 8D10004 & PNNL-AGG-415 \\
\hline \multirow{3}{*}{$\begin{array}{l}\text { HEIS No. } \\
14133-76-7\end{array}$} & B1T2P2B & \multicolumn{3}{|c|}{ Lab ID: $\quad$ 0803014-09 } & & & \\
\hline & Technetium-99 & $<0.00002$ & ug/g dry & 0.00002 & $4 / 10 / 08$ & 8D10004 & PNNL-AGG-415 \\
\hline & Uranium 238 & $<0.0006$ & ug/g dry & 0.0006 & $4 / 10 / 08$ & 8D10004 & PNNL-AGG-415 \\
\hline \multirow{3}{*}{$\begin{array}{l}\text { HEIS No. } \\
\text { 14133-76-7 }\end{array}$} & B1T2P2A & \multicolumn{3}{|c|}{ Lab ID: $\quad$ 0803014-10 } & & & \\
\hline & Technetium-99 & $<0.00002$ & ug/g dry & 0.00002 & $4 / 10 / 08$ & 8D10004 & PNNL-AGG-415 \\
\hline & Uranium 238 & $<0.0006$ & ug/g dry & 0.0006 & $4 / 10 / 08$ & 8D10004 & PNNL-AGG-415 \\
\hline \multirow{3}{*}{$\begin{array}{l}\text { HEIS No. } \\
14133-76-7\end{array}$} & B1T2P3B & \multicolumn{3}{|c|}{ Lab ID: $\quad$ 0803014-13 } & & & \\
\hline & Technetium-99 & $<0.00002$ & ug/g dry & 0.00002 & $4 / 10 / 08$ & 8D10004 & PNNL-AGG-415 \\
\hline & Uranium 238 & $<0.0005$ & ug/g dry & 0.0005 & $4 / 10 / 08$ & 8D10004 & PNNL-AGG-415 \\
\hline \multirow{3}{*}{$\begin{array}{l}\text { HEIS No. } \\
14133-76-7\end{array}$} & В1T2P3A & \multicolumn{3}{|c|}{$\begin{array}{ll}\text { Lab ID: } & \text { 0803014-14 }\end{array}$} & & & \\
\hline & Technetium-99 & $<0.00002$ & ug/g dry & 0.00002 & $4 / 10 / 08$ & 8D10004 & PNNL-AGG-415 \\
\hline & Uranium 238 & $<0.0004$ & ug/g dry & 0.0004 & $4 / 10 / 08$ & 8D10004 & PNNL-AGG-415 \\
\hline \multirow{3}{*}{$\begin{array}{l}\text { HEIS No. } \\
14133-76-7\end{array}$} & В1T2P4B & \multicolumn{3}{|c|}{ Lab ID: $\quad$ 0803014-16 } & & & \\
\hline & Technetium-99 & $<0.00002$ & ug/g dry & 0.00002 & $4 / 10 / 08$ & 8D10004 & PNNL-AGG-415 \\
\hline & Uranium 238 & $<0.0006$ & ug/g dry & 0.0006 & $4 / 10 / 08$ & 8D10004 & PNNL-AGG-415 \\
\hline \multirow{3}{*}{$\begin{array}{l}\text { HEIS No. } \\
14133-76-7\end{array}$} & B1T2P4A & \multicolumn{3}{|c|}{ Lab ID: $\quad$ 0803014-17 } & & & \\
\hline & Technetium-99 & $<0.00002$ & ug/g dry & 0.00002 & $4 / 10 / 08$ & 8D10004 & PNNL-AGG-415 \\
\hline & Uranium 238 & $<0.0006$ & ug/g dry & 0.0006 & $4 / 10 / 08$ & 8D10004 & PNNL-AGG-415 \\
\hline \multirow{3}{*}{$\begin{array}{l}\text { HEIS No. } \\
14133-76-7\end{array}$} & B1T2P5B & \multicolumn{3}{|c|}{ Lab ID: $\quad$ 0803014-20 } & & & \\
\hline & Technetium-99 & $<0.00002$ & ug/g dry & 0.00002 & $4 / 10 / 08$ & 8D10004 & PNNL-AGG-415 \\
\hline & Uranium 238 & $<0.0006$ & ug/g dry & 0.0006 & $4 / 10 / 08$ & 8D10004 & PNNL-AGG-415 \\
\hline \multirow{3}{*}{$\begin{array}{l}\text { HEIS No. } \\
14133-76-7\end{array}$} & B1T2P5A & & b ID: & $3014-21$ & & & \\
\hline & Technetium-99 & $<0.00002$ & ug/g dry & 0.00002 & $4 / 10 / 08$ & 8D10004 & PNNL-AGG-415 \\
\hline & Uranium 238 & $<0.0006$ & ug/g dry & 0.0006 & 4/10/08 & 8D10004 & PNNL-AGG-415 \\
\hline HEIS No. & B1T2P6B & & b ID: & $3014-24$ & & & \\
\hline 14133-76-7 & Technetium-99 & $<0.00002$ & ug/g dry & 0.00002 & $4 / 10 / 08$ & 8D10004 & PNNL-AGG-415 \\
\hline & Uranium 238 & $<0.0006$ & ug/g dry & 0.0006 & 4/10/08 & 8D10004 & PNNL-AGG-415 \\
\hline HEIS No. & B1T2P6A & & b ID: & $3014-25$ & & & \\
\hline 14133-76-7 & Technetium-99 & $<0.00002$ & ug/g dry & 0.00002 & $4 / 10 / 08$ & 8D10004 & PNNL-AGG-415 \\
\hline & Uranium 238 & $<0.0006$ & ug/g dry & 0.0006 & 4/10/08 & 8D10004 & PNNL-AGG-415 \\
\hline HEIS No. & B1T2P7B & & b ID: & 3015-02 & & & \\
\hline 14133-76-7 & Technetium-99 & $<0.00002$ & ug/g dry & 0.00002 & $4 / 10 / 08$ & 8D10004 & PNNL-AGG-415 \\
\hline & Uranium 238 & 0.002 & ug/g dry & 0.0006 & $4 / 10 / 08$ & 8D10004 & PNNL-AGG-415 \\
\hline HEIS No. & B1T2P7A & & b ID: & 3015-03 & & & \\
\hline 14133-76-7 & Technetium-99 & $<0.00002$ & ug/g dry & 0.00002 & $4 / 10 / 08$ & 8D10004 & PNNL-AGG-415 \\
\hline & Uranium 238 & 0.001 & ug/g dry & 0.0006 & 4/10/08 & 8D10004 & PNNL-AGG-415 \\
\hline HEIS No. & B1T2P8B & & b ID: & 3015-06 & & & \\
\hline 14133-76-7 & Technetium-99 & $<0.00002$ & ug/g dry & 0.00002 & 4/10/08 & 8D10004 & PNNL-AGG-415 \\
\hline & Uranium 238 & $<0.0006$ & ug/g dry & 0.0006 & 4/10/08 & 8D10004 & PNNL-AGG-415 \\
\hline
\end{tabular}




\section{Radionuclides by ICP-MS/Water Extract}

\begin{tabular}{|c|c|c|c|c|c|c|c|}
\hline CAS \# & Analyte & Results & Units & EQL & Analyzed & Batch & Method \\
\hline \multirow{3}{*}{$\begin{array}{l}\text { HEIS No. } \\
14133-76-7\end{array}$} & B1T2P8A & \multicolumn{3}{|c|}{ Lab ID: $\quad$ 0803015-07 } & & & \\
\hline & Technetium-99 & $<0.00002$ & ug/g dry & 0.00002 & $4 / 10 / 08$ & 8D10004 & PNNL-AGG-415 \\
\hline & Uranium 238 & $<0.0006$ & ug/g dry & 0.0006 & $4 / 10 / 08$ & 8D10004 & PNNL-AGG-415 \\
\hline \multirow{3}{*}{$\begin{array}{l}\text { HEIS No. } \\
\text { 14133-76-7 }\end{array}$} & В1T2P9B & \multicolumn{3}{|c|}{ Lab ID: $\quad$ 0803015-10 } & & & \\
\hline & Technetium-99 & $<0.00002$ & ug/g dry & 0.00002 & $4 / 10 / 08$ & 8D10004 & PNNL-AGG-415 \\
\hline & Uranium 238 & $<0.0006$ & ug/g dry & 0.0006 & $4 / 10 / 08$ & 8D10004 & PNNL-AGG-415 \\
\hline \multirow{3}{*}{$\begin{array}{l}\text { HEIS No. } \\
14133-76-7\end{array}$} & В1T2P9A & \multicolumn{3}{|c|}{ Lab ID: $\quad$ 0803015-11 } & & & \\
\hline & Technetium-99 & $<0.00002$ & ug/g dry & 0.00002 & $4 / 10 / 08$ & 8D10004 & PNNL-AGG-415 \\
\hline & Uranium 238 & $<0.0006$ & ug/g dry & 0.0006 & $4 / 10 / 08$ & 8D10004 & PNNL-AGG-415 \\
\hline \multirow{3}{*}{$\begin{array}{l}\text { HEIS No. } \\
14133-76-7\end{array}$} & B1T2R0B & \multicolumn{3}{|c|}{ Lab ID: $\quad$ 0803015-14 } & & & \\
\hline & Technetium-99 & $<0.00002$ & ug/g dry & 0.00002 & $4 / 10 / 08$ & 8D10004 & PNNL-AGG-415 \\
\hline & Uranium 238 & $<0.0006$ & ug/g dry & 0.0006 & $4 / 10 / 08$ & 8D10004 & PNNL-AGG-415 \\
\hline \multirow{3}{*}{$\begin{array}{l}\text { HEIS No. } \\
14133-76-7\end{array}$} & B1T2R0A & \multicolumn{3}{|c|}{ Lab ID: $\quad$ 0803015-15 } & & & \\
\hline & Technetium-99 & $<0.00002$ & ug/g dry & 0.00002 & $4 / 10 / 08$ & 8D10004 & PNNL-AGG-415 \\
\hline & Uranium 238 & $<0.0006$ & ug/g dry & 0.0006 & $4 / 10 / 08$ & 8D10004 & PNNL-AGG-415 \\
\hline \multirow{3}{*}{$\begin{array}{l}\text { HEIS No. } \\
14133-76-7\end{array}$} & B1TNK6B & \multicolumn{3}{|c|}{ Lab ID: $\quad$ 0803015-18 } & & & \\
\hline & Technetium-99 & $<0.00002$ & ug/g dry & 0.00002 & $4 / 10 / 08$ & 8D10004 & PNNL-AGG-415 \\
\hline & Uranium 238 & $<0.0006$ & ug/g dry & 0.0006 & $4 / 10 / 08$ & 8D10004 & PNNL-AGG-415 \\
\hline \multirow{3}{*}{$\begin{array}{l}\text { HEIS No. } \\
\text { 14133-76-7 }\end{array}$} & B1TNK6A & \multicolumn{3}{|c|}{ Lab ID: $\quad$ 0803015-19 } & & & \\
\hline & Technetium-99 & $<0.00002$ & ug/g dry & 0.00002 & $4 / 10 / 08$ & 8D10004 & PNNL-AGG-415 \\
\hline & Uranium 238 & $<0.0006$ & ug/g dry & 0.0006 & $4 / 10 / 08$ & 8D10004 & PNNL-AGG-415 \\
\hline \multirow{3}{*}{$\begin{array}{l}\text { HEIS No. } \\
14133-76-7\end{array}$} & B1TNK7B & \multicolumn{3}{|c|}{ Lab ID: $\quad$ 0803015-22 } & & & \\
\hline & Technetium-99 & $<0.00002$ & ug/g dry & 0.00002 & $4 / 10 / 08$ & 8D10004 & PNNL-AGG-415 \\
\hline & Uranium 238 & $<0.0006$ & ug/g dry & 0.0006 & $4 / 10 / 08$ & 8D10004 & PNNL-AGG-415 \\
\hline \multirow{3}{*}{$\begin{array}{l}\text { HEIS No. } \\
14133-76-7\end{array}$} & B1TNK7A & \multicolumn{3}{|c|}{ Lab ID: $\quad$ 0803015-23 } & & & \\
\hline & Technetium-99 & $<0.00002$ & ug/g dry & 0.00002 & $4 / 10 / 08$ & 8D10004 & PNNL-AGG-415 \\
\hline & Uranium 238 & $<0.0006$ & ug/g dry & 0.0006 & $4 / 10 / 08$ & 8D10004 & PNNL-AGG-415 \\
\hline \multirow{3}{*}{$\begin{array}{l}\text { HEIS No. } \\
14133-76-7\end{array}$} & B1TNK8B & \multicolumn{3}{|c|}{ Lab ID: $\quad$ 0803015-26 } & & & \\
\hline & Technetium-99 & $<0.00002$ & ug/g dry & 0.00002 & $4 / 10 / 08$ & 8D10004 & PNNL-AGG-415 \\
\hline & Uranium 238 & $<0.0006$ & ug/g dry & 0.0006 & $4 / 10 / 08$ & 8D10004 & PNNL-AGG-415 \\
\hline \multirow{3}{*}{$\begin{array}{l}\text { HEIS No. } \\
14133-76-7\end{array}$} & B1TNK8A & \multicolumn{3}{|c|}{ Lab ID: $\quad$ 0803015-27 } & & & \\
\hline & Technetium-99 & $<0.00002$ & ug/g dry & 0.00002 & $4 / 10 / 08$ & 8D10004 & PNNL-AGG-415 \\
\hline & Uranium 238 & $<0.0006$ & ug/g dry & 0.0006 & $4 / 10 / 08$ & 8D10004 & PNNL-AGG-415 \\
\hline \multirow{3}{*}{$\begin{array}{l}\text { HEIS No. } \\
14133-76-7\end{array}$} & B1TNK9B & \multicolumn{3}{|c|}{ Lab ID: $\quad$ 0803015-30 } & & & \\
\hline & Technetium-99 & $<0.00002$ & ug/g dry & 0.00002 & $4 / 10 / 08$ & 8D10004 & PNNL-AGG-415 \\
\hline & Uranium 238 & $<0.0006$ & ug/g dry & 0.0006 & $4 / 10 / 08$ & 8D10004 & PNNL-AGG-415 \\
\hline \multirow{3}{*}{$\begin{array}{l}\text { HEIS No. } \\
14133-76-7\end{array}$} & B1TNK9A & & b ID: & $3015-31$ & & & \\
\hline & Technetium-99 & $<0.00002$ & ug/g dry & 0.00002 & 4/10/08 & 8D10004 & PNNL-AGG-415 \\
\hline & Uranium 238 & $<0.0006$ & ug/g dry & 0.0006 & $4 / 10 / 08$ & 8D10004 & PNNL-AGG-415 \\
\hline
\end{tabular}


RCRA Metals By SW846 6020/Water Extract

\begin{tabular}{|c|c|c|c|c|c|c|c|}
\hline CAS \# & Analyte & Results & Units & EQL & Analyzed & Batch & Method \\
\hline HEIS No. & B1RTF8 & \multicolumn{3}{|c|}{ Lab ID: $\quad$ 0803001-01 } & & & \\
\hline $14092-98-9$ & Chromium & $<0.002$ & ug/g dry & 0.002 & 4/14/08 & 8D07005 & PNNL-AGG-415 \\
\hline 14119-06-3 & Copper & 0.008 & ug/g dry & 0.005 & $4 / 14 / 08$ & 8D07005 & PNNL-AGG-415 \\
\hline $7440-38-2$ & Arsenic & 0.017 & ug/g dry & 0.006 & $4 / 14 / 08$ & 8D07005 & PNNL-AGG-415 \\
\hline $14687-58-2$ & Selenium & $<0.011$ & ug/g dry & 0.011 & $4 / 14 / 08$ & 8D07005 & PNNL-AGG-415 \\
\hline 14392-17-7 & Molybdenum & 0.025 & ug/g dry & 0.002 & $4 / 14 / 08$ & 8D07005 & PNNL-AGG-415 \\
\hline 14914-61-5 & Ruthenium & $<0.0008$ & ug/g dry & 0.0008 & $4 / 14 / 08$ & 8D07005 & PNNL-AGG-415 \\
\hline $14378-38-2$ & Silver & $<0.001$ & ug/g dry & 0.001 & $4 / 14 / 08$ & 8D07005 & PNNL-AGG-415 \\
\hline 14041-58-8 & Cadmium & $<0.0006$ & ug/g dry & 0.0006 & $4 / 14 / 08$ & 8D07005 & PNNL-AGG-415 \\
\hline $14265-72-6$ & Antimony & $<0.0005$ & ug/g dry & 0.0005 & $4 / 14 / 08$ & 8D07005 & PNNL-AGG-415 \\
\hline 13966-28-4 & Lead & $<0.0006$ & ug/g dry & 0.0006 & $4 / 14 / 08$ & 8D07005 & PNNL-AGG-415 \\
\hline HEIS No. & B1RTF9A & \multicolumn{3}{|c|}{ Lab ID: $\quad$ 0803001-02 } & & & \\
\hline $14092-98-9$ & Chromium & $<0.002$ & ug/g dry & 0.002 & $4 / 14 / 08$ & 8D07005 & PNNL-AGG-415 \\
\hline 14119-06-3 & Copper & 0.007 & ug/g dry & 0.005 & $4 / 14 / 08$ & 8D07005 & PNNL-AGG-415 \\
\hline $7440-38-2$ & Arsenic & 0.021 & ug/g dry & 0.006 & $4 / 14 / 08$ & 8D07005 & PNNL-AGG-415 \\
\hline $14687-58-2$ & Selenium & $<0.011$ & ug/g dry & 0.011 & $4 / 14 / 08$ & 8D07005 & PNNL-AGG-415 \\
\hline $14392-17-7$ & Molybdenum & 0.012 & ug/g dry & 0.002 & $4 / 14 / 08$ & 8D07005 & PNNL-AGG-415 \\
\hline $14914-61-5$ & Ruthenium & $<0.0008$ & ug/g dry & 0.0008 & $4 / 14 / 08$ & 8D07005 & PNNL-AGG-415 \\
\hline $14378-38-2$ & Silver & $<0.001$ & ug/g dry & 0.001 & $4 / 14 / 08$ & 8D07005 & PNNL-AGG-415 \\
\hline 14041-58-8 & Cadmium & $<0.0006$ & ug/g dry & 0.0006 & $4 / 14 / 08$ & 8D07005 & PNNL-AGG-415 \\
\hline $14265-72-6$ & Antimony & $<0.0005$ & ug/g dry & 0.0005 & $4 / 14 / 08$ & 8D07005 & PNNL-AGG-415 \\
\hline 13966-28-4 & Lead & 0.0007 & ug/g dry & 0.0006 & $4 / 14 / 08$ & 8D07005 & PNNL-AGG-415 \\
\hline HEIS No. & B1RTH1A & \multicolumn{3}{|c|}{ Lab ID: $\quad$ 0803012-02 } & & & \\
\hline $14092-98-9$ & Chromium & $<0.002$ & ug/g dry & 0.002 & $4 / 14 / 08$ & 8D07005 & PNNL-AGG-415 \\
\hline 14119-06-3 & Copper & $<0.005$ & ug/g dry & 0.005 & $4 / 14 / 08$ & 8D07005 & PNNL-AGG-415 \\
\hline $7440-38-2$ & Arsenic & $<0.006$ & ug/g dry & 0.006 & $4 / 14 / 08$ & 8D07005 & PNNL-AGG-415 \\
\hline $14687-58-2$ & Selenium & $<0.011$ & ug/g dry & 0.011 & $4 / 14 / 08$ & 8D07005 & PNNL-AGG-415 \\
\hline 14392-17-7 & Molybdenum & 0.006 & ug/g dry & 0.002 & $4 / 14 / 08$ & 8D07005 & PNNL-AGG-415 \\
\hline $14914-61-5$ & Ruthenium & $<0.0008$ & ug/g dry & 0.0008 & $4 / 14 / 08$ & 8D07005 & PNNL-AGG-415 \\
\hline $14378-38-2$ & Silver & $<0.001$ & ug/g dry & 0.001 & $4 / 14 / 08$ & 8D07005 & PNNL-AGG-415 \\
\hline 14041-58-8 & Cadmium & $<0.0006$ & ug/g dry & 0.0006 & $4 / 14 / 08$ & 8D07005 & PNNL-AGG-415 \\
\hline $14265-72-6$ & Antimony & $<0.0005$ & ug/g dry & 0.0005 & $4 / 14 / 08$ & 8D07005 & PNNL-AGG-415 \\
\hline 13966-28-4 & Lead & $<0.0006$ & ug/g dry & 0.0006 & $4 / 14 / 08$ & 8D07005 & PNNL-AGG-415 \\
\hline HEIS No. & B1RTH1 & \multicolumn{3}{|c|}{ Lab ID: $\quad$ 0803012-03 } & & & \\
\hline 14092-98-9 & Chromium & $<0.002$ & ug/g dry & 0.002 & $4 / 14 / 08$ & 8D07005 & PNNL-AGG-415 \\
\hline 14119-06-3 & Copper & $<0.005$ & ug/g dry & 0.005 & $4 / 14 / 08$ & 8D07005 & PNNL-AGG-415 \\
\hline 7440-38-2 & Arsenic & 0.030 & ug/g dry & 0.006 & $4 / 14 / 08$ & 8D07005 & PNNL-AGG-415 \\
\hline $14687-58-2$ & Selenium & $<0.011$ & ug/g dry & 0.011 & $4 / 14 / 08$ & 8D07005 & PNNL-AGG-415 \\
\hline $14392-17-7$ & Molybdenum & 0.037 & ug/g dry & 0.002 & $4 / 14 / 08$ & 8D07005 & PNNL-AGG-415 \\
\hline $14914-61-5$ & Ruthenium & $<0.0008$ & ug/g dry & 0.0008 & $4 / 14 / 08$ & 8D07005 & PNNL-AGG-415 \\
\hline $14378-38-2$ & Silver & $<0.001$ & ug/g dry & 0.001 & $4 / 14 / 08$ & 8D07005 & PNNL-AGG-415 \\
\hline 14041-58-8 & Cadmium & $<0.0006$ & ug/g dry & 0.0006 & $4 / 14 / 08$ & 8D07005 & PNNL-AGG-415 \\
\hline $14265-72-6$ & Antimony & $<0.0005$ & ug/g dry & 0.0005 & $4 / 14 / 08$ & 8D07005 & PNNL-AGG-415 \\
\hline 13966-28-4 & Lead & $<0.0006$ & ug/g dry & 0.0006 & $4 / 14 / 08$ & 8D07005 & PNNL-AGG-415 \\
\hline HEIS No. & B1RTH2B & \multicolumn{3}{|c|}{ Lab ID: $\quad$ 0803012-05 } & & & \\
\hline 14092-98-9 & Chromium & $<0.002$ & ug/g dry & 0.002 & $4 / 14 / 08$ & 8D07005 & PNNL-AGG-415 \\
\hline 14119-06-3 & Copper & $<0.005$ & ug/g dry & 0.005 & $4 / 14 / 08$ & 8D07005 & PNNL-AGG-415 \\
\hline 7440-38-2 & Arsenic & $<0.006$ & ug/g dry & 0.006 & $4 / 14 / 08$ & 8D07005 & PNNL-AGG-415 \\
\hline $14687-58-2$ & Selenium & $<0.011$ & ug/g dry & 0.011 & $4 / 14 / 08$ & 8D07005 & PNNL-AGG-415 \\
\hline
\end{tabular}


RCRA Metals By SW846 6020/Water Extract

\begin{tabular}{|c|c|c|c|c|c|c|c|}
\hline CAS \# & Analyte & Results & Units & EQL & Analyzed & Batch & Method \\
\hline HEIS No. & B1RTH2B & \multicolumn{3}{|c|}{ Lab ID: $\quad$ 0803012-05 } & & & \\
\hline $14392-17-7$ & Molybdenum & 0.027 & ug/g dry & 0.002 & 4/14/08 & 8D07005 & PNNL-AGG-415 \\
\hline $14914-61-5$ & Ruthenium & $<0.0008$ & ug/g dry & 0.0008 & $4 / 14 / 08$ & 8D07005 & PNNL-AGG-415 \\
\hline $14378-38-2$ & Silver & $<0.001$ & ug/g dry & 0.001 & $4 / 14 / 08$ & 8D07005 & PNNL-AGG-415 \\
\hline 14041-58-8 & Cadmium & $<0.0006$ & ug/g dry & 0.0006 & $4 / 14 / 08$ & 8D07005 & PNNL-AGG-415 \\
\hline $14265-72-6$ & Antimony & $<0.0005$ & ug/g dry & 0.0005 & $4 / 14 / 08$ & 8D07005 & PNNL-AGG-415 \\
\hline 13966-28-4 & Lead & $<0.0006$ & ug/g dry & 0.0006 & $4 / 14 / 08$ & 8D07005 & PNNL-AGG-415 \\
\hline HEIS No. & B1RTH2A & \multicolumn{3}{|c|}{ Lab ID: $\quad$ 0803012-06 } & & & \\
\hline $14092-98-9$ & Chromium & 0.003 & ug/g dry & 0.002 & $4 / 14 / 08$ & 8D07005 & PNNL-AGG-415 \\
\hline 14119-06-3 & Copper & Over Range & ug/g dry & 0.005 & $4 / 14 / 08$ & 8D07005 & PNNL-AGG-415 \\
\hline $7440-38-2$ & Arsenic & $<0.006$ & ug/g dry & 0.006 & $4 / 14 / 08$ & 8D07005 & PNNL-AGG-415 \\
\hline $14687-58-2$ & Selenium & $<0.011$ & ug/g dry & 0.011 & $4 / 14 / 08$ & 8D07005 & PNNL-AGG-415 \\
\hline $14392-17-7$ & Molybdenum & 0.012 & ug/g dry & 0.002 & $4 / 14 / 08$ & 8D07005 & PNNL-AGG-415 \\
\hline 14914-61-5 & Ruthenium & $<0.0008$ & ug/g dry & 0.0008 & $4 / 14 / 08$ & 8D07005 & PNNL-AGG-415 \\
\hline $14378-38-2$ & Silver & $<0.001$ & ug/g dry & 0.001 & $4 / 14 / 08$ & 8D07005 & PNNL-AGG-415 \\
\hline 14041-58-8 & Cadmium & $<0.0006$ & ug/g dry & 0.0006 & $4 / 14 / 08$ & 8D07005 & PNNL-AGG-415 \\
\hline $14265-72-6$ & Antimony & $<0.0005$ & ug/g dry & 0.0005 & $4 / 14 / 08$ & 8D07005 & PNNL-AGG-415 \\
\hline 13966-28-4 & Lead & 0.028 & ug/g dry & 0.0006 & $4 / 14 / 08$ & 8D07005 & PNNL-AGG-415 \\
\hline HEIS No. & B1RTH3B & \multicolumn{3}{|c|}{ Lab ID: $\quad$ 0803012-09 } & & & \\
\hline 14092-98-9 & Chromium & $<0.002$ & ug/g dry & 0.002 & $4 / 14 / 08$ & 8D07005 & PNNL-AGG-415 \\
\hline 14119-06-3 & Copper & $<0.005$ & ug/g dry & 0.005 & $4 / 14 / 08$ & 8D07005 & PNNL-AGG-415 \\
\hline $7440-38-2$ & Arsenic & $<0.006$ & ug/g dry & 0.006 & $4 / 14 / 08$ & 8D07005 & PNNL-AGG-415 \\
\hline $14687-58-2$ & Selenium & $<0.011$ & ug/g dry & 0.011 & $4 / 14 / 08$ & 8D07005 & PNNL-AGG-415 \\
\hline $14392-17-7$ & Molybdenum & 0.012 & ug/g dry & 0.002 & $4 / 14 / 08$ & 8D07005 & PNNL-AGG-415 \\
\hline 14914-61-5 & Ruthenium & $<0.0008$ & ug/g dry & 0.0008 & $4 / 14 / 08$ & 8D07005 & PNNL-AGG-415 \\
\hline $14378-38-2$ & Silver & $<0.001$ & ug/g dry & 0.001 & $4 / 14 / 08$ & 8D07005 & PNNL-AGG-415 \\
\hline 14041-58-8 & Cadmium & $<0.0006$ & ug/g dry & 0.0006 & $4 / 14 / 08$ & 8D07005 & PNNL-AGG-415 \\
\hline $14265-72-6$ & Antimony & $<0.0005$ & ug/g dry & 0.0005 & $4 / 14 / 08$ & 8D07005 & PNNL-AGG-415 \\
\hline 13966-28-4 & Lead & $<0.0006$ & ug/g dry & 0.0006 & $4 / 14 / 08$ & 8D07005 & PNNL-AGG-415 \\
\hline HEIS No. & B1RTH3A & \multicolumn{3}{|c|}{ Lab ID: $\quad$ 0803012-10 } & & & \\
\hline 14092-98-9 & Chromium & $<0.002$ & ug/g dry & 0.002 & $4 / 14 / 08$ & 8D07005 & PNNL-AGG-415 \\
\hline 14119-06-3 & Copper & $<0.005$ & ug/g dry & 0.005 & $4 / 14 / 08$ & 8D07005 & PNNL-AGG-415 \\
\hline 7440-38-2 & Arsenic & $<0.006$ & ug/g dry & 0.006 & $4 / 14 / 08$ & 8D07005 & PNNL-AGG-415 \\
\hline $14687-58-2$ & Selenium & $<0.011$ & ug/g dry & 0.011 & $4 / 14 / 08$ & 8D07005 & PNNL-AGG-415 \\
\hline $14392-17-7$ & Molybdenum & 0.011 & ug/g dry & 0.002 & $4 / 14 / 08$ & 8D07005 & PNNL-AGG-415 \\
\hline 14914-61-5 & Ruthenium & $<0.0008$ & ug/g dry & 0.0008 & $4 / 14 / 08$ & 8D07005 & PNNL-AGG-415 \\
\hline 14378-38-2 & Silver & $<0.001$ & ug/g dry & 0.001 & $4 / 14 / 08$ & 8D07005 & PNNL-AGG-415 \\
\hline 14041-58-8 & Cadmium & $<0.0006$ & ug/g dry & 0.0006 & $4 / 14 / 08$ & 8D07005 & PNNL-AGG-415 \\
\hline $14265-72-6$ & Antimony & $<0.0005$ & ug/g dry & 0.0005 & $4 / 14 / 08$ & 8D07005 & PNNL-AGG-415 \\
\hline 13966-28-4 & Lead & $<0.0006$ & ug/g dry & 0.0006 & $4 / 14 / 08$ & 8D07005 & PNNL-AGG-415 \\
\hline HEIS No. & B1RYR7B & \multicolumn{3}{|c|}{ Lab ID: $\quad$ 0803012-13 } & & & \\
\hline 14092-98-9 & Chromium & $<0.002$ & ug/g dry & 0.002 & $4 / 14 / 08$ & 8D07005 & PNNL-AGG-415 \\
\hline 14119-06-3 & Copper & $<0.005$ & ug/g dry & 0.005 & $4 / 14 / 08$ & 8D07005 & PNNL-AGG-415 \\
\hline $7440-38-2$ & Arsenic & $<0.006$ & ug/g dry & 0.006 & $4 / 14 / 08$ & 8D07005 & PNNL-AGG-415 \\
\hline $14687-58-2$ & Selenium & $<0.011$ & ug/g dry & 0.011 & $4 / 14 / 08$ & 8D07005 & PNNL-AGG-415 \\
\hline $14392-17-7$ & Molybdenum & 0.025 & ug/g dry & 0.002 & $4 / 14 / 08$ & 8D07005 & PNNL-AGG-415 \\
\hline $14914-61-5$ & Ruthenium & $<0.0008$ & ug/g dry & 0.0008 & $4 / 14 / 08$ & 8D07005 & PNNL-AGG-415 \\
\hline $14378-38-2$ & Silver & $<0.001$ & ug/g dry & 0.001 & $4 / 14 / 08$ & 8D07005 & PNNL-AGG-415 \\
\hline 14041-58-8 & Cadmium & $<0.0006$ & ug/g dry & 0.0006 & $4 / 14 / 08$ & 8D07005 & PNNL-AGG-415 \\
\hline
\end{tabular}


RCRA Metals By SW846 6020/Water Extract

\begin{tabular}{|c|c|c|c|c|c|c|c|}
\hline CAS \# & Analyte & Results & Units & EQL & Analyzed & Batch & Method \\
\hline HEIS No. & B1RYR7B & \multicolumn{3}{|c|}{ Lab ID: $\quad$ 0803012-13 } & & & \\
\hline $14265-72-6$ & Antimony & $<0.0005$ & ug/g dry & 0.0005 & $4 / 14 / 08$ & 8D07005 & PNNL-AGG-415 \\
\hline 13966-28-4 & Lead & $<0.0006$ & ug/g dry & 0.0006 & $4 / 14 / 08$ & 8D07005 & PNNL-AGG-415 \\
\hline HEIS No. & B1RYR7A & \multicolumn{3}{|c|}{ Lab ID: $\quad$ 0803012-14 } & & & \\
\hline 14092-98-9 & Chromium & $<0.002$ & ug/g dry & 0.002 & $4 / 14 / 08$ & 8D07005 & PNNL-AGG-415 \\
\hline 14119-06-3 & Copper & $<0.005$ & ug/g dry & 0.005 & $4 / 14 / 08$ & 8D07005 & PNNL-AGG-415 \\
\hline $7440-38-2$ & Arsenic & $<0.006$ & ug/g dry & 0.006 & $4 / 14 / 08$ & 8D07005 & PNNL-AGG-415 \\
\hline $14687-58-2$ & Selenium & $<0.011$ & ug/g dry & 0.011 & $4 / 14 / 08$ & 8D07005 & PNNL-AGG-415 \\
\hline 14392-17-7 & Molybdenum & 0.018 & ug/g dry & 0.002 & $4 / 14 / 08$ & 8D07005 & PNNL-AGG-415 \\
\hline $14914-61-5$ & Ruthenium & $<0.0008$ & ug/g dry & 0.0008 & $4 / 14 / 08$ & 8D07005 & PNNL-AGG-415 \\
\hline $14378-38-2$ & Silver & $<0.001$ & ug/g dry & 0.001 & $4 / 14 / 08$ & 8D07005 & PNNL-AGG-415 \\
\hline $14041-58-8$ & Cadmium & $<0.0006$ & ug/g dry & 0.0006 & $4 / 14 / 08$ & 8D07005 & PNNL-AGG-415 \\
\hline $14265-72-6$ & Antimony & $<0.0005$ & ug/g dry & 0.0005 & $4 / 14 / 08$ & 8D07005 & PNNL-AGG-415 \\
\hline 13966-28-4 & Lead & $<0.0006$ & ug/g dry & 0.0006 & $4 / 14 / 08$ & 8D07005 & PNNL-AGG-415 \\
\hline HEIS No. & B1RYR8B & \multicolumn{3}{|c|}{ Lab ID: $\quad$ 0803012-17 } & & & \\
\hline 14092-98-9 & Chromium & $<0.002$ & ug/g dry & 0.002 & $4 / 14 / 08$ & 8D07005 & PNNL-AGG-415 \\
\hline $14119-06-3$ & Copper & $<0.005$ & ug/g dry & 0.005 & $4 / 14 / 08$ & 8D07005 & PNNL-AGG-415 \\
\hline $7440-38-2$ & Arsenic & $<0.006$ & ug/g dry & 0.006 & $4 / 14 / 08$ & 8D07005 & PNNL-AGG-415 \\
\hline $14687-58-2$ & Selenium & $<0.011$ & ug/g dry & 0.011 & 4/14/08 & 8D07005 & PNNL-AGG-415 \\
\hline $14392-17-7$ & Molybdenum & 0.033 & ug/g dry & 0.002 & $4 / 14 / 08$ & 8D07005 & PNNL-AGG-415 \\
\hline 14914-61-5 & Ruthenium & $<0.0008$ & ug/g dry & 0.0008 & $4 / 14 / 08$ & 8D07005 & PNNL-AGG-415 \\
\hline $14378-38-2$ & Silver & $<0.001$ & ug/g dry & 0.001 & $4 / 14 / 08$ & 8D07005 & PNNL-AGG-415 \\
\hline $14041-58-8$ & Cadmium & $<0.0006$ & ug/g dry & 0.0006 & $4 / 14 / 08$ & 8D07005 & PNNL-AGG-415 \\
\hline $14265-72-6$ & Antimony & $<0.0005$ & ug/g dry & 0.0005 & $4 / 14 / 08$ & 8D07005 & PNNL-AGG-415 \\
\hline $13966-28-4$ & Lead & $<0.0006$ & ug/g dry & 0.0006 & $4 / 14 / 08$ & 8D07005 & PNNL-AGG-415 \\
\hline HEIS No. & B1RYR8A & \multicolumn{3}{|c|}{ Lab ID: $\quad$ 0803012-18 } & & & \\
\hline 14092-98-9 & Chromium & $<0.002$ & ug/g dry & 0.002 & $4 / 14 / 08$ & 8D07005 & PNNL-AGG-415 \\
\hline $14119-06-3$ & Copper & $<0.005$ & ug/g dry & 0.005 & $4 / 14 / 08$ & 8D07005 & PNNL-AGG-415 \\
\hline $7440-38-2$ & Arsenic & $<0.007$ & ug/g dry & 0.007 & $4 / 14 / 08$ & 8D07005 & PNNL-AGG-415 \\
\hline $14687-58-2$ & Selenium & $<0.012$ & ug/g dry & 0.012 & $4 / 14 / 08$ & 8D07005 & PNNL-AGG-415 \\
\hline $14392-17-7$ & Molybdenum & 0.012 & ug/g dry & 0.002 & $4 / 14 / 08$ & 8D07005 & PNNL-AGG-415 \\
\hline $14914-61-5$ & Ruthenium & $<0.0009$ & ug/g dry & 0.0009 & $4 / 14 / 08$ & 8D07005 & PNNL-AGG-415 \\
\hline $14378-38-2$ & Silver & $<0.001$ & ug/g dry & 0.001 & $4 / 14 / 08$ & 8D07005 & PNNL-AGG-415 \\
\hline $14041-58-8$ & Cadmium & $<0.0007$ & ug/g dry & 0.0007 & $4 / 14 / 08$ & 8D07005 & PNNL-AGG-415 \\
\hline $14265-72-6$ & Antimony & $<0.0006$ & ug/g dry & 0.0006 & $4 / 14 / 08$ & 8D07005 & PNNL-AGG-415 \\
\hline 13966-28-4 & Lead & $<0.0006$ & ug/g dry & 0.0006 & $4 / 14 / 08$ & 8D07005 & PNNL-AGG-415 \\
\hline HEIS No. & B1RYR9B & \multicolumn{3}{|c|}{ Lab ID: $\quad$ 0803012-21 } & & & \\
\hline 14092-98-9 & Chromium & $<0.002$ & ug/g dry & 0.002 & $4 / 14 / 08$ & 8D07005 & PNNL-AGG-415 \\
\hline 14119-06-3 & Copper & $<0.005$ & ug/g dry & 0.005 & $4 / 14 / 08$ & 8D07005 & PNNL-AGG-415 \\
\hline 7440-38-2 & Arsenic & $<0.006$ & ug/g dry & 0.006 & $4 / 14 / 08$ & 8D07005 & PNNL-AGG-415 \\
\hline $14687-58-2$ & Selenium & $<0.011$ & ug/g dry & 0.011 & $4 / 14 / 08$ & 8D07005 & PNNL-AGG-415 \\
\hline 14392-17-7 & Molybdenum & 0.030 & ug/g dry & 0.002 & $4 / 14 / 08$ & 8D07005 & PNNL-AGG-415 \\
\hline 14914-61-5 & Ruthenium & $<0.0008$ & ug/g dry & 0.0008 & $4 / 14 / 08$ & 8D07005 & PNNL-AGG-415 \\
\hline $14378-38-2$ & Silver & $<0.001$ & ug/g dry & 0.001 & $4 / 14 / 08$ & 8D07005 & PNNL-AGG-415 \\
\hline $14041-58-8$ & Cadmium & $<0.0006$ & ug/g dry & 0.0006 & $4 / 14 / 08$ & 8D07005 & PNNL-AGG-415 \\
\hline $14265-72-6$ & Antimony & $<0.0005$ & ug/g dry & 0.0005 & $4 / 14 / 08$ & 8D07005 & PNNL-AGG-415 \\
\hline 13966-28-4 & Lead & $<0.0006$ & ug/g dry & 0.0006 & $4 / 14 / 08$ & 8D07005 & PNNL-AGG-415 \\
\hline HEIS No. & B1RYR9A & \multicolumn{3}{|c|}{ Lab ID: $\quad 0803012-22$} & & & \\
\hline $14092-98-9$ & Chromium & $<0.002$ & ug/g dry & 0.002 & 4/14/08 & 8D07005 & PNNL-AGG-415 \\
\hline
\end{tabular}


RCRA Metals By SW846 6020/Water Extract

\begin{tabular}{|c|c|c|c|c|c|c|c|}
\hline CAS \# & Analyte & Results & Units & EQL & Analyzed & Batch & Method \\
\hline HEIS No. & B1RYR9A & \multicolumn{3}{|c|}{ Lab ID: $\quad$ 0803012-22 } & & & \\
\hline 14119-06-3 & Copper & $<0.005$ & ug/g dry & 0.005 & 4/14/08 & 8D07005 & PNNL-AGG-415 \\
\hline $7440-38-2$ & Arsenic & $<0.006$ & ug/g dry & 0.006 & $4 / 14 / 08$ & 8D07005 & PNNL-AGG-415 \\
\hline $14687-58-2$ & Selenium & $<0.011$ & ug/g dry & 0.011 & $4 / 14 / 08$ & 8D07005 & PNNL-AGG-415 \\
\hline $14392-17-7$ & Molybdenum & 0.016 & ug/g dry & 0.002 & $4 / 14 / 08$ & 8D07005 & PNNL-AGG-415 \\
\hline 14914-61-5 & Ruthenium & $<0.0008$ & ug/g dry & 0.0008 & $4 / 14 / 08$ & 8D07005 & PNNL-AGG-415 \\
\hline 14378-38-2 & Silver & $<0.001$ & ug/g dry & 0.001 & $4 / 14 / 08$ & 8D07005 & PNNL-AGG-415 \\
\hline 14041-58-8 & Cadmium & $<0.0006$ & ug/g dry & 0.0006 & $4 / 14 / 08$ & 8D07005 & PNNL-AGG-415 \\
\hline $14265-72-6$ & Antimony & $<0.0005$ & ug/g dry & 0.0005 & $4 / 14 / 08$ & 8D07005 & PNNL-AGG-415 \\
\hline 13966-28-4 & Lead & $<0.0006$ & ug/g dry & 0.0006 & $4 / 14 / 08$ & 8D07005 & PNNL-AGG-415 \\
\hline HEIS No. & B1RYT0B & \multicolumn{3}{|c|}{ Lab ID: $\quad$ 0803014-01 } & & & \\
\hline $14092-98-9$ & Chromium & $<0.002$ & ug/g dry & 0.002 & $4 / 14 / 08$ & 8D07005 & PNNL-AGG-415 \\
\hline 14119-06-3 & Copper & $<0.005$ & ug/g dry & 0.005 & $4 / 14 / 08$ & 8D07005 & PNNL-AGG-415 \\
\hline 7440-38-2 & Arsenic & 0.127 & ug/g dry & 0.006 & $4 / 14 / 08$ & 8D07005 & PNNL-AGG-415 \\
\hline $14687-58-2$ & Selenium & $<0.011$ & ug/g dry & 0.011 & $4 / 14 / 08$ & 8D07005 & PNNL-AGG-415 \\
\hline 14392-17-7 & Molybdenum & 0.025 & ug/g dry & 0.002 & $4 / 14 / 08$ & 8D07005 & PNNL-AGG-415 \\
\hline $14914-61-5$ & Ruthenium & $<0.0008$ & ug/g dry & 0.0008 & $4 / 14 / 08$ & 8D07005 & PNNL-AGG-415 \\
\hline $14378-38-2$ & Silver & $<0.001$ & ug/g dry & 0.001 & $4 / 14 / 08$ & 8D07005 & PNNL-AGG-415 \\
\hline 14041-58-8 & Cadmium & $<0.0006$ & ug/g dry & 0.0006 & $4 / 14 / 08$ & 8D07005 & PNNL-AGG-415 \\
\hline $14265-72-6$ & Antimony & 0.0009 & ug/g dry & 0.0005 & $4 / 14 / 08$ & 8D07005 & PNNL-AGG-415 \\
\hline 13966-28-4 & Lead & 0.001 & ug/g dry & 0.0006 & $4 / 14 / 08$ & 8D07005 & PNNL-AGG-415 \\
\hline HEIS No. & B1RYT0A & \multicolumn{3}{|c|}{ Lab ID: $\quad$ 0803014-02 } & & & \\
\hline $14092-98-9$ & Chromium & $<0.002$ & ug/g dry & 0.002 & $4 / 14 / 08$ & 8D07005 & PNNL-AGG-415 \\
\hline 14119-06-3 & Copper & 0.045 & ug/g dry & 0.005 & $4 / 14 / 08$ & 8D07005 & PNNL-AGG-415 \\
\hline $7440-38-2$ & Arsenic & 0.094 & ug/g dry & 0.006 & $4 / 14 / 08$ & 8D07005 & PNNL-AGG-415 \\
\hline $14687-58-2$ & Selenium & $<0.011$ & ug/g dry & 0.011 & $4 / 14 / 08$ & 8D07005 & PNNL-AGG-415 \\
\hline $14392-17-7$ & Molybdenum & 0.029 & ug/g dry & 0.002 & $4 / 14 / 08$ & 8D07005 & PNNL-AGG-415 \\
\hline 14914-61-5 & Ruthenium & $<0.0008$ & ug/g dry & 0.0008 & $4 / 14 / 08$ & 8D07005 & PNNL-AGG-415 \\
\hline $14378-38-2$ & Silver & $<0.001$ & ug/g dry & 0.001 & $4 / 14 / 08$ & 8D07005 & PNNL-AGG-415 \\
\hline $14041-58-8$ & Cadmium & $<0.0006$ & ug/g dry & 0.0006 & $4 / 14 / 08$ & 8D07005 & PNNL-AGG-415 \\
\hline $14265-72-6$ & Antimony & 0.001 & ug/g dry & 0.0005 & $4 / 14 / 08$ & 8D07005 & PNNL-AGG-415 \\
\hline 13966-28-4 & Lead & $<0.0006$ & ug/g dry & 0.0006 & $4 / 14 / 08$ & 8D07005 & PNNL-AGG-415 \\
\hline HEIS No. & B1T2P1B & \multicolumn{3}{|c|}{ Lab ID: $\quad$ 0803014-05 } & & & \\
\hline $14092-98-9$ & Chromium & $<0.002$ & ug/g dry & 0.002 & $4 / 14 / 08$ & 8D14002 & PNNL-AGG-415 \\
\hline $14119-06-3$ & Copper & $<0.005$ & ug/g dry & 0.005 & $4 / 14 / 08$ & 8D14002 & PNNL-AGG-415 \\
\hline $7440-38-2$ & Arsenic & $<0.006$ & ug/g dry & 0.006 & $4 / 14 / 08$ & 8D14002 & PNNL-AGG-415 \\
\hline $14687-58-2$ & Selenium & $<0.011$ & ug/g dry & 0.011 & $4 / 14 / 08$ & 8D14002 & PNNL-AGG-415 \\
\hline $14392-17-7$ & Molybdenum & 0.036 & ug/g dry & 0.002 & $4 / 14 / 08$ & 8D14002 & PNNL-AGG-415 \\
\hline $14914-61-5$ & Ruthenium & $<0.0008$ & ug/g dry & 0.0008 & $4 / 14 / 08$ & 8D14002 & PNNL-AGG-415 \\
\hline 14378-38-2 & Silver & $<0.001$ & ug/g dry & 0.001 & $4 / 14 / 08$ & 8D14002 & PNNL-AGG-415 \\
\hline 14041-58-8 & Cadmium & $<0.0006$ & ug/g dry & 0.0006 & $4 / 14 / 08$ & 8D14002 & PNNL-AGG-415 \\
\hline $14265-72-6$ & Antimony & $<0.0005$ & ug/g dry & 0.0005 & $4 / 14 / 08$ & 8D14002 & PNNL-AGG-415 \\
\hline 13966-28-4 & Lead & $<0.0006$ & ug/g dry & 0.0006 & $4 / 14 / 08$ & 8D14002 & PNNL-AGG-415 \\
\hline HEIS No. & B1T2P1A & \multicolumn{3}{|c|}{ Lab ID: $\quad$ 0803014-06 } & & & \\
\hline 14092-98-9 & Chromium & $<0.002$ & ug/g dry & 0.002 & $4 / 14 / 08$ & 8D14002 & PNNL-AGG-415 \\
\hline 14119-06-3 & Copper & $<0.005$ & ug/g dry & 0.005 & $4 / 14 / 08$ & 8D14002 & PNNL-AGG-415 \\
\hline 7440-38-2 & Arsenic & $<0.006$ & ug/g dry & 0.006 & $4 / 14 / 08$ & 8D14002 & PNNL-AGG-415 \\
\hline $14687-58-2$ & Selenium & $<0.011$ & ug/g dry & 0.011 & $4 / 14 / 08$ & 8D14002 & PNNL-AGG-415 \\
\hline 14392-17-7 & Molybdenum & 0.025 & ug/g dry & 0.002 & $4 / 14 / 08$ & 8D14002 & PNNL-AGG-415 \\
\hline
\end{tabular}


RCRA Metals By SW846 6020/Water Extract

\begin{tabular}{|c|c|c|c|c|c|c|c|}
\hline CAS \# & Analyte & Results & Units & EQL & Analyzed & Batch & Method \\
\hline HEIS No. & B1T2P1A & \multicolumn{3}{|c|}{ Lab ID: $\quad$ 0803014-06 } & & & \\
\hline $14914-61-5$ & Ruthenium & $<0.0008$ & ug/g dry & 0.0008 & $4 / 14 / 08$ & 8D14002 & PNNL-AGG-415 \\
\hline $14378-38-2$ & Silver & $<0.001$ & ug/g dry & 0.001 & $4 / 14 / 08$ & 8D14002 & PNNL-AGG-415 \\
\hline $14041-58-8$ & Cadmium & $<0.0006$ & ug/g dry & 0.0006 & $4 / 14 / 08$ & 8D14002 & PNNL-AGG-415 \\
\hline $14265-72-6$ & Antimony & $<0.0005$ & ug/g dry & 0.0005 & $4 / 14 / 08$ & 8D14002 & PNNL-AGG-415 \\
\hline $13966-28-4$ & Lead & $<0.0006$ & ug/g dry & 0.0006 & $4 / 14 / 08$ & 8D14002 & PNNL-AGG-415 \\
\hline HEIS No. & B1T2P2B & \multicolumn{3}{|c|}{ Lab ID: $\quad$ 0803014-09 } & & & \\
\hline 14092-98-9 & Chromium & $<0.002$ & ug/g dry & 0.002 & 4/14/08 & 8D14002 & PNNL-AGG-415 \\
\hline $14119-06-3$ & Copper & $<0.005$ & ug/g dry & 0.005 & $4 / 14 / 08$ & 8D14002 & PNNL-AGG-415 \\
\hline $7440-38-2$ & Arsenic & $<0.006$ & ug/g dry & 0.006 & $4 / 14 / 08$ & 8D14002 & PNNL-AGG-415 \\
\hline $14687-58-2$ & Selenium & $<0.011$ & ug/g dry & 0.011 & 4/14/08 & 8D14002 & PNNL-AGG-415 \\
\hline 14392-17-7 & Molybdenum & 0.020 & ug/g dry & 0.002 & $4 / 14 / 08$ & 8D14002 & PNNL-AGG-415 \\
\hline $14914-61-5$ & Ruthenium & $<0.0008$ & ug/g dry & 0.0008 & $4 / 14 / 08$ & 8D14002 & PNNL-AGG-415 \\
\hline $14378-38-2$ & Silver & $<0.001$ & ug/g dry & 0.001 & $4 / 14 / 08$ & 8D14002 & PNNL-AGG-415 \\
\hline $14041-58-8$ & Cadmium & $<0.0006$ & ug/g dry & 0.0006 & $4 / 14 / 08$ & 8D14002 & PNNL-AGG-415 \\
\hline $14265-72-6$ & Antimony & $<0.0005$ & ug/g dry & 0.0005 & 4/14/08 & 8D14002 & PNNL-AGG-415 \\
\hline $13966-28-4$ & Lead & $<0.0006$ & ug/g dry & 0.0006 & $4 / 14 / 08$ & 8D14002 & PNNL-AGG-415 \\
\hline HEIS No. & B1T2P2A & \multicolumn{3}{|c|}{ Lab ID: $\quad$ 0803014-10 } & & & \\
\hline $14092-98-9$ & Chromium & $<0.002$ & ug/g dry & 0.002 & $4 / 14 / 08$ & 8D14002 & PNNL-AGG-415 \\
\hline 14119-06-3 & Copper & $<0.005$ & ug/g dry & 0.005 & $4 / 14 / 08$ & 8D14002 & PNNL-AGG-415 \\
\hline $7440-38-2$ & Arsenic & $<0.006$ & ug/g dry & 0.006 & $4 / 14 / 08$ & 8D14002 & PNNL-AGG-415 \\
\hline $14687-58-2$ & Selenium & $<0.011$ & ug/g dry & 0.011 & $4 / 14 / 08$ & 8D14002 & PNNL-AGG-415 \\
\hline 14392-17-7 & Molybdenum & 0.011 & ug/g dry & 0.002 & $4 / 14 / 08$ & 8D14002 & PNNL-AGG-415 \\
\hline $14914-61-5$ & Ruthenium & $<0.0008$ & ug/g dry & 0.0008 & $4 / 14 / 08$ & 8D14002 & PNNL-AGG-415 \\
\hline $14378-38-2$ & Silver & $<0.001$ & ug/g dry & 0.001 & $4 / 14 / 08$ & 8D14002 & PNNL-AGG-415 \\
\hline $14041-58-8$ & Cadmium & $<0.0006$ & ug/g dry & 0.0006 & $4 / 14 / 08$ & 8D14002 & PNNL-AGG-415 \\
\hline $14265-72-6$ & Antimony & $<0.0005$ & ug/g dry & 0.0005 & $4 / 14 / 08$ & 8D14002 & PNNL-AGG-415 \\
\hline 13966-28-4 & Lead & $<0.0006$ & ug/g dry & 0.0006 & $4 / 14 / 08$ & 8D14002 & PNNL-AGG-415 \\
\hline HEIS No. & В1T2P3B & \multicolumn{3}{|c|}{ Lab ID: $\quad$ 0803014-13 } & & & \\
\hline 14092-98-9 & Chromium & $<0.002$ & ug/g dry & 0.002 & $4 / 14 / 08$ & 8D14002 & PNNL-AGG-415 \\
\hline $14119-06-3$ & Copper & $<0.004$ & ug/g dry & 0.004 & $4 / 14 / 08$ & 8D14002 & PNNL-AGG-415 \\
\hline $7440-38-2$ & Arsenic & $<0.006$ & ug/g dry & 0.006 & $4 / 14 / 08$ & 8D14002 & PNNL-AGG-415 \\
\hline $14687-58-2$ & Selenium & $<0.010$ & ug/g dry & 0.010 & $4 / 14 / 08$ & 8D14002 & PNNL-AGG-415 \\
\hline $14392-17-7$ & Molybdenum & 0.008 & ug/g dry & 0.001 & $4 / 14 / 08$ & 8D14002 & PNNL-AGG-415 \\
\hline $14914-61-5$ & Ruthenium & $<0.0007$ & ug/g dry & 0.0007 & $4 / 14 / 08$ & 8D14002 & PNNL-AGG-415 \\
\hline $14378-38-2$ & Silver & $<0.001$ & ug/g dry & 0.001 & 4/14/08 & 8D14002 & PNNL-AGG-415 \\
\hline $14041-58-8$ & Cadmium & $<0.0006$ & ug/g dry & 0.0006 & $4 / 14 / 08$ & 8D14002 & PNNL-AGG-415 \\
\hline $14265-72-6$ & Antimony & $<0.0005$ & ug/g dry & 0.0005 & $4 / 14 / 08$ & 8D14002 & PNNL-AGG-415 \\
\hline $13966-28-4$ & Lead & $<0.0005$ & ug/g dry & 0.0005 & $4 / 14 / 08$ & 8D14002 & PNNL-AGG-415 \\
\hline HEIS No. & B1T2P3A & \multicolumn{3}{|c|}{ Lab ID: $\quad$ 0803014-14 } & & & \\
\hline $14092-98-9$ & Chromium & $<0.002$ & ug/g dry & 0.002 & $4 / 14 / 08$ & 8D14002 & PNNL-AGG-415 \\
\hline $14119-06-3$ & Copper & $<0.004$ & ug/g dry & 0.004 & $4 / 14 / 08$ & 8D14002 & PNNL-AGG-415 \\
\hline $7440-38-2$ & Arsenic & $<0.005$ & ug/g dry & 0.005 & $4 / 14 / 08$ & 8D14002 & PNNL-AGG-415 \\
\hline $14687-58-2$ & Selenium & $<0.009$ & ug/g dry & 0.009 & $4 / 14 / 08$ & 8D14002 & PNNL-AGG-415 \\
\hline $14392-17-7$ & Molybdenum & 0.010 & ug/g dry & 0.001 & $4 / 14 / 08$ & 8D14002 & PNNL-AGG-415 \\
\hline $14914-61-5$ & Ruthenium & $<0.0006$ & ug/g dry & 0.0006 & $4 / 14 / 08$ & 8D14002 & PNNL-AGG-415 \\
\hline $14378-38-2$ & Silver & $<0.0008$ & ug/g dry & 0.0008 & $4 / 14 / 08$ & 8D14002 & PNNL-AGG-415 \\
\hline $14041-58-8$ & Cadmium & $<0.0005$ & ug/g dry & 0.0005 & $4 / 14 / 08$ & 8D14002 & PNNL-AGG-415 \\
\hline $14265-72-6$ & Antimony & $<0.0004$ & ug/g dry & 0.0004 & $4 / 14 / 08$ & 8D14002 & PNNL-AGG-415 \\
\hline
\end{tabular}


RCRA Metals By SW846 6020/Water Extract

\begin{tabular}{|c|c|c|c|c|c|c|c|}
\hline CAS \# & Analyte & Results & Units & EQL & Analyzed & Batch & Method \\
\hline HEIS No. & В1Т2Р3А & \multicolumn{3}{|c|}{ Lab ID: $\quad$ 0803014-14 } & & & \\
\hline $13966-28-4$ & Lead & $<0.0004$ & ug/g dry & 0.0004 & $4 / 14 / 08$ & 8D14002 & PNNL-AGG-415 \\
\hline HEIS No. & В1T2P4B & \multicolumn{3}{|c|}{ Lab ID: $\quad$ 0803014-16 } & & & \\
\hline $14092-98-9$ & Chromium & $<0.002$ & ug/g dry & 0.002 & $4 / 14 / 08$ & 8D14002 & PNNL-AGG-415 \\
\hline $14119-06-3$ & Copper & $<0.005$ & ug/g dry & 0.005 & $4 / 14 / 08$ & 8D14002 & PNNL-AGG-415 \\
\hline 7440-38-2 & Arsenic & $<0.006$ & ug/g dry & 0.006 & $4 / 14 / 08$ & 8D14002 & PNNL-AGG-415 \\
\hline $14687-58-2$ & Selenium & $<0.011$ & ug/g dry & 0.011 & $4 / 14 / 08$ & 8D14002 & PNNL-AGG-415 \\
\hline $14392-17-7$ & Molybdenum & 0.032 & ug/g dry & 0.002 & $4 / 14 / 08$ & 8D14002 & PNNL-AGG-415 \\
\hline $14914-61-5$ & Ruthenium & $<0.0008$ & ug/g dry & 0.0008 & $4 / 14 / 08$ & 8D14002 & PNNL-AGG-415 \\
\hline $14378-38-2$ & Silver & $<0.001$ & ug/g dry & 0.001 & $4 / 14 / 08$ & 8D14002 & PNNL-AGG-415 \\
\hline $14041-58-8$ & Cadmium & $<0.0006$ & ug/g dry & 0.0006 & $4 / 14 / 08$ & 8D14002 & PNNL-AGG-415 \\
\hline $14265-72-6$ & Antimony & $<0.0005$ & ug/g dry & 0.0005 & $4 / 14 / 08$ & 8D14002 & PNNL-AGG-415 \\
\hline 13966-28-4 & Lead & $<0.0006$ & ug/g dry & 0.0006 & $4 / 14 / 08$ & 8D14002 & PNNL-AGG-415 \\
\hline HEIS No. & В1T2P4A & \multicolumn{3}{|c|}{ Lab ID: $\quad$ 0803014-17 } & & & \\
\hline $14092-98-9$ & Chromium & $<0.002$ & ug/g dry & 0.002 & $4 / 14 / 08$ & 8D14002 & PNNL-AGG-415 \\
\hline 14119-06-3 & Copper & $<0.005$ & ug/g dry & 0.005 & $4 / 14 / 08$ & 8D14002 & PNNL-AGG-415 \\
\hline 7440-38-2 & Arsenic & $<0.006$ & ug/g dry & 0.006 & $4 / 14 / 08$ & 8D14002 & PNNL-AGG-415 \\
\hline $14687-58-2$ & Selenium & $<0.011$ & ug/g dry & 0.011 & $4 / 14 / 08$ & 8D14002 & PNNL-AGG-415 \\
\hline $14392-17-7$ & Molybdenum & 0.032 & ug/g dry & 0.002 & $4 / 14 / 08$ & 8D14002 & PNNL-AGG-415 \\
\hline $14914-61-5$ & Ruthenium & $<0.0008$ & ug/g dry & 0.0008 & $4 / 14 / 08$ & 8D14002 & PNNL-AGG-415 \\
\hline $14378-38-2$ & Silver & $<0.001$ & ug/g dry & 0.001 & $4 / 14 / 08$ & 8D14002 & PNNL-AGG-415 \\
\hline $14041-58-8$ & Cadmium & $<0.0006$ & ug/g dry & 0.0006 & $4 / 14 / 08$ & 8D14002 & PNNL-AGG-415 \\
\hline $14265-72-6$ & Antimony & $<0.0005$ & ug/g dry & 0.0005 & $4 / 14 / 08$ & 8D14002 & PNNL-AGG-415 \\
\hline 13966-28-4 & Lead & $<0.0006$ & ug/g dry & 0.0006 & $4 / 14 / 08$ & 8D14002 & PNNL-AGG-415 \\
\hline HEIS No. & B1T2P5B & \multicolumn{3}{|c|}{ Lab ID: $\quad$ 0803014-20 } & & & \\
\hline 14092-98-9 & Chromium & $<0.002$ & ug/g dry & 0.002 & 4/14/08 & 8D14002 & PNNL-AGG-415 \\
\hline 14119-06-3 & Copper & $<0.005$ & ug/g dry & 0.005 & $4 / 14 / 08$ & 8D14002 & PNNL-AGG-415 \\
\hline 7440-38-2 & Arsenic & $<0.006$ & ug/g dry & 0.006 & $4 / 14 / 08$ & 8D14002 & PNNL-AGG-415 \\
\hline $14687-58-2$ & Selenium & $<0.011$ & ug/g dry & 0.011 & $4 / 14 / 08$ & 8D14002 & PNNL-AGG-415 \\
\hline $14392-17-7$ & Molybdenum & 0.039 & ug/g dry & 0.002 & $4 / 14 / 08$ & 8D14002 & PNNL-AGG-415 \\
\hline $14914-61-5$ & Ruthenium & $<0.0008$ & ug/g dry & 0.0008 & $4 / 14 / 08$ & 8D14002 & PNNL-AGG-415 \\
\hline $14378-38-2$ & Silver & $<0.001$ & ug/g dry & 0.001 & $4 / 14 / 08$ & 8D14002 & PNNL-AGG-415 \\
\hline $14041-58-8$ & Cadmium & $<0.0006$ & ug/g dry & 0.0006 & $4 / 14 / 08$ & 8D14002 & PNNL-AGG-415 \\
\hline $14265-72-6$ & Antimony & $<0.0005$ & ug/g dry & 0.0005 & $4 / 14 / 08$ & 8D14002 & PNNL-AGG-415 \\
\hline 13966-28-4 & Lead & $<0.0006$ & ug/g dry & 0.0006 & $4 / 14 / 08$ & 8D14002 & PNNL-AGG-415 \\
\hline HEIS No. & B1T2P5A & \multicolumn{3}{|c|}{ Lab ID: $\quad$ 0803014-21 } & & & \\
\hline 14092-98-9 & Chromium & $<0.002$ & ug/g dry & 0.002 & $4 / 14 / 08$ & 8D14002 & PNNL-AGG-415 \\
\hline 14119-06-3 & Copper & $<0.005$ & ug/g dry & 0.005 & $4 / 14 / 08$ & 8D14002 & PNNL-AGG-415 \\
\hline $7440-38-2$ & Arsenic & $<0.006$ & ug/g dry & 0.006 & 4/14/08 & 8D14002 & PNNL-AGG-415 \\
\hline $14687-58-2$ & Selenium & 0.014 & ug/g dry & 0.011 & $4 / 14 / 08$ & 8D14002 & PNNL-AGG-415 \\
\hline $14392-17-7$ & Molybdenum & 0.047 & ug/g dry & 0.002 & $4 / 14 / 08$ & 8D14002 & PNNL-AGG-415 \\
\hline 14914-61-5 & Ruthenium & $<0.0008$ & ug/g dry & 0.0008 & $4 / 14 / 08$ & 8D14002 & PNNL-AGG-415 \\
\hline $14378-38-2$ & Silver & $<0.001$ & ug/g dry & 0.001 & $4 / 14 / 08$ & 8D14002 & PNNL-AGG-415 \\
\hline $14041-58-8$ & Cadmium & $<0.0006$ & ug/g dry & 0.0006 & $4 / 14 / 08$ & 8D14002 & PNNL-AGG-415 \\
\hline $14265-72-6$ & Antimony & $<0.0005$ & ug/g dry & 0.0005 & $4 / 14 / 08$ & 8D14002 & PNNL-AGG-415 \\
\hline 13966-28-4 & Lead & $<0.0006$ & ug/g dry & 0.0006 & $4 / 14 / 08$ & 8D14002 & PNNL-AGG-415 \\
\hline HEIS No. & В1T2P6B & \multicolumn{3}{|c|}{ Lab ID: $\quad$ 0803014-24 } & & & \\
\hline $14092-98-9$ & Chromium & $<0.002$ & ug/g dry & 0.002 & $4 / 14 / 08$ & 8D14002 & PNNL-AGG-415 \\
\hline $14119-06-3$ & Copper & $<0.005$ & ug/g dry & 0.005 & $4 / 14 / 08$ & 8D14002 & PNNL-AGG-415 \\
\hline
\end{tabular}


RCRA Metals By SW846 6020/Water Extract

\begin{tabular}{|c|c|c|c|c|c|c|c|}
\hline CAS \# & Analyte & Results & Units & EQL & Analyzed & Batch & Method \\
\hline HEIS No. & B1T2P6B & \multicolumn{3}{|c|}{ Lab ID: $\quad$ 0803014-24 } & & & \\
\hline $7440-38-2$ & Arsenic & $<0.006$ & ug/g dry & 0.006 & $4 / 14 / 08$ & 8D14002 & PNNL-AGG-415 \\
\hline $14687-58-2$ & Selenium & $<0.011$ & ug/g dry & 0.011 & $4 / 14 / 08$ & 8D14002 & PNNL-AGG-415 \\
\hline $14392-17-7$ & Molybdenum & 0.032 & ug/g dry & 0.002 & $4 / 14 / 08$ & 8D14002 & PNNL-AGG-415 \\
\hline $14914-61-5$ & Ruthenium & $<0.0008$ & ug/g dry & 0.0008 & $4 / 14 / 08$ & 8D14002 & PNNL-AGG-415 \\
\hline 14378-38-2 & Silver & $<0.001$ & ug/g dry & 0.001 & $4 / 14 / 08$ & 8D14002 & PNNL-AGG-415 \\
\hline 14041-58-8 & Cadmium & $<0.0006$ & ug/g dry & 0.0006 & $4 / 14 / 08$ & 8D14002 & PNNL-AGG-415 \\
\hline $14265-72-6$ & Antimony & $<0.0005$ & ug/g dry & 0.0005 & $4 / 14 / 08$ & 8D14002 & PNNL-AGG-415 \\
\hline 13966-28-4 & Lead & $<0.0006$ & ug/g dry & 0.0006 & $4 / 14 / 08$ & 8D14002 & PNNL-AGG-415 \\
\hline HEIS No. & B1T2P6A & \multicolumn{3}{|c|}{ Lab ID: $\quad$ 0803014-25 } & & & \\
\hline 14092-98-9 & Chromium & $<0.002$ & ug/g dry & 0.002 & $4 / 14 / 08$ & 8D14002 & PNNL-AGG-415 \\
\hline 14119-06-3 & Copper & $<0.005$ & ug/g dry & 0.005 & $4 / 14 / 08$ & 8D14002 & PNNL-AGG-415 \\
\hline $7440-38-2$ & Arsenic & $<0.006$ & ug/g dry & 0.006 & $4 / 14 / 08$ & 8D14002 & PNNL-AGG-415 \\
\hline $14687-58-2$ & Selenium & $<0.011$ & ug/g dry & 0.011 & $4 / 14 / 08$ & 8D14002 & PNNL-AGG-415 \\
\hline $14392-17-7$ & Molybdenum & 0.018 & ug/g dry & 0.002 & $4 / 14 / 08$ & 8D14002 & PNNL-AGG-415 \\
\hline $14914-61-5$ & Ruthenium & $<0.0008$ & ug/g dry & 0.0008 & $4 / 14 / 08$ & 8D14002 & PNNL-AGG-415 \\
\hline $14378-38-2$ & Silver & $<0.001$ & ug/g dry & 0.001 & $4 / 14 / 08$ & 8D14002 & PNNL-AGG-415 \\
\hline $14041-58-8$ & Cadmium & $<0.0006$ & ug/g dry & 0.0006 & $4 / 14 / 08$ & 8D14002 & PNNL-AGG-415 \\
\hline $14265-72-6$ & Antimony & $<0.0005$ & ug/g dry & 0.0005 & $4 / 14 / 08$ & 8D14002 & PNNL-AGG-415 \\
\hline 13966-28-4 & Lead & $<0.0006$ & ug/g dry & 0.0006 & $4 / 14 / 08$ & 8D14002 & PNNL-AGG-415 \\
\hline HEIS No. & B1T2P7B & \multicolumn{3}{|c|}{ Lab ID: $\quad$ 0803015-02 } & & & \\
\hline $14092-98-9$ & Chromium & $<0.002$ & ug/g dry & 0.002 & $4 / 14 / 08$ & 8D14002 & PNNL-AGG-415 \\
\hline 14119-06-3 & Copper & $<0.005$ & ug/g dry & 0.005 & $4 / 14 / 08$ & 8D14002 & PNNL-AGG-415 \\
\hline 7440-38-2 & Arsenic & 0.007 & ug/g dry & 0.006 & $4 / 14 / 08$ & 8D14002 & PNNL-AGG-415 \\
\hline 14687-58-2 & Selenium & $<0.011$ & ug/g dry & 0.011 & $4 / 14 / 08$ & 8D14002 & PNNL-AGG-415 \\
\hline $14392-17-7$ & Molybdenum & 0.108 & ug/g dry & 0.002 & $4 / 14 / 08$ & 8D14002 & PNNL-AGG-415 \\
\hline $14914-61-5$ & Ruthenium & $<0.0008$ & ug/g dry & 0.0008 & $4 / 14 / 08$ & 8D14002 & PNNL-AGG-415 \\
\hline 14378-38-2 & Silver & $<0.001$ & ug/g dry & 0.001 & $4 / 14 / 08$ & 8D14002 & PNNL-AGG-415 \\
\hline 14041-58-8 & Cadmium & $<0.0006$ & ug/g dry & 0.0006 & $4 / 14 / 08$ & 8D14002 & PNNL-AGG-415 \\
\hline $14265-72-6$ & Antimony & $<0.0005$ & ug/g dry & 0.0005 & $4 / 14 / 08$ & 8D14002 & PNNL-AGG-415 \\
\hline 13966-28-4 & Lead & $<0.0006$ & ug/g dry & 0.0006 & $4 / 14 / 08$ & 8D14002 & PNNL-AGG-415 \\
\hline HEIS No. & B1T2P7A & \multicolumn{3}{|c|}{ Lab ID: $\quad$ 0803015-03 } & & & \\
\hline 14092-98-9 & Chromium & $<0.002$ & ug/g dry & 0.002 & $4 / 14 / 08$ & 8D14002 & PNNL-AGG-415 \\
\hline 14119-06-3 & Copper & $<0.005$ & ug/g dry & 0.005 & $4 / 14 / 08$ & 8D14002 & PNNL-AGG-415 \\
\hline 7440-38-2 & Arsenic & $<0.006$ & ug/g dry & 0.006 & $4 / 14 / 08$ & 8D14002 & PNNL-AGG-415 \\
\hline $14687-58-2$ & Selenium & $<0.011$ & ug/g dry & 0.011 & $4 / 14 / 08$ & 8D14002 & PNNL-AGG-415 \\
\hline 14392-17-7 & Molybdenum & 0.105 & ug/g dry & 0.002 & $4 / 14 / 08$ & 8D14002 & PNNL-AGG-415 \\
\hline $14914-61-5$ & Ruthenium & $<0.0008$ & ug/g dry & 0.0008 & $4 / 14 / 08$ & 8D14002 & PNNL-AGG-415 \\
\hline $14378-38-2$ & Silver & $<0.001$ & ug/g dry & 0.001 & $4 / 14 / 08$ & 8D14002 & PNNL-AGG-415 \\
\hline 14041-58-8 & Cadmium & $<0.0006$ & ug/g dry & 0.0006 & $4 / 14 / 08$ & 8D14002 & PNNL-AGG-415 \\
\hline $14265-72-6$ & Antimony & $<0.0005$ & ug/g dry & 0.0005 & $4 / 14 / 08$ & 8D14002 & PNNL-AGG-415 \\
\hline 13966-28-4 & Lead & $<0.0006$ & ug/g dry & 0.0006 & $4 / 14 / 08$ & 8D14002 & PNNL-AGG-415 \\
\hline HEIS No. & B1T2P8B & \multicolumn{3}{|c|}{ Lab ID: $\quad$ 0803015-06 } & & & \\
\hline 14092-98-9 & Chromium & $<0.002$ & ug/g dry & 0.002 & $4 / 14 / 08$ & 8D14002 & PNNL-AGG-415 \\
\hline 14119-06-3 & Copper & $<0.005$ & ug/g dry & 0.005 & $4 / 14 / 08$ & 8D14002 & PNNL-AGG-415 \\
\hline $7440-38-2$ & Arsenic & $<0.006$ & ug/g dry & 0.006 & $4 / 14 / 08$ & 8D14002 & PNNL-AGG-415 \\
\hline $14687-58-2$ & Selenium & $<0.011$ & ug/g dry & 0.011 & $4 / 14 / 08$ & 8D14002 & PNNL-AGG-415 \\
\hline $14392-17-7$ & Molybdenum & 0.009 & ug/g dry & 0.002 & $4 / 14 / 08$ & 8D14002 & PNNL-AGG-415 \\
\hline $14914-61-5$ & Ruthenium & $<0.0008$ & ug/g dry & 0.0008 & $4 / 14 / 08$ & 8D14002 & PNNL-AGG-415 \\
\hline
\end{tabular}


RCRA Metals By SW846 6020/Water Extract

\begin{tabular}{|c|c|c|c|c|c|c|c|}
\hline CAS \# & Analyte & Results & Units & EQL & Analyzed & Batch & Method \\
\hline HEIS No. & B1T2P8B & \multicolumn{3}{|c|}{ Lab ID: $\quad$ 0803015-06 } & & & \\
\hline $14378-38-2$ & Silver & $<0.001$ & ug/g dry & 0.001 & $4 / 14 / 08$ & 8D14002 & PNNL-AGG-415 \\
\hline $14041-58-8$ & Cadmium & $<0.0006$ & ug/g dry & 0.0006 & $4 / 14 / 08$ & 8D14002 & PNNL-AGG-415 \\
\hline $14265-72-6$ & Antimony & $<0.0005$ & ug/g dry & 0.0005 & 4/14/08 & 8D14002 & PNNL-AGG-415 \\
\hline 13966-28-4 & Lead & $<0.0006$ & ug/g dry & 0.0006 & 4/14/08 & 8D14002 & PNNL-AGG-415 \\
\hline HEIS No. & B1T2P8A & \multicolumn{3}{|c|}{ Lab ID: $\quad$ 0803015-07 } & & & \\
\hline 14092-98-9 & Chromium & $<0.002$ & ug/g dry & 0.002 & $4 / 14 / 08$ & 8D14002 & PNNL-AGG-415 \\
\hline 14119-06-3 & Copper & $<0.005$ & ug/g dry & 0.005 & $4 / 14 / 08$ & 8D14002 & PNNL-AGG-415 \\
\hline 7440-38-2 & Arsenic & $<0.006$ & ug/g dry & 0.006 & $4 / 14 / 08$ & 8D14002 & PNNL-AGG-415 \\
\hline $14687-58-2$ & Selenium & $<0.011$ & ug/g dry & 0.011 & $4 / 14 / 08$ & 8D14002 & PNNL-AGG-415 \\
\hline $14392-17-7$ & Molybdenum & 0.008 & ug/g dry & 0.002 & $4 / 14 / 08$ & 8D14002 & PNNL-AGG-415 \\
\hline $14914-61-5$ & Ruthenium & $<0.0008$ & ug/g dry & 0.0008 & $4 / 14 / 08$ & 8D14002 & PNNL-AGG-415 \\
\hline $14378-38-2$ & Silver & $<0.001$ & ug/g dry & 0.001 & $4 / 14 / 08$ & 8D14002 & PNNL-AGG-415 \\
\hline $14041-58-8$ & Cadmium & $<0.0006$ & ug/g dry & 0.0006 & $4 / 14 / 08$ & 8D14002 & PNNL-AGG-415 \\
\hline $14265-72-6$ & Antimony & $<0.0005$ & ug/g dry & 0.0005 & $4 / 14 / 08$ & 8D14002 & PNNL-AGG-415 \\
\hline 13966-28-4 & Lead & $<0.0006$ & ug/g dry & 0.0006 & $4 / 14 / 08$ & 8D14002 & PNNL-AGG-415 \\
\hline HEIS No. & В1T2P9B & \multicolumn{3}{|c|}{ Lab ID: $\quad$ 0803015-10 } & & & \\
\hline 14092-98-9 & Chromium & $<0.002$ & ug/g dry & 0.002 & 4/15/08 & 8D14002 & PNNL-AGG-415 \\
\hline $14119-06-3$ & Copper & $<0.005$ & ug/g dry & 0.005 & $4 / 15 / 08$ & 8D14002 & PNNL-AGG-415 \\
\hline $7440-38-2$ & Arsenic & $<0.006$ & ug/g dry & 0.006 & $4 / 15 / 08$ & 8D14002 & PNNL-AGG-415 \\
\hline $14687-58-2$ & Selenium & $<0.011$ & ug/g dry & 0.011 & $4 / 15 / 08$ & 8D14002 & PNNL-AGG-415 \\
\hline $14392-17-7$ & Molybdenum & 0.011 & ug/g dry & 0.002 & $4 / 15 / 08$ & 8D14002 & PNNL-AGG-415 \\
\hline $14914-61-5$ & Ruthenium & $<0.0008$ & ug/g dry & 0.0008 & $4 / 15 / 08$ & 8D14002 & PNNL-AGG-415 \\
\hline $14378-38-2$ & Silver & $<0.001$ & ug/g dry & 0.001 & $4 / 15 / 08$ & 8D14002 & PNNL-AGG-415 \\
\hline $14041-58-8$ & Cadmium & $<0.0006$ & ug/g dry & 0.0006 & $4 / 15 / 08$ & 8D14002 & PNNL-AGG-415 \\
\hline $14265-72-6$ & Antimony & $<0.0005$ & ug/g dry & 0.0005 & $4 / 15 / 08$ & 8D14002 & PNNL-AGG-415 \\
\hline 13966-28-4 & Lead & $<0.0006$ & ug/g dry & 0.0006 & $4 / 15 / 08$ & 8D14002 & PNNL-AGG-415 \\
\hline HEIS No. & B1T2P9A & \multicolumn{3}{|c|}{ Lab ID: $\quad$ 0803015-11 } & & & \\
\hline $14092-98-9$ & Chromium & $<0.002$ & ug/g dry & 0.002 & $4 / 15 / 08$ & 8D14002 & PNNL-AGG-415 \\
\hline 14119-06-3 & Copper & $<0.005$ & ug/g dry & 0.005 & $4 / 15 / 08$ & 8D14002 & PNNL-AGG-415 \\
\hline $7440-38-2$ & Arsenic & $<0.006$ & ug/g dry & 0.006 & $4 / 15 / 08$ & 8D14002 & PNNL-AGG-415 \\
\hline $14687-58-2$ & Selenium & $<0.011$ & ug/g dry & 0.011 & $4 / 15 / 08$ & 8D14002 & PNNL-AGG-415 \\
\hline $14392-17-7$ & Molybdenum & 0.009 & ug/g dry & 0.002 & $4 / 15 / 08$ & 8D14002 & PNNL-AGG-415 \\
\hline $14914-61-5$ & Ruthenium & $<0.0008$ & ug/g dry & 0.0008 & $4 / 15 / 08$ & 8D14002 & PNNL-AGG-415 \\
\hline $14378-38-2$ & Silver & $<0.001$ & ug/g dry & 0.001 & $4 / 15 / 08$ & 8D14002 & PNNL-AGG-415 \\
\hline $14041-58-8$ & Cadmium & $<0.0006$ & ug/g dry & 0.0006 & $4 / 15 / 08$ & 8D14002 & PNNL-AGG-415 \\
\hline $14265-72-6$ & Antimony & $<0.0005$ & ug/g dry & 0.0005 & $4 / 15 / 08$ & 8D14002 & PNNL-AGG-415 \\
\hline $13966-28-4$ & Lead & $<0.0006$ & ug/g dry & 0.0006 & $4 / 15 / 08$ & 8D14002 & PNNL-AGG-415 \\
\hline HEIS No. & B1T2R0B & \multicolumn{3}{|c|}{ Lab ID: $\quad$ 0803015-14 } & & & \\
\hline $14092-98-9$ & Chromium & $<0.002$ & ug/g dry & 0.002 & $4 / 15 / 08$ & 8D14002 & PNNL-AGG-415 \\
\hline 14119-06-3 & Copper & $<0.005$ & ug/g dry & 0.005 & $4 / 15 / 08$ & 8D14002 & PNNL-AGG-415 \\
\hline $7440-38-2$ & Arsenic & $<0.006$ & ug/g dry & 0.006 & $4 / 15 / 08$ & 8D14002 & PNNL-AGG-415 \\
\hline $14687-58-2$ & Selenium & $<0.011$ & ug/g dry & 0.011 & $4 / 15 / 08$ & 8D14002 & PNNL-AGG-415 \\
\hline $14392-17-7$ & Molybdenum & 0.022 & ug/g dry & 0.002 & $4 / 15 / 08$ & 8D14002 & PNNL-AGG-415 \\
\hline $14914-61-5$ & Ruthenium & $<0.0008$ & ug/g dry & 0.0008 & $4 / 15 / 08$ & 8D14002 & PNNL-AGG-415 \\
\hline $14378-38-2$ & Silver & $<0.001$ & ug/g dry & 0.001 & 4/15/08 & 8D14002 & PNNL-AGG-415 \\
\hline $14041-58-8$ & Cadmium & $<0.0006$ & ug/g dry & 0.0006 & $4 / 15 / 08$ & 8D14002 & PNNL-AGG-415 \\
\hline $14265-72-6$ & Antimony & $<0.0005$ & ug/g dry & 0.0005 & $4 / 15 / 08$ & 8D14002 & PNNL-AGG-415 \\
\hline 13966-28-4 & Lead & $<0.0006$ & ug/g dry & 0.0006 & $4 / 15 / 08$ & 8D14002 & PNNL-AGG-415 \\
\hline
\end{tabular}


RCRA Metals By SW846 6020/Water Extract

\begin{tabular}{|c|c|c|c|c|c|c|c|}
\hline CAS \# & Analyte & Results & Units & EQL & Analyzed & Batch & Method \\
\hline HEIS No. & B1T2R0A & \multicolumn{3}{|c|}{ Lab ID: $\quad$ 0803015-15 } & & & \\
\hline $14092-98-9$ & Chromium & $<0.002$ & ug/g dry & 0.002 & 4/15/08 & 8D14002 & PNNL-AGG-415 \\
\hline 14119-06-3 & Copper & $<0.005$ & ug/g dry & 0.005 & $4 / 15 / 08$ & 8D14002 & PNNL-AGG-415 \\
\hline $7440-38-2$ & Arsenic & $<0.006$ & ug/g dry & 0.006 & $4 / 15 / 08$ & 8D14002 & PNNL-AGG-415 \\
\hline $14687-58-2$ & Selenium & $<0.011$ & ug/g dry & 0.011 & $4 / 15 / 08$ & 8D14002 & PNNL-AGG-415 \\
\hline 14392-17-7 & Molybdenum & 0.016 & ug/g dry & 0.002 & $4 / 15 / 08$ & 8D14002 & PNNL-AGG-415 \\
\hline 14914-61-5 & Ruthenium & $<0.0008$ & ug/g dry & 0.0008 & $4 / 15 / 08$ & 8D14002 & PNNL-AGG-415 \\
\hline 14378-38-2 & Silver & $<0.001$ & ug/g dry & 0.001 & $4 / 15 / 08$ & 8D14002 & PNNL-AGG-415 \\
\hline 14041-58-8 & Cadmium & $<0.0006$ & ug/g dry & 0.0006 & $4 / 15 / 08$ & 8D14002 & PNNL-AGG-415 \\
\hline $14265-72-6$ & Antimony & $<0.0005$ & ug/g dry & 0.0005 & $4 / 15 / 08$ & 8D14002 & PNNL-AGG-415 \\
\hline 13966-28-4 & Lead & $<0.0006$ & ug/g dry & 0.0006 & $4 / 15 / 08$ & 8D14002 & PNNL-AGG-415 \\
\hline HEIS No. & B1TNK6B & \multicolumn{3}{|c|}{ Lab ID: $\quad$ 0803015-18 } & & & \\
\hline $14092-98-9$ & Chromium & $<0.002$ & ug/g dry & 0.002 & $4 / 15 / 08$ & 8D14002 & PNNL-AGG-415 \\
\hline 14119-06-3 & Copper & $<0.005$ & ug/g dry & 0.005 & $4 / 15 / 08$ & 8D14002 & PNNL-AGG-415 \\
\hline $7440-38-2$ & Arsenic & $<0.006$ & ug/g dry & 0.006 & $4 / 15 / 08$ & 8D14002 & PNNL-AGG-415 \\
\hline $14687-58-2$ & Selenium & $<0.011$ & ug/g dry & 0.011 & $4 / 15 / 08$ & 8D14002 & PNNL-AGG-415 \\
\hline $14392-17-7$ & Molybdenum & 0.013 & ug/g dry & 0.002 & $4 / 15 / 08$ & 8D14002 & PNNL-AGG-415 \\
\hline $14914-61-5$ & Ruthenium & $<0.0008$ & ug/g dry & 0.0008 & $4 / 15 / 08$ & 8D14002 & PNNL-AGG-415 \\
\hline $14378-38-2$ & Silver & $<0.001$ & ug/g dry & 0.001 & $4 / 15 / 08$ & 8D14002 & PNNL-AGG-415 \\
\hline 14041-58-8 & Cadmium & $<0.0006$ & ug/g dry & 0.0006 & $4 / 15 / 08$ & 8D14002 & PNNL-AGG-415 \\
\hline $14265-72-6$ & Antimony & $<0.0005$ & ug/g dry & 0.0005 & $4 / 15 / 08$ & 8D14002 & PNNL-AGG-415 \\
\hline 13966-28-4 & Lead & $<0.0006$ & ug/g dry & 0.0006 & $4 / 15 / 08$ & 8D14002 & PNNL-AGG-415 \\
\hline HEIS No. & B1TNK6A & \multicolumn{3}{|c|}{ Lab ID: $\quad$ 0803015-19 } & & & \\
\hline 14092-98-9 & Chromium & $<0.002$ & ug/g dry & 0.002 & $4 / 15 / 08$ & 8D14002 & PNNL-AGG-415 \\
\hline 14119-06-3 & Copper & $<0.005$ & ug/g dry & 0.005 & $4 / 15 / 08$ & 8D14002 & PNNL-AGG-415 \\
\hline $7440-38-2$ & Arsenic & $<0.006$ & ug/g dry & 0.006 & $4 / 15 / 08$ & 8D14002 & PNNL-AGG-415 \\
\hline $14687-58-2$ & Selenium & $<0.011$ & ug/g dry & 0.011 & $4 / 15 / 08$ & 8D14002 & PNNL-AGG-415 \\
\hline 14392-17-7 & Molybdenum & 0.009 & ug/g dry & 0.002 & $4 / 15 / 08$ & 8D14002 & PNNL-AGG-415 \\
\hline 14914-61-5 & Ruthenium & $<0.0008$ & ug/g dry & 0.0008 & $4 / 15 / 08$ & 8D14002 & PNNL-AGG-415 \\
\hline $14378-38-2$ & Silver & $<0.001$ & ug/g dry & 0.001 & $4 / 15 / 08$ & 8D14002 & PNNL-AGG-415 \\
\hline 14041-58-8 & Cadmium & $<0.0006$ & ug/g dry & 0.0006 & $4 / 15 / 08$ & 8D14002 & PNNL-AGG-415 \\
\hline $14265-72-6$ & Antimony & $<0.0005$ & ug/g dry & 0.0005 & $4 / 15 / 08$ & 8D14002 & PNNL-AGG-415 \\
\hline 13966-28-4 & Lead & $<0.0006$ & ug/g dry & 0.0006 & $4 / 15 / 08$ & 8D14002 & PNNL-AGG-415 \\
\hline HEIS No. & B1TNK7B & \multicolumn{3}{|c|}{ Lab ID: $\quad$ 0803015-22 } & & & \\
\hline 14092-98-9 & Chromium & $<0.002$ & ug/g dry & 0.002 & $4 / 15 / 08$ & 8D14002 & PNNL-AGG-415 \\
\hline 14119-06-3 & Copper & $<0.005$ & ug/g dry & 0.005 & $4 / 15 / 08$ & 8D14002 & PNNL-AGG-415 \\
\hline 7440-38-2 & Arsenic & $<0.006$ & ug/g dry & 0.006 & $4 / 15 / 08$ & 8D14002 & PNNL-AGG-415 \\
\hline $14687-58-2$ & Selenium & $<0.011$ & ug/g dry & 0.011 & $4 / 15 / 08$ & 8D14002 & PNNL-AGG-415 \\
\hline $14392-17-7$ & Molybdenum & 0.008 & ug/g dry & 0.002 & $4 / 15 / 08$ & 8D14002 & PNNL-AGG-415 \\
\hline $14914-61-5$ & Ruthenium & $<0.0008$ & ug/g dry & 0.0008 & $4 / 15 / 08$ & 8D14002 & PNNL-AGG-415 \\
\hline $14378-38-2$ & Silver & $<0.001$ & ug/g dry & 0.001 & $4 / 15 / 08$ & 8D14002 & PNNL-AGG-415 \\
\hline 14041-58-8 & Cadmium & $<0.0006$ & ug/g dry & 0.0006 & $4 / 15 / 08$ & 8D14002 & PNNL-AGG-415 \\
\hline $14265-72-6$ & Antimony & $<0.0005$ & ug/g dry & 0.0005 & $4 / 15 / 08$ & 8D14002 & PNNL-AGG-415 \\
\hline 13966-28-4 & Lead & $<0.0006$ & ug/g dry & 0.0006 & $4 / 15 / 08$ & 8D14002 & PNNL-AGG-415 \\
\hline HEIS No. & B1TNK7A & \multicolumn{3}{|c|}{ Lab ID: $\quad$ 0803015-23 } & & & \\
\hline 14092-98-9 & Chromium & $<0.002$ & ug/g dry & 0.002 & $4 / 15 / 08$ & 8D14002 & PNNL-AGG-415 \\
\hline 14119-06-3 & Copper & $<0.005$ & ug/g dry & 0.005 & $4 / 15 / 08$ & 8D14002 & PNNL-AGG-415 \\
\hline 7440-38-2 & Arsenic & $<0.006$ & ug/g dry & 0.006 & $4 / 15 / 08$ & 8D14002 & PNNL-AGG-415 \\
\hline $14687-58-2$ & Selenium & $<0.011$ & ug/g dry & 0.011 & $4 / 15 / 08$ & 8D14002 & PNNL-AGG-415 \\
\hline
\end{tabular}


RCRA Metals By SW846 6020/Water Extract

\begin{tabular}{|c|c|c|c|c|c|c|c|}
\hline CAS \# & Analyte & Results & Units & EQL & Analyzed & Batch & Method \\
\hline HEIS No. & B1TNK7A & \multicolumn{3}{|c|}{ Lab ID: $\quad$ 0803015-23 } & & & \\
\hline $14392-17-7$ & Molybdenum & 0.008 & ug/g dry & 0.002 & 4/15/08 & 8D14002 & PNNL-AGG-415 \\
\hline $14914-61-5$ & Ruthenium & $<0.0008$ & ug/g dry & 0.0008 & $4 / 15 / 08$ & 8D14002 & PNNL-AGG-415 \\
\hline 14378-38-2 & Silver & $<0.001$ & ug/g dry & 0.001 & $4 / 15 / 08$ & 8D14002 & PNNL-AGG-415 \\
\hline 14041-58-8 & Cadmium & $<0.0006$ & ug/g dry & 0.0006 & $4 / 15 / 08$ & 8D14002 & PNNL-AGG-415 \\
\hline $14265-72-6$ & Antimony & $<0.0005$ & ug/g dry & 0.0005 & $4 / 15 / 08$ & 8D14002 & PNNL-AGG-415 \\
\hline 13966-28-4 & Lead & $<0.0006$ & ug/g dry & 0.0006 & $4 / 15 / 08$ & 8D14002 & PNNL-AGG-415 \\
\hline HEIS No. & B1TNK8B & \multicolumn{3}{|c|}{ Lab ID: $\quad$ 0803015-26 } & & & \\
\hline $14092-98-9$ & Chromium & $<0.002$ & ug/g dry & 0.002 & $4 / 15 / 08$ & 8D14002 & PNNL-AGG-415 \\
\hline 14119-06-3 & Copper & $<0.005$ & ug/g dry & 0.005 & $4 / 15 / 08$ & 8D14002 & PNNL-AGG-415 \\
\hline $7440-38-2$ & Arsenic & $<0.006$ & ug/g dry & 0.006 & $4 / 15 / 08$ & 8D14002 & PNNL-AGG-415 \\
\hline $14687-58-2$ & Selenium & $<0.011$ & ug/g dry & 0.011 & $4 / 15 / 08$ & 8D14002 & PNNL-AGG-415 \\
\hline $14392-17-7$ & Molybdenum & 0.015 & ug/g dry & 0.002 & $4 / 15 / 08$ & 8D14002 & PNNL-AGG-415 \\
\hline $14914-61-5$ & Ruthenium & $<0.0008$ & ug/g dry & 0.0008 & $4 / 15 / 08$ & 8D14002 & PNNL-AGG-415 \\
\hline $14378-38-2$ & Silver & $<0.001$ & ug/g dry & 0.001 & $4 / 15 / 08$ & 8D14002 & PNNL-AGG-415 \\
\hline 14041-58-8 & Cadmium & $<0.0006$ & ug/g dry & 0.0006 & $4 / 15 / 08$ & 8D14002 & PNNL-AGG-415 \\
\hline $14265-72-6$ & Antimony & $<0.0005$ & ug/g dry & 0.0005 & $4 / 15 / 08$ & 8D14002 & PNNL-AGG-415 \\
\hline 13966-28-4 & Lead & $<0.0006$ & ug/g dry & 0.0006 & $4 / 15 / 08$ & 8D14002 & PNNL-AGG-415 \\
\hline HEIS No. & B1TNK8A & \multicolumn{3}{|c|}{ Lab ID: $\quad$ 0803015-27 } & & & \\
\hline 14092-98-9 & Chromium & $<0.002$ & ug/g dry & 0.002 & $4 / 15 / 08$ & 8D14002 & PNNL-AGG-415 \\
\hline 14119-06-3 & Copper & $<0.005$ & ug/g dry & 0.005 & $4 / 15 / 08$ & 8D14002 & PNNL-AGG-415 \\
\hline $7440-38-2$ & Arsenic & $<0.006$ & ug/g dry & 0.006 & $4 / 15 / 08$ & 8D14002 & PNNL-AGG-415 \\
\hline $14687-58-2$ & Selenium & $<0.011$ & ug/g dry & 0.011 & $4 / 15 / 08$ & 8D14002 & PNNL-AGG-415 \\
\hline $14392-17-7$ & Molybdenum & 0.013 & ug/g dry & 0.002 & $4 / 15 / 08$ & 8D14002 & PNNL-AGG-415 \\
\hline 14914-61-5 & Ruthenium & $<0.0008$ & ug/g dry & 0.0008 & $4 / 15 / 08$ & 8D14002 & PNNL-AGG-415 \\
\hline $14378-38-2$ & Silver & $<0.001$ & ug/g dry & 0.001 & $4 / 15 / 08$ & 8D14002 & PNNL-AGG-415 \\
\hline 14041-58-8 & Cadmium & $<0.0006$ & ug/g dry & 0.0006 & $4 / 15 / 08$ & 8D14002 & PNNL-AGG-415 \\
\hline $14265-72-6$ & Antimony & $<0.0005$ & ug/g dry & 0.0005 & $4 / 15 / 08$ & 8D14002 & PNNL-AGG-415 \\
\hline 13966-28-4 & Lead & $<0.0006$ & ug/g dry & 0.0006 & $4 / 15 / 08$ & 8D14002 & PNNL-AGG-415 \\
\hline HEIS No. & B1TNK9B & \multicolumn{3}{|c|}{ Lab ID: $\quad$ 0803015-30 } & & & \\
\hline 14092-98-9 & Chromium & $<0.002$ & ug/g dry & 0.002 & $4 / 15 / 08$ & 8D14002 & PNNL-AGG-415 \\
\hline 14119-06-3 & Copper & $<0.005$ & ug/g dry & 0.005 & $4 / 15 / 08$ & 8D14002 & PNNL-AGG-415 \\
\hline 7440-38-2 & Arsenic & $<0.007$ & ug/g dry & 0.007 & $4 / 15 / 08$ & 8D14002 & PNNL-AGG-415 \\
\hline $14687-58-2$ & Selenium & $<0.012$ & ug/g dry & 0.012 & $4 / 15 / 08$ & 8D14002 & PNNL-AGG-415 \\
\hline $14392-17-7$ & Molybdenum & 0.015 & ug/g dry & 0.002 & $4 / 15 / 08$ & 8D14002 & PNNL-AGG-415 \\
\hline $14914-61-5$ & Ruthenium & $<0.0009$ & ug/g dry & 0.0009 & $4 / 15 / 08$ & 8D14002 & PNNL-AGG-415 \\
\hline 14378-38-2 & Silver & $<0.001$ & ug/g dry & 0.001 & $4 / 15 / 08$ & 8D14002 & PNNL-AGG-415 \\
\hline 14041-58-8 & Cadmium & $<0.0007$ & ug/g dry & 0.0007 & $4 / 15 / 08$ & 8D14002 & PNNL-AGG-415 \\
\hline $14265-72-6$ & Antimony & $<0.0006$ & ug/g dry & 0.0006 & $4 / 15 / 08$ & 8D14002 & PNNL-AGG-415 \\
\hline 13966-28-4 & Lead & $<0.0006$ & ug/g dry & 0.0006 & $4 / 15 / 08$ & 8D14002 & PNNL-AGG-415 \\
\hline HEIS No. & B1TNK9A & \multicolumn{3}{|c|}{ Lab ID: $\quad$ 0803015-31 } & & & \\
\hline 14092-98-9 & Chromium & $<0.002$ & ug/g dry & 0.002 & $4 / 15 / 08$ & 8D14002 & PNNL-AGG-415 \\
\hline 14119-06-3 & Copper & $<0.005$ & ug/g dry & 0.005 & $4 / 15 / 08$ & 8D14002 & PNNL-AGG-415 \\
\hline $7440-38-2$ & Arsenic & $<0.006$ & ug/g dry & 0.006 & $4 / 15 / 08$ & 8D14002 & PNNL-AGG-415 \\
\hline $14687-58-2$ & Selenium & $<0.011$ & ug/g dry & 0.011 & $4 / 15 / 08$ & 8D14002 & PNNL-AGG-415 \\
\hline $14392-17-7$ & Molybdenum & 0.013 & ug/g dry & 0.002 & $4 / 15 / 08$ & 8D14002 & PNNL-AGG-415 \\
\hline $14914-61-5$ & Ruthenium & $<0.0008$ & ug/g dry & 0.0008 & $4 / 15 / 08$ & 8D14002 & PNNL-AGG-415 \\
\hline $14378-38-2$ & Silver & $<0.001$ & ug/g dry & 0.001 & $4 / 15 / 08$ & 8D14002 & PNNL-AGG-415 \\
\hline 14041-58-8 & Cadmium & $<0.0006$ & ug/g dry & 0.0006 & 4/15/08 & 8D14002 & PNNL-AGG-415 \\
\hline
\end{tabular}


RCRA Metals By SW846 6020/Water Extract

\begin{tabular}{|lccccccc|}
\hline CAS \# & Analyte & Results & Units & EQL & Analyzed & Batch & Method \\
\hline HEIS No. & B1TNK9A & \multicolumn{2}{c|}{ Lab ID: } & $\mathbf{0 8 0 3 0 1 5}-31$ & & \\
$14265-72-6$ & Antimony & $<0.0005$ & ug/g dry & 0.0005 & $4 / 15 / 08$ & 8D14002 & PNNL-AGG-415 \\
$13966-28-4$ & Lead & $<0.0006$ & ug/g dry & 0.0006 & 4/15/08 & 8D14002 & PNNL-AGG-415 \\
\hline
\end{tabular}


RCRA Metals By SW846 6020/Acid Extract

\begin{tabular}{|c|c|c|c|c|c|c|c|}
\hline CAS \# & Analyte & Results & Units & EQL & Analyzed & Batch & Method \\
\hline HEIS No. & B1RTF8 & \multicolumn{3}{|c|}{ Lab ID: $\quad$ 0803001-01 } & & & \\
\hline 14119-06-3 & Copper & 10.5 & ug/g dry & 0.553 & $5 / 12 / 08$ & 8D21003 & PNNL-AGG-415 \\
\hline $7440-38-2$ & Arsenic & 1.28 & ug/g dry & 0.386 & $5 / 12 / 08$ & 8D21003 & PNNL-AGG-415 \\
\hline $14687-58-2$ & Selenium & $<1.05$ & ug/g dry & 1.05 & $5 / 12 / 08$ & 8D21003 & PNNL-AGG-415 \\
\hline $14392-20-2$ & Molybdenum & 0.429 & ug/g dry & 0.082 & $5 / 12 / 08$ & 8D21003 & PNNL-AGG-415 \\
\hline 14914-61-5 & Ruthenium & $<0.041$ & ug/g dry & 0.041 & $5 / 12 / 08$ & 8D21003 & PNNL-AGG-415 \\
\hline HEIS No. & B1RTF9A & \multicolumn{3}{|c|}{ Lab ID: $\quad$ 0803001-02 } & & & \\
\hline 14119-06-3 & Copper & 11.1 & ug/g dry & 0.485 & $5 / 12 / 08$ & 8D21003 & PNNL-AGG-415 \\
\hline 7440-38-2 & Arsenic & 1.16 & ug/g dry & 0.338 & $5 / 12 / 08$ & 8D21003 & PNNL-AGG-415 \\
\hline $14687-58-2$ & Selenium & $<0.924$ & ug/g dry & 0.924 & $5 / 12 / 08$ & 8D21003 & PNNL-AGG-415 \\
\hline $14392-20-2$ & Molybdenum & 0.408 & ug/g dry & 0.072 & $5 / 12 / 08$ & 8D21003 & PNNL-AGG-415 \\
\hline $14914-61-5$ & Ruthenium & $<0.036$ & ug/g dry & 0.036 & $5 / 12 / 08$ & 8D21003 & PNNL-AGG-415 \\
\hline HEIS No. & B1RTH1A & \multicolumn{3}{|c|}{ Lab ID: $\quad$ 0803012-02 } & & & \\
\hline 14119-06-3 & Copper & 9.56 & ug/g dry & 0.462 & $5 / 12 / 08$ & 8D21003 & PNNL-AGG-415 \\
\hline $7440-38-2$ & Arsenic & 0.584 & ug/g dry & 0.322 & $5 / 12 / 08$ & 8D21003 & PNNL-AGG-415 \\
\hline $14687-58-2$ & Selenium & $<0.880$ & ug/g dry & 0.880 & $5 / 12 / 08$ & 8D21003 & PNNL-AGG-415 \\
\hline $14392-20-2$ & Molybdenum & 0.178 & ug/g dry & 0.068 & $5 / 12 / 08$ & 8D21003 & PNNL-AGG-415 \\
\hline $14914-61-5$ & Ruthenium & $<0.035$ & ug/g dry & 0.035 & $5 / 12 / 08$ & 8D21003 & PNNL-AGG-415 \\
\hline HEIS No. & B1RTH1 & \multicolumn{3}{|c|}{ Lab ID: $\quad$ 0803012-03 } & & & \\
\hline $14119-06-3$ & Copper & 9.18 & ug/g dry & 0.497 & $5 / 12 / 08$ & 8D21003 & PNNL-AGG-415 \\
\hline $7440-38-2$ & Arsenic & 0.912 & ug/g dry & 0.347 & $5 / 12 / 08$ & 8D21003 & PNNL-AGG-415 \\
\hline $14687-58-2$ & Selenium & $<0.948$ & ug/g dry & 0.948 & $5 / 12 / 08$ & 8D21003 & PNNL-AGG-415 \\
\hline $14392-20-2$ & Molybdenum & 0.210 & ug/g dry & 0.074 & $5 / 12 / 08$ & 8D21003 & PNNL-AGG-415 \\
\hline $14914-61-5$ & Ruthenium & $<0.037$ & ug/g dry & 0.037 & $5 / 12 / 08$ & 8D21003 & PNNL-AGG-415 \\
\hline HEIS No. & B1RTH2B & \multicolumn{3}{|c|}{ Lab ID: $\quad$ 0803012-05 } & & & \\
\hline 14119-06-3 & Copper & 7.82 & ug/g dry & 0.516 & $5 / 12 / 08$ & 8D21003 & PNNL-AGG-415 \\
\hline $7440-38-2$ & Arsenic & 1.64 & ug/g dry & 0.360 & $5 / 12 / 08$ & 8D21003 & PNNL-AGG-415 \\
\hline $14687-58-2$ & Selenium & $<0.985$ & ug/g dry & 0.985 & $5 / 12 / 08$ & 8D21003 & PNNL-AGG-415 \\
\hline $14914-61-5$ & Ruthenium & $<0.039$ & ug/g dry & 0.039 & $5 / 12 / 08$ & 8D21003 & PNNL-AGG-415 \\
\hline HEIS No. & B1RTH2A & \multicolumn{3}{|c|}{ Lab ID: $\quad$ 0803012-06 } & & & \\
\hline 14119-06-3 & Copper & 7.26 & ug/g dry & 0.573 & $5 / 12 / 08$ & 8D21003 & PNNL-AGG-415 \\
\hline $7440-38-2$ & Arsenic & 1.20 & ug/g dry & 0.400 & $5 / 12 / 08$ & 8D21003 & PNNL-AGG-415 \\
\hline $14687-58-2$ & Selenium & $<1.09$ & ug/g dry & 1.09 & $5 / 12 / 08$ & 8D21003 & PNNL-AGG-415 \\
\hline $14392-20-2$ & Molybdenum & 0.140 & ug/g dry & 0.085 & $5 / 12 / 08$ & 8D21003 & PNNL-AGG-415 \\
\hline $14914-61-5$ & Ruthenium & $<0.043$ & ug/g dry & 0.043 & $5 / 12 / 08$ & 8D21003 & PNNL-AGG-415 \\
\hline HEIS No. & B1RTH3B & \multicolumn{3}{|c|}{ Lab ID: $\quad$ 0803012-09 } & & & \\
\hline 14119-06-3 & Copper & 7.80 & ug/g dry & 0.541 & $5 / 12 / 08$ & 8D21003 & PNNL-AGG-415 \\
\hline $7440-38-2$ & Arsenic & 1.40 & ug/g dry & 0.377 & $5 / 12 / 08$ & 8D21003 & PNNL-AGG-415 \\
\hline $14687-58-2$ & Selenium & $<1.03$ & ug/g dry & 1.03 & $5 / 12 / 08$ & 8D21003 & PNNL-AGG-415 \\
\hline $14392-20-2$ & Molybdenum & 0.163 & ug/g dry & 0.080 & $5 / 12 / 08$ & 8D21003 & PNNL-AGG-415 \\
\hline $14914-61-5$ & Ruthenium & $<0.041$ & ug/g dry & 0.041 & $5 / 12 / 08$ & 8D21003 & PNNL-AGG-415 \\
\hline HEIS No. & B1RTH3A & \multicolumn{3}{|c|}{ Lab ID: $\quad$ 0803012-10 } & & & \\
\hline $14119-06-3$ & Copper & 14.6 & ug/g dry & 0.561 & $5 / 12 / 08$ & 8D21003 & PNNL-AGG-415 \\
\hline 7440-38-2 & Arsenic & 2.08 & ug/g dry & 0.391 & $5 / 12 / 08$ & 8D21003 & PNNL-AGG-415 \\
\hline $14687-58-2$ & Selenium & $<1.07$ & ug/g dry & 1.07 & $5 / 12 / 08$ & 8D21003 & PNNL-AGG-415 \\
\hline $14392-20-2$ & Molybdenum & 0.259 & ug/g dry & 0.083 & $5 / 12 / 08$ & 8D21003 & PNNL-AGG-415 \\
\hline $14914-61-5$ & Ruthenium & $<0.042$ & ug/g dry & 0.042 & $5 / 12 / 08$ & 8D21003 & PNNL-AGG-415 \\
\hline HEIS No. & B1RYR7B & \multicolumn{2}{|c|}{ Lab ID: } & 0803012-13 & & & \\
\hline
\end{tabular}


RCRA Metals By SW846 6020/Acid Extract

\begin{tabular}{|c|c|c|c|c|c|c|c|}
\hline CAS \# & Analyte & Results & Units & EQL & Analyzed & Batch & Method \\
\hline HEIS No. & B1RYR7B & \multicolumn{3}{|c|}{ Lab ID: $\quad$ 0803012-13 } & & & \\
\hline 14119-06-3 & Copper & 7.46 & ug/g dry & 0.551 & $5 / 12 / 08$ & 8D21003 & PNNL-AGG-415 \\
\hline $7440-38-2$ & Arsenic & 2.00 & ug/g dry & 0.385 & $5 / 12 / 08$ & 8D21003 & PNNL-AGG-415 \\
\hline $14687-58-2$ & Selenium & $<1.05$ & ug/g dry & 1.05 & $5 / 12 / 08$ & 8D21003 & PNNL-AGG-415 \\
\hline $14392-20-2$ & Molybdenum & 0.245 & ug/g dry & 0.082 & $5 / 12 / 08$ & 8D21003 & PNNL-AGG-415 \\
\hline 14914-61-5 & Ruthenium & $<0.041$ & ug/g dry & 0.041 & $5 / 12 / 08$ & $8 \mathrm{D} 21003$ & PNNL-AGG-415 \\
\hline HEIS No. & B1RYR7A & \multicolumn{3}{|c|}{ Lab ID: $\quad$ 0803012-14 } & & & \\
\hline 14119-06-3 & Copper & 7.72 & ug/g dry & 0.580 & $5 / 12 / 08$ & 8D21003 & PNNL-AGG-415 \\
\hline $7440-38-2$ & Arsenic & 1.47 & ug/g dry & 0.405 & $5 / 12 / 08$ & 8D21003 & PNNL-AGG-415 \\
\hline $14687-58-2$ & Selenium & $<1.11$ & ug/g dry & 1.11 & $5 / 12 / 08$ & 8D21003 & PNNL-AGG-415 \\
\hline $14392-20-2$ & Molybdenum & 0.270 & ug/g dry & 0.086 & $5 / 12 / 08$ & 8D21003 & PNNL-AGG-415 \\
\hline $14914-61-5$ & Ruthenium & $<0.044$ & ug/g dry & 0.044 & $5 / 12 / 08$ & 8D21003 & PNNL-AGG-415 \\
\hline HEIS No. & B1RYR8B & \multicolumn{3}{|c|}{ Lab ID: $\quad$ 0803012-17 } & & & \\
\hline 14119-06-3 & Copper & 7.76 & ug/g dry & 0.638 & $5 / 12 / 08$ & 8D21003 & PNNL-AGG-415 \\
\hline $7440-38-2$ & Arsenic & 1.92 & ug/g dry & 0.445 & $5 / 12 / 08$ & 8D21003 & PNNL-AGG-415 \\
\hline $14687-58-2$ & Selenium & $<1.22$ & ug/g dry & 1.22 & $5 / 12 / 08$ & 8D21003 & PNNL-AGG-415 \\
\hline $14392-20-2$ & Molybdenum & 0.164 & ug/g dry & 0.095 & $5 / 12 / 08$ & 8D21003 & PNNL-AGG-415 \\
\hline 14914-61-5 & Ruthenium & $<0.048$ & ug/g dry & 0.048 & $5 / 12 / 08$ & 8D21003 & PNNL-AGG-415 \\
\hline HEIS No. & B1RYR8A & \multicolumn{3}{|c|}{ Lab ID: $\quad$ 0803012-18 } & & & \\
\hline 14119-06-3 & Copper & 6.91 & ug/g dry & 0.513 & $5 / 12 / 08$ & 8D21003 & PNNL-AGG-415 \\
\hline $7440-38-2$ & Arsenic & 1.41 & ug/g dry & 0.358 & $5 / 12 / 08$ & 8D21003 & PNNL-AGG-415 \\
\hline $14687-58-2$ & Selenium & $<0.979$ & ug/g dry & 0.979 & $5 / 12 / 08$ & 8D21003 & PNNL-AGG-415 \\
\hline $14392-20-2$ & Molybdenum & 0.161 & ug/g dry & 0.076 & $5 / 12 / 08$ & $8 \mathrm{D} 21003$ & PNNL-AGG-415 \\
\hline $14914-61-5$ & Ruthenium & $<0.039$ & ug/g dry & 0.039 & $5 / 12 / 08$ & 8D21003 & PNNL-AGG-415 \\
\hline HEIS No. & B1RYR9B & \multicolumn{3}{|c|}{ Lab ID: $\quad$ 0803012-21 } & & & \\
\hline 14119-06-3 & Copper & 8.50 & ug/g dry & 0.546 & $5 / 12 / 08$ & 8D21003 & PNNL-AGG-415 \\
\hline $7440-38-2$ & Arsenic & 1.86 & ug/g dry & 0.381 & $5 / 12 / 08$ & 8D21003 & PNNL-AGG-415 \\
\hline $14687-58-2$ & Selenium & $<1.04$ & ug/g dry & 1.04 & $5 / 12 / 08$ & 8D21003 & PNNL-AGG-415 \\
\hline $14392-20-2$ & Molybdenum & 0.326 & ug/g dry & 0.081 & $5 / 12 / 08$ & 8D21003 & PNNL-AGG-415 \\
\hline 14914-61-5 & Ruthenium & $<0.041$ & ug/g dry & 0.041 & $5 / 12 / 08$ & 8D21003 & PNNL-AGG-415 \\
\hline HEIS No. & B1RYR9A & \multicolumn{3}{|c|}{ Lab ID: $\quad$ 0803012-22 } & & & \\
\hline 14119-06-3 & Copper & 7.80 & ug/g dry & 0.527 & $5 / 12 / 08$ & 8D21003 & PNNL-AGG-415 \\
\hline $7440-38-2$ & Arsenic & 1.46 & ug/g dry & 0.368 & $5 / 12 / 08$ & 8D21003 & PNNL-AGG-415 \\
\hline 14687-58-2 & Selenium & $<1.01$ & ug/g dry & 1.01 & $5 / 12 / 08$ & 8D21003 & PNNL-AGG-415 \\
\hline $14392-20-2$ & Molybdenum & 0.236 & ug/g dry & 0.078 & $5 / 12 / 08$ & 8D21003 & PNNL-AGG-415 \\
\hline 14914-61-5 & Ruthenium & $<0.040$ & ug/g dry & 0.040 & $5 / 12 / 08$ & 8D21003 & PNNL-AGG-415 \\
\hline HEIS No. & B1RYT0B & \multicolumn{3}{|c|}{ Lab ID: $\quad$ 0803014-01 } & & & \\
\hline 14119-06-3 & Copper & 14.0 & ug/g dry & 0.637 & $5 / 12 / 08$ & 8D21003 & PNNL-AGG-415 \\
\hline $7440-38-2$ & Arsenic & 5.71 & ug/g dry & 0.445 & $5 / 12 / 08$ & 8D21003 & PNNL-AGG-415 \\
\hline $14687-58-2$ & Selenium & $<1.22$ & ug/g dry & 1.22 & $5 / 12 / 08$ & 8D21003 & PNNL-AGG-415 \\
\hline $14392-20-2$ & Molybdenum & 0.163 & ug/g dry & 0.094 & $5 / 12 / 08$ & 8D21003 & PNNL-AGG-415 \\
\hline $14914-61-5$ & Ruthenium & $<0.048$ & ug/g dry & 0.048 & $5 / 12 / 08$ & 8D21003 & PNNL-AGG-415 \\
\hline HEIS No. & B1RYT0A & \multicolumn{3}{|c|}{ Lab ID: $\quad$ 0803014-02 } & & & \\
\hline 14119-06-3 & Copper & 10.3 & ug/g dry & 0.594 & $5 / 12 / 08$ & 8D21003 & PNNL-AGG-415 \\
\hline 7440-38-2 & Arsenic & 2.67 & ug/g dry & 0.415 & $5 / 12 / 08$ & 8D21003 & PNNL-AGG-415 \\
\hline $14687-58-2$ & Selenium & $<1.13$ & ug/g dry & 1.13 & $5 / 12 / 08$ & 8D21003 & PNNL-AGG-415 \\
\hline 14392-20-2 & Molybdenum & 0.121 & ug/g dry & 0.088 & $5 / 12 / 08$ & 8D21003 & PNNL-AGG-415 \\
\hline $14914-61-5$ & Ruthenium & $<0.045$ & ug/g dry & 0.045 & $5 / 12 / 08$ & 8D21003 & PNNL-AGG-415 \\
\hline
\end{tabular}


RCRA Metals By SW846 6020/Acid Extract

\begin{tabular}{|c|c|c|c|c|c|c|c|}
\hline CAS \# & Analyte & Results & Units & EQL & Analyzed & Batch & Method \\
\hline HEIS No. & B1T2P1B & \multicolumn{3}{|c|}{ Lab ID: $\quad$ 0803014-05 } & & & \\
\hline 14119-06-3 & Copper & 9.99 & ug/g dry & 0.570 & $5 / 12 / 08$ & 8D21003 & PNNL-AGG-415 \\
\hline $7440-38-2$ & Arsenic & 1.86 & ug/g dry & 0.397 & $5 / 12 / 08$ & 8D21003 & PNNL-AGG-415 \\
\hline $14687-58-2$ & Selenium & $<1.09$ & ug/g dry & 1.09 & $5 / 12 / 08$ & 8D21003 & PNNL-AGG-415 \\
\hline $14914-61-5$ & Ruthenium & $<0.043$ & ug/g dry & 0.043 & $5 / 12 / 08$ & 8D21003 & PNNL-AGG-415 \\
\hline HEIS No. & B1T2P1A & \multicolumn{3}{|c|}{ Lab ID: $\quad$ 0803014-06 } & & & \\
\hline 14119-06-3 & Copper & 8.59 & ug/g dry & 0.556 & $5 / 13 / 08$ & 8D21003 & PNNL-AGG-415 \\
\hline 7440-38-2 & Arsenic & 1.86 & ug/g dry & 0.388 & $5 / 13 / 08$ & 8D21003 & PNNL-AGG-415 \\
\hline $14687-58-2$ & Selenium & $<1.06$ & ug/g dry & 1.06 & $5 / 13 / 08$ & 8D21003 & PNNL-AGG-415 \\
\hline $14392-17-7$ & Molybdenum & 0.363 & ug/g dry & 0.217 & $5 / 13 / 08$ & 8D21003 & PNNL-AGG-415 \\
\hline 14914-61-5 & Ruthenium & $<0.042$ & ug/g dry & 0.042 & $5 / 13 / 08$ & 8D21003 & PNNL-AGG-415 \\
\hline $14336-64-2$ & Cadmium & 0.055 & ug/g dry & 0.046 & $5 / 13 / 08$ & 8D21003 & PNNL-AGG-415 \\
\hline $14265-72-6$ & Antimony & $<0.074$ & ug/g dry & 0.074 & $5 / 13 / 08$ & 8D21003 & PNNL-AGG-415 \\
\hline 13966-28-4 & Lead & 2.43 & ug/g dry & 0.034 & $5 / 13 / 08$ & 8D21003 & PNNL-AGG-415 \\
\hline HEIS No. & B1T2P2B & \multicolumn{3}{|c|}{ Lab ID: $\quad$ 0803014-09 } & & & \\
\hline 14119-06-3 & Copper & 10.9 & ug/g dry & 0.648 & $5 / 13 / 08$ & 8D21003 & PNNL-AGG-415 \\
\hline 7440-38-2 & Arsenic & 1.95 & ug/g dry & 0.452 & $5 / 13 / 08$ & 8D21003 & PNNL-AGG-415 \\
\hline $14687-58-2$ & Selenium & $<1.24$ & ug/g dry & 1.24 & $5 / 13 / 08$ & 8D21003 & PNNL-AGG-415 \\
\hline 14392-17-7 & Molybdenum & 0.261 & ug/g dry & 0.253 & $5 / 13 / 08$ & 8D21003 & PNNL-AGG-415 \\
\hline $14914-61-5$ & Ruthenium & $<0.049$ & ug/g dry & 0.049 & $5 / 13 / 08$ & 8D21003 & PNNL-AGG-415 \\
\hline $14336-64-2$ & Cadmium & 0.054 & ug/g dry & 0.053 & $5 / 13 / 08$ & 8D21003 & PNNL-AGG-415 \\
\hline $14265-72-6$ & Antimony & $<0.086$ & ug/g dry & 0.086 & $5 / 13 / 08$ & 8D21003 & PNNL-AGG-415 \\
\hline 13966-28-4 & Lead & 1.98 & ug/g dry & 0.040 & $5 / 13 / 08$ & 8D21003 & PNNL-AGG-415 \\
\hline HEIS No. & B1T2P2A & \multicolumn{3}{|c|}{ Lab ID: $\quad$ 0803014-10 } & & & \\
\hline 14119-06-3 & Copper & 9.67 & ug/g dry & 0.637 & $5 / 13 / 08$ & 8D21003 & PNNL-AGG-415 \\
\hline 7440-38-2 & Arsenic & 1.77 & ug/g dry & 0.445 & $5 / 13 / 08$ & 8D21003 & PNNL-AGG-415 \\
\hline $14687-58-2$ & Selenium & $<1.21$ & ug/g dry & 1.21 & $5 / 13 / 08$ & 8D21003 & PNNL-AGG-415 \\
\hline $14392-17-7$ & Molybdenum & 0.317 & ug/g dry & 0.249 & $5 / 13 / 08$ & 8D21003 & PNNL-AGG-415 \\
\hline $14914-61-5$ & Ruthenium & $<0.048$ & ug/g dry & 0.048 & $5 / 13 / 08$ & 8D21003 & PNNL-AGG-415 \\
\hline $14336-64-2$ & Cadmium & 0.058 & ug/g dry & 0.052 & $5 / 13 / 08$ & 8D21003 & PNNL-AGG-415 \\
\hline $14265-72-6$ & Antimony & $<0.084$ & ug/g dry & 0.084 & $5 / 13 / 08$ & 8D21003 & PNNL-AGG-415 \\
\hline 13966-28-4 & Lead & 2.33 & ug/g dry & 0.039 & $5 / 13 / 08$ & 8D21003 & PNNL-AGG-415 \\
\hline HEIS No. & B1T2P3B & \multicolumn{3}{|c|}{ Lab ID: $\quad$ 0803014-13 } & & & \\
\hline 14119-06-3 & Copper & 8.97 & ug/g dry & 0.563 & $5 / 13 / 08$ & 8D21003 & PNNL-AGG-415 \\
\hline $7440-38-2$ & Arsenic & 1.37 & ug/g dry & 0.393 & $5 / 13 / 08$ & 8D21003 & PNNL-AGG-415 \\
\hline $14687-58-2$ & Selenium & $<1.07$ & ug/g dry & 1.07 & $5 / 13 / 08$ & 8D21003 & PNNL-AGG-415 \\
\hline $14392-17-7$ & Molybdenum & 0.321 & ug/g dry & 0.220 & $5 / 13 / 08$ & 8D21003 & PNNL-AGG-415 \\
\hline 14914-61-5 & Ruthenium & $<0.042$ & ug/g dry & 0.042 & $5 / 13 / 08$ & 8D21003 & PNNL-AGG-415 \\
\hline $14336-64-2$ & Cadmium & 0.050 & ug/g dry & 0.046 & $5 / 13 / 08$ & 8D21003 & PNNL-AGG-415 \\
\hline $14265-72-6$ & Antimony & $<0.075$ & ug/g dry & 0.075 & $5 / 13 / 08$ & 8D21003 & PNNL-AGG-415 \\
\hline 13966-28-4 & Lead & 1.94 & ug/g dry & 0.035 & $5 / 13 / 08$ & 8D21003 & PNNL-AGG-415 \\
\hline HEIS No. & B1T2P3A & \multicolumn{3}{|c|}{ Lab ID: $\quad$ 0803014-14 } & & & \\
\hline 14119-06-3 & Copper & 9.81 & ug/g dry & 0.575 & $5 / 13 / 08$ & 8D21003 & PNNL-AGG-415 \\
\hline 7440-38-2 & Arsenic & 1.29 & ug/g dry & 0.401 & $5 / 13 / 08$ & 8D21003 & PNNL-AGG-415 \\
\hline $14687-58-2$ & Selenium & $<1.10$ & ug/g dry & 1.10 & $5 / 13 / 08$ & 8D21003 & PNNL-AGG-415 \\
\hline $14392-17-7$ & Molybdenum & 0.456 & ug/g dry & 0.224 & $5 / 13 / 08$ & 8D21003 & PNNL-AGG-415 \\
\hline $14914-61-5$ & Ruthenium & $<0.043$ & ug/g dry & 0.043 & $5 / 13 / 08$ & 8D21003 & PNNL-AGG-415 \\
\hline $14336-64-2$ & Cadmium & 0.072 & ug/g dry & 0.047 & $5 / 13 / 08$ & 8D21003 & PNNL-AGG-415 \\
\hline $14265-72-6$ & Antimony & $<0.076$ & ug/g dry & 0.076 & $5 / 13 / 08$ & 8D21003 & PNNL-AGG-415 \\
\hline
\end{tabular}


RCRA Metals By SW846 6020/Acid Extract

\begin{tabular}{|c|c|c|c|c|c|c|c|}
\hline CAS \# & Analyte & Results & Units & EQL & Analyzed & Batch & Method \\
\hline HEIS No. & B1T2P3A & \multicolumn{3}{|c|}{ Lab ID: $\quad$ 0803014-14 } & & & \\
\hline 13966-28-4 & Lead & 2.29 & ug/g dry & 0.035 & $5 / 13 / 08$ & 8D21003 & PNNL-AGG-415 \\
\hline HEIS No. & B1T2P4B & \multicolumn{3}{|c|}{ Lab ID: $\quad 0803014-16$} & & & \\
\hline 14119-06-3 & Copper & 7.08 & ug/g dry & 0.598 & $5 / 13 / 08$ & 8D21003 & PNNL-AGG-415 \\
\hline $7440-38-2$ & Arsenic & 1.93 & ug/g dry & 0.417 & $5 / 13 / 08$ & 8D21003 & PNNL-AGG-415 \\
\hline $14687-58-2$ & Selenium & $<1.14$ & ug/g dry & 1.14 & $5 / 13 / 08$ & 8D21003 & PNNL-AGG-415 \\
\hline $14392-17-7$ & Molybdenum & 0.347 & ug/g dry & 0.234 & $5 / 13 / 08$ & 8D21003 & PNNL-AGG-415 \\
\hline $14914-61-5$ & Ruthenium & $<0.045$ & ug/g dry & 0.045 & $5 / 13 / 08$ & 8D21003 & PNNL-AGG-415 \\
\hline $14336-64-2$ & Cadmium & 0.057 & ug/g dry & 0.049 & $5 / 13 / 08$ & 8D21003 & PNNL-AGG-415 \\
\hline $14265-72-6$ & Antimony & $<0.079$ & ug/g dry & 0.079 & $5 / 13 / 08$ & 8D21003 & PNNL-AGG-415 \\
\hline $13966-28-4$ & Lead & 2.69 & ug/g dry & 0.037 & $5 / 13 / 08$ & 8D21003 & PNNL-AGG-415 \\
\hline HEIS No. & B1T2P4A & \multicolumn{3}{|c|}{ Lab ID: $\quad$ 0803014-17 } & & & \\
\hline $14119-06-3$ & Copper & 7.63 & ug/g dry & 0.588 & $5 / 13 / 08$ & 8D21003 & PNNL-AGG-415 \\
\hline $7440-38-2$ & Arsenic & 1.83 & ug/g dry & 0.410 & $5 / 13 / 08$ & 8D21003 & PNNL-AGG-415 \\
\hline $14687-58-2$ & Selenium & $<1.12$ & ug/g dry & 1.12 & $5 / 13 / 08$ & 8D21003 & PNNL-AGG-415 \\
\hline $14392-17-7$ & Molybdenum & 0.357 & ug/g dry & 0.230 & $5 / 13 / 08$ & 8D21003 & PNNL-AGG-415 \\
\hline 14914-61-5 & Ruthenium & $<0.044$ & ug/g dry & 0.044 & $5 / 13 / 08$ & 8D21003 & PNNL-AGG-415 \\
\hline $14336-64-2$ & Cadmium & 0.050 & ug/g dry & 0.048 & $5 / 13 / 08$ & 8D21003 & PNNL-AGG-415 \\
\hline $14265-72-6$ & Antimony & $<0.078$ & ug/g dry & 0.078 & $5 / 13 / 08$ & 8D21003 & PNNL-AGG-415 \\
\hline 13966-28-4 & Lead & 2.56 & ug/g dry & 0.036 & $5 / 13 / 08$ & 8D21003 & PNNL-AGG-415 \\
\hline HEIS No. & B1T2P5B & \multicolumn{3}{|c|}{ Lab ID: $\quad$ 0803014-20 } & & & \\
\hline $14119-06-3$ & Copper & 7.99 & ug/g dry & 0.638 & $5 / 13 / 08$ & 8D21003 & PNNL-AGG-415 \\
\hline $7440-38-2$ & Arsenic & 1.90 & ug/g dry & 0.445 & $5 / 13 / 08$ & 8D21003 & PNNL-AGG-415 \\
\hline $14687-58-2$ & Selenium & $<1.22$ & ug/g dry & 1.22 & $5 / 13 / 08$ & 8D21003 & PNNL-AGG-415 \\
\hline $14392-17-7$ & Molybdenum & 0.388 & ug/g dry & 0.249 & $5 / 13 / 08$ & 8D21003 & PNNL-AGG-415 \\
\hline $14914-61-5$ & Ruthenium & $<0.048$ & ug/g dry & 0.048 & $5 / 13 / 08$ & 8D21003 & PNNL-AGG-415 \\
\hline $14336-64-2$ & Cadmium & 0.064 & ug/g dry & 0.052 & $5 / 13 / 08$ & 8D21003 & PNNL-AGG-415 \\
\hline $14265-72-6$ & Antimony & $<0.085$ & ug/g dry & 0.085 & $5 / 13 / 08$ & 8D21003 & PNNL-AGG-415 \\
\hline $13966-28-4$ & Lead & 2.77 & ug/g dry & 0.039 & $5 / 13 / 08$ & 8D21003 & PNNL-AGG-415 \\
\hline HEIS No. & B1T2P5A & \multicolumn{3}{|c|}{ Lab ID: $\quad$ 0803014-21 } & & & \\
\hline $14119-06-3$ & Copper & 8.05 & ug/g dry & 0.663 & $5 / 13 / 08$ & 8D21003 & PNNL-AGG-415 \\
\hline $7440-38-2$ & Arsenic & 2.08 & ug/g dry & 0.462 & $5 / 13 / 08$ & 8D21003 & PNNL-AGG-415 \\
\hline $14687-58-2$ & Selenium & $<1.26$ & ug/g dry & 1.26 & $5 / 13 / 08$ & 8D21003 & PNNL-AGG-415 \\
\hline $14392-17-7$ & Molybdenum & 0.300 & ug/g dry & 0.259 & $5 / 13 / 08$ & 8D21003 & PNNL-AGG-415 \\
\hline $14914-61-5$ & Ruthenium & $<0.050$ & ug/g dry & 0.050 & $5 / 13 / 08$ & 8D21003 & PNNL-AGG-415 \\
\hline $14336-64-2$ & Cadmium & 0.069 & ug/g dry & 0.054 & $5 / 13 / 08$ & 8D21003 & PNNL-AGG-415 \\
\hline $14265-72-6$ & Antimony & $<0.088$ & ug/g dry & 0.088 & $5 / 13 / 08$ & 8D21003 & PNNL-AGG-415 \\
\hline 13966-28-4 & Lead & 3.19 & ug/g dry & 0.041 & $5 / 13 / 08$ & 8D21003 & PNNL-AGG-415 \\
\hline HEIS No. & B1T2P6B & \multicolumn{3}{|c|}{ Lab ID: $\quad$ 0803014-24 } & & & \\
\hline $14119-06-3$ & Copper & 8.73 & ug/g dry & 0.638 & $5 / 13 / 08$ & 8D21003 & PNNL-AGG-415 \\
\hline 7440-38-2 & Arsenic & 2.01 & ug/g dry & 0.445 & $5 / 13 / 08$ & 8D21003 & PNNL-AGG-415 \\
\hline $14687-58-2$ & Selenium & $<1.22$ & ug/g dry & 1.22 & $5 / 13 / 08$ & 8D21003 & PNNL-AGG-415 \\
\hline $14392-17-7$ & Molybdenum & 0.606 & ug/g dry & 0.249 & $5 / 13 / 08$ & 8D21003 & PNNL-AGG-415 \\
\hline $14914-61-5$ & Ruthenium & $<0.048$ & ug/g dry & 0.048 & $5 / 13 / 08$ & 8D21003 & PNNL-AGG-415 \\
\hline 14336-64-2 & Cadmium & 0.078 & ug/g dry & 0.052 & $5 / 13 / 08$ & 8D21003 & PNNL-AGG-415 \\
\hline $14265-72-6$ & Antimony & $<0.085$ & ug/g dry & 0.085 & $5 / 13 / 08$ & 8D21003 & PNNL-AGG-415 \\
\hline 13966-28-4 & Lead & 2.71 & ug/g dry & 0.039 & $5 / 13 / 08$ & 8D21003 & PNNL-AGG-415 \\
\hline HEIS No. & B1T2P6A & \multicolumn{3}{|c|}{ Lab ID: $\quad$ 0803014-25 } & & & \\
\hline 14119-06-3 & Copper & 8.29 & ug/g dry & 0.721 & $5 / 13 / 08$ & 8D21003 & PNNL-AGG-415 \\
\hline
\end{tabular}


RCRA Metals By SW846 6020/Acid Extract

\begin{tabular}{|c|c|c|c|c|c|c|c|}
\hline CAS \# & Analyte & Results & Units & EQL & Analyzed & Batch & Method \\
\hline HEIS No. & B1T2P6A & \multicolumn{3}{|c|}{ Lab ID: $\quad$ 0803014-25 } & & & \\
\hline $7440-38-2$ & Arsenic & 1.78 & ug/g dry & 0.503 & $5 / 13 / 08$ & 8D21003 & PNNL-AGG-415 \\
\hline $14687-58-2$ & Selenium & $<1.37$ & ug/g dry & 1.37 & $5 / 13 / 08$ & 8D21003 & PNNL-AGG-415 \\
\hline $14392-17-7$ & Molybdenum & 0.515 & ug/g dry & 0.282 & 5/13/08 & 8D21003 & PNNL-AGG-415 \\
\hline 14914-61-5 & Ruthenium & $<0.054$ & ug/g dry & 0.054 & $5 / 13 / 08$ & 8D21003 & PNNL-AGG-415 \\
\hline 14336-64-2 & Cadmium & $<0.059$ & ug/g dry & 0.059 & $5 / 13 / 08$ & 8D21003 & PNNL-AGG-415 \\
\hline $14265-72-6$ & Antimony & $<0.096$ & ug/g dry & 0.096 & $5 / 13 / 08$ & 8D21003 & PNNL-AGG-415 \\
\hline 13966-28-4 & Lead & 2.60 & ug/g dry & 0.044 & $5 / 13 / 08$ & 8D21003 & PNNL-AGG-415 \\
\hline HEIS No. & B1T2P7B & \multicolumn{3}{|c|}{ Lab ID: $\quad$ 0803015-02 } & & & \\
\hline 14119-06-3 & Copper & 9.96 & ug/g dry & 0.658 & $5 / 13 / 08$ & 8D21003 & PNNL-AGG-415 \\
\hline $7440-38-2$ & Arsenic & 1.88 & ug/g dry & 0.459 & $5 / 13 / 08$ & 8D21003 & PNNL-AGG-415 \\
\hline $14687-58-2$ & Selenium & $<1.26$ & ug/g dry & 1.26 & $5 / 13 / 08$ & 8D21003 & PNNL-AGG-415 \\
\hline $14392-17-7$ & Molybdenum & $<0.257$ & ug/g dry & 0.257 & $5 / 13 / 08$ & 8D21003 & PNNL-AGG-415 \\
\hline $14914-61-5$ & Ruthenium & $<0.049$ & ug/g dry & 0.049 & $5 / 13 / 08$ & 8D21003 & PNNL-AGG-415 \\
\hline $14336-64-2$ & Cadmium & 0.061 & ug/g dry & 0.054 & $5 / 13 / 08$ & 8D21003 & PNNL-AGG-415 \\
\hline $14265-72-6$ & Antimony & $<0.087$ & ug/g dry & 0.087 & $5 / 13 / 08$ & 8D21003 & PNNL-AGG-415 \\
\hline 13966-28-4 & Lead & 3.57 & ug/g dry & 0.041 & $5 / 13 / 08$ & 8D21003 & PNNL-AGG-415 \\
\hline HEIS No. & B1T2P7A & \multicolumn{3}{|c|}{ Lab ID: $\quad$ 0803015-03 } & & & \\
\hline 14119-06-3 & Copper & 10.5 & ug/g dry & 0.642 & $5 / 13 / 08$ & 8D21003 & PNNL-AGG-415 \\
\hline $7440-38-2$ & Arsenic & 1.19 & ug/g dry & 0.448 & $5 / 13 / 08$ & 8D21003 & PNNL-AGG-415 \\
\hline $14687-58-2$ & Selenium & $<1.22$ & ug/g dry & 1.22 & $5 / 13 / 08$ & 8D21003 & PNNL-AGG-415 \\
\hline $14392-17-7$ & Molybdenum & 0.366 & ug/g dry & 0.251 & $5 / 13 / 08$ & 8D21003 & PNNL-AGG-415 \\
\hline 14914-61-5 & Ruthenium & $<0.048$ & ug/g dry & 0.048 & $5 / 13 / 08$ & 8D21003 & PNNL-AGG-415 \\
\hline $14336-64-2$ & Cadmium & 0.059 & ug/g dry & 0.053 & $5 / 13 / 08$ & 8D21003 & PNNL-AGG-415 \\
\hline $14265-72-6$ & Antimony & $<0.085$ & ug/g dry & 0.085 & $5 / 13 / 08$ & 8D21003 & PNNL-AGG-415 \\
\hline 13966-28-4 & Lead & 2.15 & ug/g dry & 0.040 & $5 / 13 / 08$ & 8D21003 & PNNL-AGG-415 \\
\hline HEIS No. & B1T2P8B & \multicolumn{3}{|c|}{ Lab ID: $\quad$ 0803015-06 } & & & \\
\hline 14119-06-3 & Copper & 7.56 & ug/g dry & 0.566 & $5 / 13 / 08$ & 8D21003 & PNNL-AGG-415 \\
\hline $7440-38-2$ & Arsenic & 1.32 & ug/g dry & 0.395 & $5 / 13 / 08$ & 8D21003 & PNNL-AGG-415 \\
\hline 14687-58-2 & Selenium & $<1.08$ & ug/g dry & 1.08 & $5 / 13 / 08$ & 8D21003 & PNNL-AGG-415 \\
\hline $14392-17-7$ & Molybdenum & 0.222 & ug/g dry & 0.221 & $5 / 13 / 08$ & 8D21003 & PNNL-AGG-415 \\
\hline 14914-61-5 & Ruthenium & $<0.042$ & ug/g dry & 0.042 & $5 / 13 / 08$ & 8D21003 & PNNL-AGG-415 \\
\hline $14336-64-2$ & Cadmium & 0.060 & ug/g dry & 0.046 & $5 / 13 / 08$ & 8D21003 & PNNL-AGG-415 \\
\hline $14265-72-6$ & Antimony & $<0.075$ & ug/g dry & 0.075 & $5 / 13 / 08$ & 8D21003 & PNNL-AGG-415 \\
\hline 13966-28-4 & Lead & 2.76 & ug/g dry & 0.035 & $5 / 13 / 08$ & 8D21003 & PNNL-AGG-415 \\
\hline HEIS No. & B1T2P8A & \multicolumn{3}{|c|}{ Lab ID: $\quad$ 0803015-07 } & & & \\
\hline 14119-06-3 & Copper & 7.95 & ug/g dry & 0.570 & $5 / 13 / 08$ & 8D21003 & PNNL-AGG-415 \\
\hline 7440-38-2 & Arsenic & 1.20 & ug/g dry & 0.397 & $5 / 13 / 08$ & 8D21003 & PNNL-AGG-415 \\
\hline $14687-58-2$ & Selenium & $<1.09$ & ug/g dry & 1.09 & $5 / 13 / 08$ & 8D21003 & PNNL-AGG-415 \\
\hline 14914-61-5 & Ruthenium & $<0.043$ & ug/g dry & 0.043 & $5 / 13 / 08$ & 8D21003 & PNNL-AGG-415 \\
\hline HEIS No. & B1T2P9B & \multicolumn{3}{|c|}{ Lab ID: $\quad$ 0803015-10 } & & & \\
\hline 14119-06-3 & Copper & 7.85 & ug/g dry & 0.530 & $5 / 13 / 08$ & 8D21003 & PNNL-AGG-415 \\
\hline $7440-38-2$ & Arsenic & 1.59 & ug/g dry & 0.370 & $5 / 13 / 08$ & 8D21003 & PNNL-AGG-415 \\
\hline $14687-58-2$ & Selenium & $<1.01$ & ug/g dry & 1.01 & $5 / 13 / 08$ & 8D21003 & PNNL-AGG-415 \\
\hline $14392-17-7$ & Molybdenum & 0.252 & ug/g dry & 0.207 & $5 / 13 / 08$ & 8D21003 & PNNL-AGG-415 \\
\hline 14914-61-5 & Ruthenium & $<0.040$ & ug/g dry & 0.040 & $5 / 13 / 08$ & 8D21003 & PNNL-AGG-415 \\
\hline 14336-64-2 & Cadmium & 0.051 & ug/g dry & 0.043 & $5 / 13 / 08$ & 8D21003 & PNNL-AGG-415 \\
\hline $14265-72-6$ & Antimony & $<0.070$ & ug/g dry & 0.070 & $5 / 13 / 08$ & 8D21003 & PNNL-AGG-415 \\
\hline 13966-28-4 & Lead & 2.07 & ug/g dry & 0.033 & $5 / 13 / 08$ & 8D21003 & PNNL-AGG-415 \\
\hline
\end{tabular}


RCRA Metals By SW846 6020/Acid Extract

\begin{tabular}{|c|c|c|c|c|c|c|c|}
\hline CAS \# & Analyte & Results & Units & EQL & Analyzed & Batch & Method \\
\hline HEIS No. & В1T2P9A & \multicolumn{3}{|c|}{ Lab ID: $\quad$ 0803015-11 } & & & \\
\hline 14119-06-3 & Copper & 7.55 & ug/g dry & 0.501 & $5 / 13 / 08$ & 8D21003 & PNNL-AGG-415 \\
\hline $7440-38-2$ & Arsenic & 1.40 & ug/g dry & 0.350 & 5/13/08 & 8D21003 & PNNL-AGG-415 \\
\hline $14687-58-2$ & Selenium & $<0.956$ & ug/g dry & 0.956 & $5 / 13 / 08$ & 8D21003 & PNNL-AGG-415 \\
\hline 14392-17-7 & Molybdenum & 0.213 & ug/g dry & 0.196 & $5 / 13 / 08$ & 8D21003 & PNNL-AGG-415 \\
\hline 14914-61-5 & Ruthenium & $<0.038$ & ug/g dry & 0.038 & $5 / 13 / 08$ & 8D21003 & PNNL-AGG-415 \\
\hline $14336-64-2$ & Cadmium & 0.045 & ug/g dry & 0.041 & $5 / 13 / 08$ & 8D21003 & PNNL-AGG-415 \\
\hline $14265-72-6$ & Antimony & $<0.066$ & ug/g dry & 0.066 & $5 / 13 / 08$ & 8D21003 & PNNL-AGG-415 \\
\hline 13966-28-4 & Lead & 1.94 & ug/g dry & 0.031 & $5 / 13 / 08$ & 8D21003 & PNNL-AGG-415 \\
\hline HEIS No. & B1T2R0B & \multicolumn{3}{|c|}{ Lab ID: $\quad$ 0803015-14 } & & & \\
\hline 14119-06-3 & Copper & 8.89 & ug/g dry & 0.735 & 5/13/08 & 8D21003 & PNNL-AGG-415 \\
\hline $7440-38-2$ & Arsenic & 2.03 & ug/g dry & 0.513 & $5 / 13 / 08$ & 8D21003 & PNNL-AGG-415 \\
\hline 14687-58-2 & Selenium & $<1.40$ & ug/g dry & 1.40 & $5 / 13 / 08$ & 8D21003 & PNNL-AGG-415 \\
\hline $14392-17-7$ & Molybdenum & $<0.287$ & ug/g dry & 0.287 & $5 / 13 / 08$ & 8D21003 & PNNL-AGG-415 \\
\hline 14914-61-5 & Ruthenium & $<0.055$ & ug/g dry & 0.055 & $5 / 13 / 08$ & 8D21003 & PNNL-AGG-415 \\
\hline $14336-64-2$ & Cadmium & 0.061 & ug/g dry & 0.060 & $5 / 13 / 08$ & 8D21003 & PNNL-AGG-415 \\
\hline $14265-72-6$ & Antimony & $<0.097$ & ug/g dry & 0.097 & $5 / 13 / 08$ & 8D21003 & PNNL-AGG-415 \\
\hline 13966-28-4 & Lead & 2.11 & ug/g dry & 0.045 & $5 / 13 / 08$ & 8D21003 & PNNL-AGG-415 \\
\hline HEIS No. & B1T2R0A & \multicolumn{3}{|c|}{ Lab ID: $\quad$ 0803015-15 } & & & \\
\hline 14119-06-3 & Copper & 8.24 & ug/g dry & 0.526 & $5 / 13 / 08$ & 8D21003 & PNNL-AGG-415 \\
\hline 7440-38-2 & Arsenic & 1.34 & ug/g dry & 0.367 & $5 / 13 / 08$ & 8D21003 & PNNL-AGG-415 \\
\hline $14687-58-2$ & Selenium & $<1.00$ & ug/g dry & 1.00 & $5 / 13 / 08$ & 8D21003 & PNNL-AGG-415 \\
\hline 14392-17-7 & Molybdenum & 0.289 & ug/g dry & 0.205 & $5 / 13 / 08$ & 8D21003 & PNNL-AGG-415 \\
\hline 14914-61-5 & Ruthenium & $<0.039$ & ug/g dry & 0.039 & $5 / 13 / 08$ & 8D21003 & PNNL-AGG-415 \\
\hline 14336-64-2 & Cadmium & 0.055 & ug/g dry & 0.043 & $5 / 13 / 08$ & 8D21003 & PNNL-AGG-415 \\
\hline $14265-72-6$ & Antimony & $<0.070$ & ug/g dry & 0.070 & $5 / 13 / 08$ & 8D21003 & PNNL-AGG-415 \\
\hline 13966-28-4 & Lead & 2.20 & ug/g dry & 0.032 & $5 / 13 / 08$ & 8D21003 & PNNL-AGG-415 \\
\hline HEIS No. & B1TNK6B & \multicolumn{3}{|c|}{ Lab ID: $\quad$ 0803015-18 } & & & \\
\hline 14119-06-3 & Copper & 7.14 & ug/g dry & 0.626 & $5 / 13 / 08$ & 8D21003 & PNNL-AGG-415 \\
\hline 7440-38-2 & Arsenic & 1.60 & ug/g dry & 0.437 & $5 / 13 / 08$ & 8D21003 & PNNL-AGG-415 \\
\hline $14687-58-2$ & Selenium & $<1.19$ & ug/g dry & 1.19 & $5 / 13 / 08$ & 8D21003 & PNNL-AGG-415 \\
\hline $14392-17-7$ & Molybdenum & $<0.245$ & ug/g dry & 0.245 & $5 / 13 / 08$ & 8D21003 & PNNL-AGG-415 \\
\hline 14914-61-5 & Ruthenium & $<0.047$ & ug/g dry & 0.047 & $5 / 13 / 08$ & 8D21003 & PNNL-AGG-415 \\
\hline $14336-64-2$ & Cadmium & $<0.051$ & ug/g dry & 0.051 & $5 / 13 / 08$ & 8D21003 & PNNL-AGG-415 \\
\hline $14265-72-6$ & Antimony & $<0.083$ & ug/g dry & 0.083 & $5 / 13 / 08$ & 8D21003 & PNNL-AGG-415 \\
\hline 13966-28-4 & Lead & 2.28 & ug/g dry & 0.039 & $5 / 13 / 08$ & 8D21003 & PNNL-AGG-415 \\
\hline HEIS No. & B1TNK6A & \multicolumn{3}{|c|}{ Lab ID: $\quad$ 0803015-19 } & & & \\
\hline 14119-06-3 & Copper & 8.19 & ug/g dry & 0.602 & $5 / 13 / 08$ & 8D21003 & PNNL-AGG-415 \\
\hline 7440-38-2 & Arsenic & 1.48 & ug/g dry & 0.420 & $5 / 13 / 08$ & 8D21003 & PNNL-AGG-415 \\
\hline 14687-58-2 & Selenium & $<1.15$ & ug/g dry & 1.15 & $5 / 13 / 08$ & 8D21003 & PNNL-AGG-415 \\
\hline $14392-17-7$ & Molybdenum & $<0.235$ & ug/g dry & 0.235 & $5 / 13 / 08$ & 8D21003 & PNNL-AGG-415 \\
\hline $14914-61-5$ & Ruthenium & $<0.045$ & ug/g dry & 0.045 & $5 / 13 / 08$ & 8D21003 & PNNL-AGG-415 \\
\hline $14336-64-2$ & Cadmium & 0.055 & ug/g dry & 0.049 & $5 / 13 / 08$ & 8D21003 & PNNL-AGG-415 \\
\hline $14265-72-6$ & Antimony & $<0.080$ & ug/g dry & 0.080 & $5 / 13 / 08$ & 8D21003 & PNNL-AGG-415 \\
\hline 13966-28-4 & Lead & 1.75 & ug/g dry & 0.037 & $5 / 13 / 08$ & 8D21003 & PNNL-AGG-415 \\
\hline HEIS No. & B1TNK7B & \multicolumn{3}{|c|}{ Lab ID: $\quad$ 0803015-22 } & & & \\
\hline 14119-06-3 & Copper & 7.94 & ug/g dry & 0.576 & $5 / 13 / 08$ & 8D21003 & PNNL-AGG-415 \\
\hline 7440-38-2 & Arsenic & 2.00 & ug/g dry & 0.402 & $5 / 13 / 08$ & 8D21003 & PNNL-AGG-415 \\
\hline $14687-58-2$ & Selenium & $<1.10$ & ug/g dry & 1.10 & $5 / 13 / 08$ & 8D21003 & PNNL-AGG-415 \\
\hline
\end{tabular}


RCRA Metals By SW846 6020/Acid Extract

\begin{tabular}{|c|c|c|c|c|c|c|c|}
\hline CAS \# & Analyte & Results & Units & EQL & Analyzed & Batch & Method \\
\hline HEIS No. & B1TNK7B & \multicolumn{3}{|c|}{ Lab ID: $\quad$ 0803015-22 } & & & \\
\hline $14392-17-7$ & Molybdenum & 0.236 & ug/g dry & 0.225 & $5 / 13 / 08$ & 8D21003 & PNNL-AGG-415 \\
\hline 14914-61-5 & Ruthenium & $<0.043$ & ug/g dry & 0.043 & $5 / 13 / 08$ & 8D21003 & PNNL-AGG-415 \\
\hline 14336-64-2 & Cadmium & 0.066 & ug/g dry & 0.047 & $5 / 13 / 08$ & 8D21003 & PNNL-AGG-415 \\
\hline $14265-72-6$ & Antimony & $<0.076$ & ug/g dry & 0.076 & $5 / 13 / 08$ & 8D21003 & PNNL-AGG-415 \\
\hline $13966-28-4$ & Lead & 2.44 & ug/g dry & 0.035 & $5 / 13 / 08$ & 8D21003 & PNNL-AGG-415 \\
\hline HEIS No. & B1TNK7A & \multicolumn{3}{|c|}{ Lab ID: $\quad$ 0803015-23 } & & & \\
\hline 14119-06-3 & Copper & 8.06 & ug/g dry & 0.537 & $5 / 13 / 08$ & 8D21003 & PNNL-AGG-415 \\
\hline 7440-38-2 & Arsenic & 1.53 & ug/g dry & 0.375 & $5 / 13 / 08$ & 8D21003 & PNNL-AGG-415 \\
\hline $14687-58-2$ & Selenium & $<1.02$ & ug/g dry & 1.02 & $5 / 13 / 08$ & 8D21003 & PNNL-AGG-415 \\
\hline $14392-17-7$ & Molybdenum & $<0.210$ & ug/g dry & 0.210 & $5 / 13 / 08$ & 8D21003 & PNNL-AGG-415 \\
\hline $14914-61-5$ & Ruthenium & $<0.040$ & ug/g dry & 0.040 & $5 / 13 / 08$ & 8D21003 & PNNL-AGG-415 \\
\hline $14336-64-2$ & Cadmium & 0.056 & ug/g dry & 0.044 & $5 / 13 / 08$ & 8D21003 & PNNL-AGG-415 \\
\hline $14265-72-6$ & Antimony & $<0.071$ & ug/g dry & 0.071 & $5 / 13 / 08$ & 8D21003 & PNNL-AGG-415 \\
\hline $13966-28-4$ & Lead & 2.09 & ug/g dry & 0.033 & $5 / 13 / 08$ & 8D21003 & PNNL-AGG-415 \\
\hline HEIS No. & B1TNK8B & \multicolumn{3}{|c|}{ Lab ID: $\quad$ 0803015-26 } & & & \\
\hline 14119-06-3 & Copper & 7.71 & ug/g dry & 0.612 & $5 / 13 / 08$ & 8D21003 & PNNL-AGG-415 \\
\hline $7440-38-2$ & Arsenic & 1.80 & ug/g dry & 0.427 & $5 / 13 / 08$ & 8D21003 & PNNL-AGG-415 \\
\hline $14687-58-2$ & Selenium & $<1.17$ & ug/g dry & 1.17 & $5 / 13 / 08$ & 8D21003 & PNNL-AGG-415 \\
\hline $14392-17-7$ & Molybdenum & 0.463 & ug/g dry & 0.239 & $5 / 13 / 08$ & 8D21003 & PNNL-AGG-415 \\
\hline $14914-61-5$ & Ruthenium & $<0.046$ & ug/g dry & 0.046 & $5 / 13 / 08$ & 8D21003 & PNNL-AGG-415 \\
\hline $14336-64-2$ & Cadmium & 0.056 & ug/g dry & 0.050 & $5 / 13 / 08$ & 8D21003 & PNNL-AGG-415 \\
\hline $14265-72-6$ & Antimony & $<0.081$ & ug/g dry & 0.081 & $5 / 13 / 08$ & 8D21003 & PNNL-AGG-415 \\
\hline 13966-28-4 & Lead & 2.34 & ug/g dry & 0.038 & $5 / 13 / 08$ & 8D21003 & PNNL-AGG-415 \\
\hline HEIS No. & B1TNK8A & \multicolumn{3}{|c|}{ Lab ID: $\quad 0803015-27$} & & & \\
\hline 14119-06-3 & Copper & 6.77 & ug/g dry & 0.654 & $5 / 13 / 08$ & 8D21003 & PNNL-AGG-415 \\
\hline 7440-38-2 & Arsenic & 1.67 & ug/g dry & 0.456 & $5 / 13 / 08$ & 8D21003 & PNNL-AGG-415 \\
\hline 14687-58-2 & Selenium & $<1.25$ & ug/g dry & 1.25 & $5 / 13 / 08$ & 8D21003 & PNNL-AGG-415 \\
\hline 14392-17-7 & Molybdenum & 0.354 & ug/g dry & 0.255 & $5 / 13 / 08$ & 8D21003 & PNNL-AGG-415 \\
\hline $14914-61-5$ & Ruthenium & $<0.049$ & ug/g dry & 0.049 & $5 / 13 / 08$ & 8D21003 & PNNL-AGG-415 \\
\hline $14336-64-2$ & Cadmium & 0.055 & ug/g dry & 0.054 & $5 / 13 / 08$ & 8D21003 & PNNL-AGG-415 \\
\hline $14265-72-6$ & Antimony & $<0.087$ & ug/g dry & 0.087 & $5 / 13 / 08$ & 8D21003 & PNNL-AGG-415 \\
\hline 13966-28-4 & Lead & 2.29 & ug/g dry & 0.040 & $5 / 13 / 08$ & 8D21003 & PNNL-AGG-415 \\
\hline HEIS No. & B1TNK9B & \multicolumn{3}{|c|}{ Lab ID: $\quad$ 0803015-30 } & & & \\
\hline 14119-06-3 & Copper & 8.58 & ug/g dry & 0.639 & $5 / 13 / 08$ & 8D21003 & PNNL-AGG-415 \\
\hline 7440-38-2 & Arsenic & 1.86 & ug/g dry & 0.446 & $5 / 13 / 08$ & 8D21003 & PNNL-AGG-415 \\
\hline $14687-58-2$ & Selenium & $<1.22$ & ug/g dry & 1.22 & $5 / 13 / 08$ & 8D21003 & PNNL-AGG-415 \\
\hline 14392-17-7 & Molybdenum & 0.340 & ug/g dry & 0.250 & $5 / 13 / 08$ & 8D21003 & PNNL-AGG-415 \\
\hline $14914-61-5$ & Ruthenium & $<0.048$ & ug/g dry & 0.048 & $5 / 13 / 08$ & 8D21003 & PNNL-AGG-415 \\
\hline $14336-64-2$ & Cadmium & 0.063 & ug/g dry & 0.052 & $5 / 13 / 08$ & 8D21003 & PNNL-AGG-415 \\
\hline $14265-72-6$ & Antimony & $<0.085$ & ug/g dry & 0.085 & $5 / 13 / 08$ & 8D21003 & PNNL-AGG-415 \\
\hline 13966-28-4 & Lead & 2.29 & ug/g dry & 0.039 & $5 / 13 / 08$ & 8D21003 & PNNL-AGG-415 \\
\hline HEIS No. & B1TNK9A & \multicolumn{3}{|c|}{ Lab ID: $\quad$ 0803015-31 } & & & \\
\hline 14119-06-3 & Copper & 8.61 & ug/g dry & 0.649 & $5 / 13 / 08$ & 8D21003 & PNNL-AGG-415 \\
\hline $7440-38-2$ & Arsenic & 1.84 & ug/g dry & 0.453 & $5 / 13 / 08$ & 8D21003 & PNNL-AGG-415 \\
\hline $14687-58-2$ & Selenium & $<1.24$ & ug/g dry & 1.24 & $5 / 13 / 08$ & 8D21003 & PNNL-AGG-415 \\
\hline $14392-17-7$ & Molybdenum & 0.336 & ug/g dry & 0.253 & $5 / 13 / 08$ & 8D21003 & PNNL-AGG-415 \\
\hline 14914-61-5 & Ruthenium & $<0.049$ & ug/g dry & 0.049 & $5 / 13 / 08$ & 8D21003 & PNNL-AGG-415 \\
\hline $14336-64-2$ & Cadmium & 0.061 & ug/g dry & 0.053 & $5 / 13 / 08$ & 8D21003 & PNNL-AGG-415 \\
\hline
\end{tabular}


RCRA Metals By SW846 6020/Acid Extract

\begin{tabular}{|lccccccc|}
\hline CAS \# & Analyte & Results & Units & EQL & Analyzed & Batch & Method \\
\hline HEIS No. & B1TNK9A & \multicolumn{2}{c|}{ Lab ID: } & $\mathbf{0 8 0 3 0 1 5}-31$ & & \\
$14265-72-6$ & Antimony & $<0.086$ & ug/g dry & 0.086 & 5/13/08 & 8D21003 & PNNL-AGG-415 \\
$13966-28-4$ & Lead & 2.43 & ug/g dry & 0.040 & 5/13/08 & 8D21003 & PNNL-AGG-415 \\
\hline
\end{tabular}




\section{Carbon Analysis/Soil}

\begin{tabular}{|c|c|c|c|c|c|}
\hline $\begin{array}{l}\text { Total Orga } \\
\text { Lab ID }\end{array}$ & $\begin{array}{l}\text { C Carbon } \\
\text { HEIS No. }\end{array}$ & $\begin{array}{l}\text { ic Carb } \\
\text { Results }\end{array}$ & EQL & Analyzed & Batch \\
\hline 0803001-01 & B1RTF8 & 254 & 200 & 4/09/08 & [CALC] \\
\hline 0803001-02 & B1RTF9A & 269 & 200 & 4/09/08 & [CALC] \\
\hline 0803012-01 & B1RTH0 & 2740 & 200 & 4/10/08 & [CALC] \\
\hline 0803012-02 & B1RTH1A & 392 & 200 & 4/09/08 & [CALC] \\
\hline 0803012-03 & B1RTH1 & $<200$ & 200 & 4/09/08 & [CALC] \\
\hline 0803012-05 & B1RTH2B & 514 & 200 & 4/09/08 & [CALC] \\
\hline 0803012-06 & B1RTH2A & 398 & 200 & 4/09/08 & [CALC] \\
\hline 0803012-09 & B1RTH3B & $<200$ & 200 & 4/09/08 & [CALC] \\
\hline 0803012-10 & B1RTH3A & $<200$ & 200 & 4/09/08 & [CALC] \\
\hline 0803012-13 & B1RYR7B & $<200$ & 200 & 4/09/08 & [CALC] \\
\hline 0803012-14 & B1RYR7A & 211 & 200 & 4/10/08 & [CALC] \\
\hline 0803012-17 & B1RYR8B & $<200$ & 200 & $4 / 10 / 08$ & [CALC] \\
\hline 0803012-18 & B1RYR8A & $<200$ & 200 & $4 / 10 / 08$ & [CALC] \\
\hline 0803012-21 & B1RYR9B & $<200$ & 200 & $4 / 10 / 08$ & [CALC] \\
\hline 0803012-22 & B1RYR9A & $<200$ & 200 & $4 / 10 / 08$ & [CALC] \\
\hline 0803014-01 & B1RYT0B & $<200$ & 200 & 4/10/08 & [CALC] \\
\hline 0803014-02 & B1RYT0A & $<200$ & 200 & $4 / 10 / 08$ & [CALC] \\
\hline 0803014-05 & B1T2P1B & $<200$ & 200 & $4 / 10 / 08$ & [CALC] \\
\hline 0803014-06 & B1T2P1A & $<200$ & 200 & $4 / 10 / 08$ & [CALC] \\
\hline 0803014-09 & В1Т2Р2B & $<200$ & 200 & 4/10/08 & [CALC] \\
\hline 0803014-10 & B1T2P2A & 215 & 200 & 4/10/08 & [CALC] \\
\hline 0803014-13 & В1Т2Р3B & $<200$ & 200 & 4/10/08 & [CALC] \\
\hline 0803014-14 & B1T2P3A & $<200$ & 200 & $4 / 11 / 08$ & [CALC] \\
\hline 0803014-16 & B1T2P4B & $<200$ & 200 & $4 / 11 / 08$ & [CALC] \\
\hline 0803014-17 & B1T2P4A & $<200$ & 200 & 4/11/08 & [CALC] \\
\hline 0803014-20 & В1Т2Р5B & $<200$ & 200 & $4 / 11 / 08$ & [CALC] \\
\hline 0803014-21 & B1T2P5A & 216 & 200 & $4 / 11 / 08$ & [CALC] \\
\hline 0803014-24 & B1T2P6B & $<200$ & 200 & $4 / 11 / 08$ & [CALC] \\
\hline 0803014-25 & B1T2P6A & $<200$ & 200 & 4/11/08 & [CALC] \\
\hline 0803015-02 & B1T2P7B & 293 & 200 & 4/11/08 & [CALC] \\
\hline 0803015-03 & B1T2P7A & 363 & 200 & $4 / 11 / 08$ & [CALC] \\
\hline 0803015-06 & B1T2P8B & $<200$ & 200 & $4 / 11 / 08$ & [CALC] \\
\hline 0803015-07 & B1T2P8A & 295 & 200 & $4 / 14 / 08$ & [CALC] \\
\hline 0803015-10 & В1Т2Р9В & $<200$ & 200 & 4/14/08 & [CALC] \\
\hline 0803015-11 & B1T2P9A & 201 & 200 & $4 / 14 / 08$ & [CALC] \\
\hline 0803015-14 & B1T2R0B & $<200$ & 200 & $4 / 14 / 08$ & [CALC] \\
\hline 0803015-15 & B1T2R0A & $<200$ & 200 & $4 / 14 / 08$ & [CALC] \\
\hline 0803015-18 & B1TNK6B & $<200$ & 200 & 4/14/08 & [CALC] \\
\hline 0803015-19 & B1TNK6A & 333 & 200 & $4 / 14 / 08$ & [CALC] \\
\hline 0803015-22 & B1TNK7B & $<200$ & 200 & $4 / 14 / 08$ & [CALC] \\
\hline 0803015-23 & B1TNK7A & $<200$ & 200 & $4 / 14 / 08$ & [CALC] \\
\hline 0803015-26 & B1TNK8B & 235 & 200 & $4 / 14 / 08$ & [CALC] \\
\hline 0803015-27 & B1TNK8A & $<200$ & 200 & $4 / 14 / 08$ & [CALC] \\
\hline 0803015-30 & B1TNK9B & 243 & 200 & $4 / 14 / 08$ & [CALC] \\
\hline 0803015-31 & B1TNK9A & 220 & 200 & 4/14/08 & [CALC] \\
\hline
\end{tabular}




\section{Carbon Analysis/Soil}

\begin{tabular}{|c|c|c|c|c|c|}
\hline $\begin{array}{l}\text { Total Car } \\
\text { Lab ID }\end{array}$ & $\begin{array}{l}\text { (ug/g) by } \\
\text { HEIS No. }\end{array}$ & Results & EQL & Analyzed & Batch \\
\hline 0803001-01 & B1RTF8 & 1230 & 200 & $4 / 01 / 08$ & $8 C 17009$ \\
\hline 0803001-02 & B1RTF9A & 796 & 200 & $4 / 01 / 08$ & 8C17009 \\
\hline 0803012-01 & B1RTH0 & 3240 & 200 & $4 / 01 / 08$ & 8C17009 \\
\hline 0803012-02 & B1RTH1A & 883 & 200 & $4 / 01 / 08$ & 8C17009 \\
\hline 0803012-03 & B1RTH1 & 1060 & 200 & $4 / 01 / 08$ & 8C17009 \\
\hline 0803012-05 & B1RTH2B & 2630 & 200 & $4 / 01 / 08$ & 8C17009 \\
\hline 0803012-06 & B1RTH2A & 2170 & 200 & $4 / 01 / 08$ & 8C17009 \\
\hline 0803012-09 & B1RTH3B & 2350 & 200 & $4 / 01 / 08$ & 8C17009 \\
\hline 0803012-10 & B1RTH3A & 2160 & 200 & $4 / 01 / 08$ & 8C17009 \\
\hline 0803012-13 & B1RYR7B & 2660 & 200 & $4 / 01 / 08$ & 8C17009 \\
\hline 0803012-14 & B1RYR7A & 2120 & 200 & $4 / 01 / 08$ & 8C17009 \\
\hline 0803012-17 & B1RYR8B & 2560 & 200 & $4 / 01 / 08$ & 8C17009 \\
\hline 0803012-18 & B1RYR8A & 2360 & 200 & $4 / 01 / 08$ & 8C17009 \\
\hline 0803012-21 & B1RYR9B & 2010 & 200 & $4 / 01 / 08$ & 8C17009 \\
\hline 0803012-22 & B1RYR9A & 2120 & 200 & $4 / 02 / 08$ & 8C17009 \\
\hline 0803014-01 & B1RYT0B & 3520 & 200 & $4 / 02 / 08$ & 8C17009 \\
\hline 0803014-02 & B1RYT0A & 1120 & 200 & $4 / 02 / 08$ & 8C17009 \\
\hline 0803014-05 & B1T2P1B & 2340 & 200 & $4 / 02 / 08$ & 8C17009 \\
\hline 0803014-06 & B1T2P1A & 2230 & 200 & $4 / 02 / 08$ & 8C17009 \\
\hline 0803014-09 & В1T2P2B & 3140 & 200 & $4 / 02 / 08$ & 8C17009 \\
\hline 0803014-10 & B1T2P2A & 2360 & 200 & $4 / 02 / 08$ & 8C17009 \\
\hline 0803014-13 & В1Т2Р3В & 2070 & 200 & $4 / 02 / 08$ & 8C17009 \\
\hline 0803014-14 & В1Т2Р3А & 2160 & 200 & $4 / 02 / 08$ & 8C17009 \\
\hline 0803014-16 & В1Т2Р4B & 2520 & 200 & $4 / 02 / 08$ & 8C17009 \\
\hline 0803014-17 & B1T2P4A & 2490 & 200 & $4 / 02 / 08$ & 8C17009 \\
\hline 0803014-20 & В1T2Р5B & 2610 & 200 & $4 / 02 / 08$ & 8C17009 \\
\hline 0803014-21 & В1T2P5A & 2980 & 200 & $4 / 02 / 08$ & 8C17009 \\
\hline 0803014-24 & В1Т2Р6B & 2130 & 200 & $4 / 02 / 08$ & 8C17009 \\
\hline 0803014-25 & B1T2P6A & 2170 & 200 & $4 / 02 / 08$ & 8C17009 \\
\hline 0803015-02 & В1Т2Р7B & 2180 & 200 & $4 / 02 / 08$ & 8C17009 \\
\hline 0803015-03 & B1T2P7A & 1640 & 200 & $4 / 02 / 08$ & 8C17009 \\
\hline 0803015-06 & В1Т2Р8B & 2040 & 200 & $4 / 02 / 08$ & 8C17009 \\
\hline 0803015-07 & B1T2P8A & 2230 & 200 & $4 / 03 / 08$ & 8C17009 \\
\hline 0803015-10 & В1Т2Р9В & 2380 & 200 & $4 / 03 / 08$ & 8C17009 \\
\hline 0803015-11 & В1Т2Р9A & 2070 & 200 & $4 / 03 / 08$ & 8C17009 \\
\hline 0803015-14 & B1T2R0B & 2050 & 200 & $4 / 03 / 08$ & 8C17009 \\
\hline 0803015-15 & B1T2R0A & 1760 & 200 & $4 / 03 / 08$ & 8C17009 \\
\hline 0803015-18 & B1TNK6B & 1960 & 200 & $4 / 03 / 08$ & 8C17009 \\
\hline 0803015-19 & B1TNK6A & 2060 & 200 & $4 / 03 / 08$ & 8C17009 \\
\hline 0803015-22 & B1TNK7B & 2200 & 200 & $4 / 03 / 08$ & 8C17009 \\
\hline 0803015-23 & B1TNK7A & 2290 & 200 & $4 / 03 / 08$ & 8C17009 \\
\hline 0803015-26 & B1TNK8B & 2160 & 200 & $4 / 03 / 08$ & 8C17009 \\
\hline 0803015-27 & B1TNK8A & 2340 & 200 & $4 / 03 / 08$ & 8C17009 \\
\hline 0803015-30 & B1TNK9B & 1810 & 200 & $4 / 03 / 08$ & 8C17009 \\
\hline 0803015-31 & B1TNK9A & 1770 & 200 & $4 / 03 / 08$ & 8C17009 \\
\hline
\end{tabular}




\begin{tabular}{|c|c|c|c|c|c|}
\hline & & Carbon & & & \\
\hline Total Inor & nic Carbon & -001 & & & \\
\hline Lab ID & HEIS No. & Results & EQL & Analyzed & Batch \\
\hline 0803001-01 & B1RTF8 & 976 & 200 & 4/09/08 & 8E14001 \\
\hline 0803001-02 & B1RTF9A & 527 & 200 & 4/09/08 & 8E14001 \\
\hline 0803012-01 & B1RTH0 & 503 & 200 & 4/10/08 & 8E14001 \\
\hline 0803012-02 & B1RTH1A & 491 & 200 & 4/09/08 & 8E14001 \\
\hline 0803012-03 & B1RTH1 & 890 & 200 & 4/09/08 & 8E14001 \\
\hline 0803012-05 & B1RTH2B & 2110 & 200 & 4/09/08 & 8E14001 \\
\hline 0803012-06 & B1RTH2A & 1770 & 200 & 4/09/08 & 8E14001 \\
\hline 0803012-09 & B1RTH3B & 2330 & 200 & 4/09/08 & 8E14001 \\
\hline 0803012-10 & B1RTH3A & 2090 & 200 & 4/09/08 & 8E14001 \\
\hline 0803012-13 & B1RYR7B & 2520 & 200 & 4/09/08 & 8E14001 \\
\hline 0803012-14 & B1RYR7A & 1910 & 200 & $4 / 10 / 08$ & 8E14001 \\
\hline 0803012-17 & B1RYR8B & 2420 & 200 & $4 / 10 / 08$ & 8E14001 \\
\hline 0803012-18 & B1RYR8A & 2160 & 200 & $4 / 10 / 08$ & 8E14001 \\
\hline 0803012-21 & B1RYR9B & 1980 & 200 & $4 / 10 / 08$ & 8E14001 \\
\hline 0803012-22 & B1RYR9A & 1940 & 200 & $4 / 10 / 08$ & 8E14001 \\
\hline 0803014-01 & B1RYT0B & 3650 & 200 & $4 / 10 / 08$ & 8E14001 \\
\hline 0803014-02 & B1RYT0A & 940 & 200 & $4 / 10 / 08$ & 8E14001 \\
\hline 0803014-05 & В1T2P1B & 2300 & 200 & $4 / 10 / 08$ & 8E14001 \\
\hline 0803014-06 & B1T2P1A & 2370 & 200 & $4 / 10 / 08$ & 8E14001 \\
\hline 0803014-09 & B1T2P2B & 2960 & 200 & $4 / 10 / 08$ & 8E14001 \\
\hline 0803014-10 & B1T2P2A & 2150 & 200 & $4 / 10 / 08$ & 8E14001 \\
\hline 0803014-13 & В1Т2Р3В & 2070 & 200 & $4 / 10 / 08$ & 8E14001 \\
\hline 0803014-14 & B1T2P3A & 2240 & 200 & $4 / 11 / 08$ & 8E14001 \\
\hline 0803014-16 & В1Т2Р4B & 2490 & 200 & $4 / 11 / 08$ & 8E14001 \\
\hline 0803014-17 & B1T2P4A & 2550 & 200 & $4 / 11 / 08$ & 8E14001 \\
\hline 0803014-20 & В1Т2Р5B & 2430 & 200 & $4 / 11 / 08$ & 8E14001 \\
\hline 0803014-21 & В1T2P5A & 2770 & 200 & $4 / 11 / 08$ & 8E14001 \\
\hline 0803014-24 & В1Т2Р6B & 1940 & 200 & $4 / 11 / 08$ & 8E14001 \\
\hline 0803014-25 & В1T2P6A & 2080 & 200 & $4 / 11 / 08$ & 8E14001 \\
\hline 0803015-02 & B1T2P7B & 1880 & 200 & $4 / 11 / 08$ & 8E14001 \\
\hline 0803015-03 & B1T2P7A & 1280 & 200 & $4 / 11 / 08$ & 8E14001 \\
\hline 0803015-06 & B1T2P8B & 1950 & 200 & $4 / 11 / 08$ & 8E14001 \\
\hline 0803015-07 & B1T2P8A & 1930 & 200 & $4 / 14 / 08$ & 8E14001 \\
\hline 0803015-10 & В1Т2Р9B & 2290 & 200 & $4 / 14 / 08$ & 8E14001 \\
\hline 0803015-11 & B1T2P9A & 1870 & 200 & $4 / 14 / 08$ & 8E14001 \\
\hline 0803015-14 & B1T2R0B & 2010 & 200 & $4 / 14 / 08$ & 8E14001 \\
\hline 0803015-15 & B1T2R0A & 1740 & 200 & $4 / 14 / 08$ & 8E14001 \\
\hline 0803015-18 & B1TNK6B & 1840 & 200 & $4 / 14 / 08$ & 8E14001 \\
\hline 0803015-19 & B1TNK6A & 1730 & 200 & $4 / 14 / 08$ & 8E14001 \\
\hline 0803015-22 & B1TNK7B & 2070 & 200 & $4 / 14 / 08$ & 8E14001 \\
\hline 0803015-23 & B1TNK7A & 2170 & 200 & $4 / 14 / 08$ & 8E14001 \\
\hline 0803015-26 & B1TNK8B & 1930 & 200 & $4 / 14 / 08$ & 8E14001 \\
\hline 0803015-27 & B1TNK8A & 2260 & 200 & $4 / 14 / 08$ & 8E14001 \\
\hline 0803015-30 & B1TNK9B & 1570 & 200 & $4 / 14 / 08$ & 8E14001 \\
\hline 0803015-31 & B1TNK9A & 1550 & 200 & $4 / 14 / 08$ & 8E14001 \\
\hline
\end{tabular}


GEA/Soil

\begin{tabular}{|c|c|c|c|c|c|c|c|c|}
\hline CAS \# & Analyte & Results & Units & MDA & UNC & Analyzed & Batch & Method \\
\hline HEIS No. & B1RTF8 & \multicolumn{3}{|c|}{ Lab ID: $\quad$ 0803001-01 } & & & & \\
\hline 13966-32-0 & Sodium-22 & $<0.236$ & $\mathrm{pCi} / \mathrm{g}$ & 0.236 & & 3/31/08 & 8C17008 & AGG-RRL-001 \\
\hline 13966-00-2 & Potassium-40 & 13.5 & $\mathrm{pCi} / \mathrm{g}$ & 2.69 & 0.972 & 3/31/08 & 8C17008 & AGG-RRL-001 \\
\hline $14392-02-0$ & Chromium-51 & $<2.12$ & $\mathrm{pCi} / \mathrm{g}$ & 2.12 & & 3/31/08 & 8C17008 & AGG-RRL-001 \\
\hline 13966-31-9 & Manganese-54 & $<0.222$ & $\mathrm{pCi} / \mathrm{g}$ & 0.222 & & 3/31/08 & 8C17008 & AGG-RRL-001 \\
\hline 13981-50-5 & Cobalt-57 & $<0.22$ & $\mathrm{pCi} / \mathrm{g}$ & 0.22 & & 3/31/08 & 8C17008 & AGG-RRL-001 \\
\hline 14596-12-4 & Iron-59 & $<0.396$ & $\mathrm{pCi} / \mathrm{g}$ & 0.396 & & 3/31/08 & 8C17008 & AGG-RRL-001 \\
\hline 10198-40-0 & Cobalt-60 & $<0.186$ & $\mathrm{pCi} / \mathrm{g}$ & 0.186 & & 3/31/08 & 8C17008 & AGG-RRL-001 \\
\hline 13982-39-3 & Zinc-65 & $<0.533$ & $\mathrm{pCi} / \mathrm{g}$ & 0.533 & & 3/31/08 & 8C17008 & AGG-RRL-001 \\
\hline 14265-71-5 & Selenium-75 & $<0.347$ & $\mathrm{pCi} / \mathrm{g}$ & 0.347 & & 3/31/08 & 8C17008 & AGG-RRL-001 \\
\hline 17056-36-9 & Rubidium-83 & $<0.448$ & $\mathrm{pCi} / \mathrm{g}$ & 0.448 & & $3 / 31 / 08$ & 8C17008 & AGG-RRL-001 \\
\hline 13967-73-2 & Strontium-85 & $<0.297$ & $\mathrm{pCi} / \mathrm{g}$ & 0.297 & & 3/31/08 & 8C17008 & AGG-RRL-001 \\
\hline 14932-53-7 & Rubidium-86 & $<2.58$ & $\mathrm{pCi} / \mathrm{g}$ & 2.58 & & $3 / 31 / 08$ & 8C17008 & AGG-RRL-001 \\
\hline 13982-36-0 & Yttrium-88 & $<0.176$ & $\mathrm{pCi} / \mathrm{g}$ & 0.176 & & $3 / 31 / 08$ & 8C17008 & AGG-RRL-001 \\
\hline 14681-63-1 & Niobium-94 & $<0.213$ & $\mathrm{pCi} / \mathrm{g}$ & 0.213 & & 3/31/08 & 8C17008 & AGG-RRL-001 \\
\hline \multirow[t]{3}{*}{ 13967-76-5 } & Niobium-95 & $<0.215$ & $\mathrm{pCi} / \mathrm{g}$ & 0.215 & & 3/31/08 & 8C17008 & AGG-RRL-001 \\
\hline & Niobium-95m & $<0.988$ & $\mathrm{pCi} / \mathrm{g}$ & 0.988 & & 3/31/08 & 8C17008 & AGG-RRL-001 \\
\hline & Technetium-95m & $<0.352$ & $\mathrm{pCi} / \mathrm{g}$ & 0.352 & & 3/31/08 & 8C17008 & AGG-RRL-001 \\
\hline \multirow[t]{2}{*}{ 13967-71-0 } & Zirconium-95 & $<0.382$ & $\mathrm{pCi} / \mathrm{g}$ & 0.382 & & 3/31/08 & 8C17008 & AGG-RRL-001 \\
\hline & Technetium-99m & $<0.21$ & $\mathrm{pCi} / \mathrm{g}$ & 0.21 & & 3/31/08 & 8C17008 & AGG-RRL-001 \\
\hline 13968-53-1 & Ruthenium-103 & $<0.218$ & $\mathrm{pCi} / \mathrm{g}$ & 0.218 & & 3/31/08 & 8C17008 & AGG-RRL-001 \\
\hline 13967-48-1 & Ruthenium-106 & $<2.23$ & $\mathrm{pCi} / \mathrm{g}$ & 2.23 & & 3/31/08 & 8C17008 & AGG-RRL-001 \\
\hline 14391-65-2 & Silver-108m & $<0.23$ & $\mathrm{pCi} / \mathrm{g}$ & 0.23 & & 3/31/08 & 8C17008 & AGG-RRL-001 \\
\hline \multirow[t]{2}{*}{ 14109-32-1 } & Cadmium-109 & $<5.97$ & $\mathrm{pCi} / \mathrm{g}$ & 5.97 & & 3/31/08 & 8C17008 & AGG-RRL-001 \\
\hline & Silver-110 & $<0.235$ & $\mathrm{pCi} / \mathrm{g}$ & 0.235 & & 3/31/08 & 8C17008 & AGG-RRL-001 \\
\hline 14391-76-5 & Silver-110m & $<0.235$ & $\mathrm{pCi} / \mathrm{g}$ & 0.235 & & 3/31/08 & 8C17008 & AGG-RRL-001 \\
\hline 13966-06-8 & Tin-113 & $<0.31$ & $\mathrm{pCi} / \mathrm{g}$ & 0.31 & & 3/31/08 & 8C17008 & AGG-RRL-001 \\
\hline 14683-10-4 & Antimony-124 & $<0.235$ & $\mathrm{pCi} / \mathrm{g}$ & 0.235 & & 3/31/08 & 8C17008 & AGG-RRL-001 \\
\hline $14234-35-6$ & Antimony-125 & $<0.702$ & $\mathrm{pCi} / \mathrm{g}$ & 0.702 & & 3/31/08 & 8C17008 & AGG-RRL-001 \\
\hline 15756-32-8 & Antimony-126 & $<0.223$ & $\mathrm{pCi} / \mathrm{g}$ & 0.223 & & 3/31/08 & 8C17008 & AGG-RRL-001 \\
\hline 15832-50-5 & Tin-126 & $<0.465$ & $\mathrm{pCi} / \mathrm{g}$ & 0.465 & & 3/31/08 & 8C17008 & AGG-RRL-001 \\
\hline 10043-66-0 & Iodine-131 & $<0.261$ & $\mathrm{pCi} / \mathrm{g}$ & 0.261 & & 3/31/08 & 8C17008 & AGG-RRL-001 \\
\hline 13981-41-4 & Barium-133 & $<0.417$ & $\mathrm{pCi} / \mathrm{g}$ & 0.417 & & 3/31/08 & 8C17008 & AGG-RRL-001 \\
\hline 13967-70-9 & Cesium-134 & $<0.249$ & $\mathrm{pCi} / \mathrm{g}$ & 0.249 & & 3/31/08 & 8C17008 & AGG-RRL-001 \\
\hline $10045-97-3$ & Cesium-137 & $<0.252$ & $\mathrm{pCi} / \mathrm{g}$ & 0.252 & & 3/31/08 & 8C17008 & AGG-RRL-001 \\
\hline 13982-30-4 & Cerium-139 & $<0.252$ & $\mathrm{pCi} / \mathrm{g}$ & 0.252 & & 3/31/08 & 8C17008 & AGG-RRL-001 \\
\hline 14762-78-8 & Cerium-144 & $<1.74$ & $\mathrm{pCi} / \mathrm{g}$ & 1.74 & & 3/31/08 & 8C17008 & AGG-RRL-001 \\
\hline 14683-23-9 & Europium-152 & $<0.836$ & $\mathrm{pCi} / \mathrm{g}$ & 0.836 & & 3/31/08 & 8C17008 & AGG-RRL-001 \\
\hline 14276-65-4 & Gadolinium-153 & $<0.663$ & $\mathrm{pCi} / \mathrm{g}$ & 0.663 & & 3/31/08 & 8C17008 & AGG-RRL-001 \\
\hline 15585-10-1 & Europium-154 & $<0.462$ & $\mathrm{pCi} / \mathrm{g}$ & 0.462 & & 3/31/08 & 8C17008 & AGG-RRL-001 \\
\hline 14391-16-3 & Europium-155 & $<0.658$ & $\mathrm{pCi} / \mathrm{g}$ & 0.658 & & 3/31/08 & 8C17008 & AGG-RRL-001 \\
\hline 13982-78-0 & Mercury-203 & $<0.273$ & $\mathrm{pCi} / \mathrm{g}$ & 0.273 & & 3/31/08 & 8C17008 & AGG-RRL-001 \\
\hline 14913-50-9 & Thallium-208 & $<0.214$ & $\mathrm{pCi} / \mathrm{g}$ & 0.214 & 0.0469 & 3/31/08 & 8C17008 & AGG-RRL-001 \\
\hline 14331-79-4 & Bismuth-210 & $<0.42$ & $\mathrm{pCi} / \mathrm{g}$ & 0.42 & & 3/31/08 & 8C17008 & AGG-RRL-001 \\
\hline \multirow[t]{3}{*}{$14255-04-0$} & Lead-210 & $<83.3$ & $\mathrm{pCi} / \mathrm{g}$ & 83.3 & & 3/31/08 & 8C17008 & AGG-RRL-001 \\
\hline & Bismuth-211 & $<5.2$ & $\mathrm{pCi} / \mathrm{g}$ & 5.2 & & 3/31/08 & 8C17008 & AGG-RRL-001 \\
\hline & Lead-211 & $<7.11$ & $\mathrm{pCi} / \mathrm{g}$ & 7.11 & & 3/31/08 & 8C17008 & AGG-RRL-001 \\
\hline 15092-94-1 & Lead-212 & $<0.61$ & $\mathrm{pCi} / \mathrm{g}$ & 0.61 & 0.135 & 3/31/08 & 8C17008 & AGG-RRL-001 \\
\hline 14733-03-0 & Bismuth-214 & $<0.592$ & $\mathrm{pCi} / \mathrm{g}$ & 0.592 & & 3/31/08 & 8C17008 & AGG-RRL-001 \\
\hline $15067-28-4$ & Lead-214 & $<0.675$ & $\mathrm{pCi} / \mathrm{g}$ & 0.675 & & 3/31/08 & 8C17008 & AGG-RRL-001 \\
\hline
\end{tabular}


GEA/Soil

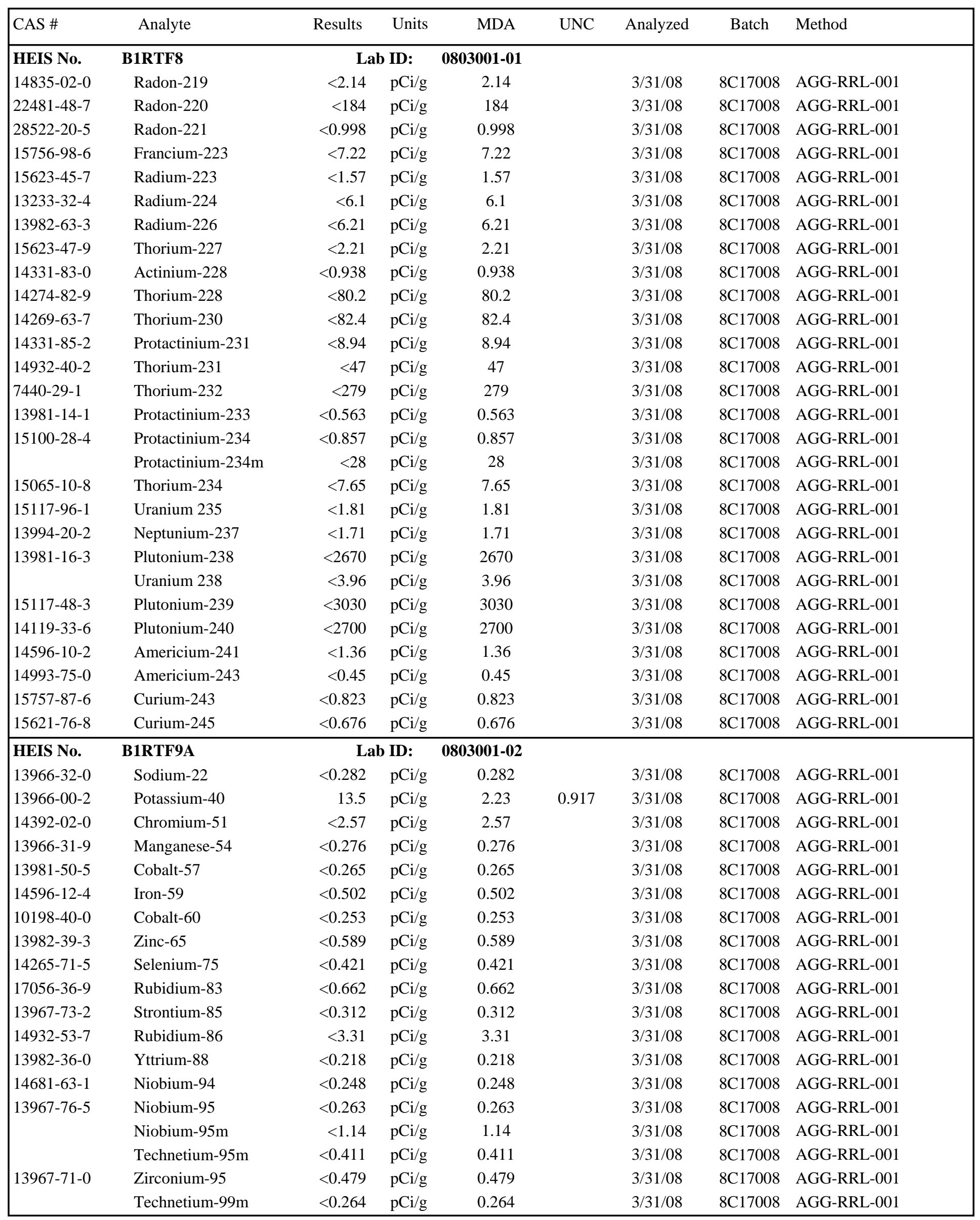


GEA/Soil

\begin{tabular}{|c|c|c|c|c|c|c|c|c|}
\hline CAS \# & Analyte & Results & Units & MDA & UNC & Analyzed & Batch & Method \\
\hline HEIS No. & B1RTF9A & \multicolumn{2}{|c|}{ Lab ID: } & 0803001-02 & & & & \\
\hline 13968-53-1 & Ruthenium-103 & $<0.31$ & $\mathrm{pCi} / \mathrm{g}$ & 0.31 & & 3/31/08 & 8C17008 & AGG-RRL-001 \\
\hline 13967-48-1 & Ruthenium-106 & $<2.83$ & $\mathrm{pCi} / \mathrm{g}$ & 2.83 & & 3/31/08 & 8C17008 & AGG-RRL-001 \\
\hline 14391-65-2 & Silver-108m & $<0.26$ & $\mathrm{pCi} / \mathrm{g}$ & 0.26 & & 3/31/08 & 8C17008 & AGG-RRL-001 \\
\hline \multirow[t]{2}{*}{ 14109-32-1 } & Cadmium-109 & $<7.42$ & $\mathrm{pCi} / \mathrm{g}$ & 7.42 & & 3/31/08 & 8C17008 & AGG-RRL-001 \\
\hline & Silver-110 & $<0.259$ & $\mathrm{pCi} / \mathrm{g}$ & 0.259 & & 3/31/08 & 8C17008 & AGG-RRL-001 \\
\hline 14391-76-5 & Silver-110m & $<0.259$ & $\mathrm{pCi} / \mathrm{g}$ & 0.259 & & 3/31/08 & 8C17008 & AGG-RRL-001 \\
\hline 13966-06-8 & Tin-113 & $<0.372$ & $\mathrm{pCi} / \mathrm{g}$ & 0.372 & & 3/31/08 & 8C17008 & AGG-RRL-001 \\
\hline 14683-10-4 & Antimony-124 & $<0.31$ & $\mathrm{pCi} / \mathrm{g}$ & 0.31 & & 3/31/08 & 8C17008 & AGG-RRL-001 \\
\hline $14234-35-6$ & Antimony-125 & $<0.805$ & $\mathrm{pCi} / \mathrm{g}$ & 0.805 & & 3/31/08 & 8C17008 & AGG-RRL-001 \\
\hline 15756-32-8 & Antimony-126 & $<0.249$ & $\mathrm{pCi} / \mathrm{g}$ & 0.249 & & 3/31/08 & 8C17008 & AGG-RRL-001 \\
\hline $15832-50-5$ & Tin-126 & $<0.608$ & $\mathrm{pCi} / \mathrm{g}$ & 0.608 & & 3/31/08 & 8C17008 & AGG-RRL-001 \\
\hline 10043-66-0 & Iodine-131 & $<0.317$ & $\mathrm{pCi} / \mathrm{g}$ & 0.317 & & 3/31/08 & 8C17008 & AGG-RRL-001 \\
\hline 13981-41-4 & Barium-133 & $<0.452$ & $\mathrm{pCi} / \mathrm{g}$ & 0.452 & & 3/31/08 & 8C17008 & AGG-RRL-001 \\
\hline 13967-70-9 & Cesium-134 & $<0.312$ & $\mathrm{pCi} / \mathrm{g}$ & 0.312 & & 3/31/08 & 8C17008 & AGG-RRL-001 \\
\hline 10045-97-3 & Cesium-137 & $<0.287$ & $\mathrm{pCi} / \mathrm{g}$ & 0.287 & & 3/31/08 & 8C17008 & AGG-RRL-001 \\
\hline 13982-30-4 & Cerium-139 & $<0.315$ & $\mathrm{pCi} / \mathrm{g}$ & 0.315 & & 3/31/08 & 8C17008 & AGG-RRL-001 \\
\hline 14762-78-8 & Cerium-144 & $<2.15$ & $\mathrm{pCi} / \mathrm{g}$ & 2.15 & & 3/31/08 & 8C17008 & AGG-RRL-001 \\
\hline 14683-23-9 & Europium-152 & $<1.06$ & $\mathrm{pCi} / \mathrm{g}$ & 1.06 & & 3/31/08 & 8C17008 & AGG-RRL-001 \\
\hline 14276-65-4 & Gadolinium-153 & $<0.794$ & $\mathrm{pCi} / \mathrm{g}$ & 0.794 & & 3/31/08 & 8C17008 & AGG-RRL-001 \\
\hline 15585-10-1 & Europium-154 & $<0.561$ & $\mathrm{pCi} / \mathrm{g}$ & 0.561 & & 3/31/08 & 8C17008 & AGG-RRL-001 \\
\hline 14391-16-3 & Europium-155 & $<0.862$ & $\mathrm{pCi} / \mathrm{g}$ & 0.862 & & 3/31/08 & 8C17008 & AGG-RRL-001 \\
\hline 13982-78-0 & Mercury-203 & $<0.322$ & $\mathrm{pCi} / \mathrm{g}$ & 0.322 & & 3/31/08 & 8C17008 & AGG-RRL-001 \\
\hline 14913-50-9 & Thallium-208 & $<0.335$ & $\mathrm{pCi} / \mathrm{g}$ & 0.335 & & 3/31/08 & 8C17008 & AGG-RRL-001 \\
\hline 14331-79-4 & Bismuth-210 & $<0.507$ & $\mathrm{pCi} / \mathrm{g}$ & 0.507 & & 3/31/08 & 8C17008 & AGG-RRL-001 \\
\hline \multirow[t]{3}{*}{ 14255-04-0 } & Lead-210 & $<36$ & $\mathrm{pCi} / \mathrm{g}$ & 36 & & 3/31/08 & 8C17008 & AGG-RRL-001 \\
\hline & Bismuth-211 & $<5.79$ & $\mathrm{pCi} / \mathrm{g}$ & 5.79 & & 3/31/08 & 8C17008 & AGG-RRL-001 \\
\hline & Lead-211 & $<7.92$ & $\mathrm{pCi} / \mathrm{g}$ & 7.92 & & 3/31/08 & 8C17008 & AGG-RRL-001 \\
\hline 15092-94-1 & Lead-212 & $<0.551$ & $\mathrm{pCi} / \mathrm{g}$ & 0.551 & 0.124 & 3/31/08 & 8C17008 & AGG-RRL-001 \\
\hline 14733-03-0 & Bismuth-214 & $<0.649$ & $\mathrm{pCi} / \mathrm{g}$ & 0.649 & & 3/31/08 & 8C17008 & AGG-RRL-001 \\
\hline 15067-28-4 & Lead-214 & $<0.734$ & $\mathrm{pCi} / \mathrm{g}$ & 0.734 & & 3/31/08 & 8C17008 & AGG-RRL-001 \\
\hline 14835-02-0 & Radon-219 & $<2.54$ & $\mathrm{pCi} / \mathrm{g}$ & 2.54 & & 3/31/08 & 8C17008 & AGG-RRL-001 \\
\hline 22481-48-7 & Radon-220 & $<241$ & $\mathrm{pCi} / \mathrm{g}$ & 241 & & 3/31/08 & 8C17008 & AGG-RRL-001 \\
\hline 28522-20-5 & Radon-221 & $<1.23$ & $\mathrm{pCi} / \mathrm{g}$ & 1.23 & & 3/31/08 & 8C17008 & AGG-RRL-001 \\
\hline 15756-98-6 & Francium-223 & $<8.46$ & $\mathrm{pCi} / \mathrm{g}$ & 8.46 & & 3/31/08 & 8C17008 & AGG-RRL-001 \\
\hline $15623-45-7$ & Radium-223 & $<1.85$ & $\mathrm{pCi} / \mathrm{g}$ & 1.85 & & 3/31/08 & 8C17008 & AGG-RRL-001 \\
\hline 13233-32-4 & Radium-224 & $<7.19$ & $\mathrm{pCi} / \mathrm{g}$ & 7.19 & & 3/31/08 & 8C17008 & AGG-RRL-001 \\
\hline 13982-63-3 & Radium-226 & $<7.61$ & $\mathrm{pCi} / \mathrm{g}$ & 7.61 & & 3/31/08 & 8C17008 & AGG-RRL-001 \\
\hline $15623-47-9$ & Thorium-227 & $<2.55$ & $\mathrm{pCi} / \mathrm{g}$ & 2.55 & & 3/31/08 & 8C17008 & AGG-RRL-001 \\
\hline 14331-83-0 & Actinium-228 & $<1.11$ & $\mathrm{pCi} / \mathrm{g}$ & 1.11 & & 3/31/08 & 8C17008 & AGG-RRL-001 \\
\hline $14274-82-9$ & Thorium-228 & $<95.4$ & $\mathrm{pCi} / \mathrm{g}$ & 95.4 & & 3/31/08 & 8C17008 & AGG-RRL-001 \\
\hline $14269-63-7$ & Thorium-230 & $<98.5$ & $\mathrm{pCi} / \mathrm{g}$ & 98.5 & & 3/31/08 & 8C17008 & AGG-RRL-001 \\
\hline 14331-85-2 & Protactinium-231 & $<11.6$ & $\mathrm{pCi} / \mathrm{g}$ & 11.6 & & 3/31/08 & 8C17008 & AGG-RRL-001 \\
\hline $14932-40-2$ & Thorium-231 & $<57.3$ & $\mathrm{pCi} / \mathrm{g}$ & 57.3 & & 3/31/08 & 8C17008 & AGG-RRL-001 \\
\hline 7440-29-1 & Thorium-232 & $<240$ & $\mathrm{pCi} / \mathrm{g}$ & 240 & & $3 / 31 / 08$ & 8C17008 & AGG-RRL-001 \\
\hline 13981-14-1 & Protactinium-233 & $<0.695$ & $\mathrm{pCi} / \mathrm{g}$ & 0.695 & & 3/31/08 & 8C17008 & AGG-RRL-001 \\
\hline \multirow[t]{2}{*}{$15100-28-4$} & Protactinium-234 & $<1.04$ & $\mathrm{pCi} / \mathrm{g}$ & 1.04 & & 3/31/08 & 8C17008 & AGG-RRL-001 \\
\hline & Protactinium-234m & $<32.1$ & $\mathrm{pCi} / \mathrm{g}$ & 32.1 & & 3/31/08 & 8C17008 & AGG-RRL-001 \\
\hline 15065-10-8 & Thorium-234 & $<8.54$ & $\mathrm{pCi} / \mathrm{g}$ & 8.54 & & 3/31/08 & 8C17008 & AGG-RRL-001 \\
\hline 15117-96-1 & Uranium 235 & $<2.27$ & $\mathrm{pCi} / \mathrm{g}$ & 2.27 & & 3/31/08 & 8C17008 & AGG-RRL-001 \\
\hline
\end{tabular}


GEA/Soil

\begin{tabular}{|c|c|c|c|c|c|c|c|c|}
\hline CAS \# & Analyte & Results & Units & MDA & UNC & Analyzed & Batch & Method \\
\hline HEIS No. & B1RTF9A & \multicolumn{2}{|c|}{ Lab ID: } & 0803001-02 & & & & \\
\hline 13994-20-2 & Neptunium-237 & $<2.25$ & $\mathrm{pCi} / \mathrm{g}$ & 2.25 & & 3/31/08 & 8C17008 & AGG-RRL-001 \\
\hline \multirow[t]{2}{*}{ 13981-16-3 } & Plutonium-238 & $<3280$ & $\mathrm{pCi} / \mathrm{g}$ & 3280 & & 3/31/08 & 8C17008 & AGG-RRL-001 \\
\hline & Uranium 238 & $<4.71$ & $\mathrm{pCi} / \mathrm{g}$ & 4.71 & & 3/31/08 & 8C17008 & AGG-RRL-001 \\
\hline 15117-48-3 & Plutonium-239 & $<3370$ & $\mathrm{pCi} / \mathrm{g}$ & 3370 & & 3/31/08 & 8C17008 & AGG-RRL-001 \\
\hline 14119-33-6 & Plutonium-240 & $<3310$ & $\mathrm{pCi} / \mathrm{g}$ & 3310 & & 3/31/08 & 8C17008 & AGG-RRL-001 \\
\hline 14596-10-2 & Americium-241 & $<1.23$ & $\mathrm{pCi} / \mathrm{g}$ & 1.23 & & 3/31/08 & 8C17008 & AGG-RRL-001 \\
\hline 14993-75-0 & Americium-243 & $<0.546$ & $\mathrm{pCi} / \mathrm{g}$ & 0.546 & & 3/31/08 & 8C17008 & AGG-RRL-001 \\
\hline 15757-87-6 & Curium-243 & $<1.01$ & $\mathrm{pCi} / \mathrm{g}$ & 1.01 & & 3/31/08 & 8C17008 & AGG-RRL-001 \\
\hline 15621-76-8 & Curium-245 & $<0.828$ & $\mathrm{pCi} / \mathrm{g}$ & 0.828 & & 3/31/08 & 8C17008 & AGG-RRL-001 \\
\hline HEIS No. & B1RTH0 & \multicolumn{2}{|c|}{ Lab ID: } & 0803012-01 & & & & \\
\hline 13966-32-0 & Sodium-22 & $<0.821$ & $\mathrm{pCi} / \mathrm{g}$ & 0.821 & & 3/31/08 & 8C17008 & AGG-RRL-001 \\
\hline 13966-00-2 & Potassium-40 & 21.0 & $\mathrm{pCi} / \mathrm{g}$ & 6.81 & 2.23 & 3/31/08 & 8C17008 & AGG-RRL-001 \\
\hline 14392-02-0 & Chromium-51 & $<6.17$ & $\mathrm{pCi} / \mathrm{g}$ & 6.17 & & 3/31/08 & 8C17008 & AGG-RRL-001 \\
\hline 13966-31-9 & Manganese-54 & $<0.637$ & $\mathrm{pCi} / \mathrm{g}$ & 0.637 & & 3/31/08 & 8C17008 & AGG-RRL-001 \\
\hline 13981-50-5 & Cobalt-57 & $<0.654$ & $\mathrm{pCi} / \mathrm{g}$ & 0.654 & & 3/31/08 & 8C17008 & AGG-RRL-001 \\
\hline $14596-12-4$ & Iron-59 & $<1.22$ & $\mathrm{pCi} / \mathrm{g}$ & 1.22 & & 3/31/08 & 8C17008 & AGG-RRL-001 \\
\hline 10198-40-0 & Cobalt-60 & $<0.672$ & $\mathrm{pCi} / \mathrm{g}$ & 0.672 & & 3/31/08 & 8C17008 & AGG-RRL-001 \\
\hline 13982-39-3 & Zinc-65 & $<1.7$ & $\mathrm{pCi} / \mathrm{g}$ & 1.7 & & 3/31/08 & 8C17008 & AGG-RRL-001 \\
\hline $14265-71-5$ & Selenium-75 & $<1.12$ & $\mathrm{pCi} / \mathrm{g}$ & 1.12 & & 3/31/08 & 8C17008 & AGG-RRL-001 \\
\hline 17056-36-9 & Rubidium-83 & $<1.32$ & $\mathrm{pCi} / \mathrm{g}$ & 1.32 & & 3/31/08 & 8C17008 & AGG-RRL-001 \\
\hline 13967-73-2 & Strontium-85 & $<0.871$ & $\mathrm{pCi} / \mathrm{g}$ & 0.871 & & 3/31/08 & 8C17008 & AGG-RRL-001 \\
\hline $14932-53-7$ & Rubidium-86 & $<7.66$ & $\mathrm{pCi} / \mathrm{g}$ & 7.66 & & 3/31/08 & 8C17008 & AGG-RRL-001 \\
\hline 13982-36-0 & Yttrium-88 & $<0.673$ & $\mathrm{pCi} / \mathrm{g}$ & 0.673 & & 3/31/08 & 8C17008 & AGG-RRL-001 \\
\hline 14681-63-1 & Niobium-94 & $<0.618$ & pCi/g & 0.618 & & 3/31/08 & 8C17008 & AGG-RRL-001 \\
\hline \multirow[t]{3}{*}{ 13967-76-5 } & Niobium-95 & $<0.7$ & $\mathrm{pCi} / \mathrm{g}$ & 0.7 & & 3/31/08 & 8C17008 & AGG-RRL-001 \\
\hline & Niobium-95m & $<3.06$ & $\mathrm{pCi} / \mathrm{g}$ & 3.06 & & 3/31/08 & 8C17008 & AGG-RRL-001 \\
\hline & Technetium-95m & $<1.09$ & $\mathrm{pCi} / \mathrm{g}$ & 1.09 & & 3/31/08 & 8C17008 & AGG-RRL-001 \\
\hline \multirow[t]{2}{*}{ 13967-71-0 } & Zirconium-95 & $<1.15$ & $\mathrm{pCi} / \mathrm{g}$ & 1.15 & & 3/31/08 & 8C17008 & AGG-RRL-001 \\
\hline & Technetium-99m & $<0.652$ & $\mathrm{pCi} / \mathrm{g}$ & 0.652 & & 3/31/08 & 8C17008 & AGG-RRL-001 \\
\hline 13968-53-1 & Ruthenium-103 & $<0.678$ & $\mathrm{pCi} / \mathrm{g}$ & 0.678 & & 3/31/08 & 8C17008 & AGG-RRL-001 \\
\hline 13967-48-1 & Ruthenium-106 & $<6.67$ & $\mathrm{pCi} / \mathrm{g}$ & 6.67 & & 3/31/08 & 8C17008 & AGG-RRL-001 \\
\hline 14391-65-2 & Silver-108m & $<0.719$ & $\mathrm{pCi} / \mathrm{g}$ & 0.719 & & 3/31/08 & 8C17008 & AGG-RRL-001 \\
\hline \multirow[t]{2}{*}{ 14109-32-1 } & Cadmium-109 & $<17.6$ & $\mathrm{pCi} / \mathrm{g}$ & 17.6 & & 3/31/08 & 8C17008 & AGG-RRL-001 \\
\hline & Silver-110 & $<0.67$ & $\mathrm{pCi} / \mathrm{g}$ & 0.67 & & 3/31/08 & 8C17008 & AGG-RRL-001 \\
\hline 14391-76-5 & Silver-110m & $<0.671$ & $\mathrm{pCi} / \mathrm{g}$ & 0.671 & & 3/31/08 & 8C17008 & AGG-RRL-001 \\
\hline 13966-06-8 & Tin-113 & $<1$ & $\mathrm{pCi} / \mathrm{g}$ & 1 & & 3/31/08 & 8C17008 & AGG-RRL-001 \\
\hline $14683-10-4$ & Antimony-124 & $<0.719$ & $\mathrm{pCi} / \mathrm{g}$ & 0.719 & & 3/31/08 & 8C17008 & AGG-RRL-001 \\
\hline $14234-35-6$ & Antimony-125 & $<2.31$ & $\mathrm{pCi} / \mathrm{g}$ & 2.31 & & 3/31/08 & 8C17008 & AGG-RRL-001 \\
\hline 15756-32-8 & Antimony-126 & $<0.612$ & $\mathrm{pCi} / \mathrm{g}$ & 0.612 & & 3/31/08 & 8C17008 & AGG-RRL-001 \\
\hline $15832-50-5$ & Tin-126 & $<1.39$ & $\mathrm{pCi} / \mathrm{g}$ & 1.39 & & 3/31/08 & 8C17008 & AGG-RRL-001 \\
\hline 10043-66-0 & Iodine-131 & $<0.811$ & $\mathrm{pCi} / \mathrm{g}$ & 0.811 & & 3/31/08 & 8C17008 & AGG-RRL-001 \\
\hline 13981-41-4 & Barium-133 & $<1.33$ & $\mathrm{pCi} / \mathrm{g}$ & 1.33 & & 3/31/08 & 8C17008 & AGG-RRL-001 \\
\hline 13967-70-9 & Cesium-134 & $<0.749$ & $\mathrm{pCi} / \mathrm{g}$ & 0.749 & & 3/31/08 & 8C17008 & AGG-RRL-001 \\
\hline $10045-97-3$ & Cesium-137 & $<0.778$ & $\mathrm{pCi} / \mathrm{g}$ & 0.778 & & 3/31/08 & 8C17008 & AGG-RRL-001 \\
\hline 13982-30-4 & Cerium-139 & $<0.799$ & $\mathrm{pCi} / \mathrm{g}$ & 0.799 & & 3/31/08 & 8C17008 & AGG-RRL-001 \\
\hline 14762-78-8 & Cerium-144 & $<5.18$ & $\mathrm{pCi} / \mathrm{g}$ & 5.18 & & 3/31/08 & 8C17008 & AGG-RRL-001 \\
\hline 14683-23-9 & Europium-152 & $<2.47$ & $\mathrm{pCi} / \mathrm{g}$ & 2.47 & & 3/31/08 & 8C17008 & AGG-RRL-001 \\
\hline $14276-65-4$ & Gadolinium-153 & $<1.99$ & $\mathrm{pCi} / \mathrm{g}$ & 1.99 & & 3/31/08 & 8C17008 & AGG-RRL-001 \\
\hline
\end{tabular}


GEA/Soil

\begin{tabular}{|c|c|c|c|c|c|c|c|c|}
\hline CAS \# & Analyte & Results & Units & MDA & UNC & Analyzed & Batch & Method \\
\hline HEIS No. & B1RTH0 & \multicolumn{2}{|c|}{ Lab ID: } & 0803012-01 & \multirow{40}{*}{0.354} & & & \\
\hline 15585-10-1 & Europium-154 & $<1.38$ & $\mathrm{pCi} / \mathrm{g}$ & 1.38 & & 3/31/08 & 8C17008 & AGG-RRL-001 \\
\hline 14391-16-3 & Europium-155 & $<1.95$ & $\mathrm{pCi} / \mathrm{g}$ & 1.95 & & $3 / 31 / 08$ & 8C17008 & AGG-RRL-001 \\
\hline 13982-78-0 & Mercury-203 & $<0.856$ & $\mathrm{pCi} / \mathrm{g}$ & 0.856 & & 3/31/08 & 8C17008 & AGG-RRL-001 \\
\hline 14913-50-9 & Thallium-208 & $<0.79$ & $\mathrm{pCi} / \mathrm{g}$ & 0.79 & & 3/31/08 & 8C17008 & AGG-RRL-001 \\
\hline 14331-79-4 & Bismuth-210 & $<1.35$ & $\mathrm{pCi} / \mathrm{g}$ & 1.35 & & 3/31/08 & 8C17008 & AGG-RRL-001 \\
\hline \multirow[t]{3}{*}{$14255-04-0$} & Lead-210 & $<265$ & $\mathrm{pCi} / \mathrm{g}$ & 265 & & 3/31/08 & 8C17008 & AGG-RRL-001 \\
\hline & Bismuth-211 & $<15.8$ & $\mathrm{pCi} / \mathrm{g}$ & 15.8 & & 3/31/08 & 8C17008 & AGG-RRL-001 \\
\hline & Lead-211 & $<21.7$ & $\mathrm{pCi} / \mathrm{g}$ & 21.7 & & 3/31/08 & 8C17008 & AGG-RRL-001 \\
\hline 15092-94-1 & Lead-212 & $<1.61$ & $\mathrm{pCi} / \mathrm{g}$ & 1.61 & & 3/31/08 & 8C17008 & AGG-RRL-001 \\
\hline 14733-03-0 & Bismuth-214 & $<1.96$ & $\mathrm{pCi} / \mathrm{g}$ & 1.96 & & 3/31/08 & 8C17008 & AGG-RRL-001 \\
\hline $15067-28-4$ & Lead-214 & $<2.11$ & $\mathrm{pCi} / \mathrm{g}$ & 2.11 & & 3/31/08 & 8C17008 & AGG-RRL-001 \\
\hline $14835-02-0$ & Radon-219 & $<6.74$ & $\mathrm{pCi} / \mathrm{g}$ & 6.74 & & 3/31/08 & 8C17008 & AGG-RRL-001 \\
\hline 22481-48-7 & Radon-220 & $<577$ & $\mathrm{pCi} / \mathrm{g}$ & 577 & & $3 / 31 / 08$ & 8C17008 & AGG-RRL-001 \\
\hline 28522-20-5 & Radon-221 & $<3.03$ & $\mathrm{pCi} / \mathrm{g}$ & 3.03 & & 3/31/08 & 8C17008 & AGG-RRL-001 \\
\hline 15756-98-6 & Francium-223 & $<22.2$ & $\mathrm{pCi} / \mathrm{g}$ & 22.2 & & 3/31/08 & 8C17008 & AGG-RRL-001 \\
\hline 15623-45-7 & Radium-223 & $<4.93$ & $\mathrm{pCi} / \mathrm{g}$ & 4.93 & & 3/31/08 & 8C17008 & AGG-RRL-001 \\
\hline 13233-32-4 & Radium-224 & $<20$ & $\mathrm{pCi} / \mathrm{g}$ & 20 & & 3/31/08 & 8C17008 & AGG-RRL-001 \\
\hline 13982-63-3 & Radium-226 & $<18.6$ & $\mathrm{pCi} / \mathrm{g}$ & 18.6 & & 3/31/08 & 8C17008 & AGG-RRL-001 \\
\hline 15623-47-9 & Thorium-227 & $<6.85$ & $\mathrm{pCi} / \mathrm{g}$ & 6.85 & & $3 / 31 / 08$ & 8C17008 & AGG-RRL-001 \\
\hline 14331-83-0 & Actinium-228 & $<2.79$ & $\mathrm{pCi} / \mathrm{g}$ & 2.79 & & 3/31/08 & 8C17008 & AGG-RRL-001 \\
\hline 14274-82-9 & Thorium-228 & $<258$ & $\mathrm{pCi} / \mathrm{g}$ & 258 & & $3 / 31 / 08$ & 8C17008 & AGG-RRL-001 \\
\hline 14269-63-7 & Thorium-230 & $<257$ & $\mathrm{pCi} / \mathrm{g}$ & 257 & & 3/31/08 & 8C17008 & AGG-RRL-001 \\
\hline 14331-85-2 & Protactinium-231 & $<27.8$ & $\mathrm{pCi} / \mathrm{g}$ & 27.8 & & 3/31/08 & 8C17008 & AGG-RRL-001 \\
\hline $14932-40-2$ & Thorium-231 & $<137$ & $\mathrm{pCi} / \mathrm{g}$ & 137 & & 3/31/08 & 8C17008 & AGG-RRL-001 \\
\hline $7440-29-1$ & Thorium-232 & $<818$ & $\mathrm{pCi} / \mathrm{g}$ & 818 & & 3/31/08 & 8C17008 & AGG-RRL-001 \\
\hline 13981-14-1 & Protactinium-233 & $<1.74$ & $\mathrm{pCi} / \mathrm{g}$ & 1.74 & & $3 / 31 / 08$ & 8C17008 & AGG-RRL-001 \\
\hline \multirow[t]{2}{*}{$15100-28-4$} & Protactinium-234 & $<2.53$ & $\mathrm{pCi} / \mathrm{g}$ & 2.53 & & 3/31/08 & 8C17008 & AGG-RRL-001 \\
\hline & Protactinium-234m & $<84.3$ & $\mathrm{pCi} / \mathrm{g}$ & 84.3 & & 3/31/08 & 8C17008 & AGG-RRL-001 \\
\hline 15065-10-8 & Thorium-234 & $<23$ & $\mathrm{pCi} / \mathrm{g}$ & 23 & & 3/31/08 & 8C17008 & AGG-RRL-001 \\
\hline 15117-96-1 & Uranium 235 & $<5.48$ & $\mathrm{pCi} / \mathrm{g}$ & 5.48 & & 3/31/08 & 8C17008 & AGG-RRL-001 \\
\hline 13994-20-2 & Neptunium-237 & $<5.09$ & $\mathrm{pCi} / \mathrm{g}$ & 5.09 & & 3/31/08 & 8C17008 & AGG-RRL-001 \\
\hline \multirow[t]{2}{*}{ 13981-16-3 } & Plutonium-238 & $<7800$ & $\mathrm{pCi} / \mathrm{g}$ & 7800 & & 3/31/08 & 8C17008 & AGG-RRL-001 \\
\hline & Uranium 238 & $<11.9$ & $\mathrm{pCi} / \mathrm{g}$ & 11.9 & & 3/31/08 & 8C17008 & AGG-RRL-001 \\
\hline $15117-48-3$ & Plutonium-239 & $<9080$ & $\mathrm{pCi} / \mathrm{g}$ & 9080 & & 3/31/08 & 8C17008 & AGG-RRL-001 \\
\hline 14119-33-6 & Plutonium-240 & $<7990$ & $\mathrm{pCi} / \mathrm{g}$ & 7990 & & 3/31/08 & 8C17008 & AGG-RRL-001 \\
\hline 14596-10-2 & Americium-241 & $<3.98$ & $\mathrm{pCi} / \mathrm{g}$ & 3.98 & & 3/31/08 & 8C17008 & AGG-RRL-001 \\
\hline $14993-75-0$ & Americium-243 & $<1.41$ & $\mathrm{pCi} / \mathrm{g}$ & 1.41 & & 3/31/08 & 8C17008 & AGG-RRL-001 \\
\hline 15757-87-6 & Curium-243 & $<2.44$ & $\mathrm{pCi} / \mathrm{g}$ & 2.44 & & 3/31/08 & 8C17008 & AGG-RRL-001 \\
\hline 15621-76-8 & Curium-245 & $<2$ & $\mathrm{pCi} / \mathrm{g}$ & 2 & & $3 / 31 / 08$ & 8C17008 & AGG-RRL-001 \\
\hline HEIS No. & B1RTH1A & \multicolumn{2}{|c|}{ Lab ID: } & 0803012-02 & \multirow{9}{*}{0.991} & & & \\
\hline 13966-32-0 & Sodium-22 & $<0.253$ & $\mathrm{pCi} / \mathrm{g}$ & 0.253 & & 3/31/08 & 8C17008 & AGG-RRL-001 \\
\hline 13966-00-2 & Potassium-40 & 14.2 & $\mathrm{pCi} / \mathrm{g}$ & 2.61 & & $3 / 31 / 08$ & 8C17008 & AGG-RRL-001 \\
\hline 14392-02-0 & Chromium-51 & $<2.12$ & $\mathrm{pCi} / \mathrm{g}$ & 2.12 & & 3/31/08 & 8C17008 & AGG-RRL-001 \\
\hline 13966-31-9 & Manganese-54 & $<0.217$ & $\mathrm{pCi} / \mathrm{g}$ & 0.217 & & 3/31/08 & 8C17008 & AGG-RRL-001 \\
\hline 13981-50-5 & Cobalt-57 & $<0.221$ & $\mathrm{pCi} / \mathrm{g}$ & 0.221 & & 3/31/08 & 8C17008 & AGG-RRL-001 \\
\hline $14596-12-4$ & Iron-59 & $<0.419$ & $\mathrm{pCi} / \mathrm{g}$ & 0.419 & & 3/31/08 & 8C17008 & AGG-RRL-001 \\
\hline 10198-40-0 & Cobalt-60 & $<0.218$ & $\mathrm{pCi} / \mathrm{g}$ & 0.218 & & 3/31/08 & 8C17008 & AGG-RRL-001 \\
\hline 13982-39-3 & Zinc-65 & $<0.5$ & $\mathrm{pCi} / \mathrm{g}$ & 0.5 & & 3/31/08 & 8C17008 & AGG-RRL-001 \\
\hline
\end{tabular}


GEA/Soil

\begin{tabular}{|c|c|c|c|c|c|c|c|c|}
\hline CAS \# & Analyte & Results & Units & MDA & UNC & Analyzed & Batch & Method \\
\hline HEIS No. & B1RTH1A & \multicolumn{2}{|c|}{ Lab ID: } & 0803012-02 & & & & \\
\hline 14265-71-5 & Selenium-75 & $<0.366$ & $\mathrm{pCi} / \mathrm{g}$ & 0.366 & & 3/31/08 & 8C17008 & AGG-RRL-001 \\
\hline 17056-36-9 & Rubidium-83 & $<0.442$ & $\mathrm{pCi} / \mathrm{g}$ & 0.442 & & 3/31/08 & 8C17008 & AGG-RRL-001 \\
\hline 13967-73-2 & Strontium-85 & $<0.286$ & $\mathrm{pCi} / \mathrm{g}$ & 0.286 & & 3/31/08 & 8C17008 & AGG-RRL-001 \\
\hline 14932-53-7 & Rubidium-86 & $<2.57$ & $\mathrm{pCi} / \mathrm{g}$ & 2.57 & & 3/31/08 & 8C17008 & AGG-RRL-001 \\
\hline 13982-36-0 & Yttrium-88 & $<0.177$ & $\mathrm{pCi} / \mathrm{g}$ & 0.177 & & 3/31/08 & 8C17008 & AGG-RRL-001 \\
\hline 14681-63-1 & Niobium-94 & $<0.192$ & $\mathrm{pCi} / \mathrm{g}$ & 0.192 & & 3/31/08 & 8C17008 & AGG-RRL-001 \\
\hline \multirow[t]{3}{*}{ 13967-76-5 } & Niobium-95 & $<0.244$ & $\mathrm{pCi} / \mathrm{g}$ & 0.244 & & 3/31/08 & 8C17008 & AGG-RRL-001 \\
\hline & Niobium-95m & $<1.06$ & $\mathrm{pCi} / \mathrm{g}$ & 1.06 & & 3/31/08 & 8C17008 & AGG-RRL-001 \\
\hline & Technetium-95m & $<0.36$ & $\mathrm{pCi} / \mathrm{g}$ & 0.36 & & 3/31/08 & 8C17008 & AGG-RRL-001 \\
\hline \multirow[t]{2}{*}{ 13967-71-0 } & Zirconium-95 & $<0.37$ & $\mathrm{pCi} / \mathrm{g}$ & 0.37 & & 3/31/08 & 8C17008 & AGG-RRL-001 \\
\hline & Technetium-99m & $<0.226$ & $\mathrm{pCi} / \mathrm{g}$ & 0.226 & & 3/31/08 & 8C17008 & AGG-RRL-001 \\
\hline 13968-53-1 & Ruthenium-103 & $<0.231$ & $\mathrm{pCi} / \mathrm{g}$ & 0.231 & & 3/31/08 & 8C17008 & AGG-RRL-001 \\
\hline 13967-48-1 & Ruthenium-106 & $<2.12$ & $\mathrm{pCi} / \mathrm{g}$ & 2.12 & & 3/31/08 & 8C17008 & AGG-RRL-001 \\
\hline 14391-65-2 & Silver-108m & $<0.221$ & $\mathrm{pCi} / \mathrm{g}$ & 0.221 & & 3/31/08 & 8C17008 & AGG-RRL-001 \\
\hline \multirow[t]{2}{*}{ 14109-32-1 } & Cadmium-109 & $<6.06$ & $\mathrm{pCi} / \mathrm{g}$ & 6.06 & & 3/31/08 & 8C17008 & AGG-RRL-001 \\
\hline & Silver-110 & $<0.249$ & $\mathrm{pCi} / \mathrm{g}$ & 0.249 & & 3/31/08 & 8C17008 & AGG-RRL-001 \\
\hline 14391-76-5 & Silver-110m & $<0.249$ & $\mathrm{pCi} / \mathrm{g}$ & 0.249 & & 3/31/08 & 8C17008 & AGG-RRL-001 \\
\hline 13966-06-8 & Tin-113 & $<0.332$ & $\mathrm{pCi} / \mathrm{g}$ & 0.332 & & 3/31/08 & 8C17008 & AGG-RRL-001 \\
\hline 14683-10-4 & Antimony-124 & $<0.219$ & $\mathrm{pCi} / \mathrm{g}$ & 0.219 & & 3/31/08 & 8C17008 & AGG-RRL-001 \\
\hline 14234-35-6 & Antimony-125 & $<0.699$ & $\mathrm{pCi} / \mathrm{g}$ & 0.699 & & 3/31/08 & 8C17008 & AGG-RRL-001 \\
\hline 15756-32-8 & Antimony-126 & $<0.232$ & $\mathrm{pCi} / \mathrm{g}$ & 0.232 & & 3/31/08 & 8C17008 & AGG-RRL-001 \\
\hline 15832-50-5 & Tin-126 & $<0.477$ & $\mathrm{pCi} / \mathrm{g}$ & 0.477 & & 3/31/08 & 8C17008 & AGG-RRL-001 \\
\hline 10043-66-0 & Iodine-131 & $<0.25$ & $\mathrm{pCi} / \mathrm{g}$ & 0.25 & & 3/31/08 & 8C17008 & AGG-RRL-001 \\
\hline 13981-41-4 & Barium-133 & $<0.401$ & $\mathrm{pCi} / \mathrm{g}$ & 0.401 & & 3/31/08 & 8C17008 & AGG-RRL-001 \\
\hline 13967-70-9 & Cesium-134 & $<0.273$ & $\mathrm{pCi} / \mathrm{g}$ & 0.273 & & 3/31/08 & 8C17008 & AGG-RRL-001 \\
\hline $10045-97-3$ & Cesium-137 & $<0.28$ & $\mathrm{pCi} / \mathrm{g}$ & 0.28 & & 3/31/08 & 8C17008 & AGG-RRL-001 \\
\hline 13982-30-4 & Cerium-139 & $<0.259$ & $\mathrm{pCi} / \mathrm{g}$ & 0.259 & & 3/31/08 & 8C17008 & AGG-RRL-001 \\
\hline 14762-78-8 & Cerium-144 & $<1.84$ & $\mathrm{pCi} / \mathrm{g}$ & 1.84 & & 3/31/08 & 8C17008 & AGG-RRL-001 \\
\hline 14683-23-9 & Europium-152 & $<0.812$ & $\mathrm{pCi} / \mathrm{g}$ & 0.812 & & 3/31/08 & 8C17008 & AGG-RRL-001 \\
\hline 14276-65-4 & Gadolinium-153 & $<0.65$ & $\mathrm{pCi} / \mathrm{g}$ & 0.65 & & 3/31/08 & 8C17008 & AGG-RRL-001 \\
\hline 15585-10-1 & Europium-154 & $<0.469$ & $\mathrm{pCi} / \mathrm{g}$ & 0.469 & & 3/31/08 & 8C17008 & AGG-RRL-001 \\
\hline 14391-16-3 & Europium-155 & $<0.671$ & $\mathrm{pCi} / \mathrm{g}$ & 0.671 & & 3/31/08 & 8C17008 & AGG-RRL-001 \\
\hline 13982-78-0 & Mercury-203 & $<0.282$ & $\mathrm{pCi} / \mathrm{g}$ & 0.282 & & 3/31/08 & 8C17008 & AGG-RRL-001 \\
\hline 14913-50-9 & Thallium-208 & $<0.218$ & $\mathrm{pCi} / \mathrm{g}$ & 0.218 & 0.0477 & 3/31/08 & 8C17008 & AGG-RRL-001 \\
\hline 14331-79-4 & Bismuth-210 & $<0.431$ & $\mathrm{pCi} / \mathrm{g}$ & 0.431 & & 3/31/08 & 8C17008 & AGG-RRL-001 \\
\hline \multirow[t]{3}{*}{$14255-04-0$} & Lead-210 & $<87.1$ & $\mathrm{pCi} / \mathrm{g}$ & 87.1 & & 3/31/08 & 8C17008 & AGG-RRL-001 \\
\hline & Bismuth-211 & $<5$ & $\mathrm{pCi} / \mathrm{g}$ & 5 & & 3/31/08 & 8C17008 & AGG-RRL-001 \\
\hline & Lead-211 & $<6.83$ & $\mathrm{pCi} / \mathrm{g}$ & 6.83 & & 3/31/08 & 8C17008 & AGG-RRL-001 \\
\hline 15092-94-1 & Lead-212 & $<0.585$ & $\mathrm{pCi} / \mathrm{g}$ & 0.585 & 0.129 & 3/31/08 & 8C17008 & AGG-RRL-001 \\
\hline 14733-03-0 & Bismuth-214 & $<0.582$ & $\mathrm{pCi} / \mathrm{g}$ & 0.582 & & 3/31/08 & 8C17008 & AGG-RRL-001 \\
\hline $15067-28-4$ & Lead-214 & $<0.638$ & $\mathrm{pCi} / \mathrm{g}$ & 0.638 & & 3/31/08 & 8C17008 & AGG-RRL-001 \\
\hline $14835-02-0$ & Radon-219 & $<2.22$ & $\mathrm{pCi} / \mathrm{g}$ & 2.22 & & 3/31/08 & 8C17008 & AGG-RRL-001 \\
\hline 22481-48-7 & Radon-220 & $<195$ & $\mathrm{pCi} / \mathrm{g}$ & 195 & & 3/31/08 & 8C17008 & AGG-RRL-001 \\
\hline 28522-20-5 & Radon-221 & $<1.06$ & $\mathrm{pCi} / \mathrm{g}$ & 1.06 & & 3/31/08 & 8C17008 & AGG-RRL-001 \\
\hline 15756-98-6 & Francium-223 & $<7.7$ & $\mathrm{pCi} / \mathrm{g}$ & 7.7 & & 3/31/08 & 8C17008 & AGG-RRL-001 \\
\hline $15623-45-7$ & Radium-223 & $<1.61$ & $\mathrm{pCi} / \mathrm{g}$ & 1.61 & & 3/31/08 & 8C17008 & AGG-RRL-001 \\
\hline 13233-32-4 & Radium-224 & $<7$ & $\mathrm{pCi} / \mathrm{g}$ & 7 & & 3/31/08 & 8C17008 & AGG-RRL-001 \\
\hline 13982-63-3 & Radium-226 & $<6.49$ & $\mathrm{pCi} / \mathrm{g}$ & 6.49 & & 3/31/08 & 8C17008 & AGG-RRL-001 \\
\hline $15623-47-9$ & Thorium-227 & $<2.36$ & $\mathrm{pCi} / \mathrm{g}$ & 2.36 & & 3/31/08 & 8C17008 & AGG-RRL-001 \\
\hline
\end{tabular}


GEA/Soil

\begin{tabular}{|c|c|c|c|c|c|c|c|c|}
\hline CAS \# & Analyte & Results & Units & MDA & UNC & Analyzed & Batch & Method \\
\hline HEIS No. & B1RTH1A & \multicolumn{2}{|c|}{ Lab ID: } & 0803012-02 & & & & \\
\hline $14331-83-0$ & Actinium-228 & $<0.975$ & $\mathrm{pCi} / \mathrm{g}$ & 0.975 & & $3 / 31 / 08$ & 8C17008 & AGG-RRL-001 \\
\hline 14274-82-9 & Thorium-228 & $<82.2$ & $\mathrm{pCi} / \mathrm{g}$ & 82.2 & & 3/31/08 & 8C17008 & AGG-RRL-001 \\
\hline 14269-63-7 & Thorium-230 & $<85$ & $\mathrm{pCi} / \mathrm{g}$ & 85 & & 3/31/08 & 8C17008 & AGG-RRL-001 \\
\hline $14331-85-2$ & Protactinium-231 & $<9.67$ & $\mathrm{pCi} / \mathrm{g}$ & 9.67 & & 3/31/08 & 8C17008 & AGG-RRL-001 \\
\hline $14932-40-2$ & Thorium-231 & $<45.9$ & $\mathrm{pCi} / \mathrm{g}$ & 45.9 & & 3/31/08 & 8C17008 & AGG-RRL-001 \\
\hline 7440-29-1 & Thorium-232 & $<273$ & $\mathrm{pCi} / \mathrm{g}$ & 273 & & $3 / 31 / 08$ & 8C17008 & AGG-RRL-001 \\
\hline 13981-14-1 & Protactinium-233 & $<0.61$ & $\mathrm{pCi} / \mathrm{g}$ & 0.61 & & 3/31/08 & 8C17008 & AGG-RRL-001 \\
\hline \multirow[t]{2}{*}{$15100-28-4$} & Protactinium-234 & $<0.828$ & $\mathrm{pCi} / \mathrm{g}$ & 0.828 & & $3 / 31 / 08$ & 8C17008 & AGG-RRL-001 \\
\hline & Protactinium-234m & $<27.4$ & $\mathrm{pCi} / \mathrm{g}$ & 27.4 & & 3/31/08 & 8C17008 & AGG-RRL-001 \\
\hline $15065-10-8$ & Thorium-234 & $<7.72$ & $\mathrm{pCi} / \mathrm{g}$ & 7.72 & & 3/31/08 & 8C17008 & AGG-RRL-001 \\
\hline 15117-96-1 & Uranium 235 & $<1.89$ & $\mathrm{pCi} / \mathrm{g}$ & 1.89 & & 3/31/08 & 8C17008 & AGG-RRL-001 \\
\hline 13994-20-2 & Neptunium-237 & $<1.75$ & $\mathrm{pCi} / \mathrm{g}$ & 1.75 & & 3/31/08 & 8C17008 & AGG-RRL-001 \\
\hline \multirow[t]{2}{*}{ 13981-16-3 } & Plutonium-238 & $<2550$ & $\mathrm{pCi} / \mathrm{g}$ & 2550 & & 3/31/08 & 8C17008 & AGG-RRL-001 \\
\hline & Uranium 238 & $<4$ & $\mathrm{pCi} / \mathrm{g}$ & 4 & & 3/31/08 & 8C17008 & AGG-RRL-001 \\
\hline $15117-48-3$ & Plutonium-239 & $<3230$ & $\mathrm{pCi} / \mathrm{g}$ & 3230 & & 3/31/08 & 8C17008 & AGG-RRL-001 \\
\hline $14119-33-6$ & Plutonium-240 & $<2690$ & $\mathrm{pCi} / \mathrm{g}$ & 2690 & & 3/31/08 & 8C17008 & AGG-RRL-001 \\
\hline $14596-10-2$ & Americium-241 & $<1.32$ & $\mathrm{pCi} / \mathrm{g}$ & 1.32 & & 3/31/08 & 8C17008 & AGG-RRL-001 \\
\hline $14993-75-0$ & Americium-243 & $<0.481$ & $\mathrm{pCi} / \mathrm{g}$ & 0.481 & & 3/31/08 & 8C17008 & AGG-RRL-001 \\
\hline 15757-87-6 & Curium-243 & $<0.819$ & $\mathrm{pCi} / \mathrm{g}$ & 0.819 & & 3/31/08 & 8C17008 & AGG-RRL-001 \\
\hline 15621-76-8 & Curium-245 & $<0.673$ & $\mathrm{pCi} / \mathrm{g}$ & 0.673 & & 3/31/08 & 8C17008 & AGG-RRL-001 \\
\hline HEIS No. & B1RTH1 & \multicolumn{2}{|c|}{ Lab ID: } & 0803012-03 & & & & \\
\hline $13966-32-0$ & Sodium-22 & $<0.321$ & $\mathrm{pCi} / \mathrm{g}$ & 0.321 & & 3/31/08 & 8C17008 & AGG-RRL-001 \\
\hline 13966-00-2 & Potassium-40 & 12.8 & $\mathrm{pCi} / \mathrm{g}$ & 3.05 & 1.05 & 3/31/08 & 8C17008 & AGG-RRL-001 \\
\hline $14392-02-0$ & Chromium-51 & $<2.93$ & $\mathrm{pCi} / \mathrm{g}$ & 2.93 & & 3/31/08 & 8C17008 & AGG-RRL-001 \\
\hline 13966-31-9 & Manganese-54 & $<0.315$ & $\mathrm{pCi} / \mathrm{g}$ & 0.315 & & 3/31/08 & 8C17008 & AGG-RRL-001 \\
\hline 13981-50-5 & Cobalt-57 & $<0.307$ & $\mathrm{pCi} / \mathrm{g}$ & 0.307 & & 3/31/08 & 8C17008 & AGG-RRL-001 \\
\hline $14596-12-4$ & Iron-59 & $<0.533$ & $\mathrm{pCi} / \mathrm{g}$ & 0.533 & & 3/31/08 & 8C17008 & AGG-RRL-001 \\
\hline 10198-40-0 & Cobalt-60 & $<0.327$ & $\mathrm{pCi} / \mathrm{g}$ & 0.327 & & 3/31/08 & 8C17008 & AGG-RRL-001 \\
\hline 13982-39-3 & Zinc-65 & $<0.672$ & $\mathrm{pCi} / \mathrm{g}$ & 0.672 & & 3/31/08 & 8C17008 & AGG-RRL-001 \\
\hline $14265-71-5$ & Selenium-75 & $<0.489$ & $\mathrm{pCi} / \mathrm{g}$ & 0.489 & & 3/31/08 & 8C17008 & AGG-RRL-001 \\
\hline 17056-36-9 & Rubidium-83 & $<0.758$ & $\mathrm{pCi} / \mathrm{g}$ & 0.758 & & 3/31/08 & 8C17008 & AGG-RRL-001 \\
\hline 13967-73-2 & Strontium-85 & $<0.349$ & $\mathrm{pCi} / \mathrm{g}$ & 0.349 & & 3/31/08 & 8C17008 & AGG-RRL-001 \\
\hline $14932-53-7$ & Rubidium-86 & $<4.04$ & $\mathrm{pCi} / \mathrm{g}$ & 4.04 & & 3/31/08 & 8C17008 & AGG-RRL-001 \\
\hline $13982-36-0$ & Yttrium-88 & $<0.273$ & $\mathrm{pCi} / \mathrm{g}$ & 0.273 & & 3/31/08 & 8C17008 & AGG-RRL-001 \\
\hline 14681-63-1 & Niobium-94 & $<0.304$ & $\mathrm{pCi} / \mathrm{g}$ & 0.304 & & 3/31/08 & 8C17008 & AGG-RRL-001 \\
\hline \multirow[t]{3}{*}{ 13967-76-5 } & Niobium-95 & $<0.31$ & $\mathrm{pCi} / \mathrm{g}$ & 0.31 & & 3/31/08 & 8C17008 & AGG-RRL-001 \\
\hline & Niobium-95m & $<1.37$ & $\mathrm{pCi} / \mathrm{g}$ & 1.37 & & 3/31/08 & 8C17008 & AGG-RRL-001 \\
\hline & Technetium-95m & $<0.481$ & $\mathrm{pCi} / \mathrm{g}$ & 0.481 & & 3/31/08 & 8C17008 & AGG-RRL-001 \\
\hline \multirow[t]{2}{*}{ 13967-71-0 } & Zirconium-95 & $<0.529$ & $\mathrm{pCi} / \mathrm{g}$ & 0.529 & & 3/31/08 & 8C17008 & AGG-RRL-001 \\
\hline & Technetium-99m & $<0.31$ & $\mathrm{pCi} / \mathrm{g}$ & 0.31 & & 3/31/08 & 8C17008 & AGG-RRL-001 \\
\hline 13968-53-1 & Ruthenium-103 & $<0.36$ & $\mathrm{pCi} / \mathrm{g}$ & 0.36 & & 3/31/08 & 8C17008 & AGG-RRL-001 \\
\hline 13967-48-1 & Ruthenium-106 & $<3.29$ & $\mathrm{pCi} / \mathrm{g}$ & 3.29 & & 3/31/08 & 8C17008 & AGG-RRL-001 \\
\hline $14391-65-2$ & Silver-108m & $<0.312$ & $\mathrm{pCi} / \mathrm{g}$ & 0.312 & & 3/31/08 & 8C17008 & AGG-RRL-001 \\
\hline \multirow[t]{2}{*}{ 14109-32-1 } & Cadmium-109 & $<8.8$ & $\mathrm{pCi} / \mathrm{g}$ & 8.8 & & 3/31/08 & 8C17008 & AGG-RRL-001 \\
\hline & Silver-110 & $<0.294$ & $\mathrm{pCi} / \mathrm{g}$ & 0.294 & & 3/31/08 & 8C17008 & AGG-RRL-001 \\
\hline 14391-76-5 & Silver-110m & $<0.295$ & $\mathrm{pCi} / \mathrm{g}$ & 0.295 & & 3/31/08 & 8C17008 & AGG-RRL-001 \\
\hline 13966-06-8 & Tin-113 & $<0.432$ & $\mathrm{pCi} / \mathrm{g}$ & 0.432 & & 3/31/08 & 8C17008 & AGG-RRL-001 \\
\hline $14683-10-4$ & Antimony-124 & $<0.336$ & $\mathrm{pCi} / \mathrm{g}$ & 0.336 & & 3/31/08 & 8C17008 & AGG-RRL-001 \\
\hline
\end{tabular}


GEA/Soil

\begin{tabular}{|c|c|c|c|c|c|c|c|c|}
\hline CAS \# & Analyte & Results & Units & MDA & UNC & Analyzed & Batch & Method \\
\hline HEIS No. & B1RTH1 & \multicolumn{3}{|c|}{ Lab ID: $\quad$ 0803012-03 } & & & & \\
\hline $14234-35-6$ & Antimony-125 & $<0.947$ & $\mathrm{pCi} / \mathrm{g}$ & 0.947 & & $3 / 31 / 08$ & 8C17008 & AGG-RRL-001 \\
\hline $15756-32-8$ & Antimony-126 & $<0.279$ & $\mathrm{pCi} / \mathrm{g}$ & 0.279 & & 3/31/08 & 8C17008 & AGG-RRL-001 \\
\hline $15832-50-5$ & Tin-126 & $<0.717$ & $\mathrm{pCi} / \mathrm{g}$ & 0.717 & & $3 / 31 / 08$ & 8C17008 & AGG-RRL-001 \\
\hline $10043-66-0$ & Iodine-131 & $<0.372$ & $\mathrm{pCi} / \mathrm{g}$ & 0.372 & & 3/31/08 & 8C17008 & AGG-RRL-001 \\
\hline $13981-41-4$ & Barium-133 & $<0.517$ & $\mathrm{pCi} / \mathrm{g}$ & 0.517 & & 3/31/08 & 8C17008 & AGG-RRL-001 \\
\hline 13967-70-9 & Cesium-134 & $<0.333$ & $\mathrm{pCi} / \mathrm{g}$ & 0.333 & & 3/31/08 & 8C17008 & AGG-RRL-001 \\
\hline $10045-97-3$ & Cesium-137 & $<0.327$ & $\mathrm{pCi} / \mathrm{g}$ & 0.327 & & $3 / 31 / 08$ & 8C17008 & AGG-RRL-001 \\
\hline 13982-30-4 & Cerium-139 & $<0.354$ & $\mathrm{pCi} / \mathrm{g}$ & 0.354 & & $3 / 31 / 08$ & 8C17008 & AGG-RRL-001 \\
\hline $14762-78-8$ & Cerium-144 & $<2.51$ & $\mathrm{pCi} / \mathrm{g}$ & 2.51 & & $3 / 31 / 08$ & 8C17008 & AGG-RRL-001 \\
\hline 14683-23-9 & Europium-152 & $<1.23$ & $\mathrm{pCi} / \mathrm{g}$ & 1.23 & & 3/31/08 & 8C17008 & AGG-RRL-001 \\
\hline 14276-65-4 & Gadolinium-153 & $<0.966$ & $\mathrm{pCi} / \mathrm{g}$ & 0.966 & & $3 / 31 / 08$ & 8C17008 & AGG-RRL-001 \\
\hline $15585-10-1$ & Europium-154 & $<0.651$ & $\mathrm{pCi} / \mathrm{g}$ & 0.651 & & $3 / 31 / 08$ & 8C17008 & AGG-RRL-001 \\
\hline $14391-16-3$ & Europium-155 & $<1.03$ & $\mathrm{pCi} / \mathrm{g}$ & 1.03 & & 3/31/08 & 8C17008 & AGG-RRL-001 \\
\hline 13982-78-0 & Mercury-203 & $<0.372$ & $\mathrm{pCi} / \mathrm{g}$ & 0.372 & & $3 / 31 / 08$ & 8C17008 & AGG-RRL-001 \\
\hline $14913-50-9$ & Thallium-208 & $<0.386$ & $\mathrm{pCi} / \mathrm{g}$ & 0.386 & & 3/31/08 & 8C17008 & AGG-RRL-001 \\
\hline 14331-79-4 & Bismuth-210 & $<0.584$ & $\mathrm{pCi} / \mathrm{g}$ & 0.584 & & $3 / 31 / 08$ & 8C17008 & AGG-RRL-001 \\
\hline \multirow[t]{3}{*}{ 14255-04-0 } & Lead-210 & $<42.4$ & $\mathrm{pCi} / \mathrm{g}$ & 42.4 & & 3/31/08 & 8C17008 & AGG-RRL-001 \\
\hline & Bismuth-211 & $<6.95$ & $\mathrm{pCi} / \mathrm{g}$ & 6.95 & & $3 / 31 / 08$ & 8C17008 & AGG-RRL-001 \\
\hline & Lead-211 & $<9.5$ & $\mathrm{pCi} / \mathrm{g}$ & 9.5 & & $3 / 31 / 08$ & 8C17008 & AGG-RRL-001 \\
\hline $15092-94-1$ & Lead-212 & $<0.799$ & $\mathrm{pCi} / \mathrm{g}$ & 0.799 & & $3 / 31 / 08$ & 8C17008 & AGG-RRL-001 \\
\hline $14733-03-0$ & Bismuth-214 & $<0.737$ & $\mathrm{pCi} / \mathrm{g}$ & 0.737 & & 3/31/08 & 8C17008 & AGG-RRL-001 \\
\hline $15067-28-4$ & Lead-214 & $<0.848$ & $\mathrm{pCi} / \mathrm{g}$ & 0.848 & & $3 / 31 / 08$ & 8C17008 & AGG-RRL-001 \\
\hline $14835-02-0$ & Radon-219 & $<2.96$ & pCi/g & 2.96 & & $3 / 31 / 08$ & 8C17008 & AGG-RRL-001 \\
\hline 22481-48-7 & Radon-220 & $<283$ & $\mathrm{pCi} / \mathrm{g}$ & 283 & & 3/31/08 & 8C17008 & AGG-RRL-001 \\
\hline 28522-20-5 & Radon-221 & $<1.44$ & $\mathrm{pCi} / \mathrm{g}$ & 1.44 & & $3 / 31 / 08$ & 8C17008 & AGG-RRL-001 \\
\hline 15756-98-6 & Francium-223 & $<10.1$ & $\mathrm{pCi} / \mathrm{g}$ & 10.1 & & $3 / 31 / 08$ & 8C17008 & AGG-RRL-001 \\
\hline $15623-45-7$ & Radium-223 & $<2.14$ & $\mathrm{pCi} / \mathrm{g}$ & 2.14 & & $3 / 31 / 08$ & 8C17008 & AGG-RRL-001 \\
\hline 13233-32-4 & Radium-224 & $<8.57$ & $\mathrm{pCi} / \mathrm{g}$ & 8.57 & & $3 / 31 / 08$ & 8C17008 & AGG-RRL-001 \\
\hline 13982-63-3 & Radium-226 & $<8.88$ & $\mathrm{pCi} / \mathrm{g}$ & 8.88 & & 3/31/08 & 8C17008 & AGG-RRL-001 \\
\hline $15623-47-9$ & Thorium-227 & $<3.05$ & $\mathrm{pCi} / \mathrm{g}$ & 3.05 & & $3 / 31 / 08$ & 8C17008 & AGG-RRL-001 \\
\hline 14331-83-0 & Actinium-228 & $<1.24$ & $\mathrm{pCi} / \mathrm{g}$ & 1.24 & & $3 / 31 / 08$ & 8C17008 & AGG-RRL-001 \\
\hline 14274-82-9 & Thorium-228 & $<112$ & $\mathrm{pCi} / \mathrm{g}$ & 112 & & 3/31/08 & 8C17008 & AGG-RRL-001 \\
\hline $14269-63-7$ & Thorium-230 & $<117$ & $\mathrm{pCi} / \mathrm{g}$ & 117 & & $3 / 31 / 08$ & 8C17008 & AGG-RRL-001 \\
\hline 14331-85-2 & Protactinium-231 & $<13.2$ & $\mathrm{pCi} / \mathrm{g}$ & 13.2 & & 3/31/08 & 8C17008 & AGG-RRL-001 \\
\hline $14932-40-2$ & Thorium-231 & $<68.7$ & $\mathrm{pCi} / \mathrm{g}$ & 68.7 & & 3/31/08 & 8C17008 & AGG-RRL-001 \\
\hline 7440-29-1 & Thorium-232 & $<294$ & $\mathrm{pCi} / \mathrm{g}$ & 294 & & 3/31/08 & 8C17008 & AGG-RRL-001 \\
\hline 13981-14-1 & Protactinium-233 & $<0.809$ & $\mathrm{pCi} / \mathrm{g}$ & 0.809 & & $3 / 31 / 08$ & 8C17008 & AGG-RRL-001 \\
\hline \multirow[t]{2}{*}{$15100-28-4$} & Protactinium-234 & $<1.26$ & $\mathrm{pCi} / \mathrm{g}$ & 1.26 & & $3 / 31 / 08$ & 8C17008 & AGG-RRL-001 \\
\hline & Protactinium-234m & $<37.7$ & $\mathrm{pCi} / \mathrm{g}$ & 37.7 & & $3 / 31 / 08$ & 8C17008 & AGG-RRL-001 \\
\hline $15065-10-8$ & Thorium-234 & $<10.3$ & $\mathrm{pCi} / \mathrm{g}$ & 10.3 & & $3 / 31 / 08$ & 8C17008 & AGG-RRL-001 \\
\hline 15117-96-1 & Uranium 235 & $<2.66$ & $\mathrm{pCi} / \mathrm{g}$ & 2.66 & & $3 / 31 / 08$ & 8C17008 & AGG-RRL-001 \\
\hline 13994-20-2 & Neptunium-237 & $<2.67$ & pCi/g & 2.67 & & $3 / 31 / 08$ & 8C17008 & AGG-RRL-001 \\
\hline \multirow[t]{2}{*}{ 13981-16-3 } & Plutonium-238 & $<3960$ & $\mathrm{pCi} / \mathrm{g}$ & 3960 & & 3/31/08 & 8C17008 & AGG-RRL-001 \\
\hline & Uranium 238 & $<5.62$ & $\mathrm{pCi} / \mathrm{g}$ & 5.62 & & $3 / 31 / 08$ & 8C17008 & AGG-RRL-001 \\
\hline $15117-48-3$ & Plutonium-239 & $<4000$ & $\mathrm{pCi} / \mathrm{g}$ & 4000 & & 3/31/08 & 8C17008 & AGG-RRL-001 \\
\hline 14119-33-6 & Plutonium-240 & $<3930$ & $\mathrm{pCi} / \mathrm{g}$ & 3930 & & $3 / 31 / 08$ & 8C17008 & AGG-RRL-001 \\
\hline $14596-10-2$ & Americium-241 & $<1.5$ & $\mathrm{pCi} / \mathrm{g}$ & 1.5 & & $3 / 31 / 08$ & 8C17008 & AGG-RRL-001 \\
\hline $14993-75-0$ & Americium-243 & $<0.65$ & $\mathrm{pCi} / \mathrm{g}$ & 0.65 & & $3 / 31 / 08$ & 8C17008 & AGG-RRL-001 \\
\hline $15757-87-6$ & Curium-243 & $<1.2$ & $\mathrm{pCi} / \mathrm{g}$ & 1.2 & & $3 / 31 / 08$ & 8C17008 & AGG-RRL-001 \\
\hline
\end{tabular}


GEA/Soil

\begin{tabular}{|c|c|c|c|c|c|c|c|c|}
\hline CAS \# & Analyte & Results & Units & MDA & UNC & Analyzed & Batch & Method \\
\hline HEIS No. & B1RTH1 & \multicolumn{2}{|c|}{ Lab ID: } & \multicolumn{2}{|l|}{ 0803012-03 } & & & \\
\hline 15621-76-8 & Curium-245 & $<0.986$ & $\mathrm{pCi} / \mathrm{g}$ & 0.986 & & 3/31/08 & 8C17008 & AGG-RRL-001 \\
\hline HEIS No. & B1RTH2B & \multicolumn{2}{|c|}{ Lab ID: } & 0803012-05 & & & & \\
\hline 13966-32-0 & Sodium-22 & $<0.398$ & $\mathrm{pCi} / \mathrm{g}$ & 0.398 & & 3/31/08 & 8C17008 & AGG-RRL-001 \\
\hline 13966-00-2 & Potassium-40 & 19.4 & $\mathrm{pCi} / \mathrm{g}$ & 2.39 & 1.22 & 3/31/08 & 8C17008 & AGG-RRL-001 \\
\hline $14392-02-0$ & Chromium-51 & $<3.39$ & $\mathrm{pCi} / \mathrm{g}$ & 3.39 & & 3/31/08 & 8C17008 & AGG-RRL-001 \\
\hline 13966-31-9 & Manganese-54 & $<0.349$ & $\mathrm{pCi} / \mathrm{g}$ & 0.349 & & 3/31/08 & 8C17008 & AGG-RRL-001 \\
\hline 13981-50-5 & Cobalt-57 & $<0.35$ & $\mathrm{pCi} / \mathrm{g}$ & 0.35 & & 3/31/08 & 8C17008 & AGG-RRL-001 \\
\hline 14596-12-4 & Iron-59 & $<0.653$ & $\mathrm{pCi} / \mathrm{g}$ & 0.653 & & 3/31/08 & 8C17008 & AGG-RRL-001 \\
\hline $10198-40-0$ & Cobalt-60 & $<0.357$ & $\mathrm{pCi} / \mathrm{g}$ & 0.357 & & 3/31/08 & 8C17008 & AGG-RRL-001 \\
\hline 13982-39-3 & Zinc-65 & $<0.823$ & $\mathrm{pCi} / \mathrm{g}$ & 0.823 & & 3/31/08 & 8C17008 & AGG-RRL-001 \\
\hline 14265-71-5 & Selenium-75 & $<0.556$ & $\mathrm{pCi} / \mathrm{g}$ & 0.556 & & 3/31/08 & 8C17008 & AGG-RRL-001 \\
\hline 17056-36-9 & Rubidium-83 & $<0.898$ & $\mathrm{pCi} / \mathrm{g}$ & 0.898 & & 3/31/08 & 8C17008 & AGG-RRL-001 \\
\hline 13967-73-2 & Strontium-85 & $<0.418$ & $\mathrm{pCi} / \mathrm{g}$ & 0.418 & & 3/31/08 & 8C17008 & AGG-RRL-001 \\
\hline $14932-53-7$ & Rubidium-86 & $<4.79$ & $\mathrm{pCi} / \mathrm{g}$ & 4.79 & & $3 / 31 / 08$ & 8C17008 & AGG-RRL-001 \\
\hline $13982-36-0$ & Yttrium-88 & $<0.311$ & $\mathrm{pCi} / \mathrm{g}$ & 0.311 & & 3/31/08 & 8C17008 & AGG-RRL-001 \\
\hline 14681-63-1 & Niobium-94 & $<0.353$ & $\mathrm{pCi} / \mathrm{g}$ & 0.353 & & 3/31/08 & 8C17008 & AGG-RRL-001 \\
\hline \multirow{3}{*}{ 13967-76-5 } & Niobium-95 & $<0.34$ & $\mathrm{pCi} / \mathrm{g}$ & 0.34 & & 3/31/08 & 8C17008 & AGG-RRL-001 \\
\hline & Niobium-95m & $<1.56$ & $\mathrm{pCi} / \mathrm{g}$ & 1.56 & & 3/31/08 & 8C17008 & AGG-RRL-001 \\
\hline & Technetium-95m & $<0.547$ & $\mathrm{pCi} / \mathrm{g}$ & 0.547 & & 3/31/08 & 8C17008 & AGG-RRL-001 \\
\hline \multirow[t]{2}{*}{ 13967-71-0 } & Zirconium-95 & $<0.607$ & $\mathrm{pCi} / \mathrm{g}$ & 0.607 & & 3/31/08 & 8C17008 & AGG-RRL-001 \\
\hline & Technetium-99m & $<0.354$ & $\mathrm{pCi} / \mathrm{g}$ & 0.354 & & 3/31/08 & 8C17008 & AGG-RRL-001 \\
\hline 13968-53-1 & Ruthenium-103 & $<0.415$ & $\mathrm{pCi} / \mathrm{g}$ & 0.415 & & 3/31/08 & 8C17008 & AGG-RRL-001 \\
\hline 13967-48-1 & Ruthenium-106 & $<3.92$ & $\mathrm{pCi} / \mathrm{g}$ & 3.92 & & 3/31/08 & 8C17008 & AGG-RRL-001 \\
\hline 14391-65-2 & Silver-108m & $<0.344$ & $\mathrm{pCi} / \mathrm{g}$ & 0.344 & & 3/31/08 & 8C17008 & AGG-RRL-001 \\
\hline \multirow[t]{2}{*}{$14109-32-1$} & Cadmium-109 & $<9.97$ & $\mathrm{pCi} / \mathrm{g}$ & 9.97 & & 3/31/08 & 8C17008 & AGG-RRL-001 \\
\hline & Silver-110 & $<0.356$ & $\mathrm{pCi} / \mathrm{g}$ & 0.356 & & 3/31/08 & 8C17008 & AGG-RRL-001 \\
\hline 14391-76-5 & Silver-110m & $<0.356$ & $\mathrm{pCi} / \mathrm{g}$ & 0.356 & & $3 / 31 / 08$ & 8C17008 & AGG-RRL-001 \\
\hline 13966-06-8 & Tin-113 & $<0.507$ & $\mathrm{pCi} / \mathrm{g}$ & 0.507 & & 3/31/08 & 8C17008 & AGG-RRL-001 \\
\hline $14683-10-4$ & Antimony-124 & $<0.417$ & $\mathrm{pCi} / \mathrm{g}$ & 0.417 & & 3/31/08 & 8C17008 & AGG-RRL-001 \\
\hline $14234-35-6$ & Antimony-125 & $<1.1$ & $\mathrm{pCi} / \mathrm{g}$ & 1.1 & & 3/31/08 & 8C17008 & AGG-RRL-001 \\
\hline 15756-32-8 & Antimony-126 & $<0.344$ & $\mathrm{pCi} / \mathrm{g}$ & 0.344 & & 3/31/08 & 8C17008 & AGG-RRL-001 \\
\hline $15832-50-5$ & Tin-126 & $<0.814$ & $\mathrm{pCi} / \mathrm{g}$ & 0.814 & & $3 / 31 / 08$ & 8C17008 & AGG-RRL-001 \\
\hline 10043-66-0 & Iodine-131 & $<0.442$ & $\mathrm{pCi} / \mathrm{g}$ & 0.442 & & 3/31/08 & 8C17008 & AGG-RRL-001 \\
\hline 13981-41-4 & Barium-133 & $<0.612$ & $\mathrm{pCi} / \mathrm{g}$ & 0.612 & & 3/31/08 & 8C17008 & AGG-RRL-001 \\
\hline 13967-70-9 & Cesium-134 & $<0.419$ & $\mathrm{pCi} / \mathrm{g}$ & 0.419 & & $3 / 31 / 08$ & 8C17008 & AGG-RRL-001 \\
\hline $10045-97-3$ & Cesium-137 & $<0.397$ & $\mathrm{pCi} / \mathrm{g}$ & 0.397 & & 3/31/08 & 8C17008 & AGG-RRL-001 \\
\hline 13982-30-4 & Cerium-139 & $<0.408$ & $\mathrm{pCi} / \mathrm{g}$ & 0.408 & & 3/31/08 & 8C17008 & AGG-RRL-001 \\
\hline 14762-78-8 & Cerium-144 & $<2.82$ & $\mathrm{pCi} / \mathrm{g}$ & 2.82 & & 3/31/08 & 8C17008 & AGG-RRL-001 \\
\hline 14683-23-9 & Europium-152 & $<1.45$ & $\mathrm{pCi} / \mathrm{g}$ & 1.45 & & 3/31/08 & 8C17008 & AGG-RRL-001 \\
\hline 14276-65-4 & Gadolinium-153 & $<1.12$ & $\mathrm{pCi} / \mathrm{g}$ & 1.12 & & 3/31/08 & 8C17008 & AGG-RRL-001 \\
\hline 15585-10-1 & Europium-154 & $<0.738$ & $\mathrm{pCi} / \mathrm{g}$ & 0.738 & & 3/31/08 & 8C17008 & AGG-RRL-001 \\
\hline $14391-16-3$ & Europium-155 & $<1.15$ & $\mathrm{pCi} / \mathrm{g}$ & 1.15 & & 3/31/08 & 8C17008 & AGG-RRL-001 \\
\hline 13982-78-0 & Mercury-203 & $<0.438$ & $\mathrm{pCi} / \mathrm{g}$ & 0.438 & & 3/31/08 & 8C17008 & AGG-RRL-001 \\
\hline $14913-50-9$ & Thallium-208 & $<0.453$ & $\mathrm{pCi} / \mathrm{g}$ & 0.453 & & 3/31/08 & 8C17008 & AGG-RRL-001 \\
\hline 14331-79-4 & Bismuth-210 & $<0.666$ & $\mathrm{pCi} / \mathrm{g}$ & 0.666 & & 3/31/08 & 8C17008 & AGG-RRL-001 \\
\hline \multirow[t]{3}{*}{$14255-04-0$} & Lead-210 & $<48.8$ & $\mathrm{pCi} / \mathrm{g}$ & 48.8 & & 3/31/08 & 8C17008 & AGG-RRL-001 \\
\hline & Bismuth-211 & $<7.9$ & $\mathrm{pCi} / \mathrm{g}$ & 7.9 & & 3/31/08 & 8C17008 & AGG-RRL-001 \\
\hline & Lead-211 & $<10.8$ & $\mathrm{pCi} / \mathrm{g}$ & 10.8 & & 3/31/08 & 8C17008 & AGG-RRL-001 \\
\hline
\end{tabular}


GEA/Soil

\begin{tabular}{|c|c|c|c|c|c|c|c|c|}
\hline CAS \# & Analyte & Results & Units & MDA & UNC & Analyzed & Batch & Method \\
\hline HEIS No. & B1RTH2B & \multicolumn{2}{|c|}{ Lab ID: } & 0803012-05 & & & & \\
\hline 15092-94-1 & Lead-212 & $<0.906$ & $\mathrm{pCi} / \mathrm{g}$ & 0.906 & & 3/31/08 & 8C17008 & AGG-RRL-001 \\
\hline 14733-03-0 & Bismuth-214 & $<0.898$ & $\mathrm{pCi} / \mathrm{g}$ & 0.898 & & $3 / 31 / 08$ & 8C17008 & AGG-RRL-001 \\
\hline $15067-28-4$ & Lead-214 & $<0.987$ & $\mathrm{pCi} / \mathrm{g}$ & 0.987 & & 3/31/08 & 8C17008 & AGG-RRL-001 \\
\hline $14835-02-0$ & Radon-219 & $<3.44$ & $\mathrm{pCi} / \mathrm{g}$ & 3.44 & & 3/31/08 & 8C17008 & AGG-RRL-001 \\
\hline 22481-48-7 & Radon-220 & $<315$ & $\mathrm{pCi} / \mathrm{g}$ & 315 & & 3/31/08 & 8C17008 & AGG-RRL-001 \\
\hline 28522-20-5 & Radon-221 & $<1.59$ & $\mathrm{pCi} / \mathrm{g}$ & 1.59 & & 3/31/08 & 8C17008 & AGG-RRL-001 \\
\hline 15756-98-6 & Francium-223 & $<11.5$ & $\mathrm{pCi} / \mathrm{g}$ & 11.5 & & 3/31/08 & 8C17008 & AGG-RRL-001 \\
\hline 15623-45-7 & Radium-223 & $<2.51$ & $\mathrm{pCi} / \mathrm{g}$ & 2.51 & & 3/31/08 & 8C17008 & AGG-RRL-001 \\
\hline 13233-32-4 & Radium-224 & $<9.77$ & $\mathrm{pCi} / \mathrm{g}$ & 9.77 & & 3/31/08 & 8C17008 & AGG-RRL-001 \\
\hline $13982-63-3$ & Radium-226 & $<9.79$ & $\mathrm{pCi} / \mathrm{g}$ & 9.79 & & 3/31/08 & 8C17008 & AGG-RRL-001 \\
\hline 15623-47-9 & Thorium-227 & $<3.49$ & $\mathrm{pCi} / \mathrm{g}$ & 3.49 & & 3/31/08 & 8C17008 & AGG-RRL-001 \\
\hline $14331-83-0$ & Actinium-228 & $<1.55$ & $\mathrm{pCi} / \mathrm{g}$ & 1.55 & & 3/31/08 & 8C17008 & AGG-RRL-001 \\
\hline $14274-82-9$ & Thorium-228 & $<127$ & $\mathrm{pCi} / \mathrm{g}$ & 127 & & 3/31/08 & 8C17008 & AGG-RRL-001 \\
\hline $14269-63-7$ & Thorium-230 & $<134$ & $\mathrm{pCi} / \mathrm{g}$ & 134 & & 3/31/08 & 8C17008 & AGG-RRL-001 \\
\hline 14331-85-2 & Protactinium-231 & $<15.7$ & $\mathrm{pCi} / \mathrm{g}$ & 15.7 & & 3/31/08 & 8C17008 & AGG-RRL-001 \\
\hline $14932-40-2$ & Thorium-231 & $<79.7$ & $\mathrm{pCi} / \mathrm{g}$ & 79.7 & & 3/31/08 & 8C17008 & AGG-RRL-001 \\
\hline 7440-29-1 & Thorium-232 & $<343$ & $\mathrm{pCi} / \mathrm{g}$ & 343 & & 3/31/08 & 8C17008 & AGG-RRL-001 \\
\hline 13981-14-1 & Protactinium-233 & $<0.927$ & $\mathrm{pCi} / \mathrm{g}$ & 0.927 & & 3/31/08 & 8C17008 & AGG-RRL-001 \\
\hline \multirow[t]{2}{*}{ 15100-28-4 } & Protactinium-234 & $<1.46$ & $\mathrm{pCi} / \mathrm{g}$ & 1.46 & & $3 / 31 / 08$ & 8C17008 & AGG-RRL-001 \\
\hline & Protactinium-234m & $<43.7$ & $\mathrm{pCi} / \mathrm{g}$ & 43.7 & & 3/31/08 & 8C17008 & AGG-RRL-001 \\
\hline $15065-10-8$ & Thorium-234 & $<11.9$ & $\mathrm{pCi} / \mathrm{g}$ & 11.9 & & 3/31/08 & 8C17008 & AGG-RRL-001 \\
\hline 15117-96-1 & Uranium 235 & $<3.01$ & $\mathrm{pCi} / \mathrm{g}$ & 3.01 & & 3/31/08 & 8C17008 & AGG-RRL-001 \\
\hline 13994-20-2 & Neptunium-237 & $<2.99$ & $\mathrm{pCi} / \mathrm{g}$ & 2.99 & & 3/31/08 & 8C17008 & AGG-RRL-001 \\
\hline \multirow[t]{2}{*}{ 13981-16-3 } & Plutonium-238 & $<4590$ & $\mathrm{pCi} / \mathrm{g}$ & 4590 & & 3/31/08 & 8C17008 & AGG-RRL-001 \\
\hline & Uranium 238 & $<6.39$ & $\mathrm{pCi} / \mathrm{g}$ & 6.39 & & 3/31/08 & 8C17008 & AGG-RRL-001 \\
\hline $15117-48-3$ & Plutonium-239 & $<4750$ & $\mathrm{pCi} / \mathrm{g}$ & 4750 & & $3 / 31 / 08$ & 8C17008 & AGG-RRL-001 \\
\hline 14119-33-6 & Plutonium-240 & $<4580$ & $\mathrm{pCi} / \mathrm{g}$ & 4580 & & 3/31/08 & 8C17008 & AGG-RRL-001 \\
\hline 14596-10-2 & Americium-241 & $<1.76$ & $\mathrm{pCi} / \mathrm{g}$ & 1.76 & & 3/31/08 & 8C17008 & AGG-RRL-001 \\
\hline 14993-75-0 & Americium-243 & $<0.726$ & $\mathrm{pCi} / \mathrm{g}$ & 0.726 & & 3/31/08 & 8C17008 & AGG-RRL-001 \\
\hline 15757-87-6 & Curium-243 & $<1.4$ & $\mathrm{pCi} / \mathrm{g}$ & 1.4 & & 3/31/08 & 8C17008 & AGG-RRL-001 \\
\hline 15621-76-8 & Curium-245 & $<1.15$ & $\mathrm{pCi} / \mathrm{g}$ & 1.15 & & $3 / 31 / 08$ & 8C17008 & AGG-RRL-001 \\
\hline HEIS No. & B1RTH2A & \multicolumn{2}{|c|}{ Lab ID: } & 0803012-06 & & & & \\
\hline $13966-32-0$ & Sodium-22 & $<0.37$ & $\mathrm{pCi} / \mathrm{g}$ & 0.37 & & 3/31/08 & 8C17008 & AGG-RRL-001 \\
\hline 13966-00-2 & Potassium-40 & 18.5 & $\mathrm{pCi} / \mathrm{g}$ & 3.19 & 1.26 & 3/31/08 & 8C17008 & AGG-RRL-001 \\
\hline $14392-02-0$ & Chromium-51 & $<2.6$ & $\mathrm{pCi} / \mathrm{g}$ & 2.6 & & 3/31/08 & 8C17008 & AGG-RRL-001 \\
\hline 13966-31-9 & Manganese-54 & $<0.289$ & $\mathrm{pCi} / \mathrm{g}$ & 0.289 & & 3/31/08 & 8C17008 & AGG-RRL-001 \\
\hline 13981-50-5 & Cobalt-57 & $<0.293$ & $\mathrm{pCi} / \mathrm{g}$ & 0.293 & & 3/31/08 & 8C17008 & AGG-RRL-001 \\
\hline $14596-12-4$ & Iron-59 & $<0.589$ & $\mathrm{pCi} / \mathrm{g}$ & 0.589 & & 3/31/08 & 8C17008 & AGG-RRL-001 \\
\hline $10198-40-0$ & Cobalt-60 & $<0.299$ & $\mathrm{pCi} / \mathrm{g}$ & 0.299 & & 3/31/08 & 8C17008 & AGG-RRL-001 \\
\hline 13982-39-3 & Zinc-65 & $<0.649$ & $\mathrm{pCi} / \mathrm{g}$ & 0.649 & & 3/31/08 & 8C17008 & AGG-RRL-001 \\
\hline 14265-71-5 & Selenium-75 & $<0.472$ & $\mathrm{pCi} / \mathrm{g}$ & 0.472 & & 3/31/08 & 8C17008 & AGG-RRL-001 \\
\hline 17056-36-9 & Rubidium-83 & $<0.553$ & $\mathrm{pCi} / \mathrm{g}$ & 0.553 & & 3/31/08 & 8C17008 & AGG-RRL-001 \\
\hline 13967-73-2 & Strontium-85 & $<0.357$ & $\mathrm{pCi} / \mathrm{g}$ & 0.357 & & 3/31/08 & 8C17008 & AGG-RRL-001 \\
\hline 14932-53-7 & Rubidium-86 & $<3.07$ & $\mathrm{pCi} / \mathrm{g}$ & 3.07 & & 3/31/08 & 8C17008 & AGG-RRL-001 \\
\hline $13982-36-0$ & Yttrium-88 & $<0.224$ & $\mathrm{pCi} / \mathrm{g}$ & 0.224 & & 3/31/08 & 8C17008 & AGG-RRL-001 \\
\hline 14681-63-1 & Niobium-94 & $<0.285$ & $\mathrm{pCi} / \mathrm{g}$ & 0.285 & & 3/31/08 & 8C17008 & AGG-RRL-001 \\
\hline \multirow[t]{2}{*}{ 13967-76-5 } & Niobium-95 & $<0.283$ & $\mathrm{pCi} / \mathrm{g}$ & 0.283 & & 3/31/08 & 8C17008 & AGG-RRL-001 \\
\hline & Niobium-95m & $<1.31$ & $\mathrm{pCi} / \mathrm{g}$ & 1.31 & & 3/31/08 & 8C17008 & AGG-RRL-001 \\
\hline
\end{tabular}


GEA/Soil

\begin{tabular}{|c|c|c|c|c|c|c|c|c|}
\hline CAS \# & Analyte & Results & Units & MDA & UNC & Analyzed & Batch & Method \\
\hline \multirow[t]{2}{*}{ HEIS No. } & B1RTH2A & \multicolumn{2}{|c|}{ Lab ID: } & 0803012-06 & & & & \\
\hline & Technetium-95m & $<0.458$ & $\mathrm{pCi} / \mathrm{g}$ & 0.458 & & 3/31/08 & 8C17008 & AGG-RRL-001 \\
\hline \multirow[t]{2}{*}{ 13967-71-0 } & Zirconium-95 & $<0.479$ & $\mathrm{pCi} / \mathrm{g}$ & 0.479 & & 3/31/08 & 8C17008 & AGG-RRL-001 \\
\hline & Technetium-99m & $<0.276$ & $\mathrm{pCi} / \mathrm{g}$ & 0.276 & & 3/31/08 & 8C17008 & AGG-RRL-001 \\
\hline 13968-53-1 & Ruthenium-103 & $<0.309$ & $\mathrm{pCi} / \mathrm{g}$ & 0.309 & & 3/31/08 & 8C17008 & AGG-RRL-001 \\
\hline 13967-48-1 & Ruthenium-106 & $<2.66$ & $\mathrm{pCi} / \mathrm{g}$ & 2.66 & & 3/31/08 & 8C17008 & AGG-RRL-001 \\
\hline 14391-65-2 & Silver-108m & $<0.278$ & $\mathrm{pCi} / \mathrm{g}$ & 0.278 & & 3/31/08 & 8C17008 & AGG-RRL-001 \\
\hline \multirow[t]{2}{*}{ 14109-32-1 } & Cadmium-109 & $<7.67$ & $\mathrm{pCi} / \mathrm{g}$ & 7.67 & & 3/31/08 & 8C17008 & AGG-RRL-001 \\
\hline & Silver-110 & $<0.303$ & $\mathrm{pCi} / \mathrm{g}$ & 0.303 & & 3/31/08 & 8C17008 & AGG-RRL-001 \\
\hline 14391-76-5 & Silver-110m & $<0.304$ & $\mathrm{pCi} / \mathrm{g}$ & 0.304 & & 3/31/08 & 8C17008 & AGG-RRL-001 \\
\hline 13966-06-8 & Tin-113 & $<0.433$ & $\mathrm{pCi} / \mathrm{g}$ & 0.433 & & 3/31/08 & 8C17008 & AGG-RRL-001 \\
\hline $14683-10-4$ & Antimony-124 & $<0.318$ & $\mathrm{pCi} / \mathrm{g}$ & 0.318 & & 3/31/08 & 8C17008 & AGG-RRL-001 \\
\hline $14234-35-6$ & Antimony-125 & $<0.894$ & $\mathrm{pCi} / \mathrm{g}$ & 0.894 & & 3/31/08 & 8C17008 & AGG-RRL-001 \\
\hline 15756-32-8 & Antimony-126 & $<0.285$ & $\mathrm{pCi} / \mathrm{g}$ & 0.285 & & 3/31/08 & 8C17008 & AGG-RRL-001 \\
\hline 15832-50-5 & Tin-126 & $<0.615$ & $\mathrm{pCi} / \mathrm{g}$ & 0.615 & & 3/31/08 & 8C17008 & AGG-RRL-001 \\
\hline 10043-66-0 & Iodine-131 & $<0.35$ & $\mathrm{pCi} / \mathrm{g}$ & 0.35 & & 3/31/08 & 8C17008 & AGG-RRL-001 \\
\hline 13981-41-4 & Barium-133 & $<0.548$ & $\mathrm{pCi} / \mathrm{g}$ & 0.548 & & 3/31/08 & 8C17008 & AGG-RRL-001 \\
\hline 13967-70-9 & Cesium-134 & $<0.323$ & $\mathrm{pCi} / \mathrm{g}$ & 0.323 & & 3/31/08 & 8C17008 & AGG-RRL-001 \\
\hline 10045-97-3 & Cesium-137 & $<0.335$ & $\mathrm{pCi} / \mathrm{g}$ & 0.335 & & 3/31/08 & 8C17008 & AGG-RRL-001 \\
\hline 13982-30-4 & Cerium-139 & $<0.345$ & $\mathrm{pCi} / \mathrm{g}$ & 0.345 & & 3/31/08 & 8C17008 & AGG-RRL-001 \\
\hline 14762-78-8 & Cerium-144 & $<2.29$ & $\mathrm{pCi} / \mathrm{g}$ & 2.29 & & 3/31/08 & 8C17008 & AGG-RRL-001 \\
\hline 14683-23-9 & Europium-152 & $<1.07$ & $\mathrm{pCi} / \mathrm{g}$ & 1.07 & & 3/31/08 & 8C17008 & AGG-RRL-001 \\
\hline $14276-65-4$ & Gadolinium-153 & $<0.86$ & $\mathrm{pCi} / \mathrm{g}$ & 0.86 & & 3/31/08 & 8C17008 & AGG-RRL-001 \\
\hline 15585-10-1 & Europium-154 & $<0.622$ & $\mathrm{pCi} / \mathrm{g}$ & 0.622 & & 3/31/08 & 8C17008 & AGG-RRL-001 \\
\hline 14391-16-3 & Europium-155 & $<0.858$ & $\mathrm{pCi} / \mathrm{g}$ & 0.858 & & 3/31/08 & 8C17008 & AGG-RRL-001 \\
\hline 13982-78-0 & Mercury-203 & $<0.356$ & $\mathrm{pCi} / \mathrm{g}$ & 0.356 & & 3/31/08 & 8C17008 & AGG-RRL-001 \\
\hline 14913-50-9 & Thallium-208 & $<0.223$ & $\mathrm{pCi} / \mathrm{g}$ & 0.223 & 0.0525 & 3/31/08 & 8C17008 & AGG-RRL-001 \\
\hline 14331-79-4 & Bismuth-210 & $<0.566$ & $\mathrm{pCi} / \mathrm{g}$ & 0.566 & & 3/31/08 & 8C17008 & AGG-RRL-001 \\
\hline \multirow[t]{3}{*}{$14255-04-0$} & Lead-210 & $<110$ & $\mathrm{pCi} / \mathrm{g}$ & 110 & & 3/31/08 & 8C17008 & AGG-RRL-001 \\
\hline & Bismuth-211 & $<6.64$ & $\mathrm{pCi} / \mathrm{g}$ & 6.64 & & 3/31/08 & 8C17008 & AGG-RRL-001 \\
\hline & Lead-211 & $<9.07$ & $\mathrm{pCi} / \mathrm{g}$ & 9.07 & & 3/31/08 & 8C17008 & AGG-RRL-001 \\
\hline 15092-94-1 & Lead-212 & 0.839 & $\mathrm{pCi} / \mathrm{g}$ & 0.484 & 28.4 & 3/31/08 & 8C17008 & AGG-RRL-001 \\
\hline 14733-03-0 & Bismuth-214 & $<0.75$ & $\mathrm{pCi} / \mathrm{g}$ & 0.75 & & 3/31/08 & 8C17008 & AGG-RRL-001 \\
\hline $15067-28-4$ & Lead-214 & $<0.877$ & $\mathrm{pCi} / \mathrm{g}$ & 0.877 & & 3/31/08 & 8C17008 & AGG-RRL-001 \\
\hline $14835-02-0$ & Radon-219 & $<2.8$ & $\mathrm{pCi} / \mathrm{g}$ & 2.8 & & 3/31/08 & 8C17008 & AGG-RRL-001 \\
\hline 22481-48-7 & Radon-220 & $<244$ & $\mathrm{pCi} / \mathrm{g}$ & 244 & & 3/31/08 & 8C17008 & AGG-RRL-001 \\
\hline 28522-20-5 & Radon-221 & $<1.31$ & $\mathrm{pCi} / \mathrm{g}$ & 1.31 & & 3/31/08 & 8C17008 & AGG-RRL-001 \\
\hline 15756-98-6 & Francium-223 & $<9.54$ & $\mathrm{pCi} / \mathrm{g}$ & 9.54 & & 3/31/08 & 8C17008 & AGG-RRL-001 \\
\hline $15623-45-7$ & Radium-223 & $<2.08$ & $\mathrm{pCi} / \mathrm{g}$ & 2.08 & & 3/31/08 & 8C17008 & AGG-RRL-001 \\
\hline 13233-32-4 & Radium-224 & $<5.76$ & $\mathrm{pCi} / \mathrm{g}$ & 5.76 & 79.6 & 3/31/08 & 8C17008 & AGG-RRL-001 \\
\hline 13982-63-3 & Radium-226 & $<8.15$ & $\mathrm{pCi} / \mathrm{g}$ & 8.15 & & 3/31/08 & 8C17008 & AGG-RRL-001 \\
\hline 15623-47-9 & Thorium-227 & $<2.93$ & $\mathrm{pCi} / \mathrm{g}$ & 2.93 & & 3/31/08 & 8C17008 & AGG-RRL-001 \\
\hline 14331-83-0 & Actinium-228 & $<1.3$ & $\mathrm{pCi} / \mathrm{g}$ & 1.3 & & 3/31/08 & 8C17008 & AGG-RRL-001 \\
\hline $14274-82-9$ & Thorium-228 & $<106$ & $\mathrm{pCi} / \mathrm{g}$ & 106 & & 3/31/08 & 8C17008 & AGG-RRL-001 \\
\hline 14269-63-7 & Thorium-230 & $<112$ & $\mathrm{pCi} / \mathrm{g}$ & 112 & & 3/31/08 & 8C17008 & AGG-RRL-001 \\
\hline 14331-85-2 & Protactinium-231 & $<11.6$ & $\mathrm{pCi} / \mathrm{g}$ & 11.6 & & 3/31/08 & 8C17008 & AGG-RRL-001 \\
\hline $14932-40-2$ & Thorium-231 & $<60.1$ & $\mathrm{pCi} / \mathrm{g}$ & 60.1 & & 3/31/08 & 8C17008 & AGG-RRL-001 \\
\hline 7440-29-1 & Thorium-232 & $<347$ & $\mathrm{pCi} / \mathrm{g}$ & 347 & & 3/31/08 & 8C17008 & AGG-RRL-001 \\
\hline 13981-14-1 & Protactinium-233 & $<0.724$ & $\mathrm{pCi} / \mathrm{g}$ & 0.724 & & 3/31/08 & 8C17008 & AGG-RRL-001 \\
\hline $15100-28-4$ & Protactinium-234 & $<1.09$ & $\mathrm{pCi} / \mathrm{g}$ & 1.09 & & 3/31/08 & 8C17008 & AGG-RRL-001 \\
\hline
\end{tabular}


GEA/Soil

\begin{tabular}{|c|c|c|c|c|c|c|c|c|}
\hline CAS \# & Analyte & Results & Units & MDA & UNC & Analyzed & Batch & Method \\
\hline \multirow[t]{2}{*}{ HEIS No. } & B1RTH2A & \multicolumn{2}{|c|}{ Lab ID: } & 0803012-06 & & & & \\
\hline & Protactinium-234m & $<38$ & $\mathrm{pCi} / \mathrm{g}$ & 38 & & 3/31/08 & 8C17008 & AGG-RRL-001 \\
\hline $15065-10-8$ & Thorium-234 & $<9.95$ & $\mathrm{pCi} / \mathrm{g}$ & 9.95 & & 3/31/08 & 8C17008 & AGG-RRL-001 \\
\hline 15117-96-1 & Uranium 235 & $<2.33$ & $\mathrm{pCi} / \mathrm{g}$ & 2.33 & & 3/31/08 & 8C17008 & AGG-RRL-001 \\
\hline 13994-20-2 & Neptunium-237 & $<2.23$ & $\mathrm{pCi} / \mathrm{g}$ & 2.23 & & 3/31/08 & 8C17008 & AGG-RRL-001 \\
\hline \multirow[t]{2}{*}{ 13981-16-3 } & Plutonium-238 & $<3370$ & $\mathrm{pCi} / \mathrm{g}$ & 3370 & & 3/31/08 & 8C17008 & AGG-RRL-001 \\
\hline & Uranium 238 & $<5.15$ & $\mathrm{pCi} / \mathrm{g}$ & 5.15 & & 3/31/08 & 8C17008 & AGG-RRL-001 \\
\hline 15117-48-3 & Plutonium-239 & $<3980$ & $\mathrm{pCi} / \mathrm{g}$ & 3980 & & 3/31/08 & 8C17008 & AGG-RRL-001 \\
\hline 14119-33-6 & Plutonium-240 & $<3520$ & $\mathrm{pCi} / \mathrm{g}$ & 3520 & & 3/31/08 & 8C17008 & AGG-RRL-001 \\
\hline 14596-10-2 & Americium-241 & $<1.7$ & $\mathrm{pCi} / \mathrm{g}$ & 1.7 & & 3/31/08 & 8C17008 & AGG-RRL-001 \\
\hline 14993-75-0 & Americium-243 & $<0.595$ & $\mathrm{pCi} / \mathrm{g}$ & 0.595 & & 3/31/08 & 8C17008 & AGG-RRL-001 \\
\hline 15757-87-6 & Curium-243 & $<1.07$ & $\mathrm{pCi} / \mathrm{g}$ & 1.07 & & 3/31/08 & 8C17008 & AGG-RRL-001 \\
\hline 15621-76-8 & Curium-245 & $<0.883$ & $\mathrm{pCi} / \mathrm{g}$ & 0.883 & & 3/31/08 & 8C17008 & AGG-RRL-001 \\
\hline HEIS No. & B1RTH3B & \multicolumn{2}{|c|}{ Lab ID: } & 0803012-09 & & & & \\
\hline 13966-32-0 & Sodium-22 & $<0.355$ & $\mathrm{pCi} / \mathrm{g}$ & 0.355 & & 3/31/08 & 8C17008 & AGG-RRL-001 \\
\hline 13966-00-2 & Potassium-40 & 17.1 & $\mathrm{pCi} / \mathrm{g}$ & 2.94 & 1.15 & 3/31/08 & 8C17008 & AGG-RRL-001 \\
\hline 14392-02-0 & Chromium-51 & $<2.87$ & $\mathrm{pCi} / \mathrm{g}$ & 2.87 & & 3/31/08 & 8C17008 & AGG-RRL-001 \\
\hline 13966-31-9 & Manganese-54 & $<0.344$ & $\mathrm{pCi} / \mathrm{g}$ & 0.344 & & 3/31/08 & 8C17008 & AGG-RRL-001 \\
\hline 13981-50-5 & Cobalt-57 & $<0.311$ & $\mathrm{pCi} / \mathrm{g}$ & 0.311 & & 3/31/08 & 8C17008 & AGG-RRL-001 \\
\hline $14596-12-4$ & Iron-59 & $<0.549$ & $\mathrm{pCi} / \mathrm{g}$ & 0.549 & & 3/31/08 & 8C17008 & AGG-RRL-001 \\
\hline 10198-40-0 & Cobalt-60 & $<0.329$ & $\mathrm{pCi} / \mathrm{g}$ & 0.329 & & 3/31/08 & 8C17008 & AGG-RRL-001 \\
\hline 13982-39-3 & Zinc-65 & $<0.689$ & $\mathrm{pCi} / \mathrm{g}$ & 0.689 & & 3/31/08 & 8C17008 & AGG-RRL-001 \\
\hline 14265-71-5 & Selenium-75 & $<0.519$ & $\mathrm{pCi} / \mathrm{g}$ & 0.519 & & 3/31/08 & 8C17008 & AGG-RRL-001 \\
\hline 17056-36-9 & Rubidium-83 & $<0.809$ & $\mathrm{pCi} / \mathrm{g}$ & 0.809 & & 3/31/08 & 8C17008 & AGG-RRL-001 \\
\hline 13967-73-2 & Strontium-85 & $<0.367$ & pCi/g & 0.367 & & 3/31/08 & 8C17008 & AGG-RRL-001 \\
\hline $14932-53-7$ & Rubidium-86 & $<4$ & $\mathrm{pCi} / \mathrm{g}$ & 4 & & 3/31/08 & 8C17008 & AGG-RRL-001 \\
\hline 13982-36-0 & Yttrium-88 & $<0.265$ & $\mathrm{pCi} / \mathrm{g}$ & 0.265 & & 3/31/08 & 8C17008 & AGG-RRL-001 \\
\hline 14681-63-1 & Niobium-94 & $<0.329$ & $\mathrm{pCi} / \mathrm{g}$ & 0.329 & & 3/31/08 & 8C17008 & AGG-RRL-001 \\
\hline \multirow[t]{3}{*}{ 13967-76-5 } & Niobium-95 & $<0.295$ & $\mathrm{pCi} / \mathrm{g}$ & 0.295 & & 3/31/08 & 8C17008 & AGG-RRL-001 \\
\hline & Niobium-95m & $<1.35$ & $\mathrm{pCi} / \mathrm{g}$ & 1.35 & & 3/31/08 & 8C17008 & AGG-RRL-001 \\
\hline & Technetium-95m & $<0.493$ & $\mathrm{pCi} / \mathrm{g}$ & 0.493 & & 3/31/08 & 8C17008 & AGG-RRL-001 \\
\hline \multirow[t]{2}{*}{ 13967-71-0 } & Zirconium-95 & $<0.553$ & $\mathrm{pCi} / \mathrm{g}$ & 0.553 & & 3/31/08 & 8C17008 & AGG-RRL-001 \\
\hline & Technetium-99m & $<0.309$ & $\mathrm{pCi} / \mathrm{g}$ & 0.309 & & 3/31/08 & 8C17008 & AGG-RRL-001 \\
\hline 13968-53-1 & Ruthenium-103 & $<0.359$ & $\mathrm{pCi} / \mathrm{g}$ & 0.359 & & 3/31/08 & 8C17008 & AGG-RRL-001 \\
\hline 13967-48-1 & Ruthenium-106 & $<3.43$ & $\mathrm{pCi} / \mathrm{g}$ & 3.43 & & 3/31/08 & 8C17008 & AGG-RRL-001 \\
\hline 14391-65-2 & Silver-108m & $<0.312$ & $\mathrm{pCi} / \mathrm{g}$ & 0.312 & & 3/31/08 & 8C17008 & AGG-RRL-001 \\
\hline \multirow[t]{2}{*}{ 14109-32-1 } & Cadmium-109 & $<8.87$ & $\mathrm{pCi} / \mathrm{g}$ & 8.87 & & 3/31/08 & 8C17008 & AGG-RRL-001 \\
\hline & Silver-110 & $<0.323$ & $\mathrm{pCi} / \mathrm{g}$ & 0.323 & & 3/31/08 & 8C17008 & AGG-RRL-001 \\
\hline 14391-76-5 & Silver-110m & $<0.323$ & $\mathrm{pCi} / \mathrm{g}$ & 0.323 & & 3/31/08 & 8C17008 & AGG-RRL-001 \\
\hline 13966-06-8 & Tin-113 & $<0.428$ & $\mathrm{pCi} / \mathrm{g}$ & 0.428 & & 3/31/08 & 8C17008 & AGG-RRL-001 \\
\hline 14683-10-4 & Antimony-124 & $<0.368$ & $\mathrm{pCi} / \mathrm{g}$ & 0.368 & & 3/31/08 & 8C17008 & AGG-RRL-001 \\
\hline $14234-35-6$ & Antimony-125 & $<0.975$ & pCi/g & 0.975 & & 3/31/08 & 8C17008 & AGG-RRL-001 \\
\hline 15756-32-8 & Antimony-126 & $<0.312$ & $\mathrm{pCi} / \mathrm{g}$ & 0.312 & & 3/31/08 & 8C17008 & AGG-RRL-001 \\
\hline $15832-50-5$ & Tin-126 & $<0.726$ & $\mathrm{pCi} / \mathrm{g}$ & 0.726 & & 3/31/08 & 8C17008 & AGG-RRL-001 \\
\hline 10043-66-0 & Iodine-131 & $<0.385$ & $\mathrm{pCi} / \mathrm{g}$ & 0.385 & & 3/31/08 & 8C17008 & AGG-RRL-001 \\
\hline 13981-41-4 & Barium-133 & $<0.533$ & $\mathrm{pCi} / \mathrm{g}$ & 0.533 & & 3/31/08 & 8C17008 & AGG-RRL-001 \\
\hline 13967-70-9 & Cesium-134 & $<0.367$ & $\mathrm{pCi} / \mathrm{g}$ & 0.367 & & 3/31/08 & 8C17008 & AGG-RRL-001 \\
\hline 10045-97-3 & Cesium-137 & $<0.366$ & $\mathrm{pCi} / \mathrm{g}$ & 0.366 & & 3/31/08 & 8C17008 & AGG-RRL-001 \\
\hline 13982-30-4 & Cerium-139 & $<0.367$ & $\mathrm{pCi} / \mathrm{g}$ & 0.367 & & 3/31/08 & 8C17008 & AGG-RRL-001 \\
\hline
\end{tabular}


GEA/Soil

\begin{tabular}{|c|c|c|c|c|c|c|c|c|}
\hline CAS \# & Analyte & Results & Units & MDA & UNC & Analyzed & Batch & Method \\
\hline HEIS No. & B1RTH3B & \multicolumn{3}{|c|}{ Lab ID: $\quad$ 0803012-09 } & & & & \\
\hline $14762-78-8$ & Cerium-144 & $<2.53$ & $\mathrm{pCi} / \mathrm{g}$ & 2.53 & & $3 / 31 / 08$ & 8C17008 & AGG-RRL-001 \\
\hline $14683-23-9$ & Europium-152 & $<1.23$ & $\mathrm{pCi} / \mathrm{g}$ & 1.23 & & 3/31/08 & 8C17008 & AGG-RRL-001 \\
\hline $14276-65-4$ & Gadolinium-153 & $<0.986$ & $\mathrm{pCi} / \mathrm{g}$ & 0.986 & & 3/31/08 & 8C17008 & AGG-RRL-001 \\
\hline 15585-10-1 & Europium-154 & $<0.66$ & $\mathrm{pCi} / \mathrm{g}$ & 0.66 & & $3 / 31 / 08$ & 8C17008 & AGG-RRL-001 \\
\hline $14391-16-3$ & Europium-155 & $<1.03$ & $\mathrm{pCi} / \mathrm{g}$ & 1.03 & & 3/31/08 & 8C17008 & AGG-RRL-001 \\
\hline 13982-78-0 & Mercury-203 & $<0.384$ & $\mathrm{pCi} / \mathrm{g}$ & 0.384 & & 3/31/08 & 8C17008 & AGG-RRL-001 \\
\hline $14913-50-9$ & Thallium-208 & $<0.397$ & $\mathrm{pCi} / \mathrm{g}$ & 0.397 & & 3/31/08 & 8C17008 & AGG-RRL-001 \\
\hline 14331-79-4 & Bismuth-210 & $<0.618$ & $\mathrm{pCi} / \mathrm{g}$ & 0.618 & & $3 / 31 / 08$ & 8C17008 & AGG-RRL-001 \\
\hline \multirow[t]{3}{*}{$14255-04-0$} & Lead-210 & $<43.3$ & $\mathrm{pCi} / \mathrm{g}$ & 43.3 & & 3/31/08 & 8C17008 & AGG-RRL-001 \\
\hline & Bismuth-211 & $<7.03$ & $\mathrm{pCi} / \mathrm{g}$ & 7.03 & & 3/31/08 & 8C17008 & AGG-RRL-001 \\
\hline & Lead-211 & $<9.61$ & $\mathrm{pCi} / \mathrm{g}$ & 9.61 & & $3 / 31 / 08$ & 8C17008 & AGG-RRL-001 \\
\hline 15092-94-1 & Lead-212 & $<0.78$ & $\mathrm{pCi} / \mathrm{g}$ & 0.78 & & $3 / 31 / 08$ & 8C17008 & AGG-RRL-001 \\
\hline $14733-03-0$ & Bismuth-214 & $<0.775$ & $\mathrm{pCi} / \mathrm{g}$ & 0.775 & & 3/31/08 & 8C17008 & AGG-RRL-001 \\
\hline $15067-28-4$ & Lead-214 & $<0.866$ & $\mathrm{pCi} / \mathrm{g}$ & 0.866 & & 3/31/08 & 8C17008 & AGG-RRL-001 \\
\hline $14835-02-0$ & Radon-219 & $<3.07$ & $\mathrm{pCi} / \mathrm{g}$ & 3.07 & & 3/31/08 & 8C17008 & AGG-RRL-001 \\
\hline 22481-48-7 & Radon-220 & $<297$ & $\mathrm{pCi} / \mathrm{g}$ & 297 & & 3/31/08 & 8C17008 & AGG-RRL-001 \\
\hline 28522-20-5 & Radon-221 & $<1.46$ & $\mathrm{pCi} / \mathrm{g}$ & 1.46 & & 3/31/08 & 8C17008 & AGG-RRL-001 \\
\hline 15756-98-6 & Francium-223 & $<9.96$ & $\mathrm{pCi} / \mathrm{g}$ & 9.96 & & 3/31/08 & 8C17008 & AGG-RRL-001 \\
\hline $15623-45-7$ & Radium-223 & $<2.26$ & $\mathrm{pCi} / \mathrm{g}$ & 2.26 & & 3/31/08 & 8C17008 & AGG-RRL-001 \\
\hline $13233-32-4$ & Radium-224 & $<8.52$ & $\mathrm{pCi} / \mathrm{g}$ & 8.52 & & 3/31/08 & 8C17008 & AGG-RRL-001 \\
\hline $13982-63-3$ & Radium-226 & $<8.98$ & $\mathrm{pCi} / \mathrm{g}$ & 8.98 & & 3/31/08 & 8C17008 & AGG-RRL-001 \\
\hline $15623-47-9$ & Thorium-227 & $<3.01$ & $\mathrm{pCi} / \mathrm{g}$ & 3.01 & & 3/31/08 & 8C17008 & AGG-RRL-001 \\
\hline $14331-83-0$ & Actinium-228 & $<1.41$ & $\mathrm{pCi} / \mathrm{g}$ & 1.41 & & 3/31/08 & 8C17008 & AGG-RRL-001 \\
\hline 14274-82-9 & Thorium-228 & $<116$ & $\mathrm{pCi} / \mathrm{g}$ & 116 & & 3/31/08 & 8C17008 & AGG-RRL-001 \\
\hline $14269-63-7$ & Thorium-230 & $<119$ & $\mathrm{pCi} / \mathrm{g}$ & 119 & & 3/31/08 & 8C17008 & AGG-RRL-001 \\
\hline $14331-85-2$ & Protactinium-231 & $<13.3$ & $\mathrm{pCi} / \mathrm{g}$ & 13.3 & & 3/31/08 & 8C17008 & AGG-RRL-001 \\
\hline $14932-40-2$ & Thorium-231 & $<70.1$ & $\mathrm{pCi} / \mathrm{g}$ & 70.1 & & 3/31/08 & 8C17008 & AGG-RRL-001 \\
\hline 7440-29-1 & Thorium-232 & $<298$ & $\mathrm{pCi} / \mathrm{g}$ & 298 & & 3/31/08 & 8C17008 & AGG-RRL-001 \\
\hline 13981-14-1 & Protactinium-233 & $<0.798$ & $\mathrm{pCi} / \mathrm{g}$ & 0.798 & & 3/31/08 & 8C17008 & AGG-RRL-001 \\
\hline \multirow[t]{2}{*}{$15100-28-4$} & Protactinium-234 & $<1.29$ & $\mathrm{pCi} / \mathrm{g}$ & 1.29 & & 3/31/08 & 8C17008 & AGG-RRL-001 \\
\hline & Protactinium-234m & $<38.5$ & $\mathrm{pCi} / \mathrm{g}$ & 38.5 & & $3 / 31 / 08$ & 8C17008 & AGG-RRL-001 \\
\hline $15065-10-8$ & Thorium-234 & $<10.5$ & $\mathrm{pCi} / \mathrm{g}$ & 10.5 & & $3 / 31 / 08$ & 8C17008 & AGG-RRL-001 \\
\hline 15117-96-1 & Uranium 235 & $<2.66$ & $\mathrm{pCi} / \mathrm{g}$ & 2.66 & & 3/31/08 & 8C17008 & AGG-RRL-001 \\
\hline 13994-20-2 & Neptunium-237 & $<2.69$ & $\mathrm{pCi} / \mathrm{g}$ & 2.69 & & $3 / 31 / 08$ & 8C17008 & AGG-RRL-001 \\
\hline \multirow[t]{2}{*}{ 13981-16-3 } & Plutonium-238 & $<4030$ & $\mathrm{pCi} / \mathrm{g}$ & 4030 & & 3/31/08 & 8C17008 & AGG-RRL-001 \\
\hline & Uranium 238 & $<5.68$ & $\mathrm{pCi} / \mathrm{g}$ & 5.68 & & 3/31/08 & 8C17008 & AGG-RRL-001 \\
\hline $15117-48-3$ & Plutonium-239 & $<4040$ & $\mathrm{pCi} / \mathrm{g}$ & 4040 & & 3/31/08 & 8C17008 & AGG-RRL-001 \\
\hline 14119-33-6 & Plutonium-240 & $<4040$ & $\mathrm{pCi} / \mathrm{g}$ & 4040 & & 3/31/08 & 8C17008 & AGG-RRL-001 \\
\hline $14596-10-2$ & Americium-241 & $<1.52$ & $\mathrm{pCi} / \mathrm{g}$ & 1.52 & & $3 / 31 / 08$ & 8C17008 & AGG-RRL-001 \\
\hline $14993-75-0$ & Americium-243 & $<0.651$ & $\mathrm{pCi} / \mathrm{g}$ & 0.651 & & 3/31/08 & 8C17008 & AGG-RRL-001 \\
\hline $15757-87-6$ & Curium-243 & $<1.24$ & $\mathrm{pCi} / \mathrm{g}$ & 1.24 & & 3/31/08 & 8C17008 & AGG-RRL-001 \\
\hline 15621-76-8 & Curium-245 & $<1.02$ & $\mathrm{pCi} / \mathrm{g}$ & 1.02 & & $3 / 31 / 08$ & 8C17008 & AGG-RRL-001 \\
\hline HEIS No. & B1RTH3A & \multicolumn{2}{|c|}{ Lab ID: } & 0803012-10 & & & & \\
\hline 13966-32-0 & Sodium-22 & $<0.321$ & $\mathrm{pCi} / \mathrm{g}$ & 0.321 & & $3 / 31 / 08$ & 8C17008 & AGG-RRL-001 \\
\hline $13966-00-2$ & Potassium-40 & 17.9 & $\mathrm{pCi} / \mathrm{g}$ & 2.57 & 1.15 & 3/31/08 & 8C17008 & AGG-RRL-001 \\
\hline $14392-02-0$ & Chromium-51 & $<2.58$ & $\mathrm{pCi} / \mathrm{g}$ & 2.58 & & 3/31/08 & 8C17008 & AGG-RRL-001 \\
\hline 13966-31-9 & Manganese-54 & $<0.27$ & $\mathrm{pCi} / \mathrm{g}$ & 0.27 & & 3/31/08 & 8C17008 & AGG-RRL-001 \\
\hline 13981-50-5 & Cobalt-57 & $<0.271$ & $\mathrm{pCi} / \mathrm{g}$ & 0.271 & & 3/31/08 & 8C17008 & AGG-RRL-001 \\
\hline
\end{tabular}


GEA/Soil

\begin{tabular}{|c|c|c|c|c|c|c|c|c|}
\hline CAS \# & Analyte & Results & Units & MDA & UNC & Analyzed & Batch & Method \\
\hline HEIS No. & B1RTH3A & \multicolumn{3}{|c|}{ Lab ID: $\quad$ 0803012-10 } & & & & \\
\hline $14596-12-4$ & Iron-59 & $<0.532$ & $\mathrm{pCi} / \mathrm{g}$ & 0.532 & & 3/31/08 & 8C17008 & AGG-RRL-001 \\
\hline 10198-40-0 & Cobalt-60 & $<0.28$ & $\mathrm{pCi} / \mathrm{g}$ & 0.28 & & 3/31/08 & 8C17008 & AGG-RRL-001 \\
\hline 13982-39-3 & Zinc-65 & $<0.618$ & $\mathrm{pCi} / \mathrm{g}$ & 0.618 & & 3/31/08 & 8C17008 & AGG-RRL-001 \\
\hline $14265-71-5$ & Selenium-75 & $<0.442$ & $\mathrm{pCi} / \mathrm{g}$ & 0.442 & & 3/31/08 & 8C17008 & AGG-RRL-001 \\
\hline 17056-36-9 & Rubidium-83 & $<0.524$ & $\mathrm{pCi} / \mathrm{g}$ & 0.524 & & 3/31/08 & 8C17008 & AGG-RRL-001 \\
\hline 13967-73-2 & Strontium-85 & $<0.357$ & $\mathrm{pCi} / \mathrm{g}$ & 0.357 & & 3/31/08 & 8C17008 & AGG-RRL-001 \\
\hline 14932-53-7 & Rubidium-86 & $<3.06$ & $\mathrm{pCi} / \mathrm{g}$ & 3.06 & & 3/31/08 & 8C17008 & AGG-RRL-001 \\
\hline 13982-36-0 & Yttrium-88 & $<0.218$ & $\mathrm{pCi} / \mathrm{g}$ & 0.218 & & 3/31/08 & 8C17008 & AGG-RRL-001 \\
\hline 14681-63-1 & Niobium-94 & $<0.25$ & $\mathrm{pCi} / \mathrm{g}$ & 0.25 & & 3/31/08 & 8C17008 & AGG-RRL-001 \\
\hline \multirow[t]{3}{*}{ 13967-76-5 } & Niobium-95 & $<0.281$ & $\mathrm{pCi} / \mathrm{g}$ & 0.281 & & $3 / 31 / 08$ & 8C17008 & AGG-RRL-001 \\
\hline & Niobium-95m & $<1.24$ & $\mathrm{pCi} / \mathrm{g}$ & 1.24 & & 3/31/08 & 8C17008 & AGG-RRL-001 \\
\hline & Technetium-95m & $<0.428$ & $\mathrm{pCi} / \mathrm{g}$ & 0.428 & & 3/31/08 & 8C17008 & AGG-RRL-001 \\
\hline \multirow[t]{2}{*}{ 13967-71-0 } & Zirconium-95 & $<0.48$ & $\mathrm{pCi} / \mathrm{g}$ & 0.48 & & 3/31/08 & 8C17008 & AGG-RRL-001 \\
\hline & Technetium-99m & $<0.272$ & $\mathrm{pCi} / \mathrm{g}$ & 0.272 & & 3/31/08 & 8C17008 & AGG-RRL-001 \\
\hline 13968-53-1 & Ruthenium-103 & $<0.282$ & $\mathrm{pCi} / \mathrm{g}$ & 0.282 & & 3/31/08 & 8C17008 & AGG-RRL-001 \\
\hline 13967-48-1 & Ruthenium-106 & $<2.71$ & $\mathrm{pCi} / \mathrm{g}$ & 2.71 & & 3/31/08 & 8C17008 & AGG-RRL-001 \\
\hline 14391-65-2 & Silver-108m & $<0.264$ & $\mathrm{pCi} / \mathrm{g}$ & 0.264 & & 3/31/08 & 8C17008 & AGG-RRL-001 \\
\hline \multirow[t]{2}{*}{ 14109-32-1 } & Cadmium-109 & $<7.27$ & $\mathrm{pCi} / \mathrm{g}$ & 7.27 & & 3/31/08 & 8C17008 & AGG-RRL-001 \\
\hline & Silver-110 & $<0.264$ & $\mathrm{pCi} / \mathrm{g}$ & 0.264 & & 3/31/08 & 8C17008 & AGG-RRL-001 \\
\hline 14391-76-5 & Silver-110m & $<0.264$ & $\mathrm{pCi} / \mathrm{g}$ & 0.264 & & 3/31/08 & 8C17008 & AGG-RRL-001 \\
\hline 13966-06-8 & Tin-113 & $<0.393$ & $\mathrm{pCi} / \mathrm{g}$ & 0.393 & & 3/31/08 & 8C17008 & AGG-RRL-001 \\
\hline 14683-10-4 & Antimony-124 & $<0.261$ & $\mathrm{pCi} / \mathrm{g}$ & 0.261 & & 3/31/08 & 8C17008 & AGG-RRL-001 \\
\hline $14234-35-6$ & Antimony-125 & $<0.819$ & $\mathrm{pCi} / \mathrm{g}$ & 0.819 & & 3/31/08 & 8C17008 & AGG-RRL-001 \\
\hline 15756-32-8 & Antimony-126 & $<0.263$ & $\mathrm{pCi} / \mathrm{g}$ & 0.263 & & 3/31/08 & 8C17008 & AGG-RRL-001 \\
\hline 15832-50-5 & Tin-126 & $<0.579$ & $\mathrm{pCi} / \mathrm{g}$ & 0.579 & & 3/31/08 & 8C17008 & AGG-RRL-001 \\
\hline 10043-66-0 & Iodine-131 & $<0.288$ & $\mathrm{pCi} / \mathrm{g}$ & 0.288 & & 3/31/08 & 8C17008 & AGG-RRL-001 \\
\hline 13981-41-4 & Barium-133 & $<0.507$ & $\mathrm{pCi} / \mathrm{g}$ & 0.507 & & 3/31/08 & 8C17008 & AGG-RRL-001 \\
\hline 13967-70-9 & Cesium-134 & $<0.306$ & $\mathrm{pCi} / \mathrm{g}$ & 0.306 & & 3/31/08 & 8C17008 & AGG-RRL-001 \\
\hline $10045-97-3$ & Cesium-137 & $<0.299$ & $\mathrm{pCi} / \mathrm{g}$ & 0.299 & & 3/31/08 & 8C17008 & AGG-RRL-001 \\
\hline 13982-30-4 & Cerium-139 & $<0.32$ & $\mathrm{pCi} / \mathrm{g}$ & 0.32 & & 3/31/08 & 8C17008 & AGG-RRL-001 \\
\hline 14762-78-8 & Cerium-144 & $<2.16$ & $\mathrm{pCi} / \mathrm{g}$ & 2.16 & & 3/31/08 & 8C17008 & AGG-RRL-001 \\
\hline 14683-23-9 & Europium-152 & $<1.02$ & $\mathrm{pCi} / \mathrm{g}$ & 1.02 & & 3/31/08 & 8C17008 & AGG-RRL-001 \\
\hline $14276-65-4$ & Gadolinium-153 & $<0.812$ & $\mathrm{pCi} / \mathrm{g}$ & 0.812 & & 3/31/08 & 8C17008 & AGG-RRL-001 \\
\hline 15585-10-1 & Europium-154 & $<0.58$ & $\mathrm{pCi} / \mathrm{g}$ & 0.58 & & 3/31/08 & 8C17008 & AGG-RRL-001 \\
\hline 14391-16-3 & Europium-155 & $<0.81$ & $\mathrm{pCi} / \mathrm{g}$ & 0.81 & & 3/31/08 & 8C17008 & AGG-RRL-001 \\
\hline 13982-78-0 & Mercury-203 & $<0.337$ & $\mathrm{pCi} / \mathrm{g}$ & 0.337 & & 3/31/08 & 8C17008 & AGG-RRL-001 \\
\hline 14913-50-9 & Thallium-208 & $<0.245$ & $\mathrm{pCi} / \mathrm{g}$ & 0.245 & 0.0569 & 3/31/08 & 8C17008 & AGG-RRL-001 \\
\hline 14331-79-4 & Bismuth-210 & $<0.528$ & $\mathrm{pCi} / \mathrm{g}$ & 0.528 & & 3/31/08 & 8C17008 & AGG-RRL-001 \\
\hline \multirow[t]{3}{*}{$14255-04-0$} & Lead-210 & $<104$ & $\mathrm{pCi} / \mathrm{g}$ & 104 & & 3/31/08 & 8C17008 & AGG-RRL-001 \\
\hline & Bismuth-211 & $<5.87$ & $\mathrm{pCi} / \mathrm{g}$ & 5.87 & & 3/31/08 & 8C17008 & AGG-RRL-001 \\
\hline & Lead-211 & $<8.03$ & $\mathrm{pCi} / \mathrm{g}$ & 8.03 & & 3/31/08 & 8C17008 & AGG-RRL-001 \\
\hline 15092-94-1 & Lead-212 & $<0.565$ & $\mathrm{pCi} / \mathrm{g}$ & 0.565 & 0.124 & 3/31/08 & 8C17008 & AGG-RRL-001 \\
\hline 14733-03-0 & Bismuth-214 & $<0.668$ & $\mathrm{pCi} / \mathrm{g}$ & 0.668 & & 3/31/08 & 8C17008 & AGG-RRL-001 \\
\hline $15067-28-4$ & Lead-214 & $<0.83$ & $\mathrm{pCi} / \mathrm{g}$ & 0.83 & & 3/31/08 & 8C17008 & AGG-RRL-001 \\
\hline 14835-02-0 & Radon-219 & $<2.62$ & $\mathrm{pCi} / \mathrm{g}$ & 2.62 & & 3/31/08 & 8C17008 & AGG-RRL-001 \\
\hline 22481-48-7 & Radon-220 & $<228$ & $\mathrm{pCi} / \mathrm{g}$ & 228 & & 3/31/08 & 8C17008 & AGG-RRL-001 \\
\hline 28522-20-5 & Radon-221 & $<1.22$ & $\mathrm{pCi} / \mathrm{g}$ & 1.22 & & 3/31/08 & 8C17008 & AGG-RRL-001 \\
\hline 15756-98-6 & Francium-223 & $<9.03$ & $\mathrm{pCi} / \mathrm{g}$ & 9.03 & & 3/31/08 & 8C17008 & AGG-RRL-001 \\
\hline $15623-45-7$ & Radium-223 & $<1.93$ & $\mathrm{pCi} / \mathrm{g}$ & 1.93 & & 3/31/08 & 8C17008 & AGG-RRL-001 \\
\hline
\end{tabular}


GEA/Soil

\begin{tabular}{|c|c|c|c|c|c|c|c|c|}
\hline CAS \# & Analyte & Results & Units & MDA & UNC & Analyzed & Batch & Method \\
\hline HEIS No. & B1RTH3A & \multicolumn{2}{|c|}{ Lab ID: } & 0803012-10 & & & & \\
\hline $13233-32-4$ & Radium-224 & $<8.01$ & $\mathrm{pCi} / \mathrm{g}$ & 8.01 & & $3 / 31 / 08$ & 8C17008 & AGG-RRL-001 \\
\hline 13982-63-3 & Radium-226 & $<7.48$ & $\mathrm{pCi} / \mathrm{g}$ & 7.48 & & 3/31/08 & 8C17008 & AGG-RRL-001 \\
\hline $15623-47-9$ & Thorium-227 & $<2.78$ & $\mathrm{pCi} / \mathrm{g}$ & 2.78 & & 3/31/08 & 8C17008 & AGG-RRL-001 \\
\hline 14331-83-0 & Actinium-228 & $<1.3$ & $\mathrm{pCi} / \mathrm{g}$ & 1.3 & & 3/31/08 & 8C17008 & AGG-RRL-001 \\
\hline $14274-82-9$ & Thorium-228 & $<99.7$ & $\mathrm{pCi} / \mathrm{g}$ & 99.7 & & 3/31/08 & 8C17008 & AGG-RRL-001 \\
\hline $14269-63-7$ & Thorium-230 & $<104$ & $\mathrm{pCi} / \mathrm{g}$ & 104 & & 3/31/08 & 8C17008 & AGG-RRL-001 \\
\hline $14331-85-2$ & Protactinium-231 & $<11.5$ & $\mathrm{pCi} / \mathrm{g}$ & 11.5 & & 3/31/08 & 8C17008 & AGG-RRL-001 \\
\hline $14932-40-2$ & Thorium-231 & $<57.4$ & $\mathrm{pCi} / \mathrm{g}$ & 57.4 & & 3/31/08 & 8C17008 & AGG-RRL-001 \\
\hline 7440-29-1 & Thorium-232 & $<320$ & $\mathrm{pCi} / \mathrm{g}$ & 320 & & 3/31/08 & 8C17008 & AGG-RRL-001 \\
\hline 13981-14-1 & Protactinium-233 & $<0.683$ & $\mathrm{pCi} / \mathrm{g}$ & 0.683 & & 3/31/08 & 8C17008 & AGG-RRL-001 \\
\hline \multirow[t]{2}{*}{$15100-28-4$} & Protactinium-234 & $<1.03$ & $\mathrm{pCi} / \mathrm{g}$ & 1.03 & & 3/31/08 & 8C17008 & AGG-RRL-001 \\
\hline & Protactinium-234m & $<31.4$ & $\mathrm{pCi} / \mathrm{g}$ & 31.4 & & 3/31/08 & 8C17008 & AGG-RRL-001 \\
\hline 15065-10-8 & Thorium-234 & $<9.11$ & $\mathrm{pCi} / \mathrm{g}$ & 9.11 & & 3/31/08 & 8C17008 & AGG-RRL-001 \\
\hline 15117-96-1 & Uranium 235 & $<2.3$ & $\mathrm{pCi} / \mathrm{g}$ & 2.3 & & 3/31/08 & 8C17008 & AGG-RRL-001 \\
\hline 13994-20-2 & Neptunium-237 & $<2.11$ & $\mathrm{pCi} / \mathrm{g}$ & 2.11 & & 3/31/08 & 8C17008 & AGG-RRL-001 \\
\hline \multirow[t]{2}{*}{ 13981-16-3 } & Plutonium-238 & $<3230$ & $\mathrm{pCi} / \mathrm{g}$ & 3230 & & 3/31/08 & 8C17008 & AGG-RRL-001 \\
\hline & Uranium 238 & $<4.72$ & $\mathrm{pCi} / \mathrm{g}$ & 4.72 & & 3/31/08 & 8C17008 & AGG-RRL-001 \\
\hline $15117-48-3$ & Plutonium-239 & $<3740$ & $\mathrm{pCi} / \mathrm{g}$ & 3740 & & 3/31/08 & 8C17008 & AGG-RRL-001 \\
\hline 14119-33-6 & Plutonium-240 & $<3300$ & $\mathrm{pCi} / \mathrm{g}$ & 3300 & & 3/31/08 & 8C17008 & AGG-RRL-001 \\
\hline $14596-10-2$ & Americium-241 & $<1.58$ & $\mathrm{pCi} / \mathrm{g}$ & 1.58 & & 3/31/08 & 8C17008 & AGG-RRL-001 \\
\hline $14993-75-0$ & Americium-243 & $<0.575$ & $\mathrm{pCi} / \mathrm{g}$ & 0.575 & & 3/31/08 & 8C17008 & AGG-RRL-001 \\
\hline $15757-87-6$ & Curium-243 & $<1.01$ & $\mathrm{pCi} / \mathrm{g}$ & 1.01 & & 3/31/08 & 8C17008 & AGG-RRL-001 \\
\hline 15621-76-8 & Curium-245 & $<0.827$ & $\mathrm{pCi} / \mathrm{g}$ & 0.827 & & 3/31/08 & 8C17008 & AGG-RRL-001 \\
\hline HEIS No. & B1RYR7B & \multicolumn{2}{|c|}{ Lab ID: } & 0803012-13 & & & & \\
\hline 13966-32-0 & Sodium-22 & $<0.341$ & $\mathrm{pCi} / \mathrm{g}$ & 0.341 & & 3/31/08 & 8C17008 & AGG-RRL-001 \\
\hline 13966-00-2 & Potassium- 40 & 18.7 & $\mathrm{pCi} / \mathrm{g}$ & 2.27 & 1.11 & 3/31/08 & 8C17008 & AGG-RRL-001 \\
\hline $14392-02-0$ & Chromium-51 & $<2.88$ & $\mathrm{pCi} / \mathrm{g}$ & 2.88 & & 3/31/08 & 8C17008 & AGG-RRL-001 \\
\hline 13966-31-9 & Manganese-54 & $<0.314$ & $\mathrm{pCi} / \mathrm{g}$ & 0.314 & & 3/31/08 & 8C17008 & AGG-RRL-001 \\
\hline $13981-50-5$ & Cobalt-57 & $<0.313$ & $\mathrm{pCi} / \mathrm{g}$ & 0.313 & & 3/31/08 & 8C17008 & AGG-RRL-001 \\
\hline $14596-12-4$ & Iron-59 & $<0.605$ & $\mathrm{pCi} / \mathrm{g}$ & 0.605 & & 3/31/08 & 8C17008 & AGG-RRL-001 \\
\hline $10198-40-0$ & Cobalt-60 & $<0.288$ & $\mathrm{pCi} / \mathrm{g}$ & 0.288 & & 3/31/08 & 8C17008 & AGG-RRL-001 \\
\hline 13982-39-3 & Zinc-65 & $<0.717$ & $\mathrm{pCi} / \mathrm{g}$ & 0.717 & & 3/31/08 & 8C17008 & AGG-RRL-001 \\
\hline $14265-71-5$ & Selenium-75 & $<0.51$ & $\mathrm{pCi} / \mathrm{g}$ & 0.51 & & 3/31/08 & 8C17008 & AGG-RRL-001 \\
\hline $17056-36-9$ & Rubidium-83 & $<0.738$ & $\mathrm{pCi} / \mathrm{g}$ & 0.738 & & 3/31/08 & 8C17008 & AGG-RRL-001 \\
\hline 13967-73-2 & Strontium-85 & $<0.343$ & $\mathrm{pCi} / \mathrm{g}$ & 0.343 & & $3 / 31 / 08$ & 8C17008 & AGG-RRL-001 \\
\hline $14932-53-7$ & Rubidium-86 & $<3.95$ & $\mathrm{pCi} / \mathrm{g}$ & 3.95 & & $3 / 31 / 08$ & 8C17008 & AGG-RRL-001 \\
\hline 13982-36-0 & Yttrium-88 & $<0.253$ & $\mathrm{pCi} / \mathrm{g}$ & 0.253 & & 3/31/08 & 8C17008 & AGG-RRL-001 \\
\hline $14681-63-1$ & Niobium-94 & $<0.281$ & $\mathrm{pCi} / \mathrm{g}$ & 0.281 & & 3/31/08 & 8C17008 & AGG-RRL-001 \\
\hline \multirow[t]{3}{*}{$13967-76-5$} & Niobium-95 & $<0.304$ & $\mathrm{pCi} / \mathrm{g}$ & 0.304 & & 3/31/08 & 8C17008 & AGG-RRL-001 \\
\hline & Niobium-95m & $<1.34$ & $\mathrm{pCi} / \mathrm{g}$ & 1.34 & & 3/31/08 & 8C17008 & AGG-RRL-001 \\
\hline & Technetium-95m & $<0.462$ & $\mathrm{pCi} / \mathrm{g}$ & 0.462 & & 3/31/08 & 8C17008 & AGG-RRL-001 \\
\hline \multirow[t]{2}{*}{$13967-71-0$} & Zirconium-95 & $<0.519$ & $\mathrm{pCi} / \mathrm{g}$ & 0.519 & & 3/31/08 & 8C17008 & AGG-RRL-001 \\
\hline & Technetium-99m & $<0.301$ & $\mathrm{pCi} / \mathrm{g}$ & 0.301 & & 3/31/08 & 8C17008 & AGG-RRL-001 \\
\hline 13968-53-1 & Ruthenium-103 & $<0.347$ & $\mathrm{pCi} / \mathrm{g}$ & 0.347 & & 3/31/08 & 8C17008 & AGG-RRL-001 \\
\hline $13967-48-1$ & Ruthenium-106 & $<3.39$ & $\mathrm{pCi} / \mathrm{g}$ & 3.39 & & 3/31/08 & 8C17008 & AGG-RRL-001 \\
\hline $14391-65-2$ & Silver-108m & $<0.307$ & $\mathrm{pCi} / \mathrm{g}$ & 0.307 & & 3/31/08 & 8C17008 & AGG-RRL-001 \\
\hline \multirow[t]{2}{*}{$14109-32-1$} & Cadmium-109 & $<8.52$ & $\mathrm{pCi} / \mathrm{g}$ & 8.52 & & 3/31/08 & 8C17008 & AGG-RRL-001 \\
\hline & Silver-110 & $<0.313$ & $\mathrm{pCi} / \mathrm{g}$ & 0.313 & & 3/31/08 & 8C17008 & AGG-RRL-001 \\
\hline
\end{tabular}


GEA/Soil

\begin{tabular}{|c|c|c|c|c|c|c|c|c|}
\hline CAS \# & Analyte & Results & Units & MDA & UNC & Analyzed & Batch & Method \\
\hline HEIS No. & B1RYR7B & \multicolumn{3}{|c|}{ Lab ID: $\quad$ 0803012-13 } & & & & \\
\hline 14391-76-5 & Silver-110m & $<0.314$ & $\mathrm{pCi} / \mathrm{g}$ & 0.314 & & 3/31/08 & 8C17008 & AGG-RRL-001 \\
\hline 13966-06-8 & Tin-113 & $<0.439$ & $\mathrm{pCi} / \mathrm{g}$ & 0.439 & & 3/31/08 & 8C17008 & AGG-RRL-001 \\
\hline $14683-10-4$ & Antimony-124 & $<0.343$ & $\mathrm{pCi} / \mathrm{g}$ & 0.343 & & 3/31/08 & 8C17008 & AGG-RRL-001 \\
\hline 14234-35-6 & Antimony-125 & $<0.934$ & $\mathrm{pCi} / \mathrm{g}$ & 0.934 & & 3/31/08 & 8C17008 & AGG-RRL-001 \\
\hline 15756-32-8 & Antimony-126 & $<0.283$ & $\mathrm{pCi} / \mathrm{g}$ & 0.283 & & 3/31/08 & 8C17008 & AGG-RRL-001 \\
\hline 15832-50-5 & Tin-126 & $<0.693$ & $\mathrm{pCi} / \mathrm{g}$ & 0.693 & & 3/31/08 & 8C17008 & AGG-RRL-001 \\
\hline 10043-66-0 & Iodine-131 & $<0.368$ & $\mathrm{pCi} / \mathrm{g}$ & 0.368 & & 3/31/08 & 8C17008 & AGG-RRL-001 \\
\hline 13981-41-4 & Barium-133 & $<0.51$ & $\mathrm{pCi} / \mathrm{g}$ & 0.51 & & 3/31/08 & 8C17008 & AGG-RRL-001 \\
\hline 13967-70-9 & Cesium-134 & $<0.346$ & $\mathrm{pCi} / \mathrm{g}$ & 0.346 & & 3/31/08 & 8C17008 & AGG-RRL-001 \\
\hline 10045-97-3 & Cesium-137 & $<0.34$ & $\mathrm{pCi} / \mathrm{g}$ & 0.34 & & $3 / 31 / 08$ & 8C17008 & AGG-RRL-001 \\
\hline 13982-30-4 & Cerium-139 & $<0.351$ & $\mathrm{pCi} / \mathrm{g}$ & 0.351 & & 3/31/08 & 8C17008 & AGG-RRL-001 \\
\hline 14762-78-8 & Cerium-144 & $<2.49$ & $\mathrm{pCi} / \mathrm{g}$ & 2.49 & & $3 / 31 / 08$ & 8C17008 & AGG-RRL-001 \\
\hline 14683-23-9 & Europium-152 & $<1.17$ & $\mathrm{pCi} / \mathrm{g}$ & 1.17 & & $3 / 31 / 08$ & 8C17008 & AGG-RRL-001 \\
\hline $14276-65-4$ & Gadolinium-153 & $<0.951$ & $\mathrm{pCi} / \mathrm{g}$ & 0.951 & & 3/31/08 & 8C17008 & AGG-RRL-001 \\
\hline 15585-10-1 & Europium-154 & $<0.663$ & $\mathrm{pCi} / \mathrm{g}$ & 0.663 & & 3/31/08 & 8C17008 & AGG-RRL-001 \\
\hline 14391-16-3 & Europium-155 & $<0.982$ & $\mathrm{pCi} / \mathrm{g}$ & 0.982 & & 3/31/08 & 8C17008 & AGG-RRL-001 \\
\hline 13982-78-0 & Mercury-203 & $<0.381$ & $\mathrm{pCi} / \mathrm{g}$ & 0.381 & & 3/31/08 & 8C17008 & AGG-RRL-001 \\
\hline 14913-50-9 & Thallium-208 & $<0.368$ & $\mathrm{pCi} / \mathrm{g}$ & 0.368 & & 3/31/08 & 8C17008 & AGG-RRL-001 \\
\hline 14331-79-4 & Bismuth-210 & $<0.606$ & $\mathrm{pCi} / \mathrm{g}$ & 0.606 & & 3/31/08 & 8C17008 & AGG-RRL-001 \\
\hline \multirow[t]{3}{*}{$14255-04-0$} & Lead-210 & $<41$ & $\mathrm{pCi} / \mathrm{g}$ & 41 & & 3/31/08 & 8C17008 & AGG-RRL-001 \\
\hline & Bismuth-211 & $<6.89$ & $\mathrm{pCi} / \mathrm{g}$ & 6.89 & & 3/31/08 & 8C17008 & AGG-RRL-001 \\
\hline & Lead-211 & $<9.42$ & $\mathrm{pCi} / \mathrm{g}$ & 9.42 & & 3/31/08 & 8C17008 & AGG-RRL-001 \\
\hline 15092-94-1 & Lead-212 & $<0.78$ & $\mathrm{pCi} / \mathrm{g}$ & 0.78 & & 3/31/08 & 8C17008 & AGG-RRL-001 \\
\hline 14733-03-0 & Bismuth-214 & $<0.757$ & $\mathrm{pCi} / \mathrm{g}$ & 0.757 & & 3/31/08 & 8C17008 & AGG-RRL-001 \\
\hline $15067-28-4$ & Lead-214 & $<0.824$ & $\mathrm{pCi} / \mathrm{g}$ & 0.824 & & 3/31/08 & 8C17008 & AGG-RRL-001 \\
\hline 14835-02-0 & Radon-219 & $<2.98$ & $\mathrm{pCi} / \mathrm{g}$ & 2.98 & & 3/31/08 & 8C17008 & AGG-RRL-001 \\
\hline 22481-48-7 & Radon-220 & $<277$ & $\mathrm{pCi} / \mathrm{g}$ & 277 & & 3/31/08 & 8C17008 & AGG-RRL-001 \\
\hline 28522-20-5 & Radon-221 & $<1.4$ & $\mathrm{pCi} / \mathrm{g}$ & 1.4 & & 3/31/08 & 8C17008 & AGG-RRL-001 \\
\hline 15756-98-6 & Francium-223 & $<9.84$ & $\mathrm{pCi} / \mathrm{g}$ & 9.84 & & 3/31/08 & 8C17008 & AGG-RRL-001 \\
\hline $15623-45-7$ & Radium-223 & $<2.18$ & $\mathrm{pCi} / \mathrm{g}$ & 2.18 & & 3/31/08 & 8C17008 & AGG-RRL-001 \\
\hline 13233-32-4 & Radium-224 & $<8.47$ & $\mathrm{pCi} / \mathrm{g}$ & 8.47 & & 3/31/08 & 8C17008 & AGG-RRL-001 \\
\hline 13982-63-3 & Radium-226 & $<8.61$ & $\mathrm{pCi} / \mathrm{g}$ & 8.61 & & 3/31/08 & 8C17008 & AGG-RRL-001 \\
\hline $15623-47-9$ & Thorium-227 & $<2.99$ & $\mathrm{pCi} / \mathrm{g}$ & 2.99 & & 3/31/08 & 8C17008 & AGG-RRL-001 \\
\hline 14331-83-0 & Actinium-228 & $<1.28$ & $\mathrm{pCi} / \mathrm{g}$ & 1.28 & & 3/31/08 & 8C17008 & AGG-RRL-001 \\
\hline 14274-82-9 & Thorium-228 & $<107$ & $\mathrm{pCi} / \mathrm{g}$ & 107 & & 3/31/08 & 8C17008 & AGG-RRL-001 \\
\hline 14269-63-7 & Thorium-230 & $<114$ & $\mathrm{pCi} / \mathrm{g}$ & 114 & & 3/31/08 & 8C17008 & AGG-RRL-001 \\
\hline 14331-85-2 & Protactinium-231 & $<12.7$ & $\mathrm{pCi} / \mathrm{g}$ & 12.7 & & 3/31/08 & 8C17008 & AGG-RRL-001 \\
\hline $14932-40-2$ & Thorium-231 & $<68.1$ & $\mathrm{pCi} / \mathrm{g}$ & 68.1 & & 3/31/08 & 8C17008 & AGG-RRL-001 \\
\hline 7440-29-1 & Thorium-232 & $<284$ & $\mathrm{pCi} / \mathrm{g}$ & 284 & & 3/31/08 & 8C17008 & AGG-RRL-001 \\
\hline 13981-14-1 & Protactinium-233 & $<0.771$ & $\mathrm{pCi} / \mathrm{g}$ & 0.771 & & 3/31/08 & 8C17008 & AGG-RRL-001 \\
\hline \multirow[t]{2}{*}{$15100-28-4$} & Protactinium-234 & $<1.24$ & $\mathrm{pCi} / \mathrm{g}$ & 1.24 & & 3/31/08 & 8C17008 & AGG-RRL-001 \\
\hline & Protactinium-234m & $<36.2$ & $\mathrm{pCi} / \mathrm{g}$ & 36.2 & & 3/31/08 & 8C17008 & AGG-RRL-001 \\
\hline 15065-10-8 & Thorium-234 & $<9.96$ & $\mathrm{pCi} / \mathrm{g}$ & 9.96 & & 3/31/08 & 8C17008 & AGG-RRL-001 \\
\hline 15117-96-1 & Uranium 235 & $<2.56$ & $\mathrm{pCi} / \mathrm{g}$ & 2.56 & & $3 / 31 / 08$ & 8C17008 & AGG-RRL-001 \\
\hline 13994-20-2 & Neptunium-237 & $<2.56$ & $\mathrm{pCi} / \mathrm{g}$ & 2.56 & & 3/31/08 & 8C17008 & AGG-RRL-001 \\
\hline \multirow[t]{2}{*}{ 13981-16-3 } & Plutonium-238 & $<3910$ & $\mathrm{pCi} / \mathrm{g}$ & 3910 & & 3/31/08 & 8C17008 & AGG-RRL-001 \\
\hline & Uranium 238 & $<5.5$ & $\mathrm{pCi} / \mathrm{g}$ & 5.5 & & 3/31/08 & 8C17008 & AGG-RRL-001 \\
\hline 15117-48-3 & Plutonium-239 & $<3830$ & $\mathrm{pCi} / \mathrm{g}$ & 3830 & & 3/31/08 & 8C17008 & AGG-RRL-001 \\
\hline 14119-33-6 & Plutonium-240 & $<3930$ & $\mathrm{pCi} / \mathrm{g}$ & 3930 & & 3/31/08 & 8C17008 & AGG-RRL-001 \\
\hline
\end{tabular}


GEA/Soil

\begin{tabular}{|c|c|c|c|c|c|c|c|c|}
\hline CAS \# & Analyte & Results & Units & MDA & UNC & Analyzed & Batch & Method \\
\hline HEIS No. & B1RYR7B & \multicolumn{2}{|c|}{ Lab ID: } & 0803012-13 & & & & \\
\hline 14596-10-2 & Americium-241 & $<1.45$ & $\mathrm{pCi} / \mathrm{g}$ & 1.45 & & 3/31/08 & 8C17008 & AGG-RRL-001 \\
\hline $14993-75-0$ & Americium-243 & $<0.629$ & $\mathrm{pCi} / \mathrm{g}$ & 0.629 & & $3 / 31 / 08$ & 8C17008 & AGG-RRL-001 \\
\hline 15757-87-6 & Curium-243 & $<1.2$ & $\mathrm{pCi} / \mathrm{g}$ & 1.2 & & 3/31/08 & 8C17008 & AGG-RRL-001 \\
\hline 15621-76-8 & Curium-245 & $<0.984$ & $\mathrm{pCi} / \mathrm{g}$ & 0.984 & & 3/31/08 & 8C17008 & AGG-RRL-001 \\
\hline HEIS No. & B1RYR7A & \multicolumn{2}{|c|}{ Lab ID: } & 0803012-14 & & & & \\
\hline 13966-32-0 & Sodium-22 & $<0.315$ & $\mathrm{pCi} / \mathrm{g}$ & 0.315 & & 3/31/08 & 8C17008 & AGG-RRL-001 \\
\hline 13966-00-2 & Potassium-40 & 18.8 & $\mathrm{pCi} / \mathrm{g}$ & 3.68 & 1.32 & 3/31/08 & 8C17008 & AGG-RRL-001 \\
\hline $14392-02-0$ & Chromium-51 & $<2.71$ & $\mathrm{pCi} / \mathrm{g}$ & 2.71 & & 3/31/08 & 8C17008 & AGG-RRL-001 \\
\hline 13966-31-9 & Manganese-54 & $<0.267$ & $\mathrm{pCi} / \mathrm{g}$ & 0.267 & & 3/31/08 & 8C17008 & AGG-RRL-001 \\
\hline $13981-50-5$ & Cobalt-57 & $<0.286$ & $\mathrm{pCi} / \mathrm{g}$ & 0.286 & & 3/31/08 & 8C17008 & AGG-RRL-001 \\
\hline $14596-12-4$ & Iron-59 & $<0.556$ & $\mathrm{pCi} / \mathrm{g}$ & 0.556 & & 3/31/08 & 8C17008 & AGG-RRL-001 \\
\hline $10198-40-0$ & Cobalt-60 & $<0.274$ & $\mathrm{pCi} / \mathrm{g}$ & 0.274 & & 3/31/08 & 8C17008 & AGG-RRL-001 \\
\hline 13982-39-3 & Zinc-65 & $<0.706$ & $\mathrm{pCi} / \mathrm{g}$ & 0.706 & & 3/31/08 & 8C17008 & AGG-RRL-001 \\
\hline $14265-71-5$ & Selenium-75 & $<0.45$ & $\mathrm{pCi} / \mathrm{g}$ & 0.45 & & $3 / 31 / 08$ & 8C17008 & AGG-RRL-001 \\
\hline 17056-36-9 & Rubidium-83 & $<0.523$ & $\mathrm{pCi} / \mathrm{g}$ & 0.523 & & 3/31/08 & 8C17008 & AGG-RRL-001 \\
\hline 13967-73-2 & Strontium-85 & $<0.349$ & $\mathrm{pCi} / \mathrm{g}$ & 0.349 & & 3/31/08 & 8C17008 & AGG-RRL-001 \\
\hline 14932-53-7 & Rubidium-86 & $<3.62$ & $\mathrm{pCi} / \mathrm{g}$ & 3.62 & & 3/31/08 & 8C17008 & AGG-RRL-001 \\
\hline 13982-36-0 & Yttrium-88 & $<0.21$ & $\mathrm{pCi} / \mathrm{g}$ & 0.21 & & 3/31/08 & 8C17008 & AGG-RRL-001 \\
\hline 14681-63-1 & Niobium-94 & $<0.27$ & $\mathrm{pCi} / \mathrm{g}$ & 0.27 & & 3/31/08 & 8C17008 & AGG-RRL-001 \\
\hline \multirow{3}{*}{$13967-76-5$} & Niobium-95 & $<0.262$ & $\mathrm{pCi} / \mathrm{g}$ & 0.262 & & 3/31/08 & 8C17008 & AGG-RRL-001 \\
\hline & Niobium-95m & $<1.28$ & $\mathrm{pCi} / \mathrm{g}$ & 1.28 & & 3/31/08 & 8C17008 & AGG-RRL-001 \\
\hline & Technetium-95m & $<0.444$ & $\mathrm{pCi} / \mathrm{g}$ & 0.444 & & 3/31/08 & 8C17008 & AGG-RRL-001 \\
\hline \multirow[t]{2}{*}{$13967-71-0$} & Zirconium-95 & $<0.455$ & $\mathrm{pCi} / \mathrm{g}$ & 0.455 & & 3/31/08 & 8C17008 & AGG-RRL-001 \\
\hline & Technetium-99m & $<0.286$ & $\mathrm{pCi} / \mathrm{g}$ & 0.286 & & 3/31/08 & 8C17008 & AGG-RRL-001 \\
\hline 13968-53-1 & Ruthenium-103 & $<0.284$ & $\mathrm{pCi} / \mathrm{g}$ & 0.284 & & 3/31/08 & 8C17008 & AGG-RRL-001 \\
\hline 13967-48-1 & Ruthenium-106 & $<2.8$ & $\mathrm{pCi} / \mathrm{g}$ & 2.8 & & 3/31/08 & 8C17008 & AGG-RRL-001 \\
\hline 14391-65-2 & Silver-108m & $<0.29$ & $\mathrm{pCi} / \mathrm{g}$ & 0.29 & & $3 / 31 / 08$ & 8C17008 & AGG-RRL-001 \\
\hline \multirow[t]{2}{*}{$14109-32-1$} & Cadmium-109 & $<7.49$ & $\mathrm{pCi} / \mathrm{g}$ & 7.49 & & 3/31/08 & 8C17008 & AGG-RRL-001 \\
\hline & Silver-110 & $<0.283$ & $\mathrm{pCi} / \mathrm{g}$ & 0.283 & & 3/31/08 & 8C17008 & AGG-RRL-001 \\
\hline 14391-76-5 & Silver-110m & $<0.283$ & $\mathrm{pCi} / \mathrm{g}$ & 0.283 & & 3/31/08 & 8C17008 & AGG-RRL-001 \\
\hline 13966-06-8 & Tin-113 & $<0.405$ & $\mathrm{pCi} / \mathrm{g}$ & 0.405 & & $3 / 31 / 08$ & 8C17008 & AGG-RRL-001 \\
\hline $14683-10-4$ & Antimony-124 & $<0.28$ & $\mathrm{pCi} / \mathrm{g}$ & 0.28 & & $3 / 31 / 08$ & 8C17008 & AGG-RRL-001 \\
\hline $14234-35-6$ & Antimony-125 & $<0.916$ & $\mathrm{pCi} / \mathrm{g}$ & 0.916 & & 3/31/08 & 8C17008 & AGG-RRL-001 \\
\hline 15756-32-8 & Antimony-126 & $<0.265$ & $\mathrm{pCi} / \mathrm{g}$ & 0.265 & & 3/31/08 & 8C17008 & AGG-RRL-001 \\
\hline $15832-50-5$ & Tin-126 & $<0.597$ & $\mathrm{pCi} / \mathrm{g}$ & 0.597 & & $3 / 31 / 08$ & 8C17008 & AGG-RRL-001 \\
\hline 10043-66-0 & Iodine-131 & $<0.326$ & $\mathrm{pCi} / \mathrm{g}$ & 0.326 & & 3/31/08 & 8C17008 & AGG-RRL-001 \\
\hline 13981-41-4 & Barium-133 & $<0.533$ & $\mathrm{pCi} / \mathrm{g}$ & 0.533 & & 3/31/08 & 8C17008 & AGG-RRL-001 \\
\hline 13967-70-9 & Cesium-134 & $<0.325$ & $\mathrm{pCi} / \mathrm{g}$ & 0.325 & & 3/31/08 & 8C17008 & AGG-RRL-001 \\
\hline $10045-97-3$ & Cesium-137 & $<0.304$ & $\mathrm{pCi} / \mathrm{g}$ & 0.304 & & 3/31/08 & 8C17008 & AGG-RRL-001 \\
\hline 13982-30-4 & Cerium-139 & $<0.332$ & $\mathrm{pCi} / \mathrm{g}$ & 0.332 & & 3/31/08 & 8C17008 & AGG-RRL-001 \\
\hline $14762-78-8$ & Cerium-144 & $<2.27$ & $\mathrm{pCi} / \mathrm{g}$ & 2.27 & & 3/31/08 & 8C17008 & AGG-RRL-001 \\
\hline 14683-23-9 & Europium-152 & $<1.09$ & $\mathrm{pCi} / \mathrm{g}$ & 1.09 & & $3 / 31 / 08$ & 8C17008 & AGG-RRL-001 \\
\hline $14276-65-4$ & Gadolinium-153 & $<0.822$ & $\mathrm{pCi} / \mathrm{g}$ & 0.822 & & 3/31/08 & 8C17008 & AGG-RRL-001 \\
\hline 15585-10-1 & Europium-154 & $<0.608$ & $\mathrm{pCi} / \mathrm{g}$ & 0.608 & & 3/31/08 & 8C17008 & AGG-RRL-001 \\
\hline 14391-16-3 & Europium-155 & $<0.838$ & $\mathrm{pCi} / \mathrm{g}$ & 0.838 & & 3/31/08 & 8C17008 & AGG-RRL-001 \\
\hline 13982-78-0 & Mercury-203 & $<0.346$ & $\mathrm{pCi} / \mathrm{g}$ & 0.346 & & 3/31/08 & 8C17008 & AGG-RRL-001 \\
\hline 14913-50-9 & Thallium-208 & $<0.359$ & $\mathrm{pCi} / \mathrm{g}$ & 0.359 & & 3/31/08 & 8C17008 & AGG-RRL-001 \\
\hline 14331-79-4 & Bismuth-210 & $<0.545$ & $\mathrm{pCi} / \mathrm{g}$ & 0.545 & & 3/31/08 & 8C17008 & AGG-RRL-001 \\
\hline
\end{tabular}


GEA/Soil

\begin{tabular}{|c|c|c|c|c|c|c|c|c|}
\hline CAS \# & Analyte & Results & Units & MDA & UNC & Analyzed & Batch & Method \\
\hline HEIS No. & B1RYR7A & \multicolumn{2}{|c|}{ Lab ID: } & 0803012-14 & \multirow{35}{*}{0.122} & & & \\
\hline \multirow[t]{3}{*}{$14255-04-0$} & Lead-210 & $<110$ & $\mathrm{pCi} / \mathrm{g}$ & 110 & & $3 / 31 / 08$ & 8C17008 & AGG-RRL-001 \\
\hline & Bismuth-211 & $<6.52$ & $\mathrm{pCi} / \mathrm{g}$ & 6.52 & & 3/31/08 & 8C17008 & AGG-RRL-001 \\
\hline & Lead-211 & $<8.91$ & $\mathrm{pCi} / \mathrm{g}$ & 8.91 & & 3/31/08 & 8C17008 & AGG-RRL-001 \\
\hline $15092-94-1$ & Lead-212 & $<0.539$ & $\mathrm{pCi} / \mathrm{g}$ & 0.539 & & 3/31/08 & 8C17008 & AGG-RRL-001 \\
\hline 14733-03-0 & Bismuth-214 & $<0.772$ & $\mathrm{pCi} / \mathrm{g}$ & 0.772 & & 3/31/08 & 8C17008 & AGG-RRL-001 \\
\hline $15067-28-4$ & Lead-214 & $<0.857$ & $\mathrm{pCi} / \mathrm{g}$ & 0.857 & & $3 / 31 / 08$ & 8C17008 & AGG-RRL-001 \\
\hline $14835-02-0$ & Radon-219 & $<2.8$ & $\mathrm{pCi} / \mathrm{g}$ & 2.8 & & 3/31/08 & 8C17008 & AGG-RRL-001 \\
\hline 22481-48-7 & Radon-220 & $<246$ & $\mathrm{pCi} / \mathrm{g}$ & 246 & & $3 / 31 / 08$ & 8C17008 & AGG-RRL-001 \\
\hline 28522-20-5 & Radon-221 & $<1.32$ & $\mathrm{pCi} / \mathrm{g}$ & 1.32 & & 3/31/08 & 8C17008 & AGG-RRL-001 \\
\hline 15756-98-6 & Francium-223 & $<9.45$ & $\mathrm{pCi} / \mathrm{g}$ & 9.45 & & 3/31/08 & 8C17008 & AGG-RRL-001 \\
\hline $15623-45-7$ & Radium-223 & $<2.02$ & $\mathrm{pCi} / \mathrm{g}$ & 2.02 & & 3/31/08 & 8C17008 & AGG-RRL-001 \\
\hline 13233-32-4 & Radium-224 & $<8.34$ & $\mathrm{pCi} / \mathrm{g}$ & 8.34 & & 3/31/08 & 8C17008 & AGG-RRL-001 \\
\hline 13982-63-3 & Radium-226 & $<8.1$ & $\mathrm{pCi} / \mathrm{g}$ & 8.1 & & 3/31/08 & 8C17008 & AGG-RRL-001 \\
\hline $15623-47-9$ & Thorium-227 & $<2.87$ & $\mathrm{pCi} / \mathrm{g}$ & 2.87 & & 3/31/08 & 8C17008 & AGG-RRL-001 \\
\hline $14331-83-0$ & Actinium-228 & $<1.31$ & $\mathrm{pCi} / \mathrm{g}$ & 1.31 & & 3/31/08 & 8C17008 & AGG-RRL-001 \\
\hline $14274-82-9$ & Thorium-228 & $<103$ & $\mathrm{pCi} / \mathrm{g}$ & 103 & & 3/31/08 & 8C17008 & AGG-RRL-001 \\
\hline 14269-63-7 & Thorium-230 & $<108$ & $\mathrm{pCi} / \mathrm{g}$ & 108 & & 3/31/08 & 8C17008 & AGG-RRL-001 \\
\hline $14331-85-2$ & Protactinium-231 & $<12$ & $\mathrm{pCi} / \mathrm{g}$ & 12 & & 3/31/08 & 8C17008 & AGG-RRL-001 \\
\hline $14932-40-2$ & Thorium-231 & $<56$ & $\mathrm{pCi} / \mathrm{g}$ & 56 & & $3 / 31 / 08$ & 8C17008 & AGG-RRL-001 \\
\hline $7440-29-1$ & Thorium-232 & $<337$ & $\mathrm{pCi} / \mathrm{g}$ & 337 & & 3/31/08 & 8C17008 & AGG-RRL-001 \\
\hline 13981-14-1 & Protactinium-233 & $<0.758$ & $\mathrm{pCi} / \mathrm{g}$ & 0.758 & & 3/31/08 & 8C17008 & AGG-RRL-001 \\
\hline \multirow[t]{2}{*}{$15100-28-4$} & Protactinium-234 & $<1.06$ & $\mathrm{pCi} / \mathrm{g}$ & 1.06 & & 3/31/08 & 8C17008 & AGG-RRL-001 \\
\hline & Protactinium-234m & $<33.5$ & $\mathrm{pCi} / \mathrm{g}$ & 33.5 & & $3 / 31 / 08$ & 8C17008 & AGG-RRL-001 \\
\hline $15065-10-8$ & Thorium-234 & $<9.65$ & $\mathrm{pCi} / \mathrm{g}$ & 9.65 & & 3/31/08 & 8C17008 & AGG-RRL-001 \\
\hline $15117-96-1$ & Uranium 235 & $<2.4$ & $\mathrm{pCi} / \mathrm{g}$ & 2.4 & & 3/31/08 & 8C17008 & AGG-RRL-001 \\
\hline 13994-20-2 & Neptunium-237 & $<2.18$ & $\mathrm{pCi} / \mathrm{g}$ & 2.18 & & 3/31/08 & 8C17008 & AGG-RRL-001 \\
\hline \multirow[t]{2}{*}{ 13981-16-3 } & Plutonium-238 & $<3230$ & $\mathrm{pCi} / \mathrm{g}$ & 3230 & & 3/31/08 & 8C17008 & AGG-RRL-001 \\
\hline & Uranium 238 & $<5$ & $\mathrm{pCi} / \mathrm{g}$ & 5 & & 3/31/08 & 8C17008 & AGG-RRL-001 \\
\hline $15117-48-3$ & Plutonium-239 & $<3950$ & $\mathrm{pCi} / \mathrm{g}$ & 3950 & & 3/31/08 & 8C17008 & AGG-RRL-001 \\
\hline 14119-33-6 & Plutonium-240 & $<3310$ & $\mathrm{pCi} / \mathrm{g}$ & 3310 & & 3/31/08 & 8C17008 & AGG-RRL-001 \\
\hline $14596-10-2$ & Americium-241 & $<1.65$ & $\mathrm{pCi} / \mathrm{g}$ & 1.65 & & 3/31/08 & 8C17008 & AGG-RRL-001 \\
\hline $14993-75-0$ & Americium-243 & $<0.594$ & $\mathrm{pCi} / \mathrm{g}$ & 0.594 & & 3/31/08 & 8C17008 & AGG-RRL-001 \\
\hline $15757-87-6$ & Curium-243 & $<1.01$ & $\mathrm{pCi} / \mathrm{g}$ & 1.01 & & 3/31/08 & 8C17008 & AGG-RRL-001 \\
\hline 15621-76-8 & Curium-245 & $<0.83$ & $\mathrm{pCi} / \mathrm{g}$ & 0.83 & & $3 / 31 / 08$ & 8C17008 & AGG-RRL-001 \\
\hline HEIS No. & B1RYR8B & \multicolumn{2}{|c|}{ Lab ID: } & 0803012-17 & \multirow{14}{*}{1.49} & & & \\
\hline 13966-32-0 & Sodium-22 & $<0.425$ & $\mathrm{pCi} / \mathrm{g}$ & 0.425 & & 3/31/08 & 8C17008 & AGG-RRL-001 \\
\hline $13966-00-2$ & Potassium-40 & 21.7 & $\mathrm{pCi} / \mathrm{g}$ & 3.79 & & 3/31/08 & 8C17008 & AGG-RRL-001 \\
\hline $14392-02-0$ & Chromium-51 & $<3.06$ & $\mathrm{pCi} / \mathrm{g}$ & 3.06 & & 3/31/08 & 8C17008 & AGG-RRL-001 \\
\hline 13966-31-9 & Manganese-54 & $<0.33$ & $\mathrm{pCi} / \mathrm{g}$ & 0.33 & & 3/31/08 & 8C17008 & AGG-RRL-001 \\
\hline $13981-50-5$ & Cobalt-57 & $<0.333$ & $\mathrm{pCi} / \mathrm{g}$ & 0.333 & & $3 / 31 / 08$ & 8C17008 & AGG-RRL-001 \\
\hline $14596-12-4$ & Iron-59 & $<0.565$ & $\mathrm{pCi} / \mathrm{g}$ & 0.565 & & 3/31/08 & 8C17008 & AGG-RRL-001 \\
\hline $10198-40-0$ & Cobalt-60 & $<0.396$ & $\mathrm{pCi} / \mathrm{g}$ & 0.396 & & 3/31/08 & 8C17008 & AGG-RRL-001 \\
\hline 13982-39-3 & Zinc-65 & $<0.811$ & $\mathrm{pCi} / \mathrm{g}$ & 0.811 & & 3/31/08 & 8C17008 & AGG-RRL-001 \\
\hline $14265-71-5$ & Selenium-75 & $<0.549$ & $\mathrm{pCi} / \mathrm{g}$ & 0.549 & & 3/31/08 & 8C17008 & AGG-RRL-001 \\
\hline $17056-36-9$ & Rubidium-83 & $<0.641$ & $\mathrm{pCi} / \mathrm{g}$ & 0.641 & & 3/31/08 & 8C17008 & AGG-RRL-001 \\
\hline 13967-73-2 & Strontium-85 & $<0.446$ & $\mathrm{pCi} / \mathrm{g}$ & 0.446 & & 3/31/08 & 8C17008 & AGG-RRL-001 \\
\hline 14932-53-7 & Rubidium-86 & $<4.15$ & $\mathrm{pCi} / \mathrm{g}$ & 4.15 & & 3/31/08 & 8C17008 & AGG-RRL-001 \\
\hline 13982-36-0 & Yttrium-88 & $<0.284$ & $\mathrm{pCi} / \mathrm{g}$ & 0.284 & & 3/31/08 & 8C17008 & AGG-RRL-001 \\
\hline
\end{tabular}


GEA/Soil

\begin{tabular}{|c|c|c|c|c|c|c|c|c|}
\hline CAS \# & Analyte & Results & Units & MDA & UNC & Analyzed & Batch & Method \\
\hline HEIS No. & B1RYR8B & \multicolumn{2}{|c|}{ Lab ID: } & 0803012-17 & & & & \\
\hline 14681-63-1 & Niobium-94 & $<0.312$ & $\mathrm{pCi} / \mathrm{g}$ & 0.312 & & 3/31/08 & 8C17008 & AGG-RRL-001 \\
\hline \multirow[t]{3}{*}{ 13967-76-5 } & Niobium-95 & $<0.331$ & $\mathrm{pCi} / \mathrm{g}$ & 0.331 & & 3/31/08 & 8C17008 & AGG-RRL-001 \\
\hline & Niobium-95m & $<1.58$ & $\mathrm{pCi} / \mathrm{g}$ & 1.58 & & 3/31/08 & 8C17008 & AGG-RRL-001 \\
\hline & Technetium-95m & $<0.545$ & $\mathrm{pCi} / \mathrm{g}$ & 0.545 & & 3/31/08 & 8C17008 & AGG-RRL-001 \\
\hline \multirow[t]{2}{*}{ 13967-71-0 } & Zirconium-95 & $<0.578$ & $\mathrm{pCi} / \mathrm{g}$ & 0.578 & & 3/31/08 & 8C17008 & AGG-RRL-001 \\
\hline & Technetium-99m & $<0.331$ & $\mathrm{pCi} / \mathrm{g}$ & 0.331 & & 3/31/08 & 8C17008 & AGG-RRL-001 \\
\hline 13968-53-1 & Ruthenium-103 & $<0.347$ & $\mathrm{pCi} / \mathrm{g}$ & 0.347 & & 3/31/08 & 8C17008 & AGG-RRL-001 \\
\hline 13967-48-1 & Ruthenium-106 & $<3.21$ & $\mathrm{pCi} / \mathrm{g}$ & 3.21 & & 3/31/08 & 8C17008 & AGG-RRL-001 \\
\hline 14391-65-2 & Silver-108m & $<0.341$ & $\mathrm{pCi} / \mathrm{g}$ & 0.341 & & 3/31/08 & 8C17008 & AGG-RRL-001 \\
\hline \multirow[t]{2}{*}{ 14109-32-1 } & Cadmium-109 & $<9.25$ & $\mathrm{pCi} / \mathrm{g}$ & 9.25 & & 3/31/08 & 8C17008 & AGG-RRL-001 \\
\hline & Silver-110 & $<0.339$ & $\mathrm{pCi} / \mathrm{g}$ & 0.339 & & 3/31/08 & 8C17008 & AGG-RRL-001 \\
\hline 14391-76-5 & Silver-110m & $<0.34$ & $\mathrm{pCi} / \mathrm{g}$ & 0.34 & & 3/31/08 & 8C17008 & AGG-RRL-001 \\
\hline 13966-06-8 & Tin-113 & $<0.502$ & $\mathrm{pCi} / \mathrm{g}$ & 0.502 & & 3/31/08 & 8C17008 & AGG-RRL-001 \\
\hline 14683-10-4 & Antimony-124 & $<0.349$ & $\mathrm{pCi} / \mathrm{g}$ & 0.349 & & 3/31/08 & 8C17008 & AGG-RRL-001 \\
\hline $14234-35-6$ & Antimony-125 & $<1.11$ & $\mathrm{pCi} / \mathrm{g}$ & 1.11 & & 3/31/08 & 8C17008 & AGG-RRL-001 \\
\hline 15756-32-8 & Antimony-126 & $<0.33$ & $\mathrm{pCi} / \mathrm{g}$ & 0.33 & & 3/31/08 & 8C17008 & AGG-RRL-001 \\
\hline $15832-50-5$ & Tin-126 & $<0.74$ & $\mathrm{pCi} / \mathrm{g}$ & 0.74 & & 3/31/08 & 8C17008 & AGG-RRL-001 \\
\hline 10043-66-0 & Iodine-131 & $<0.398$ & $\mathrm{pCi} / \mathrm{g}$ & 0.398 & & 3/31/08 & 8C17008 & AGG-RRL-001 \\
\hline 13981-41-4 & Barium-133 & $<0.624$ & $\mathrm{pCi} / \mathrm{g}$ & 0.624 & & 3/31/08 & 8C17008 & AGG-RRL-001 \\
\hline 13967-70-9 & Cesium-134 & $<0.404$ & $\mathrm{pCi} / \mathrm{g}$ & 0.404 & & 3/31/08 & 8C17008 & AGG-RRL-001 \\
\hline $10045-97-3$ & Cesium-137 & $<0.373$ & $\mathrm{pCi} / \mathrm{g}$ & 0.373 & & 3/31/08 & 8C17008 & AGG-RRL-001 \\
\hline 13982-30-4 & Cerium-139 & $<0.4$ & $\mathrm{pCi} / \mathrm{g}$ & 0.4 & & 3/31/08 & 8C17008 & AGG-RRL-001 \\
\hline 14762-78-8 & Cerium-144 & $<2.68$ & $\mathrm{pCi} / \mathrm{g}$ & 2.68 & & 3/31/08 & 8C17008 & AGG-RRL-001 \\
\hline 14683-23-9 & Europium-152 & $<1.32$ & $\mathrm{pCi} / \mathrm{g}$ & 1.32 & & 3/31/08 & 8C17008 & AGG-RRL-001 \\
\hline $14276-65-4$ & Gadolinium-153 & $<0.986$ & $\mathrm{pCi} / \mathrm{g}$ & 0.986 & & 3/31/08 & 8C17008 & AGG-RRL-001 \\
\hline 15585-10-1 & Europium-154 & $<0.701$ & $\mathrm{pCi} / \mathrm{g}$ & 0.701 & & 3/31/08 & 8C17008 & AGG-RRL-001 \\
\hline 14391-16-3 & Europium-155 & $<1.03$ & $\mathrm{pCi} / \mathrm{g}$ & 1.03 & & 3/31/08 & 8C17008 & AGG-RRL-001 \\
\hline 13982-78-0 & Mercury-203 & $<0.43$ & $\mathrm{pCi} / \mathrm{g}$ & 0.43 & & 3/31/08 & 8C17008 & AGG-RRL-001 \\
\hline $14913-50-9$ & Thallium-208 & $<0.435$ & $\mathrm{pCi} / \mathrm{g}$ & 0.435 & & 3/31/08 & 8C17008 & AGG-RRL-001 \\
\hline 14331-79-4 & Bismuth-210 & $<0.663$ & $\mathrm{pCi} / \mathrm{g}$ & 0.663 & & 3/31/08 & 8C17008 & AGG-RRL-001 \\
\hline \multirow[t]{3}{*}{$14255-04-0$} & Lead-210 & $<134$ & $\mathrm{pCi} / \mathrm{g}$ & 134 & & 3/31/08 & 8C17008 & AGG-RRL-001 \\
\hline & Bismuth-211 & $<7.88$ & $\mathrm{pCi} / \mathrm{g}$ & 7.88 & & 3/31/08 & 8C17008 & AGG-RRL-001 \\
\hline & Lead-211 & $<10.8$ & $\mathrm{pCi} / \mathrm{g}$ & 10.8 & & 3/31/08 & 8C17008 & AGG-RRL-001 \\
\hline 15092-94-1 & Lead-212 & $<0.865$ & $\mathrm{pCi} / \mathrm{g}$ & 0.865 & 0.196 & 3/31/08 & 8C17008 & AGG-RRL-001 \\
\hline 14733-03-0 & Bismuth-214 & $<0.882$ & $\mathrm{pCi} / \mathrm{g}$ & 0.882 & & 3/31/08 & 8C17008 & AGG-RRL-001 \\
\hline $15067-28-4$ & Lead-214 & $<0.995$ & $\mathrm{pCi} / \mathrm{g}$ & 0.995 & & 3/31/08 & 8C17008 & AGG-RRL-001 \\
\hline 14835-02-0 & Radon-219 & $<3.39$ & $\mathrm{pCi} / \mathrm{g}$ & 3.39 & & 3/31/08 & 8C17008 & AGG-RRL-001 \\
\hline 22481-48-7 & Radon-220 & $<316$ & $\mathrm{pCi} / \mathrm{g}$ & 316 & & 3/31/08 & 8C17008 & AGG-RRL-001 \\
\hline 28522-20-5 & Radon-221 & $<1.6$ & $\mathrm{pCi} / \mathrm{g}$ & 1.6 & & 3/31/08 & 8C17008 & AGG-RRL-001 \\
\hline 15756-98-6 & Francium-223 & $<11.4$ & $\mathrm{pCi} / \mathrm{g}$ & 11.4 & & 3/31/08 & 8C17008 & AGG-RRL-001 \\
\hline $15623-45-7$ & Radium-223 & $<2.46$ & $\mathrm{pCi} / \mathrm{g}$ & 2.46 & & 3/31/08 & 8C17008 & AGG-RRL-001 \\
\hline 13233-32-4 & Radium-224 & $<10.2$ & $\mathrm{pCi} / \mathrm{g}$ & 10.2 & & 3/31/08 & 8C17008 & AGG-RRL-001 \\
\hline 13982-63-3 & Radium-226 & $<9.79$ & $\mathrm{pCi} / \mathrm{g}$ & 9.79 & & 3/31/08 & 8C17008 & AGG-RRL-001 \\
\hline $15623-47-9$ & Thorium-227 & $<3.54$ & $\mathrm{pCi} / \mathrm{g}$ & 3.54 & & 3/31/08 & 8C17008 & AGG-RRL-001 \\
\hline 14331-83-0 & Actinium-228 & $<1.52$ & $\mathrm{pCi} / \mathrm{g}$ & 1.52 & & 3/31/08 & 8C17008 & AGG-RRL-001 \\
\hline 14274-82-9 & Thorium-228 & $<123$ & $\mathrm{pCi} / \mathrm{g}$ & 123 & & 3/31/08 & 8C17008 & AGG-RRL-001 \\
\hline 14269-63-7 & Thorium-230 & $<133$ & $\mathrm{pCi} / \mathrm{g}$ & 133 & & 3/31/08 & 8C17008 & AGG-RRL-001 \\
\hline 14331-85-2 & Protactinium-231 & $<14.2$ & $\mathrm{pCi} / \mathrm{g}$ & 14.2 & & 3/31/08 & 8C17008 & AGG-RRL-001 \\
\hline $14932-40-2$ & Thorium-231 & $<68.5$ & $\mathrm{pCi} / \mathrm{g}$ & 68.5 & & 3/31/08 & 8C17008 & AGG-RRL-001 \\
\hline
\end{tabular}


GEA/Soil

\begin{tabular}{|c|c|c|c|c|c|c|c|c|}
\hline CAS \# & Analyte & Results & Units & MDA & UNC & Analyzed & Batch & Method \\
\hline HEIS No. & B1RYR8B & \multicolumn{2}{|c|}{ Lab ID: } & 0803012-17 & & & & \\
\hline 7440-29-1 & Thorium-232 & $<416$ & $\mathrm{pCi} / \mathrm{g}$ & 416 & & $3 / 31 / 08$ & 8C17008 & AGG-RRL-001 \\
\hline 13981-14-1 & Protactinium-233 & $<0.886$ & $\mathrm{pCi} / \mathrm{g}$ & 0.886 & & 3/31/08 & 8C17008 & AGG-RRL-001 \\
\hline \multirow{2}{*}{$15100-28-4$} & Protactinium-234 & $<1.25$ & $\mathrm{pCi} / \mathrm{g}$ & 1.25 & & 3/31/08 & 8C17008 & AGG-RRL-001 \\
\hline & Protactinium-234m & $<45.5$ & $\mathrm{pCi} / \mathrm{g}$ & 45.5 & & 3/31/08 & 8C17008 & AGG-RRL-001 \\
\hline $15065-10-8$ & Thorium-234 & $<11.5$ & $\mathrm{pCi} / \mathrm{g}$ & 11.5 & & 3/31/08 & 8C17008 & AGG-RRL-001 \\
\hline 15117-96-1 & Uranium 235 & $<2.82$ & $\mathrm{pCi} / \mathrm{g}$ & 2.82 & & 3/31/08 & 8C17008 & AGG-RRL-001 \\
\hline 13994-20-2 & Neptunium-237 & $<2.69$ & $\mathrm{pCi} / \mathrm{g}$ & 2.69 & & 3/31/08 & 8C17008 & AGG-RRL-001 \\
\hline \multirow[t]{2}{*}{ 13981-16-3 } & Plutonium-238 & $<3900$ & $\mathrm{pCi} / \mathrm{g}$ & 3900 & & $3 / 31 / 08$ & 8C17008 & AGG-RRL-001 \\
\hline & Uranium 238 & $<5.97$ & $\mathrm{pCi} / \mathrm{g}$ & 5.97 & & 3/31/08 & 8C17008 & AGG-RRL-001 \\
\hline 15117-48-3 & Plutonium-239 & $<4720$ & $\mathrm{pCi} / \mathrm{g}$ & 4720 & & 3/31/08 & 8C17008 & AGG-RRL-001 \\
\hline 14119-33-6 & Plutonium-240 & $<4040$ & $\mathrm{pCi} / \mathrm{g}$ & 4040 & & 3/31/08 & 8C17008 & AGG-RRL-001 \\
\hline $14596-10-2$ & Americium-241 & $<2.05$ & $\mathrm{pCi} / \mathrm{g}$ & 2.05 & & 3/31/08 & 8C17008 & AGG-RRL-001 \\
\hline 14993-75-0 & Americium-243 & $<0.711$ & $\mathrm{pCi} / \mathrm{g}$ & 0.711 & & 3/31/08 & 8C17008 & AGG-RRL-001 \\
\hline 15757-87-6 & Curium-243 & $<1.23$ & $\mathrm{pCi} / \mathrm{g}$ & 1.23 & & 3/31/08 & 8C17008 & AGG-RRL-001 \\
\hline 15621-76-8 & Curium-245 & $<1.01$ & $\mathrm{pCi} / \mathrm{g}$ & 1.01 & & 3/31/08 & 8C17008 & AGG-RRL-001 \\
\hline HEIS No. & B1RYR8A & \multicolumn{2}{|c|}{ Lab ID: } & 0803012-18 & & & & \\
\hline 13966-32-0 & Sodium-22 & $<0.383$ & $\mathrm{pCi} / \mathrm{g}$ & 0.383 & & $3 / 31 / 08$ & 8C17008 & AGG-RRL-001 \\
\hline 13966-00-2 & Potassium-40 & 20.1 & $\mathrm{pCi} / \mathrm{g}$ & 3.49 & 1.34 & 3/31/08 & 8C17008 & AGG-RRL-001 \\
\hline $14392-02-0$ & Chromium-51 & $<3.31$ & $\mathrm{pCi} / \mathrm{g}$ & 3.31 & & 3/31/08 & 8C17008 & AGG-RRL-001 \\
\hline 13966-31-9 & Manganese-54 & $<0.367$ & $\mathrm{pCi} / \mathrm{g}$ & 0.367 & & 3/31/08 & 8C17008 & AGG-RRL-001 \\
\hline 13981-50-5 & Cobalt-57 & $<0.354$ & $\mathrm{pCi} / \mathrm{g}$ & 0.354 & & 3/31/08 & 8C17008 & AGG-RRL-001 \\
\hline $14596-12-4$ & Iron-59 & $<0.615$ & $\mathrm{pCi} / \mathrm{g}$ & 0.615 & & 3/31/08 & 8C17008 & AGG-RRL-001 \\
\hline $10198-40-0$ & Cobalt-60 & $<0.359$ & $\mathrm{pCi} / \mathrm{g}$ & 0.359 & & 3/31/08 & 8C17008 & AGG-RRL-001 \\
\hline 13982-39-3 & Zinc-65 & $<0.782$ & $\mathrm{pCi} / \mathrm{g}$ & 0.782 & & 3/31/08 & 8C17008 & AGG-RRL-001 \\
\hline 14265-71-5 & Selenium-75 & $<0.551$ & $\mathrm{pCi} / \mathrm{g}$ & 0.551 & & 3/31/08 & 8C17008 & AGG-RRL-001 \\
\hline $17056-36-9$ & Rubidium-83 & $<0.853$ & $\mathrm{pCi} / \mathrm{g}$ & 0.853 & & 3/31/08 & 8C17008 & AGG-RRL-001 \\
\hline 13967-73-2 & Strontium-85 & $<0.401$ & $\mathrm{pCi} / \mathrm{g}$ & 0.401 & & 3/31/08 & 8C17008 & AGG-RRL-001 \\
\hline 14932-53-7 & Rubidium-86 & $<4.33$ & $\mathrm{pCi} / \mathrm{g}$ & 4.33 & & 3/31/08 & 8C17008 & AGG-RRL-001 \\
\hline 13982-36-0 & Yttrium-88 & $<0.309$ & $\mathrm{pCi} / \mathrm{g}$ & 0.309 & & 3/31/08 & 8C17008 & AGG-RRL-001 \\
\hline 14681-63-1 & Niobium-94 & $<0.353$ & $\mathrm{pCi} / \mathrm{g}$ & 0.353 & & 3/31/08 & 8C17008 & AGG-RRL-001 \\
\hline \multirow[t]{3}{*}{ 13967-76-5 } & Niobium-95 & $<0.339$ & $\mathrm{pCi} / \mathrm{g}$ & 0.339 & & 3/31/08 & 8C17008 & AGG-RRL-001 \\
\hline & Niobium-95m & $<1.57$ & $\mathrm{pCi} / \mathrm{g}$ & 1.57 & & 3/31/08 & 8C17008 & AGG-RRL-001 \\
\hline & Technetium-95m & $<0.529$ & $\mathrm{pCi} / \mathrm{g}$ & 0.529 & & 3/31/08 & 8C17008 & AGG-RRL-001 \\
\hline \multirow[t]{2}{*}{ 13967-71-0 } & Zirconium-95 & $<0.61$ & $\mathrm{pCi} / \mathrm{g}$ & 0.61 & & 3/31/08 & 8C17008 & AGG-RRL-001 \\
\hline & Technetium-99m & $<0.353$ & $\mathrm{pCi} / \mathrm{g}$ & 0.353 & & 3/31/08 & 8C17008 & AGG-RRL-001 \\
\hline 13968-53-1 & Ruthenium-103 & $<0.404$ & $\mathrm{pCi} / \mathrm{g}$ & 0.404 & & 3/31/08 & 8C17008 & AGG-RRL-001 \\
\hline $13967-48-1$ & Ruthenium-106 & $<3.95$ & $\mathrm{pCi} / \mathrm{g}$ & 3.95 & & 3/31/08 & 8C17008 & AGG-RRL-001 \\
\hline $14391-65-2$ & Silver-108m & $<0.336$ & $\mathrm{pCi} / \mathrm{g}$ & 0.336 & & 3/31/08 & 8C17008 & AGG-RRL-001 \\
\hline \multirow[t]{2}{*}{ 14109-32-1 } & Cadmium-109 & $<10.1$ & $\mathrm{pCi} / \mathrm{g}$ & 10.1 & & 3/31/08 & 8C17008 & AGG-RRL-001 \\
\hline & Silver-110 & $<0.374$ & $\mathrm{pCi} / \mathrm{g}$ & 0.374 & & 3/31/08 & 8C17008 & AGG-RRL-001 \\
\hline 14391-76-5 & Silver-110m & $<0.375$ & $\mathrm{pCi} / \mathrm{g}$ & 0.375 & & 3/31/08 & 8C17008 & AGG-RRL-001 \\
\hline 13966-06-8 & Tin-113 & $<0.493$ & $\mathrm{pCi} / \mathrm{g}$ & 0.493 & & 3/31/08 & 8C17008 & AGG-RRL-001 \\
\hline $14683-10-4$ & Antimony-124 & $<0.4$ & $\mathrm{pCi} / \mathrm{g}$ & 0.4 & & 3/31/08 & 8C17008 & AGG-RRL-001 \\
\hline $14234-35-6$ & Antimony-125 & $<1.05$ & $\mathrm{pCi} / \mathrm{g}$ & 1.05 & & 3/31/08 & 8C17008 & AGG-RRL-001 \\
\hline 15756-32-8 & Antimony-126 & $<0.353$ & $\mathrm{pCi} / \mathrm{g}$ & 0.353 & & 3/31/08 & 8C17008 & AGG-RRL-001 \\
\hline $15832-50-5$ & Tin-126 & $<0.826$ & $\mathrm{pCi} / \mathrm{g}$ & 0.826 & & 3/31/08 & 8C17008 & AGG-RRL-001 \\
\hline 10043-66-0 & Iodine-131 & $<0.43$ & $\mathrm{pCi} / \mathrm{g}$ & 0.43 & & 3/31/08 & 8C17008 & AGG-RRL-001 \\
\hline 13981-41-4 & Barium-133 & $<0.6$ & $\mathrm{pCi} / \mathrm{g}$ & 0.6 & & 3/31/08 & 8C17008 & AGG-RRL-001 \\
\hline
\end{tabular}


GEA/Soil

\begin{tabular}{|c|c|c|c|c|c|c|c|c|}
\hline CAS \# & Analyte & Results & Units & MDA & UNC & Analyzed & Batch & Method \\
\hline HEIS No. & B1RYR8A & \multicolumn{3}{|c|}{ Lab ID: $\quad$ 0803012-18 } & & & & \\
\hline 13967-70-9 & Cesium-134 & $<0.401$ & $\mathrm{pCi} / \mathrm{g}$ & 0.401 & & $3 / 31 / 08$ & 8C17008 & AGG-RRL-001 \\
\hline $10045-97-3$ & Cesium-137 & $<0.416$ & $\mathrm{pCi} / \mathrm{g}$ & 0.416 & & 3/31/08 & 8C17008 & AGG-RRL-001 \\
\hline 13982-30-4 & Cerium-139 & $<0.408$ & $\mathrm{pCi} / \mathrm{g}$ & 0.408 & & 3/31/08 & 8C17008 & AGG-RRL-001 \\
\hline $14762-78-8$ & Cerium-144 & $<2.84$ & $\mathrm{pCi} / \mathrm{g}$ & 2.84 & & 3/31/08 & 8C17008 & AGG-RRL-001 \\
\hline $14683-23-9$ & Europium-152 & $<1.41$ & $\mathrm{pCi} / \mathrm{g}$ & 1.41 & & $3 / 31 / 08$ & 8C17008 & AGG-RRL-001 \\
\hline $14276-65-4$ & Gadolinium-153 & $<1.1$ & $\mathrm{pCi} / \mathrm{g}$ & 1.1 & & 3/31/08 & 8C17008 & AGG-RRL-001 \\
\hline 15585-10-1 & Europium-154 & $<0.752$ & $\mathrm{pCi} / \mathrm{g}$ & 0.752 & & $3 / 31 / 08$ & 8C17008 & AGG-RRL-001 \\
\hline 14391-16-3 & Europium-155 & $<1.18$ & $\mathrm{pCi} / \mathrm{g}$ & 1.18 & & $3 / 31 / 08$ & 8C17008 & AGG-RRL-001 \\
\hline $13982-78-0$ & Mercury-203 & $<0.437$ & $\mathrm{pCi} / \mathrm{g}$ & 0.437 & & 3/31/08 & 8C17008 & AGG-RRL-001 \\
\hline 14913-50-9 & Thallium-208 & $<0.438$ & $\mathrm{pCi} / \mathrm{g}$ & 0.438 & & 3/31/08 & 8C17008 & AGG-RRL-001 \\
\hline 14331-79-4 & Bismuth-210 & $<0.66$ & $\mathrm{pCi} / \mathrm{g}$ & 0.66 & & 3/31/08 & 8C17008 & AGG-RRL-001 \\
\hline \multirow[t]{3}{*}{$14255-04-0$} & Lead-210 & $<49.5$ & $\mathrm{pCi} / \mathrm{g}$ & 49.5 & & 3/31/08 & 8C17008 & AGG-RRL-001 \\
\hline & Bismuth-211 & $<7.83$ & $\mathrm{pCi} / \mathrm{g}$ & 7.83 & & 3/31/08 & 8C17008 & AGG-RRL-001 \\
\hline & Lead-211 & $<10.7$ & $\mathrm{pCi} / \mathrm{g}$ & 10.7 & & 3/31/08 & 8C17008 & AGG-RRL-001 \\
\hline $15092-94-1$ & Lead-212 & $<0.904$ & $\mathrm{pCi} / \mathrm{g}$ & 0.904 & & 3/31/08 & 8C17008 & AGG-RRL-001 \\
\hline $14733-03-0$ & Bismuth-214 & $<0.867$ & $\mathrm{pCi} / \mathrm{g}$ & 0.867 & & 3/31/08 & 8C17008 & AGG-RRL-001 \\
\hline $15067-28-4$ & Lead-214 & $<0.962$ & $\mathrm{pCi} / \mathrm{g}$ & 0.962 & & 3/31/08 & 8C17008 & AGG-RRL-001 \\
\hline $14835-02-0$ & Radon-219 & $<3.33$ & $\mathrm{pCi} / \mathrm{g}$ & 3.33 & & 3/31/08 & 8C17008 & AGG-RRL-001 \\
\hline 22481-48-7 & Radon-220 & $<317$ & $\mathrm{pCi} / \mathrm{g}$ & 317 & & 3/31/08 & 8C17008 & AGG-RRL-001 \\
\hline 28522-20-5 & Radon-221 & $<1.6$ & $\mathrm{pCi} / \mathrm{g}$ & 1.6 & & 3/31/08 & 8C17008 & AGG-RRL-001 \\
\hline $15756-98-6$ & Francium-223 & $<11.6$ & $\mathrm{pCi} / \mathrm{g}$ & 11.6 & & 3/31/08 & 8C17008 & AGG-RRL-001 \\
\hline $15623-45-7$ & Radium-223 & $<2.4$ & $\mathrm{pCi} / \mathrm{g}$ & 2.4 & & 3/31/08 & 8C17008 & AGG-RRL-001 \\
\hline $13233-32-4$ & Radium-224 & $<9.77$ & $\mathrm{pCi} / \mathrm{g}$ & 9.77 & & $3 / 31 / 08$ & 8C17008 & AGG-RRL-001 \\
\hline 13982-63-3 & Radium-226 & $<9.9$ & $\mathrm{pCi} / \mathrm{g}$ & 9.9 & & 3/31/08 & 8C17008 & AGG-RRL-001 \\
\hline $15623-47-9$ & Thorium-227 & $<3.5$ & $\mathrm{pCi} / \mathrm{g}$ & 3.5 & & 3/31/08 & 8C17008 & AGG-RRL-001 \\
\hline $14331-83-0$ & Actinium-228 & $<1.57$ & $\mathrm{pCi} / \mathrm{g}$ & 1.57 & & 3/31/08 & 8C17008 & AGG-RRL-001 \\
\hline 14274-82-9 & Thorium-228 & $<125$ & $\mathrm{pCi} / \mathrm{g}$ & 125 & & 3/31/08 & 8C17008 & AGG-RRL-001 \\
\hline $14269-63-7$ & Thorium-230 & $<137$ & $\mathrm{pCi} / \mathrm{g}$ & 137 & & 3/31/08 & 8C17008 & AGG-RRL-001 \\
\hline $14331-85-2$ & Protactinium-231 & $<14.7$ & $\mathrm{pCi} / \mathrm{g}$ & 14.7 & & 3/31/08 & 8C17008 & AGG-RRL-001 \\
\hline $14932-40-2$ & Thorium-231 & $<77.5$ & $\mathrm{pCi} / \mathrm{g}$ & 77.5 & & $3 / 31 / 08$ & 8C17008 & AGG-RRL-001 \\
\hline 7440-29-1 & Thorium-232 & $<344$ & $\mathrm{pCi} / \mathrm{g}$ & 344 & & 3/31/08 & 8C17008 & AGG-RRL-001 \\
\hline 13981-14-1 & Protactinium-233 & $<0.893$ & $\mathrm{pCi} / \mathrm{g}$ & 0.893 & & 3/31/08 & 8C17008 & AGG-RRL-001 \\
\hline \multirow[t]{2}{*}{$15100-28-4$} & Protactinium-234 & $<1.44$ & $\mathrm{pCi} / \mathrm{g}$ & 1.44 & & 3/31/08 & 8C17008 & AGG-RRL-001 \\
\hline & Protactinium-234m & $<41.9$ & $\mathrm{pCi} / \mathrm{g}$ & 41.9 & & $3 / 31 / 08$ & 8C17008 & AGG-RRL-001 \\
\hline 15065-10-8 & Thorium-234 & $<12$ & $\mathrm{pCi} / \mathrm{g}$ & 12 & & 3/31/08 & 8C17008 & AGG-RRL-001 \\
\hline 15117-96-1 & Uranium 235 & $<2.97$ & $\mathrm{pCi} / \mathrm{g}$ & 2.97 & & $3 / 31 / 08$ & 8C17008 & AGG-RRL-001 \\
\hline $13994-20-2$ & Neptunium-237 & $<3.06$ & $\mathrm{pCi} / \mathrm{g}$ & 3.06 & & 3/31/08 & 8C17008 & AGG-RRL-001 \\
\hline \multirow[t]{2}{*}{ 13981-16-3 } & Plutonium-238 & $<4500$ & $\mathrm{pCi} / \mathrm{g}$ & 4500 & & 3/31/08 & 8C17008 & AGG-RRL-001 \\
\hline & Uranium 238 & $<6.34$ & $\mathrm{pCi} / \mathrm{g}$ & 6.34 & & $3 / 31 / 08$ & 8C17008 & AGG-RRL-001 \\
\hline $15117-48-3$ & Plutonium-239 & $<4650$ & $\mathrm{pCi} / \mathrm{g}$ & 4650 & & 3/31/08 & 8C17008 & AGG-RRL-001 \\
\hline 14119-33-6 & Plutonium-240 & $<4470$ & $\mathrm{pCi} / \mathrm{g}$ & 4470 & & 3/31/08 & 8C17008 & AGG-RRL-001 \\
\hline $14596-10-2$ & Americium-241 & $<1.75$ & $\mathrm{pCi} / \mathrm{g}$ & 1.75 & & $3 / 31 / 08$ & 8C17008 & AGG-RRL-001 \\
\hline $14993-75-0$ & Americium-243 & $<0.749$ & $\mathrm{pCi} / \mathrm{g}$ & 0.749 & & $3 / 31 / 08$ & 8C17008 & AGG-RRL-001 \\
\hline $15757-87-6$ & Curium-243 & $<1.37$ & $\mathrm{pCi} / \mathrm{g}$ & 1.37 & & 3/31/08 & 8C17008 & AGG-RRL-001 \\
\hline $15621-76-8$ & Curium-245 & $<1.13$ & $\mathrm{pCi} / \mathrm{g}$ & 1.13 & & 3/31/08 & 8C17008 & AGG-RRL-001 \\
\hline HEIS No. & B1RYR9B & \multicolumn{2}{|c|}{ Lab ID: } & 0803012-21 & & & & \\
\hline 13966-32-0 & Sodium-22 & $<0.33$ & $\mathrm{pCi} / \mathrm{g}$ & 0.33 & & 3/31/08 & 8C17008 & AGG-RRL-001 \\
\hline 13966-00-2 & Potassium- 40 & 20.1 & $\mathrm{pCi} / \mathrm{g}$ & 2.34 & 1.11 & 3/31/08 & 8C17008 & AGG-RRL-001 \\
\hline
\end{tabular}


GEA/Soil

\begin{tabular}{|c|c|c|c|c|c|c|c|c|}
\hline CAS \# & Analyte & Results & Units & MDA & UNC & Analyzed & Batch & Method \\
\hline HEIS No. & B1RYR9B & \multicolumn{3}{|c|}{ Lab ID: $\quad$ 0803012-21 } & & & & \\
\hline 14392-02-0 & Chromium-51 & $<2.67$ & $\mathrm{pCi} / \mathrm{g}$ & 2.67 & & $3 / 31 / 08$ & 8C17008 & AGG-RRL-001 \\
\hline 13966-31-9 & Manganese-54 & $<0.281$ & $\mathrm{pCi} / \mathrm{g}$ & 0.281 & & 3/31/08 & 8C17008 & AGG-RRL-001 \\
\hline $13981-50-5$ & Cobalt-57 & $<0.284$ & $\mathrm{pCi} / \mathrm{g}$ & 0.284 & & 3/31/08 & 8C17008 & AGG-RRL-001 \\
\hline $14596-12-4$ & Iron-59 & $<0.561$ & $\mathrm{pCi} / \mathrm{g}$ & 0.561 & & 3/31/08 & 8C17008 & AGG-RRL-001 \\
\hline 10198-40-0 & Cobalt-60 & $<0.276$ & $\mathrm{pCi} / \mathrm{g}$ & 0.276 & & $3 / 31 / 08$ & 8C17008 & AGG-RRL-001 \\
\hline 13982-39-3 & Zinc-65 & $<0.651$ & $\mathrm{pCi} / \mathrm{g}$ & 0.651 & & 3/31/08 & 8C17008 & AGG-RRL-001 \\
\hline $14265-71-5$ & Selenium-75 & $<0.45$ & $\mathrm{pCi} / \mathrm{g}$ & 0.45 & & $3 / 31 / 08$ & 8C17008 & AGG-RRL-001 \\
\hline 17056-36-9 & Rubidium-83 & $<0.682$ & $\mathrm{pCi} / \mathrm{g}$ & 0.682 & & 3/31/08 & 8C17008 & AGG-RRL-001 \\
\hline 13967-73-2 & Strontium-85 & $<0.321$ & $\mathrm{pCi} / \mathrm{g}$ & 0.321 & & $3 / 31 / 08$ & 8C17008 & AGG-RRL-001 \\
\hline 14932-53-7 & Rubidium-86 & $<3.56$ & $\mathrm{pCi} / \mathrm{g}$ & 3.56 & & $3 / 31 / 08$ & 8C17008 & AGG-RRL-001 \\
\hline 13982-36-0 & Yttrium-88 & $<0.229$ & $\mathrm{pCi} / \mathrm{g}$ & 0.229 & & 3/31/08 & 8C17008 & AGG-RRL-001 \\
\hline 14681-63-1 & Niobium-94 & $<0.279$ & $\mathrm{pCi} / \mathrm{g}$ & 0.279 & & $3 / 31 / 08$ & 8C17008 & AGG-RRL-001 \\
\hline \multirow[t]{3}{*}{$13967-76-5$} & Niobium-95 & $<0.279$ & pCi/g & 0.279 & & $3 / 31 / 08$ & 8C17008 & AGG-RRL-001 \\
\hline & Niobium-95m & $<1.23$ & $\mathrm{pCi} / \mathrm{g}$ & 1.23 & & $3 / 31 / 08$ & 8C17008 & AGG-RRL-001 \\
\hline & Technetium-95m & $<0.437$ & $\mathrm{pCi} / \mathrm{g}$ & 0.437 & & $3 / 31 / 08$ & 8C17008 & AGG-RRL-001 \\
\hline \multirow[t]{2}{*}{ 13967-71-0 } & Zirconium-95 & $<0.488$ & $\mathrm{pCi} / \mathrm{g}$ & 0.488 & & $3 / 31 / 08$ & 8C17008 & AGG-RRL-001 \\
\hline & Technetium-99m & $<0.281$ & $\mathrm{pCi} / \mathrm{g}$ & 0.281 & & 3/31/08 & 8C17008 & AGG-RRL-001 \\
\hline 13968-53-1 & Ruthenium-103 & $<0.326$ & $\mathrm{pCi} / \mathrm{g}$ & 0.326 & & 3/31/08 & 8C17008 & AGG-RRL-001 \\
\hline 13967-48-1 & Ruthenium-106 & $<3.15$ & $\mathrm{pCi} / \mathrm{g}$ & 3.15 & & 3/31/08 & 8C17008 & AGG-RRL-001 \\
\hline 14391-65-2 & Silver-108m & $<0.293$ & $\mathrm{pCi} / \mathrm{g}$ & 0.293 & & 3/31/08 & 8C17008 & AGG-RRL-001 \\
\hline \multirow[t]{2}{*}{ 14109-32-1 } & Cadmium-109 & $<8.07$ & $\mathrm{pCi} / \mathrm{g}$ & 8.07 & & 3/31/08 & 8C17008 & AGG-RRL-001 \\
\hline & Silver-110 & $<0.296$ & $\mathrm{pCi} / \mathrm{g}$ & 0.296 & & 3/31/08 & 8C17008 & AGG-RRL-001 \\
\hline $14391-76-5$ & Silver-110m & $<0.296$ & $\mathrm{pCi} / \mathrm{g}$ & 0.296 & & 3/31/08 & 8C17008 & AGG-RRL-001 \\
\hline 13966-06-8 & Tin-113 & $<0.388$ & $\mathrm{pCi} / \mathrm{g}$ & 0.388 & & 3/31/08 & 8C17008 & AGG-RRL-001 \\
\hline $14683-10-4$ & Antimony-124 & $<0.332$ & $\mathrm{pCi} / \mathrm{g}$ & 0.332 & & 3/31/08 & 8C17008 & AGG-RRL-001 \\
\hline $14234-35-6$ & Antimony-125 & $<0.907$ & $\mathrm{pCi} / \mathrm{g}$ & 0.907 & & 3/31/08 & 8C17008 & AGG-RRL-001 \\
\hline 15756-32-8 & Antimony-126 & $<0.27$ & $\mathrm{pCi} / \mathrm{g}$ & 0.27 & & 3/31/08 & 8C17008 & AGG-RRL-001 \\
\hline $15832-50-5$ & Tin-126 & $<0.662$ & $\mathrm{pCi} / \mathrm{g}$ & 0.662 & & $3 / 31 / 08$ & 8C17008 & AGG-RRL-001 \\
\hline 10043-66-0 & Iodine-131 & $<0.345$ & $\mathrm{pCi} / \mathrm{g}$ & 0.345 & & 3/31/08 & 8C17008 & AGG-RRL-001 \\
\hline $13981-41-4$ & Barium-133 & $<0.479$ & $\mathrm{pCi} / \mathrm{g}$ & 0.479 & & 3/31/08 & 8C17008 & AGG-RRL-001 \\
\hline 13967-70-9 & Cesium-134 & $<0.324$ & $\mathrm{pCi} / \mathrm{g}$ & 0.324 & & 3/31/08 & 8C17008 & AGG-RRL-001 \\
\hline $10045-97-3$ & Cesium-137 & $<0.326$ & $\mathrm{pCi} / \mathrm{g}$ & 0.326 & & 3/31/08 & 8C17008 & AGG-RRL-001 \\
\hline 13982-30-4 & Cerium-139 & $<0.324$ & $\mathrm{pCi} / \mathrm{g}$ & 0.324 & & $3 / 31 / 08$ & 8C17008 & AGG-RRL-001 \\
\hline 14762-78-8 & Cerium-144 & $<2.32$ & $\mathrm{pCi} / \mathrm{g}$ & 2.32 & & 3/31/08 & 8C17008 & AGG-RRL-001 \\
\hline 14683-23-9 & Europium-152 & $<1.12$ & $\mathrm{pCi} / \mathrm{g}$ & 1.12 & & 3/31/08 & 8C17008 & AGG-RRL-001 \\
\hline $14276-65-4$ & Gadolinium-153 & $<0.87$ & $\mathrm{pCi} / \mathrm{g}$ & 0.87 & & $3 / 31 / 08$ & 8C17008 & AGG-RRL-001 \\
\hline 15585-10-1 & Europium-154 & $<0.601$ & $\mathrm{pCi} / \mathrm{g}$ & 0.601 & & 3/31/08 & 8C17008 & AGG-RRL-001 \\
\hline 14391-16-3 & Europium-155 & $<0.939$ & $\mathrm{pCi} / \mathrm{g}$ & 0.939 & & 3/31/08 & 8C17008 & AGG-RRL-001 \\
\hline 13982-78-0 & Mercury-203 & $<0.34$ & $\mathrm{pCi} / \mathrm{g}$ & 0.34 & & $3 / 31 / 08$ & 8C17008 & AGG-RRL-001 \\
\hline $14913-50-9$ & Thallium-208 & $<0.359$ & $\mathrm{pCi} / \mathrm{g}$ & 0.359 & & $3 / 31 / 08$ & 8C17008 & AGG-RRL-001 \\
\hline 14331-79-4 & Bismuth-210 & $<0.543$ & $\mathrm{pCi} / \mathrm{g}$ & 0.543 & & $3 / 31 / 08$ & 8C17008 & AGG-RRL-001 \\
\hline \multirow[t]{3}{*}{$14255-04-0$} & Lead-210 & $<38.7$ & $\mathrm{pCi} / \mathrm{g}$ & 38.7 & & 3/31/08 & 8C17008 & AGG-RRL-001 \\
\hline & Bismuth-211 & $<6.01$ & $\mathrm{pCi} / \mathrm{g}$ & 6.01 & & $3 / 31 / 08$ & 8C17008 & AGG-RRL-001 \\
\hline & Lead-211 & $<8.22$ & $\mathrm{pCi} / \mathrm{g}$ & 8.22 & & 3/31/08 & 8C17008 & AGG-RRL-001 \\
\hline 15092-94-1 & Lead-212 & $<0.709$ & $\mathrm{pCi} / \mathrm{g}$ & 0.709 & & 3/31/08 & 8C17008 & AGG-RRL-001 \\
\hline 14733-03-0 & Bismuth-214 & $<0.707$ & $\mathrm{pCi} / \mathrm{g}$ & 0.707 & & $3 / 31 / 08$ & 8C17008 & AGG-RRL-001 \\
\hline $15067-28-4$ & Lead-214 & $<0.771$ & $\mathrm{pCi} / \mathrm{g}$ & 0.771 & & 3/31/08 & 8C17008 & AGG-RRL-001 \\
\hline $14835-02-0$ & Radon-219 & $<2.72$ & $\mathrm{pCi} / \mathrm{g}$ & 2.72 & & 3/31/08 & 8C17008 & AGG-RRL-001 \\
\hline 22481-48-7 & Radon-220 & $<279$ & $\mathrm{pCi} / \mathrm{g}$ & 279 & & 3/31/08 & 8C17008 & AGG-RRL-001 \\
\hline
\end{tabular}


GEA/Soil

\begin{tabular}{|c|c|c|c|c|c|c|c|c|}
\hline CAS \# & Analyte & Results & Units & MDA & UNC & Analyzed & Batch & Method \\
\hline HEIS No. & B1RYR9B & \multicolumn{2}{|c|}{ Lab ID: } & 0803012-21 & & & & \\
\hline $28522-20-5$ & Radon-221 & $<1.31$ & $\mathrm{pCi} / \mathrm{g}$ & 1.31 & & 3/31/08 & 8C17008 & AGG-RRL-001 \\
\hline 15756-98-6 & Francium-223 & $<9.05$ & $\mathrm{pCi} / \mathrm{g}$ & 9.05 & & $3 / 31 / 08$ & 8C17008 & AGG-RRL-001 \\
\hline 15623-45-7 & Radium-223 & $<1.98$ & $\mathrm{pCi} / \mathrm{g}$ & 1.98 & & 3/31/08 & 8C17008 & AGG-RRL-001 \\
\hline 13233-32-4 & Radium-224 & $<7.7$ & $\mathrm{pCi} / \mathrm{g}$ & 7.7 & & 3/31/08 & 8C17008 & AGG-RRL-001 \\
\hline 13982-63-3 & Radium-226 & $<8.08$ & $\mathrm{pCi} / \mathrm{g}$ & 8.08 & & 3/31/08 & 8C17008 & AGG-RRL-001 \\
\hline $15623-47-9$ & Thorium-227 & $<2.74$ & $\mathrm{pCi} / \mathrm{g}$ & 2.74 & & 3/31/08 & 8C17008 & AGG-RRL-001 \\
\hline 14331-83-0 & Actinium-228 & $<1.2$ & $\mathrm{pCi} / \mathrm{g}$ & 1.2 & & 3/31/08 & 8C17008 & AGG-RRL-001 \\
\hline 14274-82-9 & Thorium-228 & $<101$ & $\mathrm{pCi} / \mathrm{g}$ & 101 & & 3/31/08 & 8C17008 & AGG-RRL-001 \\
\hline 14269-63-7 & Thorium-230 & $<109$ & $\mathrm{pCi} / \mathrm{g}$ & 109 & & 3/31/08 & 8C17008 & AGG-RRL-001 \\
\hline 14331-85-2 & Protactinium-231 & $<12.1$ & $\mathrm{pCi} / \mathrm{g}$ & 12.1 & & 3/31/08 & 8C17008 & AGG-RRL-001 \\
\hline $14932-40-2$ & Thorium-231 & $<61.6$ & $\mathrm{pCi} / \mathrm{g}$ & 61.6 & & 3/31/08 & 8C17008 & AGG-RRL-001 \\
\hline 7440-29-1 & Thorium-232 & $<269$ & $\mathrm{pCi} / \mathrm{g}$ & 269 & & 3/31/08 & 8C17008 & AGG-RRL-001 \\
\hline 13981-14-1 & Protactinium-233 & $<0.725$ & $\mathrm{pCi} / \mathrm{g}$ & 0.725 & & 3/31/08 & 8C17008 & AGG-RRL-001 \\
\hline \multirow[t]{2}{*}{$15100-28-4$} & Protactinium-234 & $<1.14$ & $\mathrm{pCi} / \mathrm{g}$ & 1.14 & & 3/31/08 & 8C17008 & AGG-RRL-001 \\
\hline & Protactinium-234m & $<33.4$ & $\mathrm{pCi} / \mathrm{g}$ & 33.4 & & 3/31/08 & 8C17008 & AGG-RRL-001 \\
\hline $15065-10-8$ & Thorium-234 & $<9.44$ & $\mathrm{pCi} / \mathrm{g}$ & 9.44 & & 3/31/08 & 8C17008 & AGG-RRL-001 \\
\hline 15117-96-1 & Uranium 235 & $<2.38$ & $\mathrm{pCi} / \mathrm{g}$ & 2.38 & & 3/31/08 & 8C17008 & AGG-RRL-001 \\
\hline 13994-20-2 & Neptunium-237 & $<2.45$ & $\mathrm{pCi} / \mathrm{g}$ & 2.45 & & 3/31/08 & 8C17008 & AGG-RRL-001 \\
\hline \multirow[t]{2}{*}{ 13981-16-3 } & Plutonium-238 & $<3550$ & $\mathrm{pCi} / \mathrm{g}$ & 3550 & & $3 / 31 / 08$ & 8C17008 & AGG-RRL-001 \\
\hline & Uranium 238 & $<5.07$ & $\mathrm{pCi} / \mathrm{g}$ & 5.07 & & 3/31/08 & 8C17008 & AGG-RRL-001 \\
\hline $15117-48-3$ & Plutonium-239 & $<3670$ & $\mathrm{pCi} / \mathrm{g}$ & 3670 & & $3 / 31 / 08$ & 8C17008 & AGG-RRL-001 \\
\hline 14119-33-6 & Plutonium-240 & $<3530$ & $\mathrm{pCi} / \mathrm{g}$ & 3530 & & 3/31/08 & 8C17008 & AGG-RRL-001 \\
\hline 14596-10-2 & Americium-241 & $<1.38$ & $\mathrm{pCi} / \mathrm{g}$ & 1.38 & & 3/31/08 & 8C17008 & AGG-RRL-001 \\
\hline 14993-75-0 & Americium-243 & $<0.595$ & $\mathrm{pCi} / \mathrm{g}$ & 0.595 & & 3/31/08 & 8C17008 & AGG-RRL-001 \\
\hline 15757-87-6 & Curium-243 & $<1.08$ & $\mathrm{pCi} / \mathrm{g}$ & 1.08 & & 3/31/08 & 8C17008 & AGG-RRL-001 \\
\hline 15621-76-8 & Curium-245 & $<0.89$ & $\mathrm{pCi} / \mathrm{g}$ & 0.89 & & $3 / 31 / 08$ & 8C17008 & AGG-RRL-001 \\
\hline HEIS No. & B1RYR9A & \multicolumn{2}{|c|}{ Lab ID: } & 0803012-22 & & & & \\
\hline 13966-32-0 & Sodium-22 & $<0.282$ & $\mathrm{pCi} / \mathrm{g}$ & 0.282 & & 3/31/08 & 8C17008 & AGG-RRL-001 \\
\hline 13966-00-2 & Potassium-40 & 18.9 & $\mathrm{pCi} / \mathrm{g}$ & 2.68 & 1.14 & 3/31/08 & 8C17008 & AGG-RRL-001 \\
\hline $14392-02-0$ & Chromium-51 & $<2.36$ & $\mathrm{pCi} / \mathrm{g}$ & 2.36 & & 3/31/08 & 8C17008 & AGG-RRL-001 \\
\hline 13966-31-9 & Manganese-54 & $<0.249$ & $\mathrm{pCi} / \mathrm{g}$ & 0.249 & & 3/31/08 & 8C17008 & AGG-RRL-001 \\
\hline 13981-50-5 & Cobalt-57 & $<0.244$ & $\mathrm{pCi} / \mathrm{g}$ & 0.244 & & $3 / 31 / 08$ & 8C17008 & AGG-RRL-001 \\
\hline $14596-12-4$ & Iron-59 & $<0.446$ & $\mathrm{pCi} / \mathrm{g}$ & 0.446 & & 3/31/08 & 8C17008 & AGG-RRL-001 \\
\hline $10198-40-0$ & Cobalt-60 & $<0.23$ & $\mathrm{pCi} / \mathrm{g}$ & 0.23 & & 3/31/08 & 8C17008 & AGG-RRL-001 \\
\hline 13982-39-3 & Zinc-65 & $<0.607$ & $\mathrm{pCi} / \mathrm{g}$ & 0.607 & & 3/31/08 & 8C17008 & AGG-RRL-001 \\
\hline $14265-71-5$ & Selenium-75 & $<0.389$ & $\mathrm{pCi} / \mathrm{g}$ & 0.389 & & 3/31/08 & 8C17008 & AGG-RRL-001 \\
\hline $17056-36-9$ & Rubidium-83 & $<0.452$ & $\mathrm{pCi} / \mathrm{g}$ & 0.452 & & 3/31/08 & 8C17008 & AGG-RRL-001 \\
\hline 13967-73-2 & Strontium-85 & $<0.312$ & $\mathrm{pCi} / \mathrm{g}$ & 0.312 & & 3/31/08 & 8C17008 & AGG-RRL-001 \\
\hline 14932-53-7 & Rubidium-86 & $<2.92$ & $\mathrm{pCi} / \mathrm{g}$ & 2.92 & & 3/31/08 & 8C17008 & AGG-RRL-001 \\
\hline 13982-36-0 & Yttrium-88 & $<0.204$ & $\mathrm{pCi} / \mathrm{g}$ & 0.204 & & 3/31/08 & 8C17008 & AGG-RRL-001 \\
\hline 14681-63-1 & Niobium-94 & $<0.212$ & $\mathrm{pCi} / \mathrm{g}$ & 0.212 & & 3/31/08 & 8C17008 & AGG-RRL-001 \\
\hline \multirow[t]{3}{*}{$13967-76-5$} & Niobium-95 & $<0.249$ & $\mathrm{pCi} / \mathrm{g}$ & 0.249 & & 3/31/08 & 8C17008 & AGG-RRL-001 \\
\hline & Niobium-95m & $<1.15$ & $\mathrm{pCi} / \mathrm{g}$ & 1.15 & & 3/31/08 & 8C17008 & AGG-RRL-001 \\
\hline & Technetium-95m & $<0.377$ & $\mathrm{pCi} / \mathrm{g}$ & 0.377 & & 3/31/08 & 8C17008 & AGG-RRL-001 \\
\hline \multirow[t]{2}{*}{ 13967-71-0 } & Zirconium-95 & $<0.421$ & $\mathrm{pCi} / \mathrm{g}$ & 0.421 & & 3/31/08 & 8C17008 & AGG-RRL-001 \\
\hline & Technetium-99m & $<0.247$ & $\mathrm{pCi} / \mathrm{g}$ & 0.247 & & 3/31/08 & 8C17008 & AGG-RRL-001 \\
\hline 13968-53-1 & Ruthenium-103 & $<0.239$ & $\mathrm{pCi} / \mathrm{g}$ & 0.239 & & 3/31/08 & 8C17008 & AGG-RRL-001 \\
\hline 13967-48-1 & Ruthenium-106 & $<2.45$ & $\mathrm{pCi} / \mathrm{g}$ & 2.45 & & 3/31/08 & 8C17008 & AGG-RRL-001 \\
\hline
\end{tabular}


GEA/Soil

\begin{tabular}{|c|c|c|c|c|c|c|c|c|}
\hline CAS \# & Analyte & Results & Units & MDA & UNC & Analyzed & Batch & Method \\
\hline HEIS No. & B1RYR9A & \multicolumn{3}{|c|}{ Lab ID: $\quad$ 0803012-22 } & & & & \\
\hline 14391-65-2 & Silver-108m & $<0.242$ & $\mathrm{pCi} / \mathrm{g}$ & 0.242 & & 3/31/08 & 8C17008 & AGG-RRL-001 \\
\hline \multirow[t]{2}{*}{ 14109-32-1 } & Cadmium-109 & $<6.53$ & $\mathrm{pCi} / \mathrm{g}$ & 6.53 & & $3 / 31 / 08$ & 8C17008 & AGG-RRL-001 \\
\hline & Silver-110 & $<0.237$ & $\mathrm{pCi} / \mathrm{g}$ & 0.237 & & 3/31/08 & 8C17008 & AGG-RRL-001 \\
\hline 14391-76-5 & Silver-110m & $<0.237$ & $\mathrm{pCi} / \mathrm{g}$ & 0.237 & & 3/31/08 & 8C17008 & AGG-RRL-001 \\
\hline 13966-06-8 & Tin-113 & $<0.358$ & $\mathrm{pCi} / \mathrm{g}$ & 0.358 & & 3/31/08 & 8C17008 & AGG-RRL-001 \\
\hline 14683-10-4 & Antimony-124 & $<0.238$ & $\mathrm{pCi} / \mathrm{g}$ & 0.238 & & 3/31/08 & 8C17008 & AGG-RRL-001 \\
\hline 14234-35-6 & Antimony-125 & $<0.706$ & $\mathrm{pCi} / \mathrm{g}$ & 0.706 & & 3/31/08 & 8C17008 & AGG-RRL-001 \\
\hline 15756-32-8 & Antimony-126 & $<0.243$ & $\mathrm{pCi} / \mathrm{g}$ & 0.243 & & 3/31/08 & 8C17008 & AGG-RRL-001 \\
\hline 15832-50-5 & Tin-126 & $<0.517$ & $\mathrm{pCi} / \mathrm{g}$ & 0.517 & & 3/31/08 & 8C17008 & AGG-RRL-001 \\
\hline 10043-66-0 & Iodine-131 & $<0.289$ & $\mathrm{pCi} / \mathrm{g}$ & 0.289 & & $3 / 31 / 08$ & 8C17008 & AGG-RRL-001 \\
\hline 13981-41-4 & Barium-133 & $<0.478$ & $\mathrm{pCi} / \mathrm{g}$ & 0.478 & & 3/31/08 & 8C17008 & AGG-RRL-001 \\
\hline 13967-70-9 & Cesium-134 & $<0.287$ & $\mathrm{pCi} / \mathrm{g}$ & 0.287 & & $3 / 31 / 08$ & 8C17008 & AGG-RRL-001 \\
\hline 10045-97-3 & Cesium-137 & $<0.282$ & $\mathrm{pCi} / \mathrm{g}$ & 0.282 & & 3/31/08 & 8C17008 & AGG-RRL-001 \\
\hline 13982-30-4 & Cerium-139 & $<0.286$ & $\mathrm{pCi} / \mathrm{g}$ & 0.286 & & 3/31/08 & 8C17008 & AGG-RRL-001 \\
\hline 14762-78-8 & Cerium-144 & $<1.94$ & $\mathrm{pCi} / \mathrm{g}$ & 1.94 & & 3/31/08 & 8C17008 & AGG-RRL-001 \\
\hline 14683-23-9 & Europium-152 & $<0.898$ & $\mathrm{pCi} / \mathrm{g}$ & 0.898 & & 3/31/08 & 8C17008 & AGG-RRL-001 \\
\hline $14276-65-4$ & Gadolinium-153 & $<0.722$ & $\mathrm{pCi} / \mathrm{g}$ & 0.722 & & 3/31/08 & 8C17008 & AGG-RRL-001 \\
\hline 15585-10-1 & Europium-154 & $<0.514$ & $\mathrm{pCi} / \mathrm{g}$ & 0.514 & & 3/31/08 & 8C17008 & AGG-RRL-001 \\
\hline 14391-16-3 & Europium-155 & $<0.721$ & $\mathrm{pCi} / \mathrm{g}$ & 0.721 & & 3/31/08 & 8C17008 & AGG-RRL-001 \\
\hline 13982-78-0 & Mercury-203 & $<0.305$ & $\mathrm{pCi} / \mathrm{g}$ & 0.305 & & 3/31/08 & 8C17008 & AGG-RRL-001 \\
\hline 14913-50-9 & Thallium-208 & $<0.272$ & $\mathrm{pCi} / \mathrm{g}$ & 0.272 & 0.0614 & 3/31/08 & 8C17008 & AGG-RRL-001 \\
\hline 14331-79-4 & Bismuth-210 & $<0.461$ & $\mathrm{pCi} / \mathrm{g}$ & 0.461 & & 3/31/08 & 8C17008 & AGG-RRL-001 \\
\hline \multirow[t]{3}{*}{$14255-04-0$} & Lead-210 & $<91.9$ & $\mathrm{pCi} / \mathrm{g}$ & 91.9 & & 3/31/08 & 8C17008 & AGG-RRL-001 \\
\hline & Bismuth-211 & $<5.71$ & $\mathrm{pCi} / \mathrm{g}$ & 5.71 & & 3/31/08 & 8C17008 & AGG-RRL-001 \\
\hline & Lead-211 & $<7.8$ & $\mathrm{pCi} / \mathrm{g}$ & 7.8 & & 3/31/08 & 8C17008 & AGG-RRL-001 \\
\hline 15092-94-1 & Lead-212 & $<0.525$ & $\mathrm{pCi} / \mathrm{g}$ & 0.525 & 0.117 & 3/31/08 & 8C17008 & AGG-RRL-001 \\
\hline 14733-03-0 & Bismuth-214 & $<0.644$ & $\mathrm{pCi} / \mathrm{g}$ & 0.644 & & 3/31/08 & 8C17008 & AGG-RRL-001 \\
\hline $15067-28-4$ & Lead-214 & $<0.751$ & $\mathrm{pCi} / \mathrm{g}$ & 0.751 & & 3/31/08 & 8C17008 & AGG-RRL-001 \\
\hline $14835-02-0$ & Radon-219 & $<2.34$ & $\mathrm{pCi} / \mathrm{g}$ & 2.34 & & 3/31/08 & 8C17008 & AGG-RRL-001 \\
\hline 22481-48-7 & Radon-220 & $<206$ & $\mathrm{pCi} / \mathrm{g}$ & 206 & & 3/31/08 & 8C17008 & AGG-RRL-001 \\
\hline 28522-20-5 & Radon-221 & $<1.14$ & $\mathrm{pCi} / \mathrm{g}$ & 1.14 & & 3/31/08 & 8C17008 & AGG-RRL-001 \\
\hline 15756-98-6 & Francium-223 & $<8.41$ & $\mathrm{pCi} / \mathrm{g}$ & 8.41 & & 3/31/08 & 8C17008 & AGG-RRL-001 \\
\hline $15623-45-7$ & Radium-223 & $<1.7$ & $\mathrm{pCi} / \mathrm{g}$ & 1.7 & & 3/31/08 & 8C17008 & AGG-RRL-001 \\
\hline 13233-32-4 & Radium-224 & $<7.2$ & $\mathrm{pCi} / \mathrm{g}$ & 7.2 & & 3/31/08 & 8C17008 & AGG-RRL-001 \\
\hline 13982-63-3 & Radium-226 & $<6.1$ & $\mathrm{pCi} / \mathrm{g}$ & 6.1 & 1.33 & 3/31/08 & 8C17008 & AGG-RRL-001 \\
\hline $15623-47-9$ & Thorium-227 & $<2.57$ & $\mathrm{pCi} / \mathrm{g}$ & 2.57 & & 3/31/08 & 8C17008 & AGG-RRL-001 \\
\hline 14331-83-0 & Actinium-228 & $<1.07$ & $\mathrm{pCi} / \mathrm{g}$ & 1.07 & & 3/31/08 & 8C17008 & AGG-RRL-001 \\
\hline 14274-82-9 & Thorium-228 & $<89.1$ & $\mathrm{pCi} / \mathrm{g}$ & 89.1 & & $3 / 31 / 08$ & 8C17008 & AGG-RRL-001 \\
\hline $14269-63-7$ & Thorium-230 & $<94.5$ & $\mathrm{pCi} / \mathrm{g}$ & 94.5 & & 3/31/08 & 8C17008 & AGG-RRL-001 \\
\hline $14331-85-2$ & Protactinium-231 & $<10$ & $\mathrm{pCi} / \mathrm{g}$ & 10 & & 3/31/08 & 8C17008 & AGG-RRL-001 \\
\hline $14932-40-2$ & Thorium-231 & $<50.5$ & $\mathrm{pCi} / \mathrm{g}$ & 50.5 & & 3/31/08 & 8C17008 & AGG-RRL-001 \\
\hline 7440-29-1 & Thorium-232 & $<294$ & $\mathrm{pCi} / \mathrm{g}$ & 294 & & $3 / 31 / 08$ & 8C17008 & AGG-RRL-001 \\
\hline 13981-14-1 & Protactinium-233 & $<0.649$ & $\mathrm{pCi} / \mathrm{g}$ & 0.649 & & 3/31/08 & 8C17008 & AGG-RRL-001 \\
\hline \multirow[t]{2}{*}{$15100-28-4$} & Protactinium-234 & $<0.924$ & $\mathrm{pCi} / \mathrm{g}$ & 0.924 & & 3/31/08 & 8C17008 & AGG-RRL-001 \\
\hline & Protactinium-234m & $<30.4$ & $\mathrm{pCi} / \mathrm{g}$ & 30.4 & & 3/31/08 & 8C17008 & AGG-RRL-001 \\
\hline 15065-10-8 & Thorium-234 & $<8.39$ & $\mathrm{pCi} / \mathrm{g}$ & 8.39 & & 3/31/08 & 8C17008 & AGG-RRL-001 \\
\hline 15117-96-1 & Uranium 235 & $<2.11$ & $\mathrm{pCi} / \mathrm{g}$ & 2.11 & & 3/31/08 & 8C17008 & AGG-RRL-001 \\
\hline 13994-20-2 & Neptunium-237 & $<1.88$ & $\mathrm{pCi} / \mathrm{g}$ & 1.88 & & 3/31/08 & 8C17008 & AGG-RRL-001 \\
\hline 13981-16-3 & Plutonium-238 & $<2940$ & $\mathrm{pCi} / \mathrm{g}$ & 2940 & & 3/31/08 & 8C17008 & AGG-RRL-001 \\
\hline
\end{tabular}


GEA/Soil

\begin{tabular}{|c|c|c|c|c|c|c|c|c|}
\hline CAS \# & Analyte & Results & Units & MDA & UNC & Analyzed & Batch & Method \\
\hline \multirow[t]{2}{*}{ HEIS No. } & B1RYR9A & \multicolumn{2}{|c|}{ Lab ID: } & \multicolumn{2}{|l|}{$0803012-22$} & & & \\
\hline & Uranium 238 & $<4.34$ & $\mathrm{pCi} / \mathrm{g}$ & 4.34 & & $3 / 31 / 08$ & 8C17008 & AGG-RRL-001 \\
\hline $15117-48-3$ & Plutonium-239 & $<3300$ & $\mathrm{pCi} / \mathrm{g}$ & 3300 & & 3/31/08 & 8C17008 & AGG-RRL-001 \\
\hline $14119-33-6$ & Plutonium-240 & $<2970$ & $\mathrm{pCi} / \mathrm{g}$ & 2970 & & 3/31/08 & 8C17008 & AGG-RRL-001 \\
\hline $14596-10-2$ & Americium-241 & $<1.46$ & $\mathrm{pCi} / \mathrm{g}$ & 1.46 & & 3/31/08 & 8C17008 & AGG-RRL-001 \\
\hline $14993-75-0$ & Americium-243 & $<0.539$ & $\mathrm{pCi} / \mathrm{g}$ & 0.539 & & 3/31/08 & 8C17008 & AGG-RRL-001 \\
\hline $15757-87-6$ & Curium-243 & $<0.905$ & $\mathrm{pCi} / \mathrm{g}$ & 0.905 & & 3/31/08 & 8C17008 & AGG-RRL-001 \\
\hline $15621-76-8$ & Curium-245 & $<0.744$ & $\mathrm{pCi} / \mathrm{g}$ & 0.744 & & 3/31/08 & 8C17008 & AGG-RRL-001 \\
\hline HEIS No. & B1RYT0B & \multicolumn{2}{|c|}{ Lab ID: } & 0803014-01 & & & & \\
\hline 13966-32-0 & Sodium-22 & $<0.31$ & $\mathrm{pCi} / \mathrm{g}$ & 0.31 & & $3 / 31 / 08$ & 8C17008 & AGG-RRL-001 \\
\hline 13966-00-2 & Potassium-40 & 18.4 & $\mathrm{pCi} / \mathrm{g}$ & 2.61 & 1.17 & 3/31/08 & 8C17008 & AGG-RRL-001 \\
\hline $14392-02-0$ & Chromium-51 & $<2.57$ & $\mathrm{pCi} / \mathrm{g}$ & 2.57 & & 3/31/08 & 8C17008 & AGG-RRL-001 \\
\hline 13966-31-9 & Manganese-54 & $<0.292$ & $\mathrm{pCi} / \mathrm{g}$ & 0.292 & & 3/31/08 & 8C17008 & AGG-RRL-001 \\
\hline 13981-50-5 & Cobalt-57 & $<0.274$ & $\mathrm{pCi} / \mathrm{g}$ & 0.274 & & 3/31/08 & 8C17008 & AGG-RRL-001 \\
\hline $14596-12-4$ & Iron-59 & $<0.581$ & $\mathrm{pCi} / \mathrm{g}$ & 0.581 & & 3/31/08 & 8C17008 & AGG-RRL-001 \\
\hline $10198-40-0$ & Cobalt-60 & $<0.256$ & $\mathrm{pCi} / \mathrm{g}$ & 0.256 & & 3/31/08 & 8C17008 & AGG-RRL-001 \\
\hline 13982-39-3 & Zinc-65 & $<0.692$ & $\mathrm{pCi} / \mathrm{g}$ & 0.692 & & 3/31/08 & 8C17008 & AGG-RRL-001 \\
\hline $14265-71-5$ & Selenium-75 & $<0.462$ & $\mathrm{pCi} / \mathrm{g}$ & 0.462 & & 3/31/08 & 8C17008 & AGG-RRL-001 \\
\hline 17056-36-9 & Rubidium-83 & $<0.535$ & $\mathrm{pCi} / \mathrm{g}$ & 0.535 & & 3/31/08 & 8C17008 & AGG-RRL-001 \\
\hline 13967-73-2 & Strontium-85 & $<0.354$ & $\mathrm{pCi} / \mathrm{g}$ & 0.354 & & 3/31/08 & 8C17008 & AGG-RRL-001 \\
\hline 14932-53-7 & Rubidium-86 & $<3.58$ & $\mathrm{pCi} / \mathrm{g}$ & 3.58 & & 3/31/08 & 8C17008 & AGG-RRL-001 \\
\hline $13982-36-0$ & Yttrium-88 & $<0.232$ & $\mathrm{pCi} / \mathrm{g}$ & 0.232 & & $3 / 31 / 08$ & 8C17008 & AGG-RRL-001 \\
\hline $14681-63-1$ & Niobium-94 & $<0.253$ & $\mathrm{pCi} / \mathrm{g}$ & 0.253 & & 3/31/08 & 8C17008 & AGG-RRL-001 \\
\hline \multirow[t]{3}{*}{$13967-76-5$} & Niobium-95 & $<0.287$ & $\mathrm{pCi} / \mathrm{g}$ & 0.287 & & 3/31/08 & 8C17008 & AGG-RRL-001 \\
\hline & Niobium-95m & $<1.36$ & $\mathrm{pCi} / \mathrm{g}$ & 1.36 & & $3 / 31 / 08$ & 8C17008 & AGG-RRL-001 \\
\hline & Technetium-95m & $<0.445$ & $\mathrm{pCi} / \mathrm{g}$ & 0.445 & & 3/31/08 & 8C17008 & AGG-RRL-001 \\
\hline \multirow[t]{2}{*}{ 13967-71-0 } & Zirconium-95 & $<0.461$ & $\mathrm{pCi} / \mathrm{g}$ & 0.461 & & 3/31/08 & 8C17008 & AGG-RRL-001 \\
\hline & Technetium-99m & $<0.278$ & $\mathrm{pCi} / \mathrm{g}$ & 0.278 & & 3/31/08 & 8C17008 & AGG-RRL-001 \\
\hline 13968-53-1 & Ruthenium-103 & $<0.282$ & $\mathrm{pCi} / \mathrm{g}$ & 0.282 & & 3/31/08 & 8C17008 & AGG-RRL-001 \\
\hline $13967-48-1$ & Ruthenium-106 & $<2.73$ & $\mathrm{pCi} / \mathrm{g}$ & 2.73 & & 3/31/08 & 8C17008 & AGG-RRL-001 \\
\hline $14391-65-2$ & Silver-108m & $<0.283$ & $\mathrm{pCi} / \mathrm{g}$ & 0.283 & & 3/31/08 & 8C17008 & AGG-RRL-001 \\
\hline \multirow[t]{2}{*}{$14109-32-1$} & Cadmium-109 & $<7.66$ & $\mathrm{pCi} / \mathrm{g}$ & 7.66 & & 3/31/08 & 8C17008 & AGG-RRL-001 \\
\hline & Silver-110 & $<0.271$ & $\mathrm{pCi} / \mathrm{g}$ & 0.271 & & 3/31/08 & 8C17008 & AGG-RRL-001 \\
\hline $14391-76-5$ & Silver-110m & $<0.271$ & $\mathrm{pCi} / \mathrm{g}$ & 0.271 & & 3/31/08 & 8C17008 & AGG-RRL-001 \\
\hline 13966-06-8 & Tin-113 & $<0.386$ & $\mathrm{pCi} / \mathrm{g}$ & 0.386 & & 3/31/08 & 8C17008 & AGG-RRL-001 \\
\hline $14683-10-4$ & Antimony-124 & $<0.279$ & $\mathrm{pCi} / \mathrm{g}$ & 0.279 & & $3 / 31 / 08$ & 8C17008 & AGG-RRL-001 \\
\hline $14234-35-6$ & Antimony-125 & $<0.891$ & $\mathrm{pCi} / \mathrm{g}$ & 0.891 & & $3 / 31 / 08$ & 8C17008 & AGG-RRL-001 \\
\hline 15756-32-8 & Antimony-126 & $<0.267$ & $\mathrm{pCi} / \mathrm{g}$ & 0.267 & & 3/31/08 & 8C17008 & AGG-RRL-001 \\
\hline $15832-50-5$ & Tin-126 & $<0.603$ & $\mathrm{pCi} / \mathrm{g}$ & 0.603 & & 3/31/08 & 8C17008 & AGG-RRL-001 \\
\hline 10043-66-0 & Iodine-131 & $<0.316$ & $\mathrm{pCi} / \mathrm{g}$ & 0.316 & & $3 / 31 / 08$ & 8C17008 & AGG-RRL-001 \\
\hline 13981-41-4 & Barium-133 & $<0.544$ & $\mathrm{pCi} / \mathrm{g}$ & 0.544 & & $3 / 31 / 08$ & 8C17008 & AGG-RRL-001 \\
\hline 13967-70-9 & Cesium-134 & $<0.289$ & $\mathrm{pCi} / \mathrm{g}$ & 0.289 & & 3/31/08 & 8C17008 & AGG-RRL-001 \\
\hline $10045-97-3$ & Cesium-137 & $<0.305$ & $\mathrm{pCi} / \mathrm{g}$ & 0.305 & & 3/31/08 & 8C17008 & AGG-RRL-001 \\
\hline 13982-30-4 & Cerium-139 & $<0.318$ & $\mathrm{pCi} / \mathrm{g}$ & 0.318 & & 3/31/08 & 8C17008 & AGG-RRL-001 \\
\hline 14762-78-8 & Cerium-144 & $<2.24$ & $\mathrm{pCi} / \mathrm{g}$ & 2.24 & & 3/31/08 & 8C17008 & AGG-RRL-001 \\
\hline $14683-23-9$ & Europium-152 & $<1.05$ & $\mathrm{pCi} / \mathrm{g}$ & 1.05 & & 3/31/08 & 8C17008 & AGG-RRL-001 \\
\hline $14276-65-4$ & Gadolinium-153 & $<0.822$ & $\mathrm{pCi} / \mathrm{g}$ & 0.822 & & 3/31/08 & 8C17008 & AGG-RRL-001 \\
\hline 15585-10-1 & Europium-154 & $<0.582$ & $\mathrm{pCi} / \mathrm{g}$ & 0.582 & & 3/31/08 & 8C17008 & AGG-RRL-001 \\
\hline $14391-16-3$ & Europium-155 & $<0.849$ & $\mathrm{pCi} / \mathrm{g}$ & 0.849 & & 3/31/08 & 8C17008 & AGG-RRL-001 \\
\hline
\end{tabular}


GEA/Soil

\begin{tabular}{|c|c|c|c|c|c|c|c|c|}
\hline CAS \# & Analyte & Results & Units & MDA & UNC & Analyzed & Batch & Method \\
\hline HEIS No. & B1RYT0B & $\mathbf{L a}$ & ID: & 0803014-01 & & & & \\
\hline $13982-78-0$ & Mercury-203 & $<0.355$ & $\mathrm{pCi} / \mathrm{g}$ & 0.355 & & $3 / 31 / 08$ & 8C17008 & AGG-RRL-001 \\
\hline 14913-50-9 & Thallium-208 & $<0.243$ & $\mathrm{pCi} / \mathrm{g}$ & 0.243 & 0.0568 & 3/31/08 & 8C17008 & AGG-RRL-001 \\
\hline $14331-79-4$ & Bismuth-210 & $<0.558$ & $\mathrm{pCi} / \mathrm{g}$ & 0.558 & & 3/31/08 & 8C17008 & AGG-RRL-001 \\
\hline \multirow[t]{3}{*}{$14255-04-0$} & Lead-210 & $<105$ & $\mathrm{pCi} / \mathrm{g}$ & 105 & & 3/31/08 & 8C17008 & AGG-RRL-001 \\
\hline & Bismuth-211 & $<6.51$ & $\mathrm{pCi} / \mathrm{g}$ & 6.51 & & 3/31/08 & 8C17008 & AGG-RRL-001 \\
\hline & Lead-211 & $<8.9$ & $\mathrm{pCi} / \mathrm{g}$ & 8.9 & & 3/31/08 & 8C17008 & AGG-RRL-001 \\
\hline 15092-94-1 & Lead-212 & 1.02 & $\mathrm{pCi} / \mathrm{g}$ & 0.609 & 0.148 & 3/31/08 & 8C17008 & AGG-RRL-001 \\
\hline 14733-03-0 & Bismuth-214 & $<0.73$ & $\mathrm{pCi} / \mathrm{g}$ & 0.73 & & $3 / 31 / 08$ & 8C17008 & AGG-RRL-001 \\
\hline $15067-28-4$ & Lead-214 & $<0.896$ & $\mathrm{pCi} / \mathrm{g}$ & 0.896 & & 3/31/08 & 8C17008 & AGG-RRL-001 \\
\hline $14835-02-0$ & Radon-219 & $<2.89$ & $\mathrm{pCi} / \mathrm{g}$ & 2.89 & & 3/31/08 & 8C17008 & AGG-RRL-001 \\
\hline 22481-48-7 & Radon-220 & $<236$ & $\mathrm{pCi} / \mathrm{g}$ & 236 & & 3/31/08 & 8C17008 & AGG-RRL-001 \\
\hline 28522-20-5 & Radon-221 & $<1.28$ & $\mathrm{pCi} / \mathrm{g}$ & 1.28 & & 3/31/08 & 8C17008 & AGG-RRL-001 \\
\hline 15756-98-6 & Francium-223 & $<9.91$ & $\mathrm{pCi} / \mathrm{g}$ & 9.91 & & 3/31/08 & 8C17008 & AGG-RRL-001 \\
\hline $15623-45-7$ & Radium-223 & $<2.05$ & $\mathrm{pCi} / \mathrm{g}$ & 2.05 & & 3/31/08 & 8C17008 & AGG-RRL-001 \\
\hline $13233-32-4$ & Radium-224 & $<8.46$ & $\mathrm{pCi} / \mathrm{g}$ & 8.46 & & 3/31/08 & 8C17008 & AGG-RRL-001 \\
\hline 13982-63-3 & Radium-226 & $<7.86$ & $\mathrm{pCi} / \mathrm{g}$ & 7.86 & & 3/31/08 & 8C17008 & AGG-RRL-001 \\
\hline $15623-47-9$ & Thorium-227 & $<3.03$ & $\mathrm{pCi} / \mathrm{g}$ & 3.03 & & 3/31/08 & 8C17008 & AGG-RRL-001 \\
\hline 14331-83-0 & Actinium-228 & $<1.18$ & $\mathrm{pCi} / \mathrm{g}$ & 1.18 & & 3/31/08 & 8C17008 & AGG-RRL-001 \\
\hline $14274-82-9$ & Thorium-228 & $<101$ & $\mathrm{pCi} / \mathrm{g}$ & 101 & & 3/31/08 & 8C17008 & AGG-RRL-001 \\
\hline $14269-63-7$ & Thorium-230 & $<106$ & $\mathrm{pCi} / \mathrm{g}$ & 106 & & 3/31/08 & 8C17008 & AGG-RRL-001 \\
\hline $14331-85-2$ & Protactinium-231 & $<11.3$ & $\mathrm{pCi} / \mathrm{g}$ & 11.3 & & 3/31/08 & 8C17008 & AGG-RRL-001 \\
\hline $14932-40-2$ & Thorium-231 & $<57.4$ & $\mathrm{pCi} / \mathrm{g}$ & 57.4 & & 3/31/08 & 8C17008 & AGG-RRL-001 \\
\hline 7440-29-1 & Thorium-232 & $<339$ & $\mathrm{pCi} / \mathrm{g}$ & 339 & & 3/31/08 & 8C17008 & AGG-RRL-001 \\
\hline 13981-14-1 & Protactinium-233 & $<0.72$ & $\mathrm{pCi} / \mathrm{g}$ & 0.72 & & 3/31/08 & 8C17008 & AGG-RRL-001 \\
\hline \multirow[t]{2}{*}{$15100-28-4$} & Protactinium-234 & $<1.04$ & $\mathrm{pCi} / \mathrm{g}$ & 1.04 & & 3/31/08 & 8C17008 & AGG-RRL-001 \\
\hline & Protactinium-234m & $<34.5$ & $\mathrm{pCi} / \mathrm{g}$ & 34.5 & & 3/31/08 & 8C17008 & AGG-RRL-001 \\
\hline 15065-10-8 & Thorium-234 & $<9.67$ & $\mathrm{pCi} / \mathrm{g}$ & 9.67 & & $3 / 31 / 08$ & 8C17008 & AGG-RRL-001 \\
\hline 15117-96-1 & Uranium 235 & $<2.37$ & $\mathrm{pCi} / \mathrm{g}$ & 2.37 & & 3/31/08 & 8C17008 & AGG-RRL-001 \\
\hline 13994-20-2 & Neptunium-237 & $<2.21$ & $\mathrm{pCi} / \mathrm{g}$ & 2.21 & & 3/31/08 & 8C17008 & AGG-RRL-001 \\
\hline \multirow[t]{2}{*}{ 13981-16-3 } & Plutonium-238 & $<3280$ & $\mathrm{pCi} / \mathrm{g}$ & 3280 & & 3/31/08 & 8C17008 & AGG-RRL-001 \\
\hline & Uranium 238 & $<5.01$ & $\mathrm{pCi} / \mathrm{g}$ & 5.01 & & 3/31/08 & 8C17008 & AGG-RRL-001 \\
\hline $15117-48-3$ & Plutonium-239 & $<3850$ & $\mathrm{pCi} / \mathrm{g}$ & 3850 & & 3/31/08 & 8C17008 & AGG-RRL-001 \\
\hline $14119-33-6$ & Plutonium-240 & $<3340$ & $\mathrm{pCi} / \mathrm{g}$ & 3340 & & 3/31/08 & 8C17008 & AGG-RRL-001 \\
\hline $14596-10-2$ & Americium-241 & $<1.65$ & $\mathrm{pCi} / \mathrm{g}$ & 1.65 & & $3 / 31 / 08$ & 8C17008 & AGG-RRL-001 \\
\hline 14993-75-0 & Americium-243 & $<0.601$ & $\mathrm{pCi} / \mathrm{g}$ & 0.601 & & 3/31/08 & 8C17008 & AGG-RRL-001 \\
\hline $15757-87-6$ & Curium-243 & $<1.02$ & $\mathrm{pCi} / \mathrm{g}$ & 1.02 & & 3/31/08 & 8C17008 & AGG-RRL-001 \\
\hline 15621-76-8 & Curium-245 & $<0.837$ & $\mathrm{pCi} / \mathrm{g}$ & 0.837 & & 3/31/08 & 8C17008 & AGG-RRL-001 \\
\hline HEIS No. & B1RYT0A & \multicolumn{2}{|c|}{ Lab ID: } & 0803014-02 & & & & \\
\hline 13966-32-0 & Sodium-22 & $<0.366$ & $\mathrm{pCi} / \mathrm{g}$ & 0.366 & & 3/31/08 & 8C17008 & AGG-RRL-001 \\
\hline $13966-00-2$ & Potassium-40 & 15.6 & $\mathrm{pCi} / \mathrm{g}$ & 2.83 & 1.14 & $3 / 31 / 08$ & 8C17008 & AGG-RRL-001 \\
\hline $14392-02-0$ & Chromium-51 & $<3.34$ & $\mathrm{pCi} / \mathrm{g}$ & 3.34 & & 3/31/08 & 8C17008 & AGG-RRL-001 \\
\hline 13966-31-9 & Manganese-54 & $<0.363$ & $\mathrm{pCi} / \mathrm{g}$ & 0.363 & & 3/31/08 & 8C17008 & AGG-RRL-001 \\
\hline 13981-50-5 & Cobalt-57 & $<0.352$ & $\mathrm{pCi} / \mathrm{g}$ & 0.352 & & 3/31/08 & 8C17008 & AGG-RRL-001 \\
\hline $14596-12-4$ & Iron-59 & $<0.624$ & $\mathrm{pCi} / \mathrm{g}$ & 0.624 & & 3/31/08 & 8C17008 & AGG-RRL-001 \\
\hline $10198-40-0$ & Cobalt-60 & $<0.34$ & $\mathrm{pCi} / \mathrm{g}$ & 0.34 & & 3/31/08 & 8C17008 & AGG-RRL-001 \\
\hline 13982-39-3 & Zinc-65 & $<0.767$ & $\mathrm{pCi} / \mathrm{g}$ & 0.767 & & 3/31/08 & 8C17008 & AGG-RRL-001 \\
\hline 14265-71-5 & Selenium-75 & $<0.576$ & $\mathrm{pCi} / \mathrm{g}$ & 0.576 & & 3/31/08 & 8C17008 & AGG-RRL-001 \\
\hline $17056-36-9$ & Rubidium-83 & $<0.862$ & $\mathrm{pCi} / \mathrm{g}$ & 0.862 & & 3/31/08 & 8C17008 & AGG-RRL-001 \\
\hline
\end{tabular}


GEA/Soil

\begin{tabular}{|c|c|c|c|c|c|c|c|c|}
\hline CAS \# & Analyte & Results & Units & MDA & UNC & Analyzed & Batch & Method \\
\hline HEIS No. & B1RYT0A & \multicolumn{3}{|c|}{ Lab ID: $\quad$ 0803014-02 } & & & & \\
\hline 13967-73-2 & Strontium-85 & $<0.4$ & $\mathrm{pCi} / \mathrm{g}$ & 0.4 & & 3/31/08 & 8C17008 & AGG-RRL-001 \\
\hline 14932-53-7 & Rubidium-86 & $<4.56$ & $\mathrm{pCi} / \mathrm{g}$ & 4.56 & & 3/31/08 & 8C17008 & AGG-RRL-001 \\
\hline 13982-36-0 & Yttrium-88 & $<0.3$ & $\mathrm{pCi} / \mathrm{g}$ & 0.3 & & 3/31/08 & 8C17008 & AGG-RRL-001 \\
\hline 14681-63-1 & Niobium-94 & $<0.325$ & $\mathrm{pCi} / \mathrm{g}$ & 0.325 & & 3/31/08 & 8C17008 & AGG-RRL-001 \\
\hline \multirow[t]{3}{*}{$13967-76-5$} & Niobium-95 & $<0.332$ & $\mathrm{pCi} / \mathrm{g}$ & 0.332 & & 3/31/08 & 8C17008 & AGG-RRL-001 \\
\hline & Niobium-95m & $<1.51$ & $\mathrm{pCi} / \mathrm{g}$ & 1.51 & & 3/31/08 & 8C17008 & AGG-RRL-001 \\
\hline & Technetium-95m & $<0.541$ & $\mathrm{pCi} / \mathrm{g}$ & 0.541 & & 3/31/08 & 8C17008 & AGG-RRL-001 \\
\hline \multirow[t]{2}{*}{ 13967-71-0 } & Zirconium-95 & $<0.625$ & $\mathrm{pCi} / \mathrm{g}$ & 0.625 & & 3/31/08 & 8C17008 & AGG-RRL-001 \\
\hline & Technetium-99m & $<0.341$ & $\mathrm{pCi} / \mathrm{g}$ & 0.341 & & 3/31/08 & 8C17008 & AGG-RRL-001 \\
\hline 13968-53-1 & Ruthenium-103 & $<0.385$ & $\mathrm{pCi} / \mathrm{g}$ & 0.385 & & 3/31/08 & 8C17008 & AGG-RRL-001 \\
\hline 13967-48-1 & Ruthenium-106 & $<3.96$ & $\mathrm{pCi} / \mathrm{g}$ & 3.96 & & 3/31/08 & 8C17008 & AGG-RRL-001 \\
\hline 14391-65-2 & Silver-108m & $<0.343$ & $\mathrm{pCi} / \mathrm{g}$ & 0.343 & & $3 / 31 / 08$ & 8C17008 & AGG-RRL-001 \\
\hline \multirow[t]{2}{*}{ 14109-32-1 } & Cadmium-109 & $<9.63$ & $\mathrm{pCi} / \mathrm{g}$ & 9.63 & & $3 / 31 / 08$ & 8C17008 & AGG-RRL-001 \\
\hline & Silver-110 & $<0.355$ & $\mathrm{pCi} / \mathrm{g}$ & 0.355 & & 3/31/08 & 8C17008 & AGG-RRL-001 \\
\hline 14391-76-5 & Silver-110m & $<0.356$ & $\mathrm{pCi} / \mathrm{g}$ & 0.356 & & 3/31/08 & 8C17008 & AGG-RRL-001 \\
\hline 13966-06-8 & Tin-113 & $<0.49$ & $\mathrm{pCi} / \mathrm{g}$ & 0.49 & & 3/31/08 & 8C17008 & AGG-RRL-001 \\
\hline $14683-10-4$ & Antimony-124 & $<0.417$ & $\mathrm{pCi} / \mathrm{g}$ & 0.417 & & 3/31/08 & 8C17008 & AGG-RRL-001 \\
\hline 14234-35-6 & Antimony-125 & $<1.05$ & $\mathrm{pCi} / \mathrm{g}$ & 1.05 & & 3/31/08 & 8C17008 & AGG-RRL-001 \\
\hline 15756-32-8 & Antimony-126 & $<0.339$ & $\mathrm{pCi} / \mathrm{g}$ & 0.339 & & 3/31/08 & 8C17008 & AGG-RRL-001 \\
\hline 15832-50-5 & Tin-126 & $<0.783$ & $\mathrm{pCi} / \mathrm{g}$ & 0.783 & & 3/31/08 & 8C17008 & AGG-RRL-001 \\
\hline 10043-66-0 & Iodine-131 & $<0.41$ & $\mathrm{pCi} / \mathrm{g}$ & 0.41 & & 3/31/08 & 8C17008 & AGG-RRL-001 \\
\hline 13981-41-4 & Barium-133 & $<0.579$ & $\mathrm{pCi} / \mathrm{g}$ & 0.579 & & 3/31/08 & 8C17008 & AGG-RRL-001 \\
\hline 13967-70-9 & Cesium-134 & $<0.378$ & $\mathrm{pCi} / \mathrm{g}$ & 0.378 & & 3/31/08 & 8C17008 & AGG-RRL-001 \\
\hline $10045-97-3$ & Cesium-137 & $<0.391$ & $\mathrm{pCi} / \mathrm{g}$ & 0.391 & & 3/31/08 & 8C17008 & AGG-RRL-001 \\
\hline 13982-30-4 & Cerium-139 & $<0.407$ & $\mathrm{pCi} / \mathrm{g}$ & 0.407 & & 3/31/08 & 8C17008 & AGG-RRL-001 \\
\hline 14762-78-8 & Cerium-144 & $<2.79$ & $\mathrm{pCi} / \mathrm{g}$ & 2.79 & & 3/31/08 & 8C17008 & AGG-RRL-001 \\
\hline 14683-23-9 & Europium-152 & $<1.38$ & $\mathrm{pCi} / \mathrm{g}$ & 1.38 & & 3/31/08 & 8C17008 & AGG-RRL-001 \\
\hline $14276-65-4$ & Gadolinium-153 & $<1.08$ & $\mathrm{pCi} / \mathrm{g}$ & 1.08 & & 3/31/08 & 8C17008 & AGG-RRL-001 \\
\hline 15585-10-1 & Europium-154 & $<0.742$ & $\mathrm{pCi} / \mathrm{g}$ & 0.742 & & 3/31/08 & 8C17008 & AGG-RRL-001 \\
\hline 14391-16-3 & Europium-155 & $<1.11$ & $\mathrm{pCi} / \mathrm{g}$ & 1.11 & & 3/31/08 & 8C17008 & AGG-RRL-001 \\
\hline 13982-78-0 & Mercury-203 & $<0.443$ & $\mathrm{pCi} / \mathrm{g}$ & 0.443 & & 3/31/08 & 8C17008 & AGG-RRL-001 \\
\hline 14913-50-9 & Thallium-208 & $<0.421$ & $\mathrm{pCi} / \mathrm{g}$ & 0.421 & & 3/31/08 & 8C17008 & AGG-RRL-001 \\
\hline 14331-79-4 & Bismuth-210 & $<0.688$ & $\mathrm{pCi} / \mathrm{g}$ & 0.688 & & 3/31/08 & 8C17008 & AGG-RRL-001 \\
\hline \multirow[t]{3}{*}{$14255-04-0$} & Lead-210 & $<48.7$ & $\mathrm{pCi} / \mathrm{g}$ & 48.7 & & 3/31/08 & 8C17008 & AGG-RRL-001 \\
\hline & Bismuth-211 & $<7.92$ & $\mathrm{pCi} / \mathrm{g}$ & 7.92 & & 3/31/08 & 8C17008 & AGG-RRL-001 \\
\hline & Lead-211 & $<10.8$ & $\mathrm{pCi} / \mathrm{g}$ & 10.8 & & 3/31/08 & 8C17008 & AGG-RRL-001 \\
\hline 15092-94-1 & Lead-212 & $<0.876$ & $\mathrm{pCi} / \mathrm{g}$ & 0.876 & & 3/31/08 & 8C17008 & AGG-RRL-001 \\
\hline 14733-03-0 & Bismuth-214 & $<0.894$ & $\mathrm{pCi} / \mathrm{g}$ & 0.894 & & 3/31/08 & 8C17008 & AGG-RRL-001 \\
\hline $15067-28-4$ & Lead-214 & $<0.934$ & $\mathrm{pCi} / \mathrm{g}$ & 0.934 & & 3/31/08 & 8C17008 & AGG-RRL-001 \\
\hline $14835-02-0$ & Radon-219 & $<3.39$ & $\mathrm{pCi} / \mathrm{g}$ & 3.39 & & $3 / 31 / 08$ & 8C17008 & AGG-RRL-001 \\
\hline 22481-48-7 & Radon-220 & $<328$ & $\mathrm{pCi} / \mathrm{g}$ & 328 & & 3/31/08 & 8C17008 & AGG-RRL-001 \\
\hline 28522-20-5 & Radon-221 & $<1.61$ & $\mathrm{pCi} / \mathrm{g}$ & 1.61 & & 3/31/08 & 8C17008 & AGG-RRL-001 \\
\hline 15756-98-6 & Francium-223 & $<11.1$ & $\mathrm{pCi} / \mathrm{g}$ & 11.1 & & 3/31/08 & 8C17008 & AGG-RRL-001 \\
\hline $15623-45-7$ & Radium-223 & $<2.48$ & $\mathrm{pCi} / \mathrm{g}$ & 2.48 & & 3/31/08 & 8C17008 & AGG-RRL-001 \\
\hline 13233-32-4 & Radium-224 & $<9.54$ & $\mathrm{pCi} / \mathrm{g}$ & 9.54 & & 3/31/08 & 8C17008 & AGG-RRL-001 \\
\hline 13982-63-3 & Radium-226 & $<9.94$ & $\mathrm{pCi} / \mathrm{g}$ & 9.94 & & 3/31/08 & 8C17008 & AGG-RRL-001 \\
\hline $15623-47-9$ & Thorium-227 & $<3.37$ & $\mathrm{pCi} / \mathrm{g}$ & 3.37 & & 3/31/08 & 8C17008 & AGG-RRL-001 \\
\hline 14331-83-0 & Actinium-228 & $<1.48$ & $\mathrm{pCi} / \mathrm{g}$ & 1.48 & & 3/31/08 & 8C17008 & AGG-RRL-001 \\
\hline 14274-82-9 & Thorium-228 & $<123$ & $\mathrm{pCi} / \mathrm{g}$ & 123 & & 3/31/08 & 8C17008 & AGG-RRL-001 \\
\hline
\end{tabular}


GEA/Soil

\begin{tabular}{|c|c|c|c|c|c|c|c|c|}
\hline CAS \# & Analyte & Results & Units & MDA & UNC & Analyzed & Batch & Method \\
\hline HEIS No. & B1RYT0A & \multicolumn{2}{|c|}{ Lab ID: } & 0803014-02 & & & & \\
\hline 14269-63-7 & Thorium-230 & $<130$ & $\mathrm{pCi} / \mathrm{g}$ & 130 & & 3/31/08 & 8C17008 & AGG-RRL-001 \\
\hline 14331-85-2 & Protactinium-231 & $<15.5$ & $\mathrm{pCi} / \mathrm{g}$ & 15.5 & & $3 / 31 / 08$ & 8C17008 & AGG-RRL-001 \\
\hline $14932-40-2$ & Thorium-231 & $<77.4$ & $\mathrm{pCi} / \mathrm{g}$ & 77.4 & & 3/31/08 & 8C17008 & AGG-RRL-001 \\
\hline 7440-29-1 & Thorium-232 & $<330$ & $\mathrm{pCi} / \mathrm{g}$ & 330 & & 3/31/08 & 8C17008 & AGG-RRL-001 \\
\hline 13981-14-1 & Protactinium-233 & $<0.932$ & $\mathrm{pCi} / \mathrm{g}$ & 0.932 & & 3/31/08 & 8C17008 & AGG-RRL-001 \\
\hline \multirow[t]{2}{*}{$15100-28-4$} & Protactinium-234 & $<1.41$ & $\mathrm{pCi} / \mathrm{g}$ & 1.41 & & 3/31/08 & 8C17008 & AGG-RRL-001 \\
\hline & Protactinium-234m & $<44.7$ & $\mathrm{pCi} / \mathrm{g}$ & 44.7 & & 3/31/08 & 8C17008 & AGG-RRL-001 \\
\hline 15065-10-8 & Thorium-234 & $<11.5$ & $\mathrm{pCi} / \mathrm{g}$ & 11.5 & & 3/31/08 & 8C17008 & AGG-RRL-001 \\
\hline 15117-96-1 & Uranium 235 & $<2.92$ & $\mathrm{pCi} / \mathrm{g}$ & 2.92 & & 3/31/08 & 8C17008 & AGG-RRL-001 \\
\hline 13994-20-2 & Neptunium-237 & $<2.9$ & $\mathrm{pCi} / \mathrm{g}$ & 2.9 & & 3/31/08 & 8C17008 & AGG-RRL-001 \\
\hline \multirow[t]{2}{*}{ 13981-16-3 } & Plutonium-238 & $<4450$ & $\mathrm{pCi} / \mathrm{g}$ & 4450 & & 3/31/08 & 8C17008 & AGG-RRL-001 \\
\hline & Uranium 238 & $<6.2$ & $\mathrm{pCi} / \mathrm{g}$ & 6.2 & & 3/31/08 & 8C17008 & AGG-RRL-001 \\
\hline 15117-48-3 & Plutonium-239 & $<4480$ & $\mathrm{pCi} / \mathrm{g}$ & 4480 & & 3/31/08 & 8C17008 & AGG-RRL-001 \\
\hline 14119-33-6 & Plutonium-240 & $<4500$ & $\mathrm{pCi} / \mathrm{g}$ & 4500 & & 3/31/08 & 8C17008 & AGG-RRL-001 \\
\hline 14596-10-2 & Americium-241 & $<1.69$ & $\mathrm{pCi} / \mathrm{g}$ & 1.69 & & 3/31/08 & 8C17008 & AGG-RRL-001 \\
\hline 14993-75-0 & Americium-243 & $<0.705$ & $\mathrm{pCi} / \mathrm{g}$ & 0.705 & & 3/31/08 & 8C17008 & AGG-RRL-001 \\
\hline $15757-87-6$ & Curium-243 & $<1.37$ & $\mathrm{pCi} / \mathrm{g}$ & 1.37 & & 3/31/08 & 8C17008 & AGG-RRL-001 \\
\hline 15621-76-8 & Curium-245 & $<1.13$ & $\mathrm{pCi} / \mathrm{g}$ & 1.13 & & 3/31/08 & 8C17008 & AGG-RRL-001 \\
\hline HEIS No. & B1T2P1B & \multicolumn{2}{|c|}{ Lab ID: } & 0803014-05 & & & & \\
\hline 13966-32-0 & Sodium-22 & $<0.372$ & $\mathrm{pCi} / \mathrm{g}$ & 0.372 & & 3/31/08 & 8C17008 & AGG-RRL-001 \\
\hline 13966-00-2 & Potassium-40 & 20.7 & $\mathrm{pCi} / \mathrm{g}$ & 3.19 & 1.31 & 3/31/08 & 8C17008 & AGG-RRL-001 \\
\hline $14392-02-0$ & Chromium-51 & $<3.48$ & $\mathrm{pCi} / \mathrm{g}$ & 3.48 & & 3/31/08 & 8C17008 & AGG-RRL-001 \\
\hline 13966-31-9 & Manganese-54 & $<0.392$ & $\mathrm{pCi} / \mathrm{g}$ & 0.392 & & 3/31/08 & 8C17008 & AGG-RRL-001 \\
\hline 13981-50-5 & Cobalt-57 & $<0.38$ & $\mathrm{pCi} / \mathrm{g}$ & 0.38 & & 3/31/08 & 8C17008 & AGG-RRL-001 \\
\hline $14596-12-4$ & Iron-59 & $<0.686$ & $\mathrm{pCi} / \mathrm{g}$ & 0.686 & & 3/31/08 & 8C17008 & AGG-RRL-001 \\
\hline 10198-40-0 & Cobalt-60 & $<0.357$ & $\mathrm{pCi} / \mathrm{g}$ & 0.357 & & 3/31/08 & 8C17008 & AGG-RRL-001 \\
\hline 13982-39-3 & Zinc-65 & $<0.876$ & $\mathrm{pCi} / \mathrm{g}$ & 0.876 & & $3 / 31 / 08$ & 8C17008 & AGG-RRL-001 \\
\hline 14265-71-5 & Selenium-75 & $<0.585$ & $\mathrm{pCi} / \mathrm{g}$ & 0.585 & & 3/31/08 & 8C17008 & AGG-RRL-001 \\
\hline 17056-36-9 & Rubidium-83 & $<0.899$ & $\mathrm{pCi} / \mathrm{g}$ & 0.899 & & 3/31/08 & 8C17008 & AGG-RRL-001 \\
\hline 13967-73-2 & Strontium-85 & $<0.418$ & $\mathrm{pCi} / \mathrm{g}$ & 0.418 & & 3/31/08 & 8C17008 & AGG-RRL-001 \\
\hline $14932-53-7$ & Rubidium-86 & $<4.59$ & $\mathrm{pCi} / \mathrm{g}$ & 4.59 & & 3/31/08 & 8C17008 & AGG-RRL-001 \\
\hline 13982-36-0 & Yttrium-88 & $<0.303$ & $\mathrm{pCi} / \mathrm{g}$ & 0.303 & & 3/31/08 & 8C17008 & AGG-RRL-001 \\
\hline 14681-63-1 & Niobium-94 & $<0.353$ & $\mathrm{pCi} / \mathrm{g}$ & 0.353 & & 3/31/08 & 8C17008 & AGG-RRL-001 \\
\hline \multirow[t]{3}{*}{ 13967-76-5 } & Niobium-95 & $<0.363$ & $\mathrm{pCi} / \mathrm{g}$ & 0.363 & & 3/31/08 & 8C17008 & AGG-RRL-001 \\
\hline & Niobium-95m & $<1.66$ & $\mathrm{pCi} / \mathrm{g}$ & 1.66 & & 3/31/08 & 8C17008 & AGG-RRL-001 \\
\hline & Technetium-95m & $<0.552$ & $\mathrm{pCi} / \mathrm{g}$ & 0.552 & & 3/31/08 & 8C17008 & AGG-RRL-001 \\
\hline \multirow[t]{2}{*}{$13967-71-0$} & Zirconium-95 & $<0.65$ & $\mathrm{pCi} / \mathrm{g}$ & 0.65 & & 3/31/08 & 8C17008 & AGG-RRL-001 \\
\hline & Technetium-99m & $<0.37$ & $\mathrm{pCi} / \mathrm{g}$ & 0.37 & & 3/31/08 & 8C17008 & AGG-RRL-001 \\
\hline 13968-53-1 & Ruthenium-103 & $<0.402$ & $\mathrm{pCi} / \mathrm{g}$ & 0.402 & & 3/31/08 & 8C17008 & AGG-RRL-001 \\
\hline 13967-48-1 & Ruthenium-106 & $<4.16$ & $\mathrm{pCi} / \mathrm{g}$ & 4.16 & & 3/31/08 & 8C17008 & AGG-RRL-001 \\
\hline 14391-65-2 & Silver-108m & $<0.363$ & $\mathrm{pCi} / \mathrm{g}$ & 0.363 & & 3/31/08 & 8C17008 & AGG-RRL-001 \\
\hline \multirow[t]{2}{*}{ 14109-32-1 } & Cadmium-109 & $<10.2$ & $\mathrm{pCi} / \mathrm{g}$ & 10.2 & & 3/31/08 & 8C17008 & AGG-RRL-001 \\
\hline & Silver-110 & $<0.355$ & $\mathrm{pCi} / \mathrm{g}$ & 0.355 & & 3/31/08 & 8C17008 & AGG-RRL-001 \\
\hline $14391-76-5$ & Silver-110m & $<0.356$ & $\mathrm{pCi} / \mathrm{g}$ & 0.356 & & 3/31/08 & 8C17008 & AGG-RRL-001 \\
\hline 13966-06-8 & Tin-113 & $<0.505$ & $\mathrm{pCi} / \mathrm{g}$ & 0.505 & & 3/31/08 & 8C17008 & AGG-RRL-001 \\
\hline $14683-10-4$ & Antimony-124 & $<0.441$ & $\mathrm{pCi} / \mathrm{g}$ & 0.441 & & 3/31/08 & 8C17008 & AGG-RRL-001 \\
\hline 14234-35-6 & Antimony-125 & $<1.12$ & $\mathrm{pCi} / \mathrm{g}$ & 1.12 & & 3/31/08 & 8C17008 & AGG-RRL-001 \\
\hline 15756-32-8 & Antimony-126 & $<0.335$ & $\mathrm{pCi} / \mathrm{g}$ & 0.335 & & 3/31/08 & 8C17008 & AGG-RRL-001 \\
\hline
\end{tabular}


GEA/Soil

\begin{tabular}{|c|c|c|c|c|c|c|c|c|}
\hline CAS \# & Analyte & Results & Units & MDA & UNC & Analyzed & Batch & Method \\
\hline HEIS No. & B1T2P1B & \multicolumn{3}{|c|}{ Lab ID: $\quad$ 0803014-05 } & & & & \\
\hline $15832-50-5$ & Tin-126 & $<0.837$ & $\mathrm{pCi} / \mathrm{g}$ & 0.837 & & 3/31/08 & 8C17008 & AGG-RRL-001 \\
\hline $10043-66-0$ & Iodine-131 & $<0.457$ & $\mathrm{pCi} / \mathrm{g}$ & 0.457 & & 3/31/08 & 8C17008 & AGG-RRL-001 \\
\hline 13981-41-4 & Barium-133 & $<0.638$ & $\mathrm{pCi} / \mathrm{g}$ & 0.638 & & 3/31/08 & 8C17008 & AGG-RRL-001 \\
\hline 13967-70-9 & Cesium-134 & $<0.425$ & $\mathrm{pCi} / \mathrm{g}$ & 0.425 & & 3/31/08 & 8C17008 & AGG-RRL-001 \\
\hline $10045-97-3$ & Cesium-137 & $<0.384$ & $\mathrm{pCi} / \mathrm{g}$ & 0.384 & & $3 / 31 / 08$ & 8C17008 & AGG-RRL-001 \\
\hline 13982-30-4 & Cerium-139 & $<0.425$ & $\mathrm{pCi} / \mathrm{g}$ & 0.425 & & 3/31/08 & 8C17008 & AGG-RRL-001 \\
\hline $14762-78-8$ & Cerium-144 & $<3.06$ & $\mathrm{pCi} / \mathrm{g}$ & 3.06 & & $3 / 31 / 08$ & 8C17008 & AGG-RRL-001 \\
\hline 14683-23-9 & Europium-152 & $<1.49$ & $\mathrm{pCi} / \mathrm{g}$ & 1.49 & & 3/31/08 & 8C17008 & AGG-RRL-001 \\
\hline $14276-65-4$ & Gadolinium-153 & $<1.13$ & $\mathrm{pCi} / \mathrm{g}$ & 1.13 & & 3/31/08 & 8C17008 & AGG-RRL-001 \\
\hline 15585-10-1 & Europium-154 & $<0.803$ & $\mathrm{pCi} / \mathrm{g}$ & 0.803 & & $3 / 31 / 08$ & 8C17008 & AGG-RRL-001 \\
\hline 14391-16-3 & Europium-155 & $<1.19$ & $\mathrm{pCi} / \mathrm{g}$ & 1.19 & & 3/31/08 & 8C17008 & AGG-RRL-001 \\
\hline 13982-78-0 & Mercury-203 & $<0.457$ & $\mathrm{pCi} / \mathrm{g}$ & 0.457 & & $3 / 31 / 08$ & 8C17008 & AGG-RRL-001 \\
\hline 14913-50-9 & Thallium-208 & $<0.495$ & $\mathrm{pCi} / \mathrm{g}$ & 0.495 & & $3 / 31 / 08$ & 8C17008 & AGG-RRL-001 \\
\hline 14331-79-4 & Bismuth-210 & $<0.701$ & $\mathrm{pCi} / \mathrm{g}$ & 0.701 & & 3/31/08 & 8C17008 & AGG-RRL-001 \\
\hline \multirow[t]{3}{*}{$14255-04-0$} & Lead-210 & $<49.1$ & $\mathrm{pCi} / \mathrm{g}$ & 49.1 & & 3/31/08 & 8C17008 & AGG-RRL-001 \\
\hline & Bismuth-211 & $<8.08$ & $\mathrm{pCi} / \mathrm{g}$ & 8.08 & & 3/31/08 & 8C17008 & AGG-RRL-001 \\
\hline & Lead-211 & $<11$ & $\mathrm{pCi} / \mathrm{g}$ & 11 & & 3/31/08 & 8C17008 & AGG-RRL-001 \\
\hline 15092-94-1 & Lead-212 & $<0.963$ & $\mathrm{pCi} / \mathrm{g}$ & 0.963 & & 3/31/08 & 8C17008 & AGG-RRL-001 \\
\hline 14733-03-0 & Bismuth-214 & $<0.929$ & $\mathrm{pCi} / \mathrm{g}$ & 0.929 & & 3/31/08 & 8C17008 & AGG-RRL-001 \\
\hline 15067-28-4 & Lead-214 & $<1.02$ & $\mathrm{pCi} / \mathrm{g}$ & 1.02 & & 3/31/08 & 8C17008 & AGG-RRL-001 \\
\hline $14835-02-0$ & Radon-219 & $<3.56$ & $\mathrm{pCi} / \mathrm{g}$ & 3.56 & & 3/31/08 & 8C17008 & AGG-RRL-001 \\
\hline 22481-48-7 & Radon-220 & $<349$ & $\mathrm{pCi} / \mathrm{g}$ & 349 & & 3/31/08 & 8C17008 & AGG-RRL-001 \\
\hline 28522-20-5 & Radon-221 & $<1.65$ & $\mathrm{pCi} / \mathrm{g}$ & 1.65 & & $3 / 31 / 08$ & 8C17008 & AGG-RRL-001 \\
\hline 15756-98-6 & Francium-223 & $<12.2$ & $\mathrm{pCi} / \mathrm{g}$ & 12.2 & & 3/31/08 & 8C17008 & AGG-RRL-001 \\
\hline $15623-45-7$ & Radium-223 & $<2.6$ & $\mathrm{pCi} / \mathrm{g}$ & 2.6 & & 3/31/08 & 8C17008 & AGG-RRL-001 \\
\hline 13233-32-4 & Radium-224 & $<10.4$ & $\mathrm{pCi} / \mathrm{g}$ & 10.4 & & $3 / 31 / 08$ & 8C17008 & AGG-RRL-001 \\
\hline 13982-63-3 & Radium-226 & $<10.2$ & $\mathrm{pCi} / \mathrm{g}$ & 10.2 & & 3/31/08 & 8C17008 & AGG-RRL-001 \\
\hline 15623-47-9 & Thorium-227 & $<3.72$ & $\mathrm{pCi} / \mathrm{g}$ & 3.72 & & 3/31/08 & 8C17008 & AGG-RRL-001 \\
\hline 14331-83-0 & Actinium-228 & $<1.69$ & $\mathrm{pCi} / \mathrm{g}$ & 1.69 & & 3/31/08 & 8C17008 & AGG-RRL-001 \\
\hline 14274-82-9 & Thorium-228 & $<134$ & $\mathrm{pCi} / \mathrm{g}$ & 134 & & 3/31/08 & 8C17008 & AGG-RRL-001 \\
\hline 14269-63-7 & Thorium-230 & $<139$ & $\mathrm{pCi} / \mathrm{g}$ & 139 & & 3/31/08 & 8C17008 & AGG-RRL-001 \\
\hline 14331-85-2 & Protactinium-231 & $<15.8$ & $\mathrm{pCi} / \mathrm{g}$ & 15.8 & & 3/31/08 & 8C17008 & AGG-RRL-001 \\
\hline $14932-40-2$ & Thorium-231 & $<80.8$ & $\mathrm{pCi} / \mathrm{g}$ & 80.8 & & 3/31/08 & 8C17008 & AGG-RRL-001 \\
\hline 7440-29-1 & Thorium-232 & $<347$ & $\mathrm{pCi} / \mathrm{g}$ & 347 & & 3/31/08 & 8C17008 & AGG-RRL-001 \\
\hline 13981-14-1 & Protactinium-233 & $<0.949$ & $\mathrm{pCi} / \mathrm{g}$ & 0.949 & & 3/31/08 & 8C17008 & AGG-RRL-001 \\
\hline \multirow[t]{2}{*}{$15100-28-4$} & Protactinium-234 & $<1.47$ & $\mathrm{pCi} / \mathrm{g}$ & 1.47 & & 3/31/08 & 8C17008 & AGG-RRL-001 \\
\hline & Protactinium-234m & $<43.5$ & $\mathrm{pCi} / \mathrm{g}$ & 43.5 & & 3/31/08 & 8C17008 & AGG-RRL-001 \\
\hline $15065-10-8$ & Thorium-234 & $<12.2$ & $\mathrm{pCi} / \mathrm{g}$ & 12.2 & & 3/31/08 & 8C17008 & AGG-RRL-001 \\
\hline 15117-96-1 & Uranium 235 & $<3.16$ & $\mathrm{pCi} / \mathrm{g}$ & 3.16 & & 3/31/08 & 8C17008 & AGG-RRL-001 \\
\hline 13994-20-2 & Neptunium-237 & $<3.09$ & $\mathrm{pCi} / \mathrm{g}$ & 3.09 & & 3/31/08 & 8C17008 & AGG-RRL-001 \\
\hline \multirow[t]{2}{*}{ 13981-16-3 } & Plutonium-238 & $<4630$ & $\mathrm{pCi} / \mathrm{g}$ & 4630 & & 3/31/08 & 8C17008 & AGG-RRL-001 \\
\hline & Uranium 238 & $<6.49$ & $\mathrm{pCi} / \mathrm{g}$ & 6.49 & & 3/31/08 & 8C17008 & AGG-RRL-001 \\
\hline $15117-48-3$ & Plutonium-239 & $<4790$ & $\mathrm{pCi} / \mathrm{g}$ & 4790 & & 3/31/08 & 8C17008 & AGG-RRL-001 \\
\hline 14119-33-6 & Plutonium-240 & $<4680$ & $\mathrm{pCi} / \mathrm{g}$ & 4680 & & 3/31/08 & 8C17008 & AGG-RRL-001 \\
\hline $14596-10-2$ & Americium-241 & $<1.78$ & $\mathrm{pCi} / \mathrm{g}$ & 1.78 & & 3/31/08 & 8C17008 & AGG-RRL-001 \\
\hline 14993-75-0 & Americium-243 & $<0.76$ & $\mathrm{pCi} / \mathrm{g}$ & 0.76 & & 3/31/08 & 8C17008 & AGG-RRL-001 \\
\hline 15757-87-6 & Curium-243 & $<1.43$ & $\mathrm{pCi} / \mathrm{g}$ & 1.43 & & 3/31/08 & 8C17008 & AGG-RRL-001 \\
\hline 15621-76-8 & Curium-245 & $<1.17$ & $\mathrm{pCi} / \mathrm{g}$ & 1.17 & & 3/31/08 & 8C17008 & AGG-RRL-001 \\
\hline
\end{tabular}


GEA/Soil

\begin{tabular}{|c|c|c|c|c|c|c|c|c|}
\hline CAS \# & Analyte & Results & Units & MDA & UNC & Analyzed & Batch & Method \\
\hline HEIS No. & B1T2P1A & \multicolumn{3}{|c|}{ Lab ID: $\quad$ 0803014-06 } & & & & \\
\hline 13966-32-0 & Sodium-22 & $<0.367$ & $\mathrm{pCi} / \mathrm{g}$ & 0.367 & & 3/31/08 & 8C17008 & AGG-RRL-001 \\
\hline 13966-00-2 & Potassium-40 & 20.7 & $\mathrm{pCi} / \mathrm{g}$ & 2.92 & 1.84 & 3/31/08 & 8C17008 & AGG-RRL-001 \\
\hline $14392-02-0$ & Chromium-51 & $<3.14$ & $\mathrm{pCi} / \mathrm{g}$ & 3.14 & & 3/31/08 & 8C17008 & AGG-RRL-001 \\
\hline 13966-31-9 & Manganese-54 & $<0.334$ & $\mathrm{pCi} / \mathrm{g}$ & 0.334 & & 3/31/08 & 8C17008 & AGG-RRL-001 \\
\hline 13981-50-5 & Cobalt-57 & $<0.334$ & $\mathrm{pCi} / \mathrm{g}$ & 0.334 & & 3/31/08 & 8C17008 & AGG-RRL-001 \\
\hline 14596-12-4 & Iron-59 & $<0.623$ & $\mathrm{pCi} / \mathrm{g}$ & 0.623 & & 3/31/08 & 8C17008 & AGG-RRL-001 \\
\hline 10198-40-0 & Cobalt-60 & $<0.377$ & $\mathrm{pCi} / \mathrm{g}$ & 0.377 & & 3/31/08 & 8C17008 & AGG-RRL-001 \\
\hline 13982-39-3 & Zinc-65 & $<0.805$ & $\mathrm{pCi} / \mathrm{g}$ & 0.805 & & 3/31/08 & 8C17008 & AGG-RRL-001 \\
\hline 14265-71-5 & Selenium-75 & $<0.547$ & $\mathrm{pCi} / \mathrm{g}$ & 0.547 & & 3/31/08 & 8C17008 & AGG-RRL-001 \\
\hline 17056-36-9 & Rubidium-83 & $<0.636$ & $\mathrm{pCi} / \mathrm{g}$ & 0.636 & & $3 / 31 / 08$ & 8C17008 & AGG-RRL-001 \\
\hline 13967-73-2 & Strontium-85 & $<0.407$ & $\mathrm{pCi} / \mathrm{g}$ & 0.407 & & 3/31/08 & 8C17008 & AGG-RRL-001 \\
\hline 14932-53-7 & Rubidium-86 & $<3.85$ & $\mathrm{pCi} / \mathrm{g}$ & 3.85 & & $3 / 31 / 08$ & 8C17008 & AGG-RRL-001 \\
\hline 13982-36-0 & Yttrium-88 & $<0.291$ & $\mathrm{pCi} / \mathrm{g}$ & 0.291 & & $3 / 31 / 08$ & 8C17008 & AGG-RRL-001 \\
\hline 14681-63-1 & Niobium-94 & $<0.343$ & $\mathrm{pCi} / \mathrm{g}$ & 0.343 & & 3/31/08 & 8C17008 & AGG-RRL-001 \\
\hline \multirow[t]{3}{*}{ 13967-76-5 } & Niobium-95 & $<0.34$ & $\mathrm{pCi} / \mathrm{g}$ & 0.34 & & 3/31/08 & 8C17008 & AGG-RRL-001 \\
\hline & Niobium-95m & $<1.62$ & $\mathrm{pCi} / \mathrm{g}$ & 1.62 & & 3/31/08 & 8C17008 & AGG-RRL-001 \\
\hline & Technetium-95m & $<0.524$ & $\mathrm{pCi} / \mathrm{g}$ & 0.524 & & 3/31/08 & 8C17008 & AGG-RRL-001 \\
\hline \multirow[t]{2}{*}{ 13967-71-0 } & Zirconium-95 & $<0.6$ & $\mathrm{pCi} / \mathrm{g}$ & 0.6 & & 3/31/08 & 8C17008 & AGG-RRL-001 \\
\hline & Technetium-99m & $<0.338$ & $\mathrm{pCi} / \mathrm{g}$ & 0.338 & & 3/31/08 & 8C17008 & AGG-RRL-001 \\
\hline 13968-53-1 & Ruthenium-103 & $<0.329$ & $\mathrm{pCi} / \mathrm{g}$ & 0.329 & & 3/31/08 & 8C17008 & AGG-RRL-001 \\
\hline 13967-48-1 & Ruthenium-106 & $<3.24$ & $\mathrm{pCi} / \mathrm{g}$ & 3.24 & & 3/31/08 & 8C17008 & AGG-RRL-001 \\
\hline 14391-65-2 & Silver-108m & $<0.343$ & $\mathrm{pCi} / \mathrm{g}$ & 0.343 & & 3/31/08 & 8C17008 & AGG-RRL-001 \\
\hline \multirow[t]{2}{*}{ 14109-32-1 } & Cadmium-109 & $<8.96$ & $\mathrm{pCi} / \mathrm{g}$ & 8.96 & & 3/31/08 & 8C17008 & AGG-RRL-001 \\
\hline & Silver-110 & $<0.344$ & $\mathrm{pCi} / \mathrm{g}$ & 0.344 & & 3/31/08 & 8C17008 & AGG-RRL-001 \\
\hline 14391-76-5 & Silver-110m & $<0.345$ & $\mathrm{pCi} / \mathrm{g}$ & 0.345 & & 3/31/08 & 8C17008 & AGG-RRL-001 \\
\hline 13966-06-8 & Tin-113 & $<0.467$ & $\mathrm{pCi} / \mathrm{g}$ & 0.467 & & 3/31/08 & 8C17008 & AGG-RRL-001 \\
\hline 14683-10-4 & Antimony-124 & $<0.361$ & $\mathrm{pCi} / \mathrm{g}$ & 0.361 & & 3/31/08 & 8C17008 & AGG-RRL-001 \\
\hline $14234-35-6$ & Antimony-125 & $<1.02$ & $\mathrm{pCi} / \mathrm{g}$ & 1.02 & & 3/31/08 & 8C17008 & AGG-RRL-001 \\
\hline 15756-32-8 & Antimony-126 & $<0.351$ & $\mathrm{pCi} / \mathrm{g}$ & 0.351 & & 3/31/08 & 8C17008 & AGG-RRL-001 \\
\hline 15832-50-5 & Tin-126 & $<0.702$ & $\mathrm{pCi} / \mathrm{g}$ & 0.702 & & 3/31/08 & 8C17008 & AGG-RRL-001 \\
\hline 10043-66-0 & Iodine-131 & $<0.395$ & $\mathrm{pCi} / \mathrm{g}$ & 0.395 & & 3/31/08 & 8C17008 & AGG-RRL-001 \\
\hline 13981-41-4 & Barium-133 & $<0.629$ & $\mathrm{pCi} / \mathrm{g}$ & 0.629 & & 3/31/08 & 8C17008 & AGG-RRL-001 \\
\hline 13967-70-9 & Cesium-134 & $<0.364$ & $\mathrm{pCi} / \mathrm{g}$ & 0.364 & & 3/31/08 & 8C17008 & AGG-RRL-001 \\
\hline $10045-97-3$ & Cesium-137 & $<0.398$ & $\mathrm{pCi} / \mathrm{g}$ & 0.398 & & 3/31/08 & 8C17008 & AGG-RRL-001 \\
\hline 13982-30-4 & Cerium-139 & $<0.39$ & $\mathrm{pCi} / \mathrm{g}$ & 0.39 & & 3/31/08 & 8C17008 & AGG-RRL-001 \\
\hline 14762-78-8 & Cerium-144 & $<2.77$ & $\mathrm{pCi} / \mathrm{g}$ & 2.77 & & 3/31/08 & 8C17008 & AGG-RRL-001 \\
\hline 14683-23-9 & Europium-152 & $<1.34$ & $\mathrm{pCi} / \mathrm{g}$ & 1.34 & & 3/31/08 & 8C17008 & AGG-RRL-001 \\
\hline $14276-65-4$ & Gadolinium-153 & $<0.999$ & $\mathrm{pCi} / \mathrm{g}$ & 0.999 & & 3/31/08 & 8C17008 & AGG-RRL-001 \\
\hline 15585-10-1 & Europium-154 & $<0.718$ & $\mathrm{pCi} / \mathrm{g}$ & 0.718 & & 3/31/08 & 8C17008 & AGG-RRL-001 \\
\hline 14391-16-3 & Europium-155 & $<0.99$ & $\mathrm{pCi} / \mathrm{g}$ & 0.99 & & 3/31/08 & 8C17008 & AGG-RRL-001 \\
\hline 13982-78-0 & Mercury-203 & $<0.44$ & $\mathrm{pCi} / \mathrm{g}$ & 0.44 & & 3/31/08 & 8C17008 & AGG-RRL-001 \\
\hline 14913-50-9 & Thallium-208 & $<0.414$ & $\mathrm{pCi} / \mathrm{g}$ & 0.414 & & 3/31/08 & 8C17008 & AGG-RRL-001 \\
\hline 14331-79-4 & Bismuth-210 & $<0.666$ & $\mathrm{pCi} / \mathrm{g}$ & 0.666 & & 3/31/08 & 8C17008 & AGG-RRL-001 \\
\hline \multirow[t]{3}{*}{$14255-04-0$} & Lead-210 & $<131$ & $\mathrm{pCi} / \mathrm{g}$ & 131 & & 3/31/08 & 8C17008 & AGG-RRL-001 \\
\hline & Bismuth-211 & $<8.19$ & $\mathrm{pCi} / \mathrm{g}$ & 8.19 & & 3/31/08 & 8C17008 & AGG-RRL-001 \\
\hline & Lead-211 & $<11.2$ & $\mathrm{pCi} / \mathrm{g}$ & 11.2 & & 3/31/08 & 8C17008 & AGG-RRL-001 \\
\hline 15092-94-1 & Lead-212 & $<0.861$ & $\mathrm{pCi} / \mathrm{g}$ & 0.861 & 0.192 & 3/31/08 & 8C17008 & AGG-RRL-001 \\
\hline 14733-03-0 & Bismuth-214 & $<0.882$ & $\mathrm{pCi} / \mathrm{g}$ & 0.882 & & 3/31/08 & 8C17008 & AGG-RRL-001 \\
\hline $15067-28-4$ & Lead-214 & $<1.01$ & $\mathrm{pCi} / \mathrm{g}$ & 1.01 & & 3/31/08 & 8C17008 & AGG-RRL-001 \\
\hline
\end{tabular}


GEA/Soil

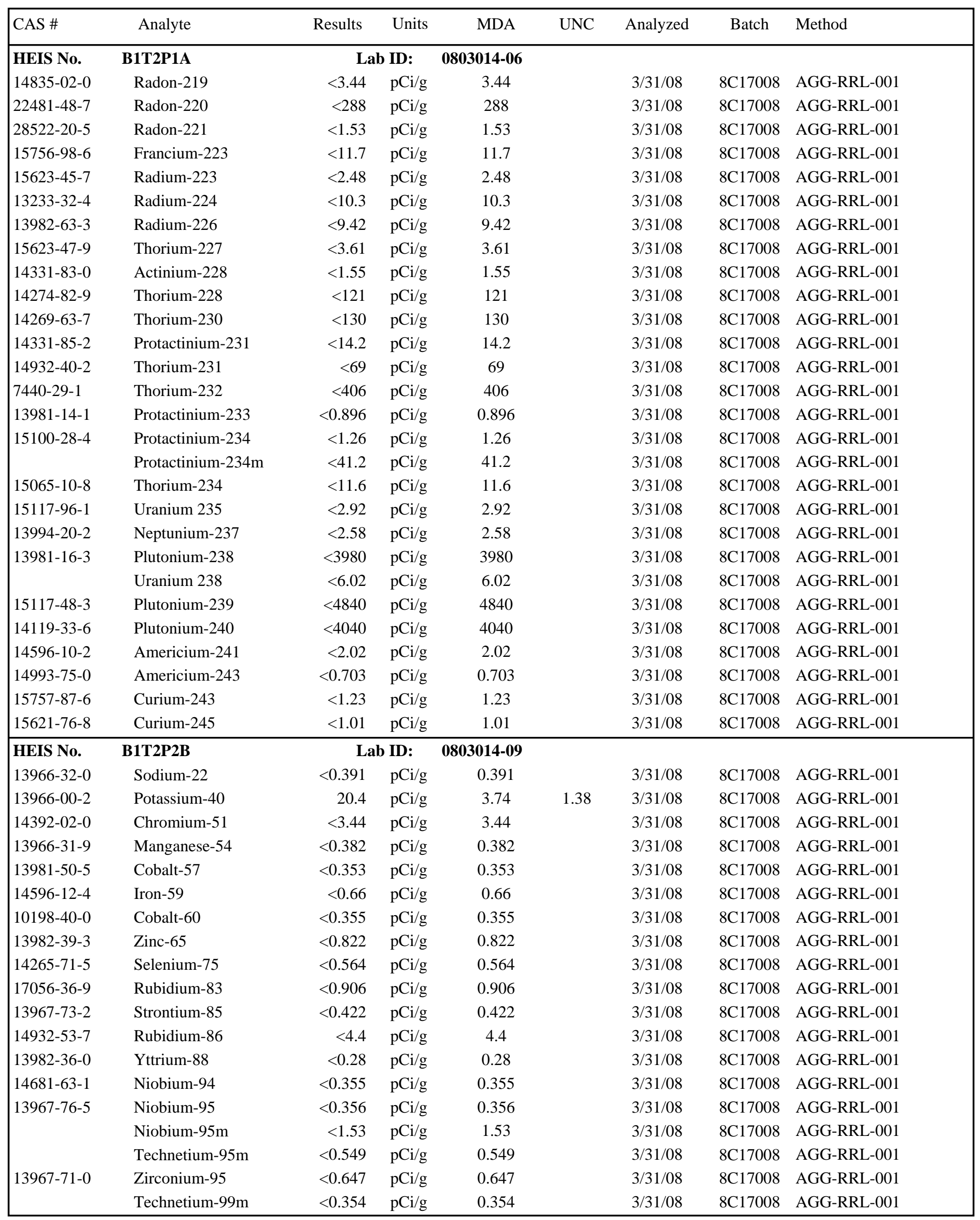


GEA/Soil

\begin{tabular}{|c|c|c|c|c|c|c|c|c|}
\hline CAS \# & Analyte & Results & Units & MDA & UNC & Analyzed & Batch & Method \\
\hline HEIS No. & B1T2P2B & \multicolumn{2}{|c|}{ Lab ID: } & 0803014-09 & & & & \\
\hline 13968-53-1 & Ruthenium-103 & $<0.393$ & $\mathrm{pCi} / \mathrm{g}$ & 0.393 & & 3/31/08 & 8C17008 & AGG-RRL-001 \\
\hline 13967-48-1 & Ruthenium-106 & $<3.84$ & $\mathrm{pCi} / \mathrm{g}$ & 3.84 & & 3/31/08 & 8C17008 & AGG-RRL-001 \\
\hline 14391-65-2 & Silver-108m & $<0.354$ & $\mathrm{pCi} / \mathrm{g}$ & 0.354 & & 3/31/08 & 8C17008 & AGG-RRL-001 \\
\hline \multirow[t]{2}{*}{ 14109-32-1 } & Cadmium-109 & $<9.78$ & $\mathrm{pCi} / \mathrm{g}$ & 9.78 & & 3/31/08 & 8C17008 & AGG-RRL-001 \\
\hline & Silver-110 & $<0.356$ & $\mathrm{pCi} / \mathrm{g}$ & 0.356 & & 3/31/08 & 8C17008 & AGG-RRL-001 \\
\hline 14391-76-5 & Silver-110m & $<0.357$ & $\mathrm{pCi} / \mathrm{g}$ & 0.357 & & 3/31/08 & 8C17008 & AGG-RRL-001 \\
\hline 13966-06-8 & Tin-113 & $<0.472$ & $\mathrm{pCi} / \mathrm{g}$ & 0.472 & & 3/31/08 & 8C17008 & AGG-RRL-001 \\
\hline 14683-10-4 & Antimony-124 & $<0.411$ & $\mathrm{pCi} / \mathrm{g}$ & 0.411 & & 3/31/08 & 8C17008 & AGG-RRL-001 \\
\hline $14234-35-6$ & Antimony-125 & $<1.09$ & $\mathrm{pCi} / \mathrm{g}$ & 1.09 & & 3/31/08 & 8C17008 & AGG-RRL-001 \\
\hline 15756-32-8 & Antimony-126 & $<0.338$ & $\mathrm{pCi} / \mathrm{g}$ & 0.338 & & 3/31/08 & 8C17008 & AGG-RRL-001 \\
\hline 15832-50-5 & Tin-126 & $<0.797$ & $\mathrm{pCi} / \mathrm{g}$ & 0.797 & & 3/31/08 & 8C17008 & AGG-RRL-001 \\
\hline 10043-66-0 & Iodine-131 & $<0.433$ & $\mathrm{pCi} / \mathrm{g}$ & 0.433 & & 3/31/08 & 8C17008 & AGG-RRL-001 \\
\hline 13981-41-4 & Barium-133 & $<0.601$ & $\mathrm{pCi} / \mathrm{g}$ & 0.601 & & 3/31/08 & 8C17008 & AGG-RRL-001 \\
\hline 13967-70-9 & Cesium-134 & $<0.406$ & $\mathrm{pCi} / \mathrm{g}$ & 0.406 & & 3/31/08 & 8C17008 & AGG-RRL-001 \\
\hline 10045-97-3 & Cesium-137 & $<0.391$ & $\mathrm{pCi} / \mathrm{g}$ & 0.391 & & 3/31/08 & 8C17008 & AGG-RRL-001 \\
\hline 13982-30-4 & Cerium-139 & $<0.405$ & $\mathrm{pCi} / \mathrm{g}$ & 0.405 & & 3/31/08 & 8C17008 & AGG-RRL-001 \\
\hline 14762-78-8 & Cerium-144 & $<2.87$ & $\mathrm{pCi} / \mathrm{g}$ & 2.87 & & 3/31/08 & 8C17008 & AGG-RRL-001 \\
\hline 14683-23-9 & Europium-152 & $<1.44$ & $\mathrm{pCi} / \mathrm{g}$ & 1.44 & & 3/31/08 & 8C17008 & AGG-RRL-001 \\
\hline 14276-65-4 & Gadolinium-153 & $<1.08$ & $\mathrm{pCi} / \mathrm{g}$ & 1.08 & & 3/31/08 & 8C17008 & AGG-RRL-001 \\
\hline 15585-10-1 & Europium-154 & $<0.757$ & $\mathrm{pCi} / \mathrm{g}$ & 0.757 & & 3/31/08 & 8C17008 & AGG-RRL-001 \\
\hline 14391-16-3 & Europium-155 & $<1.14$ & $\mathrm{pCi} / \mathrm{g}$ & 1.14 & & 3/31/08 & 8C17008 & AGG-RRL-001 \\
\hline 13982-78-0 & Mercury-203 & $<0.443$ & $\mathrm{pCi} / \mathrm{g}$ & 0.443 & & 3/31/08 & 8C17008 & AGG-RRL-001 \\
\hline 14913-50-9 & Thallium-208 & $<0.467$ & $\mathrm{pCi} / \mathrm{g}$ & 0.467 & & 3/31/08 & 8C17008 & AGG-RRL-001 \\
\hline 14331-79-4 & Bismuth-210 & $<0.672$ & $\mathrm{pCi} / \mathrm{g}$ & 0.672 & & 3/31/08 & 8C17008 & AGG-RRL-001 \\
\hline \multirow[t]{3}{*}{ 14255-04-0 } & Lead-210 & $<47.7$ & $\mathrm{pCi} / \mathrm{g}$ & 47.7 & & 3/31/08 & 8C17008 & AGG-RRL-001 \\
\hline & Bismuth-211 & $<7.69$ & $\mathrm{pCi} / \mathrm{g}$ & 7.69 & & 3/31/08 & 8C17008 & AGG-RRL-001 \\
\hline & Lead-211 & $<10.5$ & $\mathrm{pCi} / \mathrm{g}$ & 10.5 & & 3/31/08 & 8C17008 & AGG-RRL-001 \\
\hline 15092-94-1 & Lead-212 & $<0.891$ & $\mathrm{pCi} / \mathrm{g}$ & 0.891 & & 3/31/08 & 8C17008 & AGG-RRL-001 \\
\hline 14733-03-0 & Bismuth-214 & $<0.85$ & $\mathrm{pCi} / \mathrm{g}$ & 0.85 & & 3/31/08 & 8C17008 & AGG-RRL-001 \\
\hline 15067-28-4 & Lead-214 & $<0.98$ & $\mathrm{pCi} / \mathrm{g}$ & 0.98 & & 3/31/08 & 8C17008 & AGG-RRL-001 \\
\hline 14835-02-0 & Radon-219 & $<3.44$ & $\mathrm{pCi} / \mathrm{g}$ & 3.44 & & 3/31/08 & 8C17008 & AGG-RRL-001 \\
\hline 22481-48-7 & Radon-220 & $<335$ & $\mathrm{pCi} / \mathrm{g}$ & 335 & & 3/31/08 & 8C17008 & AGG-RRL-001 \\
\hline 28522-20-5 & Radon-221 & $<1.58$ & $\mathrm{pCi} / \mathrm{g}$ & 1.58 & & 3/31/08 & 8C17008 & AGG-RRL-001 \\
\hline 15756-98-6 & Francium-223 & $<11.3$ & $\mathrm{pCi} / \mathrm{g}$ & 11.3 & & 3/31/08 & 8C17008 & AGG-RRL-001 \\
\hline $15623-45-7$ & Radium-223 & $<2.48$ & $\mathrm{pCi} / \mathrm{g}$ & 2.48 & & 3/31/08 & 8C17008 & AGG-RRL-001 \\
\hline 13233-32-4 & Radium-224 & $<9.71$ & $\mathrm{pCi} / \mathrm{g}$ & 9.71 & & 3/31/08 & 8C17008 & AGG-RRL-001 \\
\hline 13982-63-3 & Radium-226 & $<9.77$ & $\mathrm{pCi} / \mathrm{g}$ & 9.77 & & 3/31/08 & 8C17008 & AGG-RRL-001 \\
\hline $15623-47-9$ & Thorium-227 & $<3.42$ & $\mathrm{pCi} / \mathrm{g}$ & 3.42 & & 3/31/08 & 8C17008 & AGG-RRL-001 \\
\hline 14331-83-0 & Actinium-228 & $<1.48$ & $\mathrm{pCi} / \mathrm{g}$ & 1.48 & & 3/31/08 & 8C17008 & AGG-RRL-001 \\
\hline $14274-82-9$ & Thorium-228 & $<128$ & $\mathrm{pCi} / \mathrm{g}$ & 128 & & 3/31/08 & 8C17008 & AGG-RRL-001 \\
\hline $14269-63-7$ & Thorium-230 & $<131$ & $\mathrm{pCi} / \mathrm{g}$ & 131 & & 3/31/08 & 8C17008 & AGG-RRL-001 \\
\hline 14331-85-2 & Protactinium-231 & $<15.4$ & $\mathrm{pCi} / \mathrm{g}$ & 15.4 & & 3/31/08 & 8C17008 & AGG-RRL-001 \\
\hline $14932-40-2$ & Thorium-231 & $<77.6$ & $\mathrm{pCi} / \mathrm{g}$ & 77.6 & & 3/31/08 & 8C17008 & AGG-RRL-001 \\
\hline 7440-29-1 & Thorium-232 & $<327$ & $\mathrm{pCi} / \mathrm{g}$ & 327 & & $3 / 31 / 08$ & 8C17008 & AGG-RRL-001 \\
\hline 13981-14-1 & Protactinium-233 & $<0.932$ & $\mathrm{pCi} / \mathrm{g}$ & 0.932 & & 3/31/08 & 8C17008 & AGG-RRL-001 \\
\hline \multirow[t]{2}{*}{$15100-28-4$} & Protactinium-234 & $<1.41$ & $\mathrm{pCi} / \mathrm{g}$ & 1.41 & & 3/31/08 & 8C17008 & AGG-RRL-001 \\
\hline & Protactinium-234m & $<39.3$ & $\mathrm{pCi} / \mathrm{g}$ & 39.3 & & 3/31/08 & 8C17008 & AGG-RRL-001 \\
\hline 15065-10-8 & Thorium-234 & $<11.6$ & $\mathrm{pCi} / \mathrm{g}$ & 11.6 & & 3/31/08 & 8C17008 & AGG-RRL-001 \\
\hline 15117-96-1 & Uranium 235 & $<3.03$ & $\mathrm{pCi} / \mathrm{g}$ & 3.03 & & 3/31/08 & 8C17008 & AGG-RRL-001 \\
\hline
\end{tabular}


GEA/Soil

\begin{tabular}{|c|c|c|c|c|c|c|c|c|}
\hline CAS \# & Analyte & Results & Units & MDA & UNC & Analyzed & Batch & Method \\
\hline HEIS No. & В1T2P2B & \multicolumn{2}{|c|}{ Lab ID: } & 0803014-09 & & & & \\
\hline 13994-20-2 & Neptunium-237 & $<2.96$ & pCi/g & 2.96 & & 3/31/08 & 8C17008 & AGG-RRL-001 \\
\hline \multirow[t]{2}{*}{ 13981-16-3 } & Plutonium-238 & $<4470$ & $\mathrm{pCi} / \mathrm{g}$ & 4470 & & 3/31/08 & 8C17008 & AGG-RRL-001 \\
\hline & Uranium 238 & $<6.32$ & $\mathrm{pCi} / \mathrm{g}$ & 6.32 & & 3/31/08 & 8C17008 & AGG-RRL-001 \\
\hline $15117-48-3$ & Plutonium-239 & $<4470$ & $\mathrm{pCi} / \mathrm{g}$ & 4470 & & 3/31/08 & 8C17008 & AGG-RRL-001 \\
\hline 14119-33-6 & Plutonium-240 & $<4500$ & $\mathrm{pCi} / \mathrm{g}$ & 4500 & & 3/31/08 & 8C17008 & AGG-RRL-001 \\
\hline 14596-10-2 & Americium-241 & $<1.68$ & $\mathrm{pCi} / \mathrm{g}$ & 1.68 & & 3/31/08 & 8C17008 & AGG-RRL-001 \\
\hline 14993-75-0 & Americium-243 & $<0.72$ & $\mathrm{pCi} / \mathrm{g}$ & 0.72 & & 3/31/08 & 8C17008 & AGG-RRL-001 \\
\hline 15757-87-6 & Curium-243 & $<1.38$ & $\mathrm{pCi} / \mathrm{g}$ & 1.38 & & 3/31/08 & 8C17008 & AGG-RRL-001 \\
\hline 15621-76-8 & Curium-245 & $<1.13$ & $\mathrm{pCi} / \mathrm{g}$ & 1.13 & & 3/31/08 & 8C17008 & AGG-RRL-001 \\
\hline HEIS No. & B1T2P2A & \multicolumn{2}{|c|}{ Lab ID: } & 0803014-10 & & & & \\
\hline 13966-32-0 & Sodium-22 & $<0.35$ & $\mathrm{pCi} / \mathrm{g}$ & 0.35 & & 3/31/08 & 8C17008 & AGG-RRL-001 \\
\hline 13966-00-2 & Potassium-40 & 20.4 & $\mathrm{pCi} / \mathrm{g}$ & 3.06 & 1.29 & 3/31/08 & 8C17008 & AGG-RRL-001 \\
\hline 14392-02-0 & Chromium-51 & $<2.85$ & $\mathrm{pCi} / \mathrm{g}$ & 2.85 & & 3/31/08 & 8C17008 & AGG-RRL-001 \\
\hline 13966-31-9 & Manganese-54 & $<0.282$ & $\mathrm{pCi} / \mathrm{g}$ & 0.282 & & 3/31/08 & 8C17008 & AGG-RRL-001 \\
\hline 13981-50-5 & Cobalt-57 & $<0.285$ & $\mathrm{pCi} / \mathrm{g}$ & 0.285 & & 3/31/08 & 8C17008 & AGG-RRL-001 \\
\hline $14596-12-4$ & Iron-59 & $<0.547$ & $\mathrm{pCi} / \mathrm{g}$ & 0.547 & & 3/31/08 & 8C17008 & AGG-RRL-001 \\
\hline 10198-40-0 & Cobalt-60 & $<0.31$ & $\mathrm{pCi} / \mathrm{g}$ & 0.31 & & 3/31/08 & 8C17008 & AGG-RRL-001 \\
\hline 13982-39-3 & Zinc-65 & $<0.691$ & $\mathrm{pCi} / \mathrm{g}$ & 0.691 & & 3/31/08 & 8C17008 & AGG-RRL-001 \\
\hline $14265-71-5$ & Selenium-75 & $<0.467$ & $\mathrm{pCi} / \mathrm{g}$ & 0.467 & & 3/31/08 & 8C17008 & AGG-RRL-001 \\
\hline 17056-36-9 & Rubidium-83 & $<0.507$ & $\mathrm{pCi} / \mathrm{g}$ & 0.507 & & 3/31/08 & 8C17008 & AGG-RRL-001 \\
\hline 13967-73-2 & Strontium-85 & $<0.374$ & $\mathrm{pCi} / \mathrm{g}$ & 0.374 & & 3/31/08 & 8C17008 & AGG-RRL-001 \\
\hline 14932-53-7 & Rubidium-86 & $<3.31$ & $\mathrm{pCi} / \mathrm{g}$ & 3.31 & & 3/31/08 & 8C17008 & AGG-RRL-001 \\
\hline 13982-36-0 & Yttrium-88 & $<0.264$ & $\mathrm{pCi} / \mathrm{g}$ & 0.264 & & 3/31/08 & 8C17008 & AGG-RRL-001 \\
\hline 14681-63-1 & Niobium-94 & $<0.266$ & $\mathrm{pCi} / \mathrm{g}$ & 0.266 & & 3/31/08 & 8C17008 & AGG-RRL-001 \\
\hline \multirow[t]{3}{*}{ 13967-76-5 } & Niobium-95 & $<0.28$ & $\mathrm{pCi} / \mathrm{g}$ & 0.28 & & 3/31/08 & 8C17008 & AGG-RRL-001 \\
\hline & Niobium-95m & $<1.36$ & $\mathrm{pCi} / \mathrm{g}$ & 1.36 & & 3/31/08 & 8C17008 & AGG-RRL-001 \\
\hline & Technetium-95m & $<0.458$ & $\mathrm{pCi} / \mathrm{g}$ & 0.458 & & 3/31/08 & 8C17008 & AGG-RRL-001 \\
\hline \multirow[t]{2}{*}{ 13967-71-0 } & Zirconium-95 & $<0.511$ & $\mathrm{pCi} / \mathrm{g}$ & 0.511 & & 3/31/08 & 8C17008 & AGG-RRL-001 \\
\hline & Technetium-99m & $<0.287$ & $\mathrm{pCi} / \mathrm{g}$ & 0.287 & & 3/31/08 & 8C17008 & AGG-RRL-001 \\
\hline 13968-53-1 & Ruthenium-103 & $<0.304$ & $\mathrm{pCi} / \mathrm{g}$ & 0.304 & & 3/31/08 & 8C17008 & AGG-RRL-001 \\
\hline 13967-48-1 & Ruthenium-106 & $<2.71$ & $\mathrm{pCi} / \mathrm{g}$ & 2.71 & & 3/31/08 & 8C17008 & AGG-RRL-001 \\
\hline 14391-65-2 & Silver-108m & $<0.278$ & $\mathrm{pCi} / \mathrm{g}$ & 0.278 & & 3/31/08 & 8C17008 & AGG-RRL-001 \\
\hline \multirow[t]{2}{*}{ 14109-32-1 } & Cadmium-109 & $<7.66$ & $\mathrm{pCi} / \mathrm{g}$ & 7.66 & & 3/31/08 & 8C17008 & AGG-RRL-001 \\
\hline & Silver-110 & $<0.29$ & $\mathrm{pCi} / \mathrm{g}$ & 0.29 & & 3/31/08 & 8C17008 & AGG-RRL-001 \\
\hline 14391-76-5 & Silver-110m & $<0.29$ & $\mathrm{pCi} / \mathrm{g}$ & 0.29 & & 3/31/08 & 8C17008 & AGG-RRL-001 \\
\hline 13966-06-8 & Tin-113 & $<0.418$ & $\mathrm{pCi} / \mathrm{g}$ & 0.418 & & 3/31/08 & 8C17008 & AGG-RRL-001 \\
\hline $14683-10-4$ & Antimony-124 & $<0.288$ & $\mathrm{pCi} / \mathrm{g}$ & 0.288 & & 3/31/08 & 8C17008 & AGG-RRL-001 \\
\hline $14234-35-6$ & Antimony-125 & $<0.91$ & $\mathrm{pCi} / \mathrm{g}$ & 0.91 & & 3/31/08 & 8C17008 & AGG-RRL-001 \\
\hline 15756-32-8 & Antimony-126 & $<0.269$ & $\mathrm{pCi} / \mathrm{g}$ & 0.269 & & 3/31/08 & 8C17008 & AGG-RRL-001 \\
\hline 15832-50-5 & Tin-126 & $<0.607$ & $\mathrm{pCi} / \mathrm{g}$ & 0.607 & & 3/31/08 & 8C17008 & AGG-RRL-001 \\
\hline 10043-66-0 & Iodine-131 & $<0.34$ & $\mathrm{pCi} / \mathrm{g}$ & 0.34 & & 3/31/08 & 8C17008 & AGG-RRL-001 \\
\hline 13981-41-4 & Barium-133 & $<0.551$ & $\mathrm{pCi} / \mathrm{g}$ & 0.551 & & 3/31/08 & 8C17008 & AGG-RRL-001 \\
\hline 13967-70-9 & Cesium-134 & $<0.346$ & $\mathrm{pCi} / \mathrm{g}$ & 0.346 & & 3/31/08 & 8C17008 & AGG-RRL-001 \\
\hline 10045-97-3 & Cesium-137 & $<0.323$ & $\mathrm{pCi} / \mathrm{g}$ & 0.323 & & 3/31/08 & 8C17008 & AGG-RRL-001 \\
\hline 13982-30-4 & Cerium-139 & $<0.331$ & $\mathrm{pCi} / \mathrm{g}$ & 0.331 & & 3/31/08 & 8C17008 & AGG-RRL-001 \\
\hline 14762-78-8 & Cerium-144 & $<2.28$ & $\mathrm{pCi} / \mathrm{g}$ & 2.28 & & 3/31/08 & 8C17008 & AGG-RRL-001 \\
\hline 14683-23-9 & Europium-152 & $<1.13$ & $\mathrm{pCi} / \mathrm{g}$ & 1.13 & & 3/31/08 & 8C17008 & AGG-RRL-001 \\
\hline $14276-65-4$ & Gadolinium-153 & $<0.846$ & $\mathrm{pCi} / \mathrm{g}$ & 0.846 & & 3/31/08 & 8C17008 & AGG-RRL-001 \\
\hline
\end{tabular}


GEA/Soil

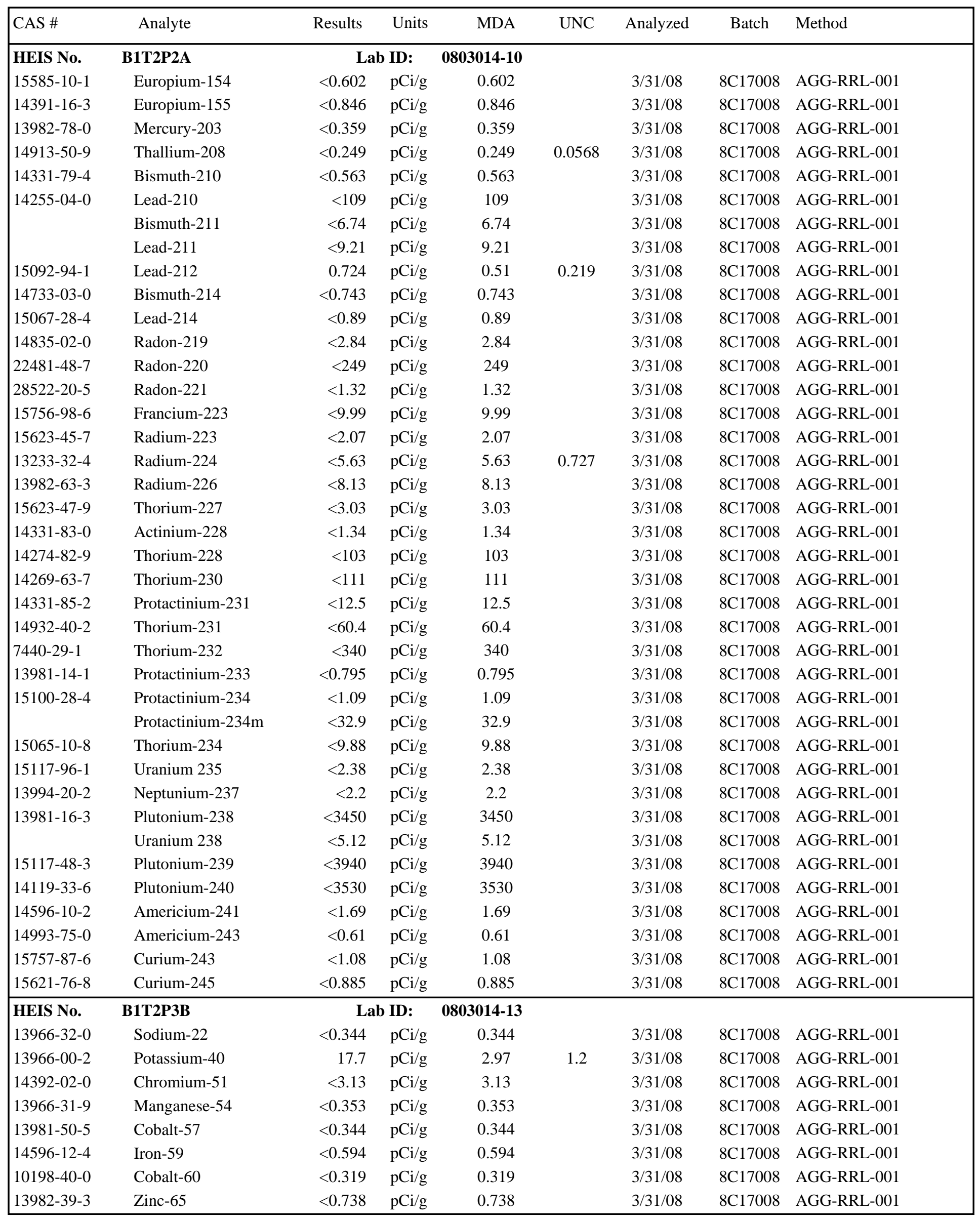


GEA/Soil

\begin{tabular}{|c|c|c|c|c|c|c|c|c|}
\hline CAS \# & Analyte & Results & Units & MDA & UNC & Analyzed & Batch & Method \\
\hline HEIS No. & В1Т2Р3В & \multicolumn{2}{|c|}{ Lab ID: } & 0803014-13 & & & & \\
\hline $14265-71-5$ & Selenium-75 & $<0.542$ & $\mathrm{pCi} / \mathrm{g}$ & 0.542 & & 3/31/08 & 8C17008 & AGG-RRL-001 \\
\hline 17056-36-9 & Rubidium-83 & $<0.822$ & $\mathrm{pCi} / \mathrm{g}$ & 0.822 & & 3/31/08 & 8C17008 & AGG-RRL-001 \\
\hline 13967-73-2 & Strontium-85 & $<0.394$ & $\mathrm{pCi} / \mathrm{g}$ & 0.394 & & 3/31/08 & 8C17008 & AGG-RRL-001 \\
\hline 14932-53-7 & Rubidium-86 & $<4.26$ & $\mathrm{pCi} / \mathrm{g}$ & 4.26 & & 3/31/08 & 8C17008 & AGG-RRL-001 \\
\hline 13982-36-0 & Yttrium-88 & $<0.278$ & $\mathrm{pCi} / \mathrm{g}$ & 0.278 & & 3/31/08 & 8C17008 & AGG-RRL-001 \\
\hline 14681-63-1 & Niobium-94 & $<0.323$ & $\mathrm{pCi} / \mathrm{g}$ & 0.323 & & 3/31/08 & 8C17008 & AGG-RRL-001 \\
\hline \multirow[t]{3}{*}{ 13967-76-5 } & Niobium-95 & $<0.343$ & $\mathrm{pCi} / \mathrm{g}$ & 0.343 & & 3/31/08 & 8C17008 & AGG-RRL-001 \\
\hline & Niobium-95m & $<1.48$ & $\mathrm{pCi} / \mathrm{g}$ & 1.48 & & 3/31/08 & 8C17008 & AGG-RRL-001 \\
\hline & Technetium-95m & $<0.51$ & $\mathrm{pCi} / \mathrm{g}$ & 0.51 & & 3/31/08 & 8C17008 & AGG-RRL-001 \\
\hline \multirow[t]{2}{*}{ 13967-71-0 } & Zirconium-95 & $<0.609$ & $\mathrm{pCi} / \mathrm{g}$ & 0.609 & & 3/31/08 & 8C17008 & AGG-RRL-001 \\
\hline & Technetium-99m & $<0.342$ & $\mathrm{pCi} / \mathrm{g}$ & 0.342 & & 3/31/08 & 8C17008 & AGG-RRL-001 \\
\hline 13968-53-1 & Ruthenium-103 & $<0.404$ & $\mathrm{pCi} / \mathrm{g}$ & 0.404 & & 3/31/08 & 8C17008 & AGG-RRL-001 \\
\hline 13967-48-1 & Ruthenium-106 & $<3.75$ & $\mathrm{pCi} / \mathrm{g}$ & 3.75 & & 3/31/08 & 8C17008 & AGG-RRL-001 \\
\hline 14391-65-2 & Silver-108m & $<0.345$ & $\mathrm{pCi} / \mathrm{g}$ & 0.345 & & 3/31/08 & 8C17008 & AGG-RRL-001 \\
\hline \multirow[t]{2}{*}{ 14109-32-1 } & Cadmium-109 & $<9.39$ & $\mathrm{pCi} / \mathrm{g}$ & 9.39 & & 3/31/08 & 8C17008 & AGG-RRL-001 \\
\hline & Silver-110 & $<0.354$ & $\mathrm{pCi} / \mathrm{g}$ & 0.354 & & 3/31/08 & 8C17008 & AGG-RRL-001 \\
\hline 14391-76-5 & Silver-110m & $<0.355$ & $\mathrm{pCi} / \mathrm{g}$ & 0.355 & & 3/31/08 & 8C17008 & AGG-RRL-001 \\
\hline 13966-06-8 & Tin-113 & $<0.456$ & $\mathrm{pCi} / \mathrm{g}$ & 0.456 & & 3/31/08 & 8C17008 & AGG-RRL-001 \\
\hline 14683-10-4 & Antimony-124 & $<0.398$ & $\mathrm{pCi} / \mathrm{g}$ & 0.398 & & 3/31/08 & 8C17008 & AGG-RRL-001 \\
\hline 14234-35-6 & Antimony-125 & $<1.07$ & $\mathrm{pCi} / \mathrm{g}$ & 1.07 & & 3/31/08 & 8C17008 & AGG-RRL-001 \\
\hline 15756-32-8 & Antimony-126 & $<0.331$ & $\mathrm{pCi} / \mathrm{g}$ & 0.331 & & 3/31/08 & 8C17008 & AGG-RRL-001 \\
\hline 15832-50-5 & Tin-126 & $<0.766$ & $\mathrm{pCi} / \mathrm{g}$ & 0.766 & & 3/31/08 & 8C17008 & AGG-RRL-001 \\
\hline 10043-66-0 & Iodine-131 & $<0.404$ & $\mathrm{pCi} / \mathrm{g}$ & 0.404 & & 3/31/08 & 8C17008 & AGG-RRL-001 \\
\hline 13981-41-4 & Barium-133 & $<0.563$ & $\mathrm{pCi} / \mathrm{g}$ & 0.563 & & 3/31/08 & 8C17008 & AGG-RRL-001 \\
\hline 13967-70-9 & Cesium-134 & $<0.386$ & $\mathrm{pCi} / \mathrm{g}$ & 0.386 & & 3/31/08 & 8C17008 & AGG-RRL-001 \\
\hline $10045-97-3$ & Cesium-137 & $<0.395$ & $\mathrm{pCi} / \mathrm{g}$ & 0.395 & & 3/31/08 & 8C17008 & AGG-RRL-001 \\
\hline 13982-30-4 & Cerium-139 & $<0.389$ & $\mathrm{pCi} / \mathrm{g}$ & 0.389 & & 3/31/08 & 8C17008 & AGG-RRL-001 \\
\hline 14762-78-8 & Cerium-144 & $<2.79$ & $\mathrm{pCi} / \mathrm{g}$ & 2.79 & & 3/31/08 & 8C17008 & AGG-RRL-001 \\
\hline 14683-23-9 & Europium-152 & $<1.33$ & $\mathrm{pCi} / \mathrm{g}$ & 1.33 & & 3/31/08 & 8C17008 & AGG-RRL-001 \\
\hline 14276-65-4 & Gadolinium-153 & $<1.05$ & $\mathrm{pCi} / \mathrm{g}$ & 1.05 & & 3/31/08 & 8C17008 & AGG-RRL-001 \\
\hline 15585-10-1 & Europium-154 & $<0.727$ & $\mathrm{pCi} / \mathrm{g}$ & 0.727 & & 3/31/08 & 8C17008 & AGG-RRL-001 \\
\hline 14391-16-3 & Europium-155 & $<1.08$ & $\mathrm{pCi} / \mathrm{g}$ & 1.08 & & 3/31/08 & 8C17008 & AGG-RRL-001 \\
\hline 13982-78-0 & Mercury-203 & $<0.404$ & $\mathrm{pCi} / \mathrm{g}$ & 0.404 & & 3/31/08 & 8C17008 & AGG-RRL-001 \\
\hline 14913-50-9 & Thallium-208 & $<0.415$ & $\mathrm{pCi} / \mathrm{g}$ & 0.415 & & 3/31/08 & 8C17008 & AGG-RRL-001 \\
\hline 14331-79-4 & Bismuth-210 & $<0.646$ & $\mathrm{pCi} / \mathrm{g}$ & 0.646 & & 3/31/08 & 8C17008 & AGG-RRL-001 \\
\hline \multirow[t]{3}{*}{$14255-04-0$} & Lead-210 & $<46.3$ & $\mathrm{pCi} / \mathrm{g}$ & 46.3 & & 3/31/08 & 8C17008 & AGG-RRL-001 \\
\hline & Bismuth-211 & $<7.6$ & $\mathrm{pCi} / \mathrm{g}$ & 7.6 & & 3/31/08 & 8C17008 & AGG-RRL-001 \\
\hline & Lead-211 & $<10.4$ & $\mathrm{pCi} / \mathrm{g}$ & 10.4 & & 3/31/08 & 8C17008 & AGG-RRL-001 \\
\hline 15092-94-1 & Lead-212 & $<0.858$ & $\mathrm{pCi} / \mathrm{g}$ & 0.858 & & 3/31/08 & 8C17008 & AGG-RRL-001 \\
\hline 14733-03-0 & Bismuth-214 & $<0.857$ & $\mathrm{pCi} / \mathrm{g}$ & 0.857 & & 3/31/08 & 8C17008 & AGG-RRL-001 \\
\hline $15067-28-4$ & Lead-214 & $<0.928$ & $\mathrm{pCi} / \mathrm{g}$ & 0.928 & & 3/31/08 & 8C17008 & AGG-RRL-001 \\
\hline $14835-02-0$ & Radon-219 & $<3.26$ & $\mathrm{pCi} / \mathrm{g}$ & 3.26 & & 3/31/08 & 8C17008 & AGG-RRL-001 \\
\hline 22481-48-7 & Radon-220 & $<298$ & $\mathrm{pCi} / \mathrm{g}$ & 298 & & 3/31/08 & 8C17008 & AGG-RRL-001 \\
\hline 28522-20-5 & Radon-221 & $<1.53$ & $\mathrm{pCi} / \mathrm{g}$ & 1.53 & & 3/31/08 & 8C17008 & AGG-RRL-001 \\
\hline 15756-98-6 & Francium-223 & $<10.9$ & $\mathrm{pCi} / \mathrm{g}$ & 10.9 & & 3/31/08 & 8C17008 & AGG-RRL-001 \\
\hline $15623-45-7$ & Radium-223 & $<2.37$ & $\mathrm{pCi} / \mathrm{g}$ & 2.37 & & 3/31/08 & 8C17008 & AGG-RRL-001 \\
\hline 13233-32-4 & Radium-224 & $<9.29$ & $\mathrm{pCi} / \mathrm{g}$ & 9.29 & & 3/31/08 & 8C17008 & AGG-RRL-001 \\
\hline 13982-63-3 & Radium-226 & $<9.42$ & $\mathrm{pCi} / \mathrm{g}$ & 9.42 & & 3/31/08 & 8C17008 & AGG-RRL-001 \\
\hline $15623-47-9$ & Thorium-227 & $<3.3$ & $\mathrm{pCi} / \mathrm{g}$ & 3.3 & & 3/31/08 & 8C17008 & AGG-RRL-001 \\
\hline
\end{tabular}


GEA/Soil

\begin{tabular}{|c|c|c|c|c|c|c|c|c|}
\hline CAS \# & Analyte & Results & Units & MDA & UNC & Analyzed & Batch & Method \\
\hline HEIS No. & В1T2P3В & \multicolumn{2}{|c|}{ Lab ID: } & 0803014-13 & & & & \\
\hline $14331-83-0$ & Actinium-228 & $<1.42$ & $\mathrm{pCi} / \mathrm{g}$ & 1.42 & & $3 / 31 / 08$ & 8C17008 & AGG-RRL-001 \\
\hline 14274-82-9 & Thorium-228 & $<121$ & $\mathrm{pCi} / \mathrm{g}$ & 121 & & 3/31/08 & 8C17008 & AGG-RRL-001 \\
\hline $14269-63-7$ & Thorium-230 & $<127$ & $\mathrm{pCi} / \mathrm{g}$ & 127 & & 3/31/08 & 8C17008 & AGG-RRL-001 \\
\hline $14331-85-2$ & Protactinium-231 & $<14.2$ & $\mathrm{pCi} / \mathrm{g}$ & 14.2 & & 3/31/08 & 8C17008 & AGG-RRL-001 \\
\hline $14932-40-2$ & Thorium-231 & $<75.1$ & $\mathrm{pCi} / \mathrm{g}$ & 75.1 & & 3/31/08 & 8C17008 & AGG-RRL-001 \\
\hline 7440-29-1 & Thorium-232 & $<325$ & $\mathrm{pCi} / \mathrm{g}$ & 325 & & $3 / 31 / 08$ & 8C17008 & AGG-RRL-001 \\
\hline 13981-14-1 & Protactinium-233 & $<0.871$ & $\mathrm{pCi} / \mathrm{g}$ & 0.871 & & 3/31/08 & 8C17008 & AGG-RRL-001 \\
\hline \multirow[t]{2}{*}{$15100-28-4$} & Protactinium-234 & $<1.37$ & $\mathrm{pCi} / \mathrm{g}$ & 1.37 & & $3 / 31 / 08$ & 8C17008 & AGG-RRL-001 \\
\hline & Protactinium-234m & $<39.9$ & $\mathrm{pCi} / \mathrm{g}$ & 39.9 & & 3/31/08 & 8C17008 & AGG-RRL-001 \\
\hline $15065-10-8$ & Thorium-234 & $<11.2$ & $\mathrm{pCi} / \mathrm{g}$ & 11.2 & & 3/31/08 & 8C17008 & AGG-RRL-001 \\
\hline 15117-96-1 & Uranium 235 & $<2.89$ & $\mathrm{pCi} / \mathrm{g}$ & 2.89 & & 3/31/08 & 8C17008 & AGG-RRL-001 \\
\hline 13994-20-2 & Neptunium-237 & $<2.82$ & $\mathrm{pCi} / \mathrm{g}$ & 2.82 & & 3/31/08 & 8C17008 & AGG-RRL-001 \\
\hline \multirow[t]{2}{*}{ 13981-16-3 } & Plutonium-238 & $<4330$ & $\mathrm{pCi} / \mathrm{g}$ & 4330 & & 3/31/08 & 8C17008 & AGG-RRL-001 \\
\hline & Uranium 238 & $<5.99$ & $\mathrm{pCi} / \mathrm{g}$ & 5.99 & & 3/31/08 & 8C17008 & AGG-RRL-001 \\
\hline 15117-48-3 & Plutonium-239 & $<4390$ & $\mathrm{pCi} / \mathrm{g}$ & 4390 & & 3/31/08 & 8C17008 & AGG-RRL-001 \\
\hline 14119-33-6 & Plutonium-240 & $<4310$ & $\mathrm{pCi} / \mathrm{g}$ & 4310 & & 3/31/08 & 8C17008 & AGG-RRL-001 \\
\hline $14596-10-2$ & Americium-241 & $<1.66$ & $\mathrm{pCi} / \mathrm{g}$ & 1.66 & & 3/31/08 & 8C17008 & AGG-RRL-001 \\
\hline $14993-75-0$ & Americium-243 & $<0.686$ & $\mathrm{pCi} / \mathrm{g}$ & 0.686 & & 3/31/08 & 8C17008 & AGG-RRL-001 \\
\hline 15757-87-6 & Curium-243 & $<1.32$ & $\mathrm{pCi} / \mathrm{g}$ & 1.32 & & 3/31/08 & 8C17008 & AGG-RRL-001 \\
\hline 15621-76-8 & Curium-245 & $<1.08$ & $\mathrm{pCi} / \mathrm{g}$ & 1.08 & & 3/31/08 & 8C17008 & AGG-RRL-001 \\
\hline HEIS No. & B1T2P3A & \multicolumn{2}{|c|}{ Lab ID: } & 0803014-14 & & & & \\
\hline $13966-32-0$ & Sodium-22 & $<0.336$ & $\mathrm{pCi} / \mathrm{g}$ & 0.336 & & 3/31/08 & 8C17008 & AGG-RRL-001 \\
\hline 13966-00-2 & Potassium-40 & 19.0 & $\mathrm{pCi} / \mathrm{g}$ & 3.17 & 1.27 & 3/31/08 & 8C17008 & AGG-RRL-001 \\
\hline $14392-02-0$ & Chromium-51 & $<2.62$ & $\mathrm{pCi} / \mathrm{g}$ & 2.62 & & 3/31/08 & 8C17008 & AGG-RRL-001 \\
\hline 13966-31-9 & Manganese-54 & $<0.281$ & $\mathrm{pCi} / \mathrm{g}$ & 0.281 & & 3/31/08 & 8C17008 & AGG-RRL-001 \\
\hline 13981-50-5 & Cobalt-57 & $<0.286$ & $\mathrm{pCi} / \mathrm{g}$ & 0.286 & & 3/31/08 & 8C17008 & AGG-RRL-001 \\
\hline $14596-12-4$ & Iron-59 & $<0.593$ & $\mathrm{pCi} / \mathrm{g}$ & 0.593 & & 3/31/08 & 8C17008 & AGG-RRL-001 \\
\hline 10198-40-0 & Cobalt-60 & $<0.296$ & $\mathrm{pCi} / \mathrm{g}$ & 0.296 & & 3/31/08 & 8C17008 & AGG-RRL-001 \\
\hline 13982-39-3 & Zinc-65 & $<0.706$ & $\mathrm{pCi} / \mathrm{g}$ & 0.706 & & 3/31/08 & 8C17008 & AGG-RRL-001 \\
\hline $14265-71-5$ & Selenium-75 & $<0.469$ & $\mathrm{pCi} / \mathrm{g}$ & 0.469 & & 3/31/08 & 8C17008 & AGG-RRL-001 \\
\hline 17056-36-9 & Rubidium-83 & $<0.552$ & $\mathrm{pCi} / \mathrm{g}$ & 0.552 & & 3/31/08 & 8C17008 & AGG-RRL-001 \\
\hline 13967-73-2 & Strontium-85 & $<0.353$ & $\mathrm{pCi} / \mathrm{g}$ & 0.353 & & 3/31/08 & 8C17008 & AGG-RRL-001 \\
\hline $14932-53-7$ & Rubidium-86 & $<3.25$ & $\mathrm{pCi} / \mathrm{g}$ & 3.25 & & 3/31/08 & 8C17008 & AGG-RRL-001 \\
\hline 13982-36-0 & Yttrium-88 & $<0.218$ & $\mathrm{pCi} / \mathrm{g}$ & 0.218 & & 3/31/08 & 8C17008 & AGG-RRL-001 \\
\hline $14681-63-1$ & Niobium-94 & $<0.245$ & $\mathrm{pCi} / \mathrm{g}$ & 0.245 & & 3/31/08 & 8C17008 & AGG-RRL-001 \\
\hline \multirow[t]{3}{*}{ 13967-76-5 } & Niobium-95 & $<0.279$ & $\mathrm{pCi} / \mathrm{g}$ & 0.279 & & 3/31/08 & 8C17008 & AGG-RRL-001 \\
\hline & Niobium-95m & $<1.3$ & $\mathrm{pCi} / \mathrm{g}$ & 1.3 & & 3/31/08 & 8C17008 & AGG-RRL-001 \\
\hline & Technetium-95m & $<0.45$ & $\mathrm{pCi} / \mathrm{g}$ & 0.45 & & 3/31/08 & 8C17008 & AGG-RRL-001 \\
\hline \multirow[t]{2}{*}{ 13967-71-0 } & Zirconium-95 & $<0.484$ & $\mathrm{pCi} / \mathrm{g}$ & 0.484 & & 3/31/08 & 8C17008 & AGG-RRL-001 \\
\hline & Technetium-99m & $<0.286$ & $\mathrm{pCi} / \mathrm{g}$ & 0.286 & & 3/31/08 & 8C17008 & AGG-RRL-001 \\
\hline 13968-53-1 & Ruthenium-103 & $<0.265$ & $\mathrm{pCi} / \mathrm{g}$ & 0.265 & & 3/31/08 & 8C17008 & AGG-RRL-001 \\
\hline 13967-48-1 & Ruthenium-106 & $<2.84$ & $\mathrm{pCi} / \mathrm{g}$ & 2.84 & & 3/31/08 & 8C17008 & AGG-RRL-001 \\
\hline $14391-65-2$ & Silver-108m & $<0.3$ & $\mathrm{pCi} / \mathrm{g}$ & 0.3 & & 3/31/08 & 8C17008 & AGG-RRL-001 \\
\hline \multirow[t]{2}{*}{ 14109-32-1 } & Cadmium-109 & $<7.79$ & $\mathrm{pCi} / \mathrm{g}$ & 7.79 & & 3/31/08 & 8C17008 & AGG-RRL-001 \\
\hline & Silver-110 & $<0.296$ & $\mathrm{pCi} / \mathrm{g}$ & 0.296 & & 3/31/08 & 8C17008 & AGG-RRL-001 \\
\hline 14391-76-5 & Silver-110m & $<0.297$ & $\mathrm{pCi} / \mathrm{g}$ & 0.297 & & 3/31/08 & 8C17008 & AGG-RRL-001 \\
\hline 13966-06-8 & Tin-113 & $<0.421$ & $\mathrm{pCi} / \mathrm{g}$ & 0.421 & & 3/31/08 & 8C17008 & AGG-RRL-001 \\
\hline $14683-10-4$ & Antimony-124 & $<0.284$ & $\mathrm{pCi} / \mathrm{g}$ & 0.284 & & 3/31/08 & 8C17008 & AGG-RRL-001 \\
\hline
\end{tabular}


GEA/Soil

\begin{tabular}{|c|c|c|c|c|c|c|c|c|}
\hline CAS \# & Analyte & Results & Units & MDA & UNC & Analyzed & Batch & Method \\
\hline HEIS No. & B1T2P3A & \multicolumn{2}{|c|}{ Lab ID: } & 0803014-14 & & & & \\
\hline $14234-35-6$ & Antimony-125 & $<0.924$ & $\mathrm{pCi} / \mathrm{g}$ & 0.924 & & 3/31/08 & 8C17008 & AGG-RRL-001 \\
\hline 15756-32-8 & Antimony-126 & $<0.28$ & $\mathrm{pCi} / \mathrm{g}$ & 0.28 & & 3/31/08 & 8C17008 & AGG-RRL-001 \\
\hline $15832-50-5$ & Tin-126 & $<0.616$ & $\mathrm{pCi} / \mathrm{g}$ & 0.616 & & 3/31/08 & 8C17008 & AGG-RRL-001 \\
\hline $10043-66-0$ & Iodine-131 & $<0.332$ & $\mathrm{pCi} / \mathrm{g}$ & 0.332 & & 3/31/08 & 8C17008 & AGG-RRL-001 \\
\hline 13981-41-4 & Barium-133 & $<0.531$ & $\mathrm{pCi} / \mathrm{g}$ & 0.531 & & 3/31/08 & 8C17008 & AGG-RRL-001 \\
\hline 13967-70-9 & Cesium-134 & $<0.302$ & $\mathrm{pCi} / \mathrm{g}$ & 0.302 & & 3/31/08 & 8C17008 & AGG-RRL-001 \\
\hline $10045-97-3$ & Cesium-137 & $<0.338$ & $\mathrm{pCi} / \mathrm{g}$ & 0.338 & & 3/31/08 & 8C17008 & AGG-RRL-001 \\
\hline 13982-30-4 & Cerium-139 & $<0.342$ & $\mathrm{pCi} / \mathrm{g}$ & 0.342 & & 3/31/08 & 8C17008 & AGG-RRL-001 \\
\hline 14762-78-8 & Cerium-144 & $<2.26$ & $\mathrm{pCi} / \mathrm{g}$ & 2.26 & & 3/31/08 & 8C17008 & AGG-RRL-001 \\
\hline 14683-23-9 & Europium-152 & $<1.11$ & $\mathrm{pCi} / \mathrm{g}$ & 1.11 & & 3/31/08 & 8C17008 & AGG-RRL-001 \\
\hline $14276-65-4$ & Gadolinium-153 & $<0.848$ & $\mathrm{pCi} / \mathrm{g}$ & 0.848 & & 3/31/08 & 8C17008 & AGG-RRL-001 \\
\hline 15585-10-1 & Europium-154 & $<0.606$ & $\mathrm{pCi} / \mathrm{g}$ & 0.606 & & 3/31/08 & 8C17008 & AGG-RRL-001 \\
\hline 14391-16-3 & Europium-155 & $<0.867$ & $\mathrm{pCi} / \mathrm{g}$ & 0.867 & & 3/31/08 & 8C17008 & AGG-RRL-001 \\
\hline 13982-78-0 & Mercury-203 & $<0.369$ & $\mathrm{pCi} / \mathrm{g}$ & 0.369 & & 3/31/08 & 8C17008 & AGG-RRL-001 \\
\hline 14913-50-9 & Thallium-208 & $<0.318$ & $\mathrm{pCi} / \mathrm{g}$ & 0.318 & 0.0726 & 3/31/08 & 8C17008 & AGG-RRL-001 \\
\hline 14331-79-4 & Bismuth-210 & $<0.56$ & $\mathrm{pCi} / \mathrm{g}$ & 0.56 & & 3/31/08 & 8C17008 & AGG-RRL-001 \\
\hline \multirow[t]{3}{*}{$14255-04-0$} & Lead-210 & $<109$ & $\mathrm{pCi} / \mathrm{g}$ & 109 & & 3/31/08 & 8C17008 & AGG-RRL-001 \\
\hline & Bismuth-211 & $<6.6$ & $\mathrm{pCi} / \mathrm{g}$ & 6.6 & & 3/31/08 & 8C17008 & AGG-RRL-001 \\
\hline & Lead-211 & $<9.02$ & $\mathrm{pCi} / \mathrm{g}$ & 9.02 & & 3/31/08 & 8C17008 & AGG-RRL-001 \\
\hline 15092-94-1 & Lead-212 & $<0.774$ & $\mathrm{pCi} / \mathrm{g}$ & 0.774 & & 3/31/08 & 8C17008 & AGG-RRL-001 \\
\hline 14733-03-0 & Bismuth-214 & $<0.728$ & $\mathrm{pCi} / \mathrm{g}$ & 0.728 & & 3/31/08 & 8C17008 & AGG-RRL-001 \\
\hline $15067-28-4$ & Lead-214 & $<0.884$ & $\mathrm{pCi} / \mathrm{g}$ & 0.884 & & 3/31/08 & 8C17008 & AGG-RRL-001 \\
\hline 14835-02-0 & Radon-219 & $<2.85$ & $\mathrm{pCi} / \mathrm{g}$ & 2.85 & & 3/31/08 & 8C17008 & AGG-RRL-001 \\
\hline 22481-48-7 & Radon-220 & $<251$ & $\mathrm{pCi} / \mathrm{g}$ & 251 & & 3/31/08 & 8C17008 & AGG-RRL-001 \\
\hline 28522-20-5 & Radon-221 & $<1.3$ & $\mathrm{pCi} / \mathrm{g}$ & 1.3 & & 3/31/08 & 8C17008 & AGG-RRL-001 \\
\hline 15756-98-6 & Francium-223 & $<9.54$ & $\mathrm{pCi} / \mathrm{g}$ & 9.54 & & 3/31/08 & 8C17008 & AGG-RRL-001 \\
\hline 15623-45-7 & Radium-223 & $<2.07$ & $\mathrm{pCi} / \mathrm{g}$ & 2.07 & & 3/31/08 & 8C17008 & AGG-RRL-001 \\
\hline 13233-32-4 & Radium-224 & $<8.4$ & $\mathrm{pCi} / \mathrm{g}$ & 8.4 & & 3/31/08 & 8C17008 & AGG-RRL-001 \\
\hline 13982-63-3 & Radium-226 & $<8.08$ & $\mathrm{pCi} / \mathrm{g}$ & 8.08 & & 3/31/08 & 8C17008 & AGG-RRL-001 \\
\hline $15623-47-9$ & Thorium-227 & $<2.91$ & $\mathrm{pCi} / \mathrm{g}$ & 2.91 & & 3/31/08 & 8C17008 & AGG-RRL-001 \\
\hline 14331-83-0 & Actinium-228 & $<1.28$ & $\mathrm{pCi} / \mathrm{g}$ & 1.28 & & 3/31/08 & 8C17008 & AGG-RRL-001 \\
\hline 14274-82-9 & Thorium-228 & $<103$ & $\mathrm{pCi} / \mathrm{g}$ & 103 & & 3/31/08 & 8C17008 & AGG-RRL-001 \\
\hline $14269-63-7$ & Thorium-230 & $<108$ & $\mathrm{pCi} / \mathrm{g}$ & 108 & & 3/31/08 & 8C17008 & AGG-RRL-001 \\
\hline 14331-85-2 & Protactinium-231 & $<12.5$ & $\mathrm{pCi} / \mathrm{g}$ & 12.5 & & 3/31/08 & 8C17008 & AGG-RRL-001 \\
\hline 14932-40-2 & Thorium-231 & $<59.3$ & $\mathrm{pCi} / \mathrm{g}$ & 59.3 & & 3/31/08 & 8C17008 & AGG-RRL-001 \\
\hline 7440-29-1 & Thorium-232 & $<335$ & $\mathrm{pCi} / \mathrm{g}$ & 335 & & 3/31/08 & 8C17008 & AGG-RRL-001 \\
\hline 13981-14-1 & Protactinium-233 & $<0.721$ & $\mathrm{pCi} / \mathrm{g}$ & 0.721 & & 3/31/08 & 8C17008 & AGG-RRL-001 \\
\hline \multirow[t]{2}{*}{$15100-28-4$} & Protactinium-234 & $<1.08$ & $\mathrm{pCi} / \mathrm{g}$ & 1.08 & & 3/31/08 & 8C17008 & AGG-RRL-001 \\
\hline & Protactinium-234m & $<36.6$ & $\mathrm{pCi} / \mathrm{g}$ & 36.6 & & 3/31/08 & 8C17008 & AGG-RRL-001 \\
\hline $15065-10-8$ & Thorium-234 & $<9.84$ & $\mathrm{pCi} / \mathrm{g}$ & 9.84 & & 3/31/08 & 8C17008 & AGG-RRL-001 \\
\hline 15117-96-1 & Uranium 235 & $<2.46$ & $\mathrm{pCi} / \mathrm{g}$ & 2.46 & & 3/31/08 & 8C17008 & AGG-RRL-001 \\
\hline 13994-20-2 & Neptunium-237 & $<2.26$ & $\mathrm{pCi} / \mathrm{g}$ & 2.26 & & $3 / 31 / 08$ & 8C17008 & AGG-RRL-001 \\
\hline \multirow[t]{2}{*}{ 13981-16-3 } & Plutonium-238 & $<3400$ & $\mathrm{pCi} / \mathrm{g}$ & 3400 & & 3/31/08 & 8C17008 & AGG-RRL-001 \\
\hline & Uranium 238 & $<5.09$ & $\mathrm{pCi} / \mathrm{g}$ & 5.09 & & $3 / 31 / 08$ & 8C17008 & AGG-RRL-001 \\
\hline 15117-48-3 & Plutonium-239 & $<3920$ & $\mathrm{pCi} / \mathrm{g}$ & 3920 & & 3/31/08 & 8C17008 & AGG-RRL-001 \\
\hline 14119-33-6 & Plutonium-240 & $<3410$ & $\mathrm{pCi} / \mathrm{g}$ & 3410 & & 3/31/08 & 8C17008 & AGG-RRL-001 \\
\hline 14596-10-2 & Americium-241 & $<1.66$ & $\mathrm{pCi} / \mathrm{g}$ & 1.66 & & 3/31/08 & 8C17008 & AGG-RRL-001 \\
\hline 14993-75-0 & Americium-243 & $<0.604$ & $\mathrm{pCi} / \mathrm{g}$ & 0.604 & & 3/31/08 & 8C17008 & AGG-RRL-001 \\
\hline 15757-87-6 & Curium-243 & $<1.04$ & $\mathrm{pCi} / \mathrm{g}$ & 1.04 & & 3/31/08 & 8C17008 & AGG-RRL-001 \\
\hline
\end{tabular}


GEA/Soil

\begin{tabular}{|c|c|c|c|c|c|c|c|c|}
\hline CAS \# & Analyte & Results & Units & MDA & UNC & Analyzed & Batch & Method \\
\hline HEIS No. & В1T2P3A & \multicolumn{2}{|c|}{ Lab ID: } & \multicolumn{2}{|l|}{ 0803014-14 } & & & \\
\hline 15621-76-8 & Curium-245 & $<0.854$ & $\mathrm{pCi} / \mathrm{g}$ & 0.854 & & 3/31/08 & 8C17008 & AGG-RRL-001 \\
\hline HEIS No. & B1T2P4B & \multicolumn{2}{|c|}{ Lab ID: } & 0803014-16 & & & & \\
\hline 13966-32-0 & Sodium-22 & $<0.456$ & $\mathrm{pCi} / \mathrm{g}$ & 0.456 & & 3/31/08 & 8C17008 & AGG-RRL-001 \\
\hline 13966-00-2 & Potassium-40 & 23.4 & $\mathrm{pCi} / \mathrm{g}$ & 3.28 & 1.51 & 3/31/08 & 8C17008 & AGG-RRL-001 \\
\hline $14392-02-0$ & Chromium-51 & $<3.42$ & $\mathrm{pCi} / \mathrm{g}$ & 3.42 & & 3/31/08 & 8C17008 & AGG-RRL-001 \\
\hline 13966-31-9 & Manganese-54 & $<0.328$ & $\mathrm{pCi} / \mathrm{g}$ & 0.328 & & 3/31/08 & 8C17008 & AGG-RRL-001 \\
\hline 13981-50-5 & Cobalt-57 & $<0.349$ & $\mathrm{pCi} / \mathrm{g}$ & 0.349 & & 3/31/08 & 8C17008 & AGG-RRL-001 \\
\hline 14596-12-4 & Iron-59 & $<0.631$ & $\mathrm{pCi} / \mathrm{g}$ & 0.631 & & 3/31/08 & 8C17008 & AGG-RRL-001 \\
\hline $10198-40-0$ & Cobalt-60 & $<0.339$ & $\mathrm{pCi} / \mathrm{g}$ & 0.339 & & 3/31/08 & 8C17008 & AGG-RRL-001 \\
\hline 13982-39-3 & Zinc-65 & $<0.811$ & $\mathrm{pCi} / \mathrm{g}$ & 0.811 & & 3/31/08 & 8C17008 & AGG-RRL-001 \\
\hline 14265-71-5 & Selenium-75 & $<0.552$ & $\mathrm{pCi} / \mathrm{g}$ & 0.552 & & 3/31/08 & 8C17008 & AGG-RRL-001 \\
\hline 17056-36-9 & Rubidium-83 & $<0.697$ & $\mathrm{pCi} / \mathrm{g}$ & 0.697 & & 3/31/08 & 8C17008 & AGG-RRL-001 \\
\hline 13967-73-2 & Strontium-85 & $<0.461$ & $\mathrm{pCi} / \mathrm{g}$ & 0.461 & & 3/31/08 & 8C17008 & AGG-RRL-001 \\
\hline $14932-53-7$ & Rubidium-86 & $<4.22$ & $\mathrm{pCi} / \mathrm{g}$ & 4.22 & & $3 / 31 / 08$ & 8C17008 & AGG-RRL-001 \\
\hline $13982-36-0$ & Yttrium-88 & $<0.274$ & $\mathrm{pCi} / \mathrm{g}$ & 0.274 & & 3/31/08 & 8C17008 & AGG-RRL-001 \\
\hline 14681-63-1 & Niobium-94 & $<0.356$ & $\mathrm{pCi} / \mathrm{g}$ & 0.356 & & 3/31/08 & 8C17008 & AGG-RRL-001 \\
\hline \multirow{3}{*}{ 13967-76-5 } & Niobium-95 & $<0.338$ & $\mathrm{pCi} / \mathrm{g}$ & 0.338 & & 3/31/08 & 8C17008 & AGG-RRL-001 \\
\hline & Niobium-95m & $<1.59$ & $\mathrm{pCi} / \mathrm{g}$ & 1.59 & & 3/31/08 & 8C17008 & AGG-RRL-001 \\
\hline & Technetium-95m & $<0.553$ & $\mathrm{pCi} / \mathrm{g}$ & 0.553 & & 3/31/08 & 8C17008 & AGG-RRL-001 \\
\hline \multirow[t]{2}{*}{ 13967-71-0 } & Zirconium-95 & $<0.604$ & $\mathrm{pCi} / \mathrm{g}$ & 0.604 & & 3/31/08 & 8C17008 & AGG-RRL-001 \\
\hline & Technetium-99m & $<0.351$ & $\mathrm{pCi} / \mathrm{g}$ & 0.351 & & 3/31/08 & 8C17008 & AGG-RRL-001 \\
\hline 13968-53-1 & Ruthenium-103 & $<0.366$ & $\mathrm{pCi} / \mathrm{g}$ & 0.366 & & 3/31/08 & 8C17008 & AGG-RRL-001 \\
\hline 13967-48-1 & Ruthenium-106 & $<3.34$ & $\mathrm{pCi} / \mathrm{g}$ & 3.34 & & 3/31/08 & 8C17008 & AGG-RRL-001 \\
\hline 14391-65-2 & Silver-108m & $<0.349$ & $\mathrm{pCi} / \mathrm{g}$ & 0.349 & & 3/31/08 & 8C17008 & AGG-RRL-001 \\
\hline \multirow[t]{2}{*}{$14109-32-1$} & Cadmium-109 & $<9.36$ & $\mathrm{pCi} / \mathrm{g}$ & 9.36 & & 3/31/08 & 8C17008 & AGG-RRL-001 \\
\hline & Silver-110 & $<0.359$ & $\mathrm{pCi} / \mathrm{g}$ & 0.359 & & 3/31/08 & 8C17008 & AGG-RRL-001 \\
\hline 14391-76-5 & Silver-110m & $<0.36$ & $\mathrm{pCi} / \mathrm{g}$ & 0.36 & & $3 / 31 / 08$ & 8C17008 & AGG-RRL-001 \\
\hline 13966-06-8 & Tin-113 & $<0.512$ & $\mathrm{pCi} / \mathrm{g}$ & 0.512 & & 3/31/08 & 8C17008 & AGG-RRL-001 \\
\hline $14683-10-4$ & Antimony-124 & $<0.375$ & $\mathrm{pCi} / \mathrm{g}$ & 0.375 & & 3/31/08 & 8C17008 & AGG-RRL-001 \\
\hline $14234-35-6$ & Antimony-125 & $<1.09$ & $\mathrm{pCi} / \mathrm{g}$ & 1.09 & & 3/31/08 & 8C17008 & AGG-RRL-001 \\
\hline 15756-32-8 & Antimony-126 & $<0.351$ & $\mathrm{pCi} / \mathrm{g}$ & 0.351 & & 3/31/08 & 8C17008 & AGG-RRL-001 \\
\hline $15832-50-5$ & Tin-126 & $<0.746$ & $\mathrm{pCi} / \mathrm{g}$ & 0.746 & & $3 / 31 / 08$ & 8C17008 & AGG-RRL-001 \\
\hline 10043-66-0 & Iodine-131 & $<0.413$ & $\mathrm{pCi} / \mathrm{g}$ & 0.413 & & 3/31/08 & 8C17008 & AGG-RRL-001 \\
\hline 13981-41-4 & Barium-133 & $<0.662$ & $\mathrm{pCi} / \mathrm{g}$ & 0.662 & & 3/31/08 & 8C17008 & AGG-RRL-001 \\
\hline 13967-70-9 & Cesium-134 & $<0.373$ & $\mathrm{pCi} / \mathrm{g}$ & 0.373 & & $3 / 31 / 08$ & 8C17008 & AGG-RRL-001 \\
\hline $10045-97-3$ & Cesium-137 & $<0.415$ & $\mathrm{pCi} / \mathrm{g}$ & 0.415 & & 3/31/08 & 8C17008 & AGG-RRL-001 \\
\hline 13982-30-4 & Cerium-139 & $<0.401$ & $\mathrm{pCi} / \mathrm{g}$ & 0.401 & & $3 / 31 / 08$ & 8C17008 & AGG-RRL-001 \\
\hline 14762-78-8 & Cerium-144 & $<2.81$ & $\mathrm{pCi} / \mathrm{g}$ & 2.81 & & 3/31/08 & 8C17008 & AGG-RRL-001 \\
\hline 14683-23-9 & Europium-152 & $<1.35$ & $\mathrm{pCi} / \mathrm{g}$ & 1.35 & & 3/31/08 & 8C17008 & AGG-RRL-001 \\
\hline 14276-65-4 & Gadolinium-153 & $<1.02$ & $\mathrm{pCi} / \mathrm{g}$ & 1.02 & & 3/31/08 & 8C17008 & AGG-RRL-001 \\
\hline 15585-10-1 & Europium-154 & $<0.736$ & $\mathrm{pCi} / \mathrm{g}$ & 0.736 & & 3/31/08 & 8C17008 & AGG-RRL-001 \\
\hline $14391-16-3$ & Europium-155 & $<1.04$ & $\mathrm{pCi} / \mathrm{g}$ & 1.04 & & 3/31/08 & 8C17008 & AGG-RRL-001 \\
\hline 13982-78-0 & Mercury-203 & $<0.444$ & $\mathrm{pCi} / \mathrm{g}$ & 0.444 & & 3/31/08 & 8C17008 & AGG-RRL-001 \\
\hline $14913-50-9$ & Thallium-208 & $<0.304$ & $\mathrm{pCi} / \mathrm{g}$ & 0.304 & 0.0677 & 3/31/08 & 8C17008 & AGG-RRL-001 \\
\hline 14331-79-4 & Bismuth-210 & $<0.663$ & $\mathrm{pCi} / \mathrm{g}$ & 0.663 & & 3/31/08 & 8C17008 & AGG-RRL-001 \\
\hline \multirow[t]{3}{*}{$14255-04-0$} & Lead-210 & $<138$ & $\mathrm{pCi} / \mathrm{g}$ & 138 & & 3/31/08 & 8C17008 & AGG-RRL-001 \\
\hline & Bismuth-211 & $<7.87$ & $\mathrm{pCi} / \mathrm{g}$ & 7.87 & & 3/31/08 & 8C17008 & AGG-RRL-001 \\
\hline & Lead-211 & $<10.8$ & $\mathrm{pCi} / \mathrm{g}$ & 10.8 & & 3/31/08 & 8C17008 & AGG-RRL-001 \\
\hline
\end{tabular}


GEA/Soil

\begin{tabular}{|c|c|c|c|c|c|c|c|c|}
\hline CAS \# & Analyte & Results & Units & MDA & UNC & Analyzed & Batch & Method \\
\hline HEIS No. & B1T2P4B & \multicolumn{2}{|c|}{ Lab ID: } & 0803014-16 & & & & \\
\hline 15092-94-1 & Lead-212 & $<0.826$ & $\mathrm{pCi} / \mathrm{g}$ & 0.826 & 0.186 & 3/31/08 & 8C17008 & AGG-RRL-001 \\
\hline 14733-03-0 & Bismuth-214 & $<0.903$ & $\mathrm{pCi} / \mathrm{g}$ & 0.903 & & $3 / 31 / 08$ & 8C17008 & AGG-RRL-001 \\
\hline $15067-28-4$ & Lead-214 & $<1.08$ & $\mathrm{pCi} / \mathrm{g}$ & 1.08 & & 3/31/08 & 8C17008 & AGG-RRL-001 \\
\hline $14835-02-0$ & Radon-219 & $<3.58$ & $\mathrm{pCi} / \mathrm{g}$ & 3.58 & & 3/31/08 & 8C17008 & AGG-RRL-001 \\
\hline 22481-48-7 & Radon-220 & $<294$ & $\mathrm{pCi} / \mathrm{g}$ & 294 & & 3/31/08 & 8C17008 & AGG-RRL-001 \\
\hline 28522-20-5 & Radon-221 & $<1.61$ & $\mathrm{pCi} / \mathrm{g}$ & 1.61 & & 3/31/08 & 8C17008 & AGG-RRL-001 \\
\hline 15756-98-6 & Francium-223 & $<11.7$ & $\mathrm{pCi} / \mathrm{g}$ & 11.7 & & 3/31/08 & 8C17008 & AGG-RRL-001 \\
\hline 15623-45-7 & Radium-223 & $<2.57$ & $\mathrm{pCi} / \mathrm{g}$ & 2.57 & & 3/31/08 & 8C17008 & AGG-RRL-001 \\
\hline 13233-32-4 & Radium-224 & $<10.1$ & $\mathrm{pCi} / \mathrm{g}$ & 10.1 & & 3/31/08 & 8C17008 & AGG-RRL-001 \\
\hline 13982-63-3 & Radium-226 & $<9.96$ & $\mathrm{pCi} / \mathrm{g}$ & 9.96 & & 3/31/08 & 8C17008 & AGG-RRL-001 \\
\hline 15623-47-9 & Thorium-227 & $<3.56$ & $\mathrm{pCi} / \mathrm{g}$ & 3.56 & & 3/31/08 & 8C17008 & AGG-RRL-001 \\
\hline $14331-83-0$ & Actinium-228 & $<1.57$ & $\mathrm{pCi} / \mathrm{g}$ & 1.57 & & 3/31/08 & 8C17008 & AGG-RRL-001 \\
\hline $14274-82-9$ & Thorium-228 & $<124$ & $\mathrm{pCi} / \mathrm{g}$ & 124 & & $3 / 31 / 08$ & 8C17008 & AGG-RRL-001 \\
\hline $14269-63-7$ & Thorium-230 & $<131$ & $\mathrm{pCi} / \mathrm{g}$ & 131 & & 3/31/08 & 8C17008 & AGG-RRL-001 \\
\hline 14331-85-2 & Protactinium-231 & $<14.4$ & $\mathrm{pCi} / \mathrm{g}$ & 14.4 & & 3/31/08 & 8C17008 & AGG-RRL-001 \\
\hline $14932-40-2$ & Thorium-231 & $<72.1$ & $\mathrm{pCi} / \mathrm{g}$ & 72.1 & & 3/31/08 & 8C17008 & AGG-RRL-001 \\
\hline 7440-29-1 & Thorium-232 & $<426$ & $\mathrm{pCi} / \mathrm{g}$ & 426 & & 3/31/08 & 8C17008 & AGG-RRL-001 \\
\hline 13981-14-1 & Protactinium-233 & $<0.902$ & $\mathrm{pCi} / \mathrm{g}$ & 0.902 & & 3/31/08 & 8C17008 & AGG-RRL-001 \\
\hline \multirow[t]{2}{*}{ 15100-28-4 } & Protactinium-234 & $<1.31$ & $\mathrm{pCi} / \mathrm{g}$ & 1.31 & & $3 / 31 / 08$ & 8C17008 & AGG-RRL-001 \\
\hline & Protactinium-234m & $<45.8$ & $\mathrm{pCi} / \mathrm{g}$ & 45.8 & & 3/31/08 & 8C17008 & AGG-RRL-001 \\
\hline $15065-10-8$ & Thorium-234 & $<12$ & $\mathrm{pCi} / \mathrm{g}$ & 12 & & 3/31/08 & 8C17008 & AGG-RRL-001 \\
\hline 15117-96-1 & Uranium 235 & $<2.96$ & $\mathrm{pCi} / \mathrm{g}$ & 2.96 & & 3/31/08 & 8C17008 & AGG-RRL-001 \\
\hline 13994-20-2 & Neptunium-237 & $<2.71$ & $\mathrm{pCi} / \mathrm{g}$ & 2.71 & & 3/31/08 & 8C17008 & AGG-RRL-001 \\
\hline \multirow[t]{2}{*}{ 13981-16-3 } & Plutonium-238 & $<4110$ & $\mathrm{pCi} / \mathrm{g}$ & 4110 & & 3/31/08 & 8C17008 & AGG-RRL-001 \\
\hline & Uranium 238 & $<6.22$ & $\mathrm{pCi} / \mathrm{g}$ & 6.22 & & 3/31/08 & 8C17008 & AGG-RRL-001 \\
\hline $15117-48-3$ & Plutonium-239 & $<4960$ & $\mathrm{pCi} / \mathrm{g}$ & 4960 & & $3 / 31 / 08$ & 8C17008 & AGG-RRL-001 \\
\hline 14119-33-6 & Plutonium-240 & $<4210$ & $\mathrm{pCi} / \mathrm{g}$ & 4210 & & 3/31/08 & 8C17008 & AGG-RRL-001 \\
\hline 14596-10-2 & Americium-241 & $<2.08$ & $\mathrm{pCi} / \mathrm{g}$ & 2.08 & & 3/31/08 & 8C17008 & AGG-RRL-001 \\
\hline 14993-75-0 & Americium-243 & $<0.718$ & $\mathrm{pCi} / \mathrm{g}$ & 0.718 & & 3/31/08 & 8C17008 & AGG-RRL-001 \\
\hline 15757-87-6 & Curium-243 & $<1.28$ & $\mathrm{pCi} / \mathrm{g}$ & 1.28 & & 3/31/08 & 8C17008 & AGG-RRL-001 \\
\hline 15621-76-8 & Curium-245 & $<1.05$ & $\mathrm{pCi} / \mathrm{g}$ & 1.05 & & $3 / 31 / 08$ & 8C17008 & AGG-RRL-001 \\
\hline HEIS No. & B1T2P4A & \multicolumn{2}{|c|}{ Lab ID: } & 0803014-17 & & & & \\
\hline $13966-32-0$ & Sodium-22 & $<0.518$ & $\mathrm{pCi} / \mathrm{g}$ & 0.518 & & 3/31/08 & 8C17008 & AGG-RRL-001 \\
\hline 13966-00-2 & Potassium-40 & $<9.39$ & $\mathrm{pCi} / \mathrm{g}$ & 9.39 & & 3/31/08 & 8C17008 & AGG-RRL-001 \\
\hline $14392-02-0$ & Chromium-51 & $<3.96$ & $\mathrm{pCi} / \mathrm{g}$ & 3.96 & & 3/31/08 & 8C17008 & AGG-RRL-001 \\
\hline 13966-31-9 & Manganese-54 & $<0.424$ & $\mathrm{pCi} / \mathrm{g}$ & 0.424 & & 3/31/08 & 8C17008 & AGG-RRL-001 \\
\hline 13981-50-5 & Cobalt-57 & $<0.42$ & $\mathrm{pCi} / \mathrm{g}$ & 0.42 & & 3/31/08 & 8C17008 & AGG-RRL-001 \\
\hline $14596-12-4$ & Iron-59 & $<0.809$ & $\mathrm{pCi} / \mathrm{g}$ & 0.809 & & 3/31/08 & 8C17008 & AGG-RRL-001 \\
\hline $10198-40-0$ & Cobalt-60 & $<0.452$ & $\mathrm{pCi} / \mathrm{g}$ & 0.452 & & 3/31/08 & 8C17008 & AGG-RRL-001 \\
\hline 13982-39-3 & Zinc-65 & $<0.956$ & $\mathrm{pCi} / \mathrm{g}$ & 0.956 & & 3/31/08 & 8C17008 & AGG-RRL-001 \\
\hline 14265-71-5 & Selenium-75 & $<0.687$ & $\mathrm{pCi} / \mathrm{g}$ & 0.687 & & 3/31/08 & 8C17008 & AGG-RRL-001 \\
\hline 17056-36-9 & Rubidium-83 & $<1.06$ & $\mathrm{pCi} / \mathrm{g}$ & 1.06 & & 3/31/08 & 8C17008 & AGG-RRL-001 \\
\hline 13967-73-2 & Strontium-85 & $<0.503$ & $\mathrm{pCi} / \mathrm{g}$ & 0.503 & & 3/31/08 & 8C17008 & AGG-RRL-001 \\
\hline 14932-53-7 & Rubidium-86 & $<5.71$ & $\mathrm{pCi} / \mathrm{g}$ & 5.71 & & 3/31/08 & 8C17008 & AGG-RRL-001 \\
\hline $13982-36-0$ & Yttrium-88 & $<0.377$ & $\mathrm{pCi} / \mathrm{g}$ & 0.377 & & 3/31/08 & 8C17008 & AGG-RRL-001 \\
\hline 14681-63-1 & Niobium-94 & $<0.425$ & $\mathrm{pCi} / \mathrm{g}$ & 0.425 & & 3/31/08 & 8C17008 & AGG-RRL-001 \\
\hline \multirow[t]{2}{*}{ 13967-76-5 } & Niobium-95 & $<0.429$ & $\mathrm{pCi} / \mathrm{g}$ & 0.429 & & 3/31/08 & 8C17008 & AGG-RRL-001 \\
\hline & Niobium-95m & $<1.89$ & $\mathrm{pCi} / \mathrm{g}$ & 1.89 & & 3/31/08 & 8C17008 & AGG-RRL-001 \\
\hline
\end{tabular}


GEA/Soil

\begin{tabular}{|c|c|c|c|c|c|c|c|c|}
\hline CAS \# & Analyte & Results & Units & MDA & UNC & Analyzed & Batch & Method \\
\hline \multirow[t]{2}{*}{ HEIS No. } & B1T2P4A & \multicolumn{2}{|c|}{ Lab ID: } & 0803014-17 & & & & \\
\hline & Technetium-95m & $<0.641$ & $\mathrm{pCi} / \mathrm{g}$ & 0.641 & & 3/31/08 & 8C17008 & AGG-RRL-001 \\
\hline \multirow[t]{2}{*}{ 13967-71-0 } & Zirconium-95 & $<0.758$ & $\mathrm{pCi} / \mathrm{g}$ & 0.758 & & 3/31/08 & 8C17008 & AGG-RRL-001 \\
\hline & Technetium-99m & $<0.429$ & $\mathrm{pCi} / \mathrm{g}$ & 0.429 & & 3/31/08 & 8C17008 & AGG-RRL-001 \\
\hline 13968-53-1 & Ruthenium-103 & $<0.497$ & $\mathrm{pCi} / \mathrm{g}$ & 0.497 & & 3/31/08 & 8C17008 & AGG-RRL-001 \\
\hline 13967-48-1 & Ruthenium-106 & $<4.73$ & $\mathrm{pCi} / \mathrm{g}$ & 4.73 & & 3/31/08 & 8C17008 & AGG-RRL-001 \\
\hline 14391-65-2 & Silver-108m & $<0.429$ & $\mathrm{pCi} / \mathrm{g}$ & 0.429 & & 3/31/08 & 8C17008 & AGG-RRL-001 \\
\hline \multirow[t]{2}{*}{ 14109-32-1 } & Cadmium-109 & $<11.9$ & $\mathrm{pCi} / \mathrm{g}$ & 11.9 & & 3/31/08 & 8C17008 & AGG-RRL-001 \\
\hline & Silver-110 & $<0.431$ & $\mathrm{pCi} / \mathrm{g}$ & 0.431 & & 3/31/08 & 8C17008 & AGG-RRL-001 \\
\hline 14391-76-5 & Silver-110m & $<0.433$ & $\mathrm{pCi} / \mathrm{g}$ & 0.433 & & 3/31/08 & 8C17008 & AGG-RRL-001 \\
\hline 13966-06-8 & Tin-113 & $<0.593$ & $\mathrm{pCi} / \mathrm{g}$ & 0.593 & & 3/31/08 & 8C17008 & AGG-RRL-001 \\
\hline $14683-10-4$ & Antimony-124 & $<0.514$ & $\mathrm{pCi} / \mathrm{g}$ & 0.514 & & 3/31/08 & 8C17008 & AGG-RRL-001 \\
\hline 14234-35-6 & Antimony-125 & $<1.33$ & $\mathrm{pCi} / \mathrm{g}$ & 1.33 & & 3/31/08 & 8C17008 & AGG-RRL-001 \\
\hline 15756-32-8 & Antimony-126 & $<0.404$ & $\mathrm{pCi} / \mathrm{g}$ & 0.404 & & 3/31/08 & 8C17008 & AGG-RRL-001 \\
\hline 15832-50-5 & Tin-126 & $<0.966$ & $\mathrm{pCi} / \mathrm{g}$ & 0.966 & & 3/31/08 & 8C17008 & AGG-RRL-001 \\
\hline 10043-66-0 & Iodine-131 & $<0.527$ & $\mathrm{pCi} / \mathrm{g}$ & 0.527 & & 3/31/08 & 8C17008 & AGG-RRL-001 \\
\hline 13981-41-4 & Barium-133 & $<0.745$ & $\mathrm{pCi} / \mathrm{g}$ & 0.745 & & 3/31/08 & 8C17008 & AGG-RRL-001 \\
\hline 13967-70-9 & Cesium-134 & $<0.488$ & $\mathrm{pCi} / \mathrm{g}$ & 0.488 & & 3/31/08 & 8C17008 & AGG-RRL-001 \\
\hline 10045-97-3 & Cesium-137 & $<0.479$ & $\mathrm{pCi} / \mathrm{g}$ & 0.479 & & 3/31/08 & 8C17008 & AGG-RRL-001 \\
\hline 13982-30-4 & Cerium-139 & $<0.501$ & $\mathrm{pCi} / \mathrm{g}$ & 0.501 & & 3/31/08 & 8C17008 & AGG-RRL-001 \\
\hline 14762-78-8 & Cerium-144 & $<3.47$ & $\mathrm{pCi} / \mathrm{g}$ & 3.47 & & 3/31/08 & 8C17008 & AGG-RRL-001 \\
\hline 14683-23-9 & Europium-152 & $<1.7$ & $\mathrm{pCi} / \mathrm{g}$ & 1.7 & & 3/31/08 & 8C17008 & AGG-RRL-001 \\
\hline 14276-65-4 & Gadolinium-153 & $<1.31$ & $\mathrm{pCi} / \mathrm{g}$ & 1.31 & & 3/31/08 & 8C17008 & AGG-RRL-001 \\
\hline 15585-10-1 & Europium-154 & $<0.889$ & $\mathrm{pCi} / \mathrm{g}$ & 0.889 & & 3/31/08 & 8C17008 & AGG-RRL-001 \\
\hline 14391-16-3 & Europium-155 & $<1.37$ & $\mathrm{pCi} / \mathrm{g}$ & 1.37 & & 3/31/08 & 8C17008 & AGG-RRL-001 \\
\hline 13982-78-0 & Mercury-203 & $<0.544$ & $\mathrm{pCi} / \mathrm{g}$ & 0.544 & & 3/31/08 & 8C17008 & AGG-RRL-001 \\
\hline 14913-50-9 & Thallium-208 & $<0.529$ & $\mathrm{pCi} / \mathrm{g}$ & 0.529 & & 3/31/08 & 8C17008 & AGG-RRL-001 \\
\hline 14331-79-4 & Bismuth-210 & $<0.828$ & $\mathrm{pCi} / \mathrm{g}$ & 0.828 & & 3/31/08 & 8C17008 & AGG-RRL-001 \\
\hline \multirow[t]{3}{*}{$14255-04-0$} & Lead-210 & $<61.4$ & $\mathrm{pCi} / \mathrm{g}$ & 61.4 & & 3/31/08 & 8C17008 & AGG-RRL-001 \\
\hline & Bismuth-211 & $<9.45$ & $\mathrm{pCi} / \mathrm{g}$ & 9.45 & & 3/31/08 & 8C17008 & AGG-RRL-001 \\
\hline & Lead-211 & $<12.9$ & $\mathrm{pCi} / \mathrm{g}$ & 12.9 & & 3/31/08 & 8C17008 & AGG-RRL-001 \\
\hline 15092-94-1 & Lead-212 & $<1.1$ & $\mathrm{pCi} / \mathrm{g}$ & 1.1 & & 3/31/08 & 8C17008 & AGG-RRL-001 \\
\hline 14733-03-0 & Bismuth-214 & $<1.09$ & $\mathrm{pCi} / \mathrm{g}$ & 1.09 & & 3/31/08 & 8C17008 & AGG-RRL-001 \\
\hline $15067-28-4$ & Lead-214 & $<1.18$ & $\mathrm{pCi} / \mathrm{g}$ & 1.18 & & 3/31/08 & 8C17008 & AGG-RRL-001 \\
\hline $14835-02-0$ & Radon-219 & $<4.2$ & $\mathrm{pCi} / \mathrm{g}$ & 4.2 & & 3/31/08 & 8C17008 & AGG-RRL-001 \\
\hline 22481-48-7 & Radon-220 & $<397$ & $\mathrm{pCi} / \mathrm{g}$ & 397 & & 3/31/08 & 8C17008 & AGG-RRL-001 \\
\hline 28522-20-5 & Radon-221 & $<1.99$ & $\mathrm{pCi} / \mathrm{g}$ & 1.99 & & 3/31/08 & 8C17008 & AGG-RRL-001 \\
\hline 15756-98-6 & Francium-223 & $<13.9$ & $\mathrm{pCi} / \mathrm{g}$ & 13.9 & & 3/31/08 & 8C17008 & AGG-RRL-001 \\
\hline $15623-45-7$ & Radium-223 & $<3.03$ & $\mathrm{pCi} / \mathrm{g}$ & 3.03 & & 3/31/08 & 8C17008 & AGG-RRL-001 \\
\hline 13233-32-4 & Radium-224 & $<12$ & $\mathrm{pCi} / \mathrm{g}$ & 12 & & 3/31/08 & 8C17008 & AGG-RRL-001 \\
\hline 13982-63-3 & Radium-226 & $<12.2$ & $\mathrm{pCi} / \mathrm{g}$ & 12.2 & & 3/31/08 & 8C17008 & AGG-RRL-001 \\
\hline 15623-47-9 & Thorium-227 & $<4.22$ & $\mathrm{pCi} / \mathrm{g}$ & 4.22 & & 3/31/08 & 8C17008 & AGG-RRL-001 \\
\hline 14331-83-0 & Actinium-228 & $<1.71$ & $\mathrm{pCi} / \mathrm{g}$ & 1.71 & & 3/31/08 & 8C17008 & AGG-RRL-001 \\
\hline $14274-82-9$ & Thorium-228 & $<152$ & $\mathrm{pCi} / \mathrm{g}$ & 152 & & 3/31/08 & 8C17008 & AGG-RRL-001 \\
\hline 14269-63-7 & Thorium-230 & $<157$ & $\mathrm{pCi} / \mathrm{g}$ & 157 & & 3/31/08 & 8C17008 & AGG-RRL-001 \\
\hline 14331-85-2 & Protactinium-231 & $<18.6$ & $\mathrm{pCi} / \mathrm{g}$ & 18.6 & & 3/31/08 & 8C17008 & AGG-RRL-001 \\
\hline $14932-40-2$ & Thorium-231 & $<93.7$ & $\mathrm{pCi} / \mathrm{g}$ & 93.7 & & 3/31/08 & 8C17008 & AGG-RRL-001 \\
\hline 7440-29-1 & Thorium-232 & $<403$ & $\mathrm{pCi} / \mathrm{g}$ & 403 & & 3/31/08 & 8C17008 & AGG-RRL-001 \\
\hline 13981-14-1 & Protactinium-233 & $<1.07$ & $\mathrm{pCi} / \mathrm{g}$ & 1.07 & & 3/31/08 & 8C17008 & AGG-RRL-001 \\
\hline $15100-28-4$ & Protactinium-234 & $<1.71$ & $\mathrm{pCi} / \mathrm{g}$ & 1.71 & & 3/31/08 & 8C17008 & AGG-RRL-001 \\
\hline
\end{tabular}


GEA/Soil

\begin{tabular}{|c|c|c|c|c|c|c|c|c|}
\hline CAS \# & Analyte & Results & Units & MDA & UNC & Analyzed & Batch & Method \\
\hline \multirow[t]{2}{*}{ HEIS No. } & B1T2P4A & \multicolumn{2}{|c|}{ Lab ID: } & 0803014-17 & & & & \\
\hline & Protactinium-234m & $<49.7$ & $\mathrm{pCi} / \mathrm{g}$ & 49.7 & & 3/31/08 & 8C17008 & AGG-RRL-001 \\
\hline $15065-10-8$ & Thorium-234 & $<14$ & $\mathrm{pCi} / \mathrm{g}$ & 14 & & 3/31/08 & 8C17008 & AGG-RRL-001 \\
\hline 15117-96-1 & Uranium 235 & $<3.67$ & $\mathrm{pCi} / \mathrm{g}$ & 3.67 & & 3/31/08 & 8C17008 & AGG-RRL-001 \\
\hline 13994-20-2 & Neptunium-237 & $<3.58$ & $\mathrm{pCi} / \mathrm{g}$ & 3.58 & & 3/31/08 & 8C17008 & AGG-RRL-001 \\
\hline \multirow[t]{2}{*}{ 13981-16-3 } & Plutonium-238 & $<5390$ & $\mathrm{pCi} / \mathrm{g}$ & 5390 & & 3/31/08 & 8C17008 & AGG-RRL-001 \\
\hline & Uranium 238 & $<7.6$ & $\mathrm{pCi} / \mathrm{g}$ & 7.6 & & 3/31/08 & 8C17008 & AGG-RRL-001 \\
\hline 15117-48-3 & Plutonium-239 & $<5600$ & $\mathrm{pCi} / \mathrm{g}$ & 5600 & & 3/31/08 & 8C17008 & AGG-RRL-001 \\
\hline 14119-33-6 & Plutonium-240 & $<5390$ & $\mathrm{pCi} / \mathrm{g}$ & 5390 & & 3/31/08 & 8C17008 & AGG-RRL-001 \\
\hline 14596-10-2 & Americium-241 & $<2.06$ & $\mathrm{pCi} / \mathrm{g}$ & 2.06 & & 3/31/08 & 8C17008 & AGG-RRL-001 \\
\hline 14993-75-0 & Americium-243 & $<0.864$ & $\mathrm{pCi} / \mathrm{g}$ & 0.864 & & 3/31/08 & 8C17008 & AGG-RRL-001 \\
\hline 15757-87-6 & Curium-243 & $<1.65$ & $\mathrm{pCi} / \mathrm{g}$ & 1.65 & & 3/31/08 & 8C17008 & AGG-RRL-001 \\
\hline 15621-76-8 & Curium-245 & $<1.35$ & $\mathrm{pCi} / \mathrm{g}$ & 1.35 & & 3/31/08 & 8C17008 & AGG-RRL-001 \\
\hline HEIS No. & B1T2P5B & \multicolumn{2}{|c|}{ Lab ID: } & 0803014-20 & & & & \\
\hline 13966-32-0 & Sodium-22 & $<0.43$ & $\mathrm{pCi} / \mathrm{g}$ & 0.43 & & 3/31/08 & 8C17008 & AGG-RRL-001 \\
\hline 13966-00-2 & Potassium-40 & 21.1 & $\mathrm{pCi} / \mathrm{g}$ & 3.91 & 1.54 & 3/31/08 & 8C17008 & AGG-RRL-001 \\
\hline 14392-02-0 & Chromium-51 & $<3.61$ & $\mathrm{pCi} / \mathrm{g}$ & 3.61 & & 3/31/08 & 8C17008 & AGG-RRL-001 \\
\hline 13966-31-9 & Manganese-54 & $<0.353$ & $\mathrm{pCi} / \mathrm{g}$ & 0.353 & & 3/31/08 & 8C17008 & AGG-RRL-001 \\
\hline 13981-50-5 & Cobalt-57 & $<0.357$ & $\mathrm{pCi} / \mathrm{g}$ & 0.357 & & 3/31/08 & 8C17008 & AGG-RRL-001 \\
\hline $14596-12-4$ & Iron-59 & $<0.743$ & $\mathrm{pCi} / \mathrm{g}$ & 0.743 & & 3/31/08 & 8C17008 & AGG-RRL-001 \\
\hline 10198-40-0 & Cobalt-60 & $<0.338$ & $\mathrm{pCi} / \mathrm{g}$ & 0.338 & & 3/31/08 & 8C17008 & AGG-RRL-001 \\
\hline 13982-39-3 & Zinc-65 & $<0.967$ & $\mathrm{pCi} / \mathrm{g}$ & 0.967 & & 3/31/08 & 8C17008 & AGG-RRL-001 \\
\hline $14265-71-5$ & Selenium-75 & $<0.623$ & $\mathrm{pCi} / \mathrm{g}$ & 0.623 & & 3/31/08 & 8C17008 & AGG-RRL-001 \\
\hline 17056-36-9 & Rubidium-83 & $<0.709$ & $\mathrm{pCi} / \mathrm{g}$ & 0.709 & & 3/31/08 & 8C17008 & AGG-RRL-001 \\
\hline 13967-73-2 & Strontium-85 & $<0.486$ & pCi/g & 0.486 & & 3/31/08 & 8C17008 & AGG-RRL-001 \\
\hline 14932-53-7 & Rubidium-86 & $<4.67$ & $\mathrm{pCi} / \mathrm{g}$ & 4.67 & & 3/31/08 & 8C17008 & AGG-RRL-001 \\
\hline 13982-36-0 & Yttrium-88 & $<0.386$ & $\mathrm{pCi} / \mathrm{g}$ & 0.386 & & 3/31/08 & 8C17008 & AGG-RRL-001 \\
\hline 14681-63-1 & Niobium-94 & $<0.353$ & $\mathrm{pCi} / \mathrm{g}$ & 0.353 & & 3/31/08 & 8C17008 & AGG-RRL-001 \\
\hline \multirow[t]{3}{*}{ 13967-76-5 } & Niobium-95 & $<0.378$ & $\mathrm{pCi} / \mathrm{g}$ & 0.378 & & 3/31/08 & 8C17008 & AGG-RRL-001 \\
\hline & Niobium-95m & $<1.76$ & $\mathrm{pCi} / \mathrm{g}$ & 1.76 & & 3/31/08 & 8C17008 & AGG-RRL-001 \\
\hline & Technetium-95m & $<0.593$ & $\mathrm{pCi} / \mathrm{g}$ & 0.593 & & 3/31/08 & 8C17008 & AGG-RRL-001 \\
\hline \multirow[t]{2}{*}{ 13967-71-0 } & Zirconium-95 & $<0.618$ & $\mathrm{pCi} / \mathrm{g}$ & 0.618 & & 3/31/08 & 8C17008 & AGG-RRL-001 \\
\hline & Technetium-99m & $<0.371$ & $\mathrm{pCi} / \mathrm{g}$ & 0.371 & & 3/31/08 & 8C17008 & AGG-RRL-001 \\
\hline 13968-53-1 & Ruthenium-103 & $<0.392$ & $\mathrm{pCi} / \mathrm{g}$ & 0.392 & & 3/31/08 & 8C17008 & AGG-RRL-001 \\
\hline 13967-48-1 & Ruthenium-106 & $<3.66$ & $\mathrm{pCi} / \mathrm{g}$ & 3.66 & & 3/31/08 & 8C17008 & AGG-RRL-001 \\
\hline 14391-65-2 & Silver-108m & $<0.396$ & $\mathrm{pCi} / \mathrm{g}$ & 0.396 & & 3/31/08 & 8C17008 & AGG-RRL-001 \\
\hline \multirow[t]{2}{*}{ 14109-32-1 } & Cadmium-109 & $<10$ & $\mathrm{pCi} / \mathrm{g}$ & 10 & & 3/31/08 & 8C17008 & AGG-RRL-001 \\
\hline & Silver-110 & $<0.39$ & $\mathrm{pCi} / \mathrm{g}$ & 0.39 & & 3/31/08 & 8C17008 & AGG-RRL-001 \\
\hline 14391-76-5 & Silver-110m & $<0.391$ & $\mathrm{pCi} / \mathrm{g}$ & 0.391 & & 3/31/08 & 8C17008 & AGG-RRL-001 \\
\hline 13966-06-8 & Tin-113 & $<0.552$ & $\mathrm{pCi} / \mathrm{g}$ & 0.552 & & 3/31/08 & 8C17008 & AGG-RRL-001 \\
\hline 14683-10-4 & Antimony-124 & $<0.35$ & $\mathrm{pCi} / \mathrm{g}$ & 0.35 & & 3/31/08 & 8C17008 & AGG-RRL-001 \\
\hline $14234-35-6$ & Antimony-125 & $<1.23$ & pCi/g & 1.23 & & 3/31/08 & 8C17008 & AGG-RRL-001 \\
\hline 15756-32-8 & Antimony-126 & $<0.379$ & $\mathrm{pCi} / \mathrm{g}$ & 0.379 & & 3/31/08 & 8C17008 & AGG-RRL-001 \\
\hline 15832-50-5 & Tin-126 & $<0.79$ & $\mathrm{pCi} / \mathrm{g}$ & 0.79 & & 3/31/08 & 8C17008 & AGG-RRL-001 \\
\hline 10043-66-0 & Iodine-131 & $<0.459$ & $\mathrm{pCi} / \mathrm{g}$ & 0.459 & & 3/31/08 & 8C17008 & AGG-RRL-001 \\
\hline 13981-41-4 & Barium-133 & $<0.731$ & $\mathrm{pCi} / \mathrm{g}$ & 0.731 & & 3/31/08 & 8C17008 & AGG-RRL-001 \\
\hline 13967-70-9 & Cesium-134 & $<0.43$ & $\mathrm{pCi} / \mathrm{g}$ & 0.43 & & 3/31/08 & 8C17008 & AGG-RRL-001 \\
\hline 10045-97-3 & Cesium-137 & $<0.441$ & $\mathrm{pCi} / \mathrm{g}$ & 0.441 & & 3/31/08 & 8C17008 & AGG-RRL-001 \\
\hline 13982-30-4 & Cerium-139 & $<0.442$ & $\mathrm{pCi} / \mathrm{g}$ & 0.442 & & 3/31/08 & 8C17008 & AGG-RRL-001 \\
\hline
\end{tabular}


GEA/Soil

\begin{tabular}{|c|c|c|c|c|c|c|c|c|}
\hline CAS \# & Analyte & Results & Units & MDA & UNC & Analyzed & Batch & Method \\
\hline HEIS No. & B1T2P5B & \multicolumn{2}{|c|}{ Lab ID: } & 0803014-20 & \multirow{43}{*}{0.187} & & & \\
\hline $14762-78-8$ & Cerium-144 & $<2.97$ & $\mathrm{pCi} / \mathrm{g}$ & 2.97 & & $3 / 31 / 08$ & 8C17008 & AGG-RRL-001 \\
\hline $14683-23-9$ & Europium-152 & $<1.41$ & $\mathrm{pCi} / \mathrm{g}$ & 1.41 & & 3/31/08 & 8C17008 & AGG-RRL-001 \\
\hline $14276-65-4$ & Gadolinium-153 & $<1.11$ & $\mathrm{pCi} / \mathrm{g}$ & 1.11 & & 3/31/08 & 8C17008 & AGG-RRL-001 \\
\hline 15585-10-1 & Europium-154 & $<0.757$ & $\mathrm{pCi} / \mathrm{g}$ & 0.757 & & 3/31/08 & 8C17008 & AGG-RRL-001 \\
\hline $14391-16-3$ & Europium-155 & $<1.12$ & $\mathrm{pCi} / \mathrm{g}$ & 1.12 & & 3/31/08 & 8C17008 & AGG-RRL-001 \\
\hline 13982-78-0 & Mercury-203 & $<0.462$ & $\mathrm{pCi} / \mathrm{g}$ & 0.462 & & 3/31/08 & 8C17008 & AGG-RRL-001 \\
\hline 14913-50-9 & Thallium-208 & $<0.469$ & $\mathrm{pCi} / \mathrm{g}$ & 0.469 & & 3/31/08 & 8C17008 & AGG-RRL-001 \\
\hline 14331-79-4 & Bismuth-210 & $<0.752$ & $\mathrm{pCi} / \mathrm{g}$ & 0.752 & & $3 / 31 / 08$ & 8C17008 & AGG-RRL-001 \\
\hline \multirow[t]{3}{*}{$14255-04-0$} & Lead-210 & $<146$ & $\mathrm{pCi} / \mathrm{g}$ & 146 & & 3/31/08 & 8C17008 & AGG-RRL-001 \\
\hline & Bismuth-211 & $<8.58$ & $\mathrm{pCi} / \mathrm{g}$ & 8.58 & & 3/31/08 & 8C17008 & AGG-RRL-001 \\
\hline & Lead-211 & $<11.7$ & $\mathrm{pCi} / \mathrm{g}$ & 11.7 & & 3/31/08 & 8C17008 & AGG-RRL-001 \\
\hline 15092-94-1 & Lead-212 & $<0.843$ & $\mathrm{pCi} / \mathrm{g}$ & 0.843 & & 3/31/08 & 8C17008 & AGG-RRL-001 \\
\hline $14733-03-0$ & Bismuth-214 & $<0.938$ & $\mathrm{pCi} / \mathrm{g}$ & 0.938 & & 3/31/08 & 8C17008 & AGG-RRL-001 \\
\hline $15067-28-4$ & Lead-214 & $<1.14$ & $\mathrm{pCi} / \mathrm{g}$ & 1.14 & & 3/31/08 & 8C17008 & AGG-RRL-001 \\
\hline $14835-02-0$ & Radon-219 & $<3.85$ & $\mathrm{pCi} / \mathrm{g}$ & 3.85 & & 3/31/08 & 8C17008 & AGG-RRL-001 \\
\hline 22481-48-7 & Radon-220 & $<324$ & $\mathrm{pCi} / \mathrm{g}$ & 324 & & 3/31/08 & 8C17008 & AGG-RRL-001 \\
\hline 28522-20-5 & Radon-221 & $<1.73$ & $\mathrm{pCi} / \mathrm{g}$ & 1.73 & & 3/31/08 & 8C17008 & AGG-RRL-001 \\
\hline 15756-98-6 & Francium-223 & $<12.7$ & $\mathrm{pCi} / \mathrm{g}$ & 12.7 & & 3/31/08 & 8C17008 & AGG-RRL-001 \\
\hline $15623-45-7$ & Radium-223 & $<2.81$ & $\mathrm{pCi} / \mathrm{g}$ & 2.81 & & 3/31/08 & 8C17008 & AGG-RRL-001 \\
\hline $13233-32-4$ & Radium-224 & $<11.1$ & $\mathrm{pCi} / \mathrm{g}$ & 11.1 & & 3/31/08 & 8C17008 & AGG-RRL-001 \\
\hline $13982-63-3$ & Radium-226 & $<10.6$ & $\mathrm{pCi} / \mathrm{g}$ & 10.6 & & 3/31/08 & 8C17008 & AGG-RRL-001 \\
\hline $15623-47-9$ & Thorium-227 & $<3.93$ & $\mathrm{pCi} / \mathrm{g}$ & 3.93 & & 3/31/08 & 8C17008 & AGG-RRL-001 \\
\hline $14331-83-0$ & Actinium-228 & $<1.68$ & $\mathrm{pCi} / \mathrm{g}$ & 1.68 & & 3/31/08 & 8C17008 & AGG-RRL-001 \\
\hline 14274-82-9 & Thorium-228 & $<141$ & $\mathrm{pCi} / \mathrm{g}$ & 141 & & 3/31/08 & 8C17008 & AGG-RRL-001 \\
\hline $14269-63-7$ & Thorium-230 & $<144$ & $\mathrm{pCi} / \mathrm{g}$ & 144 & & 3/31/08 & 8C17008 & AGG-RRL-001 \\
\hline $14331-85-2$ & Protactinium-231 & $<15.3$ & $\mathrm{pCi} / \mathrm{g}$ & 15.3 & & 3/31/08 & 8C17008 & AGG-RRL-001 \\
\hline $14932-40-2$ & Thorium-231 & $<76.5$ & $\mathrm{pCi} / \mathrm{g}$ & 76.5 & & 3/31/08 & 8C17008 & AGG-RRL-001 \\
\hline 7440-29-1 & Thorium-232 & $<451$ & $\mathrm{pCi} / \mathrm{g}$ & 451 & & 3/31/08 & 8C17008 & AGG-RRL-001 \\
\hline 13981-14-1 & Protactinium-233 & $<0.969$ & $\mathrm{pCi} / \mathrm{g}$ & 0.969 & & 3/31/08 & 8C17008 & AGG-RRL-001 \\
\hline \multirow[t]{2}{*}{$15100-28-4$} & Protactinium-234 & $<1.42$ & $\mathrm{pCi} / \mathrm{g}$ & 1.42 & & 3/31/08 & 8C17008 & AGG-RRL-001 \\
\hline & Protactinium-234m & $<43.3$ & $\mathrm{pCi} / \mathrm{g}$ & 43.3 & & $3 / 31 / 08$ & 8C17008 & AGG-RRL-001 \\
\hline $15065-10-8$ & Thorium-234 & $<13.2$ & $\mathrm{pCi} / \mathrm{g}$ & 13.2 & & $3 / 31 / 08$ & 8C17008 & AGG-RRL-001 \\
\hline 15117-96-1 & Uranium 235 & $<3.09$ & $\mathrm{pCi} / \mathrm{g}$ & 3.09 & & 3/31/08 & 8C17008 & AGG-RRL-001 \\
\hline 13994-20-2 & Neptunium-237 & $<2.92$ & $\mathrm{pCi} / \mathrm{g}$ & 2.92 & & $3 / 31 / 08$ & 8C17008 & AGG-RRL-001 \\
\hline \multirow[t]{2}{*}{$13981-16-3$} & Plutonium-238 & $<4490$ & $\mathrm{pCi} / \mathrm{g}$ & 4490 & & 3/31/08 & 8C17008 & AGG-RRL-001 \\
\hline & Uranium 238 & $<6.82$ & $\mathrm{pCi} / \mathrm{g}$ & 6.82 & & 3/31/08 & 8C17008 & AGG-RRL-001 \\
\hline $15117-48-3$ & Plutonium-239 & $<5140$ & $\mathrm{pCi} / \mathrm{g}$ & 5140 & & 3/31/08 & 8C17008 & AGG-RRL-001 \\
\hline 14119-33-6 & Plutonium-240 & $<4500$ & $\mathrm{pCi} / \mathrm{g}$ & 4500 & & $3 / 31 / 08$ & 8C17008 & AGG-RRL-001 \\
\hline $14596-10-2$ & Americium-241 & $<2.23$ & $\mathrm{pCi} / \mathrm{g}$ & 2.23 & & $3 / 31 / 08$ & 8C17008 & AGG-RRL-001 \\
\hline $14993-75-0$ & Americium-243 & $<0.791$ & $\mathrm{pCi} / \mathrm{g}$ & 0.791 & & 3/31/08 & 8C17008 & AGG-RRL-001 \\
\hline $15757-87-6$ & Curium-243 & $<1.37$ & $\mathrm{pCi} / \mathrm{g}$ & 1.37 & & 3/31/08 & 8C17008 & AGG-RRL-001 \\
\hline 15621-76-8 & Curium-245 & $<1.13$ & $\mathrm{pCi} / \mathrm{g}$ & 1.13 & & $3 / 31 / 08$ & 8C17008 & AGG-RRL-001 \\
\hline HEIS No. & B1T2P5A & \multicolumn{2}{|c|}{ Lab ID: } & 0803014-21 & & & & \\
\hline 13966-32-0 & Sodium-22 & $<0.487$ & $\mathrm{pCi} / \mathrm{g}$ & 0.487 & & 3/31/08 & 8C17008 & AGG-RRL-001 \\
\hline $13966-00-2$ & Potassium-40 & 21.2 & $\mathrm{pCi} / \mathrm{g}$ & 3.99 & 1.57 & 3/31/08 & 8C17008 & AGG-RRL-001 \\
\hline $14392-02-0$ & Chromium-51 & $<4.62$ & $\mathrm{pCi} / \mathrm{g}$ & 4.62 & & 3/31/08 & 8C17008 & AGG-RRL-001 \\
\hline 13966-31-9 & Manganese-54 & $<0.467$ & $\mathrm{pCi} / \mathrm{g}$ & 0.467 & & 3/31/08 & 8C17008 & AGG-RRL-001 \\
\hline 13981-50-5 & Cobalt-57 & $<0.457$ & $\mathrm{pCi} / \mathrm{g}$ & 0.457 & & 3/31/08 & 8C17008 & AGG-RRL-001 \\
\hline
\end{tabular}


GEA/Soil

\begin{tabular}{|c|c|c|c|c|c|c|c|c|}
\hline CAS \# & Analyte & Results & Units & MDA & UNC & Analyzed & Batch & Method \\
\hline HEIS No. & B1T2P5A & \multicolumn{3}{|c|}{ Lab ID: $\quad$ 0803014-21 } & & & & \\
\hline $14596-12-4$ & Iron-59 & $<0.822$ & $\mathrm{pCi} / \mathrm{g}$ & 0.822 & & 3/31/08 & 8C17008 & AGG-RRL-001 \\
\hline 10198-40-0 & Cobalt-60 & $<0.49$ & $\mathrm{pCi} / \mathrm{g}$ & 0.49 & & 3/31/08 & 8C17008 & AGG-RRL-001 \\
\hline 13982-39-3 & Zinc-65 & $<1.02$ & $\mathrm{pCi} / \mathrm{g}$ & 1.02 & & 3/31/08 & 8C17008 & AGG-RRL-001 \\
\hline $14265-71-5$ & Selenium-75 & $<0.727$ & $\mathrm{pCi} / \mathrm{g}$ & 0.727 & & 3/31/08 & 8C17008 & AGG-RRL-001 \\
\hline 17056-36-9 & Rubidium-83 & $<1.16$ & $\mathrm{pCi} / \mathrm{g}$ & 1.16 & & 3/31/08 & 8C17008 & AGG-RRL-001 \\
\hline 13967-73-2 & Strontium-85 & $<0.532$ & $\mathrm{pCi} / \mathrm{g}$ & 0.532 & & 3/31/08 & 8C17008 & AGG-RRL-001 \\
\hline 14932-53-7 & Rubidium-86 & $<5.8$ & $\mathrm{pCi} / \mathrm{g}$ & 5.8 & & 3/31/08 & 8C17008 & AGG-RRL-001 \\
\hline 13982-36-0 & Yttrium-88 & $<0.391$ & $\mathrm{pCi} / \mathrm{g}$ & 0.391 & & 3/31/08 & 8C17008 & AGG-RRL-001 \\
\hline 14681-63-1 & Niobium-94 & $<0.453$ & $\mathrm{pCi} / \mathrm{g}$ & 0.453 & & 3/31/08 & 8C17008 & AGG-RRL-001 \\
\hline \multirow[t]{3}{*}{ 13967-76-5 } & Niobium-95 & $<0.458$ & $\mathrm{pCi} / \mathrm{g}$ & 0.458 & & 3/31/08 & 8C17008 & AGG-RRL-001 \\
\hline & Niobium-95m & $<2.03$ & $\mathrm{pCi} / \mathrm{g}$ & 2.03 & & 3/31/08 & 8C17008 & AGG-RRL-001 \\
\hline & Technetium-95m & $<0.697$ & $\mathrm{pCi} / \mathrm{g}$ & 0.697 & & 3/31/08 & 8C17008 & AGG-RRL-001 \\
\hline \multirow[t]{2}{*}{ 13967-71-0 } & Zirconium-95 & $<0.792$ & $\mathrm{pCi} / \mathrm{g}$ & 0.792 & & 3/31/08 & 8C17008 & AGG-RRL-001 \\
\hline & Technetium-99m & $<0.465$ & $\mathrm{pCi} / \mathrm{g}$ & 0.465 & & 3/31/08 & 8C17008 & AGG-RRL-001 \\
\hline 13968-53-1 & Ruthenium-103 & $<0.528$ & $\mathrm{pCi} / \mathrm{g}$ & 0.528 & & 3/31/08 & 8C17008 & AGG-RRL-001 \\
\hline 13967-48-1 & Ruthenium-106 & $<5.06$ & $\mathrm{pCi} / \mathrm{g}$ & 5.06 & & 3/31/08 & 8C17008 & AGG-RRL-001 \\
\hline 14391-65-2 & Silver-108m & $<0.456$ & $\mathrm{pCi} / \mathrm{g}$ & 0.456 & & 3/31/08 & 8C17008 & AGG-RRL-001 \\
\hline \multirow[t]{2}{*}{ 14109-32-1 } & Cadmium-109 & $<12.6$ & $\mathrm{pCi} / \mathrm{g}$ & 12.6 & & 3/31/08 & 8C17008 & AGG-RRL-001 \\
\hline & Silver-110 & $<0.474$ & $\mathrm{pCi} / \mathrm{g}$ & 0.474 & & 3/31/08 & 8C17008 & AGG-RRL-001 \\
\hline 14391-76-5 & Silver-110m & $<0.475$ & $\mathrm{pCi} / \mathrm{g}$ & 0.475 & & 3/31/08 & 8C17008 & AGG-RRL-001 \\
\hline 13966-06-8 & Tin-113 & $<0.634$ & $\mathrm{pCi} / \mathrm{g}$ & 0.634 & & 3/31/08 & 8C17008 & AGG-RRL-001 \\
\hline 14683-10-4 & Antimony-124 & $<0.521$ & $\mathrm{pCi} / \mathrm{g}$ & 0.521 & & 3/31/08 & 8C17008 & AGG-RRL-001 \\
\hline $14234-35-6$ & Antimony-125 & $<1.41$ & $\mathrm{pCi} / \mathrm{g}$ & 1.41 & & 3/31/08 & 8C17008 & AGG-RRL-001 \\
\hline 15756-32-8 & Antimony-126 & $<0.446$ & $\mathrm{pCi} / \mathrm{g}$ & 0.446 & & 3/31/08 & 8C17008 & AGG-RRL-001 \\
\hline 15832-50-5 & Tin-126 & $<1.03$ & $\mathrm{pCi} / \mathrm{g}$ & 1.03 & & 3/31/08 & 8C17008 & AGG-RRL-001 \\
\hline 10043-66-0 & Iodine-131 & $<0.58$ & $\mathrm{pCi} / \mathrm{g}$ & 0.58 & & 3/31/08 & 8C17008 & AGG-RRL-001 \\
\hline 13981-41-4 & Barium-133 & $<0.794$ & $\mathrm{pCi} / \mathrm{g}$ & 0.794 & & 3/31/08 & 8C17008 & AGG-RRL-001 \\
\hline 13967-70-9 & Cesium-134 & $<0.523$ & $\mathrm{pCi} / \mathrm{g}$ & 0.523 & & 3/31/08 & 8C17008 & AGG-RRL-001 \\
\hline $10045-97-3$ & Cesium-137 & $<0.522$ & $\mathrm{pCi} / \mathrm{g}$ & 0.522 & & 3/31/08 & 8C17008 & AGG-RRL-001 \\
\hline 13982-30-4 & Cerium-139 & $<0.543$ & $\mathrm{pCi} / \mathrm{g}$ & 0.543 & & 3/31/08 & 8C17008 & AGG-RRL-001 \\
\hline 14762-78-8 & Cerium-144 & $<3.71$ & $\mathrm{pCi} / \mathrm{g}$ & 3.71 & & 3/31/08 & 8C17008 & AGG-RRL-001 \\
\hline 14683-23-9 & Europium-152 & $<1.87$ & $\mathrm{pCi} / \mathrm{g}$ & 1.87 & & 3/31/08 & 8C17008 & AGG-RRL-001 \\
\hline $14276-65-4$ & Gadolinium-153 & $<1.4$ & $\mathrm{pCi} / \mathrm{g}$ & 1.4 & & 3/31/08 & 8C17008 & AGG-RRL-001 \\
\hline 15585-10-1 & Europium-154 & $<0.965$ & $\mathrm{pCi} / \mathrm{g}$ & 0.965 & & 3/31/08 & 8C17008 & AGG-RRL-001 \\
\hline 14391-16-3 & Europium-155 & $<1.47$ & $\mathrm{pCi} / \mathrm{g}$ & 1.47 & & 3/31/08 & 8C17008 & AGG-RRL-001 \\
\hline 13982-78-0 & Mercury-203 & $<0.566$ & $\mathrm{pCi} / \mathrm{g}$ & 0.566 & & 3/31/08 & 8C17008 & AGG-RRL-001 \\
\hline 14913-50-9 & Thallium-208 & $<0.608$ & $\mathrm{pCi} / \mathrm{g}$ & 0.608 & & 3/31/08 & 8C17008 & AGG-RRL-001 \\
\hline 14331-79-4 & Bismuth-210 & $<0.878$ & $\mathrm{pCi} / \mathrm{g}$ & 0.878 & & 3/31/08 & 8C17008 & AGG-RRL-001 \\
\hline \multirow[t]{3}{*}{$14255-04-0$} & Lead-210 & $<65.1$ & $\mathrm{pCi} / \mathrm{g}$ & 65.1 & & 3/31/08 & 8C17008 & AGG-RRL-001 \\
\hline & Bismuth-211 & $<9.9$ & $\mathrm{pCi} / \mathrm{g}$ & 9.9 & & 3/31/08 & 8C17008 & AGG-RRL-001 \\
\hline & Lead-211 & $<13.5$ & $\mathrm{pCi} / \mathrm{g}$ & 13.5 & & 3/31/08 & 8C17008 & AGG-RRL-001 \\
\hline 15092-94-1 & Lead-212 & $<1.17$ & $\mathrm{pCi} / \mathrm{g}$ & 1.17 & & 3/31/08 & 8C17008 & AGG-RRL-001 \\
\hline 14733-03-0 & Bismuth-214 & $<1.1$ & $\mathrm{pCi} / \mathrm{g}$ & 1.1 & & 3/31/08 & 8C17008 & AGG-RRL-001 \\
\hline $15067-28-4$ & Lead-214 & $<1.29$ & $\mathrm{pCi} / \mathrm{g}$ & 1.29 & & 3/31/08 & 8C17008 & AGG-RRL-001 \\
\hline $14835-02-0$ & Radon-219 & $<4.45$ & $\mathrm{pCi} / \mathrm{g}$ & 4.45 & & 3/31/08 & 8C17008 & AGG-RRL-001 \\
\hline 22481-48-7 & Radon-220 & $<435$ & $\mathrm{pCi} / \mathrm{g}$ & 435 & & 3/31/08 & 8C17008 & AGG-RRL-001 \\
\hline 28522-20-5 & Radon-221 & $<2.18$ & $\mathrm{pCi} / \mathrm{g}$ & 2.18 & & 3/31/08 & 8C17008 & AGG-RRL-001 \\
\hline 15756-98-6 & Francium-223 & $<14.9$ & $\mathrm{pCi} / \mathrm{g}$ & 14.9 & & 3/31/08 & 8C17008 & AGG-RRL-001 \\
\hline $15623-45-7$ & Radium-223 & $<3.24$ & $\mathrm{pCi} / \mathrm{g}$ & 3.24 & & 3/31/08 & 8C17008 & AGG-RRL-001 \\
\hline
\end{tabular}


GEA/Soil

\begin{tabular}{|c|c|c|c|c|c|c|c|c|}
\hline CAS \# & Analyte & Results & Units & MDA & UNC & Analyzed & Batch & Method \\
\hline HEIS No. & B1T2P5A & \multicolumn{2}{|c|}{ Lab ID: } & 0803014-21 & & & & \\
\hline $13233-32-4$ & Radium-224 & $<12.8$ & $\mathrm{pCi} / \mathrm{g}$ & 12.8 & & $3 / 31 / 08$ & 8C17008 & AGG-RRL-001 \\
\hline 13982-63-3 & Radium-226 & $<13.4$ & $\mathrm{pCi} / \mathrm{g}$ & 13.4 & & 3/31/08 & 8C17008 & AGG-RRL-001 \\
\hline $15623-47-9$ & Thorium-227 & $<4.55$ & $\mathrm{pCi} / \mathrm{g}$ & 4.55 & & 3/31/08 & 8C17008 & AGG-RRL-001 \\
\hline 14331-83-0 & Actinium-228 & $<1.98$ & $\mathrm{pCi} / \mathrm{g}$ & 1.98 & & 3/31/08 & 8C17008 & AGG-RRL-001 \\
\hline $14274-82-9$ & Thorium-228 & $<164$ & $\mathrm{pCi} / \mathrm{g}$ & 164 & & 3/31/08 & 8C17008 & AGG-RRL-001 \\
\hline $14269-63-7$ & Thorium-230 & $<169$ & $\mathrm{pCi} / \mathrm{g}$ & 169 & & 3/31/08 & 8C17008 & AGG-RRL-001 \\
\hline $14331-85-2$ & Protactinium-231 & $<21$ & $\mathrm{pCi} / \mathrm{g}$ & 21 & & 3/31/08 & 8C17008 & AGG-RRL-001 \\
\hline $14932-40-2$ & Thorium-231 & $<101$ & $\mathrm{pCi} / \mathrm{g}$ & 101 & & 3/31/08 & 8C17008 & AGG-RRL-001 \\
\hline $7440-29-1$ & Thorium-232 & $<419$ & $\mathrm{pCi} / \mathrm{g}$ & 419 & & 3/31/08 & 8C17008 & AGG-RRL-001 \\
\hline $13981-14-1$ & Protactinium-233 & $<1.28$ & $\mathrm{pCi} / \mathrm{g}$ & 1.28 & & 3/31/08 & 8C17008 & AGG-RRL-001 \\
\hline \multirow[t]{2}{*}{$15100-28-4$} & Protactinium-234 & $<1.83$ & $\mathrm{pCi} / \mathrm{g}$ & 1.83 & & 3/31/08 & 8C17008 & AGG-RRL-001 \\
\hline & Protactinium-234m & $<56.3$ & $\mathrm{pCi} / \mathrm{g}$ & 56.3 & & $3 / 31 / 08$ & 8C17008 & AGG-RRL-001 \\
\hline $15065-10-8$ & Thorium-234 & $<14.8$ & $\mathrm{pCi} / \mathrm{g}$ & 14.8 & & 3/31/08 & 8C17008 & AGG-RRL-001 \\
\hline 15117-96-1 & Uranium 235 & $<3.99$ & $\mathrm{pCi} / \mathrm{g}$ & 3.99 & & 3/31/08 & 8C17008 & AGG-RRL-001 \\
\hline 13994-20-2 & Neptunium-237 & $<3.84$ & $\mathrm{pCi} / \mathrm{g}$ & 3.84 & & 3/31/08 & 8C17008 & AGG-RRL-001 \\
\hline \multirow[t]{2}{*}{ 13981-16-3 } & Plutonium-238 & $<5750$ & $\mathrm{pCi} / \mathrm{g}$ & 5750 & & 3/31/08 & 8C17008 & AGG-RRL-001 \\
\hline & Uranium 238 & $<8.05$ & $\mathrm{pCi} / \mathrm{g}$ & 8.05 & & 3/31/08 & 8C17008 & AGG-RRL-001 \\
\hline $15117-48-3$ & Plutonium-239 & $<5930$ & $\mathrm{pCi} / \mathrm{g}$ & 5930 & & 3/31/08 & 8C17008 & AGG-RRL-001 \\
\hline 14119-33-6 & Plutonium-240 & $<5870$ & $\mathrm{pCi} / \mathrm{g}$ & 5870 & & 3/31/08 & 8C17008 & AGG-RRL-001 \\
\hline $14596-10-2$ & Americium-241 & $<2.16$ & $\mathrm{pCi} / \mathrm{g}$ & 2.16 & & $3 / 31 / 08$ & 8C17008 & AGG-RRL-001 \\
\hline $14993-75-0$ & Americium-243 & $<0.932$ & $\mathrm{pCi} / \mathrm{g}$ & 0.932 & & 3/31/08 & 8C17008 & AGG-RRL-001 \\
\hline $15757-87-6$ & Curium-243 & $<1.79$ & $\mathrm{pCi} / \mathrm{g}$ & 1.79 & & 3/31/08 & 8C17008 & AGG-RRL-001 \\
\hline 15621-76-8 & Curium-245 & $<1.47$ & $\mathrm{pCi} / \mathrm{g}$ & 1.47 & & $3 / 31 / 08$ & 8C17008 & AGG-RRL-001 \\
\hline HEIS No. & B1T2P6B & \multicolumn{2}{|c|}{ Lab ID: } & 0803014-24 & & & & \\
\hline 13966-32-0 & Sodium-22 & $<0.383$ & $\mathrm{pCi} / \mathrm{g}$ & 0.383 & & 3/31/08 & 8C17008 & AGG-RRL-001 \\
\hline 13966-00-2 & Potassium- 40 & 19.1 & $\mathrm{pCi} / \mathrm{g}$ & 2.89 & 1.24 & 3/31/08 & 8C17008 & AGG-RRL-001 \\
\hline $14392-02-0$ & Chromium-51 & $<2.81$ & $\mathrm{pCi} / \mathrm{g}$ & 2.81 & & 3/31/08 & 8C17008 & AGG-RRL-001 \\
\hline 13966-31-9 & Manganese-54 & $<0.318$ & $\mathrm{pCi} / \mathrm{g}$ & 0.318 & & 3/31/08 & 8C17008 & AGG-RRL-001 \\
\hline $13981-50-5$ & Cobalt-57 & $<0.288$ & $\mathrm{pCi} / \mathrm{g}$ & 0.288 & & 3/31/08 & 8C17008 & AGG-RRL-001 \\
\hline $14596-12-4$ & Iron-59 & $<0.501$ & $\mathrm{pCi} / \mathrm{g}$ & 0.501 & & 3/31/08 & 8C17008 & AGG-RRL-001 \\
\hline $10198-40-0$ & Cobalt-60 & $<0.261$ & $\mathrm{pCi} / \mathrm{g}$ & 0.261 & & 3/31/08 & 8C17008 & AGG-RRL-001 \\
\hline 13982-39-3 & Zinc-65 & $<0.767$ & $\mathrm{pCi} / \mathrm{g}$ & 0.767 & & $3 / 31 / 08$ & 8C17008 & AGG-RRL-001 \\
\hline $14265-71-5$ & Selenium-75 & $<0.455$ & $\mathrm{pCi} / \mathrm{g}$ & 0.455 & & 3/31/08 & 8C17008 & AGG-RRL-001 \\
\hline $17056-36-9$ & Rubidium-83 & $<0.558$ & $\mathrm{pCi} / \mathrm{g}$ & 0.558 & & 3/31/08 & 8C17008 & AGG-RRL-001 \\
\hline 13967-73-2 & Strontium-85 & $<0.381$ & $\mathrm{pCi} / \mathrm{g}$ & 0.381 & & $3 / 31 / 08$ & 8C17008 & AGG-RRL-001 \\
\hline $14932-53-7$ & Rubidium-86 & $<3.06$ & $\mathrm{pCi} / \mathrm{g}$ & 3.06 & & $3 / 31 / 08$ & 8C17008 & AGG-RRL-001 \\
\hline 13982-36-0 & Yttrium-88 & $<0.251$ & $\mathrm{pCi} / \mathrm{g}$ & 0.251 & & 3/31/08 & 8C17008 & AGG-RRL-001 \\
\hline $14681-63-1$ & Niobium-94 & $<0.258$ & $\mathrm{pCi} / \mathrm{g}$ & 0.258 & & 3/31/08 & 8C17008 & AGG-RRL-001 \\
\hline \multirow[t]{3}{*}{$13967-76-5$} & Niobium-95 & $<0.296$ & $\mathrm{pCi} / \mathrm{g}$ & 0.296 & & 3/31/08 & 8C17008 & AGG-RRL-001 \\
\hline & Niobium-95m & $<1.37$ & $\mathrm{pCi} / \mathrm{g}$ & 1.37 & & 3/31/08 & 8C17008 & AGG-RRL-001 \\
\hline & Technetium-95m & $<0.472$ & $\mathrm{pCi} / \mathrm{g}$ & 0.472 & & 3/31/08 & 8C17008 & AGG-RRL-001 \\
\hline \multirow[t]{2}{*}{$13967-71-0$} & Zirconium-95 & $<0.498$ & $\mathrm{pCi} / \mathrm{g}$ & 0.498 & & 3/31/08 & 8C17008 & AGG-RRL-001 \\
\hline & Technetium-99m & $<0.287$ & $\mathrm{pCi} / \mathrm{g}$ & 0.287 & & 3/31/08 & 8C17008 & AGG-RRL-001 \\
\hline 13968-53-1 & Ruthenium-103 & $<0.301$ & $\mathrm{pCi} / \mathrm{g}$ & 0.301 & & 3/31/08 & 8C17008 & AGG-RRL-001 \\
\hline $13967-48-1$ & Ruthenium-106 & $<2.6$ & $\mathrm{pCi} / \mathrm{g}$ & 2.6 & & 3/31/08 & 8C17008 & AGG-RRL-001 \\
\hline $14391-65-2$ & Silver-108m & $<0.306$ & $\mathrm{pCi} / \mathrm{g}$ & 0.306 & & 3/31/08 & 8C17008 & AGG-RRL-001 \\
\hline \multirow[t]{2}{*}{$14109-32-1$} & Cadmium-109 & $<7.94$ & $\mathrm{pCi} / \mathrm{g}$ & 7.94 & & 3/31/08 & 8C17008 & AGG-RRL-001 \\
\hline & Silver-110 & $<0.304$ & $\mathrm{pCi} / \mathrm{g}$ & 0.304 & & 3/31/08 & 8C17008 & AGG-RRL-001 \\
\hline
\end{tabular}


GEA/Soil

\begin{tabular}{|c|c|c|c|c|c|c|c|c|}
\hline CAS \# & Analyte & Results & Units & MDA & UNC & Analyzed & Batch & Method \\
\hline HEIS No. & B1T2P6B & \multicolumn{3}{|c|}{ Lab ID: $\quad$ 0803014-24 } & & & & \\
\hline 14391-76-5 & Silver-110m & $<0.304$ & $\mathrm{pCi} / \mathrm{g}$ & 0.304 & & 3/31/08 & 8C17008 & AGG-RRL-001 \\
\hline 13966-06-8 & Tin-113 & $<0.406$ & $\mathrm{pCi} / \mathrm{g}$ & 0.406 & & 3/31/08 & 8C17008 & AGG-RRL-001 \\
\hline $14683-10-4$ & Antimony-124 & $<0.293$ & $\mathrm{pCi} / \mathrm{g}$ & 0.293 & & 3/31/08 & 8C17008 & AGG-RRL-001 \\
\hline 14234-35-6 & Antimony-125 & $<0.942$ & $\mathrm{pCi} / \mathrm{g}$ & 0.942 & & 3/31/08 & 8C17008 & AGG-RRL-001 \\
\hline 15756-32-8 & Antimony-126 & $<0.276$ & $\mathrm{pCi} / \mathrm{g}$ & 0.276 & & 3/31/08 & 8C17008 & AGG-RRL-001 \\
\hline 15832-50-5 & Tin-126 & $<0.626$ & $\mathrm{pCi} / \mathrm{g}$ & 0.626 & & 3/31/08 & 8C17008 & AGG-RRL-001 \\
\hline 10043-66-0 & Iodine-131 & $<0.324$ & $\mathrm{pCi} / \mathrm{g}$ & 0.324 & & 3/31/08 & 8C17008 & AGG-RRL-001 \\
\hline 13981-41-4 & Barium-133 & $<0.548$ & $\mathrm{pCi} / \mathrm{g}$ & 0.548 & & 3/31/08 & 8C17008 & AGG-RRL-001 \\
\hline 13967-70-9 & Cesium-134 & $<0.329$ & $\mathrm{pCi} / \mathrm{g}$ & 0.329 & & 3/31/08 & 8C17008 & AGG-RRL-001 \\
\hline 10045-97-3 & Cesium-137 & $<0.324$ & $\mathrm{pCi} / \mathrm{g}$ & 0.324 & & $3 / 31 / 08$ & 8C17008 & AGG-RRL-001 \\
\hline 13982-30-4 & Cerium-139 & $<0.339$ & $\mathrm{pCi} / \mathrm{g}$ & 0.339 & & 3/31/08 & 8C17008 & AGG-RRL-001 \\
\hline 14762-78-8 & Cerium-144 & $<2.36$ & $\mathrm{pCi} / \mathrm{g}$ & 2.36 & & $3 / 31 / 08$ & 8C17008 & AGG-RRL-001 \\
\hline 14683-23-9 & Europium-152 & $<1.12$ & $\mathrm{pCi} / \mathrm{g}$ & 1.12 & & 3/31/08 & 8C17008 & AGG-RRL-001 \\
\hline $14276-65-4$ & Gadolinium-153 & $<0.858$ & $\mathrm{pCi} / \mathrm{g}$ & 0.858 & & 3/31/08 & 8C17008 & AGG-RRL-001 \\
\hline 15585-10-1 & Europium-154 & $<0.612$ & $\mathrm{pCi} / \mathrm{g}$ & 0.612 & & 3/31/08 & 8C17008 & AGG-RRL-001 \\
\hline 14391-16-3 & Europium-155 & $<0.874$ & $\mathrm{pCi} / \mathrm{g}$ & 0.874 & & 3/31/08 & 8C17008 & AGG-RRL-001 \\
\hline 13982-78-0 & Mercury-203 & $<0.372$ & $\mathrm{pCi} / \mathrm{g}$ & 0.372 & & 3/31/08 & 8C17008 & AGG-RRL-001 \\
\hline 14913-50-9 & Thallium-208 & 0.357 & $\mathrm{pCi} / \mathrm{g}$ & 0.311 & 0.0744 & 3/31/08 & 8C17008 & AGG-RRL-001 \\
\hline 14331-79-4 & Bismuth-210 & $<0.55$ & $\mathrm{pCi} / \mathrm{g}$ & 0.55 & & 3/31/08 & 8C17008 & AGG-RRL-001 \\
\hline \multirow[t]{3}{*}{ 14255-04-0 } & Lead-210 & $<105$ & $\mathrm{pCi} / \mathrm{g}$ & 105 & 23.3 & 3/31/08 & 8C17008 & AGG-RRL-001 \\
\hline & Bismuth-211 & $<6.61$ & $\mathrm{pCi} / \mathrm{g}$ & 6.61 & & 3/31/08 & 8C17008 & AGG-RRL-001 \\
\hline & Lead-211 & $<9.04$ & $\mathrm{pCi} / \mathrm{g}$ & 9.04 & & 3/31/08 & 8C17008 & AGG-RRL-001 \\
\hline 15092-94-1 & Lead-212 & 1.06 & $\mathrm{pCi} / \mathrm{g}$ & 0.698 & 0.166 & 3/31/08 & 8C17008 & AGG-RRL-001 \\
\hline 14733-03-0 & Bismuth-214 & $<0.765$ & $\mathrm{pCi} / \mathrm{g}$ & 0.765 & & 3/31/08 & 8C17008 & AGG-RRL-001 \\
\hline $15067-28-4$ & Lead-214 & $<0.874$ & $\mathrm{pCi} / \mathrm{g}$ & 0.874 & & 3/31/08 & 8C17008 & AGG-RRL-001 \\
\hline 14835-02-0 & Radon-219 & $<2.99$ & $\mathrm{pCi} / \mathrm{g}$ & 2.99 & & 3/31/08 & 8C17008 & AGG-RRL-001 \\
\hline 22481-48-7 & Radon-220 & $<261$ & $\mathrm{pCi} / \mathrm{g}$ & 261 & & 3/31/08 & 8C17008 & AGG-RRL-001 \\
\hline 28522-20-5 & Radon-221 & $<1.35$ & $\mathrm{pCi} / \mathrm{g}$ & 1.35 & & 3/31/08 & 8C17008 & AGG-RRL-001 \\
\hline 15756-98-6 & Francium-223 & $<10$ & $\mathrm{pCi} / \mathrm{g}$ & 10 & & 3/31/08 & 8C17008 & AGG-RRL-001 \\
\hline $15623-45-7$ & Radium-223 & $<2.1$ & $\mathrm{pCi} / \mathrm{g}$ & 2.1 & & 3/31/08 & 8C17008 & AGG-RRL-001 \\
\hline 13233-32-4 & Radium-224 & $<8.88$ & $\mathrm{pCi} / \mathrm{g}$ & 8.88 & & 3/31/08 & 8C17008 & AGG-RRL-001 \\
\hline 13982-63-3 & Radium-226 & $<8.35$ & $\mathrm{pCi} / \mathrm{g}$ & 8.35 & & 3/31/08 & 8C17008 & AGG-RRL-001 \\
\hline $15623-47-9$ & Thorium-227 & $<3.05$ & $\mathrm{pCi} / \mathrm{g}$ & 3.05 & & 3/31/08 & 8C17008 & AGG-RRL-001 \\
\hline 14331-83-0 & Actinium-228 & $<1.29$ & $\mathrm{pCi} / \mathrm{g}$ & 1.29 & & 3/31/08 & 8C17008 & AGG-RRL-001 \\
\hline 14274-82-9 & Thorium-228 & $<104$ & $\mathrm{pCi} / \mathrm{g}$ & 104 & & 3/31/08 & 8C17008 & AGG-RRL-001 \\
\hline 14269-63-7 & Thorium-230 & $<112$ & $\mathrm{pCi} / \mathrm{g}$ & 112 & & 3/31/08 & 8C17008 & AGG-RRL-001 \\
\hline 14331-85-2 & Protactinium-231 & $<11.9$ & $\mathrm{pCi} / \mathrm{g}$ & 11.9 & & 3/31/08 & 8C17008 & AGG-RRL-001 \\
\hline $14932-40-2$ & Thorium-231 & $<59.7$ & $\mathrm{pCi} / \mathrm{g}$ & 59.7 & & $3 / 31 / 08$ & 8C17008 & AGG-RRL-001 \\
\hline 7440-29-1 & Thorium-232 & $<346$ & $\mathrm{pCi} / \mathrm{g}$ & 346 & & 3/31/08 & 8C17008 & AGG-RRL-001 \\
\hline 13981-14-1 & Protactinium-233 & $<0.767$ & $\mathrm{pCi} / \mathrm{g}$ & 0.767 & & 3/31/08 & 8C17008 & AGG-RRL-001 \\
\hline \multirow[t]{2}{*}{$15100-28-4$} & Protactinium-234 & $<1.09$ & $\mathrm{pCi} / \mathrm{g}$ & 1.09 & & 3/31/08 & 8C17008 & AGG-RRL-001 \\
\hline & Protactinium-234m & $<36.8$ & $\mathrm{pCi} / \mathrm{g}$ & 36.8 & & 3/31/08 & 8C17008 & AGG-RRL-001 \\
\hline 15065-10-8 & Thorium-234 & $<10.1$ & $\mathrm{pCi} / \mathrm{g}$ & 10.1 & & 3/31/08 & 8C17008 & AGG-RRL-001 \\
\hline 15117-96-1 & Uranium 235 & $<2.48$ & $\mathrm{pCi} / \mathrm{g}$ & 2.48 & & $3 / 31 / 08$ & 8C17008 & AGG-RRL-001 \\
\hline 13994-20-2 & Neptunium-237 & $<2.27$ & $\mathrm{pCi} / \mathrm{g}$ & 2.27 & & 3/31/08 & 8C17008 & AGG-RRL-001 \\
\hline \multirow[t]{2}{*}{ 13981-16-3 } & Plutonium-238 & $<3440$ & $\mathrm{pCi} / \mathrm{g}$ & 3440 & & 3/31/08 & 8C17008 & AGG-RRL-001 \\
\hline & Uranium 238 & $<5.24$ & $\mathrm{pCi} / \mathrm{g}$ & 5.24 & & 3/31/08 & 8C17008 & AGG-RRL-001 \\
\hline 15117-48-3 & Plutonium-239 & $<4090$ & $\mathrm{pCi} / \mathrm{g}$ & 4090 & & 3/31/08 & 8C17008 & AGG-RRL-001 \\
\hline 14119-33-6 & Plutonium-240 & $<3470$ & $\mathrm{pCi} / \mathrm{g}$ & 3470 & & 3/31/08 & 8C17008 & AGG-RRL-001 \\
\hline
\end{tabular}


GEA/Soil

\begin{tabular}{|c|c|c|c|c|c|c|c|c|}
\hline CAS \# & Analyte & Results & Units & MDA & UNC & Analyzed & Batch & Method \\
\hline HEIS No. & B1T2P6B & \multicolumn{2}{|c|}{ Lab ID: } & 0803014-24 & & & & \\
\hline 14596-10-2 & Americium-241 & $<1.73$ & $\mathrm{pCi} / \mathrm{g}$ & 1.73 & & 3/31/08 & 8C17008 & AGG-RRL-001 \\
\hline $14993-75-0$ & Americium-243 & $<0.628$ & $\mathrm{pCi} / \mathrm{g}$ & 0.628 & & $3 / 31 / 08$ & 8C17008 & AGG-RRL-001 \\
\hline 15757-87-6 & Curium-243 & $<1.06$ & $\mathrm{pCi} / \mathrm{g}$ & 1.06 & & 3/31/08 & 8C17008 & AGG-RRL-001 \\
\hline 15621-76-8 & Curium-245 & $<0.869$ & $\mathrm{pCi} / \mathrm{g}$ & 0.869 & & 3/31/08 & 8C17008 & AGG-RRL-001 \\
\hline HEIS No. & B1T2P6A & \multicolumn{2}{|c|}{ Lab ID: } & 0803014-25 & & & & \\
\hline 13966-32-0 & Sodium-22 & $<0.412$ & $\mathrm{pCi} / \mathrm{g}$ & 0.412 & & 3/31/08 & 8C17008 & AGG-RRL-001 \\
\hline 13966-00-2 & Potassium-40 & 20.8 & $\mathrm{pCi} / \mathrm{g}$ & 2.88 & 1.27 & 3/31/08 & 8C17008 & AGG-RRL-001 \\
\hline $14392-02-0$ & Chromium-51 & $<3.39$ & $\mathrm{pCi} / \mathrm{g}$ & 3.39 & & 3/31/08 & 8C17008 & AGG-RRL-001 \\
\hline 13966-31-9 & Manganese-54 & $<0.376$ & $\mathrm{pCi} / \mathrm{g}$ & 0.376 & & 3/31/08 & 8C17008 & AGG-RRL-001 \\
\hline $13981-50-5$ & Cobalt-57 & $<0.353$ & $\mathrm{pCi} / \mathrm{g}$ & 0.353 & & 3/31/08 & 8C17008 & AGG-RRL-001 \\
\hline $14596-12-4$ & Iron-59 & $<0.591$ & $\mathrm{pCi} / \mathrm{g}$ & 0.591 & & 3/31/08 & 8C17008 & AGG-RRL-001 \\
\hline $10198-40-0$ & Cobalt-60 & $<0.356$ & $\mathrm{pCi} / \mathrm{g}$ & 0.356 & & 3/31/08 & 8C17008 & AGG-RRL-001 \\
\hline 13982-39-3 & Zinc-65 & $<0.77$ & $\mathrm{pCi} / \mathrm{g}$ & 0.77 & & 3/31/08 & 8C17008 & AGG-RRL-001 \\
\hline $14265-71-5$ & Selenium-75 & $<0.554$ & $\mathrm{pCi} / \mathrm{g}$ & 0.554 & & $3 / 31 / 08$ & 8C17008 & AGG-RRL-001 \\
\hline 17056-36-9 & Rubidium-83 & $<0.862$ & $\mathrm{pCi} / \mathrm{g}$ & 0.862 & & 3/31/08 & 8C17008 & AGG-RRL-001 \\
\hline 13967-73-2 & Strontium-85 & $<0.402$ & $\mathrm{pCi} / \mathrm{g}$ & 0.402 & & 3/31/08 & 8C17008 & AGG-RRL-001 \\
\hline 14932-53-7 & Rubidium-86 & $<4.14$ & $\mathrm{pCi} / \mathrm{g}$ & 4.14 & & 3/31/08 & 8C17008 & AGG-RRL-001 \\
\hline 13982-36-0 & Yttrium-88 & $<0.268$ & $\mathrm{pCi} / \mathrm{g}$ & 0.268 & & 3/31/08 & 8C17008 & AGG-RRL-001 \\
\hline 14681-63-1 & Niobium-94 & $<0.332$ & $\mathrm{pCi} / \mathrm{g}$ & 0.332 & & 3/31/08 & 8C17008 & AGG-RRL-001 \\
\hline \multirow{3}{*}{$13967-76-5$} & Niobium-95 & $<0.338$ & $\mathrm{pCi} / \mathrm{g}$ & 0.338 & & 3/31/08 & 8C17008 & AGG-RRL-001 \\
\hline & Niobium-95m & $<1.55$ & $\mathrm{pCi} / \mathrm{g}$ & 1.55 & & 3/31/08 & 8C17008 & AGG-RRL-001 \\
\hline & Technetium-95m & $<0.531$ & $\mathrm{pCi} / \mathrm{g}$ & 0.531 & & 3/31/08 & 8C17008 & AGG-RRL-001 \\
\hline \multirow[t]{2}{*}{$13967-71-0$} & Zirconium-95 & $<0.61$ & $\mathrm{pCi} / \mathrm{g}$ & 0.61 & & 3/31/08 & 8C17008 & AGG-RRL-001 \\
\hline & Technetium-99m & $<0.353$ & $\mathrm{pCi} / \mathrm{g}$ & 0.353 & & 3/31/08 & 8C17008 & AGG-RRL-001 \\
\hline 13968-53-1 & Ruthenium-103 & $<0.402$ & $\mathrm{pCi} / \mathrm{g}$ & 0.402 & & 3/31/08 & 8C17008 & AGG-RRL-001 \\
\hline 13967-48-1 & Ruthenium-106 & $<3.94$ & $\mathrm{pCi} / \mathrm{g}$ & 3.94 & & 3/31/08 & 8C17008 & AGG-RRL-001 \\
\hline 14391-65-2 & Silver-108m & $<0.334$ & $\mathrm{pCi} / \mathrm{g}$ & 0.334 & & $3 / 31 / 08$ & 8C17008 & AGG-RRL-001 \\
\hline \multirow[t]{2}{*}{$14109-32-1$} & Cadmium-109 & $<9.9$ & $\mathrm{pCi} / \mathrm{g}$ & 9.9 & & 3/31/08 & 8C17008 & AGG-RRL-001 \\
\hline & Silver-110 & $<0.343$ & $\mathrm{pCi} / \mathrm{g}$ & 0.343 & & 3/31/08 & 8C17008 & AGG-RRL-001 \\
\hline 14391-76-5 & Silver-110m & $<0.344$ & $\mathrm{pCi} / \mathrm{g}$ & 0.344 & & 3/31/08 & 8C17008 & AGG-RRL-001 \\
\hline 13966-06-8 & Tin-113 & $<0.491$ & $\mathrm{pCi} / \mathrm{g}$ & 0.491 & & $3 / 31 / 08$ & 8C17008 & AGG-RRL-001 \\
\hline $14683-10-4$ & Antimony-124 & $<0.405$ & $\mathrm{pCi} / \mathrm{g}$ & 0.405 & & $3 / 31 / 08$ & 8C17008 & AGG-RRL-001 \\
\hline $14234-35-6$ & Antimony-125 & $<1.02$ & $\mathrm{pCi} / \mathrm{g}$ & 1.02 & & 3/31/08 & 8C17008 & AGG-RRL-001 \\
\hline 15756-32-8 & Antimony-126 & $<0.324$ & $\mathrm{pCi} / \mathrm{g}$ & 0.324 & & 3/31/08 & 8C17008 & AGG-RRL-001 \\
\hline $15832-50-5$ & Tin-126 & $<0.806$ & $\mathrm{pCi} / \mathrm{g}$ & 0.806 & & 3/31/08 & 8C17008 & AGG-RRL-001 \\
\hline 10043-66-0 & Iodine-131 & $<0.438$ & $\mathrm{pCi} / \mathrm{g}$ & 0.438 & & 3/31/08 & 8C17008 & AGG-RRL-001 \\
\hline 13981-41-4 & Barium-133 & $<0.585$ & $\mathrm{pCi} / \mathrm{g}$ & 0.585 & & 3/31/08 & 8C17008 & AGG-RRL-001 \\
\hline 13967-70-9 & Cesium-134 & $<0.411$ & $\mathrm{pCi} / \mathrm{g}$ & 0.411 & & 3/31/08 & 8C17008 & AGG-RRL-001 \\
\hline $10045-97-3$ & Cesium-137 & $<0.377$ & $\mathrm{pCi} / \mathrm{g}$ & 0.377 & & 3/31/08 & 8C17008 & AGG-RRL-001 \\
\hline 13982-30-4 & Cerium-139 & $<0.406$ & $\mathrm{pCi} / \mathrm{g}$ & 0.406 & & 3/31/08 & 8C17008 & AGG-RRL-001 \\
\hline $14762-78-8$ & Cerium-144 & $<2.9$ & $\mathrm{pCi} / \mathrm{g}$ & 2.9 & & 3/31/08 & 8C17008 & AGG-RRL-001 \\
\hline 14683-23-9 & Europium-152 & $<1.39$ & $\mathrm{pCi} / \mathrm{g}$ & 1.39 & & $3 / 31 / 08$ & 8C17008 & AGG-RRL-001 \\
\hline $14276-65-4$ & Gadolinium-153 & $<1.08$ & $\mathrm{pCi} / \mathrm{g}$ & 1.08 & & 3/31/08 & 8C17008 & AGG-RRL-001 \\
\hline 15585-10-1 & Europium-154 & $<0.756$ & $\mathrm{pCi} / \mathrm{g}$ & 0.756 & & 3/31/08 & 8C17008 & AGG-RRL-001 \\
\hline 14391-16-3 & Europium-155 & $<1.15$ & $\mathrm{pCi} / \mathrm{g}$ & 1.15 & & 3/31/08 & 8C17008 & AGG-RRL-001 \\
\hline 13982-78-0 & Mercury-203 & $<0.428$ & $\mathrm{pCi} / \mathrm{g}$ & 0.428 & & 3/31/08 & 8C17008 & AGG-RRL-001 \\
\hline 14913-50-9 & Thallium-208 & $<0.449$ & $\mathrm{pCi} / \mathrm{g}$ & 0.449 & & 3/31/08 & 8C17008 & AGG-RRL-001 \\
\hline 14331-79-4 & Bismuth-210 & $<0.657$ & $\mathrm{pCi} / \mathrm{g}$ & 0.657 & & 3/31/08 & 8C17008 & AGG-RRL-001 \\
\hline
\end{tabular}


GEA/Soil

\begin{tabular}{|c|c|c|c|c|c|c|c|c|}
\hline CAS \# & Analyte & Results & Units & MDA & UNC & Analyzed & Batch & Method \\
\hline HEIS No. & B1T2P6A & \multicolumn{2}{|c|}{ Lab ID: } & 0803014-25 & & & & \\
\hline \multirow[t]{3}{*}{$14255-04-0$} & Lead-210 & $<46.9$ & $\mathrm{pCi} / \mathrm{g}$ & 46.9 & & $3 / 31 / 08$ & 8C17008 & AGG-RRL-001 \\
\hline & Bismuth-211 & $<7.81$ & $\mathrm{pCi} / \mathrm{g}$ & 7.81 & & 3/31/08 & 8C17008 & AGG-RRL-001 \\
\hline & Lead-211 & $<10.7$ & $\mathrm{pCi} / \mathrm{g}$ & 10.7 & & 3/31/08 & 8C17008 & AGG-RRL-001 \\
\hline $15092-94-1$ & Lead-212 & $<0.903$ & $\mathrm{pCi} / \mathrm{g}$ & 0.903 & & 3/31/08 & 8C17008 & AGG-RRL-001 \\
\hline $14733-03-0$ & Bismuth-214 & $<0.87$ & $\mathrm{pCi} / \mathrm{g}$ & 0.87 & & 3/31/08 & 8C17008 & AGG-RRL-001 \\
\hline $15067-28-4$ & Lead-214 & $<0.947$ & $\mathrm{pCi} / \mathrm{g}$ & 0.947 & & 3/31/08 & 8C17008 & AGG-RRL-001 \\
\hline $14835-02-0$ & Radon-219 & $<3.31$ & $\mathrm{pCi} / \mathrm{g}$ & 3.31 & & 3/31/08 & 8C17008 & AGG-RRL-001 \\
\hline 22481-48-7 & Radon-220 & $<308$ & $\mathrm{pCi} / \mathrm{g}$ & 308 & & $3 / 31 / 08$ & 8C17008 & AGG-RRL-001 \\
\hline 28522-20-5 & Radon-221 & $<1.63$ & $\mathrm{pCi} / \mathrm{g}$ & 1.63 & & 3/31/08 & 8C17008 & AGG-RRL-001 \\
\hline 15756-98-6 & Francium-223 & $<11.4$ & $\mathrm{pCi} / \mathrm{g}$ & 11.4 & & 3/31/08 & 8C17008 & AGG-RRL-001 \\
\hline $15623-45-7$ & Radium-223 & $<2.44$ & $\mathrm{pCi} / \mathrm{g}$ & 2.44 & & 3/31/08 & 8C17008 & AGG-RRL-001 \\
\hline 13233-32-4 & Radium-224 & $<9.75$ & $\mathrm{pCi} / \mathrm{g}$ & 9.75 & & 3/31/08 & 8C17008 & AGG-RRL-001 \\
\hline 13982-63-3 & Radium-226 & $<10$ & $\mathrm{pCi} / \mathrm{g}$ & 10 & & 3/31/08 & 8C17008 & AGG-RRL-001 \\
\hline $15623-47-9$ & Thorium-227 & $<3.47$ & $\mathrm{pCi} / \mathrm{g}$ & 3.47 & & 3/31/08 & 8C17008 & AGG-RRL-001 \\
\hline $14331-83-0$ & Actinium-228 & $<1.48$ & $\mathrm{pCi} / \mathrm{g}$ & 1.48 & & 3/31/08 & 8C17008 & AGG-RRL-001 \\
\hline $14274-82-9$ & Thorium-228 & $<125$ & $\mathrm{pCi} / \mathrm{g}$ & 125 & & 3/31/08 & 8C17008 & AGG-RRL-001 \\
\hline 14269-63-7 & Thorium-230 & $<132$ & $\mathrm{pCi} / \mathrm{g}$ & 132 & & 3/31/08 & 8C17008 & AGG-RRL-001 \\
\hline $14331-85-2$ & Protactinium-231 & $<15.4$ & $\mathrm{pCi} / \mathrm{g}$ & 15.4 & & 3/31/08 & 8C17008 & AGG-RRL-001 \\
\hline $14932-40-2$ & Thorium-231 & $<76.8$ & $\mathrm{pCi} / \mathrm{g}$ & 76.8 & & $3 / 31 / 08$ & 8C17008 & AGG-RRL-001 \\
\hline $7440-29-1$ & Thorium-232 & $<328$ & $\mathrm{pCi} / \mathrm{g}$ & 328 & & 3/31/08 & 8C17008 & AGG-RRL-001 \\
\hline 13981-14-1 & Protactinium-233 & $<0.93$ & $\mathrm{pCi} / \mathrm{g}$ & 0.93 & & 3/31/08 & 8C17008 & AGG-RRL-001 \\
\hline \multirow[t]{2}{*}{$15100-28-4$} & Protactinium-234 & $<1.41$ & $\mathrm{pCi} / \mathrm{g}$ & 1.41 & & 3/31/08 & 8C17008 & AGG-RRL-001 \\
\hline & Protactinium-234m & $<42.4$ & $\mathrm{pCi} / \mathrm{g}$ & 42.4 & & $3 / 31 / 08$ & 8C17008 & AGG-RRL-001 \\
\hline $15065-10-8$ & Thorium-234 & $<11.5$ & $\mathrm{pCi} / \mathrm{g}$ & 11.5 & & 3/31/08 & 8C17008 & AGG-RRL-001 \\
\hline $15117-96-1$ & Uranium 235 & $<2.98$ & $\mathrm{pCi} / \mathrm{g}$ & 2.98 & & 3/31/08 & 8C17008 & AGG-RRL-001 \\
\hline 13994-20-2 & Neptunium-237 & $<2.99$ & $\mathrm{pCi} / \mathrm{g}$ & 2.99 & & 3/31/08 & 8C17008 & AGG-RRL-001 \\
\hline \multirow[t]{2}{*}{ 13981-16-3 } & Plutonium-238 & $<4460$ & $\mathrm{pCi} / \mathrm{g}$ & 4460 & & 3/31/08 & 8C17008 & AGG-RRL-001 \\
\hline & Uranium 238 & $<6.3$ & $\mathrm{pCi} / \mathrm{g}$ & 6.3 & & 3/31/08 & 8C17008 & AGG-RRL-001 \\
\hline $15117-48-3$ & Plutonium-239 & $<4510$ & $\mathrm{pCi} / \mathrm{g}$ & 4510 & & 3/31/08 & 8C17008 & AGG-RRL-001 \\
\hline 14119-33-6 & Plutonium-240 & $<4430$ & $\mathrm{pCi} / \mathrm{g}$ & 4430 & & 3/31/08 & 8C17008 & AGG-RRL-001 \\
\hline $14596-10-2$ & Americium-241 & $<1.67$ & $\mathrm{pCi} / \mathrm{g}$ & 1.67 & & 3/31/08 & 8C17008 & AGG-RRL-001 \\
\hline $14993-75-0$ & Americium-243 & $<0.722$ & $\mathrm{pCi} / \mathrm{g}$ & 0.722 & & 3/31/08 & 8C17008 & AGG-RRL-001 \\
\hline $15757-87-6$ & Curium-243 & $<1.35$ & $\mathrm{pCi} / \mathrm{g}$ & 1.35 & & 3/31/08 & 8C17008 & AGG-RRL-001 \\
\hline 15621-76-8 & Curium-245 & $<1.11$ & $\mathrm{pCi} / \mathrm{g}$ & 1.11 & & $3 / 31 / 08$ & 8C17008 & AGG-RRL-001 \\
\hline HEIS No. & B1T2P7B & \multicolumn{2}{|c|}{ Lab ID: } & 0803015-02 & & & & \\
\hline 13966-32-0 & Sodium-22 & $<0.338$ & $\mathrm{pCi} / \mathrm{g}$ & 0.338 & & $3 / 31 / 08$ & 8C17008 & AGG-RRL-001 \\
\hline $13966-00-2$ & Potassium-40 & 15.0 & $\mathrm{pCi} / \mathrm{g}$ & 2.43 & 1.31 & 3/31/08 & 8C17008 & AGG-RRL-001 \\
\hline $14392-02-0$ & Chromium-51 & $<2.65$ & $\mathrm{pCi} / \mathrm{g}$ & 2.65 & & 3/31/08 & 8C17008 & AGG-RRL-001 \\
\hline 13966-31-9 & Manganese-54 & $<0.29$ & $\mathrm{pCi} / \mathrm{g}$ & 0.29 & & $3 / 31 / 08$ & 8C17008 & AGG-RRL-001 \\
\hline $13981-50-5$ & Cobalt-57 & $<0.267$ & $\mathrm{pCi} / \mathrm{g}$ & 0.267 & & $3 / 31 / 08$ & 8C17008 & AGG-RRL-001 \\
\hline $14596-12-4$ & Iron-59 & $<0.523$ & $\mathrm{pCi} / \mathrm{g}$ & 0.523 & & 3/31/08 & 8C17008 & AGG-RRL-001 \\
\hline $10198-40-0$ & Cobalt-60 & $<0.27$ & $\mathrm{pCi} / \mathrm{g}$ & 0.27 & & 3/31/08 & 8C17008 & AGG-RRL-001 \\
\hline 13982-39-3 & Zinc-65 & $<0.671$ & $\mathrm{pCi} / \mathrm{g}$ & 0.671 & & 3/31/08 & 8C17008 & AGG-RRL-001 \\
\hline $14265-71-5$ & Selenium-75 & $<0.455$ & $\mathrm{pCi} / \mathrm{g}$ & 0.455 & & 3/31/08 & 8C17008 & AGG-RRL-001 \\
\hline $17056-36-9$ & Rubidium-83 & $<0.559$ & $\mathrm{pCi} / \mathrm{g}$ & 0.559 & & 3/31/08 & 8C17008 & AGG-RRL-001 \\
\hline 13967-73-2 & Strontium-85 & $<0.346$ & $\mathrm{pCi} / \mathrm{g}$ & 0.346 & & 3/31/08 & 8C17008 & AGG-RRL-001 \\
\hline 14932-53-7 & Rubidium-86 & $<3.14$ & $\mathrm{pCi} / \mathrm{g}$ & 3.14 & & 3/31/08 & 8C17008 & AGG-RRL-001 \\
\hline 13982-36-0 & Yttrium-88 & $<0.208$ & $\mathrm{pCi} / \mathrm{g}$ & 0.208 & & 3/31/08 & 8C17008 & AGG-RRL-001 \\
\hline
\end{tabular}


GEA/Soil

\begin{tabular}{|c|c|c|c|c|c|c|c|c|}
\hline CAS \# & Analyte & Results & Units & MDA & UNC & Analyzed & Batch & Method \\
\hline HEIS No. & B1T2P7B & \multicolumn{2}{|c|}{ Lab ID: } & 0803015-02 & & & & \\
\hline 14681-63-1 & Niobium-94 & $<0.28$ & $\mathrm{pCi} / \mathrm{g}$ & 0.28 & & 3/31/08 & 8C17008 & AGG-RRL-001 \\
\hline \multirow[t]{3}{*}{ 13967-76-5 } & Niobium-95 & $<0.277$ & $\mathrm{pCi} / \mathrm{g}$ & 0.277 & & 3/31/08 & 8C17008 & AGG-RRL-001 \\
\hline & Niobium-95m & $<1.34$ & $\mathrm{pCi} / \mathrm{g}$ & 1.34 & & 3/31/08 & 8C17008 & AGG-RRL-001 \\
\hline & Technetium-95m & $<0.434$ & $\mathrm{pCi} / \mathrm{g}$ & 0.434 & & 3/31/08 & 8C17008 & AGG-RRL-001 \\
\hline \multirow[t]{2}{*}{ 13967-71-0 } & Zirconium-95 & $<0.46$ & $\mathrm{pCi} / \mathrm{g}$ & 0.46 & & 3/31/08 & 8C17008 & AGG-RRL-001 \\
\hline & Technetium-99m & $<0.276$ & $\mathrm{pCi} / \mathrm{g}$ & 0.276 & & 3/31/08 & 8C17008 & AGG-RRL-001 \\
\hline 13968-53-1 & Ruthenium-103 & $<0.288$ & $\mathrm{pCi} / \mathrm{g}$ & 0.288 & & 3/31/08 & 8C17008 & AGG-RRL-001 \\
\hline 13967-48-1 & Ruthenium-106 & $<2.72$ & $\mathrm{pCi} / \mathrm{g}$ & 2.72 & & 3/31/08 & 8C17008 & AGG-RRL-001 \\
\hline 14391-65-2 & Silver-108m & $<0.286$ & $\mathrm{pCi} / \mathrm{g}$ & 0.286 & & 3/31/08 & 8C17008 & AGG-RRL-001 \\
\hline \multirow[t]{2}{*}{ 14109-32-1 } & Cadmium-109 & $<7.52$ & $\mathrm{pCi} / \mathrm{g}$ & 7.52 & & 3/31/08 & 8C17008 & AGG-RRL-001 \\
\hline & Silver-110 & $<0.303$ & $\mathrm{pCi} / \mathrm{g}$ & 0.303 & & 3/31/08 & 8C17008 & AGG-RRL-001 \\
\hline 14391-76-5 & Silver-110m & $<0.303$ & $\mathrm{pCi} / \mathrm{g}$ & 0.303 & & 3/31/08 & 8C17008 & AGG-RRL-001 \\
\hline 13966-06-8 & Tin-113 & $<0.407$ & $\mathrm{pCi} / \mathrm{g}$ & 0.407 & & 3/31/08 & 8C17008 & AGG-RRL-001 \\
\hline 14683-10-4 & Antimony-124 & $<0.281$ & $\mathrm{pCi} / \mathrm{g}$ & 0.281 & & 3/31/08 & 8C17008 & AGG-RRL-001 \\
\hline $14234-35-6$ & Antimony-125 & $<0.887$ & $\mathrm{pCi} / \mathrm{g}$ & 0.887 & & 3/31/08 & 8C17008 & AGG-RRL-001 \\
\hline 15756-32-8 & Antimony-126 & $<0.279$ & $\mathrm{pCi} / \mathrm{g}$ & 0.279 & & 3/31/08 & 8C17008 & AGG-RRL-001 \\
\hline $15832-50-5$ & Tin-126 & $<0.595$ & $\mathrm{pCi} / \mathrm{g}$ & 0.595 & & 3/31/08 & 8C17008 & AGG-RRL-001 \\
\hline 10043-66-0 & Iodine-131 & $<0.315$ & $\mathrm{pCi} / \mathrm{g}$ & 0.315 & & 3/31/08 & 8C17008 & AGG-RRL-001 \\
\hline 13981-41-4 & Barium-133 & $<0.527$ & $\mathrm{pCi} / \mathrm{g}$ & 0.527 & & 3/31/08 & 8C17008 & AGG-RRL-001 \\
\hline 13967-70-9 & Cesium-134 & $<0.325$ & $\mathrm{pCi} / \mathrm{g}$ & 0.325 & & 3/31/08 & 8C17008 & AGG-RRL-001 \\
\hline $10045-97-3$ & Cesium-137 & $<0.333$ & $\mathrm{pCi} / \mathrm{g}$ & 0.333 & & 3/31/08 & 8C17008 & AGG-RRL-001 \\
\hline 13982-30-4 & Cerium-139 & $<0.321$ & $\mathrm{pCi} / \mathrm{g}$ & 0.321 & & 3/31/08 & 8C17008 & AGG-RRL-001 \\
\hline 14762-78-8 & Cerium-144 & $<2.24$ & $\mathrm{pCi} / \mathrm{g}$ & 2.24 & & 3/31/08 & 8C17008 & AGG-RRL-001 \\
\hline 14683-23-9 & Europium-152 & $<1.06$ & $\mathrm{pCi} / \mathrm{g}$ & 1.06 & & 3/31/08 & 8C17008 & AGG-RRL-001 \\
\hline $14276-65-4$ & Gadolinium-153 & $<0.798$ & $\mathrm{pCi} / \mathrm{g}$ & 0.798 & & 3/31/08 & 8C17008 & AGG-RRL-001 \\
\hline 15585-10-1 & Europium-154 & $<0.57$ & $\mathrm{pCi} / \mathrm{g}$ & 0.57 & & 3/31/08 & 8C17008 & AGG-RRL-001 \\
\hline 14391-16-3 & Europium-155 & $<0.837$ & $\mathrm{pCi} / \mathrm{g}$ & 0.837 & & 3/31/08 & 8C17008 & AGG-RRL-001 \\
\hline 13982-78-0 & Mercury-203 & $<0.353$ & $\mathrm{pCi} / \mathrm{g}$ & 0.353 & & 3/31/08 & 8C17008 & AGG-RRL-001 \\
\hline $14913-50-9$ & Thallium-208 & $<0.25$ & $\mathrm{pCi} / \mathrm{g}$ & 0.25 & 0.059 & 3/31/08 & 8C17008 & AGG-RRL-001 \\
\hline 14331-79-4 & Bismuth-210 & $<0.552$ & $\mathrm{pCi} / \mathrm{g}$ & 0.552 & & 3/31/08 & 8C17008 & AGG-RRL-001 \\
\hline \multirow[t]{3}{*}{$14255-04-0$} & Lead-210 & $<106$ & $\mathrm{pCi} / \mathrm{g}$ & 106 & & 3/31/08 & 8C17008 & AGG-RRL-001 \\
\hline & Bismuth-211 & $<6.35$ & $\mathrm{pCi} / \mathrm{g}$ & 6.35 & & 3/31/08 & 8C17008 & AGG-RRL-001 \\
\hline & Lead-211 & $<8.68$ & $\mathrm{pCi} / \mathrm{g}$ & 8.68 & & 3/31/08 & 8C17008 & AGG-RRL-001 \\
\hline 15092-94-1 & Lead-212 & 0.712 & $\mathrm{pCi} / \mathrm{g}$ & 0.49 & 0.3 & 3/31/08 & 8C17008 & AGG-RRL-001 \\
\hline 14733-03-0 & Bismuth-214 & $<0.75$ & $\mathrm{pCi} / \mathrm{g}$ & 0.75 & & 3/31/08 & 8C17008 & AGG-RRL-001 \\
\hline $15067-28-4$ & Lead-214 & $<0.863$ & $\mathrm{pCi} / \mathrm{g}$ & 0.863 & & 3/31/08 & 8C17008 & AGG-RRL-001 \\
\hline 14835-02-0 & Radon-219 & $<2.82$ & $\mathrm{pCi} / \mathrm{g}$ & 2.82 & & 3/31/08 & 8C17008 & AGG-RRL-001 \\
\hline 22481-48-7 & Radon-220 & $<230$ & $\mathrm{pCi} / \mathrm{g}$ & 230 & & 3/31/08 & 8C17008 & AGG-RRL-001 \\
\hline 28522-20-5 & Radon-221 & $<1.28$ & $\mathrm{pCi} / \mathrm{g}$ & 1.28 & & 3/31/08 & 8C17008 & AGG-RRL-001 \\
\hline 15756-98-6 & Francium-223 & $<9.65$ & $\mathrm{pCi} / \mathrm{g}$ & 9.65 & & 3/31/08 & 8C17008 & AGG-RRL-001 \\
\hline $15623-45-7$ & Radium-223 & $<2.05$ & $\mathrm{pCi} / \mathrm{g}$ & 2.05 & & 3/31/08 & 8C17008 & AGG-RRL-001 \\
\hline 13233-32-4 & Radium-224 & $<4.9$ & $\mathrm{pCi} / \mathrm{g}$ & 4.9 & 0.925 & 3/31/08 & 8C17008 & AGG-RRL-001 \\
\hline 13982-63-3 & Radium-226 & $<7.95$ & $\mathrm{pCi} / \mathrm{g}$ & 7.95 & & 3/31/08 & 8C17008 & AGG-RRL-001 \\
\hline $15623-47-9$ & Thorium-227 & $<2.99$ & $\mathrm{pCi} / \mathrm{g}$ & 2.99 & & 3/31/08 & 8C17008 & AGG-RRL-001 \\
\hline 14331-83-0 & Actinium-228 & $<1.27$ & $\mathrm{pCi} / \mathrm{g}$ & 1.27 & & 3/31/08 & 8C17008 & AGG-RRL-001 \\
\hline 14274-82-9 & Thorium-228 & $<103$ & $\mathrm{pCi} / \mathrm{g}$ & 103 & & 3/31/08 & 8C17008 & AGG-RRL-001 \\
\hline 14269-63-7 & Thorium-230 & $<106$ & $\mathrm{pCi} / \mathrm{g}$ & 106 & & 3/31/08 & 8C17008 & AGG-RRL-001 \\
\hline 14331-85-2 & Protactinium-231 & $<11.4$ & $\mathrm{pCi} / \mathrm{g}$ & 11.4 & & 3/31/08 & 8C17008 & AGG-RRL-001 \\
\hline $14932-40-2$ & Thorium-231 & $<55.8$ & $\mathrm{pCi} / \mathrm{g}$ & 55.8 & & 3/31/08 & 8C17008 & AGG-RRL-001 \\
\hline
\end{tabular}


GEA/Soil

\begin{tabular}{|c|c|c|c|c|c|c|c|c|}
\hline CAS \# & Analyte & Results & Units & MDA & UNC & Analyzed & Batch & Method \\
\hline HEIS No. & В1T2P7B & \multicolumn{2}{|c|}{ Lab ID: } & 0803015-02 & & & & \\
\hline 7440-29-1 & Thorium-232 & $<330$ & $\mathrm{pCi} / \mathrm{g}$ & 330 & & $3 / 31 / 08$ & 8C17008 & AGG-RRL-001 \\
\hline 13981-14-1 & Protactinium-233 & $<0.731$ & $\mathrm{pCi} / \mathrm{g}$ & 0.731 & & 3/31/08 & 8C17008 & AGG-RRL-001 \\
\hline \multirow{2}{*}{$15100-28-4$} & Protactinium-234 & $<1.02$ & $\mathrm{pCi} / \mathrm{g}$ & 1.02 & & 3/31/08 & 8C17008 & AGG-RRL-001 \\
\hline & Protactinium-234m & $<35.7$ & $\mathrm{pCi} / \mathrm{g}$ & 35.7 & & 3/31/08 & 8C17008 & AGG-RRL-001 \\
\hline $15065-10-8$ & Thorium-234 & $<9.57$ & $\mathrm{pCi} / \mathrm{g}$ & 9.57 & & 3/31/08 & 8C17008 & AGG-RRL-001 \\
\hline 15117-96-1 & Uranium 235 & $<2.32$ & $\mathrm{pCi} / \mathrm{g}$ & 2.32 & & 3/31/08 & 8C17008 & AGG-RRL-001 \\
\hline 13994-20-2 & Neptunium-237 & $<2.18$ & $\mathrm{pCi} / \mathrm{g}$ & 2.18 & & 3/31/08 & 8C17008 & AGG-RRL-001 \\
\hline \multirow[t]{2}{*}{ 13981-16-3 } & Plutonium-238 & $<3200$ & $\mathrm{pCi} / \mathrm{g}$ & 3200 & & $3 / 31 / 08$ & 8C17008 & AGG-RRL-001 \\
\hline & Uranium 238 & $<4.95$ & $\mathrm{pCi} / \mathrm{g}$ & 4.95 & & 3/31/08 & 8C17008 & AGG-RRL-001 \\
\hline 15117-48-3 & Plutonium-239 & $<3880$ & $\mathrm{pCi} / \mathrm{g}$ & 3880 & & 3/31/08 & 8C17008 & AGG-RRL-001 \\
\hline 14119-33-6 & Plutonium-240 & $<3270$ & $\mathrm{pCi} / \mathrm{g}$ & 3270 & & 3/31/08 & 8C17008 & AGG-RRL-001 \\
\hline $14596-10-2$ & Americium-241 & $<1.64$ & $\mathrm{pCi} / \mathrm{g}$ & 1.64 & & 3/31/08 & 8C17008 & AGG-RRL-001 \\
\hline 14993-75-0 & Americium-243 & $<0.601$ & $\mathrm{pCi} / \mathrm{g}$ & 0.601 & & 3/31/08 & 8C17008 & AGG-RRL-001 \\
\hline 15757-87-6 & Curium-243 & $<0.997$ & $\mathrm{pCi} / \mathrm{g}$ & 0.997 & & 3/31/08 & 8C17008 & AGG-RRL-001 \\
\hline 15621-76-8 & Curium-245 & $<0.819$ & $\mathrm{pCi} / \mathrm{g}$ & 0.819 & & 3/31/08 & 8C17008 & AGG-RRL-001 \\
\hline HEIS No. & B1T2P7A & \multicolumn{2}{|c|}{ Lab ID: } & 0803015-03 & & & & \\
\hline 13966-32-0 & Sodium-22 & $<0.364$ & $\mathrm{pCi} / \mathrm{g}$ & 0.364 & & $3 / 31 / 08$ & 8C17008 & AGG-RRL-001 \\
\hline 13966-00-2 & Potassium-40 & 15.1 & $\mathrm{pCi} / \mathrm{g}$ & 2.91 & 1.11 & 3/31/08 & 8C17008 & AGG-RRL-001 \\
\hline $14392-02-0$ & Chromium-51 & $<3.07$ & $\mathrm{pCi} / \mathrm{g}$ & 3.07 & & 3/31/08 & 8C17008 & AGG-RRL-001 \\
\hline 13966-31-9 & Manganese-54 & $<0.324$ & $\mathrm{pCi} / \mathrm{g}$ & 0.324 & & 3/31/08 & 8C17008 & AGG-RRL-001 \\
\hline 13981-50-5 & Cobalt-57 & $<0.317$ & $\mathrm{pCi} / \mathrm{g}$ & 0.317 & & 3/31/08 & 8C17008 & AGG-RRL-001 \\
\hline $14596-12-4$ & Iron-59 & $<0.597$ & $\mathrm{pCi} / \mathrm{g}$ & 0.597 & & 3/31/08 & 8C17008 & AGG-RRL-001 \\
\hline $10198-40-0$ & Cobalt-60 & $<0.358$ & $\mathrm{pCi} / \mathrm{g}$ & 0.358 & & 3/31/08 & 8C17008 & AGG-RRL-001 \\
\hline 13982-39-3 & Zinc-65 & $<0.732$ & $\mathrm{pCi} / \mathrm{g}$ & 0.732 & & 3/31/08 & 8C17008 & AGG-RRL-001 \\
\hline 14265-71-5 & Selenium-75 & $<0.505$ & $\mathrm{pCi} / \mathrm{g}$ & 0.505 & & 3/31/08 & 8C17008 & AGG-RRL-001 \\
\hline $17056-36-9$ & Rubidium-83 & $<0.78$ & $\mathrm{pCi} / \mathrm{g}$ & 0.78 & & 3/31/08 & 8C17008 & AGG-RRL-001 \\
\hline 13967-73-2 & Strontium-85 & $<0.369$ & $\mathrm{pCi} / \mathrm{g}$ & 0.369 & & 3/31/08 & 8C17008 & AGG-RRL-001 \\
\hline 14932-53-7 & Rubidium-86 & $<4.04$ & $\mathrm{pCi} / \mathrm{g}$ & 4.04 & & 3/31/08 & 8C17008 & AGG-RRL-001 \\
\hline 13982-36-0 & Yttrium-88 & $<0.261$ & $\mathrm{pCi} / \mathrm{g}$ & 0.261 & & 3/31/08 & 8C17008 & AGG-RRL-001 \\
\hline 14681-63-1 & Niobium-94 & $<0.318$ & $\mathrm{pCi} / \mathrm{g}$ & 0.318 & & 3/31/08 & 8C17008 & AGG-RRL-001 \\
\hline \multirow[t]{3}{*}{ 13967-76-5 } & Niobium-95 & $<0.327$ & $\mathrm{pCi} / \mathrm{g}$ & 0.327 & & 3/31/08 & 8C17008 & AGG-RRL-001 \\
\hline & Niobium-95m & $<1.37$ & $\mathrm{pCi} / \mathrm{g}$ & 1.37 & & 3/31/08 & 8C17008 & AGG-RRL-001 \\
\hline & Technetium-95m & $<0.512$ & $\mathrm{pCi} / \mathrm{g}$ & 0.512 & & 3/31/08 & 8C17008 & AGG-RRL-001 \\
\hline \multirow[t]{2}{*}{ 13967-71-0 } & Zirconium-95 & $<0.588$ & $\mathrm{pCi} / \mathrm{g}$ & 0.588 & & 3/31/08 & 8C17008 & AGG-RRL-001 \\
\hline & Technetium-99m & $<0.323$ & $\mathrm{pCi} / \mathrm{g}$ & 0.323 & & 3/31/08 & 8C17008 & AGG-RRL-001 \\
\hline 13968-53-1 & Ruthenium-103 & $<0.379$ & $\mathrm{pCi} / \mathrm{g}$ & 0.379 & & 3/31/08 & 8C17008 & AGG-RRL-001 \\
\hline $13967-48-1$ & Ruthenium-106 & $<3.59$ & $\mathrm{pCi} / \mathrm{g}$ & 3.59 & & 3/31/08 & 8C17008 & AGG-RRL-001 \\
\hline $14391-65-2$ & Silver-108m & $<0.327$ & $\mathrm{pCi} / \mathrm{g}$ & 0.327 & & 3/31/08 & 8C17008 & AGG-RRL-001 \\
\hline \multirow[t]{2}{*}{ 14109-32-1 } & Cadmium-109 & $<9.17$ & $\mathrm{pCi} / \mathrm{g}$ & 9.17 & & 3/31/08 & 8C17008 & AGG-RRL-001 \\
\hline & Silver-110 & $<0.344$ & $\mathrm{pCi} / \mathrm{g}$ & 0.344 & & 3/31/08 & 8C17008 & AGG-RRL-001 \\
\hline 14391-76-5 & Silver-110m & $<0.344$ & $\mathrm{pCi} / \mathrm{g}$ & 0.344 & & 3/31/08 & 8C17008 & AGG-RRL-001 \\
\hline 13966-06-8 & Tin-113 & $<0.443$ & $\mathrm{pCi} / \mathrm{g}$ & 0.443 & & 3/31/08 & 8C17008 & AGG-RRL-001 \\
\hline $14683-10-4$ & Antimony-124 & $<0.383$ & $\mathrm{pCi} / \mathrm{g}$ & 0.383 & & 3/31/08 & 8C17008 & AGG-RRL-001 \\
\hline $14234-35-6$ & Antimony-125 & $<1.01$ & $\mathrm{pCi} / \mathrm{g}$ & 1.01 & & 3/31/08 & 8C17008 & AGG-RRL-001 \\
\hline 15756-32-8 & Antimony-126 & $<0.323$ & $\mathrm{pCi} / \mathrm{g}$ & 0.323 & & 3/31/08 & 8C17008 & AGG-RRL-001 \\
\hline $15832-50-5$ & Tin-126 & $<0.75$ & $\mathrm{pCi} / \mathrm{g}$ & 0.75 & & 3/31/08 & 8C17008 & AGG-RRL-001 \\
\hline 10043-66-0 & Iodine-131 & $<0.415$ & $\mathrm{pCi} / \mathrm{g}$ & 0.415 & & 3/31/08 & 8C17008 & AGG-RRL-001 \\
\hline 13981-41-4 & Barium-133 & $<0.569$ & $\mathrm{pCi} / \mathrm{g}$ & 0.569 & & 3/31/08 & 8C17008 & AGG-RRL-001 \\
\hline
\end{tabular}


GEA/Soil

\begin{tabular}{|c|c|c|c|c|c|c|c|c|}
\hline CAS \# & Analyte & Results & Units & MDA & UNC & Analyzed & Batch & Method \\
\hline HEIS No. & B1T2P7A & \multicolumn{3}{|c|}{ Lab ID: $\quad$ 0803015-03 } & & & & \\
\hline 13967-70-9 & Cesium-134 & $<0.349$ & $\mathrm{pCi} / \mathrm{g}$ & 0.349 & & $3 / 31 / 08$ & 8C17008 & AGG-RRL-001 \\
\hline $10045-97-3$ & Cesium-137 & $<0.387$ & $\mathrm{pCi} / \mathrm{g}$ & 0.387 & & 3/31/08 & 8C17008 & AGG-RRL-001 \\
\hline 13982-30-4 & Cerium-139 & $<0.379$ & $\mathrm{pCi} / \mathrm{g}$ & 0.379 & & 3/31/08 & 8C17008 & AGG-RRL-001 \\
\hline $14762-78-8$ & Cerium-144 & $<2.59$ & $\mathrm{pCi} / \mathrm{g}$ & 2.59 & & 3/31/08 & 8C17008 & AGG-RRL-001 \\
\hline $14683-23-9$ & Europium-152 & $<1.33$ & $\mathrm{pCi} / \mathrm{g}$ & 1.33 & & $3 / 31 / 08$ & 8C17008 & AGG-RRL-001 \\
\hline $14276-65-4$ & Gadolinium-153 & $<0.994$ & $\mathrm{pCi} / \mathrm{g}$ & 0.994 & & 3/31/08 & 8C17008 & AGG-RRL-001 \\
\hline 15585-10-1 & Europium-154 & $<0.666$ & $\mathrm{pCi} / \mathrm{g}$ & 0.666 & & $3 / 31 / 08$ & 8C17008 & AGG-RRL-001 \\
\hline 14391-16-3 & Europium-155 & $<1.07$ & $\mathrm{pCi} / \mathrm{g}$ & 1.07 & & $3 / 31 / 08$ & 8C17008 & AGG-RRL-001 \\
\hline $13982-78-0$ & Mercury-203 & $<0.402$ & $\mathrm{pCi} / \mathrm{g}$ & 0.402 & & 3/31/08 & 8C17008 & AGG-RRL-001 \\
\hline 14913-50-9 & Thallium-208 & $<0.399$ & $\mathrm{pCi} / \mathrm{g}$ & 0.399 & & 3/31/08 & 8C17008 & AGG-RRL-001 \\
\hline 14331-79-4 & Bismuth-210 & $<0.606$ & $\mathrm{pCi} / \mathrm{g}$ & 0.606 & & 3/31/08 & 8C17008 & AGG-RRL-001 \\
\hline \multirow[t]{3}{*}{$14255-04-0$} & Lead-210 & $<44.3$ & $\mathrm{pCi} / \mathrm{g}$ & 44.3 & & 3/31/08 & 8C17008 & AGG-RRL-001 \\
\hline & Bismuth-211 & $<7.23$ & $\mathrm{pCi} / \mathrm{g}$ & 7.23 & & 3/31/08 & 8C17008 & AGG-RRL-001 \\
\hline & Lead-211 & $<9.88$ & $\mathrm{pCi} / \mathrm{g}$ & 9.88 & & 3/31/08 & 8C17008 & AGG-RRL-001 \\
\hline $15092-94-1$ & Lead-212 & $<0.801$ & $\mathrm{pCi} / \mathrm{g}$ & 0.801 & & 3/31/08 & 8C17008 & AGG-RRL-001 \\
\hline $14733-03-0$ & Bismuth-214 & $<0.829$ & $\mathrm{pCi} / \mathrm{g}$ & 0.829 & & 3/31/08 & 8C17008 & AGG-RRL-001 \\
\hline $15067-28-4$ & Lead-214 & $<0.92$ & $\mathrm{pCi} / \mathrm{g}$ & 0.92 & & 3/31/08 & 8C17008 & AGG-RRL-001 \\
\hline $14835-02-0$ & Radon-219 & $<3.06$ & $\mathrm{pCi} / \mathrm{g}$ & 3.06 & & 3/31/08 & 8C17008 & AGG-RRL-001 \\
\hline 22481-48-7 & Radon-220 & $<312$ & $\mathrm{pCi} / \mathrm{g}$ & 312 & & 3/31/08 & 8C17008 & AGG-RRL-001 \\
\hline 28522-20-5 & Radon-221 & $<1.53$ & $\mathrm{pCi} / \mathrm{g}$ & 1.53 & & 3/31/08 & 8C17008 & AGG-RRL-001 \\
\hline $15756-98-6$ & Francium-223 & $<10$ & $\mathrm{pCi} / \mathrm{g}$ & 10 & & 3/31/08 & 8C17008 & AGG-RRL-001 \\
\hline $15623-45-7$ & Radium-223 & $<2.23$ & $\mathrm{pCi} / \mathrm{g}$ & 2.23 & & 3/31/08 & 8C17008 & AGG-RRL-001 \\
\hline $13233-32-4$ & Radium-224 & $<8.71$ & $\mathrm{pCi} / \mathrm{g}$ & 8.71 & & $3 / 31 / 08$ & 8C17008 & AGG-RRL-001 \\
\hline 13982-63-3 & Radium-226 & $<9.42$ & $\mathrm{pCi} / \mathrm{g}$ & 9.42 & & 3/31/08 & 8C17008 & AGG-RRL-001 \\
\hline $15623-47-9$ & Thorium-227 & $<3.05$ & $\mathrm{pCi} / \mathrm{g}$ & 3.05 & & 3/31/08 & 8C17008 & AGG-RRL-001 \\
\hline $14331-83-0$ & Actinium-228 & $<1.29$ & $\mathrm{pCi} / \mathrm{g}$ & 1.29 & & 3/31/08 & 8C17008 & AGG-RRL-001 \\
\hline $14274-82-9$ & Thorium-228 & $<115$ & $\mathrm{pCi} / \mathrm{g}$ & 115 & & 3/31/08 & 8C17008 & AGG-RRL-001 \\
\hline $14269-63-7$ & Thorium-230 & $<123$ & $\mathrm{pCi} / \mathrm{g}$ & 123 & & 3/31/08 & 8C17008 & AGG-RRL-001 \\
\hline $14331-85-2$ & Protactinium-231 & $<14.2$ & $\mathrm{pCi} / \mathrm{g}$ & 14.2 & & 3/31/08 & 8C17008 & AGG-RRL-001 \\
\hline $14932-40-2$ & Thorium-231 & $<70.6$ & $\mathrm{pCi} / \mathrm{g}$ & 70.6 & & $3 / 31 / 08$ & 8C17008 & AGG-RRL-001 \\
\hline $7440-29-1$ & Thorium-232 & $<307$ & $\mathrm{pCi} / \mathrm{g}$ & 307 & & 3/31/08 & 8C17008 & AGG-RRL-001 \\
\hline 13981-14-1 & Protactinium-233 & $<0.854$ & $\mathrm{pCi} / \mathrm{g}$ & 0.854 & & 3/31/08 & 8C17008 & AGG-RRL-001 \\
\hline \multirow[t]{2}{*}{$15100-28-4$} & Protactinium-234 & $<1.3$ & $\mathrm{pCi} / \mathrm{g}$ & 1.3 & & 3/31/08 & 8C17008 & AGG-RRL-001 \\
\hline & Protactinium-234m & $<37.5$ & $\mathrm{pCi} / \mathrm{g}$ & 37.5 & & $3 / 31 / 08$ & 8C17008 & AGG-RRL-001 \\
\hline $15065-10-8$ & Thorium-234 & $<10.9$ & $\mathrm{pCi} / \mathrm{g}$ & 10.9 & & 3/31/08 & 8C17008 & AGG-RRL-001 \\
\hline 15117-96-1 & Uranium 235 & $<2.78$ & $\mathrm{pCi} / \mathrm{g}$ & 2.78 & & 3/31/08 & 8C17008 & AGG-RRL-001 \\
\hline $13994-20-2$ & Neptunium-237 & $<2.78$ & $\mathrm{pCi} / \mathrm{g}$ & 2.78 & & 3/31/08 & 8C17008 & AGG-RRL-001 \\
\hline \multirow[t]{2}{*}{ 13981-16-3 } & Plutonium-238 & $<4090$ & $\mathrm{pCi} / \mathrm{g}$ & 4090 & & 3/31/08 & 8C17008 & AGG-RRL-001 \\
\hline & Uranium 238 & $<5.77$ & $\mathrm{pCi} / \mathrm{g}$ & 5.77 & & 3/31/08 & 8C17008 & AGG-RRL-001 \\
\hline $15117-48-3$ & Plutonium-239 & $<4240$ & $\mathrm{pCi} / \mathrm{g}$ & 4240 & & 3/31/08 & 8C17008 & AGG-RRL-001 \\
\hline 14119-33-6 & Plutonium-240 & $<4080$ & $\mathrm{pCi} / \mathrm{g}$ & 4080 & & 3/31/08 & 8C17008 & AGG-RRL-001 \\
\hline $14596-10-2$ & Americium-241 & $<1.57$ & $\mathrm{pCi} / \mathrm{g}$ & 1.57 & & $3 / 31 / 08$ & 8C17008 & AGG-RRL-001 \\
\hline $14993-75-0$ & Americium-243 & $<0.674$ & $\mathrm{pCi} / \mathrm{g}$ & 0.674 & & $3 / 31 / 08$ & 8C17008 & AGG-RRL-001 \\
\hline $15757-87-6$ & Curium-243 & $<1.25$ & $\mathrm{pCi} / \mathrm{g}$ & 1.25 & & 3/31/08 & 8C17008 & AGG-RRL-001 \\
\hline $15621-76-8$ & Curium-245 & $<1.02$ & $\mathrm{pCi} / \mathrm{g}$ & 1.02 & & 3/31/08 & 8C17008 & AGG-RRL-001 \\
\hline HEIS No. & B1T2P8B & \multicolumn{2}{|c|}{ Lab ID: } & 0803015-06 & & & & \\
\hline 13966-32-0 & Sodium-22 & $<0.334$ & $\mathrm{pCi} / \mathrm{g}$ & 0.334 & & 3/31/08 & 8C17008 & AGG-RRL-001 \\
\hline 13966-00-2 & Potassium- 40 & 21.4 & $\mathrm{pCi} / \mathrm{g}$ & 2.37 & 1.21 & 3/31/08 & 8C17008 & AGG-RRL-001 \\
\hline
\end{tabular}


GEA/Soil

\begin{tabular}{|c|c|c|c|c|c|c|c|c|}
\hline CAS \# & Analyte & Results & Units & MDA & UNC & Analyzed & Batch & Method \\
\hline HEIS No. & В1T2P8B & \multicolumn{3}{|c|}{ Lab ID: $\quad$ 0803015-06 } & & & & \\
\hline 14392-02-0 & Chromium-51 & $<3.06$ & $\mathrm{pCi} / \mathrm{g}$ & 3.06 & & 3/31/08 & 8C17008 & AGG-RRL-001 \\
\hline 13966-31-9 & Manganese-54 & $<0.319$ & $\mathrm{pCi} / \mathrm{g}$ & 0.319 & & $3 / 31 / 08$ & 8C17008 & AGG-RRL-001 \\
\hline $13981-50-5$ & Cobalt-57 & $<0.313$ & $\mathrm{pCi} / \mathrm{g}$ & 0.313 & & 3/31/08 & 8C17008 & AGG-RRL-001 \\
\hline $14596-12-4$ & Iron-59 & $<0.637$ & $\mathrm{pCi} / \mathrm{g}$ & 0.637 & & 3/31/08 & 8C17008 & AGG-RRL-001 \\
\hline 10198-40-0 & Cobalt-60 & $<0.312$ & $\mathrm{pCi} / \mathrm{g}$ & 0.312 & & 3/31/08 & 8C17008 & AGG-RRL-001 \\
\hline 13982-39-3 & Zinc-65 & $<0.784$ & $\mathrm{pCi} / \mathrm{g}$ & 0.784 & & 3/31/08 & 8C17008 & AGG-RRL-001 \\
\hline 14265-71-5 & Selenium-75 & $<0.501$ & $\mathrm{pCi} / \mathrm{g}$ & 0.501 & & 3/31/08 & 8C17008 & AGG-RRL-001 \\
\hline 17056-36-9 & Rubidium-83 & $<0.745$ & $\mathrm{pCi} / \mathrm{g}$ & 0.745 & & 3/31/08 & 8C17008 & AGG-RRL-001 \\
\hline 13967-73-2 & Strontium-85 & $<0.359$ & $\mathrm{pCi} / \mathrm{g}$ & 0.359 & & 3/31/08 & 8C17008 & AGG-RRL-001 \\
\hline $14932-53-7$ & Rubidium-86 & $<3.95$ & $\mathrm{pCi} / \mathrm{g}$ & 3.95 & & 3/31/08 & 8C17008 & AGG-RRL-001 \\
\hline 13982-36-0 & Yttrium-88 & $<0.24$ & $\mathrm{pCi} / \mathrm{g}$ & 0.24 & & 3/31/08 & 8C17008 & AGG-RRL-001 \\
\hline 14681-63-1 & Niobium-94 & $<0.324$ & $\mathrm{pCi} / \mathrm{g}$ & 0.324 & & $3 / 31 / 08$ & 8C17008 & AGG-RRL-001 \\
\hline \multirow[t]{3}{*}{$13967-76-5$} & Niobium-95 & $<0.301$ & $\mathrm{pCi} / \mathrm{g}$ & 0.301 & & $3 / 31 / 08$ & 8C17008 & AGG-RRL-001 \\
\hline & Niobium-95m & $<1.41$ & $\mathrm{pCi} / \mathrm{g}$ & 1.41 & & 3/31/08 & 8C17008 & AGG-RRL-001 \\
\hline & Technetium-95m & $<0.472$ & $\mathrm{pCi} / \mathrm{g}$ & 0.472 & & 3/31/08 & 8C17008 & AGG-RRL-001 \\
\hline \multirow[t]{2}{*}{ 13967-71-0 } & Zirconium-95 & $<0.528$ & $\mathrm{pCi} / \mathrm{g}$ & 0.528 & & 3/31/08 & 8C17008 & AGG-RRL-001 \\
\hline & Technetium-99m & $<0.315$ & $\mathrm{pCi} / \mathrm{g}$ & 0.315 & & 3/31/08 & 8C17008 & AGG-RRL-001 \\
\hline 13968-53-1 & Ruthenium-103 & $<0.367$ & $\mathrm{pCi} / \mathrm{g}$ & 0.367 & & 3/31/08 & 8C17008 & AGG-RRL-001 \\
\hline 13967-48-1 & Ruthenium-106 & $<3.72$ & $\mathrm{pCi} / \mathrm{g}$ & 3.72 & & 3/31/08 & 8C17008 & AGG-RRL-001 \\
\hline 14391-65-2 & Silver-108m & $<0.316$ & $\mathrm{pCi} / \mathrm{g}$ & 0.316 & & 3/31/08 & 8C17008 & AGG-RRL-001 \\
\hline \multirow[t]{2}{*}{ 14109-32-1 } & Cadmium-109 & $<9.04$ & $\mathrm{pCi} / \mathrm{g}$ & 9.04 & & 3/31/08 & 8C17008 & AGG-RRL-001 \\
\hline & Silver-110 & $<0.304$ & $\mathrm{pCi} / \mathrm{g}$ & 0.304 & & 3/31/08 & 8C17008 & AGG-RRL-001 \\
\hline 14391-76-5 & Silver-110m & $<0.304$ & $\mathrm{pCi} / \mathrm{g}$ & 0.304 & & 3/31/08 & 8C17008 & AGG-RRL-001 \\
\hline 13966-06-8 & Tin-113 & $<0.432$ & $\mathrm{pCi} / \mathrm{g}$ & 0.432 & & 3/31/08 & 8C17008 & AGG-RRL-001 \\
\hline 14683-10-4 & Antimony-124 & $<0.384$ & $\mathrm{pCi} / \mathrm{g}$ & 0.384 & & 3/31/08 & 8C17008 & AGG-RRL-001 \\
\hline $14234-35-6$ & Antimony-125 & $<0.967$ & $\mathrm{pCi} / \mathrm{g}$ & 0.967 & & 3/31/08 & 8C17008 & AGG-RRL-001 \\
\hline 15756-32-8 & Antimony-126 & $<0.282$ & $\mathrm{pCi} / \mathrm{g}$ & 0.282 & & 3/31/08 & 8C17008 & AGG-RRL-001 \\
\hline 15832-50-5 & Tin-126 & $<0.737$ & $\mathrm{pCi} / \mathrm{g}$ & 0.737 & & 3/31/08 & 8C17008 & AGG-RRL-001 \\
\hline 10043-66-0 & Iodine-131 & $<0.383$ & $\mathrm{pCi} / \mathrm{g}$ & 0.383 & & 3/31/08 & 8C17008 & AGG-RRL-001 \\
\hline 13981-41-4 & Barium-133 & $<0.528$ & $\mathrm{pCi} / \mathrm{g}$ & 0.528 & & 3/31/08 & 8C17008 & AGG-RRL-001 \\
\hline 13967-70-9 & Cesium-134 & $<0.384$ & $\mathrm{pCi} / \mathrm{g}$ & 0.384 & & 3/31/08 & 8C17008 & AGG-RRL-001 \\
\hline 10045-97-3 & Cesium-137 & $<0.338$ & $\mathrm{pCi} / \mathrm{g}$ & 0.338 & & 3/31/08 & 8C17008 & AGG-RRL-001 \\
\hline 13982-30-4 & Cerium-139 & $<0.37$ & $\mathrm{pCi} / \mathrm{g}$ & 0.37 & & 3/31/08 & 8C17008 & AGG-RRL-001 \\
\hline 14762-78-8 & Cerium-144 & $<2.53$ & $\mathrm{pCi} / \mathrm{g}$ & 2.53 & & 3/31/08 & 8C17008 & AGG-RRL-001 \\
\hline 14683-23-9 & Europium-152 & $<1.23$ & $\mathrm{pCi} / \mathrm{g}$ & 1.23 & & 3/31/08 & 8C17008 & AGG-RRL-001 \\
\hline $14276-65-4$ & Gadolinium-153 & $<0.976$ & $\mathrm{pCi} / \mathrm{g}$ & 0.976 & & 3/31/08 & 8C17008 & AGG-RRL-001 \\
\hline 15585-10-1 & Europium-154 & $<0.662$ & $\mathrm{pCi} / \mathrm{g}$ & 0.662 & & 3/31/08 & 8C17008 & AGG-RRL-001 \\
\hline 14391-16-3 & Europium-155 & $<1.05$ & $\mathrm{pCi} / \mathrm{g}$ & 1.05 & & 3/31/08 & 8C17008 & AGG-RRL-001 \\
\hline 13982-78-0 & Mercury-203 & $<0.386$ & $\mathrm{pCi} / \mathrm{g}$ & 0.386 & & 3/31/08 & 8C17008 & AGG-RRL-001 \\
\hline 14913-50-9 & Thallium-208 & $<0.417$ & $\mathrm{pCi} / \mathrm{g}$ & 0.417 & & 3/31/08 & 8C17008 & AGG-RRL-001 \\
\hline 14331-79-4 & Bismuth-210 & $<0.598$ & $\mathrm{pCi} / \mathrm{g}$ & 0.598 & & 3/31/08 & 8C17008 & AGG-RRL-001 \\
\hline \multirow[t]{3}{*}{$14255-04-0$} & Lead-210 & $<43.3$ & $\mathrm{pCi} / \mathrm{g}$ & 43.3 & & 3/31/08 & 8C17008 & AGG-RRL-001 \\
\hline & Bismuth-211 & $<6.93$ & $\mathrm{pCi} / \mathrm{g}$ & 6.93 & & 3/31/08 & 8C17008 & AGG-RRL-001 \\
\hline & Lead-211 & $<9.47$ & $\mathrm{pCi} / \mathrm{g}$ & 9.47 & & 3/31/08 & 8C17008 & AGG-RRL-001 \\
\hline 15092-94-1 & Lead-212 & $<0.811$ & $\mathrm{pCi} / \mathrm{g}$ & 0.811 & & 3/31/08 & 8C17008 & AGG-RRL-001 \\
\hline 14733-03-0 & Bismuth-214 & $<0.806$ & $\mathrm{pCi} / \mathrm{g}$ & 0.806 & & 3/31/08 & 8C17008 & AGG-RRL-001 \\
\hline $15067-28-4$ & Lead-214 & $<0.855$ & $\mathrm{pCi} / \mathrm{g}$ & 0.855 & & 3/31/08 & 8C17008 & AGG-RRL-001 \\
\hline 14835-02-0 & Radon-219 & $<3.1$ & $\mathrm{pCi} / \mathrm{g}$ & 3.1 & & 3/31/08 & 8C17008 & AGG-RRL-001 \\
\hline 22481-48-7 & Radon-220 & $<292$ & $\mathrm{pCi} / \mathrm{g}$ & 292 & & 3/31/08 & 8C17008 & AGG-RRL-001 \\
\hline
\end{tabular}


GEA/Soil

\begin{tabular}{|c|c|c|c|c|c|c|c|c|}
\hline CAS \# & Analyte & Results & Units & MDA & UNC & Analyzed & Batch & Method \\
\hline HEIS No. & B1T2P8B & \multicolumn{2}{|c|}{ Lab ID: } & 0803015-06 & & & & \\
\hline 28522-20-5 & Radon-221 & $<1.45$ & $\mathrm{pCi} / \mathrm{g}$ & 1.45 & & $3 / 31 / 08$ & 8C17008 & AGG-RRL-001 \\
\hline $15756-98-6$ & Francium-223 & $<10.4$ & $\mathrm{pCi} / \mathrm{g}$ & 10.4 & & 3/31/08 & 8C17008 & AGG-RRL-001 \\
\hline $15623-45-7$ & Radium-223 & $<2.23$ & $\mathrm{pCi} / \mathrm{g}$ & 2.23 & & 3/31/08 & 8C17008 & AGG-RRL-001 \\
\hline 13233-32-4 & Radium-224 & $<8.78$ & $\mathrm{pCi} / \mathrm{g}$ & 8.78 & & 3/31/08 & 8C17008 & AGG-RRL-001 \\
\hline 13982-63-3 & Radium-226 & $<8.94$ & $\mathrm{pCi} / \mathrm{g}$ & 8.94 & & 3/31/08 & 8C17008 & AGG-RRL-001 \\
\hline $15623-47-9$ & Thorium-227 & $<3.16$ & $\mathrm{pCi} / \mathrm{g}$ & 3.16 & & 3/31/08 & 8C17008 & AGG-RRL-001 \\
\hline $14331-83-0$ & Actinium-228 & $<1.33$ & $\mathrm{pCi} / \mathrm{g}$ & 1.33 & & 3/31/08 & 8C17008 & AGG-RRL-001 \\
\hline 14274-82-9 & Thorium-228 & $<111$ & $\mathrm{pCi} / \mathrm{g}$ & 111 & & $3 / 31 / 08$ & 8C17008 & AGG-RRL-001 \\
\hline $14269-63-7$ & Thorium-230 & $<119$ & $\mathrm{pCi} / \mathrm{g}$ & 119 & & 3/31/08 & 8C17008 & AGG-RRL-001 \\
\hline $14331-85-2$ & Protactinium-231 & $<14.2$ & $\mathrm{pCi} / \mathrm{g}$ & 14.2 & & 3/31/08 & 8C17008 & AGG-RRL-001 \\
\hline $14932-40-2$ & Thorium-231 & $<69.8$ & $\mathrm{pCi} / \mathrm{g}$ & 69.8 & & 3/31/08 & 8C17008 & AGG-RRL-001 \\
\hline 7440-29-1 & Thorium-232 & $<296$ & $\mathrm{pCi} / \mathrm{g}$ & 296 & & 3/31/08 & 8C17008 & AGG-RRL-001 \\
\hline 13981-14-1 & Protactinium-233 & $<0.857$ & $\mathrm{pCi} / \mathrm{g}$ & 0.857 & & 3/31/08 & 8C17008 & AGG-RRL-001 \\
\hline \multirow[t]{2}{*}{$15100-28-4$} & Protactinium-234 & $<1.28$ & $\mathrm{pCi} / \mathrm{g}$ & 1.28 & & 3/31/08 & 8C17008 & AGG-RRL-001 \\
\hline & Protactinium-234m & $<38.8$ & $\mathrm{pCi} / \mathrm{g}$ & 38.8 & & 3/31/08 & 8C17008 & AGG-RRL-001 \\
\hline 15065-10-8 & Thorium-234 & $<10.3$ & $\mathrm{pCi} / \mathrm{g}$ & 10.3 & & 3/31/08 & 8C17008 & AGG-RRL-001 \\
\hline $15117-96-1$ & Uranium 235 & $<2.68$ & $\mathrm{pCi} / \mathrm{g}$ & 2.68 & & 3/31/08 & 8C17008 & AGG-RRL-001 \\
\hline $13994-20-2$ & Neptunium-237 & $<2.73$ & $\mathrm{pCi} / \mathrm{g}$ & 2.73 & & 3/31/08 & 8C17008 & AGG-RRL-001 \\
\hline \multirow[t]{2}{*}{ 13981-16-3 } & Plutonium-238 & $<4030$ & $\mathrm{pCi} / \mathrm{g}$ & 4030 & & 3/31/08 & 8C17008 & AGG-RRL-001 \\
\hline & Uranium 238 & $<5.7$ & $\mathrm{pCi} / \mathrm{g}$ & 5.7 & & 3/31/08 & 8C17008 & AGG-RRL-001 \\
\hline $15117-48-3$ & Plutonium-239 & $<4090$ & $\mathrm{pCi} / \mathrm{g}$ & 4090 & & 3/31/08 & 8C17008 & AGG-RRL-001 \\
\hline 14119-33-6 & Plutonium-240 & $<4060$ & $\mathrm{pCi} / \mathrm{g}$ & 4060 & & 3/31/08 & 8C17008 & AGG-RRL-001 \\
\hline $14596-10-2$ & Americium-241 & $<1.52$ & $\mathrm{pCi} / \mathrm{g}$ & 1.52 & & $3 / 31 / 08$ & 8C17008 & AGG-RRL-001 \\
\hline $14993-75-0$ & Americium-243 & $<0.656$ & $\mathrm{pCi} / \mathrm{g}$ & 0.656 & & 3/31/08 & 8C17008 & AGG-RRL-001 \\
\hline $15757-87-6$ & Curium-243 & $<1.24$ & $\mathrm{pCi} / \mathrm{g}$ & 1.24 & & 3/31/08 & 8C17008 & AGG-RRL-001 \\
\hline $15621-76-8$ & Curium-245 & $<1.02$ & $\mathrm{pCi} / \mathrm{g}$ & 1.02 & & 3/31/08 & 8C17008 & AGG-RRL-001 \\
\hline HEIS No. & B1T2P8A & \multicolumn{2}{|c|}{ Lab ID: } & 0803015-07 & & & & \\
\hline 13966-32-0 & Sodium-22 & $<0.296$ & $\mathrm{pCi} / \mathrm{g}$ & 0.296 & & 3/31/08 & 8C17008 & AGG-RRL-001 \\
\hline $13966-00-2$ & Potassium-40 & 18.7 & $\mathrm{pCi} / \mathrm{g}$ & 3.53 & 1.28 & 3/31/08 & 8C17008 & AGG-RRL-001 \\
\hline $14392-02-0$ & Chromium-51 & $<2.51$ & $\mathrm{pCi} / \mathrm{g}$ & 2.51 & & 3/31/08 & 8C17008 & AGG-RRL-001 \\
\hline 13966-31-9 & Manganese-54 & $<0.272$ & $\mathrm{pCi} / \mathrm{g}$ & 0.272 & & 3/31/08 & 8C17008 & AGG-RRL-001 \\
\hline 13981-50-5 & Cobalt-57 & $<0.279$ & $\mathrm{pCi} / \mathrm{g}$ & 0.279 & & $3 / 31 / 08$ & 8C17008 & AGG-RRL-001 \\
\hline $14596-12-4$ & Iron-59 & $<0.502$ & $\mathrm{pCi} / \mathrm{g}$ & 0.502 & & 3/31/08 & 8C17008 & AGG-RRL-001 \\
\hline $10198-40-0$ & Cobalt-60 & $<0.276$ & $\mathrm{pCi} / \mathrm{g}$ & 0.276 & & 3/31/08 & 8C17008 & AGG-RRL-001 \\
\hline 13982-39-3 & Zinc-65 & $<0.702$ & $\mathrm{pCi} / \mathrm{g}$ & 0.702 & & $3 / 31 / 08$ & 8C17008 & AGG-RRL-001 \\
\hline $14265-71-5$ & Selenium-75 & $<0.431$ & $\mathrm{pCi} / \mathrm{g}$ & 0.431 & & $3 / 31 / 08$ & 8C17008 & AGG-RRL-001 \\
\hline $17056-36-9$ & Rubidium-83 & $<0.547$ & $\mathrm{pCi} / \mathrm{g}$ & 0.547 & & 3/31/08 & 8C17008 & AGG-RRL-001 \\
\hline 13967-73-2 & Strontium-85 & $<0.353$ & $\mathrm{pCi} / \mathrm{g}$ & 0.353 & & 3/31/08 & 8C17008 & AGG-RRL-001 \\
\hline 14932-53-7 & Rubidium-86 & $<3.04$ & $\mathrm{pCi} / \mathrm{g}$ & 3.04 & & $3 / 31 / 08$ & 8C17008 & AGG-RRL-001 \\
\hline $13982-36-0$ & Yttrium-88 & $<0.245$ & $\mathrm{pCi} / \mathrm{g}$ & 0.245 & & $3 / 31 / 08$ & 8C17008 & AGG-RRL-001 \\
\hline $14681-63-1$ & Niobium-94 & $<0.251$ & $\mathrm{pCi} / \mathrm{g}$ & 0.251 & & 3/31/08 & 8C17008 & AGG-RRL-001 \\
\hline \multirow[t]{3}{*}{$13967-76-5$} & Niobium-95 & $<0.267$ & $\mathrm{pCi} / \mathrm{g}$ & 0.267 & & 3/31/08 & 8C17008 & AGG-RRL-001 \\
\hline & Niobium-95m & $<1.27$ & $\mathrm{pCi} / \mathrm{g}$ & 1.27 & & 3/31/08 & 8C17008 & AGG-RRL-001 \\
\hline & Technetium-95m & $<0.433$ & $\mathrm{pCi} / \mathrm{g}$ & 0.433 & & 3/31/08 & 8C17008 & AGG-RRL-001 \\
\hline \multirow[t]{2}{*}{ 13967-71-0 } & Zirconium-95 & $<0.466$ & $\mathrm{pCi} / \mathrm{g}$ & 0.466 & & 3/31/08 & 8C17008 & AGG-RRL-001 \\
\hline & Technetium-99m & $<0.279$ & $\mathrm{pCi} / \mathrm{g}$ & 0.279 & & 3/31/08 & 8C17008 & AGG-RRL-001 \\
\hline 13968-53-1 & Ruthenium-103 & $<0.304$ & $\mathrm{pCi} / \mathrm{g}$ & 0.304 & & 3/31/08 & 8C17008 & AGG-RRL-001 \\
\hline $13967-48-1$ & Ruthenium-106 & $<2.66$ & $\mathrm{pCi} / \mathrm{g}$ & 2.66 & & 3/31/08 & 8C17008 & AGG-RRL-001 \\
\hline
\end{tabular}


GEA/Soil

\begin{tabular}{|c|c|c|c|c|c|c|c|c|}
\hline CAS \# & Analyte & Results & Units & MDA & UNC & Analyzed & Batch & Method \\
\hline HEIS No. & B1T2P8A & \multicolumn{2}{|c|}{ Lab ID: } & 0803015-07 & & & & \\
\hline $14391-65-2$ & Silver-108m & $<0.28$ & $\mathrm{pCi} / \mathrm{g}$ & 0.28 & & 3/31/08 & 8C17008 & AGG-RRL-001 \\
\hline \multirow[t]{2}{*}{ 14109-32-1 } & Cadmium-109 & $<7.33$ & $\mathrm{pCi} / \mathrm{g}$ & 7.33 & & 3/31/08 & 8C17008 & AGG-RRL-001 \\
\hline & Silver-110 & $<0.265$ & $\mathrm{pCi} / \mathrm{g}$ & 0.265 & & 3/31/08 & 8C17008 & AGG-RRL-001 \\
\hline 14391-76-5 & Silver-110m & $<0.266$ & $\mathrm{pCi} / \mathrm{g}$ & 0.266 & & 3/31/08 & 8C17008 & AGG-RRL-001 \\
\hline 13966-06-8 & Tin-113 & $<0.39$ & $\mathrm{pCi} / \mathrm{g}$ & 0.39 & & 3/31/08 & 8C17008 & AGG-RRL-001 \\
\hline 14683-10-4 & Antimony-124 & $<0.285$ & $\mathrm{pCi} / \mathrm{g}$ & 0.285 & & 3/31/08 & 8C17008 & AGG-RRL-001 \\
\hline $14234-35-6$ & Antimony-125 & $<0.844$ & $\mathrm{pCi} / \mathrm{g}$ & 0.844 & & 3/31/08 & 8C17008 & AGG-RRL-001 \\
\hline 15756-32-8 & Antimony-126 & $<0.266$ & $\mathrm{pCi} / \mathrm{g}$ & 0.266 & & 3/31/08 & 8C17008 & AGG-RRL-001 \\
\hline 15832-50-5 & Tin-126 & $<0.577$ & $\mathrm{pCi} / \mathrm{g}$ & 0.577 & & 3/31/08 & 8C17008 & AGG-RRL-001 \\
\hline 10043-66-0 & Iodine-131 & $<0.31$ & $\mathrm{pCi} / \mathrm{g}$ & 0.31 & & 3/31/08 & 8C17008 & AGG-RRL-001 \\
\hline 13981-41-4 & Barium-133 & $<0.526$ & $\mathrm{pCi} / \mathrm{g}$ & 0.526 & & 3/31/08 & 8C17008 & AGG-RRL-001 \\
\hline 13967-70-9 & Cesium-134 & $<0.305$ & $\mathrm{pCi} / \mathrm{g}$ & 0.305 & & 3/31/08 & 8C17008 & AGG-RRL-001 \\
\hline 10045-97-3 & Cesium-137 & $<0.299$ & $\mathrm{pCi} / \mathrm{g}$ & 0.299 & & 3/31/08 & 8C17008 & AGG-RRL-001 \\
\hline 13982-30-4 & Cerium-139 & $<0.313$ & $\mathrm{pCi} / \mathrm{g}$ & 0.313 & & 3/31/08 & 8C17008 & AGG-RRL-001 \\
\hline 14762-78-8 & Cerium-144 & $<2.25$ & $\mathrm{pCi} / \mathrm{g}$ & 2.25 & & 3/31/08 & 8C17008 & AGG-RRL-001 \\
\hline 14683-23-9 & Europium-152 & $<1.07$ & $\mathrm{pCi} / \mathrm{g}$ & 1.07 & & 3/31/08 & 8C17008 & AGG-RRL-001 \\
\hline $14276-65-4$ & Gadolinium-153 & $<0.802$ & $\mathrm{pCi} / \mathrm{g}$ & 0.802 & & 3/31/08 & 8C17008 & AGG-RRL-001 \\
\hline 15585-10-1 & Europium-154 & $<0.596$ & $\mathrm{pCi} / \mathrm{g}$ & 0.596 & & 3/31/08 & 8C17008 & AGG-RRL-001 \\
\hline 14391-16-3 & Europium-155 & $<0.804$ & $\mathrm{pCi} / \mathrm{g}$ & 0.804 & & 3/31/08 & 8C17008 & AGG-RRL-001 \\
\hline 13982-78-0 & Mercury-203 & $<0.338$ & $\mathrm{pCi} / \mathrm{g}$ & 0.338 & & 3/31/08 & 8C17008 & AGG-RRL-001 \\
\hline 14913-50-9 & Thallium-208 & $<0.269$ & $\mathrm{pCi} / \mathrm{g}$ & 0.269 & 0.0625 & 3/31/08 & 8C17008 & AGG-RRL-001 \\
\hline 14331-79-4 & Bismuth-210 & $<0.523$ & $\mathrm{pCi} / \mathrm{g}$ & 0.523 & & 3/31/08 & 8C17008 & AGG-RRL-001 \\
\hline \multirow[t]{3}{*}{$14255-04-0$} & Lead-210 & $<104$ & $\mathrm{pCi} / \mathrm{g}$ & 104 & & 3/31/08 & 8C17008 & AGG-RRL-001 \\
\hline & Bismuth-211 & $<6.25$ & $\mathrm{pCi} / \mathrm{g}$ & 6.25 & & 3/31/08 & 8C17008 & AGG-RRL-001 \\
\hline & Lead-211 & $<8.55$ & $\mathrm{pCi} / \mathrm{g}$ & 8.55 & & 3/31/08 & 8C17008 & AGG-RRL-001 \\
\hline 15092-94-1 & Lead-212 & $<0.843$ & $\mathrm{pCi} / \mathrm{g}$ & 0.843 & 0.189 & 3/31/08 & 8C17008 & AGG-RRL-001 \\
\hline 14733-03-0 & Bismuth-214 & $<0.699$ & $\mathrm{pCi} / \mathrm{g}$ & 0.699 & & 3/31/08 & 8C17008 & AGG-RRL-001 \\
\hline $15067-28-4$ & Lead-214 & $<0.823$ & $\mathrm{pCi} / \mathrm{g}$ & 0.823 & & 3/31/08 & 8C17008 & AGG-RRL-001 \\
\hline $14835-02-0$ & Radon-219 & $<2.79$ & $\mathrm{pCi} / \mathrm{g}$ & 2.79 & & 3/31/08 & 8C17008 & AGG-RRL-001 \\
\hline 22481-48-7 & Radon-220 & $<239$ & $\mathrm{pCi} / \mathrm{g}$ & 239 & & 3/31/08 & 8C17008 & AGG-RRL-001 \\
\hline 28522-20-5 & Radon-221 & $<1.19$ & $\mathrm{pCi} / \mathrm{g}$ & 1.19 & & 3/31/08 & 8C17008 & AGG-RRL-001 \\
\hline 15756-98-6 & Francium-223 & $<9.25$ & $\mathrm{pCi} / \mathrm{g}$ & 9.25 & & 3/31/08 & 8C17008 & AGG-RRL-001 \\
\hline $15623-45-7$ & Radium-223 & $<2.03$ & $\mathrm{pCi} / \mathrm{g}$ & 2.03 & & 3/31/08 & 8C17008 & AGG-RRL-001 \\
\hline 13233-32-4 & Radium-224 & $<8.05$ & $\mathrm{pCi} / \mathrm{g}$ & 8.05 & & 3/31/08 & 8C17008 & AGG-RRL-001 \\
\hline 13982-63-3 & Radium-226 & $<7.39$ & $\mathrm{pCi} / \mathrm{g}$ & 7.39 & & 3/31/08 & 8C17008 & AGG-RRL-001 \\
\hline $15623-47-9$ & Thorium-227 & $<2.84$ & $\mathrm{pCi} / \mathrm{g}$ & 2.84 & & 3/31/08 & 8C17008 & AGG-RRL-001 \\
\hline 14331-83-0 & Actinium-228 & $<1.29$ & $\mathrm{pCi} / \mathrm{g}$ & 1.29 & & 3/31/08 & 8C17008 & AGG-RRL-001 \\
\hline 14274-82-9 & Thorium-228 & $<97$ & $\mathrm{pCi} / \mathrm{g}$ & 97 & & $3 / 31 / 08$ & 8C17008 & AGG-RRL-001 \\
\hline $14269-63-7$ & Thorium-230 & $<105$ & $\mathrm{pCi} / \mathrm{g}$ & 105 & & 3/31/08 & 8C17008 & AGG-RRL-001 \\
\hline $14331-85-2$ & Protactinium-231 & $<11.6$ & $\mathrm{pCi} / \mathrm{g}$ & 11.6 & & 3/31/08 & 8C17008 & AGG-RRL-001 \\
\hline $14932-40-2$ & Thorium-231 & $<57.7$ & $\mathrm{pCi} / \mathrm{g}$ & 57.7 & & 3/31/08 & 8C17008 & AGG-RRL-001 \\
\hline 7440-29-1 & Thorium-232 & $<327$ & $\mathrm{pCi} / \mathrm{g}$ & 327 & & $3 / 31 / 08$ & 8C17008 & AGG-RRL-001 \\
\hline 13981-14-1 & Protactinium-233 & $<0.684$ & $\mathrm{pCi} / \mathrm{g}$ & 0.684 & & 3/31/08 & 8C17008 & AGG-RRL-001 \\
\hline \multirow[t]{2}{*}{$15100-28-4$} & Protactinium-234 & $<1.03$ & $\mathrm{pCi} / \mathrm{g}$ & 1.03 & & 3/31/08 & 8C17008 & AGG-RRL-001 \\
\hline & Protactinium-234m & $<34.7$ & $\mathrm{pCi} / \mathrm{g}$ & 34.7 & & 3/31/08 & 8C17008 & AGG-RRL-001 \\
\hline 15065-10-8 & Thorium-234 & $<9.34$ & $\mathrm{pCi} / \mathrm{g}$ & 9.34 & & 3/31/08 & 8C17008 & AGG-RRL-001 \\
\hline 15117-96-1 & Uranium 235 & $<2.34$ & $\mathrm{pCi} / \mathrm{g}$ & 2.34 & & 3/31/08 & 8C17008 & AGG-RRL-001 \\
\hline 13994-20-2 & Neptunium-237 & $<2.09$ & $\mathrm{pCi} / \mathrm{g}$ & 2.09 & & 3/31/08 & 8C17008 & AGG-RRL-001 \\
\hline 13981-16-3 & Plutonium-238 & $<3260$ & $\mathrm{pCi} / \mathrm{g}$ & 3260 & & 3/31/08 & 8C17008 & AGG-RRL-001 \\
\hline
\end{tabular}


GEA/Soil

\begin{tabular}{|c|c|c|c|c|c|c|c|c|}
\hline CAS \# & Analyte & Results & Units & MDA & UNC & Analyzed & Batch & Method \\
\hline \multirow[t]{2}{*}{ HEIS No. } & B1T2P8A & \multicolumn{2}{|c|}{ Lab ID: } & \multicolumn{2}{|l|}{ 0803015-07 } & & & \\
\hline & Uranium 238 & $<4.84$ & $\mathrm{pCi} / \mathrm{g}$ & 4.84 & & $3 / 31 / 08$ & 8C17008 & AGG-RRL-001 \\
\hline $15117-48-3$ & Plutonium-239 & $<3870$ & $\mathrm{pCi} / \mathrm{g}$ & 3870 & & 3/31/08 & 8C17008 & AGG-RRL-001 \\
\hline $14119-33-6$ & Plutonium-240 & $<3340$ & $\mathrm{pCi} / \mathrm{g}$ & 3340 & & 3/31/08 & 8C17008 & AGG-RRL-001 \\
\hline $14596-10-2$ & Americium-241 & $<1.57$ & $\mathrm{pCi} / \mathrm{g}$ & 1.57 & & 3/31/08 & 8C17008 & AGG-RRL-001 \\
\hline $14993-75-0$ & Americium-243 & $<0.573$ & $\mathrm{pCi} / \mathrm{g}$ & 0.573 & & 3/31/08 & 8C17008 & AGG-RRL-001 \\
\hline $15757-87-6$ & Curium-243 & $<1.02$ & $\mathrm{pCi} / \mathrm{g}$ & 1.02 & & 3/31/08 & 8C17008 & AGG-RRL-001 \\
\hline $15621-76-8$ & Curium-245 & $<0.836$ & $\mathrm{pCi} / \mathrm{g}$ & 0.836 & & 3/31/08 & 8C17008 & AGG-RRL-001 \\
\hline HEIS No. & B1T2P9B & \multicolumn{2}{|c|}{ Lab ID: } & 0803015-10 & & & & \\
\hline 13966-32-0 & Sodium-22 & $<0.331$ & $\mathrm{pCi} / \mathrm{g}$ & 0.331 & & $3 / 31 / 08$ & 8C17008 & AGG-RRL-001 \\
\hline 13966-00-2 & Potassium-40 & 21.0 & $\mathrm{pCi} / \mathrm{g}$ & 2.96 & 1.29 & 3/31/08 & 8C17008 & AGG-RRL-001 \\
\hline $14392-02-0$ & Chromium-51 & $<2.77$ & $\mathrm{pCi} / \mathrm{g}$ & 2.77 & & 3/31/08 & 8C17008 & AGG-RRL-001 \\
\hline 13966-31-9 & Manganese-54 & $<0.296$ & $\mathrm{pCi} / \mathrm{g}$ & 0.296 & & 3/31/08 & 8C17008 & AGG-RRL-001 \\
\hline 13981-50-5 & Cobalt-57 & $<0.274$ & $\mathrm{pCi} / \mathrm{g}$ & 0.274 & & 3/31/08 & 8C17008 & AGG-RRL-001 \\
\hline $14596-12-4$ & Iron-59 & $<0.533$ & $\mathrm{pCi} / \mathrm{g}$ & 0.533 & & 3/31/08 & 8C17008 & AGG-RRL-001 \\
\hline $10198-40-0$ & Cobalt-60 & $<0.286$ & $\mathrm{pCi} / \mathrm{g}$ & 0.286 & & 3/31/08 & 8C17008 & AGG-RRL-001 \\
\hline 13982-39-3 & Zinc-65 & $<0.652$ & $\mathrm{pCi} / \mathrm{g}$ & 0.652 & & 3/31/08 & 8C17008 & AGG-RRL-001 \\
\hline $14265-71-5$ & Selenium-75 & $<0.472$ & $\mathrm{pCi} / \mathrm{g}$ & 0.472 & & 3/31/08 & 8C17008 & AGG-RRL-001 \\
\hline 17056-36-9 & Rubidium-83 & $<0.534$ & $\mathrm{pCi} / \mathrm{g}$ & 0.534 & & 3/31/08 & 8C17008 & AGG-RRL-001 \\
\hline 13967-73-2 & Strontium-85 & $<0.377$ & $\mathrm{pCi} / \mathrm{g}$ & 0.377 & & 3/31/08 & 8C17008 & AGG-RRL-001 \\
\hline 14932-53-7 & Rubidium-86 & $<3.3$ & $\mathrm{pCi} / \mathrm{g}$ & 3.3 & & $3 / 31 / 08$ & 8C17008 & AGG-RRL-001 \\
\hline $13982-36-0$ & Yttrium-88 & $<0.216$ & $\mathrm{pCi} / \mathrm{g}$ & 0.216 & & 3/31/08 & 8C17008 & AGG-RRL-001 \\
\hline $14681-63-1$ & Niobium-94 & $<0.269$ & $\mathrm{pCi} / \mathrm{g}$ & 0.269 & & 3/31/08 & 8C17008 & AGG-RRL-001 \\
\hline \multirow[t]{3}{*}{$13967-76-5$} & Niobium-95 & $<0.276$ & $\mathrm{pCi} / \mathrm{g}$ & 0.276 & & 3/31/08 & 8C17008 & AGG-RRL-001 \\
\hline & Niobium-95m & $<1.32$ & $\mathrm{pCi} / \mathrm{g}$ & 1.32 & & $3 / 31 / 08$ & 8C17008 & AGG-RRL-001 \\
\hline & Technetium-95m & $<0.447$ & $\mathrm{pCi} / \mathrm{g}$ & 0.447 & & 3/31/08 & 8C17008 & AGG-RRL-001 \\
\hline \multirow[t]{2}{*}{ 13967-71-0 } & Zirconium-95 & $<0.515$ & $\mathrm{pCi} / \mathrm{g}$ & 0.515 & & 3/31/08 & 8C17008 & AGG-RRL-001 \\
\hline & Technetium-99m & $<0.288$ & $\mathrm{pCi} / \mathrm{g}$ & 0.288 & & 3/31/08 & 8C17008 & AGG-RRL-001 \\
\hline 13968-53-1 & Ruthenium-103 & $<0.306$ & $\mathrm{pCi} / \mathrm{g}$ & 0.306 & & 3/31/08 & 8C17008 & AGG-RRL-001 \\
\hline $13967-48-1$ & Ruthenium-106 & $<2.85$ & $\mathrm{pCi} / \mathrm{g}$ & 2.85 & & 3/31/08 & 8C17008 & AGG-RRL-001 \\
\hline $14391-65-2$ & Silver-108m & $<0.289$ & $\mathrm{pCi} / \mathrm{g}$ & 0.289 & & 3/31/08 & 8C17008 & AGG-RRL-001 \\
\hline \multirow[t]{2}{*}{$14109-32-1$} & Cadmium-109 & $<7.38$ & $\mathrm{pCi} / \mathrm{g}$ & 7.38 & & 3/31/08 & 8C17008 & AGG-RRL-001 \\
\hline & Silver-110 & $<0.305$ & $\mathrm{pCi} / \mathrm{g}$ & 0.305 & & 3/31/08 & 8C17008 & AGG-RRL-001 \\
\hline $14391-76-5$ & Silver-110m & $<0.306$ & $\mathrm{pCi} / \mathrm{g}$ & 0.306 & & 3/31/08 & 8C17008 & AGG-RRL-001 \\
\hline 13966-06-8 & Tin-113 & $<0.406$ & $\mathrm{pCi} / \mathrm{g}$ & 0.406 & & 3/31/08 & 8C17008 & AGG-RRL-001 \\
\hline $14683-10-4$ & Antimony-124 & $<0.29$ & $\mathrm{pCi} / \mathrm{g}$ & 0.29 & & $3 / 31 / 08$ & 8C17008 & AGG-RRL-001 \\
\hline $14234-35-6$ & Antimony-125 & $<0.911$ & $\mathrm{pCi} / \mathrm{g}$ & 0.911 & & 3/31/08 & 8C17008 & AGG-RRL-001 \\
\hline 15756-32-8 & Antimony-126 & $<0.289$ & $\mathrm{pCi} / \mathrm{g}$ & 0.289 & & 3/31/08 & 8C17008 & AGG-RRL-001 \\
\hline $15832-50-5$ & Tin-126 & $<0.583$ & $\mathrm{pCi} / \mathrm{g}$ & 0.583 & & 3/31/08 & 8C17008 & AGG-RRL-001 \\
\hline 10043-66-0 & Iodine-131 & $<0.349$ & $\mathrm{pCi} / \mathrm{g}$ & 0.349 & & $3 / 31 / 08$ & 8C17008 & AGG-RRL-001 \\
\hline 13981-41-4 & Barium-133 & $<0.555$ & $\mathrm{pCi} / \mathrm{g}$ & 0.555 & & $3 / 31 / 08$ & 8C17008 & AGG-RRL-001 \\
\hline 13967-70-9 & Cesium-134 & $<0.319$ & $\mathrm{pCi} / \mathrm{g}$ & 0.319 & & 3/31/08 & 8C17008 & AGG-RRL-001 \\
\hline $10045-97-3$ & Cesium-137 & $<0.347$ & $\mathrm{pCi} / \mathrm{g}$ & 0.347 & & 3/31/08 & 8C17008 & AGG-RRL-001 \\
\hline 13982-30-4 & Cerium-139 & $<0.326$ & $\mathrm{pCi} / \mathrm{g}$ & 0.326 & & 3/31/08 & 8C17008 & AGG-RRL-001 \\
\hline 14762-78-8 & Cerium-144 & $<2.25$ & $\mathrm{pCi} / \mathrm{g}$ & 2.25 & & 3/31/08 & 8C17008 & AGG-RRL-001 \\
\hline $14683-23-9$ & Europium-152 & $<1.08$ & $\mathrm{pCi} / \mathrm{g}$ & 1.08 & & 3/31/08 & 8C17008 & AGG-RRL-001 \\
\hline $14276-65-4$ & Gadolinium-153 & $<0.852$ & $\mathrm{pCi} / \mathrm{g}$ & 0.852 & & 3/31/08 & 8C17008 & AGG-RRL-001 \\
\hline 15585-10-1 & Europium-154 & $<0.576$ & $\mathrm{pCi} / \mathrm{g}$ & 0.576 & & 3/31/08 & 8C17008 & AGG-RRL-001 \\
\hline $14391-16-3$ & Europium-155 & $<0.812$ & $\mathrm{pCi} / \mathrm{g}$ & 0.812 & & 3/31/08 & 8C17008 & AGG-RRL-001 \\
\hline
\end{tabular}


GEA/Soil

\begin{tabular}{|c|c|c|c|c|c|c|c|c|}
\hline CAS \# & Analyte & Results & Units & MDA & UNC & Analyzed & Batch & Method \\
\hline HEIS No. & В1T2P9B & $\mathbf{L a}$ & ID: & 0803015-10 & & & & \\
\hline $13982-78-0$ & Mercury-203 & $<0.355$ & $\mathrm{pCi} / \mathrm{g}$ & 0.355 & & $3 / 31 / 08$ & 8C17008 & AGG-RRL-001 \\
\hline 14913-50-9 & Thallium-208 & $<0.353$ & $\mathrm{pCi} / \mathrm{g}$ & 0.353 & & 3/31/08 & 8C17008 & AGG-RRL-001 \\
\hline $14331-79-4$ & Bismuth-210 & $<0.555$ & $\mathrm{pCi} / \mathrm{g}$ & 0.555 & & 3/31/08 & 8C17008 & AGG-RRL-001 \\
\hline \multirow[t]{3}{*}{$14255-04-0$} & Lead-210 & $<108$ & $\mathrm{pCi} / \mathrm{g}$ & 108 & & 3/31/08 & 8C17008 & AGG-RRL-001 \\
\hline & Bismuth-211 & $<6.45$ & $\mathrm{pCi} / \mathrm{g}$ & 6.45 & & 3/31/08 & 8C17008 & AGG-RRL-001 \\
\hline & Lead-211 & $<8.82$ & $\mathrm{pCi} / \mathrm{g}$ & 8.82 & & 3/31/08 & 8C17008 & AGG-RRL-001 \\
\hline $15092-94-1$ & Lead-212 & $<0.644$ & $\mathrm{pCi} / \mathrm{g}$ & 0.644 & 0.145 & 3/31/08 & 8C17008 & AGG-RRL-001 \\
\hline 14733-03-0 & Bismuth-214 & $<0.717$ & $\mathrm{pCi} / \mathrm{g}$ & 0.717 & & $3 / 31 / 08$ & 8C17008 & AGG-RRL-001 \\
\hline $15067-28-4$ & Lead-214 & $<0.869$ & $\mathrm{pCi} / \mathrm{g}$ & 0.869 & & 3/31/08 & 8C17008 & AGG-RRL-001 \\
\hline $14835-02-0$ & Radon-219 & $<2.72$ & $\mathrm{pCi} / \mathrm{g}$ & 2.72 & & 3/31/08 & 8C17008 & AGG-RRL-001 \\
\hline 22481-48-7 & Radon-220 & $<258$ & $\mathrm{pCi} / \mathrm{g}$ & 258 & & 3/31/08 & 8C17008 & AGG-RRL-001 \\
\hline 28522-20-5 & Radon-221 & $<1.32$ & $\mathrm{pCi} / \mathrm{g}$ & 1.32 & & 3/31/08 & 8C17008 & AGG-RRL-001 \\
\hline 15756-98-6 & Francium-223 & $<9.57$ & $\mathrm{pCi} / \mathrm{g}$ & 9.57 & & 3/31/08 & 8C17008 & AGG-RRL-001 \\
\hline $15623-45-7$ & Radium-223 & $<2.02$ & $\mathrm{pCi} / \mathrm{g}$ & 2.02 & & 3/31/08 & 8C17008 & AGG-RRL-001 \\
\hline $13233-32-4$ & Radium-224 & $<8.34$ & $\mathrm{pCi} / \mathrm{g}$ & 8.34 & & 3/31/08 & 8C17008 & AGG-RRL-001 \\
\hline 13982-63-3 & Radium-226 & $<8.15$ & $\mathrm{pCi} / \mathrm{g}$ & 8.15 & & 3/31/08 & 8C17008 & AGG-RRL-001 \\
\hline $15623-47-9$ & Thorium-227 & $<2.94$ & $\mathrm{pCi} / \mathrm{g}$ & 2.94 & & 3/31/08 & 8C17008 & AGG-RRL-001 \\
\hline $14331-83-0$ & Actinium-228 & $<1.3$ & $\mathrm{pCi} / \mathrm{g}$ & 1.3 & & 3/31/08 & 8C17008 & AGG-RRL-001 \\
\hline $14274-82-9$ & Thorium-228 & $<102$ & $\mathrm{pCi} / \mathrm{g}$ & 102 & & 3/31/08 & 8C17008 & AGG-RRL-001 \\
\hline $14269-63-7$ & Thorium-230 & $<112$ & $\mathrm{pCi} / \mathrm{g}$ & 112 & & 3/31/08 & 8C17008 & AGG-RRL-001 \\
\hline $14331-85-2$ & Protactinium-231 & $<11.3$ & $\mathrm{pCi} / \mathrm{g}$ & 11.3 & & 3/31/08 & 8C17008 & AGG-RRL-001 \\
\hline $14932-40-2$ & Thorium-231 & $<60.3$ & $\mathrm{pCi} / \mathrm{g}$ & 60.3 & & 3/31/08 & 8C17008 & AGG-RRL-001 \\
\hline 7440-29-1 & Thorium-232 & $<341$ & $\mathrm{pCi} / \mathrm{g}$ & 341 & & 3/31/08 & 8C17008 & AGG-RRL-001 \\
\hline 13981-14-1 & Protactinium-233 & $<0.724$ & $\mathrm{pCi} / \mathrm{g}$ & 0.724 & & 3/31/08 & 8C17008 & AGG-RRL-001 \\
\hline \multirow[t]{2}{*}{$15100-28-4$} & Protactinium-234 & $<1.08$ & $\mathrm{pCi} / \mathrm{g}$ & 1.08 & & 3/31/08 & 8C17008 & AGG-RRL-001 \\
\hline & Protactinium-234m & $<33.2$ & $\mathrm{pCi} / \mathrm{g}$ & 33.2 & & 3/31/08 & 8C17008 & AGG-RRL-001 \\
\hline 15065-10-8 & Thorium-234 & $<9.79$ & $\mathrm{pCi} / \mathrm{g}$ & 9.79 & & $3 / 31 / 08$ & 8C17008 & AGG-RRL-001 \\
\hline 15117-96-1 & Uranium 235 & $<2.45$ & $\mathrm{pCi} / \mathrm{g}$ & 2.45 & & 3/31/08 & 8C17008 & AGG-RRL-001 \\
\hline 13994-20-2 & Neptunium-237 & $<2.11$ & $\mathrm{pCi} / \mathrm{g}$ & 2.11 & & 3/31/08 & 8C17008 & AGG-RRL-001 \\
\hline \multirow[t]{2}{*}{ 13981-16-3 } & Plutonium-238 & $<3360$ & $\mathrm{pCi} / \mathrm{g}$ & 3360 & & 3/31/08 & 8C17008 & AGG-RRL-001 \\
\hline & Uranium 238 & $<5.07$ & $\mathrm{pCi} / \mathrm{g}$ & 5.07 & & 3/31/08 & 8C17008 & AGG-RRL-001 \\
\hline $15117-48-3$ & Plutonium-239 & $<3860$ & $\mathrm{pCi} / \mathrm{g}$ & 3860 & & 3/31/08 & 8C17008 & AGG-RRL-001 \\
\hline $14119-33-6$ & Plutonium-240 & $<3440$ & $\mathrm{pCi} / \mathrm{g}$ & 3440 & & 3/31/08 & 8C17008 & AGG-RRL-001 \\
\hline $14596-10-2$ & Americium-241 & $<1.69$ & $\mathrm{pCi} / \mathrm{g}$ & 1.69 & & $3 / 31 / 08$ & 8C17008 & AGG-RRL-001 \\
\hline 14993-75-0 & Americium-243 & $<0.603$ & $\mathrm{pCi} / \mathrm{g}$ & 0.603 & & 3/31/08 & 8C17008 & AGG-RRL-001 \\
\hline $15757-87-6$ & Curium-243 & $<1.05$ & $\mathrm{pCi} / \mathrm{g}$ & 1.05 & & 3/31/08 & 8C17008 & AGG-RRL-001 \\
\hline 15621-76-8 & Curium-245 & $<0.862$ & $\mathrm{pCi} / \mathrm{g}$ & 0.862 & & 3/31/08 & 8C17008 & AGG-RRL-001 \\
\hline HEIS No. & B1T2P9A & \multicolumn{2}{|c|}{ Lab ID: } & 0803015-11 & & & & \\
\hline 13966-32-0 & Sodium-22 & $<0.384$ & $\mathrm{pCi} / \mathrm{g}$ & 0.384 & & 3/31/08 & 8C17008 & AGG-RRL-001 \\
\hline $13966-00-2$ & Potassium-40 & 19.0 & $\mathrm{pCi} / \mathrm{g}$ & 2.73 & 1.18 & $3 / 31 / 08$ & 8C17008 & AGG-RRL-001 \\
\hline $14392-02-0$ & Chromium-51 & $<3.12$ & $\mathrm{pCi} / \mathrm{g}$ & 3.12 & & 3/31/08 & 8C17008 & AGG-RRL-001 \\
\hline 13966-31-9 & Manganese-54 & $<0.333$ & $\mathrm{pCi} / \mathrm{g}$ & 0.333 & & 3/31/08 & 8C17008 & AGG-RRL-001 \\
\hline 13981-50-5 & Cobalt-57 & $<0.322$ & $\mathrm{pCi} / \mathrm{g}$ & 0.322 & & 3/31/08 & 8C17008 & AGG-RRL-001 \\
\hline $14596-12-4$ & Iron-59 & $<0.598$ & $\mathrm{pCi} / \mathrm{g}$ & 0.598 & & 3/31/08 & 8C17008 & AGG-RRL-001 \\
\hline $10198-40-0$ & Cobalt-60 & $<0.34$ & $\mathrm{pCi} / \mathrm{g}$ & 0.34 & & 3/31/08 & 8C17008 & AGG-RRL-001 \\
\hline 13982-39-3 & Zinc-65 & $<0.741$ & $\mathrm{pCi} / \mathrm{g}$ & 0.741 & & 3/31/08 & 8C17008 & AGG-RRL-001 \\
\hline 14265-71-5 & Selenium-75 & $<0.506$ & $\mathrm{pCi} / \mathrm{g}$ & 0.506 & & 3/31/08 & 8C17008 & AGG-RRL-001 \\
\hline $17056-36-9$ & Rubidium-83 & $<0.771$ & $\mathrm{pCi} / \mathrm{g}$ & 0.771 & & 3/31/08 & 8C17008 & AGG-RRL-001 \\
\hline
\end{tabular}


GEA/Soil

\begin{tabular}{|c|c|c|c|c|c|c|c|c|}
\hline CAS \# & Analyte & Results & Units & MDA & UNC & Analyzed & Batch & Method \\
\hline HEIS No. & B1T2P9A & \multicolumn{2}{|c|}{ Lab ID: } & 0803015-11 & & & & \\
\hline 13967-73-2 & Strontium-85 & $<0.358$ & $\mathrm{pCi} / \mathrm{g}$ & 0.358 & & 3/31/08 & 8C17008 & AGG-RRL-001 \\
\hline 14932-53-7 & Rubidium-86 & $<4.16$ & $\mathrm{pCi} / \mathrm{g}$ & 4.16 & & 3/31/08 & 8C17008 & AGG-RRL-001 \\
\hline 13982-36-0 & Yttrium-88 & $<0.266$ & $\mathrm{pCi} / \mathrm{g}$ & 0.266 & & 3/31/08 & 8C17008 & AGG-RRL-001 \\
\hline 14681-63-1 & Niobium-94 & $<0.32$ & $\mathrm{pCi} / \mathrm{g}$ & 0.32 & & 3/31/08 & 8C17008 & AGG-RRL-001 \\
\hline \multirow[t]{3}{*}{ 13967-76-5 } & Niobium-95 & $<0.296$ & $\mathrm{pCi} / \mathrm{g}$ & 0.296 & & 3/31/08 & 8C17008 & AGG-RRL-001 \\
\hline & Niobium-95m & $<1.42$ & $\mathrm{pCi} / \mathrm{g}$ & 1.42 & & 3/31/08 & 8C17008 & AGG-RRL-001 \\
\hline & Technetium-95m & $<0.472$ & $\mathrm{pCi} / \mathrm{g}$ & 0.472 & & 3/31/08 & 8C17008 & AGG-RRL-001 \\
\hline \multirow[t]{2}{*}{ 13967-71-0 } & Zirconium-95 & $<0.514$ & $\mathrm{pCi} / \mathrm{g}$ & 0.514 & & 3/31/08 & 8C17008 & AGG-RRL-001 \\
\hline & Technetium-99m & $<0.321$ & $\mathrm{pCi} / \mathrm{g}$ & 0.321 & & 3/31/08 & 8C17008 & AGG-RRL-001 \\
\hline 13968-53-1 & Ruthenium-103 & $<0.339$ & $\mathrm{pCi} / \mathrm{g}$ & 0.339 & & 3/31/08 & 8C17008 & AGG-RRL-001 \\
\hline 13967-48-1 & Ruthenium-106 & $<3.49$ & $\mathrm{pCi} / \mathrm{g}$ & 3.49 & & 3/31/08 & 8C17008 & AGG-RRL-001 \\
\hline 14391-65-2 & Silver-108m & $<0.298$ & $\mathrm{pCi} / \mathrm{g}$ & 0.298 & & 3/31/08 & 8C17008 & AGG-RRL-001 \\
\hline \multirow[t]{2}{*}{ 14109-32-1 } & Cadmium-109 & $<8.89$ & $\mathrm{pCi} / \mathrm{g}$ & 8.89 & & 3/31/08 & 8C17008 & AGG-RRL-001 \\
\hline & Silver-110 & $<0.321$ & $\mathrm{pCi} / \mathrm{g}$ & 0.321 & & 3/31/08 & 8C17008 & AGG-RRL-001 \\
\hline 14391-76-5 & Silver-110m & $<0.322$ & $\mathrm{pCi} / \mathrm{g}$ & 0.322 & & 3/31/08 & 8C17008 & AGG-RRL-001 \\
\hline 13966-06-8 & Tin-113 & $<0.449$ & $\mathrm{pCi} / \mathrm{g}$ & 0.449 & & 3/31/08 & 8C17008 & AGG-RRL-001 \\
\hline 14683-10-4 & Antimony-124 & $<0.381$ & $\mathrm{pCi} / \mathrm{g}$ & 0.381 & & 3/31/08 & 8C17008 & AGG-RRL-001 \\
\hline 14234-35-6 & Antimony-125 & $<0.958$ & $\mathrm{pCi} / \mathrm{g}$ & 0.958 & & 3/31/08 & 8C17008 & AGG-RRL-001 \\
\hline 15756-32-8 & Antimony-126 & $<0.309$ & $\mathrm{pCi} / \mathrm{g}$ & 0.309 & & 3/31/08 & 8C17008 & AGG-RRL-001 \\
\hline 15832-50-5 & Tin-126 & $<0.727$ & $\mathrm{pCi} / \mathrm{g}$ & 0.727 & & 3/31/08 & 8C17008 & AGG-RRL-001 \\
\hline 10043-66-0 & Iodine-131 & $<0.377$ & $\mathrm{pCi} / \mathrm{g}$ & 0.377 & & 3/31/08 & 8C17008 & AGG-RRL-001 \\
\hline 13981-41-4 & Barium-133 & $<0.518$ & $\mathrm{pCi} / \mathrm{g}$ & 0.518 & & 3/31/08 & 8C17008 & AGG-RRL-001 \\
\hline 13967-70-9 & Cesium-134 & $<0.359$ & $\mathrm{pCi} / \mathrm{g}$ & 0.359 & & 3/31/08 & 8C17008 & AGG-RRL-001 \\
\hline $10045-97-3$ & Cesium-137 & $<0.355$ & $\mathrm{pCi} / \mathrm{g}$ & 0.355 & & 3/31/08 & 8C17008 & AGG-RRL-001 \\
\hline 13982-30-4 & Cerium-139 & $<0.367$ & $\mathrm{pCi} / \mathrm{g}$ & 0.367 & & 3/31/08 & 8C17008 & AGG-RRL-001 \\
\hline 14762-78-8 & Cerium-144 & $<2.59$ & $\mathrm{pCi} / \mathrm{g}$ & 2.59 & & 3/31/08 & 8C17008 & AGG-RRL-001 \\
\hline 14683-23-9 & Europium-152 & $<1.21$ & $\mathrm{pCi} / \mathrm{g}$ & 1.21 & & 3/31/08 & 8C17008 & AGG-RRL-001 \\
\hline $14276-65-4$ & Gadolinium-153 & $<0.97$ & $\mathrm{pCi} / \mathrm{g}$ & 0.97 & & 3/31/08 & 8C17008 & AGG-RRL-001 \\
\hline 15585-10-1 & Europium-154 & $<0.679$ & $\mathrm{pCi} / \mathrm{g}$ & 0.679 & & 3/31/08 & 8C17008 & AGG-RRL-001 \\
\hline 14391-16-3 & Europium-155 & $<1.03$ & $\mathrm{pCi} / \mathrm{g}$ & 1.03 & & 3/31/08 & 8C17008 & AGG-RRL-001 \\
\hline 13982-78-0 & Mercury-203 & $<0.381$ & $\mathrm{pCi} / \mathrm{g}$ & 0.381 & & 3/31/08 & 8C17008 & AGG-RRL-001 \\
\hline 14913-50-9 & Thallium-208 & $<0.41$ & $\mathrm{pCi} / \mathrm{g}$ & 0.41 & & 3/31/08 & 8C17008 & AGG-RRL-001 \\
\hline 14331-79-4 & Bismuth-210 & $<0.603$ & $\mathrm{pCi} / \mathrm{g}$ & 0.603 & & 3/31/08 & 8C17008 & AGG-RRL-001 \\
\hline \multirow[t]{3}{*}{$14255-04-0$} & Lead-210 & $<42.2$ & $\mathrm{pCi} / \mathrm{g}$ & 42.2 & & 3/31/08 & 8C17008 & AGG-RRL-001 \\
\hline & Bismuth-211 & $<6.89$ & $\mathrm{pCi} / \mathrm{g}$ & 6.89 & & 3/31/08 & 8C17008 & AGG-RRL-001 \\
\hline & Lead-211 & $<9.41$ & $\mathrm{pCi} / \mathrm{g}$ & 9.41 & & 3/31/08 & 8C17008 & AGG-RRL-001 \\
\hline 15092-94-1 & Lead-212 & $<0.816$ & $\mathrm{pCi} / \mathrm{g}$ & 0.816 & & 3/31/08 & 8C17008 & AGG-RRL-001 \\
\hline 14733-03-0 & Bismuth-214 & $<0.796$ & $\mathrm{pCi} / \mathrm{g}$ & 0.796 & & 3/31/08 & 8C17008 & AGG-RRL-001 \\
\hline $15067-28-4$ & Lead-214 & $<0.84$ & $\mathrm{pCi} / \mathrm{g}$ & 0.84 & & 3/31/08 & 8C17008 & AGG-RRL-001 \\
\hline $14835-02-0$ & Radon-219 & $<2.98$ & $\mathrm{pCi} / \mathrm{g}$ & 2.98 & & 3/31/08 & 8C17008 & AGG-RRL-001 \\
\hline 22481-48-7 & Radon-220 & $<311$ & $\mathrm{pCi} / \mathrm{g}$ & 311 & & 3/31/08 & 8C17008 & AGG-RRL-001 \\
\hline 28522-20-5 & Radon-221 & $<1.47$ & $\mathrm{pCi} / \mathrm{g}$ & 1.47 & & 3/31/08 & 8C17008 & AGG-RRL-001 \\
\hline 15756-98-6 & Francium-223 & $<10.4$ & $\mathrm{pCi} / \mathrm{g}$ & 10.4 & & 3/31/08 & 8C17008 & AGG-RRL-001 \\
\hline $15623-45-7$ & Radium-223 & $<2.2$ & $\mathrm{pCi} / \mathrm{g}$ & 2.2 & & 3/31/08 & 8C17008 & AGG-RRL-001 \\
\hline 13233-32-4 & Radium-224 & $<8.91$ & $\mathrm{pCi} / \mathrm{g}$ & 8.91 & & 3/31/08 & 8C17008 & AGG-RRL-001 \\
\hline 13982-63-3 & Radium-226 & $<9.05$ & $\mathrm{pCi} / \mathrm{g}$ & 9.05 & & 3/31/08 & 8C17008 & AGG-RRL-001 \\
\hline $15623-47-9$ & Thorium-227 & $<3.17$ & $\mathrm{pCi} / \mathrm{g}$ & 3.17 & & 3/31/08 & 8C17008 & AGG-RRL-001 \\
\hline 14331-83-0 & Actinium-228 & $<1.31$ & $\mathrm{pCi} / \mathrm{g}$ & 1.31 & & 3/31/08 & 8C17008 & AGG-RRL-001 \\
\hline 14274-82-9 & Thorium-228 & $<111$ & $\mathrm{pCi} / \mathrm{g}$ & 111 & & 3/31/08 & 8C17008 & AGG-RRL-001 \\
\hline
\end{tabular}


GEA/Soil

\begin{tabular}{|c|c|c|c|c|c|c|c|c|}
\hline CAS \# & Analyte & Results & Units & MDA & UNC & Analyzed & Batch & Method \\
\hline HEIS No. & B1T2P9A & \multicolumn{2}{|c|}{ Lab ID: } & 0803015-11 & & & & \\
\hline 14269-63-7 & Thorium-230 & $<116$ & $\mathrm{pCi} / \mathrm{g}$ & 116 & & 3/31/08 & 8C17008 & AGG-RRL-001 \\
\hline 14331-85-2 & Protactinium-231 & $<13.5$ & $\mathrm{pCi} / \mathrm{g}$ & 13.5 & & $3 / 31 / 08$ & 8C17008 & AGG-RRL-001 \\
\hline $14932-40-2$ & Thorium-231 & $<70.1$ & $\mathrm{pCi} / \mathrm{g}$ & 70.1 & & 3/31/08 & 8C17008 & AGG-RRL-001 \\
\hline 7440-29-1 & Thorium-232 & $<288$ & $\mathrm{pCi} / \mathrm{g}$ & 288 & & 3/31/08 & 8C17008 & AGG-RRL-001 \\
\hline 13981-14-1 & Protactinium-233 & $<0.855$ & $\mathrm{pCi} / \mathrm{g}$ & 0.855 & & 3/31/08 & 8C17008 & AGG-RRL-001 \\
\hline \multirow[t]{2}{*}{$15100-28-4$} & Protactinium-234 & $<1.27$ & $\mathrm{pCi} / \mathrm{g}$ & 1.27 & & 3/31/08 & 8C17008 & AGG-RRL-001 \\
\hline & Protactinium-234m & $<37.8$ & $\mathrm{pCi} / \mathrm{g}$ & 37.8 & & 3/31/08 & 8C17008 & AGG-RRL-001 \\
\hline $15065-10-8$ & Thorium-234 & $<10.2$ & $\mathrm{pCi} / \mathrm{g}$ & 10.2 & & 3/31/08 & 8C17008 & AGG-RRL-001 \\
\hline 15117-96-1 & Uranium 235 & $<2.73$ & $\mathrm{pCi} / \mathrm{g}$ & 2.73 & & 3/31/08 & 8C17008 & AGG-RRL-001 \\
\hline 13994-20-2 & Neptunium-237 & $<2.69$ & $\mathrm{pCi} / \mathrm{g}$ & 2.69 & & 3/31/08 & 8C17008 & AGG-RRL-001 \\
\hline \multirow[t]{2}{*}{ 13981-16-3 } & Plutonium-238 & $<4000$ & $\mathrm{pCi} / \mathrm{g}$ & 4000 & & 3/31/08 & 8C17008 & AGG-RRL-001 \\
\hline & Uranium 238 & $<5.62$ & $\mathrm{pCi} / \mathrm{g}$ & 5.62 & & 3/31/08 & 8C17008 & AGG-RRL-001 \\
\hline $15117-48-3$ & Plutonium-239 & $<3990$ & $\mathrm{pCi} / \mathrm{g}$ & 3990 & & $3 / 31 / 08$ & 8C17008 & AGG-RRL-001 \\
\hline 14119-33-6 & Plutonium-240 & $<4050$ & $\mathrm{pCi} / \mathrm{g}$ & 4050 & & 3/31/08 & 8C17008 & AGG-RRL-001 \\
\hline 14596-10-2 & Americium-241 & $<1.48$ & $\mathrm{pCi} / \mathrm{g}$ & 1.48 & & 3/31/08 & 8C17008 & AGG-RRL-001 \\
\hline 14993-75-0 & Americium-243 & $<0.646$ & $\mathrm{pCi} / \mathrm{g}$ & 0.646 & & 3/31/08 & 8C17008 & AGG-RRL-001 \\
\hline 15757-87-6 & Curium-243 & $<1.24$ & $\mathrm{pCi} / \mathrm{g}$ & 1.24 & & 3/31/08 & 8C17008 & AGG-RRL-001 \\
\hline 15621-76-8 & Curium-245 & $<1.02$ & $\mathrm{pCi} / \mathrm{g}$ & 1.02 & & $3 / 31 / 08$ & 8C17008 & AGG-RRL-001 \\
\hline HEIS No. & B1T2R0B & \multicolumn{2}{|c|}{ Lab ID: } & 0803015-14 & & & & \\
\hline 13966-32-0 & Sodium-22 & $<0.407$ & $\mathrm{pCi} / \mathrm{g}$ & 0.407 & & 3/31/08 & 8C17008 & AGG-RRL-001 \\
\hline 13966-00-2 & Potassium-40 & 18.5 & $\mathrm{pCi} / \mathrm{g}$ & 3.46 & 1.31 & 3/31/08 & 8C17008 & AGG-RRL-001 \\
\hline $14392-02-0$ & Chromium-51 & $<3.52$ & $\mathrm{pCi} / \mathrm{g}$ & 3.52 & & 3/31/08 & 8C17008 & AGG-RRL-001 \\
\hline 13966-31-9 & Manganese-54 & $<0.399$ & $\mathrm{pCi} / \mathrm{g}$ & 0.399 & & 3/31/08 & 8C17008 & AGG-RRL-001 \\
\hline 13981-50-5 & Cobalt-57 & $<0.359$ & $\mathrm{pCi} / \mathrm{g}$ & 0.359 & & 3/31/08 & 8C17008 & AGG-RRL-001 \\
\hline $14596-12-4$ & Iron-59 & $<0.705$ & $\mathrm{pCi} / \mathrm{g}$ & 0.705 & & 3/31/08 & 8C17008 & AGG-RRL-001 \\
\hline 10198-40-0 & Cobalt-60 & $<0.338$ & $\mathrm{pCi} / \mathrm{g}$ & 0.338 & & 3/31/08 & 8C17008 & AGG-RRL-001 \\
\hline 13982-39-3 & Zinc-65 & $<0.845$ & $\mathrm{pCi} / \mathrm{g}$ & 0.845 & & $3 / 31 / 08$ & 8C17008 & AGG-RRL-001 \\
\hline $14265-71-5$ & Selenium-75 & $<0.584$ & $\mathrm{pCi} / \mathrm{g}$ & 0.584 & & 3/31/08 & 8C17008 & AGG-RRL-001 \\
\hline 17056-36-9 & Rubidium-83 & $<0.9$ & $\mathrm{pCi} / \mathrm{g}$ & 0.9 & & 3/31/08 & 8C17008 & AGG-RRL-001 \\
\hline 13967-73-2 & Strontium-85 & $<0.418$ & $\mathrm{pCi} / \mathrm{g}$ & 0.418 & & 3/31/08 & 8C17008 & AGG-RRL-001 \\
\hline $14932-53-7$ & Rubidium-86 & $<4.41$ & $\mathrm{pCi} / \mathrm{g}$ & 4.41 & & 3/31/08 & 8C17008 & AGG-RRL-001 \\
\hline 13982-36-0 & Yttrium-88 & $<0.298$ & $\mathrm{pCi} / \mathrm{g}$ & 0.298 & & 3/31/08 & 8C17008 & AGG-RRL-001 \\
\hline 14681-63-1 & Niobium-94 & $<0.365$ & $\mathrm{pCi} / \mathrm{g}$ & 0.365 & & 3/31/08 & 8C17008 & AGG-RRL-001 \\
\hline \multirow[t]{3}{*}{ 13967-76-5 } & Niobium-95 & $<0.371$ & $\mathrm{pCi} / \mathrm{g}$ & 0.371 & & 3/31/08 & 8C17008 & AGG-RRL-001 \\
\hline & Niobium-95m & $<1.59$ & $\mathrm{pCi} / \mathrm{g}$ & 1.59 & & 3/31/08 & 8C17008 & AGG-RRL-001 \\
\hline & Technetium-95m & $<0.56$ & $\mathrm{pCi} / \mathrm{g}$ & 0.56 & & 3/31/08 & 8C17008 & AGG-RRL-001 \\
\hline \multirow[t]{2}{*}{ 13967-71-0 } & Zirconium-95 & $<0.669$ & $\mathrm{pCi} / \mathrm{g}$ & 0.669 & & 3/31/08 & 8C17008 & AGG-RRL-001 \\
\hline & Technetium-99m & $<0.366$ & $\mathrm{pCi} / \mathrm{g}$ & 0.366 & & 3/31/08 & 8C17008 & AGG-RRL-001 \\
\hline 13968-53-1 & Ruthenium-103 & $<0.419$ & $\mathrm{pCi} / \mathrm{g}$ & 0.419 & & 3/31/08 & 8C17008 & AGG-RRL-001 \\
\hline 13967-48-1 & Ruthenium-106 & $<4$ & $\mathrm{pCi} / \mathrm{g}$ & 4 & & 3/31/08 & 8C17008 & AGG-RRL-001 \\
\hline 14391-65-2 & Silver-108m & $<0.365$ & $\mathrm{pCi} / \mathrm{g}$ & 0.365 & & 3/31/08 & 8C17008 & AGG-RRL-001 \\
\hline \multirow[t]{2}{*}{$14109-32-1$} & Cadmium-109 & $<10.3$ & $\mathrm{pCi} / \mathrm{g}$ & 10.3 & & 3/31/08 & 8C17008 & AGG-RRL-001 \\
\hline & Silver-110 & $<0.384$ & $\mathrm{pCi} / \mathrm{g}$ & 0.384 & & 3/31/08 & 8C17008 & AGG-RRL-001 \\
\hline 14391-76-5 & Silver-110m & $<0.385$ & $\mathrm{pCi} / \mathrm{g}$ & 0.385 & & 3/31/08 & 8C17008 & AGG-RRL-001 \\
\hline 13966-06-8 & Tin-113 & $<0.511$ & $\mathrm{pCi} / \mathrm{g}$ & 0.511 & & 3/31/08 & 8C17008 & AGG-RRL-001 \\
\hline $14683-10-4$ & Antimony-124 & $<0.421$ & $\mathrm{pCi} / \mathrm{g}$ & 0.421 & & 3/31/08 & 8C17008 & AGG-RRL-001 \\
\hline 14234-35-6 & Antimony-125 & $<1.12$ & $\mathrm{pCi} / \mathrm{g}$ & 1.12 & & 3/31/08 & 8C17008 & AGG-RRL-001 \\
\hline 15756-32-8 & Antimony-126 & $<0.368$ & $\mathrm{pCi} / \mathrm{g}$ & 0.368 & & 3/31/08 & 8C17008 & AGG-RRL-001 \\
\hline
\end{tabular}


GEA/Soil

\begin{tabular}{|c|c|c|c|c|c|c|c|c|}
\hline CAS \# & Analyte & Results & Units & MDA & UNC & Analyzed & Batch & Method \\
\hline HEIS No. & B1T2R0B & \multicolumn{3}{|c|}{ Lab ID: $\quad$ 0803015-14 } & & & & \\
\hline $15832-50-5$ & Tin-126 & $<0.839$ & $\mathrm{pCi} / \mathrm{g}$ & 0.839 & & 3/31/08 & 8C17008 & AGG-RRL-001 \\
\hline $10043-66-0$ & Iodine-131 & $<0.449$ & $\mathrm{pCi} / \mathrm{g}$ & 0.449 & & 3/31/08 & 8C17008 & AGG-RRL-001 \\
\hline 13981-41-4 & Barium-133 & $<0.628$ & $\mathrm{pCi} / \mathrm{g}$ & 0.628 & & 3/31/08 & 8C17008 & AGG-RRL-001 \\
\hline 13967-70-9 & Cesium-134 & $<0.418$ & $\mathrm{pCi} / \mathrm{g}$ & 0.418 & & 3/31/08 & 8C17008 & AGG-RRL-001 \\
\hline $10045-97-3$ & Cesium-137 & $<0.421$ & $\mathrm{pCi} / \mathrm{g}$ & 0.421 & & $3 / 31 / 08$ & 8C17008 & AGG-RRL-001 \\
\hline 13982-30-4 & Cerium-139 & $<0.426$ & $\mathrm{pCi} / \mathrm{g}$ & 0.426 & & 3/31/08 & 8C17008 & AGG-RRL-001 \\
\hline $14762-78-8$ & Cerium-144 & $<2.98$ & $\mathrm{pCi} / \mathrm{g}$ & 2.98 & & $3 / 31 / 08$ & 8C17008 & AGG-RRL-001 \\
\hline 14683-23-9 & Europium-152 & $<1.45$ & $\mathrm{pCi} / \mathrm{g}$ & 1.45 & & 3/31/08 & 8C17008 & AGG-RRL-001 \\
\hline $14276-65-4$ & Gadolinium-153 & $<1.13$ & $\mathrm{pCi} / \mathrm{g}$ & 1.13 & & $3 / 31 / 08$ & 8C17008 & AGG-RRL-001 \\
\hline 15585-10-1 & Europium-154 & $<0.764$ & $\mathrm{pCi} / \mathrm{g}$ & 0.764 & & $3 / 31 / 08$ & 8C17008 & AGG-RRL-001 \\
\hline 14391-16-3 & Europium-155 & $<1.18$ & $\mathrm{pCi} / \mathrm{g}$ & 1.18 & & 3/31/08 & 8C17008 & AGG-RRL-001 \\
\hline 13982-78-0 & Mercury-203 & $<0.446$ & $\mathrm{pCi} / \mathrm{g}$ & 0.446 & & $3 / 31 / 08$ & 8C17008 & AGG-RRL-001 \\
\hline 14913-50-9 & Thallium-208 & $<0.484$ & $\mathrm{pCi} / \mathrm{g}$ & 0.484 & & $3 / 31 / 08$ & 8C17008 & AGG-RRL-001 \\
\hline 14331-79-4 & Bismuth-210 & $<0.693$ & $\mathrm{pCi} / \mathrm{g}$ & 0.693 & & 3/31/08 & 8C17008 & AGG-RRL-001 \\
\hline \multirow[t]{3}{*}{$14255-04-0$} & Lead-210 & $<49.8$ & $\mathrm{pCi} / \mathrm{g}$ & 49.8 & & 3/31/08 & 8C17008 & AGG-RRL-001 \\
\hline & Bismuth-211 & $<8.24$ & $\mathrm{pCi} / \mathrm{g}$ & 8.24 & & 3/31/08 & 8C17008 & AGG-RRL-001 \\
\hline & Lead-211 & $<11.3$ & $\mathrm{pCi} / \mathrm{g}$ & 11.3 & & 3/31/08 & 8C17008 & AGG-RRL-001 \\
\hline 15092-94-1 & Lead-212 & $<0.934$ & $\mathrm{pCi} / \mathrm{g}$ & 0.934 & & 3/31/08 & 8C17008 & AGG-RRL-001 \\
\hline 14733-03-0 & Bismuth-214 & $<0.91$ & $\mathrm{pCi} / \mathrm{g}$ & 0.91 & & 3/31/08 & 8C17008 & AGG-RRL-001 \\
\hline 15067-28-4 & Lead-214 & $<1$ & $\mathrm{pCi} / \mathrm{g}$ & 1 & & 3/31/08 & 8C17008 & AGG-RRL-001 \\
\hline $14835-02-0$ & Radon-219 & $<3.48$ & $\mathrm{pCi} / \mathrm{g}$ & 3.48 & & 3/31/08 & 8C17008 & AGG-RRL-001 \\
\hline 22481-48-7 & Radon-220 & $<351$ & $\mathrm{pCi} / \mathrm{g}$ & 351 & & 3/31/08 & 8C17008 & AGG-RRL-001 \\
\hline 28522-20-5 & Radon-221 & $<1.61$ & $\mathrm{pCi} / \mathrm{g}$ & 1.61 & & $3 / 31 / 08$ & 8C17008 & AGG-RRL-001 \\
\hline 15756-98-6 & Francium-223 & $<11.7$ & $\mathrm{pCi} / \mathrm{g}$ & 11.7 & & 3/31/08 & 8C17008 & AGG-RRL-001 \\
\hline $15623-45-7$ & Radium-223 & $<2.55$ & $\mathrm{pCi} / \mathrm{g}$ & 2.55 & & 3/31/08 & 8C17008 & AGG-RRL-001 \\
\hline 13233-32-4 & Radium-224 & $<10.1$ & $\mathrm{pCi} / \mathrm{g}$ & 10.1 & & $3 / 31 / 08$ & 8C17008 & AGG-RRL-001 \\
\hline 13982-63-3 & Radium-226 & $<9.94$ & $\mathrm{pCi} / \mathrm{g}$ & 9.94 & & 3/31/08 & 8C17008 & AGG-RRL-001 \\
\hline 15623-47-9 & Thorium-227 & $<3.56$ & $\mathrm{pCi} / \mathrm{g}$ & 3.56 & & 3/31/08 & 8C17008 & AGG-RRL-001 \\
\hline 14331-83-0 & Actinium-228 & $<1.53$ & $\mathrm{pCi} / \mathrm{g}$ & 1.53 & & 3/31/08 & 8C17008 & AGG-RRL-001 \\
\hline 14274-82-9 & Thorium-228 & $<130$ & $\mathrm{pCi} / \mathrm{g}$ & 130 & & 3/31/08 & 8C17008 & AGG-RRL-001 \\
\hline 14269-63-7 & Thorium-230 & $<133$ & $\mathrm{pCi} / \mathrm{g}$ & 133 & & 3/31/08 & 8C17008 & AGG-RRL-001 \\
\hline 14331-85-2 & Protactinium-231 & $<15.6$ & $\mathrm{pCi} / \mathrm{g}$ & 15.6 & & 3/31/08 & 8C17008 & AGG-RRL-001 \\
\hline $14932-40-2$ & Thorium-231 & $<80.4$ & $\mathrm{pCi} / \mathrm{g}$ & 80.4 & & 3/31/08 & 8C17008 & AGG-RRL-001 \\
\hline 7440-29-1 & Thorium-232 & $<331$ & $\mathrm{pCi} / \mathrm{g}$ & 331 & & 3/31/08 & 8C17008 & AGG-RRL-001 \\
\hline 13981-14-1 & Protactinium-233 & $<0.975$ & $\mathrm{pCi} / \mathrm{g}$ & 0.975 & & 3/31/08 & 8C17008 & AGG-RRL-001 \\
\hline \multirow[t]{2}{*}{$15100-28-4$} & Protactinium-234 & $<1.47$ & $\mathrm{pCi} / \mathrm{g}$ & 1.47 & & 3/31/08 & 8C17008 & AGG-RRL-001 \\
\hline & Protactinium-234m & $<44.7$ & $\mathrm{pCi} / \mathrm{g}$ & 44.7 & & 3/31/08 & 8C17008 & AGG-RRL-001 \\
\hline $15065-10-8$ & Thorium-234 & $<11.7$ & $\mathrm{pCi} / \mathrm{g}$ & 11.7 & & 3/31/08 & 8C17008 & AGG-RRL-001 \\
\hline 15117-96-1 & Uranium 235 & $<3.13$ & $\mathrm{pCi} / \mathrm{g}$ & 3.13 & & 3/31/08 & 8C17008 & AGG-RRL-001 \\
\hline 13994-20-2 & Neptunium-237 & $<3.08$ & $\mathrm{pCi} / \mathrm{g}$ & 3.08 & & 3/31/08 & 8C17008 & AGG-RRL-001 \\
\hline \multirow[t]{2}{*}{ 13981-16-3 } & Plutonium-238 & $<4660$ & $\mathrm{pCi} / \mathrm{g}$ & 4660 & & 3/31/08 & 8C17008 & AGG-RRL-001 \\
\hline & Uranium 238 & $<6.53$ & $\mathrm{pCi} / \mathrm{g}$ & 6.53 & & 3/31/08 & 8C17008 & AGG-RRL-001 \\
\hline $15117-48-3$ & Plutonium-239 & $<4670$ & $\mathrm{pCi} / \mathrm{g}$ & 4670 & & 3/31/08 & 8C17008 & AGG-RRL-001 \\
\hline 14119-33-6 & Plutonium-240 & $<4570$ & $\mathrm{pCi} / \mathrm{g}$ & 4570 & & 3/31/08 & 8C17008 & AGG-RRL-001 \\
\hline $14596-10-2$ & Americium-241 & $<1.69$ & $\mathrm{pCi} / \mathrm{g}$ & 1.69 & & 3/31/08 & 8C17008 & AGG-RRL-001 \\
\hline 14993-75-0 & Americium-243 & $<0.731$ & $\mathrm{pCi} / \mathrm{g}$ & 0.731 & & 3/31/08 & 8C17008 & AGG-RRL-001 \\
\hline 15757-87-6 & Curium-243 & $<1.4$ & $\mathrm{pCi} / \mathrm{g}$ & 1.4 & & 3/31/08 & 8C17008 & AGG-RRL-001 \\
\hline 15621-76-8 & Curium-245 & $<1.15$ & $\mathrm{pCi} / \mathrm{g}$ & 1.15 & & 3/31/08 & 8C17008 & AGG-RRL-001 \\
\hline
\end{tabular}


GEA/Soil

\begin{tabular}{|c|c|c|c|c|c|c|c|c|}
\hline CAS \# & Analyte & Results & Units & MDA & UNC & Analyzed & Batch & Method \\
\hline HEIS No. & B1T2R0A & \multicolumn{3}{|c|}{ Lab ID: $\quad$ 0803015-15 } & & & & \\
\hline 13966-32-0 & Sodium-22 & $<0.336$ & $\mathrm{pCi} / \mathrm{g}$ & 0.336 & & 3/31/08 & 8C17008 & AGG-RRL-001 \\
\hline 13966-00-2 & Potassium-40 & 19.4 & $\mathrm{pCi} / \mathrm{g}$ & 2.62 & 1.24 & $3 / 31 / 08$ & 8C17008 & AGG-RRL-001 \\
\hline $14392-02-0$ & Chromium-51 & $<2.71$ & $\mathrm{pCi} / \mathrm{g}$ & 2.71 & & 3/31/08 & 8C17008 & AGG-RRL-001 \\
\hline 13966-31-9 & Manganese-54 & $<0.258$ & $\mathrm{pCi} / \mathrm{g}$ & 0.258 & & 3/31/08 & 8C17008 & AGG-RRL-001 \\
\hline 13981-50-5 & Cobalt-57 & $<0.293$ & $\mathrm{pCi} / \mathrm{g}$ & 0.293 & & 3/31/08 & 8C17008 & AGG-RRL-001 \\
\hline 14596-12-4 & Iron-59 & $<0.549$ & $\mathrm{pCi} / \mathrm{g}$ & 0.549 & & 3/31/08 & 8C17008 & AGG-RRL-001 \\
\hline 10198-40-0 & Cobalt-60 & $<0.282$ & $\mathrm{pCi} / \mathrm{g}$ & 0.282 & & 3/31/08 & 8C17008 & AGG-RRL-001 \\
\hline 13982-39-3 & Zinc-65 & $<0.764$ & $\mathrm{pCi} / \mathrm{g}$ & 0.764 & & 3/31/08 & 8C17008 & AGG-RRL-001 \\
\hline 14265-71-5 & Selenium-75 & $<0.466$ & $\mathrm{pCi} / \mathrm{g}$ & 0.466 & & 3/31/08 & 8C17008 & AGG-RRL-001 \\
\hline 17056-36-9 & Rubidium-83 & $<0.585$ & $\mathrm{pCi} / \mathrm{g}$ & 0.585 & & $3 / 31 / 08$ & 8C17008 & AGG-RRL-001 \\
\hline 13967-73-2 & Strontium-85 & $<0.368$ & $\mathrm{pCi} / \mathrm{g}$ & 0.368 & & 3/31/08 & 8C17008 & AGG-RRL-001 \\
\hline 14932-53-7 & Rubidium-86 & $<3.66$ & $\mathrm{pCi} / \mathrm{g}$ & 3.66 & & $3 / 31 / 08$ & 8C17008 & AGG-RRL-001 \\
\hline 13982-36-0 & Yttrium-88 & $<0.279$ & $\mathrm{pCi} / \mathrm{g}$ & 0.279 & & $3 / 31 / 08$ & 8C17008 & AGG-RRL-001 \\
\hline 14681-63-1 & Niobium-94 & $<0.295$ & $\mathrm{pCi} / \mathrm{g}$ & 0.295 & & 3/31/08 & 8C17008 & AGG-RRL-001 \\
\hline \multirow[t]{3}{*}{ 13967-76-5 } & Niobium-95 & $<0.302$ & $\mathrm{pCi} / \mathrm{g}$ & 0.302 & & 3/31/08 & 8C17008 & AGG-RRL-001 \\
\hline & Niobium-95m & $<1.36$ & $\mathrm{pCi} / \mathrm{g}$ & 1.36 & & 3/31/08 & 8C17008 & AGG-RRL-001 \\
\hline & Technetium-95m & $<0.459$ & $\mathrm{pCi} / \mathrm{g}$ & 0.459 & & 3/31/08 & 8C17008 & AGG-RRL-001 \\
\hline \multirow[t]{2}{*}{ 13967-71-0 } & Zirconium-95 & $<0.473$ & $\mathrm{pCi} / \mathrm{g}$ & 0.473 & & 3/31/08 & 8C17008 & AGG-RRL-001 \\
\hline & Technetium-99m & $<0.294$ & $\mathrm{pCi} / \mathrm{g}$ & 0.294 & & 3/31/08 & 8C17008 & AGG-RRL-001 \\
\hline 13968-53-1 & Ruthenium-103 & $<0.299$ & $\mathrm{pCi} / \mathrm{g}$ & 0.299 & & 3/31/08 & 8C17008 & AGG-RRL-001 \\
\hline 13967-48-1 & Ruthenium-106 & $<2.66$ & $\mathrm{pCi} / \mathrm{g}$ & 2.66 & & 3/31/08 & 8C17008 & AGG-RRL-001 \\
\hline 14391-65-2 & Silver-108m & $<0.307$ & $\mathrm{pCi} / \mathrm{g}$ & 0.307 & & 3/31/08 & 8C17008 & AGG-RRL-001 \\
\hline \multirow[t]{2}{*}{ 14109-32-1 } & Cadmium-109 & $<7.96$ & $\mathrm{pCi} / \mathrm{g}$ & 7.96 & & 3/31/08 & 8C17008 & AGG-RRL-001 \\
\hline & Silver-110 & $<0.343$ & $\mathrm{pCi} / \mathrm{g}$ & 0.343 & & 3/31/08 & 8C17008 & AGG-RRL-001 \\
\hline 14391-76-5 & Silver-110m & $<0.344$ & $\mathrm{pCi} / \mathrm{g}$ & 0.344 & & 3/31/08 & 8C17008 & AGG-RRL-001 \\
\hline 13966-06-8 & Tin-113 & $<0.406$ & $\mathrm{pCi} / \mathrm{g}$ & 0.406 & & 3/31/08 & 8C17008 & AGG-RRL-001 \\
\hline 14683-10-4 & Antimony-124 & $<0.308$ & $\mathrm{pCi} / \mathrm{g}$ & 0.308 & & 3/31/08 & 8C17008 & AGG-RRL-001 \\
\hline $14234-35-6$ & Antimony-125 & $<0.93$ & $\mathrm{pCi} / \mathrm{g}$ & 0.93 & & 3/31/08 & 8C17008 & AGG-RRL-001 \\
\hline 15756-32-8 & Antimony-126 & $<0.327$ & $\mathrm{pCi} / \mathrm{g}$ & 0.327 & & 3/31/08 & 8C17008 & AGG-RRL-001 \\
\hline 15832-50-5 & Tin-126 & $<0.62$ & $\mathrm{pCi} / \mathrm{g}$ & 0.62 & & 3/31/08 & 8C17008 & AGG-RRL-001 \\
\hline 10043-66-0 & Iodine-131 & $<0.351$ & $\mathrm{pCi} / \mathrm{g}$ & 0.351 & & 3/31/08 & 8C17008 & AGG-RRL-001 \\
\hline 13981-41-4 & Barium-133 & $<0.56$ & $\mathrm{pCi} / \mathrm{g}$ & 0.56 & & 3/31/08 & 8C17008 & AGG-RRL-001 \\
\hline 13967-70-9 & Cesium-134 & $<0.345$ & $\mathrm{pCi} / \mathrm{g}$ & 0.345 & & 3/31/08 & 8C17008 & AGG-RRL-001 \\
\hline $10045-97-3$ & Cesium-137 & $<0.381$ & $\mathrm{pCi} / \mathrm{g}$ & 0.381 & & 3/31/08 & 8C17008 & AGG-RRL-001 \\
\hline 13982-30-4 & Cerium-139 & $<0.337$ & $\mathrm{pCi} / \mathrm{g}$ & 0.337 & & 3/31/08 & 8C17008 & AGG-RRL-001 \\
\hline 14762-78-8 & Cerium-144 & $<2.39$ & $\mathrm{pCi} / \mathrm{g}$ & 2.39 & & 3/31/08 & 8C17008 & AGG-RRL-001 \\
\hline 14683-23-9 & Europium-152 & $<1.09$ & $\mathrm{pCi} / \mathrm{g}$ & 1.09 & & 3/31/08 & 8C17008 & AGG-RRL-001 \\
\hline $14276-65-4$ & Gadolinium-153 & $<0.857$ & $\mathrm{pCi} / \mathrm{g}$ & 0.857 & & 3/31/08 & 8C17008 & AGG-RRL-001 \\
\hline 15585-10-1 & Europium-154 & $<0.619$ & $\mathrm{pCi} / \mathrm{g}$ & 0.619 & & 3/31/08 & 8C17008 & AGG-RRL-001 \\
\hline 14391-16-3 & Europium-155 & $<0.872$ & $\mathrm{pCi} / \mathrm{g}$ & 0.872 & & 3/31/08 & 8C17008 & AGG-RRL-001 \\
\hline 13982-78-0 & Mercury-203 & $<0.37$ & $\mathrm{pCi} / \mathrm{g}$ & 0.37 & & 3/31/08 & 8C17008 & AGG-RRL-001 \\
\hline 14913-50-9 & Thallium-208 & $<0.302$ & $\mathrm{pCi} / \mathrm{g}$ & 0.302 & 0.0667 & 3/31/08 & 8C17008 & AGG-RRL-001 \\
\hline 14331-79-4 & Bismuth-210 & $<0.563$ & $\mathrm{pCi} / \mathrm{g}$ & 0.563 & & 3/31/08 & 8C17008 & AGG-RRL-001 \\
\hline \multirow[t]{3}{*}{$14255-04-0$} & Lead-210 & $<112$ & $\mathrm{pCi} / \mathrm{g}$ & 112 & & 3/31/08 & 8C17008 & AGG-RRL-001 \\
\hline & Bismuth-211 & $<6.78$ & $\mathrm{pCi} / \mathrm{g}$ & 6.78 & & 3/31/08 & 8C17008 & AGG-RRL-001 \\
\hline & Lead-211 & $<9.26$ & $\mathrm{pCi} / \mathrm{g}$ & 9.26 & & 3/31/08 & 8C17008 & AGG-RRL-001 \\
\hline 15092-94-1 & Lead-212 & 0.624 & $\mathrm{pCi} / \mathrm{g}$ & 0.541 & 0.238 & 3/31/08 & 8C17008 & AGG-RRL-001 \\
\hline 14733-03-0 & Bismuth-214 & $<0.759$ & $\mathrm{pCi} / \mathrm{g}$ & 0.759 & & 3/31/08 & 8C17008 & AGG-RRL-001 \\
\hline $15067-28-4$ & Lead-214 & 0.946 & $\mathrm{pCi} / \mathrm{g}$ & 0.671 & 0.529 & 3/31/08 & 8C17008 & AGG-RRL-001 \\
\hline
\end{tabular}


GEA/Soil

\begin{tabular}{|c|c|c|c|c|c|c|c|c|}
\hline CAS \# & Analyte & Results & Units & MDA & UNC & Analyzed & Batch & Method \\
\hline HEIS No. & B1T2R0A & \multicolumn{2}{|c|}{ Lab ID: } & 0803015-15 & & & & \\
\hline $14835-02-0$ & Radon-219 & $<2.9$ & $\mathrm{pCi} / \mathrm{g}$ & 2.9 & & 3/31/08 & 8C17008 & AGG-RRL-001 \\
\hline 22481-48-7 & Radon-220 & $<266$ & $\mathrm{pCi} / \mathrm{g}$ & 266 & & $3 / 31 / 08$ & 8C17008 & AGG-RRL-001 \\
\hline 28522-20-5 & Radon-221 & $<1.33$ & $\mathrm{pCi} / \mathrm{g}$ & 1.33 & & 3/31/08 & 8C17008 & AGG-RRL-001 \\
\hline 15756-98-6 & Francium-223 & $<9.9$ & $\mathrm{pCi} / \mathrm{g}$ & 9.9 & & 3/31/08 & 8C17008 & AGG-RRL-001 \\
\hline 15623-45-7 & Radium-223 & $<2.08$ & $\mathrm{pCi} / \mathrm{g}$ & 2.08 & & 3/31/08 & 8C17008 & AGG-RRL-001 \\
\hline 13233-32-4 & Radium-224 & $<5.64$ & $\mathrm{pCi} / \mathrm{g}$ & 5.64 & 0.83 & 3/31/08 & 8C17008 & AGG-RRL-001 \\
\hline 13982-63-3 & Radium-226 & $<8.27$ & $\mathrm{pCi} / \mathrm{g}$ & 8.27 & & $3 / 31 / 08$ & 8C17008 & AGG-RRL-001 \\
\hline 15623-47-9 & Thorium-227 & $<3.04$ & $\mathrm{pCi} / \mathrm{g}$ & 3.04 & & 3/31/08 & 8C17008 & AGG-RRL-001 \\
\hline 14331-83-0 & Actinium-228 & $<1.38$ & $\mathrm{pCi} / \mathrm{g}$ & 1.38 & & 3/31/08 & 8C17008 & AGG-RRL-001 \\
\hline $14274-82-9$ & Thorium-228 & $<108$ & $\mathrm{pCi} / \mathrm{g}$ & 108 & & 3/31/08 & 8C17008 & AGG-RRL-001 \\
\hline 14269-63-7 & Thorium-230 & $<112$ & $\mathrm{pCi} / \mathrm{g}$ & 112 & & 3/31/08 & 8C17008 & AGG-RRL-001 \\
\hline 14331-85-2 & Protactinium-231 & $<12.7$ & $\mathrm{pCi} / \mathrm{g}$ & 12.7 & & 3/31/08 & 8C17008 & AGG-RRL-001 \\
\hline $14932-40-2$ & Thorium-231 & $<59.9$ & $\mathrm{pCi} / \mathrm{g}$ & 59.9 & & 3/31/08 & 8C17008 & AGG-RRL-001 \\
\hline $7440-29-1$ & Thorium-232 & $<361$ & $\mathrm{pCi} / \mathrm{g}$ & 361 & & 3/31/08 & 8C17008 & AGG-RRL-001 \\
\hline 13981-14-1 & Protactinium-233 & $<0.798$ & $\mathrm{pCi} / \mathrm{g}$ & 0.798 & & 3/31/08 & 8C17008 & AGG-RRL-001 \\
\hline \multirow[t]{2}{*}{$15100-28-4$} & Protactinium-234 & $<1.1$ & $\mathrm{pCi} / \mathrm{g}$ & 1.1 & & 3/31/08 & 8C17008 & AGG-RRL-001 \\
\hline & Protactinium-234m & $<41.1$ & $\mathrm{pCi} / \mathrm{g}$ & 41.1 & & $3 / 31 / 08$ & 8C17008 & AGG-RRL-001 \\
\hline $15065-10-8$ & Thorium-234 & $<10.2$ & $\mathrm{pCi} / \mathrm{g}$ & 10.2 & & 3/31/08 & 8C17008 & AGG-RRL-001 \\
\hline 15117-96-1 & Uranium 235 & $<2.51$ & $\mathrm{pCi} / \mathrm{g}$ & 2.51 & & $3 / 31 / 08$ & 8C17008 & AGG-RRL-001 \\
\hline 13994-20-2 & Neptunium-237 & $<2.27$ & $\mathrm{pCi} / \mathrm{g}$ & 2.27 & & 3/31/08 & 8C17008 & AGG-RRL-001 \\
\hline \multirow[t]{2}{*}{ 13981-16-3 } & Plutonium-238 & $<3470$ & $\mathrm{pCi} / \mathrm{g}$ & 3470 & & $3 / 31 / 08$ & 8C17008 & AGG-RRL-001 \\
\hline & Uranium 238 & $<5.28$ & $\mathrm{pCi} / \mathrm{g}$ & 5.28 & & 3/31/08 & 8C17008 & AGG-RRL-001 \\
\hline 15117-48-3 & Plutonium-239 & $<4050$ & $\mathrm{pCi} / \mathrm{g}$ & 4050 & & 3/31/08 & 8C17008 & AGG-RRL-001 \\
\hline 14119-33-6 & Plutonium-240 & $<3550$ & $\mathrm{pCi} / \mathrm{g}$ & 3550 & & 3/31/08 & 8C17008 & AGG-RRL-001 \\
\hline $14596-10-2$ & Americium-241 & $<1.77$ & $\mathrm{pCi} / \mathrm{g}$ & 1.77 & & 3/31/08 & 8C17008 & AGG-RRL-001 \\
\hline 14993-75-0 & Americium-243 & $<0.619$ & $\mathrm{pCi} / \mathrm{g}$ & 0.619 & & $3 / 31 / 08$ & 8C17008 & AGG-RRL-001 \\
\hline 15757-87-6 & Curium-243 & $<1.08$ & $\mathrm{pCi} / \mathrm{g}$ & 1.08 & & 3/31/08 & 8C17008 & AGG-RRL-001 \\
\hline 15621-76-8 & Curium-245 & $<0.889$ & $\mathrm{pCi} / \mathrm{g}$ & 0.889 & & $3 / 31 / 08$ & 8C17008 & AGG-RRL-001 \\
\hline HEIS No. & B1TNK6B & \multicolumn{2}{|c|}{ Lab ID: } & 0803015-18 & & & & \\
\hline 13966-32-0 & Sodium-22 & $<0.388$ & $\mathrm{pCi} / \mathrm{g}$ & 0.388 & & 3/31/08 & 8C17008 & AGG-RRL-001 \\
\hline 13966-00-2 & Potassium-40 & 20.1 & $\mathrm{pCi} / \mathrm{g}$ & 3.21 & 1.32 & 3/31/08 & 8C17008 & AGG-RRL-001 \\
\hline 14392-02-0 & Chromium-51 & $<3.09$ & $\mathrm{pCi} / \mathrm{g}$ & 3.09 & & $3 / 31 / 08$ & 8C17008 & AGG-RRL-001 \\
\hline 13966-31-9 & Manganese-54 & $<0.316$ & $\mathrm{pCi} / \mathrm{g}$ & 0.316 & & 3/31/08 & 8C17008 & AGG-RRL-001 \\
\hline 13981-50-5 & Cobalt-57 & $<0.294$ & $\mathrm{pCi} / \mathrm{g}$ & 0.294 & & 3/31/08 & 8C17008 & AGG-RRL-001 \\
\hline $14596-12-4$ & Iron-59 & $<0.607$ & $\mathrm{pCi} / \mathrm{g}$ & 0.607 & & $3 / 31 / 08$ & 8C17008 & AGG-RRL-001 \\
\hline 10198-40-0 & Cobalt-60 & $<0.292$ & $\mathrm{pCi} / \mathrm{g}$ & 0.292 & & 3/31/08 & 8C17008 & AGG-RRL-001 \\
\hline 13982-39-3 & Zinc-65 & $<0.718$ & $\mathrm{pCi} / \mathrm{g}$ & 0.718 & & 3/31/08 & 8C17008 & AGG-RRL-001 \\
\hline $14265-71-5$ & Selenium-75 & $<0.472$ & $\mathrm{pCi} / \mathrm{g}$ & 0.472 & & 3/31/08 & 8C17008 & AGG-RRL-001 \\
\hline 17056-36-9 & Rubidium-83 & $<0.573$ & $\mathrm{pCi} / \mathrm{g}$ & 0.573 & & 3/31/08 & 8C17008 & AGG-RRL-001 \\
\hline 13967-73-2 & Strontium-85 & $<0.376$ & $\mathrm{pCi} / \mathrm{g}$ & 0.376 & & 3/31/08 & 8C17008 & AGG-RRL-001 \\
\hline $14932-53-7$ & Rubidium-86 & $<3.27$ & $\mathrm{pCi} / \mathrm{g}$ & 3.27 & & 3/31/08 & 8C17008 & AGG-RRL-001 \\
\hline 13982-36-0 & Yttrium-88 & $<0.24$ & $\mathrm{pCi} / \mathrm{g}$ & 0.24 & & 3/31/08 & 8C17008 & AGG-RRL-001 \\
\hline 14681-63-1 & Niobium-94 & $<0.296$ & $\mathrm{pCi} / \mathrm{g}$ & 0.296 & & 3/31/08 & 8C17008 & AGG-RRL-001 \\
\hline \multirow[t]{3}{*}{$13967-76-5$} & Niobium-95 & $<0.317$ & $\mathrm{pCi} / \mathrm{g}$ & 0.317 & & 3/31/08 & 8C17008 & AGG-RRL-001 \\
\hline & Niobium-95m & $<1.4$ & $\mathrm{pCi} / \mathrm{g}$ & 1.4 & & 3/31/08 & 8C17008 & AGG-RRL-001 \\
\hline & Technetium-95m & $<0.483$ & $\mathrm{pCi} / \mathrm{g}$ & 0.483 & & 3/31/08 & 8C17008 & AGG-RRL-001 \\
\hline \multirow[t]{2}{*}{ 13967-71-0 } & Zirconium-95 & $<0.491$ & $\mathrm{pCi} / \mathrm{g}$ & 0.491 & & 3/31/08 & 8C17008 & AGG-RRL-001 \\
\hline & Technetium-99m & $<0.301$ & $\mathrm{pCi} / \mathrm{g}$ & 0.301 & & 3/31/08 & 8C17008 & AGG-RRL-001 \\
\hline
\end{tabular}


GEA/Soil

\begin{tabular}{|c|c|c|c|c|c|c|c|c|}
\hline CAS \# & Analyte & Results & Units & MDA & UNC & Analyzed & Batch & Method \\
\hline HEIS No. & B1TNK6B & \multicolumn{2}{|c|}{ Lab ID: } & 0803015-18 & & & & \\
\hline 13968-53-1 & Ruthenium-103 & $<0.318$ & $\mathrm{pCi} / \mathrm{g}$ & 0.318 & & 3/31/08 & 8C17008 & AGG-RRL-001 \\
\hline 13967-48-1 & Ruthenium-106 & $<2.73$ & $\mathrm{pCi} / \mathrm{g}$ & 2.73 & & 3/31/08 & 8C17008 & AGG-RRL-001 \\
\hline 14391-65-2 & Silver-108m & $<0.29$ & $\mathrm{pCi} / \mathrm{g}$ & 0.29 & & 3/31/08 & 8C17008 & AGG-RRL-001 \\
\hline \multirow[t]{2}{*}{ 14109-32-1 } & Cadmium-109 & $<7.87$ & $\mathrm{pCi} / \mathrm{g}$ & 7.87 & & 3/31/08 & 8C17008 & AGG-RRL-001 \\
\hline & Silver-110 & $<0.297$ & $\mathrm{pCi} / \mathrm{g}$ & 0.297 & & 3/31/08 & 8C17008 & AGG-RRL-001 \\
\hline 14391-76-5 & Silver-110m & $<0.298$ & $\mathrm{pCi} / \mathrm{g}$ & 0.298 & & 3/31/08 & 8C17008 & AGG-RRL-001 \\
\hline 13966-06-8 & Tin-113 & $<0.451$ & $\mathrm{pCi} / \mathrm{g}$ & 0.451 & & 3/31/08 & 8C17008 & AGG-RRL-001 \\
\hline 14683-10-4 & Antimony-124 & $<0.303$ & $\mathrm{pCi} / \mathrm{g}$ & 0.303 & & 3/31/08 & 8C17008 & AGG-RRL-001 \\
\hline $14234-35-6$ & Antimony-125 & $<0.921$ & $\mathrm{pCi} / \mathrm{g}$ & 0.921 & & 3/31/08 & 8C17008 & AGG-RRL-001 \\
\hline 15756-32-8 & Antimony-126 & $<0.304$ & $\mathrm{pCi} / \mathrm{g}$ & 0.304 & & 3/31/08 & 8C17008 & AGG-RRL-001 \\
\hline 15832-50-5 & Tin-126 & $<0.614$ & $\mathrm{pCi} / \mathrm{g}$ & 0.614 & & 3/31/08 & 8C17008 & AGG-RRL-001 \\
\hline 10043-66-0 & Iodine-131 & $<0.36$ & $\mathrm{pCi} / \mathrm{g}$ & 0.36 & & 3/31/08 & 8C17008 & AGG-RRL-001 \\
\hline 13981-41-4 & Barium-133 & $<0.58$ & $\mathrm{pCi} / \mathrm{g}$ & 0.58 & & 3/31/08 & 8C17008 & AGG-RRL-001 \\
\hline 13967-70-9 & Cesium-134 & $<0.346$ & $\mathrm{pCi} / \mathrm{g}$ & 0.346 & & 3/31/08 & 8C17008 & AGG-RRL-001 \\
\hline 10045-97-3 & Cesium-137 & $<0.359$ & $\mathrm{pCi} / \mathrm{g}$ & 0.359 & & 3/31/08 & 8C17008 & AGG-RRL-001 \\
\hline 13982-30-4 & Cerium-139 & $<0.355$ & $\mathrm{pCi} / \mathrm{g}$ & 0.355 & & 3/31/08 & 8C17008 & AGG-RRL-001 \\
\hline 14762-78-8 & Cerium-144 & $<2.33$ & $\mathrm{pCi} / \mathrm{g}$ & 2.33 & & 3/31/08 & 8C17008 & AGG-RRL-001 \\
\hline 14683-23-9 & Europium-152 & $<1.13$ & $\mathrm{pCi} / \mathrm{g}$ & 1.13 & & 3/31/08 & 8C17008 & AGG-RRL-001 \\
\hline 14276-65-4 & Gadolinium-153 & $<0.837$ & $\mathrm{pCi} / \mathrm{g}$ & 0.837 & & 3/31/08 & 8C17008 & AGG-RRL-001 \\
\hline 15585-10-1 & Europium-154 & $<0.62$ & $\mathrm{pCi} / \mathrm{g}$ & 0.62 & & 3/31/08 & 8C17008 & AGG-RRL-001 \\
\hline 14391-16-3 & Europium-155 & $<0.862$ & $\mathrm{pCi} / \mathrm{g}$ & 0.862 & & 3/31/08 & 8C17008 & AGG-RRL-001 \\
\hline 13982-78-0 & Mercury-203 & $<0.366$ & $\mathrm{pCi} / \mathrm{g}$ & 0.366 & & 3/31/08 & 8C17008 & AGG-RRL-001 \\
\hline 14913-50-9 & Thallium-208 & $<0.359$ & $\mathrm{pCi} / \mathrm{g}$ & 0.359 & 0.0827 & 3/31/08 & 8C17008 & AGG-RRL-001 \\
\hline 14331-79-4 & Bismuth-210 & $<0.565$ & $\mathrm{pCi} / \mathrm{g}$ & 0.565 & & 3/31/08 & 8C17008 & AGG-RRL-001 \\
\hline \multirow[t]{3}{*}{ 14255-04-0 } & Lead-210 & $<117$ & $\mathrm{pCi} / \mathrm{g}$ & 117 & & 3/31/08 & 8C17008 & AGG-RRL-001 \\
\hline & Bismuth-211 & $<6.81$ & $\mathrm{pCi} / \mathrm{g}$ & 6.81 & & 3/31/08 & 8C17008 & AGG-RRL-001 \\
\hline & Lead-211 & $<9.31$ & $\mathrm{pCi} / \mathrm{g}$ & 9.31 & & 3/31/08 & 8C17008 & AGG-RRL-001 \\
\hline 15092-94-1 & Lead-212 & 0.676 & $\mathrm{pCi} / \mathrm{g}$ & 0.558 & 0.25 & 3/31/08 & 8C17008 & AGG-RRL-001 \\
\hline 14733-03-0 & Bismuth-214 & $<0.746$ & $\mathrm{pCi} / \mathrm{g}$ & 0.746 & & 3/31/08 & 8C17008 & AGG-RRL-001 \\
\hline 15067-28-4 & Lead-214 & 1.11 & $\mathrm{pCi} / \mathrm{g}$ & 0.882 & 0.602 & 3/31/08 & 8C17008 & AGG-RRL-001 \\
\hline 14835-02-0 & Radon-219 & $<2.96$ & $\mathrm{pCi} / \mathrm{g}$ & 2.96 & & 3/31/08 & 8C17008 & AGG-RRL-001 \\
\hline 22481-48-7 & Radon-220 & $<278$ & $\mathrm{pCi} / \mathrm{g}$ & 278 & & 3/31/08 & 8C17008 & AGG-RRL-001 \\
\hline 28522-20-5 & Radon-221 & $<1.4$ & $\mathrm{pCi} / \mathrm{g}$ & 1.4 & & 3/31/08 & 8C17008 & AGG-RRL-001 \\
\hline 15756-98-6 & Francium-223 & $<10.2$ & $\mathrm{pCi} / \mathrm{g}$ & 10.2 & & 3/31/08 & 8C17008 & AGG-RRL-001 \\
\hline $15623-45-7$ & Radium-223 & $<2.12$ & $\mathrm{pCi} / \mathrm{g}$ & 2.12 & & 3/31/08 & 8C17008 & AGG-RRL-001 \\
\hline 13233-32-4 & Radium-224 & $<4.98$ & $\mathrm{pCi} / \mathrm{g}$ & 4.98 & 0.957 & 3/31/08 & 8C17008 & AGG-RRL-001 \\
\hline 13982-63-3 & Radium-226 & $<8.59$ & $\mathrm{pCi} / \mathrm{g}$ & 8.59 & & 3/31/08 & 8C17008 & AGG-RRL-001 \\
\hline $15623-47-9$ & Thorium-227 & $<3.12$ & $\mathrm{pCi} / \mathrm{g}$ & 3.12 & & 3/31/08 & 8C17008 & AGG-RRL-001 \\
\hline 14331-83-0 & Actinium-228 & $<1.46$ & $\mathrm{pCi} / \mathrm{g}$ & 1.46 & & 3/31/08 & 8C17008 & AGG-RRL-001 \\
\hline $14274-82-9$ & Thorium-228 & $<108$ & $\mathrm{pCi} / \mathrm{g}$ & 108 & & 3/31/08 & 8C17008 & AGG-RRL-001 \\
\hline $14269-63-7$ & Thorium-230 & $<115$ & $\mathrm{pCi} / \mathrm{g}$ & 115 & & 3/31/08 & 8C17008 & AGG-RRL-001 \\
\hline 14331-85-2 & Protactinium-231 & $<12.4$ & $\mathrm{pCi} / \mathrm{g}$ & 12.4 & & 3/31/08 & 8C17008 & AGG-RRL-001 \\
\hline $14932-40-2$ & Thorium-231 & $<60.5$ & $\mathrm{pCi} / \mathrm{g}$ & 60.5 & & 3/31/08 & 8C17008 & AGG-RRL-001 \\
\hline 7440-29-1 & Thorium-232 & $<358$ & $\mathrm{pCi} / \mathrm{g}$ & 358 & & $3 / 31 / 08$ & 8C17008 & AGG-RRL-001 \\
\hline 13981-14-1 & Protactinium-233 & $<0.778$ & $\mathrm{pCi} / \mathrm{g}$ & 0.778 & & 3/31/08 & 8C17008 & AGG-RRL-001 \\
\hline \multirow[t]{2}{*}{$15100-28-4$} & Protactinium-234 & $<1.1$ & $\mathrm{pCi} / \mathrm{g}$ & 1.1 & & 3/31/08 & 8C17008 & AGG-RRL-001 \\
\hline & Protactinium-234m & $<35.6$ & $\mathrm{pCi} / \mathrm{g}$ & 35.6 & & 3/31/08 & 8C17008 & AGG-RRL-001 \\
\hline 15065-10-8 & Thorium-234 & $<9.97$ & $\mathrm{pCi} / \mathrm{g}$ & 9.97 & & 3/31/08 & 8C17008 & AGG-RRL-001 \\
\hline 15117-96-1 & Uranium 235 & $<2.62$ & $\mathrm{pCi} / \mathrm{g}$ & 2.62 & & 3/31/08 & 8C17008 & AGG-RRL-001 \\
\hline
\end{tabular}


GEA/Soil

\begin{tabular}{|c|c|c|c|c|c|c|c|c|}
\hline CAS \# & Analyte & Results & Units & MDA & UNC & Analyzed & Batch & Method \\
\hline HEIS No. & B1TNK6B & \multicolumn{2}{|c|}{ Lab ID: } & 0803015-18 & & & & \\
\hline 13994-20-2 & Neptunium-237 & $<2.24$ & $\mathrm{pCi} / \mathrm{g}$ & 2.24 & & 3/31/08 & 8C17008 & AGG-RRL-001 \\
\hline \multirow[t]{2}{*}{ 13981-16-3 } & Plutonium-238 & $<3430$ & $\mathrm{pCi} / \mathrm{g}$ & 3430 & & 3/31/08 & 8C17008 & AGG-RRL-001 \\
\hline & Uranium 238 & $<5.16$ & $\mathrm{pCi} / \mathrm{g}$ & 5.16 & & 3/31/08 & 8C17008 & AGG-RRL-001 \\
\hline $15117-48-3$ & Plutonium-239 & $<4050$ & $\mathrm{pCi} / \mathrm{g}$ & 4050 & & 3/31/08 & 8C17008 & AGG-RRL-001 \\
\hline 14119-33-6 & Plutonium-240 & $<3540$ & $\mathrm{pCi} / \mathrm{g}$ & 3540 & & 3/31/08 & 8C17008 & AGG-RRL-001 \\
\hline 14596-10-2 & Americium-241 & $<1.77$ & $\mathrm{pCi} / \mathrm{g}$ & 1.77 & & 3/31/08 & 8C17008 & AGG-RRL-001 \\
\hline 14993-75-0 & Americium-243 & $<0.643$ & $\mathrm{pCi} / \mathrm{g}$ & 0.643 & & 3/31/08 & 8C17008 & AGG-RRL-001 \\
\hline 15757-87-6 & Curium-243 & $<1.08$ & $\mathrm{pCi} / \mathrm{g}$ & 1.08 & & 3/31/08 & 8C17008 & AGG-RRL-001 \\
\hline 15621-76-8 & Curium-245 & $<0.887$ & $\mathrm{pCi} / \mathrm{g}$ & 0.887 & & 3/31/08 & 8C17008 & AGG-RRL-001 \\
\hline HEIS No. & B1TNK6A & \multicolumn{2}{|c|}{ Lab ID: } & 0803015-19 & & & & \\
\hline 13966-32-0 & Sodium-22 & $<0.372$ & $\mathrm{pCi} / \mathrm{g}$ & 0.372 & & 3/31/08 & 8C17008 & AGG-RRL-001 \\
\hline 13966-00-2 & Potassium-40 & 17.8 & $\mathrm{pCi} / \mathrm{g}$ & 3.84 & 1.3 & 3/31/08 & 8C17008 & AGG-RRL-001 \\
\hline 14392-02-0 & Chromium-51 & $<3.11$ & $\mathrm{pCi} / \mathrm{g}$ & 3.11 & & 3/31/08 & 8C17008 & AGG-RRL-001 \\
\hline 13966-31-9 & Manganese-54 & $<0.327$ & $\mathrm{pCi} / \mathrm{g}$ & 0.327 & & 3/31/08 & 8C17008 & AGG-RRL-001 \\
\hline 13981-50-5 & Cobalt-57 & $<0.332$ & $\mathrm{pCi} / \mathrm{g}$ & 0.332 & & 3/31/08 & 8C17008 & AGG-RRL-001 \\
\hline $14596-12-4$ & Iron-59 & $<0.683$ & $\mathrm{pCi} / \mathrm{g}$ & 0.683 & & 3/31/08 & 8C17008 & AGG-RRL-001 \\
\hline 10198-40-0 & Cobalt-60 & $<0.33$ & $\mathrm{pCi} / \mathrm{g}$ & 0.33 & & 3/31/08 & 8C17008 & AGG-RRL-001 \\
\hline 13982-39-3 & Zinc-65 & $<0.807$ & $\mathrm{pCi} / \mathrm{g}$ & 0.807 & & 3/31/08 & 8C17008 & AGG-RRL-001 \\
\hline $14265-71-5$ & Selenium-75 & $<0.516$ & $\mathrm{pCi} / \mathrm{g}$ & 0.516 & & 3/31/08 & 8C17008 & AGG-RRL-001 \\
\hline 17056-36-9 & Rubidium-83 & $<0.79$ & $\mathrm{pCi} / \mathrm{g}$ & 0.79 & & 3/31/08 & 8C17008 & AGG-RRL-001 \\
\hline 13967-73-2 & Strontium-85 & $<0.366$ & $\mathrm{pCi} / \mathrm{g}$ & 0.366 & & 3/31/08 & 8C17008 & AGG-RRL-001 \\
\hline 14932-53-7 & Rubidium-86 & $<4.03$ & $\mathrm{pCi} / \mathrm{g}$ & 4.03 & & 3/31/08 & 8C17008 & AGG-RRL-001 \\
\hline 13982-36-0 & Yttrium-88 & $<0.309$ & $\mathrm{pCi} / \mathrm{g}$ & 0.309 & & 3/31/08 & 8C17008 & AGG-RRL-001 \\
\hline 14681-63-1 & Niobium-94 & $<0.322$ & $\mathrm{pCi} / \mathrm{g}$ & 0.322 & & 3/31/08 & 8C17008 & AGG-RRL-001 \\
\hline \multirow[t]{3}{*}{ 13967-76-5 } & Niobium-95 & $<0.326$ & $\mathrm{pCi} / \mathrm{g}$ & 0.326 & & 3/31/08 & 8C17008 & AGG-RRL-001 \\
\hline & Niobium-95m & $<1.44$ & $\mathrm{pCi} / \mathrm{g}$ & 1.44 & & 3/31/08 & 8C17008 & AGG-RRL-001 \\
\hline & Technetium-95m & $<0.492$ & $\mathrm{pCi} / \mathrm{g}$ & 0.492 & & 3/31/08 & 8C17008 & AGG-RRL-001 \\
\hline \multirow[t]{2}{*}{ 13967-71-0 } & Zirconium-95 & $<0.573$ & $\mathrm{pCi} / \mathrm{g}$ & 0.573 & & 3/31/08 & 8C17008 & AGG-RRL-001 \\
\hline & Technetium-99m & $<0.332$ & $\mathrm{pCi} / \mathrm{g}$ & 0.332 & & 3/31/08 & 8C17008 & AGG-RRL-001 \\
\hline 13968-53-1 & Ruthenium-103 & $<0.361$ & $\mathrm{pCi} / \mathrm{g}$ & 0.361 & & 3/31/08 & 8C17008 & AGG-RRL-001 \\
\hline 13967-48-1 & Ruthenium-106 & $<3.68$ & $\mathrm{pCi} / \mathrm{g}$ & 3.68 & & 3/31/08 & 8C17008 & AGG-RRL-001 \\
\hline 14391-65-2 & Silver-108m & $<0.317$ & $\mathrm{pCi} / \mathrm{g}$ & 0.317 & & 3/31/08 & 8C17008 & AGG-RRL-001 \\
\hline \multirow[t]{2}{*}{ 14109-32-1 } & Cadmium-109 & $<9.25$ & $\mathrm{pCi} / \mathrm{g}$ & 9.25 & & 3/31/08 & 8C17008 & AGG-RRL-001 \\
\hline & Silver-110 & $<0.319$ & $\mathrm{pCi} / \mathrm{g}$ & 0.319 & & 3/31/08 & 8C17008 & AGG-RRL-001 \\
\hline 14391-76-5 & Silver-110m & $<0.319$ & $\mathrm{pCi} / \mathrm{g}$ & 0.319 & & 3/31/08 & 8C17008 & AGG-RRL-001 \\
\hline 13966-06-8 & Tin-113 & $<0.46$ & $\mathrm{pCi} / \mathrm{g}$ & 0.46 & & 3/31/08 & 8C17008 & AGG-RRL-001 \\
\hline $14683-10-4$ & Antimony-124 & $<0.366$ & $\mathrm{pCi} / \mathrm{g}$ & 0.366 & & 3/31/08 & 8C17008 & AGG-RRL-001 \\
\hline $14234-35-6$ & Antimony-125 & $<0.944$ & $\mathrm{pCi} / \mathrm{g}$ & 0.944 & & 3/31/08 & 8C17008 & AGG-RRL-001 \\
\hline 15756-32-8 & Antimony-126 & $<0.302$ & $\mathrm{pCi} / \mathrm{g}$ & 0.302 & & 3/31/08 & 8C17008 & AGG-RRL-001 \\
\hline 15832-50-5 & Tin-126 & $<0.753$ & $\mathrm{pCi} / \mathrm{g}$ & 0.753 & & 3/31/08 & 8C17008 & AGG-RRL-001 \\
\hline 10043-66-0 & Iodine-131 & $<0.401$ & $\mathrm{pCi} / \mathrm{g}$ & 0.401 & & 3/31/08 & 8C17008 & AGG-RRL-001 \\
\hline 13981-41-4 & Barium-133 & $<0.558$ & $\mathrm{pCi} / \mathrm{g}$ & 0.558 & & 3/31/08 & 8C17008 & AGG-RRL-001 \\
\hline 13967-70-9 & Cesium-134 & $<0.371$ & $\mathrm{pCi} / \mathrm{g}$ & 0.371 & & 3/31/08 & 8C17008 & AGG-RRL-001 \\
\hline 10045-97-3 & Cesium-137 & $<0.349$ & $\mathrm{pCi} / \mathrm{g}$ & 0.349 & & 3/31/08 & 8C17008 & AGG-RRL-001 \\
\hline 13982-30-4 & Cerium-139 & $<0.372$ & $\mathrm{pCi} / \mathrm{g}$ & 0.372 & & 3/31/08 & 8C17008 & AGG-RRL-001 \\
\hline 14762-78-8 & Cerium-144 & $<2.68$ & $\mathrm{pCi} / \mathrm{g}$ & 2.68 & & 3/31/08 & 8C17008 & AGG-RRL-001 \\
\hline 14683-23-9 & Europium-152 & $<1.31$ & $\mathrm{pCi} / \mathrm{g}$ & 1.31 & & 3/31/08 & 8C17008 & AGG-RRL-001 \\
\hline $14276-65-4$ & Gadolinium-153 & $<1$ & $\mathrm{pCi} / \mathrm{g}$ & 1 & & 3/31/08 & 8C17008 & AGG-RRL-001 \\
\hline
\end{tabular}


GEA/Soil

\begin{tabular}{|c|c|c|c|c|c|c|c|c|}
\hline CAS \# & Analyte & Results & Units & MDA & UNC & Analyzed & Batch & Method \\
\hline HEIS No. & B1TNK6A & \multicolumn{3}{|c|}{ Lab ID: $\quad$ 0803015-19 } & & & & \\
\hline $15585-10-1$ & Europium-154 & $<0.706$ & $\mathrm{pCi} / \mathrm{g}$ & 0.706 & & $3 / 31 / 08$ & 8C17008 & AGG-RRL-001 \\
\hline 14391-16-3 & Europium-155 & $<1.07$ & $\mathrm{pCi} / \mathrm{g}$ & 1.07 & & 3/31/08 & 8C17008 & AGG-RRL-001 \\
\hline 13982-78-0 & Mercury-203 & $<0.41$ & $\mathrm{pCi} / \mathrm{g}$ & 0.41 & & 3/31/08 & 8C17008 & AGG-RRL-001 \\
\hline 14913-50-9 & Thallium-208 & $<0.414$ & $\mathrm{pCi} / \mathrm{g}$ & 0.414 & & $3 / 31 / 08$ & 8C17008 & AGG-RRL-001 \\
\hline 14331-79-4 & Bismuth-210 & $<0.615$ & $\mathrm{pCi} / \mathrm{g}$ & 0.615 & & $3 / 31 / 08$ & 8C17008 & AGG-RRL-001 \\
\hline \multirow[t]{3}{*}{$14255-04-0$} & Lead-210 & $<46$ & $\mathrm{pCi} / \mathrm{g}$ & 46 & & $3 / 31 / 08$ & 8C17008 & AGG-RRL-001 \\
\hline & Bismuth-211 & $<7.08$ & $\mathrm{pCi} / \mathrm{g}$ & 7.08 & & $3 / 31 / 08$ & 8C17008 & AGG-RRL-001 \\
\hline & Lead-211 & $<9.67$ & $\mathrm{pCi} / \mathrm{g}$ & 9.67 & & $3 / 31 / 08$ & 8C17008 & AGG-RRL-001 \\
\hline $15092-94-1$ & Lead-212 & $<0.837$ & $\mathrm{pCi} / \mathrm{g}$ & 0.837 & & 3/31/08 & 8C17008 & AGG-RRL-001 \\
\hline 14733-03-0 & Bismuth-214 & $<0.789$ & $\mathrm{pCi} / \mathrm{g}$ & 0.789 & & 3/31/08 & 8C17008 & AGG-RRL-001 \\
\hline $15067-28-4$ & Lead-214 & $<0.913$ & $\mathrm{pCi} / \mathrm{g}$ & 0.913 & & 3/31/08 & 8C17008 & AGG-RRL-001 \\
\hline $14835-02-0$ & Radon-219 & $<3.18$ & $\mathrm{pCi} / \mathrm{g}$ & 3.18 & & 3/31/08 & 8C17008 & AGG-RRL-001 \\
\hline $22481-48-7$ & Radon-220 & $<311$ & $\mathrm{pCi} / \mathrm{g}$ & 311 & & 3/31/08 & 8C17008 & AGG-RRL-001 \\
\hline 28522-20-5 & Radon-221 & $<1.5$ & $\mathrm{pCi} / \mathrm{g}$ & 1.5 & & 3/31/08 & 8C17008 & AGG-RRL-001 \\
\hline $15756-98-6$ & Francium-223 & $<10.6$ & $\mathrm{pCi} / \mathrm{g}$ & 10.6 & & 3/31/08 & 8C17008 & AGG-RRL-001 \\
\hline $15623-45-7$ & Radium-223 & $<2.27$ & $\mathrm{pCi} / \mathrm{g}$ & 2.27 & & 3/31/08 & 8C17008 & AGG-RRL-001 \\
\hline $13233-32-4$ & Radium-224 & $<9.08$ & $\mathrm{pCi} / \mathrm{g}$ & 9.08 & & 3/31/08 & 8C17008 & AGG-RRL-001 \\
\hline 13982-63-3 & Radium-226 & $<9.24$ & $\mathrm{pCi} / \mathrm{g}$ & 9.24 & & 3/31/08 & 8C17008 & AGG-RRL-001 \\
\hline $15623-47-9$ & Thorium-227 & $<3.22$ & $\mathrm{pCi} / \mathrm{g}$ & 3.22 & & 3/31/08 & 8C17008 & AGG-RRL-001 \\
\hline $14331-83-0$ & Actinium-228 & $<1.43$ & $\mathrm{pCi} / \mathrm{g}$ & 1.43 & & $3 / 31 / 08$ & 8C17008 & AGG-RRL-001 \\
\hline $14274-82-9$ & Thorium-228 & $<115$ & $\mathrm{pCi} / \mathrm{g}$ & 115 & & 3/31/08 & 8C17008 & AGG-RRL-001 \\
\hline $14269-63-7$ & Thorium-230 & $<122$ & $\mathrm{pCi} / \mathrm{g}$ & 122 & & 3/31/08 & 8C17008 & AGG-RRL-001 \\
\hline $14331-85-2$ & Protactinium-231 & $<13.6$ & $\mathrm{pCi} / \mathrm{g}$ & 13.6 & & 3/31/08 & 8C17008 & AGG-RRL-001 \\
\hline $14932-40-2$ & Thorium-231 & $<72.1$ & $\mathrm{pCi} / \mathrm{g}$ & 72.1 & & 3/31/08 & 8C17008 & AGG-RRL-001 \\
\hline $7440-29-1$ & Thorium-232 & $<305$ & $\mathrm{pCi} / \mathrm{g}$ & 305 & & 3/31/08 & 8C17008 & AGG-RRL-001 \\
\hline 13981-14-1 & Protactinium-233 & $<0.834$ & $\mathrm{pCi} / \mathrm{g}$ & 0.834 & & 3/31/08 & 8C17008 & AGG-RRL-001 \\
\hline \multirow[t]{2}{*}{$15100-28-4$} & Protactinium-234 & $<1.31$ & $\mathrm{pCi} / \mathrm{g}$ & 1.31 & & 3/31/08 & 8C17008 & AGG-RRL-001 \\
\hline & Protactinium-234m & $<37.1$ & $\mathrm{pCi} / \mathrm{g}$ & 37.1 & & $3 / 31 / 08$ & 8C17008 & AGG-RRL-001 \\
\hline 15065-10-8 & Thorium-234 & $<10.6$ & $\mathrm{pCi} / \mathrm{g}$ & 10.6 & & 3/31/08 & 8C17008 & AGG-RRL-001 \\
\hline 15117-96-1 & Uranium 235 & $<2.8$ & $\mathrm{pCi} / \mathrm{g}$ & 2.8 & & $3 / 31 / 08$ & 8C17008 & AGG-RRL-001 \\
\hline 13994-20-2 & Neptunium-237 & $<2.79$ & $\mathrm{pCi} / \mathrm{g}$ & 2.79 & & 3/31/08 & 8C17008 & AGG-RRL-001 \\
\hline \multirow[t]{2}{*}{ 13981-16-3 } & Plutonium-238 & $<4120$ & $\mathrm{pCi} / \mathrm{g}$ & 4120 & & 3/31/08 & 8C17008 & AGG-RRL-001 \\
\hline & Uranium 238 & $<5.83$ & $\mathrm{pCi} / \mathrm{g}$ & 5.83 & & 3/31/08 & 8C17008 & AGG-RRL-001 \\
\hline $15117-48-3$ & Plutonium-239 & $<4180$ & $\mathrm{pCi} / \mathrm{g}$ & 4180 & & $3 / 31 / 08$ & 8C17008 & AGG-RRL-001 \\
\hline 14119-33-6 & Plutonium-240 & $<4180$ & $\mathrm{pCi} / \mathrm{g}$ & 4180 & & 3/31/08 & 8C17008 & AGG-RRL-001 \\
\hline $14596-10-2$ & Americium-241 & $<1.56$ & $\mathrm{pCi} / \mathrm{g}$ & 1.56 & & 3/31/08 & 8C17008 & AGG-RRL-001 \\
\hline $14993-75-0$ & Americium-243 & $<0.674$ & $\mathrm{pCi} / \mathrm{g}$ & 0.674 & & 3/31/08 & 8C17008 & AGG-RRL-001 \\
\hline $15757-87-6$ & Curium-243 & $<1.28$ & $\mathrm{pCi} / \mathrm{g}$ & 1.28 & & 3/31/08 & 8C17008 & AGG-RRL-001 \\
\hline 15621-76-8 & Curium-245 & $<1.05$ & $\mathrm{pCi} / \mathrm{g}$ & 1.05 & & $3 / 31 / 08$ & 8C17008 & AGG-RRL-001 \\
\hline HEIS No. & B1TNK7B & \multicolumn{2}{|c|}{ Lab ID: } & 0803015-22 & & & & \\
\hline 13966-32-0 & Sodium-22 & $<0.336$ & $\mathrm{pCi} / \mathrm{g}$ & 0.336 & & $3 / 31 / 08$ & 8C17008 & AGG-RRL-001 \\
\hline 13966-00-2 & Potassium-40 & 20.9 & $\mathrm{pCi} / \mathrm{g}$ & 2.39 & 1.34 & $3 / 31 / 08$ & 8C17008 & AGG-RRL-001 \\
\hline $14392-02-0$ & Chromium-51 & $<2.89$ & $\mathrm{pCi} / \mathrm{g}$ & 2.89 & & 3/31/08 & 8C17008 & AGG-RRL-001 \\
\hline 13966-31-9 & Manganese-54 & $<0.284$ & $\mathrm{pCi} / \mathrm{g}$ & 0.284 & & 3/31/08 & 8C17008 & AGG-RRL-001 \\
\hline 13981-50-5 & Cobalt-57 & $<0.292$ & $\mathrm{pCi} / \mathrm{g}$ & 0.292 & & 3/31/08 & 8C17008 & AGG-RRL-001 \\
\hline $14596-12-4$ & Iron-59 & $<0.561$ & $\mathrm{pCi} / \mathrm{g}$ & 0.561 & & 3/31/08 & 8C17008 & AGG-RRL-001 \\
\hline 10198-40-0 & Cobalt-60 & $<0.289$ & $\mathrm{pCi} / \mathrm{g}$ & 0.289 & & 3/31/08 & 8C17008 & AGG-RRL-001 \\
\hline 13982-39-3 & Zinc-65 & $<0.712$ & $\mathrm{pCi} / \mathrm{g}$ & 0.712 & & 3/31/08 & 8C17008 & AGG-RRL-001 \\
\hline
\end{tabular}


GEA/Soil

\begin{tabular}{|c|c|c|c|c|c|c|c|c|}
\hline CAS \# & Analyte & Results & Units & MDA & UNC & Analyzed & Batch & Method \\
\hline HEIS No. & B1TNK7B & \multicolumn{2}{|c|}{ Lab ID: } & 0803015-22 & & & & \\
\hline 14265-71-5 & Selenium-75 & $<0.465$ & $\mathrm{pCi} / \mathrm{g}$ & 0.465 & & 3/31/08 & 8C17008 & AGG-RRL-001 \\
\hline 17056-36-9 & Rubidium-83 & $<0.575$ & $\mathrm{pCi} / \mathrm{g}$ & 0.575 & & 3/31/08 & 8C17008 & AGG-RRL-001 \\
\hline 13967-73-2 & Strontium-85 & $<0.356$ & $\mathrm{pCi} / \mathrm{g}$ & 0.356 & & 3/31/08 & 8C17008 & AGG-RRL-001 \\
\hline 14932-53-7 & Rubidium-86 & $<3.51$ & $\mathrm{pCi} / \mathrm{g}$ & 3.51 & & 3/31/08 & 8C17008 & AGG-RRL-001 \\
\hline 13982-36-0 & Yttrium-88 & $<0.237$ & $\mathrm{pCi} / \mathrm{g}$ & 0.237 & & 3/31/08 & 8C17008 & AGG-RRL-001 \\
\hline 14681-63-1 & Niobium-94 & $<0.277$ & $\mathrm{pCi} / \mathrm{g}$ & 0.277 & & 3/31/08 & 8C17008 & AGG-RRL-001 \\
\hline \multirow[t]{3}{*}{ 13967-76-5 } & Niobium-95 & $<0.282$ & $\mathrm{pCi} / \mathrm{g}$ & 0.282 & & 3/31/08 & 8C17008 & AGG-RRL-001 \\
\hline & Niobium-95m & $<1.35$ & $\mathrm{pCi} / \mathrm{g}$ & 1.35 & & 3/31/08 & 8C17008 & AGG-RRL-001 \\
\hline & Technetium-95m & $<0.456$ & $\mathrm{pCi} / \mathrm{g}$ & 0.456 & & 3/31/08 & 8C17008 & AGG-RRL-001 \\
\hline \multirow[t]{2}{*}{ 13967-71-0 } & Zirconium-95 & $<0.503$ & $\mathrm{pCi} / \mathrm{g}$ & 0.503 & & 3/31/08 & 8C17008 & AGG-RRL-001 \\
\hline & Technetium-99m & $<0.287$ & $\mathrm{pCi} / \mathrm{g}$ & 0.287 & & 3/31/08 & 8C17008 & AGG-RRL-001 \\
\hline 13968-53-1 & Ruthenium-103 & $<0.291$ & $\mathrm{pCi} / \mathrm{g}$ & 0.291 & & 3/31/08 & 8C17008 & AGG-RRL-001 \\
\hline 13967-48-1 & Ruthenium-106 & $<2.68$ & $\mathrm{pCi} / \mathrm{g}$ & 2.68 & & 3/31/08 & 8C17008 & AGG-RRL-001 \\
\hline 14391-65-2 & Silver-108m & $<0.289$ & $\mathrm{pCi} / \mathrm{g}$ & 0.289 & & 3/31/08 & 8C17008 & AGG-RRL-001 \\
\hline \multirow[t]{2}{*}{ 14109-32-1 } & Cadmium-109 & $<7.76$ & $\mathrm{pCi} / \mathrm{g}$ & 7.76 & & 3/31/08 & 8C17008 & AGG-RRL-001 \\
\hline & Silver-110 & $<0.29$ & $\mathrm{pCi} / \mathrm{g}$ & 0.29 & & 3/31/08 & 8C17008 & AGG-RRL-001 \\
\hline 14391-76-5 & Silver-110m & $<0.291$ & $\mathrm{pCi} / \mathrm{g}$ & 0.291 & & 3/31/08 & 8C17008 & AGG-RRL-001 \\
\hline 13966-06-8 & Tin-113 & $<0.429$ & $\mathrm{pCi} / \mathrm{g}$ & 0.429 & & 3/31/08 & 8C17008 & AGG-RRL-001 \\
\hline 14683-10-4 & Antimony-124 & $<0.288$ & $\mathrm{pCi} / \mathrm{g}$ & 0.288 & & 3/31/08 & 8C17008 & AGG-RRL-001 \\
\hline 14234-35-6 & Antimony-125 & $<0.926$ & $\mathrm{pCi} / \mathrm{g}$ & 0.926 & & 3/31/08 & 8C17008 & AGG-RRL-001 \\
\hline 15756-32-8 & Antimony-126 & $<0.287$ & $\mathrm{pCi} / \mathrm{g}$ & 0.287 & & 3/31/08 & 8C17008 & AGG-RRL-001 \\
\hline 15832-50-5 & Tin-126 & $<0.618$ & $\mathrm{pCi} / \mathrm{g}$ & 0.618 & & 3/31/08 & 8C17008 & AGG-RRL-001 \\
\hline 10043-66-0 & Iodine-131 & $<0.319$ & $\mathrm{pCi} / \mathrm{g}$ & 0.319 & & 3/31/08 & 8C17008 & AGG-RRL-001 \\
\hline 13981-41-4 & Barium-133 & $<0.528$ & $\mathrm{pCi} / \mathrm{g}$ & 0.528 & & 3/31/08 & 8C17008 & AGG-RRL-001 \\
\hline 13967-70-9 & Cesium-134 & $<0.327$ & $\mathrm{pCi} / \mathrm{g}$ & 0.327 & & 3/31/08 & 8C17008 & AGG-RRL-001 \\
\hline $10045-97-3$ & Cesium-137 & $<0.331$ & $\mathrm{pCi} / \mathrm{g}$ & 0.331 & & 3/31/08 & 8C17008 & AGG-RRL-001 \\
\hline 13982-30-4 & Cerium-139 & $<0.334$ & $\mathrm{pCi} / \mathrm{g}$ & 0.334 & & 3/31/08 & 8C17008 & AGG-RRL-001 \\
\hline 14762-78-8 & Cerium-144 & $<2.29$ & $\mathrm{pCi} / \mathrm{g}$ & 2.29 & & 3/31/08 & 8C17008 & AGG-RRL-001 \\
\hline 14683-23-9 & Europium-152 & $<1.04$ & $\mathrm{pCi} / \mathrm{g}$ & 1.04 & & 3/31/08 & 8C17008 & AGG-RRL-001 \\
\hline 14276-65-4 & Gadolinium-153 & $<0.832$ & $\mathrm{pCi} / \mathrm{g}$ & 0.832 & & 3/31/08 & 8C17008 & AGG-RRL-001 \\
\hline 15585-10-1 & Europium-154 & $<0.608$ & $\mathrm{pCi} / \mathrm{g}$ & 0.608 & & 3/31/08 & 8C17008 & AGG-RRL-001 \\
\hline 14391-16-3 & Europium-155 & $<0.869$ & $\mathrm{pCi} / \mathrm{g}$ & 0.869 & & 3/31/08 & 8C17008 & AGG-RRL-001 \\
\hline 13982-78-0 & Mercury-203 & $<0.353$ & $\mathrm{pCi} / \mathrm{g}$ & 0.353 & & 3/31/08 & 8C17008 & AGG-RRL-001 \\
\hline 14913-50-9 & Thallium-208 & $<0.306$ & $\mathrm{pCi} / \mathrm{g}$ & 0.306 & 0.0682 & 3/31/08 & 8C17008 & AGG-RRL-001 \\
\hline 14331-79-4 & Bismuth-210 & $<0.558$ & $\mathrm{pCi} / \mathrm{g}$ & 0.558 & & 3/31/08 & 8C17008 & AGG-RRL-001 \\
\hline \multirow[t]{3}{*}{$14255-04-0$} & Lead-210 & $<111$ & $\mathrm{pCi} / \mathrm{g}$ & 111 & & 3/31/08 & 8C17008 & AGG-RRL-001 \\
\hline & Bismuth-211 & $<6.49$ & $\mathrm{pCi} / \mathrm{g}$ & 6.49 & & 3/31/08 & 8C17008 & AGG-RRL-001 \\
\hline & Lead-211 & $<8.87$ & $\mathrm{pCi} / \mathrm{g}$ & 8.87 & & 3/31/08 & 8C17008 & AGG-RRL-001 \\
\hline 15092-94-1 & Lead-212 & $<0.747$ & $\mathrm{pCi} / \mathrm{g}$ & 0.747 & 0.164 & 3/31/08 & 8C17008 & AGG-RRL-001 \\
\hline 14733-03-0 & Bismuth-214 & $<0.753$ & $\mathrm{pCi} / \mathrm{g}$ & 0.753 & & 3/31/08 & 8C17008 & AGG-RRL-001 \\
\hline $15067-28-4$ & Lead-214 & $<0.856$ & $\mathrm{pCi} / \mathrm{g}$ & 0.856 & & 3/31/08 & 8C17008 & AGG-RRL-001 \\
\hline $14835-02-0$ & Radon-219 & $<2.81$ & $\mathrm{pCi} / \mathrm{g}$ & 2.81 & & 3/31/08 & 8C17008 & AGG-RRL-001 \\
\hline 22481-48-7 & Radon-220 & $<247$ & $\mathrm{pCi} / \mathrm{g}$ & 247 & & 3/31/08 & 8C17008 & AGG-RRL-001 \\
\hline 28522-20-5 & Radon-221 & $<1.34$ & $\mathrm{pCi} / \mathrm{g}$ & 1.34 & & 3/31/08 & 8C17008 & AGG-RRL-001 \\
\hline 15756-98-6 & Francium-223 & $<9.91$ & $\mathrm{pCi} / \mathrm{g}$ & 9.91 & & 3/31/08 & 8C17008 & AGG-RRL-001 \\
\hline $15623-45-7$ & Radium-223 & $<2.07$ & $\mathrm{pCi} / \mathrm{g}$ & 2.07 & & 3/31/08 & 8C17008 & AGG-RRL-001 \\
\hline 13233-32-4 & Radium-224 & $<8.69$ & $\mathrm{pCi} / \mathrm{g}$ & 8.69 & & 3/31/08 & 8C17008 & AGG-RRL-001 \\
\hline 13982-63-3 & Radium-226 & $<8.32$ & $\mathrm{pCi} / \mathrm{g}$ & 8.32 & & 3/31/08 & 8C17008 & AGG-RRL-001 \\
\hline $15623-47-9$ & Thorium-227 & $<3.03$ & $\mathrm{pCi} / \mathrm{g}$ & 3.03 & & 3/31/08 & 8C17008 & AGG-RRL-001 \\
\hline
\end{tabular}


GEA/Soil

\begin{tabular}{|c|c|c|c|c|c|c|c|c|}
\hline CAS \# & Analyte & Results & Units & MDA & UNC & Analyzed & Batch & Method \\
\hline HEIS No. & B1TNK7B & \multicolumn{2}{|c|}{ Lab ID: } & 0803015-22 & & & & \\
\hline $14331-83-0$ & Actinium-228 & $<1.26$ & $\mathrm{pCi} / \mathrm{g}$ & 1.26 & & $3 / 31 / 08$ & 8C17008 & AGG-RRL-001 \\
\hline 14274-82-9 & Thorium-228 & $<106$ & $\mathrm{pCi} / \mathrm{g}$ & 106 & & 3/31/08 & 8C17008 & AGG-RRL-001 \\
\hline $14269-63-7$ & Thorium-230 & $<108$ & $\mathrm{pCi} / \mathrm{g}$ & 108 & & 3/31/08 & 8C17008 & AGG-RRL-001 \\
\hline $14331-85-2$ & Protactinium-231 & $<11.8$ & $\mathrm{pCi} / \mathrm{g}$ & 11.8 & & 3/31/08 & 8C17008 & AGG-RRL-001 \\
\hline $14932-40-2$ & Thorium-231 & $<59.8$ & $\mathrm{pCi} / \mathrm{g}$ & 59.8 & & 3/31/08 & 8C17008 & AGG-RRL-001 \\
\hline 7440-29-1 & Thorium-232 & $<354$ & $\mathrm{pCi} / \mathrm{g}$ & 354 & & $3 / 31 / 08$ & 8C17008 & AGG-RRL-001 \\
\hline 13981-14-1 & Protactinium-233 & $<0.768$ & $\mathrm{pCi} / \mathrm{g}$ & 0.768 & & 3/31/08 & 8C17008 & AGG-RRL-001 \\
\hline \multirow[t]{2}{*}{$15100-28-4$} & Protactinium-234 & $<1.06$ & $\mathrm{pCi} / \mathrm{g}$ & 1.06 & & $3 / 31 / 08$ & 8C17008 & AGG-RRL-001 \\
\hline & Protactinium-234m & $<38$ & $\mathrm{pCi} / \mathrm{g}$ & 38 & & 3/31/08 & 8C17008 & AGG-RRL-001 \\
\hline $15065-10-8$ & Thorium-234 & $<9.72$ & $\mathrm{pCi} / \mathrm{g}$ & 9.72 & & 3/31/08 & 8C17008 & AGG-RRL-001 \\
\hline 15117-96-1 & Uranium 235 & $<2.43$ & $\mathrm{pCi} / \mathrm{g}$ & 2.43 & & 3/31/08 & 8C17008 & AGG-RRL-001 \\
\hline 13994-20-2 & Neptunium-237 & $<2.26$ & $\mathrm{pCi} / \mathrm{g}$ & 2.26 & & 3/31/08 & 8C17008 & AGG-RRL-001 \\
\hline \multirow[t]{2}{*}{ 13981-16-3 } & Plutonium-238 & $<3380$ & $\mathrm{pCi} / \mathrm{g}$ & 3380 & & 3/31/08 & 8C17008 & AGG-RRL-001 \\
\hline & Uranium 238 & $<5.03$ & $\mathrm{pCi} / \mathrm{g}$ & 5.03 & & 3/31/08 & 8C17008 & AGG-RRL-001 \\
\hline 15117-48-3 & Plutonium-239 & $<4010$ & $\mathrm{pCi} / \mathrm{g}$ & 4010 & & 3/31/08 & 8C17008 & AGG-RRL-001 \\
\hline 14119-33-6 & Plutonium-240 & $<3470$ & $\mathrm{pCi} / \mathrm{g}$ & 3470 & & 3/31/08 & 8C17008 & AGG-RRL-001 \\
\hline $14596-10-2$ & Americium-241 & $<1.73$ & $\mathrm{pCi} / \mathrm{g}$ & 1.73 & & 3/31/08 & 8C17008 & AGG-RRL-001 \\
\hline $14993-75-0$ & Americium-243 & $<0.604$ & $\mathrm{pCi} / \mathrm{g}$ & 0.604 & & 3/31/08 & 8C17008 & AGG-RRL-001 \\
\hline 15757-87-6 & Curium-243 & $<1.06$ & $\mathrm{pCi} / \mathrm{g}$ & 1.06 & & 3/31/08 & 8C17008 & AGG-RRL-001 \\
\hline 15621-76-8 & Curium-245 & $<0.87$ & $\mathrm{pCi} / \mathrm{g}$ & 0.87 & & 3/31/08 & 8C17008 & AGG-RRL-001 \\
\hline HEIS No. & B1TNK7A & \multicolumn{2}{|c|}{ Lab ID: } & 0803015-23 & & & & \\
\hline $13966-32-0$ & Sodium-22 & $<0.367$ & $\mathrm{pCi} / \mathrm{g}$ & 0.367 & & 3/31/08 & 8C17008 & AGG-RRL-001 \\
\hline 13966-00-2 & Potassium-40 & 19.9 & $\mathrm{pCi} / \mathrm{g}$ & 3.24 & 1.28 & 3/31/08 & 8C17008 & AGG-RRL-001 \\
\hline $14392-02-0$ & Chromium-51 & $<3.17$ & $\mathrm{pCi} / \mathrm{g}$ & 3.17 & & 3/31/08 & 8C17008 & AGG-RRL-001 \\
\hline 13966-31-9 & Manganese-54 & $<0.333$ & $\mathrm{pCi} / \mathrm{g}$ & 0.333 & & 3/31/08 & 8C17008 & AGG-RRL-001 \\
\hline 13981-50-5 & Cobalt-57 & $<0.331$ & $\mathrm{pCi} / \mathrm{g}$ & 0.331 & & 3/31/08 & 8C17008 & AGG-RRL-001 \\
\hline $14596-12-4$ & Iron-59 & $<0.622$ & $\mathrm{pCi} / \mathrm{g}$ & 0.622 & & 3/31/08 & 8C17008 & AGG-RRL-001 \\
\hline 10198-40-0 & Cobalt-60 & $<0.335$ & $\mathrm{pCi} / \mathrm{g}$ & 0.335 & & 3/31/08 & 8C17008 & AGG-RRL-001 \\
\hline 13982-39-3 & Zinc-65 & $<0.778$ & $\mathrm{pCi} / \mathrm{g}$ & 0.778 & & 3/31/08 & 8C17008 & AGG-RRL-001 \\
\hline $14265-71-5$ & Selenium-75 & $<0.534$ & $\mathrm{pCi} / \mathrm{g}$ & 0.534 & & 3/31/08 & 8C17008 & AGG-RRL-001 \\
\hline 17056-36-9 & Rubidium-83 & $<0.854$ & $\mathrm{pCi} / \mathrm{g}$ & 0.854 & & 3/31/08 & 8C17008 & AGG-RRL-001 \\
\hline 13967-73-2 & Strontium-85 & $<0.402$ & $\mathrm{pCi} / \mathrm{g}$ & 0.402 & & 3/31/08 & 8C17008 & AGG-RRL-001 \\
\hline $14932-53-7$ & Rubidium-86 & $<4.37$ & $\mathrm{pCi} / \mathrm{g}$ & 4.37 & & 3/31/08 & 8C17008 & AGG-RRL-001 \\
\hline 13982-36-0 & Yttrium-88 & $<0.261$ & $\mathrm{pCi} / \mathrm{g}$ & 0.261 & & 3/31/08 & 8C17008 & AGG-RRL-001 \\
\hline $14681-63-1$ & Niobium-94 & $<0.334$ & $\mathrm{pCi} / \mathrm{g}$ & 0.334 & & 3/31/08 & 8C17008 & AGG-RRL-001 \\
\hline \multirow[t]{3}{*}{ 13967-76-5 } & Niobium-95 & $<0.333$ & $\mathrm{pCi} / \mathrm{g}$ & 0.333 & & 3/31/08 & 8C17008 & AGG-RRL-001 \\
\hline & Niobium-95m & $<1.47$ & $\mathrm{pCi} / \mathrm{g}$ & 1.47 & & 3/31/08 & 8C17008 & AGG-RRL-001 \\
\hline & Technetium-95m & $<0.505$ & $\mathrm{pCi} / \mathrm{g}$ & 0.505 & & 3/31/08 & 8C17008 & AGG-RRL-001 \\
\hline \multirow[t]{2}{*}{ 13967-71-0 } & Zirconium-95 & $<0.601$ & $\mathrm{pCi} / \mathrm{g}$ & 0.601 & & 3/31/08 & 8C17008 & AGG-RRL-001 \\
\hline & Technetium-99m & $<0.332$ & $\mathrm{pCi} / \mathrm{g}$ & 0.332 & & 3/31/08 & 8C17008 & AGG-RRL-001 \\
\hline 13968-53-1 & Ruthenium-103 & $<0.392$ & $\mathrm{pCi} / \mathrm{g}$ & 0.392 & & 3/31/08 & 8C17008 & AGG-RRL-001 \\
\hline 13967-48-1 & Ruthenium-106 & $<3.82$ & $\mathrm{pCi} / \mathrm{g}$ & 3.82 & & 3/31/08 & 8C17008 & AGG-RRL-001 \\
\hline $14391-65-2$ & Silver-108m & $<0.333$ & $\mathrm{pCi} / \mathrm{g}$ & 0.333 & & 3/31/08 & 8C17008 & AGG-RRL-001 \\
\hline \multirow[t]{2}{*}{ 14109-32-1 } & Cadmium-109 & $<9.5$ & $\mathrm{pCi} / \mathrm{g}$ & 9.5 & & 3/31/08 & 8C17008 & AGG-RRL-001 \\
\hline & Silver-110 & $<0.346$ & $\mathrm{pCi} / \mathrm{g}$ & 0.346 & & 3/31/08 & 8C17008 & AGG-RRL-001 \\
\hline 14391-76-5 & Silver-110m & $<0.347$ & $\mathrm{pCi} / \mathrm{g}$ & 0.347 & & 3/31/08 & 8C17008 & AGG-RRL-001 \\
\hline 13966-06-8 & Tin-113 & $<0.441$ & $\mathrm{pCi} / \mathrm{g}$ & 0.441 & & 3/31/08 & 8C17008 & AGG-RRL-001 \\
\hline $14683-10-4$ & Antimony-124 & $<0.391$ & $\mathrm{pCi} / \mathrm{g}$ & 0.391 & & 3/31/08 & 8C17008 & AGG-RRL-001 \\
\hline
\end{tabular}


GEA/Soil

\begin{tabular}{|c|c|c|c|c|c|c|c|c|}
\hline CAS \# & Analyte & Results & Units & MDA & UNC & Analyzed & Batch & Method \\
\hline HEIS No. & B1TNK7A & \multicolumn{3}{|c|}{ Lab ID: $\quad$ 0803015-23 } & & & & \\
\hline $14234-35-6$ & Antimony-125 & $<1.04$ & $\mathrm{pCi} / \mathrm{g}$ & 1.04 & & $3 / 31 / 08$ & 8C17008 & AGG-RRL-001 \\
\hline $15756-32-8$ & Antimony-126 & $<0.328$ & $\mathrm{pCi} / \mathrm{g}$ & 0.328 & & 3/31/08 & 8C17008 & AGG-RRL-001 \\
\hline $15832-50-5$ & Tin-126 & $<0.776$ & $\mathrm{pCi} / \mathrm{g}$ & 0.776 & & $3 / 31 / 08$ & 8C17008 & AGG-RRL-001 \\
\hline 10043-66-0 & Iodine-131 & $<0.412$ & $\mathrm{pCi} / \mathrm{g}$ & 0.412 & & 3/31/08 & 8C17008 & AGG-RRL-001 \\
\hline $13981-41-4$ & Barium-133 & $<0.573$ & $\mathrm{pCi} / \mathrm{g}$ & 0.573 & & 3/31/08 & 8C17008 & AGG-RRL-001 \\
\hline 13967-70-9 & Cesium-134 & $<0.365$ & $\mathrm{pCi} / \mathrm{g}$ & 0.365 & & 3/31/08 & 8C17008 & AGG-RRL-001 \\
\hline $10045-97-3$ & Cesium-137 & $<0.386$ & $\mathrm{pCi} / \mathrm{g}$ & 0.386 & & $3 / 31 / 08$ & 8C17008 & AGG-RRL-001 \\
\hline 13982-30-4 & Cerium-139 & $<0.38$ & $\mathrm{pCi} / \mathrm{g}$ & 0.38 & & $3 / 31 / 08$ & 8C17008 & AGG-RRL-001 \\
\hline $14762-78-8$ & Cerium-144 & $<2.73$ & $\mathrm{pCi} / \mathrm{g}$ & 2.73 & & $3 / 31 / 08$ & 8C17008 & AGG-RRL-001 \\
\hline 14683-23-9 & Europium-152 & $<1.36$ & $\mathrm{pCi} / \mathrm{g}$ & 1.36 & & 3/31/08 & 8C17008 & AGG-RRL-001 \\
\hline 14276-65-4 & Gadolinium-153 & $<1.03$ & $\mathrm{pCi} / \mathrm{g}$ & 1.03 & & $3 / 31 / 08$ & 8C17008 & AGG-RRL-001 \\
\hline $15585-10-1$ & Europium-154 & $<0.705$ & $\mathrm{pCi} / \mathrm{g}$ & 0.705 & & $3 / 31 / 08$ & 8C17008 & AGG-RRL-001 \\
\hline $14391-16-3$ & Europium-155 & $<1.11$ & $\mathrm{pCi} / \mathrm{g}$ & 1.11 & & 3/31/08 & 8C17008 & AGG-RRL-001 \\
\hline 13982-78-0 & Mercury-203 & $<0.412$ & $\mathrm{pCi} / \mathrm{g}$ & 0.412 & & $3 / 31 / 08$ & 8C17008 & AGG-RRL-001 \\
\hline $14913-50-9$ & Thallium-208 & $<0.407$ & $\mathrm{pCi} / \mathrm{g}$ & 0.407 & & 3/31/08 & 8C17008 & AGG-RRL-001 \\
\hline 14331-79-4 & Bismuth-210 & $<0.641$ & $\mathrm{pCi} / \mathrm{g}$ & 0.641 & & $3 / 31 / 08$ & 8C17008 & AGG-RRL-001 \\
\hline \multirow[t]{3}{*}{ 14255-04-0 } & Lead-210 & $<45.8$ & $\mathrm{pCi} / \mathrm{g}$ & 45.8 & & 3/31/08 & 8C17008 & AGG-RRL-001 \\
\hline & Bismuth-211 & $<7.3$ & $\mathrm{pCi} / \mathrm{g}$ & 7.3 & & $3 / 31 / 08$ & 8C17008 & AGG-RRL-001 \\
\hline & Lead-211 & $<9.97$ & $\mathrm{pCi} / \mathrm{g}$ & 9.97 & & $3 / 31 / 08$ & 8C17008 & AGG-RRL-001 \\
\hline $15092-94-1$ & Lead-212 & $<0.854$ & $\mathrm{pCi} / \mathrm{g}$ & 0.854 & & $3 / 31 / 08$ & 8C17008 & AGG-RRL-001 \\
\hline $14733-03-0$ & Bismuth-214 & $<0.848$ & $\mathrm{pCi} / \mathrm{g}$ & 0.848 & & 3/31/08 & 8C17008 & AGG-RRL-001 \\
\hline $15067-28-4$ & Lead-214 & $<0.931$ & $\mathrm{pCi} / \mathrm{g}$ & 0.931 & & $3 / 31 / 08$ & 8C17008 & AGG-RRL-001 \\
\hline $14835-02-0$ & Radon-219 & $<3.22$ & pCi/g & 3.22 & & $3 / 31 / 08$ & 8C17008 & AGG-RRL-001 \\
\hline 22481-48-7 & Radon-220 & $<302$ & $\mathrm{pCi} / \mathrm{g}$ & 302 & & 3/31/08 & 8C17008 & AGG-RRL-001 \\
\hline 28522-20-5 & Radon-221 & $<1.51$ & $\mathrm{pCi} / \mathrm{g}$ & 1.51 & & $3 / 31 / 08$ & 8C17008 & AGG-RRL-001 \\
\hline 15756-98-6 & Francium-223 & $<10.9$ & $\mathrm{pCi} / \mathrm{g}$ & 10.9 & & $3 / 31 / 08$ & 8C17008 & AGG-RRL-001 \\
\hline $15623-45-7$ & Radium-223 & $<2.34$ & $\mathrm{pCi} / \mathrm{g}$ & 2.34 & & $3 / 31 / 08$ & 8C17008 & AGG-RRL-001 \\
\hline 13233-32-4 & Radium-224 & $<9.29$ & $\mathrm{pCi} / \mathrm{g}$ & 9.29 & & 3/31/08 & 8C17008 & AGG-RRL-001 \\
\hline 13982-63-3 & Radium-226 & $<9.31$ & $\mathrm{pCi} / \mathrm{g}$ & 9.31 & & 3/31/08 & 8C17008 & AGG-RRL-001 \\
\hline $15623-47-9$ & Thorium-227 & $<3.29$ & $\mathrm{pCi} / \mathrm{g}$ & 3.29 & & $3 / 31 / 08$ & 8C17008 & AGG-RRL-001 \\
\hline 14331-83-0 & Actinium-228 & $<1.38$ & $\mathrm{pCi} / \mathrm{g}$ & 1.38 & & $3 / 31 / 08$ & 8C17008 & AGG-RRL-001 \\
\hline 14274-82-9 & Thorium-228 & $<119$ & $\mathrm{pCi} / \mathrm{g}$ & 119 & & $3 / 31 / 08$ & 8C17008 & AGG-RRL-001 \\
\hline $14269-63-7$ & Thorium-230 & $<127$ & $\mathrm{pCi} / \mathrm{g}$ & 127 & & $3 / 31 / 08$ & 8C17008 & AGG-RRL-001 \\
\hline 14331-85-2 & Protactinium-231 & $<14.3$ & $\mathrm{pCi} / \mathrm{g}$ & 14.3 & & $3 / 31 / 08$ & 8C17008 & AGG-RRL-001 \\
\hline $14932-40-2$ & Thorium-231 & $<73.1$ & $\mathrm{pCi} / \mathrm{g}$ & 73.1 & & 3/31/08 & 8C17008 & AGG-RRL-001 \\
\hline 7440-29-1 & Thorium-232 & $<319$ & $\mathrm{pCi} / \mathrm{g}$ & 319 & & 3/31/08 & 8C17008 & AGG-RRL-001 \\
\hline 13981-14-1 & Protactinium-233 & $<0.858$ & $\mathrm{pCi} / \mathrm{g}$ & 0.858 & & $3 / 31 / 08$ & 8C17008 & AGG-RRL-001 \\
\hline \multirow[t]{2}{*}{$15100-28-4$} & Protactinium-234 & $<1.34$ & $\mathrm{pCi} / \mathrm{g}$ & 1.34 & & $3 / 31 / 08$ & 8C17008 & AGG-RRL-001 \\
\hline & Protactinium-234m & $<40.2$ & $\mathrm{pCi} / \mathrm{g}$ & 40.2 & & $3 / 31 / 08$ & 8C17008 & AGG-RRL-001 \\
\hline $15065-10-8$ & Thorium-234 & $<11.1$ & $\mathrm{pCi} / \mathrm{g}$ & 11.1 & & $3 / 31 / 08$ & 8C17008 & AGG-RRL-001 \\
\hline 15117-96-1 & Uranium 235 & $<2.85$ & $\mathrm{pCi} / \mathrm{g}$ & 2.85 & & $3 / 31 / 08$ & 8C17008 & AGG-RRL-001 \\
\hline 13994-20-2 & Neptunium-237 & $<2.88$ & pCi/g & 2.88 & & $3 / 31 / 08$ & 8C17008 & AGG-RRL-001 \\
\hline \multirow[t]{2}{*}{ 13981-16-3 } & Plutonium-238 & $<4210$ & $\mathrm{pCi} / \mathrm{g}$ & 4210 & & 3/31/08 & 8C17008 & AGG-RRL-001 \\
\hline & Uranium 238 & $<6.01$ & $\mathrm{pCi} / \mathrm{g}$ & 6.01 & & $3 / 31 / 08$ & 8C17008 & AGG-RRL-001 \\
\hline $15117-48-3$ & Plutonium-239 & $<4380$ & $\mathrm{pCi} / \mathrm{g}$ & 4380 & & 3/31/08 & 8C17008 & AGG-RRL-001 \\
\hline 14119-33-6 & Plutonium-240 & $<4200$ & $\mathrm{pCi} / \mathrm{g}$ & 4200 & & $3 / 31 / 08$ & 8C17008 & AGG-RRL-001 \\
\hline $14596-10-2$ & Americium-241 & $<1.63$ & $\mathrm{pCi} / \mathrm{g}$ & 1.63 & & $3 / 31 / 08$ & 8C17008 & AGG-RRL-001 \\
\hline $14993-75-0$ & Americium-243 & $<0.695$ & $\mathrm{pCi} / \mathrm{g}$ & 0.695 & & $3 / 31 / 08$ & 8C17008 & AGG-RRL-001 \\
\hline $15757-87-6$ & Curium-243 & $<1.29$ & $\mathrm{pCi} / \mathrm{g}$ & 1.29 & & $3 / 31 / 08$ & 8C17008 & AGG-RRL-001 \\
\hline
\end{tabular}


GEA/Soil

\begin{tabular}{|c|c|c|c|c|c|c|c|c|}
\hline CAS \# & Analyte & Results & Units & MDA & UNC & Analyzed & Batch & Method \\
\hline HEIS No. & B1TNK7A & \multicolumn{2}{|c|}{ Lab ID: } & \multicolumn{2}{|l|}{ 0803015-23 } & & & \\
\hline 15621-76-8 & Curium-245 & $<1.06$ & $\mathrm{pCi} / \mathrm{g}$ & 1.06 & & 3/31/08 & 8C17008 & AGG-RRL-001 \\
\hline HEIS No. & B1TNK8B & \multicolumn{2}{|c|}{ Lab ID: } & 0803015-26 & & & & \\
\hline 13966-32-0 & Sodium-22 & $<0.379$ & $\mathrm{pCi} / \mathrm{g}$ & 0.379 & & 3/31/08 & 8C17008 & AGG-RRL-001 \\
\hline $13966-00-2$ & Potassium-40 & 20.8 & $\mathrm{pCi} / \mathrm{g}$ & 2.86 & 1.28 & 3/31/08 & 8C17008 & AGG-RRL-001 \\
\hline $14392-02-0$ & Chromium-51 & $<3.32$ & $\mathrm{pCi} / \mathrm{g}$ & 3.32 & & 3/31/08 & 8C17008 & AGG-RRL-001 \\
\hline 13966-31-9 & Manganese-54 & $<0.344$ & $\mathrm{pCi} / \mathrm{g}$ & 0.344 & & 3/31/08 & 8C17008 & AGG-RRL-001 \\
\hline 13981-50-5 & Cobalt-57 & $<0.356$ & $\mathrm{pCi} / \mathrm{g}$ & 0.356 & & 3/31/08 & 8C17008 & AGG-RRL-001 \\
\hline $14596-12-4$ & Iron-59 & $<0.694$ & $\mathrm{pCi} / \mathrm{g}$ & 0.694 & & 3/31/08 & 8C17008 & AGG-RRL-001 \\
\hline $10198-40-0$ & Cobalt-60 & $<0.33$ & $\mathrm{pCi} / \mathrm{g}$ & 0.33 & & 3/31/08 & 8C17008 & AGG-RRL-001 \\
\hline 13982-39-3 & Zinc-65 & $<0.788$ & $\mathrm{pCi} / \mathrm{g}$ & 0.788 & & 3/31/08 & 8C17008 & AGG-RRL-001 \\
\hline 14265-71-5 & Selenium-75 & $<0.544$ & $\mathrm{pCi} / \mathrm{g}$ & 0.544 & & 3/31/08 & 8C17008 & AGG-RRL-001 \\
\hline 17056-36-9 & Rubidium-83 & $<0.87$ & $\mathrm{pCi} / \mathrm{g}$ & 0.87 & & 3/31/08 & 8C17008 & AGG-RRL-001 \\
\hline 13967-73-2 & Strontium-85 & $<0.406$ & $\mathrm{pCi} / \mathrm{g}$ & 0.406 & & 3/31/08 & 8C17008 & AGG-RRL-001 \\
\hline $14932-53-7$ & Rubidium-86 & $<4.48$ & $\mathrm{pCi} / \mathrm{g}$ & 4.48 & & 3/31/08 & 8C17008 & AGG-RRL-001 \\
\hline $13982-36-0$ & Yttrium-88 & $<0.283$ & $\mathrm{pCi} / \mathrm{g}$ & 0.283 & & 3/31/08 & 8C17008 & AGG-RRL-001 \\
\hline 14681-63-1 & Niobium-94 & $<0.345$ & $\mathrm{pCi} / \mathrm{g}$ & 0.345 & & 3/31/08 & 8C17008 & AGG-RRL-001 \\
\hline \multirow{3}{*}{ 13967-76-5 } & Niobium-95 & $<0.359$ & $\mathrm{pCi} / \mathrm{g}$ & 0.359 & & 3/31/08 & 8C17008 & AGG-RRL-001 \\
\hline & Niobium-95m & $<1.56$ & $\mathrm{pCi} / \mathrm{g}$ & 1.56 & & 3/31/08 & 8C17008 & AGG-RRL-001 \\
\hline & Technetium-95m & $<0.529$ & $\mathrm{pCi} / \mathrm{g}$ & 0.529 & & 3/31/08 & 8C17008 & AGG-RRL-001 \\
\hline \multirow[t]{2}{*}{ 13967-71-0 } & Zirconium-95 & $<0.636$ & $\mathrm{pCi} / \mathrm{g}$ & 0.636 & & 3/31/08 & 8C17008 & AGG-RRL-001 \\
\hline & Technetium-99m & $<0.35$ & $\mathrm{pCi} / \mathrm{g}$ & 0.35 & & 3/31/08 & 8C17008 & AGG-RRL-001 \\
\hline 13968-53-1 & Ruthenium-103 & $<0.408$ & $\mathrm{pCi} / \mathrm{g}$ & 0.408 & & 3/31/08 & 8C17008 & AGG-RRL-001 \\
\hline 13967-48-1 & Ruthenium-106 & $<3.89$ & $\mathrm{pCi} / \mathrm{g}$ & 3.89 & & 3/31/08 & 8C17008 & AGG-RRL-001 \\
\hline 14391-65-2 & Silver-108m & $<0.357$ & $\mathrm{pCi} / \mathrm{g}$ & 0.357 & & 3/31/08 & 8C17008 & AGG-RRL-001 \\
\hline \multirow[t]{2}{*}{$14109-32-1$} & Cadmium-109 & $<9.97$ & $\mathrm{pCi} / \mathrm{g}$ & 9.97 & & 3/31/08 & 8C17008 & AGG-RRL-001 \\
\hline & Silver-110 & $<0.354$ & $\mathrm{pCi} / \mathrm{g}$ & 0.354 & & 3/31/08 & 8C17008 & AGG-RRL-001 \\
\hline 14391-76-5 & Silver-110m & $<0.355$ & $\mathrm{pCi} / \mathrm{g}$ & 0.355 & & 3/31/08 & 8C17008 & AGG-RRL-001 \\
\hline 13966-06-8 & Tin-113 & $<0.5$ & $\mathrm{pCi} / \mathrm{g}$ & 0.5 & & 3/31/08 & 8C17008 & AGG-RRL-001 \\
\hline $14683-10-4$ & Antimony-124 & $<0.405$ & $\mathrm{pCi} / \mathrm{g}$ & 0.405 & & 3/31/08 & 8C17008 & AGG-RRL-001 \\
\hline $14234-35-6$ & Antimony-125 & $<1.1$ & $\mathrm{pCi} / \mathrm{g}$ & 1.1 & & 3/31/08 & 8C17008 & AGG-RRL-001 \\
\hline 15756-32-8 & Antimony-126 & $<0.345$ & $\mathrm{pCi} / \mathrm{g}$ & 0.345 & & 3/31/08 & 8C17008 & AGG-RRL-001 \\
\hline $15832-50-5$ & Tin-126 & $<0.814$ & $\mathrm{pCi} / \mathrm{g}$ & 0.814 & & 3/31/08 & 8C17008 & AGG-RRL-001 \\
\hline 10043-66-0 & Iodine-131 & $<0.426$ & $\mathrm{pCi} / \mathrm{g}$ & 0.426 & & 3/31/08 & 8C17008 & AGG-RRL-001 \\
\hline 13981-41-4 & Barium-133 & $<0.595$ & $\mathrm{pCi} / \mathrm{g}$ & 0.595 & & 3/31/08 & 8C17008 & AGG-RRL-001 \\
\hline 13967-70-9 & Cesium-134 & $<0.41$ & $\mathrm{pCi} / \mathrm{g}$ & 0.41 & & 3/31/08 & 8C17008 & AGG-RRL-001 \\
\hline $10045-97-3$ & Cesium-137 & $<0.404$ & $\mathrm{pCi} / \mathrm{g}$ & 0.404 & & 3/31/08 & 8C17008 & AGG-RRL-001 \\
\hline 13982-30-4 & Cerium-139 & $<0.395$ & $\mathrm{pCi} / \mathrm{g}$ & 0.395 & & 3/31/08 & 8C17008 & AGG-RRL-001 \\
\hline 14762-78-8 & Cerium-144 & $<2.9$ & $\mathrm{pCi} / \mathrm{g}$ & 2.9 & & 3/31/08 & 8C17008 & AGG-RRL-001 \\
\hline 14683-23-9 & Europium-152 & $<1.42$ & $\mathrm{pCi} / \mathrm{g}$ & 1.42 & & 3/31/08 & 8C17008 & AGG-RRL-001 \\
\hline 14276-65-4 & Gadolinium-153 & $<1.09$ & $\mathrm{pCi} / \mathrm{g}$ & 1.09 & & 3/31/08 & 8C17008 & AGG-RRL-001 \\
\hline 15585-10-1 & Europium-154 & $<0.752$ & $\mathrm{pCi} / \mathrm{g}$ & 0.752 & & 3/31/08 & 8C17008 & AGG-RRL-001 \\
\hline $14391-16-3$ & Europium-155 & $<1.16$ & $\mathrm{pCi} / \mathrm{g}$ & 1.16 & & 3/31/08 & 8C17008 & AGG-RRL-001 \\
\hline 13982-78-0 & Mercury-203 & $<0.429$ & $\mathrm{pCi} / \mathrm{g}$ & 0.429 & & 3/31/08 & 8C17008 & AGG-RRL-001 \\
\hline $14913-50-9$ & Thallium-208 & $<0.442$ & $\mathrm{pCi} / \mathrm{g}$ & 0.442 & & 3/31/08 & 8C17008 & AGG-RRL-001 \\
\hline 14331-79-4 & Bismuth-210 & $<0.648$ & $\mathrm{pCi} / \mathrm{g}$ & 0.648 & & 3/31/08 & 8C17008 & AGG-RRL-001 \\
\hline \multirow[t]{3}{*}{$14255-04-0$} & Lead-210 & $<47.4$ & $\mathrm{pCi} / \mathrm{g}$ & 47.4 & & 3/31/08 & 8C17008 & AGG-RRL-001 \\
\hline & Bismuth-211 & $<8.07$ & $\mathrm{pCi} / \mathrm{g}$ & 8.07 & & 3/31/08 & 8C17008 & AGG-RRL-001 \\
\hline & Lead-211 & $<11$ & $\mathrm{pCi} / \mathrm{g}$ & 11 & & 3/31/08 & 8C17008 & AGG-RRL-001 \\
\hline
\end{tabular}


GEA/Soil

\begin{tabular}{|c|c|c|c|c|c|c|c|c|}
\hline CAS \# & Analyte & Results & Units & MDA & UNC & Analyzed & Batch & Method \\
\hline HEIS No. & B1TNK8B & \multicolumn{2}{|c|}{ Lab ID: } & 0803015-26 & & & & \\
\hline 15092-94-1 & Lead-212 & $<0.908$ & $\mathrm{pCi} / \mathrm{g}$ & 0.908 & & 3/31/08 & 8C17008 & AGG-RRL-001 \\
\hline 14733-03-0 & Bismuth-214 & $<0.86$ & $\mathrm{pCi} / \mathrm{g}$ & 0.86 & & $3 / 31 / 08$ & 8C17008 & AGG-RRL-001 \\
\hline $15067-28-4$ & Lead-214 & $<0.969$ & $\mathrm{pCi} / \mathrm{g}$ & 0.969 & & 3/31/08 & 8C17008 & AGG-RRL-001 \\
\hline $14835-02-0$ & Radon-219 & $<3.29$ & $\mathrm{pCi} / \mathrm{g}$ & 3.29 & & 3/31/08 & 8C17008 & AGG-RRL-001 \\
\hline 22481-48-7 & Radon-220 & $<351$ & $\mathrm{pCi} / \mathrm{g}$ & 351 & & 3/31/08 & 8C17008 & AGG-RRL-001 \\
\hline 28522-20-5 & Radon-221 & $<1.6$ & $\mathrm{pCi} / \mathrm{g}$ & 1.6 & & 3/31/08 & 8C17008 & AGG-RRL-001 \\
\hline 15756-98-6 & Francium-223 & $<11.4$ & $\mathrm{pCi} / \mathrm{g}$ & 11.4 & & 3/31/08 & 8C17008 & AGG-RRL-001 \\
\hline 15623-45-7 & Radium-223 & $<2.4$ & $\mathrm{pCi} / \mathrm{g}$ & 2.4 & & 3/31/08 & 8C17008 & AGG-RRL-001 \\
\hline 13233-32-4 & Radium-224 & $<9.79$ & $\mathrm{pCi} / \mathrm{g}$ & 9.79 & & 3/31/08 & 8C17008 & AGG-RRL-001 \\
\hline $13982-63-3$ & Radium-226 & $<9.87$ & $\mathrm{pCi} / \mathrm{g}$ & 9.87 & & 3/31/08 & 8C17008 & AGG-RRL-001 \\
\hline 15623-47-9 & Thorium-227 & $<3.49$ & $\mathrm{pCi} / \mathrm{g}$ & 3.49 & & 3/31/08 & 8C17008 & AGG-RRL-001 \\
\hline $14331-83-0$ & Actinium-228 & $<1.51$ & $\mathrm{pCi} / \mathrm{g}$ & 1.51 & & 3/31/08 & 8C17008 & AGG-RRL-001 \\
\hline $14274-82-9$ & Thorium-228 & $<126$ & $\mathrm{pCi} / \mathrm{g}$ & 126 & & 3/31/08 & 8C17008 & AGG-RRL-001 \\
\hline $14269-63-7$ & Thorium-230 & $<130$ & $\mathrm{pCi} / \mathrm{g}$ & 130 & & 3/31/08 & 8C17008 & AGG-RRL-001 \\
\hline 14331-85-2 & Protactinium-231 & $<14.9$ & $\mathrm{pCi} / \mathrm{g}$ & 14.9 & & 3/31/08 & 8C17008 & AGG-RRL-001 \\
\hline $14932-40-2$ & Thorium-231 & $<77$ & $\mathrm{pCi} / \mathrm{g}$ & 77 & & 3/31/08 & 8C17008 & AGG-RRL-001 \\
\hline 7440-29-1 & Thorium-232 & $<320$ & $\mathrm{pCi} / \mathrm{g}$ & 320 & & 3/31/08 & 8C17008 & AGG-RRL-001 \\
\hline 13981-14-1 & Protactinium-233 & $<0.904$ & $\mathrm{pCi} / \mathrm{g}$ & 0.904 & & 3/31/08 & 8C17008 & AGG-RRL-001 \\
\hline \multirow[t]{2}{*}{ 15100-28-4 } & Protactinium-234 & $<1.41$ & $\mathrm{pCi} / \mathrm{g}$ & 1.41 & & $3 / 31 / 08$ & 8C17008 & AGG-RRL-001 \\
\hline & Protactinium-234m & $<44$ & $\mathrm{pCi} / \mathrm{g}$ & 44 & & 3/31/08 & 8C17008 & AGG-RRL-001 \\
\hline $15065-10-8$ & Thorium-234 & $<11.3$ & $\mathrm{pCi} / \mathrm{g}$ & 11.3 & & 3/31/08 & 8C17008 & AGG-RRL-001 \\
\hline 15117-96-1 & Uranium 235 & $<2.97$ & $\mathrm{pCi} / \mathrm{g}$ & 2.97 & & 3/31/08 & 8C17008 & AGG-RRL-001 \\
\hline 13994-20-2 & Neptunium-237 & $<3.01$ & $\mathrm{pCi} / \mathrm{g}$ & 3.01 & & 3/31/08 & 8C17008 & AGG-RRL-001 \\
\hline \multirow[t]{2}{*}{ 13981-16-3 } & Plutonium-238 & $<4450$ & $\mathrm{pCi} / \mathrm{g}$ & 4450 & & 3/31/08 & 8C17008 & AGG-RRL-001 \\
\hline & Uranium 238 & $<6.29$ & $\mathrm{pCi} / \mathrm{g}$ & 6.29 & & 3/31/08 & 8C17008 & AGG-RRL-001 \\
\hline $15117-48-3$ & Plutonium-239 & $<4430$ & $\mathrm{pCi} / \mathrm{g}$ & 4430 & & $3 / 31 / 08$ & 8C17008 & AGG-RRL-001 \\
\hline 14119-33-6 & Plutonium-240 & $<4420$ & $\mathrm{pCi} / \mathrm{g}$ & 4420 & & 3/31/08 & 8C17008 & AGG-RRL-001 \\
\hline 14596-10-2 & Americium-241 & $<1.64$ & $\mathrm{pCi} / \mathrm{g}$ & 1.64 & & 3/31/08 & 8C17008 & AGG-RRL-001 \\
\hline 14993-75-0 & Americium-243 & $<0.718$ & $\mathrm{pCi} / \mathrm{g}$ & 0.718 & & 3/31/08 & 8C17008 & AGG-RRL-001 \\
\hline 15757-87-6 & Curium-243 & $<1.35$ & $\mathrm{pCi} / \mathrm{g}$ & 1.35 & & 3/31/08 & 8C17008 & AGG-RRL-001 \\
\hline 15621-76-8 & Curium-245 & $<1.11$ & $\mathrm{pCi} / \mathrm{g}$ & 1.11 & & $3 / 31 / 08$ & 8C17008 & AGG-RRL-001 \\
\hline HEIS No. & B1TNK8A & \multicolumn{2}{|c|}{ Lab ID: } & 0803015-27 & & & & \\
\hline $13966-32-0$ & Sodium-22 & $<0.335$ & $\mathrm{pCi} / \mathrm{g}$ & 0.335 & & 3/31/08 & 8C17008 & AGG-RRL-001 \\
\hline 13966-00-2 & Potassium-40 & 21.6 & $\mathrm{pCi} / \mathrm{g}$ & 2.64 & 1.31 & 3/31/08 & 8C17008 & AGG-RRL-001 \\
\hline $14392-02-0$ & Chromium-51 & $<2.87$ & $\mathrm{pCi} / \mathrm{g}$ & 2.87 & & 3/31/08 & 8C17008 & AGG-RRL-001 \\
\hline 13966-31-9 & Manganese-54 & $<0.279$ & $\mathrm{pCi} / \mathrm{g}$ & 0.279 & & 3/31/08 & 8C17008 & AGG-RRL-001 \\
\hline 13981-50-5 & Cobalt-57 & $<0.304$ & $\mathrm{pCi} / \mathrm{g}$ & 0.304 & & 3/31/08 & 8C17008 & AGG-RRL-001 \\
\hline $14596-12-4$ & Iron-59 & $<0.607$ & $\mathrm{pCi} / \mathrm{g}$ & 0.607 & & 3/31/08 & 8C17008 & AGG-RRL-001 \\
\hline $10198-40-0$ & Cobalt-60 & $<0.289$ & $\mathrm{pCi} / \mathrm{g}$ & 0.289 & & 3/31/08 & 8C17008 & AGG-RRL-001 \\
\hline 13982-39-3 & Zinc-65 & $<0.719$ & $\mathrm{pCi} / \mathrm{g}$ & 0.719 & & 3/31/08 & 8C17008 & AGG-RRL-001 \\
\hline 14265-71-5 & Selenium-75 & $<0.495$ & $\mathrm{pCi} / \mathrm{g}$ & 0.495 & & 3/31/08 & 8C17008 & AGG-RRL-001 \\
\hline 17056-36-9 & Rubidium-83 & $<0.605$ & $\mathrm{pCi} / \mathrm{g}$ & 0.605 & & 3/31/08 & 8C17008 & AGG-RRL-001 \\
\hline 13967-73-2 & Strontium-85 & $<0.399$ & $\mathrm{pCi} / \mathrm{g}$ & 0.399 & & 3/31/08 & 8C17008 & AGG-RRL-001 \\
\hline 14932-53-7 & Rubidium-86 & $<3.75$ & $\mathrm{pCi} / \mathrm{g}$ & 3.75 & & 3/31/08 & 8C17008 & AGG-RRL-001 \\
\hline $13982-36-0$ & Yttrium-88 & $<0.215$ & $\mathrm{pCi} / \mathrm{g}$ & 0.215 & & 3/31/08 & 8C17008 & AGG-RRL-001 \\
\hline 14681-63-1 & Niobium-94 & $<0.292$ & $\mathrm{pCi} / \mathrm{g}$ & 0.292 & & 3/31/08 & 8C17008 & AGG-RRL-001 \\
\hline \multirow[t]{2}{*}{ 13967-76-5 } & Niobium-95 & $<0.288$ & $\mathrm{pCi} / \mathrm{g}$ & 0.288 & & 3/31/08 & 8C17008 & AGG-RRL-001 \\
\hline & Niobium-95m & $<1.43$ & $\mathrm{pCi} / \mathrm{g}$ & 1.43 & & 3/31/08 & 8C17008 & AGG-RRL-001 \\
\hline
\end{tabular}


GEA/Soil

\begin{tabular}{|c|c|c|c|c|c|c|c|c|}
\hline CAS \# & Analyte & Results & Units & MDA & UNC & Analyzed & Batch & Method \\
\hline \multirow[t]{2}{*}{ HEIS No. } & B1TNK8A & \multicolumn{2}{|c|}{ Lab ID: } & 0803015-27 & & & & \\
\hline & Technetium-95m & $<0.492$ & $\mathrm{pCi} / \mathrm{g}$ & 0.492 & & 3/31/08 & 8C17008 & AGG-RRL-001 \\
\hline \multirow[t]{2}{*}{ 13967-71-0 } & Zirconium-95 & $<0.512$ & $\mathrm{pCi} / \mathrm{g}$ & 0.512 & & 3/31/08 & 8C17008 & AGG-RRL-001 \\
\hline & Technetium-99m & $<0.31$ & $\mathrm{pCi} / \mathrm{g}$ & 0.31 & & 3/31/08 & 8C17008 & AGG-RRL-001 \\
\hline 13968-53-1 & Ruthenium-103 & $<0.324$ & $\mathrm{pCi} / \mathrm{g}$ & 0.324 & & 3/31/08 & 8C17008 & AGG-RRL-001 \\
\hline 13967-48-1 & Ruthenium-106 & $<2.9$ & $\mathrm{pCi} / \mathrm{g}$ & 2.9 & & 3/31/08 & 8C17008 & AGG-RRL-001 \\
\hline 14391-65-2 & Silver-108m & $<0.304$ & $\mathrm{pCi} / \mathrm{g}$ & 0.304 & & 3/31/08 & 8C17008 & AGG-RRL-001 \\
\hline \multirow[t]{2}{*}{ 14109-32-1 } & Cadmium-109 & $<8.26$ & $\mathrm{pCi} / \mathrm{g}$ & 8.26 & & 3/31/08 & 8C17008 & AGG-RRL-001 \\
\hline & Silver-110 & $<0.327$ & $\mathrm{pCi} / \mathrm{g}$ & 0.327 & & 3/31/08 & 8C17008 & AGG-RRL-001 \\
\hline 14391-76-5 & Silver-110m & $<0.328$ & $\mathrm{pCi} / \mathrm{g}$ & 0.328 & & 3/31/08 & 8C17008 & AGG-RRL-001 \\
\hline 13966-06-8 & Tin-113 & $<0.442$ & $\mathrm{pCi} / \mathrm{g}$ & 0.442 & & 3/31/08 & 8C17008 & AGG-RRL-001 \\
\hline $14683-10-4$ & Antimony-124 & $<0.308$ & $\mathrm{pCi} / \mathrm{g}$ & 0.308 & & 3/31/08 & 8C17008 & AGG-RRL-001 \\
\hline $14234-35-6$ & Antimony-125 & $<0.924$ & $\mathrm{pCi} / \mathrm{g}$ & 0.924 & & 3/31/08 & 8C17008 & AGG-RRL-001 \\
\hline 15756-32-8 & Antimony-126 & $<0.294$ & $\mathrm{pCi} / \mathrm{g}$ & 0.294 & & 3/31/08 & 8C17008 & AGG-RRL-001 \\
\hline 15832-50-5 & Tin-126 & $<0.654$ & $\mathrm{pCi} / \mathrm{g}$ & 0.654 & & 3/31/08 & 8C17008 & AGG-RRL-001 \\
\hline 10043-66-0 & Iodine-131 & $<0.36$ & $\mathrm{pCi} / \mathrm{g}$ & 0.36 & & 3/31/08 & 8C17008 & AGG-RRL-001 \\
\hline 13981-41-4 & Barium-133 & $<0.553$ & $\mathrm{pCi} / \mathrm{g}$ & 0.553 & & 3/31/08 & 8C17008 & AGG-RRL-001 \\
\hline 13967-70-9 & Cesium-134 & $<0.356$ & $\mathrm{pCi} / \mathrm{g}$ & 0.356 & & 3/31/08 & 8C17008 & AGG-RRL-001 \\
\hline 10045-97-3 & Cesium-137 & $<0.361$ & $\mathrm{pCi} / \mathrm{g}$ & 0.361 & & 3/31/08 & 8C17008 & AGG-RRL-001 \\
\hline 13982-30-4 & Cerium-139 & $<0.349$ & $\mathrm{pCi} / \mathrm{g}$ & 0.349 & & 3/31/08 & 8C17008 & AGG-RRL-001 \\
\hline 14762-78-8 & Cerium-144 & $<2.38$ & $\mathrm{pCi} / \mathrm{g}$ & 2.38 & & 3/31/08 & 8C17008 & AGG-RRL-001 \\
\hline 14683-23-9 & Europium-152 & $<1.12$ & $\mathrm{pCi} / \mathrm{g}$ & 1.12 & & 3/31/08 & 8C17008 & AGG-RRL-001 \\
\hline $14276-65-4$ & Gadolinium-153 & $<0.903$ & $\mathrm{pCi} / \mathrm{g}$ & 0.903 & & 3/31/08 & 8C17008 & AGG-RRL-001 \\
\hline 15585-10-1 & Europium-154 & $<0.639$ & $\mathrm{pCi} / \mathrm{g}$ & 0.639 & & 3/31/08 & 8C17008 & AGG-RRL-001 \\
\hline 14391-16-3 & Europium-155 & $<0.922$ & $\mathrm{pCi} / \mathrm{g}$ & 0.922 & & 3/31/08 & 8C17008 & AGG-RRL-001 \\
\hline 13982-78-0 & Mercury-203 & $<0.364$ & $\mathrm{pCi} / \mathrm{g}$ & 0.364 & & 3/31/08 & 8C17008 & AGG-RRL-001 \\
\hline 14913-50-9 & Thallium-208 & $<0.38$ & $\mathrm{pCi} / \mathrm{g}$ & 0.38 & & 3/31/08 & 8C17008 & AGG-RRL-001 \\
\hline 14331-79-4 & Bismuth-210 & $<0.599$ & $\mathrm{pCi} / \mathrm{g}$ & 0.599 & & 3/31/08 & 8C17008 & AGG-RRL-001 \\
\hline \multirow[t]{3}{*}{$14255-04-0$} & Lead-210 & $<116$ & $\mathrm{pCi} / \mathrm{g}$ & 116 & & 3/31/08 & 8C17008 & AGG-RRL-001 \\
\hline & Bismuth-211 & $<7.1$ & $\mathrm{pCi} / \mathrm{g}$ & 7.1 & & 3/31/08 & 8C17008 & AGG-RRL-001 \\
\hline & Lead-211 & $<9.7$ & $\mathrm{pCi} / \mathrm{g}$ & 9.7 & & 3/31/08 & 8C17008 & AGG-RRL-001 \\
\hline 15092-94-1 & Lead-212 & 0.591 & $\mathrm{pCi} / \mathrm{g}$ & 0.587 & 0.284 & 3/31/08 & 8C17008 & AGG-RRL-001 \\
\hline 14733-03-0 & Bismuth-214 & $<0.803$ & $\mathrm{pCi} / \mathrm{g}$ & 0.803 & & 3/31/08 & 8C17008 & AGG-RRL-001 \\
\hline $15067-28-4$ & Lead-214 & $<0.896$ & $\mathrm{pCi} / \mathrm{g}$ & 0.896 & & 3/31/08 & 8C17008 & AGG-RRL-001 \\
\hline $14835-02-0$ & Radon-219 & $<3.01$ & $\mathrm{pCi} / \mathrm{g}$ & 3.01 & & 3/31/08 & 8C17008 & AGG-RRL-001 \\
\hline 22481-48-7 & Radon-220 & $<260$ & $\mathrm{pCi} / \mathrm{g}$ & 260 & & 3/31/08 & 8C17008 & AGG-RRL-001 \\
\hline 28522-20-5 & Radon-221 & $<1.38$ & $\mathrm{pCi} / \mathrm{g}$ & 1.38 & & 3/31/08 & 8C17008 & AGG-RRL-001 \\
\hline 15756-98-6 & Francium-223 & $<10.3$ & $\mathrm{pCi} / \mathrm{g}$ & 10.3 & & 3/31/08 & 8C17008 & AGG-RRL-001 \\
\hline $15623-45-7$ & Radium-223 & $<2.21$ & $\mathrm{pCi} / \mathrm{g}$ & 2.21 & & 3/31/08 & 8C17008 & AGG-RRL-001 \\
\hline 13233-32-4 & Radium-224 & $<5.22$ & $\mathrm{pCi} / \mathrm{g}$ & 5.22 & 1.18 & 3/31/08 & 8C17008 & AGG-RRL-001 \\
\hline 13982-63-3 & Radium-226 & $<8.51$ & $\mathrm{pCi} / \mathrm{g}$ & 8.51 & & 3/31/08 & 8C17008 & AGG-RRL-001 \\
\hline 15623-47-9 & Thorium-227 & $<3.2$ & $\mathrm{pCi} / \mathrm{g}$ & 3.2 & & 3/31/08 & 8C17008 & AGG-RRL-001 \\
\hline 14331-83-0 & Actinium-228 & $<1.33$ & $\mathrm{pCi} / \mathrm{g}$ & 1.33 & & 3/31/08 & 8C17008 & AGG-RRL-001 \\
\hline $14274-82-9$ & Thorium-228 & $<113$ & $\mathrm{pCi} / \mathrm{g}$ & 113 & & 3/31/08 & 8C17008 & AGG-RRL-001 \\
\hline 14269-63-7 & Thorium-230 & $<116$ & $\mathrm{pCi} / \mathrm{g}$ & 116 & & 3/31/08 & 8C17008 & AGG-RRL-001 \\
\hline 14331-85-2 & Protactinium-231 & $<12.7$ & $\mathrm{pCi} / \mathrm{g}$ & 12.7 & & 3/31/08 & 8C17008 & AGG-RRL-001 \\
\hline $14932-40-2$ & Thorium-231 & $<61.7$ & $\mathrm{pCi} / \mathrm{g}$ & 61.7 & & 3/31/08 & 8C17008 & AGG-RRL-001 \\
\hline 7440-29-1 & Thorium-232 & $<368$ & $\mathrm{pCi} / \mathrm{g}$ & 368 & & 3/31/08 & 8C17008 & AGG-RRL-001 \\
\hline 13981-14-1 & Protactinium-233 & $<0.769$ & $\mathrm{pCi} / \mathrm{g}$ & 0.769 & & 3/31/08 & 8C17008 & AGG-RRL-001 \\
\hline $15100-28-4$ & Protactinium-234 & $<1.14$ & $\mathrm{pCi} / \mathrm{g}$ & 1.14 & & 3/31/08 & 8C17008 & AGG-RRL-001 \\
\hline
\end{tabular}


GEA/Soil

\begin{tabular}{|c|c|c|c|c|c|c|c|c|}
\hline CAS \# & Analyte & Results & Units & MDA & UNC & Analyzed & Batch & Method \\
\hline \multirow[t]{2}{*}{ HEIS No. } & B1TNK8A & \multicolumn{2}{|c|}{ Lab ID: } & 0803015-27 & & & & \\
\hline & Protactinium-234m & $<35.5$ & $\mathrm{pCi} / \mathrm{g}$ & 35.5 & & 3/31/08 & 8C17008 & AGG-RRL-001 \\
\hline $15065-10-8$ & Thorium-234 & $<10.6$ & $\mathrm{pCi} / \mathrm{g}$ & 10.6 & & 3/31/08 & 8C17008 & AGG-RRL-001 \\
\hline 15117-96-1 & Uranium 235 & $<2.64$ & $\mathrm{pCi} / \mathrm{g}$ & 2.64 & & 3/31/08 & 8C17008 & AGG-RRL-001 \\
\hline 13994-20-2 & Neptunium-237 & $<2.4$ & $\mathrm{pCi} / \mathrm{g}$ & 2.4 & & 3/31/08 & 8C17008 & AGG-RRL-001 \\
\hline \multirow[t]{2}{*}{ 13981-16-3 } & Plutonium-238 & $<3590$ & $\mathrm{pCi} / \mathrm{g}$ & 3590 & & 3/31/08 & 8C17008 & AGG-RRL-001 \\
\hline & Uranium 238 & $<5.49$ & $\mathrm{pCi} / \mathrm{g}$ & 5.49 & & 3/31/08 & 8C17008 & AGG-RRL-001 \\
\hline 15117-48-3 & Plutonium-239 & $<4160$ & $\mathrm{pCi} / \mathrm{g}$ & 4160 & & 3/31/08 & 8C17008 & AGG-RRL-001 \\
\hline 14119-33-6 & Plutonium-240 & $<3610$ & $\mathrm{pCi} / \mathrm{g}$ & 3610 & & 3/31/08 & 8C17008 & AGG-RRL-001 \\
\hline 14596-10-2 & Americium-241 & $<1.79$ & $\mathrm{pCi} / \mathrm{g}$ & 1.79 & & 3/31/08 & 8C17008 & AGG-RRL-001 \\
\hline 14993-75-0 & Americium-243 & $<0.323$ & $\mathrm{pCi} / \mathrm{g}$ & 0.323 & & 3/31/08 & 8C17008 & AGG-RRL-001 \\
\hline 15757-87-6 & Curium-243 & $<1.1$ & $\mathrm{pCi} / \mathrm{g}$ & 1.1 & & 3/31/08 & 8C17008 & AGG-RRL-001 \\
\hline 15621-76-8 & Curium-245 & $<0.904$ & $\mathrm{pCi} / \mathrm{g}$ & 0.904 & & 3/31/08 & 8C17008 & AGG-RRL-001 \\
\hline HEIS No. & B1TNK9B & \multicolumn{2}{|c|}{ Lab ID: } & 0803015-30 & & & & \\
\hline 13966-32-0 & Sodium-22 & $<0.393$ & $\mathrm{pCi} / \mathrm{g}$ & 0.393 & & 3/31/08 & 8C17008 & AGG-RRL-001 \\
\hline 13966-00-2 & Potassium-40 & 19.5 & $\mathrm{pCi} / \mathrm{g}$ & 2.64 & 1.2 & 3/31/08 & 8C17008 & AGG-RRL-001 \\
\hline 14392-02-0 & Chromium-51 & $<3.23$ & $\mathrm{pCi} / \mathrm{g}$ & 3.23 & & 3/31/08 & 8C17008 & AGG-RRL-001 \\
\hline 13966-31-9 & Manganese-54 & $<0.345$ & $\mathrm{pCi} / \mathrm{g}$ & 0.345 & & 3/31/08 & 8C17008 & AGG-RRL-001 \\
\hline 13981-50-5 & Cobalt-57 & $<0.331$ & $\mathrm{pCi} / \mathrm{g}$ & 0.331 & & 3/31/08 & 8C17008 & AGG-RRL-001 \\
\hline $14596-12-4$ & Iron-59 & $<0.662$ & $\mathrm{pCi} / \mathrm{g}$ & 0.662 & & 3/31/08 & 8C17008 & AGG-RRL-001 \\
\hline 10198-40-0 & Cobalt-60 & $<0.299$ & $\mathrm{pCi} / \mathrm{g}$ & 0.299 & & 3/31/08 & 8C17008 & AGG-RRL-001 \\
\hline 13982-39-3 & Zinc-65 & $<0.83$ & $\mathrm{pCi} / \mathrm{g}$ & 0.83 & & 3/31/08 & 8C17008 & AGG-RRL-001 \\
\hline 14265-71-5 & Selenium-75 & $<0.526$ & $\mathrm{pCi} / \mathrm{g}$ & 0.526 & & 3/31/08 & 8C17008 & AGG-RRL-001 \\
\hline 17056-36-9 & Rubidium-83 & $<0.798$ & $\mathrm{pCi} / \mathrm{g}$ & 0.798 & & 3/31/08 & 8C17008 & AGG-RRL-001 \\
\hline 13967-73-2 & Strontium-85 & $<0.374$ & pCi/g & 0.374 & & 3/31/08 & 8C17008 & AGG-RRL-001 \\
\hline $14932-53-7$ & Rubidium-86 & $<4.4$ & $\mathrm{pCi} / \mathrm{g}$ & 4.4 & & 3/31/08 & 8C17008 & AGG-RRL-001 \\
\hline 13982-36-0 & Yttrium-88 & $<0.327$ & $\mathrm{pCi} / \mathrm{g}$ & 0.327 & & 3/31/08 & 8C17008 & AGG-RRL-001 \\
\hline 14681-63-1 & Niobium-94 & $<0.334$ & $\mathrm{pCi} / \mathrm{g}$ & 0.334 & & 3/31/08 & 8C17008 & AGG-RRL-001 \\
\hline \multirow[t]{3}{*}{ 13967-76-5 } & Niobium-95 & $<0.303$ & $\mathrm{pCi} / \mathrm{g}$ & 0.303 & & 3/31/08 & 8C17008 & AGG-RRL-001 \\
\hline & Niobium-95m & $<1.48$ & $\mathrm{pCi} / \mathrm{g}$ & 1.48 & & 3/31/08 & 8C17008 & AGG-RRL-001 \\
\hline & Technetium-95m & $<0.513$ & $\mathrm{pCi} / \mathrm{g}$ & 0.513 & & 3/31/08 & 8C17008 & AGG-RRL-001 \\
\hline \multirow[t]{2}{*}{ 13967-71-0 } & Zirconium-95 & $<0.521$ & $\mathrm{pCi} / \mathrm{g}$ & 0.521 & & 3/31/08 & 8C17008 & AGG-RRL-001 \\
\hline & Technetium-99m & $<0.331$ & $\mathrm{pCi} / \mathrm{g}$ & 0.331 & & 3/31/08 & 8C17008 & AGG-RRL-001 \\
\hline 13968-53-1 & Ruthenium-103 & $<0.375$ & $\mathrm{pCi} / \mathrm{g}$ & 0.375 & & 3/31/08 & 8C17008 & AGG-RRL-001 \\
\hline 13967-48-1 & Ruthenium-106 & $<3.65$ & $\mathrm{pCi} / \mathrm{g}$ & 3.65 & & 3/31/08 & 8C17008 & AGG-RRL-001 \\
\hline 14391-65-2 & Silver-108m & $<0.335$ & $\mathrm{pCi} / \mathrm{g}$ & 0.335 & & 3/31/08 & 8C17008 & AGG-RRL-001 \\
\hline \multirow[t]{2}{*}{ 14109-32-1 } & Cadmium-109 & $<9.33$ & $\mathrm{pCi} / \mathrm{g}$ & 9.33 & & 3/31/08 & 8C17008 & AGG-RRL-001 \\
\hline & Silver-110 & $<0.331$ & $\mathrm{pCi} / \mathrm{g}$ & 0.331 & & 3/31/08 & 8C17008 & AGG-RRL-001 \\
\hline 14391-76-5 & Silver-110m & $<0.332$ & $\mathrm{pCi} / \mathrm{g}$ & 0.332 & & 3/31/08 & 8C17008 & AGG-RRL-001 \\
\hline 13966-06-8 & Tin-113 & $<0.449$ & $\mathrm{pCi} / \mathrm{g}$ & 0.449 & & 3/31/08 & 8C17008 & AGG-RRL-001 \\
\hline 14683-10-4 & Antimony-124 & $<0.383$ & $\mathrm{pCi} / \mathrm{g}$ & 0.383 & & 3/31/08 & 8C17008 & AGG-RRL-001 \\
\hline $14234-35-6$ & Antimony-125 & $<1.04$ & pCi/g & 1.04 & & 3/31/08 & 8C17008 & AGG-RRL-001 \\
\hline 15756-32-8 & Antimony-126 & $<0.319$ & $\mathrm{pCi} / \mathrm{g}$ & 0.319 & & 3/31/08 & 8C17008 & AGG-RRL-001 \\
\hline 15832-50-5 & Tin-126 & $<0.76$ & $\mathrm{pCi} / \mathrm{g}$ & 0.76 & & 3/31/08 & 8C17008 & AGG-RRL-001 \\
\hline 10043-66-0 & Iodine-131 & $<0.416$ & $\mathrm{pCi} / \mathrm{g}$ & 0.416 & & 3/31/08 & 8C17008 & AGG-RRL-001 \\
\hline 13981-41-4 & Barium-133 & $<0.579$ & $\mathrm{pCi} / \mathrm{g}$ & 0.579 & & 3/31/08 & 8C17008 & AGG-RRL-001 \\
\hline 13967-70-9 & Cesium-134 & $<0.37$ & $\mathrm{pCi} / \mathrm{g}$ & 0.37 & & 3/31/08 & 8C17008 & AGG-RRL-001 \\
\hline 10045-97-3 & Cesium-137 & $<0.373$ & $\mathrm{pCi} / \mathrm{g}$ & 0.373 & & 3/31/08 & 8C17008 & AGG-RRL-001 \\
\hline 13982-30-4 & Cerium-139 & $<0.379$ & $\mathrm{pCi} / \mathrm{g}$ & 0.379 & & 3/31/08 & 8C17008 & AGG-RRL-001 \\
\hline
\end{tabular}


GEA/Soil

\begin{tabular}{|c|c|c|c|c|c|c|c|c|}
\hline CAS \# & Analyte & Results & Units & MDA & UNC & Analyzed & Batch & Method \\
\hline HEIS No. & B1TNK9B & \multicolumn{3}{|c|}{ Lab ID: $\quad$ 0803015-30 } & & & & \\
\hline $14762-78-8$ & Cerium-144 & $<2.71$ & $\mathrm{pCi} / \mathrm{g}$ & 2.71 & & $3 / 31 / 08$ & 8C17008 & AGG-RRL-001 \\
\hline $14683-23-9$ & Europium-152 & $<1.38$ & $\mathrm{pCi} / \mathrm{g}$ & 1.38 & & 3/31/08 & 8C17008 & AGG-RRL-001 \\
\hline $14276-65-4$ & Gadolinium-153 & $<1.01$ & $\mathrm{pCi} / \mathrm{g}$ & 1.01 & & 3/31/08 & 8C17008 & AGG-RRL-001 \\
\hline 15585-10-1 & Europium-154 & $<0.701$ & $\mathrm{pCi} / \mathrm{g}$ & 0.701 & & $3 / 31 / 08$ & 8C17008 & AGG-RRL-001 \\
\hline $14391-16-3$ & Europium-155 & $<1.09$ & $\mathrm{pCi} / \mathrm{g}$ & 1.09 & & 3/31/08 & 8C17008 & AGG-RRL-001 \\
\hline 13982-78-0 & Mercury-203 & $<0.412$ & $\mathrm{pCi} / \mathrm{g}$ & 0.412 & & 3/31/08 & 8C17008 & AGG-RRL-001 \\
\hline 14913-50-9 & Thallium-208 & $<0.409$ & $\mathrm{pCi} / \mathrm{g}$ & 0.409 & & $3 / 31 / 08$ & 8C17008 & AGG-RRL-001 \\
\hline 14331-79-4 & Bismuth-210 & $<0.633$ & $\mathrm{pCi} / \mathrm{g}$ & 0.633 & & $3 / 31 / 08$ & 8C17008 & AGG-RRL-001 \\
\hline \multirow[t]{3}{*}{$14255-04-0$} & Lead-210 & $<44.9$ & $\mathrm{pCi} / \mathrm{g}$ & 44.9 & & 3/31/08 & 8C17008 & AGG-RRL-001 \\
\hline & Bismuth-211 & $<7.36$ & $\mathrm{pCi} / \mathrm{g}$ & 7.36 & & 3/31/08 & 8C17008 & AGG-RRL-001 \\
\hline & Lead-211 & $<10.1$ & $\mathrm{pCi} / \mathrm{g}$ & 10.1 & & $3 / 31 / 08$ & 8C17008 & AGG-RRL-001 \\
\hline 15092-94-1 & Lead-212 & $<0.855$ & $\mathrm{pCi} / \mathrm{g}$ & 0.855 & & $3 / 31 / 08$ & 8C17008 & AGG-RRL-001 \\
\hline $14733-03-0$ & Bismuth-214 & $<0.821$ & $\mathrm{pCi} / \mathrm{g}$ & 0.821 & & 3/31/08 & 8C17008 & AGG-RRL-001 \\
\hline $15067-28-4$ & Lead-214 & $<0.953$ & $\mathrm{pCi} / \mathrm{g}$ & 0.953 & & 3/31/08 & 8C17008 & AGG-RRL-001 \\
\hline $14835-02-0$ & Radon-219 & $<3.2$ & $\mathrm{pCi} / \mathrm{g}$ & 3.2 & & 3/31/08 & 8C17008 & AGG-RRL-001 \\
\hline 22481-48-7 & Radon-220 & $<304$ & $\mathrm{pCi} / \mathrm{g}$ & 304 & & 3/31/08 & 8C17008 & AGG-RRL-001 \\
\hline 28522-20-5 & Radon-221 & $<1.52$ & $\mathrm{pCi} / \mathrm{g}$ & 1.52 & & 3/31/08 & 8C17008 & AGG-RRL-001 \\
\hline 15756-98-6 & Francium-223 & $<10.9$ & $\mathrm{pCi} / \mathrm{g}$ & 10.9 & & 3/31/08 & 8C17008 & AGG-RRL-001 \\
\hline $15623-45-7$ & Radium-223 & $<2.34$ & $\mathrm{pCi} / \mathrm{g}$ & 2.34 & & 3/31/08 & 8C17008 & AGG-RRL-001 \\
\hline $13233-32-4$ & Radium-224 & $<9.25$ & $\mathrm{pCi} / \mathrm{g}$ & 9.25 & & $3 / 31 / 08$ & 8C17008 & AGG-RRL-001 \\
\hline $13982-63-3$ & Radium-226 & $<9.39$ & $\mathrm{pCi} / \mathrm{g}$ & 9.39 & & 3/31/08 & 8C17008 & AGG-RRL-001 \\
\hline $15623-47-9$ & Thorium-227 & $<3.32$ & $\mathrm{pCi} / \mathrm{g}$ & 3.32 & & 3/31/08 & 8C17008 & AGG-RRL-001 \\
\hline $14331-83-0$ & Actinium-228 & $<1.43$ & $\mathrm{pCi} / \mathrm{g}$ & 1.43 & & 3/31/08 & 8C17008 & AGG-RRL-001 \\
\hline 14274-82-9 & Thorium-228 & $<119$ & $\mathrm{pCi} / \mathrm{g}$ & 119 & & 3/31/08 & 8C17008 & AGG-RRL-001 \\
\hline $14269-63-7$ & Thorium-230 & $<127$ & $\mathrm{pCi} / \mathrm{g}$ & 127 & & 3/31/08 & 8C17008 & AGG-RRL-001 \\
\hline $14331-85-2$ & Protactinium-231 & $<14.4$ & $\mathrm{pCi} / \mathrm{g}$ & 14.4 & & 3/31/08 & 8C17008 & AGG-RRL-001 \\
\hline $14932-40-2$ & Thorium-231 & $<72.7$ & $\mathrm{pCi} / \mathrm{g}$ & 72.7 & & 3/31/08 & 8C17008 & AGG-RRL-001 \\
\hline 7440-29-1 & Thorium-232 & $<318$ & $\mathrm{pCi} / \mathrm{g}$ & 318 & & 3/31/08 & 8C17008 & AGG-RRL-001 \\
\hline 13981-14-1 & Protactinium-233 & $<0.861$ & $\mathrm{pCi} / \mathrm{g}$ & 0.861 & & 3/31/08 & 8C17008 & AGG-RRL-001 \\
\hline \multirow[t]{2}{*}{$15100-28-4$} & Protactinium-234 & $<1.32$ & $\mathrm{pCi} / \mathrm{g}$ & 1.32 & & 3/31/08 & 8C17008 & AGG-RRL-001 \\
\hline & Protactinium-234m & $<42.9$ & $\mathrm{pCi} / \mathrm{g}$ & 42.9 & & $3 / 31 / 08$ & 8C17008 & AGG-RRL-001 \\
\hline $15065-10-8$ & Thorium-234 & $<11.1$ & $\mathrm{pCi} / \mathrm{g}$ & 11.1 & & $3 / 31 / 08$ & 8C17008 & AGG-RRL-001 \\
\hline 15117-96-1 & Uranium 235 & $<2.85$ & $\mathrm{pCi} / \mathrm{g}$ & 2.85 & & 3/31/08 & 8C17008 & AGG-RRL-001 \\
\hline 13994-20-2 & Neptunium-237 & $<2.83$ & $\mathrm{pCi} / \mathrm{g}$ & 2.83 & & $3 / 31 / 08$ & 8C17008 & AGG-RRL-001 \\
\hline \multirow[t]{2}{*}{ 13981-16-3 } & Plutonium-238 & $<4180$ & $\mathrm{pCi} / \mathrm{g}$ & 4180 & & 3/31/08 & 8C17008 & AGG-RRL-001 \\
\hline & Uranium 238 & $<5.88$ & $\mathrm{pCi} / \mathrm{g}$ & 5.88 & & 3/31/08 & 8C17008 & AGG-RRL-001 \\
\hline $15117-48-3$ & Plutonium-239 & $<4310$ & $\mathrm{pCi} / \mathrm{g}$ & 4310 & & 3/31/08 & 8C17008 & AGG-RRL-001 \\
\hline 14119-33-6 & Plutonium-240 & $<4180$ & $\mathrm{pCi} / \mathrm{g}$ & 4180 & & 3/31/08 & 8C17008 & AGG-RRL-001 \\
\hline $14596-10-2$ & Americium-241 & $<1.63$ & $\mathrm{pCi} / \mathrm{g}$ & 1.63 & & $3 / 31 / 08$ & 8C17008 & AGG-RRL-001 \\
\hline $14993-75-0$ & Americium-243 & $<0.698$ & $\mathrm{pCi} / \mathrm{g}$ & 0.698 & & $3 / 31 / 08$ & 8C17008 & AGG-RRL-001 \\
\hline $15757-87-6$ & Curium-243 & $<1.28$ & $\mathrm{pCi} / \mathrm{g}$ & 1.28 & & 3/31/08 & 8C17008 & AGG-RRL-001 \\
\hline 15621-76-8 & Curium-245 & $<1.05$ & $\mathrm{pCi} / \mathrm{g}$ & 1.05 & & $3 / 31 / 08$ & 8C17008 & AGG-RRL-001 \\
\hline HEIS No. & B1TNK9A & \multicolumn{2}{|c|}{ Lab ID: } & 0803015-31 & & & & \\
\hline 13966-32-0 & Sodium-22 & $<0.335$ & $\mathrm{pCi} / \mathrm{g}$ & 0.335 & & $3 / 31 / 08$ & 8C17008 & AGG-RRL-001 \\
\hline $13966-00-2$ & Potassium-40 & 19.4 & $\mathrm{pCi} / \mathrm{g}$ & 3.34 & 1.28 & 3/31/08 & 8C17008 & AGG-RRL-001 \\
\hline $14392-02-0$ & Chromium-51 & $<2.67$ & $\mathrm{pCi} / \mathrm{g}$ & 2.67 & & 3/31/08 & 8C17008 & AGG-RRL-001 \\
\hline 13966-31-9 & Manganese-54 & $<0.284$ & $\mathrm{pCi} / \mathrm{g}$ & 0.284 & & 3/31/08 & 8C17008 & AGG-RRL-001 \\
\hline $13981-50-5$ & Cobalt-57 & $<0.275$ & $\mathrm{pCi} / \mathrm{g}$ & 0.275 & & 3/31/08 & 8C17008 & AGG-RRL-001 \\
\hline
\end{tabular}


GEA/Soil

\begin{tabular}{|c|c|c|c|c|c|c|c|c|}
\hline CAS \# & Analyte & Results & Units & MDA & UNC & Analyzed & Batch & Method \\
\hline HEIS No. & B1TNK9A & \multicolumn{2}{|c|}{ Lab ID: } & 0803015-31 & & & & \\
\hline $14596-12-4$ & Iron-59 & $<0.568$ & $\mathrm{pCi} / \mathrm{g}$ & 0.568 & & 3/31/08 & 8C17008 & AGG-RRL-001 \\
\hline $10198-40-0$ & Cobalt-60 & $<0.288$ & $\mathrm{pCi} / \mathrm{g}$ & 0.288 & & 3/31/08 & 8C17008 & AGG-RRL-001 \\
\hline 13982-39-3 & Zinc-65 & $<0.707$ & $\mathrm{pCi} / \mathrm{g}$ & 0.707 & & 3/31/08 & 8C17008 & AGG-RRL-001 \\
\hline $14265-71-5$ & Selenium-75 & $<0.451$ & $\mathrm{pCi} / \mathrm{g}$ & 0.451 & & 3/31/08 & 8C17008 & AGG-RRL-001 \\
\hline 17056-36-9 & Rubidium-83 & $<0.566$ & $\mathrm{pCi} / \mathrm{g}$ & 0.566 & & 3/31/08 & 8C17008 & AGG-RRL-001 \\
\hline 13967-73-2 & Strontium-85 & $<0.363$ & $\mathrm{pCi} / \mathrm{g}$ & 0.363 & & 3/31/08 & 8C17008 & AGG-RRL-001 \\
\hline 14932-53-7 & Rubidium-86 & $<3.32$ & $\mathrm{pCi} / \mathrm{g}$ & 3.32 & & 3/31/08 & 8C17008 & AGG-RRL-001 \\
\hline 13982-36-0 & Yttrium-88 & $<0.212$ & $\mathrm{pCi} / \mathrm{g}$ & 0.212 & & 3/31/08 & 8C17008 & AGG-RRL-001 \\
\hline 14681-63-1 & Niobium-94 & $<0.27$ & $\mathrm{pCi} / \mathrm{g}$ & 0.27 & & 3/31/08 & 8C17008 & AGG-RRL-001 \\
\hline \multirow[t]{3}{*}{ 13967-76-5 } & Niobium-95 & $<0.253$ & $\mathrm{pCi} / \mathrm{g}$ & 0.253 & & 3/31/08 & 8C17008 & AGG-RRL-001 \\
\hline & Niobium-95m & $<1.32$ & $\mathrm{pCi} / \mathrm{g}$ & 1.32 & & 3/31/08 & 8C17008 & AGG-RRL-001 \\
\hline & Technetium-95m & $<0.456$ & $\mathrm{pCi} / \mathrm{g}$ & 0.456 & & 3/31/08 & 8C17008 & AGG-RRL-001 \\
\hline \multirow[t]{2}{*}{ 13967-71-0 } & Zirconium-95 & $<0.456$ & $\mathrm{pCi} / \mathrm{g}$ & 0.456 & & 3/31/08 & 8C17008 & AGG-RRL-001 \\
\hline & Technetium-99m & $<0.282$ & $\mathrm{pCi} / \mathrm{g}$ & 0.282 & & 3/31/08 & 8C17008 & AGG-RRL-001 \\
\hline 13968-53-1 & Ruthenium-103 & $<0.279$ & $\mathrm{pCi} / \mathrm{g}$ & 0.279 & & 3/31/08 & 8C17008 & AGG-RRL-001 \\
\hline 13967-48-1 & Ruthenium-106 & $<2.78$ & $\mathrm{pCi} / \mathrm{g}$ & 2.78 & & 3/31/08 & 8C17008 & AGG-RRL-001 \\
\hline 14391-65-2 & Silver-108m & $<0.295$ & $\mathrm{pCi} / \mathrm{g}$ & 0.295 & & 3/31/08 & 8C17008 & AGG-RRL-001 \\
\hline \multirow[t]{2}{*}{ 14109-32-1 } & Cadmium-109 & $<7.45$ & $\mathrm{pCi} / \mathrm{g}$ & 7.45 & & 3/31/08 & 8C17008 & AGG-RRL-001 \\
\hline & Silver-110 & $<0.279$ & $\mathrm{pCi} / \mathrm{g}$ & 0.279 & & 3/31/08 & 8C17008 & AGG-RRL-001 \\
\hline 14391-76-5 & Silver-110m & $<0.28$ & $\mathrm{pCi} / \mathrm{g}$ & 0.28 & & 3/31/08 & 8C17008 & AGG-RRL-001 \\
\hline 13966-06-8 & Tin-113 & $<0.4$ & $\mathrm{pCi} / \mathrm{g}$ & 0.4 & & 3/31/08 & 8C17008 & AGG-RRL-001 \\
\hline $14683-10-4$ & Antimony-124 & $<0.27$ & $\mathrm{pCi} / \mathrm{g}$ & 0.27 & & 3/31/08 & 8C17008 & AGG-RRL-001 \\
\hline $14234-35-6$ & Antimony-125 & $<0.838$ & $\mathrm{pCi} / \mathrm{g}$ & 0.838 & & 3/31/08 & 8C17008 & AGG-RRL-001 \\
\hline 15756-32-8 & Antimony-126 & $<0.261$ & $\mathrm{pCi} / \mathrm{g}$ & 0.261 & & 3/31/08 & 8C17008 & AGG-RRL-001 \\
\hline 15832-50-5 & Tin-126 & $<0.588$ & $\mathrm{pCi} / \mathrm{g}$ & 0.588 & & 3/31/08 & 8C17008 & AGG-RRL-001 \\
\hline 10043-66-0 & Iodine-131 & $<0.324$ & $\mathrm{pCi} / \mathrm{g}$ & 0.324 & & 3/31/08 & 8C17008 & AGG-RRL-001 \\
\hline 13981-41-4 & Barium-133 & $<0.506$ & $\mathrm{pCi} / \mathrm{g}$ & 0.506 & & 3/31/08 & 8C17008 & AGG-RRL-001 \\
\hline 13967-70-9 & Cesium-134 & $<0.313$ & $\mathrm{pCi} / \mathrm{g}$ & 0.313 & & 3/31/08 & 8C17008 & AGG-RRL-001 \\
\hline $10045-97-3$ & Cesium-137 & $<0.298$ & $\mathrm{pCi} / \mathrm{g}$ & 0.298 & & 3/31/08 & 8C17008 & AGG-RRL-001 \\
\hline 13982-30-4 & Cerium-139 & $<0.321$ & $\mathrm{pCi} / \mathrm{g}$ & 0.321 & & 3/31/08 & 8C17008 & AGG-RRL-001 \\
\hline 14762-78-8 & Cerium-144 & $<2.25$ & $\mathrm{pCi} / \mathrm{g}$ & 2.25 & & 3/31/08 & 8C17008 & AGG-RRL-001 \\
\hline 14683-23-9 & Europium-152 & $<1.06$ & $\mathrm{pCi} / \mathrm{g}$ & 1.06 & & 3/31/08 & 8C17008 & AGG-RRL-001 \\
\hline $14276-65-4$ & Gadolinium-153 & $<0.843$ & $\mathrm{pCi} / \mathrm{g}$ & 0.843 & & 3/31/08 & 8C17008 & AGG-RRL-001 \\
\hline 15585-10-1 & Europium-154 & $<0.582$ & $\mathrm{pCi} / \mathrm{g}$ & 0.582 & & 3/31/08 & 8C17008 & AGG-RRL-001 \\
\hline 14391-16-3 & Europium-155 & $<0.829$ & $\mathrm{pCi} / \mathrm{g}$ & 0.829 & & 3/31/08 & 8C17008 & AGG-RRL-001 \\
\hline 13982-78-0 & Mercury-203 & $<0.347$ & $\mathrm{pCi} / \mathrm{g}$ & 0.347 & & 3/31/08 & 8C17008 & AGG-RRL-001 \\
\hline 14913-50-9 & Thallium-208 & $<0.287$ & $\mathrm{pCi} / \mathrm{g}$ & 0.287 & 0.0634 & 3/31/08 & 8C17008 & AGG-RRL-001 \\
\hline 14331-79-4 & Bismuth-210 & $<0.543$ & $\mathrm{pCi} / \mathrm{g}$ & 0.543 & & 3/31/08 & 8C17008 & AGG-RRL-001 \\
\hline \multirow[t]{3}{*}{$14255-04-0$} & Lead-210 & $<106$ & $\mathrm{pCi} / \mathrm{g}$ & 106 & & 3/31/08 & 8C17008 & AGG-RRL-001 \\
\hline & Bismuth-211 & $<6.33$ & $\mathrm{pCi} / \mathrm{g}$ & 6.33 & & 3/31/08 & 8C17008 & AGG-RRL-001 \\
\hline & Lead-211 & $<8.65$ & $\mathrm{pCi} / \mathrm{g}$ & 8.65 & & 3/31/08 & 8C17008 & AGG-RRL-001 \\
\hline 15092-94-1 & Lead-212 & $<0.699$ & $\mathrm{pCi} / \mathrm{g}$ & 0.699 & 0.156 & 3/31/08 & 8C17008 & AGG-RRL-001 \\
\hline 14733-03-0 & Bismuth-214 & $<0.747$ & $\mathrm{pCi} / \mathrm{g}$ & 0.747 & & 3/31/08 & 8C17008 & AGG-RRL-001 \\
\hline $15067-28-4$ & Lead-214 & $<0.838$ & $\mathrm{pCi} / \mathrm{g}$ & 0.838 & & 3/31/08 & 8C17008 & AGG-RRL-001 \\
\hline $14835-02-0$ & Radon-219 & $<2.78$ & $\mathrm{pCi} / \mathrm{g}$ & 2.78 & & 3/31/08 & 8C17008 & AGG-RRL-001 \\
\hline 22481-48-7 & Radon-220 & $<260$ & $\mathrm{pCi} / \mathrm{g}$ & 260 & & 3/31/08 & 8C17008 & AGG-RRL-001 \\
\hline 28522-20-5 & Radon-221 & $<1.26$ & $\mathrm{pCi} / \mathrm{g}$ & 1.26 & & 3/31/08 & 8C17008 & AGG-RRL-001 \\
\hline 15756-98-6 & Francium-223 & $<9.69$ & $\mathrm{pCi} / \mathrm{g}$ & 9.69 & & 3/31/08 & 8C17008 & AGG-RRL-001 \\
\hline $15623-45-7$ & Radium-223 & $<2.01$ & $\mathrm{pCi} / \mathrm{g}$ & 2.01 & & 3/31/08 & 8C17008 & AGG-RRL-001 \\
\hline
\end{tabular}


GEA/Soil

\begin{tabular}{|c|c|c|c|c|c|c|c|c|}
\hline CAS \# & Analyte & Results & Units & MDA & UNC & Analyzed & Batch & Method \\
\hline HEIS No. & B1TNK9A & \multicolumn{2}{|c|}{ Lab ID: } & 0803015-31 & & & & \\
\hline $13233-32-4$ & Radium-224 & $<8.46$ & $\mathrm{pCi} / \mathrm{g}$ & 8.46 & & 3/31/08 & 8C17008 & AGG-RRL-001 \\
\hline 13982-63-3 & Radium-226 & $<7.78$ & $\mathrm{pCi} / \mathrm{g}$ & 7.78 & & 3/31/08 & 8C17008 & AGG-RRL-001 \\
\hline $15623-47-9$ & Thorium-227 & $<2.95$ & $\mathrm{pCi} / \mathrm{g}$ & 2.95 & & 3/31/08 & 8C17008 & AGG-RRL-001 \\
\hline $14331-83-0$ & Actinium-228 & $<1.28$ & $\mathrm{pCi} / \mathrm{g}$ & 1.28 & & 3/31/08 & 8C17008 & AGG-RRL-001 \\
\hline $14274-82-9$ & Thorium-228 & $<102$ & $\mathrm{pCi} / \mathrm{g}$ & 102 & & 3/31/08 & 8C17008 & AGG-RRL-001 \\
\hline 14269-63-7 & Thorium-230 & $<107$ & $\mathrm{pCi} / \mathrm{g}$ & 107 & & 3/31/08 & 8C17008 & AGG-RRL-001 \\
\hline $14331-85-2$ & Protactinium-231 & $<11.8$ & $\mathrm{pCi} / \mathrm{g}$ & 11.8 & & 3/31/08 & 8C17008 & AGG-RRL-001 \\
\hline $14932-40-2$ & Thorium-231 & $<57.8$ & $\mathrm{pCi} / \mathrm{g}$ & 57.8 & & 3/31/08 & 8C17008 & AGG-RRL-001 \\
\hline 7440-29-1 & Thorium-232 & $<338$ & $\mathrm{pCi} / \mathrm{g}$ & 338 & & 3/31/08 & 8C17008 & AGG-RRL-001 \\
\hline 13981-14-1 & Protactinium-233 & $<0.738$ & $\mathrm{pCi} / \mathrm{g}$ & 0.738 & & 3/31/08 & 8C17008 & AGG-RRL-001 \\
\hline \multirow[t]{2}{*}{$15100-28-4$} & Protactinium-234 & $<1.07$ & $\mathrm{pCi} / \mathrm{g}$ & 1.07 & & 3/31/08 & 8C17008 & AGG-RRL-001 \\
\hline & Protactinium-234m & $<37.3$ & $\mathrm{pCi} / \mathrm{g}$ & 37.3 & & 3/31/08 & 8C17008 & AGG-RRL-001 \\
\hline $15065-10-8$ & Thorium-234 & $<9.65$ & $\mathrm{pCi} / \mathrm{g}$ & 9.65 & & 3/31/08 & 8C17008 & AGG-RRL-001 \\
\hline 15117-96-1 & Uranium 235 & $<2.42$ & $\mathrm{pCi} / \mathrm{g}$ & 2.42 & & 3/31/08 & 8C17008 & AGG-RRL-001 \\
\hline 13994-20-2 & Neptunium-237 & $<2.16$ & $\mathrm{pCi} / \mathrm{g}$ & 2.16 & & 3/31/08 & 8C17008 & AGG-RRL-001 \\
\hline \multirow[t]{2}{*}{ 13981-16-3 } & Plutonium-238 & $<3320$ & $\mathrm{pCi} / \mathrm{g}$ & 3320 & & 3/31/08 & 8C17008 & AGG-RRL-001 \\
\hline & Uranium 238 & $<5$ & $\mathrm{pCi} / \mathrm{g}$ & 5 & & 3/31/08 & 8C17008 & AGG-RRL-001 \\
\hline $15117-48-3$ & Plutonium-239 & $<3920$ & $\mathrm{pCi} / \mathrm{g}$ & 3920 & & 3/31/08 & 8C17008 & AGG-RRL-001 \\
\hline 14119-33-6 & Plutonium-240 & $<3360$ & $\mathrm{pCi} / \mathrm{g}$ & 3360 & & 3/31/08 & 8C17008 & AGG-RRL-001 \\
\hline 14596-10-2 & Americium-241 & $<1.69$ & $\mathrm{pCi} / \mathrm{g}$ & 1.69 & & 3/31/08 & 8C17008 & AGG-RRL-001 \\
\hline $14993-75-0$ & Americium-243 & $<0.594$ & $\mathrm{pCi} / \mathrm{g}$ & 0.594 & & 3/31/08 & 8C17008 & AGG-RRL-001 \\
\hline $15757-87-6$ & Curium-243 & $<1.03$ & $\mathrm{pCi} / \mathrm{g}$ & 1.03 & & 3/31/08 & 8C17008 & AGG-RRL-001 \\
\hline $15621-76-8$ & Curium-245 & $<0.842$ & $\mathrm{pCi} / \mathrm{g}$ & 0.842 & & 3/31/08 & 8C17008 & AGG-RRL-001 \\
\hline
\end{tabular}


Total Alpha Total Beta/Acid Extract

\begin{tabular}{|c|c|c|c|c|c|c|c|}
\hline CAS \# & Analyte & Results & MDA & UNC & Analyzed & Batch & Method \\
\hline HEIS No. & B1RTF8 & \multicolumn{3}{|c|}{ Lab ID: $\quad$ 0803001-01 } & & & \\
\hline $12587-47-2$ & Gross Beta & $<50$ pCi/g dry & 50 & & $4 / 11 / 08$ & 8D10001 & AGG-RRL-002 \\
\hline $12587-46-1$ & Gross Alpha & $<17.6 \mathrm{pCi} / \mathrm{g}$ dry & 17.6 & & $4 / 11 / 08$ & 8D10001 & AGG-RRL-002 \\
\hline HEIS No. & B1RTF9A & \multicolumn{3}{|c|}{ Lab ID: $\quad 0803001-02$} & & & \\
\hline $12587-47-2$ & Gross Beta & $<50$ pCi/g dry & 50 & & $4 / 11 / 08$ & 8D10001 & AGG-RRL-002 \\
\hline $12587-46-1$ & Gross Alpha & $<17.6 \mathrm{pCi} / \mathrm{g}$ dry & 17.6 & & $4 / 11 / 08$ & 8D10001 & AGG-RRL-002 \\
\hline HEIS No. & B1RTH1A & \multicolumn{3}{|c|}{ Lab ID: $\quad$ 0803012-02 } & & & \\
\hline $12587-47-2$ & Gross Beta & $<50 \mathrm{pCi} / \mathrm{g}$ dry & 50 & & $4 / 11 / 08$ & 8D10001 & AGG-RRL-002 \\
\hline $12587-46-1$ & Gross Alpha & $<17.6 \mathrm{pCi} / \mathrm{g}$ dry & 17.6 & & $4 / 11 / 08$ & 8D10001 & AGG-RRL-002 \\
\hline HEIS No. & B1RTH1 & \multicolumn{3}{|c|}{ Lab ID: $\quad$ 0803012-03 } & & & \\
\hline $12587-47-2$ & Gross Beta & $<50$ pCi/g dry & 50 & & $4 / 11 / 08$ & 8D10001 & AGG-RRL-002 \\
\hline $12587-46-1$ & Gross Alpha & $<17.6 \mathrm{pCi} / \mathrm{g}$ dry & 17.6 & & $4 / 11 / 08$ & 8D10001 & AGG-RRL-002 \\
\hline HEIS No. & B1RTH2B & \multicolumn{3}{|c|}{ Lab ID: $\quad$ 0803012-05 } & & & \\
\hline $12587-47-2$ & Gross Beta & $<50$ pCi/g dry & 50 & & $4 / 11 / 08$ & 8D10001 & AGG-RRL-002 \\
\hline $12587-46-1$ & Gross Alpha & $<17.6$ pCi/g dry & 17.6 & & $4 / 11 / 08$ & 8D10001 & AGG-RRL-002 \\
\hline HEIS No. & B1RTH2A & \multicolumn{3}{|c|}{ Lab ID: $\quad$ 0803012-06 } & & & \\
\hline $12587-47-2$ & Gross Beta & $<50$ pCi/g dry & 50 & & $4 / 11 / 08$ & 8D10001 & AGG-RRL-002 \\
\hline $12587-46-1$ & Gross Alpha & $<17.6 \mathrm{pCi} / \mathrm{g}$ dry & 17.6 & & $4 / 11 / 08$ & 8D10001 & AGG-RRL-002 \\
\hline HEIS No. & B1RTH3B & \multicolumn{3}{|c|}{ Lab ID: $\quad$ 0803012-09 } & & & \\
\hline $12587-47-2$ & Gross Beta & $<50$ pCi/g dry & 50 & & $4 / 11 / 08$ & 8D10001 & AGG-RRL-002 \\
\hline $12587-46-1$ & Gross Alpha & $<17.6 \mathrm{pCi} / \mathrm{g}$ dry & 17.6 & & $4 / 11 / 08$ & 8D10001 & AGG-RRL-002 \\
\hline HEIS No. & B1RTH3A & \multicolumn{3}{|c|}{ Lab ID: $\quad$ 0803012-10 } & & & \\
\hline $12587-47-2$ & Gross Beta & $<50$ pCi/g dry & 50 & & $4 / 11 / 08$ & 8D10001 & AGG-RRL-002 \\
\hline $12587-46-1$ & Gross Alpha & $<17.6 \mathrm{pCi} / \mathrm{g}$ dry & 17.6 & & $4 / 11 / 08$ & 8D10001 & AGG-RRL-002 \\
\hline HEIS No. & B1RYR7B & \multicolumn{3}{|c|}{ Lab ID: $\quad$ 0803012-13 } & & & \\
\hline $12587-47-2$ & Gross Beta & $<50$ pCi/g dry & 50 & & $4 / 11 / 08$ & 8D10001 & AGG-RRL-002 \\
\hline $12587-46-1$ & Gross Alpha & $<17.6 \mathrm{pCi} / \mathrm{g}$ dry & 17.6 & & $4 / 11 / 08$ & 8D10001 & AGG-RRL-002 \\
\hline HEIS No. & B1RYR7A & \multicolumn{3}{|c|}{ Lab ID: $\quad$ 0803012-14 } & & & \\
\hline $12587-47-2$ & Gross Beta & $<50 \mathrm{pCi} / \mathrm{g}$ dry & 50 & & $4 / 11 / 08$ & 8D10001 & AGG-RRL-002 \\
\hline $12587-46-1$ & Gross Alpha & $<17.6 \mathrm{pCi} / \mathrm{g}$ dry & 17.6 & & $4 / 11 / 08$ & 8D10001 & AGG-RRL-002 \\
\hline HEIS No. & B1RYR8B & \multicolumn{3}{|c|}{ Lab ID: $\quad$ 0803012-17 } & & & \\
\hline $12587-47-2$ & Gross Beta & $<50$ pCi/g dry & 50 & & $4 / 11 / 08$ & 8D10001 & AGG-RRL-002 \\
\hline $12587-46-1$ & Gross Alpha & $<17.6$ pCi/g dry & 17.6 & & $4 / 11 / 08$ & 8D10001 & AGG-RRL-002 \\
\hline HEIS No. & B1RYR8A & \multicolumn{3}{|c|}{ Lab ID: $\quad$ 0803012-18 } & & & \\
\hline $12587-47-2$ & Gross Beta & $<50$ pCi/g dry & 50 & & $4 / 11 / 08$ & 8D10001 & AGG-RRL-002 \\
\hline $12587-46-1$ & Gross Alpha & $<17.6 \mathrm{pCi} / \mathrm{g}$ dry & 17.6 & & $4 / 11 / 08$ & 8D10001 & AGG-RRL-002 \\
\hline HEIS No. & B1RYR9B & \multicolumn{3}{|c|}{ Lab ID: $\quad$ 0803012-21 } & & & \\
\hline $12587-47-2$ & Gross Beta & $<50$ pCi/g dry & 50 & & $4 / 11 / 08$ & 8D10001 & AGG-RRL-002 \\
\hline $12587-46-1$ & Gross Alpha & $<17.6$ pCi/g dry & 17.6 & & $4 / 11 / 08$ & 8D10001 & AGG-RRL-002 \\
\hline HEIS No. & B1RYR9A & \multicolumn{3}{|c|}{ Lab ID: $\quad$ 0803012-22 } & & & \\
\hline $12587-47-2$ & Gross Beta & $<50$ pCi/g dry & 50 & & $4 / 11 / 08$ & 8D10001 & AGG-RRL-002 \\
\hline $12587-46-1$ & Gross Alpha & $<17.6$ pCi/g dry & 17.6 & & $4 / 11 / 08$ & 8D10001 & AGG-RRL-002 \\
\hline HEIS No. & B1RYT0B & \multicolumn{3}{|c|}{ Lab ID: $\quad$ 0803014-01 } & & & \\
\hline $12587-47-2$ & Gross Beta & $<50$ pCi/g dry & 50 & & $4 / 11 / 08$ & 8D10001 & AGG-RRL-002 \\
\hline $12587-46-1$ & Gross Alpha & $<17.6 \mathrm{pCi} / \mathrm{g}$ dry & 17.6 & & $4 / 11 / 08$ & 8D10001 & AGG-RRL-002 \\
\hline HEIS No. & B1RYT0A & \multicolumn{3}{|c|}{ Lab ID: $\quad$ 0803014-02 } & & & \\
\hline $12587-47-2$ & Gross Beta & $<50$ pCi/g dry & 50 & & 4/11/08 & 8D10001 & AGG-RRL-002 \\
\hline
\end{tabular}


Total Alpha Total Beta/Acid Extract

\begin{tabular}{|c|c|c|c|c|c|c|c|}
\hline CAS \# & Analyte & Results & MDA & UNC & Analyzed & Batch & Method \\
\hline HEIS No. & B1RYT0A & \multicolumn{2}{|c|}{ Lab ID: $\quad$ 0803014-02 } & & & & \\
\hline 12587-46-1 & Gross Alpha & $<17.6 \mathrm{pCi} / \mathrm{g}$ dry & 17.6 & & $4 / 11 / 08$ & 8D10001 & AGG-RRL-002 \\
\hline HEIS No. & B1T2P1B & \multicolumn{2}{|c|}{ Lab ID: $\quad$ 0803014-05 } & & & & \\
\hline $12587-47-2$ & Gross Beta & $<50$ pCi/g dry & 50 & & $4 / 11 / 08$ & 8D10001 & AGG-RRL-002 \\
\hline 12587-46-1 & Gross Alpha & $<17.6$ pCi/g dry & 17.6 & & $4 / 11 / 08$ & 8D10001 & AGG-RRL-002 \\
\hline HEIS No. & B1T2P1A & \multicolumn{2}{|c|}{ Lab ID: $\quad$ 0803014-06 } & & & & \\
\hline $12587-47-2$ & Gross Beta & $<50$ pCi/g dry & 50 & & $4 / 11 / 08$ & 8D10001 & AGG-RRL-002 \\
\hline $12587-46-1$ & Gross Alpha & $<17.6 \mathrm{pCi} / \mathrm{g}$ dry & 17.6 & & $4 / 11 / 08$ & 8D10001 & AGG-RRL-002 \\
\hline HEIS No. & B1T2P2B & \multicolumn{2}{|c|}{ Lab ID: $\quad$ 0803014-09 } & & & & \\
\hline $12587-47-2$ & Gross Beta & $<50$ pCi/g dry & 50 & & $4 / 11 / 08$ & 8D10001 & AGG-RRL-002 \\
\hline $12587-46-1$ & Gross Alpha & $<17.6 \mathrm{pCi} / \mathrm{g}$ dry & 17.6 & & $4 / 11 / 08$ & 8D10001 & AGG-RRL-002 \\
\hline HEIS No. & B1T2P2A & \multicolumn{2}{|c|}{ Lab ID: $\quad$ 0803014-10 } & & & & \\
\hline $12587-47-2$ & Gross Beta & $<50 \mathrm{pCi} / \mathrm{g}$ dry & 50 & & $4 / 11 / 08$ & 8D10001 & AGG-RRL-002 \\
\hline $12587-46-1$ & Gross Alpha & $<17.6 \mathrm{pCi} / \mathrm{g}$ dry & 17.6 & & $4 / 11 / 08$ & 8D10001 & AGG-RRL-002 \\
\hline HEIS No. & B1T2P3B & \multicolumn{2}{|c|}{ Lab ID: $\quad$ 0803014-13 } & & & & \\
\hline $12587-47-2$ & Gross Beta & $<50$ pCi/g dry & 50 & & $4 / 11 / 08$ & 8D10001 & AGG-RRL-002 \\
\hline $12587-46-1$ & Gross Alpha & $<17.6 \mathrm{pCi} / \mathrm{g}$ dry & 17.6 & & $4 / 11 / 08$ & 8D10001 & AGG-RRL-002 \\
\hline HEIS No. & B1T2P3A & \multicolumn{2}{|c|}{ Lab ID: $\quad$ 0803014-14 } & & & & \\
\hline $12587-47-2$ & Gross Beta & $<50 \mathrm{pCi} / \mathrm{g}$ dry & 50 & & $4 / 11 / 08$ & 8D10001 & AGG-RRL-002 \\
\hline $12587-46-1$ & Gross Alpha & $<17.6 \mathrm{pCi} / \mathrm{g}$ dry & 17.6 & & $4 / 11 / 08$ & 8D10001 & AGG-RRL-002 \\
\hline HEIS No. & B1T2P4B & \multicolumn{2}{|c|}{ Lab ID: $\quad$ 0803014-16 } & & & & \\
\hline $12587-47-2$ & Gross Beta & $<50$ pCi/g dry & 50 & & $4 / 11 / 08$ & 8D10001 & AGG-RRL-002 \\
\hline $12587-46-1$ & Gross Alpha & $<17.6$ pCi/g dry & 17.6 & & $4 / 11 / 08$ & 8D10001 & AGG-RRL-002 \\
\hline HEIS No. & B1T2P4A & \multicolumn{2}{|c|}{ Lab ID: $\quad$ 0803014-17 } & & & & \\
\hline $12587-47-2$ & Gross Beta & $<50$ pCi/g dry & 50 & & $4 / 11 / 08$ & 8D10001 & AGG-RRL-002 \\
\hline $12587-46-1$ & Gross Alpha & $<17.6$ pCi/g dry & 17.6 & & $4 / 11 / 08$ & 8D10001 & AGG-RRL-002 \\
\hline HEIS No. & B1T2P5B & \multicolumn{2}{|c|}{ Lab ID: $\quad$ 0803014-20 } & & & & \\
\hline $12587-47-2$ & Gross Beta & $<50$ pCi/g dry & 50 & & $4 / 11 / 08$ & 8D10001 & AGG-RRL-002 \\
\hline $12587-46-1$ & Gross Alpha & $<17.6 \mathrm{pCi} / \mathrm{g}$ dry & 17.6 & & $4 / 11 / 08$ & 8D10001 & AGG-RRL-002 \\
\hline HEIS No. & B1T2P5A & \multicolumn{2}{|c|}{ Lab ID: $\quad$ 0803014-21 } & & & & \\
\hline $12587-47-2$ & Gross Beta & $<50$ pCi/g dry & 50 & & $4 / 11 / 08$ & 8D10001 & AGG-RRL-002 \\
\hline $12587-46-1$ & Gross Alpha & $<17.6$ pCi/g dry & 17.6 & & $4 / 11 / 08$ & 8D10001 & AGG-RRL-002 \\
\hline HEIS No. & B1T2P6B & \multicolumn{2}{|c|}{ Lab ID: $\quad$ 0803014-24 } & & & & \\
\hline $12587-47-2$ & Gross Beta & $<50$ pCi/g dry & 50 & & $4 / 11 / 08$ & 8D10001 & AGG-RRL-002 \\
\hline $12587-46-1$ & Gross Alpha & $<17.6 \mathrm{pCi} / \mathrm{g}$ dry & 17.6 & & $4 / 11 / 08$ & 8D10001 & AGG-RRL-002 \\
\hline HEIS No. & B1T2P6A & \multicolumn{2}{|c|}{ Lab ID: $\quad$ 0803014-25 } & & & & \\
\hline $12587-47-2$ & Gross Beta & $<50$ pCi/g dry & 50 & & $4 / 11 / 08$ & 8D10001 & AGG-RRL-002 \\
\hline $12587-46-1$ & Gross Alpha & $<17.6 \mathrm{pCi} / \mathrm{g}$ dry & 17.6 & & $4 / 11 / 08$ & 8D10001 & AGG-RRL-002 \\
\hline HEIS No. & B1T2P7B & \multicolumn{2}{|c|}{ Lab ID: $\quad$ 0803015-02 } & & & & \\
\hline $12587-47-2$ & Gross Beta & $<50$ pCi/g dry & 50 & & $4 / 11 / 08$ & 8D10001 & AGG-RRL-002 \\
\hline $12587-46-1$ & Gross Alpha & $<17.6$ pCi/g dry & 17.6 & & $4 / 11 / 08$ & 8D10001 & AGG-RRL-002 \\
\hline HEIS No. & B1T2P7A & \multicolumn{2}{|c|}{ Lab ID: $\quad$ 0803015-03 } & & & & \\
\hline $12587-47-2$ & Gross Beta & $<50$ pCi/g dry & 50 & & $4 / 11 / 08$ & 8D10001 & AGG-RRL-002 \\
\hline $12587-46-1$ & Gross Alpha & $<17.6 \mathrm{pCi} / \mathrm{g}$ dry & 17.6 & & $4 / 11 / 08$ & 8D10001 & AGG-RRL-002 \\
\hline HEIS No. & B1T2P8B & \multicolumn{2}{|c|}{ Lab ID: $\quad$ 0803015-06 } & & & & \\
\hline $12587-47-2$ & Gross Beta & $<50$ pCi/g dry & 50 & & $4 / 11 / 08$ & 8D10001 & AGG-RRL-002 \\
\hline 12587-46-1 & Gross Alpha & $<17.6$ pCi/g dry & 17.6 & & $4 / 11 / 08$ & 8D10001 & AGG-RRL-002 \\
\hline
\end{tabular}


Total Alpha Total Beta/Acid Extract

\begin{tabular}{|c|c|c|c|c|c|c|c|c|}
\hline CAS \# & Analyte & Results & Units & MDA & UNC & Analyzed & Batch & Method \\
\hline HEIS No. & B1T2P8A & \multicolumn{4}{|c|}{ Lab ID: $\quad$ 0803015-07 } & & & \\
\hline $12587-47-2$ & Gross Beta & $<50$ & pCi/g dry & 50 & & $4 / 11 / 08$ & 8D10001 & AGG-RRL-002 \\
\hline $12587-46-1$ & Gross Alpha & $<17.6$ & pCi/g dry & 17.6 & & $4 / 11 / 08$ & 8D10001 & AGG-RRL-002 \\
\hline HEIS No. & В1T2P9B & \multicolumn{4}{|c|}{ Lab ID: $\quad$ 0803015-10 } & & & \\
\hline $12587-47-2$ & Gross Beta & $<50$ & pCi/g dry & 50 & & $4 / 11 / 08$ & 8D10001 & AGG-RRL-002 \\
\hline $12587-46-1$ & Gross Alpha & $<17.6$ & pCi/g dry & 17.6 & & $4 / 11 / 08$ & 8D10001 & AGG-RRL-002 \\
\hline HEIS No. & B1T2P9A & \multicolumn{4}{|c|}{ Lab ID: $\quad$ 0803015-11 } & & & \\
\hline $12587-47-2$ & Gross Beta & $<50$ & pCi/g dry & 50 & & $4 / 11 / 08$ & 8D10001 & AGG-RRL-002 \\
\hline $12587-46-1$ & Gross Alpha & $<17.6$ & pCi/g dry & 17.6 & & 4/11/08 & 8D10001 & AGG-RRL-002 \\
\hline HEIS No. & B1T2R0B & \multicolumn{4}{|c|}{ Lab ID: $\quad$ 0803015-14 } & & & \\
\hline $12587-47-2$ & Gross Beta & $<50$ & pCi/g dry & 50 & & $4 / 11 / 08$ & 8D10001 & AGG-RRL-002 \\
\hline $12587-46-1$ & Gross Alpha & $<17.6$ & pCi/g dry & 17.6 & & $4 / 11 / 08$ & 8D10001 & AGG-RRL-002 \\
\hline HEIS No. & B1T2R0A & \multicolumn{4}{|c|}{ Lab ID: $\quad$ 0803015-15 } & & & \\
\hline $12587-47-2$ & Gross Beta & $<50$ & pCi/g dry & 50 & & $4 / 11 / 08$ & 8D10001 & AGG-RRL-002 \\
\hline $12587-46-1$ & Gross Alpha & $<17.6$ & pCi/g dry & 17.6 & & $4 / 11 / 08$ & 8D10001 & AGG-RRL-002 \\
\hline HEIS No. & B1TNK6B & \multicolumn{4}{|c|}{ Lab ID: $\quad$ 0803015-18 } & & & \\
\hline $12587-47-2$ & Gross Beta & $<50$ & pCi/g dry & 50 & & $4 / 11 / 08$ & 8D10001 & AGG-RRL-002 \\
\hline $12587-46-1$ & Gross Alpha & $<17.6$ & pCi/g dry & 17.6 & & $4 / 11 / 08$ & 8D10001 & AGG-RRL-002 \\
\hline HEIS No. & B1TNK6A & \multicolumn{4}{|c|}{ Lab ID: $\quad$ 0803015-19 } & & & \\
\hline $12587-47-2$ & Gross Beta & $<50$ & pCi/g dry & 50 & & $4 / 11 / 08$ & 8D10001 & AGG-RRL-002 \\
\hline $12587-46-1$ & Gross Alpha & $<17.6$ & pCi/g dry & 17.6 & & 4/11/08 & 8D10001 & AGG-RRL-002 \\
\hline HEIS No. & B1TNK7B & \multicolumn{4}{|c|}{ Lab ID: $\quad$ 0803015-22 } & & & \\
\hline $12587-47-2$ & Gross Beta & $<50$ & pCi/g dry & 50 & & $4 / 11 / 08$ & 8D10001 & AGG-RRL-002 \\
\hline $12587-46-1$ & Gross Alpha & $<17.6$ & pCi/g dry & 17.6 & & 4/11/08 & 8D10001 & AGG-RRL-002 \\
\hline HEIS No. & B1TNK7A & \multicolumn{4}{|c|}{ Lab ID: $\quad$ 0803015-23 } & & & \\
\hline $12587-47-2$ & Gross Beta & $<50$ & pCi/g dry & 50 & & $4 / 11 / 08$ & 8D10001 & AGG-RRL-002 \\
\hline $12587-46-1$ & Gross Alpha & $<17.6$ & pCi/g dry & 17.6 & & 4/11/08 & 8D10001 & AGG-RRL-002 \\
\hline HEIS No. & B1TNK8B & \multicolumn{4}{|c|}{ Lab ID: $\quad$ 0803015-26 } & & & \\
\hline $12587-47-2$ & Gross Beta & $<50$ & pCi/g dry & 50 & & $4 / 11 / 08$ & 8D10001 & AGG-RRL-002 \\
\hline $12587-46-1$ & Gross Alpha & $<17.6$ & pCi/g dry & 17.6 & & $4 / 11 / 08$ & 8D10001 & AGG-RRL-002 \\
\hline HEIS No. & B1TNK8A & \multicolumn{4}{|c|}{ Lab ID: $\quad$ 0803015-27 } & & & \\
\hline $12587-47-2$ & Gross Beta & $<50$ & pCi/g dry & 50 & & 4/11/08 & 8D10001 & AGG-RRL-002 \\
\hline $12587-46-1$ & Gross Alpha & $<17.6$ & pCi/g dry & 17.6 & & 4/11/08 & 8D10001 & AGG-RRL-002 \\
\hline HEIS No. & B1TNK9B & \multicolumn{4}{|c|}{ Lab ID: $\quad$ 0803015-30 } & & & \\
\hline $12587-47-2$ & Gross Beta & $<50$ & pCi/g dry & 50 & & $4 / 11 / 08$ & 8D10001 & AGG-RRL-002 \\
\hline $12587-46-1$ & Gross Alpha & $<17.6$ & pCi/g dry & 17.6 & & $4 / 11 / 08$ & 8D10001 & AGG-RRL-002 \\
\hline HEIS No. & B1TNK9A & \multicolumn{4}{|c|}{ Lab ID: $\quad$ 0803015-31 } & & & \\
\hline $12587-47-2$ & Gross Beta & $<50$ & pCi/g dry & 50 & & 4/11/08 & 8D10001 & AGG-RRL-002 \\
\hline $12587-46-1$ & Gross Alpha & $<17.6$ & pCi/g dry & 17.6 & & 4/11/08 & 8D10001 & AGG-RRL-002 \\
\hline
\end{tabular}


Total Alpha Total Beta/Water Extract

\begin{tabular}{|c|c|c|c|c|c|c|c|}
\hline CAS \# & Analyte & Results & MDA & UNC & Analyzed & Batch & Method \\
\hline HEIS No. & B1RTF8 & \multicolumn{3}{|c|}{ Lab ID: $\quad$ 0803001-01 } & & & \\
\hline $12587-47-2$ & Gross Beta & $<14.3 \mathrm{pCi} / \mathrm{g}$ dry & 14.3 & & 4/08/08 & 8D09004 & AGG-RRL-002 \\
\hline $12587-46-1$ & Gross Alpha & $<4.53 \mathrm{pCi} / \mathrm{g}$ dry & 4.53 & & $4 / 08 / 08$ & 8D09004 & AGG-RRL-002 \\
\hline HEIS No. & B1RTF9A & \multicolumn{3}{|c|}{ Lab ID: $\quad$ 0803001-02 } & & & \\
\hline $12587-47-2$ & Gross Beta & $<14.3 \mathrm{pCi} / \mathrm{g}$ dry & 14.3 & & 4/08/08 & 8D09004 & AGG-RRL-002 \\
\hline $12587-46-1$ & Gross Alpha & $<4.53 \mathrm{pCi} / \mathrm{g}$ dry & 4.53 & & 4/08/08 & 8D09004 & AGG-RRL-002 \\
\hline HEIS No. & B1RTH1A & \multicolumn{3}{|c|}{ Lab ID: $\quad$ 0803012-02 } & & & \\
\hline $12587-47-2$ & Gross Beta & $<14.3 \mathrm{pCi} / \mathrm{g}$ dry & 14.3 & & $4 / 08 / 08$ & 8D09004 & AGG-RRL-002 \\
\hline $12587-46-1$ & Gross Alpha & $<4.53 \mathrm{pCi} / \mathrm{g}$ dry & 4.53 & & $4 / 08 / 08$ & 8D09004 & AGG-RRL-002 \\
\hline HEIS No. & B1RTH1 & \multicolumn{3}{|c|}{ Lab ID: $\quad$ 0803012-03 } & & & \\
\hline $12587-47-2$ & Gross Beta & $<14.3 \mathrm{pCi} / \mathrm{g}$ dry & 14.3 & & 4/08/08 & 8D09004 & AGG-RRL-002 \\
\hline $12587-46-1$ & Gross Alpha & $<4.53 \mathrm{pCi} / \mathrm{g}$ dry & 4.53 & & 4/08/08 & 8D09004 & AGG-RRL-002 \\
\hline HEIS No. & B1RTH2B & \multicolumn{3}{|c|}{ Lab ID: $\quad$ 0803012-05 } & & & \\
\hline $12587-47-2$ & Gross Beta & $<14.3 \mathrm{pCi} / \mathrm{g}$ dry & 14.3 & & $4 / 08 / 08$ & 8D09004 & AGG-RRL-002 \\
\hline $12587-46-1$ & Gross Alpha & $<4.53 \mathrm{pCi} / \mathrm{g}$ dry & 4.53 & & 4/08/08 & 8D09004 & AGG-RRL-002 \\
\hline HEIS No. & B1RTH2A & \multicolumn{3}{|c|}{ Lab ID: $\quad$ 0803012-06 } & & & \\
\hline $12587-47-2$ & Gross Beta & $<14.3 \mathrm{pCi} / \mathrm{g}$ dry & 14.3 & & 4/08/08 & 8D09004 & AGG-RRL-002 \\
\hline $12587-46-1$ & Gross Alpha & $<4.53 \mathrm{pCi} / \mathrm{g}$ dry & 4.53 & & 4/08/08 & 8D09004 & AGG-RRL-002 \\
\hline HEIS No. & B1RTH3B & \multicolumn{3}{|c|}{ Lab ID: $\quad$ 0803012-09 } & & & \\
\hline $12587-47-2$ & Gross Beta & $<14.3 \mathrm{pCi} / \mathrm{g}$ dry & 14.3 & & 4/08/08 & 8D09004 & AGG-RRL-002 \\
\hline $12587-46-1$ & Gross Alpha & $<4.53 \mathrm{pCi} / \mathrm{g}$ dry & 4.53 & & $4 / 08 / 08$ & 8D09004 & AGG-RRL-002 \\
\hline HEIS No. & B1RTH3A & \multicolumn{3}{|c|}{ Lab ID: $\quad$ 0803012-10 } & & & \\
\hline $12587-47-2$ & Gross Beta & $<14.3 \mathrm{pCi} / \mathrm{g}$ dry & 14.3 & & $4 / 08 / 08$ & 8D09004 & AGG-RRL-002 \\
\hline $12587-46-1$ & Gross Alpha & $<4.53 \mathrm{pCi} / \mathrm{g}$ dry & 4.53 & & 4/08/08 & 8D09004 & AGG-RRL-002 \\
\hline HEIS No. & B1RYR7B & \multicolumn{3}{|c|}{ Lab ID: $\quad$ 0803012-13 } & & & \\
\hline $12587-47-2$ & Gross Beta & $<14.3$ pCi/g dry & 14.3 & & 4/08/08 & 8D09004 & AGG-RRL-002 \\
\hline $12587-46-1$ & Gross Alpha & $<4.53 \mathrm{pCi} / \mathrm{g}$ dry & 4.53 & & 4/08/08 & 8D09004 & AGG-RRL-002 \\
\hline HEIS No. & B1RYR7A & \multicolumn{3}{|c|}{ Lab ID: $\quad$ 0803012-14 } & & & \\
\hline $12587-47-2$ & Gross Beta & $<14.3 \mathrm{pCi} / \mathrm{g}$ dry & 14.3 & & 4/08/08 & 8D09004 & AGG-RRL-002 \\
\hline $12587-46-1$ & Gross Alpha & $<4.53 \mathrm{pCi} / \mathrm{g}$ dry & 4.53 & & 4/08/08 & 8D09004 & AGG-RRL-002 \\
\hline HEIS No. & B1RYR8B & \multicolumn{3}{|c|}{ Lab ID: $\quad$ 0803012-17 } & & & \\
\hline $12587-47-2$ & Gross Beta & $<14.3 \mathrm{pCi} / \mathrm{g}$ dry & 14.3 & & 4/08/08 & 8D09004 & AGG-RRL-002 \\
\hline $12587-46-1$ & Gross Alpha & $<4.53 \mathrm{pCi} / \mathrm{g}$ dry & 4.53 & & 4/08/08 & 8D09004 & AGG-RRL-002 \\
\hline HEIS No. & B1RYR8A & \multicolumn{3}{|c|}{ Lab ID: $\quad$ 0803012-18 } & & & \\
\hline $12587-47-2$ & Gross Beta & $<14.3 \mathrm{pCi} / \mathrm{g}$ dry & 14.3 & & 4/08/08 & 8D09004 & AGG-RRL-002 \\
\hline $12587-46-1$ & Gross Alpha & $<4.53 \mathrm{pCi} / \mathrm{g}$ dry & 4.53 & & $4 / 08 / 08$ & 8D09004 & AGG-RRL-002 \\
\hline HEIS No. & B1RYR9B & \multicolumn{3}{|c|}{ Lab ID: $\quad$ 0803012-21 } & & & \\
\hline $12587-47-2$ & Gross Beta & $<14.3 \mathrm{pCi} / \mathrm{g}$ dry & 14.3 & & 4/08/08 & 8D09004 & AGG-RRL-002 \\
\hline $12587-46-1$ & Gross Alpha & $<4.53 \mathrm{pCi} / \mathrm{g}$ dry & 4.53 & & 4/08/08 & 8D09004 & AGG-RRL-002 \\
\hline HEIS No. & B1RYR9A & \multicolumn{3}{|c|}{ Lab ID: $\quad$ 0803012-22 } & & & \\
\hline $12587-47-2$ & Gross Beta & $<14.3 \mathrm{pCi} / \mathrm{g}$ dry & 14.3 & & $4 / 08 / 08$ & 8D09004 & AGG-RRL-002 \\
\hline $12587-46-1$ & Gross Alpha & $<4.53 \mathrm{pCi} / \mathrm{g}$ dry & 4.53 & & 4/08/08 & 8D09004 & AGG-RRL-002 \\
\hline HEIS No. & B1RYT0B & \multicolumn{3}{|c|}{ Lab ID: $\quad$ 0803014-01 } & & & \\
\hline $12587-47-2$ & Gross Beta & $<14.3 \mathrm{pCi} / \mathrm{g}$ dry & 14.3 & & $4 / 08 / 08$ & 8D09004 & AGG-RRL-002 \\
\hline $12587-46-1$ & Gross Alpha & $<4.53 \mathrm{pCi} / \mathrm{g}$ dry & 4.53 & & 4/08/08 & 8D09004 & AGG-RRL-002 \\
\hline HEIS No. & B1RYT0A & \multicolumn{3}{|c|}{ Lab ID: $\quad$ 0803014-02 } & & & \\
\hline $12587-47-2$ & Gross Beta & $<14.3 \mathrm{pCi} / \mathrm{g}$ dry & 14.3 & & 4/08/08 & 8D09004 & AGG-RRL-002 \\
\hline
\end{tabular}


Total Alpha Total Beta/Water Extract

\begin{tabular}{|c|c|c|c|c|c|c|c|}
\hline CAS \# & Analyte & Results & MDA & UNC & Analyzed & Batch & Method \\
\hline HEIS No. & B1RYT0A & \multicolumn{3}{|c|}{ Lab ID: $\quad$ 0803014-02 } & & & \\
\hline 12587-46-1 & Gross Alpha & $<4.53 \mathrm{pCi} / \mathrm{g}$ dry & 4.53 & & $4 / 08 / 08$ & 8D09004 & AGG-RRL-002 \\
\hline HEIS No. & B1T2P1B & \multicolumn{3}{|c|}{ Lab ID: $\quad$ 0803014-05 } & & & \\
\hline $12587-47-2$ & Gross Beta & $<14.3 \mathrm{pCi} / \mathrm{g}$ dry & 14.3 & & $4 / 08 / 08$ & 8D09004 & AGG-RRL-002 \\
\hline 12587-46-1 & Gross Alpha & $<4.53 \mathrm{pCi} / \mathrm{g}$ dry & 4.53 & & 4/08/08 & 8D09004 & AGG-RRL-002 \\
\hline HEIS No. & B1T2P1A & \multicolumn{3}{|c|}{ Lab ID: $\quad$ 0803014-06 } & & & \\
\hline $12587-47-2$ & Gross Beta & $<14.3 \mathrm{pCi} / \mathrm{g}$ dry & 14.3 & & $4 / 08 / 08$ & 8D09004 & AGG-RRL-002 \\
\hline $12587-46-1$ & Gross Alpha & $<4.53 \mathrm{pCi} / \mathrm{g}$ dry & 4.53 & & $4 / 08 / 08$ & 8D09004 & AGG-RRL-002 \\
\hline HEIS No. & B1T2P2B & \multicolumn{3}{|c|}{ Lab ID: $\quad$ 0803014-09 } & & & \\
\hline $12587-47-2$ & Gross Beta & $<14.3 \mathrm{pCi} / \mathrm{g}$ dry & 14.3 & & 4/08/08 & 8D09004 & AGG-RRL-002 \\
\hline $12587-46-1$ & Gross Alpha & $<4.53 \mathrm{pCi} / \mathrm{g}$ dry & 4.53 & & 4/08/08 & 8D09004 & AGG-RRL-002 \\
\hline HEIS No. & B1T2P2A & \multicolumn{3}{|c|}{ Lab ID: $\quad$ 0803014-10 } & & & \\
\hline $12587-47-2$ & Gross Beta & $<14.3 \mathrm{pCi} / \mathrm{g}$ dry & 14.3 & & $4 / 08 / 08$ & 8D09004 & AGG-RRL-002 \\
\hline $12587-46-1$ & Gross Alpha & $<4.53 \mathrm{pCi} / \mathrm{g}$ dry & 4.53 & & 4/08/08 & 8D09004 & AGG-RRL-002 \\
\hline HEIS No. & B1T2P3B & \multicolumn{3}{|c|}{ Lab ID: $\quad$ 0803014-13 } & & & \\
\hline $12587-47-2$ & Gross Beta & $<14.3 \mathrm{pCi} / \mathrm{g}$ dry & 14.3 & & 4/08/08 & 8D09004 & AGG-RRL-002 \\
\hline $12587-46-1$ & Gross Alpha & $<4.53 \mathrm{pCi} / \mathrm{g}$ dry & 4.53 & & $4 / 08 / 08$ & 8D09004 & AGG-RRL-002 \\
\hline HEIS No. & B1T2P3A & \multicolumn{3}{|c|}{ Lab ID: $\quad$ 0803014-14 } & & & \\
\hline $12587-47-2$ & Gross Beta & $<14.3 \mathrm{pCi} / \mathrm{g}$ dry & 14.3 & & 4/08/08 & 8D09004 & AGG-RRL-002 \\
\hline $12587-46-1$ & Gross Alpha & $<4.53 \mathrm{pCi} / \mathrm{g}$ dry & 4.53 & & $4 / 08 / 08$ & 8D09004 & AGG-RRL-002 \\
\hline HEIS No. & B1T2P4B & \multicolumn{3}{|c|}{ Lab ID: $\quad$ 0803014-16 } & & & \\
\hline $12587-47-2$ & Gross Beta & $<14.3 \mathrm{pCi} / \mathrm{g}$ dry & 14.3 & & 4/08/08 & 8D09004 & AGG-RRL-002 \\
\hline $12587-46-1$ & Gross Alpha & $<4.53 \mathrm{pCi} / \mathrm{g}$ dry & 4.53 & & $4 / 08 / 08$ & 8D09004 & AGG-RRL-002 \\
\hline HEIS No. & B1T2P4A & \multicolumn{3}{|c|}{ Lab ID: $\quad$ 0803014-17 } & & & \\
\hline $12587-47-2$ & Gross Beta & $<14.3 \mathrm{pCi} / \mathrm{g}$ dry & 14.3 & & 4/08/08 & 8D09004 & AGG-RRL-002 \\
\hline $12587-46-1$ & Gross Alpha & $<4.53 \mathrm{pCi} / \mathrm{g}$ dry & 4.53 & & 4/08/08 & 8D09004 & AGG-RRL-002 \\
\hline HEIS No. & B1T2P5B & \multicolumn{3}{|c|}{ Lab ID: $\quad$ 0803014-20 } & & & \\
\hline $12587-47-2$ & Gross Beta & $<14.3 \mathrm{pCi} / \mathrm{g}$ dry & 14.3 & & 4/08/08 & 8D09004 & AGG-RRL-002 \\
\hline $12587-46-1$ & Gross Alpha & $<4.53 \mathrm{pCi} / \mathrm{g}$ dry & 4.53 & & 4/08/08 & 8D09004 & AGG-RRL-002 \\
\hline HEIS No. & B1T2P5A & \multicolumn{3}{|c|}{ Lab ID: $\quad$ 0803014-21 } & & & \\
\hline $12587-47-2$ & Gross Beta & $<14.3 \mathrm{pCi} / \mathrm{g}$ dry & 14.3 & & $4 / 08 / 08$ & 8D09004 & AGG-RRL-002 \\
\hline $12587-46-1$ & Gross Alpha & $<4.53 \mathrm{pCi} / \mathrm{g}$ dry & 4.53 & & 4/08/08 & 8D09004 & AGG-RRL-002 \\
\hline HEIS No. & B1T2P6B & \multicolumn{3}{|c|}{ Lab ID: $\quad$ 0803014-24 } & & & \\
\hline $12587-47-2$ & Gross Beta & $<14.3 \mathrm{pCi} / \mathrm{g}$ dry & 14.3 & & 4/08/08 & 8D09004 & AGG-RRL-002 \\
\hline $12587-46-1$ & Gross Alpha & $<4.53 \mathrm{pCi} / \mathrm{g}$ dry & 4.53 & & 4/08/08 & 8D09004 & AGG-RRL-002 \\
\hline HEIS No. & B1T2P6A & \multicolumn{3}{|c|}{ Lab ID: $\quad$ 0803014-25 } & & & \\
\hline $12587-47-2$ & Gross Beta & $<14.3 \mathrm{pCi} / \mathrm{g}$ dry & 14.3 & & 4/08/08 & 8D09004 & AGG-RRL-002 \\
\hline $12587-46-1$ & Gross Alpha & $<4.53 \mathrm{pCi} / \mathrm{g}$ dry & 4.53 & & 4/08/08 & 8D09004 & AGG-RRL-002 \\
\hline HEIS No. & B1T2P7B & \multicolumn{3}{|c|}{ Lab ID: $\quad$ 0803015-02 } & & & \\
\hline $12587-47-2$ & Gross Beta & $<14.3 \mathrm{pCi} / \mathrm{g}$ dry & 14.3 & & 4/08/08 & 8D09004 & AGG-RRL-002 \\
\hline $12587-46-1$ & Gross Alpha & $<4.53 \mathrm{pCi} / \mathrm{g}$ dry & 4.53 & & $4 / 08 / 08$ & 8D09004 & AGG-RRL-002 \\
\hline HEIS No. & B1T2P7A & \multicolumn{3}{|c|}{ Lab ID: $\quad$ 0803015-03 } & & & \\
\hline $12587-47-2$ & Gross Beta & $<14.3 \mathrm{pCi} / \mathrm{g}$ dry & 14.3 & & 4/08/08 & 8D09004 & AGG-RRL-002 \\
\hline $12587-46-1$ & Gross Alpha & $<4.53 \mathrm{pCi} / \mathrm{g}$ dry & 4.53 & & 4/08/08 & 8D09004 & AGG-RRL-002 \\
\hline HEIS No. & B1T2P8B & \multicolumn{3}{|c|}{ Lab ID: $\quad$ 0803015-06 } & & & \\
\hline $12587-47-2$ & Gross Beta & $<14.3 \mathrm{pCi} / \mathrm{g}$ dry & 14.3 & & 4/08/08 & 8D09004 & AGG-RRL-002 \\
\hline 12587-46-1 & Gross Alpha & $<4.53 \mathrm{pCi} / \mathrm{g}$ dry & 4.53 & & 4/08/08 & 8D09004 & AGG-RRL-002 \\
\hline
\end{tabular}


Total Alpha Total Beta/Water Extract

\begin{tabular}{|c|c|c|c|c|c|c|c|c|}
\hline CAS \# & Analyte & Results & Units & MDA & UNC & Analyzed & Batch & Method \\
\hline HEIS No. & B1T2P8A & \multicolumn{4}{|c|}{ Lab ID: $\quad$ 0803015-07 } & & & \\
\hline $12587-47-2$ & Gross Beta & $<14.3$ & pCi/g dry & 14.3 & & 4/08/08 & 8D09004 & AGG-RRL-002 \\
\hline $12587-46-1$ & Gross Alpha & $<4.53$ & pCi/g dry & 4.53 & & $4 / 08 / 08$ & 8D09004 & AGG-RRL-002 \\
\hline HEIS No. & В1Т2Р9B & \multicolumn{4}{|c|}{ Lab ID: $\quad$ 0803015-10 } & & & \\
\hline $12587-47-2$ & Gross Beta & $<14.3$ & pCi/g dry & 14.3 & & 4/08/08 & 8D09004 & AGG-RRL-002 \\
\hline $12587-46-1$ & Gross Alpha & $<4.53$ & pCi/g dry & 4.53 & & $4 / 08 / 08$ & 8D09004 & AGG-RRL-002 \\
\hline HEIS No. & B1T2P9A & \multicolumn{4}{|c|}{ Lab ID: $\quad$ 0803015-11 } & & & \\
\hline $12587-47-2$ & Gross Beta & $<14.3$ & pCi/g dry & 14.3 & & 4/08/08 & 8D09004 & AGG-RRL-002 \\
\hline $12587-46-1$ & Gross Alpha & $<4.53$ & pCi/g dry & 4.53 & & $4 / 08 / 08$ & 8D09004 & AGG-RRL-002 \\
\hline HEIS No. & B1T2R0B & \multicolumn{4}{|c|}{ Lab ID: $\quad$ 0803015-14 } & & & \\
\hline $12587-47-2$ & Gross Beta & $<14.3$ & pCi/g dry & 14.3 & & $4 / 08 / 08$ & 8D09004 & AGG-RRL-002 \\
\hline 12587-46-1 & Gross Alpha & $<4.53$ & pCi/g dry & 4.53 & & $4 / 08 / 08$ & 8D09004 & AGG-RRL-002 \\
\hline HEIS No. & B1T2R0A & \multicolumn{4}{|c|}{ Lab ID: $\quad$ 0803015-15 } & & & \\
\hline $12587-47-2$ & Gross Beta & $<14.3$ & pCi/g dry & 14.3 & & $4 / 08 / 08$ & 8D09004 & AGG-RRL-002 \\
\hline $12587-46-1$ & Gross Alpha & $<4.53$ & pCi/g dry & 4.53 & & $4 / 08 / 08$ & 8D09004 & AGG-RRL-002 \\
\hline HEIS No. & B1TNK6B & \multicolumn{4}{|c|}{ Lab ID: $\quad 0803015-18$} & & & \\
\hline $12587-47-2$ & Gross Beta & $<14.3$ & pCi/g dry & 14.3 & & 4/08/08 & 8D09004 & AGG-RRL-002 \\
\hline $12587-46-1$ & Gross Alpha & $<4.53$ & pCi/g dry & 4.53 & & $4 / 08 / 08$ & 8D09004 & AGG-RRL-002 \\
\hline HEIS No. & B1TNK6A & \multicolumn{4}{|c|}{ Lab ID: $\quad$ 0803015-19 } & & & \\
\hline $12587-47-2$ & Gross Beta & $<14.3$ & pCi/g dry & 14.3 & & $4 / 08 / 08$ & 8D09004 & AGG-RRL-002 \\
\hline $12587-46-1$ & Gross Alpha & $<4.53$ & pCi/g dry & 4.53 & & $4 / 08 / 08$ & 8D09004 & AGG-RRL-002 \\
\hline HEIS No. & B1TNK7B & \multicolumn{4}{|c|}{ Lab ID: $\quad$ 0803015-22 } & & & \\
\hline $12587-47-2$ & Gross Beta & $<14.3$ & pCi/g dry & 14.3 & & $4 / 08 / 08$ & 8D09004 & AGG-RRL-002 \\
\hline $12587-46-1$ & Gross Alpha & $<4.53$ & pCi/g dry & 4.53 & & 4/08/08 & 8D09004 & AGG-RRL-002 \\
\hline HEIS No. & B1TNK7A & \multicolumn{4}{|c|}{ Lab ID: $\quad$ 0803015-23 } & & & \\
\hline $12587-47-2$ & Gross Beta & $<14.3$ & pCi/g dry & 14.3 & & $4 / 08 / 08$ & 8D09004 & AGG-RRL-002 \\
\hline $12587-46-1$ & Gross Alpha & $<4.53$ & pCi/g dry & 4.53 & & $4 / 08 / 08$ & 8D09004 & AGG-RRL-002 \\
\hline HEIS No. & B1TNK8B & \multicolumn{4}{|c|}{ Lab ID: $\quad$ 0803015-26 } & & & \\
\hline $12587-47-2$ & Gross Beta & $<14.3$ & pCi/g dry & 14.3 & & 4/08/08 & 8D09004 & AGG-RRL-002 \\
\hline $12587-46-1$ & Gross Alpha & $<4.53$ & pCi/g dry & 4.53 & & $4 / 08 / 08$ & 8D09004 & AGG-RRL-002 \\
\hline HEIS No. & B1TNK8A & \multicolumn{4}{|c|}{ Lab ID: $\quad$ 0803015-27 } & & & \\
\hline $12587-47-2$ & Gross Beta & $<14.3$ & pCi/g dry & 14.3 & & $4 / 08 / 08$ & 8D09004 & AGG-RRL-002 \\
\hline $12587-46-1$ & Gross Alpha & $<4.53$ & pCi/g dry & 4.53 & & $4 / 08 / 08$ & 8D09004 & AGG-RRL-002 \\
\hline HEIS No. & B1TNK9B & \multicolumn{4}{|c|}{ Lab ID: $\quad$ 0803015-30 } & & & \\
\hline $12587-47-2$ & Gross Beta & $<14.3$ & pCi/g dry & 14.3 & & 4/08/08 & 8D09004 & AGG-RRL-002 \\
\hline $12587-46-1$ & Gross Alpha & $<4.53$ & pCi/g dry & 4.53 & & $4 / 08 / 08$ & 8D09004 & AGG-RRL-002 \\
\hline HEIS No. & B1TNK9A & \multicolumn{4}{|c|}{ Lab ID: $\quad$ 0803015-31 } & & & \\
\hline $12587-47-2$ & Gross Beta & $<14.3$ & pCi/g dry & 14.3 & & 4/08/08 & 8D09004 & AGG-RRL-002 \\
\hline $12587-46-1$ & Gross Alpha & $<4.53$ & pCi/g dry & 4.53 & & 4/08/08 & 8D09004 & AGG-RRL-002 \\
\hline
\end{tabular}


Wet Chemistry - Quality Control

Environmental Science Laboratory

\begin{tabular}{|lrrrrrrrrrrr}
\hline & & Reporting & & Spike & Source & & \%REC & & RPD & & \\
Analyte & Result & Limit & Units & Level & Result & \%REC & Limits & RPD & Limit & Notes \\
\hline
\end{tabular}

Batch 8D01004 - 1:1 Water Extract (pH_EC_Alk)

\begin{tabular}{|c|c|c|c|c|c|c|}
\hline \multicolumn{4}{|l|}{ Blank (8D01004-BLK1) } & \multicolumn{3}{|l|}{ Prepared: 04/01/08 Analyzed: 04/02/08 } \\
\hline Specific Conductance (EC) & 0.0110 & 0.0100 & $\mathrm{mS} / \mathrm{cm}$ & & & \\
\hline Duplicate (8D01004-DUP1) & \multicolumn{3}{|c|}{ Source: 0803012-05 } & \multicolumn{3}{|l|}{ Prepared: 04/01/08 Analyzed: 04/02/08 } \\
\hline $\mathrm{pH}$ & 7.72 & & pH Units & 7.84 & 1.54 & 20 \\
\hline Specific Conductance (EC) & 0.301 & 0.00958 & $\mathrm{mS} / \mathrm{cm}$ & 0.303 & 0.662 & 20 \\
\hline Duplicate (8D01004-DUP2) & \multicolumn{3}{|c|}{ Source: 0803012-14 } & \multicolumn{3}{|l|}{ Prepared: 04/01/08 Analyzed: 04/02/08 } \\
\hline $\mathrm{pH}$ & 7.74 & & pH Units & 7.92 & 2.30 & 20 \\
\hline \multicolumn{7}{|c|}{ Batch 8D03004 - 1:1 Water Extract (pH_EC_Alk) } \\
\hline Blank (8D03004-BLK1) & & & & Prepared \& Analyzed: 04/03/08 & & \\
\hline $\mathrm{pH}$ & 5.08 & & pH Units & & & \\
\hline Specific Conductance (EC) & 0.0100 & 0.0100 & $\mathrm{mS} / \mathrm{cm}$ & & & \\
\hline Blank (8D03004-BLK2) & & & & Prepared \& Analyzed: 04/03/08 & & \\
\hline $\mathrm{pH}$ & 5.39 & & pH Units & & & \\
\hline Specific Conductance (EC) & 0.0100 & 0.0100 & $\mathrm{mS} / \mathrm{cm}$ & & & \\
\hline Duplicate (8D03004-DUP1) & \multicolumn{3}{|c|}{ Source: 0803014-05 } & \multicolumn{3}{|l|}{ Prepared \& Analyzed: 04/03/08 } \\
\hline $\mathrm{pH}$ & 7.81 & & pH Units & 7.84 & 0.383 & 20 \\
\hline Specific Conductance (EC) & 0.254 & 0.00970 & $\mathrm{mS} / \mathrm{cm}$ & 0.255 & 0.393 & 20 \\
\hline Duplicate (8D03004-DUP2) & \multicolumn{3}{|c|}{ Source: 0803015-07 } & \multicolumn{3}{|l|}{ Prepared \& Analyzed: 04/03/08 } \\
\hline Specific Conductance (EC) & 0.392 & 0.00966 & $\mathrm{mS} / \mathrm{cm}$ & 0.253 & 43.1 & 20 \\
\hline $\mathrm{pH}$ & 7.58 & & pH Units & 7.77 & 2.48 & 20 \\
\hline
\end{tabular}


Wet Chemistry - Quality Control

Environmental Science Laboratory

\begin{tabular}{|lrrrrrrrrrr}
\hline & & Reporting & & Spike & Source & & \%REC & & RPD & \\
Analyte & Result & Limit & Units & Level & Result & \%REC & Limits & RPD & Limit & Notes \\
\hline
\end{tabular}

Batch 8D03004 - 1:1 Water Extract (pH_EC_Alk)

\begin{tabular}{lllll} 
Duplicate (8D03004-DUP3) & Source: 0803014-10 & Prepared \& Analyzed: 04/03/08 & \\
\hline $\mathrm{pH}$ & 7.70 & $\mathrm{pH}$ Units & 7.67 & 0.390
\end{tabular}

Batch 8D04002 - 1:1 Water Extract (pH_EC_Alk)

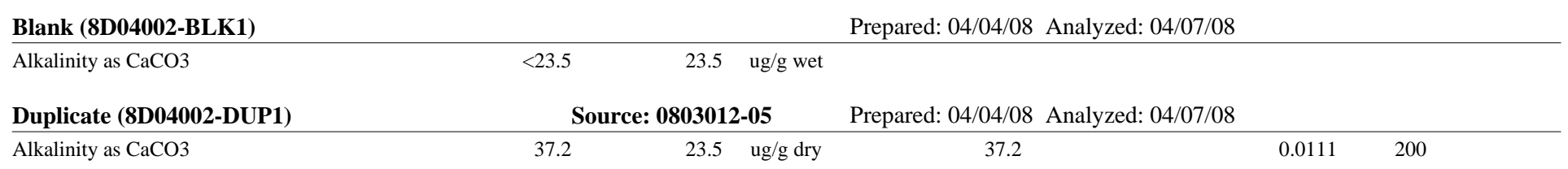

Batch 8D04003 - 1:1 Water Extract (pH_EC_Alk)

\begin{tabular}{|c|c|c|c|c|c|c|c|}
\hline \multirow{2}{*}{$\begin{array}{l}\text { Blank (8D04003-BLK1) } \\
\text { Alkalinity as } \mathrm{CaCO} 3\end{array}$} & \multicolumn{7}{|c|}{ Prepared: 04/04/08 Analyzed: 04/14/08 } \\
\hline & $<23.5$ & 23.5 & ug/g wet & & & & \\
\hline Blank (8D04003-BLK2) & \multicolumn{7}{|c|}{ Prepared: 04/04/08 Analyzed: 04/14/08 } \\
\hline Alkalinity as $\mathrm{CaCO} 3$ & $<23.5$ & 23.5 & ug/g wet & & & & \\
\hline Duplicate (8D04003-DUP1) & \multicolumn{3}{|c|}{ Source: 0803014-05 } & \multicolumn{4}{|c|}{ Prepared: 04/04/08 Analyzed: 04/14/08 } \\
\hline Alkalinity as $\mathrm{CaCO} 3$ & 35.0 & 23.5 & ug/g dry & 36.5 & & 4.27 & 200 \\
\hline Duplicate (8D04003-DUP2) & \multicolumn{3}{|c|}{ Source: 0803015-07 } & Prepared: 04/04/08 & Analyzed: 04/14/08 & & \\
\hline Alkalinity as $\mathrm{CaCO} 3$ & 27.4 & 23.5 & ug/g dry & 30.4 & & 10.6 & 200 \\
\hline
\end{tabular}


Anions by Ion Chromatography - Quality Control

Environmental Science Laboratory

\begin{tabular}{|lrrrrrrrrrrr}
\hline & & Reporting & & Spike & Source & & & \%REC & & RPD & \\
Analyte & Result & Limit & Units & Level & Result & \%REC & Limits & RPD & Limit & Notes \\
\hline
\end{tabular}

Batch 8D02003 - 1:1 Water Extract (IC)

\begin{tabular}{|c|c|c|c|c|c|c|c|c|c|}
\hline \multirow{2}{*}{$\begin{array}{l}\text { Blank (8D02003-BLK1) } \\
\text { Fluoride }\end{array}$} & \multicolumn{9}{|c|}{ Prepared: 04/02/08 Analyzed: 04/03/08 } \\
\hline & $<0.200$ & 0.200 & ug/g wet & & & & & & \\
\hline Chloride & $<0.500$ & 0.500 & $"$ & & & & & & \\
\hline Nitrite & $<1.00$ & 1.00 & " & & & & & & \\
\hline Bromide & $<1.00$ & 1.00 & $"$ & & & & & & \\
\hline Nitrate & $<1.00$ & 1.00 & $"$ & & & & & & \\
\hline Sulfate & $<1.50$ & 1.50 & $"$ & & & & & & \\
\hline Phosphate & $<1.50$ & 1.50 & $"$ & & & & & & \\
\hline LCS (8D02003-BS1) & \multicolumn{9}{|c|}{ Prepared: 04/02/08 Analyzed: 04/03/08 } \\
\hline Fluoride & 2.09 & 0.200 & ug/g wet & 2.000 & & 105 & $80-120$ & & \\
\hline Chloride & 4.81 & 0.500 & $"$ & 5.000 & & 96.1 & $80-120$ & & \\
\hline Nitrite & 10.7 & 1.00 & $"$ & 10.03 & & 106 & $80-120$ & & \\
\hline Bromide & 9.68 & 1.00 & " & 10.00 & & 96.8 & $80-120$ & & \\
\hline Nitrate & 10.3 & 1.00 & " & 10.03 & & 102 & $80-120$ & & \\
\hline Sulfate & 14.9 & 1.50 & $"$ & 15.07 & & 98.8 & $80-120$ & & \\
\hline Phosphate & 14.7 & 1.50 & " & 15.07 & & 97.4 & $80-120$ & & \\
\hline Duplicate (8D02003-DUP1) & \multicolumn{3}{|c|}{ Source: 0803012-05 } & \multicolumn{6}{|c|}{ Prepared: 04/02/08 Analyzed: 04/03/08 } \\
\hline Fluoride & 0.312 & 0.200 & ug/g dry & & 0.294 & & & 5.93 & 20 \\
\hline Chloride & 6.49 & 0.500 & $"$ & & 6.56 & & & 1.10 & 20 \\
\hline Nitrite & $<1.00$ & 1.00 & " & & ND & & & & 20 \\
\hline Bromide & $<1.00$ & 1.00 & " & & ND & & & & 20 \\
\hline Nitrate & 5.99 & 1.00 & " & & 5.91 & & & 1.42 & 20 \\
\hline Sulfate & 97.3 & 1.50 & $"$ & & 98.2 & & & 0.922 & 20 \\
\hline Phosphate & $<1.50$ & 1.50 & $"$ & & ND & & & & 20 \\
\hline Post Spike (8D02003-PS1) & \multicolumn{3}{|c|}{ Source: 0803014-02 } & \multicolumn{4}{|c|}{ Prepared \& Analyzed: 04/02/08 } & & \\
\hline Fluoride & 2.04 & & $\mathrm{ug} / \mathrm{mL}$ & 0.769 & 1.24 & 104 & $75-125$ & & \\
\hline Chloride & 3.06 & & $"$ & 1.92 & 1.12 & 101 & $75-125$ & & \\
\hline Nitrite & 4.24 & & $"$ & 3.85 & 0.319 & 102 & $75-125$ & & \\
\hline Bromide & 3.9 & & $"$ & 3.85 & 0.352 & 92.4 & $75-125$ & & \\
\hline Nitrate & 11.7 & & $"$ & 3.85 & 7.89 & 99.3 & $75-125$ & & \\
\hline Sulfate & 22 & & $"$ & 5.77 & 16 & 103 & $75-125$ & & \\
\hline Phosphate & 6.09 & & " & 5.77 & 0.568 & 95.8 & $75-125$ & & \\
\hline
\end{tabular}


Anions by Ion Chromatography - Quality Control

Environmental Science Laboratory

\begin{tabular}{|lrrrrrrrrrrr}
\hline & & Reporting & & Spike & Source & & & \%REC & & RPD & \\
Analyte & Result & Limit & Units & Level & Result & \%REC & Limits & RPD & Limit & Notes \\
\hline
\end{tabular}

Batch 8D03001 - 1:1 Water Extract (IC)

\begin{tabular}{|c|c|c|c|c|c|c|}
\hline \multirow{2}{*}{$\begin{array}{l}\text { Blank (8D03001-BLK1) } \\
\text { Fluoride }\end{array}$} & \multicolumn{6}{|c|}{ Prepared: 04/03/08 Analyzed: 04/04/08 } \\
\hline & $<0.200$ & 0.200 & ug/g wet & & & \\
\hline Chloride & $<0.500$ & 0.500 & " & & & \\
\hline Nitrite & $<1.00$ & 1.00 & $"$ & & & \\
\hline Bromide & $<1.00$ & 1.00 & $"$ & & & \\
\hline Nitrate & $<1.00$ & 1.00 & $"$ & & & \\
\hline Sulfate & $<1.50$ & 1.50 & $"$ & & & \\
\hline Phosphate & $<1.50$ & 1.50 & $"$ & & & \\
\hline Blank (8D03001-BLK2) & \multicolumn{6}{|c|}{ Prepared: 04/03/08 Analyzed: 04/04/08 } \\
\hline Fluoride & $<0.200$ & 0.200 & ug/g wet & & & \\
\hline Chloride & $<0.500$ & 0.500 & " & & & \\
\hline Nitrite & $<1.00$ & 1.00 & $"$ & & & \\
\hline Bromide & $<1.00$ & 1.00 & " & & & \\
\hline Nitrate & $<1.00$ & 1.00 & $"$ & & & \\
\hline Sulfate & $<1.50$ & 1.50 & " & & & \\
\hline Phosphate & $<1.50$ & 1.50 & " & & & \\
\hline LCS (8D03001-BS1) & \multicolumn{6}{|c|}{ Prepared: 04/03/08 Analyzed: 04/04/08 } \\
\hline Fluoride & 2.07 & 0.207 & ug/g wet & 2.070 & 99.8 & $80-120$ \\
\hline Chloride & 5.02 & 0.517 & $"$ & 5.174 & 97.1 & $80-120$ \\
\hline Nitrite & 10.9 & 1.03 & $"$ & 10.38 & 105 & $80-120$ \\
\hline Bromide & 9.96 & 1.03 & $"$ & 10.35 & 96.2 & $80-120$ \\
\hline Nitrate & 10.3 & 1.03 & " & 10.38 & 99.4 & $80-120$ \\
\hline Sulfate & 15.4 & 1.55 & $"$ & 15.59 & 98.9 & $80-120$ \\
\hline Phosphate & 15.0 & 1.55 & $"$ & 15.59 & 96.2 & $80-120$ \\
\hline LCS (8D03001-BS2) & \multicolumn{6}{|c|}{ Prepared: 04/03/08 Analyzed: 04/04/08 } \\
\hline Fluoride & 2.12 & 0.207 & ug/g wet & 2.070 & 103 & $80-120$ \\
\hline Chloride & 5.02 & 0.517 & $"$ & 5.174 & 97.0 & $80-120$ \\
\hline Nitrite & 10.8 & 1.03 & $"$ & 10.38 & 104 & $80-120$ \\
\hline Bromide & 9.94 & 1.03 & $"$ & 10.35 & 96.1 & $80-120$ \\
\hline Nitrate & 10.2 & 1.03 & $"$ & 10.38 & 98.5 & $80-120$ \\
\hline Sulfate & 15.4 & 1.55 & $"$ & 15.59 & 98.9 & $80-120$ \\
\hline Phosphate & 15.1 & 1.55 & " & 15.59 & 96.5 & $80-120$ \\
\hline
\end{tabular}


Anions by Ion Chromatography - Quality Control

Environmental Science Laboratory

\begin{tabular}{|c|c|c|c|c|c|c|c|c|c|c|}
\hline Analyte & Result & $\begin{array}{c}\text { orting } \\
\text { Limit }\end{array}$ & Units & $\begin{array}{l}\text { Spike } \\
\text { Level }\end{array}$ & $\begin{array}{l}\text { Source } \\
\text { Result }\end{array}$ & \%REC & $\begin{array}{l}\text { \%REC } \\
\text { Limits }\end{array}$ & RPD & $\begin{array}{l}\text { RPD } \\
\text { Limit }\end{array}$ & Notes \\
\hline
\end{tabular}

Batch 8D03001 - 1:1 Water Extract (IC)

\begin{tabular}{|c|c|c|c|c|c|c|c|c|c|}
\hline \multirow{2}{*}{$\begin{array}{l}\text { Duplicate (8D03001-DUP1) } \\
\text { Fluoride }\end{array}$} & \multicolumn{3}{|c|}{ Source: 0803014-05 } & \multicolumn{4}{|c|}{ Prepared: 04/03/08 Analyzed: 04/04/08 } & \multirow[b]{2}{*}{0.580} & \multirow[b]{2}{*}{20} \\
\hline & 0.346 & 0.200 & ug/g dry & & 0.344 & & & & \\
\hline Chloride & 8.03 & 0.500 & " & & 8.04 & & & 0.162 & 20 \\
\hline Nitrite & $<1.00$ & 1.00 & $"$ & & ND & & & & 20 \\
\hline Bromide & $<1.00$ & 1.00 & $"$ & & ND & & & & 20 \\
\hline Nitrate & 5.04 & 1.00 & $"$ & & 4.95 & & & 1.70 & 20 \\
\hline Sulfate & 69.9 & 1.50 & $"$ & & 70.5 & & & 0.874 & 20 \\
\hline Phosphate & $<1.50$ & 1.50 & $"$ & & ND & & & & 20 \\
\hline Duplicate (8D03001-DUP2) & \multicolumn{3}{|c|}{ Source: 0803015-07 } & \multicolumn{2}{|c|}{ Prepared: 04/03/08 } & Analyzec & $4 / 04 / 08$ & & \\
\hline Fluoride & 0.262 & 0.200 & ug/g dry & & 0.249 & & & 5.09 & 20 \\
\hline Chloride & 2.47 & 0.500 & $"$ & & 2.11 & & & 15.9 & 20 \\
\hline Nitrite & $<1.00$ & 1.00 & $"$ & & ND & & & & 20 \\
\hline Bromide & $<1.00$ & 1.00 & $"$ & & ND & & & & 20 \\
\hline Nitrate & 8.39 & 1.00 & $"$ & & 8.64 & & & 2.95 & 20 \\
\hline Sulfate & 150 & 1.50 & " & & 78.4 & & & 62.5 & 20 \\
\hline Phosphate & $<1.50$ & 1.50 & $"$ & & ND & & & & 20 \\
\hline Post Spike (8D03001-PS1) & \multicolumn{3}{|c|}{ Source: 0803014-06 } & \multicolumn{2}{|c|}{ Prepared: 04/03/08 } & \multicolumn{2}{|c|}{ Analyzed: 04/04/08 } & & \\
\hline Fluoride & 1.09 & & $\mathrm{ug} / \mathrm{mL}$ & 0.8 & 0.311 & 97 & $75-125$ & & \\
\hline Chloride & 8.58 & & $"$ & 2 & 6.99 & 79.5 & $75-125$ & & \\
\hline Nitrite & 3.8 & & $"$ & 4 & 0.312 & 87.2 & $75-125$ & & \\
\hline Bromide & 3.77 & & $"$ & 4 & 0.518 & 81.3 & $75-125$ & & \\
\hline Nitrate & 7.41 & & $"$ & 4 & 3.89 & 88.2 & $75-125$ & & \\
\hline Sulfate & 67.6 & & $"$ & 5.77 & 64.6 & 50.9 & $75-125$ & & \\
\hline Phosphate & 5.33 & & $"$ & 6 & 0.272 & 84.3 & $75-125$ & & \\
\hline Post Spike (8D03001-PS2) & \multicolumn{3}{|c|}{ Source: $0803015-31$} & \multicolumn{2}{|c|}{ Prepared: 04/03/08 } & \multicolumn{2}{|c|}{ Analyzed: 04/04/08 } & & \\
\hline Fluoride & 1.12 & & $\mathrm{ug} / \mathrm{mL}$ & 0.8 & 0.371 & 93.8 & $75-125$ & & \\
\hline Chloride & 7.81 & & $"$ & 2 & 6.27 & 76.8 & $75-125$ & & \\
\hline Nitrite & 3.55 & & $"$ & 4 & 0.297 & 81.4 & $75-125$ & & \\
\hline Bromide & 3.79 & & $"$ & 4 & 0.508 & 82 & $75-125$ & & \\
\hline Nitrate & 9.26 & & $"$ & 4 & 5.73 & 88.3 & $75-125$ & & \\
\hline Sulfate & 136 & & $"$ & 5.77 & 137 & NR & $75-125$ & & \\
\hline Phosphate & 5.29 & & $"$ & 6 & 0.266 & 83.8 & $75-125$ & & \\
\hline
\end{tabular}


Total Metals by SW846 6010B/Water Extract - Quality Control

Environmental Science Laboratory

\begin{tabular}{|c|c|c|c|c|c|c|c|c|c|c|}
\hline & & porting & & Spike & Source & & \%REC & & RPD & \\
\hline Analyte & Result & Limit & Units & Level & Result & $\%$ REC & Limits & RPD & Limit & Notes \\
\hline
\end{tabular}

Batch 8D28005 - 1:1 Water Extract (ICP/ICPMS)

\begin{tabular}{|c|c|c|c|c|}
\hline Blank (8D28005-BLK1) & & & & Prepared: 04/28/08 Analyzed: 05/07/08 \\
\hline Aluminum & $<0.086$ & 0.086 & ug/g wet & \\
\hline Arsenic & $<0.360$ & 0.360 & " & \\
\hline Boron & $<1.94$ & 1.94 & $"$ & \\
\hline Barium & $<0.009$ & 0.009 & " & \\
\hline Beryllium & $<0.028$ & 0.028 & $"$ & \\
\hline Bismuth & $<0.188$ & 0.188 & $"$ & \\
\hline Calcium & $<0.387$ & 0.387 & $"$ & \\
\hline Cadmium & $<0.027$ & 0.027 & " & \\
\hline Cobalt & $<0.096$ & 0.096 & $"$ & \\
\hline Chromium & $<0.033$ & 0.033 & $"$ & \\
\hline Copper & $<0.080$ & 0.080 & $"$ & \\
\hline Iron & $<0.142$ & 0.142 & $"$ & \\
\hline Potassium & $<2.33$ & 2.33 & $"$ & \\
\hline Lithium & $<0.540$ & 0.540 & $"$ & \\
\hline Magnesium & $<0.083$ & 0.083 & $"$ & \\
\hline Manganese & $<0.017$ & 0.017 & $"$ & \\
\hline Molybdenum & $<0.139$ & 0.139 & $"$ & \\
\hline Nickel & $<0.093$ & 0.093 & $"$ & \\
\hline Phosphorus & $<1.03$ & 1.03 & $"$ & \\
\hline Lead & $<0.432$ & 0.432 & $"$ & \\
\hline Selenium & $<1.95$ & 1.95 & $"$ & \\
\hline Strontium & $<0.052$ & 0.052 & $"$ & \\
\hline Thallium & $<1.03$ & 1.03 & $"$ & \\
\hline Vanadium & $<0.045$ & 0.045 & $"$ & \\
\hline Zinc & $<0.093$ & 0.093 & $"$ & \\
\hline Sodium & $<0.669$ & 0.669 & $"$ & \\
\hline Silicon & $<1.50$ & 1.50 & " & \\
\hline Sulfur & $<3.08$ & 3.08 & $"$ & \\
\hline Titanium & $<0.009$ & 0.009 & $"$ & \\
\hline Zirconium & $<0.150$ & 0.150 & $"$ & \\
\hline Silver & $<0.077$ & 0.077 & $"$ & \\
\hline Rhenium & $<0.152$ & 0.152 & $"$ & \\
\hline Antimony & $<0.633$ & 0.633 & $"$ & \\
\hline
\end{tabular}


Total Metals by SW846 6010B/Water Extract - Quality Control

Environmental Science Laboratory

\begin{tabular}{|lcccccccccccc}
\hline & & Reporting & & Spike & Source & & \%REC & & RPD & & \\
Analyte & Result & Limit & Units & Level & Result & \%REC & Limits & RPD & Limit & Notes \\
\hline
\end{tabular}

Batch 8D28005 - 1:1 Water Extract (ICP/ICPMS)

\begin{tabular}{|c|c|c|c|c|c|c|}
\hline \multirow{2}{*}{$\begin{array}{l}\text { LCS (8D28005-BS1) } \\
\text { Aluminum }\end{array}$} & \multicolumn{6}{|c|}{ Prepared: 04/28/08 Analyzed: 05/07/08 } \\
\hline & 4.60 & 0.086 & ug/g wet & 4.992 & 92.1 & $80-120$ \\
\hline Arsenic & 4.82 & 0.360 & $"$ & 4.992 & 96.5 & $80-120$ \\
\hline Boron & 4.84 & 1.94 & $"$ & 4.992 & 97.0 & $80-120$ \\
\hline Barium & 4.88 & 0.009 & $"$ & 4.992 & 97.8 & $80-120$ \\
\hline Beryllium & 4.87 & 0.028 & $"$ & 4.992 & 97.7 & $80-120$ \\
\hline Bismuth & $<0.188$ & 0.188 & $"$ & & & $80-120$ \\
\hline Calcium & 4.70 & 0.387 & $"$ & 4.992 & 94.2 & $80-120$ \\
\hline Cadmium & 5.01 & 0.027 & $"$ & 4.992 & 100 & $80-120$ \\
\hline Cobalt & 4.77 & 0.096 & $"$ & 4.992 & 95.5 & $80-120$ \\
\hline Chromium & 5.04 & 0.033 & " & 4.992 & 101 & $80-120$ \\
\hline Copper & 4.67 & 0.080 & $"$ & 4.992 & 93.5 & $80-120$ \\
\hline Iron & 4.90 & 0.142 & $"$ & 4.992 & 98.2 & $80-120$ \\
\hline Potassium & 48.6 & 2.33 & " & 49.92 & 97.3 & $80-120$ \\
\hline Lithium & $<0.540$ & 0.540 & $"$ & & & $80-120$ \\
\hline Magnesium & 4.69 & 0.083 & $"$ & 4.992 & 93.9 & $80-120$ \\
\hline Manganese & 4.76 & 0.017 & $"$ & 4.992 & 95.4 & $80-120$ \\
\hline Molybdenum & 4.75 & 0.139 & $"$ & 4.992 & 95.1 & $80-120$ \\
\hline Nickel & 4.87 & 0.093 & $"$ & 4.992 & 97.6 & $80-120$ \\
\hline Phosphorus & $<1.03$ & 1.03 & $"$ & & & $80-120$ \\
\hline Lead & 4.80 & 0.432 & $"$ & 4.992 & 96.1 & $80-120$ \\
\hline Selenium & 4.94 & 1.95 & $"$ & 4.992 & 98.9 & $80-120$ \\
\hline Strontium & $<0.052$ & 0.052 & $"$ & & & $80-120$ \\
\hline Thallium & 4.77 & 1.03 & $"$ & 4.992 & 95.5 & $80-120$ \\
\hline Vanadium & 4.74 & 0.045 & $"$ & 4.992 & 95.0 & $80-120$ \\
\hline Zinc & 4.89 & 0.093 & $"$ & 4.992 & 97.9 & $80-120$ \\
\hline Sodium & 5.06 & 0.669 & " & 4.992 & 101 & $80-120$ \\
\hline Silicon & 2.78 & 1.50 & $"$ & 2.496 & 111 & $80-120$ \\
\hline Sulfur & $<3.08$ & 3.08 & $"$ & & & $80-120$ \\
\hline Titanium & 4.75 & 0.009 & " & 4.992 & 95.2 & $80-120$ \\
\hline Zirconium & $<0.150$ & 0.150 & $"$ & & & $80-120$ \\
\hline Silver & 4.82 & 0.077 & $"$ & 4.992 & 96.5 & $80-120$ \\
\hline Rhenium & $<0.152$ & 0.152 & $"$ & & & $80-120$ \\
\hline Antimony & 4.82 & 0.633 & " & 4.992 & 96.5 & $80-120$ \\
\hline
\end{tabular}


Total Metals by SW846 6010B/Water Extract - Quality Control

Environmental Science Laboratory

\begin{tabular}{|c|c|c|c|c|c|c|c|c|c|c|}
\hline & & porting & & Spike & Source & & \%REC & & RPD & \\
\hline Analyte & Result & Limit & Units & Level & Result & $\%$ REC & Limits & RPD & Limit & Notes \\
\hline
\end{tabular}

Batch 8D28005 - 1:1 Water Extract (ICP/ICPMS)

\begin{tabular}{|c|c|c|c|c|c|c|}
\hline \multirow{2}{*}{$\begin{array}{l}\text { Duplicate (8D28005-DUP1) } \\
\text { Aluminum }\end{array}$} & \multicolumn{3}{|c|}{ Source: 0803012-05 } & \multirow{2}{*}{$\begin{array}{r}\text { Prepared: } 04 / 28 / 08 \\
0.0937\end{array}$} & \multicolumn{2}{|l|}{ Analyzed: 05/07/08 } \\
\hline & 0.106 & 0.086 & ug/g dry & & 11.8 & 20 \\
\hline Arsenic & $<0.360$ & 0.360 & $"$ & ND & & 20 \\
\hline Boron & $<1.94$ & 1.94 & $"$ & ND & & 20 \\
\hline Barium & $<0.009$ & 0.009 & $"$ & 0.0243 & & 20 \\
\hline Beryllium & $<0.028$ & 0.028 & $"$ & ND & & 20 \\
\hline Bismuth & $<0.188$ & 0.188 & $"$ & ND & & 20 \\
\hline Calcium & 21.1 & 0.387 & " & 22.9 & 8.07 & 20 \\
\hline Cadmium & $<0.027$ & 0.027 & " & ND & & 20 \\
\hline Cobalt & $<0.096$ & 0.096 & " & ND & & 20 \\
\hline Chromium & $<0.033$ & 0.033 & " & ND & & 20 \\
\hline Copper & $<0.080$ & 0.080 & " & ND & & 20 \\
\hline Iron & $<0.142$ & 0.142 & " & ND & & 20 \\
\hline Potassium & 6.74 & 2.33 & $"$ & 7.25 & 7.32 & 20 \\
\hline Lithium & $<0.540$ & 0.540 & $"$ & ND & & 20 \\
\hline Magnesium & 8.01 & 0.083 & $"$ & 8.50 & 6.03 & 20 \\
\hline Manganese & $<0.017$ & 0.017 & $"$ & ND & & 20 \\
\hline Molybdenum & $<0.139$ & 0.139 & $"$ & ND & & 20 \\
\hline Nickel & $<0.093$ & 0.093 & " & ND & & 20 \\
\hline Phosphorus & $<1.03$ & 1.03 & " & ND & & 20 \\
\hline Lead & $<0.432$ & 0.432 & $"$ & ND & & 20 \\
\hline Selenium & $<1.95$ & 1.95 & $"$ & ND & & 20 \\
\hline Strontium & 0.0898 & 0.052 & $"$ & 0.0947 & 5.30 & 20 \\
\hline Thallium & $<1.03$ & 1.03 & $"$ & ND & & 20 \\
\hline Vanadium & $<0.045$ & 0.045 & $"$ & ND & & 20 \\
\hline Zinc & $<0.093$ & 0.093 & $"$ & 0.825 & & 20 \\
\hline Sodium & 17.4 & 0.669 & " & 19.0 & 8.40 & 20 \\
\hline Silicon & 6.14 & 1.50 & $"$ & 5.75 & 6.62 & 20 \\
\hline Sulfur & 32.7 & 3.08 & $"$ & 35.1 & 7.32 & 20 \\
\hline Titanium & $<0.009$ & 0.009 & $"$ & ND & & 20 \\
\hline Zirconium & $<0.150$ & 0.150 & $"$ & ND & & 20 \\
\hline Silver & $<0.077$ & 0.077 & $"$ & ND & & 20 \\
\hline Rhenium & $<0.152$ & 0.152 & $"$ & ND & & 20 \\
\hline Antimony & $<0.633$ & 0.633 & $"$ & ND & & 20 \\
\hline
\end{tabular}


Total Metals by SW846 6010B/Water Extract - Quality Control

Environmental Science Laboratory

\begin{tabular}{|lrrrrrrrrrrr}
\hline & & Reporting & & Spike & Source & & \%REC & & RPD \\
Analyte & Result & Limit & Units & Level & Result & \%REC & Limits & RPD & Limit & Notes \\
\hline
\end{tabular}

Batch 8D28005 - 1:1 Water Extract (ICP/ICPMS)

\begin{tabular}{|c|c|c|c|c|c|c|}
\hline \multirow{2}{*}{$\begin{array}{l}\text { Post Spike (8D28005-PS1) } \\
\text { Aluminum }\end{array}$} & \multicolumn{2}{|c|}{ Source: 0803012-05 } & \multicolumn{2}{|c|}{ Prepared: 04/28/08 } & \multicolumn{2}{|c|}{ Analyzed: 05/07/08 } \\
\hline & 506 & $\mathrm{ug} / \mathrm{L}$ & 546 & 31.2 & 86.9 & $75-125$ \\
\hline Arsenic & 487 & $"$ & 546 & 0.831 & 89.1 & $75-125$ \\
\hline Boron & 494 & $"$ & 546 & 9.09 & 88.9 & $75-125$ \\
\hline Barium & 264 & $"$ & 273 & 8.1 & 93.8 & $75-125$ \\
\hline Beryllium & 239 & $"$ & 273 & ND & 88.4 & $75-125$ \\
\hline Bismuth & 449 & $"$ & 546 & 7.77 & 80.8 & $75-125$ \\
\hline Calcium & 8220 & $"$ & 546 & 7630 & 108 & $75-125$ \\
\hline Cadmium & 228 & $"$ & 273 & ND & 85.4 & $75-125$ \\
\hline Cobalt & 226 & $"$ & 273 & ND & 84.4 & $75-125$ \\
\hline Chromium & 113 & $"$ & 137 & ND & 87.5 & $75-125$ \\
\hline Copper & 503 & $"$ & 546 & ND & 92.3 & $75-125$ \\
\hline Iron & 497 & $"$ & 546 & 8.13 & 89.5 & $75-125$ \\
\hline Potassium & 3580 & $"$ & 1370 & 2420 & 85.3 & $75-125$ \\
\hline Lithium & 510 & $"$ & 546 & 3.28 & 92.7 & $75-125$ \\
\hline Magnesium & 3360 & $"$ & 546 & 2830 & 95.8 & $75-125$ \\
\hline Manganese & 237 & $"$ & 273 & $\mathrm{ND}$ & 88 & $75-125$ \\
\hline Molybdenum & 492 & $"$ & 546 & 19.2 & 86.5 & $75-125$ \\
\hline Nickel & 468 & $"$ & 546 & ND & 86.6 & $75-125$ \\
\hline Phosphorus & 1220 & $"$ & 1370 & 18.1 & 88.2 & 75-125 \\
\hline Lead & 459 & $"$ & 546 & ND & 85.9 & $75-125$ \\
\hline Selenium & 482 & $"$ & 546 & 24.5 & 83.8 & $75-125$ \\
\hline Strontium & 512 & $"$ & 546 & 31.6 & 88 & $75-125$ \\
\hline Thallium & 457 & $"$ & 546 & ND & 89.8 & $75-125$ \\
\hline Vanadium & 238 & $"$ & 273 & ND & 88.4 & $75-125$ \\
\hline Zinc & 480 & $"$ & 273 & 275 & 75.3 & $75-125$ \\
\hline Sodium & 6770 & $"$ & 546 & 6320 & 82.4 & $75-125$ \\
\hline Silicon & 2460 & $"$ & 546 & 1920 & 99.6 & $75-125$ \\
\hline Sulfur & 12200 & $"$ & 1090 & 11700 & 41.1 & $75-125$ \\
\hline Titanium & 233 & $"$ & 273 & 1.6 & 84.8 & $75-125$ \\
\hline Zirconium & 247 & $"$ & 273 & 8.05 & 87.5 & $75-125$ \\
\hline Silver & 478 & $"$ & 546 & $\mathrm{ND}$ & 87.8 & $75-125$ \\
\hline Rhenium & 471 & $"$ & 546 & 4.19 & 85.4 & $75-125$ \\
\hline Antimony & 486 & $"$ & 546 & 8.52 & 87.4 & $75-125$ \\
\hline
\end{tabular}


Total Metals by SW846 6010B/Water Extract - Quality Control

Environmental Science Laboratory

\begin{tabular}{|c|c|c|c|c|c|c|c|c|c|c|}
\hline & & porting & & Spike & Source & & \%REC & & RPD & \\
\hline Analyte & Result & Limit & Units & Level & Result & $\%$ REC & Limits & RPD & Limit & Notes \\
\hline
\end{tabular}

Batch 8D28006 - 1:1 Water Extract (ICP/ICPMS)

\begin{tabular}{|c|c|c|c|}
\hline \multicolumn{4}{|c|}{ Blank (8D28006-BLK1) } \\
\hline Aluminum & $<0.086$ & 0.086 & ug/g wet \\
\hline Arsenic & $<0.360$ & 0.360 & $"$ \\
\hline Boron & $<1.94$ & 1.94 & $"$ \\
\hline Barium & $<0.009$ & 0.009 & $"$ \\
\hline Beryllium & $<0.028$ & 0.028 & $"$ \\
\hline Bismuth & $<0.188$ & 0.188 & $"$ \\
\hline Calcium & $<0.387$ & 0.387 & $"$ \\
\hline Cadmium & $<0.027$ & 0.027 & $"$ \\
\hline Cobalt & $<0.096$ & 0.096 & $"$ \\
\hline Chromium & $<0.033$ & 0.033 & $"$ \\
\hline Copper & $<0.080$ & 0.080 & $"$ \\
\hline Iron & $<0.142$ & 0.142 & $"$ \\
\hline Potassium & $<2.33$ & 2.33 & $"$ \\
\hline Lithium & $<0.540$ & 0.540 & $"$ \\
\hline Magnesium & $<0.083$ & 0.083 & $"$ \\
\hline Manganese & $<0.017$ & 0.017 & $"$ \\
\hline Molybdenum & $<0.139$ & 0.139 & $"$ \\
\hline Nickel & $<0.093$ & 0.093 & $"$ \\
\hline Phosphorus & $<1.03$ & 1.03 & $"$ \\
\hline Lead & $<0.432$ & 0.432 & $"$ \\
\hline Selenium & $<1.95$ & 1.95 & $"$ \\
\hline Strontium & $<0.052$ & 0.052 & $"$ \\
\hline Thallium & $<1.03$ & 1.03 & $"$ \\
\hline Vanadium & $<0.045$ & 0.045 & $"$ \\
\hline Sodium & $<0.669$ & 0.669 & $"$ \\
\hline Silicon & $<1.50$ & 1.50 & $"$ \\
\hline Sulfur & $<3.08$ & 3.08 & $"$ \\
\hline Titanium & $<0.009$ & 0.009 & $"$ \\
\hline Zirconium & $<0.150$ & 0.150 & $"$ \\
\hline Silver & $<0.077$ & 0.077 & $"$ \\
\hline Rhenium & $<0.152$ & 0.152 & $"$ \\
\hline Antimony & $<0.633$ & 0.633 & $"$ \\
\hline
\end{tabular}


Total Metals by SW846 6010B/Water Extract - Quality Control

Environmental Science Laboratory

\begin{tabular}{|c|c|c|c|c|c|c|c|c|c|c|}
\hline & & porting & & Spike & Source & & \%REC & & RPD & \\
\hline Analyte & Result & Limit & Units & Level & Result & $\%$ REC & Limits & RPD & Limit & Notes \\
\hline
\end{tabular}

Batch 8D28006 - 1:1 Water Extract (ICP/ICPMS)

\begin{tabular}{|c|c|c|c|c|}
\hline \multirow{2}{*}{$\begin{array}{l}\text { Blank (8D28006-BLK2) } \\
\text { Aluminum }\end{array}$} & \multirow[b]{2}{*}{$<0.086$} & \multicolumn{3}{|r|}{ Prepared: 04/28/08 Analyzed: 05/07/08 } \\
\hline & & 0.086 & ug/g wet & \\
\hline Arsenic & $<0.360$ & 0.360 & $"$ & \\
\hline Boron & $<1.94$ & 1.94 & $"$ & \\
\hline Barium & $<0.009$ & 0.009 & $"$ & \\
\hline Beryllium & $<0.028$ & 0.028 & $"$ & \\
\hline Bismuth & $<0.188$ & 0.188 & $"$ & \\
\hline Calcium & $<0.387$ & 0.387 & $"$ & \\
\hline Cadmium & $<0.027$ & 0.027 & $"$ & \\
\hline Cobalt & $<0.096$ & 0.096 & $"$ & \\
\hline Chromium & $<0.033$ & 0.033 & $"$ & \\
\hline Copper & $<0.080$ & 0.080 & $"$ & \\
\hline Iron & $<0.142$ & 0.142 & $"$ & \\
\hline Potassium & $<2.33$ & 2.33 & $"$ & \\
\hline Lithium & $<0.540$ & 0.540 & $"$ & \\
\hline Magnesium & $<0.083$ & 0.083 & $"$ & \\
\hline Manganese & $<0.017$ & 0.017 & $"$ & \\
\hline Molybdenum & $<0.139$ & 0.139 & $"$ & \\
\hline Nickel & $<0.093$ & 0.093 & $"$ & \\
\hline Phosphorus & $<1.03$ & 1.03 & $"$ & \\
\hline Lead & $<0.432$ & 0.432 & $"$ & \\
\hline Selenium & $<1.95$ & 1.95 & $"$ & \\
\hline Strontium & $<0.052$ & 0.052 & $"$ & \\
\hline Thallium & $<1.03$ & 1.03 & $"$ & \\
\hline Vanadium & $<0.045$ & 0.045 & $"$ & \\
\hline Sodium & $<0.669$ & 0.669 & $"$ & \\
\hline Silicon & $<1.50$ & 1.50 & $"$ & \\
\hline Sulfur & $<3.08$ & 3.08 & $"$ & \\
\hline Titanium & $<0.009$ & 0.009 & $"$ & \\
\hline Zirconium & $<0.150$ & 0.150 & $"$ & \\
\hline Silver & $<0.077$ & 0.077 & $"$ & \\
\hline Rhenium & $<0.152$ & 0.152 & $"$ & \\
\hline Antimony & $<0.633$ & 0.633 & $"$ & \\
\hline
\end{tabular}


Total Metals by SW846 6010B/Water Extract - Quality Control

Environmental Science Laboratory

\begin{tabular}{|lcccccccccccc}
\hline & & Reporting & & Spike & Source & & \%REC & & RPD & & \\
Analyte & Result & Limit & Units & Level & Result & \%REC & Limits & RPD & Limit & Notes \\
\hline
\end{tabular}

Batch 8D28006 - 1:1 Water Extract (ICP/ICPMS)

\begin{tabular}{|c|c|c|c|c|c|c|}
\hline \multirow{2}{*}{$\frac{\text { LCS (8D28006-BS1) }}{\text { Aluminum }}$} & \multicolumn{6}{|c|}{ Prepared: 04/28/08 Analyzed: 05/07/08 } \\
\hline & 4.58 & 0.086 & ug/g wet & 4.990 & 91.7 & $80-120$ \\
\hline Arsenic & 4.85 & 0.360 & $"$ & 4.990 & 97.3 & $80-120$ \\
\hline Boron & 4.81 & 1.94 & $"$ & 4.990 & 96.4 & $80-120$ \\
\hline Barium & 4.72 & 0.009 & $"$ & 4.990 & 94.5 & $80-120$ \\
\hline Beryllium & 4.85 & 0.028 & $"$ & 4.990 & 97.2 & $80-120$ \\
\hline Bismuth & $<0.188$ & 0.188 & $"$ & & & $80-120$ \\
\hline Calcium & 4.57 & 0.387 & $"$ & 4.990 & 91.5 & $80-120$ \\
\hline Cadmium & 5.00 & 0.027 & $"$ & 4.990 & 100 & $80-120$ \\
\hline Cobalt & 4.74 & 0.096 & $"$ & 4.990 & 95.0 & $80-120$ \\
\hline Chromium & 4.99 & 0.033 & $"$ & 4.990 & 100 & $80-120$ \\
\hline Copper & 4.63 & 0.080 & $"$ & 4.990 & 92.8 & $80-120$ \\
\hline Iron & 4.88 & 0.142 & $"$ & 4.990 & 97.9 & $80-120$ \\
\hline Potassium & 47.3 & 2.33 & $"$ & 49.90 & 94.8 & $80-120$ \\
\hline Lithium & $<0.540$ & 0.540 & $"$ & & & $80-120$ \\
\hline Magnesium & 4.68 & 0.083 & $"$ & 4.990 & 93.8 & $80-120$ \\
\hline Manganese & 4.74 & 0.017 & $"$ & 4.990 & 94.9 & $80-120$ \\
\hline Molybdenum & 4.79 & 0.139 & $"$ & 4.990 & 95.9 & $80-120$ \\
\hline Nickel & 4.85 & 0.093 & $"$ & 4.990 & 97.2 & $80-120$ \\
\hline Phosphorus & $<1.03$ & 1.03 & $"$ & & & $80-120$ \\
\hline Lead & 4.80 & 0.432 & $"$ & 4.990 & 96.1 & $80-120$ \\
\hline Selenium & 4.94 & 1.95 & $"$ & 4.990 & 99.1 & $80-120$ \\
\hline Strontium & $<0.052$ & 0.052 & $"$ & & & $80-120$ \\
\hline Thallium & 4.69 & 1.03 & $"$ & 4.990 & 93.9 & $80-120$ \\
\hline Vanadium & 4.74 & 0.045 & $"$ & 4.990 & 94.9 & $80-120$ \\
\hline Zinc & 4.93 & 0.093 & $"$ & 4.990 & 98.7 & $80-120$ \\
\hline Sodium & 4.92 & 0.669 & $"$ & 4.990 & 98.5 & $80-120$ \\
\hline Silicon & 2.76 & 1.50 & $"$ & 2.495 & 110 & $80-120$ \\
\hline Sulfur & $<3.08$ & 3.08 & $"$ & & & $80-120$ \\
\hline Titanium & 4.67 & 0.009 & $"$ & 4.990 & 93.5 & $80-120$ \\
\hline Zirconium & $<0.150$ & 0.150 & $"$ & & & $80-120$ \\
\hline Silver & 4.80 & 0.077 & $"$ & 4.990 & 96.2 & $80-120$ \\
\hline Rhenium & $<0.152$ & 0.152 & $"$ & & & $80-120$ \\
\hline Antimony & 4.79 & 0.633 & " & 4.990 & 96.1 & $80-120$ \\
\hline
\end{tabular}


Total Metals by SW846 6010B/Water Extract - Quality Control

Environmental Science Laboratory

\begin{tabular}{|lcccccccccccc}
\hline & & Reporting & & Spike & Source & & \%REC & & RPD & & \\
Analyte & Result & Limit & Units & Level & Result & \%REC & Limits & RPD & Limit & Notes \\
\hline
\end{tabular}

Batch 8D28006 - 1:1 Water Extract (ICP/ICPMS)

\begin{tabular}{|c|c|c|c|c|c|c|}
\hline \multirow{2}{*}{$\frac{\text { LCS (8D28006-BS2) }}{\text { Aluminum }}$} & \multicolumn{6}{|c|}{ Prepared: 04/28/08 Analyzed: 05/07/08 } \\
\hline & 4.45 & 0.086 & ug/g wet & 4.992 & 89.1 & $80-120$ \\
\hline Arsenic & 4.67 & 0.360 & $"$ & 4.992 & 93.6 & $80-120$ \\
\hline Boron & 4.73 & 1.94 & $"$ & 4.992 & 94.7 & $80-120$ \\
\hline Barium & 4.77 & 0.009 & $"$ & 4.992 & 95.5 & $80-120$ \\
\hline Beryllium & 4.77 & 0.028 & $"$ & 4.992 & 95.6 & $80-120$ \\
\hline Bismuth & $<0.188$ & 0.188 & $"$ & & & $80-120$ \\
\hline Calcium & 4.60 & 0.387 & $"$ & 4.992 & 92.2 & $80-120$ \\
\hline Cadmium & 4.88 & 0.027 & $"$ & 4.992 & 97.8 & $80-120$ \\
\hline Cobalt & 4.65 & 0.096 & $"$ & 4.992 & 93.2 & $80-120$ \\
\hline Chromium & 4.91 & 0.033 & $"$ & 4.992 & 98.3 & $80-120$ \\
\hline Copper & 4.51 & 0.080 & $"$ & 4.992 & 90.4 & $80-120$ \\
\hline Iron & 4.80 & 0.142 & $"$ & 4.992 & 96.1 & $80-120$ \\
\hline Potassium & 47.7 & 2.33 & $"$ & 49.92 & 95.5 & $80-120$ \\
\hline Lithium & $<0.540$ & 0.540 & $"$ & & & $80-120$ \\
\hline Magnesium & 4.57 & 0.083 & $"$ & 4.992 & 91.5 & $80-120$ \\
\hline Manganese & 4.62 & 0.017 & $"$ & 4.992 & 92.5 & $80-120$ \\
\hline Molybdenum & 4.66 & 0.139 & $"$ & 4.992 & 93.4 & $80-120$ \\
\hline Nickel & 4.75 & 0.093 & $"$ & 4.992 & 95.2 & $80-120$ \\
\hline Phosphorus & $<1.03$ & 1.03 & $"$ & & & $80-120$ \\
\hline Lead & 4.67 & 0.432 & $"$ & 4.992 & 93.5 & $80-120$ \\
\hline Selenium & 4.83 & 1.95 & $"$ & 4.992 & 96.7 & $80-120$ \\
\hline Strontium & $<0.052$ & 0.052 & $"$ & & & $80-120$ \\
\hline Thallium & 4.60 & 1.03 & $"$ & 4.992 & 92.2 & $80-120$ \\
\hline Vanadium & 4.64 & 0.045 & $"$ & 4.992 & 92.9 & $80-120$ \\
\hline Zinc & 4.84 & 0.093 & $"$ & 4.992 & 96.9 & $80-120$ \\
\hline Sodium & 4.94 & 0.669 & $"$ & 4.992 & 98.9 & $80-120$ \\
\hline Silicon & 2.70 & 1.50 & $"$ & 2.496 & 108 & $80-120$ \\
\hline Sulfur & $<3.08$ & 3.08 & $"$ & & & $80-120$ \\
\hline Titanium & 4.55 & 0.009 & $"$ & 4.992 & 91.2 & $80-120$ \\
\hline Zirconium & $<0.150$ & 0.150 & $"$ & & & $80-120$ \\
\hline Silver & 4.70 & 0.077 & $"$ & 4.992 & 94.1 & $80-120$ \\
\hline Rhenium & $<0.152$ & 0.152 & $"$ & & & $80-120$ \\
\hline Antimony & 4.73 & 0.633 & " & 4.992 & 94.7 & $80-120$ \\
\hline
\end{tabular}


Total Metals by SW846 6010B/Water Extract - Quality Control

Environmental Science Laboratory

\begin{tabular}{|c|c|c|c|c|c|c|c|c|c|c|}
\hline & & porting & & Spike & Source & & \%REC & & RPD & \\
\hline Analyte & Result & Limit & Units & Level & Result & $\%$ REC & Limits & RPD & Limit & Notes \\
\hline
\end{tabular}

Batch 8D28006 - 1:1 Water Extract (ICP/ICPMS)

\begin{tabular}{|c|c|c|c|c|c|c|}
\hline \multirow{2}{*}{$\begin{array}{l}\text { Duplicate (8D28006-DUP1) } \\
\text { Aluminum }\end{array}$} & \multicolumn{3}{|c|}{ Source: 0803014-05 } & \multirow{2}{*}{$\begin{array}{r}\text { Prepared: } 04 / 28 / 08 \\
0.205\end{array}$} & \multicolumn{2}{|l|}{ Analyzed: 05/07/08 } \\
\hline & 0.171 & 0.086 & ug/g dry & & 18.0 & 20 \\
\hline Arsenic & $<0.360$ & 0.360 & $"$ & ND & & 20 \\
\hline Boron & $<1.94$ & 1.94 & $"$ & ND & & 20 \\
\hline Barium & $<0.009$ & 0.009 & $"$ & ND & & 20 \\
\hline Beryllium & $<0.028$ & 0.028 & $"$ & ND & & 20 \\
\hline Bismuth & $<0.188$ & 0.188 & $"$ & ND & & 20 \\
\hline Calcium & 18.1 & 0.387 & " & 17.4 & 3.51 & 20 \\
\hline Cadmium & $<0.027$ & 0.027 & " & ND & & 20 \\
\hline Cobalt & $<0.096$ & 0.096 & " & ND & & 20 \\
\hline Chromium & $<0.033$ & 0.033 & " & ND & & 20 \\
\hline Copper & $<0.080$ & 0.080 & $"$ & ND & & 20 \\
\hline Iron & $<0.142$ & 0.142 & " & ND & & 20 \\
\hline Potassium & 7.10 & 2.33 & $"$ & 6.93 & 2.39 & 20 \\
\hline Lithium & $<0.540$ & 0.540 & $"$ & ND & & 20 \\
\hline Magnesium & 6.33 & 0.083 & $"$ & 6.30 & 0.531 & 20 \\
\hline Manganese & $<0.017$ & 0.017 & $"$ & ND & & 20 \\
\hline Molybdenum & $<0.139$ & 0.139 & $"$ & ND & & 20 \\
\hline Nickel & $<0.093$ & 0.093 & " & ND & & 20 \\
\hline Phosphorus & $<1.03$ & 1.03 & " & ND & & 20 \\
\hline Lead & $<0.432$ & 0.432 & $"$ & ND & & 20 \\
\hline Selenium & $<1.95$ & 1.95 & $"$ & ND & & 20 \\
\hline Strontium & 0.0766 & 0.052 & $"$ & 0.0748 & 2.37 & 20 \\
\hline Thallium & $<1.03$ & 1.03 & $"$ & ND & & 20 \\
\hline Vanadium & $<0.045$ & 0.045 & $"$ & ND & & 20 \\
\hline Zinc & $<0.093$ & 0.093 & $"$ & ND & & 20 \\
\hline Sodium & 16.1 & 0.669 & " & 15.2 & 5.51 & 20 \\
\hline Silicon & 6.18 & 1.50 & $"$ & 6.15 & 0.438 & 20 \\
\hline Sulfur & 24.3 & 3.07 & $"$ & 23.7 & 2.54 & 20 \\
\hline Titanium & $<0.009$ & 0.009 & $"$ & 0.0102 & & 20 \\
\hline Zirconium & $<0.150$ & 0.150 & $"$ & ND & & 20 \\
\hline Silver & $<0.077$ & 0.077 & $"$ & ND & & 20 \\
\hline Rhenium & $<0.152$ & 0.152 & $"$ & ND & & 20 \\
\hline Antimony & $<0.633$ & 0.633 & $"$ & ND & & 20 \\
\hline
\end{tabular}


Total Metals by SW846 6010B/Water Extract - Quality Control

Environmental Science Laboratory

\begin{tabular}{|c|c|c|c|c|c|c|c|c|c|c|}
\hline & & porting & & Spike & Source & & \%REC & & RPD & \\
\hline Analyte & Result & Limit & Units & Level & Result & $\%$ REC & Limits & RPD & Limit & Notes \\
\hline
\end{tabular}

Batch 8D28006 - 1:1 Water Extract (ICP/ICPMS)

\begin{tabular}{|c|c|c|c|c|c|c|}
\hline \multirow{2}{*}{$\begin{array}{l}\text { Duplicate (8D28006-DUP2) } \\
\text { Aluminum }\end{array}$} & \multicolumn{3}{|c|}{ Source: 0803015-07 } & \multirow{2}{*}{$\begin{array}{r}\text { Prepared: } 04 / 28 / 08 \\
0.146\end{array}$} & \multicolumn{2}{|l|}{ Analyzed: 05/07/08 } \\
\hline & $<0.086$ & 0.086 & ug/g dry & & & 20 \\
\hline Arsenic & $<0.360$ & 0.360 & $"$ & ND & & 20 \\
\hline Boron & $<1.94$ & 1.94 & $"$ & ND & & 20 \\
\hline Barium & $<0.009$ & 0.009 & $"$ & ND & & 20 \\
\hline Beryllium & $<0.028$ & 0.028 & $"$ & ND & & 20 \\
\hline Bismuth & $<0.188$ & 0.188 & $"$ & ND & & 20 \\
\hline Calcium & 38.6 & 0.387 & $"$ & 20.5 & 61.2 & 20 \\
\hline Cadmium & $<0.027$ & 0.027 & $"$ & ND & & 20 \\
\hline Cobalt & $<0.096$ & 0.096 & $"$ & ND & & 20 \\
\hline Chromium & $<0.033$ & 0.033 & $"$ & ND & & 20 \\
\hline Copper & $<0.080$ & 0.080 & $"$ & ND & & 20 \\
\hline Iron & $<0.142$ & 0.142 & $"$ & ND & & 20 \\
\hline Potassium & 5.65 & 2.33 & $"$ & 4.70 & 18.4 & 20 \\
\hline Lithium & $<0.540$ & 0.540 & $"$ & ND & & 20 \\
\hline Magnesium & 12.4 & 0.083 & $"$ & 7.30 & 51.7 & 20 \\
\hline Manganese & $<0.017$ & 0.017 & $"$ & ND & & 20 \\
\hline Molybdenum & $<0.139$ & 0.139 & $"$ & ND & & 20 \\
\hline Nickel & $<0.093$ & 0.093 & $"$ & ND & & 20 \\
\hline Phosphorus & $<1.03$ & 1.03 & $"$ & ND & & 20 \\
\hline Lead & $<0.432$ & 0.432 & $"$ & ND & & 20 \\
\hline Selenium & $<1.95$ & 1.95 & $"$ & ND & & 20 \\
\hline Strontium & 0.131 & 0.052 & $"$ & 0.0847 & 43.0 & 20 \\
\hline Thallium & $<1.03$ & 1.03 & $"$ & ND & & 20 \\
\hline Vanadium & $<0.045$ & 0.045 & $"$ & ND & & 20 \\
\hline Zinc & $<0.093$ & 0.093 & $"$ & ND & & 20 \\
\hline Sodium & 13.7 & 0.669 & $"$ & 12.9 & 5.96 & 20 \\
\hline Silicon & 6.87 & 1.50 & $"$ & 7.16 & 4.16 & 20 \\
\hline Sulfur & 51.1 & 3.07 & $"$ & 26.9 & 62.0 & 20 \\
\hline Titanium & $<0.009$ & 0.009 & $"$ & ND & & 20 \\
\hline Zirconium & $<0.150$ & 0.150 & $"$ & ND & & 20 \\
\hline Silver & $<0.077$ & 0.077 & $"$ & ND & & 20 \\
\hline Rhenium & $<0.152$ & 0.152 & $"$ & ND & & 20 \\
\hline Antimony & $<0.633$ & 0.633 & $"$ & ND & & 20 \\
\hline
\end{tabular}


Total Metals by SW846 6010B/Water Extract - Quality Control

Environmental Science Laboratory

\begin{tabular}{|c|c|c|c|c|c|c|c|c|c|c|}
\hline & & porting & & Spike & Source & & \%REC & & RPD & \\
\hline Analyte & Result & Limit & Units & Level & Result & $\%$ REC & Limits & RPD & Limit & Notes \\
\hline
\end{tabular}

Batch 8D28006 - 1:1 Water Extract (ICP/ICPMS)

\begin{tabular}{|c|c|c|c|c|c|c|}
\hline \multirow{2}{*}{$\begin{array}{l}\text { Post Spike (8D28006-PS1) } \\
\text { Aluminum }\end{array}$} & \multicolumn{2}{|c|}{ Source: 0803014-05 } & \multicolumn{2}{|c|}{ Prepared: 04/28/08 } & \multicolumn{2}{|c|}{ Analyzed: 05/07/08 } \\
\hline & 552 & $\mathrm{ug} / \mathrm{L}$ & 532 & 68.2 & 90.9 & $75-125$ \\
\hline Arsenic & 489 & $"$ & 532 & 1.28 & 91.7 & $75-125$ \\
\hline Boron & 498 & $"$ & 532 & 9.21 & 91.8 & $75-125$ \\
\hline Barium & 262 & $"$ & 266 & ND & 98.9 & $75-125$ \\
\hline Beryllium & 241 & $"$ & 266 & ND & 91.2 & $75-125$ \\
\hline Bismuth & 447 & $"$ & 532 & ND & 84.9 & $75-125$ \\
\hline Calcium & 6800 & $"$ & 532 & 5810 & 186 & $75-125$ \\
\hline Cadmium & 230 & $"$ & 266 & ND & 88.2 & $75-125$ \\
\hline Cobalt & 228 & $"$ & 266 & ND & 87 & $75-125$ \\
\hline Chromium & 115 & $"$ & 133 & ND & 90.2 & $75-125$ \\
\hline Copper & 514 & $"$ & 532 & ND & 96.5 & $75-125$ \\
\hline Iron & 532 & $"$ & 532 & 37.7 & 92.9 & $75-125$ \\
\hline Potassium & 3650 & $"$ & 1330 & 2310 & 101 & $75-125$ \\
\hline Lithium & 524 & $"$ & 532 & 5.88 & 97.4 & $75-125$ \\
\hline Magnesium & 2680 & $"$ & 532 & 2100 & 108 & $75-125$ \\
\hline Manganese & 239 & $"$ & 266 & ND & 90.8 & $75-125$ \\
\hline Molybdenum & 503 & $"$ & 532 & 21.4 & 90.4 & $75-125$ \\
\hline Nickel & 472 & $"$ & 532 & ND & 89.4 & $75-125$ \\
\hline Phosphorus & 1220 & $"$ & 1330 & 16.5 & 90.2 & 75-125 \\
\hline Lead & 469 & $"$ & 532 & $\mathrm{ND}$ & 89.1 & 75-125 \\
\hline Selenium & 462 & $"$ & 532 & 3.01 & 86.2 & $75-125$ \\
\hline Strontium & 525 & $"$ & 532 & 24.9 & 94 & $75-125$ \\
\hline Thallium & 454 & $"$ & 532 & ND & 90 & $75-125$ \\
\hline Vanadium & 243 & $"$ & 266 & ND & 91.8 & $75-125$ \\
\hline Sodium & 5950 & $"$ & 532 & 5070 & 164 & $75-125$ \\
\hline Silicon & 2630 & $"$ & 532 & 2050 & 109 & $75-125$ \\
\hline Sulfur & 8800 & $"$ & 1060 & 7890 & 85.2 & $75-125$ \\
\hline Titanium & 237 & $"$ & 266 & 3.38 & 87.9 & $75-125$ \\
\hline Zirconium & 251 & $"$ & 266 & 8.23 & 91.1 & $75-125$ \\
\hline Silver & 488 & $"$ & 532 & ND & 91.8 & $75-125$ \\
\hline Rhenium & 479 & $"$ & 532 & 6.05 & 89 & $75-125$ \\
\hline Antimony & 488 & $"$ & 532 & 19.9 & 87.9 & $75-125$ \\
\hline
\end{tabular}


Total Metals by SW846 6010B/Water Extract - Quality Control

Environmental Science Laboratory

\begin{tabular}{|c|c|c|c|c|c|c|c|c|c|c|}
\hline & & porting & & Spike & Source & & \%REC & & RPD & \\
\hline Analyte & Result & Limit & Units & Level & Result & $\%$ REC & Limits & RPD & Limit & Notes \\
\hline
\end{tabular}

Batch 8D28006 - 1:1 Water Extract (ICP/ICPMS)

\begin{tabular}{|c|c|c|c|c|c|c|}
\hline \multirow{2}{*}{$\begin{array}{l}\text { Post Spike (8D28006-PS2) } \\
\text { Aluminum }\end{array}$} & \multicolumn{2}{|c|}{ Source: 0803015-07 } & \multicolumn{2}{|c|}{ Prepared: 04/28/08 } & \multicolumn{2}{|c|}{ Analyzed: 05/07/08 } \\
\hline & 542 & $\mathrm{ug} / \mathrm{L}$ & 536 & 48.8 & 92 & $75-125$ \\
\hline Arsenic & 513 & $"$ & 536 & ND & 96.1 & $75-125$ \\
\hline Boron & 508 & $"$ & 536 & 5.2 & 93.7 & $75-125$ \\
\hline Barium & 266 & $"$ & 268 & 0.58 & 99 & $75-125$ \\
\hline Beryllium & 245 & $"$ & 268 & ND & 92.2 & $75-125$ \\
\hline Bismuth & 463 & $"$ & 536 & ND & 88.1 & $75-125$ \\
\hline Calcium & 7580 & $"$ & 536 & 6850 & 136 & $75-125$ \\
\hline Cadmium & 234 & $"$ & 268 & ND & 89.2 & $75-125$ \\
\hline Cobalt & 233 & $"$ & 268 & ND & 88.6 & $75-125$ \\
\hline Chromium & 117 & $"$ & 134 & ND & 92.5 & $75-125$ \\
\hline Copper & 521 & $"$ & 536 & ND & 97.5 & $75-125$ \\
\hline Iron & 520 & $"$ & 536 & 14.1 & 94.4 & $75-125$ \\
\hline Potassium & 2850 & $"$ & 1340 & 1570 & 95.7 & $75-125$ \\
\hline Lithium & 529 & $"$ & 536 & 9.24 & 96.9 & $75-125$ \\
\hline Magnesium & 2970 & $"$ & 536 & 2430 & 100 & $75-125$ \\
\hline Manganese & 244 & $"$ & 268 & ND & 92.6 & $75-125$ \\
\hline Molybdenum & 500 & $"$ & 536 & 10.6 & 91.2 & $75-125$ \\
\hline Nickel & 483 & $"$ & 536 & ND & 91 & $75-125$ \\
\hline Phosphorus & 1230 & $"$ & 1340 & 21 & 90.5 & 75-125 \\
\hline Lead & 477 & $"$ & 536 & $\mathrm{ND}$ & 90.8 & 75-125 \\
\hline Selenium & 494 & $"$ & 536 & 9.76 & 90.2 & $75-125$ \\
\hline Strontium & 531 & $"$ & 536 & 28.2 & 93.7 & $75-125$ \\
\hline Thallium & 461 & $"$ & 536 & ND & 89.1 & $75-125$ \\
\hline Vanadium & 249 & $"$ & 268 & 2.93 & 91.8 & $75-125$ \\
\hline Sodium & 4900 & $"$ & 536 & 4310 & 111 & $75-125$ \\
\hline Silicon & 2960 & $"$ & 536 & 2390 & 107 & $75-125$ \\
\hline Sulfur & 9910 & $"$ & 1070 & 8960 & 88.8 & $75-125$ \\
\hline Titanium & 241 & $"$ & 268 & 1.91 & 89.2 & $75-125$ \\
\hline Zirconium & 255 & $"$ & 268 & 8.26 & 91.9 & $75-125$ \\
\hline Silver & 496 & $"$ & 536 & ND & 92.7 & $75-125$ \\
\hline Rhenium & 485 & $"$ & 536 & 3.72 & 89.7 & $75-125$ \\
\hline Antimony & 498 & $"$ & 536 & 3 & 92.4 & $75-125$ \\
\hline
\end{tabular}


Total Metals by SW846 6010B/Acid Extract - Quality Control

Environmental Science Laboratory

\begin{tabular}{|c|c|c|c|c|c|c|c|c|c|c|}
\hline & & porting & & Spike & Source & & \%REC & & RPD & \\
\hline Analyte & Result & Limit & Units & Level & Result & $\%$ REC & Limits & RPD & Limit & Notes \\
\hline
\end{tabular}

Batch 8E12001 - ASTM D 5198 (ICP/ICPMS)

\begin{tabular}{|c|c|c|c|}
\hline \multicolumn{4}{|c|}{ Blank (8E12001-BLK1) } \\
\hline Aluminum & $<0.573$ & 0.573 & ug/g wet \\
\hline Arsenic & $<1.39$ & 1.39 & $"$ \\
\hline Boron & $<12.1$ & 12.1 & $"$ \\
\hline Barium & $<0.055$ & 0.055 & $"$ \\
\hline Beryllium & $<0.022$ & 0.022 & $"$ \\
\hline Bismuth & $<0.290$ & 0.290 & $"$ \\
\hline Calcium & $<2.13$ & 2.13 & $"$ \\
\hline Cadmium & $<0.033$ & 0.033 & $"$ \\
\hline Cobalt & $<0.119$ & 0.119 & $"$ \\
\hline Chromium & $<0.048$ & 0.048 & $"$ \\
\hline Copper & $<0.508$ & 0.508 & $"$ \\
\hline Iron & $<1.46$ & 1.46 & $"$ \\
\hline Potassium & $<1.35$ & 1.35 & $"$ \\
\hline Lithium & $<0.207$ & 0.207 & $"$ \\
\hline Magnesium & $<0.451$ & 0.451 & $"$ \\
\hline Manganese & $<0.017$ & 0.017 & $"$ \\
\hline Molybdenum & $<0.323$ & 0.323 & $"$ \\
\hline Nickel & $<0.117$ & 0.117 & $"$ \\
\hline Phosphorus & $<1.07$ & 1.07 & $"$ \\
\hline Lead & $<0.453$ & 0.453 & $"$ \\
\hline Selenium & $<1.99$ & 1.99 & $"$ \\
\hline Strontium & $<0.021$ & 0.021 & $"$ \\
\hline Thallium & $<0.644$ & 0.644 & $"$ \\
\hline Vanadium & $<0.053$ & 0.053 & $"$ \\
\hline Zinc & $<0.089$ & 0.089 & $"$ \\
\hline Sodium & $<15.3$ & 15.3 & $"$ \\
\hline Silicon & $<19.9$ & 19.9 & $"$ \\
\hline Sulfur & $<3.38$ & 3.38 & $"$ \\
\hline Titanium & $<0.450$ & 0.450 & $"$ \\
\hline Zirconium & $<0.290$ & 0.290 & $"$ \\
\hline Rhenium & $<0.251$ & 0.251 & $"$ \\
\hline Antimony & $<1.60$ & 1.60 & $"$ \\
\hline
\end{tabular}


Total Metals by SW846 6010B/Acid Extract - Quality Control

Environmental Science Laboratory

\begin{tabular}{|c|c|c|c|c|c|c|c|c|c|c|}
\hline & & porting & & Spike & Source & & \%REC & & RPD & \\
\hline Analyte & Result & Limit & Units & Level & Result & $\%$ REC & Limits & RPD & Limit & Notes \\
\hline
\end{tabular}

Batch 8E12001 - ASTM D 5198 (ICP/ICPMS)

\begin{tabular}{|c|c|c|c|}
\hline \multicolumn{4}{|c|}{ Blank (8E12001-BLK2) } \\
\hline Aluminum & $<0.495$ & 0.495 & ug/g wet \\
\hline Arsenic & $<1.20$ & 1.20 & $"$ \\
\hline Boron & $<10.5$ & 10.5 & $"$ \\
\hline Barium & $<0.047$ & 0.047 & $"$ \\
\hline Beryllium & $<0.019$ & 0.019 & $"$ \\
\hline Bismuth & $<0.250$ & 0.250 & $"$ \\
\hline Calcium & $<1.84$ & 1.84 & $"$ \\
\hline Cadmium & $<0.028$ & 0.028 & $"$ \\
\hline Cobalt & $<0.103$ & 0.103 & $"$ \\
\hline Chromium & $<0.041$ & 0.041 & $"$ \\
\hline Copper & $<0.439$ & 0.439 & $"$ \\
\hline Iron & $<1.26$ & 1.26 & $"$ \\
\hline Potassium & $<1.17$ & 1.17 & $"$ \\
\hline Lithium & $<0.178$ & 0.178 & $"$ \\
\hline Magnesium & $<0.390$ & 0.390 & $"$ \\
\hline Manganese & $<0.015$ & 0.015 & $"$ \\
\hline Molybdenum & $<0.279$ & 0.279 & $"$ \\
\hline Nickel & $<0.101$ & 0.101 & $"$ \\
\hline Phosphorus & $<0.928$ & 0.928 & $"$ \\
\hline Lead & $<0.392$ & 0.392 & $"$ \\
\hline Selenium & $<1.72$ & 1.72 & $"$ \\
\hline Strontium & $<0.018$ & 0.018 & $"$ \\
\hline Thallium & $<0.557$ & 0.557 & $"$ \\
\hline Vanadium & $<0.046$ & 0.046 & $"$ \\
\hline Zinc & 0.181 & 0.077 & $"$ \\
\hline Sodium & $<13.2$ & 13.2 & $"$ \\
\hline Silicon & $<17.2$ & 17.2 & $"$ \\
\hline Sulfur & $<2.92$ & 2.92 & $"$ \\
\hline Titanium & $<0.389$ & 0.389 & " \\
\hline Zirconium & $<0.251$ & 0.251 & $"$ \\
\hline Rhenium & $<0.217$ & 0.217 & $"$ \\
\hline Antimony & $<1.38$ & 1.38 & $"$ \\
\hline
\end{tabular}


Total Metals by SW846 6010B/Acid Extract - Quality Control

Environmental Science Laboratory

\begin{tabular}{|c|c|c|c|c|c|c|c|c|c|c|}
\hline & & porting & & Spike & Source & & \%REC & & RPD & \\
\hline Analyte & Result & Limit & Units & Level & Result & $\%$ REC & Limits & RPD & Limit & Notes \\
\hline
\end{tabular}

Batch 8E12001 - ASTM D 5198 (ICP/ICPMS)

\begin{tabular}{|c|c|c|c|}
\hline \multicolumn{4}{|c|}{ Blank (8E12001-BLK3) } \\
\hline Aluminum & $<0.494$ & 0.494 & ug/g wet \\
\hline Arsenic & $<1.20$ & 1.20 & $"$ \\
\hline Boron & $<10.4$ & 10.4 & $"$ \\
\hline Barium & $<0.047$ & 0.047 & $"$ \\
\hline Beryllium & $<0.019$ & 0.019 & $"$ \\
\hline Bismuth & $<0.249$ & 0.249 & $"$ \\
\hline Calcium & $<1.83$ & 1.83 & $"$ \\
\hline Cadmium & $<0.028$ & 0.028 & $"$ \\
\hline Cobalt & $<0.102$ & 0.102 & $"$ \\
\hline Chromium & $<0.041$ & 0.041 & $"$ \\
\hline Copper & $<0.437$ & 0.437 & $"$ \\
\hline Iron & $<1.25$ & 1.25 & $"$ \\
\hline Potassium & $<1.16$ & 1.16 & $"$ \\
\hline Lithium & $<0.178$ & 0.178 & $"$ \\
\hline Magnesium & $<0.388$ & 0.388 & $"$ \\
\hline Manganese & $<0.015$ & 0.015 & $"$ \\
\hline Molybdenum & $<0.278$ & 0.278 & $"$ \\
\hline Nickel & $<0.101$ & 0.101 & $"$ \\
\hline Phosphorus & $<0.925$ & 0.925 & $"$ \\
\hline Lead & $<0.390$ & 0.390 & $"$ \\
\hline Selenium & $<1.71$ & 1.71 & $"$ \\
\hline Strontium & $<0.018$ & 0.018 & $"$ \\
\hline Thallium & $<0.555$ & 0.555 & $"$ \\
\hline Vanadium & $<0.046$ & 0.046 & $"$ \\
\hline Zinc & $<0.077$ & 0.077 & $"$ \\
\hline Sodium & $<13.2$ & 13.2 & $"$ \\
\hline Silicon & $<17.2$ & 17.2 & $"$ \\
\hline Sulfur & $<2.91$ & 2.91 & $"$ \\
\hline Titanium & $<0.387$ & 0.387 & $"$ \\
\hline Zirconium & $<0.250$ & 0.250 & $"$ \\
\hline Rhenium & $<0.216$ & 0.216 & $"$ \\
\hline Antimony & $<1.37$ & 1.37 & $"$ \\
\hline
\end{tabular}


Total Metals by SW846 6010B/Acid Extract - Quality Control

Environmental Science Laboratory

\begin{tabular}{|lrrrrrrrrrr}
\hline & & Reporting & & Spike & Source & & \%REC & & RPD \\
Analyte & Result & Limit & Units & Level & Result & \%REC & Limits & RPD & Limit & Notes \\
\hline
\end{tabular}

Batch 8E12001 - ASTM D 5198 (ICP/ICPMS)

\begin{tabular}{|c|c|c|c|c|c|c|}
\hline LCS (8E12001-BS1) & & & & Prepared: 05/12/08 & Analyzec & $5 / 22 / 08$ \\
\hline Aluminum & 4.81 & 0.495 & ug/g wet & 5.000 & 96.2 & $80-120$ \\
\hline Arsenic & 5.11 & 1.20 & " & 5.000 & 102 & $80-120$ \\
\hline Boron & 4.82 & 2.99 & $"$ & 5.000 & 96.4 & $80-120$ \\
\hline Barium & 5.42 & 0.047 & $"$ & 5.000 & 108 & $80-120$ \\
\hline Beryllium & 4.65 & 0.019 & $"$ & 5.000 & 93.0 & $80-120$ \\
\hline Bismuth & $<0.250$ & 0.250 & $"$ & & & $80-120$ \\
\hline Calcium & 5.16 & 1.84 & $"$ & 5.000 & 103 & $80-120$ \\
\hline Cadmium & 5.27 & 0.028 & $"$ & 5.000 & 105 & $80-120$ \\
\hline Cobalt & 5.28 & 0.103 & $"$ & 5.000 & 106 & $80-120$ \\
\hline Chromium & 4.97 & 0.041 & $"$ & 5.000 & 99.3 & $80-120$ \\
\hline Copper & 5.14 & 0.438 & $"$ & 5.000 & 103 & $80-120$ \\
\hline Iron & 5.40 & 1.26 & $"$ & 5.000 & 108 & $80-120$ \\
\hline Potassium & 43.2 & 1.17 & $"$ & 50.00 & 86.4 & $80-120$ \\
\hline Lithium & $<0.178$ & 0.178 & $"$ & & & $80-120$ \\
\hline Magnesium & 4.81 & 0.389 & $"$ & 5.000 & 96.3 & $80-120$ \\
\hline Manganese & 5.39 & 0.015 & $"$ & 5.000 & 108 & $80-120$ \\
\hline Molybdenum & 4.79 & 0.279 & $"$ & 5.000 & 95.9 & $80-120$ \\
\hline Nickel & 5.33 & 0.101 & $"$ & 5.000 & 107 & $80-120$ \\
\hline Phosphorus & $<0.927$ & 0.927 & $"$ & & & $80-120$ \\
\hline Lead & 5.36 & 0.391 & $"$ & 5.000 & 107 & $80-120$ \\
\hline Selenium & 5.05 & 1.72 & $"$ & 5.000 & 101 & $80-120$ \\
\hline Strontium & $<0.018$ & 0.018 & $"$ & & & $80-120$ \\
\hline Thallium & 5.21 & 0.556 & $"$ & 5.000 & 104 & $80-120$ \\
\hline Vanadium & 5.30 & 0.046 & $"$ & 5.000 & 106 & $80-120$ \\
\hline Zinc & 4.78 & 0.077 & $"$ & 5.000 & 95.6 & $80-120$ \\
\hline Sodium & $<13.2$ & 13.2 & $"$ & 5.000 & & $80-120$ \\
\hline Silicon & $<17.2$ & 17.2 & $"$ & 2.500 & & $80-120$ \\
\hline Sulfur & $<2.92$ & 2.92 & $"$ & & & $80-120$ \\
\hline Titanium & 5.29 & 0.388 & $"$ & 5.000 & 106 & $80-120$ \\
\hline Zirconium & $<0.251$ & 0.251 & $"$ & & & $80-120$ \\
\hline Rhenium & $<0.217$ & 0.217 & $"$ & & & $80-120$ \\
\hline Antimony & 5.05 & 1.38 & $"$ & 5.000 & 101 & $80-120$ \\
\hline
\end{tabular}


Total Metals by SW846 6010B/Acid Extract - Quality Control

Environmental Science Laboratory

\begin{tabular}{|lrrrrrrrrrr}
\hline & & Reporting & & Spike & Source & & \%REC & & RPD \\
Analyte & Result & Limit & Units & Level & Result & \%REC & Limits & RPD & Limit & Notes \\
\hline
\end{tabular}

Batch 8E12001 - ASTM D 5198 (ICP/ICPMS)

\begin{tabular}{|c|c|c|c|c|c|c|}
\hline \multirow{2}{*}{$\begin{array}{l}\text { LCS (8E12001-BS2) } \\
\text { Aluminum }\end{array}$} & \multicolumn{6}{|c|}{ Prepared: 05/12/08 Analyzed: 05/22/08 } \\
\hline & 4.71 & 0.492 & ug/g wet & 5.000 & 94.2 & $80-120$ \\
\hline Arsenic & 4.90 & 1.19 & $"$ & 5.000 & 98.0 & $80-120$ \\
\hline Boron & 4.72 & 2.97 & $"$ & 5.000 & 94.5 & $80-120$ \\
\hline Barium & 5.26 & 0.047 & $"$ & 5.000 & 105 & $80-120$ \\
\hline Beryllium & 4.61 & 0.019 & $"$ & 5.000 & 92.3 & $80-120$ \\
\hline Bismuth & $<0.248$ & 0.248 & $"$ & & & $80-120$ \\
\hline Calcium & 5.07 & 1.83 & $"$ & 5.000 & 101 & $80-120$ \\
\hline Cadmium & 5.15 & 0.028 & $"$ & 5.000 & 103 & $80-120$ \\
\hline Cobalt & 5.11 & 0.102 & $"$ & 5.000 & 102 & $80-120$ \\
\hline Chromium & 5.01 & 0.041 & $"$ & 5.000 & 100 & $80-120$ \\
\hline Copper & 5.07 & 0.435 & $"$ & 5.000 & 101 & $80-120$ \\
\hline Iron & 5.30 & 1.25 & $"$ & 5.000 & 106 & $80-120$ \\
\hline Potassium & 43.3 & 1.16 & $"$ & 50.00 & 86.7 & $80-120$ \\
\hline Lithium & $<0.177$ & 0.177 & $"$ & & & $80-120$ \\
\hline Magnesium & 4.73 & 0.387 & $"$ & 5.000 & 94.6 & $80-120$ \\
\hline Manganese & 5.28 & 0.015 & $"$ & 5.000 & 106 & $80-120$ \\
\hline Molybdenum & 4.78 & 0.277 & $"$ & 5.000 & 95.6 & $80-120$ \\
\hline Nickel & 5.16 & 0.101 & $"$ & 5.000 & 103 & $80-120$ \\
\hline Phosphorus & $<0.921$ & 0.921 & $"$ & & & $80-120$ \\
\hline Lead & 5.12 & 0.389 & $"$ & 5.000 & 102 & $80-120$ \\
\hline Selenium & 4.61 & 1.71 & $"$ & 5.000 & 92.2 & $80-120$ \\
\hline Strontium & $<0.018$ & 0.018 & $"$ & & & $80-120$ \\
\hline Thallium & 5.01 & 0.552 & $"$ & 5.000 & 100 & $80-120$ \\
\hline Vanadium & 5.21 & 0.045 & $"$ & 5.000 & 104 & $80-120$ \\
\hline Zinc & 4.78 & 0.077 & $"$ & 5.000 & 95.6 & $80-120$ \\
\hline Sodium & $<13.1$ & 13.1 & $"$ & 5.000 & & $80-120$ \\
\hline Silicon & $<17.1$ & 17.1 & $"$ & 2.500 & & $80-120$ \\
\hline Sulfur & $<2.90$ & 2.90 & $"$ & & & $80-120$ \\
\hline Titanium & 5.13 & 0.386 & $"$ & 5.000 & 103 & $80-120$ \\
\hline Zirconium & $<0.249$ & 0.249 & $"$ & & & $80-120$ \\
\hline Rhenium & $<0.215$ & 0.215 & $"$ & & & $80-120$ \\
\hline Antimony & 4.98 & 1.37 & $"$ & 5.000 & 99.6 & $80-120$ \\
\hline
\end{tabular}


Total Metals by SW846 6010B/Acid Extract - Quality Control

Environmental Science Laboratory

\begin{tabular}{|lrrrrrrrrrr}
\hline & & Reporting & & Spike & Source & & \%REC & & RPD \\
Analyte & Result & Limit & Units & Level & Result & \%REC & Limits & RPD & Limit & Notes \\
\hline
\end{tabular}

Batch 8E12001 - ASTM D 5198 (ICP/ICPMS)

\begin{tabular}{|c|c|c|c|c|c|c|}
\hline \multirow{2}{*}{$\begin{array}{l}\text { LCS (8E12001-BS3) } \\
\text { Aluminum }\end{array}$} & \multicolumn{6}{|c|}{ Prepared: 05/12/08 Analyzed: 05/22/08 } \\
\hline & 4.96 & 0.490 & ug/g wet & 5.000 & 99.2 & $80-120$ \\
\hline Arsenic & 5.16 & 1.19 & $"$ & 5.000 & 103 & $80-120$ \\
\hline Boron & 5.01 & 2.96 & $"$ & 5.000 & 100 & $80-120$ \\
\hline Barium & 5.51 & 0.047 & $"$ & 5.000 & 110 & $80-120$ \\
\hline Beryllium & 4.59 & 0.018 & $"$ & 5.000 & 91.7 & $80-120$ \\
\hline Bismuth & $<0.247$ & 0.247 & $"$ & & & $80-120$ \\
\hline Calcium & 5.33 & 1.82 & $"$ & 5.000 & 107 & $80-120$ \\
\hline Cadmium & 5.39 & 0.028 & $"$ & 5.000 & 108 & $80-120$ \\
\hline Cobalt & 5.38 & 0.102 & $"$ & 5.000 & 108 & $80-120$ \\
\hline Chromium & 4.92 & 0.041 & $"$ & 5.000 & 98.4 & $80-120$ \\
\hline Copper & 5.27 & 0.434 & $"$ & 5.000 & 105 & $80-120$ \\
\hline Iron & 5.53 & 1.24 & $"$ & 5.000 & 111 & $80-120$ \\
\hline Potassium & 42.9 & 1.15 & $"$ & 50.00 & 85.9 & $80-120$ \\
\hline Lithium & $<0.176$ & 0.176 & $"$ & & & $80-120$ \\
\hline Magnesium & 4.91 & 0.385 & $"$ & 5.000 & 98.3 & $80-120$ \\
\hline Manganese & 5.51 & 0.015 & $"$ & 5.000 & 110 & $80-120$ \\
\hline Molybdenum & 4.76 & 0.276 & $"$ & 5.000 & 95.2 & $80-120$ \\
\hline Nickel & 5.44 & 0.100 & $"$ & 5.000 & 109 & $80-120$ \\
\hline Phosphorus & $<0.917$ & 0.917 & $"$ & & & $80-120$ \\
\hline Lead & 5.40 & 0.387 & $"$ & 5.000 & 108 & $80-120$ \\
\hline Selenium & 4.99 & 1.70 & $"$ & 5.000 & 99.9 & $80-120$ \\
\hline Strontium & $<0.018$ & 0.018 & $"$ & & & $80-120$ \\
\hline Thallium & 5.37 & 0.550 & $"$ & 5.000 & 107 & $80-120$ \\
\hline Vanadium & 5.43 & 0.045 & $"$ & 5.000 & 109 & $80-120$ \\
\hline Zinc & 4.74 & 0.076 & $"$ & 5.000 & 94.7 & $80-120$ \\
\hline Sodium & $<13.0$ & 13.0 & $"$ & 5.000 & & $80-120$ \\
\hline Silicon & $<17.0$ & 17.0 & $"$ & 2.500 & & $80-120$ \\
\hline Sulfur & $<2.88$ & 2.88 & $"$ & & & $80-120$ \\
\hline Titanium & 5.38 & 0.384 & $"$ & 5.000 & 108 & $80-120$ \\
\hline Zirconium & $<0.248$ & 0.248 & $"$ & & & $80-120$ \\
\hline Rhenium & $<0.215$ & 0.215 & $"$ & & & $80-120$ \\
\hline Antimony & 5.22 & 1.36 & $"$ & 5.000 & 104 & $80-120$ \\
\hline
\end{tabular}


Total Metals by SW846 6010B/Acid Extract - Quality Control

Environmental Science Laboratory

\begin{tabular}{|lrrrrrrrrrr}
\hline & & Reporting & & Spike & Source & & \%REC & & RPD & \\
Analyte & Result & Limit & Units & Level & Result & \%REC & Limits & RPD & Limit & Notes \\
\hline
\end{tabular}

Batch 8E12001 - ASTM D 5198 (ICP/ICPMS)

\begin{tabular}{|c|c|c|c|c|c|c|}
\hline \multirow{2}{*}{$\begin{array}{l}\text { Duplicate (8E12001-DUP1) } \\
\text { Aluminum }\end{array}$} & \multicolumn{3}{|c|}{ Source: 0803012-05 } & \multirow{2}{*}{$\begin{array}{r}\text { Prepared: 05/12/08 } \\
4560\end{array}$} & \multirow{2}{*}{ Analyzed: 05/22/08 } & \multirow[b]{2}{*}{20} \\
\hline & 4270 & 33.3 & ug/g dry & & & \\
\hline Arsenic & $<80.8$ & 80.8 & $"$ & ND & & 20 \\
\hline Boron & $<704$ & 704 & $"$ & ND & & 20 \\
\hline Barium & 41.0 & 3.17 & $"$ & 39.8 & 3.09 & 20 \\
\hline Beryllium & $<1.26$ & 1.26 & $"$ & ND & & 20 \\
\hline Bismuth & $<16.8$ & 16.8 & $"$ & ND & & 20 \\
\hline Calcium & 6590 & 124 & $"$ & 7330 & 10.6 & 20 \\
\hline Cadmium & $<1.89$ & 1.89 & $"$ & ND & & 20 \\
\hline Cobalt & $<6.91$ & 6.91 & $"$ & ND & & 20 \\
\hline Chromium & 7.75 & 2.77 & $"$ & 7.03 & 9.73 & 20 \\
\hline Copper & $<29.5$ & 29.5 & $"$ & ND & & 20 \\
\hline Iron & 10600 & 84.5 & $"$ & 11200 & 5.98 & 20 \\
\hline Potassium & 675 & 78.5 & $"$ & 669 & 0.892 & 20 \\
\hline Lithium & $<12.0$ & 12.0 & $"$ & ND & & 20 \\
\hline Magnesium & 3590 & 26.2 & $"$ & 3480 & 2.91 & 20 \\
\hline Manganese & 199 & 0.990 & $"$ & 217 & 8.43 & 20 \\
\hline Molybdenum & $<18.8$ & 18.8 & $"$ & ND & & 20 \\
\hline Nickel & 9.21 & 6.80 & $"$ & 7.64 & 18.6 & 20 \\
\hline Phosphorus & 469 & 62.3 & $"$ & 453 & 3.32 & 20 \\
\hline Lead & $<26.3$ & 26.3 & $"$ & ND & & 20 \\
\hline Selenium & $<116$ & 116 & $"$ & ND & & 20 \\
\hline Strontium & 22.6 & 1.20 & $"$ & 20.4 & 10.2 & 20 \\
\hline Thallium & $<37.4$ & 37.4 & $"$ & ND & & 20 \\
\hline Vanadium & 12.4 & 3.08 & $"$ & 13.6 & 9.75 & 20 \\
\hline Zinc & 22.9 & 5.19 & $"$ & 23.8 & 3.99 & 20 \\
\hline Sodium & $<887$ & 887 & $"$ & ND & & 20 \\
\hline Silicon & $<1160$ & 1160 & $"$ & ND & & 20 \\
\hline Sulfur & $<196$ & 196 & $"$ & ND & & 20 \\
\hline Titanium & 499 & 26.1 & $"$ & 537 & 7.33 & 20 \\
\hline Zirconium & $<16.8$ & 16.8 & $"$ & ND & & 20 \\
\hline Rhenium & $<14.6$ & 14.6 & $"$ & ND & & 20 \\
\hline Antimony & $<92.6$ & 92.6 & $"$ & ND & & 20 \\
\hline
\end{tabular}


Total Metals by SW846 6010B/Acid Extract - Quality Control

Environmental Science Laboratory

\begin{tabular}{|lrrrrrrrrrr}
\hline & & Reporting & & Spike & Source & & \%REC & & RPD & \\
Analyte & Result & Limit & Units & Level & Result & \%REC & Limits & RPD & Limit & Notes \\
\hline
\end{tabular}

Batch 8E12001 - ASTM D 5198 (ICP/ICPMS)

\begin{tabular}{|c|c|c|c|c|c|c|}
\hline \multirow{2}{*}{$\begin{array}{l}\text { Duplicate (8E12001-DUP2) } \\
\text { Aluminum }\end{array}$} & \multicolumn{3}{|c|}{ Source: 0803014-05 } & \multirow{2}{*}{$\begin{array}{r}\text { Prepared: 05/12/08 } \\
4760\end{array}$} & \multicolumn{2}{|l|}{ Analyzed: 05/22/08 } \\
\hline & 5050 & 35.8 & ug/g dry & & 5.89 & 20 \\
\hline Arsenic & $<86.9$ & 86.9 & $"$ & ND & & 20 \\
\hline Boron & $<757$ & 757 & $"$ & ND & & 20 \\
\hline Barium & 43.3 & 3.41 & $"$ & 43.4 & 0.308 & 20 \\
\hline Beryllium & $<1.35$ & 1.35 & $"$ & ND & & 20 \\
\hline Bismuth & $<18.1$ & 18.1 & $"$ & ND & & 20 \\
\hline Calcium & 8610 & 133 & $"$ & 8140 & 5.69 & 20 \\
\hline Cadmium & $<2.03$ & 2.03 & $"$ & ND & & 20 \\
\hline Cobalt & $<7.42$ & 7.42 & $"$ & ND & & 20 \\
\hline Chromium & 13.4 & 2.97 & $"$ & 13.4 & 0.440 & 20 \\
\hline Copper & $<31.7$ & 31.7 & $"$ & ND & & 20 \\
\hline Iron & 12200 & 90.9 & $"$ & 11600 & 4.67 & 20 \\
\hline Potassium & 772 & 84.4 & $"$ & 764 & 1.04 & 20 \\
\hline Lithium & $<12.9$ & 12.9 & $"$ & ND & & 20 \\
\hline Magnesium & 4200 & 28.1 & $"$ & 3980 & 5.25 & 20 \\
\hline Manganese & 240 & 1.06 & $"$ & 233 & 2.83 & 20 \\
\hline Molybdenum & $<20.2$ & 20.2 & $"$ & ND & & 20 \\
\hline Nickel & 19.7 & 7.32 & $"$ & 20.1 & 2.03 & 20 \\
\hline Phosphorus & 535 & 67.0 & $"$ & 504 & 5.98 & 20 \\
\hline Lead & $<28.3$ & 28.3 & $"$ & ND & & 20 \\
\hline Selenium & $<124$ & 124 & $"$ & ND & & 20 \\
\hline Strontium & 21.6 & 1.29 & $"$ & 22.2 & 2.54 & 20 \\
\hline Thallium & $<40.2$ & 40.2 & $"$ & ND & & 20 \\
\hline Vanadium & 13.6 & 3.31 & $"$ & 12.6 & 7.10 & 20 \\
\hline Zinc & 27.0 & 5.58 & $"$ & 26.7 & 1.22 & 20 \\
\hline Sodium & $<953$ & 953 & $"$ & ND & & 20 \\
\hline Silicon & $<1240$ & 1240 & $"$ & ND & & 20 \\
\hline Sulfur & $<211$ & 211 & $"$ & ND & & 20 \\
\hline Titanium & 527 & 28.1 & $"$ & 499 & 5.60 & 20 \\
\hline Zirconium & $<18.1$ & 18.1 & $"$ & ND & & 20 \\
\hline Rhenium & $<15.7$ & 15.7 & $"$ & ND & & 20 \\
\hline Antimony & $<99.6$ & 99.6 & $"$ & ND & & 20 \\
\hline
\end{tabular}


Total Metals by SW846 6010B/Acid Extract - Quality Control

Environmental Science Laboratory

\begin{tabular}{|lrrrrrrrrrr}
\hline & & Reporting & & Spike & Source & & \%REC & & RPD & \\
Analyte & Result & Limit & Units & Level & Result & \%REC & Limits & RPD & Limit & Notes \\
\hline
\end{tabular}

Batch 8E12001 - ASTM D 5198 (ICP/ICPMS)

\begin{tabular}{|c|c|c|c|c|c|c|}
\hline \multirow{2}{*}{$\begin{array}{l}\text { Duplicate (8E12001-DUP3) } \\
\text { Aluminum }\end{array}$} & \multicolumn{3}{|c|}{ Source: 0803015-07 } & \multirow{2}{*}{$\begin{array}{r}\text { Prepared: 05/12/08 } \\
4760\end{array}$} & \multicolumn{2}{|l|}{ Analyzed: 05/22/08 } \\
\hline & 4300 & 33.8 & ug/g dry & & 10.2 & 20 \\
\hline Arsenic & $<82.0$ & 82.0 & $"$ & ND & & 20 \\
\hline Boron & $<714$ & 714 & $"$ & ND & & 20 \\
\hline Barium & 44.9 & 3.22 & $"$ & 55.7 & 21.4 & 20 \\
\hline Beryllium & $<1.27$ & 1.27 & $"$ & ND & & 20 \\
\hline Bismuth & $<17.0$ & 17.0 & $"$ & ND & & 20 \\
\hline Calcium & 7810 & 125 & $"$ & 9190 & 16.2 & 20 \\
\hline Cadmium & $<1.92$ & 1.92 & $"$ & ND & & 20 \\
\hline Cobalt & $<7.00$ & 7.00 & $"$ & ND & & 20 \\
\hline Chromium & 4.26 & 2.81 & $"$ & 4.98 & 15.6 & 20 \\
\hline Copper & $<29.9$ & 29.9 & $"$ & ND & & 20 \\
\hline Iron & 11500 & 85.8 & $"$ & 11500 & 0.810 & 20 \\
\hline Potassium & 686 & 79.6 & $"$ & 692 & 0.945 & 20 \\
\hline Lithium & $<12.2$ & 12.2 & $"$ & ND & & 20 \\
\hline Magnesium & 3190 & 26.5 & $"$ & 3430 & 7.23 & 20 \\
\hline Manganese & 210 & 1.00 & $"$ & 204 & 2.70 & 20 \\
\hline Molybdenum & $<19.0$ & 19.0 & $"$ & ND & & 20 \\
\hline Nickel & $<6.90$ & 6.90 & $"$ & 6.94 & & 20 \\
\hline Phosphorus & 582 & 63.2 & $"$ & 538 & 7.79 & 20 \\
\hline Lead & $<26.7$ & 26.7 & $"$ & ND & & 20 \\
\hline Selenium & $<117$ & 117 & $"$ & ND & & 20 \\
\hline Strontium & 21.6 & 1.22 & $"$ & 21.6 & 0.0826 & 20 \\
\hline Thallium & $<37.9$ & 37.9 & $"$ & ND & & 20 \\
\hline Vanadium & 15.5 & 3.12 & $"$ & 15.4 & 0.726 & 20 \\
\hline Zinc & 25.6 & 5.26 & $"$ & 24.8 & 3.16 & 20 \\
\hline Sodium & $<899$ & 899 & $"$ & ND & & 20 \\
\hline Silicon & $<1170$ & 1170 & $"$ & ND & & 20 \\
\hline Sulfur & $<199$ & 199 & $"$ & ND & & 20 \\
\hline Titanium & 576 & 26.5 & $"$ & 548 & 5.04 & 20 \\
\hline Zirconium & $<17.1$ & 17.1 & $"$ & ND & & 20 \\
\hline Rhenium & $<14.8$ & 14.8 & $"$ & ND & & 20 \\
\hline Antimony & $<94.0$ & 94.0 & $"$ & ND & & 20 \\
\hline
\end{tabular}


Total Metals by SW846 6010B/Acid Extract - Quality Control

Environmental Science Laboratory

\begin{tabular}{|c|c|c|c|c|c|c|c|c|c|c|}
\hline & & porting & & Spike & Source & & \%REC & & RPD & \\
\hline Analyte & Result & Limit & Units & Level & Result & $\%$ REC & Limits & RPD & Limit & Notes \\
\hline
\end{tabular}

Batch 8E12001 - ASTM D 5198 (ICP/ICPMS)

\begin{tabular}{|c|c|c|c|c|c|c|}
\hline \multirow{2}{*}{$\begin{array}{l}\text { Post Spike (8E12001-PS1) } \\
\text { Aluminum }\end{array}$} & \multicolumn{2}{|c|}{ Source: 0803012-05 } & \multicolumn{2}{|c|}{ Prepared: 05/12/08 } & \multicolumn{2}{|c|}{ Analyzed: 05/22/08 } \\
\hline & 15800 & $\mathrm{ug} / \mathrm{L}$ & 500 & 15200 & 127 & $75-125$ \\
\hline Arsenic & 438 & $"$ & 500 & ND & 96.6 & $75-125$ \\
\hline Boron & 494 & $"$ & 500 & ND & 99.2 & $75-125$ \\
\hline Barium & 355 & $"$ & 250 & 132 & 89 & $75-125$ \\
\hline Beryllium & 231 & $"$ & 250 & ND & 93.4 & $75-125$ \\
\hline Bismuth & 492 & $"$ & 500 & 36.5 & 91.1 & $75-125$ \\
\hline Calcium & 25800 & $"$ & 500 & 24400 & 273 & $75-125$ \\
\hline Cadmium & 245 & $"$ & 250 & ND & 99.7 & $75-125$ \\
\hline Cobalt & 255 & $"$ & 250 & 16.6 & 95.2 & $75-125$ \\
\hline Chromium & 138 & $"$ & 125 & 23.4 & 91.3 & $75-125$ \\
\hline Copper & 492 & $"$ & 500 & 20.7 & 94.4 & $75-125$ \\
\hline Iron & 38300 & $"$ & 500 & 37400 & 182 & $75-125$ \\
\hline Potassium & 3260 & $"$ & 1250 & 2230 & 82.7 & $75-125$ \\
\hline Lithium & 487 & $"$ & 500 & 17.7 & 93.8 & $75-125$ \\
\hline Magnesium & 12200 & $"$ & 500 & 11600 & 128 & $75-125$ \\
\hline Manganese & 976 & $"$ & 250 & 722 & 101 & $75-125$ \\
\hline Molybdenum & 460 & $"$ & 500 & 15 & 89 & $75-125$ \\
\hline Nickel & 508 & $"$ & 500 & 25.5 & 96.4 & $75-125$ \\
\hline Phosphorus & 2740 & $"$ & 1250 & 1510 & 98.6 & $75-125$ \\
\hline Lead & 488 & $"$ & 500 & ND & 101 & $75-125$ \\
\hline Selenium & 483 & $"$ & 500 & ND & 99.6 & $75-125$ \\
\hline Strontium & 499 & $"$ & 500 & 68 & 86.3 & $75-125$ \\
\hline Thallium & 435 & $"$ & 500 & ND & 96.3 & $75-125$ \\
\hline Vanadium & 289 & $"$ & 250 & 45.4 & 97.5 & $75-125$ \\
\hline Zinc & 320 & $"$ & 250 & 79.4 & 96.3 & $75-125$ \\
\hline Sodium & 1000 & $"$ & 500 & 587 & 83.1 & $75-125$ \\
\hline Silicon & 479 & $"$ & 500 & ND & 102 & $75-125$ \\
\hline Sulfur & 934 & $"$ & 1000 & ND & 94.5 & $75-125$ \\
\hline Titanium & 2040 & $"$ & 250 & 1790 & 102 & $75-125$ \\
\hline Zirconium & 259 & $"$ & 250 & 26 & 93.2 & $75-125$ \\
\hline Rhenium & 482 & $"$ & 500 & ND & 96.8 & $75-125$ \\
\hline Antimony & 450 & " & 500 & ND & 93.2 & $75-125$ \\
\hline
\end{tabular}


Total Metals by SW846 6010B/Acid Extract - Quality Control

Environmental Science Laboratory

\begin{tabular}{|c|c|c|c|c|c|c|c|c|c|c|}
\hline & & porting & & Spike & Source & & \%REC & & RPD & \\
\hline Analyte & Result & Limit & Units & Level & Result & $\%$ REC & Limits & RPD & Limit & Notes \\
\hline
\end{tabular}

Batch 8E12001 - ASTM D 5198 (ICP/ICPMS)

\begin{tabular}{|c|c|c|c|c|c|c|}
\hline \multirow{2}{*}{$\begin{array}{l}\text { Post Spike (8E12001-PS2) } \\
\text { Aluminum }\end{array}$} & \multicolumn{2}{|c|}{ Source: 0803014-05 } & \multicolumn{2}{|c|}{ Prepared: 05/12/08 } & \multicolumn{2}{|c|}{ Analyzed: 05/22/08 } \\
\hline & 14600 & $\mathrm{ug} / \mathrm{L}$ & 500 & 14400 & 37.2 & $75-125$ \\
\hline Arsenic & 458 & $"$ & 500 & ND & 98.9 & $75-125$ \\
\hline Boron & 518 & $"$ & 500 & 1.9 & 103 & $75-125$ \\
\hline Barium & 363 & $"$ & 250 & 131 & 92.6 & $75-125$ \\
\hline Beryllium & 236 & $"$ & 250 & ND & 95.3 & $75-125$ \\
\hline Bismuth & 521 & $"$ & 500 & 29.7 & 98.4 & $75-125$ \\
\hline Calcium & 26100 & $"$ & 500 & 24600 & 313 & $75-125$ \\
\hline Cadmium & 255 & $"$ & 250 & ND & 103 & $75-125$ \\
\hline Cobalt & 262 & $"$ & 250 & 16.6 & 98 & $75-125$ \\
\hline Chromium & 158 & $"$ & 125 & 40.3 & 93.9 & $75-125$ \\
\hline Copper & 520 & $"$ & 500 & 27.4 & 98.5 & $75-125$ \\
\hline Iron & 35000 & $"$ & 500 & 35000 & NR & $75-125$ \\
\hline Potassium & 3380 & $"$ & 1250 & 2310 & 85.8 & $75-125$ \\
\hline Lithium & 491 & $"$ & 500 & 18.5 & 94.4 & $75-125$ \\
\hline Magnesium & 12300 & $"$ & 500 & 12000 & 63.4 & $75-125$ \\
\hline Manganese & 962 & $"$ & 250 & 703 & 104 & $75-125$ \\
\hline Molybdenum & 479 & $"$ & 500 & 19.2 & 92 & $75-125$ \\
\hline Nickel & 558 & $"$ & 500 & 60.6 & 99.4 & $75-125$ \\
\hline Phosphorus & 2770 & $"$ & 1250 & 1520 & 99.9 & $75-125$ \\
\hline Lead & 505 & $"$ & 500 & ND & 104 & $75-125$ \\
\hline Selenium & 453 & $"$ & 500 & ND & 92.4 & $75-125$ \\
\hline Strontium & 507 & $"$ & 500 & 67 & 87.9 & $75-125$ \\
\hline Thallium & 448 & $"$ & 500 & ND & 99.7 & $75-125$ \\
\hline Vanadium & 294 & $"$ & 250 & 38.1 & 102 & $75-125$ \\
\hline Zinc & 328 & $"$ & 250 & 80.7 & 98.8 & $75-125$ \\
\hline Sodium & 878 & $"$ & 500 & 450 & 85.5 & $75-125$ \\
\hline Silicon & 518 & $"$ & 500 & ND & 107 & $75-125$ \\
\hline Sulfur & 862 & $"$ & 1000 & ND & 94.5 & $75-125$ \\
\hline Titanium & 1720 & $"$ & 250 & 1510 & 86.4 & $75-125$ \\
\hline Zirconium & 265 & $"$ & 250 & 22.5 & 96.9 & $75-125$ \\
\hline Rhenium & 501 & $"$ & 500 & ND & 100 & $75-125$ \\
\hline Antimony & 483 & " & 500 & ND & 99.4 & $75-125$ \\
\hline
\end{tabular}


Total Metals by SW846 6010B/Acid Extract - Quality Control

Environmental Science Laboratory

\begin{tabular}{|c|c|c|c|c|c|c|c|c|c|c|}
\hline & & porting & & Spike & Source & & \%REC & & RPD & \\
\hline Analyte & Result & Limit & Units & Level & Result & $\%$ REC & Limits & RPD & Limit & Notes \\
\hline
\end{tabular}

Batch 8E12001 - ASTM D 5198 (ICP/ICPMS)

\begin{tabular}{|c|c|c|c|c|c|c|}
\hline \multirow{2}{*}{$\begin{array}{l}\text { Post Spike (8E12001-PS3) } \\
\text { Aluminum }\end{array}$} & \multicolumn{2}{|c|}{ Source: 0803015-07 } & \multicolumn{2}{|c|}{ Prepared: 05/12/08 } & \multicolumn{2}{|c|}{ Analyzed: 05/22/08 } \\
\hline & 14600 & $\mathrm{ug} / \mathrm{L}$ & 500 & 14400 & 51.1 & $75-125$ \\
\hline Arsenic & 467 & $"$ & 500 & ND & 99.1 & $75-125$ \\
\hline Boron & 513 & $"$ & 500 & ND & 103 & $75-125$ \\
\hline Barium & 380 & $"$ & 250 & 168 & 84.7 & $75-125$ \\
\hline Beryllium & 238 & $"$ & 250 & ND & 96.3 & $75-125$ \\
\hline Bismuth & 515 & $"$ & 500 & 34 & 96.2 & $75-125$ \\
\hline Calcium & 27500 & " & 500 & 27800 & NR & $75-125$ \\
\hline Cadmium & 251 & $"$ & 250 & ND & 102 & $75-125$ \\
\hline Cobalt & 261 & $"$ & 250 & 17.3 & 97.4 & $75-125$ \\
\hline Chromium & 134 & " & 125 & 15 & 95.2 & $75-125$ \\
\hline Copper & 512 & " & 500 & 20.6 & 98.2 & $75-125$ \\
\hline Iron & 34600 & $"$ & 500 & 34600 & NR & $75-125$ \\
\hline Potassium & 3270 & " & 1250 & 2090 & 94.7 & $75-125$ \\
\hline Lithium & 491 & " & 500 & 13.4 & 95.5 & $75-125$ \\
\hline Magnesium & 10700 & $"$ & 500 & 10300 & 66.9 & $75-125$ \\
\hline Manganese & 872 & $"$ & 250 & 617 & 102 & $75-125$ \\
\hline Molybdenum & 491 & $"$ & 500 & 14 & 95.5 & $75-125$ \\
\hline Nickel & 516 & $"$ & 500 & 21 & 99.1 & $75-125$ \\
\hline Phosphorus & 2870 & $"$ & 1250 & 1630 & 99.8 & $75-125$ \\
\hline Lead & 492 & $"$ & 500 & ND & 102 & $75-125$ \\
\hline Selenium & 480 & $"$ & 500 & 18.1 & 92.4 & $75-125$ \\
\hline Strontium & 473 & $"$ & 500 & 65.2 & 81.6 & $75-125$ \\
\hline Thallium & 432 & $"$ & 500 & ND & 99.4 & $75-125$ \\
\hline Vanadium & 300 & " & 250 & 46.5 & 101 & $75-125$ \\
\hline Zinc & 329 & $"$ & 250 & 74.9 & 102 & $75-125$ \\
\hline Sodium & 1010 & " & 500 & 583 & 84.7 & $75-125$ \\
\hline Silicon & 500 & $"$ & 500 & ND & 106 & $75-125$ \\
\hline Sulfur & 964 & $"$ & 1000 & ND & 101 & $75-125$ \\
\hline Titanium & 1860 & " & 250 & 1650 & 84 & $75-125$ \\
\hline Zirconium & 268 & $"$ & 250 & 28.7 & 95.5 & $75-125$ \\
\hline Rhenium & 494 & " & 500 & ND & 99.2 & $75-125$ \\
\hline Antimony & 478 & " & 500 & ND & 99.6 & $75-125$ \\
\hline
\end{tabular}


Radionuclides by ICP-MS/Acid Extract - Quality Control

Environmental Science Laboratory

\begin{tabular}{|c|c|c|c|c|c|c|c|c|c|c|}
\hline Analyte & Result & $\begin{array}{c}\text { orting } \\
\text { Limit }\end{array}$ & Units & $\begin{array}{l}\text { Spike } \\
\text { Level }\end{array}$ & $\begin{array}{l}\text { Source } \\
\text { Result }\end{array}$ & \%REC & $\begin{array}{l}\text { \%REC } \\
\text { Limits }\end{array}$ & RPD & $\begin{array}{l}\text { RPD } \\
\text { Limit }\end{array}$ & Notes \\
\hline
\end{tabular}

Batch 8D16002 - ASTM D 5198 (ICP/ICPMS)

Blank (8D16002-BLK1)

Technetium-99

Uranium 238

Blank (8D16002-BLK2)

Technetium-99

Uranium 238

Blank (8D16002-BLK3)

Technetium-99

Uranium 238

Duplicate (8D16002-DUP1)

Technetium-99

Uranium 238

Duplicate (8D16002-DUP2)

Technetium-99

Uranium 238

Duplicate (8D16002-DUP3)

Technetium-99

Uranium 238

Post Spike (8D16002-PS1)

Technetium-99

Uranium 238

Post Spike (8D16002-PS2)

Technetium-99

Uranium 238

$\begin{array}{ccc}<0.048 & 0.048 & \text { ug/g wet } \\ <0.351 & 0.351 & "\end{array}$

Prepared \& Analyzed: 04/16/08

Prepared \& Analyzed: 04/16/08

$<0.042 \quad 0.042 \quad$ ug/g wet

$<0.303 \quad 0.303 \quad "$

Prepared \& Analyzed: 04/16/08

$\begin{array}{ccc}<0.001 & 0.001 & \text { ug/g wet } \\ <0.009 & 0.009 & "\end{array}$

Source: 0803012-05

\begin{tabular}{|c|c|c|}
\hline$<0.004$ & 0.004 ug/g dry & ND \\
\hline
\end{tabular}

$0.660 \quad 0.029 \quad "$

Source: 0803014-05

Prepared \& Analyzed: 04/16/08

$\begin{array}{cccccc}<0.004 & 0.004 & \text { ug/g dry } & \text { ND } & 20 \\ 0.336 & 0.031 & " & 0.359 & 6.45 & 20\end{array}$

Source: 0803015-07 Prepared \& Analyzed: 04/16/08

$\begin{array}{cccccc}<0.004 & 0.004 & \text { ug/g dry } & \text { ND } & 20 \\ 0.368 & 0.030 & " & 0.405 & 9.73 & 20\end{array}$

Source: $0803012-05$

$\begin{array}{llllll}0.505 & \text { ug/L } & 0.5 & 0.00196 & 101 & \text { Prepared \& Analyzed: 04/16/08 }\end{array}$

$\begin{array}{llllll}1.81 & \text { " } & 0.5 & 1.38 & 86.4 & 75-125\end{array}$

\begin{tabular}{cccccc}
\multicolumn{2}{c}{ Source: $\mathbf{0 8 0 3 0 1 4 - 0 5}$} & \multicolumn{4}{c}{ Prepared \& Analyzed: $04 / 16 / 08$} \\
\hline 0.469 & ug/L & 0.5 & 0.00298 & 93.2 & $75-125$ \\
1.5 & $"$ & 0.5 & 1.08 & 83.3 & $75-125$
\end{tabular}


Radionuclides by ICP-MS/Acid Extract - Quality Control

Environmental Science Laboratory

\begin{tabular}{|lrrrrrrrrrrr}
\hline & & Reporting & & Spike & Source & & \%REC & & RPD & & \\
Analyte & Result & Limit & Units & Level & Result & \%REC & Limits & RPD & Limit & Notes
\end{tabular}

Batch 8D16002 - ASTM D 5198 (ICP/ICPMS)

Post Spike (8D16002-PS3)

Technetium-99

Source: 0803015-07

Prepared \& Analyzed: 04/16/08

Uranium 238

\begin{tabular}{cccccc}
\multicolumn{2}{c}{ Source: 0803015-07 } & \multicolumn{5}{c}{ Prepared \& Analyzed: 04/16/08 } \\
\hline 0.424 & ug/L & 0.5 & 0.00191 & 84.5 & $75-125$ \\
1.69 & $"$ & 0.5 & 1.22 & 92.3 & $75-125$
\end{tabular}




\section{Radionuclides by ICP-MS/Water Extract - Quality Control \\ Environmental Science Laboratory}

\begin{tabular}{|c|c|c|c|c|c|c|c|c|c|c|}
\hline Analyte & Result & $\begin{array}{c}\text { orting } \\
\text { Limit }\end{array}$ & Units & $\begin{array}{l}\text { Spike } \\
\text { Level }\end{array}$ & $\begin{array}{l}\text { Source } \\
\text { Result }\end{array}$ & \%REC & $\begin{array}{l}\text { \%REC } \\
\text { Limits }\end{array}$ & RPD & $\begin{array}{l}\text { RPD } \\
\text { Limit }\end{array}$ & Notes \\
\hline
\end{tabular}

Batch 8D10003 - 1:1 Water Extract (ICP/ICPMS)

Blank (8D10003-BLK1)

Technetium-99

Uranium 238

Duplicate (8D10003-DUP1)

Technetium-99

Uranium 238

Post Spike (8D10003-PS1)

Technetium-99

Uranium 238

$\begin{array}{crc}<0.00002 & 0.00002 & \text { ug/g wet } \\ <0.0006 & 0.0006 & "\end{array}$

Prepared \& Analyzed: 04/10/08

Source: 0803012-05

$0.0000249 \quad 0.00002 \mathrm{ug} / \mathrm{g}$ dry $\quad$ Prepared \& Analyzed: 04/10/08

$<0.0006 \quad 0.0006 \quad "$

ND

20

20

Source: 0803012-05 Prepared \& Analyzed: 04/10/08

$\begin{array}{llllll}0.471 & \mathrm{ug} / \mathrm{L} & 0.5 & 0.00379 & 93.4 & 75-125\end{array}$

Batch 8D10004 - 1:1 Water Extract (ICP/ICPMS)

\begin{tabular}{|c|c|c|c|c|c|c|}
\hline \multirow{2}{*}{$\begin{array}{l}\text { Blank (8D10004-BLK1) } \\
\text { Technetium-99 }\end{array}$} & \multicolumn{6}{|c|}{ Prepared \& Analyzed: 04/10/08 } \\
\hline & $<0.00002$ & 0.00002 & ug/g wet & & & \\
\hline Uranium 238 & $<0.0006$ & 0.0006 & $"$ & & & \\
\hline Blank (8D10004-BLK2) & \multicolumn{6}{|c|}{ Prepared \& Analyzed: 04/10/08 } \\
\hline Technetium-99 & $<0.00002$ & 0.00002 & ug/g wet & & & \\
\hline Uranium 238 & $<0.0006$ & 0.0006 & $"$ & & & \\
\hline Duplicate (8D10004-DUP1) & \multicolumn{3}{|c|}{ Source: 0803014-05 } & \multicolumn{3}{|l|}{ Prepared \& Analyzed: 04/10/08 } \\
\hline Technetium-99 & 0.0000634 & 0.00002 & ug/g dry & 0.0000554 & 13.5 & 20 \\
\hline Uranium 238 & $<0.0006$ & 0.0006 & $"$ & ND & & 20 \\
\hline Duplicate (8D10004-DUP2) & \multicolumn{3}{|c|}{ Source: 0803015-07 } & Prepared \& Analyzed: 04/10/08 & & \\
\hline Technetium-99 & $<0.00002$ & 0.00002 & ug/g dry & ND & & 20 \\
\hline Uranium 238 & $<0.0006$ & 0.0006 & $"$ & ND & & 20 \\
\hline
\end{tabular}




\section{Radionuclides by ICP-MS/Water Extract - Quality Control \\ Environmental Science Laboratory}

\begin{tabular}{|c|c|c|c|c|c|c|c|c|c|c|}
\hline & & orting & & Spike & Source & & $\%$ REC & & RPD & \\
\hline Analyte & Result & Limit & Units & Level & Result & \%REC & Limits & RPD & Limit & Notes \\
\hline
\end{tabular}

Batch 8D10004 - 1:1 Water Extract (ICP/ICPMS)

\begin{tabular}{|c|c|c|c|c|c|c|}
\hline Post Spike (8D10004-PS1) & \multicolumn{2}{|c|}{ Source: 0803014-05 } & \multicolumn{4}{|c|}{ Prepared \& Analyzed: 04/10/08 } \\
\hline Technetium-99 & 0.493 & ug/L & 0.5 & 0.0111 & 96.4 & $75-125$ \\
\hline Uranium 238 & 0.537 & " & 0.5 & 0.0428 & 98.9 & $75-125$ \\
\hline Post Spike (8D10004-PS2) & \multicolumn{2}{|c|}{ Source: 0803015-07 } & \multicolumn{4}{|c|}{ Prepared \& Analyzed: 04/10/08 } \\
\hline Technetium-99 & 0.473 & ug/L & 0.5 & 0.0013 & 94.4 & $75-125$ \\
\hline Uranium 238 & 0.544 & $"$ & 0.5 & 0.0604 & 96.7 & $75-125$ \\
\hline
\end{tabular}


RCRA Metals By SW846 6020/Water Extract - Quality Control

Environmental Science Laboratory

\begin{tabular}{|lrrrrrrrrrr}
\hline & & Reporting & & Spike & Source & & \%REC & & RPD & \\
Analyte & Result & Limit & Units & Level & Result & \%REC & Limits & RPD & Limit & Notes \\
\hline
\end{tabular}

Batch 8D07005 - 1:1 Water Extract (ICP/ICPMS)

Blank (8D07005-BLK1)

Chromium

Copper

Arsenic

Selenium

Molybdenum

Ruthenium

Silver

Cadmium

Antimony

Lead

\begin{tabular}{|c|c|c|}
\hline$<0.002$ & 0.002 & ug/g wet \\
\hline$<0.005$ & 0.005 & " \\
\hline$<0.006$ & 0.006 & $"$ \\
\hline$<0.011$ & 0.011 & $"$ \\
\hline$<0.002$ & 0.002 & $"$ \\
\hline$<0.0008$ & 0.0008 & $"$ \\
\hline$<0.001$ & 0.001 & " \\
\hline$<0.0006$ & 0.0006 & $"$ \\
\hline$<0.0005$ & 0.0005 & " \\
\hline$<0.0006$ & 0.0006 & $"$ \\
\hline
\end{tabular}

Prepared: 04/07/08 Analyzed: 04/14/08

LCS (8D07005-BS1)

\begin{tabular}{l} 
Chromium \\
Copper \\
Arsenic \\
Selenium \\
Molybdenum \\
Ruthenium \\
Silver \\
Cadmium \\
Antimony \\
Lead \\
Duplicate $(8 D$ \\
\hline Chromium \\
Copper \\
Arsenic \\
Selenium \\
Molybdenum \\
Ruthenium \\
Silver \\
Cadmium \\
Antimony \\
Lead
\end{tabular}

S1)

4.45

4.45

4.88

4.61
4.89

4.89

4.73

$<0.082$

4.08

4.58

4.50

4.75

$\begin{array}{rcr}0.206 & \text { ug/g wet } & 5.000 \\ 0.484 & \text { " } & 5.000 \\ 0.625 & \text { " } & 5.000 \\ 1.10 & \text { " } & 5.000 \\ 0.160 & \text { " } & 5.000 \\ 0.082 & \text { " } & \\ 0.107 & \text { " } & 5.000 \\ 0.062 & \text { " } & 5.000 \\ 0.054 & \text { " } & 5.000 \\ 0.056 & \text { " } & 5.000\end{array}$

Prepared: 04/07/08 Analyzed: 04/14/08

Source: 0803012-05 Prepared: 04/07/08 Analyzed: 04/14/08

\begin{tabular}{|c|c|c|c|c|c|}
\hline$<0.002$ & 0.002 & ug/g dry & ND & & 20 \\
\hline$<0.005$ & 0.005 & $"$ & ND & & 20 \\
\hline$<0.006$ & 0.006 & " & ND & & 20 \\
\hline$<0.011$ & 0.011 & $"$ & ND & & 20 \\
\hline 0.0261 & 0.002 & $"$ & 0.0265 & 1.80 & 20 \\
\hline$<0.0008$ & 0.0008 & " & ND & & 20 \\
\hline$<0.001$ & 0.001 & $"$ & ND & & 20 \\
\hline$<0.0006$ & 0.0006 & " & ND & & 20 \\
\hline$<0.0005$ & 0.0005 & $"$ & ND & & 20 \\
\hline$<0.0006$ & 0.0006 & $"$ & ND & & 20 \\
\hline
\end{tabular}


RCRA Metals By SW846 6020/Water Extract - Quality Control

Environmental Science Laboratory

\begin{tabular}{|lrrrrrrrrrrr}
\hline & & Reporting & & Spike & Source & & \%REC & & RPD \\
Analyte & Result & Limit & Units & Level & Result & \%REC & Limits & RPD & Limit & Notes \\
\hline
\end{tabular}

Batch 8D07005 - 1:1 Water Extract (ICP/ICPMS)

\begin{tabular}{|c|c|c|c|c|c|c|c|}
\hline \multirow{2}{*}{$\begin{array}{l}\text { Post Spike (8D07005-PS1) } \\
\text { Chromium }\end{array}$} & \multicolumn{3}{|c|}{ Source: 0803012-05 } & \multicolumn{2}{|c|}{ Prepared: 04/07/08 } & \multicolumn{2}{|c|}{ Analyzed: 04/14/08 } \\
\hline & 4.62 & 0.002 & ug/g dry & 5 & 0.212 & 88.3 & $75-125$ \\
\hline Copper & 4.94 & 0.005 & " & 5 & 0.176 & 95.4 & $75-125$ \\
\hline Arsenic & 5.85 & 0.006 & $"$ & 5 & 0.68 & 103 & $75-125$ \\
\hline Selenium & 5.51 & 0.011 & $"$ & 5 & 0.217 & 106 & $75-125$ \\
\hline Molybdenum & 10.2 & 0.002 & $"$ & 5 & 5.31 & 97.2 & $75-125$ \\
\hline Ruthenium & 4.47 & 0.0008 & $"$ & 5 & 0.012 & 89.2 & $75-125$ \\
\hline Silver & 4.53 & 0.001 & $"$ & 5 & 0.00774 & 90.5 & $75-125$ \\
\hline Cadmium & 4.65 & 0.0006 & $"$ & 5 & 0.0276 & 92.5 & $75-125$ \\
\hline Antimony & 4.74 & 0.0005 & $"$ & 5 & 0.0592 & 93.6 & $75-125$ \\
\hline Lead & 4.75 & 0.0006 & $"$ & 5 & 0.0273 & 94.5 & $75-125$ \\
\hline
\end{tabular}

Batch 8D14002 - 1:1 Water Extract (ICP/ICPMS)

\begin{tabular}{|c|c|c|c|c|}
\hline Blank (8D14002-BLK1) & & & & Prepared \& Analyzed: 04/14/08 \\
\hline Chromium & $<0.002$ & 0.002 & ug/g wet & \\
\hline Copper & $<0.005$ & 0.005 & $"$ & \\
\hline Arsenic & $<0.006$ & 0.006 & $"$ & \\
\hline Selenium & $<0.011$ & 0.011 & $"$ & \\
\hline Molybdenum & $<0.002$ & 0.002 & $"$ & \\
\hline Ruthenium & $<0.0008$ & 0.0008 & $"$ & \\
\hline Silver & $<0.001$ & 0.001 & $"$ & \\
\hline Cadmium & $<0.0006$ & 0.0006 & $"$ & \\
\hline Antimony & $<0.0005$ & 0.0005 & $"$ & \\
\hline Lead & $<0.0006$ & 0.0006 & $"$ & \\
\hline Blank (8D14002-BLK2) & & & & Prepared \& Analyzed: 04/14/08 \\
\hline Chromium & $<0.002$ & 0.002 & ug/g wet & \\
\hline Copper & $<0.005$ & 0.005 & $"$ & \\
\hline Arsenic & $<0.006$ & 0.006 & $"$ & \\
\hline Selenium & $<0.011$ & 0.011 & $"$ & \\
\hline Molybdenum & $<0.002$ & 0.002 & $"$ & \\
\hline Ruthenium & $<0.0008$ & 0.0008 & $"$ & \\
\hline Silver & $<0.001$ & 0.001 & $"$ & \\
\hline Cadmium & $<0.0006$ & 0.0006 & $"$ & \\
\hline Antimony & $<0.0005$ & 0.0005 & $"$ & \\
\hline Lead & $<0.0006$ & 0.0006 & $"$ & \\
\hline
\end{tabular}


RCRA Metals By SW846 6020/Water Extract - Quality Control

Environmental Science Laboratory

\begin{tabular}{|lrrrrrrrrrrr}
\hline & & Reporting & & Spike & Source & & \%REC & & RPD \\
Analyte & Result & Limit & Units & Level & Result & \%REC & Limits & RPD & Limit & Notes \\
\hline
\end{tabular}

Batch 8D14002 - 1:1 Water Extract (ICP/ICPMS)

\begin{tabular}{|c|c|c|c|c|c|c|c|c|}
\hline \multirow{2}{*}{$\begin{array}{l}\text { LCS (8D14002-BS1) } \\
\text { Chromium }\end{array}$} & \multicolumn{8}{|c|}{ Prepared: 04/14/08 Analyzed: 04/15/08 } \\
\hline & 5.10 & 0.206 & ug/g wet & 5.003 & 102 & $80-120$ & & \\
\hline Copper & 5.00 & 0.484 & $"$ & 5.003 & 99.9 & $80-120$ & & \\
\hline Arsenic & 4.97 & 0.625 & $"$ & 5.003 & 99.4 & $80-120$ & & \\
\hline Selenium & 5.12 & 1.10 & $"$ & 5.003 & 102 & $80-120$ & & \\
\hline Molybdenum & 4.80 & 0.160 & $"$ & 5.003 & 96.0 & $80-120$ & & \\
\hline Ruthenium & $<0.082$ & 0.082 & $"$ & & & $80-120$ & & \\
\hline Silver & 4.66 & 0.107 & $"$ & 5.003 & 93.2 & $80-120$ & & \\
\hline Cadmium & 4.79 & 0.062 & $"$ & 5.003 & 95.8 & $80-120$ & & \\
\hline Antimony & 4.82 & 0.054 & $"$ & 5.003 & 96.3 & $80-120$ & & \\
\hline Lead & 4.90 & 0.056 & $"$ & 5.003 & 98.0 & $80-120$ & & \\
\hline LCS (8D14002-BS2) & \multicolumn{8}{|c|}{ Prepared: 04/14/08 Analyzed: 04/15/08 } \\
\hline Chromium & 4.78 & 0.206 & ug/g wet & 5.002 & 95.6 & $80-120$ & & \\
\hline Copper & 4.60 & 0.484 & $"$ & 5.002 & 92.0 & $80-120$ & & \\
\hline Arsenic & 4.63 & 0.625 & $"$ & 5.002 & 92.5 & $80-120$ & & \\
\hline Selenium & 4.83 & 1.10 & $"$ & 5.002 & 96.5 & $80-120$ & & \\
\hline Molybdenum & 4.57 & 0.160 & $"$ & 5.002 & 91.3 & $80-120$ & & \\
\hline Ruthenium & $<0.082$ & 0.082 & $"$ & & & $80-120$ & & \\
\hline Silver & 4.37 & 0.107 & $"$ & 5.002 & 87.4 & $80-120$ & & \\
\hline Cadmium & 4.56 & 0.062 & $"$ & 5.002 & 91.1 & $80-120$ & & \\
\hline Antimony & 4.53 & 0.054 & $"$ & 5.002 & 90.5 & $80-120$ & & \\
\hline Lead & 4.59 & 0.056 & " & 5.002 & 91.7 & $80-120$ & & \\
\hline Duplicate (8D14002-DUP1) & \multicolumn{3}{|c|}{ Source: 0803014-05 } & \multicolumn{3}{|c|}{ Prepared \& Analyzed: 04/14/08 } & & \\
\hline Chromium & 0.00211 & 0.002 & ug/g dry & & & & & 20 \\
\hline Copper & $<0.005$ & 0.005 & $"$ & & & & & 20 \\
\hline Arsenic & $<0.006$ & 0.006 & $"$ & & & & & 20 \\
\hline Selenium & $<0.011$ & 0.011 & $"$ & & & & & 20 \\
\hline Molybdenum & 0.0360 & 0.002 & $"$ & & & & 0.441 & 20 \\
\hline Ruthenium & $<0.0008$ & 0.0008 & " & & & & & 20 \\
\hline Silver & $<0.001$ & 0.001 & $"$ & & & & & 20 \\
\hline Cadmium & $<0.0006$ & 0.0006 & $"$ & & & & & 20 \\
\hline Antimony & $<0.0005$ & 0.0005 & $"$ & & & & & 20 \\
\hline Lead & $<0.0006$ & 0.0006 & $"$ & & & & & 20 \\
\hline
\end{tabular}


RCRA Metals By SW846 6020/Water Extract - Quality Control

Environmental Science Laboratory

\begin{tabular}{|lrrrrrrrrrrr}
\hline & & Reporting & & Spike & Source & & \%REC & & RPD \\
Analyte & Result & Limit & Units & Level & Result & \%REC & Limits & RPD & Limit & Notes \\
\hline
\end{tabular}

Batch 8D14002 - 1:1 Water Extract (ICP/ICPMS)

\begin{tabular}{|c|c|c|c|c|c|c|c|c|c|}
\hline \multirow{2}{*}{$\begin{array}{l}\text { Duplicate (8D14002-DUP2) } \\
\text { Chromium }\end{array}$} & \multicolumn{3}{|c|}{ Source: 0803015-07 } & \multicolumn{6}{|c|}{ Prepared \& Analyzed: 04/14/08 } \\
\hline & $<0.002$ & 0.002 & ug/g dry & & ND & & & & 20 \\
\hline Copper & $<0.005$ & 0.005 & $"$ & & ND & & & & 20 \\
\hline Arsenic & $<0.006$ & 0.006 & $"$ & & ND & & & & 20 \\
\hline Selenium & $<0.011$ & 0.011 & " & & ND & & & & 20 \\
\hline Molybdenum & 0.00770 & 0.002 & $"$ & & 0.00796 & & & 3.31 & 20 \\
\hline Ruthenium & $<0.0008$ & 0.0008 & $"$ & & ND & & & & 20 \\
\hline Silver & $<0.001$ & 0.001 & $"$ & & ND & & & & 20 \\
\hline Cadmium & $<0.0006$ & 0.0006 & $"$ & & ND & & & & 20 \\
\hline Antimony & $<0.0005$ & 0.0005 & $"$ & & ND & & & & 20 \\
\hline Lead & $<0.0006$ & 0.0006 & $"$ & & ND & & & & 20 \\
\hline Post Spike (8D14002-PS1) & \multicolumn{3}{|c|}{ Source: 0803014-05 } & \multicolumn{4}{|c|}{ Prepared \& Analyzed: 04/14/08 } & & \\
\hline Chromium & 4.64 & & ug/L & 5 & 0.408 & 84.6 & $75-125$ & & \\
\hline Copper & 4.89 & & $"$ & 5 & 0.233 & 93.1 & $75-125$ & & \\
\hline Arsenic & 5.78 & & $"$ & 5 & 0.694 & 102 & $75-125$ & & \\
\hline Selenium & 5.42 & & $"$ & 5 & 0.093 & 107 & $75-125$ & & \\
\hline Molybdenum & 12.5 & & $"$ & 5 & 7.24 & 106 & $75-125$ & & \\
\hline Ruthenium & 4.62 & & $"$ & 5 & 0.0106 & 92.1 & $75-125$ & & \\
\hline Silver & 4.55 & & $"$ & 5 & 0.00565 & 90.8 & $75-125$ & & \\
\hline Cadmium & 4.72 & & $"$ & 5 & 0.0202 & 94.1 & $75-125$ & & \\
\hline Antimony & 4.87 & & $"$ & 5 & 0.0603 & 96.2 & $75-125$ & & \\
\hline Lead & 4.76 & & $"$ & 5 & 0.0575 & 94.1 & $75-125$ & & \\
\hline Post Spike (8D14002-PS2) & \multicolumn{3}{|c|}{ Source: 0803015-07 } & \multicolumn{4}{|c|}{ Prepared \& Analyzed: 04/14/08 } & & \\
\hline Chromium & 4.54 & & ug/L & 5 & 0.209 & 86.6 & $75-125$ & & \\
\hline Copper & 4.74 & & " & 5 & 0.125 & 92.2 & $75-125$ & & \\
\hline Arsenic & 5.93 & & $"$ & 5 & 0.869 & 101 & $75-125$ & & \\
\hline Selenium & 5.44 & & " & 5 & 0.0309 & 108 & $75-125$ & & \\
\hline Molybdenum & 6.34 & & $"$ & 5 & 1.59 & 94.9 & $75-125$ & & \\
\hline Ruthenium & 4.5 & & " & 5 & 0.0091 & 89.9 & $75-125$ & & \\
\hline Silver & 4.47 & & $"$ & 5 & 0.00505 & 89.3 & $75-125$ & & \\
\hline Cadmium & 4.57 & & $"$ & 5 & 0.00927 & 91.2 & $75-125$ & & \\
\hline Antimony & 4.79 & & $"$ & 5 & 0.031 & 95.3 & $75-125$ & & \\
\hline Lead & 4.8 & & $"$ & 5 & 0.0224 & 95.5 & $75-125$ & & \\
\hline
\end{tabular}


RCRA Metals By SW846 6020/Acid Extract - Quality Control

Environmental Science Laboratory

\begin{tabular}{|lrrrrrrrrrrr}
\hline & & Reporting & & Spike & Source & & \%REC & & RPD \\
Analyte & Result & Limit & Units & Level & Result & \%REC & Limits & RPD & Limit & Notes \\
\hline
\end{tabular}

Batch 8D21003 - ASTM D 5198 (ICP/ICPMS)

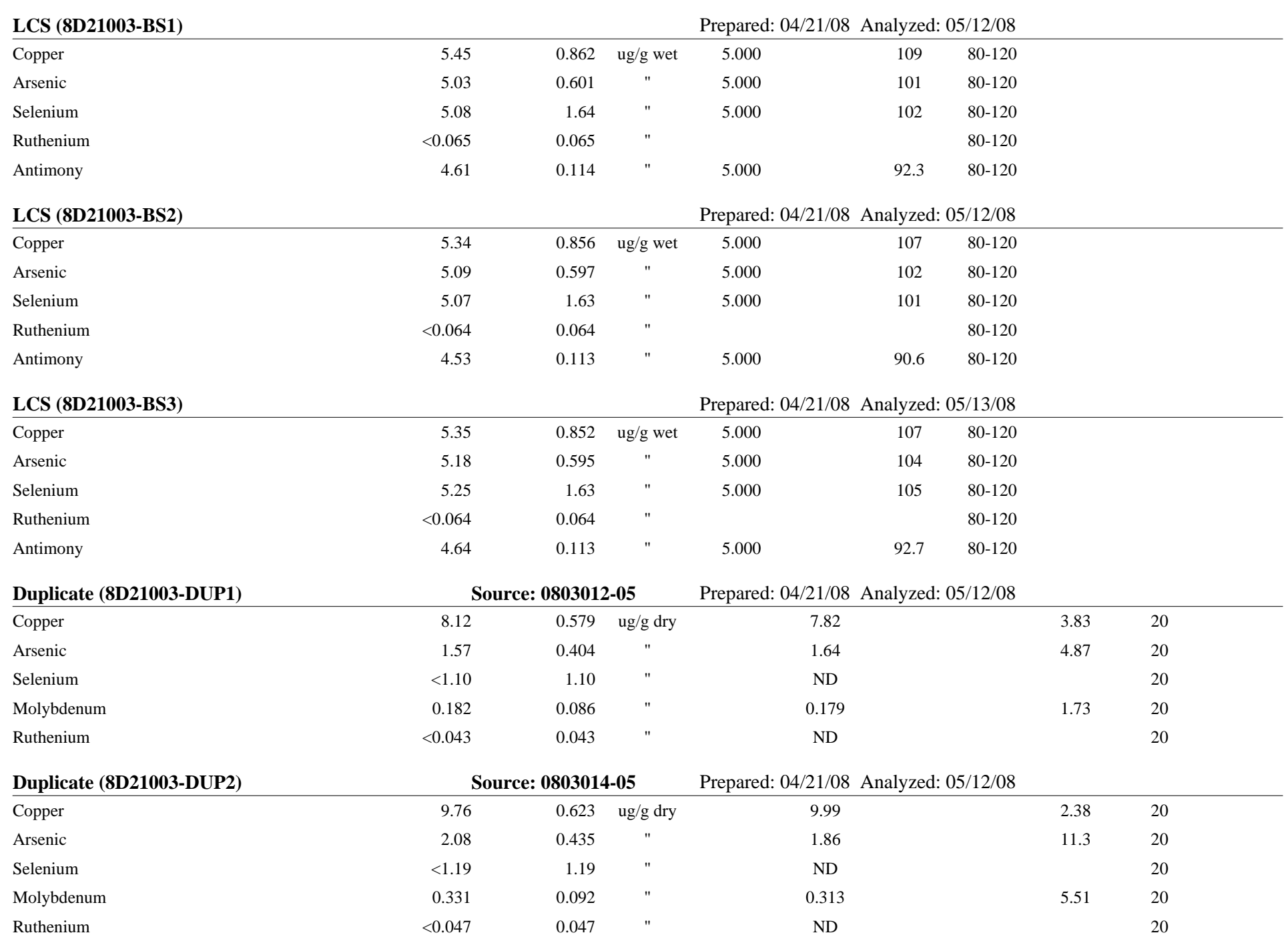


RCRA Metals By SW846 6020/Acid Extract - Quality Control

Environmental Science Laboratory

\begin{tabular}{|lrrrrrrrrrr}
\hline & & Reporting & & Spike & Source & & \%REC & & RPD & \\
Analyte & Result & Limit & Units & Level & Result & \%REC & Limits & RPD & Limit & Notes \\
\hline
\end{tabular}

Batch 8D21003 - ASTM D 5198 (ICP/ICPMS)

\begin{tabular}{|c|c|c|c|c|c|c|}
\hline Post Spike (8D21003-PS1) & \multicolumn{2}{|c|}{ Source: 0803012-05 } & \multicolumn{4}{|c|}{ Prepared: 04/21/08 Analyzed: 05/12/08 } \\
\hline Copper & 30.6 & ug/L & 5 & 26 & 91.5 & $75-125$ \\
\hline Arsenic & 9.96 & " & 5 & 5.48 & 89.7 & $75-125$ \\
\hline Ruthenium & 3.8 & $"$ & 5 & 0.00924 & 75.8 & $75-125$ \\
\hline Post Spike (8D21003-PS2) & \multicolumn{2}{|c|}{ Source: 0803014-05 } & \multicolumn{4}{|c|}{ Prepared: 04/21/08 Analyzed: 05/12/08 } \\
\hline Copper & 34.2 & $\mathrm{ug} / \mathrm{L}$ & 5 & 30.2 & 80 & $75-125$ \\
\hline Arsenic & 10.1 & " & 5 & 5.61 & 89.9 & $75-125$ \\
\hline Ruthenium & 3.81 & $"$ & 5 & 0.01 & 76 & $75-125$ \\
\hline
\end{tabular}


Total Alpha Total Beta/Acid Extract - Quality Control

Environmental Science Laboratory

\begin{tabular}{|lrrrrrrrrrrr}
\hline & & Reporting & & Spike & Source & & & \%REC & & RPD & \\
Analyte & Result & Limit & Units & Level & Result & \%REC & Limits & RPD & Limit & Notes \\
\hline
\end{tabular}

\section{Batch 8D10001 - ASTM D 5198 (RadChem)}

\begin{tabular}{|c|c|c|c|c|c|}
\hline \multicolumn{4}{|l|}{ Blank (8D10001-BLK1) } & \multicolumn{2}{|l|}{ Prepared: 04/10/08 Analyzed: 04/11/08 } \\
\hline Gross Beta & $<500$ & 500 & $\mathrm{pCi} / \mathrm{g}$ wet & & \\
\hline Gross Alpha & $<176$ & 176 & " & & \\
\hline Blank (8D10001-BLK2) & & & & Prepared: 04/10/08 Analyzed: 04/11/08 & \\
\hline Gross Beta & $<500$ & 500 & $\mathrm{pCi} / \mathrm{g}$ wet & & \\
\hline Gross Alpha & $<176$ & 176 & " & & \\
\hline Blank (8D10001-BLK3) & & & & Prepared: 04/10/08 Analyzed: 04/11/08 & \\
\hline Gross Beta & $<14.3$ & 14.3 & $\mathrm{pCi} / \mathrm{g}$ wet & & \\
\hline Gross Alpha & $<5.02$ & 5.02 & $"$ & & \\
\hline Duplicate (8D10001-DUP1) & & 30301 & -05 & Prepared: 04/10/08 Analyzed: 04/11/08 & \\
\hline Gross Beta & $<48.1$ & 48.1 & pCi/g dry & ND & 200 \\
\hline Gross Alpha & $<16.9$ & 16.9 & $"$ & ND & 200 \\
\hline Duplicate (8D10001-DUP2) & & 30301 & -05 & Prepared: 04/10/08 Analyzed: 04/11/08 & \\
\hline Gross Beta & $<51.7$ & 51.7 & $\mathrm{pCi} / \mathrm{g}$ dry & ND & 200 \\
\hline Gross Alpha & $<18.2$ & 18.2 & $"$ & ND & 200 \\
\hline Duplicate (8D10001-DUP3) & & 30301 & -07 & Prepared: 04/10/08 Analyzed: 04/11/08 & \\
\hline Gross Beta & $<48.8$ & 48.8 & pCi/g dry & ND & 200 \\
\hline Gross Alpha & $<17.2$ & 17.2 & $"$ & ND & 200 \\
\hline
\end{tabular}




\section{Total Alpha Total Beta/Water Extract - Quality Control \\ Environmental Science Laboratory}

\begin{tabular}{|lrrrrrrrrrrr}
\hline & & Reporting & & Spike & Source & & \%REC & & RPD & & \\
Analyte & Result & Limit & Units & Level & Result & \%REC & Limits & RPD & Limit & Notes \\
\hline
\end{tabular}

Batch 8D09004 - 1:1 Water Extract (RadChem)

\begin{tabular}{|c|c|c|c|c|c|c|}
\hline \multicolumn{4}{|l|}{ Blank (8D09004-BLK1) } & \multicolumn{3}{|c|}{ Prepared: 04/07/08 Analyzed: 04/08/08 } \\
\hline Gross Beta & $<14.3$ & 14.3 & pCi/g wet & & & \\
\hline Gross Alpha & $<4.53$ & 4.53 & $"$ & & & \\
\hline Blank (8D09004-BLK2) & & & & Prepared: 04/07/08 & Analyzed: 04/08/08 & \\
\hline Gross Beta & $<14.3$ & 14.3 & $\mathrm{pCi} / \mathrm{g}$ wet & & & \\
\hline Gross Alpha & $<4.53$ & 4.53 & $"$ & & & \\
\hline Blank (8D09004-BLK3) & & & & Prepared: 04/07/08 & Analyzed: 04/08/08 & \\
\hline Gross Beta & $<14.3$ & 14.3 & $\mathrm{pCi} / \mathrm{g}$ wet & & & \\
\hline Gross Alpha & $<4.53$ & 4.53 & " & & & \\
\hline Duplicate (8D09004-DUP1) & & 30301 & -05 & Prepared: 04/07/08 & Analyzed: 04/08/08 & \\
\hline Gross Beta & $<14.3$ & 14.3 & pCi/g dry & ND & & 200 \\
\hline Gross Alpha & $<4.53$ & 4.53 & $"$ & ND & & 200 \\
\hline Duplicate (8D09004-DUP2) & & 30301 & -05 & Prepared: 04/07/08 & Analyzed: 04/08/08 & \\
\hline Gross Beta & $<14.3$ & 14.3 & $\mathrm{pCi} / \mathrm{g}$ dry & ND & & 200 \\
\hline Gross Alpha & $<4.53$ & 4.53 & $"$ & ND & & 200 \\
\hline Duplicate (8D09004-DUP3) & & 30301 & -07 & Prepared: 04/07/08 & Analyzed: 04/08/08 & \\
\hline Gross Beta & $<14.3$ & 14.3 & pCi/g dry & ND & & 200 \\
\hline Gross Alpha & $<4.53$ & 4.53 & $"$ & ND & & 200 \\
\hline
\end{tabular}




\section{- ALCTOR}

HALL, FM

\section{SAMPLING LOCATION}

ICE CHEST NO.

$$
\text { C5952A }
$$

$$
\sin L-441
$$

SHIPPED TO

PNNL Building 325

MATRIX*

$\mathrm{OL}=$ OTHER LIQUID

OS = OTHER SOLIO

$S=$ SOIL

$W=$ WATER

\section{SAMPLE NO.}

\section{COMPANY CONTACT}

SYDNOR, HA

\section{PROJECT DESIGNATION}

"C" Tank Farm Soil Sampling - Boreholes C5944-C5966

\section{FIELD LOGBOOK NO.}

ACTUAL SAMPLE DEPTH

DTS-SAWS-H97

OFFSITE PROPERTY NO.

\section{TELEPHONE NO.}

$9.5^{\prime}-10.0^{\prime}$
PROJECT COORDINATOR

TRENT, S]

\section{SAF NO.}

V08-001

COA
PRICE CODE $8 \mathrm{~N}$

AIR QUALITY

METHOD OF SHIPMENT

GOVERNMENT VEHICLE

BILL OF LADING/AIR BILL NO.

LAB ID

MATRIX*

\section{SPECIAL HANDLING AND/OR STORAGE}

B1RTHO

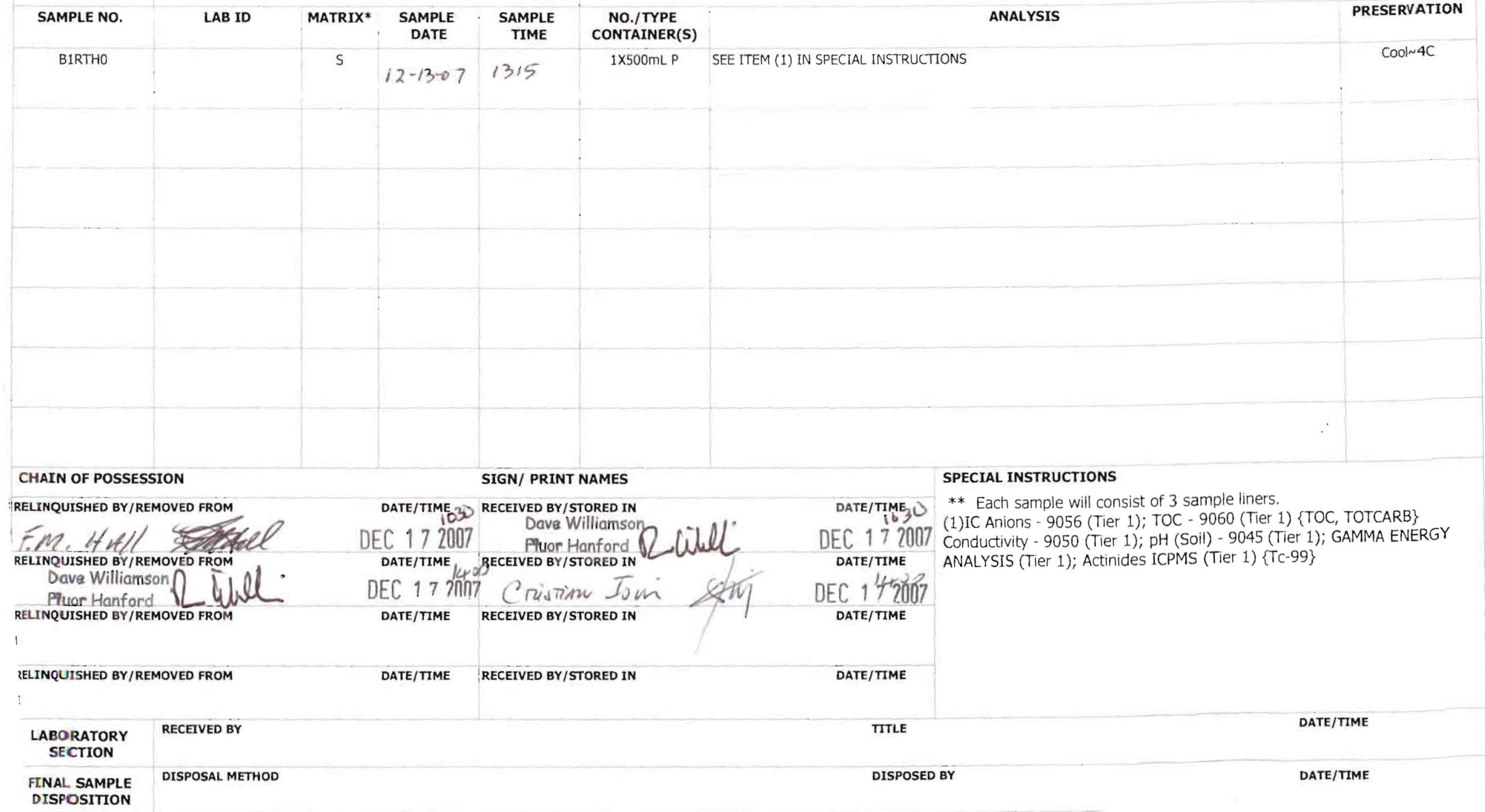

\section{POSSIBLE SAMPLE HAZARDS / REMARKS}

Contains Radioactive Material at concentrations that are not regulated for transportation per 49 CFR but are not releasable per DOE Order $5400.5(1990 / 1993)$ 


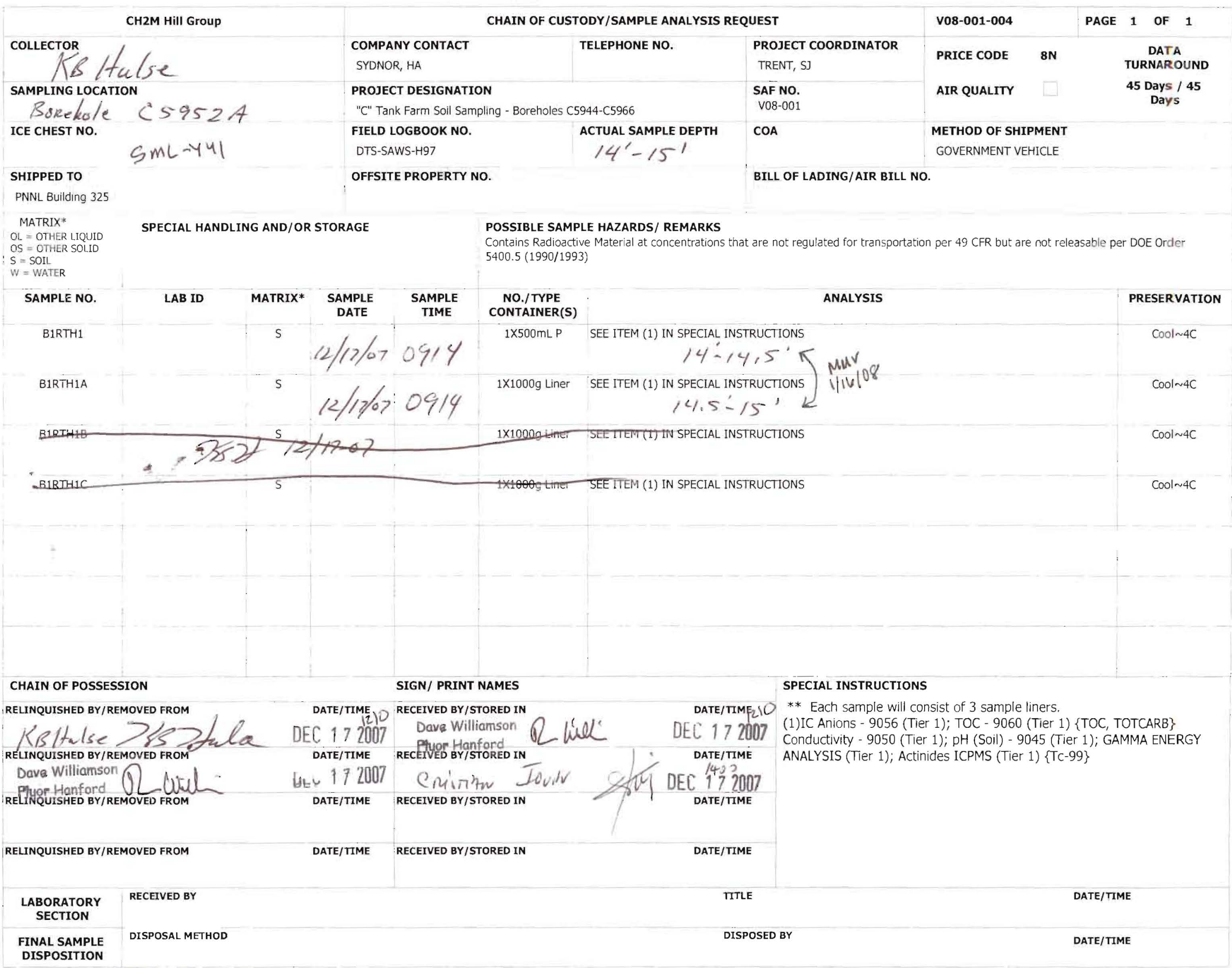




\section{CH2M Hill Group}

Fluor Hantord

SAMPLING LOCATION

ICE CHEST NO

$$
\text { C } 5952 A
$$

SHIPPED TO

PNNL Building 325

MATRIX*

$O L=$ OTHER LIQUID

OS = OTHER SOLTD

$S=$ SOIL

\section{SAMPLE NO.}

LAB ID

SPECIAL HANDLING AND/OR STORAGE

COMPANY CONTACT

SYDNOR, HA

PROJECT DESIGNATION

"C" Tank Farm Soil Sampling - Boreholes C5944-C5966

\section{FIELD LOGBOOK NO.}

DTS-SAWS-H97

OFFSITE PROPERTY NO.
ACTUAL SAMPLE DEPTH

\section{CHAIN OF CUSTODY/SAMPLE ANALYSIS REQUEST}

TELEPHONE NO.

PROJECT COORDINATOR

TRENT, SJ

SAF NO.

VO8-001

COA

$$
60^{\circ} \cdot 62
$$

\begin{tabular}{|c|c|c|}
\hline V08-001-005 & PAGE 1 OF 1 \\
\hline PRICE CODE & $8 \mathrm{~N}$ & $\begin{array}{c}\text { DATA } \\
\text { TURNAROUND }\end{array}$ \\
AIR QUALITY & $\square$ & $\begin{array}{c}45 \text { Days / } 45 \\
\text { Days }\end{array}$ \\
\hline
\end{tabular}

METHOD OF SHIPMENT

GOVERNMENT VEHICLE

BILL OF LADING/AIR BILL NO.

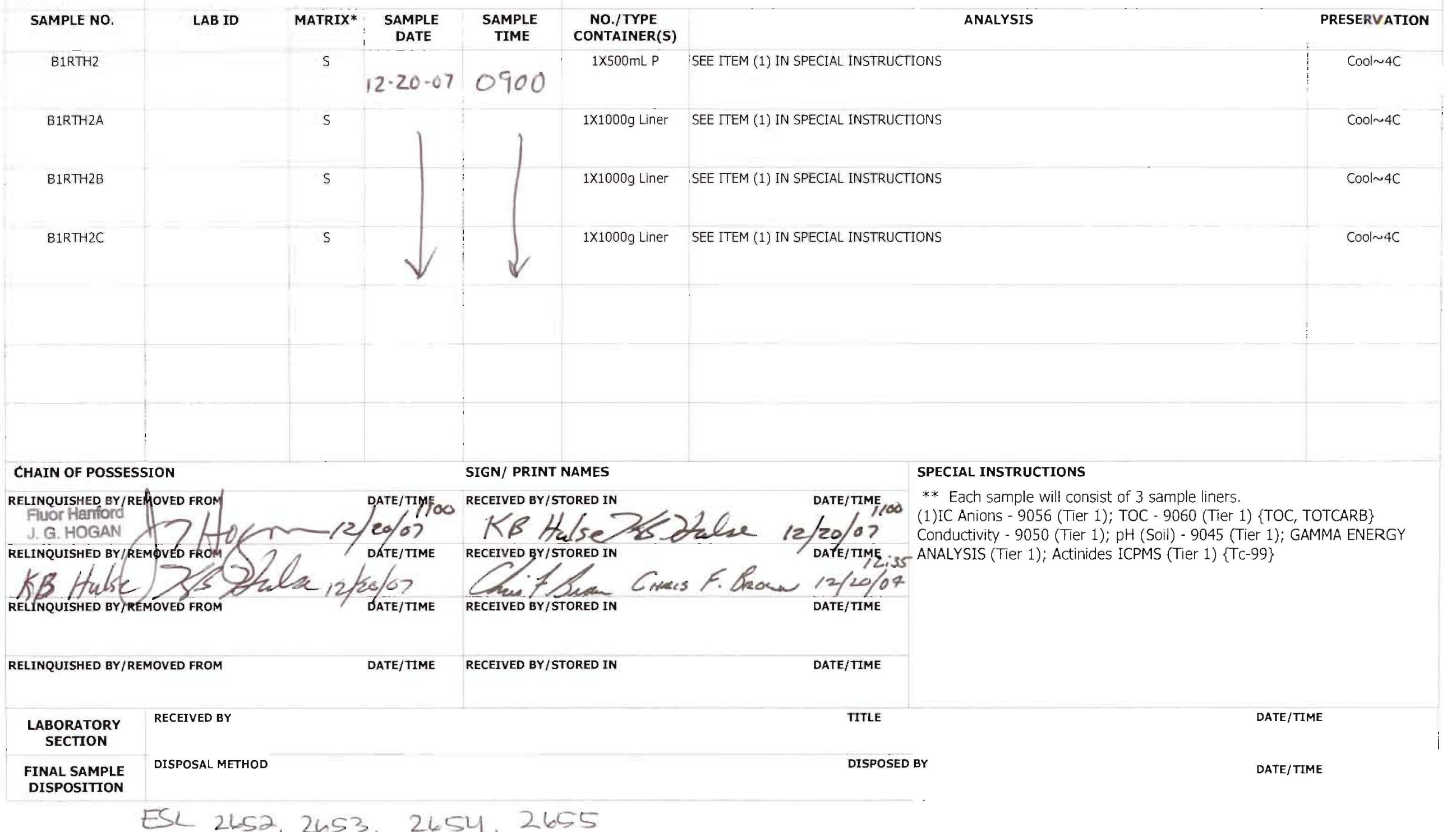




\section{SAMPLING LOCATION}

$$
\text { C } 5952 \text { A }
$$

ICE CHEST NO.

$$
5 m L-600
$$

SHIPPED TO

PNNL Building 325

MATRIX*

$\mathrm{OL}=$ OTHER LIQUID

OS = OTHER SOLID

$\mathrm{S}=\mathrm{SOIL}$

\section{SAMPLE NO.}

LAB ID

\begin{tabular}{|ccc} 
MATRIX* & $\begin{array}{c}\text { SAMPLE } \\
\text { DATE }\end{array}$ & $\begin{array}{c}\text { SAMPLE } \\
\text { TIME }\end{array}$ \\
\hdashline 5 & $12 / 21 / 07$ & 0855 \\
\cline { 1 - 2 } & &
\end{tabular}

B1RTH3A

SPECIAL HANDLING AND/OR STORAGE

\section{COMPANY CONTACT}

SYDNOR, HA

\section{PROJECT DESIGNATION}

"C" Tank Farm Soil Sampling - Boreholes C5944-C5966

\section{FIELD LOGBOOK NO.}

DTS-SAWS-H97

\section{ACTUAL SAMPLE DEPTH}

OFFSITE PROPERTY NO.

POSSIBLE SAMPLE HAZARDS/ REMARKS $5400.5(1990 / 1993)$

\section{NO./TYPE} CONTAINER(S)

B1RTH3

$1 \times 500 \mathrm{~mL} P$ v08-001-006

PROJECT COORDINATOR

TRENT, SJ

SAF NO.

V08-001

COA

$$
80^{\prime}-82^{\prime}
$$

\begin{tabular}{|c|c|c|}
\hline PRICE CODE & $8 \mathrm{~N}$ & $\begin{array}{c}\text { DATA } \\
\text { TURNAROUND }\end{array}$ \\
\hline AIR QUALITY & & $\begin{array}{c}45 \text { Days / } 45 \\
\text { Days }\end{array}$ \\
\hline
\end{tabular}

\section{BILL OF LADING/AIR BILL NO.}

METHOD OF SHIPMENT

GOVERNMENT VEHICLE

Contains Radioactive Material at concentrations that are not regulated for transportation per 49 CFR but are not releasable per DOE Order

ANALYSIS

PRESERVATION

SEE TTEM (1) IN SPECIAL INSTRUCTIONS

Cool 4C

$1 \times 1000 \mathrm{~g}$ Liner SEE ITEM (1) IN SPECIAL INSTRUCTIONS

Cool $\sim 4 C$

$1 \times 1000 \mathrm{~g}$ Liner SEE ITEM (1) IN SPECIAL INSTRUCTIONS

Cool 4C

$1 \times 1000 \mathrm{~g}$ Liner SEE ITEM (1) IN SPECIAL INSTRUCTIONS

Cool $\sim 4 C$

\section{CHAIN OF POSSESSION}

RELINQUISHED BY/REMOVED FROM

ISB tals

Se

RELINOUISHED BY/REMOVED FROM

RELINQUISHED BY/REMOVED FROM

\section{SIGN/ PRINT NAMES}

DATE/TIME :RECEIVED BY/STORED IN

\begin{tabular}{|l|l|}
\hline $\begin{array}{c}\text { LABORATORY } \\
\text { SECTION }\end{array}$ & RECEIVED BY \\
\hline FINAL SAMPLE & DISPOSAL METHOD \\
\hline
\end{tabular}

TITLE

DISPOSED BY

\section{SPECIAL INSTRUCTIONS}

DATE/TIME

** Each sample will consist of 3 sample liners.

(1)IC Anions - 9056 (Tier 1); TOC - 9060 (Tier 1) \{TOC, TOTCARB\}

Conductivity - 9050 (Tier 1); pH (Soil) - 9045 (Tier 1); GAMMA ENERGY ANALYSIS (Tier 1); Actinides ICPMS (Tier 1) \{Tc-99\}

DISPOSITION 


\section{CHAIN OF CUSTODY/SAMPLE ANALYSIS REQUEST}

COMPANY CONTACT

SYDNOR, HA

$13 \mathrm{Hta} / \mathrm{C}$

SAMPLING LOCATION

$\therefore B+R Y A 7$ ICE CHEST NO.

UM $\checkmark$ PROJECT DESIGNATION

\section{SHIPPED TO}

PNNL Building 325

MATRIX*

$\mathrm{OL}=$ OTHER LQUID

OS = OTHER SOLID

$S=$ SOIL

\section{SAMPLE NO.}

LAB ID

SPECIAL HANDLING AND/OR STORAGE

FIELD LOGBOOK NO.

DTS-SAWS-H97

OFFSITE PROPERTY NO.
TELEPHONE NO.

PROJECT COORDINATOR
TRENT, SJ
SAF NO.
VO8-001
COA

ACTUAL SAMPLE DEPTH COA v08-001-007

PAGE 1 OF 1

PRICE CODE

$8 N$

DATA

AIR QUALITY

45 Days / 45

Days

METHOD OF SHIPMENT

GOVERNMENT VEHICLE

BILL OF LADING/AIR BILL NO.

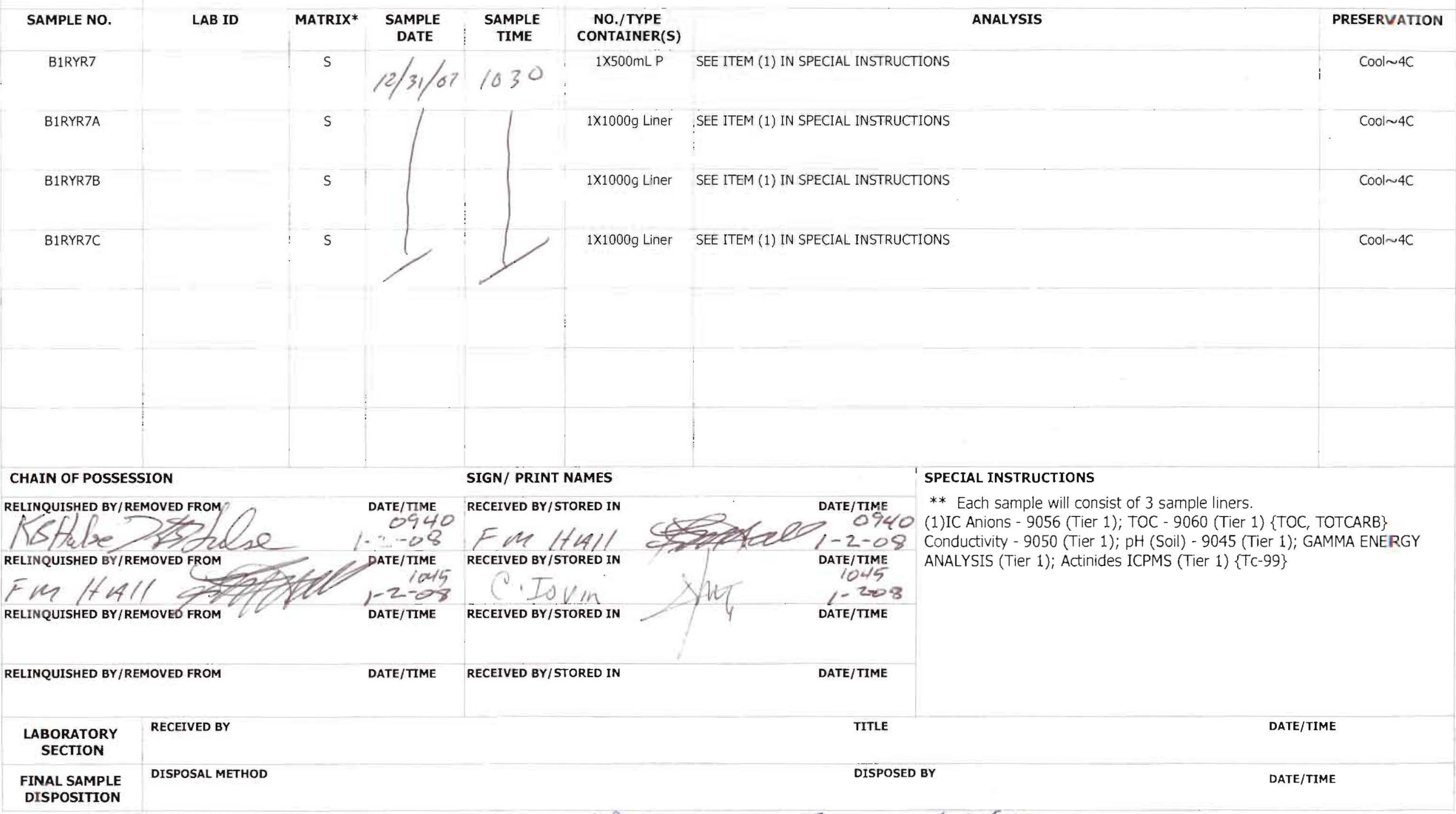

POSSIBLE SAMPLE HAZARDS/ REMARKS

Contains Radioactive Material at concentrations that are not regulated for transportation per 49 CFR but are not releasable per DOE Order $5400.5(1990 / 1993)$ 


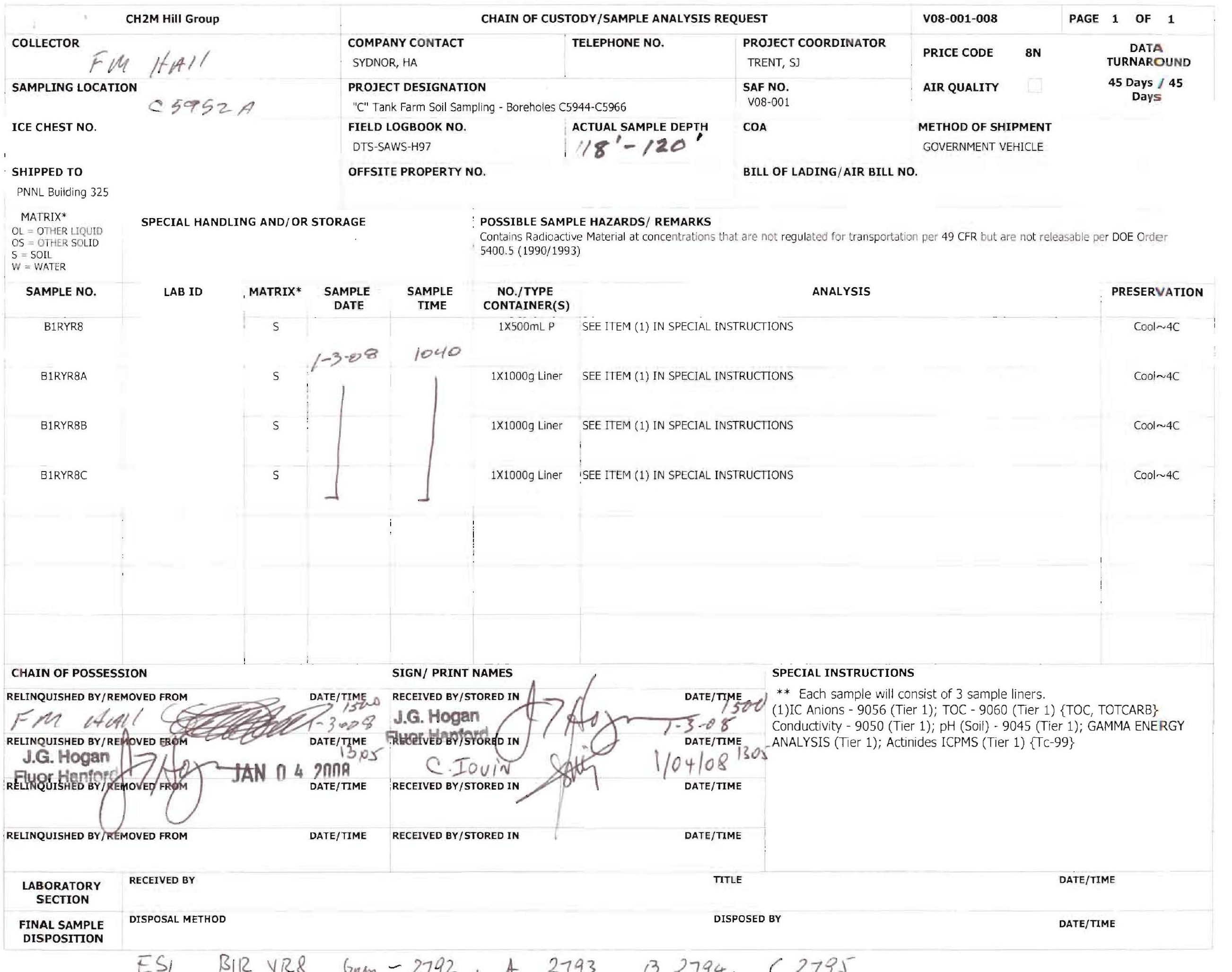




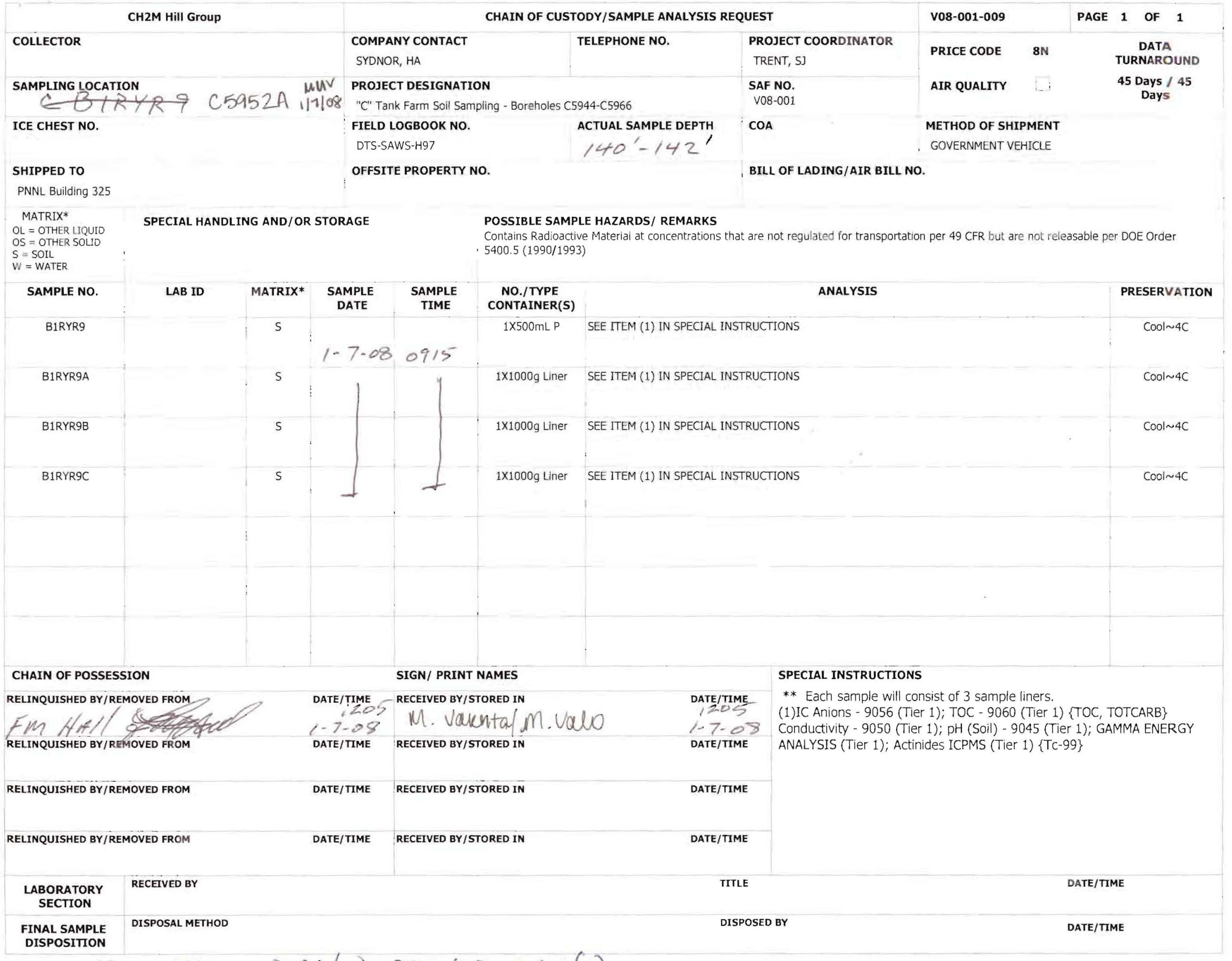




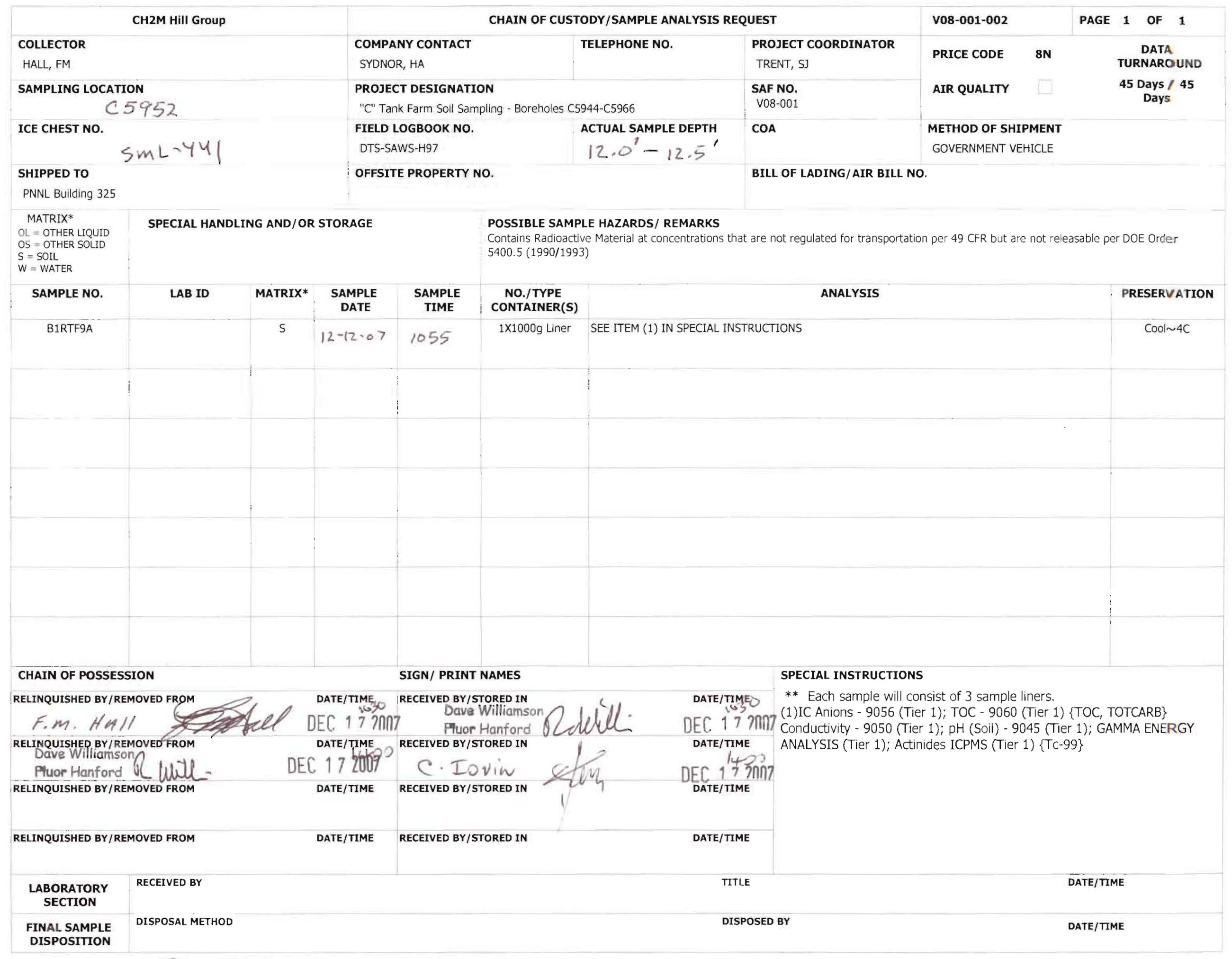

tesi 2640 


\section{CH2M Hill Group}

COLLECTOR

HALL, FM

SAMPLING LOCATION

ICE CHEST NO.

$$
\mathrm{C} 5952
$$

$$
5 m L-441
$$

SHIPPED TO

PNNL Building 325

MATRIX*

OL $=$ OTHER LUQID
OS = OTHER SOLID

$S=$ SOIL

$W=$ WATER

SAMPLE NO.

SPECIAL HANDLING AND/OR STORAGE

\section{COMPANY CONTACT}

SYDNOR, HA

\section{PROJECT DESIGNATION}

"C" Tank Farm Soil Sampling - Boreholes C5944-C5966

\section{FIELD LOGBOOK NO.}

DTS-SAWS-H97

OFFSITE PROPERTY NO.

LAB ID
CHAIN OF CUSTODY/SAMPLE ANALYSIS REQUEST

TELEPHONE NO.

PROJECT COORDINATOR

TRENT, S]

SAF NO.

V08-001

COA

$$
9.5^{\prime}=10.5^{\prime}
$$

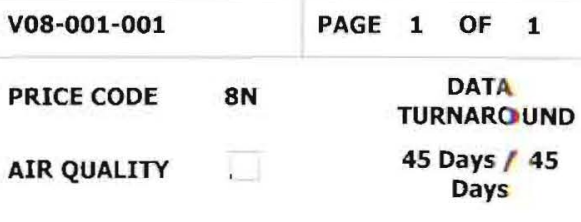

METHOD OF SHIPMENT

GOVERNMENT VEHICLE

BILL OF LADING/AIR BILL NO.

B1RTF8

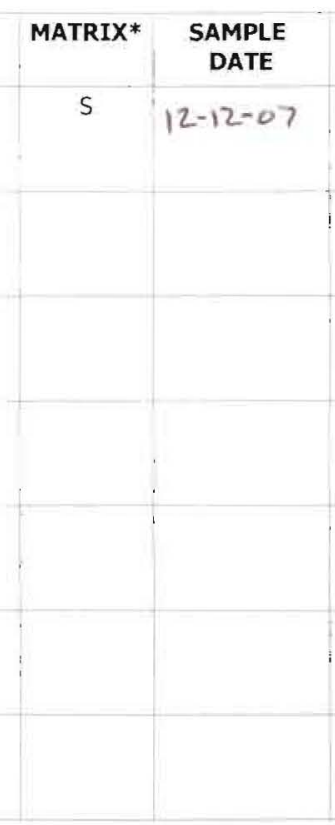

CHAIN OF POSSESSION

RELINQUISHED BY/REMOVED FROM

FM. HAII

RELINQUISHED BY/REMOVEDD FROM

Dave Williom Re

Pluor Hanford

RELINQUISHED BY/REMOVED FROM

\section{3}

DATE/TIME
SIGN/ PRINT NAMES

RECEIVED BY/STORED IN

POSSIBLE SAMPLE HAZARDS/ REMARKS

Contains Radioactive Material at concentrations that are not regulated for transportation per 49 CFR but are not releasable per DOE Order $5400.5(1990 / 1993)$

SAMPLE
TIME

\section{NO./TYPE}

CONTAINER(S)
$1 \times 500 \mathrm{~mL} P \quad$ SEE ITEM (1) IN SPECIAL INSTRUCTIONS
ANALYSIS

PRESERVATION

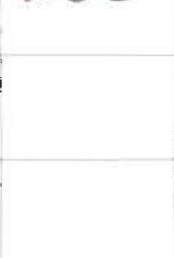

\begin{tabular}{|c|c|}
\hline $\begin{array}{c}\text { LABORATORY } \\
\text { SECTION }\end{array}$ & RECEIVED BY \\
\hline $\begin{array}{c}\text { FINAL SAMPLE } \\
\text { DISPOSITION }\end{array}$ & DISPOSAL METHOD \\
\hline
\end{tabular}

TITLE

DATE/TIME

DISPOSITION

FSl 2639 


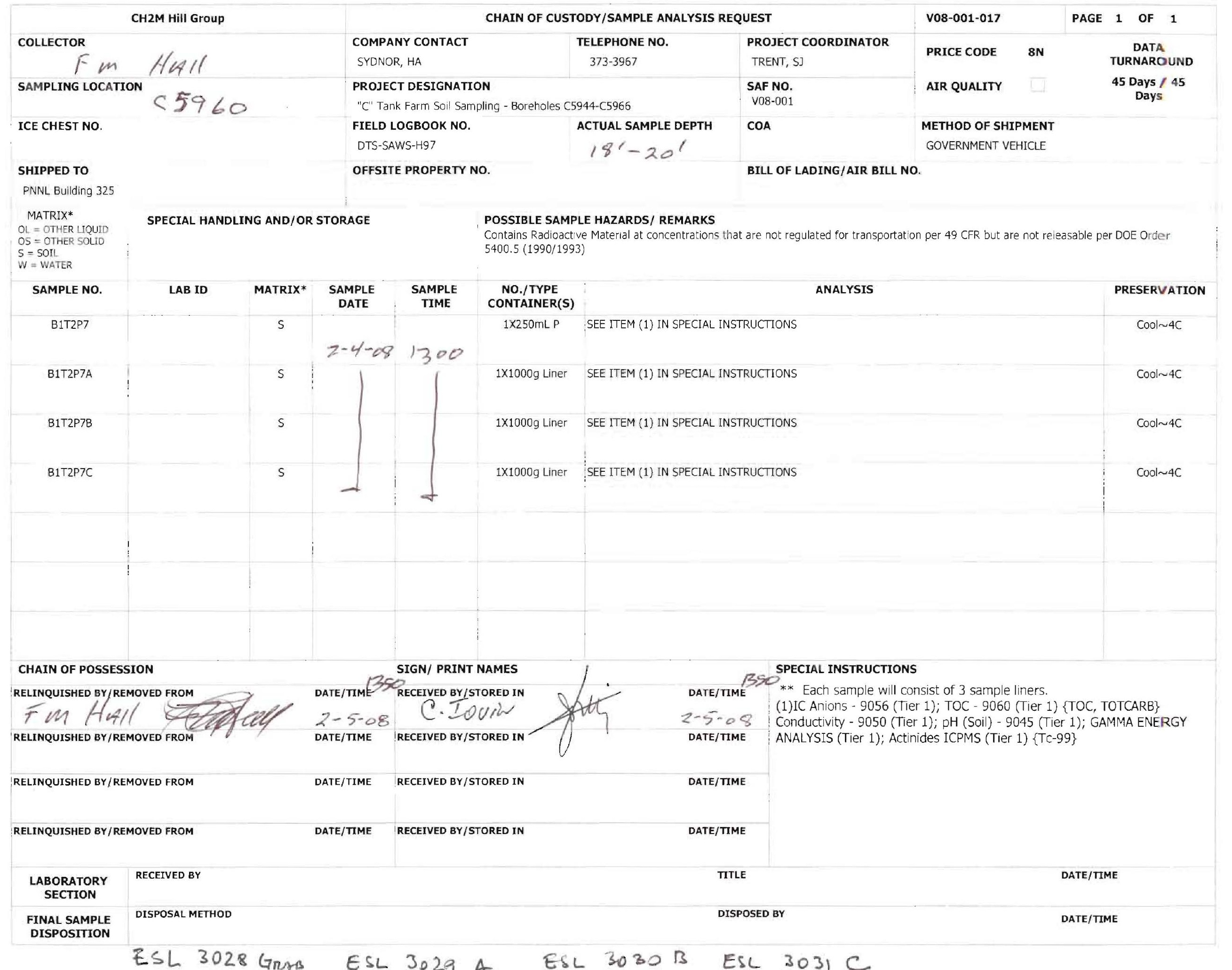




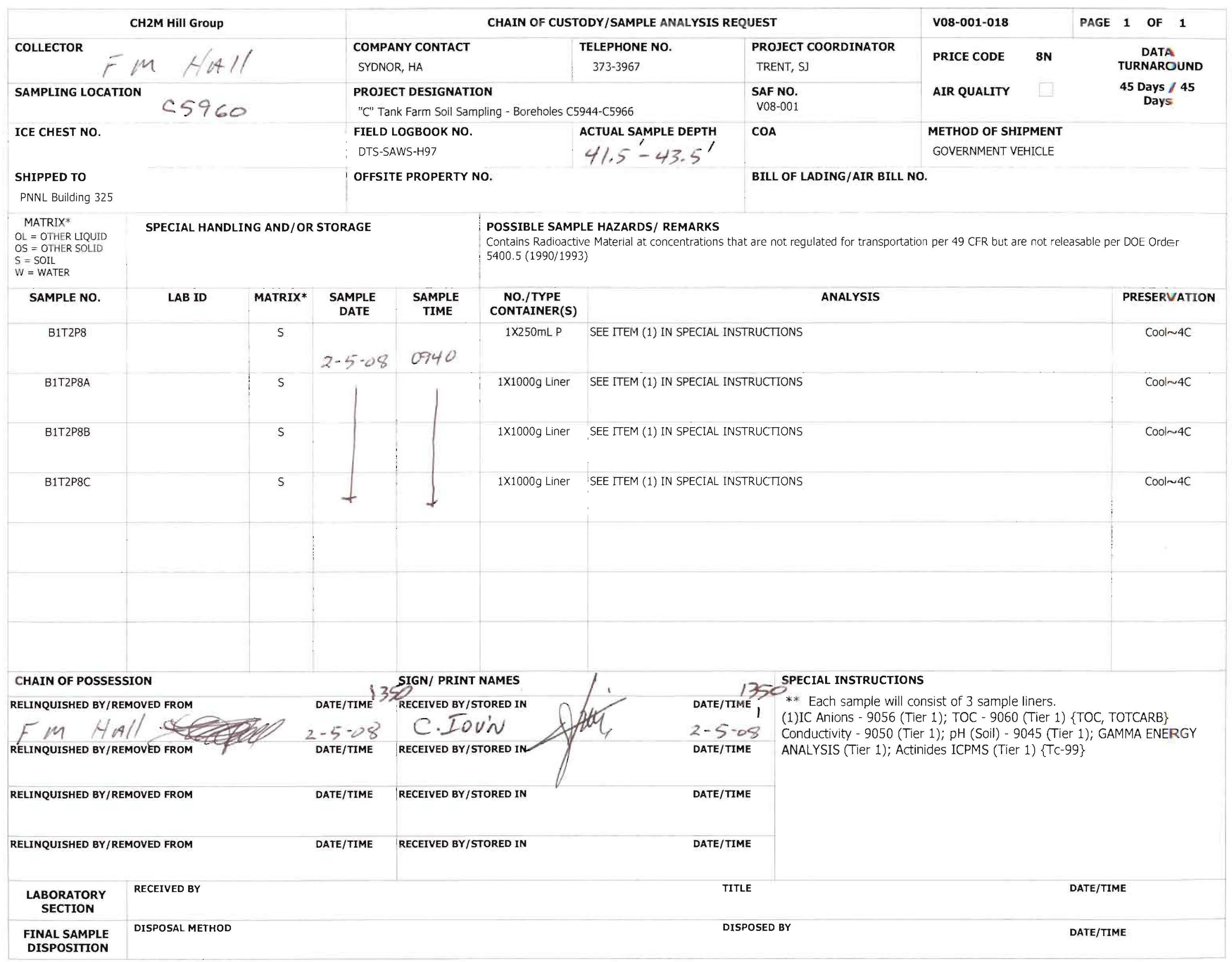




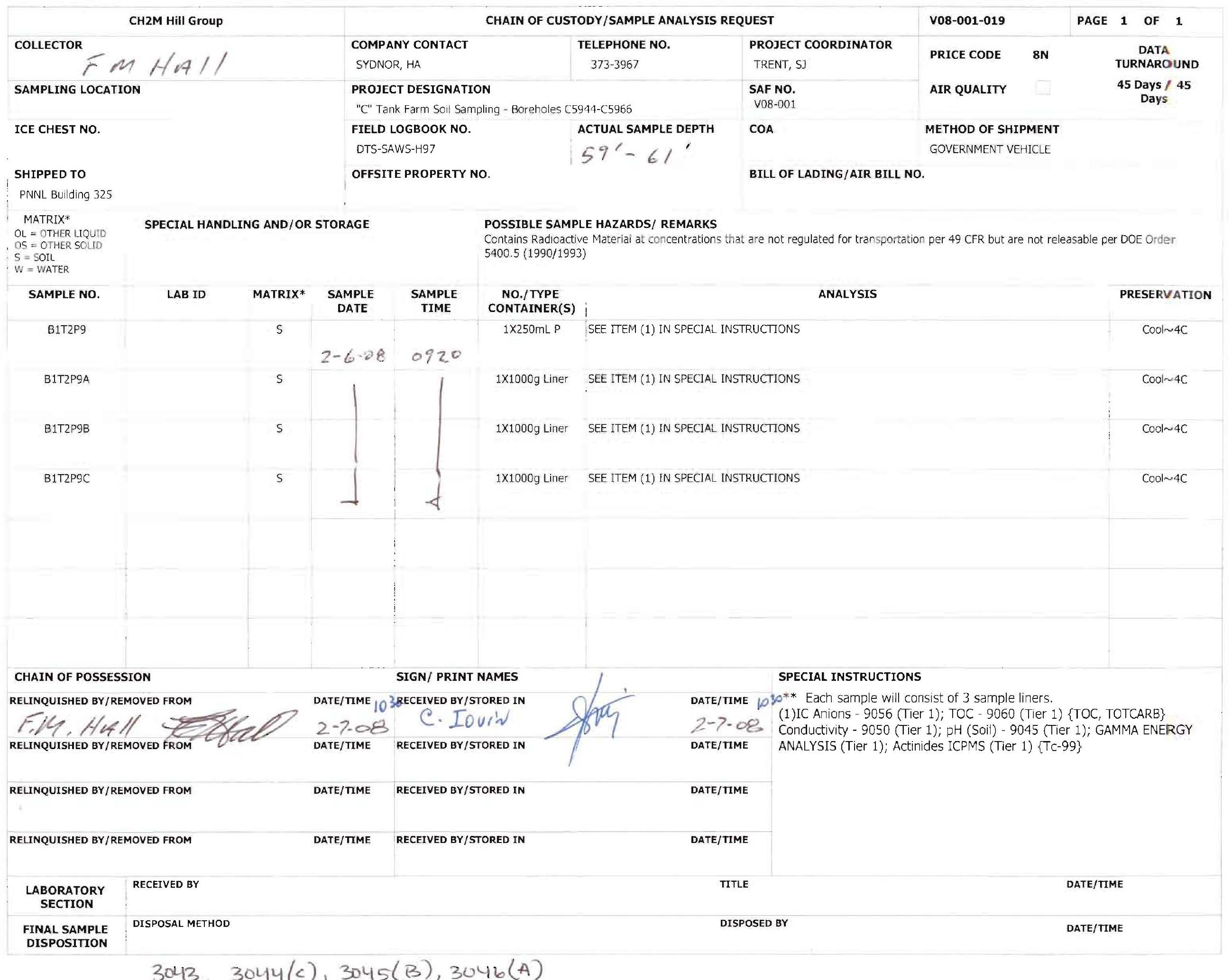




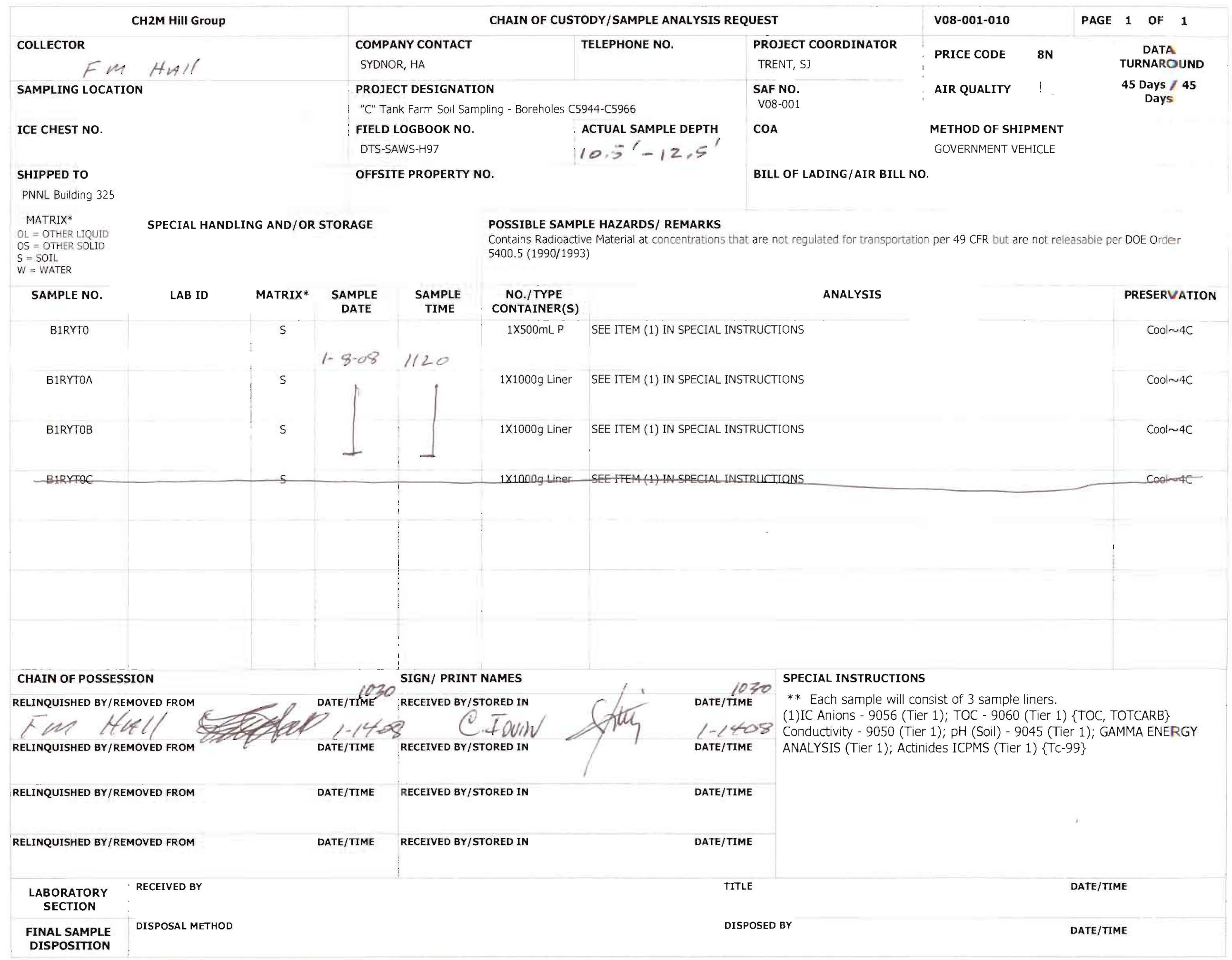




\begin{tabular}{|c|c|c|c|c|c|c|c|}
\hline CH2M Hill Group & \multicolumn{3}{|c|}{ CHAIN OF CUSTODY/SAMPLE ANALYSIS REQUEST } & \multicolumn{2}{|l|}{ V08-001-011 } & PAGE & OF \\
\hline $\begin{array}{l}\text { COLLECTOR } \\
F M H A M\end{array}$ & $\begin{array}{l}\text { COMPANY CONTACT } \\
\text { SYDNOR, HA }\end{array}$ & TELEPHONE NO. & $\begin{array}{l}\text { PROJECT COORDINATOR } \\
\text { TRENT, SJ }\end{array}$ & \multirow{2}{*}{$\begin{array}{l}\text { PRICE CODE } \\
\text { AIR QUALITY }\end{array}$} & $8 \mathbf{N}$ & \multicolumn{2}{|r|}{$\begin{array}{l}\text { DATA } \\
\text { TURNAROUND }\end{array}$} \\
\hline $\begin{array}{l}\text { SAMPLING LOCATION } \\
C 5958\end{array}$ & \multicolumn{2}{|c|}{$\begin{array}{l}\text { PROJECT DESIGNATION } \\
\text { "C" Tank Farm Soil Sampling - Boreholes C5944-C5966 }\end{array}$} & $\begin{array}{l}\text { SAF NO. } \\
\text { V08-001 }\end{array}$ & & $\square$ & & $\begin{array}{l}45 \text { Days / } 45 \\
\text { Days }\end{array}$ \\
\hline ICE CHEST NO. & $\begin{array}{l}\text { FIELD LOGBOOK NO. } \\
\text { DTS-SAWS-H97 }\end{array}$ & $\begin{array}{l}\text { ACTUAL SAMPLE DEPTH } \\
54^{\prime}-56^{\prime}\end{array}$ & COA & \multicolumn{4}{|c|}{ GOVERNMENT VEHICLE } \\
\hline
\end{tabular}

PNNL Building 325

MATRIX*

$\mathrm{OL}=$ OTHER LIQUID

SPECIAL HANDLING AND/OR STORAGE

POSSIBLE SAMPLE HAZARDS/ REMARKS

$S=$ SOIL

$W=$ WATER

\section{SAMPLE NO.}

LAB ID

\begin{tabular}{c} 
MATRIX* \\
\hline $\mathrm{S}$ \\
\hline
\end{tabular}

B1T2P1

B1T2P1A

s

B1T2P1B

SAMPLE

DATE

SAMPLE

TIME

Contains Radioactive

Material at concentrations that are not regulated for transportation per 49 CFR but are not releasable per DOE Order

NO./TYPE

CONTAINER(S)

ANALYSIS

PRESERVATION

$1-10-080915$

B1T2P1C

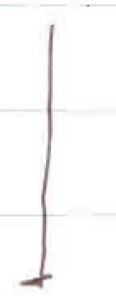

1X250mL Liner

1 1X1000g Liner SEE ITEM (1) IN SPECIAL INSTRUCTIONS

Cool 4C

$1 \times 1000 \mathrm{~g}$ Liner SEE TTEM (1) IN SPECIAL INSTRUCTIONS

Cool 4C

Cool 4C

$1 \times 1000 \mathrm{~g}$ Liner SEE ITEM (1) IN SPECIAL INSTRUCTIONS

Cool $4 \mathrm{C}$

\section{CHAIN OF POSSESSION}

RELINQUISHED BY/REMOVED FROM

ton $4 M 11$

RELINQUISHED BY/REMOVED FROM

RELINQUISHED BY/REMOVED FROM

RELINQUISHED BY/REMOVED FROM

\begin{abstract}
SIGN/ PRINT NAMES
DATE/TME
\end{abstract}

$1-14-08$

DATE/TIME

(2) $10 \mathrm{~N}, \mathrm{~W}$

ECEIVED BY/STORED IN

DATE/TIME

RECEIVED BY/STORED IN

DATE/TIME

\section{SPECIAL INSTRUCTIONS}

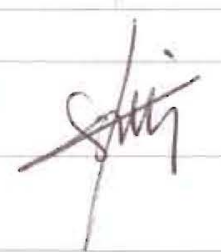

DATE/TIME 30 ** Each sample will consist of 3 sample liners.
(1)IC Anions - 9056 (Tier 1); TOC - 9060 (Tier 1) \{TOC, TOTCARB $\}$

$1-14-08$ Conductivity - 9050 (Tier 1); pH (Soil) - 9045 (Tier 1); GAMMA ENERGY

DATE/TIME ANALYSIS (Tier 1); Actinides ICPMS (Tier 1) $\{$ TC-99\}

DATE/TIME

DATE/TIME

\begin{tabular}{|c|c|c|c|}
\hline $\begin{array}{l}\text { LABORATORY } \\
\text { SECTION }\end{array}$ & RECEIVED BY & TITLE & DATE/TIME \\
\hline $\begin{array}{l}\text { FINAL SAMPLE } \\
\text { DISPOSITION }\end{array}$ & DISPOSAL METHOD & DISPOSED BY & DATE/TTME \\
\hline
\end{tabular}

ES $2817 G$ FSI $9828 A$ ESL 282913 ESL $2830 \mathrm{C}$ 


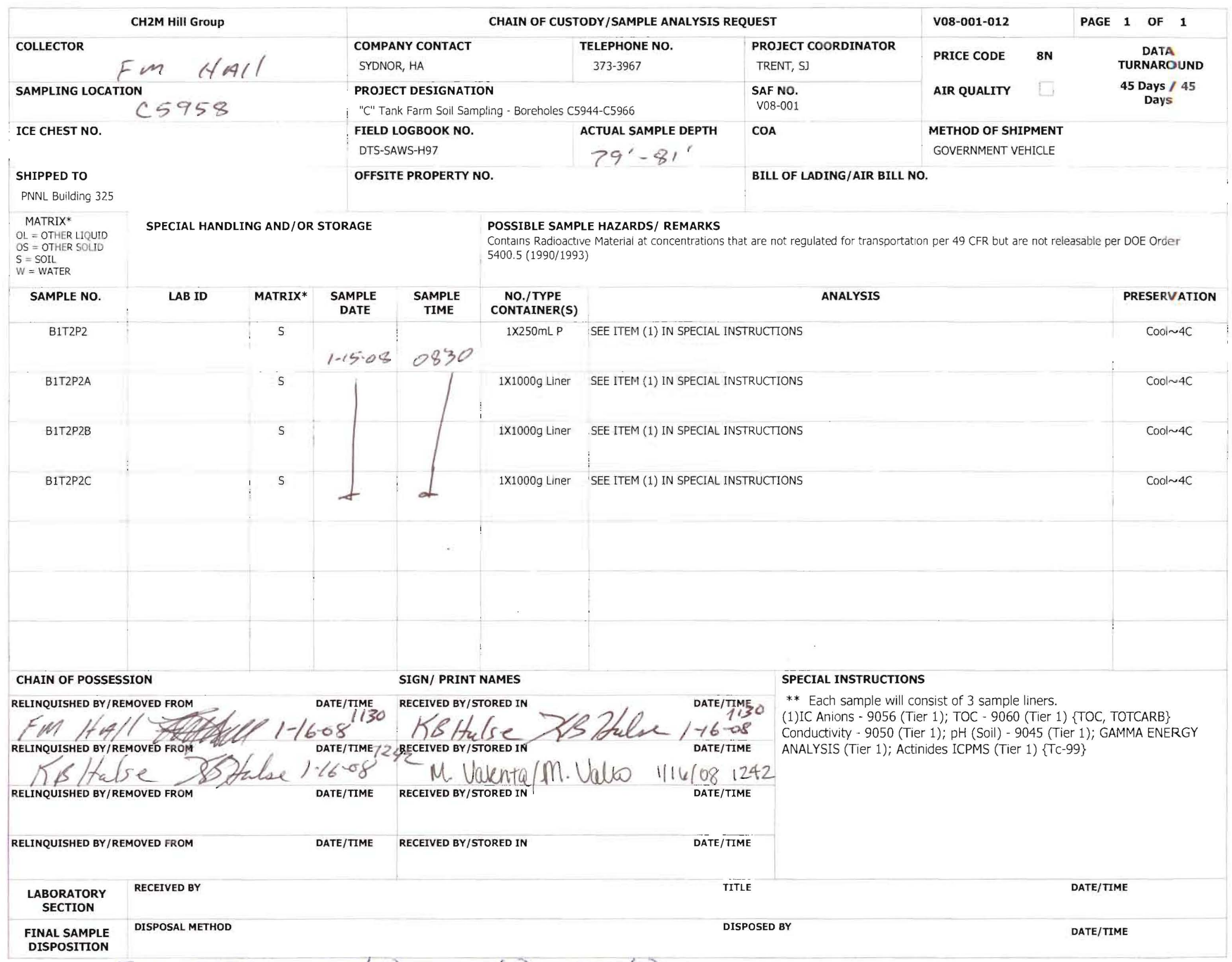




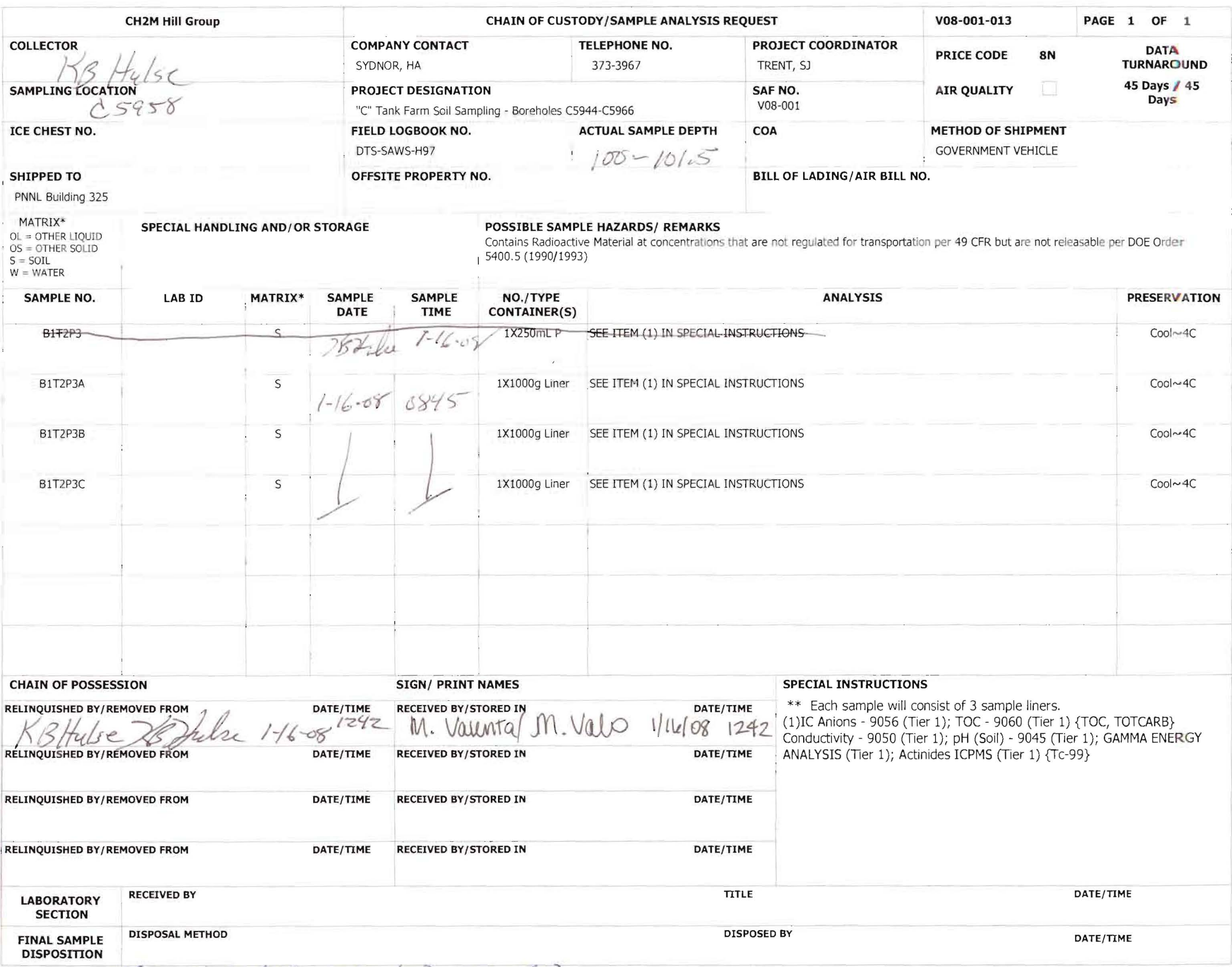




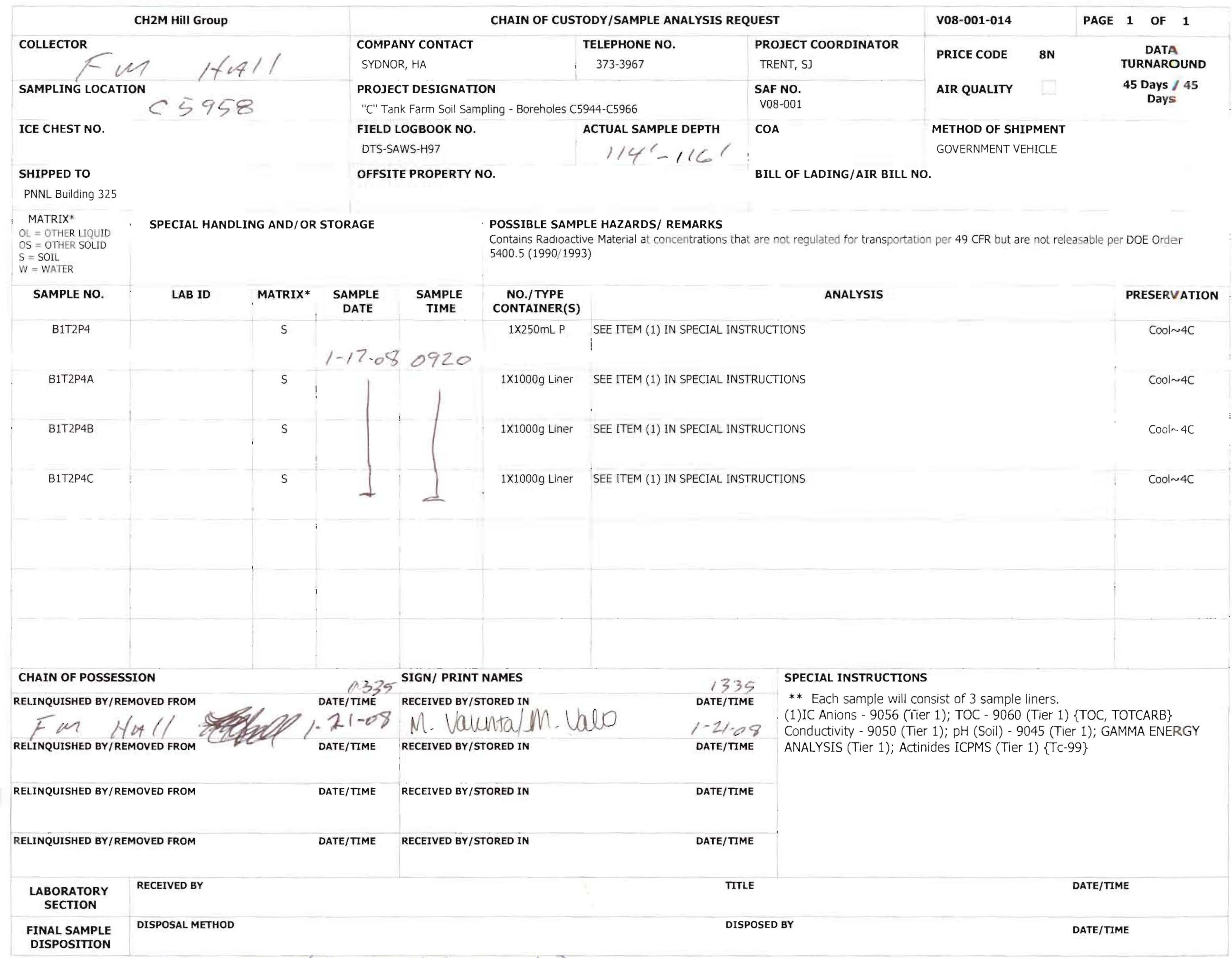




\section{COLLECTOR} FM $/ 1411$

SAMPLING LOCATION

C 5958

ICE CHEST NO.

SHIPPED TO

PNNL Building 325

MATRIX*

OL = OTHER LIQUID

OS = OTHER SOLID

$S=$ SOIL

\section{SAMPLE NO.}

B1T2P5

B1T2P5A

LAB ID

SPECIAL HANDLING AND/OR STORAGE

COMPANY CONTACT

SYDNOR, HA

\section{PROJECT DESIGNATION}

"C" Tank Farm Soil Sampling - Boreholes C5944-C5966

\section{FIELD LOGBOOK NO.}

DTS-SAWS-H97

OFFSITE PROPERTY NO. $5400.5(1990 / 1993)$

\section{NO./TYPE}

DATE TIME

\section{$1 \times 250 \mathrm{~mL} P$}

TELEPHONE NO.

PROJECT COORDINATOR

TRENT, SJ

SAF NO.

V08-001

COA

$$
\begin{aligned}
& \text { ACTUAL SAMPLE DEPTH } \\
& 1 / 6^{-} / 18^{\prime}
\end{aligned}
$$

BILL OF LADING / AIR BILL NO.

\section{v08-001-015}

PRICE CODE

PAGE 1 OF 1

AIR QUALITY

$8 \mathbf{N}$

DATA

TURNAROUND

45 Days / 45

Days

\section{METHOD OF SHIPMENT}

GOVERNMENT VEHICLE

\section{POSSIBLE SAMPLE HAZARDS/ REMARKS}

Contains Radioactive Material at concentrations that are not regulated for transportation per 49 CFR but are not releasable per DOE Order

CONTAINER(S)

ANALYSIS

PRESERVATION

SEE ITEM (1) IN SPECIAL INSTRUCTIONS

Cool 4C

$1 \times 1000 \mathrm{~g}$ Liner SEE ITEM (1) IN SPECIAL INSTRUCTIONS

Cool $\sim 4 C$

$1 \times 1000 \mathrm{~g}$ Liner SEE ITEM (1) IN SPECIAL INSTRUCTIONS

Cool $4 \mathrm{C}$

$1 \times 1000 \mathrm{~g}$ Liner SEE ITEM (1) IN SPECIAL INSTRUCTIONS

Cool $\sim 4 C$

\section{CHAIN OF POSSESSION}

RELINQUISHED BY/REMOVED FROM

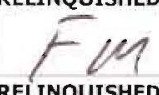

REINQUISHED BY/REMOVED

RELINQUISHED BY/REMOVED FROM

RELINQUISHED BY/REMOVED FROM

\section{SIGN/ PRINT NAMES}

DATE/TIME RECEIVED BY/STORED IN

, -2l-g M. Valenta / M. Walo

DATE/TIME RECEIVED BY/STORED IN

RECEIVED BY/STORED IN

DATE/TIME

RECEIVED BY/STORED IN

\section{SPECIAL INSTRUCTIONS}

** Each sample will consist of 3 sample liners.

(1)IC Anions - 9056 (Tier 1); TOC - 9060 (Tier 1) \{TOC, TOTCARB

Conductivity - 9050 (Tier 1); pH (Soil) - 9045 (Tier 1); GAMMA ENERGY

\begin{tabular}{|c|c|c|c|}
\hline $\begin{array}{l}\text { LABORATORY } \\
\text { SECTION }\end{array}$ & RECEIVED BY & TITLE & DATE/TIME \\
\hline $\begin{array}{l}\text { FINAL SAMPLE } \\
\text { DISPOSITION }\end{array}$ & DISPOSAL METHOD & DISPOSED BY & DATE/TIME \\
\hline
\end{tabular}

ANALYSIS (Tier 1); Actinides ICPMS (Tier 1) \{TC-99\} 


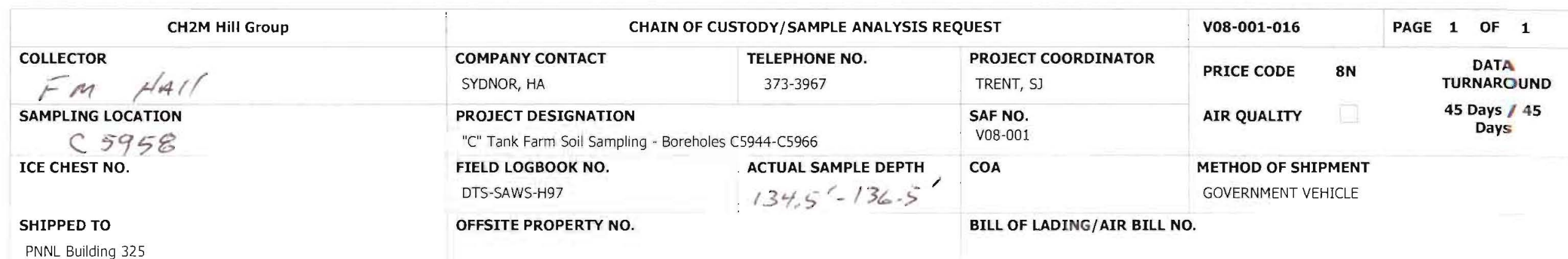

PNNL Building 325

MATRIX*

$\mathrm{OL}=$ OTHER LIQUID

OS = OTHER SOLID

$S=$ SOIL

SAMPLE NO.

LAB ID

B1T2PG

B1T2P6A

B1T2P6B

B1T2P6C
SPECIAL HANDLING AND/OR STORAGE

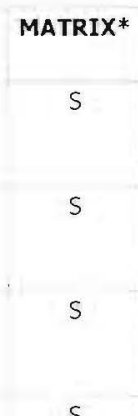

SAMPLE

\section{POSSIBLE SAMPLE HAZARDS/ REMARKS}

Contains Radioactive Materia! at concentrations that are not regulated for transportation per 49 CFR but are not releasable per DOE Order $5400.5(1990 / 1993)$

\begin{tabular}{|c|c|}
\hline $\begin{array}{c}\text { NO./TYPE } \\
\text { CONTAINER(S) }\end{array}$ & \\
\hline $1 \times 250 \mathrm{~mL} P$ & SEE ITEM (1) IN SPECIAL INSTRUCTIONS \\
\hline $1 \times 1000 \mathrm{~g}$ Liner & SEE ITEM (1) IN SPECIAL INSTRUCTIONS \\
\hline $1 \times 1000 \mathrm{~g}$ Liner & SEE ITEM (1) IN SPECIAL INSTRUCTIONS \\
\hline $1 \times 1000 \mathrm{q}$ Liner & SEE ITEM (1) IN SPECIAL INSTRUCTIONS \\
\hline
\end{tabular}

ANALYSIS

PRESERVATION

SAMPLE
TIME

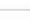

Cool $4 \mathrm{C}$

$$
1-21.080940
$$

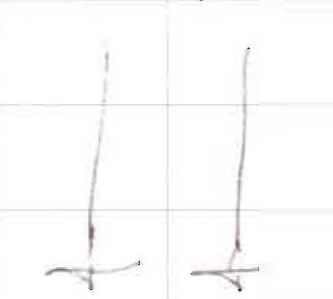

1xiong Liner

Cool $\sim 4 C$

Cool 4C

Cool 4C

\section{CHAIN OF POSSESSION}

RELINQUISHED BY/REMOVED FROM

$$
\text { FM H } 411
$$

RELINOUISHED BY/REMOVED FROM

RELINQUISHED BY/REMOVED FROM

RELINQUISHED BY/REMOVED FROM
1333 DATE/TIME

$(-2 /-08$ DATE/TIME

DATE/TIME

DATE/TTME

\section{SIGN/ PRINT NAMES}

RECEIVED BY/STORED IN

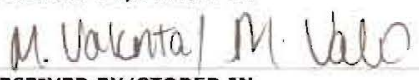

RECEIVED BY/STORED IN

RECEIVED BY/STORED IN

RECEIVED BY/STORED IN

\section{SPECIAL INSTRUCTIONS}

DATE/TIME $\quad * *$ Each sample will consist of 3 sample liners.

AU. O8 (1)IC Anions - 9056 (Tier 1); TOC - 9060 (Tier 1) \{TOC, TOTCARB

Conductivity - 9050 (Tier 1); pH (Soil) - 9045 (Tier 1); GAMMA ENERGY

ANALYSIS (Tier 1); Actinides ICPMS (Tier 1) \{TC-99\}

\begin{tabular}{|c|c|}
\hline $\begin{array}{c}\text { LABORATORY } \\
\text { SECTION }\end{array}$ & RECEIVED BY \\
\hline $\begin{array}{c}\text { FINAL SAMPLE } \\
\text { DISPOSIITON }\end{array}$ & DISPOSAL METHOD \\
\hline
\end{tabular}

TITLE

DATE/TIME

DISPOSITION

DISPOSED BY 


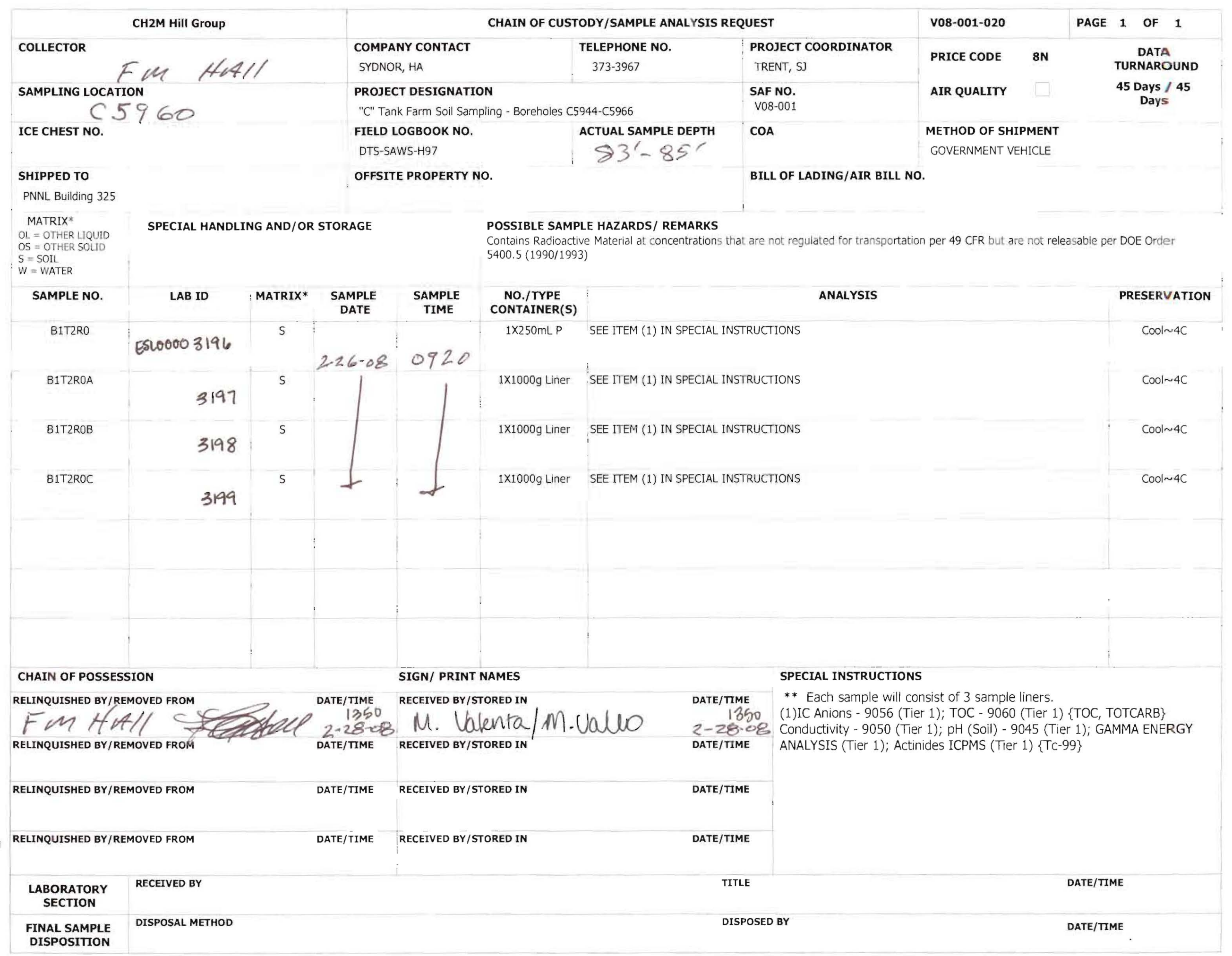




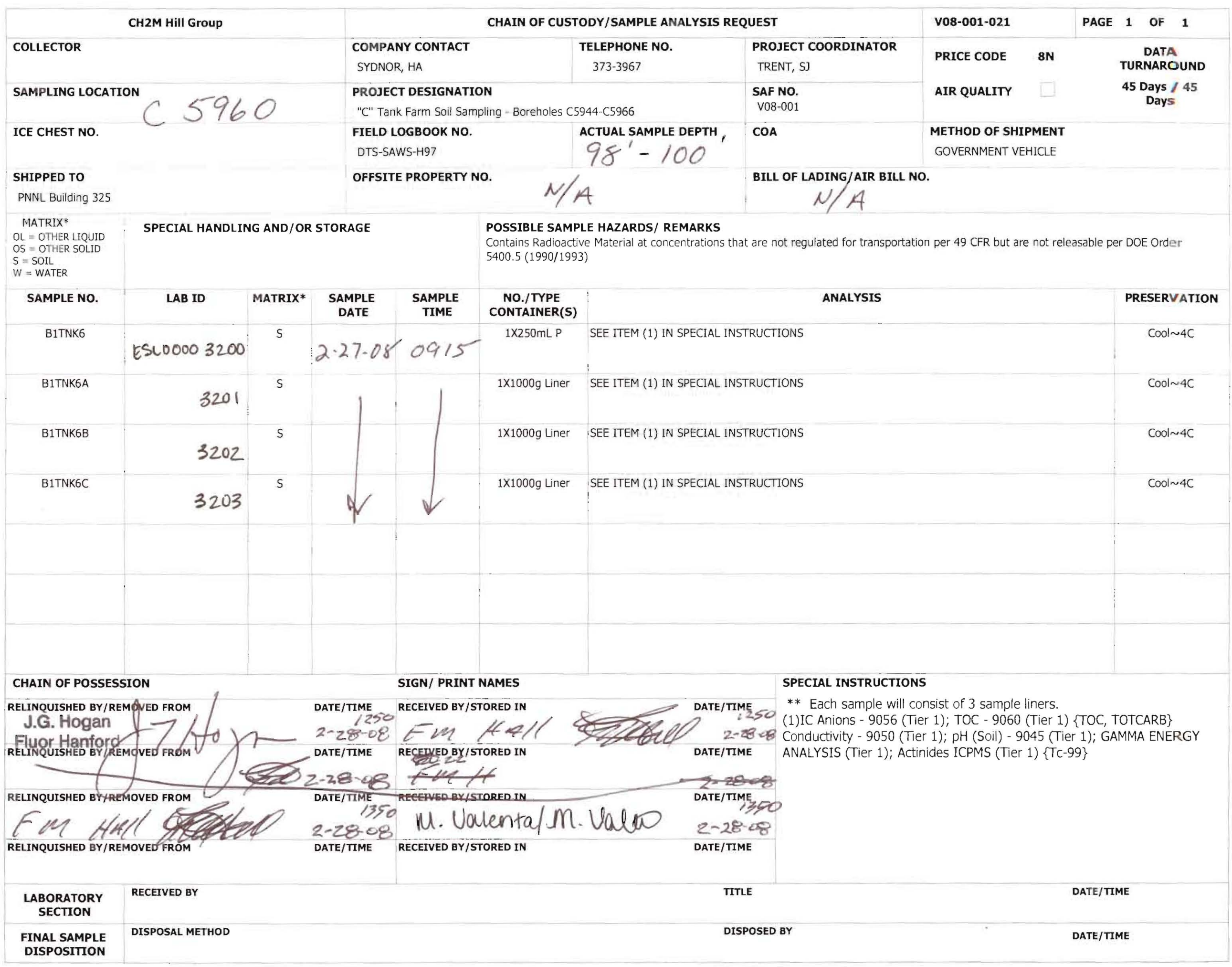




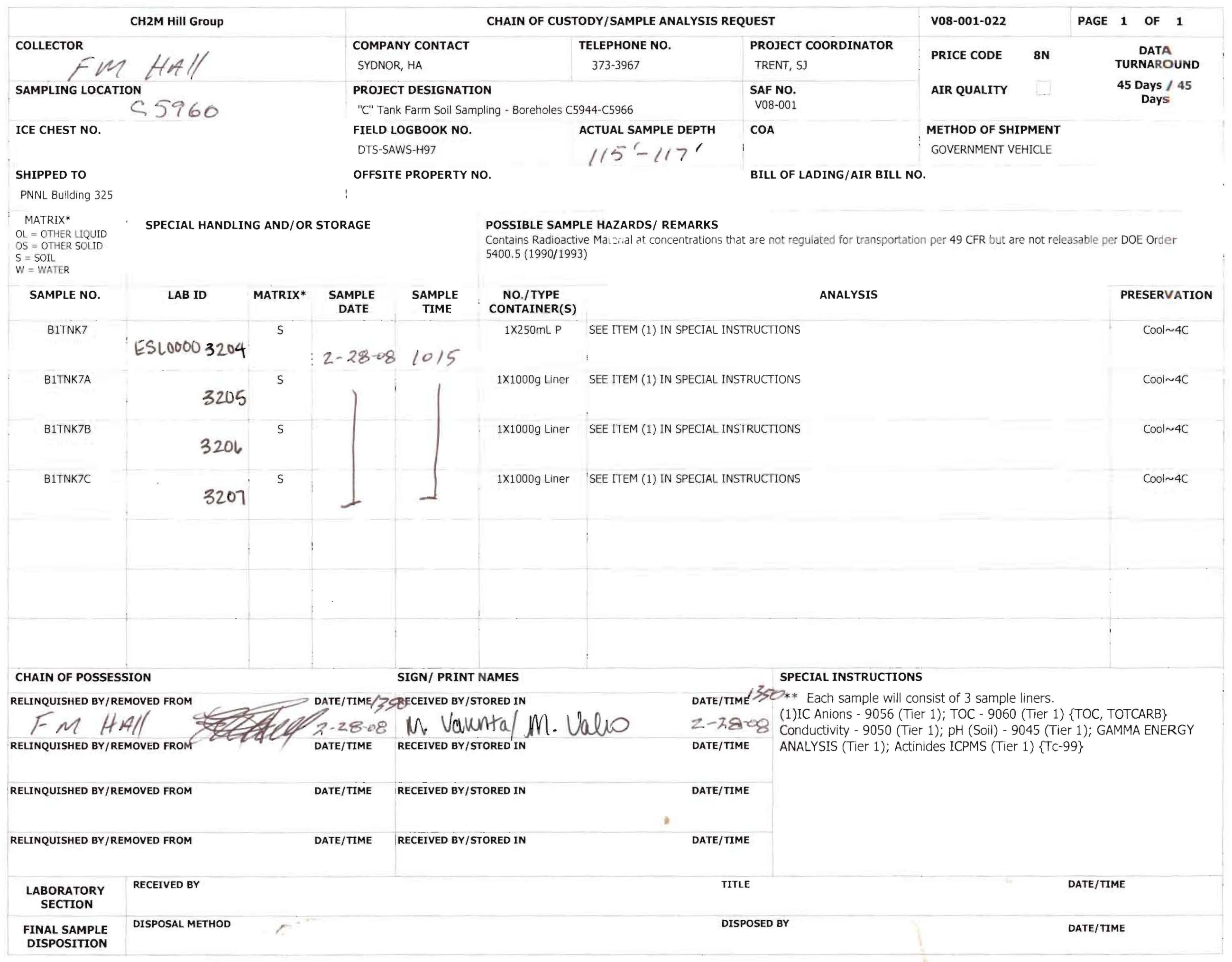




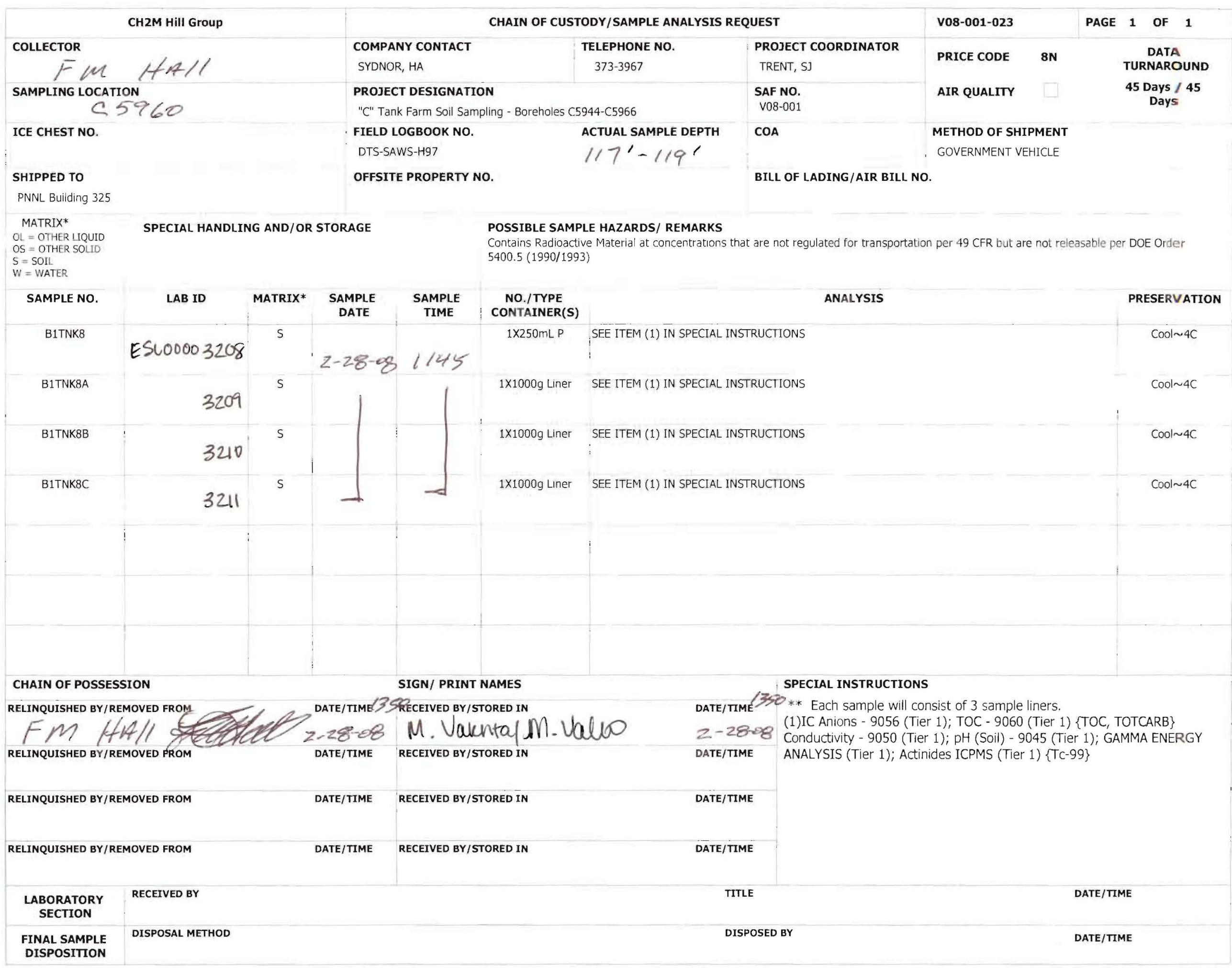




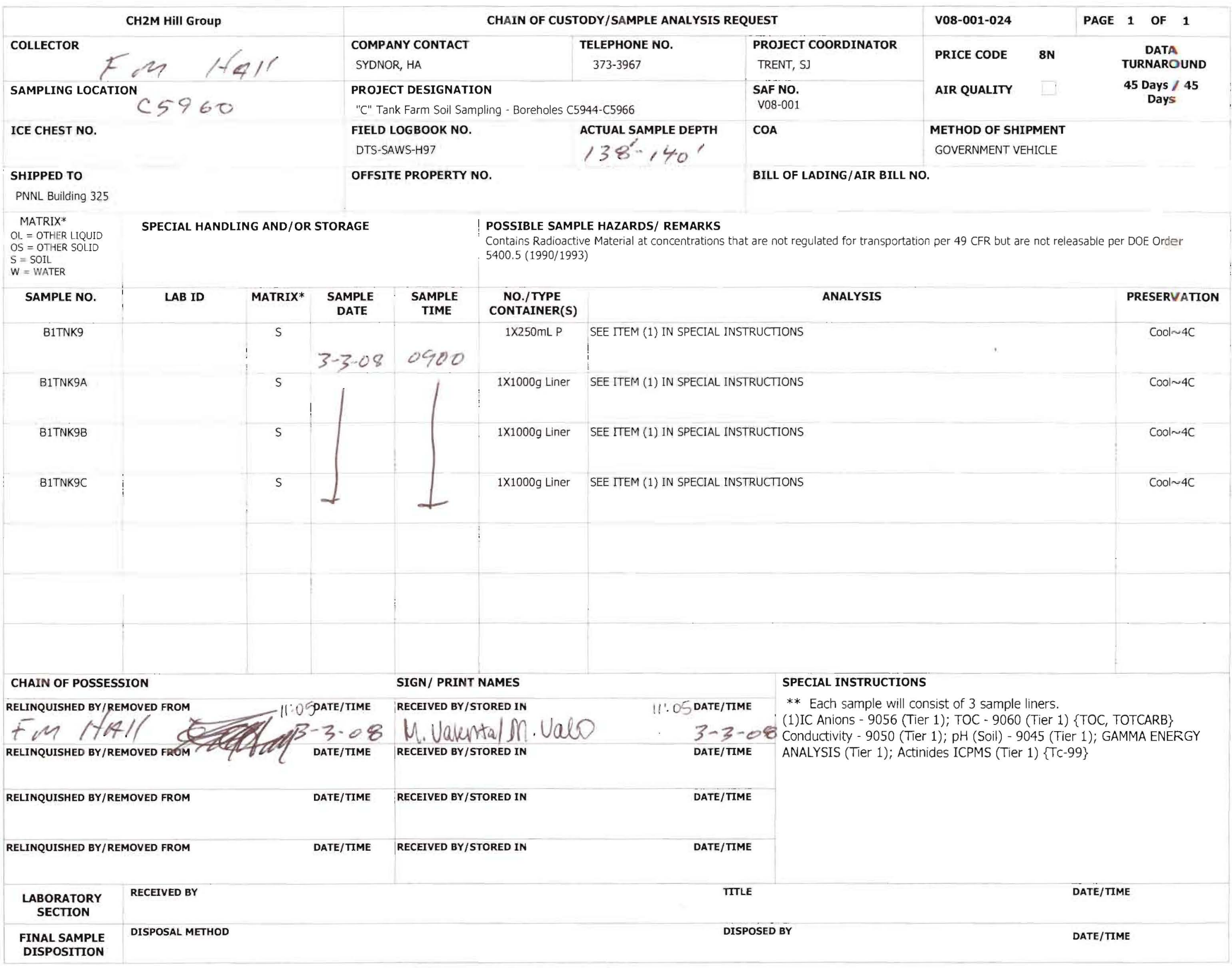

